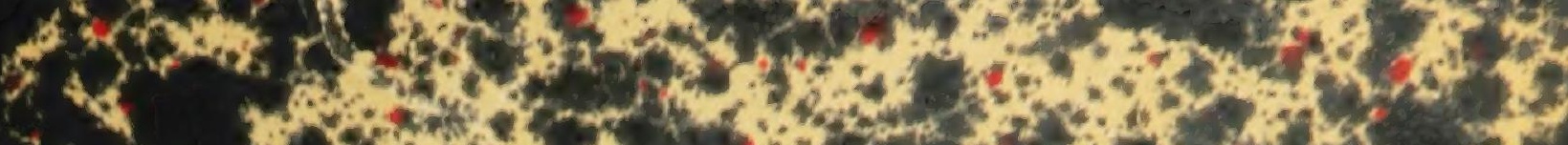
s. A. I.

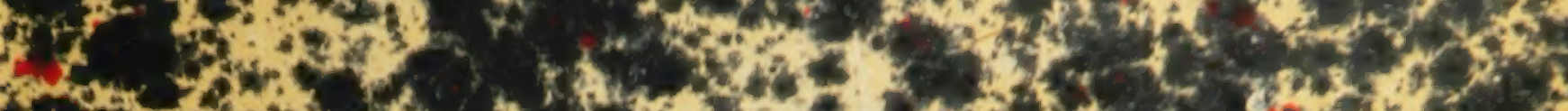

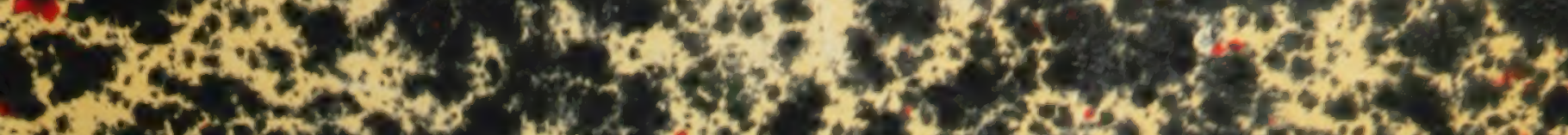
20.

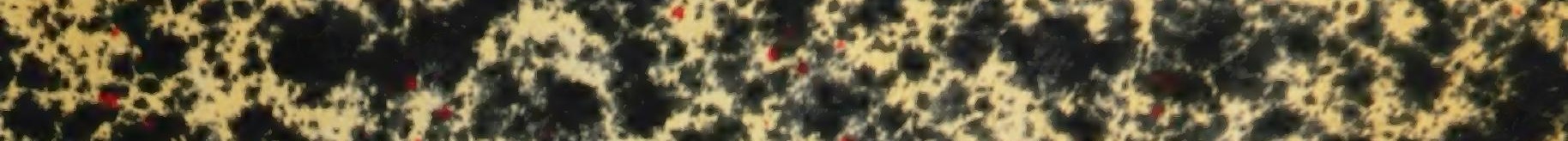

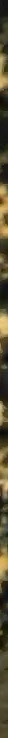




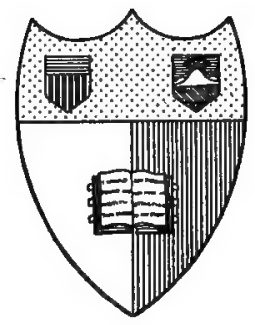

Garuell Unimeraity Wihrary

3thara, Nem 19uxk

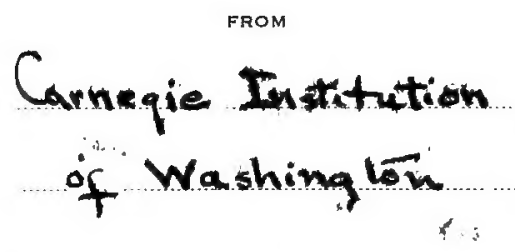




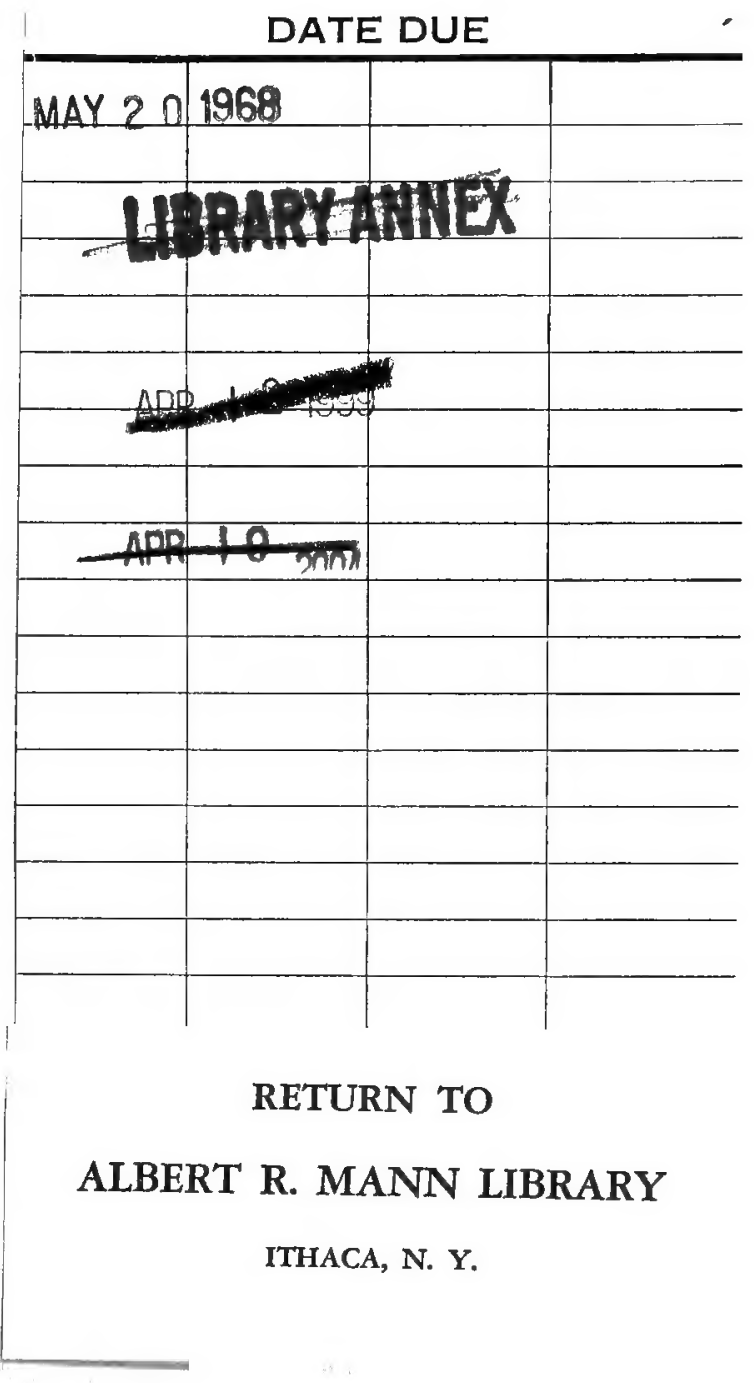




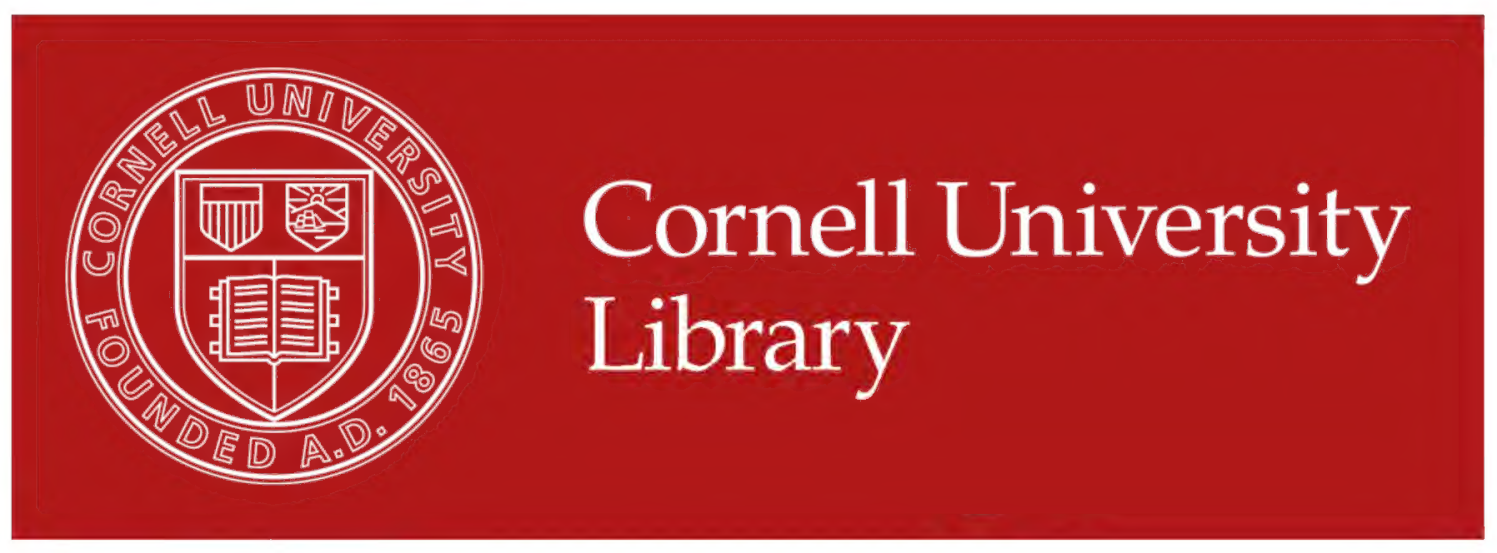

\section{The original of this book is in the Cornell University Library.}

There are no known copyright restrictions in the United States on the use of the text. 


\title{
A BIOCHEMIC BASIS FOR THE STUDY OF PROBLEMS OF TAXONOMY, HEREDITY, EVOLUTION, ETC., WITH ESPECIAL REFERENCE TO THE STARCHES AND TISSUES OF PARENT-STOCKS AND HYBRID-STOCKS AND THE STARCHES AND HEMOGLOBINS OF VARIETIES, SPECIES, AND GENERA.
}

\author{
BY \\ EDWARD TYSON REICHERT, M.D., Sc.D. \\ Professor of Physiology in the University of Pennsylvania \\ Research Associate of the Carnegie Institution of Wushington
}

IN TWO PARTS

PART I

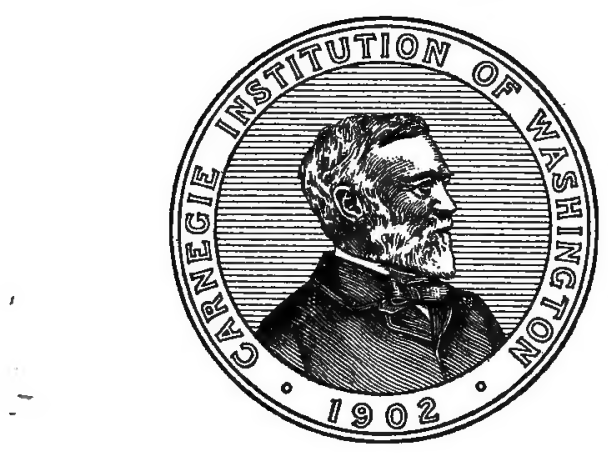

WASHINGTON, D. C.

Published by the Carnegie Institution of Washington 



\section{A BIOCHEMIC BASIS FOR THE STUDY OF PROBLEMS OF TAXONOMY, HEREDITY, EVOLUTION, ETC., WITH ESPECIAL REFERENCE TO THE STARCHES AND TISSUES OF PARENT-STOCKS AND HYBRID-STOCKS AND THE STARCHES AND HEMOGLOBINS OF VARIETIES, SPECIES, AND GENERA.}

BY

EDWARD TYSON REICHERT, M.D., Sc.D.

Professor of Physiology in the University of Pennsylvania

Research Associate of the Carnegie Institution of Washington

IN TWO PARTS

PART I

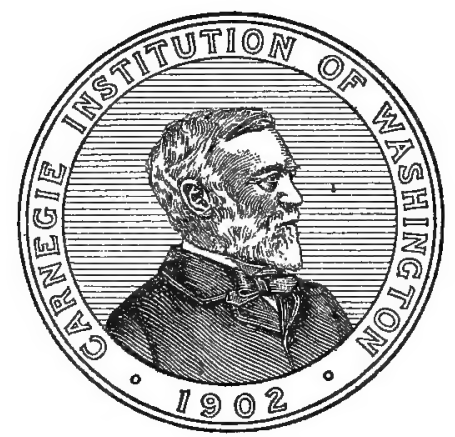

WASHINGTON, D. C.

Published by the Carnegre Institution of Washington 1919

L

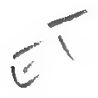




$$
\begin{aligned}
& . k K \\
& 898 \\
& 03 \\
& R 351 \\
& p t .1
\end{aligned}
$$

A 462820

CARNEGIE INSTITUTION OF WASHINGTON

\section{Publication No. 270, Part I}

PRESS OF J. B. LIPPINCOTT COMPANY

$$
\text { PHILADELPHIA }
$$

$$
\begin{aligned}
& \text { i }:(1: 21) \div \\
& +x^{\prime}, 1 / 111 \\
& \text { (1):1 }
\end{aligned}
$$




\title{
TABLE OF CONTENTS
}

\author{
PART I.
}

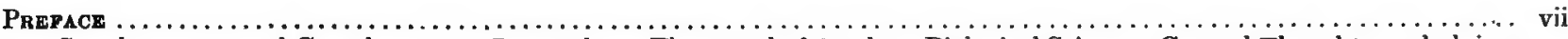

Supplementary and Complementary Researches. The Trend of Modern Biological Sciences. General Thoughts underlying these Researches. Inter-relationships between Molecular Configuration of Various Substances and Protoplasm. Biologic Propositions. Relations of Various Substances to Biologic Classification. Differences in the Methods Employed in these Researches. Forecast of Further Research. Unit-Characters and Unit-Character-Phases of Starches and Plant Tissues. Physics and Physical Chemistry in their Bearings on the Development of Biologic Sciences.

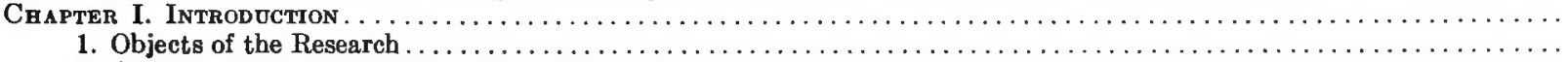

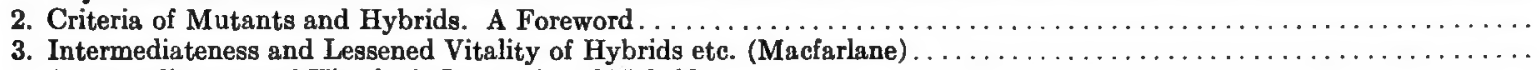

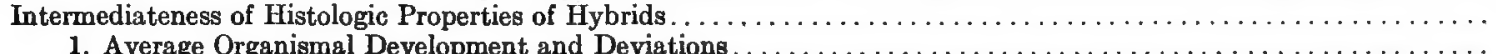

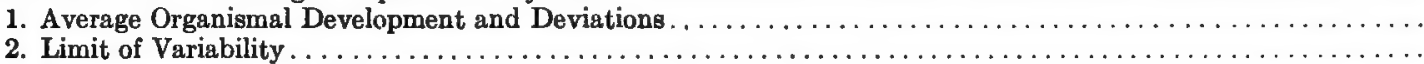

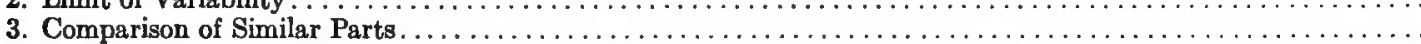

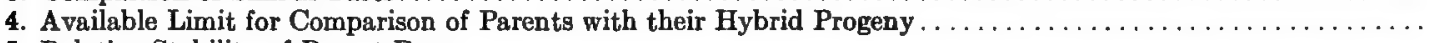

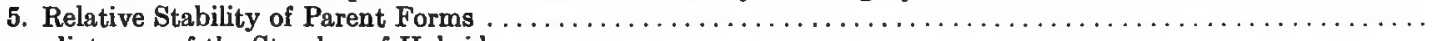

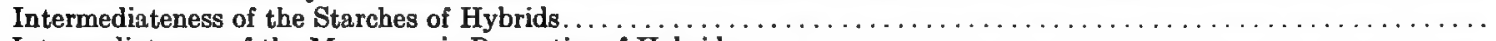

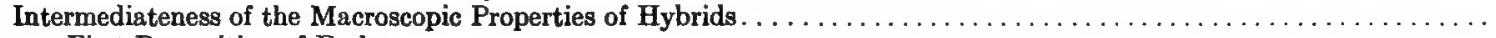

First Proposition of Focke.

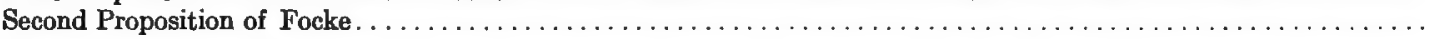

Third Proposition of Focke.

4. Pertial or Complete Sterility of Hybrids..

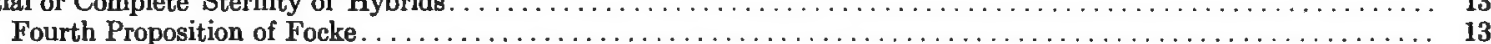

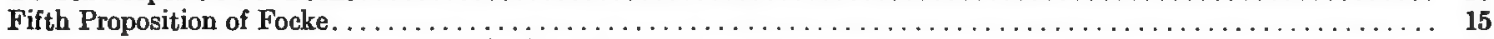

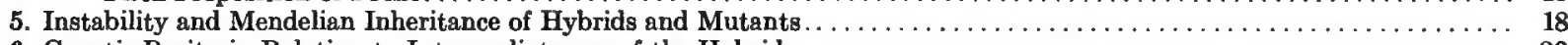

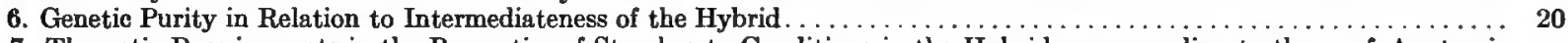

7. Theoretic Requirements in the Properties of Starches to Conditions in the Hybrid corresponding to those of Anatomic

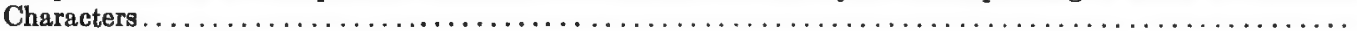

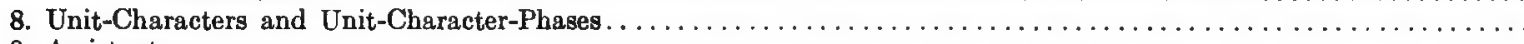

9. Assistants

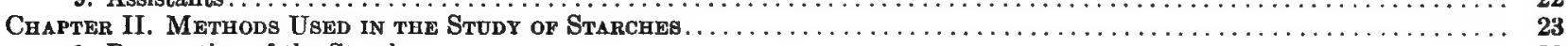

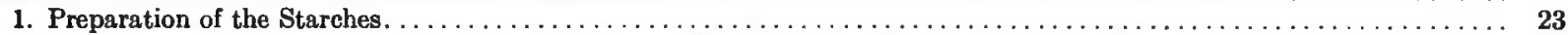

2. Simultaneous Studies of Starches of the Parents and Hybrid and of the Members of a Genus.................

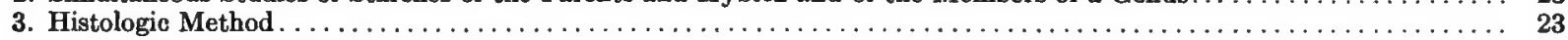

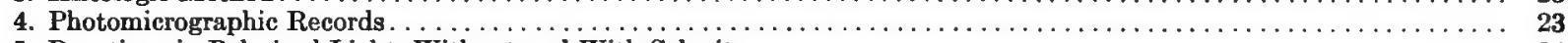

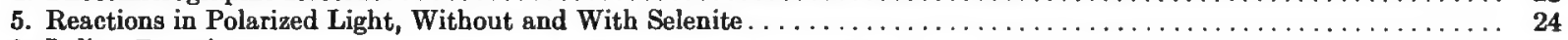

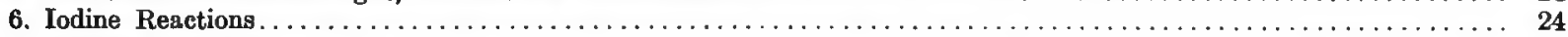

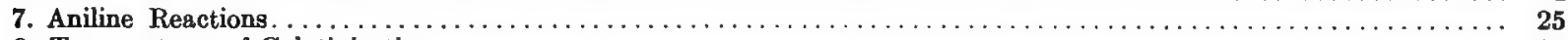

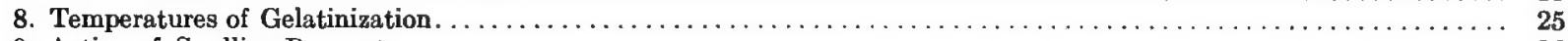

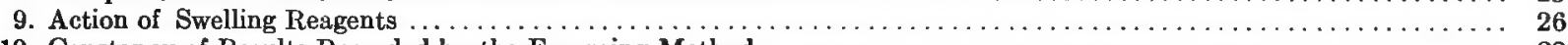

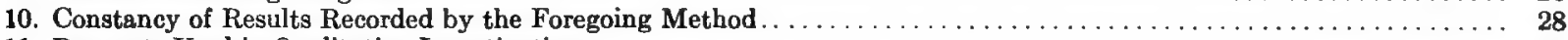

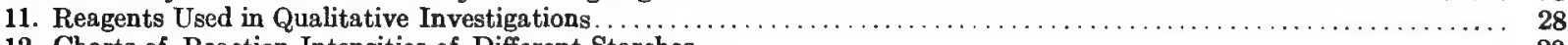

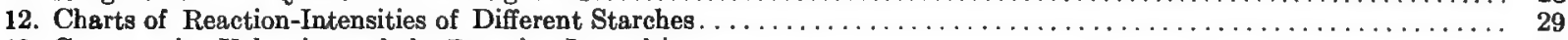

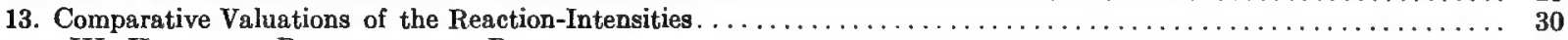

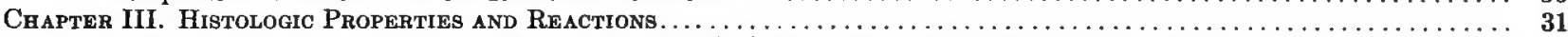

Comparisons of the More Important Data of the Histologie Properties and the Polariscopic, Iodine, Aniline, Temperature,

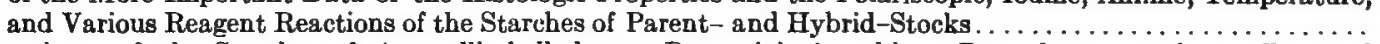

1. Comparisons of the Starches of Amaryllis belladonna, Brunsvigia josephinæ, Brunsdonna sanderce alba, and Brunsdonna sanderce.

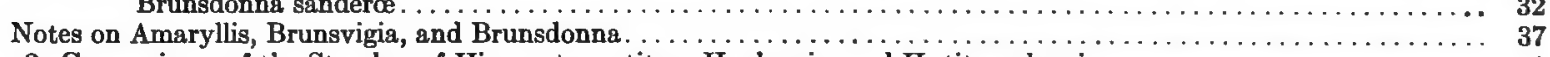

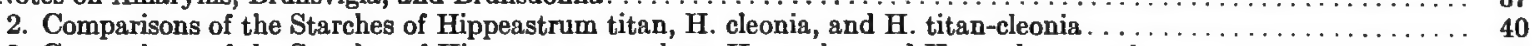

3. Comparisons of the Starches of Hippeastrum ossultan, $\mathbf{H}$. pyrrha, and H. ossultan-pyrrha $\ldots \ldots \ldots \ldots \ldots \ldots \ldots$

4. Comparisons of the Starches of Hippeastrum dæones, $H$. zephyr, and $H$. dæones-zephyr $\ldots \ldots \ldots \ldots \ldots \ldots \ldots \ldots$

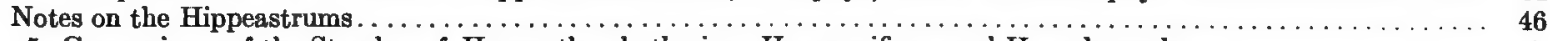

5. Comparisons of the Starches of Hæmanthus katherinæ, $H$. magnificus, and $H$. andromeda $\ldots \ldots \ldots \ldots \ldots \ldots \ldots \ldots$

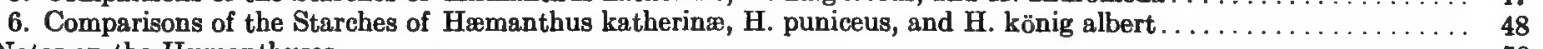

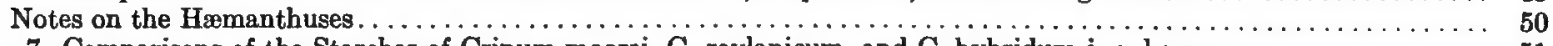

7. Comparisons of the Starches of Crinum moorei, C. zeylanicum, and C. hybridum j. c. harvey $\ldots \ldots \ldots \ldots \ldots \ldots \ldots \ldots$

8. Comparisons of the Starches of Crinum zeylanicum, C. longifolium, and C. kircape.....................

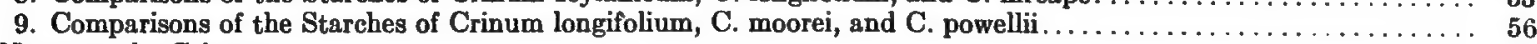

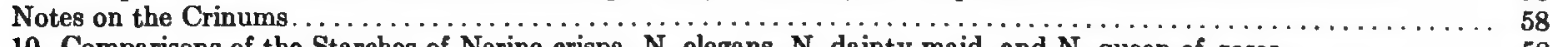

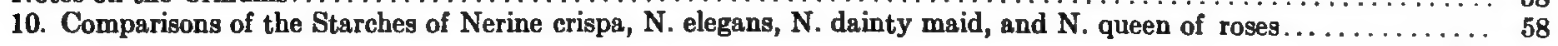


11. Comparisons of the Starches of Nerine bowdeni, N. sarniensis var. corusca major, N. giantess, and N. abundance 62 12. Comparisons of the Starches of Nerine sarniensis var. corusca major, $\mathbf{N}$. curvifolia var. fothergilli major, and N. glory of sarnia.

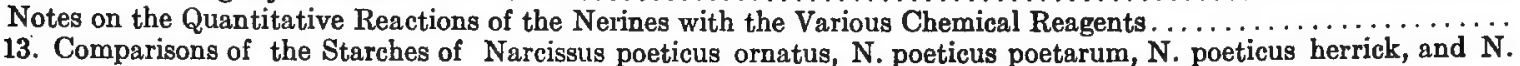

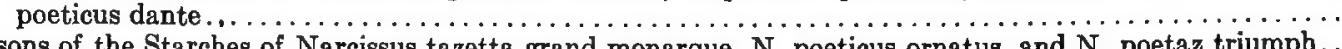

14. Comparisons of the Starches of Narcissus tazetta grand monarque, N. poeticus ornatus, and $\mathrm{N}$. poetaz triumph..

15. Comparisons of the Starches of Narcissus gloria mundi, N. poeticus ornatus, and N. fiery cross......

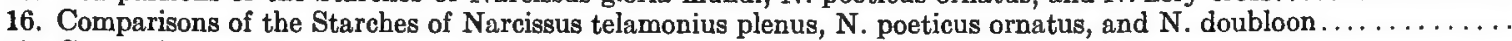

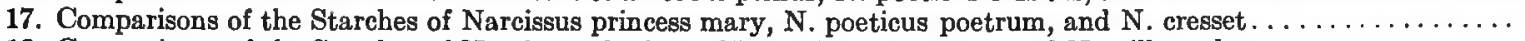

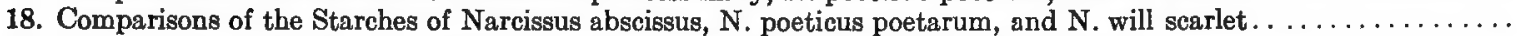

19. Comparisons of the Starches of Narcissus albicans, N. abscissus, and N. bicolor apricot. . . . . . . . . . . . . . . .

20. Comparisons of the Starches of Narcissus empress, N. albicans, and N. madame de graaff .

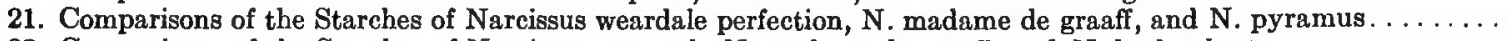

22. Comparisons of the Starches of Narcissus monarch, N. madame de graaff, and $N$. lord roberts............

23. Comparisons of the Starches of Narcissus leedsii minnie hume, N. triandrus-albus, and N. agnes harvey........

24. Comparisons of the Starches of Narcissus emperor, N. triandrus albus, and N. j. t. bennett poe ..........

Notes on the Narcissi

25. Comparisons of the Starches of Lilium martagon album, $\mathbf{L}$. maculatum, and $\mathbf{L}$. marhan $\ldots \ldots \ldots \ldots \ldots \ldots \ldots \ldots$

26. Comparisons of the Starches of Lilium martagon, $\mathrm{L}$. maculatum, and $\mathrm{L}$. dalhansoni $\ldots \ldots \ldots \ldots \ldots \ldots \ldots \ldots \ldots$

27. Comparisons of the Starches of Lilium tenuifolium, L. martagon album, and L. golden gleam $\ldots \ldots \ldots \ldots \ldots \ldots \ldots$

28. Comparisons of the Starches of Lilium chalcedonicum, $L$. candidum, and L. testaceum. . . . . . . . . 98

29. Comparisons of the Starches of Lilium pardalinum, L. parryi, and L. burbanki...................

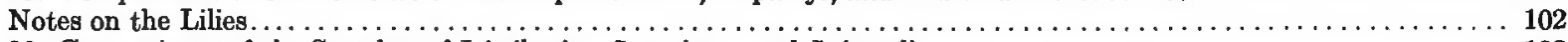

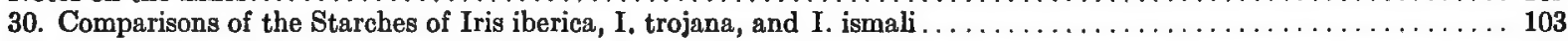

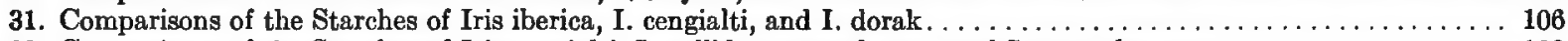

32. Comparisons of the Starches of Iris cengialti, I. pallida queen of may, and I. mrs. alan grey . . . . . . . . . 108

33. Comparisons of the Starches of Iris persica var. purpurea, I. sindjarensis, and I. pursind. . . . . . . . . . 110

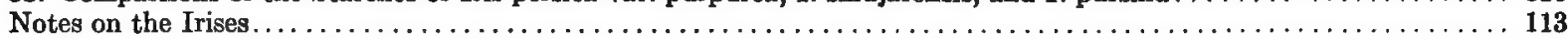

34. Comparisons of the Starches of Gladiolus cardinalis, G. tristis, and G. colvillei . . . . . . . . . . . . . . . 114

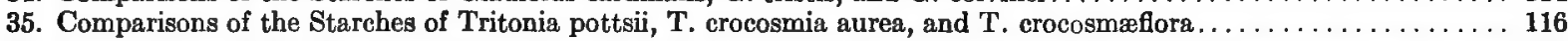

36. Comparisons of the Starches of Begonia single crimson scarlet, B. socotrana, and B. mrs. heal . . . . . . . . 118

37. Comparisons of the Starches of Begonia double light rose, B. socotrana, and B. ensign . . . . . . . . . . . . . 120

38. Comparisons of the Starches of Begonia double white, B. socotrana, and B. julius . . . . . . . . . . . . 122

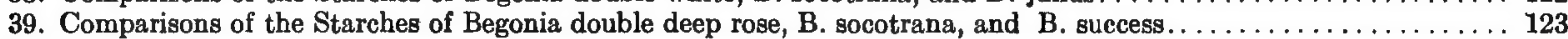

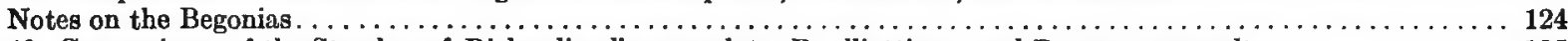

40. Comparisons of the Starches of Richardia albo-maculata, $R$. elliottiana, and $R$. mrs. roosevelt . . . . . . . 125

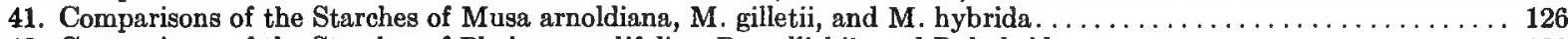

42. Comparisons of the Starches of Phaius grandifolius, $P$. wallichii, and $P$. hybridus . . . . . . . . . . . . . . . .

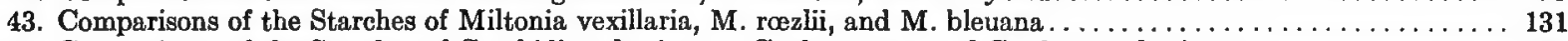

44. Comparisons of the Starches of Cymbidium Iowianum, C. eburneum, and C. eburneo-lowianum . . . . . . . . 133

45. Comparisons of the Starches of Calanthe rosea, C. vestita var, rubro-oculata, and C. veitchii . . . . . . . . 135

46. Comparisons of the Starches of Calanthe vestita var. rubro-oculata, C. regnieri, and C. bryan . . . . . . . . 137

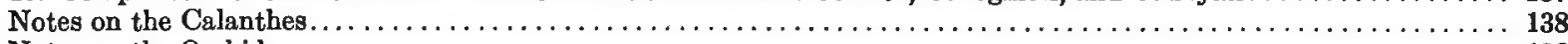

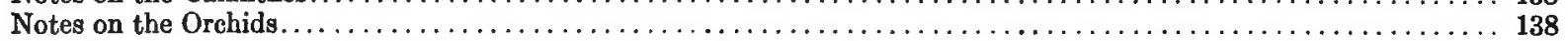

Chapter IV. General and Special Congiderations of the Reaction-Intengities of the Starches of Parent-STocke

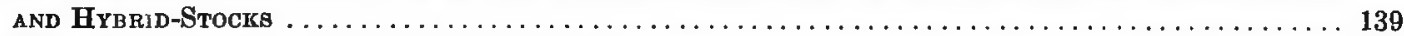

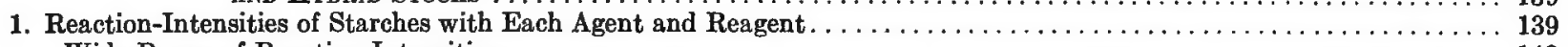

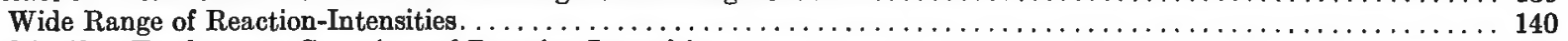

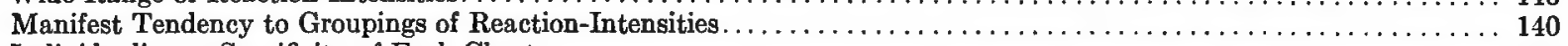

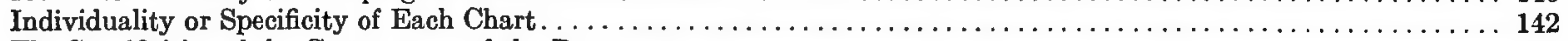

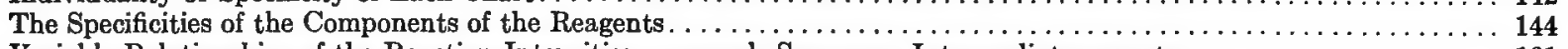

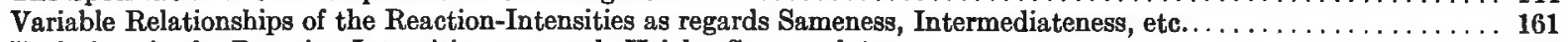

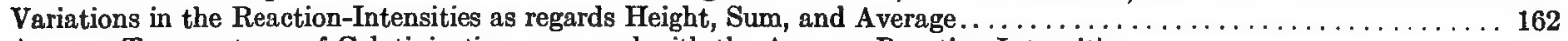

Average Temperatures of Gelatinization compared with the Average Reaction-Intensities . . . . . . . . . . . . . . 164

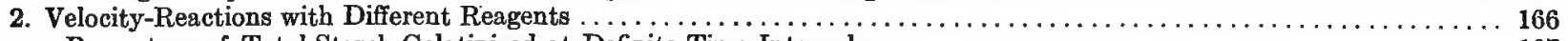

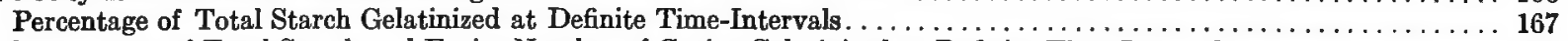

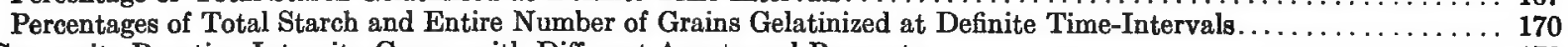

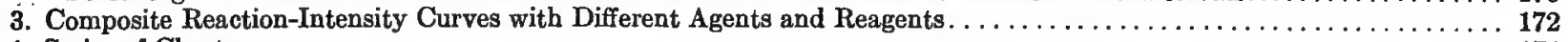

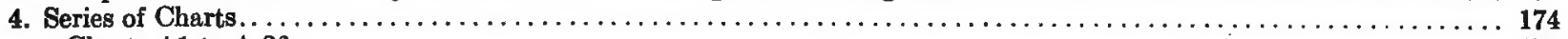

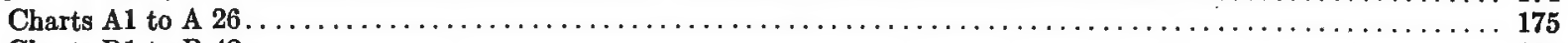

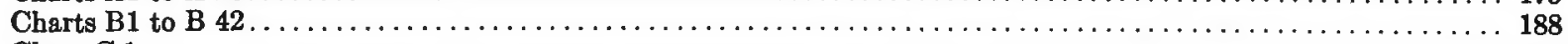

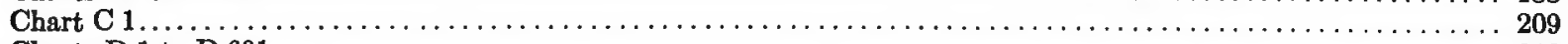

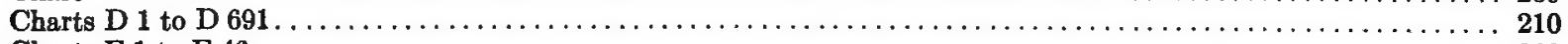

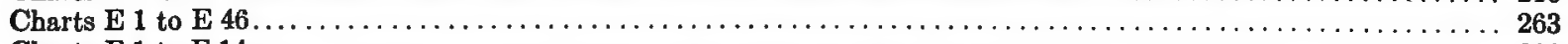

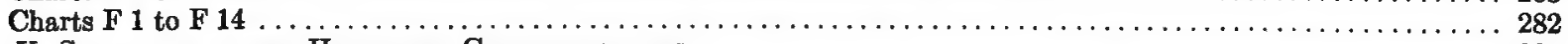

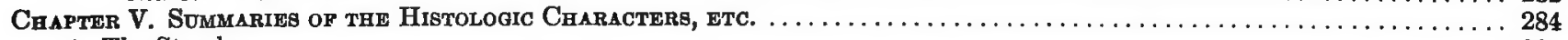

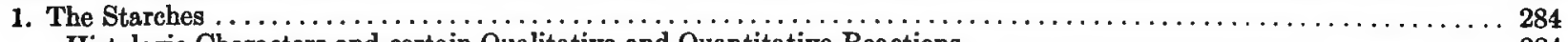

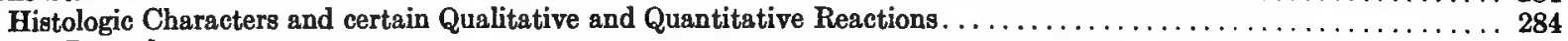

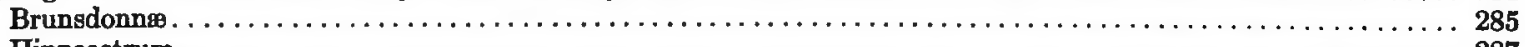

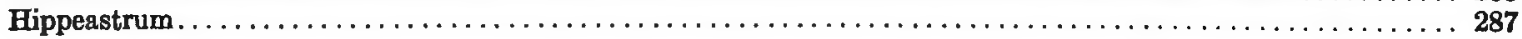


1. The Starches-Continued.

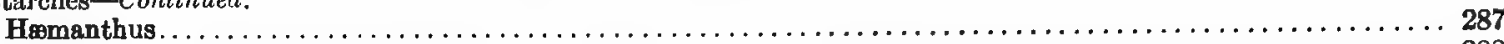

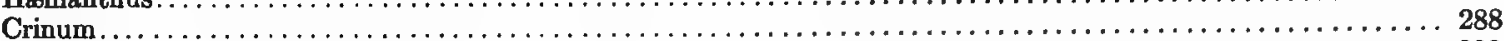

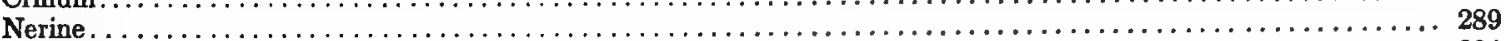

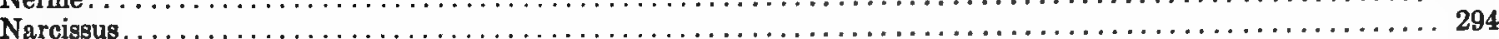

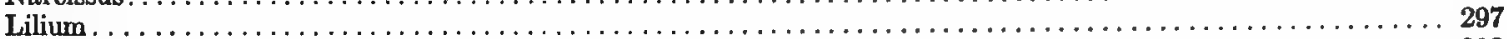

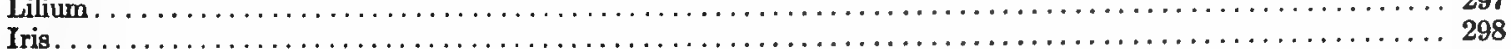

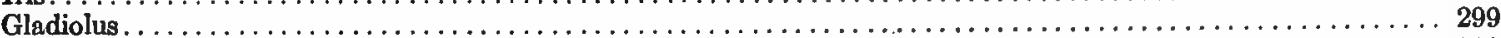

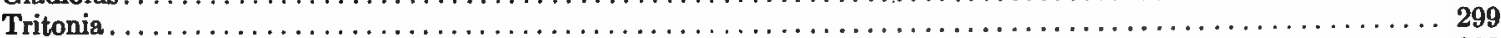

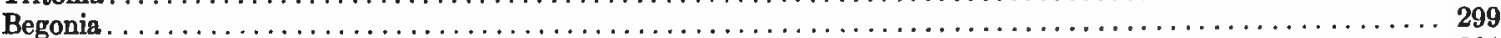

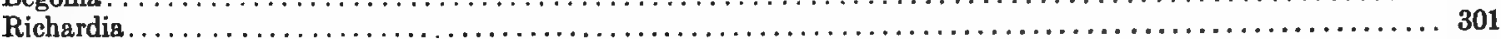

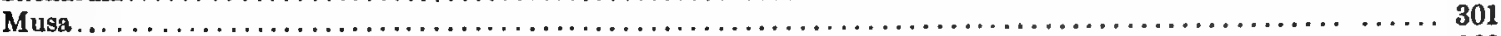

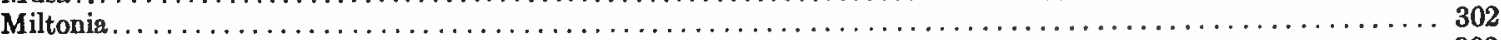

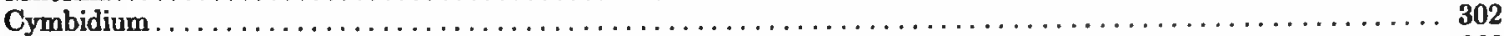

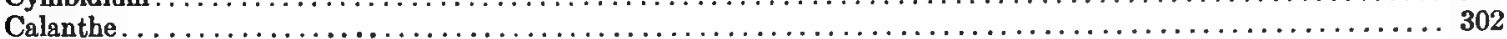

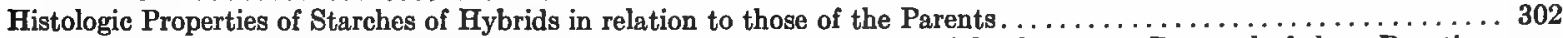

Qualitative and Quantitative Reactions of Starches of Hybrids with especial reference to Reversal of these Reactions

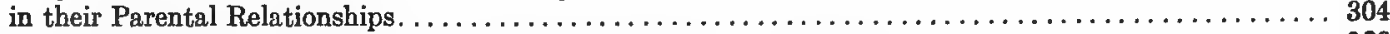

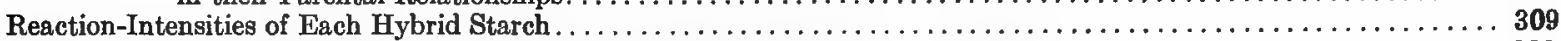

Reaction-Intensities of Each Hybrid Starch with Different Agents and Reagents............................

Reaction-Intensities of Each Hybrid Starch in Relation to Sameness and Inclination to Each Parent and Both Parents. 322

Reaction-Intensities of All of the Hybrid Starches with Each Agent and Reagent and as Regards Sameness and Inelination of their Properties in Relation to One or the Other Parent or Both Parents. .............. 323

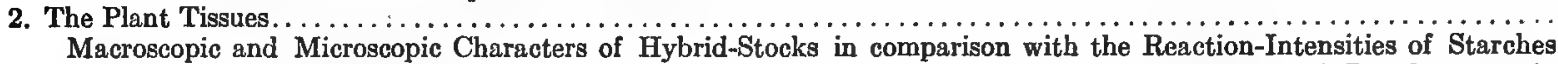
of Hybrid-Stocks as Regards Sameness, Intermediateness, Excess, and Deficit of Development in

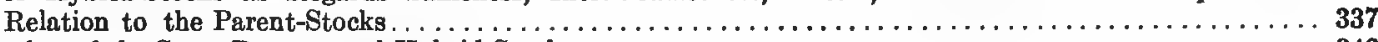

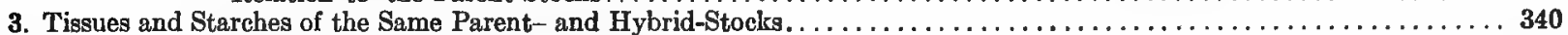

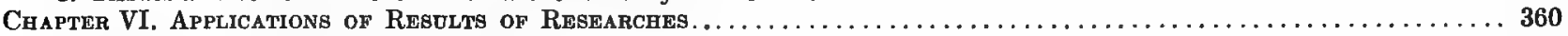

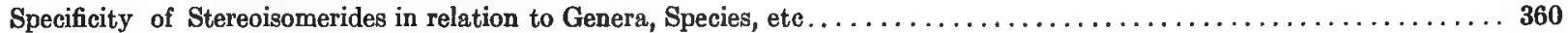

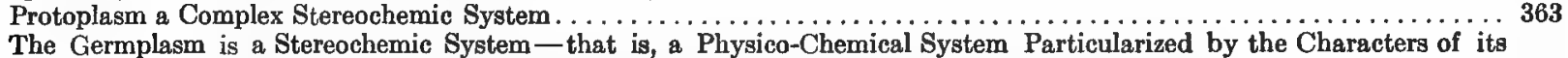
Stereoisomers and the Arrangements of its Components in the Three Dimensions of Space..........364

Protoplasmic Stereochemic System applied to the Explanation of the Mechanism of Variations, Sports, Fluctuations, etc.. 367

Protoplasmic Stereochemic System applied to the Genesis of Species. . . . . . . . . . . . . . . . . . . . . . . . 368

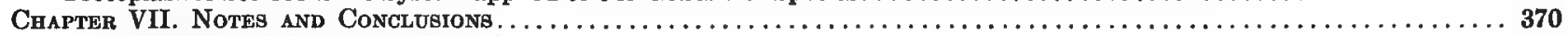

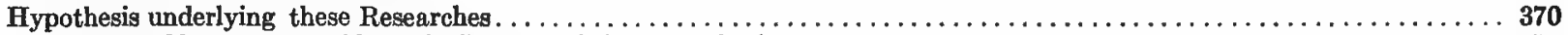

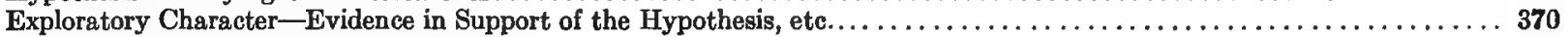

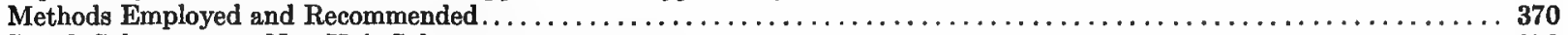

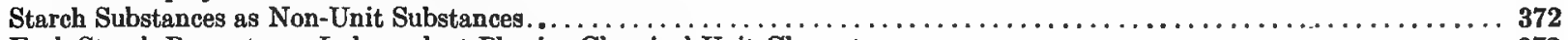

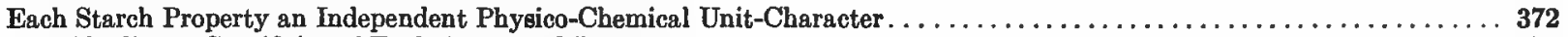

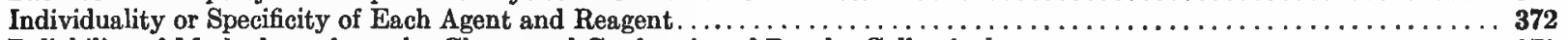

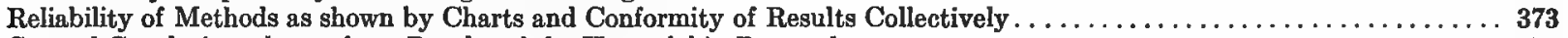

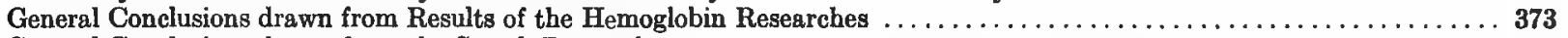

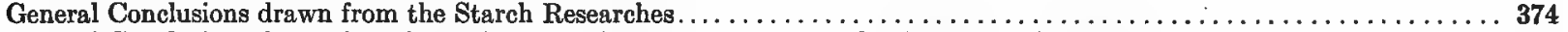

General Conclusions drawn from Investigations of the Macroscopic and Microscopic Characters of Plants............ 374

The Relative Potentialities of the Seed Parent and the Pollen Parent in influencing the Characters of the Hybrid ........ 374

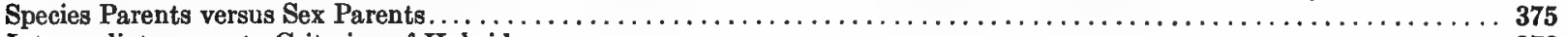

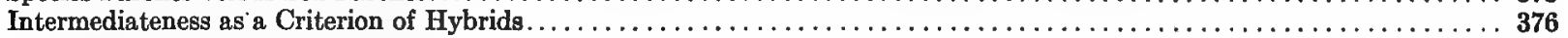

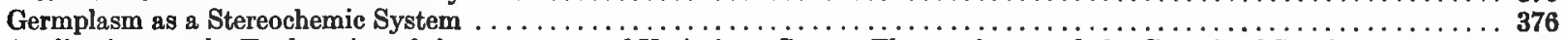

Applications to the Explanation of the occurrence of Variations, Sports, Fluctuations, and the Genesis of Species........ 376

Scientific Basis for Classification of Plants and Animals and for the Study of Protoplasm. . . . . . . . . . . . . . . . 376

PART II.

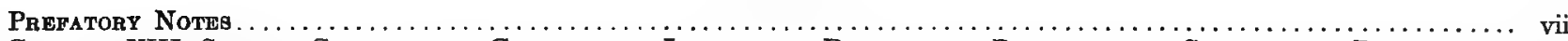
Chapter Vili. Special, General, and Comparative Laboratory Data of the Propertieg op Starches of Parent- and

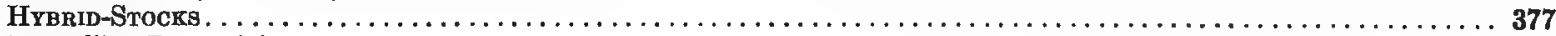

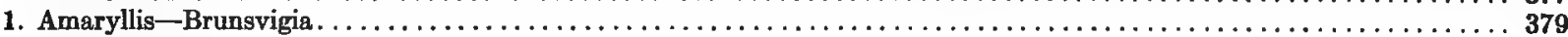

1. Starches of Amaryllis belladonna, Brunsvigia josephinæ, Brunsdonna sanderce alba, and B. sanderœ.........379

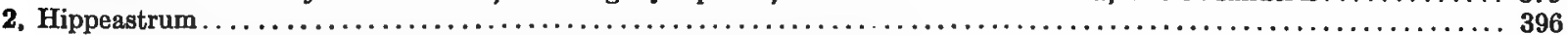

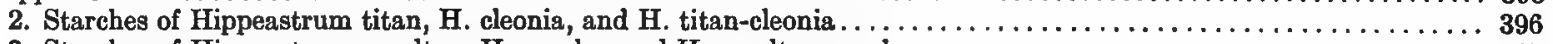

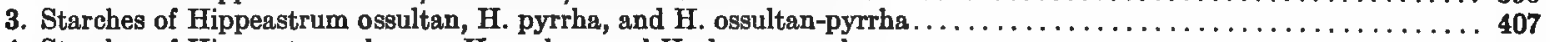

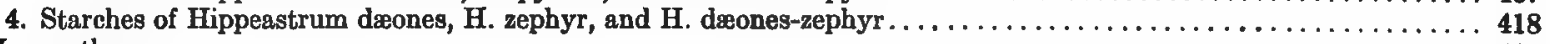

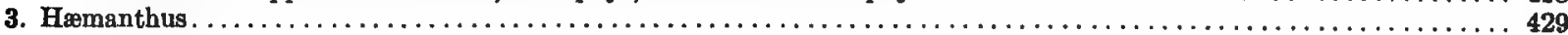

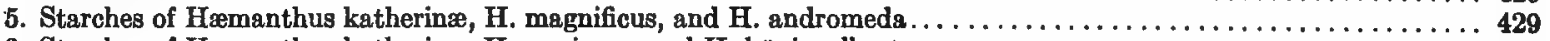

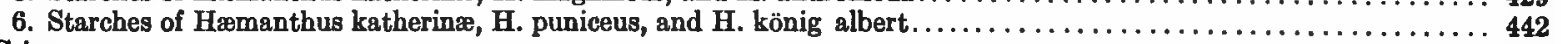

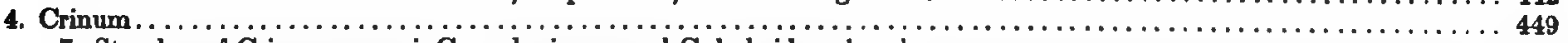

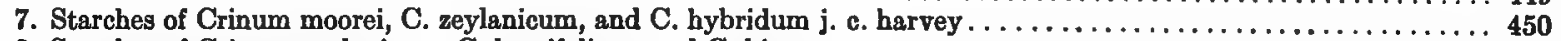

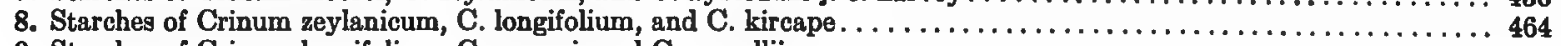

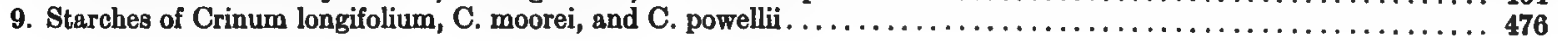




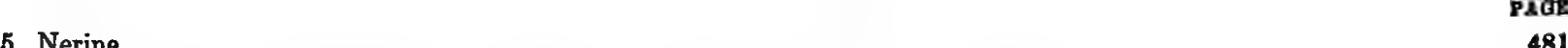

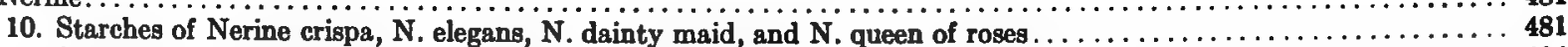

11. Starches of Nerine bowdeni, N. sarniensis var. corusca major, $\mathbf{N}$. giantess, and $\mathbf{N}$. abundance............494

12. Starches of Nerine sarniensis var. corusca major. N. curvifolia var. fothergilli major, N. glory of sarnia ....... 508

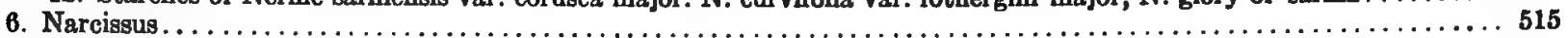

13. Starches of $\mathrm{Narcissus}$ poeticus ornatus, $\mathrm{N}$. poeticus poetarum, $\mathrm{N}$. poeticus herrick, and $\mathrm{N}$. poeticus dante...... 515

14. Starches of Narcissus tazetta grand monarque, $N$. poeticus ornatus, and $N$. poetaz triumph.............527

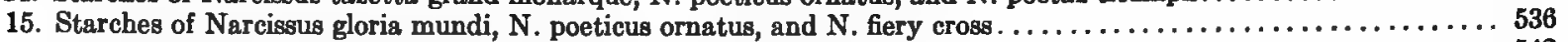

16. Starches of Narcissus telamonius plenus, $\mathbf{N}$. poeticus ornatus, and $\mathbf{N}$, doubloon . . . . . . . . . . . . . . 542

17. Starches of Narcissus princess mary, $\mathbf{N}$. poeticus poetarum, and $\mathbf{N}$. cresset. . . . . . . . . . . . . . . . 548

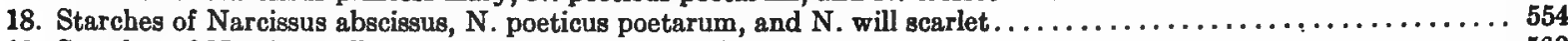

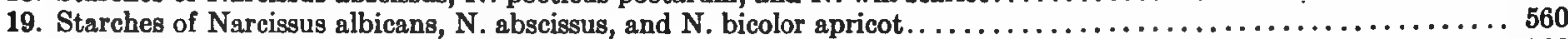

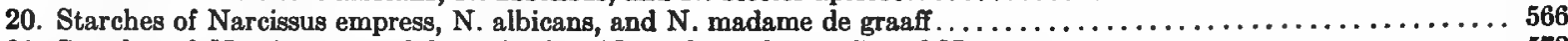

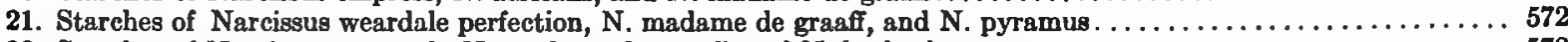

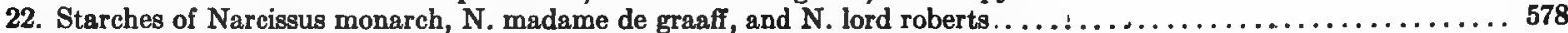

23. Starches of Narcissus leedsii minnie hume, $\mathbf{N}$. triandrus albus, and $\mathrm{N}$. agnes harvey . . . . . . . . . . . . 584

24. Starches of Narcissus emperor, N. triandrus albus, and N. j. t. bennett poe................... 591

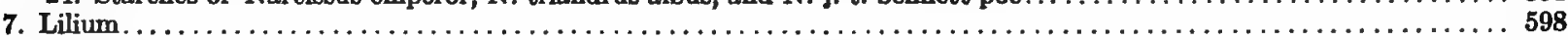

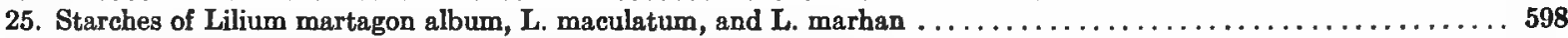

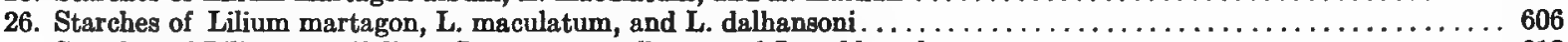

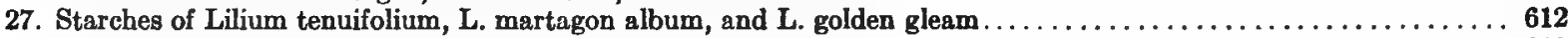

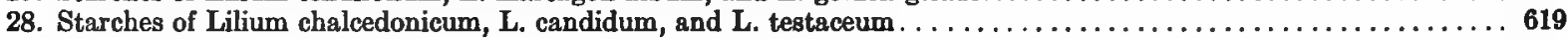

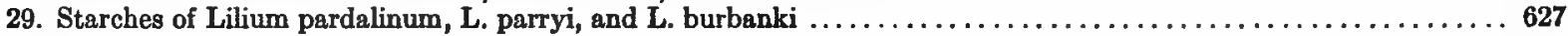

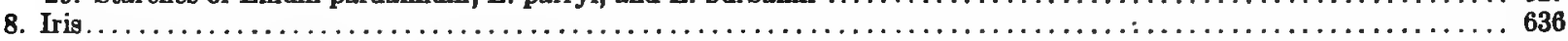

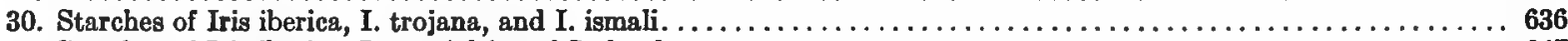

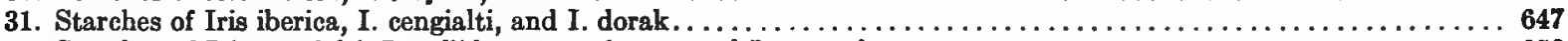

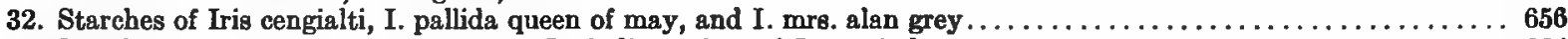

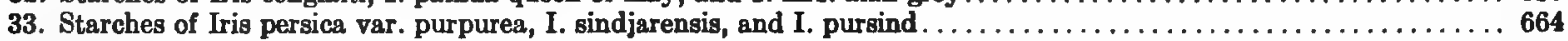

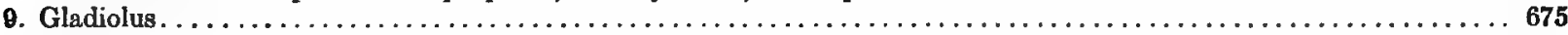

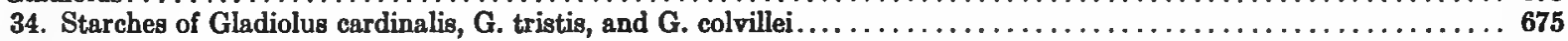

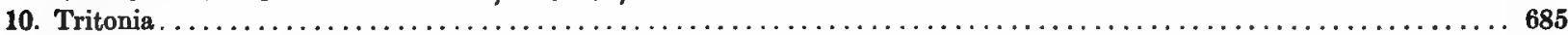

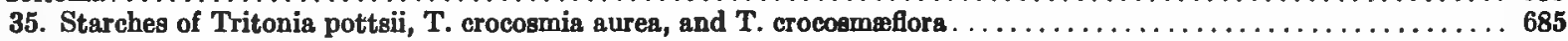

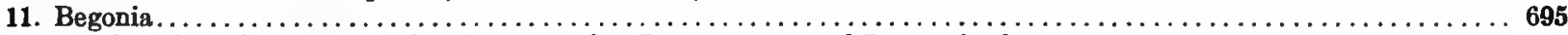

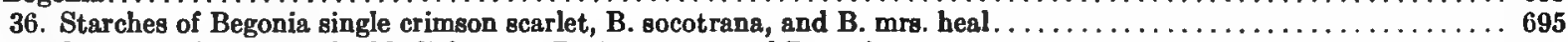

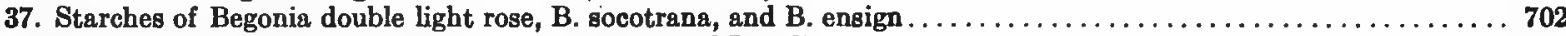

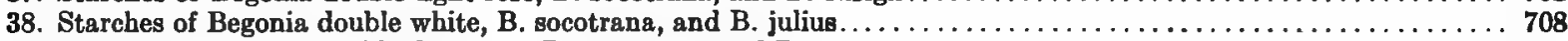

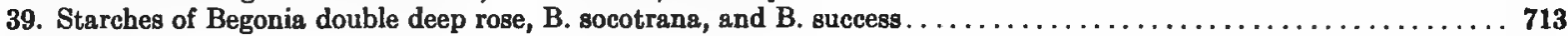

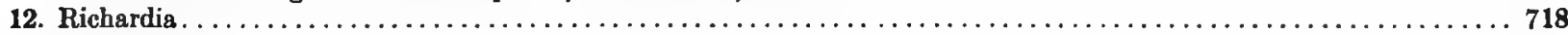

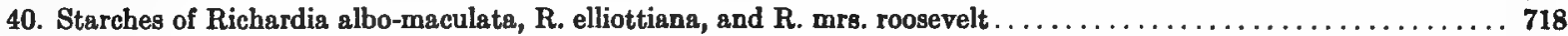

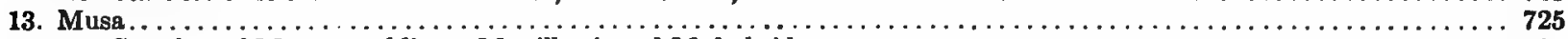

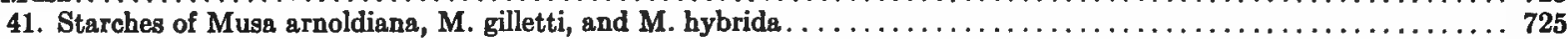

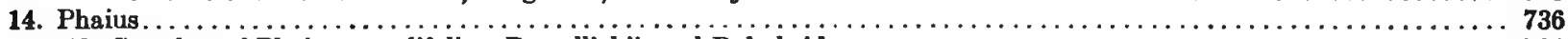

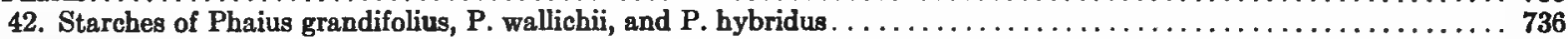

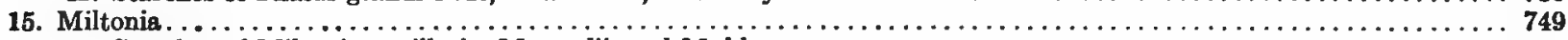

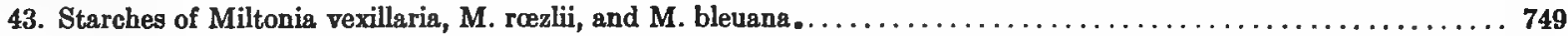

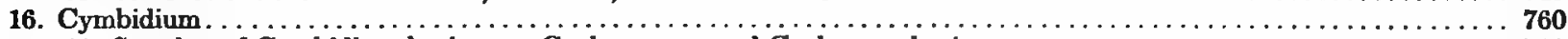

44. Starches of Cymbidium lowianum, C. eburneum, and C. eburneo-lowianum.................. 760

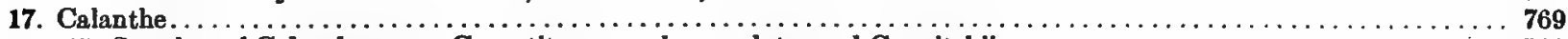

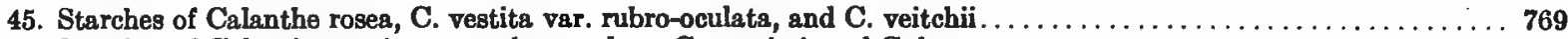

46. Starches of Calanthe vestita var. rubro-oculata, C. regnieri, and C. bryan $\ldots \ldots \ldots \ldots \ldots \ldots \ldots \ldots \ldots \ldots \ldots \ldots$

Chapter IX. Macroscopic and Microscopic Characters of Parent-Stocks and Hirbeid

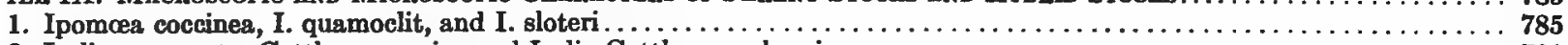

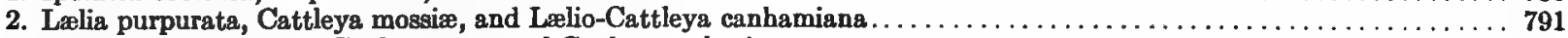

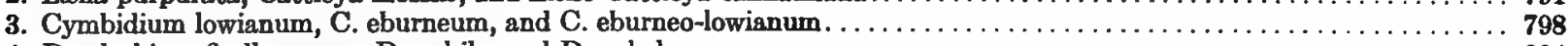

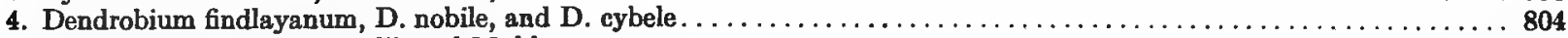

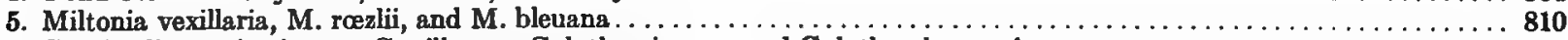

6. Cypripedium spicerianum, C. villosum, C. lathamianum, and C. lathamianum inversum $\ldots \ldots \ldots \ldots \ldots \ldots \ldots \ldots \ldots \ldots$

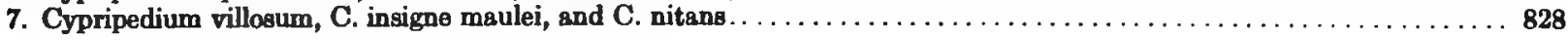




\section{PREFACE.}

This memoir is complementary and supplementary to publication No. 116 of the Carnegie Institution of Washington, entitled "The Differentiation and Specificity of Corresponding Proteins and other Vital Substances in relation to Biological Classification and Organic Evolution: The Crystallography of Hemoglobins," and publication No. 173 of the same series, entitled "The Differentiation and Specificity of Starches in relation of Genera, Species, etc.: Stereochemistry applied to Protoplasmic Processes and Products, and as a strictly scientific basis for the Classification of Plants and Animals." Like its predecessors, this is a report of an exploratory investigation. In the preface of No. 173 there appeared the following statement of the thoughts that underlie these studies, and of their support up to that time by the results of experimental inquiry:

"The present memoir, which is purely in the nature of a report of a preliminary investigation, is complementary and supplementary to Publication No. 116 of this Institution, entitled 'The Differentiation and Specificity of Corresponding Proteins and other Vital Substances in Relation to Biological Classification and Organic Evolution: The Crystallography of Hemoglobins,' in the preface of which the following statement was made of the hypothesis upon which the research was founded, and of the support of the hypothesis by the results of the inquiry:

" "The trend of modern biological science seems to be irresistibly toward the explanation of all vital phenomena on a physico-chemical basis, and this movement has already brought about the development of a physicochemical physiology, a physico-chemical pathology, and a physico-chemical therapeutics. The striking parallelisms that have been shown to exist in the properties and reactions of colloidal and crystalloidal.matter in vitro and in the living organism lead to the assumption that protoplasm may be looked upon as consisting essentially of an extremely complex solution of interacting and interdependent colloids and crystalloids, and therefore that the phenomena of life are manifestations of colloidal and crystalloidal interactions in a peculiarly organized solution. We imagine this solution to consist mainly of proteins with various organic and inorganic substances. The constant presence of protein, fat, carbohydrate, and inorganic salts, together with the existence of protein-fat, protein-carbohydrate, and protein-inorganic salt combinations, justifies the belief that not only such substances, but also such combinations, are absolutely essential to the existence of life.

" 'The very important fact that the physical, nutritive, or toxic properties of given substances may be greatly altered by a very slight change in the arrange- ment of the atoms or groups of molecules may be assumed to be conclusive evidence that a trifing modification in the chemical constitution of a vital substance may give rise to even a profound alteration in its physiological properties. This, coupled with the fact that differences in centesimal composition have proved very inadequate to explain the differences in the phenomena of living matter, implies that a much greater degree of importance is to be attached to peculiarities of chemical constitution than is universally recognized.

" "The possibilities of an inconceivable number of constitutional differences in any given protein are instanced in the fact that the serum albumin molecule may, as has been estimated, have as many as 1,000 million stereoisomers. If we assume that serum globulin, myoalbumin, and other of the highest proteins may each have a similar number, and that the simpler proteins and the fats and carbohydrates, and perhaps other complex organic substances, may each have only a fraction of this number, it can readily be conceived how, primarily by differences in chemical constitution of vital substances, and secondarily by differences in chemical composition, there might be brought about all of those differences which serve to characterize genera, species, and individuals. Furthermore, since the factors which give rise to constitutional changes in one vital substance would probably operate at the same time to cause related changes in certain others, the alterations in one may logically be assumed to serve as a common index of all.

" 'In accordance with the foregoing statement, it can readily be understood how environment, for instance, might so affect the individual's metabolic processes as to give rise to modifications of the constitutions of certain corresponding proteins and other vital molecules which, even though they be of too subtle a character for the chemist to detect by his present methods, may nevertheless be sufficient to cause not only physiological and morphological differentiations in the individual, but also become manifested physiologically and morphologically in the offspring.

" "Furthermore, if the corresponding proteins and other complex organic structural units of the different forms of protoplasm are not identical in chemical constitution, it would seem to follow, as a corollary, that the homologous organic metabolites should have specific dependent differences. If this be so, it is obvious that such differences should constitute a preëminently important means of determining the structural and physiological peculiarities of protoplasm.

" "It was such germinal thoughts that led to the present research, which I began upon the hypothesis that if it should be found that corresponding vital substances are not identical, the alterations in one would doubtless be associated with related changes in others, and that if definite relationships could be shown to exist between these differences and peculiarities of the living organism, a fundamental principle of the utmost importance would be established in the explanation of 
heredity, mutations, the influences of food and environment, the differentiation of sex, and other great problems of biology, normal and pathological.

" 'To what extent this hypothesis is well founded may be judged from this partial report of the results of our investigations: It has been conclusively shown not only that corresponding hemoglobins are not identical, but also that their peculiarities are of positive generic specificity, and even much more sensitive in their differentiations than the "zoöprecipitin test." Moreover, it has been found that one can with some certainty predict by these peculiarities, without previous knowledge of the species from which the hemoglobins were derived, whether or not interbreeding is probable or possible, and also certain characteristics of habit, etc., as will be seen by the context. The question of interbreeding has, for instance, seemed perfectly clear in the case of Canidx and Muridx, and no difficulty was experienced in forecasting similarities and dissimilarities of habit in Sciuridæ, Muridæ, Felidæ, etc., not because hemoglobin is per se the determining factor, but because, according to this hypothesis, it serves as an index (gross though it be, with our present very limited knowledge) of those physicochemical properties which serve directly or indirectly to differentiate genera, species, and individuals. In other words, vital peculiarities may be resolved to a physicochemical basis.'

"Before and since the inception of the foregoing research, data have been slowly accumulating which point more and more strongly to the extremely important interrelationships that exist between the intramolecular configurations of various substances that play active rôles in life's processes and the configurations of protoplasm. Hence, any progress in the application of stereochemistry to metabolic processes brings us closer to an understanding of those peculiar mechanisms of protoplasm which give rise to the phenomena which in the aggregate -constitute life in its normal and abnormal manifestations.

"Hemoglobin, next to protoplasm, is unquestionably the most important organic substance of vertebrate life, and in conjunction with the stroma with which it is associated is an active functionating protein, the main function of which is the conveyance of oxygen from the external organs of respiration to the internal organs of respiration or the tissues generally. Starch is similarly an extremely important constituent of a vast number of forms of plant life, but its rôle in vital processes, while, on the whole, as essential to the continuance of life, is of an entirely different character. Moreover, the general and special characters of these substances in relation to those of the bodies which originate them, and the mechanisms of their formation, are likewise strikingly different. Hemoglobin constitutes nearly the whole of the erythrocyte or red-blood corpuscle, and that portion of the erythrocyte which is not this substance may properly be regarded as being in the nature of an adjunct, but nevertheless essential. In early embryonic life the erythrocytes are nucleated and probably derived directly from the mesoblastic elements, and they increase in num- ber by mitosis. Later, proliferation occurs in all parts of the circulation, in certain capillary areas more than others, especially in those of the liver, spleen, and bonemarrow. During the progress of fetal development the erythrocytes, primarily spherical and nucleated, in time lose their nuclei, and become smaller, and take on the peculiar disk or cup-shaped form of postnatal life. After birth the red bone-marrow is the chief or sole seat of formation of erythrocytes. It is the common conception that in this structure these corpuscles arise from nucleated red cells which exist at first as colorless, nucleated erythroblasts, and subsequently as smaller, denser, colored, nucleated normoblasts. The former, which are looked upon as the hereditary representatives of the embryonal erythrocytes, are generally conceived to be converted into normoblasts by mitosis, and the latter in turn to become ordinary erythrocytes upon the disappearance of the nuclei by solution or extrusion. It is, however, more likely, as suggested in 1882 by Malassez, and very recently (1912) by the investigations of Emmel by means of plasma cultures, that the erythrocyte of late fetal and post fetal life is formed from the cytoplasm of the erythroblast by a simple process of budding and detachment.* According to either conception the erythrocyte is a separated portion of the mother substance that has been set free in a highly specialized life-sustaining medium, but in a distinctly modified form, inasmuch as it has a much higher hemoglobin content and is lacking in the amoboid activities and power of reproduction of the parent substance, the latter differences being readily accounted for in the absence of nuclear matter. Starch, on the other hand, is a synthetic product of metabolic activity which bears no resemblance to the protoplasm that gave rise to it, and which is destined to serve an entirely different purpose from that of hemoglobin in the life-history of the organism. With hemoglobin as it exists associated with the stroma in the erythrocytes we are dealing with an active, living, functionating, highly specialized form of protoplasm; with starch, we deal with an absolutely inert, non-living, non-functionating, extremely complex carbohydrate in the nature of a storedup pabulum, and a synthetic product of plastids which are specialized forms of protoplasm. In the hemoglobin research it was shown that the hemoglobin molecule is modified in specific relationship to genus, species, etc., which may be taken to mean that the form of protoplasm that is expressed by the term erythrocyte is correspondingly stereochemically modified; with starch it has been found, as will be seen by the context, that the molecule is likewise changed in specific relationship to genera, species, etc., which accordingly may also be taken to mean that during synthesis the products of activity are altered in their molecular peculiarities in specific rela-

*See Science 1912, Xxxv, 873; 1914, $x \times x I x, 334$. Kite (Proc. Soc. Exp. Biol. Med., 1914, xI, 112) and Oliver (Science, 1914, xu, 648) have found that erythrocytes can be so modified structurally and vitally as to have ciliate or flagellate processes, and Oliver has shown that some of the latter exhibit a high degree of irritability in relation to mechanical stimulus. 
tionship to the stereochemic modifications of the forms of protoplasm which produce them. In other words, one may lay down the dictum that each and every form of protoplasm existent in any organism is stereochemically peculiarly modified in specific relationship to that organism, and that, as a corollary, the products of synthesis will be modified in conformity with the molecular pecu liarities of the protoplasm giving rise to them. It follows, therefore, that if the plastids of any given plant be of different stereochemic structure from those of others, the starch produced will show corresponding stereochemic variations, and hence be absolutely diagnostic in relation to the plant. Abundant evidence will be found in the pages which follow in justification of this statement. Moreover, if such differences are diagnostic, it is evident that they constitute a strictly scientific basis for the classification of plants.

"The research on starches was undertaken with three primary objects in view: First, to determine if the hypothesis underlying the hemoglobin investigation would be supported by the stereochemic peculiarities of other complex synthetic metabolites; second, to add materially to our knowledge of one of the most important substances in the life-history of both plant and animal kingdoms; and third, to throw open fields of investigation which offer extraordinary promise, particularly in adding to our knowledge of the all-important properties of protoplasm."

Since the beginning of these researches, facts have been accumulating steadily along various channels of investigation which are in support of the propositions: That all vital phenomena are or will be found to be explicable upon a physico-chemical basis; that the line of demarcation between chemical and biochemical laws and phenomena is fast disappearing; that it is becoming recognized that the genesis of living matter, individuals, sex, varieties, species, and genera is being resolved to studies of the genesis of chemical compounds and interactions, and of the laws and applications of physical chemistry; and that the specificities of stereoisomerides in relation to various tissues, organs, and organisms is one of the most extraordinary and fundamental phenomena of living matter, and inseparable from specificities of molecular constitutions and vital characteristics of various forms of protoplasm.

In the introduction of the Hemoglobin memoir references were made to certain differences that have been noted in corresponding substances, plant and animal, in relation to biological classification; and in the corresponding chapter of the Starch memoir many instances were cited of various substances, inorganic and organic, that appear in stereoisomeric forms and exhibit marked physical, nutritive, and toxic differences in accordance with peculiarities of molecular configuration. Among such substances, those of bio- logic origin are of preëminent interest because of their direct or indirect dependence upon protoplasm for their existence and peculiarities, and many investigations bearing upon them have been carried out (during especially the last decade) that are of such particular importance in their bearings upon the objects of these investigations as to demand here at least casual notices. It has already been noted that some years ago Hoppe-Seyler and others found that the pepsing of warm-blooded and cold-blooded animals are not identical, and that Wróblewsky and others recorded differences in the pepsins of different animals. Now, it is of interest to note that these differentiations have been added to by Hedin (Zeit. f. physiolog: Chemie, 1911, IxxxI, 187 ; 1911, LxxIv, $242 ; 1912$, LXXXI, 175), who found in comparative studies of renninogens from species of different genera that either rennase or antirennase can be prepared at will from the same renninogen, and that the antirennase is inhibitory to the rennase of the same species but not to the rennase of other species, therefore showing distinct generic specificity. Moreover, it is probable, as Hedin pointed out, that the invertases from different yeasts, bacteria, molds, etc., are not identical. Scherman and Schlesinger (Proc. Soc. Exp. Biol. and Med., 1915, xur, 118) have ro ported that the amylases from pancreas and malt are not identical. Malt amylase they found to be most active in a somewhat acid solution, while the optimum solution for pancreatic amylase is slightly alkaline, and the amylase of pancreas was less than half as active as that of malt. The investigations of Dudley and Woodman (Biochem. Jour., 1915, Ix, 97 ) indicate that the casein of sheep differs from that of the cow; and the studies by Dakin and Dudley (Biochem. Jour., 1913, xv, 271) in digestion, Schmidt (Proc. Soc. Exp. Biol, and Med., 1917, xIV, 104) in immunization, Ten Broeck (Biolog. Chem., 1914, xvIr, 369) in antigenic tests, and Underwood and Hendrix (Biolog. Chem., 1915, xxIr, 453) in toxicity experiments have shown that "racemic" casein is not identical with casein.

The specificities of the hemoglobins and starches in relation to the animal or plant source, as set forth in the preceding memoirs, has had abundant support by various biologic reactions (complement-fixation,. agglutinin, precipitin, anaphylactic). It seems evident that all of these reactions or tests have a biochemic basis; that they are dependent upon peculiarities of chemical constitution or structure of protein molecules; and that they are "group" reactions in the sense that they are restricted to the same or to similar proteins of the same individual or closely 
related or allied species or genera. Since Magendi in 1839 found that when egg albumin is injected into rabbits the animals become so sensitized that death is caused by a second injection, an enormous amount of work has been done in similar and allied experiments. The literature that has accumulated is so exceedingly voluminous and of such a character that even a review of the most important of the investigations is quite impossible within the allotted limits of space of this report. But there are several researches that have appeared since the publication of the preceding memoirs' which, like the foregoing, are of such especial importance in connection with the present investigations that they, as in the case of several others above referred to, should receive at least a passing notice. For instance, Bradley and Sansun (Jour. Biolog. Chem., 1914, xviII, 497) found that guinea pigs that are sensitized to beef or dog hemoglobin, fail to react, or react only slightly, to hemoglobins of other origins. They tried the hemoglobins of the dog, beef, cat, rabbit, rat, turtle, pig, horse, calf, goat, sheep, pigeon, and chicken, and of man, and they found reasons for the conclusion that the hemoglobins from different sources are chemically different.

The studies of Wells and of Wells and Osborne of the biological reactions of vegetable proteins (Jour. Infect. Dis., 1911, vIII, 66 ; 1913, xII, 341; 1914, XIV, 377 ; 1915, XVIr, 259 ; and 1916, XIX, 183) show among various findings of variable degrees of importance that chemically similar proteins from the seeds of different genera react anaphylactically with one another, while chemically dissimilar proteins from the same seeds in many cases fail to do so. Blakeslee and Gortner (Carnegie Institution of Washington Year-Book, No. 12, 1913, 99) record evidence in their investigations of the precipitin reactions of the proteins of mold that is consistent with the conclusion that there are not only "species proteins" but also "sex proteins" (see Chapter VI, pages 366 and 367) ; and Gohlke and $\mathrm{Mez}$, and Lange (Umschau, 1914; Scientific Amer. Sup. 1914, No. 2016, 122) have recorded most significant data in the determination of plant relationships by means of sero-diagnosis. Taxonomic relationships of a number of families were studied and references are also made by Gohlke to the differentiations of plant albumins by Kowarski and to the experiments of Magnus and Friedenthal which showed a relationship between truffles and yeast. Legrand (Revue Generale des Sciences, 1918; Scientific American Supplement, 1918, No. 2238, 322) has brought together a large number of diversified facts in support of zoologic biochemic specificities.
Comparing the results of the various "biologic tests" with those recorded by means of the methods used in the starch and hemoglobin researches, it seems to be conclusively demonstrated, as far as these investigations have gone, that the latter are capable of practically unlimited development by addition and improvement. The studies of the starches and hemoglobins are not more than merely started, and there remain virtually untouched (for exceptionally inviting and extensive investigation) albumins, globulins, proteoses, glycogens, fats, cholesterols, alkaloids, enzymes, hormones, and a host of other substances that undoubtedly appear in animal and plant life in stereoisomeric forms that are specifically modified in relation to the protoplasmic source. When one pictures what these three exploratory researches have brought forth and what they suggest as being in part the outcome of further inquiry the imagination becomes bewildered by the marvellous richness of what is thus forecasted.

The methods used in the preceding research have in the present investigation been extended and so improved as to yield records that are satisfactory in quantity, kind, and accuracy; and in reference thereto, it seems needless at this juncture to do more than present certain excerpts from reports by the writer that have appeared in the Year Books of the Carnegie Institution of Washington or elsewhere, as follows?

"The investigations with the starches were necessarily carried on by methods that are quite different from those employed in the study of the hemoglobins. Although the starch granule is a spherocrystal that lends itself to crystallographic study, very little can be learned of its molecular characters that is of usefulness in the differentiation of various starches. Other methods, however, offer very satisfactory means of study, especially those which elicit molecular differences by means of peculiarities of gelatinization. These methods, all microscopic, have included inquiries into histological characters; polariscopic, iodine, and aniline reactions; temperatures of gelatinization; and quantitative and qualitative gelatinization reactions with a variety of chemical reagents which represent a wide range of difference in molecular composition.

" Each starch property, whether it be manifested in peculiarities in size, form, hilum, lamellation or fissuration, or in reactions with light, or in color reactions with iodine or anilines, or in gelatinization reactions with heat or chemical reagents, is an expression of an independent physico-chemical unit-character that is an index of specific peculiarities of intramolecular configuration, the sum of which is in turn an index which expresses specific peculiarities of the constitution of the protoplasm that synthetized the starch molecule. The unitcharacter represented by the form of the starch grain is independent of that of size; that of lamellation independent of that of fissuration, etc. This is evident in the fact that in different starches variations in one may not 
be associated with variations in another, and that when variations in different properties are coincidently observed they may be of like or unlike character. Gelatinizability is one of the most conspicuous properties of starch and it represents a primary physico-chemical unitcharacter, which character may be studied in as many quantitative and qualitative phases as there are kinds of starches and kinds of gelatinizing reagents, the phenomena of gelatinization by heat being distinguishable from those by a given chemical reagent, and those by one reagent from those by another, and those of one starch by a given reagent from those of another starch. The gelatinization of the starch grain is certainly not, as is commonly supposed, a manifestation of a simple process of imbibition of water, such as occurs in the swelling of particles of dry gelatin or albumin, but in fact a very definite chemical process corresponding to that which occurs in the swelling of liquid crystals, and which must vary in character in accordance with the reagent entering into the reaction. It therefore follows, as a corollary, that the property of gelatinizability of any specimen of starch may be expressed in as many independent physico-chemical unit-character-phases as there are reagents to elicit them. By these methods both physico-chemical unit-characters and unit-character phases can be reduced to figures, from which charts can be constructed which show in the case of each starch that the sum total of these values is as distinctive of the kind of starch and plant source as are botanical characters of the plant.

"Individualities of one or the other of the parental starches may or may not be observed in the starch of the offspring, and if present they may or may not appear in modified form. Moreover, the starch of the offspring may exhibit peculiarities that are not seen in either of the parental starches, and when two or more sets of hybrids have resulted from separate crosses of the same parental stock, each lot of hybrids may not only exhibit in common distinctive variations from parental characters but also independent individualities, and, as a corollary, differ, from each other in well-defined respects. Hence, not only may a given hybrid be definitely attached to definite parentage, but also the hybrids of separate crosses may be recognized as such.

"The studies of the starches of parent- and hybridstocks have been supplemented by corresponding and somewhat laborious histological examinations of plant tissues associated with some macroscopical inquiry. The results of this supplementary research are in striking accord with those of the starch investigations, and both are in entire harmony with universally recognized principles of the plant and animal breeder and with the dictum underlying these researches, 'vital peculiarities may be resolved to a physico-chemical basis'-with which may be coupled a second dictum, 'corresponding complex organic substances exist in stereoisomeric forms that are modified specifically in relation to and diagnostic of the protoplasmic source." "
While the present research treats almost solely of the properties of parent-stocks and hybrid-stocks; and correspondingly of heredity, it will be found that the results can be utilized in very broad applications to biology. Apart from the derogation of intermediateness as a criterion of hybrids, there is perhaps no single feature of the report that will appeal more immediately to biologists in general than the facts that have been collated that indicate a far greater degree of importance of hybridization in the genesis of species and evolution than has thus far been recognized. Moreover, to every student who has kept abreast of the developments of modern biologic science it must be evident that the great advances now foreshadowed seem to be inseparably associated with physics and physical chemistry; and from the results of these researches on the physical chemistry of starches and hemoglobins it seems that it may with safety be predicted that the principles and methods herein presented will serve as one of the essential starting-points that will certainly lead to results of great if not epochal importance. What physics promises in explanation of the phenomena of organic growth and form, physical chemistry promises in the explanation of organic function.

Finally, an apologetic word may not be amiss. This investigation like its two predecessors has been pursued amidst the endless interruptions and disconcertions that are inseparable from the exactions of professorial duties and other unavoidable conditions, and not infrequently it has of necessity been set aside for weeks or months. This obviously has not only somewhat but seriously interfered with that continuity of work and thought that is so important in the successful pursuit of elaborate investigations in unexplored fields of inquiry. On this account there will appear not a little evidence of a lack of uniformity of treatment of corresponding parts of the work; an absence here and there of sufficient and careful detail, correlation, and analysis; and a failure not infrequently to discuss with sufficient fullness many facts in their biologic relationships and applications. Moreover, inasmuch as the writer is not a botanist, some facts that may be of especial botanic interest may not have been given adequate treatment, while some of minor interest may have been unduly accentuated.

\section{Edward Trson Reichert.}

From the S. Weir Mitchell Laboratory of Physiology, University of Pennsylvania. 


\begin{tabular}{c}
\hline \hline PART I. \\
SUMMARIES AND COMPARISONS 0F THE PROPERTIES 0F THE STARCHES AND OF \\
THE TISSUES 0F PARENT-STOCKS AND HYBRID-STOCKS. APPLICATIONS \\
OF THE RESULTS OF THE RESEARCHES TO THE GERM-PLASM, \\
VARIATIONS, FLUCTUATIONS, SPORTS, MUTANTS, SPECIES, \\
TAXONOMY, HEREDITY, ETC. NOTES AND CONCLUSIONS. \\
BY EDWARD TYSON REICHERT, M.D., Sc.D.
\end{tabular}





\section{CHAPTER I.}

\section{- INTRODUCTION.}

\section{Objects of this Research.}

In both of the preceding researches satisfactory evidence was recorded to justify the conclusion that complex organic substances exist in different stereoisomeric forms in different organisms, and that the differences are specific in relation to genera, species, and varieties, and in general in striking accord with the accepted data of the systematist. Naturally it seemed to be a matter of the greatest fundamental importance to determine to what recognizable degree these physico-chemical properties are transmitted from seed and pollen parents in altered or unaltered form in the hybrid; if it is possible to predict the heritability of this or that property; whether or not new physico-chemical properties appear in the hybrid; and if the phenomena of physico-chemical inheritance are not only consistent with but also in explanation of the data of the systematist and with the experience of the plant breeder.

\section{Criteria of Hybrids and Mutants.}

\section{A FOREWORD.}

Beginning with the elementary investigations of Linnæus, data pertaining to the comparative peculiarities of parents and of hybrids have been accumulating, and at present, notwithstanding that thousands of such sets are known in literature, only very few of them have been recorded in a way that renders them of more than general value in formulating laws of inheritance. Standards for the recognition of hybrids and mutants, respectively, have found widespread acceptance, yet one may well hesitate to inquire if in the restrictedness of our analyses and comparisons, the narrowness of our conceptions, and the manifest prejudices and errors of judgment we have not been fostering many views that have led to general misunderstanding and illusory conclusions.

The universally recognized primary or essential distinguishing characters of hybrids are: Intermediateness of the first generation; lessened vitality that may be expressed in many ways; partial or complete sterility, especially as regards the pollen; instability and Mendelian inheritance in the second and succeeding generations. But if we were to carefully examine a large number of diversified characters of say a dozen hybrids selected at random, what percentage of these characters would be found to be intermediate, and what percentages of these intermediate characters would be of mid-intermediate value or nearly the same as in one or the other parent? Are there not many hybrids that are nearly or quite as fertile as their parents, or if their fertility is subnormal in the first generation may it not become normal during subsequent generations? Are there not many hybrids that show little or no tendency toward Mendelian inheritance, or which, in other words, breed true? Is it not common to find in hybrids unimpaired vitality and a luxuriance of growth even exceeding that of the parents?

The primary or essential distinguishing characteristics of mutants are set forth in the laws formulated by $\mathrm{DeVries:}$

(1) New elementary species arise suddenly, without transitional forms.

(2) New elementary species are, as a rule, absolutely constant from the moment they arise.

(3) Most of the new forms that have appeared are elementary species, and not varieties in the strict sense of the term.

(4) New elementary species appear in large numbers at the same time or at any rate during the same period.

(5) The new characters have nothing to do with individual variability.

(6) The mutations, to which the origin of new elementary species is due, appear to be indefinite, that is to say, the changes may affect all organs and seem to take place in almost every conceivable direction.

Do not all of these laws conform in all essential respects with the data in many hybrids? Is not partial or complete sterility common among mutants? Do not mutants when crossed give rise as commonly as hybrids to offspring which exhibit Mendelian phenomena? In a word, has a definite line of demarcation been established between hybrids and mutants? In the present research mutants, as such, are of only indirect interest, but if they are hybrids, as is held by many, they are obviously of direct and fundamental importance.

One need not turn many pages of the vast literature of heredity before becoming bewildered by the conflicting statements of recognized authorities and noting that many of even the more important deductions rest upon false premises. In the following elementary sketch the botanist, zoologist, evolutionist, and others who are very familiar with the subject of heredity will not find anything new, either in facts or deductions, the sole purpose of the presentation being to lay before the general reader data-to show the antipodal views of different authorities; to indicate with what reserve we should accept certain well-known laws, rules, criteria, and conceptions; and to point to what should, in a general sense, be expected in heredity upon the bases of recognized facts of hybridization and mutation. 


\section{Intermediateness and Lessened Vitality of Hybrids, etc.}

The gross structural characters of plants have attracted the attention of mankind from time immemorial, and for generations they have constituted the essential means by which plants have been differentiated and classified; yet beneath them there lay an infinitude of microscopical, chemical, physical, and physico-chemical properties of tissues and various protoplasmic substances which will undoubtedly be found to be of far greater significance in differentiation, not only in taxonomy and phylogeny, but also in the elucidation of various problems that constantly confront the botanist. The scientific value of the histological method of plant study to the systematist was satisfactorily demonstrated in 1883 by Radlkofer in "Uber die Methoden in der botanischen Systematik insbesondere dieanatomische Methode." This method he holds is applicable to the study of species, and since his time it has been successfully extended to varieties and hybrids. A century ago De Candolle found the microscope useful in plant classification, and Radlkofer predicted in his memoir that the energies of the systematist would for the next century be devoted to the histological method. Previous to the investigations of the latter, much work on the micro-anatomical and the microchemical peculiarities of plants was recorded, and since then literature of this character has accumulated to an enormous volume, as is evident at a glance through the encyclopedic pages of Solereder's "Systematische Anatomie der Dicotyledonen " that appeared in 1898. While such researches have proved to be of value in taxonomy, in the explanation of many problems that baffled the oldschool systematist, and in throwing open new avenues of thought and investigation, but little has been systematized that seems to be of immediate practical usefulness to the plant-breeder and to the student of evolution. Time will undoubtedly show, with the sifting out of these records in conjunction with recent work, a wealth of material that far exceeds in value even the greatest expectations.

All of our knowledge of hybrids dates from a period scarcely more than two centuries ago. It was near the end of the seventeenth century when the existence of sexual organs of plants was recognized, and it was sometime shortly antedating 1719 that Thomas Fairchild, a London gardener, produced a hybrid (Fairchild Sweet William) by the fertilization of Dianthus caryophyllus (the clove pink) with $D$. barbatus (the common Sweet William). This was followed by investigations of parents and hybrids by Linnæus. To Kölreuter, however, whose laborious experiments in hybridization began in 1760 by crossing Nicotiana rustica with $N$. panicu lata, must be given the credit for laying a working foundation that has proved of the greatest value in arousing interest and active investigation in this exceptionally important field of research. What had been recorded of both naturallv and artificially produced hybrids up to 35 years ago was summarized and commented upon by Focke (Die Pflanzen-Mischlinge: ein Beitrag zur Biologie der Gewächse, 1881). Probably as many as 2,000 hybrids are here referred to. Since then the number has been considerably added to in botanical literature. Such investigations, up to the time of the appearance of the memoir by Macfarlane on "A Comparison of the Minute Structure of Plant Hybrids with that of their Parents, and its Bearing on Biological Problems" that appeared in 1892, were confined practically wholly to the grosser phenomena of plant life, such as the parentage, size, vigor, rapidity of growth, length of life, appearance of malformations, fertility, etc.-in a word, gross characters such as have been and continue to be the tools of the old-school systematist.

\section{Intermediateness of Histologic Properties or Hrarids.}

Macfarlane in referring to the earlier microscopical investigations states that Henslow (Cambridge Phil. Trans., 1831) made a microscopic comparison of a hybrid Digitalis with its parents and showed that in the size and shape of the hairs and other structures the hybrid is intermediate between the parents; that Wichura (Bastardefruchtung, 1865) with Salix, and Kerner (Monographia Pulmonar., 1878) with Pulmonaria, likewise found the hybrid to be intermediate; and that Wettstein (Sitz. der. Kaiser. Akad. der Wissen., 1888), in comparing the leaves of four coniferous hybrids observed in transverse sections of the leaves that each hybrid in the number of stomata, depth of the epidermal cells, and number and arrangement of the sclerenchyma elements of the bundles is exactly intermediate between their parents.

In investigations of the minute characters of over 60 hybrids in comparison with their parents, Macfarlane found it necessary to adopt certain precautionary measures in order to secure safe comparative results. Inasmuch as they have served as our guide in the anatomical part of the present research they are here quoted in full:

\section{Average Organigmal Development and Deviations.}

"It is now recognized by botanists that every species exhibits a sum-total of naked-eye characters which distinguish it with greater or less precision from allied species. These are duly given in every local Flora. But further, specific features-alike macroscopic and microscopic-which are of great importance, are passed over. Radlkofer (Akad. der Wissenschaften, Munich, 1883) has already insisted that the anatomical method must be applied to the study of species, and I have pointed out that this is equally true of subspecies and varieties (Trans. Bot. Soc. Edin., vol. xIX, 1891). But it is the sum-total or accumulation of minute peculiarities which gives specific identity to any organism, and it is to be expected that evident or naked-eye variations will often have their commencement in trivial structural deviations, which, being perpetuated and exaggerated it may be in size, will ultimately appeal to the naked 
eye. It was this, well illustrated in the group Cirripedia, which forced Darwin slowly but surely to frame and enunciate his evolution hypothesis.

"As plant after plant has passed under my observation, I have been greatly impressed, not only with the average similarity in development that each shows, but even more with the constant tendency there is for individuals to vary from that average either in under or over development, it may be only of some part or area or of some large organ. As illustrations on a somewhat large scale, I may refer to the number, position on the stem, and size of leaves, a line of inquiry which has been entirely overlooked by systematists, but which can afford characters of considerable value. Thus Hedychium gardnerianum, when well grown and not overcrowded in a hot-house, sends up flowering shoots which bear on the average 13 lamina-producing leaves, beside one or two basal scales. $H$. coronarium bears 21 , while the hybrid H. sadlerianum bears $1 \%$. But not unfrequently from overcrowding, lack of light and nourishment, or other unfavorable surroundings, the number in each may be considerably reduced. Conversely, when very favorable vegetative conditions occur, these are accompanied with greater luxuriance.

"A shoot of Saxifraga aizoon, with freedom for growth, produces annually 23 to 26 leaves; S. geum, 40 to 45 ; and their hybrid, $S$. andrewsii, 30 to 32 .

"During the autumn of 1890 I happened to go over a large bed of sunflowers, and, in by far the greater number, $2^{7}$ to 28 leaves were formed between the cotyledons and terminal capitulum. A few instructive cases of variability from the average were noted. The bed was one which sloped to the sun and some plants at the back that were slightly overshadowed by trees had been starved in their light and moisture supply. Their leaves were reduced to 20 or 21 . On the other hand, one in a favorable situation produced 31 leaves.

"But minute changes are correlated with these grosser variations, such as an increase or decrease in the stomata over a given area or in the length and number of hairs, etc. In the choice of material, therefore, for hybrid investigation one should either be acquainted with the parent individuals and the conditions under which they were grown or try to choose an average specimen of each for study.

\section{Limit of VARIABILITy.}

"A wide field of patient and laborious work is open in the direction of ascertaining how far the individuals of a species may differ microscopically without losing specific identity. As yet this field may be said to be untrodden. The contributions that have recently been made (Bot. Central., Bd. xIV, XLVI) by Schumann are exactly on the lines desiderated and form a valuable study in tissue variability, but if we are to get an exact estimate alike of species and hybrid production the knowledge must be forthcoming. Thus Lapageria rosea is a parent form which I have chosen for pretty exhaustive description, and though I have tried to select material from what I regard as an average strain, this may still differ from the parent plant used, as several varieties are known to be in cultivation. This may partially explain why it is that hybrids at times exhibit a slight divergence toward one parent. Again, I shall have to refer at some length to the remarkable change of color exhibited by the flowers of Dianthus grievei, from white on first opening to rich crimson or crimson-purple on fading. The one parent, $D$. alpinus, shows scarcely any trace of such floral change, but among the numerous varieties of $D$. barbatus in cultivation one exhibits the above peculiarity in an equally or even more striking manner.

"Now, every varietal form inherits certain common specific peculiarities, and also the points that stamp it as a variety, so that one would err in comparing the ordinary species with the hybrid. But the very fact that varieties are often inconstant in their varietal details, and do not hand these down in all cases so steadily as a marked species, are reasons for our giving a certain latitude in comparison with the hybrid, but equally are reasons for our desiring an exact knowledge of how far a specific form may vary.

\section{Comparison of Simmar Parts.}

"In my earlier investigations it was sometimes found that a certain part or organ of a hybrid did not exhibit intermediate blending of the structure of both parents, but a decided leaning to one. This was at first regarded as an instance of variation from average hybridity, but more careful and exhaustive comparison showed that the apparently exceptional conditions arose from choice of material that did not agree in age, position, or opportunities for growth. Thus I stated in the "Gardeners' Chronicle' (April 1890) that while Saxifraga aizoon had many stomata on its upper leaf surface and $S$. geum had none, $S$. andrewsii resembled the latter in this respect. Now, I had expected to find some on the leaf chosen from the hybrid, which was one of the lowest of an annual shoot, those of the parents being from the upper parts of shoots. On returning to the matter more recently, it was found that the closely intermediate character of the hybrid was established when leaves of the same relative position and age were chosen. Thus, since $S$. aizoon produces on the average 25 leaves annually, the hybrid 32 , and $S$. geum 40 , if the tenth leaf from the base be chosen in the first, we should select the fourteenth in the hybrid and the eighteenth in the other parent. The same principle of judicious selection of material must be applied not only in dealing with large organs but also in minuter details, such as bundle elements, matrix cells, and sclerenchyma, as well as starch grains, chloroplasts, and other cell products.

\section{Afatlable limit for Comparison of Parents with their Hybrid Progeny.}

"During the last decade problems bearing on the relative potency of the male and female elements in the development of an organism have been greatly discussed. The present investigation not only throws great light on these, but will enable us to compare more accurately than hitherto the capabilities of each sex element. It is manifest, however, that when a hybrid is the product of parents that are widely divergent in histological details the comparison will be easy, but when we attempt to compare a hybrid with two parents which are regarded as species, but whose chief specific differences are those of coloring and size, it is almost or quite impossible to 
detect microscopically any blending of patent characters, even though these may occur. Some may demur to accepting conclusions drawn from comparison of the hybrids of two parents that are even moderately removed from each other in affinity, particularly since we know that such are frequently less fertile than the pure product of either parents, or are entirely sterile. The objection will afterwards be considered, but here I may premise that, as a rule, whether the parents are remotely or closely related their evenly blended peculiarities appear, if comparison is at all possible.

"To the above general conclusion, however, we must make an important exception. In not a few cases, which will afterwards be cited, a separation or prepotency of the sexual molecules of each parent seems clearly to be indicated.

\section{Relative Stability of Parent Forms.}

"Some species, both in the wild state and under cultivation, show a greater degree of stability, or want of variation tendencies, than do others. This is probably to be explained by an average structure having been slowly but steadily evolved through crossing and recrossing of an aggregate of like individuals with survival of those best fitted for a set of environmental conditions that remained constant through long periods of time. These, therefore, even when removed to rather disadvantageous surroundings, do not readily exhibit change. As examples, I may name Erica tetralix, $E$. cinerea, and Philesia buxifolia. One finds that the opposite is equally true of not a few species. Thus, if a series of individuals of Geum rivale or Dianthus barbatus (cultivated) be compared microscopically, considerable variation is traceable.

"But even species which are considered to vary little, if compared from wide areas, may present unexpected changes. An interesting illustration is furnished by a plant just cited as one of the most invariable, viz, Erica tetralix. I have shown elsewhere * that this species resolves itself into four subspecies, three of which are found in Connemara, and these, so far as they have been experimented on, remain true under cultivation. It is necessary, therefore, in the selection of a hybrid to know the exact type of each parent, if not the actual parent, and to examine such alongside the hybrid offspring."

Macfarlane made detailed studies of the microscopic peculiarities of nine sets of parent-stocks and hybridstocks, including the following:

1. Lalageria rosea, Philesia buxifolia, $\mathbf{P}$. veitchii.

2. Dianthus alpinus, D. barbatus, D. grievei.

3. Geum rivale, G. urbanum, G. intermedium.

4. Ribes grossularia, $R$. nigrum, $R$ culverwellii.

5. Saxifraga geum, S. aizoon, S. andrewsii.

6. Erica tetralix, E. ciliaris, E. watsoni.

7. Mensiesia empetriformis, Rhododendron chamæcistus, Bryanthus erectus.

8. Masdevallia amabilis, M. veitchiana, M. chelsoni.

9. Cypripedium spicerianum, C. insigne, C. leeanum.

He also recorded many data respecting other hybrids and parents, including in the text only some special features which seemed to deserve consideration, to-

*Trans. Bot. Soc. Edin., xIx, 1891. gether with a rather full account of the characters of a graft hybrid, Cytisus adami. The following is Macfarlane's "General Summary of Results on Seed Hybrids":

"It has been demonstrated that in hair production, if the parents possess one or more kinds that are fundamentally similar, but which differ in size, number, and position, the hybrid reproduces these in an intermediate way. Illustrations of this were presented by Geum intermedium, Erica watsoni, Cypripedium leeanum, and Masdevallia chelsoni. But if only one parent possesses hairs over a given region the hybrid usually inherits these to half the extent, as in the petals of Dianthus barbatus and some floral parts of Bryanthus erectus. If the hairs of two parents are pretty dissimilar, instead of blending of these in one, the hybrid reproduces each, though reduced in size and number by half. The gland hairs of Saxifraga andrewsii, the simple and gland hairs of Ribes culverwellii, and those on the vegetative organs of Bryanthus erectus are examples. The peculiar case of air distribution in relation to color formation noticed in the sepal of Cypripedium leeanum may also be noted here.

"In the formation of nectaries as traced in Phitageria, Dianthus, Saxifraga, Ribes, etc., the above principles also hold.

"The distribution of stomata over any epidermal area has been proved to be a mean between the extremes of the parents, if the stomata of the parents occur over one surface or both, and if the leaves are similar in consistence, but, as in Hedychirum sadlerianum, and to a less degree in Saxifraga andrewsii, if the stomatic distribution and leaf consistence differ in the parents, this may give rise to correspondingly different results in the hybrid.

"In amount of cuticular deposit, and arrangement of it into ridges or other localized growths, hybrids have been proved intermediate between the parents. We may merely recall here the case of Philageria stem, which inherited cuticular ridges from Lapageria, though reduced to half the size, since the Philesia parent was devoid of them.

"As Wichura has already proved for the vegetative leaves of hybrid willows, the venation of hybrid leaves is very uniformly intermediate between those of the parents. Figures are given with this paper of the vegetative leaves of Philageria and Saxifraga, and of the petals of Dianthus and Geum. The relation of the bundles to special terminations, as in the water stomata of Saxifraga, is in conformity with the venation.

"But the growth of tissue in a hybrid which is to determine the outline or angular position which any organ or part of one will assume is intermediate between those of the parents when the latter show traceable differences. Thus the sepals and petals, as also the styles and style-arms, of Geum intermedium, the floral parts as a whole of Saxifraga andrewsii and $R$ ibes culverwellii, the frilling of some of the floral parts of Bryanthus and Cypripedium leeanum are pronounced cases, while minor ones have been referred to.

" Turning to minuter anatomical details, every hybrid has yielded a large series of examples which prove that the size, outline, amount of thickening, and localization of growth of cell walls, is, as a rule, intermediate 
between those of the parents. We have repeatedly stated that as the outcome of growth localization, intercellular spaces of a hybrid are modified in size and shape as are the cells which surround them. Now this clearly demonstrates that the living protoplasm which has formed the cells is so organized in its molecular or micellar constitution that in every cell and over every infinitesimally minute area on its surface where cellulose is to be laid down the balanced effect of both parents is felt.

"Equally in the laying down of secondary wall thickenings, whether of a cuticularized, lignified, or colloid nature, numerous citations have been made where the amount and mode of deposition is evenly between the extremes of the parents. Perhaps the most striking case is that of the bundle-sheath cells of Philageria and its parents, where usually five lignified lamellæ are traceable in each cell of Lapageria, eleven or twelve in Philesia, and eight or nine in Philageria.

"In summarizing as to protoplasm and its modifications as plastids, where considerable differences can be traced in the plastids of two parents the hybrid gives excellent results. Only in a few parent plants have these differences been sufficiently marked to allow of compari6on with the hybrid. The leucoplasts in the epidermal cells of the parents of Dianthus lindsayi are very different in size, while most of the leucoplasts in the hybrid are exactly intermediate, but from careful measurement of lantern projection images of these it has been found that some very nearly resemble those of the female parent. The chromoplasts of the petal cells in Geum intermedium and of the sepal cells in Masdevallia chetsoni are additional illustrations. Those of the former are very variable in size and number, but this is probably to be explained from its inheriting half of its hereditary features from Geum rivale, which is equally variable as a species. Leaves of corresponding age and position from Saxifraga andrewsii and its parents have furnished chloroplasts of small size and dark green color in one parent, of large size and soft emerald green color in the other, and an intermediate type in the hybrid, though some diverge towards the "Geum" parent in having large chloroplasts.

"But the average size, shape, and lamellar deposition in starches of Hedychium hybrids are perhaps the most interesting cases adduced. When we remember that these are bodies formed temporarily as reserve food, and that they are built up by addition of successive micellæ through the agency of minute protoplasmic masses or leucoplasts, we have a direct proof that these leucoplasts are themselves fundamentally modified. Their activity in the cells of the hybrid is evinced by the building up of starch grains which, though only of temporary duration in the history of the plant, are so accurately constructed as to be an exact combination in appearance of $a$ half corpuscle of each parent.

"Finally, we may recall the facts advanced as to color, flowering period, chemical combinations, and growth vigor, which, though scanty and fragmentary in their nature, all point to the conclusion that hybrids are intermediate between their parents in general life phenomena."

In reviewing this summary one is struck by the records of universality of intermediateness by blended or exclusive inheritance of every property. In not a single instance is any character developed in either direction beyond the extremes of development of the corresponding character of the parents. However, these conclusions are doubtless to be taken as being general or broad rather than as dogmatic, inasmuch as here and there in the text of the memoir there are records of departures beyond parental extremes, as in Philageria veitchii, in connection with which it is stated it is generally to be noticed that both upper and lower epidermal cells of the hybrid are equal to, if not larger than, the largest of either parent. "Those of the one parent (Lapageria rosea) are on an average larger than those of the other parent (Philesia folia), while in the hybrid they may be larger than in either"; also, in the hybrid Bryanthus erectus, in which " the power of conglomerate erystal formation is not only inherited from the male parent (Menziesia empetriformis var.) but also appears on a more exaggerated scale, there being at least 50 per cent more crydtals in a given area of the hybrid pit than in the parent"; and also, as is quite common, in the greater luxuriance of growth of the hybrid than of the parents, as instanced in Philageria veitchii, Geum intermedium, Bryanthus erectus, etc., which peculiarity is attributed by Macfarlane to an increase in the size rather than increased multiplication of the cells of the hybrid over the parents; but in either case it is obvious that there is higher development of the hybrid in relation to the parents; moreover, even where intermediateness has been recorded, it has been recognized in some instances that the characters of the hybrid "very nearly resemble those of female parent," etc. In support of Macfarlane, Davis (American Naturalist, 1911, XLV, 193; 1912, XLVI, 377), in studies of the offspring of different species of Oenothera, found that in gross morphological characters the hybrids are intermediate between the parents, and he has since recorded that in histological characters they exhibit the same peculiarity. Holden (Science, 1913, xxxvIII, 932) states that spontaneous hybrids that are recognized as varietal modifications of species can often be diagnosed by their internal anatomy, both vegetative and reproductive, referring particularly to the intermediate histological characters of the tissues and to abortive pollen. A number of references are given by Holden to the results of the investigations of Betula and Equisetum, instancing in the hybrid transitional features between the parents in internal and external anatomy associated with abortive spores of hybrids. Reference might be made, did space permit or were it necessary, to various other articles which also are in support of the conception that hybrids are in morphological and anatomical characters, distinguished by "intermediateness."

\section{Intermediateness of the Starches of Hybrids.}

Macfarlane (loc. cit.) made notes of the starches of Ribes culverwellii and its parents, of Bryanthus erectus and its parents, and of Hedychium hybrids and their parents. He records that in Ribes grossularia (parent) the largest grains are $7 \mu$ and the average $4 \mu$; in $R$. nig- 
rum (parent) $3 \mu$ and the average $1.5 \mu$; and in $R$. culverwellii (hybrid) $5 \mu$ and the average $3 \mu$. In Menziesia empetriformis var. the largest starch grains are $6 \mu$, and in all cases they are larger than in the other parent Rhododendron chamacistus; while in the hybrid Bryanthus erectus the grains are $4 \mu$ across at their largest, though most are from 2 to $3 \mu$, the size being intermediate but falling rather toward the latter parent. Macfarlane states:

"Hedychium gardnerianum, the one parent of $H$. sadlerianum, forms strong rhizomes, whose storing cells are large, but scantily filled with starch in all that I have examined. Each starch grain is a small, flat, triangular plate, measuring 10 to $12 \mu$ from hilum to base, and the lamination is not very distinct. $H$. coronarium, the other parent, forms smaller and fewer rhizomes, and the starch-storing cells are from half to three-fourths the size of the last, but these are densely filled, particularly in the central parenchyma, with large starch granules. Each is ovate, or in some cases is tapered rather finely to a point at the hilum. They are from 32 to $60 \mu$ long, measuring as before, and the lamination is very marked. The cells of the hybrid are on the average between those of the parents; but if one may judge by opacity of cells the amount of stored starch approaches more closely to that of the latter parent. The grains may best be described if we suppose a rather reduced one of the first parent to be set on the reduced basal half of one of the latter. The lamination also is more pronounced than in the first, less so than in the second.

"A second cross was effected by Mr. Lindsay with $H$. coronarium, and examination of the rhizome starches proves that the second hybrid approaches very closely to the species parent. But the grains of $H$. lindsayi illustrate microscopically a phenomenon which has been repeatedly referred to, viz, the greater variability and instability of a second over a first hybrid; for many of the grains (in some specimens the majority) have fantastic shapes, appearing as if undergoing rapid disintegration by leucoplasts, or perhaps more truly as if the latter were incapable of building up the shells of starch in a regular and uniform manner.

"A set of crosses has been effected between $H$. elatum and $H$. coronarium. The grains of the first are like those of $H$. gardnerianum, except that they are larger (18 to $24 \mu$ ), and that the lamination is coarse. The grains of the hybrid are larger than those of $H$. sadlerianum, and exhibit even more evident lamellæ. They measure on the average, $40 \mu$, but vary from 30 to $50 \mu$. Not infrequently all the above hybrids have (mixed up with grains more typically intermediate) some grains which can scarcely, if at all, be distinguished from the small ones peculiar to one parent, while very rarely I have observed grains so large and rounded as to pass for those of $H$. coronarium. Now, when describing the epidermal leucoplasts of Dianthus grievei it was stated that, though the average was nearly $3 \mu$, some measured $2.5 \mu$ or slightly less, others as much as $3.5 \mu$. The occurrence of these, and similar minute differences in protoplasmic masses, or in formed materials like starch grains which are due to manufacture by these masses, induced me to prepare a set of micro-photographs, and to project lantern trans- parencies of these on a 7-foot screen. Thus it was possible to study their dimensions more exactly than under the microscope. It was then found that while the shape, appearance, and size of most starch grains of Hedychium, of Dianthus leucoplasts, and of Geum and Masdavallia chromoplasts were intermediate, examples might be got which reverted powerfully to one parent, and, so far as they have yet been studied, the reversion was most frequently towards the parent with the more minute cellcontents."

The results of the studies of starches are therefore in entire accord with Macfarlane's conclusions pertaining to the tissues in showing intermediateness of the hybrid, with a tendency at times to a leaning to one parent.

Investigations of the starches of varieties and of parents and hybrids of varieties of round and wrinkled peas have been made by Gregory (The New Phytologist, 1903, II, 226), Weldon (Biometrica, 1902, I, 246), and Darbishire (Proc. Roy. Soc., B., 1908, Lxxx, 122 ; Breeding and the Mendelian Discovery, 1912, 124).

Gregory (The New Phytologist, 1903, II, 226) found that the starches of round and wrinkled peas occur in two very different types. In the round seeds the peripheral cell-layers of the cotyledons contained a few oval starch-grains which did not exceed $0.06 \mathrm{~mm}$. in the greatest diameter. In the third layer the grains reached 0.2 $\mathrm{mm}$. in length, while the more deeply situated cells were crowded with oval grains measuring as much as $0.34 \mathrm{~mm}$. in the greatest dimension. The grains were regular in shape, with a definite center surrounded by well-marked lines of stratification. In the wrinkled peas the grains of the peripheral layers were of about the same size as those of the round peas, but were of a different type, occurring in irregular spheres with several centers, thus forming a compound grain which has a strong tendency to break up into smaller parts. In the cells which lie deeply these compound grains never attain a greater length than $0.1 \mathrm{~mm}$. in the greatest dimension. Table 1 gives a list of the seeds examined.

TABle 1.

\begin{tabular}{|c|c|c|}
\hline Race. & $\begin{array}{c}\text { Seed } \\
\text { character. }\end{array}$ & $\begin{array}{l}\text { Form of } \\
\text { starch- } \\
\text { grain. }\end{array}$ \\
\hline 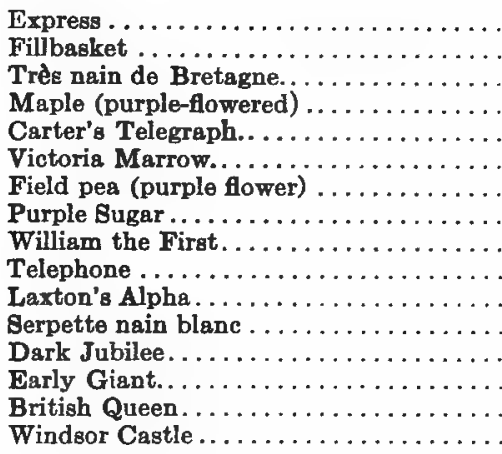 & $\begin{array}{l}\text { Round. } \\
\text { Do. } \\
\text { Do. } \\
\text { Do. } \\
\text { Do. } \\
\text { Do. } \\
\text { Indent. } \\
\text { Do. } \\
\text { See below. } \\
\text { Wrinkled. } \\
\text { Do. } \\
\text { Do. } \\
\text { Do. } \\
\text { Do. } \\
\text { Do. } \\
\text { Do. }\end{array}$ & $\begin{array}{l}\text { Large. } \\
\text { Do. } \\
\text { Do. } \\
\text { Do. } \\
\text { Do. } \\
\text { Do. } \\
\text { Do. } \\
\text { Do. } \\
\text { Small. } \\
\text { Do. } \\
\text { Do. } \\
\text { Do. } \\
\text { Do. } \\
\text { Do. } \\
\text { Do. } \\
\text { Do. }\end{array}$ \\
\hline
\end{tabular}

Gregory notes that seeds of intermediate and dubious shapes were not uncommon in certain of the races. The 
depressions in these seeds were sometimes mere pitting, as in Victoria Marrow; or they may be so marked that the seed would be described as wrinkled. The latter were especially common in William the First, but microscopic examination showed at once that these seeds are really of the round type. There are, therefore, states Gregory, two entirely different types of wrinkling, and while it is clear that the process by which wrinkling is produced is connected with shrinkage on drying, the regularity of the shrinking of the round type and its irregularity in the two other types can not at present be explained. There occasionally occur among the offspring of hybrids between round and wrinkled types seeds of dubious shape which it is difficult, on superficial examination, to classify as round or wrinkled. The existence of such seeds and types of doubtful shape was taken by Weldon to indicate irregularities of Mendelian segregation and dominance, but Gregory states that no seed has been found which upon histological examination allowed of any doubt as to its true character, and consequently that occasionally pitting and spurious wrinkling must be distinguished from the true wrinkling of the wrinkled types.

The nature of the starch-grain in the hybrid, and how the characters of the starch-grains segregate, if they do so at all, in subsequent generations, are points which suggested themselves to Darbishire, who states that they are matters on which we are ignorant. He found that the starch-grains of the round pea, such as of the "Eclipse," appear as single potato-shaped grains, with an average length of $0.0322 \mathrm{~mm}$. and an average breadth of $0.0213 \mathrm{~mm}$. The length-breadth-index (i.e., $100 \times$ breadth $\div$ length) is 66.14 . Besides these potato-shaped grains, there are extremely few very much smaller grains which are round. The grains of wrinkled peas like the "British Queen" are compound, each consisting of a number of pieces which vary between 2 and 8 . These pieces are held together by a refrangent yellow substance which does not color blue with iodine, and they are likely to break apart. The commonest types are those with 4, 5, or 6 components; grains with 7 or 8 are rarer; grains with 2 or 3 are intermediate in frequency between those with 4,5 , or 6 on the one hand and 7 or 8 on the other. While the grains with 7 to 8 pieces are not much larger than those with 4,5 , or 6 ; grains with 2 or 3 are always conspicuously smaller than those with 4,5 , or 6 . The average length is $0.0269 \mathrm{~mm}$., the average breadth $0.0248 \mathrm{~mm}$., and the length-breadth-index is 92.19 . In these peas are a number of very small single grains which can be distinguished from the pieces of the compound grains by the fact of their being circular and always smaller than the grains consisting of two pieces. Very rarely will be found isolated potato-shaped grains.

The grains of the $F_{1}$ cotyledons produced by crossing the round with the wrinkled pea are nearly round; the majority of the grains are single and the remainder compound; the compoundness exhibited by the compound grains in $\mathrm{F}_{1}$ seeds is intermediate between singleness and the degree of compoundness in the grains of wrinkled peas, for while in the latter the number of pieces varies between 2 and 8 and the commonest is 6 , in the $F_{1}$ grain it varies between 2 and 4 and the commonest is 3 . The differences in the measurements of the three starches are shown in table 2 , by which it will be seen that in shape the $F_{1}$ grain is intermediate between the potato-shaped grain and the compound grain, but nearer the latter.

TABLE 2 .

\begin{tabular}{|c|c|c|c|}
\hline & $\begin{array}{l}\text { Round. } \\
\text { potato- } \\
\text { shaped } \\
\text { grain. }\end{array}$ & $\begin{array}{l}\mathbf{F}_{1 .} \\
\text { round } \\
\text { grain. }\end{array}$ & $\begin{array}{l}\text { Wrinkled. } \\
\text { compound } \\
\text { grain. }\end{array}$ \\
\hline Average length. & $\begin{array}{c}m m . \\
0.0322\end{array}$ & $\begin{array}{c}m m . \\
0.0276\end{array}$ & $\begin{array}{c}m m . \\
0.0269\end{array}$ \\
\hline 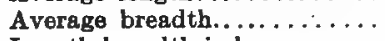 & 0.0213 & 0.0236 & 0.0248 \\
\hline Length-breadth-index . . . . . . & 66.14 & 86.5 & 92.19 \\
\hline
\end{tabular}

Darbishire also examined the grains of $F_{5}$. These he did not measure, but he states that no differences could be seen between the potato-shaped, compound, and round grains from the three types already described. He notes that the evidence points to the fact that the heterozygote round peas in generations subsequent to $F_{1}$ are characterized by the passession of irregular round or round grains, and homozygote round peas by potato-shaped grains. Darbishire records that if the association of round grains with heterozygote round and of potato-shaped grains with homozygote round holds good for the $F_{2}$ generation, we have a means of distinguishing between DD round and DR round in $\mathrm{F}_{3}$, instead of, as at present, having to wait until their progeny are mature in the following year. Another point demonstrated by the nature of grains in $F_{1}$, and borne out by those of $F_{5}$, is that the shape of the grain is inherited separately from its composition-if we may use this term to cover the singleness or compoundness of the grain. In the round pea the grains are single and long; in the wrinkled peas they are compound and round; in the hybrid they may be either single or compound, but are more round than long. In $F_{5}$ there are round grains exhibiting much compoundness and others exhibiting little. Possibly there are potato-shaped grains either with no compounds or with few, and intermediate grains either with few compounds or with many. The wrinkled peas of this generation contained, as was to be expected, compound grains, but some of them had in addition, very sparingly potato-shaped grains. Darbishire also studied the absorptive capacities of the three starches in relation to water. The following facts are summed up from the results of his investigations:

1. Although roundness is dominant over wrinkledness in peas, the round starch-grain of the $F_{1}$ generation is a blend between the type of grain of the round pea (the potato-shaped) and the type of grain of the wrinkled pea (the compound) in respect of three characters: $(a)$ it is intermediate in shape as measured by its lengthbreadth-index, that of the potato-shaped grain being 66.14, that of the compound grains 92.19 , and that of the round grain $8.5 ;(b)$ it is intermediate in the distribu- 
tion of compoundness, inasmuch as some of the round grains are compound and some single; $(c)$ it is intermediate in the degree of compoundness, inasmuch as amongst those round grains which are compound the most common number of constituent pieces is 3 , whereas in compound grains it is 6 .

2. In a subsequent generation $\left(F_{6}\right)$ the homozygote round peas contain potato-shaped grains and the heterozygote round peas contain round or intermediate grains. But both round and intermediate grains may be associated either with a high or a low degree of compoundness.

3. Potato-shaped grains occasionally occur in wrinkled peas in $F_{5}$, and the evidence suggests that the existence of these grains in wrinkled peas tends to make them less wrinkled.

4. A wrinkled pea takes up more water when it germinates than a round one. The hybrid between a round and a wrinkled pea is intermediate in respect to this character between its two parents.

5. But the intermediateness of the hybrid in absorption capacity is not occasioned by the intermediateness of the starch-grain of the hybrid, because both $\mathrm{F}_{2}$ peas containing round grains and peas containing potatoshaped grains have the same absorption capacity as the $F_{1}$ pea.

6. When, therefore, a round pea is crossed with a wrinkled pea, four separately heritable characters are dealt with: (a) the shape of the pea, whether round or wrinkled; $(b)$ the absorption capacity of the pea as regards water, whether low or high; (c) the shape of the starch-grain, whether long or round; $(d)$ the constitution of the starch-grain, whether single or compound.

The results of these researches are not only confirmatory of the records of Macfarlane in showing intermediateness in the microscopical properties of the starch of the hybrid, but also go further by demonstrating other forms of intermediateness.

\section{Intermediateness of the Macroscopic Properties OF HYBRIDS.}

No criterion of hybrids is more widely recognized than intermediateness of naked-eye characters. References have been made incidentally in preceding sections to these peculiarities, but inasmuch as macroscopic characters have been the essential tools of the systematist it is here that we must look for the data that constitute the great foundation stones upon which rests the doctrine of intermediateness. Macfarlane in summarizing the gross characters of parent-stocks and hybrids states that "color, flowering period, chemical combinations, and growth-vigor, which, though scanty and fragmentary in their nature, they all point to the conclusion that hybrids are intermediate between their parents in general life phenomena." Masters (quoted by Macfarlane, ibid., page 209) in comparing the bigeneric hybrid Philageria veitchii with its parents Lapageria rosea and Philesia buxifolia states:

"In habit our plant [the hybrid] is, of the two, more akin to the female parent (Lapageria) than to the male. Its foliage is singularly intermediate, but at the same time nearest like that of the pollen parent (Phi- lesia). In the characters of the flower-stalk, calyx, and corolla, it is more like Philesia than Lapageria, but in the stamens it approximates to the mother-plant, and diverges from the characters of the male. In color it is also more like the mother-plant than it is like Philesia. The fruit we have not seen. The characteristics of both parents are so curiously blended that we fear this plant will lend much aid to those investigators who are striving to determine what is the effect on the offspring of pollen or seed parent, respectively. On the whole, it would seem as though the organs of vegetation, including the calyx and corolla, were more like those of the male (Philesia), while in the stamens and pistil the progeny favor the mother."

From the foregoing data in this and preceding sections one is led to the belief that intermediate inheritance in the first generation is almost so universal as to be all but a law, but such a conception is inconsistent with a considerable mass of literature pertaining to both plants and animals. Focke (loc. cit.), in his Fourth Lecture, summarizes under five propositions a most important collection of data pertaining to the characters of hundreds of hybrids and their offspring. Inasmuch as these facts are of great interest, fundamental importance, and broad applicability, and as scant recognition seems to be given to this work, and as the book is rarely found in our libraries, a translation of his lecture is here given practically in full:

\section{Propositions of Focke.}

First Proposition. Stmple Primary Hybrins (A x B).

If individuals which have sprung collectively from the crossing of two pure species of races are produced and grown under similar conditions they resemble one another exactly, or are, as a rule, hardly to be differentiated from one another just as in specimens belonging to one and the same species.

The principle thus formulated seems in many experiments to be sufficiently well-grounded, but it has many exceptions. Several instances in hybrids indicate such similarity only of individuals produced from the same impregnated part (seed pod, etc.). In any event, the rule proves trustworthy only in cases in which similarity of conditions of production and growth are present.

It is difficult to answer satisfactorily a most strenuously debated question if one or the other sex has the stronger influence on the form of the offspring. The hybrids of the two species or races, $A$ and $B$, are like one another no matter whether $A$ in the crossing was the male or the female progenitor. Kölreuter, Gärtner, Naudin, and Wichura in common could find no differences between the products of the two crossings $A \& \times$ $\mathrm{B} \mathrm{o}^{\mathrm{x}}$ and $\mathrm{B} \& \times \mathrm{A}^{\mathrm{Q}} \mathrm{O}$ More than 100 years after Kölreuter noticed the similarity between the crosses Nicotiana rustica $\circ \times \mathbf{N}$. paniculata $\sigma^{\pi}$ and $N$. paniculata i $\times N$. rustica $\delta$, and one of the most observant botanists of our time, Timbal-Lagrave, was astonished by a similar experience. All the rules and assumed principles by which botanists try to determine by the morphological characteristics of the hybrid which is the pollen and which is the seed parent prove to be entirely theoretical and of no value. It has been established by many experiments that in the case of pure species in the 
vegetable kingdom in general the male and female procreative elements are of equal potency. The rule of the similarity of reciprocal hybrids, as in all other rules in the study of hybrids, is not without exceptions. It is self-evident that a certain dissimilarity of reciprocal hybrids can be correctly attributed only to the stronger influence of the male or of the female elements if the experiments are carefully carried out in the same way, and if they have, after many repetitions, always given rise to the same results. Nearly all of the reports up to this time leave much to be desired in these respects and room for justifiable doubt. The following statements on the dissimilarity of reciprocal hybrids are worth consideration:

$a$. The female element influences most strongly all parts of the morphology of Pelargonium fulgidum $\times$ $P$. grandiflorum, $P$. peltatum $\times P$. zonale, Epilobium hirsulum $\times E$. tournefortii. In many Digitalis hybrids it influences most strongly the coloring of the flowers, and in several the forms of the corolla also. In $N y m p h o e a$ rubra $\times N$. dentata the cotyledons are always much more like those of the female parent species.

$b$. The female element exercises apparently a predominating influence on the capacity of resistance to cold of Rhododendron (hybrid of $R$. arboreum), of Lycium, and possibly also of Crinum (hybrid of $C$. capense).

$c$. The influence of the male element is predominant in all parts of the morphology of Papaver caucasicum $X$ $P$. somniferum and Cypripedium barbatum $\times C$. villosum (ob constant?). It exercises a powerful influence on the flower coloration of Petunia.

d. Gärtner has several times noticed variations in the fertility of the seed of the offespring in reciprocal hybrids, as in Dianthus barbatus $\times D$. superbus. Gärtner's experiments are, however, hardly sufficient to prove the uniformity of these findings in the hybrids concerned. (In literature there may be found many speculations advanced on the influences of the male and female element on the properties of a hybrid, but supported by the description of only one hybrid.) It is evident there can be no basis for comparison unless the forms resulting from $\mathrm{A} \& \times \mathrm{B} i \hat{\text { and }} \mathrm{B}$ \% $\times \mathrm{A}$ o are both known.

Departures of an isolated specimen of a hybrid from the typical form are much more frequently noticed and are entirely independent of the rôles played by the parent forms in their production. Not infrequently, important differences appear in seedlings from a single crossing that are grown under absolutely similar conditions. These variations show themselves in various ways.

a. Individuals resulting from a given hybridization show among themselves unimportant differences, especially in the coloring of the flowers and other similarly easily altered characteristics, as in the hybrids of $V e r$ bascum phoniceum, Salix cuprea $\times S$. daphnoides.

$b$. The hybrid appears in two different types, each showing a different combination of the characters of the parent species. As a rule, the one type is closer to one, and the other to the other, parent species; the frequency of the appearance of both types is often very variable. Gärtner designated the type which appears less frequently as the exceptional type ("Ausnahmetypus"). Instances may be seen among Cistus, Dianthus, Geum,
Oenothera, Lobelia, Verbascum thapsus $\times V$. nigrum, Nicotiana quadrivalvis $\times N$. tabacum macrophylla.

c. The hybrid appears in several different types. Gärtner gives several examples of this, but there are only three known forms by a polymorphic union.

$d$. The hybrid shows one typical form of a midintermediateness, together with a number of varying forms that are usually closer to one or the other parent, among which no well-marked types can be distinguished. Such is the behavior of Medicago falcata $\times M$. sativa, and similarly of Melandryum album $\times M$. rubrum.

$e$. The hybrid is polymorphous from the beginning. The observations up to the present leave it doubtful whether one should in these circumstances distinguish between varying forms or between several fixed types with similar combinations of properties. Examples: Abutilon, hybrids of Pelargonium glaucum L'Hér., $P$. radula $\times P$. myrrhifolium, Passiflora, Hieracium, Nepenthes, Narcissus. Gärtner has offered the hypothesis that hybrids between different species are always of the same form and that the hybrids between varieties are polymorphic. If by "varieties" garden forms or garden hybrids are understood, this rule is correct; but if, on the other hand, one understands constant races of pure descent it is decidedly incorrect.

Comparisons of hybrids which arise from the same species, but which are produced and grown in different places, exhibit many other results. Spontaneous or natural hybrids are, as a rule, more variable than those produced artificially, as for example, Verbascum lychnitis $X$ $\nabla$. thapsus and $V$. lychnitis $\times \nabla$. nigrum. My own hybrids between Digitalis purpurea and $D$. lutea were very much like one another when I sowed the seed, but a great variety of forms appeared if the seeds had by chance sown themselves. It may be that in these cases there is no real causal connection between the varieties of the forms and the methods of sowing; but, on the other hand, it is a fact that different cultivators in crossing the same species have very often obtained different products. Hence, while similarity of the forms of all the plants of one crossing appears to be without doubt the rule in experiments in cultivation, similarity appears to be the exception in nature. It remains to be determined how great an influence dissimilar nutrition of the parentspecies or of the hybrid embryos may have on the variability of form of the hybrids.

\section{Second Proposition.}

The properties of the hybrids are derived from the properties of the parents. For the most part the hybrids differ from their parents,only in size and luxuriance of growth and in their generative powers.

The methods and modes in which the properties of the parent species are combined in the hybrids are very variable. In general, a blending or mutual penetration of the different properties is found, often in such a way that in one respect the one and in another the other parent form appears to predominate. That is to say, in many instances the hybrid resembles one parent more in the leaves, and the other parent more in the flowers. Now and then an exceptional variety of the hybrid (the "Ausnahmetypus" of Gärtner) appears in which the properties are inversely apportioned. Many hybrids at first more nearly resemble one, and later more nearly 
the other parent form; or in the Spring their leaves resemble the one, and in the Autumn the other type (Cistus; Populus) ; or the flower-coloring is altered during the fall of the bloom (as in Melandryum album $\times M$. rubrum, Epilobium roseum $\times E$. montanum, lantana) or in the Autumn (as in Nicotiana rustica $\times$ N. tabacum, Tropaolum, Lobelia, etc.), sometimes also in different years (as in Bletia crispa $\times B$. cinnabarina, Galium cinereum $\times G$. verum). In the crossing of races, rarely of hybrids in a strict sense, one finds now and then the properties of the parents unblended and side by side (as in Cucumis melo, the thorniness of the Datura fruits, the flower-coloring of $R$ hododendron rhodora $\times R$. calendulaceum, $R$. ponticum $\times R$. flavum, Anagallis, Linaria vulgaris $\times L$. purpurea, Calceolaria, Mimulus, Mirabilis). The flowercoloration often behaves in unexpected ways. The hybrids of Verbascum phoniceum, while having similarity of form, are very variable in the flower colorings. In Helianthemum hybrids variously colored flowers have been found on the same stem.

Frequently, from the crossing of nearly related races, especially color varieties, plants are produced which are exactly like or closely resemble one of the parent races, as in Brassica rapa var., Linum, Pisum, Phaseolus, Ana gallis, Atropa, Datura strammonium, Salvia hormium, etc. In the second generation the influence of the other parent race is usually first disclosed by a part of the seedlings reverting to it completely, or only in certain definite properties. Only in Atropa a reversion to the unstable yellow form has not been noted.

In many cases the hybrid is so like one of the parent forms that it could be considered as a very slight variation of the same. In the crossing of widely separated species the overwhelming influence of one parent species shows itself in the hybrids in a striking manner. Thus, the cross of Dianthus armeria $\times D$. deltoides is much nearer to $D$. deltoides, of $D$. caryophyllus $\times D$. chinensis to $D$. caryophyllus, of Melandryum rubrum $\times M$. noctiflorum, to $N$. rubrum, of Verbascum blattaria $\times V$. nigrum to $V$. nigrum, and of Digitalis lutea $\times D$. purpurea to $D$. Iutea, than to the second species.

Occasionally the hybrids of the first generation show properties which are entirely different from those of both parent species. This is particularly noticeable in the colors of the flowers. The most noteworthy example of this is the blue-blossomed hybrids of the white Datura ferox with the equally white species $D$. lovis and $D$. strammonium bertolonii. Instances of unexpected blossom-coloration are numerous in hybrids of species with colored flowers, in which the hybrids in no way show the coloring which one would expect from a mixture of the pigments of the parents, as in Clematis recta $X$ C. integrifolia, Aquilegia atropurpurea $\times A$. canadensis (and others), Anemone patens $\times A$. vernalis, Begonia dregei $\times B$. sutherlandi (and others), Nicotiana suaveolens $\times N$. glutinosa, Verbascum pulverulentum $\times N$. thapsiforme, and in hybrids of $C$. phaniceum which are especially good examples. In the crossing of races properties appear many times which do not resemble the parent forms but other races of the same species, as in Papaver somniferum and Datura strammonium. The hybrid Nicotiana mustica $\times N$. paniculata shows at times the flower coloration of $N$. texana, a foreign subspecies of
N. rustica. Other properties which in the hybrids are developed to a greater degree than in the parent forms are, for example, the greater stickiness of several hybrids of Nicotiana ( $N$. rustica $\times N$. paniculata); the apparently greater abundance of honey in the hybrid of $N$. mustica $\times N$. paniculata; the stronger of the nauseating odor of the hybrids of Melandryum viscosum; and, according to Kuntze, the alleged much larger quantity of quinine (?) in the hybrids of Cinchona.

In later generations the offspring of the hybrids show still further variations from the properties of the parent species.

\section{Third Proposition.}

Hybrids between different races and species are, as a rule, differentiated from specimens of a pure race by their vegetative power. Hybrids between widely separated species are frequently very weak, especially when young, so that the raising of the seedlings is rarely successful. Hybrids between more closely related species and races are, on the other hand, uncommonly luaruriant and strong, these qualities mostly showing themselves in size, quickness of growth, early blooming, lusuriance of bloom, longer duration of life, great power of reproduction, exceptional size of some particular organs, and in analogous pecu liarities.

In support of this proposition it will be necessary to refer to several examples: Delicate seedlings, it is stated, follow from the crossing of Nymphoa alba with foreign species, Hibiscus, Rhododendron rhodora with other species, $R$. sinenses with Eurhodendren, Convolvulus, hybrids resulting from species of Salix where a species and a hybrid or two hybrids are crossed, Crinum and Narcissus. The fact that embryo plants from the fertilized seeds of hybrids are delicate and difficult to raise is, moreover, frequently noted. Dwarfed growth is seldom noted in hybrids, except in some of the hybrids of Nicotiana, especially $N$. quadrivalio $\times N$. tabacum macrophylla. Giant growth is, on the other hand, more frequent, as in $L y$ cium, Datura, Isoloma, Mirabilis. In size, the hybrids usually exceed both parent species, or are of a height that is the average of the heights of the parents, as in many hybrids of Nicotiana, Verbascum, Digitalis. Development often proceeds with striking rapidity. Klotzsch emphasizes the rapidity of growth of his hybrids of Ulmus, Alnus, Quercus, and Pinus. They often flower earlier than the parent species, as in Papaver dubium $X$ $P$. somniferum; in many Dianthus hybrids (Focke's cross, $D$. arenarius + $\times D$. plumarius $\delta$, showed no inclination to flower earlier than the parents); Rhododendron arboreum $\times$ Rh. catawbiense, Lycium, Nicotiana rustica $\times N$. paniculata, Digitalis, Wichura's sixfold Salix-hybrid, Gladiolus, Hippeastrum vittatum $\times$ $H$. regince, and so forth, and particularly many hybrids of Verbascum. On the other hand, there are also several hybrids which do not flower at all or only after a long time, as in the genera Cereus and Rhododendron. Of the earlier ripening of seeds unconnected with earlier flowering, I know, at present of but one example, in Nuphar. Very frequently, an extraordinary wealth of bloom has been noticed, as in Capsella, Helianthemum, Tropcolum passiflora, Begonia, Rhododendron, Nicotiana $(N$. rustica $\times N$. paniculata, $N$. glutinosa $\times N$. tabacum, and others) ; Verbascum, Digitalis, many Gesneracea, Mirabilis, and Cyripedium. The flowers are very frequently larger in hybrids. In the crossing of 
two species whose flowers are of different size, those of the hybrid are frequently of the same size or approximate the size of the bloom of the species having the larger flowers. Examples of uncommonly large flowers are seen in Dianthus arenarius $\times D$. superbus, Rubus casius $\times R$. bellardii, hybrids of Rosa gallica, Begonia boliviensis and Isoloma tydoum.

A high. vegetative power is very common in hybrids, as in Nymphrea, Rubus ccesius, Nicotiana suaveolens $X$ $N$. latissima, Linaria striata $\times L$. vulgaris and Potamogeton. A greater duration of life has been noted in connection with several hybrids of Nicotiana and Digitalis. An increased resistance to cold has been noted especially in Nicotiana suaveolens $\times N$. tabacum latiss.; while, on the other hand, Salix viminalis $\times S$. purpurea is more sensitive to cold than either parent species.

These facts point in part to an apparent lessened vitality of hybrids in consequence of their abnormal mode of production; and in part in some instances to an extraordinary vegetative power. The cause of this last phenomenon, which is observed less frequently than lessened vitality, has been in some degree only recently understood. Noteworthy experiments of Knight, Lecoq, and others have been published, but it has been through the painstaking researches of Charles Darwin that the ease with which a cross between different individuals and races of one and the same species is effected was first clearly explained. The increase of the vegetative power in hybrids is clearly a phenomenon that closely corresponds with the peculiar conditions of hybrid production, and needs not a special explanation. It was at first thought that lessened fertility was compensated for by greater vegetative luxuriance, an hypothesis that Gärtner has shown to be untenable, as is evident by the fact that many of the most fertile hybrids (Durata, Mirabilis) are also notable for the largest growth.

\section{Partial or Complete Sterility of Hybrids.}

Subnormal fertility of hybrids, especially as regards the pollen, has long been recognized as one of the most important criteria of hybrids. It seems, however, that this character like intermediateness has been an almost unbridled conception and hence greatly overvalued as a distinguishing feature. Focke in his summary gives us a wealth of facts in this connection:

\section{Fourth Proposition.}

Hybrids between different species show in their anthers $a$ smaller number of normal pollen-grains and a smaller number of normal seed than in plants of pure descent. Frequently they produce neither pollen nor seed. In hybridization between nearly related races this weakening of the power of searual reproduction is not present. The flowers of sterile or nearly sterile hybrids usually remain fresh for a long time.

No property of hybrids has attracted so much attention as the lessening of the ability of sexual reproduction. Kölreuter believes that this peculiarity permits a sharp border-line to be drawn between species and varieties. Since then many botanists have accepted the same view, and lately B. Naudin, Decaisne, and Caspary have adopted it in a more or less modified form. Knight and Klotzsch, and before them Godron, hold that the pollen of hybrids is entirely impotent, which contention had already been disproved by Kölreuter's accurate researches. Kölreuter is accredited with the promulgation of the doctrine of complete sterility of hybrids, but this erroneous charge is to be explained only through an ignorance or misunderstanding of the Latin texts: Kölreuter does not speak of complete sterility, but only of a lessened fertility, as a universal property of hybrids.

In different plant genera the fertility of hybrids is very varied. Fertility is observed in a very low degree in the hybrids Papaver, Viola, Verbascum, and Digitalis; it is more common in Anemone, Nicotiana, Mentha, Crinum, Cucurbitacece, and Passifloracece; and it is more common than sterility in Aquilegia, Dianthus, Pelargonium, Geum, Epilobium, Fuschia, Cotyledon, Begonia, Cirsium, Erica, Rhododendron, Calceolaria, Quercus, Salix, Gladiolus, Cypripedium, and Hippeastrum. In the genera Vitis, Prunus, Fragaria, and Pirus, hybrids of closely related species are used as seed-bearing plants; and in Cereus the hybrids of widely separated species show undiminished fertility.

The sterility of hybrids is expressed at times by their showing no inclination to flower, which peculiarity has been noticed especially in several hybrids of Rhododendron, Epilobium, Cereus, and Hymenocallis; but these are exceptions, inasmuch as hybrids usually flower more abundantly and earlier than true species.

In hybrids with unisexual flowers the male flowers fall off when in the bud, as in Cucurbitacece and Begonia (hybrids of $B$. froebeli A. DC.). In bisexual flowers the stamens are stunted, as noted in several hybrids of Pelargonium and Digitalis (D. lutea $\times D$. purpurea $f$. tubiflora Lindl.). The most common sequel of hybrid production is a deficient development of the pollen-grains in hybrid plants. Commonly the anthers of hybrids are sterile and do not contain any pollen; or they are small and do not open. Such deficiency of pollen is noted in Rubus idceus $\times R$. odoratus, Ribes aureum $\times$ $R$. sanguineum, and Alopecurus geniculatus $\times$ A. pratensis. In other cases the stamens produce small powdery grains which do not swell with moisture, which are of varying size and shape, and with which are usually mixed a few single, well-formed, embryo-forming pollen grains. The number of normal grains is, however, frequently larger, and comprises 10,20 , or more per cent of the total number. Large, rough grains which swell with moisture, together with small well-formed grains, are present often in greater or less number among the stunted grains. In hybrids of closely related species, as in Melandryum album $\times M$. rubrum, but little irregularity is usually found in the form of the pollen-grains. In one hybrid, Sinningia, the pollen was better in the second year of flowering than in the first.

In the hybrids of unquestionably different species a normal formation of the stamens is seldom met with. Assertions in support of this still need confirmation, in part, therefore I refer to Nymphoa lotus $\times N$. rubra, Begonia rubrovenia $\times$ B. xanthina, Isoloma tydoum $\times$ I. sciadocalyx $\times$ Salix purpurea $\times$ S. repens; pollen grains which are all of nearly the same form are found in Salix aurita, and $S$. caprea and $S$. viminalis $\times S$. repens.

On the other hand, a deficient development of stamens appears less frequently in race crossings. Possibly, fur- 
ther research will show that it actually appears more often. The only two examples that I know are in my Anagallis cross-breeds. It is doubtful whether Raphanus sativus and $R$. raphanistrum should be considered as representing species or races. It seems, however, that some individual hybrids of closely related species are entirely sterile, as in Capsella rubella $\times C$. bursa pastoris, Viola alba $\times \nabla$. scotophylla, Papaver dubium $\times$ $P$. rhoeas.

Fertility of the female organs is not, as a rule, so much weakened in hybrids as is that of the male organs. It is, however, usually impaired to a great degree. Many hybrids never develop fruit. Assertions as to the absolute sterility of hybrids can not, however, be advanced without manifold researches. From the crossing Rubus casius $\times R$. idaus one sees many thousand flowers remain sterile and only here and there individuals produce fruit. See also Digitalis lutea $\times D$. purpurea, Lobelia fulgens $\times L$. syphilitica, Crinum capense $\times C$. scabrum. A morphologically recognizable imperfection of the ovule has heretofore rarely been seen, unless by Bornet in Cistus. To obtain conclusive information as to the female fertility of a hybrid, the stigma should be fertilized with pollen from the parent species, which fertilization universally brings forth better fruit than the pollen of the hybrid which is weakened in its fertilizing power. In some cases hybrids having the pollen which has a subnormal potency produce normal fruit with parental pollen, as in Luffa.

Several hybrids drop their unwithered flowers with fully formed calyx and stamens, as in Ribes, Nicotiana rustica $\times N$. paniculata and other hybrid Nicotianas.

As a rule, the corolla withers in a normal manner after a longer existence than in the parent species, or it will be thrown off as in the parent species; but following this there is no setting of fruit or a setting of only poor fruit. In many cases the fruit while externally well formed is seedless. In many other cases the fruit is set, but in smaller number and with fewer seeds than in the parent species. In hybrids of very closely related species the number of seeds appears to be somewhat less than in the parents. Examples of this, according to Gärtner, are Melandryum album $\times M$. rubrum, and Lobelia cardinalis $X L$. fulgens. It is also true in race-crossings of Verbascum.

Hybrids of essentially different species seldom show an undiminished fertility. However, no striking lessening of fertility has been observed in Brassica napus $X$ $B$. oleracea, Dianthus chinensis $\times D$. plumarius sibiricus, Pelargonium pinnatum $\times P$. hirsutum, Abutilon, Medicago, several Cereus and Begonias, Hieracium auranticum $\times H$. echioides, Nicotiana alata $\times N$. langsdorffi, several hybrids of Erica, Calceolaria, Isoloma, Veronica, and several Orchidaceæ. Also, among many wild-growing hybrids one finds fruits and seeds in great quantities, as in many Rosa, Epilobias, Fuchsias, Cirsiei, Hieraciei, Salices, Lobelia, and so forth. In such cases, therefore, it is not sufficient to ascertain whether the plants in question are primary hybrids or whether, as is usually the case, they belong to later generations or have arisen from back-crossings.

In order to produce seeds or to obtain a luxuriant progeny some hybrid plants require fertilization with the pollen of others, as in hybrids of Cistus, Begonia, Gladiolus, and Hippeastrum.

In many hybrid plants only the first flowers produce seeds, as in Aquilegia, Dianthus, Silene, Lavateria Thuringiaca $\times T$. pseudolbia, and Rubus foliosus $\times R$. sprengelii. In other cases the first flowerings are usually sterile while the later flowerings are frequently fertile, as in Datura, Nicotiana rustica $\times N$. paniculata, $N$. rustica $\times N$. quadrivalvis, and Mirabilis. In long-lived plants, the flowers in general are sterile during the first year, while later, when the plant has reached a definite age, they produce fruit. This is noted in Rubus idous $\times R$. cosius, $R$. bellardii $\times R$. casius, Calceolaria integrifolia $\times C$. plantaginea, and Crinum capense $\times C$. scabrum.

The fertility of the ovule is, as a rule, diminished to a somewhat less extent than the fertility of the pollen, but there are some known examples of an opposite character, as in Nymphoa lotus $\times N$. rubra, Ciconium $\times$ Dibrachya in the genus Pelargonium, Lobelia fulgens $\times L$. syphititica, Verbascum thapsiforme $\times V$. nigrum, Narcissus montanus, and so forth. These are certainly only of an occasional occurrence.

The long persistence of the blossoms (especially those with stamens) in many sterile hybrids corresponds with the longer duration of unfertilized or incompletely fertilized flowers. Frequently the fruit of sterile hybrids, especially after fertilization with the pollen of the parents, develops more or less strongly without producing any seed, or producing only imperfect seeds. Especially well-developed but seedless fruits are found in the Cactaceæ, Passifolaceæ, Cucurbitaceæ, and Orchidaceæ. Gärtner has studied carefully these phenomena, but in the study of hybrids they hardly possess a great value. Apart from this they furnish an important demonstration of the correctness of the principle that the normal development of the pericarp follows upon the stimulation when the germinating pollen is discharged on the stigma, but which is, nevertheless, entirely independent of the ripening of the egg cells and the development of the embryo and the seeds.

The rule in general is that hybrids of closely related races are on an average more fertile than those of definitely separated species. The rule can also be stated, as shown above, that closely related species can more easily produce hybrids than widely separated species. Both rules, however, have only conditional values, for if it should be concluded from this that the more easily hybrids are produced the more fertile they are, one would fall into error. There is no known or traceable connection between the ease of production and fertility of the hybrids.

From the teleological standpoint the sterility of hybrids was formerly considered the means whereby species were kept separate. Just what advantage such separation is (unless it be for the conveniences of the systematists) was never demonstrated. On the other hand, it may now be asked whether or not the genesis and differentiation of species are not brought about by the lessened fertility of mongrels between well-marked races of the parent type. The notable similarity between illegitimates and hybrid offspring do not offer a basis for further investigations of the causes of sterility. A better explanation is probably afforded by the hybrids of $E q u i$ 
setum and Musci, in which the production of sexual spores is as defective as is the production of pollen grains in the hybrids of Aerogams. The obstacle to the regular propagation of hybrids appears consequently to lie in the development of those individual cells which have the power to propagate the type of the parent form, and these particular cells may or may not have the power of sexual reproduction. At all events, more evidence must be gathered before such a conception of a proposition of such great biological importance is justifiable. As an hypothesis this gives no explanation, but it may prepare the way for the understandng of the conditions already noted, since it unites under one heading a number of different yet manifestly analogous phenomena in the animal and vegetable kingdoms.

\section{Fufth Proposition.}

Malformation and odd forms, espevially of the flowers, are in hybrid plants much more common than in specimens of plants of pure descent. As in Papaver, Dianthus, Pelargonium, Nicotiana, Digitalis, double flowers also appear to be produced with especial ease in hybrids.

The Descendants of Hybrids.-Hybrid plants are more easily and more successfully fertilized by the pollen of the parent species than by their own pollen. Exceptions to this rule are rarely seen (as for instance in Hieracium echioides $\times H$. aurantiacum), but sufficient experiments in this direction have not yet been made. By their own pollen is understood the pollen of hybrids resulting from the crossing of the same species, and not only that of the identical specimens themselves. If hybrid plants grow in the neighborhood of their parent species they must frequently be fertilized by these species; and in this case many intermediate forms between the hybrid and the parents will appear in their progeny. It has never been determined whether or not fertilization of the parents could take place by the pollen of the hybrid. The common statement, that the progeny of a hybrid are very variable, is therefore of but little value. Occasionally also a hybrid is more easily fertilized by the pollen of a third species than by its own as in Nicotiana rustica $\times N$. paniculata and Linaria purpurea $\times L$. genistafolia.

Progeny of Hybrids Fertilized by their Own Pollen. $(\mathrm{A} \times \mathrm{B})$ o $\times(\mathrm{A} \times \mathrm{B})$ o.-(1) If fertile hybrids are protected from pollenization by the parent plants or by plants of a different species, one will obtain hybrid plants of a second generation. It is my opinion that the progeny of hybrids exhibit marked differences in the duration of life. In long-lived plants the blending and stronger union of the two types united in the hybrid is frequently more complete, so that the progeny inherit the characteristics of this new intermediate type. The progeny of annual or biennial hybrid plants are, as a rule, particularly variable and rich in different forms, as in Pisum, Phaseolus, Lactuca, Tragopogon, Datura, Nicotiana alata $\times N$. langsdorffi, and so forth. Exceptions are found in Brassica, Oenothera, Nicotiana rustica $X$ N. paniculata, and Verbascum austriacum $\times \nabla$. nigrum. The progeny of perennial plants behave in general in a similar way, but the instances in which the intermediate type remains constant appear to be the more frequent. Many of the hybrids often breed, moderately, true, as in Aquilegia, Dianthus, Lavatera, Geum, Cereus, Begonia, Cirsium, Hieracium, Primula, Linara, Veronica,
Lamium, and Hippeastrum. The progeny of hybrid shrubs and trees are in the majority of cases moderately stable, as in Esculus, Amygdalus, Prunus, Erica, Quercus, and Salix; the progeny of many Fuschiae and Calceolario are constant. Some Rhododendron hybrids breed true and a portion variably. The progeny of the hybrids of Vitis, Pirus, and Cratagus appear to be very variable.

2. The different forms in which many primary hybrids appear are usually not stable in their offspring. In Dianthus the less-frequent forms ("Ausnahmetypen," according to Gärtner) usually revert to the normal hybrid form. Mendel found that the different primary forms of the Hieracium hybrids breed true.

3. C. F. v. Gärtner and other botanists have advanced the proposition that the progeny of hybrids become weaker and less fertile from generation to generation. It is true that their vegetative power, which at first is increased, is progressively decreased by self-fertilization. Gärtner's researches were, moreover, instituted on a very small scale, so that not only very close inbreeding but also the many circumstances which cause deterioration in garden-plants of which only a few specimens are cultivated influenced his hybrids. Gärtner himself noticed exceptions in Aquilegia, Dianthus barbatus $\times D$. chinenss, and $D$. armeria. $\times D$. deltoides. Hybrids of nearly related species are often grown perenially with ease, as in Brassica, Melandryum, Medicago, Petunia. Many gardeners assert with great positiveness that many hybrids can be propagated by means of seeds through many generations, as in Lychnis, Erica, Primula auricula $\times P$. hirsuta, and Datura.* Many observations on wild plants seem to confirm these views. The theory has also been advanced that the fertility of hybrids is increased in later generations. It does not appear that such a rule can have a universal validity. It is much nearer the truth that many times fertile hybrids appear and that they can easily increase under favorable environment because of increased fertility. Fertile offspring of hybrids are, in fact, often products of back-crossings.

4. Complete reversions to the parent forms without influence of the parental pollen arise, except in rare instances, only in hybrids of nearly related races. In such hybrids true reversion appears only in a small number of plants, as in Phaseolus.

5. From the variable progeny of fertile hybrids several dominant types are often produced in three to four generations. If these new types are protected from crossing they tend to become constant. Scientific researches which confirm these statements have been carried out in but small numbers, especially by Lecoq in Mirabilis, by Godron in Linaria and particularly in Datura. Gardeners have produced many new races with wellmarked characteristics by crossing different species, and many permanent wild intermediate forms have probably originated in this way, as for example, Brassica, Lychnis, Zinnia, Primula, Petunia, Nicotiana commutata, Pentstemon, Mentha, and Lamium. The new types of hybrid progeny depart frequently in individual properties from

* "Botanists say that species so produced" (i. e., hybrids) "revert to either of their parents in the third or fourth generation, or become sterile altogether. This is plausible enough in theory, in the closet, but will not do in the potting bench." Beaton, quoted by Loudon, Arbeit II, p. 944 . 
both parent forms. My Nicotiana $\times N$. paniculata had in the second and third generations mostly much narrower leaves than in the parent species.

6. The sterility and inconstancy of the offspring of hybrids has often misled botanists into conclusions which are not supported by experience. As may be seen by the facts already set forth, it is absolutely incorrect if it is concluded that all hybrids must necessarily die out quickly because of the many and various properties which are combined in them. The variable forms resulting from a crossing are the material from which not only gardeners produce their new varieties, but which are also biologically valuable in that they furnish new species in the economy of nature.

(c) Back-crossings of Hybrids with Parent Species

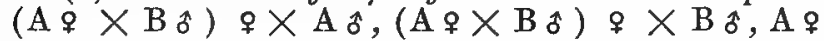
$X(\mathrm{~A} \times \mathrm{B}) \hat{\delta}$. As long as great stress was laid on the rôle which the pollen of the seed-parent species played in the production of a hybrid a careful distinction was made that advancing hybrid forms approached the male parent species and reverting hybrid forms approached the female species. These distinctions are, however, according to the mass of recent experiments, of very secondary or of no significance.

On fertilization of a hybrid with parental pollen there appear, as a rule, a moderately variable progeny. Intermediate forms between the hybrid and the parent are the most numerous and most fertile. With these are a smaller number of individuals which are similar to the primary hybrid or to' the parent species, and both kinds are usually of lessened fertility.

The three-fourths hybrid $(\mathrm{A} \times \mathrm{B})$ i $\times \mathrm{A}$ \& are often moderately fertile with their own pollen and seem to produce stable races more readily than the primary hybrid, as in Eigilops speltaformis. Gärtner noted many times that in later generations of three-fourths hybrids the pollen was nearer normal and the fertility greater, as in Dianthus (chinensis $\times$ barbatus) $\times D$. barbatus, and also in other three-fourths hybrids of Dianthus, Lavatera, and Nicotiana.

If the three-fourths hybrid $(\mathrm{A} \times \mathrm{B})$ \& $\times \mathrm{A}$ of be fertilized with the pollen of $A$, there will be produced a seven-eighths hybrid or the third hybrid generation which, as a rule, is very similar to the parent species represented as seven-eighths of the product, but which, in individual specimens, still shows material differences in form and fertility. The last trace of the one original parent species is obliterated in the fourth, fifth, or even in the sixth hybrid generation.

Kölreuter and Gärtner have effected the transformation from one parent species to the other in many instances. They found that for the transformation to be complete three to six generations are required, usually four to five. Manifestly, the greater or lesser duration of the period of transformation depends in part on collateral conditions. Godron found that Melandryum album $\times M$. rubrum fertilized with its own pollen reverts in the second generation to the parent species, while Gärtner considered three to four generations necessary to carry one species over to the other through fertilization with parental pollen.

In general, the products of the fertilization of one parent species with hybrid pollen, as $\mathrm{A}$ \% $\times(\mathrm{A} \times \mathrm{B})$ o, are similar to those of the reverse fertilization, but observers agree that the variety of forms is greater if the hybrid is used as the male factor, as in Dianthus and Salix.

As in the direct progeny, so also in back-crossings of hybrids, new properties frequently appear which are absent in the present forms, but which are often found in related species or races.

Hybrids of Several Species. Triple Hybrids.-Kölreuter, during the first year of his research, succeeded in combining three entirely different Nicotiana species in one hybrid form. The only formulas according to which such a combination can be made are: $(\mathrm{A} \times \mathrm{B})$ \& $\times$

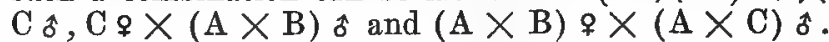
In the genera Dianthus, Pelargonium, Begonia, Rhododendron, Nicotiana, Achimenes, Calceolaria, Salix, Hippeastrum, Gladiolus, and several others, many such combinations have been produced without especial difficulty. Differentiation must be made between combinations of three entirely different species, and combinations in which two or all three of the factors are closely related. There are several manifestly different species which in hybridization with one another act almost like races of the same species, as Melandryum album and $M$. rubrum; Vitis vinifera, V. cordifolia, V. astivalis and V. labrusca; Lobelia fulgens, L. splendens and $L$. cardinalis; Rhododendron ponticum, $R$. arboreum and $R$. catawbiense; Rhododendron flavum, $R$. viscosum, $R$. nudiflorum and $R$. calendulaceum; Berberis aquifolium and nearly related species.

Hybrids produced by crossing the hybrids of two species of these groups with a third species of the same genus can as little be considered true triple hybrids as hybrids of three of the narrow groups belonging to the Vitis, Lobelia, and Rhododendron species. True triple hybrids formed from three essentially separate species usually produce a moderate variety of forms, especially if the male parent is a hybrid. On the other hand, in the combination which is easiest to produce, and which is formed on the formula $(\mathrm{A} \times \mathrm{B})$ o $\times \mathrm{C}$ s , the type of $\mathrm{C}$ usually predominates, as in Nicotiana ( $N$. rustica $\times N$. paniculata $) \& \times N$. langsdorffi $\delta$, Achimenes. $(A$. grandiflora $\times A$. candida) $\$ \times A$. longiflora $\hat{\sigma}$, and several of the Gesneracea.

The hybrids of Erica when crossed produce as uniform a progeny as do the pure species. Several Salix hybrids behave in a similar manner.

Triple hybrids in many genera (Pelargonium, Begonia, Rhododendron, Achimenes, Isoloma, Cypripedium, Gladiolus) are for these reasons very valuable to gardeners. If they produce seed their progeny are very unstable.

Hybrids of Four to Six Species.-If the hybrids between very nearly related species ( $\nabla$ itis, Rhododendron, and so forth) are not considered, hybrids from four or more parent forms are moderately rare. They are found especially in the genera Dianthus, Pelargonium, Begonia, Rhododendron, Nicotiana, Salix, Hippeastrum, and Gladiolus. The artificial combination of different species in a single hybrid form was practised to the widest extent by Wichura, who has combined in Salix six species.

Hybrids of Combined Hybrid Offspring.-In several genera (Pelargonium, Fuchsia, Begonia, Rosa, 
Erica, Rhododendron, Achimenes, Calceolaria, Gladiolus, and Hippeastrum) gardeners have crossed the species intentionally and unintentionally in the greatest variety of ways, and from the forms obtained they have used those most desirable for further cultivation. The offspring of these complicated hybridization products are naturally almost always very varied. On the other hand, there are exceptions to this rule. Sweet particularly emphasizes the fact that the same hybrid form is obtained from the crossings of several complex Pelargonium hybrids. Such constant complex Pelargonium hybrids are, according to him, $P$. involucratum $\times P$. ignescens, and $P$. mostynce $\times P$. ignescens. It has already been mentioned that Erica and several Salix hybrids on crossing furnish offespring of constant form.

Cross-breed's and Hybrids.--According to a dictum hybrids of two different varieties of one species are designated as cross-breeds, and hybrids of two different species as hybrids. As the term varieties is vague it is necessary at this point to remember that only varieties which breed true, as well as races, or subspecies, can with certainty transmit in some degree their properties. Unstable breeds which are designated varieties are useless in the study of hybridization.

Many writers have taken great pains to discover a sharp distinction between cross-breeds and hybrids. They hold to the expectation that by researches in hybridization a border line between species and subspecies will be fixed. Gärtner, who in many places in his works has declared that the conditions of the hybrids demonstrate clearly the specific differences or similarities of the parent-forms, would soon retract if he attempted to develop any connection or continuity by the literature of variety hybrids. Herbert and Naudin have through many researches arrived at the conviction that it is impossible to draw a sharp borderline between crosses and hybrids; nevertheless, later botanists have always sought such a fixed difference.

The following propositions have been formulated:

1. The pollen of a cross-breed is normal; there are more or less numerous deformed pollen grains in a hybrid.

2. The fertility of a cross-breed is normal; that of a hybrid is distinctly subnormal.

3. Hybrids of two species having differently colored flowers bear flowers of modified coloring. Plants with irregularly dappled flowers are produced from the crossing of varieties. They behave similarly in regard to coloring, marking, and formation of fruit, and other properties.

4. Cross-breeds have a decided inclination in later generations to revert entirely to the parent forms.

These four propositions are in general correct, but give very little help to a final decision in doubtful cases. The hybrids of the red and blue Anagallis arvensis must according to the pollen be considered a hybrid, but according to the production of bicolored flowers, a crossbreed. Datura hybrids, which are manifestly characteristic hybrids in other ways, readily revert completely to the parent species. Hybrids whose fertility is apparently in no way weakened have already been specified. The rule can, therefore, be set forth that hybrids of very nearly related races usually show the properties attrib- uted to cross-breeds, but it is another matter to establish a sharp boundary line between race-cross-breeds and species-hybrids.

Several other properties of cross-breeds have been added by which they may be distinguished from specieshybrids. Gärtner has maintained that cross-breeds of a similar origin will be very unlike one another even in the first generation, while hybrids of the first generation will be of the same form. This assertion, which has been repeated by others, is entirely unjustified. The multiplicity of forms of the species-hybrids of Abutilon, Passiflora, Hieracium, and so forth has already been pointed out and, on the other hand, race-cross-breeds of the first generation are usually as similarly formed as true hybrids. Again, it is often maintained that the varieties of one and the same species if crossed with another species produce the same hybrid forms. Gärtner especially has emphasized this alleged behavior of "varieties," although he must have known that Kölreuter had already noted the transmission of flower-coloring in races of Mirabilis, Dianthus, and Verbascum, the flower-filling (Bluithenfüllung) of Aquilegia and Dianthus, and the form and leaf-shape of races of Nicotiana tabacum and Hibiscus. The white-blooming Datura ferox and $D$. strammonium typ. (a white-flowered form) with the smooth-fruited race (var. bertolonii) of the same species forms a blueflowered hybrid. Nymphaa lotus $\times N$. rubra is different from $N$. lotus $\times N$. dentata. It is unquestionable that properties of races and so-called varieties which are hereditary in pure-breeding are also transmitted to their hybrid offspring. It is self-evident that forms whose normal offspring behave in an unstable fashion will also produce polymorphous hybrids and that the unstable characteristics of varieties will entirely disappear in the products of the hybridization of pure species.

The facts in short are as follows: The nearer the morphological and systematic relationships of the parent forms the less does the procreative power of the hybrid depart from the normal. The farther the parent forms are from one another the more commonly is the fertility of the hybrid weakened. Exceptions, however, are not infrequent.

The nearer the parent forms are related to one another, the more frequently does the offspring of hybrids show reversion to the parent forms.

Hybrids of nearly related parent-forms show in their fruits the characteristic properties of the parents unblended and side by side, but in hybrids of very different parent forms this is seldom seen.

The most asymmetrically variegated flowers (Mirabilis, Camellia, Mimulus, Petunia and so forth) have, moreover, originated from the offspring of hybrids.

The propositions of Focke, although published in 1881, are not subject to modifications in principles even at the present time. Much literature on the subject of the sterility of hybrids might be quoted and some references might be made to extensions and additions of a more or less important character to the data and propositions set forth, but this seems needless for the purposes of this chapter and this research. 
5. Instability and Mendelian Inheritance of Hybrids and Mutants.

Focke's data show that instability is usually quite marked in hybrids, especially in hybrids that are the offspring of a number of species and of crossed hybrids. As has long been known, there is no characteristic of hybrids that has been found so undesirable to the plantbreeder as the tendency to vary in succeeding generations, especially in the direction of reversion to one or the other parent. The partial or complete absence of fixity following the first generation was merely a matter of speculation until the contributions of Mendel (1865 to $18 \% 0$ ), which, however, remained practically unnoticed until 1900. Mendel's discoveries and his conceptions of unit characters and their mode of inheritance have offered in an important but restricted measure explanations for the common failure of many plant and animal breeders to anticipate with any degree of certainty several results that may under certain conditions be expected by crossing and in successive generations of the offspring, especially in the case of certain kinds of parents. Mendel recognized that hybrids, as a rule, are not exactly intermediate between the parent species, and that while with some of the more striking characters intermediateness is seen, with others one of the parental characters is so preponderant that it is difficult or impassible to detect the other in the hybrid. He was the fir'st to show that in order to be able to predict with sureness certain characters of the hybrid it is essential to start with pure stock; study each character separately as an individual unit; group the characters in contrasting pairs, one of which pair tends to be transmitted entirely or almost unchanged (dominant character), while the other tends to lessened development (recessive character) or to entirely disappear, but to reappear unchanged in their progeny; look upon each pair as being independent of the others in heritability; and regard each generation of offspring as a distinct entity, but in association with the characters of preceding and succeeding generations. Mendel found that the hybrids in their various macroscopical characters, singly and collectively, either closely resemble or are almost identical with one or the other parent species, or are intermediate between the parents; that the hybrid may exhibit greater luxuriance of growth; that the hybrid seeds are often more spotted (the spots even coalescing in patches) than in the parents; that the dominant character may be parental or hybrid in character and, if the latter, maintain the same behavior in the second generation; that the hybrids resulting from reciprocal crosses are formed alike and exhibit no appreciable difference in subsequent development; and that in the first and succeeding generations bred from seeds of hybrids there appear in the offspring both dominant and recessive characters of contrasting pairs in definite average or mathematical proportions. The hybrids of varieties were found to exhibit peculiarities like those of species, but with greater variability of form and greater tendency to reversion to the original types. Mendel's statement that the results of reciprocal crossing are identical must be taken as having a very limited application, and then only in a very gross sense.

The Mendelian doctrine has found a wide though limited application in the explanation of the various phenomena of heredity, and it seems probable that when all or a large number of parental and hybrid characters of given parents and offspring are studied it will be found to be applicable to a fewer number of characters than is generally believed and of little importance in explaining the phenomena of heredity under natural conditions. In fact, the Mendelian doctrine deals with inheritance and not with origin of characters and it absolutely fails in so far as the possibility of the origination of new characters is concerned, and hence is useless in accounting for the occurrence of characters in the hybrid excepting by dominance, recession, and redistribution of preëxistent ancestral characters. Mendel, while recognizing the commonness of intermediateness of parental characters in the hybrid, made no attempt to apply or extend the doctrine to the explanation of blended inheritance. In fact, he recognized that his doctrine was not applicable to characters that blend. In recent years several investigators have suggested a Mendelian interpretation of blended inheritance. Nilsson-Ehle (Lund's Universitets Arsskrift, 1909, v, 2) holds the view that such form of inheritance is really a segregated inheritance due to the association of several independent but similar units or factors which yield a pseudo or actual blending.

The general assumption by pro-Mendelianists that unit characters are constant and changeless has been shown by Castle (American Breeder's Magazine, 1912, III, 270; American Naturalist, 1912, XLVI, 352) to be without warrant, and that, to the contrary, unit characters are variable and modifiable. It is well known that a hybrid has characters that may or may not be intermediate, and that may even be peculiar to itself, and that it is the sum of such characters that gives hybrids the characters of elementary new species, of which an illustration will be found in our histologic and microscopic study of Ipomoea sloteri in Part II, Chapter II. Plasticity of characters as regards degree of development, fixity, and genesis has long been recognized as one of the most essential fundamental properties of living matter. Development of various characters exceeding that of the parents has been frequently observed among both hybrids and mutants. Increased virulence of succeeding generations of bacteria was pointed out by Pasteur, Chamberland, Roux, and many others. Loss of characters is of too common an occurrence to demand special notice. Modifiability, genesis of new characters, and heritability of both modified and new characters have been recorded by a number of investigators.

Massini (Archiv f. Hygiene, 1907, LXI, 250) cultivated a strain of Bacteria coli mutabile that gave rise through successive partial mutations to colonies that fermented lactose and (in the course of successive genera- 
tions) this property became fixed and the race bred true. Similar phenomena have been recorded by ather experimenters. Permanent color changes were induced by Wolf (Zeit. f. ind. Abst. u. Vererb., 1909, II, 90) in Bacillus prodigiosus by propagation in culture media containing small amounts of potassium and other salts. Rosenow's (Jour. Infect. Dis., 1914, XIV, 1) investigations show mutations and transformations of the streptococcus-pneumococcus group by means of environmental conditions. Thiele and Embleton (Zeit. f. Immunitätsforsch $u$. exper. Ther., 1913, xIx, 643) brought about such morphological and physiological changes as to transform one species of bacillus into another. Revis (Proc. Roy. Soc., B, 1913, Lxxxvi, 373) from an original typical culture of Bacillus coli from a single cell produced two strains one of which appeared slightly modified but which could not be further altered, and another which underwent profound and increasing change, resulting in an organism entirely different from the original, the strain remaining of a permanent character. Jordon (Proc. Nat. Acad. Sci., 1915, I, 160) in cultures of Bacillus coli obtained mutation that "seems to fulfil the requirements $(a)$ of appearing suddenly without intermediate stages, $(b)$ of being irreversible, at least for three years and for some hundreds of testtube generations, $(c)$ of comprising change in two characters (saccharase- and raffinose-fermenting power), and $(d)$ of not involving all the cells of the parent strain." Henri (Compt. rend. Acad. Sci., 1914, CLVIII, 1032) found that metabolism was so affected in Bacillus anthracis by ultra-violet rays as to cause marked mutations. Sohmankewitsch (Zeit. f. wiss. Zool., 1875, XXV, 103; 1877, XxIX, 429), in experiments with various crustaceæ to show effects of environment, found in Daphnia and Branchipus that changes in salinity brought about marked functional and morphological alteration of charaaters commonly regarded as being specific. Woltereck (Verh. deutsch. zool. Gesellsch., 1909, 110) recorded variations in Daphnia that are heritable, and states that by selection a modified race can be bred. Literature such as the foregoing is plentiful, both as to plant and animal life.

The Mendelian doctrine is one of fixity and constancy of characters which segregate in inheritance-the very antithesis of what must be recognized as one of the most fundamental principles of evolution, i.e., plasticity and adaptability to environmental conditions that permit or lead to the formation of new characters. It is important to note that while the Mendelian doctrine is a scientific fact and of unquestionable value in explaining certain phenomena of inheritance, it is also obvious that it can not be accepted as, and never can be made, a universal principle of heredity, and that the main question pertaining to this doctrine is in regard to the conditions under which it holds good. In a word, it deals with but one of several types of mechanisms of heredity. Considerable misconception has already arisen because of absolutely false ideas that have been promulgated by hybridizers who have selected in their investi- gations only such plants as yield offspring which in their phenomena of inheritance conform to the Mendelian Law, or who have selected only such characters for examination as agree with this law and entirely ignore others which represent non-Mendelian inheritance. It is obvious that in order to obtain safe results for and against any doctrine it is essential that all of the characters, as far as possible, should be recorded and without reference to preconceived theories or hypotheses. Scarcely anything in scientific investigation can be more pernicious than an attempt to make facts fit theory, hypothesis, or doctrine, and to ignore them if they do not. One of the manifest weaknesses of studies of Mendelian phenomena is to be found in an absence of a recognized and wholly satisfactory method of standardization. It is obvious that until such is adopted the extent of applicability of the Mendelian doctrine to the explanation of phenomena of heredity must remain in considerable doubt.

Among the fundamentally important contributions to the study of heredity are those pertaining to mutations by DeVries (Mutation Theory, 1909) and by various subsequent investigators. A large literature has accumulated bearing especially upon Oenothera and certain other genera in which not only mutations but also spontaneous hybridizations have been recorded as being of frequent occurrence. Whether or not the mutants of DeVries and his school are in fact mutants or unquestionable hybrids that have arisen from spontaneous crossing is a warmly debated question. Bartlet (American Naturalist, 1915, XIIX, 129; Botanical Gazette, 1915, LIX, 810) contends that there are Oenothera mutants; that the mutant-ratio can not be explained on Mendelian grounds; that mutation is a distinct process from Mendelian segregation; and that the phenomena exhibited by the mutants Oenothera lamarckiana, $O$. biennis, and $O$. practincola can not ibe attributed to heterozygosis. Gates (The Mutation Factor in Evolution, 1915) holds the view that mutations are not merely manifestations of some type of hereditary behavior, but a process sui generis; that mutation phenomena represent a well-defined type of variability; that mutations are completely inherited in some or all of the offspring; and that cytological evidence is in accord with theoretical requirements and experimental facts in serving to controvert the Mendelian conception that mutation is only Mendelism under another guise.

On the other hand, the hybrid and Mendelian characters of mutants have led many to believe that many mutants are hybrids. Heribert-Nilsson (Zeit. f. Abst. u. Vererb., 1912, vIII, 89) holds that mutants are combinations, i.e., they represent new combinations of Mendelian characters. Renner (Flora, 1914, cVII, 115) also holds that DeVries's mutations are explicable on a Mendelian basis. Davis (Amer. Nat., 1911, XLV, 193; ibid., $1912, \mathrm{XLVI}, 37 \%$ ) found, in studies of the offspring of different species of Oenothera, that in gross morphological characters the hybrids are intermediate between the parents and that some of the hybrids resemble $O$. $l a$ marckiana, the best-known of all mutants. Jeffrey 
(Science, 1914, XxxIX, 488; Bot. Gaz., 1914, LVIII, 322 ; Amer. Nat., 1915, XLIX, 5) asserts that there seems to be absolutely no doubt upon morphological grounds and sterility that the Oenothera mutants are really hybrids. He records that an examination of a large amount of material of recognized wild species of Oenothera led him to the conclusion that spontaneous hybridism is extremely common in the genus; that in general it represents a condition of high genetical impurity; and that in orders such as Rosaceæ and Ornagraceæ there is grading of recognized species and hybrids into each other, having in common the character of partial or complete sterility. Such literature would make volumes.

\section{Genetic Purity in Relation to Intermedi- ateness of the HYBRID.}

It may be held that intermediateness of the hybrids depends upon the existence of purity of the parents and that, as a corollary, absence of intermediateness is diagnastic of parental impurity. It will be noted, however, that while Davis (loc. cit.) with carefully selected, presumably pure stock recorded intermediateness in the hybrid, Jeffrey refers to Oenothera lamarckiana as a hybrid having a similar intermediateness, yet being the offspring of spontaneous hybridism that represents a high degree of genetical impurity. In fact, there is no conclusive evidence in any of the investigations referred to that the parents were pure. The term pure is an arbitrary conception. The only test of purity we have at present is in the constancy of characters of the offspring through successive generations. Nor are purity and typicalness by any means synonymous terms. A typical specimen of a species or hybrid is one having characters which in their sum total are nearest the mean of the species or hybrids, but a typical specimen may be far from being pure inasmuch as there may be latent or undeveloped characters that may not appear except under some peculiar condition. In the investigations of Macfarlane and others quoted by him, the parent species examined may have been typical, yet there is no evidence of purity. Darbishire used for the preparation of the starch only two seeds from crosses of garden varieties of peas - the round pea "Eclipse" and the wrinkled pea "British Queen" (hardy variety) being crossed. The parents referred to in Focke's work may or may not have been pure, but there is no satisfactory evidence in either direction. Mendel was extremely careful to select specimens belonging to groups that possess constant differentiating character's, and in both of his papers he makes notes of only certain selected differentiating characters. He found, as already stated, that the hybrids, as a rule, are not exactly intermediate between their parents, and that while in the case of some of the more striking characters intermediateness is always present, in other cases one of the two parental characters is so preponderant that the corresponding character of the other parent is almost or wholly absent. He also nates in Hieracium hybrids there may be three types, one being almost exactly intermediate, a second nearer to the seed parent, and a third nearer the pollen parent. In all of these instances the parents may have been typical, yet not pure, and in Mendel's experiments they might be regarded as being both typical and pure-pure, because of the constancy of Mendelian inheritance in succeeding generations. But even here purity is questionable. Thus, in the second generation the dominants which breed true to the dominant character are looked upon as being pure, yet they may have latent or undeveloped characters that can be demonstrated only under peculiar conditions. This has been shown by Darbishire (Breeding and the Mendelian Discovery, 912, 218) in crosses of the common albino and the Japanese waltzing mice. In the second generation he found two types of albinos, one to all appearances identical with the pure albinos and the óther with waltzers. When these apparently pure albinos are mated with each other they breed true, but when mated with waltzers they were found to be very different from pure albinos, "for among the offspring of extracted albinos mated with waltzers there appeared pink-eyed and even albino mice, forms which are never produced. when pure albinos are mated with waltzers."

\section{Theoretical Requirements in the Properties of Starches to Conditions in the Hybrid Corresponding to those of Anatomic Char- ACTERS.}

It is evident from the literature quoted that the doctrine of intermediateness of the hybrid and the doctrine of Mendel are expressions of rules that have many exceptions and hence are only of limited applicability. The success of the plant and animal breeder depends upon the elimination of undesirable characters; the redistribution of characters; the variation, modification, and recombination of characters; the development of some particular characters to a degree beyond parental extremes, together with their perpetuation and even further exaggeration in subsequent generations; and the development of new and perpetuation of desirable characters. Neither the doctrine of intermediateness nor the doctrine of Mendel admits of the possibility of generating ideal organisms by crossing and selection; nor are they consistent with the development of parental characters in the hybrid beyond parental extremes; nor are they compatible with the appearance of new characters except upon the untenable assumption of such characters being latent in the parents. Both are doctrines of non-plasticity, yet the most significant phenomenon of successful breeding and the genesis of elementary species is plasticity which is manifested to a pre-eminent degree of importance in development in the offspring of characters beyond the extremes of the parents, new combinations of characters, and the appearance of new characters. No investigations on record have shown more forcefully the utter inadequateness of these doctrines and their limitations than their application to the explanation of the building up of ideal forms and the appearance of elementary species by hybridization and, on the other hand, none has better set forth the great pos- 
sibilities of the breeder than those of Burbank. In referring to the results obtained by crossing and selection in general, he states (New Creations of Plant Life, Harwood, 1912, 216) that " there is no barrier to obtaining fruits of any size, form, or flavor desired, and none to producing plants and flowers of any form, color, or fragrance. All that is needed is a knowledge to guide our efforts in the right direction, undeviating patience, and cultivated eyes to detect variations in values."

If starch characters are heritable they should, in order to meet theoretic requirements, exhibit peculiarities of inheritance corresponding to those observed in gross and microscopic anatomic plant characters. This deduction will be found to have ample justification in the results of this research. Hierein it will be found that the starches of the hybrids frequently exhibit in histologic, polariscopic, and physico-chemic properties some degree of intermediateness between the parents, usually nearer one or the other. In any given hybrid certain of the properties may be exactly or practically exactly intermediate, and other properties may be identical with the corresponding properties of one or the other parent. In many instances one or more of the characters of the hybrid, such as the relative number and the types of compound grains, the degree of fissuration, the regularity or iregularity of the forms of the grains, the characters of the hilum, the distinctness and size of the lamellæ, the polariscopic properties, the temperature of gelatinization, the aniline reactions, and the qualitative and quantitative reactions with the various chemical reagents, were developed or manifested in degrees beyond the parental extremes. Moreover, peculiarities of various kinds were observed at times in the hybrid that were not apparent in either parent. In so far as these results go they are, in general, in entire accord with the experience of the plant and animal breeder and with unquestionable statements of literature.

The doctrine of intermediateness of the microscopic characters as set forth in a preceding section is not warranted by the literature of naked-eye characters and is opposed to the results of the work with starches. This led to supplementary studies of the macroscopic and micrascopic characters of parent- and hybrid-stocks which compose Chapter IX of Part II. It seems clear upon general grounds that if characters of the starch of the hybrid may be intermediate, dominant, recessive, blended, modified, developed beyond the parental extremes, new characters developed, etc., corresponding phenomena should be exhibited by the tissues. It was expected when this part of the research was planned that in the case of each plant both starch and tissues could be studied coincidently and compared, but this was found to be impracticable; therefore the studies of the plant tissues were carried on as an independent but correlated research. Here, as with the starches, excepting Ipomcea, the specimens of both parent- and hybrid-stocks are of the first generation that has been perpetuated from year to year by the propagation of tubers, pseudo-tubers, rhizomes, bulbs, bulbils, etc. Both of the parent- and the hybrid-stocks of Ipomoea were grown from seeds which breed true. The hybrid is of the offspring of successive annual seed plantings since 1908 , and probably represents the sixth or seventh in the line of descent. The seeds were obtained from the originator of the hybrid, and the other stock from reliable plant-growers.

The different specimens of starches were prepared from a number (varying usually from 5 or 10 to 100 or more) of bulbs, rhizomes, etc., so that the preparations may be taken as representing a fair mean; but with the plants used for the supply of tissue we were dependent in each case usually upon one or two specimens which may be taken to be of about the average or fairly representative.

In selecting the material from the different plants for the microscopic preparations the precautionary measures promulgated by Macfarlane (page 4) to secure safe comparative results were as far as possible carefully followed out. Inasmuch as there is a tendency for individuals of a species, even when grown under the same conditions, to vary in one or more of their characters from the average degree and manner of development, macroscopically and microscopically, it is manifest that in a comparative examination of parents and offspring there should be studied either the actual parents and a selected typical specimen of the hybrid that exhibits the average mean properties of the hybrids, or typical specimens of both parent- and hybrid-stocks. When neither is practicable, as was the case in the present inquiry, there are probabilities that the relative values of the various characters may not be wholly correct, as for instance, a given character of the hybrid may be intermediate but nearer one or the other parent instead of being exactly mid-intermediate, or vice versa, as might be the case had the plants been very carefully selected upon the basis of the specificity of intermediateness. On the other hand, it goes without saying that in the selection of the hybrid the assumption that the one having most nearly properties that are exactly intermediate between those of the parents is a typical hybrid is certain to lead to the worst of pitfalls, because it of necessity implies that blended inheritance is a sine qua non; therefore, as a corollary, that having a given hybrid its parentage might positively be detected by the selection of species that have characteristics such as would meet the theoretical requirements of intermediateness in the hybrid. It is obvious that such a plant might be far more undesirable and even absolutely unreliable for comparative purposes than one that has the least degree of intermediateness, because the latter but not the former may typify the mean of the hybrid characteristies. The results of various investigations fully justify the statement that intermediateness may be absolutely misleading as a criterion in the recognition of hybrids.

\section{Unit-Characters and Untt-Character- Phases.}

The term character is used throughout this research in a conventional sense to signify any property that 
serves to characterize any part or property of starch or plant. Inasmuch as each such property is a unit of comparison, each may appropriately and advantageously be referred to as a unit-character. A unit-character such as the property of gelatinizability may be manifested in varied phases or modified forms which conformably are distinguished as unit-character-phases. Many of the unit-characters and unit-character-phases that have been studied in this memoir may seem to be unimportant or even trivial, but experience in various lines of inquiry has shown that the correlation of such properties may prove of the greatest importance.

Each property of starch, whether it be manifested by peculiarities of form, hilum, lamellæ, or size of the grains, or in the reactions in polarized light, or in the reactions with iodine or the anilines, or in the gelatinization reactions with heat and the various chemical reagents, is an expression of a physico-chemical unit-character that is one of many indexes of the peculiarities of intramolecular structure of starch, and is an independent unit although correlatively related to the others. These unit-characters fall into arbitrary but natural groups in accordance with the methods of investigation employed, and as a matter of convenience and facility of study they have been treated under the designations above noted. Under the designation form are included a number of unit-characters which are expressed specifically in the occurrence of varieties or types of the grains (whether as isolated, aggregates, or compound grains), their numerical proportions and the peculiarities of the components in number and arrangement of the aggregates and compound grains; the regularity of outline of the grains, and the kinds and causes of irregularities; the conspicuous forms, etc. Under the designation hilum are included characters that are specifically expressed in distinctness, form, number, fissuration, and eccentricity. Under lamella are designated properties specifically expressed in distinctness, form, fineness or coarseness, variety and distribution, and number. Under size are included the ratios of length to breadith, general dimensions of grains of different types, especially of those of common size. Under polariscopic properties are characters that are expressed by peculiarities of the figure or "cross" in regard to eccentricity, distinctness, definition, courses, and other characters of the lines; the occurrence of single or multiple figures, the degree of polarization; the appearances with selenite of the quadrants as regards especially definition, equality of size, form, and colors. Under iodine reactions are included character reactions of the raw starch grains; and after boiling the grains, the reactions of the grains, solution, grain-residues, and capsules. Under aniline reactions are included characters elicited by the degree of staining by gentian violet and safranin immediately and after a half hour. Under temperature reactions are included the temperatures of gelatinization of a majority of the grains and of all or practically all of the grains. Under various reagents are included character manifestations that are expressed hv auantitative and qualitative reactions with various gelatinizing reagents. With each reagent it is found that there are peculiarities in respect to the percentages of the entire number of grains and total starch gelatinized at definite time-intervals; and to the number and kinds of gelatinization processes, these processes varying in both particulars not only in different starches with the same reagent, but also in the same starch with different reagents. Hence, while the property of gelatinizability is a fundamental or primary unit-character, it may be manifested in as many phases or modifications (unitcharacter-phases) as there are starches and gelatinizing agents. Among all of the varied properties of starches there seems to be none so certain to show slight intramolecular differences as these unit-character-phases.

The independence of each of these unit-characters and unit-character-phases of each other will be found to be well exhibited in every one of the groups of properties comprised in the several foregoing designations. This is most strikingly shown in hybrids - for instance, in the general characters of the hilum the propenties of the hybrid may be identical with those of one parent, while in eccentricity identical with those of the other parent, or intermediate, etc.; in the qualitative reactions with chloral hydrate some of the processes of gelatinization may be more like or identical with those of one parent; others, more like or identical with those of the other parent; others, which are individual are therefore not observed in either parent, etc. Hence, it is found, in summing up the unit-characters and unit-characterphases, that certain of the characters embraced in any designation may tend in one parental direction while others tend in another, but usually it is found that in the aggregate there is a variable degree of leaning to one or the other parent. Moreover, while such group properties may in the case of one designation lean in the aggregate to one parent, those of another group may incline to the other parent, and so on. This extraordinary variability in parental relationship is particularly well shown in the qualitative reactions with the various chemical reagents. These phenomena of variability are also strikingly illustrated in both macroseopic and microscopic properties of plant structure. (See Part II, Chapter IX.)

\section{Assistants in the Research.}

In the studies of the starches, the histologic data and the polariscopic, iodine, gentian violet, safranin, and temperature of gelatinization experiments were recorded by Dr. Elizabeth E. Clark, B.A. (Bryn Mawr), M.D. (Women's Medical College of Philadelphia); and the quantitative and qualitative reactions with the various chemical reagents were studied by Miss Martha Bunting, B.L. (Swarthmore), Ph.D. (Bryn Mawr). Both of these assistants had had two years previous experience in the study of starches. The macroscopic and microscopic data of plants are due to Miss Margaret Henderson, B.S., M.A. (University of Pennsylvania), who prepared all the microscopic slides and made all of the measurements. 


\section{CHAPTER II. METHODS USED IN THE STUDY OF STARCHES.}

The methods used in the preceding research (Publication No. 173) were at its inception sufficiently satisfactory to meet the theoretical requirements of a purely preliminary and exploratory investigation, but as the work progressed it was found, as was to be expected, that radical improvements could be made in various directions. Advantage has been taken of this experience, and while the methods continue to be inexact, in the conventional sense, they are practically exact so far as satisfactory differentiation and reoggnition of different starches are concerned. For obvious reasons the descriptions of the methods given in the previous research are herein in a large measure repeated, with some omissions, modifications, and additions.

\section{Preparation of the Starches.}

The starches were prepared from bulbs, tubers, rhizomes, bulbils, and pseudobulbs, all in the resting state. The specimens were comminuted by the aid of an ordinary culinary grater. Four or five volumes of water are added to the pulp, the mass strained through four thicknesses of cheese-cloth, and the pulp then washed with sufficient water and strained as before. The starch-water preparation is decanted in cylinders and the starch is cleansed by repeated washing and decantation. Finally the starch is collected in shallow dishes, the water as far as possible drained off, and the preparation dried at a temperature of $50^{\circ} \mathrm{C}$. By this simple means starches can be prepared which are with rare exceptions practically free from gross impurities. To have carried out purification to the extent of practical demineralization would have proven of far greater disadvantage than gain.

2. Simultaneods Studies of Starches of the Parents and Hybrid and of the Members of a Genus.

For obvious reasons, in a comparative investigation such as the present it is desirable to make simultaneous examinations of all three or four starches of a set by one of the various methods of study and to take up the methods seriatim in preference to taking one starch and subjecting it to the entire series of methods before studying another specimen; the same plan commends itself when there is a number of sets belonging to the same genus.

\section{Histologic Method.}

This method has been found to be of signal usefulness, and up to recent years it has been the sole reliance in attempts to determine the kind of starch. It was, however, perfectly obvious at the very inception of these researches, and rendered clear as far back as the investi- gation of C. Nägeli in 1858, that this method, unless associated with others, could not be depended upon, and that it was liable to be absolutely misleading. Moreover, differences in form may not in the least imply differences in the starch-substance, as has been pointed out in early chapters of the preceding memoir. Magnification ranging from 85 to 400 , sometimes higher, was used, according to the size of the grains and incidental conditions. A sufficient amount of dried starch was disseminated on a slide and mounted in a very dilute Lugol's solution, care being taken not to add a larger quantity of iodine than is sufficient to accentuate the lamellæ. Since starches of different sources show wide differences in the intensity with which they become colored with iodine, it was found convenient to have on hand a number of solutions ranging from 1 to 2 per cent down. By the aid of such ordinary microscopic technique there wiere recorded the form and size of the grain; the position and form of the hilum; the form, number, and other characteristics of the lamellæ; the characteristies pertaining to the form of the grains, whether single or in doublets, triplets, aggregates, etc. In describing the grains the terms "proximal end" and "distal end" have been adopted, the former being the end nearer which the hilum is located. The "longitudinal axis" corresponds with an imaginary line, extending from the proximal end through the hilum to the distal end. In different starches and in different grains of the same kind of starch this may be the long or the short axis. The measurements of eccentricity of the hilum have reference to the distance of the hilum from the proximal end of the longitudinal axis.

\section{Photomicrographic Reords.}

Verbal descriptions of the histological characteristics of starch-grains fail to convey adequate conceptions. The notes included in the text have therefore been accompanied by photomicrographs of the grains lightly colored with iodine, as seen in the microscope. In making these photographs we used an ordinary Bausch and Lomb microscope with a $1 / 4$-inch objective and a 2 -inch eyepiece, which gave us a magnification on the field of projection of 300 diameters. For obvious reasons, many of the more minute features of the grains will not be seen in the photomicrographs. Moreover, inasmuch as no two fields are alike in case of any starch or slide, the pictures are to be taken as being grossly of an average character of a field. In recording the histological descriptions, especially as regards variations in form, many fields were examined.

The photomicrographs of the plant tissues were made by the use of a 11/2-inch objective and a 2-inch eye-piece (draw-tube in), or a $2 / 3$-inch objective and a 
2-inch eye-piece, or a $1 / 4$-inch objective and a 2 -inch eyepiece, giving magnifications on the field of projection of 72,180 , and 300 diameters, respectively.

\section{Reactions in Polarized Light Withodt and With Selenite.}

Starches have been found to exhibit not only marked differences in the degrees with which they rotate the plane of polarized light, but also differences in the characteristics of the "interference figure" or "cross," as it is generally termed. The general characteristics, distinctness, shape, regularity, and position of the interference figure, and also the approximate degree of anisotropy or intensity of polarization were readily studied. $\mathrm{By}$ the aid of selenite it was determined whether the optic properties were negative or positive, and also the size, shape, and regularity of the quadrants, as well as the intensity and pureness of the blue and yellow colors. In spherical grains with centrally located hila, the two parts of the "cross" intersect at the hilum, or mathematic center, of the grain, so that the term quadrant has a proper application; but in the case of grains having eccentric hila the position of the point of intersection of the two parts of the cross, together with their curvatures, may destroy every semblance of quadrants according to the conventional definition of this word. This term has therefore been used in a very broad sense throughout our investigation to indicate the four parts of the grain that are defined by the two parts of the cross, in preference to the great multiplicity of terms that would be required to define these parts if great accuracy were attempted. Likewise, for convenience we have referred to the "lines" of the interference figure in preference to the "arms" of the cross.

All starches are "optically negative," hence no special references have been made in the text in this particular.

The slides for polariscopic examination are prepared as follows: The end of a small spatula is thrust into the specimen of starch and moved about, withdrawn and sharply tapped several times in the center of the slide, and the slide jarred in a manner to cause a practically uniform distribution of the starch grains in a single well-disseminated layer. The margins of this layer are carefully removed so as to leave an area $12 \mathrm{~mm}$. square. An expeditious way of removing the margin so as to insure a uniform area of starch is to use as a wiper a piece of sheet celluloid having a $12-\mathrm{mm}$. slot, wiping transversely and then longitudinally. A couple of drops of balsam are carefully added at the center of the area, a cover-slip put on, and the slide placed on the stage of the polarizing micrascope. After determining the degree of polarization, the selenite plate is introduced and the specimen again examined.

In order to reduce the degree of polarization into values in comparative terms and figures it was found desirable to adopt an arbitrary scale (Chart B 2, Chapter IV), and to select three starches as standards that give wide and properly separated gradations of value. Thus, adopting a scale of 100 divided primarily into units of 5 , the starch of Solanum tuberosum was taken as having a value of 90 and "very high"; that of Narcissus poeticus ornatus as having a value of 50, or "moderate"; and that of Richardia albo-maculata as having a value of 30 , or " low." Intermediate gradations are readily expressed by both words and figures. If the starch examined has, for instance, the same degree of polarization as that of Narcissus poeticus ornatus it is given a value of moderate or 50 , but if its value be between moderate $(50)$ and high (70) it is recorded as being moderately high (60), or moderate to moderately high (55), or moderately high to high (65). In some instances intermediate values are given where it is necessary to express smaller differences, as between members of a set consisting of parents and hybrid. The different grains of any given specimen of starch vary in the degree of polarization, so that in rating the average must be estimated; as a consequence all of the records are averages. The method is of a very gross character and the personal equation in determining values may be very important and lead to more or less divergent records by different observers, but in practice it has been found that after a degree of skill has been acquired, as is common in all such gross methods of experiment, essentially or absolutely the same values are recorded when experiments are repeated several times at well-separated intervals, or made by two individuals who have had practically the same training. Owing to variations in illumination from time to time, it is quite important to use persistently, in conjunction with the starch to be examined, some starch that has been adopted as the standard of comparison, preferably one that has a close value. Thus, when studying the starches of a group, one of the starches is standardized with the starch-standard and scale adopted, as before stated, the standard recorded for this starch serving as the fundamental standard for comparison for the others of the group. This method gives very good comparative results, especially when the group consists of a few members; but it is, on the whole, the least valuable of all the methods employed in this research, and its usefulness is chiefly because of its remoteness from the characters of the other methods.

\section{Iodine Reactions.}

The use of iodine not only served to bring out certain histological peculiarities, but also valuable data in the differentiation of different kinds of starch. The typical or ordinarily observed reaction of starch with iodine is an indigo-blue, but if an excess of iodine be aroided the reaction of the grains will be found to vary usually from a blue to reddish-violet, including within these extremes all shades of violet from a purple to a reddishviolet according to the kind of starch. In fact, in the presence of minute quantities of iodine, starches are colored some shade of violet, varying with the kind of starch. With any quantity of iodine certain starchgrains yield a red reaction. In studying the iodine reac- 
tions we used $0.125,0.25$, and 2 per cent Lugol's solution. Four serial reactions were studied; two with raw starch and two with gelatinized starch. In the first two, the slides are prepared as in the polarization examinations, substituting solutions of iodine for the balsam and examining the slides in ordinary light with a fully open diaphragm and low power. In the first reaction 2 drops of 0.25 per cent Lugol's solution are placed on the starch, the slide quickly adjusted on the stage of the microscope, and the color reaction in quality and quantity at once determined, the quantitative value recorded being taken as the standard of comparison in relation to other starches. Here, as in the polarization determinations, it was found necessary to adopt an arbitrary scale and starch standards. The same scale is used as for the polarization values, but the terms light, deep, etc., were substituted for low, high, etc. Moreover, it was found necessary to modify the selection of starches to be used as standards. The starch of Solanum tuberosum was taken as having a value of 60 or "moderately deep," that of Crinum moorei as having a value of 50 or " moderate," and that of Walsonia humilis as having a value of 30 or "light," with corresponding intermediate figures and terms as in the polariscopic determinations.

The second experiment is made, using 0.125 per cent solution, often bringing out color peculiarities which may be obscured or not be observed when the reagent is stronger.

The third and fourth experiments are made with boiled starch with the object of eliciting peculiarities of reaction of the grains, solution, grain-residues, and capsules. After heating the grains until complete gelatinization occurs a variable amount of the starch passes into solution, so that both grains and solution give starch reactions. Upon boiling the preparation for 2 minutes a comparatvely large amount of the starch passes into solution, and the remains of the grains appear in the form of grain-residues which are made up of partially disintegrated grains (capsules with variable amounts of contents), together with some capsules that are almost or wholly free of starch contents.

In the third experiment 0.05 gram of starch is placed in 20 c.c. of water and carefully heated over a bunsen burner only to the point of complete gelatinization. To 2 c.c. of this preparation is added 2 c.c. of a 2 per cent Lugol's solution, and then the colorations of grains and solution are determined by microscopic examination.

In the fourth experiment the remainder of the boiled preparation is boiled for 2 minutes to further break down the starch grains; then 4 c.c. of the 2 per cent Lugol's solution added; and then microscopic determination made of the colorations of grain residues, capsules, and solution.

\section{Aniline Reactions.}

A number of anilines have been found by various investigators to be of value in the differentiation of starches from different sources, of different grains of the same kind of starch, and of different parts of individual grains. Some experimenters have employed double or triple stains. There is also no doubt that the use of double or triple stains would bring out, at times at least, many points of much histological importance, but this would have involved the carrying out of the histological examinations in such detail as to be prohibitive in a research of this character. Safranin and gentian-violet were selected, not because they are probably the best of these stains for differential purposes, but because they have been found very useful in starch examinations and as they yield single color reactions.

Aniline colors in solution, especially when in weak solution and exposed to light, are notably unstable, and in order to secure strictly comparable results a quantity of a relatively strong standard solution was prepared and kept in the dark, tightly corked. The stock solutions were composed of 0.25 gram of aniline with 150 c.c. of distilled water. From day to day dilute solutions were prepared by adding 33 c.c. of water to 2 c.c. of the stock solution; 15 c.c. of the latter solution are placed in a test-tube containing 0.07 gram of starch, the preparation agitated, 1 or 2 drops withdrawn in a minute and examined under the microscope, and a final examination made at the end of half an hour. In these color determinations the microscope is used, as in the iodine reactions, with a fully open diaphragm and low power. Owing to the relatively slow reaction, the values for comparative purposes were taken at the end of a half hour instead of immediately, as in the first iodine reaction. The method of valuation is the same as in the iodine reactions, but the starch standards for these reactions are: Solanum tuberosum, value 90, "very deep"; A maryllis belladonna, value 50, "moderate" ; Freesia refracta alba, value 30, "light."

\section{Temperatures of Gelatinization.}

While the records of various investigators indicate that there are more or less marked differences in the temperatures of gelatinization of different kinds of starches, and even in case of different grains of the same starches, the figures applying to the same kind of starch are generally so at variance that not much value is to be attached to them. The sources of fallacy in such observations, unless the determinations are made with the greatest precautions, are well known to every biochemist. We therefore carried out this work with especiall care. A long quadrangular water-bath was used, holding about 4 liters of water; one end was placed over the gas flame, and in the other end was inserted a thermometer which was calibrated in tenths centigrade, but which could readily be read in hundredths. A small quantity of starch with 10 c.c. of water was placed in a test-tube, into which was inserted, through a perforated cork, a thermometer similar to the one in the waterbath, and the test-tube immersed in a suspended wire basket in the part of the water-bath farthest from the flame. The temperature of the water was raised very 
slowly, and the water occasionally stirred, so that at no time did the two thermometers differ more than about $2^{\circ}$. As the temperature increased, specimens of the starch were examined at intervals, the tube being shaken, and a specimen obtained by inserting the end of the pipette to the bottom of the tube, a clean pipette being used to remove each specimen. Each specimen was placed on a slide, upon which was recorded both temperatures, and the slide was examined in the polarizing microscope. The temperatures at which there is an entire loss of anisotropy of a majority and of all of the grains were recorded as the temperatures of the tube. The lower temperature recorded on the slide was the record of the thermometer in the test-tube, and the higher temperature was that of the water-bath. The actual temperature of gelatinization lies somewhere between the two, and for convenience, especially for purposes of comparison, the mean of the two was for obvious reasons taken as the "temperature of gelatinization." In the records all three temperatures are given in accordance with the foregoing.

\section{Action of Swelling Reagents.}

Quite a number of swelling or gelatinizing reagents, of very diverse chemical composition and exhibiting more or less individuality of action, have been used by various experimenters in studies of the structural peculiarities of starch-grains or in the differentiation of different kinds of starch or for other incidental purposes. This method of differentiating starches seemed so promising that in the preceding research five such reagents were selected. For obvious reasons choice was made of those which differ widely in chemical composition and which yield sufficiently prompt and characteristic results. Those selected included chloral hydrate-iodine, chromic acid, pyrogallic acid, ferric chloride, and Purdy's solution. For evident reasons it is desirable to repeat some of the statements made in the preceding memoir.

The chloral hydrate-iodine solution was prepared by saturating a saturated solution of chloral hydrate with iodine. This solution, sooner or later, not only causes swelling and ultimate partial dissolution of the grains, but, owing to the presence of iodine, also yields important accompanying color reactions; and it is, on the whole, to be regarded as a very important reagent.

Chromic acid was used in the form of a 25 per cent solution, and it is the only one of the five reagents that causes, within the periods of observation, a complete disintegration of the grains. It gives rise to gas bubbles during the decomposition processes.

The pyrogallic-acid solution was prepared by making a saturated solution and diluting this with three parts of water, adding oxalic acid in the proportion of 4 per cent to hinder oxidation.

The ferric-chloride solution consisted of equal parts of a saturated solution and water. Purdy's solution was made by diluting the standard solution with an equal volume of water.
The last reagent was usually found to be the least active of the five, and it is, so far as the effects on the grains are concerned, probably essentially an aqueous solution of potassium hydroxide, and therefore likely possesses no advantages, except perhaps in keeping quaiities, over the simple aqueous solution. Oxygen or exposure to the air favors the actions of pyrogallic acid, but hinders those of chloral hydrate and ferric chloride. In the former case, the grains near the edge, or on the outside, of the cover-slip are decidedly more affected than those within, while with the latter the opposite is true.

There are some forms of commercial chloral hydrate that have very little action, which may be due to underhydration or over-hydration. The crystals put up by Schering were used throughout this investigation.

It is important that fresh solutions of the reagents be prepared at short intervals, as all tend to deteriorate, and it is well to let them stand over night before using.

In using these reagents a small amount of starch was placed in a slide as in the polarization experiments, several drops of the reagent were added, a cover-glass put on, and the progress of events examined under the microscope. In using a given reagent with a given kind of starch, it was found that there was a certain amount of variation in the effects from time to time, probably attributable to variations in temperature, so that these studies were made as far as possible under constant temperature conditions. The variations, as a rule, were unimportant. These agents give rise to gelatinization and swelling of the grain and cause the existence of the outer and inner parts of the grains to appear very conspicuous-the outer part becoming sac-like and inclosing a less dense or semi-fluid substance.

Experience taught us that not only the method but also the reagents, as regards both kind and concentration of solution, can be markedly improved. As previously stated, the method though gross seemed to meet the theoretical requirements of the research-that is, the determination whether or not starches are modified in relation to species and genera-without attempting to establish constants or strictly exact data. During the progress of the present research we used, in a limited number of experiments, certain reagents which in the text that follows are designated:

SoLUTION No. 2.

Chloral hydrate-iodine-Schering's crystals of chloral hydrate 30 grams, water 17 c.c., Lugol's solution 3 c.c.

Chromic acid 10 grams, water 40 c.c.

Pyrogallic acid 9 grams, oxalic acid 0.5 gram, water 40 c.c.

Ferric chloride 50 grams, water 5 c.c.

Ammonium nitrate 15 grams, water 10 c.c.

After a time the ferric chloride was abandoned because of difficulties in standardization and in obtaining satisfactory uniformity in the results of repeated experiments, and it was also found that other of the reagents could be used to better advantage in a modified form. A few experiments were also made.with ammonium nitrate and certain other reagents, but for various reasons were set aside. It is yet wholly problematical as to 
what reagents and what concentrations are best adapted for such studies, but the following were finally adopted in this research, although experience has shown that all or nearly all can be modified to advantage in concentration and they can be added to with great profit. Chemically pure chemicals and distilled water were used. The solutions should be made only in small quantities, and when fresh solutions are prepared they must be tested with the several selected starches, the reaction-intensities of which are known, to determine whether or not they are of exactly proper strength.

Chloral hydrate--Schering's chloral hydrate crystals 15 grams, water 5 c.c.

Chromic acid 2.5 grams, water 20 c.o.

Pyrogallic acid 4 grams, oxalic acid 0.3 gram, water 35 c.c.

Nitric acid 10 c.c. water 35 c.c.

Sulphuric acid 10 c.c., water 27 c.c.

Hydrochloric acid 9 c.c., water 10 c.c.

Potassium hydroxide 0.75 gram, water 55 c.c.

Potassium iodide 10 grams, water 30 c.c.

Potassium sulphocyanate 5 grams, water 30 c.c.

Potassium sulphide 1 gram, water 40 o.c.

Sodium hydroxide 0.5 gram, water 100 c.c.

Sodium sulphide 1 gram, water 45 c.c.

Sodium salicylate 10 grams, water 10 c.c.

Calcium nitrate 8 grams, water 16 c.c.

Uranium nitrate 8 grams, water 10 o.c.

Strontium nitrate 5 grams, water 7 c.c.

Cobalt nitrate 9 grams, water 15 c.c.

Copper nitrate 15 grams, water 30 c.c.

Cupric chloride 9 grams, water 15 c.o.

Barium chloride 5 grams, water 12 c.c.

Mercuric chloride 18 grams, ammonium chloride 10 grams, water $\mathbf{4 0}$ c.c.

Occasionally modified solutions were used in qualitative experiments to meet special conditions, note being made in the text at the proper place whenever this has been done.

In the reactions with the chemical reagents it is essential, in order to obtain uniform and wholly reliable results, that the slides should be prepared with much care as regards the quantity and distribution of the starch and the quantity of the reagent, and that immediately upon the addition of the reagent the preparation be protected so that changes due to alterations in concentration and to oxidation will not occur. The method pursued is as follows:

A square area of starch is first prepared on a slide as in the polarization reactions. This square is surrounded by a layer of purified vaseline $5 \mathrm{~mm}$. wide, applied by an artist's flat camel's hair brush. A cover-slip is now prepared by coating the margin of one surface with a corresponding band of vaseline, so that when the coverslip is placed on the slide the surfaces of two vaseline squares form an air-tight junction, preventing change in concentration of the reagent by evaporation or absorption of water and eliminating influences of the oxygen of the atmosphere. Two drops of the reagent are carefully and quickly placed on the center of the starch layer, the cover-slip instantly applied, the slide placed on the stage of the polarizing microscope, a suitable field speedily found and examined in polarized light. Usually a practically exact count is made of the number of grains in view, but if the reaction is very rapid this part of the method is modified as hereinafter stated. All these procedures are done as expeditiously as possible. In the starches of some species there are to be found variable proportions of very minute grains which for obvious reasons must be ignored in making the count. The number of grains in the field ranges usually from 150 to 200 , rarely as few as 75 to 100 or as many as 400 to 600 , the number depending largely upon and in approximate ratio to the mean size of the grains; but such differences in number do not imply corresponding differences in the total amount of starch present. In specimens in which the grains are small, the number of grains in the field will be larger than when the grains are large, and the number will vary also because of some irregularities in the distribution of the grains, a field always being selected that is well adapted for the count and for watching the processes of gelatinization. Unless gelatinization occurs very rapidly the percentages of grains and total starch gelatinized are not determined until at the end of 5 minutes from the time of the addition of the reagent, and subsequently at $15,30,45$, and 60 minute intervals, or as may be desirable. At these periods the number of grains not completely gelatinized is counted, and then the percentage of grains completely gelatinized is computed by finding the difference between the original number in the field and the number thus found. In addition to the grains completely gelatinized there will be seen grains in partial stages of gelatinization and perhaps some wholly unaffected. The amount of starch remaining ungelatinized is computed in terms of grains and is estimated by finding the number of grains that are unaffected and the proportions of starch ungelatinized in the partially gelatinized grains. Thus, in the latter case, if there remains an average of one-quarter of the starch unaffected (in some grains it may be onetenth, in others one-fifth, etc.), it will take 4 grains to represent the amount of starch in an average grain of the specimen, the number thus determined being added to the number of grains that are unaffected, the sum deducted from the original number under observation, computing by the difference the percentage of the total starch gelatinized.

When gelatinization occurs very rapidly or very slowly the foregoing method must be modified to suit conditions. Frequently complete or almost complete gelatinization occurs within $\mathbf{1 5}$ seconds after the application of the reagent. Obviously time is not permitted for a count of the number of grains in the field before determining the number of grains wholly and partially ungelatinized. By extreme alertness it is possible within 15 seconds after the addition of the reagent to have the slide on the stage of the microscope, select a field, make a count of the ungelatinized grains, and estimate the parts of grains that remain ungelatinized. The number of grains in the field can not be satisfactorily counted after gelatinization because of the swollen and distorted condition and overlapping of the grains. Hence, in these very rapid reactions the average number of grains in a field is determined beforehand and a corresponding field is selected. It follows from this that the percentage of starch gelatinized under such conditions is very grossly estimated, that no importance is to be attached to the 
figures beyond the time-limit of complete gelatinization, and that the figures have no value for comparison in cases of starches which likewise are very quickly gelatinized, unless by averages obtained from frequently repeated experiments.

When gelatinization occurs very slowly it often is easier, after having made the count in the field, to determine the number of grains gelatinized and partially gelatinized, as for instance when only 1 per cent of the total starch is gelatinized at the end of 5 minutes or 5 or 10 per cent at the end of an hour.

\section{Constancy of Results Recorded by tile Fore-} goina Merhod.

It goes without saying that such experiments should be carried out as far as possible under fixed conditions, especially as regards the quantity of starch in relation to the quantity of reagent. The variations in the quantity of starch, in so far as constant results are concerned, are absolutely negligible, as has been found not only in the records of repeated experiments, but also in the records of varieties of a species when the records should be expected to be very close because of the starches being nearly identical. The quantity of reagent used is invariably 2 drops, each reagent being kept in a 50 c.c. bottle having a glass-stoppered finger pipette dropper with a rubber tip. Under practically identical laboratory conditions as regards quantity of starch, quantity of reagent, temperature, and humidity the results recorded by repeated experiments are either identical or vary within limits that are so narrow as to be absolutely without importance. Even marked variations in temperature and humidity have not been found to be important, except in rare instances. (See note under Amaryllis-Brunsvigia-Brunsdonnce, page 34.)

Obviously, some variations, even though trifling, are to be expected, so that in order to obtain constants a given experiment should be repeated a sufficient number of times and an average taken of the records, as in the determination of melting-points. Experience has shown, however, that in so far as the requirements of the present exploratory research are concerned the results of a single experiment carefully carried out are dependable within narrow and wholly unimportant limits of error. The chief sources of error to be guarded against are leakage through the vaseline seal; the presence of contaminating substances in the starch; certain peculiarities occasionally observed in the behavior of starches towards certain reagents; and errors in estimation when the reactions are very rapid. Leakage through the vaseline seal is sedulously to be avoided, and if a leak occurs the slide and records must be discarded.

The presence of oxalate crystals in the starch is by no means uncommon, but no clear evidence has been found to lead to the belief that, unless in exceptionally large quantity, they in any way influence the course or time of gelatinization by the reagents used. In the present research in Calanthe only were there even many of these crystals; in the Phaius a few; and none or practically none in the other starches. Occasionally foreign matter in the form of undetermined debris is present which can not be gotten rid of by repeated washing, as in Tritonia pottsii. Such matter may affect the polarization, iodine, and aniline reactions to a detectable degree, but no effect has been noted in the other reactions. With the exception of this starch all have been free from such contamination. Erratic behavior of an inexplicable character has upon rare occasions been observed in the use of the sulphide and salicylate solutions. Finally, when the reactions are very rapid, while satisfactory records may not be obtained for comparison with those of other starches which gelatinize with similar rapidity, changes in the concentrations of the reagents can be made so as to lengthen the time of the reactions and thus permit of satisfactory differentiation.

Comparatively little importance is to be attached to the polarization, iodine, gentian violet, and safranin reactions when the reactions are close. Personal equation and incidental conditions are here not unimportant factors that may greatly vary the limits of error of experiment. In future investigations these agents might with profit be discarded for better means of study unless further experience brings out greater values than they have thus far shown.

\section{Reagents used in Qualitative Investigations.}

The methods used in this research are both quantitative and qualitative, chiefly the former because of the ease with which the data recorded can be reduced to figures and charts. The qualitative reactions have been studied especially by means of certain of the chemical reagents that were selected from time to time because of their especial adaptation to certain kinds of starches to elicit qualitative phenomena, some reagents acting better with some kinds of starches than with others. Incidentally here and there special qualitative records were made by the use of selenite, iodine, gentian violet, safranin, and heat. In the qualitative reactions many points of varying degrees of interest and importance were brought out that can not be studied by the quantitative methods described, some of equal or greater importance than those obtained generally by the latter methods.

In studying the starches of the Amaryllidacer we used chloral hydrate, nitric acid, potassium iodide, potassium sulphocyanate, potassium sulphide, and sodium salicylate, excepting in the Narcissi when the sodium salicylate was omitted. Additional studies were occasionally made with sodium hydroxide, sodium sulphide, cobalt nitrate, copper nitrate, cupric chloride, barium chloride, or mercuric chloride. In studying the Lilliaceæ we used chloral hydrate, chromic acid, potassium hydroxide, cobalt nitrate, and cupric chloride; in the Iridaceæ, chloral hydrate, hydrochloric acid, potassium iodide, sodium hydroxide, and sodium salicylate; in Begonia, chloral hydrate, chromic acid, pyrogallic acid, nitric acid, 
and strontium nitrate; in Richardia, chloral hydrate, chromic acid, hydrochloric acid, sodium hydroxide, and sodium salicylate; in $\mathrm{Musa}$, chloral hydrate, chromic acid, pyrogallic acid, sodium salicylate, and cobalt nitrate; in Phaius, chloral hydrate, chromic acid, nitric acid, hydrochloric acid, potassium hydroxide, potassium iodide, potassium sulphocyanate, potassium sulphide, sodium hydroxide, sodium sulphide, and sodium salicylate; in Miltonia, chloral hydrate, chromic acid, hydrochloric acid, potassium iodide, and sodium salicylate; in $\mathrm{Cym}$ bidium, chloral hydrate, chromic acid, sodium salicylate, barium chloride, and mercuric chloride; and in Calanthe, chloral hydrate, chromic acid, nitric acid, hydrochloric acid, potassium hydroxide, and sodium salicylate. Instances here and there will be found where additional reagents, or reagents of concentrations varying from the standards given, were used. The special reasons for the selections in the various cases will be found in Chapter V.

\section{Charts of Reaction-Intensities of Different STARCHES.}

It is difficult or impossible to associate the different reaction-intensities of a given starch with different reagents or those of different starches with a single reagent when expressed in figures in such a way as to form an accurate or even a reasonably approximate mental picture of their individual and related values; and, moreover, an association of this kind becomes increasingly difficult or absolutely impossible when one attempts to multiply such pictures in a comparison of the reactions of two or more starches with different reagents or of two or more reagents with a given starch. Hence, it has been found necessary to translate these figures into the forms of curves which, as will be seen, give not only strikingly clear presentations of these extremely varied reactionintensities, but also, as a corollary, permit of the readiest and most satisfactory comparisons. It was found during the development of the research that it is desirable to exhibit these peculiarities in six kinds of charts as follows :

A 1 to A 26, showing the reaction-intensities of all or many of the starches with each agent and reagent.

$B 1$ to $B^{\prime} 42$, showing the reaction-intensities of certain starches with certain agents and reagents.

C 1, showing the reaction-intensities of genera and subgenera or other generic subdivisions as regards height, sum, and average.

D 1 to D 691 , showing the velocity-reactions of different starches with different reagents.

$\mathrm{E} 1$ to $\mathrm{E} 46$, showing composite reaction-intensity curves of the starches of parent- and hybrid-stocks with different agents and reagents.

F 1 to $F$ 14, showing the percentages of macroscopic and microscopic characters of plants, and of the percentages of the reaction-intensities of starches, as regards sameness to one or the other or both parents, intermediateness, and excess and deficit of development.
Inasmuch as this research is primarily a comparative investigation of the starches of parent- and hybridstocks, the curves that represent parents and offepring have, whenever feasible or desirable, been plotted out together in order to render comparisons easy. For various reasons, hereafter stated, all of these charts have been brought together and now compose the last part of Chapter IV, page 175 , et seq.

In the groups of charts designated A, B, and $\mathrm{E}$, in the polarization, iodine, gentian-violet, and safranin reactions the abscissæ are in terms of quantitative light and color values based on an arbitrary scale of 105 in divisions of twentieths; in the temperatures of gelatinization in the centigrade scale from $40^{\circ}$ to $95^{\circ}$ in divisions of $2.5^{\circ}$; and in the gelatinization experiments with different reagents in a duplex scale, the upper portion giving the time of complete or practically complete gelatinization (95 per cent or more of the total starch), and the lower portion the percentage of the total starch gelatinized when complete or practically complete gelatinization has not occurred within 60 minutes. In Charts A 1 to A 26 the vertical lines that are projected from the plant names are extended to the abscissæ that represent the reactionintensity values. Thus, if gelatinization is complete or practically complete at the end of 5 minutes the line is carried to the 5-minute abscissa ; if 80 per cent is gelatinized at the end of the 60 -minute period the line is carried to the lower part of the scale-that is, to the abscissa designated 80 per cent of the total starch gelatinized in 60 minutes, and so on. The second form of chart, including B 1 to B 40, while having the same abscissæ as the first and fifth forms have different ordinates, and Charts B 41 and B 42 while having the same ordinates as the others of this group have wholly or partly different abscissæ to meet special conditions. In these charts the reaction-intensity values have been recorded at the proper abscissa on each ordinate and then a line projected from ordinate to ordinate to form a curve. In Charts $\mathrm{E} 1$ to $\mathrm{E} 46$ the ordinates represent the various agents and reagents, the values are recorded as in group B 1 to B 40, and in each chart the curves of the reactionintensities of parent-stocks and offspring are presented. In Chart $\mathrm{C} 1$ the abscissæ are in terms of height, sum, and average reaction-intensities, and the ordinates represent genera, subgenera, or generic subdivisions. In charts D 1 to D 670 there are given records of the progress of gelatinization in per cent-time, the curves of each set of parent-stocks and offispring being recorded on each chart, excepting in case of a few special charts. The abscissæ are in terms of percentages of total starch and the ordinates are in time-intervals of $\mathbf{5}$ minutes. While determining the percentage of total starch gelatinized at definite time-intervals simultaneous records were made at the same periods of the total number of grains completely gelatinized. When these two sets of data are reduced to curves it is found that varying differences are exhibited by the different starches, in the case of each starch with the various reagents, and by the differ- 
ent starches with each reagent, the variations in the courses and degrees of separation of the two curves being, on the whole, quite as significant in the differentiation of the starches as differences in the percentage of total starch gelatinized (see Chapter IV, page 170). In case of some starches with a given reagent the percentage of total starch and the percentage of grains completely gelatinized run closely together, or even almost parallel, while with other starches a large percentage of the total starch may be gelatinized, yet only a small percentage of grains be completely gelatinized; the same peculiarity holds good in regard to any given starch with different reagents. Obviously all such data must be of importance in the formulation of the physico-chemical characteristics of any kind of starch. In Charts F 1 to F 14 there are plotted out in some percentages of macroscopic and microscopic characters of plants, and in others those of plant and starch characters, the abscissæ and ordinates being varied to meet particular and obvious conditions.

No one kind of chart of itself presents in full starch peculiarities. In fact, a satisfactory picture of the peculiarities of any starch can be had only by combining the curves of the several kinds of charts with histological peculiarities, and the polariscopical, iodine, aniline, heat, and chemical qualitative reactions. In other words, characters not brought out by one means of investigation may be by another, etc.; hence, it is the sum-total of data that must be taken in the final analysis.

\section{Comparative Valoations of the Reaction- INTENSITIES.}

Throughout all of the reactions definite standards of comparison were adopted, varying somewhat with the different agents, yet all forming a definite coördinate system based upon common abscissæ (Chart A 1, Chapter IV). Thus, the reaction-values in the polarization, iodine, gentian violet, and safranin reactions are based upon a "light and color reaction" scale up to 105, from 0 to less than 20 being grouped as very low or very light, 20 to less than 40 as low to light, 40 to less than 60 as moderate, 60 to less than 80 as high or deep, and 80 to 105 as very high or very deep; the terms very low, low, moderate, high, and very high are applied to the polarization reactions; and very light, light, moderate, deep, and very deep to the iodine and aniline reactions, the sets of terms being synonymous in so far as comparative values are concerned. The reactive-values of the temperature of gelatinization experiments range from $42^{\circ}$ to $95^{\circ} \mathrm{C}$. ("temperature of gelatinization" scale), $82.5^{\circ}$ corresponding to $20,72.5^{\circ}$ to $40,62.5^{\circ}$ to $60,52.5^{\circ}$ to 80 , and $42.5^{\circ}$ to 100 , of the foregoing scale. The reaction-values of the reactions with the various chemical reagents are, as previously stated, in terms of complete and partial gelatinization-of complete gelatinization within a period of 60 minutes, and of percentage of total starch gelatinized in 60 minutes, the scale consisting of two parts in accordance with this division. These reactive-values based upon the light and color scale of 105, are as follows: 50 per cent of the total starch gelatinized in 60 minutes corresponding to 20 , and 90 per cent to 40 ; complete gelatinization in 45 minutes to 60 , in 25 minutes to 80 , and in 5 minutes to 100 .

Comparative reactive-intensities are grossly presented in the text by referring the reactions to five groups upon the basis of the values as they fall within the five divisions enumerated; very low, low, moderate, high, and very high. This plan has been followed in the Summaries at the end of each set of parent- and hybrid-stocks, and each group of sets that belong to a given genus. It was found, however, in the final summing up of such data to show generic differences, that the reactive-intensities could better be presented when the exact value in units in each reaction was taken instead of the group value. For instance, two starches whose values fall within the "very high" division may have very different numerical values, one a value of 80 and the other of 100 or more, according to the first scale given, etc. In making out these values each abscissa was taken as having a value of 5 , making the range of the scale from 0 to 115 , the abscissa having a value of 25 corresponding to 20,45 to 40,65 to 60,85 to 80 , and 105 to 100 of the "light and color reaction" scale. This difference is owing to the raising of the light scale 5 points higher than it should have been under usual circumstances. 


\section{CHAPTER III. HISTOLOGIC PROPERTIES AND REACTIONS.}

Comparisons of the Móre Important Data of the Histologic Properties and the Polariscopio, Iodine, Aniline, Temperature, and Various Reagent Reactions of the Starches of Parent- and Hybrid-Stocks.*

The great volume of matter that has been recorded in the laboratory investigations of the starches of parents and hybrids, and which constitutes Chapter I of Part II of this memoir, renders it desirable, for various reasons that will be obvious, to bring together in a very succinct form such of the data as seem to be the more important in showing parental and hybrid relationships and peculiarities. This has been attempted in the present chapter, but the records of the histologic properties in the laboratory notes are so condensed that in a large number of instances the summaries in this chapter will be found to be more suggestive than adequate, or even have been omitted in order to avoid an almost full restatement.

In the comparisons of the properties of parents and hybrids a definite system has been adopted throughout all of the parent-hybrid sets. In Section 1 the histologic properties and the qualitative polariscopic and iodine reactions, respectively, of the parents are with rare exceptions each first compared, and then those of the hybrid with those of the parents, and then when there are two hybrids of the same parentage their properties are compared. Much attention was given in the laboratory work to the study of qualitative reactions with several of the reagents, which reactions have been found to be of importance not only in the study of the starches of different varieties, species, and genera, but also of the starches of parents and hybrids. References are made to these reactions in this section, especially in regard to the peculiarities of the hybrid in relation to the parents. In subsequent sections the data are quantitative, lending themselves admirably to both tabulation and charting.

Section 2 records comparisons of the reaction-intensities in the polarization, iodine, gentian-violet, and temperature experiments. The data are tabulated under these headings in forms well adapted for ready comparisons, the tables being followed by brief comparative summaries of the peculiarities of the reactions of the parents and of the reactions of hybrid and parents, and of the two hybrids when such exist.

In Section 3 the reaction-intensities of the starches expressed in terms of percentage of total starch gelatinized at definite time-intervals are tabulated under head-

* For convenience the parent- and hybrid-stocks are usually referred to briefly as parents and hybrids. ings that designate the reagents used, and in a form that is well adapted to show parental and parental and hybrid relationships and variations in the reactions of the starches with each reagent. In most of the sets of parents and hybrids 21 reagents were used; in some only 5, usually the same. It would have been desirable to have employed the 21 reagents throughout, and also not only additional reagents, but certain of the reagents in two or more concentrations, but limitations of time, together with other conditions, rendered this practically prohibitory.

By reference to the text of Part II, Chapter I, it will be seen that while making these records both the percentage of the total starch and the percentage of the entire number of grains completely gelatinized were recorded at the ends of the several time-intervals. As will be pointed out later on (Chapter IV, page 170), these two percentages vary greatly in their relationships, and the differences are often of more or less diagnostic importance. It was not, however, found to be desirable to include these figures in the tables here given because any advantage gained would be more than counterbalanced by their interference with the clear-cut presentation of the figures given, nor have they been found to be of sufficient value at present to justify a separate tabulation. The figures recorded in most of the tables do not convey to the mind the same impressions that are exhibited by charts, because they are too numerous and varied; therefore, since these data are of exceptional value in the determination of similarities and dissimilarities of the starches from different plant sources they have been rendered in the form of curves (Charts D 1 to D 691, Chapter IV, page 210), which admirably picture the progress of the several reactions. These charts have been studied somewhat in detail, individually and comparatively, in Section 4 and also in Chapter IV, page $16 \%$ In these experiments records were usually made at time-intervals of $5,15,30,45$, and 60 minutes. Occasionally, when the processes of gelatinization were very rapid, records were made at $1,2,3,4$, or 5 minute intervals, and sometimes, when the processes were exceedingly slow, only at the end of 60 minutes. Rarely records were also made at 10 or 20 minutes, or other periods. Little or no importance is to be attached to differences in the intensities of reactions that are recorded in less than 5 minutes unless the figures are quite different, small differences falling within the limits of error of experiment. In the studies of the velocityreaction curves that constitute Section 4 the data pertaining to the parents were first considered and then those of parents and hybrids and of the hybrids, as in Sections 1 and 2. 
The marked variabilities that are exhibited by the reaction-intensities of the starches of the hybrids in relation to those of the parents, coupled with the importance that is almost invariably attached to intermediateness as a criterion of hybridism, led to the introduction of Section 5, which summarizes the reactionintensities of the starches of the hybrid as regards sameness, intermediateness, excess, and deficit of reaction-values in relation to one or the other parent or both parents. The statements herein are based upon the tables A 1 to A 26, and the Charts D 1 to D 670 in Chapter IV, page 210.' The quantitative relations of the reactions of the hybrid to those of the parents could not in some instances be satisfactorily determined, because usually of too rapid or too slow reactions, variant courses of reaction, or differences that are so small as to fall within the limits of error of experiment; and differences may be seen in the tables that can not be or are not satisfactorily presented in the charts, especially such as may be recorded during the first 5 minutes of the experiments. When the reactions are very rapid, any differentiation must be determined very early, and unless the records differ markedly the hybrid is credited with sameness in relation to one or the other or both parents, as the case may be. Sometimes there may be no differences early in the experiments, but marked differences occur later, in which case the values are determined late, and so on. Occasionally one or more of the curves will take on a variant course, so that the hybrid relationships to one or the other parent or both parents may be different at different periods of the experiment, in which case the relation of the hybrid must be determined by the general impression conveyed by the chart (see Chapter IV, page 168). However, in the vast majority of cases the hybrid and parental relationships are presented quite definitely. It will be seen that particular attention has been given in the statements of intermediateness to note whether or not there is mid-intermediateness, and if not, the inclination to one or the other parent or both parents, and it will be found that intermediateness is an exception rather than a rule. In each of these sections the reactionintensities have been summarized in tabular form that will be found of much value for comparative purposes.

In the preceding sections the starches of the parentstocks and hybrid-stocks have been studied in their histological properties and reactions with each of the various agents and reagents, separately and comparatively, and in a measure collectively; but as yet these reactions have not been so presented as to give a clear picture, as it were, of the reaction-intensities of each starch when collectively considered and of each starch with the others of the set. This has been attempted with a very large measure of success in Section 6. Herein representative reaction-values of each starch elicited by all of the agents and reagents used are so linked as to form a composite curve, and all three or four of the composite curves of the starches of the set are plotted out in the form of a single chart. By this means there is afforded not only a method for the study of parental and hybrid relationships, but also species, generic, and other taxonomic peculiarities. The plan of plotting out these curves is described in Chapter II, Section 12, and these curves are given further consideration, especially from the aspect of plant classification, in Chapter IV, page 172.

It is of importance to note that in the gelatinization reactions the values recorded are in terms of terminal and not progress values-that is, of the time of complete or practically complete gelatinization within 60 minutes or of the percentage of the total starch gelatinized when the process is not or practically not completed within this period. Therefore, when these values are compared with those stated in Sections 4 and 5 , where they are based on reaction-intensities observed during the progress of gelatinization, there may appear to be many discrepancies of statement-discrepancies that depend solely upon different adopted standards of valuation. For instance, turning to Chart E 1, the reaction-values of all four starches in the chromic-acid and sodium-salicylate reactions, respectively, are charted as being in each case the same-that is, in the former, complete or practically complete gelatinization in 30 minutes and in the latter in 5 minutes; while in Sections 4 and 5 these starches are differentiated in each of these reactions. The construction of these composite charts is therefore manifestly seriously faulty, because important differences recorded during the progress of the reactions are in part or wholly ignored, for which reason such charts must have only tentative and otherwise restricted values. Notwithstanding such grave defects, they have a very great measure of usefulness, and it is obvious from the context that in their application to the recognition of parents, hybrids, varieties, species, and genera they should be studied conjointly with the data of the preceding sections of this chapter.

1. Comparisons of Starches of Amaryllis belladonna, Brunstigta josephinde, Brunsdonna SANDERT ALBA, AND BRUNSDONNA SANDERE.

In form the grains of Brunsvigia josephino, in comparison with those of Amaryllis belladonna are less regular in outline and more varied in character, and unlike those of the latter are somewhat flattened. There are aggregates not found in the latter. Compound grains are more numerous and are much more varied in form. A type of compound grain is present that consists of two small components joined by incomplete secondary lamellæ, sometimes by tertiary lamellæ, that is not seen in A maryllis belladonna. Indentations of the margins of the grains may be noted which are absent in the latter. The hilum is more distinct and usually less eccentric. The lamellæ are not so fine, more distinct, much less numerous, and the outermost tend, unlike in Amaryllis belladonna, to be irregular and often not to follow the outline of the grain. In size the average is less, and the grains are broader in proportion to length than in the latter. The polariscopic figure is, on the whole, considerably less eccentric and less distinct; the lines are 
coarser and, as a rule, less oblique, and distortion and bisection are much more frequent; compound grains are much more numerous. With selenite the quadrants are less sharply defined, and impurity of both the blue and orange, due to a greenish tint, is less frequent. In the quantitative and qualitative iodine reactions the coloration is of a deeper blue and more reddish than in Amaryllis belladonna.

In histological characters the grains of Brunsdonna sandera alba are in form closer, on the whole, to those of Amaryllis belladonna, but in some respects closer to Brunsvigia josephina. A type of grain peculiar to this hybrid is noted which consists of an amorphous-looking mass composed of a number of fused grains adherent to the side or distal end of a large grajn-mass, all inclosed in 6 to 12 lamellæ. The hilum more closely resembles that of Amaryllis belladonna; the lamellæ in form and arrangement are closer to those of A maryllis belladonna, but in number they are closer to Brunsvigia josephine; in size and in proportions of length to breadth they are closer to Amaryllis belladonna; in polariscopic figures and lines and selenite reactions and in the qualitative iodine reactions they exhibit a closer relationship to Amaryllis belladonna. The qualitative rèactions with the chemical reagents are, on the whole, much closer to Amaryllis belladonna than to Brunsvigia josephino.

In histological characters the grains of Brunsdonna sandero are in form much nearer to those of Amaryllis belladonna than to those of the other parent, but they are not so near those of Amaryllis belladonna as those of the other hybrid, and not so near Brunsvigia josephince in the number and type of compound grains as those of the other hybrid. The hilum is the same as in the other hybrid, and hence nearer that of Amaryllis belladonna. It differs from the hilum of the other hybrid in being less often fissured; but it is more often fissured than in either parent. In character and eccentricity of the hilum these grains are nearer those of the parents than those of the other hybrid. The lamellæ in character and arrangement closely resemble those of the other hybrid and are closer to those of Amaryllis belladonna than to those of the other parent, but in numbers they are closer to Brunsvigia josephino. In the ratio of length to breadth of the grains, and in larger grains in length, it is nearer to Amaryllis belladonna; but in the length of the common-sized grains it is nearer to Brunsvigia josephince. In polariscopic properties in the character of the figure and appearance with selenite this hybrid is closer to Amaryllis belladonna than to the other parent, but not so close as the other hybrid. In qualitative iodine reactions it is closer to Amaryllis belladonna, but not so close as the other hybrid. In the qualitative reactions with the chemical reagents close relationship is shown to Amaryllis belladonna and to the other hybrid, but closer on the whole to this parent than to the latter. In some respects the reactions are closer to Brunsvigia josephina than to Amaryllis belladonna, showing the influences of both parents. In the chloral-hydrate, nitric-acid, potassiumsulphocyanate, and sodium-salicylate reactions it is closer to Amaryllis belladonna than to the other hybrid, but in the cobalt-nitrate, copper-nitrate, and cupric-chloride reactions it is closer to the other hybrid.
Reaotion-Intensities Eapressed by Light, Color, and Tempera-

Polarization:

A. belladonna, very high, value 97

B. josephinæ, moderately high to very high, value 85 .

B. sanderœe alba, very high, value 97 .

B. sandero, very high, value 95 .

Iodine:

A. belladonna, moderate to moderately deep, value 55 .

B. josephinæ, moderately deep, value 60 .

B. sandero alba, moderate to moderately deep, value 55 .

B. sanderce, moderate to moderately deep, value 55 .

Gentian violet:

A. belladonna, moderate to moderately deep, value 55 .

B. josephinæ, moderate to deep, value 57 .

B. sanderoe alba, moderately deep, value 60 .

B. sanderce, moderately deep, value 63 .

Safranin:

A. belladonna, moderate to moderately deep, value 55 .

B. josephinæ, moderate, value 53.

B. sanderœ alba, moderately deep, value 60 .

B. sanderœ, moderately deep, value 60 .

Temperature:

A. belladonna, majority at 70 to $71^{\circ}$, all but distal part of rare grains 72.5 to $73^{\circ}$, mean $72.7^{\circ}$.

B. josephinæ, majority at 65 to $66^{\circ}$, all but rare grains at 70 to $72^{\circ}$, mean $71^{\circ}$

B. sanderce alba, majority at 70 to $71^{\circ}$, all but distal part of rare grains 71.5 to $73^{\circ}$, mean $72.25^{\circ}$

B. sanderœ, majority at 70 to $71.5^{\circ}$, all but distal part of rare grains 72 to $72.5^{\circ}$, mean $72.25^{\circ}$.

The starch of Amaryllis belladonna in comparison with that of Brunsvigia josephince shows higher polarization and safranin reactions, and lower iodine, gentianviolet, and temperature reactions. In the polarization, iodine, safranin, and temperature reactions both hybrids are distinctly closer to Amaryllis belladonna than to the other parent-Brunsdonna sandero alba showing as a whole a slightly closer relationship than the other hybrid; in the gentian-violet reactions they show greater closeness to Brunsvigia josephina, the closer being Brunsdonna sandero alba. In the gentian-violet and safranin reactions both hybrids show higher reactivities than either parent, and the same or almost identical reactivities as those of Amaryllis belladonna in the polarization, iodine, and temperature reactions.

Table A 1 shows the reaction intensities in percentages of total starch gelatinized at definite intervals (minutes).

\section{Velocity-Reaction Curves.}

This section considers velocity-reaction curves of the starches of Amaryllis belladonna, Brunsvigia josephince, Brunsdonna sanderce alba, and Brunsdonna sanderce, showing the quantitative differences in the behavior toward different reagents at definite time-intervals. (Charts D 1 to D 21.)

The Amaryllis and Brunsvigia curves tend, in reactions with nitric acid, sulphuric acid, hydrochloric acid, potassium hydroxide, potassium iodide, potassium sulphocyanate, potassium sulphide, sodium hydroxide, sodium sulphide, uranium nitrate, cobalt nitrate, and barium chloride, to keep very close together; while in reactions with chloral hydrate, chromic acid, pyrogallic acid, sodium salicylate, calcium nitrate, strontium nitrate, copper nitrate, cupric chloride, and mercuric chloride there is a well-marked separation during some important part, or the whole, of the 60 -minute period. In the chloral-hydrate reactions the curves are very close up to the 15-minute record, at which time they begin 
to diverge, showing at the end of 60 minutes a difference of 14 per cent in the total starch gelatinized. In the reactions with chromic acid, pyrogallic acid, copper nitrate, and cupric chloride the greatest differences are noted at the end of the 5-minute period, and in the mercuric-chloride reactions at the end of 60 minutes.*

The curves of the hybrids Brunsdonna sanderae alba and $B$. sanderoe likewise tend to keep close together in more than half of the reactions, and in even a larger number than in the parents. Tendency to a well-marked separation of the two hybrid curves is seen in the reactions with sodium hydroxide, sodium sulphide, calcium nitrate, uranium nitrate, and copper nitrate. There is not a constant relationship of the parental and hybrid curves; for instance, the parental curves may be very close to one another, while the hybrid curves are well separated from them and even from each other, as in the latter case, in the sodium-sulphide reactions; or all four curves may be well separated, as in the calciumnitrate reactions; or the parental curves may be fairly

* Notes on the Reactive-Intensities of the Brunsdonnce Starches.The reactions of these starches have been found at times to be quite erratic, especially with sodium hydroxide and potassium sulphide, and they appear to be affected by variations in temperature, pressure, and humidity and certain other attendant conditions to a marked degree, whereas most if not all other starches studied are either but very little or not at all influenced by corresponding conditions. There may be considerable variation in the percentage-gelatinization at different parts of the slide, so that it is always quite important that the observations with these starches be made in center of the field even though the cover-slip be sealed in the manner stated in Chapter II. Sometimes the reaction appeared to be more rapid at the margin of the cover and at other times at the central part of the preparation. Then again, where the grains are crowded the reaction appeared to be considerably retarded. The crowding may be apparent, particularly in clumps of grains that have been massed after the addition of the reagent.

TABLe A 1.

\begin{tabular}{|c|c|c|c|c|c|c|c|c|c|c|}
\hline & $\underset{-1}{\dot{a}}$ & $\underset{\text { N }}{\text { d }}$ & $\underset{\infty}{\dot{\theta}}$ & $\underset{*}{\text { 日 }}$ & $\underset{20}{\text { 日 }}$ & 영 & $\underset{2}{d}$ & घं & 泉 & $\begin{array}{l}\text { в } \\
8\end{array}$ \\
\hline \multicolumn{11}{|l|}{ Chloral hydrate: } \\
\hline A. belladonna......... & $\cdots$ & $\cdots$ & $\cdots$ & $\cdots$ & 12 & . & 50 & 85 & 92 & 96 \\
\hline B. josephinæ......... & . & . & .. & .. & 9 & $\ldots$ & 46 & 74 & 78 & 82 \\
\hline B. sand. alba. & . & . & $\cdots$ & $\cdots$ & 10 & . & 75 & 95 & 97 & 98 \\
\hline B. sanderœ... & . & . & .. & . & 15 & . & 85 & 98 & 99 & 99 \\
\hline \multicolumn{11}{|l|}{ Chromic acid: } \\
\hline A. belladonna......... & $\cdots$ & $\cdots$ & $\cdots$ & $\cdots$ & 10 & . & 70 & 99 & . & $\cdots$ \\
\hline B. josephinæ......... & . & $\cdots$ & $\ldots$ & . & 30 & . & 85 & 99 & $\ldots$ & . \\
\hline B. sand. alba.. & .. & . & . & . & 3 & . & 80 & 100 & . & . \\
\hline B. sanderœ.... & . & . & . & . & 1 & . & 80 & 99 & . & . \\
\hline \multicolumn{11}{|l|}{ Pyrogallic acid: } \\
\hline A. belladonna ......... & $\cdots$ & $\cdots$ & . & . & $\mathbf{5}$ & $\cdots$ & 40 & 75 & 85 & 90 \\
\hline B. josephinæ. ........ & . & . & . & $\cdots$ & 32 & . & 64 & 98 & . & 99 \\
\hline B. eand. alba....... & . & . & . & - & 1 & & 2 & 10 & 12 & 12 \\
\hline B. sanderce. . . . . . . . & .. & . & . & . & 1 & . & 0.5 & 4 & 7 & 7 \\
\hline \multicolumn{11}{|l|}{ Nitric acid: . . . . . . . . } \\
\hline A. belladonna......... & 95 & 99 & $\cdots$ & $\cdots$ & $\cdots$ & $\cdots$ & $\cdots$ & $\ldots$ & $\ldots$ & . \\
\hline B. josephing... & 80 & 93 & 95 & $\therefore$ & 98 & . & . & .. & . & . \\
\hline B. sand. alba... & 73 & 88 & 98 & . & 99 & • & & . & . & . \\
\hline B. sanderce. . . . & 35 & 65 & 82 & .. & 98 & . & 99 & $\ldots$ & $\ldots$ & . \\
\hline \multicolumn{11}{|l|}{ Sulphuric acid: } \\
\hline A. belladonna.. & 95 & 100 & $\cdots$ & . & $\cdots$ & $\cdots$ & $\cdots$ & $\ldots$ & $\cdots$ & $\ldots$ \\
\hline B. josephins . . & 88 & 99 & . & $\ldots$ & . & $\cdots$ & . & $\ldots$ & $\cdots$ & . \\
\hline B. sand. alba... & 95 & 100 & . & . & . & . & $\ldots$ & $\ldots$ & $\ldots$ & . \\
\hline B. sanderce....... & 95. & 100 & . & . & . & $\ldots$ & . & $\ldots$ & . & . \\
\hline \multicolumn{11}{|l|}{ Hydrochloric acid: } \\
\hline A. belladonna... & 95 & 99 & $\ldots$ & $\ldots$ & $\cdots$ & $\cdots$ & $\ldots$ & $\cdots$ & $\cdots$ & . \\
\hline B. josephinø. ... . & 90 & 95 & 99 & . & .. & . & . & $\ldots$ & .. & . \\
\hline B. sand. alba.... & 50 & 95 & 99 & .. & . & . & . & . & . & . \\
\hline B. sanderce........... & 30 & 90 & 87 & 89 & . & . & & .. & . & . \\
\hline
\end{tabular}

TABLE A 1.-Continued.

\begin{tabular}{|c|c|c|c|c|c|c|c|c|c|c|}
\hline & 离 & $\underset{\text { घ }}{\text { घे }}$ & 离 & 昌 & 百 & $\begin{array}{l}\text { 日் } \\
\stackrel{-}{-}\end{array}$ & $\begin{array}{l}\dot{g} \\
\stackrel{2}{2}\end{array}$ & 离 & $\begin{array}{l}\text { 泉 } \\
\text { s }\end{array}$ & $\begin{array}{l}\text { घं } \\
\text { \& }\end{array}$ \\
\hline 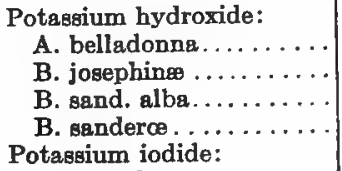 & $\begin{array}{r}100 \\
98 \\
100 \\
100\end{array}$ & $\begin{array}{l}\cdots \\
\cdots \\
\cdots\end{array}$ & $\begin{array}{l}. \\
\cdots \\
\cdots\end{array}$ & $\begin{array}{l}\cdots \\
\cdots \\
\cdots\end{array}$ & $\begin{array}{c}\ddot{\theta 9} \\
\cdots \\
\therefore\end{array}$ & $\begin{array}{l}\cdots \\
\ldots \\
\cdots\end{array}$ & $\begin{array}{l}\ldots \\
\cdots \\
\cdots\end{array}$ & $\begin{array}{l}\cdots \\
\cdots \\
\cdots\end{array}$ & $\begin{array}{l}\cdots \\
\cdots \\
\cdots \\
.\end{array}$ & $\begin{array}{l}\ldots \\
\cdots \\
\cdots\end{array}$ \\
\hline
\end{tabular}

Potassium iodide:

A. belladonna .

B. josephinm.

B.

B. sanderœ .

Potassium sulphocyanate:

A. belladonna ..........

B. josephins.

B. sand. alba............

B. sanderce..........

Potassium sulphide:

A. belladonna.........

B. јовеphinm...........

B. sand. alba..........

B. sanderœe.

Sodium hydroxide:

A. belladonna .

B. josephinse........

B. sand. alba.

B. sandero ...........

Sodium sulphide:

A. belladonna

B. josephinæ.

B. sand. alba.

B. sanderos...
Sodium salicylate:

A. belladonna.

B. josephinæ.

B. sand. alba

B. sanderc.

Calcium nitrate:

A. belladonna

B. josephinx.

B. sand. alba.

B. sanderœ...

Uranium nitrate:

A. belladonna

B. josephinæ.

B. sanderce.

Strontium nitrate:

A. belladonna.

B. јosephinæ............

B. sand. albe.

B. sanderce

Cobalt nitrate:

A. belladonna

B. josephins . . . . . . . .

B. sand. alba ..........

B. sanderce .

Copper nitrate:

A. belladonna

B. josephing.

B. sand. alba.

B. sanderœ.

Cupric chloride:

A. belladonna

B. josephins.

B. sand. alba

B. sanderœ.

Barium chloride:

A. belladonna.

B. josephinæ.

B. sand. alba...........

B. sanderce .

Mercuric chloride:

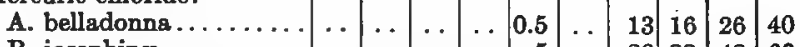

B. josephinx.........

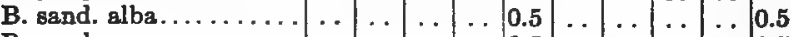

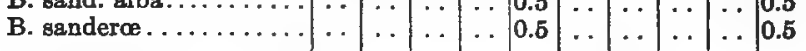


well separated but the hybrid curves very close together, as in the cupric-chloride reactions. (See following section.)

Amaryllis in some reactions shows a higher reactivity than Brunsvigia, in others the reverse, and in others no essential difference. There is higher reactivity of Amaryllis with chloral hydrate, potassium sulphide, sodium hydroxide, sodium salicylate, calcium nitrate, uranium nitrate, strontium nitrate, cobalt nitrate, copper nitrate, and cupric chloride; but a lower reactivity with chromic acid, pyrogallic acid, sodium sulphide, barium chloride, and mercuric chloride. No essential differences are noted in the reactions with nitric acid, sulphuric acid, hydrochloric acid, potassium hydroxide, and potassium iodide, because of the great rapidity of the reactions, while in the potassium-sulphocyanate reactions an important difference is noted only at the end of the 5-minute period.

Comparing the parental and hybrid curves (eliminating reactions with nitric acid, sulphuric acid, hydrochloric acid, and potassium hydroxide because of their high rapidity obscuring differences), it will be observed that the curves tend to be grouped in couples corresponding to parents and hybrids, each couple taking its own course, which may be similar or dissimilar to the course of the other couple; that the parental curves are lower than those of the hybrids in the reaction with chloral hydrate; that the parental curves are higher than those of the hybrids in the reactions with pyrogallic acid, potassium iodide, potassium sulphocyanate, sodium hydroxide, sodium sulphide, calcium nitrate, uranium nitrate, cobalt nitrate, copper nitrate, cupric chloride, barium chloride, and mercuric chloride; and that the parental curves tend to be intermediate, or approximately so, in those with potassium sulphide, sodium salicylate, and strontium nitrate. In the chromic-acid reactions all four curves run very close together, the only notable difference being seen at the end of 5 minutes, at which time the parental curves are higher than the hybrid curves, very soon after which the hybrid curves tend to intermediateness. The most remarkable feature of these curves, as a whole, is seen in most of the reactions in the more or less markedly lower degree of reactivity of the hybrids than of the parents.

The curves of the hybrids tend, as a rule, to keep close together, there being a well-marked inclination to separation in only the reactions with sodium hydroxide, sodium sulphide, calcium nitrate, uranium nitrate, and copper nitrate. In reactions of the hybrids with nitric acid, sulphuric acid, hydrochloric acid, and potassium hydroxide, gelatinization occurs so rapidly that no satisfactory differentiation can be made; but in the reactions with chloral hydrate, potassium iodide, potassium sulphocyanate, potassium sulphide, sodium hydroxide, sodium salicylate, calcium nitrate, uranium nitrate, cobalt nitrate, and copper nitrate the curves of Brunsdonna sanderce alba are lower than those of the other hybrid; and they are practically the same in the reactions with chromic acid, pyrogallic acid, strontium nitrate, cupric chloride, barium chloride, and mercuric chloride.

A marked early period of resistance that is followed by a moderate to rapid reaction is observed in these four starches in comparatively few instances. In some it is observed in all four starches, as in the chloral-hydrate reactions; in others, in one, two, or three, as the case may be, as in the reactions with chromic acid, pyrogallic acid, potassium iodide, and sodium hydroxide. In a number of the reactions either a very rapid reaction occurs at once, particularly with the mineral acids, potassium hydroxide, and potassium sulphide, or a very slow reaction, as with barium chloride and mercuric chloride. Both types of reaction may be present, as with potassium sulphocyanate; in other instances there may be various forms of combination and gradation of these types of curves.

The courses of the curves are not identical with any two reagents (excepting in the case of nitric acid, sulphuric acid, hydrochloric acid, and potassium hydroxide, in which it is shown that the reactions occur too quickly for any or at least an entirely satisfactory differentiation), so that each reagent carries with its reactions the stamp of individuality. While in case of some of the charts the curves at first glance may convey the impression of close similarity, as in the reactions with sodium sulphide, uranium nitrate, copper nitrate, and cupric chloride, even a superficial examination will show well-defined differences. The parental curves are very nearly alike in their course, but with the important exception that in the sodium-sulphide reactions the $A$ maryllis curve is the lower, while in the other three reactions it is the higher-a striking difference. The hybrid curves in the four reactions do not correspond in their courses with the peculiarities of the parental curves, and in no two are they identical. The curve of Brunsdonna sanderce alba is always the lowest, and the curves of both hybrids show a direct quantitative relationship to the parental curves in so far as when the parental curves are lower the hybrid curves are lower. While the parental curves tend to run closely together the two hybrid curves exhibit some degree of independence, not only of the parents but also of each other.

The earliest period during the 60 minutes at which the curves are best separated for differential purposes is variable with the different reagents, and in some instances no definite time can be stated, owing to extreme rapidity of the reactions, while in other instances statements must be made with reserve. Approximately, this period is noted at the end of 3 minutes in the potassiumsulphide reactions; at the end of 5 minutes in the reactions with chromic acid, potassium iodide, potassium sulphocyanate, sodium hydroxide, sodium salicylate, strontium nitrate, and cupric chloride; at the end of 15 minutes in the reactions with chloral hydrate, sodium sulphide, calcium nitrate, uranium nitrate, and copper nitrate; at the end of 30 minutes in the reactions with pyrogallic acid; and at the end of 60 minutes in the reactions with calcium nitrate, barium chloride, and mercuric chloride.

\section{REACTION-INTENSITIES OF THE HYBRIDS.}

This section treats of the reaction-intensities of the hybrids as regards sameness, intermediateness, excess, and deficit in relation to those of the parents. (Table A 1 and Charts D 1 to D 21.)

The reactivities of Brunsdonna sanderce alba are the same as those of the seed parent in reactions with polar- 
ization and iodine, sulphuric acid, and barium chloride; the same as those of the pollen parent in none; the same as those of both parents in the potassium-hydroxide reaction in which the reactions occur with great rapidity; intermediate in the temperature reactions and those of chromic acid, potassium sulphide, sodium salicylate, and strontium nitrate (in two being closer to the seed parent and in three being mid-intermediate); highest in the reactions with gentian violet, safranin, and chloral hydrate (in two being closer to the pollen parent, and in one closer to the seed parent) ; and lowest in the reactions with pyrogallic acid, nitric acid, hydrochloric acid, potassium iodide, potassium sulphocyanate, sodium hydroxide, sodium sulphide, calcium nitrate, uranium nitrate, cobalt nitrate, copper nitrate, cupric chloride, and mercuric chloride (in four being closer to the seed parent, in eight being closer to the pollen parent, and in one being as close to one as to the other parent).

The reactivities of Brunsdonna sanderce are the same as those of the seed parent in the reactions with iodine, temperature, sulphuric acid, potassium sulphide, sodium salicylate, strontium nitrate, and barium chloride; the same as those of the pollen parent in none; the same as those of both parents in the potassium-hydroxide reaction, in which the reactions oecur with great rapidity; intermediate in the polarization and strontium nitrate (in one being closer to the seed parent and in one being mid-intermediate) ; highest in the reactions with gentian violet, safranin, and chloral hydrate (in two being closer to the seed parent, and in one closer to the pollen parent) ; and lowest in the reactions with chromic acid, pyrogallic acid, nitric acid, hydrochloric acid, potassium iodide, potassium sulphocyanate, sodium hydroxide, sodium sulphide, calcium nitrate, uranium nitrate, cobalt nitrate, copper nitrate, cupric chloride, and mercuric chloride (in 3 being closer to the seed parent, in 8 closer to the pollen parent, and in 3 being as close to one as to the other parent).

The hybrids differ in their parental relationships in the polarization, the safranin and temperature reactions, and in those of chromic acid, potassium iodide, potassium sulphide, sodium salicylate, strontium nitrate, and cobalt nitrate. In the polarization reactions one is the same as the seed parent, the other intermediate, but nearer the seed parent. In the safranin reactions both are highest, but one closer to the pollen parent and the other to the seed parent. In the temperature reactions one is intermediate and closer to the seed parent, and the other the same as the seed parent. In the chromic-acid reactions one is mid-intermediate, and the other the lowest, but closer to the pollen parent. In the potassium-iodide reactions both are the lowest; one is closer to the seed parent, and the other as close to one as to the other parent. In the potassium-sulphide reactions one is midintermediate and the other the same as the seed parent. In the sodium-salicylate reactions one is intermediate and closer to the seed parent and the other the same as the seed parent. In the strontium-nitrate reactions both are intermediate, one being mid-intermediate and the other closer to the seed parent. In the cobalt-nitrate reactions both are highest, but one is closer to the pollen parent and the other as close to one as to the other parent.
The following table is a summary of the reactionintensities :

\begin{tabular}{|c|c|c|}
\hline & $\begin{array}{l}\text { B. sande- } \\
\text { rœe alba. }\end{array}$ & $\begin{array}{l}\text { B. sande- } \\
\text { rœ. }\end{array}$ \\
\hline Same as seed parent... & 4 & 6 \\
\hline Same as pollen parent.. & $\mathbf{0}$ & 0 \\
\hline Same as both parents... & 1 & 1 \\
\hline Intermediate......... & $\mathbf{5}$ & 2 \\
\hline Highest............ & 3 & 3 \\
\hline Lowest.......... & 13 & 14 \\
\hline
\end{tabular}

In none of the reactions of either hybrid is the reaction the same as that of the pollen parent, while there are 10 reactions of the 52 which are the same as those of the seed parent. The dominating influence of the seed parent, Amaryllis belladonna, on the properties of the starch of the hybrid are well marked.

\section{Composite Curves of the Reaction-intensities.}

This section treats of the composite curves of the reaction-intensities showing the differentiation of the starches of Amaryllis belladonna, Brunsvigia josephince, Brunsdonna sanderce alba, and Brunsdonna sanderce. (Chart E 1.)

The most conspicuous features of this chart may be summed up as follows:

(1) Taking the curves of Amaryllis belladonna as a standard of comparison, it will be noted that the curve of Brunsvigia josephince follows it very closely in the up-and-down courses except in the reactions with pyrogallic acid, potassium sulphide; and calcium nitrate, here and there crossing in accordance with higher or lower reactivity. Except the three reactions noted and those with uranium nitrate, copper nitrate, and cupric chloride, the curves keep close together. These departures indicate species widely separated and belonging either to a given genus or to two closely related genera, in this case the latter.

(2) It will be noted that the reactions of Amaryllis belladonna are higher than those of Brunsvigia josephince in polarization and in the reactions with safranin, chloral hydrate, potassium sulphide, sodium hydroxide, calcium nitrate, uranium nitrate, strontium nitrate, cobalt nitrate, copper nitrate, and cupric chloride; lower in those with iodine, gentian violet, temperature of gelatinization, pyrogallic acid, barium chloride, and mercuric chloride; and the same or practically the same in those with chromic acid, nitric acid, sulphuric acid, hydrochloric acid, potassium hydroxide, potassium iodide, potassium sulphocyanate, sodium sulphide, sodium salicylate, and cobalt nitrate.

(3) In Amaryllis belladonna the very high polarization and reactions with nitric acid, sulphuric acid, hydrochloric acid, potassium hydroxide, potassium iodide, potassium sulphide, sodium hydroxide, sodium salicylate, calcium nitrate, strontium nitrate; the high reactions with chromic acid, potassium sulphocyanate, uranium nitrate, copper nitrate, and cupric chloride; the moderate reactions with iodine, gentian violet, safranin, temperature, chloral hydrate, pyrogallic acid, and sodium sulphide; the low reactions with cobalt nitrate, and very low reactions with barium chloride and mercuric chloride.

(4) In Brunsvigia josephina the very high polarization and reactions with nitric acid, sulphuric acid, hydro- 
chloric acid, potassium hydroxide, potassium iodide, sodium hydroxide, sodium salicylate; the high reactions with iodine, chromic acid, pyrogallic acid, potassium sulphocyanate, and strontium nitrate; moderate reactions with gentian violet, safranin, temperature of gelatinization, potassium sulphide, sodium sulphide, calcium nitrate, and uranium nitrate; the low reactions with chloral hydrate, cobalt nitrate, copper nitrate, cupric chloride, and mercuric chloride; and the very low reactions with barium chloride.

(5) In the hybrids Brunsdonna sanderce alba and Brunsdonna sanderce the very high polarization and reactions with nitric acid, sulphuric acid, hydrochloric acid, potassium hydroxide, potassium sulphide, sodium salicylate, and strontium nitrate; the high reactions with gentian violet, safranin, chloral hydrate, and chromic acid; the moderate reactions with iodine and temperature of gelatinization; the low with potassium iodide, sodium hydroxide, calcium nitrate, and uranium nitrate; and the very low with pyrogallic acid, potassium sulphocyanate, sodium sulphide, cobalt nitrate, copper nitrate, cupric chloride, barium chloride, and mercuric chloride. The following is a summary of the reaction-intensities:

\begin{tabular}{l|c|c|c|c|c}
\hline & $\begin{array}{c}\text { Very } \\
\text { high. }\end{array}$ & High. & $\begin{array}{c}\text { Moder- } \\
\text { ate. }\end{array}$ & Low. & $\begin{array}{c}\text { Very } \\
\text { low. }\end{array}$ \\
\hline A. belladonna...... & 11 & 5 & 7 & 1 & 2 \\
B. josephinm....... & 8 & 5 & 7 & 5 & 1 \\
B. sand. alba........ & 8 & 4 & 2 & 4 & 8 \\
B. sanderø........ & 8 & 4 & 2 & 4 & 8 \\
\hline
\end{tabular}

(6) In the curves of the hybrids which show in the first place a very close correspondence with each other, and in the second place a closer correspondence, on the whole, with the curves of Amaryllis belladonna than with those of Brunsvigia josephina, the hybrid curves are for the most part either lower than or practically the same as the Amaryllis curves, in only four instances are the curves higher, and then in an unimportant degree.

Notes on Amartluis, Bronsvigia, and Bronsdonnas.

The botanist has assigned Amaryllis belladonna and Brunsvigia josephinoe to separate genera. Upon the basis of the peculiarities of their starches in their histologic properties and reactions with the various agents and reagents, it seems that these species may be regarded as being members of either closely related genera or wellseparated species of the same genus, such as representatives of subgenera; but the data are too limited to justify more than speculation. The most remarkable features of these records are: (1) in the hybrids the many extraordinary low or high reactivities, especially the former, that exceed the parental extremes, this being noted in 15 out of the 26 reactions; (2) the absence of sameness of any reaction as that of the pollen parent; (3) the sameness of the reaction as that of the seed parent in 4 reactions of one and 6 reactions of the other hybrid. The marked departures of the hybrid curves shown in excessive or deficient reactivities in comparison with the reactivities of the parents seem to be more suggestive of bigeneric parents than of parents belonging to the same genus.
BRUNSDONNA TUBERGENI, ETC.

This additional matter treats of descriptions of Brunsdonna tubergeni, Amaryllis parkeri, and $A$. parkeri alba ( $A$. belladonna lewensis alba), and comparisons of the starches of $B$. tubergeni, $A$. parkeri alba, Brunsdonna sanderce alba, and $B$. sandero.

Brunsdonna tubergeni, $A$. parkeri, and A. parkeri $a l b a$ are of especial interest in conjunction with the foregoing studies of the Amaryllis-Brunsvigia-Brunsdonna group because: the first is known to be a hybrid of Brunsvigia and Amaryllis; the second is looked upon as being probably a Brunsvigia-Amaryllis hybrid; the third is a variety of the second and is regarded as being the same as $A$. belladonna kewensis alba, the parentage of which is unknown; and the last two are known hybrids of Amaryllis-Brunsvigia, but without positive knowledge of the direction of the cross. Appertaining to the foregoing, the following data appeared in The Gardeners' Chronicle, 1909, XLV, 5\%; 1911, L, 210 :

Brunsdonna tubergeni: Mr. C. G. Tubergen, Jr., thus describes the circumstances of a cross between Brunvigia josephinoe and Amaryllis belladonna:

Principally with a view of ascertaining the parentage of the Kew variety of Amaryllis belladonna (see illustration in The Garden, November 19, 1898; also notes in The Gardeners' Chronicle, February 9, 1901, etc.), in the autumn of 1892 I artificially impregnated Brunsvigia josephina with the pollen of Amaryllis belladonna. Seeds formed freely, as the two genera, Bmunsvigia and Amaryllis, are very nearly related. As could be foreseen, with slow-growing Brunsvigia josephince as the female parent, a long time had to elapse before the seedling plants would be strong enough to reach flowering size. After 16 years of patient waiting, two of the strongest bulbs produced flower-spikes in September of last year. When the hybrid plants had been growing for a few seasons it became evident that they differed in habit from the Kew variety of Amaryllis belladonna, which produces a leaf-stem of about 4 inches high, whereas my hybrids all bear the character of Brunsvigia josephina in the foliage, leaves being formed directly above the neck of the bulbs. The infusion of belladonna blood is clearly shown in the bulbs, as these resemble those of the belladonna and produce offsets freely, whilst Brunsvigia never produces offsets. A comparison of the supplementary illustration, which was drawn by Mr. Worthington Smith from the inflorescensce sent from my garden, with the engraving in the Garden above cited, leads to the conclusion that the Kew plant can no longer be regarded as a hybrid between these species, unless it was a cross effected in the reverse way, taking Amaryllis belladona as the female plant. In that case the variety blanda must have been used, it being the only variety of $\boldsymbol{A}$. belladonna known which produces a leaf-stem. The color of the flowers of my hybrid was a clear, deep rose, suffused with carmine. A single spike produced 22 flowers.

Amaryllis parkeri (hyb.). This is assumed to be a hybrid between Brunsvigia josephino and Amaryllis belladonna. It differs in the form of the umbel from $A$. belladonna, being quite circular and carrying some 30 flowers and buds. The flowers are of a deep rose shade, with white and orange at the base and orange-colored on the exterior of the tube. It is distinct from the ordinary $A$. belladonna, possesses greater vigor, and has a stem some 3 feet in length. This plant is almost identical with the plant known as the Kew variety of $A$. belladonna, which is also $A$. parkeri, being the same cross and varying only in being a better rose color with less orange shade. Mr. Hudson informed us that his Amaryllis was shown as A. belladonna "Kew variety," because it was received under this name from an amateur cultivator in New Zealand some six years ago. This is the first season of flowering at Gunnersberry House. It may prove to be Mr Van Tubergen's plant, which he obtained from crossing Brunsvigia with Amaryllis belladonna. Mr. Tubergen's hybrid formed the subject of a supplementary illustration in The Gardeners' Chronicle, January 23, 1909. 
Amaryllis parkeri alba. This plant is evidently a variety of $A$. parkeri. It possesses a fine umbel, a large number of flowers almost pure white but with the same orange shading at the base as in the flower described above. It is a most striking and distinct novelty. The origin was not stated, but everything points to the same cross. This was shown as $A$. bella donna kewenis alba by Mr. Worsley, Mandeville House, Isleworth.

Brunsdonna sanderce alba. In this case the umbel resembled typical $A$. belladonna in formation, being one-sided rather than globular. This plant is also the result of a cross between Brunsvigia and Amaryllis belladonna, but there is not sufficient information to determine whether the parentage is the same as in the case of $A$. parkeri.

Comparative examinations of a preliminary character were made of the starches of $A$. parkeri alba, Brunsdonna tubergeni, Brunsdonna sanderoe alba, and $B$. sandera, as follows:

Histologic Properties.-All of these starches are alike in that all have very few compound grains which consist of two components, and all have very few aggregates which usually are in the form of doublets of equal size, but occasionally as triplets that are linearly arranged. The grains of $A$. parkeri alba and of Brunsdonna sanderce alba, and $B$. sanderce have about the same degree of irregularity of surface, while those of $B$. tubergeni are much more irregular than the preceding, the irregularities in all being due to the same causes. The conspicuous forms of the grains of $A$. parkeri alba and of $B$. sanderoe alba and $B$. sanderce are very much alike, but those of the first are more slender and elongated than those of the two latter. The grains of $B$. tubergeni are, as a rule, intermediate in slenderness between those of $A$. parkeri and $B$. sandera alba, and $B$. sanderce, but closer to those of the latter; and there is a conspicuousness of elliptical, irregularly triangular, and nearly round grains. The hila of the grains of $A$. parkeri alba and those of $B$. sanderce alba and $B$. sanderce show the same degree of distinctness, and in all three more distinctness than in $B$. tubergeni. The eccentricity is about the same in all four starches. The lamellæ of $A$. parkeri alba and $B$. tubergeni are more distinct and more often coarse than those of $B$. sanderce alba and $B$. sanderce, otherwise they are practically the same in all four starches except that in $B$. tubergeni, in which they are somewhat more often irregular than in the others. In size the grains of $B$. sanderce $a l b a$ and $B$. sanderce are smallest, those of $A$. parkeri alba intermediate, and those of $B$. tubergeni largest; but there are no marked differences.

Polariscopic Properties.--The polariscopic figure is very nearly the same in all four starches, but it is more often irregular in $B$. tubergeni than in the others. The degree of polarization is practically the same in all of the starches.

Iodine Reactions.-With 0.25 per cent Lugol's solution $A$. parkeri alba, $B$. sandera alba, and $B$. sanderee color about equally and from 3 to 5 units more than B. tubergeni.

Aniline Reactions.-With gentian violet $A$. parkeri alba, $B$. sanderce alba, and $B$. sanderce color about the same and about 5 units less than $B$. tubergeni. With safranin the results are practically the same as the foregoing, but there is somewhat less variation of coloring of the grains of $B$. tubergeni than of the starches.
The temperatures of gelatinization are as follows (degrees) :

\begin{tabular}{|c|c|c|c|}
\hline & Majority at- & Complete at- & Mean. \\
\hline 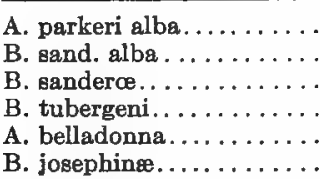 & $\begin{array}{ll}71.5 & \\
70 & \text { to } 71.5 \\
70 & \text { to } 71.5 \\
62 & \text { to } 63.5 \\
70 & \text { to } 71 \\
65 & \text { to } 66\end{array}$ & $\begin{array}{l}74.2 \text { to } 76 \\
71.5 \text { to } 73 \\
72 \text { to } 72.5 \\
64 \text { to } 65.5 \\
72.5 \text { to } 73 \\
70 \text { to } 72\end{array}$ & $\begin{array}{l}75.1 \\
72.25 \\
72.75 \\
64.75 \\
72.7 \\
71\end{array}$ \\
\hline
\end{tabular}

The reaction of $A$. parkeri alba with sulphuric acid begins immediately. Complete gelatinization occurs in about 3 per cent of the entire number of grains and 10 per cent of the total starch in 15 seconds; in about 70 per cent of the grains and 80 per cent of the total starch in 30 seconds; in about 96 per cent of the grains and 98 per cent of the total starch in 45 seconds; and in about 99 per cent of the grains and over 99 per cent of the total starch in 1 minute. The reactions of Brunsdonna sanderce alba and $B$. sanderee with sulphuric acid are given on pages 389 and 394, Part II, and Chart D 5.

The reactions of Brunsdonna tubergeni with sulphuric acid begin immediately. Complete gelatinization occurs in about 80 per cent of the entire number of grains and 90 per cent of the total starch in $30 \mathrm{sec}-$ onds; in about 99 per cent of the grains and in more than 99 per cent of the total starch in 45 seconds; and in 100 per cent of the starch in 1 minute.

The reaction of $A$. parkeri alba with potassium iodide begins in a few grains in 30 seconds. Complete gelatinization occurs in about 1 per cent of the entire number of grains and 65 per cent of the total starch in 5 minutes; in about 20 per cent of the grains and 75 per cent of the total starch in 15 minutes; in about 32 per cent of the grains and 88 per cent of the total starch in 30 minutes; in about 52 per cent of the grains and 90 per cent of the total starch in 45 minutes; and with little if any further advance in 60 minutes.

The reactions of $B$. sanderce alba and $B$. sandero with potassium iodide are given on pages 389 and 394, Part II, and Chart D 8.

The reaction of $B$. tubergeni with potassium iodide begins immediately. Complete gelatinization occurs in 59 per cent of the entire number of grains and 95 per cent of the total starch in 5 minutes; in about 95 per cent of the grains and in more than 99 per cent of the total starch in 15 minutes.

The reaction of $A$. parkeri alba with sodium hydroxide begins immediately. Complete gelatinization occurs in about 50 per cent of the entire number of grains and 92 per cent of the total starch in 2 minutes; in about 81 per cent of the grains and 97 per cent of the total starch in 5 minutes; and in about 97 per cent of the grains and over 99 per cent of the total starch in 10 minutes.

The reactions of Brunsdonna sanderce alba and $B$. sanderce with sodium hydroxide are given on pages 390 and 395, Part II, and Chart D 11.

The reaction of Brunsdonna tubergeni with sodium hydroxide begins immediately. Complete gelatinization occurs in about 84 per cent of the entire number of grains and 97 per cent of the total starch in 5 minutes.

The most important questions here involved are: (1) 
Do the properties of Brunsdonna tubergeni, Brunsdonna sanderce alba, and Brunsdonna sanderce indicate that these hybrids are the offspring of the same cross or of reciprocal crosses; and (2) what are the indications of the probable parentage of A maryllis parkeri alba?

The starch of Brunsdonna tubergeni has in comparison with the starch of $B$. sanderex alba and $B$. sanderce certain properties that are closely similar or identical and others that are more or less markedly dissimilar, the latter much predominating. The grains of the former are more irregular, and more slender and elongated; the hila are less distinct; the lamellæ are more distinct, more often coarse, and more often irregular; the grains are larger. In the polariscopic properties there are not any conspicuous differences except that the figures tend to be more irregular. In the iodine reactions the coloration is distinctly less. In the aniline reactions with both gentian violet and safranin the coloration is more marked. In most of the foregoing instances the starch of $B$. tubergeni does not differ more from the starches of $B$. sanderce alba and $B$. sanderce than do the latter from each other. In the temperatures of gelatinization the figure for $B$. tubergeni is $64.76^{\circ}$, or a difference approximately of $7.5^{\circ}$ less than the temperatures of the parental starches, these being $72.7^{\circ}$ and $71^{\circ}$, respectively. The temperatures for $B$. sanderce alb $a$ and $B$. sanderoe are $72.25^{\circ}$ and $72.75^{\circ}$, respectively. It will be noted that while the temperature for the parental starches differ only $1.7^{\circ}$, that of $B$. tubergeni differs from that of the pollen parent ( $A$. belladonna) $7.94^{\circ}$, and from that of the seed parent ( $B$. josephinoe) $6.24^{\circ}$; and that the temperatures for $B$. sanderce alba and $B$. sanderce and their parents differ very little, mostly within the narow limits of error of experiment. The very low temperature for $B$. tubergen $i$ on the one hand and the marked closeness of all of the temperatures for $B$. sanderce $a l b a$ and $B$. sanderce and their parents on the other indicate quite conclusively that $B$. tubergeni and $B$. sanderce alba must have arisen from reciprocal crosses. This conclusion is substantiated by the records (notwithstanding their limitation) of the reactions with chemical reagents. The reactions of all of the starches with sulphuric acid occur with such rapidity that no satisfactory differentiation is possible, but with both potassium iodide and sodium hydroxide there are marked and distinctly diagnostic differences. In reactions with potassium iodide the starch of $B$. tubergeni exhibits a somewhat higher reactivity than the starch of either parent, while on the other hand the starches of $B$. sanderce $a l b a$ and $B$. sanderce show very much lower reactivities, not nearly so much of the latter being gelatinized at the end of an hour as there is in case of the $B$. tubergeni and parental starches in 5 minutes. It is also to be noted that during the progress of gelatinization the curves of $B$. sanderos alba and $B$. sanderce tend to pursue the same course, they being separated at and after the 5 -minute interval by about 10 points. In the sodium hydroxide reactions similar results are recorded, the reactivity of the starch of $B$. tubergeni being very high and closely corresponding to the reactivities of the parental starches, but slightly higher than either, while the reactivities of the starches of $B$. sanderce alba and $B$. sanderce are both moderate, the reactivity of the former being distinctly lower than that of the latter.
There were studied in this research three groups of parental and hybrid starches in each of which were included two hybrids of the same cross, and it is of interest to note to what degrees in general the members of each pair compare with each other and with their parents and how these peculiarities compare with those of the Brunsdonnæ hybrids and their parents. Examining first the temperatures of gelatinization and taking up the Nerine crispa-elegans-dainty maid-queen of roses group (page 165) it will be seen that the temperature of the hybrids differ only $1.3^{\circ}$ and that they are intermediate between the parental temperatures, which latter differ $5.2^{\circ}$; in the Nerine bowdeni-sarniensis var. corusca major-giantess-abundance group the temperatures of the hybrids differ $3.35^{\circ}$ and both are lower than either of the parental temperatures, these differing $3.9^{\circ}$; and in the Narcissus poeticus-poeticus poetarum-poeticus herrickpoeticus dante group the temperatures of the hybrids differ $2^{\circ}$, that of one being intermediate between the parental temperatures and the other practically the same as that of the seed parent, while the parental temperatures differ $5.5^{\circ}$, that of the seed parent being the higher. The temperatures of each of these pairs of hybrids keep close together and close to the temperatures of the parents, as in the case of Brunsdonna sanderce alba and $B$. sandero, with wider variations in the former than in the latter, but there is no suggestion of a wide departure, such as is found in B. tubergeni, this latter indicating either difference in parentage or in the direction of the cross from that of the other Brunsdonnce.

In the reactions of the members of these groups with potassium iodide and sodium hydroxide corresponding characteristics have been recorded, that is, that the two starches of each group show close reaction-intensities. In the potassium iodide reactions of the Nerine crispaelegans-dainty maid-queen of roses group, those of the hybrids are very much alike and, on the whole, intermediate between those of the parents; and in the Nerine bowdeni-sarniensis var. corusca major-giantess-abundance group, while those of the hybrids are low and differ distinctly, at least one and probably both tend to intermediateness, and one takes more after the seed parent and the other more after the pollen parent. In the sodiumhydroxide reactions, in the first group those of the hybrids are not only very close but also close to those of the parents; and in the second group those of the hybrids are very close and lower than those of the parents. It will be seen that in the reactions of each of the several pairs of hybrids there are no such departures of the reactions of each of the couples as are observed in the case of Brunsdonna tubergeni compared with $B$. sanderce $a l b a$ and $B$. sandera. From the description of $B$. tubergeni this hybrid is more closely related in its properties to Brunsvigia josephince than to Amaryllis belladonna, while the data of $B$. sanderce alba and $B$. sandero indicate that, on the whole, both of these hybrids show a closer relationship to $A$. belladonna than to $B$. josephinc-in other words, in each case the hybrid is more closely related to the seed parent.

These data also give a clue as to the probable origin of Amaryltis parkeri alba. The starch of this plant throughout the histologic and polariscopic properties and the iodine and aniline reactions, with rare exceptions, exhibits a much closer relationship to Brunsdonna san- 
derce alba and $B$. sanderce than to $B$. tubergeni; in the temperature reactions it differs little from those of $B$. sanderce alba and $B$. sanderce, but much from those of $B$. $t$ ubergeni; while in the potassium-iodide and sodiumhydroxide reactions it is closer to $B$. tubergen $i$ than to the other hybrids. From the foregoing it seems obvious that this plant is not to be identified with either $B$. tubergeni or the sander ${ }^{2}$ hybrids, although closely related. It seems probable, as suggested by Tubergen, that the parentage of $A$. parkeri on the Amaryllis side was $A$. belladonna var. blanda (A. blanda Gawl) - the histologic and polariscopic properties and the iodine, aniline, and temperature reactions pointing to the same direction of the cross as of $B$. sanderce alba and $B$. sanderce, while the potassium iodide and sodium hydroxide reactions indicate a cross in the opposite direction; but the temperature reaction alone is almost if not conclusive. Additional studies of the reactions would undoubtedly make absolutely positive the direction of the cross if $A$. parkeri is a hybrid.

\section{Comparisons of the Starches of Hippeastrum} TITAN, H. ClEONIA, AND H. TITAN-CLEONIA.

In histologic characteristics, polariscopic figures, reactions with selenite, qualitative reactions with iodine, and qualitative reactions with the various chemical reagents these three starches are very much alike. The starch of Hippeastrum cleonia is distinguished from that of the other parent chiefly in the larger number of compound grains and aggregates; the presence of isolated grains each having a large pressure facet; more roundness but greater irregularity of the grains; somewhat less fissuration and less eccentricity of the hilum; more distinct and more regular lamellæ; somewhat larger average size of the grains; larger number of double and multiple polariscopic figures; greater frequency of equality of size, less frequency of irregularity of shape, and less often purity of color of the quadrants in the selenite reaction; and some slight differences in qualitative reactions with iodine. The starch of the hybrid is in form, hilum, and polariscopic figure more closely related to the seed parent; and in distinctness and regularity of the lamellæ, size, and iodine reactions more closely related to the other parent. In the selenite reactions certain properties lean to one or the other parent. A given character may appear more conspicuously in the hybrid than in either parent. The qualitative reactions with chloral hydrate, nitric acid, potassium iodide, potassium sulphocyanate, and sodium salicylate are closer to those of seed parent.

Reaotion-intensities Expressed by Light, Color, and TemperaPolarization:

$\mathrm{H}$. titan, high to very high, value 83 .

H. cleonia, high to very high, lower than in H. titan, value 80 .

H. titan-cleonia, high to very high, higher than in either parent,

Iodine: value 85 .

H. titan, moderate, value 52.

H. cleonia, moderately deep, deeper than in $\mathbf{H}$. titan, value 55 .

H. titan-oleonia, moderate to deep, deeper than in the parents, value 58.

Gentian violet:

H. titan, moderately light to light, value 45.

H. cleonia, moderate, deeper than in H. titan, value 50 .

H. titan-cleonia, moderate, the same as in $H$. cleonia, value 50 . Safranin:

H. titan, moderate, value 50 .

H. cleonia, moderate, a little deeper than in H.titan, value 55.

H. titan-cleonia, moderate, the same as in H. cleonia, value 55 .

Temperature of gelatinization:

H. titan, in majority at 74 to $75^{\circ}$, in all but rare grains at 77 to $77.5^{\circ}$, mean $77.25^{\circ}$.

H. cleonia, in majority at 71 to $73^{\circ}$, in all but rare grains at 73 to $74^{\circ}$, mean $73.5^{\circ}$

H. titan-cleonia, in majority at 72 to $74^{\circ}$, in all but rare grains at 73 to $74^{\circ}$, mean $73.5^{\circ}$.
The reactivity of Hippeastrum titan is higher than that of Hippeastrum cleonia in the polarization reaction, and lower in the reactions with iodine, gentian violet, safranin, and temperature. The hybrid shows in the polarization and iodine reactions the highest reactivities of all three starches; in the reactions with gentian violet, safranin, and temperature the same reactivities as those of Hippeastrum cleonia, all three reactions being higher than the corresponding reactions of the other parent.

Table A 2 shows the reaction intensities in percentages of total starch gelatinized at definite intervals (minutes).

\section{Velocity-Reaction CuRves.}

This section treats of the velocity-reaction curves of the starches of Hippeastrum titan, $H$. cleonia, and $H$. titan-cleonia, showing the quantitative differences in the behavior toward different reagents at definite time-intervals. (Charts D 22 to D 42.)

Among the conspicuous features of these charts are:

(1) The closeness of the curves of the three starches in all of the reactions. The reactions are so slow with potassium iodide, potassium sulphide, sodium sulphide, calcium nitrate, uranium nitrate, strontium nitrate, cobalt nitrate, copper nitrate, cupric chloride, barium chloride, and mercuric chloride that there is almost if not absolutely no differentiation. Omitting the foregoing reactions, the curve of Hippeastrum titan is higher than that of the other parent in the reactions with chromic acid and sulphuric acid, and lower in those with chloral hydrate, pyrogallic acid, nitric acid, potassium hydroxide, potassium sulphocyanate, sodium hydroxide, and sodium salicylate, indicating, on the whole, a lower reactivity of this starch.

(2) The curves of the hybrid show marked variations in their parental relationships, with as much of a tendency to be higher or lower than the parental curves as to intermediateness. In a few reactions the curves are the same as those of the seed parent or of the pollen parent, and in about one-third they are the same as the parental curves. (See following section.)

(3) In most of the charts in which there was a moderate to rapid reactivity there are indications of an early period of comparatively marked resistance.

(4) The best period during the 60 minutes for the differentiation of the three starches is variable, and in case of all the very slow reactions and including those with chloral hydrate, nitric acid, potassium sulphocyanate, and sodium hydroxide, the curves are best separated, if at all, at the end of 60 minutes. This period is noted at the end of 15 minutes in the reactions with chromic acid, pyrogallic acid, sulphuric acid, potassium hydroxide, and sodium salicylate; at the end of 30 minutes with hydrochloric acid; and at the end of 60 minutes with the other reagents.

\section{Reaction-Intensities of THE HrbRid.}

This section treats of the reaction-intensities of the hybrid as regards sameness, intermediateness, excess, and deficit in relation to the parents. (Table $\mathrm{A} 2$ and Charts D 22 to D 42.)

The reactivities of the hybrid are the same as those of the seed parent in the reactions with sodium sulphide and strontium nitrate; the same as those of the pollen parent with gentian violet, safranin, and temperature; the same as those of both parents with potassium sulphide, calcium nitrate, uranium nitrate, cobalt nitrate, copper nitrate, cupric chloride, barium chloride, and mercuric chloride, in all of which the reactions are exceedingly slow; intermediate with nitric acid, hydrochloric acid, potassium iodide and potassium sulpho- 
TABLE A 2.

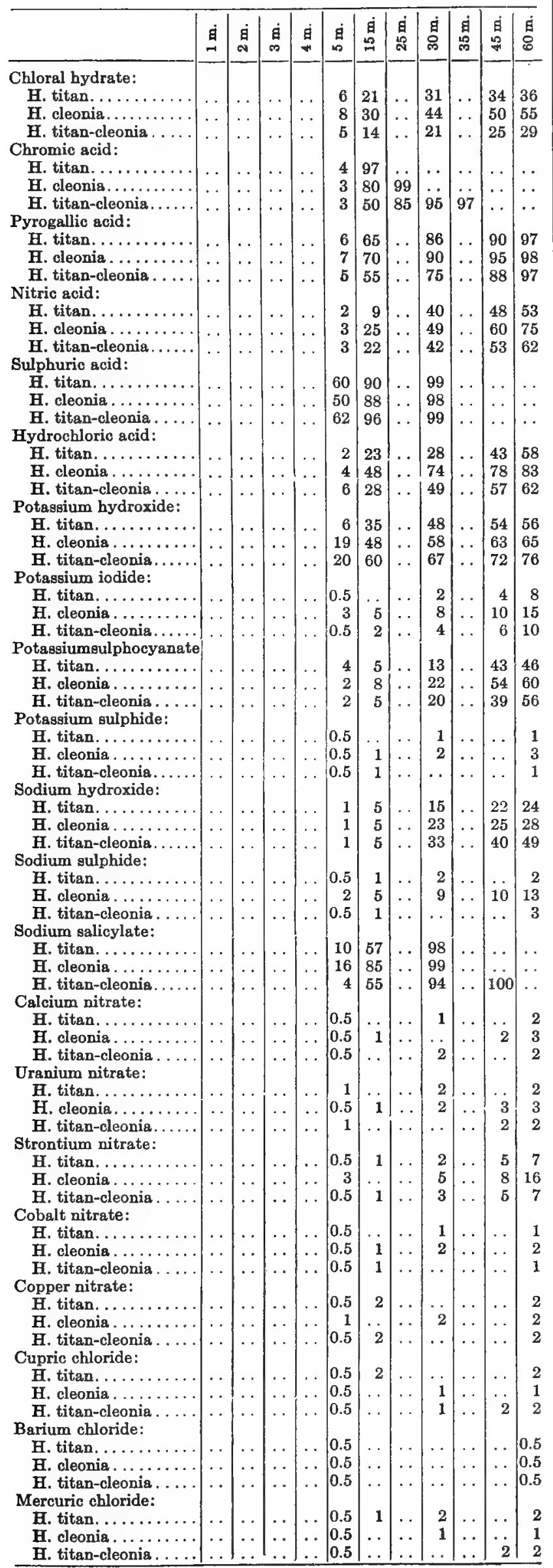

cyanate (in one being closer to the seed parent and in three mid-intermediate); highest with polarization, iodine, sulphuric acid, potassium hydroxide, and sodium hydroxide (in two being closer to the seed parent and in three closer to the pollen parent); and lowest with chloral hydrate, chromic acid, pyrogallic acid, and sodium salicylate (in three being closer to the seed parent and in one closer to the pollen parent).

The following is a summary of the reaction-intensities: Same as seed parent, 2 ; same as pollen parent, 3 ; same as both parents, 8 ; intermediate, 4 ; highest, 5 ; lowest, 4.

The seed parent shows a stronger influence than the pollen parent on the characters of the starch of the hybrid.

\section{Composite CuRves of the Reaction-intensities.}

The following section treats of the composite curves of the reaction-intensities showing the differentiation of the starches of Hippeastrum titan, $H$. cleonia, and $H$. titan-cleonia. (Chart F 2.)

Among the conspicuous features of this chart are:

(1) The closeness of all three curves, indicating a very close relationship. of all three starches and plantsources.

(2) The generally lower position of the curve of Hippeastrum titan in relation to the curve of the other parent, it being lower in the reactions with iodine, gentian violet, safranin, temperature, chloral hydrate, pyrogallic acid, nitric acid, hydrochloric acid, potassium hydroxide, potassium iodide, potassium sulphocyanate, sodium hydroxide, sodium sulphide, and strontium nitrate; higher with polarization and chromic acid; and the same or practically the same with sulphuric acid, potassium sulphide, sodium salicylate, calcium nitrate, uranium nitrate, cobalt nitrate, copper nitrate, cupric chloride, barium chloride, and mercuric chloride.

(3) The curve of Hippeastrum titan is very high in the polarization and chromic-acid reactions; high with sulphuric acid and sodium salicylate; moderate with iodine, gentian violet, safranin, and pyrogallic acid; low with temperature, nitric acid, hydrochloric, and potassium hydroxide; very low with chloral hydrate, potassium iodide, potassium sulphocyanate, potassium sulphide, sodium hydroxide, sodium sulphide, calcium nitrate, uranium nitrate, strontium nitrate, cobalt nitrate, copper nitrate, cupric chloride, barium chloride, and mercuric chloride.

(4) The curve of Hippeastrum cleonia is very high in the polarization and chromic-acid reactions; high with pyrogallic acid, sulphuric acid, and sodium salicylate; moderate in the iodine, gentian violet, and safranin; and low with temperature, chloral hydrate, nitric acid, hydrochloric acid, potassium hydroxide, and potassium sulphocyanate; and very low with potassium iodide, potassium sulphide, sodium hydroxide, sodium sulphide, calcium nitrate, uranium nitrate, strontium nitrate, cobalt nitrate, copper nitrate, cupric chloride, barium chloride, and mercuric chloride.

(5) The curve of the hybrid is very high in the polarization and sulphuric-acid reactions; high with chromic acid and sodium salicylate; moderate with iodine, gentian violet, safranin, and pyrogallic acid; low with temperature, nitric acid, hydrochloric acid, potassium hydroxide, and potassium sulphocyanate; and very low with chloral hydrate, potassium iodide, potassium sulphide, sodium hydroxide, sodium sulphide, calcium nitrate, uranium nitrate, strontium nitrate, cobalt nitrate, copper nitrate, cupric chloride, barium chloride, and mercuric chloride. 

ties:

The following is a summary of the reaction-intensi-

\begin{tabular}{l|c|c|c|c|c}
\hline & $\begin{array}{c}\text { Very } \\
\text { high. }\end{array}$ & High. & $\begin{array}{c}\text { Moder- } \\
\text { ate. }\end{array}$ & Low. & $\begin{array}{c}\text { Very } \\
\text { low. }\end{array}$ \\
\hline H. titan............ & 2 & 2 & 4 & 4 & 14 \\
H. cleonia......... & 2 & 3 & 3 & 6 & 12 \\
H. titan-cleonia.... & 2 & 2 & 4 & 5 & 13
\end{tabular}

\section{Comparisons of the Starohes of Hippeastrum} ossultan, H. PYRRHa, AND H. OsSUltan-PYRRHa.

In the histologic characteristics and polariscopic figures, reactions with selenite, qualitative reactions with iodine, and qualitative reactions with the various chemical reagents the three starches are closely alike. The starch of $H$. pyrrh $a$ in comparison with that of the seed parent has fewer compound grains and aggregates, more single grains with one or more pressure facets, and more irregularities of the grains; the hilum is more frequently and more extensively fissured and is more eccentric; the lamellæ are distinct in a larger number of grains, but as a rule less in number; the size as a rule is less, but the proportions of length to breadth are the same; and the polariscopic figures, reactions with selenite, and the qualitative reactions with iodine show minor differences which in the aggregate are of account in differentiation of the starches. The starch of the hybrid closely resembles those of the parents. It is closer to that of the seed parent in size of the grains and number of the lamellæ, but closer to the pollen parent in the form of the grains, fissuration and eccentricity of the hilum, and character of the lamellæ. In the qualitative polarization and iodine reactions it is closer to the seed parent. In the qualitative reactions with chloral hydrate, potassium iodide, and potassium sulphocyanate it is more like that of the seed parent, while in the nitric-acid and sodium-salicylate reactions more like that of the other parent.

\section{Reaction-intensities Expressed by Light, Color, and Tempera- ture Reactions.}

Polarization:

H. ossultan, high to very high, value 83 .

H. pyrrha, high to very high, higher than in H. ossultan, value 85

H. ossult.-pyrh, high to very high, higher than in either parent, Iodine: value 87.

H. ossultan, moderately light to moderate, value 45 .

$H$. pyrrha, moderate to moderately deep, deeper than in $H$. ossultan, value 55 .

H. ossult.-pyrh., moderately light to moderately deep, and intermediate between the parents, value 50 .

Gentian violet:

H. ossultan, moderate, value 50 .

H. pyrrha, moderately light to moderately deep, lighter than in H. ossultan, value 48 .

H. ossult.-pyrh., moderate to moderately deep, deeper than in Safranin: either parent, value 53 .

H. ossultan, moderate to moderately deep, value 55 .

H. pyrrha, moderate, lighter than in $H$. ossultan, value 50 .

H. ossult.-pyrh., moderate to moderately deep, deeper than in either parent, value 58 .

Temperature of gelatinization:

H. ossultan, in majority at 73 to $74^{\circ}$, in all except rare grains at 75 to $76^{\circ}$, mean $75.5^{\circ}$.

H. pyrrha, in majority at 71 to $73^{\circ}$, in all except rare grains at 73 to $74^{\circ}$, mean $73.5^{\circ}$.

H. ossult.-pyrh., in majority at 70 to $72^{\circ}$, in all but rare grains at 72 to $73^{\circ}$, mean $72.5^{\circ}$.

The reactivities of $H$. ossultan are lower than those of the other parent in the polarization, iodine, and temperature reactions and higher in those of gentian violet and safranin. The reactivities of the hybrid are higher than those of either parent in the polarization, gentian-violet, safranin and temperature reactions, and
Table A 3.

\begin{tabular}{|c|c|c|c|c|c|c|c|c|c|c|}
\hline & 豞 & ㅂ & घ્ & 家 & 星 & 昌 & $\begin{array}{l}\text { 㝵 } \\
\text { 学 }\end{array}$ & $\underset{\text { 㤩 }}{ }$ & 皃 & g் \\
\hline hlorel & & & & & & & & & & \\
\hline & . & $\cdots$ & . & $\cdots$ & 7 & 27 & . & 37 & 42 & 45 \\
\hline & & . & & & 3 & 19 & & 28 & 39 & 42 \\
\hline & . & $\ldots$ & .. & $\ldots$ & 4 & 26 & . & 36 & 40 & 43 \\
\hline Chrom & & & & & & & & & & \\
\hline & $\cdots$ & $\cdots$ & . & $\cdots$ & 1 & 25 & 96 & 99 & . & \\
\hline H. & $\ldots$ & $\ldots$ & .. & $\ldots$ & 1 & 20 & 90 & 99 & . & . \\
\hline & .. & $\cdots$ & . & $\cdots$ & 1 & 45 & 96 & 99 & . . & \\
\hline Pyroge & & & & & & & & & & \\
\hline H. o & $\cdots$ & $\cdots$ & . & . & 10 & 67 & . . & 80 & 90 & 95 \\
\hline H. $\mathrm{p}$ & $\cdots$ & $\cdots$ & . & . & 5 & 80 & . & 89 & 92 & 96 \\
\hline H. & $\ldots$ & . & . & . & & 85 & . & 93 & 86 & \\
\hline Nitric & & & & & & & & & & \\
\hline H. & $\cdots$ & $\cdots$ & . & $\cdots$ & 4 & 17 & . . & 30 & 43 & 56 \\
\hline & $\cdots$ & $\cdots$ & . . & $\ldots$ & 2 & 6 & .. & 16 & 33 & 50 \\
\hline & . & $\cdots$ & . & . & 2 & 19 & .. & 40 & 65 & 67 \\
\hline ulph & & & & & & & & & & \\
\hline & . & $\cdots$ & . & . & 45 & 95 & & 99 & . & \\
\hline H. & . & . & . & $\cdots$ & 70 & 96 & & 99 & $\cdots$ & . \\
\hline & $\cdots$ & . & .. & . & 40 & 95 & .. & 99 & $\cdots$ & $\cdots$ \\
\hline Hyd & & & & & & & & & & \\
\hline H. o & $\because$ & $\because$ & $\cdots$ & $\cdots$ & 5 & 40 & . & $\begin{array}{l}62 \\
70\end{array}$ & 80 & $\begin{array}{l}86 \\
88\end{array}$ \\
\hline & $\therefore$ & $\because$ & $\because$ & $\because$ & 5 & 41 & $\cdots$ & $\begin{array}{l}70 \\
82\end{array}$ & & 88 \\
\hline $\begin{array}{c}\text { H. } \\
\text { Potas }\end{array}$ & & & & & 6 & & & 82 & $3 y$ & \\
\hline & . . & $\cdots$ & $\cdots$ & $\cdots$ & 14 & 50 & $\ldots$ & 62 & 69 & 73 \\
\hline & .. & . & .. & .. & 8 & 51 & .. & 72 & 74 & 75 \\
\hline & . & $\ldots$ & . & $\ldots$ & 20 & 54 & . & 74 & 76 & 78 \\
\hline Potass & & & & & & & & & & \\
\hline & . & $\cdots$ & $\cdots$ & .. & 4 & 11 & . & 19 & 21 & 23 \\
\hline H. & . & $\cdots$ & $\cdots$ & . & 0.5 & 5 & . & 7 & 11 & 17 \\
\hline H. & . & $\ldots$ & .. & . & 3 & 10 & $\ldots$ & 20 & 25 & 33 \\
\hline Potass & & & & & & & & & & \\
\hline . & . & . & . & $\cdots$ & 4 & 10 & $\cdots$ & 34 & 48 & 64 \\
\hline & .. & $\ldots$ & . & $\ldots$ & 2 & 5 & $\ldots$ & 25 & 46 & 61 \\
\hline H. & $\ldots$ & $\ldots$ & $\ldots$ & $\ldots$ & 3 & 10 & $\ldots$ & 48 & 61 & 70 \\
\hline Pot & & & & & & & & & & \\
\hline H. o & $\cdots$ & $\cdots$ & . & $\cdots$ & 0.5 & 1 & . & 3 & 4 & \\
\hline & $\ldots$ & . & . & $\ldots$ & 1 & 2 & . & 3 & & \\
\hline & $\ldots$ & $\ldots$ & $\ldots$ & $\ldots$ & 0.5 & 0.5 & $\ldots$ & 3 & . & \\
\hline Sodiu & & & & & & & & & & \\
\hline H. & . & $\cdots$ & $\ldots$ & . & 10 & 31 & . & 39 & 44 & 48 \\
\hline H. & 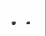 & . & .. & .. & 2 & 8 & . & 29 & 36 & 43 \\
\hline & . & . & $\cdots$ & . & 3 & 27 & .. & 35 & 43 & 45 \\
\hline Sodiu & & & & & & & & & & \\
\hline & . & . & $\ldots$ & & 2 & 3 & $\cdots$ & 5 & 8 & \\
\hline & $\ldots$ & . & $\ldots$ & . . & 1 & 3 & . & 5 & & 5 \\
\hline H. & $-\mathrm{C}_{0}$ & . & .. & . & 2 & 4 & . & 6 & 8 & 8 \\
\hline Sodiun & & & & & & & & & & \\
\hline H. & $\cdots$ & . & $\cdots$ & . & 45 & 95 & & 99 & $\cdots$ & . \\
\hline & $\cdots$ & .. & $\ldots$ & . . & 32 & 90 & & 99 & & . \\
\hline & $\ldots$ & $\ldots$ & $\ldots$ & . & 22 & 85 & $\cdots$ & 98 & 99 & \\
\hline Calciu & & & & & & & & & & \\
\hline & . & . & $\cdots$ & $\cdots$ & 1 & 3 & & 5 & & 5 \\
\hline H. & .. & $\ldots$ & .. & . & 1 & 2 & . & 3 & .. & \\
\hline H. & $\cdots$ & .. & . & $\ldots$ & 0.5 & 1 & & 2 & & 2 \\
\hline Urani & & & & & & & & & & \\
\hline H. o & . & . & . & $\cdots$ & 2 & 5 & . & 6 & 9 & 10 \\
\hline & $\cdots$ & . . & . & . & 0.5 & 1 & .. & 4 & $\ldots$ & 4 \\
\hline & .. & $\ldots$ & $\ldots$ & $\ldots$ & 0.5 & 1 & $\ldots$ & 4 & $\ldots$ & 5 \\
\hline Stront & & & & & & & & & & \\
\hline & $\ldots$ & $\ldots$ & . . & $\cdots$ & 2 & 7 & . & 10 & 11 & 12 \\
\hline H. & . & $\ldots$ & . & . & 1 & 2 & .. & 5 & 8 & 12 \\
\hline & $\ldots$ & $\ldots$ & $\ldots$ & . . & 2 & $\ldots$ & $\ldots$ & 4 & 6 & 11 \\
\hline Cobal & & & & & & & & & & \\
\hline H. o & $\cdots$ & . & $\cdots$ & - & 0.5 & 1 & . & 2 & 3 & \\
\hline & $\ldots$ & $\ldots$ & $\ldots$ & . & 0.5 & 1 & . & . & 2 & \\
\hline H. & $\cdots$ & . & $\ldots$ & .. & 0.5 & 1 & .. & $\cdots$ & . & \\
\hline Copp & & & & & & & & & & \\
\hline & . & . & . & . & 0.5 & 2 & . & 3 & 4 & \\
\hline H. I & . & . & .. & . & 0.5 & $\ldots$ & $\ldots$ & .. & & 0.5 \\
\hline & $\ldots$ & .. & . & $\ldots$ & 0.5 & 2 & & . & . & \\
\hline Cupric & & & & & & & & & & \\
\hline H. oss & . & & . & $\ldots$ & 0.5 & 2 & & 3 & 4 & \\
\hline & $\cdots$ & $\ldots$ & . & .. & 0.5 & . & . & & . & \\
\hline & $\ldots$ & .. & .. & .. & 0.5 & $\cdots$ & & 1 & $\cdots$ & \\
\hline & & & & & & & & & & \\
\hline H. o & . & . & . & . & 0.5 & . & . & 1 & 2 & \\
\hline H. & . & & . & .. & 0.5 & & . & • & .. & 0.5 \\
\hline $\begin{array}{r}\text { H. o } \\
\text { Mercu }\end{array}$ & . & $\cdots$ & $\cdots$ & . & 0.5 & $\cdots$ & $\cdots$ & .. & & 0.5 \\
\hline & . & .. & . . & .. & 0.5 & $\ldots$ & . & 1 & 2 & 2 \\
\hline & .. & .. & . & .. & & $\ldots$ & .. & $\cdots$ & & 0.6 \\
\hline H. ossult.-py & . & & & & 0.5 & & & & 1 & \\
\hline
\end{tabular}


mid-intermediate in the reaction with iodine. In the polarization and temperature reactions it is closer to the pollen parent, and in the gentian-violet and safranin reactions closer to the seed parent.

Table A 3 shows the reaction-intensities in percentages of total starch gelatinized at definite intervals (minutes).

\section{Velocity-REACTION CUR7es.}

This section treats of the velocity-reaction curves of the starches of Hippeastrum ossultan, H. pyrrha, and $H$. ossultan-pyrrha, showing the quantitative differences in the behavior toward different reagents at definite timeintervals. (Charts D 43 to D 63.)

The conspicuous features of these charts do not differ in many respects from those of the preceding set.

(1) The curves of all three starches are in all of the reactions close and, on the whole, about the same as regards the extent of separation as in the first set, in some reactions there being a little more separation and in others less. In most of the reactions there is a tendency for a slightly higher reactivity than in the $H$. titan-cleonia set. Many of the reactions are so slow that there is no important if any differentiation, as in those with potassium sulphide, sodium sulphide, calcium nitrate, uranium nitrate, strontium nitrate, cobalt nitrate, copper nitrate, cupric chloride, barium chloride, and mercuric chloride.

(2) Omitting these very slow reactions, the curve of $H$. ossultan is in the remaining 11 reactions higher than the corresponding curve of the other parent in the reactions with chloral hydrate, chromic acid, nitric acid, patassium iodide, potassium sulphocyanate, sodium hydroxide, and sodium salicylate; and lower in those with pyrogallic acid, sulphuric acid, hydrochloric acid, and potassium hydroxide.

(3) The curves of the hybrid bear varying relations to the parental curves, with very little tendency to sameness in relation to the seed parent and none to the pollen parent; with little tendency to intermediateness or to being the lowest of the three curves; with a marked tendency to be the highest of the three; and with a tendency to sameness as both parents in the reactions that take place with marked slowness. (See the following section.)

(4) An early. period of comparatively high resistance is noticed especially in the reactions with chloral hydrate, chromic acid, nitric acid, hydrochloric acid, and potassium sulphocyanate; the opposite with potassium hydroxide and sodium salicylate.

(5) The best period for the differentiation of the three starches is in case of the very slow reactions above referred to at the end of the 60 minutes, but in some of them even at this time there is very little or no difference. The curves appear to be best separated at $5 \mathrm{~min}-$ utes in the reactions with sulphuric acid, potassium hydroxide, and sodium salicylate; at 15 minutes with chloral hydrate, chromic acid, pyrogallic acid, and sodium hydroxide; at 30 minutes with nitric acid, hydrochloric acid, and potassium sulphocyanate.

Reaction-INTENSITIES OF THE HyBRID.

This section treats of the reaction-intensities of the hybrid as regards sameness, intermediateness, excess, and deficit in relation to the parents. (Table A 3 and Charts D 43 to $\mathrm{D} 63$.)

The reactivities of the hybrid are the same as those of the seed parent with sulphuric acid, sodium sulphide, and uranium nitrate; the same as those of the pollen parent in none; the same as those of both parents with potassium sulphide, calcium nitrate, strontium nitrate, cobalt nitrate, copper nitrate, cupric chloride, barium clloride, and mercuric chloride; intermediate with iodine, chloral hydrate, and sodium hydroxide (in the first being mid-intermediate and in the last two nearer the seed parent) ; highest with polarization, gentian violet, safranin, temperature, chromic acid, nitric acid, pyrogallic acid, hydrochloric acid, potassium hydroxide, potassium iodide, and potassium sulphocyanate (in six being closer to the seed parent and in five being closer to the pollen parent) ; and the lowest with sodium salicylate, it being in these nearer the pollen parent.

The following is a summary of the reaction-intensities: Same as seed parent, 3 ; same as pollen parent, 0 ; same as both parents, 9 ; intermediate, 3 ; highest, 11 ; lowest, 1 .

In not a single reaction is there sameness in relation to the pollen parent, and the stronger influence of the seed parent on the properties of the hybrid is quite marked. Intermediateness is rather exceptional, a tendency to the lowest reactivity very exceptional, and a tendency to the highest reactivity very marked.

\section{Composite Curves of the Reaction-intensities.}

This section treats of composite curves of the reaction-intensities showing the differentiation of the starches of Hippeastrum ossultan, $H$. pyrrha, and $H$. ossultan-pyrrha. (Chart E 3.)

Among the conspicuous features of this chart are:

(1) The remarkable closeness of all three curves, the differences for the most part being insignificant or actually falling within the limits of error of experiment, showing an extreme botanical closeness of the parents and extremely little variance of the hybrid from the parents. The only reactions in which the parents are readily differentiated are those with iodine, gentian violet, safranin, temperature, chromic acid, and sodium salicylate, and even in these the differences are without exception of a minor degree.

(2) In this curve of $H$. ossultan compared with that of $H$. pyrrha the reactivities are shown to be distinctly higher in the reactions with gentian violet, safranin, chromic acid, and sodium salicylate, and lower with polarization, iodine, and temperature. In the other instances the differences are unimportant or even negligible excepting in so far as they tend to indicate a generally slightly higher reactivity of $H$. ossultan.

(3) In $H$. ossultan the very high reactions with polarization, chromic acid, sulphuric acid, and sodium salicylate, the moderate reactions with iodine, safranin, gentian violet, and pyrogallic acid; the low reactious with temperature, nitric acid, hydrochloric acid, potassium hydroxide, and potassium sulphocyanate; and the very low reactions with chloral hydrate, potassium iodide, potassium sulphite, sodium hydroxide, sodium sulphide, calcium nitrate, uranium nitrate, strontium nitrate, cobalt nitrate, copper nitrate, cupric chloride, barium chloride, and mercuric chloride.

(4) In $H$. pyrrha the very high reactions with polarization, sulphuric acid, and sodium salicylate; the high reactions with chromic acid, the moderate reactions with iodine, gentian violet, safranin and pyrogallic acid; the low reactions with temperature, nitric acid, hydrochloric acid, potassium hydroxide, potassium sulphocyanate; and the very low reactions with chloral hydrate, potassium iodide, potassium sulphide, sodium hydroxide, sodium sulphide, calcium nitrate, uranium nitrate, strontium nitrate, cobalt nitrate, copper nitrate, cupric chloride, barium chloride, and mercuric chloride.

(5) In the hybrid the very high reactions with polarization, chromic acid, sulphuric acid, pyrogallic acid, and sodium salicylate; the moderate reactions with iodine, gentian violet, safranin, temperature, and hydrochloric 
acid; the low reactions with nitric acid, potassium hydroxide, and potassium sulphocyanate; and the very low reactions with chloral hydrate, potassium iodide, potassium sulphide, sodium hydroxide, sodium sulphide, calcium nitrate, uranium nitrate, strontium nitrate, cobalt nitrate, copper nitrate, cupric chloride, barium chloride, and mercuric chloride. ties:

The following is a summary of the reaction-intensi-

\begin{tabular}{l|c|c|c|c|c}
\hline & $\begin{array}{c}\text { Very } \\
\text { high. }\end{array}$ & High. & $\begin{array}{c}\text { Mod- } \\
\text { erate. }\end{array}$ & Low. & $\begin{array}{c}\text { Very } \\
\text { low. }\end{array}$ \\
\hline H. ossultan......... & 4 & 0 & 4 & 5 & 13 \\
H. pyrrha....... & 3 & 1 & 4 & 5 & 13 \\
H. ossult.-pyrh..... & 5 & 0 & 5 & 3 & 13 \\
\hline
\end{tabular}

4. Comparisons of the Starches of Hippeastrum DAOONES, H. ZEPHYR, AND H. DANONES-ZEPHYR.

In histologic characteristics, polariscopic figures, reactions with selenite, qualitative reactions with iodine, and qualitative reactions with the various chemical reagents the starches of the parents exhibit properties in common and certain individualities, but generally a very close correspondence throughout. The grains of $H$. zephyr in comparison with those of the seed parent are found to include less numbers of aggregates and compounds; they are free from the long, narrow finger-like grains found in the latter; they are more regular, the protuberances being less numerous and not so large. The hilum is less distinct and less frequently fissured. The lamellæ are less distinct, less fine, and less in number. The common size is about the same, but the large grains show some differences in ratio of length to breadth. The polariscopic, selenite, and qualitative iodine reactions exhibit some minor differences. The starch of the hybrid in comparison with the starches of the parents contains'a relatively larger number of aggregates and compounds but none of the long, narrow finger-like grains found in $H$. doeones but not in $H$. zephyr. The hilum is more frequently fissured than in either parent, and in character and eccentricity it is closer to $H$. doones. The lamellæ in character and number are nearer to $H$. docones. The common size of the grains is somewhat less than in either parent, and the size of the larger grains approaches nearer that of $H$. zeplyyr. In the qualitative polariscopic properties the leaning is in certain respects toward one parent and in other respects toward the other, and in the selenite reactions there is development of properties in excess of the development in the parents, with a leaning closer to the pollen parent. The qualitative iodine reactions are closer to $H$. zephyr. In the qualitative chemical reactions with chloral hydrate, nitric acid, potassium iodide, and potassium sulphocyanate the hybrid is closer to $H$. dacones, while in the sodium-salicylate reactions the relationship to the two parents is of equal degree.

Reaction-intensities Expressed by Light, Color, and Temperar Polarization:

H. dæones, high to very high, value 80 .

H. zephyr, high to very high, little higher than in H. dæones, value 83.

H. dæon. zeph., high to very high, higher than in the parents, Iodine: value 85 .

H. dæones, moderate to moderately deep, value 55 .

$H$. zephyr, moderate, less than in H. dæones, value 50 .

H. dæon.-zeph., moderate, same as in H. zephyr, value 50 . Gentian violet:

H. dæones, moderate to moderately deep, value 58 .

H. zephyr, moderate to moderately deep, lighter than in $\mathrm{H}$. dæones, value $5 \mathbf{5}$.

H. dæon.-zeph., moderate, lighter than in either parent, value 50 .
Safranin:

H. dæones, moderate to moderately deep, value $\mathbf{5 5}$.

H. zephyr, moderate to moderately deep, the same as in $H$. dæones, value 55 .

H. dæon.-zeph., moderate to moderately deep, the ane as in both parents, value 55 .

Temperature:

$H$. dæones, in majority at 72.5 to $74^{\circ}$, in all but rare grains at 74 to $75^{\circ}$, mean $74.5^{\circ}$.

H. zephyr, in the majority at 72 to $73^{\circ}$, in all but rare grains at 73 to $75^{\circ}$, mean $74^{\circ}$.

H. dæon.-zeph., in the majority at 72 to 73 , in all but rare grains at 72 to $73^{\circ}$, mean $72.5^{\circ}$.

The reactivities of $H$. dacones are lower than those of the other parent in the polarization and temperature reactions, higher in the iodine and gentian-violet reactions, and the same in the safranin reaction. The reactivities of the hybrid are higher than those of either parent in the polarization and temperature reactions, lower than that of either parent in the gentian-violet reaction, the same as that of the pollen parent in the iodine reaction, and the same as those of both parents in the safranin reactions. On the whole the inclination is toward the pollen parent.

Table A 4 shows the reaction-intensities in percentages of total starch gelatinized at definite intervals (minutes) :

\section{Velocity-REaction CURVES.}

The following section treats of the velocity-reaction curves of the starches of Hippeastrum docones, H. zephyr, and $H$. dcoones-zephyr, showing the quantitative differences in the behavior toward different reagents at definite time-intervals. (Charts D 64 to D 84.)

As noted in the preceding sections the three starches are very closely alike, exhibiting only minor differences, but not infrequently character developments of the hybrid that exceed the parental extremes. The most conspicuous features of these charts are:

(1) The nearness of the three curves throughout.

(2) The curve of $H$. daones is higher than the curve of $H$. zephyr in the reactions with chloral hydrate, chromic acid, pyrogallic acid, nitric acid, sulphuric acid, hydrochloric acid, potassium iodide, potassium sulphocyanate, sodium hydroxide, and sodium sulphide through the 60 minutes. It also tends to be above in the reaction with strontium nitrate. In the sodium-salicylate reaction, in which gelatinization goes on with moderate rapidity, the curves are about the same; and in the reactions with potassium sulphide, calcium nitrate, uranium nitrate, cobalt nitrate, copper nitrate, cupric chloride, barium chloride, and mercuric chloride gelatinization proceeds so slowly that there is little or no differentiation. From these data $H$. docones has, on the whole, the higher reactivity.

(3) The curves of the hybrid show varying relationships to the parental curves, in some instances being the same as that of one or the other parent or both parents, in others intermediate, and in others higher or lower than both parental curves. (See following section.)

(4) Evidence of a preliminary period of comparative resistance is apparent in several of the charts.

(5) The earliest period at which the three curves are best separated for differential purposes is variable. In the very slow reactions no differentiation seems possible even at the end of 60 minutes, the differences noted being wholly within the limits of error of observation and of no significance whatsoever. The best period for sulphuric acid is at 5 minutes; for chromic acid, pyrogallic acid, hydrochloric acid, potassium sulphocyanate, sodium hydroxide and sodium salicylate at 15 minutes; for sodium sulphide at 30 minutes; for strontium nitrate at 45 minutes; and for chloral hydrate, nitric acid, and potassium iodide at 60 minutes. 
Table A 4.

\begin{tabular}{|c|c|c|c|c|c|c|c|c|c|c|}
\hline & 正 & 音 & घั & g & 昌 & 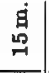 & 昌 & 咅 & $\begin{array}{l}\text { g } \\
\text { अ }\end{array}$ & $\stackrel{8}{8}$ \\
\hline \multicolumn{11}{|l|}{ Chloral hydrate: } \\
\hline H. dæones.... & $\ldots$ & $\cdots$ & . & $\cdots$ & 9 & 29 & .. & 42 & 50 & 53 \\
\hline H. zeph & & $\ldots$ & .. & $\ldots$ & 5 & 21 & .. & 32 & 36 & 39 \\
\hline H. dæones-ze & . & . & . & & & 13 & .. & 14 & 17 & 18 \\
\hline Chromic acid: & & & & & & & & & & \\
\hline II. 0 & & $\cdots$ & . & . & 3 & 90 & 99 & 100 & & \\
\hline $\mathrm{H}$. & & $\cdots$ & . & .. & 2 & 50 & 76 & 95 & 100 & \\
\hline H. & . & .. & . & .. & 30 & 80 & 90 & 100 & $\ldots$ & . \\
\hline Pyrog & & & & & & & & & & \\
\hline $\mathrm{H}$. & . & $\cdots$ & $\cdots$ & . & 15 & 70 & . & 96 & 96 & 97 \\
\hline H. $z$ & & . & . & . & 11 & 68 & . & 93 & 95 & 97 \\
\hline H. & . & $\cdots$ & . & .. & 17 & 80 & $\ldots$ & 96 & 97 & 98 \\
\hline Nitric & & & & & & & & & & \\
\hline $\mathrm{H}$. & . & . & .. & . & 7 & 32 & $\cdots$ & 70 & 73 & 78 \\
\hline H. & $\ldots$ & $\ldots$ & . & . & 6 & 12 & . & 45 & 60 & 65 \\
\hline & $\ldots$ & .. & $\ldots$ & .. & 7 & 34 & .. & 73 & 79 & 85 \\
\hline Sulpht & & & & & & & & & & \\
\hline $\mathrm{H}$. & $\cdots$ & $\cdots$ & . & . & 95 & 99 & $\cdots$ & & $\cdots$ & \\
\hline H. & . & . & .. & .. & 80 & 97 & $\ldots$ & 99 & $\cdots$ & . \\
\hline H. o & $\ldots$ & $\ldots$ & .. & .. & 81 & 97 & $\cdots$ & 99 & .. & . \\
\hline Hydro & & & & & & & & & & \\
\hline H. & $\cdots$ & $\cdots$ & .. & .. & 12 & 75 & . & 83 & 90 & 92 \\
\hline & . & .. & .. & . & 7 & 60 & . & 73 & 77 & 80 \\
\hline H. & $\ldots$ & . & $\cdots$ & . & 8 & 70 & . & 78 & 83 & 86 \\
\hline Potassium hydro & & & & & & & & & & \\
\hline H. & $\cdots$ & $\cdots$ & $\cdots$ & . & 16 & 67 & $\cdots$ & 72 & 81 & 83 \\
\hline H. 2 & .. & .. & .. & .. & 14 & 56 & .. & 72 & 74 & 75 \\
\hline$\vec{H}$. & .. & . . & . & . & 16 & 60 & $\ldots$ & 70 & 77 & 83 \\
\hline Potass & & & & & & & & & & \\
\hline H. & $\cdots$ & $\cdots$ & $\cdots$ & .. & 1 & 12 & $\cdots$ & 29 & 38 & 45 \\
\hline H. & .. & . & .. & . & 5 & 9 & . & 20 & 25 & 30 \\
\hline H. d & .. & . & . & .. & 2 & 10 & . & 27 & 33 & 42 \\
\hline Potassi & & & & & & & & & & \\
\hline H. & . & . & $\cdots$ & .. & 11 & 52 & . & 68 & 75 & 84 \\
\hline H. 2 & . & .. & $\ldots$ & .. & 6 & 12 & . & 50 & 65 & 75 \\
\hline H. d $\varepsilon$ & 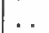 & $\ldots$ & . & $\ldots$ & 8 & 34 & . & 59 & 68 & 80 \\
\hline Potase & & & & & & & & & & \\
\hline H. d & $\cdots$ & $\ldots$ & $\ldots$ & .. & 1 & 2 & $\cdots$ & 3 & . & 4 \\
\hline & . & . & $\cdots$ & .. & 1 & 2 & . & 3 & & 4 \\
\hline & . & $\cdots$ & $\ldots$ & . & 1 & 1 & . & 2 & 3 & 4 \\
\hline Sodiur & & & & & & & & & & \\
\hline H. C & $\cdots$ & $\ldots$ & .. & . & 6 & 18 & $\therefore$ & 43 & 45 & 52 \\
\hline H. & . & .. & . & .. & 1 & 6 & . & 35 & 42 & 48 \\
\hline & .. & $\ldots$ & $\ldots$ & $\ldots$ & 2 & 11 & . & 41 & 48 & 58 \\
\hline Sodiur & & & & & & & & & & \\
\hline & & . & $\cdots$ & . & 3 & 10 & . & 16 & 23 & 27 \\
\hline H. 2 & . & $\ldots$ & & .. & 2 & 5 & .. & 10 & 14 & 15 \\
\hline H. & & . . & . & $\ldots$ & 0.5 & $\ldots$ & $\ldots$ & 1 & 10 & 14 \\
\hline Sodiur & & & & & & & & & & \\
\hline & & $\cdots$ & . & . & 25 & 76 & $\ldots$ & 96 & 99 & . \\
\hline & $\ldots$ & $\ldots$ & $\ldots$ & . & 19 & 79 & $\ldots$ & 99 & & . \\
\hline H. & . & .. & . & . & 17 & 65 & . & 95 & 99 & . \\
\hline Calci & & & & & & & & & & \\
\hline H. & $\cdots$ & . & . & . & 0.5 & 2 & .. & & 3 & 4 \\
\hline & . & $\ldots$ & . & .. & 1 & & . & 2 & 3 & 3 \\
\hline H. & .. & . . & . & . & 0.5 & 2 & . & 3 & 5 & 5 \\
\hline Urani & & & & & & & & & & \\
\hline & $\ldots$ & . & . & & 0.5 & 2 & . . & 4 & 5 & \\
\hline & $\ldots$ & $\ldots$ & $\ldots$ & .. & 2 & 3 & .. & $\ldots$ & 4 & 4 \\
\hline & . & $\ldots$ & . & .. & 1 & 2 & .. & 3 & $\ldots$ & 3 \\
\hline Stront & & & & & & & & & & \\
\hline & . & $\ldots$ & . & . & 2 & 3 & .. & 11 & 19 & 25 \\
\hline & .. & $\ldots$ & $\ldots$ & & 3 & 5 & .. & 7 & 9 & 14 \\
\hline & & .. & .. & . . & 1 & 5 & . & 9 & 13 & 25 \\
\hline Cobal & & & & & & & & & & \\
\hline & .. & $\ldots$ & $\cdots$ & . & 0.5 & 2 & & & 3 & 3 \\
\hline & . & $\ldots$ & $\ldots$ & . & 0.5 & 2 & . & 3 & $\cdots$ & 3 \\
\hline & .. & . . & & .. & 0.5 & 1 & . & 1.5 & & 1.5 \\
\hline Copp & & & & & & & & & & \\
\hline & . & . & . . & . . & 0.5 & 2 & & & 3 & 4 \\
\hline & & .. & .. & .. & 0.5 & & . & . & 1 & 2 \\
\hline & . . & $\ldots$ & $\ldots$ & .. & 0.5 & 1.5 & .. & $\ldots$ & 2 & 2 \\
\hline Cupri & & & & & & & & & & \\
\hline & . . & . & & & 0.5 & & & 1 & 3 & 3 \\
\hline & . & .. & .. & & 0.5 & 1 & .. & 2 & 3 & 3 \\
\hline & & .. & $\ldots$ & $\ldots$ & 0.5 & 1 & .. & 1.5 & & 1.5 \\
\hline Bar & & & & & & & & & & \\
\hline & & .. & . & & 0.5 & . . & . . & & & \\
\hline & & . & . & & 0.5 & .. & & & 1 & 1 \\
\hline & & $\ldots$ & . & . . & 0.5 & .. & . . & 1 & $\ldots$ & 1 \\
\hline & & & & & & & & & & \\
\hline & . & .. & . & . & 0.5 & 1 & . & . & & \\
\hline & & .. & . & & 0.5 & 1 & . . & & 2 & 2 \\
\hline H. & & .. & . & . & 1 & 2 & & & & 4 \\
\hline
\end{tabular}

REaOtion-Intensities OF THe Hybrid.

This section treats of the reaction-intensities of the hybrid as regards sameness, intermediateness, excess, and deficit in relation to the parents. (Table A 4 and Charts D 64 to D 84.)

The reactivities of the hybrid are the same as those of the seed parent in not a single reaction; the same as those of the pollen parent with iodine and sulphuric acid; the same as those of both parents with safranin, potassium sulphide, calcium nitrate, uranium nitrate, cobalt nitrate, copper nitrate, cupric chloride, barium chloride, and mercuric chloride; intermediate with hydrochloric acid, potassium hydroxide, potassium iodide, potassium sulphocyanate, sodium hydroxide, and strontium nitrate (in three reactions being closer to those of the seed parent and in three mid-intermediate); highest with polarization, temperature, chromic acid, pyrogallic acid, and nitric acid (in one being closer to the pollen parent, in three closer to the seed parent, and in one as close to one as to the other parent); and the lowest with gentian violet, chloral hydrate, sodium sulphide, and sodium salicylate (in two being closer to the pollen parent, in one closer to the seed parent, and in one as close to one as to the other parent).

The following is a summary of reaction-intensities: Same as seed parent, 0; same as pollen parent, 2; same as both parents, 9 ; intermediate, 6 ; highest, 5 ; lowest, 4 .

In none of the reactions is there sameness to the seed and in only two is there sameness to the pollen parent; and intermediateness is scarcely more frequent than development in excess or deficit of parental extremes. Parental influences on the starch of the hybrid seem to be somewhat in favor of the seed parent.

\section{Composite CuRves of the Reaction-Intensities.}

This section treats of the composite curves of the reaction-intensities showing the differentiation of the starches of Hippeastrum dacones, $H$. zephyr, and $H$. daones-zephyr. (Chart E 4.)

The most conspicuous features of this chart are:

(1) The closeness of all three curves.

(2) The curve of $H$. docones, excepting in the polarization reaction, is higher than the corresponding reactions of $H$. zephyr in the reactions with iodine, gentian violet, chloral hydrate, chromic acid, pyrogallic acid, nitric acid, sulphuric acid, hydrochloric acid, potassium hydroxide, potassium iodide, potassium sulphocyanate, sodium hydroxide, sodium sủlphide, and strontium nitrate; lower with polarization; and the same or practically the same with safranin, temperature, potassium sulphide, sodium salicylate, calcium nitrate, uranium nitrate, cobalt nitrate, copper nitrate, cupric chloride, barium chloride, and mercuric chloride.

(3) In $H$. dcoones, the very high reactions with polarization, chromic acid, and sulphuric acid; the high with pyrogallic acid and sodium salicylate; the moderate reactions with iodine, gentian violet, safranin, and hydrochloric acid; the low reactions with temperature, chloral hydrate, nitric acid, potassium hydroxide, potassium sulphocyanate, and sodium hydroxide; and the very low reactions with potassium iodide, potassium sulphide, sodium sulphide, calcium nitrate, uranium nitrate, strontium nitrate, cobalt nitrate, copper nitrate, cupric chloride, barium chloride, and mercuric chloride reactions.

(4) In $H$. zephyr, the very high reactions with polarization and sulphuric acid; the high with chromic acid, pyrogallic acid, and sodium salicylate; the moderate with iodine, gentian violet, and safranin; the low with temperature, nitric acid, hydrochloric acid, potassium hydroxide, and potasium sulphocyanate; the very low with chloral hydrate, potassium iodide, potassium sul- 
phide, sodium hydroxide, sodium sulphide, calcium nitrate, uranium nitrate, strontium nitrate, cobalt nitrate, copper nitrate, cupric chloride, barium chloride, and mercuric chloride.

(5) In the hybrid, H. daones-zephyr, the very high reactions with polarization and sulphuric acid; the high with chromic acid, pyrogallic acid, and sodium salicylate; the moderate with iodine, gentian violet, and safranin; the low with temperature, nitric acid, hydrochloric acid, potassium hydroxide, and potassium sulphocyanate; and the very low with chloral hydrate, potassium iodide, potassium sulphide, sodium hydroxide, sodium sulphide, calcium nitrate, uranium nitrate, strontium nitrate, cobalt nitrate, copper nitrate, cupric chloride, barium chloride, and mercuric chloride. ties:

The following is a summary of the reaction intensi-

\begin{tabular}{l|c|c|c|c|c}
\hline & $\begin{array}{c}\text { Very } \\
\text { high. }\end{array}$ & High. & $\begin{array}{c}\text { Mod- } \\
\text { erate. }\end{array}$ & Low. & $\begin{array}{l}\text { Very } \\
\text { low. }\end{array}$ \\
\hline H. dæones.......... & 3 & 2 & 4 & 6 & 11 \\
H. zephyr........ & 2 & 3 & 3 & 5 & 13 \\
H. dæones-zephyr... & 2 & 3 & 3 & 5 & 13 \\
\hline
\end{tabular}

Notes on the Hippeastrums.

The hippeastrums exhibit properties in general so closely alike as to suggest very closely related plants, such as in fact they are. In histological properties while all possess in common certain fundamental generic characters, each has certain individualities that are manifested in variable ways. Each hybrid is more closely related in certain histological features to one parent and in certain others to the other parent, but the directions of these variations may be the same or different in the different hybrids. Thus, in form $H$. titan-cleonia is closer to the seed parent than to the pollen parent, while in H. ossultan-pyrrha the relationship is closer to the pollen parent; in hilum two of the hybrids are closer to the seed parent and one closer to the pollen parent; in lamellæ in one hybrid in characters they are nearer the pollen parent, but in number the same as both parents, in another hybrid the number is the same as in the seed parent but in the characters closer to those of the pollen parent, and in the third hybrid characters and number are closer to seed parent; and in size one hybrid is more closely related to the seed parent, another to the pollen parent, and another in the larger grains to the pollen parent. The hybrid modifications are associated with inherent peculiarities of the parents, and inasmuch as the parents of the three sets differ the hybrids differ, and in fact they differ as much from each other as do the parents.

The uniformity or close correspondence in the courses of the velocity-reaction curves in the case of each reagent associated with a corresponding uniformity of the composite reaction curves affords striking evidence of the accuracy of the method employed in the recognition of plant relationships. In a word, there is a hippeastrum curve, which curve is modified in relation to each plant represented.

The parental relationships of the hybrids in the various reactions are as variable as those indicated in the histological peculiarities. Each of the hybrids may be in some of the reactions the same as the seed parent, in others the same as the pollen parent or as both parents, in others intermediate, and in others higher or lower than either parent. Intermediateness is far from being the rule, since in only 13 out of 78 reactions was intermediateness recorded, and in only 6 was there mid-intermediateness. In fact, reactivity of the hybrid in excess
TABLE A 5.

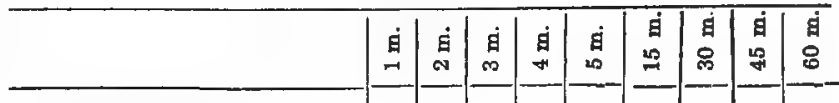

Chloral hydrate:

H. katherinæw.

H. magnificus.

Chromic acid:

H. katherinæ.

H. magnificus
H. andromeda

H. andromed:
Pyrogallic acid:

H. kathering .

H. magnificus.

H. andromeda.

Nitric acid:

H. katherinæ.

cus............

H. andromeda.

Sulphuric acid:

H. kathering .

H. magnificus. . . .

H. andromeda...........

Hydrochloric acid:

H. katherinæ.

H. magnificus..........

H. andromeda.....

Potassium hydroxide:

H. katherinæ.

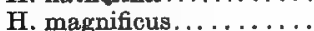

H. andromeda.

Potassium iodide:

H. katherinæ.

H. magnificus.

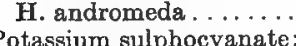

H. katherinæ.

H. magnificus.

H. andromeda....

Potassium sulphide:

H. katherinæ .

H. magnificus.

Sodium hydroxide:

H. katherinæ. .

H. magnificus...........

H. andromeda.

Sodium sulphide:

H. katherinæ

H. magnificus.

H. andromeda .

Sodium salicylate:

H. katherinæ.

H. magnificus. .

Calcium nitrate:

H. katherinæ

H. magnificus.............

H. andromeda .

Uranium nitrate:

H. katherinæ

H. magnificus.

H. andromeda.
Strontium nitrate:

Strontium nitrate:

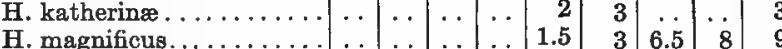

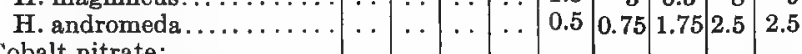

Cobalt nitrate:

H. katherinæe

H. magnificus............

$\mathrm{H}$. andromeda . . . . . . . .

Copper nitrate:

H. katherinæ

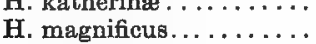

H. andromeda

Cupric chloride:

H. kathering.

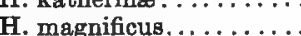

H. andromeda...........

Barium chloride:

H. katherin $\circledast$

H. magnificus .............

H. andromeda .

Mercuric chloride:

H. katherinx .

H. magnificus..........

H. andromeda $\ldots \ldots \ldots$ \begin{tabular}{l|l|l|l|l}
7 & 20 & 60 & 67 & 74
\end{tabular}

\begin{tabular}{ll|l|l|l}
4 & 14 & 15 & 17 & 17
\end{tabular}

$\begin{array}{lllll}1 & 0.5 & 23 & 92 & 97\end{array}$

\begin{tabular}{r|r|r|r|r}
3 & 19 & 27 & 86 & 97 \\
0.5 & 8 & 25 & 90 & 95
\end{tabular}

\begin{tabular}{l|l|l|l|l}
3 & 7 & 10 & 12 & 30
\end{tabular}

\begin{tabular}{l|l|l|l|l}
7 & 20 & 60 & 76 & 86
\end{tabular}

\begin{tabular}{rr|r|r|r}
1 & 3 & 8 & 12 & 26
\end{tabular}

$\begin{array}{rrrrr}1.5 & 2 & 3 & 4 & 6\end{array}$

\begin{tabular}{l|l|l|l|l}
4 & 40 & 45 & 48 & 50
\end{tabular}

\begin{tabular}{lllll|l}
10 & 35 & 79 & 90 & 94
\end{tabular}

\begin{tabular}{lllll|l|l}
10 & 75 & 87 & 97 & 99
\end{tabular}

\begin{tabular}{l|l|l|l|l}
9 & 50 & 81 & 93 & 98
\end{tabular}

\begin{tabular}{l|l|l|l|l}
1 & 3 & 10 & 12 & 15
\end{tabular}

\begin{tabular}{l|l|l|l|l}
7 & 35 & 66 & 75 & 83 \\
3 & $\ldots$ & 11 & 30 & 42
\end{tabular}

\begin{tabular}{r|r|r|r|r}
1 & $\ldots$ & 2 & $\ldots$ & 2 \\
3 & 9 & 11 & $\ldots$ & 20
\end{tabular}

\begin{tabular}{r|r|r|r|r}
3 & 6 & 7 & 9 & 11
\end{tabular}

$\begin{array}{lllllll}1.5 & \ldots & 2 & \ldots & 3\end{array}$

$\begin{array}{lllll}3 & 4.5 & 7 & 12\end{array}$

$\begin{array}{lllllll}1 & \ldots & 2.5 & \ldots & 3\end{array}$

\begin{tabular}{r|r|r|r|r}
2.5 & ii & 22 & 3 & 4 \\
\hline & 11 & 22 & 40
\end{tabular}

\begin{tabular}{rrrr|r|r}
1 & 3 & 3.5 & 4 & 4
\end{tabular}

\begin{tabular}{ll|r|r|r}
1 & $\ldots$ & 2 & $\ldots$ & 2
\end{tabular}

\begin{tabular}{rr|r|r|r} 
& 1 & 2.5 &.. & 2.5 \\
&.. &.. &.. & 1
\end{tabular}

$\begin{array}{lllllll}1 & \ldots & \ldots & \ldots & 3\end{array}$

\begin{tabular}{llllll|l}
2 & 15 & 24 & 27 & 35
\end{tabular}

$\begin{array}{llllll}0.5 & \ldots & \ldots & 2 & 2\end{array}$

\begin{tabular}{lllll|l}
2 & 5 & 7.5 & 9.5 & 9.5
\end{tabular}

\begin{tabular}{llll|l|l|l}
0.5 & $\cdots$ & 1 & 2 & 2.5
\end{tabular}

$\begin{array}{lllll}9.5 & 36 & 70 & \ddot{95} & 9 \dot{8} .5\end{array}$

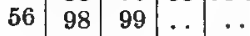

$\begin{array}{llllllll}1 & \ldots & \ldots & \ldots & 1\end{array}$

\begin{tabular}{cc|c|c|c}
2.5 & 3.5 & 5 & 5.5 & 6 \\
0.5 & $\ldots$ & 1 &. & 1
\end{tabular}

$\begin{array}{llllll}1 & \ldots & \ldots & \ldots & 1.25\end{array}$

\begin{tabular}{r|rrrrr}
2 & $\ldots$ & 3.5 & 5 & 5
\end{tabular}

\begin{tabular}{llll|l|l}
0.5 & $\ldots$ & $\ldots$ &. & 0.5
\end{tabular}

0.5

$\begin{array}{lllll}0.5 & \ldots & \ldots & \ldots & 0.5\end{array}$

\begin{tabular}{llll|l|l}
0.5 & $\ldots$ & $\ldots$ & $\ldots$ & 0.5 \\
& & & & &
\end{tabular}

$\begin{array}{lllllll}0.5 & \ldots & \ldots & \ldots & 1.5\end{array}$

\begin{tabular}{rrr|r|r}
0.5 & $\ldots$ & 1 & $\ldots$ & 1
\end{tabular}

\begin{tabular}{ll|llll}
0.5 & $\ldots$ & $\ldots$ & $\ldots$ & 0.5
\end{tabular}

$\begin{array}{lllllll}0.5 & \ldots & \ldots & \ldots & 0.5\end{array}$

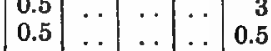

$\begin{array}{lllllll}1.25 & 1.5 & \ldots & \ldots & 1.5\end{array}$

\begin{tabular}{rr|r|r|r}
0.5 & $\cdots$ & $\cdots$ & $\cdots$ & 1 \\
0.5 & $\cdots$ & & & 0.5
\end{tabular}

\begin{tabular}{c|cc|c|c}
0.5 & $\ldots$ & $\ldots$ & $\ldots$ & 0.5 \\
1.25 & 1.5 & $\ldots$ & $\ldots$ & 0.5
\end{tabular}

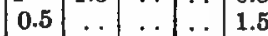

\begin{tabular}{ll|l|l|l}
0.5 & $\ldots$ & $\ldots$ & $\ldots$ & 1.5 \\
& & $\ldots$ &
\end{tabular} 
or deficit of parental extremes is more common than intermediateness, for in 21 reactions the hybrids were higher than those of either parent and in 9 lower than those of either parent. In case of all three hybrids the seed parent seems to be the more potent in influencing the characters of the starch, this potency being the most marked in $H$. ossultan-pyrrha and least marked in $H$. daones-zephyr.

\section{Comparisons of the Starches of Hzmantifus KATHERINA, H. MAGNIFICUS, AND H. ANDROMEDA.}

In histologic characteristics, in polariscopic figures, in the reactions with selenite, in the reactions with iodine, and in the qualitative reactions with the various chemical reagents it will be noted that the parent starches not only exhibit properties in common in variable degrees of development, but also individualities which collectively serve to distinguish them.

The starch grains of Homanthus magnificus contain proportionately a larger number of aggregates; there are compound grains that are not found in $H$. katherina; and the grains tend to more irregularity, to more breadth in relation to length, and to rounded ends. The hilum is more distinct and more frequently fissured, but the eccentricity is about the same; the lamellæ are less distinct; and the size is larger, with a tendency to broadness. In polariscopic figure and reactions with selenite there are various differences. The grains of the hybrid $H$. andromeda are in form in general closer to those of $H$. katherince, and in certain respects closer to those of the other parent. They are more irregular than those of either parent, and there are compound grains like those found in $H$. magnificus, but they are less numerous. In the character of the hilum and in size they are closer to those of $H$. katherince, but in lamella there does not appear to be a definite leaning toward one or the other parent. In the polariscopic figure and appearances with selenite the grains are closer to $H$. katherino, and the same is true in regard to their qualitative behavior with iodine. In the qualitative reactions with chloral hydrate, nitric acid, potassium iodide, potassium sulphocyanate, and sodium salicylate the grains show a close relationship to those of $H$. katherince, except in the case of a few grains in each reaction which show a corresponding relationship to $H$. magnificus. On the whole, the relationship is very close to $H$. katherince.

Reaction-intensities Expressed by Light, Color, and TemperaPolarization: ture Reactions.

H. katherinæ, high to very high, value 75 .

H. magnificus, very high, much higher than $H$. katherinæ, value 90 .

H. andromeda, high to very high, higher than $H$. katherinæ, Iodine: value 82 .

H. katherinæ, moderate to light, value 45.

H. magnificus, moderate, deeper than H. katherinæ, value 50 .

H. andromeda, moderate to deep, a little deeper than $H$. katherinæ, value 47

Gentian violet:

H. katherinæ, moderate to deep, value 60 .

H. magnificus, moderate to deep; not so deep as H. katherinæ, value 55.

H. andromeda, moderate to deep, slightly lighter than H. katherinæ, value 58 .

Safranin:

H. katherinæ, moderate to deep, value 60 .

H. magnificus, moderate to deep, the same as $H$. katherinm, value 60 .

H. andromeda, moderate to deep, lighter than in the parent-stock, value 58 .

Temperature:

H. katherine, majority at 79 to $81^{\circ}$, all at 82 to $84^{\circ}$, mean $83^{\circ}$.

H. magnificus, majority at 77 to $77.5^{\circ}$, all at 78 to $79^{\circ}$, mean $78.5^{\circ}$,

H. andromeda, majority at 75.5 to $80^{\circ}$, all at 81 to $82^{\circ}$, mean $81.5^{\circ}$.

The reactivities of $H$. katherince are lower than those of $H$. magnificus in the reactions with polarization, iodine, and temperature; higher with gentian violet; and the same with safranin. The reactivities of the hybrid are intermediate in the reactions with polarization, iodine, gentian violet, and temperature; and lower than those of the parents with safranin. With the exception of the last and the temperature reaction the relationship of the hybrid is practically exactly mid-intermediate; and in the temperature reaction it is closer to $H$. katherinoe.

Table A 5 shows the reaction-intensities in percentages of total starch gelatinized at definite intervals (minutes) :

\section{Velocity-REaction CURves.}

This section treats of the velocity-reaction curves of the starches of Hamanthus katherince, $H$. magnificus, and $H$. andromeda, showing the quantitative differences in the behavior toward different reagents at definite timeintervals. (Chart D 85 to D 105.)

The most conspicuous features of these charts are:

(1) The individualities of each chart in relation to the reagent, except in the cases where the reactions are so slow and the figures so close as to be within the limits of error. In the charts in which the reactions are otherwise than very slow the three curves vary in their closeness to one another within wide limits. Thus, in the reactions with chromic acid and sulphuric acid all three curves keep close together throughout the 60 minutes, but the charts are readily distinguishable from each other, especially at the 15 - and 30 -minute periods, at which times the curves are much higher in the sulphuricacid chart. The curves for chloral hydrate, nitric acid, and hydrochloric acid show a tendency during the progress of the reactions to divergence, in all three charts the curves of the hybrid being intermediate, but in two closer to the curve of $H$. katherince. The chart for sodium salicylate stands isolated, owing especially to the relatively high reactivities of the hybrid and $H$. katherino during the first 5 minutes. In all of the charts in which the three curves are sufficiently separated to make satisfactory determinations, the curve of the hybrid, with the exception of a few instances, tends definitely to intermediateness.

(2) The curves of $H$. magnificus in the reactions with chloral hydrate, pyrogallic acid, chromic acid, potassium hydroxide, potassium sulphocyanate, sodium salicylate, and sodium hydroxide, in all of which the reactivities are sufficiently marked to bring out positive differences in reactive-intensities, are the highest excepting in two cases (chloral hydrate and sodium salicylate), in both of which the curves of $H$. katherino are the highest-a curious reversal of position. In all of the charts in which positive differences have been brought out, the curve of the hybrid tends to be closer to that of $H$. katherine irrespective of the position of the latter in relation to the curve of $H$. magnificus.

(3) The curves of the hybrid, except in the reactions in which all three curves are essentially the same, tend to be the same as those of the seed parent or of some degree of intermediateness. In the latter group there is an obvious tendency to mid-intermediateness or to the seed parent.

\section{Reaction-Intensities of the Hrbrid.}

The following section treats of the reaction-intensities of the hybrid as regards sameness, intermediateness, excess, and deficit in relation to the parents. (Table A 5 and Charts D 85 to D 105.)

The reactivities of the hybrid are the same as those of the seed parent in the pyrogallic acid, potassium iodide, potassium sulphocyanate, sodium hydroxide, sodium sulphide, calcium nitrate, uranium nitrate, and 
strontium nitrate; the same as those of the pollen parent in none; the same as those of both parents in the reactions with potassium sulphide, cobalt nitrate, copper nitrate, cupric chloride, barium chloride, and mercuric chloride; intermediate with polarization, iodine, gentian violet, temperature, chloral hydrate, chromic acid, nitric acid, sulphuric acid, hydrochloric acid, potassium hydroxide, and sodium salicylate (in four being closer to the seed parent, and in seven mid-intermediate) ; highest in none; and the lowest with safranin, in which it is as close to one as to the other parent.

The following is a summary of the reaction-intensities: Same as seed parent, 8; same as pollen parent, 0 ; same as both parents, 6 ; intermediate, 11 ; highest, 0 ; lowest, 1 .

The stronger influences of the seed parent on the properties of the starch of the hybrid are very marked. Intermediateness is quite common. In no reaction is there sameness in relation to the pollen parent or the highest reactivity of the three starches, and in only one reaction is the hybrid the lowest.

\section{Composite-cURVES OF THE REACTION-INTENSITIES.}

This section deals with the composite-curves of the reaction-intensities, showing the differentiation of the starches of Homanthus katherince, $H$. magnificus, and H. andromeda. (Chart E 5.)

The most conspicuous features of the chart may be summed up as follows:

(1) The moderate to very low, generally very low, positions of the curves with few exceptions, the only important members of the latter group being the polarization and sodium-salicylate reactions, thus showing that these starches exhibit generally a high to very high resistance.

(2) The contiguity of all three curves throughout the chart and the unity of type of curve, indicating a close botanical relationship of the parents and no tendency for departure of hybrid characteristics from those of the parents.

(3) The highest position of the curve of $H$. magnificus throughout the chart, excepting in the reactions with gentian violet, safranin, chloral hydrate, chromic acid, and sodium salicylate--in the safranin and chromic acid the curves are the same or practically the same as those of $H$. katherino, and with chloral hydrate and sodium salicylate distinctly lower, they being the lowest of all three curves. The inversion of the positions of the $H$. magnificus and $H$. katherine curves in the gentian violet, chloral hydrate, and sodium salicylate reactions is most interesting and significant.

(4) In the curve of $H$. katherina the very high reaction with sodium salicylate; the high with polarization, gentian violet, and safranin; the moderate with iodine, chromic acid, and sulphuric acid; the low with chloral hydrate; the very low with temperature, pyrogallic acid, nitric acid, hydrochloric acid, potassium hydroxide, potassium iodide, potassium sulphocyanate, potassium sulphide, sodium hydroxide, sodium sulphide, calcium nitrate, uranium nitrate, strontium nitrate, copper nitrate, cupric chloride, barium chloride, and mercuric chloride.

(5) In the curve of $B$. magnificus the very high polarization reaction; the high reactions with safranin, sulphuric acid, and sodium salicylate; the moderate with iodine, gentian violet, and chromic acid; the low with temperature, pyrogallic acid, nitric acid, and hydrochloric acid; the very low with chloral hydrate, potassium hydroxide, potassium iodide, potassium sulphocyanate, potassium sulphide, sodium hydroxide, sodium sulphide, calcium nitrate, uranium nitrate, strontium nitrate, cobalt nitrate, copper nitrate, cupric chloride, barium chloride, and mercuric chloride.

(6) In the curve of the hybrid $H$. andromeda, the very high reactions with polarization and sodium salicylate; the absence of high reactions; the moderate with iodine, gentian violet, safranin, chromic acid, and sulphuric acid, the low with temperature; and the very low with chloral hydrate, pyrogallic acid, nitric acid, hydrochloric acid, potassium hydroxide, potassium iodide, potassium sulphocyanate, potassium sulphide, sodium hydroxide, sodium sulphide, calcium nitrate, uranium nitrate, strontium nitrate, cobalt nitrate, copper nitrate, cupric chloride, barium chloride, and mercuric chloride. The following is a summary of the reaction-intensities:

\begin{tabular}{l|c|c|c|c|c}
\hline & $\begin{array}{c}\text { Very } \\
\text { high. }\end{array}$ & High. & $\begin{array}{c}\text { Mod- } \\
\text { erate. }\end{array}$ & Low. & $\begin{array}{c}\text { Very } \\
\text { low. }\end{array}$ \\
\hline H. kathering....... & 1 & 3 & 3 & 1 & 18 \\
H. magnificus...... & 1 & 3 & 3 & 4 & 15 \\
H. andromeda..... & 2 & 0 & 5 & 1 & 18 \\
\hline
\end{tabular}

\section{Comparisons of the Starches of H无manthus}

ratherin 2 , H. PUNICEUS, AND H. KöNIG ALBERT.

In histologic characteristics, polariscopic figures, in the reactions with selenite and with iodine, and in the qualitative reactions with the various chemical reagents it will be noted that the parents exhibit properties in common in varying degrees of development and individualities by which collectively they can be differentiated. The most conspicuous differences in the starch of $H$. puniceus in comparison with that of Homanthus katherince are to be seen in the well-marked depressions (sometimes slightly concave) which are not present in the latter starch, less frequent rounded protuberances, less frequent secondary lamellæ, peculiar arrangements of the components of aggregates, and much more flattening of the grains. The hilum is more often demonstrable and is, on the whole, less eccentric; the primary lamellæ vary somewhat in general characters from those of $H$. lcatherina, and they are somewhat more numerous, but secondary lamellæ are less numerous; and while the sizes are much alike there is a manifest tendency for a relatively greater breadth in proportion to length. In polariscopic figure, selenite reactions, and qualitative reactions with iodine there are some minor differences. In the qualitative reactions with the chemical reagents there are similarities and individualities. The starch of the hybrid $H$. könig albert, is in form, character, and eccentricity of the hilum, lamellæ, and size more closely related to $H$. puniceus than to the other parent. In the polariscopic figures and reactions with selenite it is closer to $H$. puniceus, but in both qualitative and quantitative reactions with iodine it is closer to $H$. katherina. In the qualitative chemical reactions with chloral hydrate, nitric acid, potassium iodide, potassium sulphocyanate, potassium sulphide, and sodium salicylate it is closer, generally much closer, to $H$. Katherina.

Reaction-intensities Expressed by Light, Color, and TemperaPolarization:

H. katherinæ, high to very high, value 75 .

H. puniceus, high to very high, slightly higher than $H$. katherinæ, value 78.

H. könig albert, high to very high, slightly higher than $H$. puniceus, value 80 .

Iodine:

H. katherinæ, moderate to light, value 45 .

H. puniceus, moderate to light, lighter than in $H$. katherine, value 40.

H. könig albert, moderate to light, not so deep as in H. katherinæ, but deeper than in $H$. puniceus, value 43. 
Gentian violet:

H. katherinæ, moderate to deep; value 60 .

H. puniceus, moderately deep to deep, slightly deeper than $\mathbf{H}$. katherinæ; value 62.

H. könig albert, moderate to deep, not so deep as in the parents, value 58 .

Safranin:

H. katherinæ, moderate to deep; value 60 .

H. puniceus, moderately deep to deep, slightly deeper than in $\mathbf{H}$. katherinæ, value 62 .

H. könig albert, moderate to deep, not so deep as in the parents, value 58 .

Temperature:

H. katherinæ, majority at 79 to $80^{\circ}$, all at 82 to $84^{\circ}$, mean $83^{\circ}$.

H. puniceus, majority at 77 to $79^{\circ}$, all at 81 to $82.5^{\circ}$, mean $81.75^{\circ}$.

H. könig albert, majority at 80 to $82^{\circ}$, all at 82.5 to $84^{\circ}$, mean $83.25^{\circ}$.

The reactivity of $H$. katherino is higher than that of the other parent in the reaction with iodine and lower in those with polarization, gentian violet, safranin, and temperature. The hybrid is mid-intermediate in the iodine reaction, the highest in the polarization reaction, lowest in the gentian violet and safranin reactions, and the same as that of the seed parent in the temperature reaction. In three it is closer to or the same as the seed parent, in one closer to the pollen parent, and in one mid-intermediate.

Table A 6 shows the reaction-intensities in percentages of total starch gelatinized at definite intervals (minutes).

\section{Velocity-REACTION CURVES.}

The following section deals with velocity-reaction curves of the starches of Hamanthus katherince, $H$. puniceus, and $H$. könig albert, showing the quantitative differences in the behavior toward different reagents at definite time-intervals. (Charts D 106 to D 126.)

The most conspicuous features of these charts are:

(1) The marked tendency for the curves of $H$. katherino and the hybrid to run together, usually very closely, and well separated from the curve of $H$. puniceus. Both features are well exhibited in all of the reactions, with the exception of those with chloral hydrate, pyrogallic acid, sodium salicylate, and barium chloride. Even in these instances the closer relationship of $H$. katherina and the hybrid is evident.

(2) The tendency for the curve of the hybrid to an intermediate position between those of the parent-stocks, although distinctly closer to that of $H$. katherince, as shown in the reactions with chromic acid, pyrogallic acid, nitric acid, sulphuric acid, hydrochloric acid, and sodium salicylate. In the chloral-hydrate reaction the curve of the hybrid is curiously distinctly lower than that of either parent. In the remaining reactions, 14 in number, the starches of both $H$. katherince and the hybrid are so resistant that such differences as are recorded are slight and fall within the limits of error. From experience with other resistant starches modifications in the strengths of the reagents would doubtless elicit peculiarities in accord with the foregoing:

(3) The individuality of each of the charts with few exceptions; hence, the peculiarity of each chart in specific relation to the reagent. Some bear somewhat close resemblances, as for instance, those particularly of pyrogallic and nitric acid, and those of another group including the potassium iodide, potassium hydroxide, potassium sulphocyanate, potassium sulphide, sodium hydroxide, sodium sulphide, calcium nitrate, strontium nitrate, and cupric chloride, in which the main difference between the positions of the curves lies in the height of the curves of $H$. puniceus. The curves of the sodiumsalicylate reactions are of a markedly different character from those of other chemical reagents because of the high reactivities of all three starches. High reactivities of $H$. puniceus are also exhibited in the charts for pyro-
Table A 6.

\begin{tabular}{|c|c|c|c|c|c|c|c|c|c|}
\hline & $\underset{-1}{\text { g }}$ & 离 & 正 & 嵌 & 皇 & 豆 & 离 & 离 & $\dot{\dot{g}}$ \\
\hline Chloral hydrate: & & & & & & & & & \\
\hline & $\cdots$ & . & . & & 7 & 20 & 60 & 67 & \\
\hline H. $\mathrm{I}$ & $\ldots$ & . & . & . & 14 & 64 & 78 & 80 & \\
\hline H. könig albe & $\cdots$ & . & . & .. & 3 & 18 & 39 & 53 & \\
\hline Chromic acid: & & & & & & & & & \\
\hline & & & . & $\cdots$ & 1 & 5 & 23 & 92 & \\
\hline & .. & $\ldots$ & $\ldots$ & .. & 27 & 78 & 87 & 97 & \\
\hline & . & $\ldots$ & .. & $\ldots$ & 0.5 & 19 & 46 & 83 & \\
\hline Pyroge & & & & & & & & & \\
\hline & . & . & $\cdots$ & & 3 & 7 & 10 & 12 & \\
\hline & . & $\ldots$ & . & . & 65 & 95 & 97 & 98 & \\
\hline & $\ldots$ & $\ldots$ & . & $\ldots$ & 2 & 5 & 11 & 25 & \\
\hline Nitric & & & & & & & & & \\
\hline & . & $\ldots$ & . & . & 0.5 & 2 & 3 & 4 & \\
\hline H. & $\ldots$ & $\ldots$ & . & .. & 60 & 75 & 90 & 95 & \\
\hline & $\cdots$ & $\cdots$ & $\therefore$ & $\therefore$ & 0 & 2.5 & 11 & 14 & \\
\hline Sulphur & & & & & & & & & \\
\hline & . & $\ldots$ & . & $\cdots$ & 10 & 35 & 79 & 90 & \\
\hline & .. & . & . & . & 95 & 99 & & & \\
\hline & . & . & .. & . & 10 & 50 & 90 & 95 & \\
\hline Hyd & & & & & & & & & \\
\hline H. $\mathrm{k}$ & $\cdots$ & $\cdots$ & . & $\cdots$ & 1 & 3 & 10 & 12 & \\
\hline & $\ldots$ & . & . & .. & 80 & 91 & 95 & & 97 \\
\hline & $\ldots$ & .. & . & .. & 1 & 12 & 54 & 62 & \\
\hline Pota & & & & & & & & & \\
\hline$\ldots$ & $\ldots$ & $\ldots$ & . & . & 1 & & 2 & & \\
\hline & $\ldots$ & $\ldots$ & . & .. & 70 & 80 & 95 & 97 & \\
\hline & $\ldots$ & .. & $\ldots$ & . & 1.5 & 2 & , . & $\mathbf{3}$ & \\
\hline Pota & & & & & & & & & \\
\hline & $\ldots$ & . & . & . & 1.5 & & 2 & & \\
\hline & $\ldots$ & $\ldots$ & $\ldots$ & . & 52 & 56 & 67 & 80 & 92 \\
\hline & .. & $\ldots$ & $\ldots$ & .. & 1 & . & $\ldots$ & $\ldots$ & 1. \\
\hline Potass & & & & & & & & & \\
\hline H. & $\cdots$ & . & $\cdots$ & $\cdots$ & 2.5 & & $\ldots$ & 3 & \\
\hline H. & $\ldots$ & . & . & . & 72 & 84 & 89 & . & \\
\hline & .. & $\ldots$ & $\ldots$ & . & 12 & 2.5 & 3.5 & . & \\
\hline Potas & & & & & & & & & \\
\hline & $\cdots$ & . & . & . & 1 & & 2 & $\cdots$ & \\
\hline & $\ldots$ & $\ldots$ & $\ldots$ & .. & 45 & 60 & 66 & $\ldots$ & 70 \\
\hline & . & .. & . & . & 1 & .. & .. & . & \\
\hline Sodiu & & & & & & & & & \\
\hline & $\ldots$ & . & . & . & 1 & & $\cdots$ & & \\
\hline & $\ldots$ & .. & $\ldots$ & .. & 61 & 67 & 73 & 80 & 8 \\
\hline & $\ldots$ & . & $\ldots$ & . & 0.5 & . & $\ldots$ & 1.5 & \\
\hline Sodi & & & & & & & & & \\
\hline & $\ldots$ & . & . & . . & 0.5 & & $\ldots$ & 2 & \\
\hline & .. & $\ldots$ & . & . . & 50 & 54 & $\ldots$ & 57 & 60 \\
\hline & $\ldots$ & $\ldots$ & $\ldots$ & .. & 2 & 2.5 & .. & .. & 2. \\
\hline odi & & & & & & & & & \\
\hline & $\cdots$ & $\ldots$ & $\cdots$ & . . & 80 & 99 & & $\cdots$ & \\
\hline & . & . & $\ldots$ & . . & 60 & 96 & 100 & .. & \\
\hline & $\ldots$ & $\ldots$ & $\ldots$ & .. & 65 & 97 & 99 & .. &. \\
\hline Calcis & & & & & & & & & \\
\hline & $\ldots$ & . . & $\ldots$ & $\cdots$ & 1 & & & . & \\
\hline & . . & . & . & .. & 50 & 57 & 60 & .. & 62 \\
\hline $\bar{H}$ & $\ldots$ & $\ldots$ & $\ldots$ & . . & 0.5 & .. & . & .. & 0. \\
\hline Uran & & & & & & & & & \\
\hline & .. & $\cdots$ & . & . & 1 & $\cdots$ & & $\cdots$ & 1.2 \\
\hline & $\ldots$ & .. & . & . & 26 & 30 & 35 & . & 3 \\
\hline & $\ldots$ & $\ldots$ & $\ldots$ & .. & 0.5 & .. & $\ldots$ & .. & 0. \\
\hline Stron & & & & & & & & & \\
\hline & $\ldots$ & .. & . & . . & 2 & 3 & & & \\
\hline & .. & .. & $\ldots$ & .. & 44 & 50 & 60 & 68 & 78 \\
\hline & .. & .. & $\ldots$ & . & 0.5 & & 1 & .. & \\
\hline Cobal & & & & & & & & & \\
\hline & $\ldots$ & . & . & . & 0.5 & & & & \\
\hline & .. & .. & .. & . & 4 & 7 & 10 & 12 & 13 \\
\hline & .. & & $\ldots$ & .. & 0.5 & .. & $\ldots$ & .. & 0. \\
\hline & & & & & & & & & \\
\hline & $\ldots$ & .. & $\ldots$ & . & 0.5 & & & & \\
\hline & $\cdots$ & $\ldots$ & $\cdots$ & $\cdots$ & 11 & 14 & 15 & 16 & \\
\hline & $\ldots$ & $\ldots$ & $\ldots$ & .. & 0.5 & . & .. & . & 0. \\
\hline & & & & & & & & & \\
\hline H. 1 & $\cdots$ & . & . & & 0.5 & & & & 0. \\
\hline & & . . & .. & . & 37 & 53 & .. & 56 & 5 \\
\hline & $\ldots$ & . & .. & .. & 0.5 & & $\ldots$ & & 0. \\
\hline Bariu & & & & & & & & & \\
\hline & . & . & & & 1.25 & 1.5 & & & \\
\hline & .. & $\ldots$ & $\ldots$ & $\ldots$ & 1.5 & 2 & 6 & & \\
\hline & $\therefore$ & & . & . & 0.5 & & $\ldots$ & . & \\
\hline Mere & & & & & & & & & \\
\hline & .. & . & . & . & 1.25 & 1.5 & & & \\
\hline & .. & . & .. & .. & 7 & 15 & 17 & 20 & \\
\hline H. könig alber & & . & . & . & 0.5 & & $\cdots$ & & \\
\hline
\end{tabular}


gallic acid, nitric acid, sulphuric acid, and hydrochloric acid. It is of interest to note that while the $H$. puniceus curves are high, those of $H$. katherince and the hybrid are very low in the reactions with pyrogallic acid and nitric acid and variable from high to low in those with sulphuric acid and hydrochloric acid.

(4) The earliest period during the 60 minutes at which the three curves are best separated, and hence the best time to differentiate the starches, varies with the different reagents : with sodium salicylate at 5 minutes, with chromic acid and sulphuric acid at 15 minutes, with chloral hydrate and hydrochloric acid at 30 minutes, with pyrogallic acid at 45 minutes, and with nitric acid and the remaining reagents ( 15 in all), all of which react very slowly with $H$. katherince and the hybrid, in 60 minutes.

\section{Reaction-Intensities of the Hybrid.}

This section treats of the reaction-intensities of the hybrid as regards sameness, intermediateness, excess, and deficit in relation to the parents. (Table A 6, and Charts D 106 to D 126.)

The reactivities of the hybrid are the same as those of the seed parent with temperature, potassium hydroxide, potassium iodide, potassium sulphocyanate, potassium sulphide, sodium hydroxide, sodium sulphide, calcium nitrate, uranium nitrate, strontium nitrate, cobalt nitrate, copper nitrate, cupric chloride, barium chloride, and mercuric chloride; the same as the pollen parent in none; the same as those of both parents in none; intermediate with iodine, chromic acid, pyrogallic acid, nitric acid, sulphuric acid, hydrochloric acid, and sodium salicylate (in one being mid-intermediate, in one closer to the pollen parent, and in five closer to the seed parent); highest in the polarization reaction, and closer to the pollen parent; and the lowest in the reactions with gentian violet, safranin, and chloral hydrate, in all three being closer to the seed parent.

The following is a summary of the reaction-intensities: Same as seed parent, 15 ; same as pollen parent, 0 ; same as both parents, 0 ; intermediate, 7 ; highest, 1 ; lowest, 3.

While intermediateness is common, the inclination here and elsewhere, with three exceptions, is to the seed parent, and in over half of the cases the reactions are the same as those of the seed parent. The closeness of the hybrid to the seed parent almost throughout is very striking.

\section{Composite CURfes of Reaction-Intensities.}

The following section deals with the composite curves of the reaction-intensities, showing the differentiation of the starches of Homanthus katherino, $H$. puniceus, and H. könig albert. (Chart E 6.)

The most conspicuous features of the chart may be summed up as follows:

(1) The close correspondence of type of all three curves, excepting in the pyrogallic-acid reaction, in which those of $H$. puniceus exhibit an aberrant character, the curve rising instead of falling in order to be coincident with the curves of $H$. katherina and the hybrid. In the reactions in which both $H$. katherina and the hybrid are very resistant, which are numerous, no satisfactory relationship can be determined.

(2) The tendency of the curve of $H$. puniceus to be distinctly higher in most of the chemical reactions and therefore to be well separated from the curves of $H$. katherince and the hybrid. In the sodium-salicylate reaction all three curves impinge at practically the same point, and in the reactions with uranium nitrate, copper nitrate, cupric chloride, barium chloride, and mercuric chloride they approximate very closely or are practically identical. The stereochemic peculiarities of these three starches are strikingly suggested in the sameness of reaction with sodium salicylate, associated with the marked divergencies in the reactions, especially in the pyrogallic acid, nitric acid, sulphuric acid, hydrochloric acid, and other reactions.

(3) In $H$. katherinae, the very high reaction with sodium salicylate; the high with polarization, gentian violet, and safranin; the moderate with iodine, chromic acid, and sulphuric acid; the low with chloral hydrate; and the very low with temperature, pyrogallic acid, nitric acid, hydrochloric acid, potassium hydroxide, potassium iodide, potassium sulphocyanate, potassium sulphide, sodium hydroxide, sodium sulphide, calcium nitrate, uranium nitrate, strontium nitrate, cobalt nitrate, copper nitrate, cupric chloride, barium chloride, and mercuric chloride.

(4) In $H$. puniceus, the very high reactions with pyrogallic acid, sulphuric acid, hydrochloric acid, and sodium salicylate; the high with polarization, gentian violet, safranin, chromic acid, nitric acid, and potassium hydroxide; the moderate with iodine, potassium iodide, and potassium sulphocyanate; the low temperature, chloral hydrate, potassium sulphide, sodium hydroxide, sodium sulphide, calcium nitrate, strontium nitrate, and cupric chloride; and the very low with uranium nitrate, cobalt nitrate, copper nitrate, barium chloride, and mercuric chloride.

(5) In the hybrid, the very high reactions with poilarization and sodium salicylate; the high with sulphuric acid; the moderate with iodine, gentian violet, safranin, and chromic acid; the low with chloral hydrate and hydrochloric acid; and the very low with temperature, pyrogallic acid, nitric acid, potassium hydroxide, potassium iodide, potassium sulphocyanate, potassium sulphide, sodium hydroxide, sodium sulphide, calcium nitrate, uranium nitrate, strontium nitrate, copper nitrate, cupric chloride, barium chloride, and mercuric chloride. ties:

The following is a summary of the reaction-intensi-

\begin{tabular}{l|c|c|c|c|c}
\hline & $\begin{array}{c}\text { Very } \\
\text { high. }\end{array}$ & High. & $\begin{array}{c}\text { Mod- } \\
\text { erate. }\end{array}$ & Low. & $\begin{array}{c}\text { Very } \\
\text { low. }\end{array}$ \\
\hline H. katherinæ........ & 1 & 3 & 3 & 1 & 18 \\
H. puniceus....... & 4 & 6 & 3 & 8 & 5 \\
H. könig albert..... & 2 & 1 & 4 & 2 & 17 \\
\hline
\end{tabular}

Notes ON THE H \#MANTHUSES.

The hæmanthuses belong to a group of plants that yields starches that have distinctly low mean reactivities, all three species and their two hybrids showing this . peculiarity, only one-sixth of the total number of reac- 
tions being high to very high. It is of interest to note that in the sodium-salicylate reactions, with the exception of the reaction of $H$. magnificus, the curves are not only very high but also the same, while in this species the curve is distinctly lower than in the former. In the other reactions the curves of all of the starches show an unmistakable tendency toward coincidence in direction, the rises and falls being quite in harmony, excepting in $H$. puniceus with pyrogallic acid, in which there is a marked aberration, this curve rising while the curves of the other four fall. This peculiarity has been found in other genera, and is doubtless of both botanical and general biological significance. Comparing the curves of the three species, the curve of $H$. puniceus tends to be the highest, that of $H$. katherince the lowest, and that of $H$. magnificus intermediate, but near that of $H$. katherina.

According to Baker, $H$. katherina belongs to the subgenus Nerissa, and $H$. puniceus and $H$. magnificus to the subgenus Gyraxis, but the results of this investigation indicate that $H$. katherina and $H$. magnificus are much more closely related than are $H$. puniceus and $H$. magnificus. The curves of the former are such as to indicate different species of a subgenus, while the curve of $H$. puniceus is, as a whole, so well separated from those of the other two species as to point to this species being a member of another subgeneric group.

In comparing the influences of the parents on the properties of the offspring, it will be seen that in both sets there is a manifest greater potency of $H$. katherince than of the other parent, this being decidedly more marked in the $H$. katherina-puniceus-könig albert set than in the H. katherince-magnificus-andromeda set.

\section{Comparisons of the Starches of Crindm MOOREI, C. ZEYLANICUM, AND C. HYBRIDUM J.} c. HARVEY.

In histologic characteristics, in polariscopic figures, in the reactions with selenite, in the color reactions witl iodine, and in the qualitative reactions with the various chemical reagents it will be noted that the starches of the parents and hybrid exhibit properties in common in varying degrees of development, and also individualities which collectively are characteristic in each case. The starch grains of Crinum zeylanicum in comparison with those of $C$. moore $i$ exhibit differences in the proportions of certain of the conspicuous forms; not so much irregularity of the grains; certain protuberances and curvatures that are not observed in $C$. moorei; differences in size and definition of components of certain compound grains; and more broadening and flattening of the grains. The hilum is less refractive and has less frequently a rounded cavity; the fissures are more numerous and deeper, and a dragon-fly form may be present; a longitudinal fissure, rarely observed in C. moorei, is usually present, and it is longer, deeper, and branched; and the eccentricity is more variable. The lamellæ are finer distalward from the hilum than in $C$. moorei; there are some differences in the conspicuousness, distribution, and number of the coarse, fairly coarse, and secondary lamellæ; and the number of lamellæ is less. In size there is less variation, and the grains are, on the whole, dis- tinctly larger. In polariscopic properties, reactions with selenite, and qualitative reactions with iodine there are minor differences. There are also differences in the qualitative reactions with the chemical reagents. The grains of the hybrid are, in form, characters of the hilum and lamellæ, and in size in ratio of length to width closer to those of $C$. zeylanicum, but in length closer to C. moorei. In polariscopic figures, reactions with selenite, and qualitative reactions with iodine they are distinctly closer to those of $C$. zeylanicum. In the qualitative reactions with chloral hydrate, nitric acid, potassium hydroxide, potassium iodide, potassium sulphocyanate, potassium sulphide, sodium sulphide, sodium salicylate, copper nitrate, cupric chloride, and mercuric chloride alliances to both parental starches are noted, but the relationship to $C$. zeylanicum is markedly closer than to the other parent. The resemblances to C. moorei are most prominent in the sodium-salicylate reactions.

\section{Reaction-intensities Expressed by Light, Color, and Tempera- ture Reactions.} Polarization:

C. moorei, high to very high, value 85 .

C. zeylanicum, very high, much higher than C. moorei, value 93.

C. hybridum j. o. harvey, high to very high, higher than C. zeylaniIodine: cum, value 95.

C. moorei, moderate, value 50 .

C. zeylanicum, light to moderate, value 35 .

C. hybridum j. c. harvey, light, about the ame as C. zeylanicum value 35 .

Gentian violet:

C. moorei, moderate to deep, value 65 .

C. zeylanicum, moderate deep to deep, deeper than C. moorei, value 67.

C. hybridum j. c. harvey, moderately deep to deep, deeper than Safranin: either parent, value 70 .

C. moorei, moderately deep to deep, value 65 .

C. zeylanicum, moderately deep to deep, deeper than in C. moorei, value 67.

C. hybridum j. v. harvey, moderate to deep, the mean lighter than in either parent, value 60 .

Temperature:

C. moorei, majority at 68 to $70^{\circ}$, all but rare grains at 70 to $71^{\circ}$, mean $70.5^{\circ}$.

C. zeylanicum, majority at 77 to $78^{\circ}$, all but rare grains at 79 to $80^{\circ}$, mean 79.5 .

C. hybridum j. c. harvey, majority at 78 to $80^{\circ}$, all but rare grains at 80 to $82^{\circ}$, mean $81^{\circ}$.

The reactivities of $C$. moorei are lower than those of C. zeylanicum in the reactions with polarization, gentian violet, and safranin, and higher in those with iodine and temperature. In all of these reactions, excepting the safranin, the hybrid is closer to $C$. zeylanicum than to the other parent. In the iodine reaction it is the same as that of $C$.zeylanicum and lower than that of $C$. moorei. In the polarization and gentian violet the reactivities are higher than in either parent, and in the temperature reaction lower than in either parent. The marked differences in the temperature reactions of the parental starches and the much closer relationship of the hybrid to $C$. zeylanicum are very striking. In none of these reactions is there the least tendency to intermediateness of the hybrid, but distinctly with one exception to excess or deficit in relation to parental extremes.

Table A 7 shows the reaction-intensities in percentages of total starch gelatinized at definite intervals (minutes) : 
TABLE A 7.

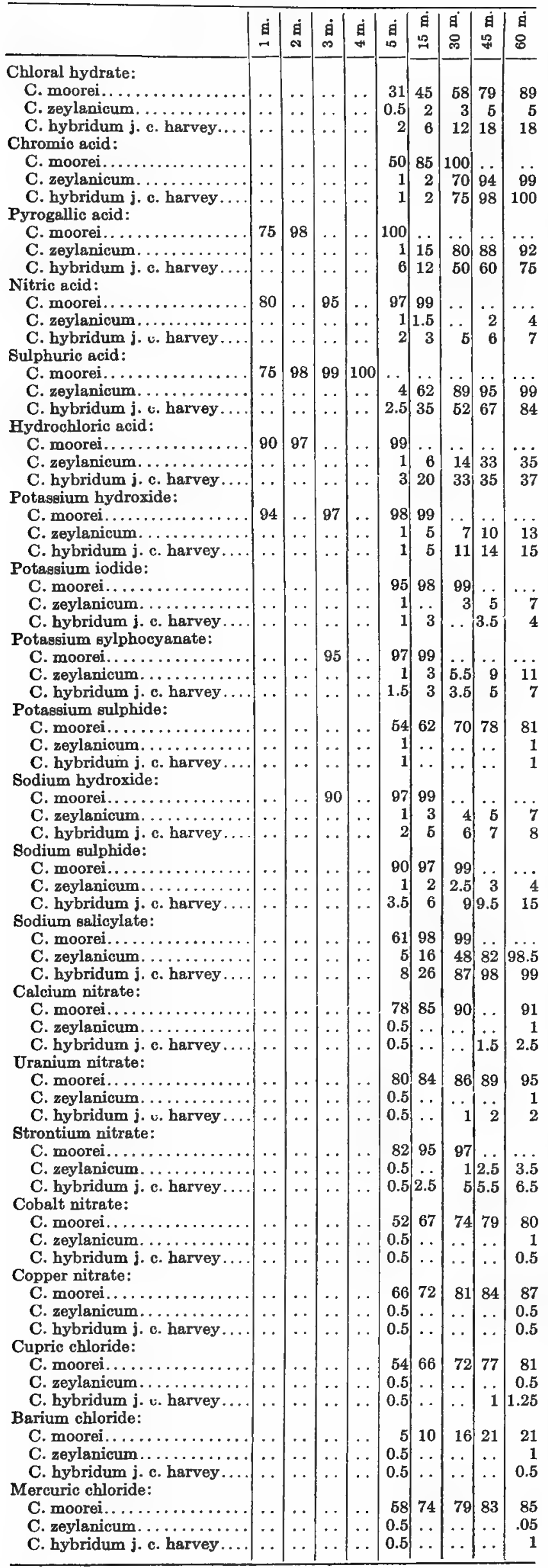

VELOCITY-Reaction CURVES.

This section treats of the velocity-reaction curves of the starches of Crinum moorei, C. zeylanicum, and $C$. hybridum, $j$. c. harvey, showing the quantitative differences in the behavior toward different reagents at definite time-intervals. (Charts D 127 to D 14\%.)

Among the most conspicuous features of this group of curves are:

(1) The marked differences between the curves of the starch of $C$. moorei on the one hand and those of C. zeylanicum and the hybrid on the other. The former is in nearly all reactions quick-reacting, while the latter is the reverse. In only 6 of the 21 reactions the former (including the reactions with chloral hydrate, chromic acid, pyrogallic acid, sulphuric acid, sodium salicylate, and barium chloride) is there an evident approximation of the curve of $C$. moorei to that of the other parent or the hybrid. In the reactions with chloral hydrate and barium chloride the approach of the curves is owing essentially (chloral hydrate) or solely (barium chloride) to the relatively low degree of reactivity of $C$. moorei with these reagents as compared with others; in those with pyrogallic acid and sulphuric acid to the relatively very high reactivity of $C$. zeylanicum and $C$. hybridum j. c. harvey; and in those with chromic acid and sodium salicylate to the combined relatively low reactivity of C. moorei and relatively high reactivity of $C$. zeylanicum and C. hybridum j. c. harvey.

(2) The marked early period of resistance followed by a moderately rapid to a rapid reaction exhibited by C. zeylanicum and the hybrid in the reactions with chromic acid, pyrogallic acid, sulphuric acid, hydrochloric acid, and sodium salicylate are in striking contrast with the very marked continued resistance that is exhibited by the records of the remaining 16 reagents during the entire 60-minute interval.

(3) A comparison of the differences in the course of the reaction-curves will elicit many points of interest. Thus, taking the acid group, and comparing the charts for chromic acid, pyrogallic acid, nitric acid, sulphuric acid, and hydrochloric acid, it will be seen, at a glance, that they so differ that the influence of any one reagent can readily be distinguished from those of others; likewise, those of potassium sulphide and sodium sulphide. On the other hand, three groups of charts, including those of (a) potassium hydroxide and sodium hydroxide, (b) calcium nitrate, uranium nitrate, strontium nitrate, cobalt nitrate, copper nitrate, cupric chloride, and mercuric chloride, and (c) nitric acid, potassium hydroxide, potassium iodide, potassium sulphocyanate, sodium hydroxide, and potassium sulphide are in each case closely alike, notwithstanding wide differences in the characters of the reagents.

(4) The earliest period during the 60 minutes at which the reaction-curves are farthest apart, and hence the best period for the differentiation of the three starches, varies markedly with the different reagents. Approximately, this optimal period occurs at the end of 15 minutes in the reactions with nitric acid, sulphuric acid, potassium iodide, and sodium hydroxide; 30 minutes with chromic acid, pyrogallic acid, hydrochloric acid, potassium hydroxide, sodium sulphide, and sodium salicylate; and 60 minutes with chloral hydrate, potassium sulphocyanate, potassium sulphide, calcium nitrate, uranium nitrate, strontium nitrate, cobalt nitrate, copper nitrate, cupric chloride, barium chloride, and mercuric chloride.

\section{Reaction-Intensities of the Hybrid.}

This section deals with the reaction-intensities of the hybrid as regards sameness, intermediateness, excess and 
deficit in relation to the parents. (Table A 7 and Charts D. $12 \%$ to D $14 \%$.)

The reactivities of the hybrid are the same as those of the seed parent in none of the reactions; the same as those of the pollen parent in the reactions with iodine, chromic acid, nitric acid, potassium hydroxide, sodium hydroxide, calcium nitrate, uranium nitrate, cobalt nitrate, copper nitrate, cupric chloride, barium chloride, and mercuric chloride; the same as those of both parents in none of the reactions ; intermediate in those with chloral hydrate, hydrochloric acid, sodium sulphide, sodium salicylate, and strontium nitrate, in all of which being closer to the pollen parent; highest with polarization and gentian violet, in both being closer to the pollen parent; and the lowest with safranin, temperature, pyrogallic acid, sulphuric acid, potassium iodide, potassium sulphocyanate, and potassium sulphide, in 6 being closer to the pollen parent and in 1 closer to the seed parent.

The following is a summary of the reaction-intensities: Same as seed parent, 0; same as pollen parent, 12; same as both parents, 0 ; intermediate, 5 ; highest, 2 ; lowest, $\%$.

Intermediateness is recorded in less than one-fifth of the reactions; excess and deficit of reactivity is almost twice as frequent as intermediateness; and sameness as the pollen parent is noted as often as intermediateness and excess and deficit combined. From these data the seed parent has exercised very little influence on the properties of the starch of the hybrid.

\section{Composite Curves of Reaction-intensitres.}

This section deals with the composite curves of the reaction-intensities, showing the differentiation of the starches of Crinum moorei, C. zeylanicum, and C. hybridum j. c. harvey. (Chart E \%.)

The most conspicuous features of the chart may be summed up as follows:

(1) The wide separation of the curve of $C$. moorei in four-fifths of the reactions from the curves of $C$. zeylanicum and the hybrid, which latter tend to run together with remarkable closeness.

(2) In $C$. moorei, the lower polarization and gentian-violet reactions coupled with higher reactions with iodine, heat, and with all of the chemical reagents as compared with $C$. zeylanicum.

(3) The differences in the relative positions of the three curves of reaction with polarization, iodine, gentian violet, and safranin; as for instance, the curves of $C$. moorei being lowest in polarization, highest in iodine, lowest in gentian-violet, and intermediate in safranin reactions, and thereafter in the chart always highest.

(4) In C. moorei, the very high reactions with polarization, pyrogallic acid, nitric acid, sulphuric acid, hydrochloric acid, potassium hydroxide, potassium iodide, potassium sulphocyanate, sodium hydroxide, sodium sulphide, sodium salicylate, and strontium nitrate; the high reactions with gentian violet, safranin, and chromic acid; the moderate reactions with iodine, temperature, calcium nitrate, and uranium nitrate; the low reactions with chloral hydrate, potassium sulphide, cobalt nitrate, copper nitrate, cupric chloride, and mercuric chloride; and the very low reaction with barium chloride.

(5) In $C$. zeylanicum the very high polarization reactions; the high reactions with gentian violet, safranin, and sulphuric acid; the moderate reactions with chromic acid, pyrogallic acid, and sodium salicylate; the low reactions with iodine and temperature; and the very low reactions with chloral hydrate, nitric acid, hydrochloric acid, potassium hydroxide, potassium iodide, potassium sulphocyanate, potassium sulphide, sodium hydroxide, sodium sulphide, calcium nitrate, uranium nitrate, stron- tium nitrate, cobalt nitrate, copper nitrate, cupric chloride, barium chloride, and mercuric chloride.

(6) In C. hybridum j. c. harvey, the very high reaction with polarization; the high with gentian violet and safranin; the moderate with chromic acid and sodium salicylate; the low with iodine, temperature, pyrogallic acid, and sulphuric acid, and the very low with chloral hydrate, nitric acid, hydrochloric acid, potassium hydroxide, potassium iodide, potassium sulphocyanate, potassium sulphide, sodium hydroxide, sodium sulphide, calcium nitrate, uranium nitrate, strontium nitrate, cobalt nitrate, copper nitrate, cupric chloride, barium chloride, and mercuric chloride. ties :

The following is a summary of the reaction-intensi-

\begin{tabular}{l|c|c|c|c|c}
\hline & $\begin{array}{c}\text { Very } \\
\text { high. }\end{array}$ & High. & $\begin{array}{c}\text { Mod- } \\
\text { erate. }\end{array}$ & Low. & $\begin{array}{c}\text { Very } \\
\text { low. }\end{array}$ \\
\hline C. moorei.................. & 12 & 3 & 4 & 6 & 1 \\
C. zeylanium......... & 1 & 3 & 3 & 2 & 17 \\
C. hybridum j. .. harvey. & 1 & 2 & 2 & 4 & 17 \\
\hline
\end{tabular}

\section{Comparisons of the Starches of Crinum} ZEYLANICUM, C. LONGIFOLIUM, AND C. KIRCAPE.

In histologic characteristics, in polariscopic figures, in the reactions with selenite, in the reactions with iodine, and in the qualitative reactions with the various chemical reagents it will be noted that the starches of the parents and hybrid exhibit not only properties in common in varying degrees of development, but also individualities which collectively are in each case characteristic of the starch. The starch of $C$. longifolium shows in comparison with that of Crinum zeylanicum a much smaller proportion of aggregates and compound grains; that irregularities are more prominent and more frequently present; and that the majority of the graing are broader, relatively and absolutely, and more flattened. The hilum is not quite so frequently fissured and is slightly less refractive; multiple hila are absent, although present in C. zeylanicum; the fissures are, as a rule, less deep; and eccentricity is somewhat greater. The lamellæ are more distinct distalward and often more discernible in this region than in a lustrous band at the distal margin, which is the reverse of what is noted in C. zeylanicum; there are some numerical differences in the lamellæ and bands of lamellæ, and also in the lengths of the bands; and the number of the lamellæ is less. The common sizes are nearly the same, the larger grains are larger, and, in case of both, the width is greater than the length-the opposite to what is seen in C. zeylanicum. In polariscopic figures, reactions with selenite, qualitative reactions with iodine, reactions with gentian violet and safranin, and qualitative reactions with the chemical reagents there are differences, some of them striking, and of variable degrees of importance in differentiation.

The starch of the hybrid in form, hilum, lamellæ, and size bears in most respects a closer relationship to that of $C$. zeylanicum than to that of the other parent, but in some instances the reverse. The same is true of the polariscopic figures and reactions with selenite. In the iodine reactions it is distinctly closer to $C$. zeylanicum. In the qualitative reactions with chloral hydrate, nitric acid, potassium hydroxide, potassium iodide, potassium sulphocyanate, sodium sulphide, sodium salicylate, copper nitrate, cupric chloride, and mercuric chloride the relationships are, on the whole, much closer to $C$. zeylanicum, but in certain respects here and there closer to C. longifolium. Marked individualities of the 
TABLE A 8.

\begin{tabular}{|c|c|c|c|c|c|c|c|c|c|}
\hline & $\stackrel{\text { a }}{-}$ & ถู่ & 喤 & $\dot{g}$ & 鬲 & $\underset{\sim}{\text { 豆 }}$ & ఏ్ & 嘼 & घ̊ \\
\hline \multicolumn{10}{|l|}{ Chloral hydrate: } \\
\hline & $\cdots$ & .. & -. & & 0.5 & 2 & 3 & $\mathbf{5}$ & 5 \\
\hline C. longifolium. & $\cdots$ & . & .. & . & 46 & 57 & 65 & 68 & 68 \\
\hline C. kircape.... & $\cdots$ & $\cdots$ & . & . & 0.5 & 1 & 3 & 4 & 4 \\
\hline \multicolumn{10}{|l|}{ Chromic acid: } \\
\hline $\begin{array}{l}\text { C. zeylanicum... } \\
\text { C. longifolium.. }\end{array}$ & $\cdots$ & $\cdots$ & $\cdots$ & $\begin{array}{l}\cdots \\
\cdots\end{array}$ & $\begin{array}{r}1 \\
45\end{array}$ & 2 & $\begin{array}{l}70 \\
99\end{array}$ & 94 & 99 \\
\hline C. kircape ...... & .. & .. & . & . & $\begin{array}{r}40 \\
1\end{array}$ & $\begin{array}{c}0 \\
5\end{array}$ & $\begin{array}{l}99 \\
80\end{array}$ & 99 & 100 \\
\hline Pyrogallic acid: & & & & & & & & & \\
\hline C. zeylanicum. & & & & $\cdots$ & 3 & 15 & 80 & 88 & 92 \\
\hline C. longifolium.. & 50 & 65 & 85 & . & 98 & & & & \\
\hline C. kircape...... & .. & .. & .. & . & 33 & 87 & 96 & 98 & 98 \\
\hline Nitric acid: & & & & & & & & & \\
\hline C. zeylanicum. & & .. & & $\cdots$ & 1 & 1.5 & & 2 & 4 \\
\hline C. longifolium & 75 & $\cdots$ & 89 & - & 92 & 96 & 99 & & \\
\hline C. kircape.... & $\cdots$ & $\cdots$ & $\cdots$ & $\cdots$ & 7 & 30 & 50 & 61 & 73 \\
\hline Sulphuric acid: & & & & & & & & & \\
\hline C. zeylanicum. . & $\cdots$ & . & $\cdots$ & $\cdots$ & 4 & 62 & 89 & 95 & 99 \\
\hline C. longifolium.. & $\cdots$ & $\cdots$ & $\cdots$ & $\cdots$ & 96 & 100 & & & $\cdots$ \\
\hline C. kircape....... & $\cdots$ & $\cdots$ & . & . & 40 & 87 & 96 & 99 & .. \\
\hline Hydrochlorie acid: & & & & & & & & & \\
\hline C. zeylanicum.. & . & $\ddot{0}$ & . & $\cdots$ & 1 & 6 & 14 & 33 & 35 \\
\hline C. longifolium... & . & 88 & . & . & 99 & . & & & \\
\hline C. kircape...... & .. & .. & . & . & 37 & 65 & 75 & 84 & 85 \\
\hline Potassium hydroxide: & & & & & & & & & \\
\hline C. zeylanicum.. & & . & & . & 1 & 5 & $7)$ & 10 & 13 \\
\hline C. longifolium. & 89 & $\because$ & . & . & 90 & 97 & 99 & & \\
\hline C. kireape....... & $\cdots$ & $\cdots$ & . & . & 11 & 52 & 65 & 67 & 70 \\
\hline Potassium iodide: & & & & & & & & & \\
\hline C. zeylanicum.. & $\cdots$ & $\cdots$ & . & . & 1 & & 3 & 5 & 7 \\
\hline C. longifolium & $\cdots$ & $\cdots$ & . & . & 90 & 97 & 98 & 99 & \\
\hline C. kircape..... & $\cdots$ & $\cdots$ & . & . & 3 & 18 & 28 & 39 & 45 \\
\hline Potassium sulphoc & & & & & & & & & \\
\hline C. zeylanicum.. & $\cdots$ & $\cdots$ & & . & 1 & 35 & 5.5 & 9 & 11 \\
\hline C. longifolium. & . & $\cdots$ & 70 & $\cdots$ & 93 & 95 & 99 & & \\
\hline C. kircape... $\because \ldots \ldots \ldots \ldots$ & . & $\cdots$ & $\cdots$ & $\cdots$ & 7 & 50 & 70 & 76 & 82 \\
\hline Potassium sulphide: & & & & & & & & & \\
\hline C. zeylanicum & $\cdots$ & $\cdots$ & $\cdots$ & $\cdots$ & 1 & & & . & 1 \\
\hline C. longifolium. & . & . & . & . & 50 & 55 & 60 & & 66 \\
\hline C. kircape....... & . & .. & .. & . & 1 & 2 & 3 & 3 & 3 \\
\hline Sodium hydroxide: & & & & & & & & & \\
\hline C. zeylanicum. . & . & $\cdots$ & & . & 1 & 3 & 4 & 5 & 7 \\
\hline C. longifolium. & . & $\cdots$ & 90 & . & 91 & 95 & 98 & & 99 \\
\hline C. kircape..... & $\cdots$ & $\cdots$ & ... & .. & 3 & 20 & 29 & 33 & 33 \\
\hline Sodium sulphide: & & & & & & & & & \\
\hline C. zeylanicum.. & $\cdots$ & . & .. & .. & 1 & $\left.2\right|^{2}$ & 2.5 & 3 & 4 \\
\hline C. longifolium... & . & . & . & . & 52 & 66 & 82 & 84 & 91 \\
\hline C. kircape................ & .. & .. & $\ldots$ & .. & 2 & 12 & 27 & 35 & 42 \\
\hline Sodium salicylate: & & & & & & & & & \\
\hline C. zeylanicum... & $\cdots$ & . & $\cdots$ & .. & 5 & 16 & 48 & 82 & 98 \\
\hline C. Iongifolium. & $\cdots$ & . & $\cdots$ & .. & 37 & 66 & 95 & 99 & \\
\hline C. kircape.. & $\cdots$ & . & $\cdots$ & .. & 3 & 9 & 40 & 69 & 78 \\
\hline Calcium nitrate: & & & & & & & & & \\
\hline C. zeylanicum .......... & $\cdots$ & . & $\cdots$ & $\cdots$ & 0.5 & & & & 1 \\
\hline C. longifolium.......... & ... & .. & .. & . & 65 & 78 & 78 & 81 & 81 \\
\hline C. kircape..... & . & . & ... & .. & 2 & 11] & 15 & 19 & 20 \\
\hline Uranium nitrate: & & & & & & & & & \\
\hline C. zeyla & $\cdots$ & . & . & $\cdots$ & 0.5 & & & & 1 \\
\hline C. longifolium. & $\cdots$ & . & $\cdots$ & . & 65 & 74 & 82 & 87 & 87 \\
\hline C. kircape.............. & . & ... & ... & . & 0.5 & 3.5 & 6 & 8 & 10 \\
\hline Strontium nitrate: & & & & & & & & & \\
\hline C. zeylanicum... & . & . & $\cdots$ & $\cdots$ & 0.5 & & 1 & 2.5 & 3.5 \\
\hline C. longifolium. & . & . & . . & $\ldots$ & 69 & 83 & 97 & 98 & 98 \\
\hline C. kircape... & .. & .. & . . & $\cdots$ & 0.5 & 3 & 6 & 15 & 32 \\
\hline Cobalt nitrate: & & & & & & & & & \\
\hline C. zeyla & $\cdots$ & . & $\cdots$ & . & 0.5 & & & & 1 \\
\hline C. longifolium. & .. & . & .. & . & 34 & 54 & 60 & 65 & 70 \\
\hline C. kires & $\cdots$ & $\cdots$ & . & $\cdots$ & 0.5 & $\cdots$ & 1 & $\cdots$ & 2 \\
\hline Copper nitrate: & & & & & & & & & \\
\hline C. zeylanicum.... & . & . & . & . & 0.5 & & & & 0.5 \\
\hline C. longifolium.... & .. & . & . & .. & 54 & 70 & 78 & 80 & 81 \\
\hline C. kircape .... & .. & . & .. & . & 0.5 & 4 & 5 & 7 & 8 \\
\hline Cuprie chloride: & & & & & & & & & \\
\hline C. zeylanicum.... & . & .. & $\cdots$ & . & 0.5 & & & & 0.5 \\
\hline C. longifolium.... & .. & . & . & . & 48 & 56 & 60 & 62 & 64 \\
\hline C. kirc & . & . & .. & , & 0.5 & $\ldots$ & 3 & 6 & 8 \\
\hline Barium chloride: & & & & & & & & & \\
\hline C. zeylanicum..... & . & .. & . & . & 0.5 & & & & 1 \\
\hline C. longifolium... & . & . & . & $\cdots$ & 3 & 8 & 16 & 19 & 20 \\
\hline C. kireape...... . & . & .. & $\ldots$ & . & $|0.5|$ & $\cdots$ & $\ldots$ & $\ldots$ & 0.5 \\
\hline Mercuric chloride: & & & & & & & & & \\
\hline C. zeylanicum.... & . & . & -. & . & 0.5 & $\because$ & $\ddot{i}$ & 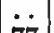 & 0.5 \\
\hline C. longifolium...... & .. & .. & .. & . & 57 & 63 & 70 & 77 & 77 \\
\hline C. kircape........ & $\cdots$ & . & . & $\cdots$ & $\cdots$ & 1 & $\cdots$ & 3 & 4 \\
\hline
\end{tabular}

hybrid are noted especially in the reactions with potassium iodide, potassium sulphide, and sodium sulphide.

Reaction-intensities Expressed by Light, Color, and TemperaPolarization:

C. zeylanicum, very high, value 93 .

C. longifolium, high to very high, much lower than C. zeylanicum, value 83.

C. kircape, high, slightly higher than C. zeylanicum, value 95 . Iodine:

C. zeylanicum, light to moderate, value 35 .

C. longifolium, light to moderate, deeper than C. zeylanicum, value 40.

C. kircape, light to moderate, slightly lighter than C. longifolium. value 38 .

Gentian violet:

C. zeylanicum, moderately deep to deep, value 67 .

C. longifolium, moderate, lighter than C. zeylanicum, value 60.

C. kircape, moderate, the same as C. longifolium, value 60 .

Safranin:

C. zeylanicum, moderately deep to deep, value 67 .

C. longifolium, moderate, lighter than C. zeylanicum, value 60 .

C. kircape, moderately deep to deep, deeper than either parent, value 70 .

Temperature:

C. zeylanicum, majority at 77 to $78^{\circ}$, all but rare grains at 79 to $80^{\circ}$, mean $79.5^{\circ}$.

C. longifolium, majority at 70 to $71^{\circ}$, all at 74 to $75^{\circ}$, mean $74.5^{\circ}$

C. kircape, majority at 75 to $76^{\circ}$, all but rare grains at 77 to $79^{\circ}$, mean $78^{\circ}$.

The reactivities of $C$. zeylanicum are higher than those of $C$. longifolium in the polarization, gentian-violet, and safranin reactions, and lower in the iodine and temperature reactions.

Interesting differences are noted in these reactions in the relations between those of the hybrid to one or the other parent. In the polarization and safranin reactions the hybrid reactions are higher than those of either parent, in both instances being nearer those of C. zeylanicum, the seed parent; in the iodine reaction it stands intermediate, but somewhat closer to C. longifolium; while in the gentian-violet reaction it is lower than in C. zeylanicum and the same as in C. longifolium. The temperature reaction is intermediate, yet distinctly closer to that of $C$. zeylanicum, the mean being $1.5^{\circ}$ lower than in C. zeylanicum and $3.5^{\circ}$ higher than in C. longifolium. The reactions, on the whole, are closer to $C$. zeylanicum.

Table A 8 shows the reaction intensities in percentages of total starch gelatinized at definite intervals (minutes).

\section{Velocity-Reaction Curves.}

This section treats of the velocity-reaction curves of the starches of Crinum zeylanicum, C. longifolium, and C. kircape, showing the qualitative differences in the behavior toward different reagents at definite time-intervals. (Charts D 148 to D 168.)

The most striking features of this group of curves are:

(1) The immediate and relatively very marked reactivity of Crinum longifolium with all of the reagents excepting barium chloride. With 7 of the 21 reagents, 90 per cent or over of the total starch was gelatinized in 5 minutes; with 3 reagents, 60 per cent or over; the lowest percentage being 34 ; the average gelatinization for all of the reagents, excepting barium chloride, being nearly 70 per cent in 5 minutes, as compared with usually an average of 0.5 to 3 per cent in case of $C$. zeylanicum and the hybrid. With the latter, in only the reactions with pyrogallic acid, sulphuric acid, and hydrochloric acid was there any marked effect during this time-interval, these reactions in case of the hybrid ranging from 33 to 40 per cent, while with $C$. zeylanicum with the same reagents there was a gelatinization of 4 per cent or less, thus showing a remarkable approach in the properties of the starch in relation to these three reagents to the properties of $C$. longifolium. In the 
reactions with nitric acid, potasium hydroxide, and potassium sulphocyanate reactivity during the first 5 minutes is distinctly higher in the hybrid than in $C$. zeylanicum.

(2) As the reactions proceed the tendency, with two exceptions, is for the hybrid curves to become well separated from those of $C$. zeylanicum, becoming intermediate, yet keeping closer to this parent than to $C$. longifolium. The starch therefore manifests the reactive properties of both parents, but is influenced distinctly more by the high resistant properties of $C$. zeylanicum than by the relatively low resistant properties of C. longifolium. The degrees of separation of the three curves vary remarkably in the different reactions. In some reactions they are to a notable extent separated, showing correspondingly wide differences in reactionintensities of all three starches, as is especially marked in the reactions with nitric acid, hydrochloric acid, potassium hydroxide, potassium iodide, potassium sulphocyanate, sodium hydroxide, and sodium sulphide; in others, the three curves tend to be comparatively close, as in especially the sulphuric-acid reaction. In others there is marked tendency for the curve of $C$. longifolium to be separated from those of $C$. zeylanicum and the hybrid, the two latter inclining markedly toward one another, as in especially the reactions with chromic acid, potassium sulphide, sodium salicylate, calcium nitrate, uranium nitrate, strontium nitrate, cobalt nitrate, copper nitrate, cupric chloride, barium chloride, and mercuric chloride. In other reactions various gradations of relationship exist between the foregoing groups. The comparative slowness of the $C$. kircape reactions appears to be due in some cases to the high resistance of the starches during particularly the earlier period of the reactions, as for instance, in those with chromic acid, potassium sulphocyanate, and sodium salicylate. In certain other reactions the resistance during the same period is low.

The best period for the differentiation of the starches is in most of the reactions at the end of 30 minutes, including here those with chromic acid, nitric acid, potassium hydroxide, potassium iodide, and sodium salicylate; in a few at the end of 15 minutes, as in those with pyrogallic acid, sulphuric acid, hydrochloric acid, and potassium sulphocyanate; in others at the end of 60 minutes, as in those with chloral hydrate, calcium nitrate, uranium nitrate, strontium nitrate, cobalt nitrate, copper nitrate, cupric chloride, barium chloride, and mercuric chloride. In some of these reactions the differences between the figures for C. zeylanicum and C. kircape are trifling and within the limits of error, as in the reactions with chloral hydrate, potassium sulphide, barium chloride, and mercuric chloride; and in certain others the variations are unimportant, as in those with chromic acid, potassium sulphide, uranium nitrate, copper nitrate, and cupric chloride.

\section{Reaction-Intensities OF THE HybRids.}

This section deals with the reaction-intensities of the hybrid as regards sameness, intermediateness, excess and deficit in relation to the parents. (Table A 8 and Charts 'D 148 to D 168.)

The reactivities of the hybrid are the same as those of the seed parent in the reactions with chloral hydrate, potassium sulphide, cobalt nitrate, and barium chloride; the same as those of the pollen parent with gentian violet; the same as those of both parents in none; intermediate in those with iodine, temperature, chromic acid, pyrogallic acid, nitric acid, sulphuric acid, hydrochloric acid, potassium hydroxide, potassium iodide, potassium sulphocyanate, sodium hydroxide, sodium sulphide, calcium nitrate, uranium nitrate, strontium nitrate, copper nitrate, cupric chloride, and mercuric chloride (in 3 being closer to those of the pollen parent; in 15 being closer to the seed parent; and in several being nearly the same); highest in the polarization and safranin reactions, in both being closer to the seed parent; and the lowest in the sodium salicylate reaction and closer to the seed parent.

The following is a summary of the reaction-intensities: Same as seed parent, 4; same as pollen parent, 1; same as both parents, 0 ; intermediate, 18 ; highest, 2 ; lowest, 1.

The tendency to intermediateness and to the seed parent is very marked, and it is obvious from these data that the pollen parent has exercised comparatively very little influence on the properties of the starch of the hybrid, the reverse of what was recorded in the preceding set, in which C. zeylanicum is the pollen parent, while in this set this species is the seed parent, from which it seems that C. zeylanicum is the potent parent, whether seed or pollen, in determining the properties of the hybrid.

\section{Composite CuRves of the Reaction-intensiteies.}

This section deals with the composite curves of the reaction-intensities, showing the differentiation of the starches of Crinum zeylanicum, $C$. longifolium, and $C$. kircape. (Chart E. 8.)

The most conspicuous features of the chart may be summed up as follows:

(1) The very distinct separation of the curves of C. zeylanicum and C. kircape from the curve of $C$. longifolium, excepting in the reactions with polarization, iodine, gentian violet, safranin, and temperature.

(2) The intermediate position of the curve of the hybrid (except in the reactions with polarization, iodine, safranin, and sodium salicylate and its relative closeness, with few exceptions, to the curve of C. zeylanicum. In the reactions with safranin, chromic acid, and pyrogallic acid the curve is closer to that of C. longifolium; and in the gentian-violet reaction it is the same as in $C$. longifotium.

(3) In $C$. zeylanicum the very high reaction with polarization; the high reactions with gentian violet, safranin, and sulphuric acid ; the moderate reactions with chromic acid, pyrogallic acid, and sodium salicylate; the low reactions with iodine and temperature reactions; and the very low reactions with chloral hydrate, nitric acid, hydrochloric acid, potassium hydroxide, potassium iodide, potassium sulphocyanate, potassium sulphide, sodium hydroxide, sodium sulphide, calcium nitrate, uranium nitrate,. strontium nitrate, cobalt nitrate, copper nitrate, cupric chloride, barium chloride, and mercuric chloride.

(4) In $C$. longifolium the very high reactions with polarization, pyrogallic acid, nitric acid, sulphuric acid, hydrochloric acid, potassium hydroxide, potassium iodide, potassium sulphocyanate, and sodium hydroxide; the high reactions with gentian violet, safranin, chromic acid, sodium salicylate, and strontium nitrate; the moderate reactions with iodine and sodium sulphide; the low reactions with temperature, chloral hydrate, potassium sulphide, calcium nitrate, uranium nitrate, cobalt nitrate, copper nitrate, cupric chloride, and mercuric chloride; and the very low reactions with barium chloride.

(5) In $C$. kircape the very high reaction with polarization; the high reactions with gentian violet, safranin, chromic acid, pyrogallic acid, and sulphuric acid; the low reactions with iodine, temperature, nitric acid, hydrochloric acid, potassium hydroxide, potassium sulphocyanate, and sodium salicylate; and the very low reactions with chloral hydrate, potassium iodide, potassium sul- 
phide, sodium hydroxide, sodium sulphide, calcium nitrate, uranium nitrate, strontium nitrate, cobalt nitrate, copper nitrate, cupric chloride, barium chloride, and mercuric chloride. ties:

The following is a summary of the reaction-intensi-

\begin{tabular}{l|c|c|c|c|c}
\hline & $\begin{array}{c}\text { Very } \\
\text { high. }\end{array}$ & High. & $\begin{array}{c}\text { Mod- } \\
\text { erate. }\end{array}$ & Low. & $\begin{array}{c}\text { Very } \\
\text { low. }\end{array}$ \\
\hline C. zeylanicum........ & 1 & 3 & 3 & 2 & 17 \\
C. longifolium...... & 9 & 5 & 2 & 9 & 1 \\
C. kircape......... & 1 & 5 & 0 & 7 & 13 \\
\hline
\end{tabular}

9. Comparisons of the Starches of Crindm LONGIFOLIUM, C. MOOREI, AND C. POWELLII.

In histologic characteristics, polariscopic figures, reactions with selenite, reactions with iodine, and qualitative reactions with various chemical reagents it will be found that the starches of the parents and hybrid exhibit not only properties in common in varying degrees of development but also individualities, the sum of which in case of each starch is distinctive of the starch. The starch of the hybrid is in form, characters of the hilum, lamellæ, and size in certain respects closer to one than the other parent, and in other respects as close to one as to the other. There are larger numbers of both aggregates and compound grains than are found in Crinum longifolium, but not quite so many as in C. moorei. The irregularities of the grains are more prominent and more numerous than in $C$. longifolium, but less than in C. moorei. An abrupt deflection of elongated, slender grains at or just distal to the slightly eccentric hilum is seen, this peculiarity being absent from $C$. longifolium, but present in C. moorei. The majority of the grains are not so broadened and flattened as in $C$. longifolium, yet more flattened than in C. moorei. In size, the grains are more evenly divided into elongated and broadened forms than in case of either parent. In polariscopic figures and appearances with selenite, and in the iodine reactions, the hybrid shows on the whole a distinctly closer relationship to $C$. moorei. In the qualitative reactions with chloral hydrate, potassium iodide, potassium sulphocyanate, potassium sulphide, sodium sulphide, sodium salicylate, copper nitrate, cupric chloride, and mercuric chloride it is, on the whole, very much closer to $C$. moorei than to $C$. longifolium. In some reactions there are certain features that are much more like those of $C$. longifolium, particularly in some of the processes with potassium iodide and sodium sulphide. In the reactions with copper nitrate, cupric chloride, and mercuric chloride the starch of the hybrid exhibits certain very interesting peculiarities, especially with reference to excess or deficit of parental extremes.

Reaction-intensities Expressed by Light, Color, and TemperaPolarization: ture Reactions.

C. longifolium, high to very high, value 83 .

C. moorei, high to very high, slightly higher than C. longifolium, value 85 .

C. powellii, high to very high, the same as C. moorei, value 85 .

Iodine:

C. longifolium, light to moderate, value 40 .

C. moorei, moderate, higher than C. longifolium, value 50 .

C. powellii, slightly to moderate, value $\mathbf{4 5}$.

Gentian violet:

C. longifolium, moderately deep to deep, value 60 .

C. moorei, moderately deep to deep, deeper than C. longifolium, value 65.

C. powellii, moderately deep to deep, the same as C. moorei, value 65 .
Safranin:

C. longifolium, moderately deep to deep, value 60 .

C. moorei, moderately deep to deep, deeper than C longifolium, value 65.

C. powellii, moderately deep to deep, the same as C. moorei, value 65 .

Temperature:

C. longifolium, majority at 70 to $71^{\circ}$, all at 74 to $75^{\circ}$; mean $74.5^{\circ}$.

C. moorei, majority at 68 to $70^{\circ}$, all but rare grains at 70 to $71^{\circ}$; mean $70.5^{\circ}$.

C. powellii, majority at 65 to $67^{\circ}$, all at 68 to $69^{\circ}$; mean 68.5 .

In all five reactions the reactivities of $C$. longifolium are lower than those of $C$. moorei in varying degree. The reactivities of the hybrid are the same as those of C. moorei in the polarization, gentian-violet, and safranin reactions; intermediate in the iodine reaction; and higher than those of either parent, but closer to C. moorei, in the temperature reaction. In four of the five reactions it is closer to the pollen parent, and in one intermediate.

Table A 9 shows the reaction-intensities in percentages of total starch gelatinized at definite intervals (minutes).

\section{Veloctty-Reaction CURtes.}

This section deals with the velocity-reaction curves of the starches of Crinum longifolium, C. moorei, and $C$. powellii, showing the quantitative differences in the behavior toward different reagents at definite timeintervals. (Charts D 169 to D 189.)

The most conspicuous features of this group of curves are :

(1) The closeness of all three curves, indicating not only a closeness of the parent stocks, but also very little modification of parental peculiarities in the hybrid.

(2) The higher reactivity of the hybrid than of either parent, excepting in the sodium salicylate reaction in which it is at first intermediate and then the same or practically the same as that of the pollen parent.

(3) The tendency for all three curves to run close together throughout the periods of the reactions.

(4) The intermediate position of the C. moorei curve throughout the series of reactions, excepting in the reactions with sodium salicylate and barium chloride. In the former it is practically the same as that of the hybrid, and in the latter practically the same as that of C. longifolium. It is of interest to note that while the curves of the parents in the reaction with barium chloride are practically the same, the curve of the hybrid is well separated (higher) from them. In many of the reactions gelatinization goes on so rapidly during the first 5 minutes that there is but little differentiation of any two or of all three, as the case may be. With proper strengths of solution marked differences could undoubtedly be elicited.

(5) The earliest period during the 60 minutes at which the three curves are so separated as to show the most marked differences between them varies with the different reagents. Approximately, this period occurs within 5 minutes in the reactions with pyrogallic acid, nitric acid, sulphuric acid, hydrochloric acid, potassium hydroxide, potassium iodide, potassium sulphocyanate, sodium hydroxide, sodium sulphide, sodium salicylate, calcium nitrate, strontium nitrate, and cobalt nitrate; within 15 minutes in those with chromic acid, uranium nitrate, mercuric chloride, copper nitrate, and cupric chloride; at $\mathbf{3 0}$ minutes with chloral hydrate and potassium sulphide; and at 60 minutes with barium chloride.

\section{Reaction-intensities of the Hybrid.}

This section treats of the reaction-intensities of the hybrid as regards sameness, intermediateness, excess, and deficit in relation to the parents. (Table A 9 and Charts D 169 to D 189.) 
Table A 9.

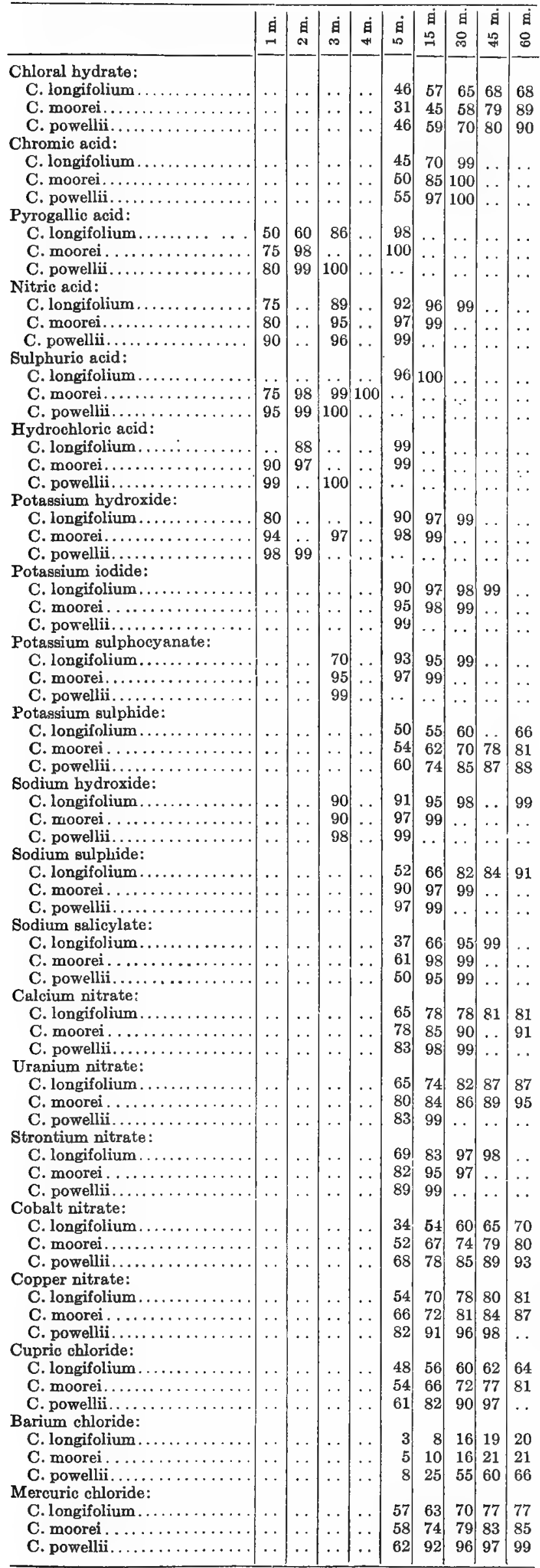

The reactivities of the hybrid are the same as thase of the seed parent in none of the reactions; the same as those of the pollen parent in the reactions with polarization, gentian violet, and safranin; the same as those of both parents in none of the reactions; intermediate with iodine and sodium salicylate, in one being midintermediate and in the other closer to the pollen parent; highest with temperature, chloral hydrate, chromic acid, pyrogallic acid, nitric acid, sulphuric acid, hydrochloric acid, potassium hydroxide, potassium iodide, potassium sulphocyanate, potassium sulphide, sodium hydroxide, sodium sulphide, calcium nitrate, uranium nitrate, strontium nitrate, cobalt nitrate, copper nitrate, cupric chloride, barium chloride, and mercuric chloride (in 19 being closer to the pollen parent, and in 2 being as close to one as the other parent) ; and the lowest in no reaction.

The following is a summary of the reaction-intensities: Same as seed parent, 0; same as pollen parent, 3; same as both parents, 0 ; intermediate, 2 ; highest, 21 ; lowest, 0 .

Intermediateness is almost absent, sameness or inclination to the seed parent entirely absent, and highest reactivity and sameness or inclination to the pollen parent very conspicuous. C. moorei, the seed parent, not only tends to higher reactivities than the other parent, but also to so markedly raise the reactivities of the hybrid as to bring the latter higher as a rule than its own. The seed parent has obviously had very little influence in determining the properties of the starch of the hybrid. In this set $C$. longifolium is the seed parent and in the preceding set the pollen parent, and in both it has been comparatively impotent in determining the parental leanings of the hybrid. (See Chapter 5, Section 4.)

\section{Composite Curves of Reaction Intensities.}

This section treats of the composite curves of the reaction-intensities showing the differentiation of the starches of Crinum longifolium, $C$. moorei, and $C$. powellii. (Chart E 9.)

The most conspicuous features of this chart are:

(1) The relatively remarkably high reactivity of the hybrid. It is higher than in either parent with few exceptions, and in the latter instances it is the same or slightly lower than that of one or the other parent.

(2) The closeness with which the hybrid and $C$. moorei curves run through most of the reactions. In 17 out of the 26 reactions the hybrid curve is closer to the C. moorei curve. In 7 instances (chromic acid, calcium nitrate, uranium nitrate, cobalt nitrate, copper nitrate, cupric chloride, and mercuric chloride) it is farther separated from the curves of the parent stocks than are the latter separated from each other. The high reactivity of the hybrid in comparison with the reactivities of the parent stocks in the reactions with calcium nitrate, uranium nitrate, copper nitrate, cupric chloride, and mercuric chloride is quite remarkable by showing a wide departure from intermediateness.

(3) In C. longifolium the very high reactions with polarization, pyrogallic acid, nitric acid, sulphuric acid, hydrochloric acid, potassium hydroxide, potassium iodide, potassium sulphocyanate, and sodium hydroxide; the high reactions with gentian violet, safranin, chromic acid, sodium salicylate, and strontium nitrate; the moderate reactions with iodine and sodium sulphide; the low reactions with chloral hydrate, temperature, potassium sulphide, calcium nitrate, uranium nitrate, cobalt nitrate, copper nitrate, cupric chloride, and mercuric chloride; and the very low reaction with barium chloride.

(4) In $C$. moorei the very high reactions with polarization, pyrogallic acid, nitric acid, sulphuric acid, hydrochloric acid, potassium hydroxide, potassium iodide, 
potassium sulphocyanate, sodium hydroxide, sodium sulphide, sodium salicylate, and strontium nitrate; the high reactions with gentian violet, safranin, and chromic acid; the moderate reactions with iodine, temperature, calcium nitrate, and uranium nitrate; the low reactions with chloral hydrate, potassium sulphide, cobalt nitrate, copper nitrate, cupric chloride, and mercuric chloride; and - the very low reaction with barium chloride.

(5) In C. powellii the very high reactions with polarization, chromic acid, pyrogallic acid, nitric acid, sulphuric acid, hydrochloric acid, potassium hydroxide, potassium iodide, potassium sulphocyanate, sodium hydroxide, sodium sulphide, sodium salicylate, calcium nitrate, uranium nitrate, and strontium nitrate; the high reactions with gentian violet, safranin, copper nitrate, cupric chloride, and mercuric chloride; the moderate reactions with iodine, temperature, and cobalt nitrate; the low reactions with chloral hydrate, potassium sulphide, and barium chloride; and the absence of any very low reaction.

The following is a summary of the reaction-intensities :

\begin{tabular}{l|c|c|c|c|c}
\hline & $\begin{array}{c}\text { Very } \\
\text { high. }\end{array}$ & High. & $\begin{array}{c}\text { Mod- } \\
\text { erate. }\end{array}$ & Low. & $\begin{array}{l}\text { Very } \\
\text { low. }\end{array}$ \\
\hline C. longifolium....... & 9 & 5 & 2 & 9 & 1 \\
C. moorei......... & 12 & 3 & 4 & 6 & 1 \\
C. powellii........ & 15 & 5 & 3 & 3 & 0 \\
\hline
\end{tabular}

\section{Notes ON THE CRINUMs.}

Among the starches studied are three from recognized species, two of which, C. moorei and C. longifolium, are more closely related botanically and horticulturally than is either to C. zeylanicum. The first two are stated to be the only hardy species of the genus, $C$. moorei being less hardy than $C$. longifolium. C. powellii, the hybrid of $C$. moorei and $C$. longifotium, is recorded as being more hardy than $C$. moorei.

In comparing the reactions of the starches of these three species as presented in Charts E $7, \mathrm{E} 8$, and E 9, several features of interest in addition to those already referred to will be noted:

(1) The wide separation of the curves of $C$. longifolium and $C$. moorei from the curve of C. zeylanicum, a departure so marked as to suggest a greater difference botanically than is recognized or that it is an expression of marked horticultural difference. The explanation seems to rest in the latter: C. longifolium and C. moorei are, as stated, hardy crinums, and they exhibit a far higher reactivity than C. zeylanicum, a tender crinum, which has a low degree of reactivity. A number of the tender crinums were studied in respect to the reactiveintensities, including the well-known species, $C$. americanum, C. erubescens, C. fimbriatulum, C. scabrum, and C. virginicum, all of which have low reactivity curves corresponding with the curve of $C$. zeylanicum. Therefore, it seems probable that among species of this genus hardiness or tenderness bears an inverse relationship to reactive-intensity. Such a relationship has been noted in other genera, as, for instance, between Amaryllis and Hippeastrum, the former being relatively hardy and the latter tender; the former being of distinctly higher mean reactivity than the latter. In accordance with the foregoing there are two generic types of curves which correspond with the two groups of hardy and tender groups of plants, respectively, and it appears from the charts that the hybrid C. kircape is in a marked measure in the nature of a connecting link between the two groups.

(2) The type of curve of $C$. longifolium and $C$. moorei, notwithstanding that these curves are far separated in all of the important reactions from the curve of $C$. zeylanicum, corresponds with that of $C$. zeylanicum. The rises and falls are strikingly coincident-coincidences that could be greatly accentuated by modifications in the strengths of the reagents.

(3) The curves of the hybrids, in the three charts exhibit certain well-defined peculiarities: In each the hybrid curve tends to follow closely one parent, that of C. hybridum j. c. harvey following the curve of $C$. zeylanicum; that of $C$. powellii the curve of $C$. moorei; and that of $C$. kircape the curve of $C$. zeylanicum. The relatively very potent influences of $C$. zeylanicum on the properties of the hybrid are strikingly evident, especially on $C$. hybridum $j$. c. harvey.

As regards sameness, intermediateness, and deficit of development in relation to the parents, the data of the three sets of starches show marked differences, as is illustrated in the following summaries:

\begin{tabular}{|c|c|c|c|}
\hline & $\begin{array}{l}\text { C. hybridum } \\
\text { j. c. harvey. }\end{array}$ & C. kircape. & C. powellii. \\
\hline $\begin{array}{l}\text { Same as, or practically the } \\
\text { same as: }\end{array}$ & & & \\
\hline Seed parent $\ldots \ldots \ldots \ldots \ldots$ & 0 & 4 & $\mathbf{0}$ \\
\hline Pollen parent $\ldots \ldots \ldots \ldots$ & 12 & 1 & $\mathbf{3}$ \\
\hline Both parents.......... & 0 & 0 & $\mathbf{0}$ \\
\hline Intermediate.......... & $\mathbf{5}$ & 18 & $\mathbf{2}$ \\
\hline Highest.......... & 2 & 2 & 21 \\
\hline Lowest.............. & 7 & 1 & $\mathbf{0}$ \\
\hline
\end{tabular}

10. Comparisons of the Starches of Nerine CRIsPa, N. elegans, N. DaInty Maid, and N. QUEEN OF ROSES.

In histologic characteristics, polariscopic figures, reactions with selenite, qualitative reactions with iodine, and qualitative reactions with the various chemical reagents, all four starches exhibit properties in common in varying degrees of development, and each starch has certain individualities. The starch of Nerine elegans in comparison with that of the other parent $N$. crispa is found to contain compound grains which have a larger number of components, and also aggregates which are not found in the latter. The grains are more regular in form, of less breadth usually in proportion to length, and in the majority of the grains the proximal end is smaller than the distal end, whereas in $N$. crispa only the minority of the grains have this feature. The hilum is not so distinct, less fissured, and slightly more eccentric. The lamellæ are, as a rule, finer but not so distinct; there are more grains that have lamellæ that are not so fine at the distal end as near the hilum; and the number of lamellæ is less. The sizes are generally less and there are differences in the ratios of length to breadth. In the polariscopic figures, reactions with selenite, and qualitative reactions with iodine there are many differences, mostly apparently of a minor charac- 
ter. In the qualitative reactions with chloral hydrate, nitric acid, potassium iodide, potassium sulphide, and sodium salicylate many differences are noted, some rather striking but mostly seemingly of minor importance. The starch of the hybrid $N$. dainty maid in comparison with the starches of the parents contains more aggregates than that of $N$. elegans and as many as in $N$. crispa; the irregularities are more numerous than in $N$. elegans and about the same as in the other parent; and while most of the grains in relative sizes of the proximal and distal ends resemble those of $N$. crispa, there are more that have the proximal end smaller than the distal end. The hilum in distinctness is closer to $N$. crispa, while in the absence of fissuration it is closer to $N$. elegans; in eccentricity it also is closer to the latter. The lamellæ are finer than those of either parent, but nearer $N$. elegans, while in general characters and arrangements they are nearer $N$. crispa; the number is less than in either parent, but nearer that of $N$. crispa. The size is somewhat closer to $N$. elegans. In the polarization, selenite, and qualitative reactions the resemblances lean to one or the other parent, but on the whole distinctly more to $N$. elegans. In the qualitative reactions with chloral hydrate, nitric acid, potassium iodide, potassium sulphocyanate, potassium sulphide, and sodium salicylate certain of the phenomena lean to one parent and certain others to the other parent, but the relationship is, on the whole, distinctly closer to $N$. elegans. In comparison with the starches of the parents the starch of the hybrid $N$. queen of roses contains a larger number of aggregates which have a larger number of component grains, and more compound grains than in either parent; and the latter are like those of $N$. elegans; the grains are less regular than those of $N$. elegans but more regular than those of $N$. crispa. The hilum is as distinct as in $N$. crispa and more distinct than in the other parent; it is rarely fissured, thus being closer to $N$. elegans; and the eccentricity is greater than in either parent, being nearer $N$. elegans: The lamellæ in characters and arrangements closely resemble those of $N$. crispa, but the number is less than in either parent and closer to that of $N$. elegans. In size the grains are smaller than those of either parent, and closer to those of $N$. elegans. In the polarization, selenite, and qualitative reactions with iodine the resemblances are closer to $N$. elegans. In the qualitative reactions with chloral hydrate, nitric acid, potassium iodide, potassium sulphocyanate, potassium sulphide, and sodium salicylate certain of the phenomena lean to one parent and certain others to the other. In the reactions with chloral hydrate and sodium salicylate they, on the whole, more closely resemble those of $N$. crispa, but those with nitric acid, potassium iodide, potassium sulphocyanate, and potassium sulphide more closely resemble those of $N$. elegans.

The two hybrids differ in certain very interesting respects, especially as regards their greater resemblances in their various properties to one or the other parent. $N$. dainty maid is in form more like $N$. crispa than $N$. elegans, but in other histological respects more like the other parent. $N$. queen of roses is in form and hilum more like $N$. elegans than $N$. crispa, but in the lamellæ it is nearer to $N$. crispa. In the polarization properties both hybrids are closer to $N$. elegans than to $N$. crispa, $N$. queen of roses being closer than $N$. dainty maid. In the iodine reactions, both quantitative and qualitative, $N$. dainty maid more closely resembles $N$. elegans; but in the other hybrid, $N$. queen of roses, the unheated grains show a closer relationship to $N$. elegans and the heated or gelatinized grains to the other parent. In the aniline reactions $N$. dainty maid is closer to $N$. elegans than to $N$. crispa; while $N$. queen of roses is closer to $N$. crispa than to $N$. elegans. In the qualitative reactions with the various chemical reagents similar curious individualities are recorded, as regards interparental and inter-hybrid and parental-hybrid reactions. The hybrids are sometimes practically alike and at others quite as different from each other as they are from the parents, or as the parents are from each other. The qualitative reactions may be closer to one or the other parent, according to the reagent. In the chloral-hydrate reactions both hybrids are closer to $N$. crispa, $N$. dainty maid being the closer. In the reactions with nitric acid, potassium iodide, potassium sulphocyanate, and potassium sulphide the hybrids are closer to $N$. elegans, $N$. dainty maid being the closer. In the sodium-salicylate reactions $N$. dainty maid is nearer to $N$. elegans, and $N$. queen of roses nearer to $N$. crispa, there being nearly as much difference between the hybrids themselves as between the hybrid $N$. queen of roses and the parent $N$. elegans.

Reaction-intensities Expressed by Light, Color, and Temperature Reactions.

Polarization:

Nerine crispa, moderate to very high, value 85 .

Nerine elegans, moderate to very high, lower than $N$. crispa, value 80 .

Nerine dainty maid, moderate to very high, same as $\mathrm{N}$. elegans, value 80.

Nerine queen of roses, moderate to very high, lower than either Iodine: parent, value 77 .

Nerine crispa, moderate, value 45 .

Nerine elegans, moderate, deeper than in N. crispa, value $\mathbf{5 5}$.

Nerine dainty maid, moderate to deep, deeper than in either parent. value 60 .

Nerine queen of roses, moderate, the same as in N. elegans, value 55. Gentian violet:

Nerine crispa, light to moderate, value 40 .

Nerine elegans, light to moderate, lighter than N. crispa, value 35.

Nerine dainty maid, light to moderate, the same as in N. elegans value 35 .

Nerine queen of roses, light to moderate, the same as in N. crispa, value 40 .

Safranin:

Nerine crispa, moderate, value 50 .

Nerine elegans, moderate, lighter than in N. crispa, value 45 .

Nerine dainty maid, moderate, the same as in N. elegans, value 50.

Nerine queen of roses, moderate, the same as in N. crispa, value 50. Temperature:

Nerine crispa, in the majority at 64 to $65^{\circ}$; in all at 70 to $71.5^{\circ}$; mean $70.7^{\circ}$.

Nerine elegans, in the majority at 68.5 to $70^{\circ}$; in all at 75 to $76.9^{\circ}$; mean $75.9^{\circ}$.

Nerine dainty maid, in the majority at 69 to $70.5^{\circ}$; in all at 72.5 to $73.8^{\circ}$; mean, $73.2^{\circ}$.

Nerine queen of roses, in the majority at 68 to $69.1^{\circ}$; in all at 71 to $72.8^{\circ}$; mean $71.9^{\circ}$.

$N$. crispa shows a higher reactivity than the other parent $N$. elegans in the reactions with polarization, gentian violet, safranin, and temperature, and a lower reactivity with iodine. Both hybrids in the polarization and iodine reactions are nearer to $N$. elegans than to the other parent, $N$. dainty maid having the same polarization reaction as this parent, but a higher iodine reaction. 
With gentian violet and safranin $N$. dainty maid is the same as $N$. elegans, while $N$. queen of roses is the same as $N$. crispa. In the temperature reactions the hybrids are intermediate, $N$. dainty maid being closer to $N$. elegans, and $N$. queen of roses closer to $N$. crispa. $N$. dainty maid is, on the whole, more closely related in these reactions to the pollen parent, and $N$. queen of roses to the seed parent.

Table A 10 shows the reaction-intensities in percentages of total starch gelatinized at definite intervals (minutes) :

Table A 10.

\begin{tabular}{|c|c|c|c|c|c|c|c|c|c|}
\hline & 白 & $\underset{\sim}{\mathbb{E}}$ & $\underset{\infty}{\text { 旦 }}$ & $\stackrel{\text { g }}{\not}$ & $\underset{\text { a }}{\text { a }}$ & $\begin{array}{l}\text { 旦 } \\
\text { 음 }\end{array}$ & 뭉 & $\begin{array}{l}9 \\
40 \\
\&\end{array}$ & $\begin{array}{l}8 \\
8\end{array}$ \\
\hline \multicolumn{10}{|l|}{ Chloral hydrate: } \\
\hline Nerine crispa . . . . . . . . & $\cdots$ & $\cdots$ & $\cdots$ & . & 12 & 37 & 65 & 67 & 72 \\
\hline Nerine elegans . . . . . . . . . & . & . & . & . & 15 & 89 & 97 & & \\
\hline Nerine dainty maid ........ & - & . & $\cdots$ & . & 13 & 77 & 90 & 92 & 95 \\
\hline Nerine queen of roses. . & .. & $\cdots$ & . & . & 70 & 99 & . & $\ldots$ & . \\
\hline \multicolumn{10}{|l|}{ Chromic acid: } \\
\hline Nerine crispa . . . . . . . . . . & $\cdots$ & $\cdots$ & $\cdots$ & - & 1 & 2 & 36 & 90 & 95 \\
\hline Nerine elegans . . . . . . . . . & . & $\cdots$ & . & . & 0.5 & 3 & 50 & 92 & 99 \\
\hline Nerine dainty maid... & . & . & . & $\cdots$ & 0.5 & 1 & 33 & 83 & 95 \\
\hline Nerine queen of roses. & . & . & . & . & 2 & 4 & 34 & 86 & 95 \\
\hline \multicolumn{10}{|l|}{ Pyrogallic acid: } \\
\hline Nerine crispa . . . . . . . . . & . & . & . & . & $1)$ & 2 & 3 & & 3 \\
\hline Nerine elegans... & $\cdots$ & $\cdots$ & $\cdots$ & $\cdots$ & 0.5 & $\cdots$ & $\cdots$ & 1 & 1 \\
\hline Nerine dainty maid.... & . & $\cdots$ & . & . & 2 & . & $\cdots$ & . & 2 \\
\hline Nerine queen of roses. . . . . . & . & . & . & . & 0.5 & .. & $\ldots$ & . & 0.5 \\
\hline \multicolumn{10}{|l|}{ Nitric acid: } \\
\hline Nerine crispa . ........ & $\cdots$ & $\cdots$ & $\cdots$ & $\cdots$ & 62 & 80 & 95 & 99 & 99 \\
\hline Nerine elegans . . . . . . & . & $\cdots$ & . & . & 88 & 96 & 99 & $\ldots$ & . \\
\hline Nerine dainty maid. ... . . . & . & $\cdots$ & . & . & 72 & 83 & 95 & 96 & 97 \\
\hline Nerine queen of roses. . . . . . & . & . & . & . & 75 & 90 & 98 & 99 & \\
\hline \multicolumn{10}{|l|}{ Sulphuric acid: } \\
\hline Nerine crispa . . & 85 & $\cdots$ & 99 & $\ldots$ & 100 & & $\cdots$ & $\cdots$ & $\cdots$ \\
\hline Nerine elegans...... & 90 & . & 99 & . & 99 & $\cdots$ & . & . & . \\
\hline Nerine dainty maid $\ldots \ldots \ldots$ & 95 & . & 99 & . & 99 & . & . & . & $\cdots$ \\
\hline Nerine queen of roses. ...... & 99 & . & 100 & . & 99 & . & . & . & . \\
\hline \multicolumn{10}{|l|}{ Hydrochloric acid: } \\
\hline Nerine crispa . . . . . . . . . & $\cdots$ & $\cdots$ & $\cdots$ & $\cdots$ & 90 & 99 & . & $\cdots$ & $\cdots$ \\
\hline Nerine elegans . . . . . . . . & $\cdots$ & $\cdots$ & . & $\cdots$ & 90 & 99 & . & $\cdots$ & . \\
\hline Nerine dainty maid. ........ & . & . & . & . & 95 & 98 & $\cdots$ & . & . \\
\hline Nerine queen of roses. . . . . . & . & . & . & . & 98 & 99 & . & . & $\ldots$ \\
\hline \multicolumn{10}{|l|}{ Potassium hydroxide: } \\
\hline Nerine crispa. . . . . . . & 97 & $\cdots$ & 99 & . & 99 & . & . & $\cdots$ & $\cdots$ \\
\hline Nerine elegans....... & 99 & . & & $\cdots$ & 99 & $\cdots$ & $\cdots$ & . & . \\
\hline Nerine dainty maid. ....... & 95 & . & 97 & . & 99 & . & .. & . & . \\
\hline Nerine queen of roses. . . . . . & 99 & $\cdots$ & $\cdots$ & . & 99 & . & .. & $\cdots$ & . \\
\hline \multicolumn{10}{|l|}{ Potassium iodide: } \\
\hline Nerine crispa ... & $\cdots$ & . & $\cdots$ & $\cdots$ & 1 & 4 & 9 & 17 & 28 \\
\hline Nerine elegans ... . . . & $\cdots$ & . & . & . & 0.5 & 1 & 2 & 3 & 8 \\
\hline Nerine dainty maid.. & . & . & . & . & 0.5 & 3 & 9 & 12 & 15 \\
\hline Nerine queen of roses. . & . & . & . & . & 1 & $\mathbf{3}$ & 6 & 11 & 19 \\
\hline \multicolumn{10}{|l|}{ Potassium sulphocyanate: } \\
\hline Nerine crispa . ......... & $\cdots$ & $\cdots$ & $\cdots$ & $\cdots$ & 3 & 10 & 42 & 61 & 70 \\
\hline Nerine elegans........ & . & . & . & $\cdots$ & 3 & 10 & 30 & 40 & 55 \\
\hline Nerine dainty maid. ....... & . & . & . & $\cdots$ & 4 & 42 & 70 & 85 & 90 \\
\hline Nerine queen of roses. . . . . . . & . & . & $\cdots$ & . & 5 & 36 & 65 & 80 & 88 \\
\hline \multicolumn{10}{|l|}{ Potassium sulphide: } \\
\hline . Nerine crispa.... . & . & $\cdots$ & $\cdots$ & $\cdots$ & 60 & 88 & 93 & 95 & 95 \\
\hline Nerine elegand... & $\cdots$ & $\cdots$ & $\cdots$ & $\cdots$ & 62 & 91 & 95 & 97 & 97 \\
\hline Nerine dainty ma & . & . & $\cdots$ & $\cdots$ & 63 & 90 & 91 & 95 & 98 \\
\hline Nerine queen of roses. . & . & . & . & . & 75 & 92 & 94 & 96 & 99 \\
\hline \multicolumn{10}{|l|}{ Sodium hydroxide: } \\
\hline Nerine crispa. . . . . . & $\cdots$ & $\cdots$ & $\cdots$ & $\cdots$ & 1 & 2 & 3 & 6 & 10 \\
\hline Nerine elegans... . . . & $\cdots$ & . & $\cdots$ & . & 3 & 5 & 8 & 12 & 15 \\
\hline Nerine dainty maid... & . & $\cdots$ & . & . & 0.5 & 4 & 7 & 16 & 18 \\
\hline Nerine queen of roses. . & . & $\cdots$ & $\cdots$ & . & 3 & $\mathbf{5}$ & 12 & 15 & 22 \\
\hline \multicolumn{10}{|l|}{ Sodium sulphide: } \\
\hline Nerine crispa...... . & $\cdots$ & $\cdots$ & $\cdots$ & $\cdots$ & 1 & $\because$ & 2 & $\cdots$ & 4 \\
\hline Nerine elegans... . . . . & . & $\cdots$ & . & . & 2 & 3 & $\cdots$ & 4 & 5 \\
\hline Nerine dainty maid... & . & $\cdots$ & . & .. & 2 & 3 & 5 & 6 & 7 \\
\hline Nerine queen of roses. ...... & . & $\cdots$ & . & . & 1 & 2 & $\mathbf{3}$ & 4 & 6 \\
\hline
\end{tabular}

TABLE A 10.-Continued.

\begin{tabular}{|c|c|c|c|c|c|c|c|c|c|}
\hline & 皇 & $\underset{\sim}{\dot{g}}$ & $\underset{\infty}{\mathbb{g}}$ & 泉 & 軺 & $\begin{array}{l}\dot{a} \\
\stackrel{2}{二}\end{array}$ & $\begin{array}{l}\text { घ่ } \\
\text { 口े }\end{array}$ & $\begin{array}{l}\text { g } \\
\text { fo }\end{array}$ & 星 \\
\hline \multicolumn{10}{|l|}{ Sodium salicylate: } \\
\hline & $\cdots$ & . & . & . & 42 & 82 & 98 & .. & \\
\hline Nerix & .. & & & . & 88 & 99 & & $\ldots$ & .. \\
\hline Nerine dain & .. & . & . & . & 75 & 98 & . & .. & .. \\
\hline Nerine queen of ro & . & . & 93 & & & $\ldots$ & & $\cdots$ & .. \\
\hline \multicolumn{10}{|l|}{ Calcium nitrate: } \\
\hline Nerine crispa.. & $\cdots$ & . & $\cdots$ & $\cdots$ & 1 & 2 & 4 & 8 & 10 \\
\hline Neri & .. & & .. & .. & 1 & 2 & & 5 & 8 \\
\hline Neri & $\ldots$ & .. & . & $\ldots$ & 2 & 4 & 6 & 10 & 15 \\
\hline Nerine queen of & . & .. & . & .. & 3 & & & $\ldots$ & $\theta$ \\
\hline \multicolumn{10}{|l|}{ Uranium nitrate: } \\
\hline Nerine crispa.. & $\cdots$ & . & . & $\because$ & 2 & 3 & 9 & 19 & 28 \\
\hline Neriy & $\cdots$ & . . & . & $\therefore$ & 0.5 & 3 & 9 & 11 & 14 \\
\hline Neri & . & .. & .. & .. & 2 & 8 & 20 & 30 & 38 \\
\hline \multirow{2}{*}{\multicolumn{10}{|c|}{ Strontium nitrate: }} \\
\hline & & & & & & & & & \\
\hline Nerine crispa .... & $\cdots$ & . & $\cdots$ & $\cdots$ & 68 & 90 & 95 & 96 & 99 \\
\hline Nerin & .. & . & $\ldots$ & $\ldots$ & 60 & 95 & 98 & 99 & 99 \\
\hline Neri & . & . & . & . & 63 & 90 & 95 & 98 & 98 \\
\hline \multirow{2}{*}{\multicolumn{10}{|c|}{ Cobalt nitrate: }} \\
\hline & & & & & & & & & \\
\hline Nerine crispa . & . & . & . & - & 0.5 & 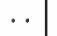 & . & 1 & \\
\hline Neri & $\ldots$ & . & . & . & 1 & & .. & 2 & 2 \\
\hline & $\ldots$ & . & . & . & 0.5 & 2 & .. & . & \\
\hline \multirow{2}{*}{\multicolumn{10}{|c|}{ Copper nitrate: }} \\
\hline & & & & & & & & & \\
\hline Nerine crispa . & .. & . & . & $\cdots$ & 0.5 & 2 & 14 & 22 & 25 \\
\hline Ner & . & . & $\ldots$ & . & 0.5 & 0.5 & 2 & 6 & 9 \\
\hline & .. & .. & . & .. & 1 & 5 & 20 & 25 & 33 \\
\hline Nerine queen of & . & .. & .. & . & 0.5 & 1 & 5 & 10 & 17 \\
\hline \multicolumn{10}{|l|}{ Cupric chloride: } \\
\hline Nerine crispa. & . & - & $\cdots$ & & 0.5 & 2 & & & 2 \\
\hline Nerin & . & .. & .. & . & 1 &. & 2 & & 2 \\
\hline & & & & 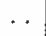 & 1 & 2 & $\cdots$ & 3 & 3 \\
\hline Nerine queen of & . & .. & $\cdots$ & . & 3 & . & . & $\ldots$ & 3 \\
\hline \multicolumn{10}{|l|}{ Barium chloride: } \\
\hline Nerine crispa. . & & & . & . & 0.5 & 2 & $\cdots$ & & 2 \\
\hline Nerine elegans. & . & . & . & . & 0.5 & 1 & . & . & 1 \\
\hline & . & . & . & . & 0.5 & 1 & . & . & 1 \\
\hline Nerine queen of $\mathrm{r}$ & .. & .. & .. & .. & 0.5 & & . & .. & 0.5 \\
\hline \multicolumn{10}{|l|}{ Mercuric chloride: } \\
\hline Nerine crispa. . . & $\cdots$ & - & . & . & 5 & & 5 & & $\mathbf{5}$ \\
\hline Nerine elegans.. & . & . & . & . & 0.5 & 1 & . & 3 & 3 \\
\hline & . & . & . & . & 0.5 & 1 & 2 & . . & 3 \\
\hline Nerine queen of roses. & .. & . & . & . & 0.5 & 2 & . & & 2 \\
\hline
\end{tabular}

\section{Velocity-Reaction CURVes.}

This section deals with the velocity-reaction curves of the starches of Nerine crispa, $N$. elegans, $N$. dainty maid, and $N$. queen of roses, showing the quantitative differences in the behavior toward different reagents at definite time-intervals. (Charts D 190 to D 210.)

Among the conspicuous features of these charts are:

(1) The marked closeness of all four curves, excepting in the reactions with chloral hydrate and potassium sulphocyanate, in which there is a marked tendency to separation, especially in the former, although in the general course of curves the characters of the reactions agree. In the reactions with pyrogallic acid, sulphuric acid, hydrochloric acid, potassium hydroxide, sodium sulphide, calcium nitrate, copper nitrate, cupric chloride, barium chloride, and mercuric chloride gelatinization occurs either with such rapidity or slowness that there is no satisfactory differentiation, such differences as are noted falling within the limits of error of experiment or being unimportant. Even in some of the other reactions the differences are small. 
(2) The curve of $N$. crispa is higher than the curve of $N$. elegans in the reactions with potassium iodide, potassium sulphocyanate, uranium nitrate, and copper nitrate; and lower with chloral hydrate, chromic acid, nitric acid, potassium sulphide, sodium hydroxide, sodium salicylate, and strontium nitrate.

(3) The curves of the hybrids show varying parental relationships, there being a well-marked tendency in the reactions of $N$. dainty maid to intermediateness and a higher position than the parental curves, with a somewhat more marked closeness to the pollen parent, while in $N$. queen of roses there is less tendency to intermediateness but a greater tendency to highness with about an equal inclination to one or the other parent.

(5) An early period of comparatively marked resistance followed by a rapid to moderate gelatinization is seldom recorded, as seen for instance in the curves for chromic acid and potassium sulphocyanate.

(6) The earliest period of the 60 minutes that is the best for the differentiation of the four starches is for the reactions with nitric acid, potassium sulphide, sodium salicylate, and strontium nitrate at 5 minutes; with the chloral hydrate at 15 minutes; with chromic acid and potassium sulphocyanate at 30 minutes; and with potassium iodide, sodium hydroxide, uranium nitrate, and copper nitrate at 60 minutes. The other reactions are either so fast or so slow that no satisfactory differentiation can be made.

\section{REACTION-INTENSITIES OF THE HYBRID.}

This section treats of the reaction-intensities of the hybrid as regards sameness, intermediateness, excess, and deficit in relation to the parent. (Table A 10, and Charts D 190 to D 210.)

The reactivities of the hybrid $N$. dainty maid are the same as those of the seed parent in the safranin reaction; the same as those of the pollen parent with polarization and gentian violet; the same as both parents with pyrogallic acid, potassium sulphide, sodium sulphide, cobalt nitrate, cupric chloride, barium chloride, and mercuric chloride; intermediate with temperature, chloral hydrate, nitric acid, potassium iodide, sodium hydroxide, sodium salicylate (in four being closer to the pollen parent, in one nearer the seed parent, and in one midintermediate ) ; highest with iodine, sulphuric acid, hydrochloric acid, potassium sulphocyanate, calcium nitrate, uranium nitrate, strontium nitrate, copper nitrate (in three being closer to the pollen parent, in four nearer the seed parent, and in one as near to one as to the other parent) ; and lowest with chromic acid and potassium hydroxide (in one being nearer to seed parent, and in one as near one as the other parent).

The reactivities of the hybrid $N$. queen of roses are the same as those of the seed parent in the reactions with gentian violet and safranin; the same as those of the pollen parent with iodine; the same as both parents with pyrogallic acid, potassium hydroxide, sodium sulphide, cobalt nitrate, cupric chloride, barium chloride, and mercuric chloride; intermediate with temperature, nitric acid, and potassium iodide (in two being closer to the seed parent, and in one mid-intermediate); highest with chloral hydrate, sulphuric acid, hydrochloric acid, potassium sulphocyanate, potassium sulphide, so- dium hydroxide, sodium salicylate, calcium nitrate, uranium nitrate, strontium nitrate, and copper nitrate (in six being nearer the pollen parent, in four nearer the seed parent, and in one as near to one as to the other parent) ; and the lowest with polarization and chromic acid (in one being nearer the pollen parent and in the other nearer the seed parent).

The following is a summary of the reaction-intensities of the hybrid as regards sameness, intermediateness, excess, and deficit in relation to the parents:

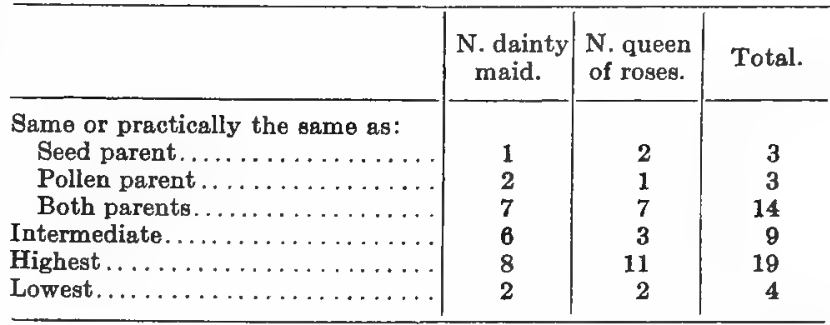

The hybrids differ from each other in the reactions with polarization, iodine, gentian violet, safranin, temperature, chloral hydrate, sodium hydroxide, strontium nitrate, calcium nitrate, and copper nitrate, in several to a minor degree. The hybrid $N$. dainty maid has a higher reactivity than the other hybrid in the reactions with polarization, iodine, calcium nitrate, and copper nitrate, and a lower reactivity in those with gentian violet, safranin, temperature, chloral hydrate, sodium hydroxide, and strontium nitrate. The most striking difference is seen in the reactions with chloral hydrate. The hybrids differ on the whole less from each other than the parents from each other, but they differ as much from the parents as do the parents from each other. The parental relationships of the two hybrids vary in the different reactions as regards sameness, intermediateness, etc., each hybrid showing relationships quite independent of those of other. Thus, in the polarization reactions $N$. dainty maid is the same as the pollen parent, while $N$. queen of roses has the lowest reactivity and is nearer the pollen parent; in the temperature reactions both are intermediate, but the former is nearer the pollen parent, and the latter nearer the seed parent; in the reactions with chloral hydrate the former is intermediate and nearer the pollen parent, and the latter highest and nearer the pollen parent, etc. (See Chapter V.)

\section{Composite CuRves of Reaction-intensities. •}

This section deals with the composite curves of the reaction-intensities, showing the differentiation of the starches of Nerine crispa, $N$. elegans, $N$. dainty maid, and $N$. queen of roses. (Chart $\mathrm{E} 10$.)

The most conspicuous features of this chart are:

(1) The very close correspondence in the rises and falls of the curves of the two parents, excepting in the reaction with chloral hydrate, in which the curve of one parent is ascending and of the other descending. As will be seen also by other charts (E 11 and E 12) some of the nerines are comparatively fast-reacting with this reagent and others the reverse. The curves run so closely as to suggest closely related plants.

( $N$. crispa is a garden variety and $N$. elegans is a hybrid of $N$. flexuosa and $N$. sarniensis var. rosea. $N$. flex- 
uosa has a high reactivity with chloral hydrate and $N$. sarniensis var. rosea a low reactivity, so that $N$. elegans takes after $N$. flexuosa in this reaction.)

(2) N. crispa, in comparison with the other parent $N$. elegans, shows higher reactions with polarization, gentian violet, safranin, temperature, potassium iodide, potassium sulphocyanate, calcium nitrate, uranium nitrate, and cupric chloride; lower reactions with iodine, chloral hydrate, nitric acid, potassium sulphide, sodium salicylate, and strontium nitrate; and the same or practically the same reactions with chromic acid, pyrogallic acid, sulphuric acid, hydrochloric acid, potassium hydroxide, sodium hydroxide, sodium sulphide, calcium nitrate, cobalt nitrate, cupric chloride, barium chloride, and mercuric chloride.

(3) The closeness of the curves of the two hybrids is striking, the only important differences in their courses being noted in the chromic-acid reactions, the reaction of $N$. dainty maid being distinctly higher than in either of the parents, and very much higher than in the other hybrid $N$. queen of roses. The reaction of $N$. dainty maid is closer to $N$. elegans, while that of $N$. queen of roses is intermediate between the parents, but very much closer to $N$. crispa. $N$. dainty maid shows higher reactivities with polarization, iodine, calcium nitrate, and copper nitrate; and lower reactivities with gentian violet, safranin, temperature, chloral hydrate, and strontium nitrate; and the same or practically the same reactivities with chromic acid, pyrogallic acid, nitric acid, sulphuric acid, hydrochloric acid, potassium hydroxide, potassium iodide, potassium sulphocyanate, potassium sulphide, sodium hydroxide, sodium sulphide, sodium salicylate, uranium nitrate, cobalt nitrate, cupric chloride, barium chloride, and mercuric chloride.

(4) In N. crispa the very high reactions with polarization, sulphuric acid, hydrochloric acid, potassium hydroxide, and sodium salicylate; the high reactions with nitric acid, potassium sulphide, and strontium nitrate; the moderate reactions with iodine, gentian violet, safranin, temperature, and chromic acid; the low reactions with chloral hydrate, and potassium sulphocyanate; and the very low reactions with pyrogallic acid, potassium iodide, sodium hydroxide, sodium sulphide, calcium nitrate, uranium nitrate, cobalt nitrate, copper nitrate, cupric chloride, barium chloride, and mercuric chloride.

(5) In $N$. elegans the very high reactions with polarization, nitric acid, sulphuric acid, hydrochloric acid, potassium hydroxide, sodium salicylate, and strontium nitrate; the high reactions with chloral hydrate, and potassium sulphide; the moderate reactions with iodine, safranin, and chromic acid; the low reactions with gentian violet, temperature, and potassium sulphocyanate; and the very low reactions with pyrogallic acid, potassium iodide, sodium hydroxide, sodium sulphide, calcium nitrate, uranium nitrate, cobalt nitrate, copper nitrate, cupric chloride, barium chloride, and mercuric chloride.

(6) In the hybrid $N$. dainty maid the very high reactions with polarization, sulphuric acid, hydrochloric acid, potassium hydroxide, and sodium salicylate; the high reactions with iodine, nitric acid, potassium sulphide, and strontium nitrate; the moderate reactions with safranin, chromic acid, and potassium sulphocyanate; the low reactions with gentian violet and temperature; and the very low reactions with pyrogallic acid, potassium iodide, sodium hydroxide, sodium sulphide, calcium nitrate, uranium nitrate, cobalt nitrate, copper nitrate, cupric chloride, barium chloride, and mercuric chloride.

(7) In the reactivities of the hybrid $N$. queen of roses the very high reactions with chloral hydrate, sulphuric acid, hydrochloric acid, potassium hydroxide, sodium salicylate, and strontium nitrate; the high reactions with polarization, nitric acid, and potassium sulphide; the moderate reactions with iodine, gentian violet, safranin, temperature, and chromic acid; the low reactions with potassium sulphocyanate; and the very low reactions with pyrogallic acid, potassium iodide, sodium hydroxide, sodium sulphide, calcium nitrate, uranium nitrate, cobalt nitrate, copper nitrate, cupric chloride, barium chloride, and mercuric chloride. ties:

The following is a summary of the reaction-intensi-

\begin{tabular}{l|c|c|c|c|c}
\hline & $\begin{array}{c}\text { Very } \\
\text { high. }\end{array}$ & High. & $\begin{array}{c}\text { Mod- } \\
\text { erate. }\end{array}$ & Low. & $\begin{array}{c}\text { Very } \\
\text { low. }\end{array}$ \\
\hline Nerine crispa...... & 5 & 3 & 5 & 2 & 11 \\
Nerine elegans.... & 7 & 2 & 3 & 3 & 11 \\
Nerine dainty maid. & 5 & 4 & 2 & 3 & 11 \\
Nerine queen of roses & 6 & 3 & 5 & 1 & 11 \\
\hline
\end{tabular}

11. Comparisons of the Starches of Nerine BOWDENI, N. SARNIENSIS VAR. CORUSCA MAJOR, N. gLaNTESS, AND N. ABUNDANCE.

In histologic characteristics, polariscopic figures, reactions with selenite, qualitative reactions with iodine, and qualitative reactions with the various chemical reagents the starches of the parents exhibit properties in common, and also individualities by which they can be differentiated. The starch of Nerine sarniensis var. corusca major in comparison with that of $N$. bowdeni contains a smaller number of compound grains and aggregates; the grains are more regular and less varied in form, and the irregularities are due much more frequently to notches and depressions at the margins; and the flattened broad forms are less flattened. The hilum is not so distinct, is less frequently fissured, and is more eccentric. The lamellæ are not quite as distinct, they are more regular, coarse lamellæ are less numerous, the arrangements of coarse and fine lamellæ differ from that which is observed in $N$. bowdeni, and the number is somewhat less. In size the grains are smaller, and there are not forms that are as broad as are found in the other parent. In the polariscopic, selenite, and iodine reactions there are many differences. In the qualitative reactions with chloral hydrate, nitric acid, potassium iodide, potassium sulphide, potassium sulphocyanate, and sodium salicylate there are also many differences, some of which are quite interesting, and all are collectively of marked value in the differentiation of the two starches. The starch of the hybrid $N$. giantess, in comparison with the starches of the parents, contains a much less number of compound grains and aggregates than that of $N$. bowdeni, but slightly more than in the starch of the other parent, and the compound grains are partly of a type that is found exclusively in $N$. bowdeni, and also partly of other types 
that are found in the starches of both parents; and in irregularity of outline they are nearer to $N$. bowdeni. The hilum in character and eccentricity is the same as that of $N$. sarniensis var. corusca major. The lamellæ in character and arrangement, and the size are also nearer those of this species. The number of lamellæ is less than in either parent. In the polariscopic figures and reactions with selenite the relationship is closer to $N$. sarniensis var. corusca major. In the qualitative iodine reactions the raw grains behave more like those of $N$. sarniensis var. corusca major, but the heated grains more like those of the other species. In the qualitative reactions with the chemical reagents the resemblances are closer to the reactions of $N$. bowdeni in the reactions with chloral hydrate and sodium salicylate, but closer to the other parent in those with nitric acid, potassium iodide, potassium sulphocyanate, and potassium sulphide. The starch of the hybrid $N$. abundance, in comparison with the starches of the parents, contains a smaller number of compound grains and aggregates than either, and only an occasional compound grain is seen of a type that was noted exclusively in $N$. bowdeni; irregularity is more than in $N$. sarniensis var. corrusca major, but considerably less than in the other parent. The form is in general nearly mid-intermediate between the forms of the parental starches, but somewhat nearer that of $N$. sarniensis var. corusca major. The hilum is in character nearer $N$. bowdeni, but in eccentricity it exceeds that of either parent and is nearer $N$. sarniensis var. corusca major. The lamellæ are in both character and arrangement nearer $N$. sarniensis var. corusca major, but the number is notably less than in either parent. The size is, on the whole, intermediate, but somewhat nearer that of $N$. bowdeni. In the polariscopic, selenite, and qualitative iodine reactions it is nearer $N$. bowdeni. In the qualitative chemical reactions with the six reagents resemblances lean to one or the other parent, but on the whole the relationship is closer to $N$. bowdeni. For the most part the hybrids bear closer relationships to each other than does either to either parent. They vary much in their parent-leanings, each independently of the other, so that while one hybrid may show a leaning to the seed parent in a given character, the other hybrid may in this same character lean as markedly toward the other parent. Thus, in form $N$. giantess is more closely related to $N$. bowdeni, but $N$. abundance is nearly mid-intermediate between the parents with an inclination to $N$. sarniensis var. corusca major. In hilum $N$. giantess is closer to $N$. sarniensis var. corusca major, while $N$. abundance is closer to $N$. bowdeni in characters and to the other parent in eccentricity. In lamellæ both are closer to $N$. sarniensis var. corusca major. In size $N$. giantess is closer to $N$. sarniensis var. corusca major, and $N$. abundance to $N$. bowdeni. In the qualitative iodine reactions $N$. giantess is in the reactions of the ungelatinized grains closer to $N$. sarniensis var. corusca major, and in the gelatinized grains closer to $N$. bowdeni; but $N$. abundance is in both respects closer to $N$. bowdeni. In the qualitative reactions with the chemical reagents $N$. giantess is with certain reagents closer to one parent and with others closer to the other parent, while $N$. abundance is closer with all reagents to $N$. bowdeni.
Reaction-intensities Expressed by Light, Color, and TemperaPolarization:

N. bowdeni, moderate to high, value 85 .

N. sarn. var. cor. maj., moderate to very high, higher than in $\mathbf{N}$. bowdeni, value 90 .

N. giantess, moderately high to very high, lower than in either parent, value 80 .

N. abundance, moderately high to very high, the same as N. giantIodine: ess, value 80 .

N. bowdeni, moderate, value 50 .

N. Sarn. var. cor. maj., moderately deep, deeper than in N. bowdeni, value 60 .

N. giantess, moderately deep, the same as N. sarn. var. cor. maj., value 60.

N. abundance, moderate, sam $\theta$ as N. bowdeni, value 50 .

Gentian violet:

$\mathrm{N}$. bowdeni, moderate, value 45.

N. sarn. var, cor. maj., light to moderate, lighter than N. bowdeni, value 40 .

N. giantess, moderate, same as in N. bowdeni, value 45.

$\mathrm{N}$. abundance, light to moderate, aame as $\mathrm{N}$. sarn. var. cor. maj., value 40.

Safranin:

N. bowdeni, moderate, value 50 .

N. sarn. var. cor. maj., moderate, much less than in N. bowdeni, value 40.

N. giantess, moderate, the same as N. bowdeni, value 50 .

$\mathrm{N}$. abundance, moderate, less than $\mathrm{N}$. bowdeni and much more than N. sarn. var. cor. maj., value 45 .

\section{Temperature:}

N. bowdeni, in majority at 67.6 to $67.9^{\circ}$, in all at 74 to $75^{\circ}$, mean $74.5^{\circ}$.

N. sarn. var. cor. maj., in majority at 70 to $71^{\circ}$, in all but rare grains at 76 to $78.8^{\circ}$, mean $78.4^{\circ}$.

N. giantess, in majority at 68.2 to $69.1^{\circ}$, in all at 70.9 to $71^{\circ}$, mean $70.95^{\circ}$.

N. abundance, in majority at 69 to $69.9^{\circ}$, in all at 73.9 to $74.8^{\circ}$, mean $74.3^{\circ}$.

$N$. bowdeni shows in the polarization and iodine reactions lower reactivities than $\bar{N}$. sarniensis var. corusca

TABLE A 11.

\begin{tabular}{|c|c|c|c|c|c|c|c|c|c|c|}
\hline & $\stackrel{\text { g }}{-}$ & ล & 昆 & 最 & 珀 & $\stackrel{g}{9}$ & 是 & 㫛 & $\begin{array}{l}\text { 豆 } \\
\text { 号 }\end{array}$ & 罟 \\
\hline Chloral hydrate: & & & & & & & & & & \\
\hline N. 1 & .. & & $\cdots$ & . & 3 & . & 26 & 39 & 52 & 56 \\
\hline N. sarn var. cor maj.. & . & .. & . & . & 20 & .. & 80 & 95 & 98 & 99 \\
\hline N. giantess . . . . . . . & . & .. & . & .. & 17 & . & 80 & 95 & 97 & 99 \\
\hline N. abundance..... & . & . & . & . & 45 & . & 82 & 97 & 98 & 99 \\
\hline Chromic acid: & & & & & & & & & & \\
\hline N. bowdeni & . & -. & . & . & 0.5 & & 2 & 75 & 95 & 98 \\
\hline N. sarn. var. cor. maj... & .. & . & . & . & 0.5 & . . & 2 & 65 & 86 & 97 \\
\hline N. giantess......... & & . & .. & .. & 0.5 & & $\mathbf{2}$ & 68 & 89 & 98 \\
\hline N. abundance. . & . & .. & . & . & 1 & . & 2 & 62 & 85 & 98 \\
\hline Pyrogallic acid: & & & & & & & & & & \\
\hline N. bowdeni........ & . & $\cdots$ & - & - & 0.5 & $\cdots$ & & .. & 1 & \\
\hline N. sarn. var. cor. maj .. & .. & .. & . & . & 1 & . & $\mathbf{3}$ & . & . & 3 \\
\hline N. giantess . . . & & - & . & . & 0.5 & . & 1 & . & . & 1 \\
\hline N. abundance. . & . & . & .. & . & 2 & . & 3 & . & . & $\mathbf{3}$ \\
\hline Nitric acid: & & & & & & & & & & \\
\hline N. bowdeni .......... & . & $\cdots$ & . & . & 58 & . & 80 & 92 & 96 & 97 \\
\hline N. sarn. var. cor. maj ... & & . & . & . . & 43 & . & 78 & 90 & 93 & 95 \\
\hline N. giantess... & . & $\cdots$ & . & . & 33 & . & 74 & 88 & 92 & 95 \\
\hline N. abundance. . & . & .. & . & . & 32 & . & 70 & 81 & 87 & 91 \\
\hline Sulphuric acid: & & & & & & & & & & \\
\hline N. bowdeni..... & 84 & . & 97 & . & 99 & . & . & -. & . & . \\
\hline N. sarn. var. cor. & 92 & . & 98 & .. & 99 & . & . & * & . & . \\
\hline N. giantess... & 92 & . & 96 & . & 97 & . & . & $\cdots$ & . & . \\
\hline N. abundance... & 86 & . & 97 & . & 99 & . & - & $\cdots$ & $\cdots$ & $\cdots$ \\
\hline $\begin{array}{l}\text { Hydrochloric acid: } \\
\text { N. bowdeni ....... }\end{array}$ & .. & .. & .. & . & 76 & & 93 & 95 & 99 & 99 \\
\hline N. sarn. var. cor maj ... & .. & .. & . & . & 77 & . & 93 & 95 & 96 & 97 \\
\hline N. giantess. . . . . & . & .. & .. & .. & 77 & . & 92 & 95 & 96 & 96 \\
\hline N. abundance. & & & . & & 75 & . & 90 & 95 & 96 & 96 \\
\hline
\end{tabular}


TaBtr A 11.-Continued.

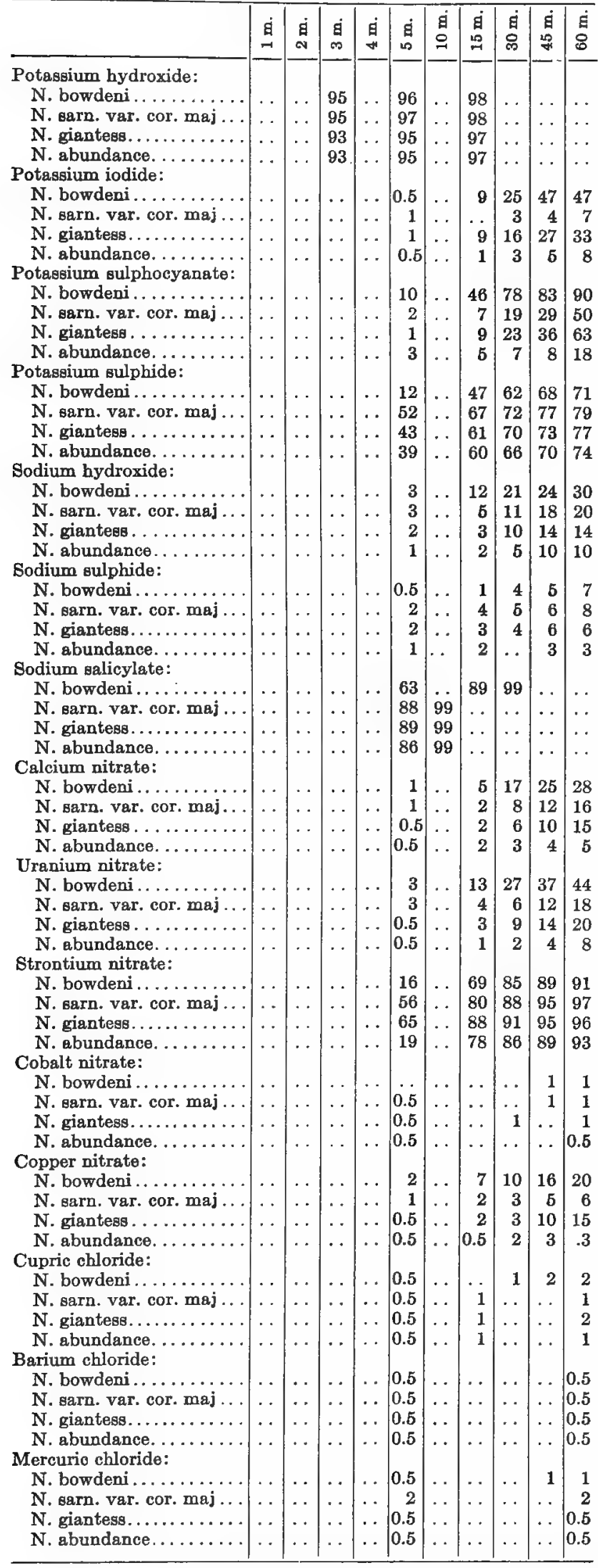

major, and in the gentian-violet, safranin, and temperature reactions higher reactivities. Both hybrids in the polarization and temperature reactions show higher reactivities than either parent, both being in both reactions closer to $N$. bowden $i$ than to the other parent, but in the temperature reaction $N$. abundance is practically the same as $N$. bowdeni. The hybrid $N$. giantess in the iodine reactions is the same as $N$. sarniensis var. corusca major, but $N$. abundance is the same as the other parents. $N$. giantess is the same as $N$. bowdeni in the gentianviolet reactions, while $N$. abundance is the same as the other parent. $N$. giantess is the same as $N$. bowdeni in the safranin reactions, while $N$. abundance is intermediate between the parents, but closer to $N$. bowdeni.

Table A 11 shows the reaction-intensities in percentages of total starch gelatinized at definite intervals (minutes).

\section{Velocity-reaction Curves.}

This section treats of the velocity-reaction curves of the starches of Nerine bowdeni, $N$. sarniensis var. corusca major, $N$. giantess, and $N$. abundance, showing the quantitative differences in the behavior toward different reagents at definite time-intervals. (Charts D'211 to D 231.)

Among the most conspicuous features of these charts are:

(1) The marked closeness and correspondence in the courses of all four curves, excepting in the reactions with chloral hydrate and potassium sulphocyanate, as was noted in the preceding set. Owing to too rapid, too slow, or too close reactions no satisfactory if any differentiation can be made in the reactions with pyrogallic acid, sulphuric acid, hydrochloric acid, potassium hydroxide, sodium sulphide, cobalt nitrate, cupric chloride, barium chloride, and mercuric chloride.

(2). The curve of $N$. bowdeni is higher than the curve of the other parent in the reactions with chromic acid, nitric acid, potassium iodide, potassium sulphocyanate, sodium hydroxide, calcium nitrate, uranium nitrate, and cupric chloride; and lower in those with chloral hydrate, potassium sulphide, sodium salicylate, and strontium nitrate.

(3) The curves of the hybrids bear varying relationships to the parental curves, and the hybrid curves themselves differ in many respects from each other. There is in $N$. giantess a distinct tendency to intermediateness and to the lowest position in relation to the parental curves, and with a decided inclination to the curves of the pollen parent; while in $N$. abundance there is a particularly marked inclination to be the highest of the three curves and to the curves of the pollen parent.

(4) An early period of high resistance followed by a rapid to moderate gelatinization is noted in very few of the experiments, but especially in the chromic-acid reaction.

(5) The earliest period during the 60 minutes that is best for the differentiation of all four starches is for chloral hydrate, nitric acid, potassium sulphide, sodium salicylate, and strontium nitrate at 5 minutes; for potassium iodide at 30 minutes; for potassium sulphocyanate, sodium hydroxide, calcium nitrate, uranium nitrate, and cupric chloride at 60 minutes. Other reactions are too slow or too fast for satisfactory differentiation. 


\section{Reaction-Intensities OF the Hybrids.}

This section treats of the reaction-intensities of the hybrids as regards sameness, intermediateness, excess, and deficit in relation to the parents. (Table A 11 and Charts D 211 to D 231.)

The reactivities of the hybrid $N$. giantess are the same as those of the seed parent in the reactions with gentian violet and safranin; the same as those of the pollen parent with iodine, chloral hydrate, sulphuric acid, sodium salicylate, calcium nitrate, and uranium nitrate; and the same as those of both parents with pyrogallic acid, potassium hydroxide, sodium sulphide, cobalt nitrate, cupric chloride, barium chloride, and mercuric chloride, in all of which the reactions are too fast or too slow for differentiation; intermediate with chromic acid, potassium iodide, potassium sulphocyanate, potassium sulphide, strontium nitrate, and copper nitrate (in three being mid-intermediate, in one nearer the seed parent, and in two nearer the pollen parent) ; highest in the temperature reaction, and nearer the seed parent; and lowest in the reactions with polarization, nitric acid, hydrochloric acid, and sodium hydroxide (in one being as near as the other parent, in one nearer the seed parent, and in one nearer the pollen parent).

The reactivities of the hybrid $N$. abundance are the same as those of the seed parent in the reactions with iodine, temperature, and sulphuric acid; the same as those of the pollen parent with gentian violet, potassium iodide, and sodium salicylate; the same as those of both parents with pyrogallic acid, potassium hydroxide, sodium sulphide, cobalt nitrate, cupric chloride, barium chloride, and mercuric chloride, in all of which the reactions are too fast or too slow for differentiation; intermediate with safranin, potassium sulphide, and strontium nitrate (in two being closer to the seed parent, and in one closer to the pollen parent); highest with temperature and chloral hydrate, in the former being closer to the seed parent and in the latter to the pollen parent; and lowest with polarization, chromic acid, nitric acid, hydrochloric acid, potassium sulphocyanate, sodium hydroxide, calcium nitrate, uranium nitrate, and copper nitrate (in one being as close to one parent as to the other, in one closer to the seed parent, and in seven closer to the pollen parent).

\section{Composite Curves of the Reaction-intensities.}

This section treats of the composite curves of the reaction-intensities, showing the differentiation of the starches of Nerine bowdeni, $N$. sarniensis var. corusca major, N. giantess, and N. abundance. (Chart E 11.)

The most conspicuous features of this chart are:

(1) The very close correspondence in the rises and falls of the curves of the parents, excepting in the reactions with chloral hydrate and potassium sulphide, the same peculiarity having been noted in the preceding set, excepting that in this set the potassium-sulphide curves retain the same relative positions, the disagreement in the latter being attributable to the relatively low reactivity of $N$. bowdeni.

(2) $N$. bowdeni has higher reactivities than the other parent ( $N$. sarniensis var. corusca major) with gentian violet, safranin, temperature, chromic acid, nitric acid, potassium iodide, potassium sulphocyanate, sodium hy- droxide, calcium nitrate, uranium nitrate, and copper nitrate; lower with polarization, iodine, chloral hydrate, sodium salicylate, and strontium nitrate; and the same or practically the same with pyrogallic acid, sulphuric acid, hydrochloric acid, potassium hydroxide, potassium sulphide, sodium sulphide, cobalt nitrate, cupric chloride, barium chloride, and mercuric chloride.

(3) In $N$. bowdeni the very high reactions with polarization, sulphuric acid, and potassium hydroxide; the high reactions with chromic acid, hydrochloric acid, and sodium salicylate; the moderate reactions with iodine, gentian violet, safranin, nitric acid, potassium sulphocyanate, and strontium nitrate; the low reactions with temperature, chloral hydrate, and potassium sulphide; the very low reactions with pyrogallic acid, potassium iodide, sodium hydroxide, sodium sulphide, calcium nitrate, uranium nitrate, cobalt nitrate, copper nitrate, cupric chloride, barium chloride, and mercuric chloride.

(4) In N. sarniensis var. corusca major the very high reactions with polarization, sulphuric acid, potassium hydroxide, and sodium salicylate; the high reactions with iodine, chloral hydrate, hydrochloric acid, and strontium nitrate; the moderate reactions with gentian violet, safranin, chromic acid, and nitric acid; the low reactions with temperature, potassium sulphocyanate, and potassium sulphide; and the very low reactions with pyrogallic acid, potassium iodide, sodium hydroxide, sodium sulphide, calcium nitrate, uranium nitrate, cobalt nitrate, copper nitrate, cupric chloride, barium chloride, and mercuric chloride.

(5) In the hybrid $N$. giantess the very high reactions with polarization, sulphuric acid, potassium hydroxide, and sodium salicylate; the high reactions with iodine, chloral hydrate, hydrochloric acid, and strontium nitrate; the moderate reactions with gentian violet, safranin, temperature, chromic acid, and nitric acid; the low reactions with potassium sulphocyanate and potassium sulphide; and the very low reactions with pyrogallic acid, potassium iodide, sodium hydroxide, sodium sulphide, calcium nitrate, uranium nitrate, cobalt nitrate, copper nitrate, cupric chloride, barium chloride, and mercuric chloride.

(6) In the hybrid $N$. abundance the very high reactions with polarization, sulphuric acid, potassium hydrozide, and sodium salicylate; the high reactions with chloral hydrate and hydrochloric acid; the moderate reactions with iodine, gentian violet, safranin, chromic acid, nitric acid, and strontium nitrate; the low reactions with temperature and potassium sulphide; and the very low reactions with pyrogallic acid, potassium iodide, potassium sulphocyanate, sodium hydroxide, sodium sulphide, calcium nitrate, uranium nitrate, cobalt nitrate, copper nitrate, cupric chloride, barium chloride, and mercuric chloride. ties:

The following is a summary of the reaction-intensi-

\begin{tabular}{|c|c|c|c|c|c}
\hline & $\begin{array}{c}\text { Very } \\
\text { high. }\end{array}$ & High. & $\begin{array}{c}\text { Mod- } \\
\text { erate. }\end{array}$ & Low. & $\begin{array}{l}\text { Very } \\
\text { low. }\end{array}$ \\
\hline N. bowdeni............ & 3 & 3 & 6 & 3 & 11 \\
N. sarn. var. cor. maj. & 4 & 4 & 4 & 3 & 11 \\
N. giantess........... & 4 & 4 & 5 & 2 & 11 \\
N. abundance........ & 4 & 3 & 5 & 2 & 12 \\
\hline
\end{tabular}


The two hybrids show in general a closer relationship in their reactivities to each other than does either to either parent. In some reactions the reactivities are the same, and in others one hybrid has a higher reactivity than the other, but in other reactions the reverse. Then again their reactivities in their parental relationships are of a most variable character in that in a given reaction both may be lower or higher than the reactions of the parents, in another reaction that of one may be higher and that of the other lower, or intermediate, or the same, etc. Thus, eliminating the seven reactions in which, owing to a too rapid or too slow reaction, the results were the same in case of all four starches, it will be noted that out of the remaining 19 reactions in only 6 were the reactions of the same relationship to the parentsin the polarization reactions the reactivities of both hybrids are the lowest and both nearer the seed parent; in the temperature reactions one is higher than either parent, but closer to the seed parent, and the other is practically the same as the seed parent; in the nitric acid reactions both are the highest, in the former nearer the seed parent and in the latter nearer the pollen parent; in the hydrochloric acid reactions the reactivities are lowest, and both as close to one as to the other parent; in the sodium-hydroxide reactions both are highest and nearer the seed parent; and in the sodium-salicylate reactions both are the same as the pollen parent. In each of the other reactions one hybrid shows a parental relationship that is different from that of the other. Thus, in the iodine reactions $N$. giantess is closer to the seed parent, while $N$. abundance is closer to the pollen parent; in the sulphuric-acid reactions $N$. giantess is closer to the pollen parent, while $N$. abundance is closer to the seed parent; in the potassium-sulphide reactions both hybrids are intermediate, but one is closer to the pollen parent and the other to the seed parent, etc. The reactivities of $N$. giantess are, on the whole, slightly higher than those of the other hybrid, and both are in this respect nearer the pollen than the seed parent, $N$. giantess being the closer.

The following is a summary of the reaction-intensities of the hybrids as regards sameness, intermediateness, excess, and deficit in relation to the parents:

\begin{tabular}{|c|c|c|}
\hline & N. giantess. & N. abundance. \\
\hline 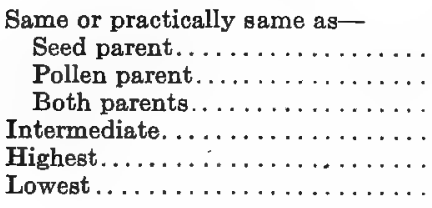 & $\begin{array}{l}2 \\
6 \\
7 \\
6 \\
1 \\
4\end{array}$ & $\begin{array}{l}3 \\
3 \\
7 \\
3 \\
1 \\
9\end{array}$ \\
\hline
\end{tabular}

In both hybrids the properties seem to be influenced much more by the pollen parent. In the first hybrid there is greater tendency to intermediateness and less tendency to lowness of reactivity than in the other hybrid. The hybrids differ sufficiently in their parental relationships to be readily distinguished notwithstanding their close similarities. (See Chapter V.)

\section{Comparisons of the Starches of Nerine SARNIENSIS VAR. CORUSCA MAAJOR, N. CURVIFOLIA VAR. FOTHERGILLI MAJOR, AND N. GLORY OF SARNIA.}

In histologic characteristics, polariscopic figures, reactions with selenite, qualitative reactions with iodine, and qualitative reactions with the various chemical reagents all three starches exhibit properties in common, and each has certain individualities, but all are closely related. The starch of $N$. curvifotia var. fothergitli major contains in comparison with the starch of the other parent a larger number of compound grains and aggregates, and the former are of more varied types. The grains are less regular and somewhat more slender and pointed. The hilum is more distinct and eccentric. The lamellæ are more distinct and less numerous, and there is difference in the grouping of the coarse lamellæ. The size is less and the grains tend to be less broad in proportion to length. In the polariscopic, selenite, and iodine reactions differences are noted. In the qualitative reactions with the chemical reagents many similarities and differences are recorded, some of the latter being quite striking, and taken collectively readily differentiate the starches. The starch of the hybrid contains fewer compound grains and aggregates than are fornd in the parents, and the types of compound grains are for the most part those observed in the starch of $N$. sarniensis var. corusca major. The grains are more regular in form than in either parent, and on the whole nearer those of $N$. sarniensis var. corusca major. The characters of the hilum are closer to those of the same parent, and the eccentricity is less than in either parent. The lamellæ are less distinct but more numerous than in either parent, and they are more closely related to those of $N$. sarniensis var. corusca major. In sizes the grains are also more closely related to the same parent. In the qualitative polarization, selenite, and iodine reactions the hybrid shows a more marked closeness to $N$. sarniensis var. corusca major. In the qualitative reactions with the chemical reagents, including :choral hydrate, nitric acid, potassium iodide, potassium sulphocyanate, potassium sulphide, and sodium salicylate, reactions in each resembling more closely those of one or the other parent are noted, but in case of each reagent the phenomena are collectively closer to those of $N$. sarniensis var. corusca major than to those of the other parent.

Reaction-intensities Expressed by Light, Color, and TemperaPolarization:

N. sarn. var. cor. maj., moderate to very high, value 90 .

N. curvi. var. foth. maj., moderate to very high, lower than $N$. sarn. var. cor. maj., value 87

N. glory of sarnia, moderate to very high, the same as N. sarn. Iodine: var. cor. maj., value 90 .

N. earn. var. cor. maj., moderately deep, value 60 .

N. curvi. var. foth. maj., moderately deep, deeper than N. sarn. var. cor. maj., value 65 .

$\mathrm{N}$. glory of sarnia, moderate, less than either parent, value 55 .

Gentian violet:

N. sarn. var. cor. maj., light to moderate, value 40 .

N. curvi. var. foth. maj., moderate, deeper than N. sarn. v. cor. maj., value 45 .

N. glory of sarnia, light to moderate, lighter than in either parent, value 35.

Safranin:

N. earn. var. cor. maj., moderate, value 40.

N. curvi. var. foth. maj., moderate, deeper than $\mathrm{N}$. sarn var. cor. maj., value 35 .

N. glory of sarnia, light to moderate, less than either parent, value 35 .

Temperature:

N. aarn. var. cor. maj., in the majority at 70 to $71^{\circ}$, in all but rare grains 76 to $78.8^{\circ}$, mean $78.4^{\circ}$.

N. curvi. var. foth maj., in the majority at 68.1 to $69^{\circ}$, in all at 73.2 to $74.3^{\circ}$, mean $73.8^{\circ}$.

N. glory of sarnia, in the majority at 70 to $72^{\circ}$ in all at 75.8 to $77^{\circ}$, mean $76.4^{\circ}$.

$N$. sarniensis var. corusca major shows in the polarization and temperature reactions higher reactivities than the other parent, but lower reactivities in those with iodine, gentian violet, and safranin. The hybrid shows the same reactivity as $N$. sarniensis var. corusca major in the polarization reaction, but less than that of the other parent; lower reactivities than the parents with iodine, 
Table A 12.

\begin{tabular}{|c|c|c|c|c|c|c|c|c|c|c|}
\hline & $\underset{\sim}{\text { घี }}$ & $\underset{\text { ำ }}{\text { ज }}$ & $\underset{\infty}{\dot{\Xi}}$ & $\begin{array}{l}\text { घ } \\
+\end{array}$ & $\underset{20}{\dot{g}}$ & $\stackrel{8}{8}$ & $\begin{array}{c}\text { घ } \\
\text { I }\end{array}$ & $\begin{array}{l}\text { घं } \\
\text { ᄋ }\end{array}$ & $\underset{\text { 19 }}{\text { व }}$ & $\begin{array}{l}\text { 日 } \\
8\end{array}$ \\
\hline al & & & & & & & & & & \\
\hline & $\cdots$ & $\cdots$ & $\cdots$ & $\cdots$ & 20 & & 80 & 95 & 98 & 90 \\
\hline N. & $\cdots$ & . & . & . & 21 & $\ldots$ & 90 & 98 & & \\
\hline & . & . & .. & . & 14 & .. & 77 & 86 & 89 & 90 \\
\hline Chro & & & & & & & & & & \\
\hline N. & $\cdots$ & $\cdots$ & $\cdots$ & $\cdots$ & 0.5 & $\cdots$ & 2 & 65 & 86 & 97 \\
\hline$N$ & $\cdots$ & . & . & $\ldots$ & 0.5 & . & 3 & 36 & 85 & 97 \\
\hline N. & . & . & $\ldots$ & $\cdots$ & 0.5 & $\ldots$ & 1 & 30 & 78 & 94 \\
\hline yrog & & & & & & & & & & \\
\hline N. & $\cdots$ & $\cdots$ & $\cdots$ & $\cdots$ & 1 & $\cdots$ & 3 & & $\cdots$ & \\
\hline & . & . & . & . & 1 & . & $\cdots$ & 2 & . & \\
\hline & $\cdots$ & . & $\cdots$ & $\cdots$ & 0.5 & $\cdots$ & . & . & . & 0.5 \\
\hline & . & & & & 43 & & 78 & 90 & 93 & 95 \\
\hline & $\ldots$ & $\ldots$ & 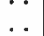 & . & 6 & $\cdots$ & 60 & 87 & 90 & 95 \\
\hline & $\ldots$ & $\ldots$ & $\cdots$ & $\cdots$ & 5 & $\cdots$ & 50 & 72 & 80 & 82 \\
\hline ulp & & & & & & & & & & \\
\hline N. & 92 & & 98 & . & 99 & $\cdots$ & . & . & $\cdots$ & \\
\hline & 97 & . & 99 & . & 99 & . & $\ldots$ & . & . & \\
\hline & 75 & . & 92 & $\cdots$ & 96 & $\cdots$ & $\cdots$ & $\cdots$ & $\cdots$ & " \\
\hline Hyd & & & & & & & & & & \\
\hline & $\cdots$ & $\cdots$ & $\cdots$ & $\cdots$ & 7 & $\cdots$ & 93 & 95 & 96 & 97 \\
\hline & $\cdots$ & $\cdots$ & $\cdots$ & $\cdots$ & 6 & $\cdots$ & 98 & 99 & & 99 \\
\hline & . & $\cdots$ & $\cdots$ & $\cdots$ & 6 & $\cdots$ & 88 & 80 & 95 & 97 \\
\hline Pota & & & 95 & & & & 98 & & & \\
\hline & $\cdots$ & $\cdots$ & $\begin{array}{l}95 \\
97\end{array}$ & . & 99 & $\cdots$ & $\begin{array}{l}98 \\
99\end{array}$ & . & & \\
\hline & 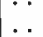 & $\cdots$ & 94 & . & 96 & & 98 & $\because$ & . & \\
\hline Potassiun & & & & & & $\because$ & & & & \\
\hline & $\cdots$ & $\cdots$ & . & $\cdots$ & 1 & . & & 3 & 4 & 7 \\
\hline & . & . & $\ldots$ & $\ldots$ & 1 & & 2 & 3 & 4 & 5 \\
\hline & $\ldots$ & , . & $\ldots$ & . & 2 & $\ldots$ & 3 & $\ldots$ & 4 & \\
\hline Pots & & & & & & & & & & \\
\hline & $\cdots$ & $\ldots$ & $\cdots$ & $\cdots$ & 2 & & 7 & 19 & 29 & 50 \\
\hline & . & . & . & $\ldots$ & 2 & . & 3 & 4 & & \\
\hline & $\cdots$ & $\ldots$ & . & $\ldots$ & 1 & $\cdots$ & 3 & 4 & 5 & 6 \\
\hline Pot: & & & & & & & & & & \\
\hline & $\cdots$ & $\ldots$ & $\cdots$ & $\cdot$ & 52 & $\cdots$ & 67 & 72 & 77 & 79 \\
\hline & $\cdots$ & . & $\cdots$ & $\cdots$ & 41 & . & 35 & 70 & 74 & 76 \\
\hline & $\ldots$ & $\ldots$ & . & . & 10 & . & 33 & 52 & 67 & 69 \\
\hline Sodi & & & & & & & & & & \\
\hline & $\cdots$ & $\ldots$ & $\cdots$ & $\cdots$ & 3 & $\cdots$ & 5 & 11 & 18 & 20 \\
\hline & $\cdots$ & $\ldots$ & . & $\ldots$ & 1 & . & 1 & 2 & 3 & 4 \\
\hline & $\ldots$ & $\ldots$ & $\ldots$ & $\cdots$ & 0.5 & $\cdots$ & 1 & $\ldots$ & 2 & 3 \\
\hline od & & & & & & & & & & \\
\hline & $\cdots$ & $\cdots$ & $\cdots$ & & 2 & $\cdots$ & 4 & 5 & 6 & 8 \\
\hline & $\cdots$ & $\cdots$ & $\cdots$ & & .5 & $\cdots$ & 2 & 3 & . & \\
\hline & $\ldots$ & $\cdots$ & $\cdots$ & $\ldots$ & .5 & $\cdots$ & $\cdots$ & $\cdots$ & $\cdots$ & \\
\hline odi & & & & & & & & & & \\
\hline & $\cdots$ & $\cdot$ & $\cdots$ & . & $\begin{array}{l}88 \\
91\end{array}$ & $\begin{array}{c}99 \\
100\end{array}$ & & . & . & \\
\hline & . & & $\cdots$ & & & $\begin{array}{c}100 \\
07\end{array}$ & & - & . & \\
\hline & . & $\cdots$ & $\cdots$ & $\cdots$ & 74 & 97 & 99 & $\ldots$ & $\cdots$ & $\cdots$ \\
\hline alc & & & & & & & & & & \\
\hline & . & $\cdots$ & $\because$ & & & $\cdots$ & 2 & 8 & 12 & 6 \\
\hline & . & & $\cdots$ & & 05 & $\cdots$ & $\cdots$ & 2 & 4 & 4 \\
\hline & $\cdots$ & & $\cdots$ & & 0.5 & $\cdots$ & $\cdots$ & 1 & 2 & \\
\hline Ura & & & . & & 3 & & & 6 & & \\
\hline & $\cdots$ & & & & 0 & & $\begin{array}{l}4 \\
2\end{array}$ & 6 & $\begin{array}{r}12 \\
3\end{array}$ & \\
\hline & & & $\cdots$ & & 0.5 & & & & & \\
\hline & & & & & 0.5 & $\cdots$ & 1 & 2 & $\cdots$ & \\
\hline $\operatorname{trc}$ & & $\ldots$ & & & 56 & & & & & \\
\hline & & $\ldots$ & $\ldots$ & 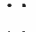 & & & 0 & 38 & 95 & 7 \\
\hline & . & & $\cdots$ & & & $\cdots$ & 8 & 6 & 93 & 97 \\
\hline & & $\cdots$ & $\cdots$ & $\cdots$ & 10 & $\ldots$ & 73 & 35 & 87 & \\
\hline & & & & & & & & & & \\
\hline & & & - & . & & $\cdots$ & $\because$ & $\ddot{2}$ & 1 & \\
\hline & . & $\because$ & 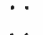 & $\cdots$ & & $\cdots$ & 1 & & & \\
\hline & . & $\cdots$ & $\cdots$ & $\ldots$ & & . & $\ldots$ & 1 & $\ldots$ & \\
\hline & & & & & & & & & & \\
\hline & $\cdots$ & $\cdots$ & $\cdots$ & $\cdots$ & 1 & $\cdots$ & 2 & 3 & 5 & 6 \\
\hline & . . & $\ldots$ & $\ldots$ & $\ldots$ & 0.5 & & 1 & 2 & . & \\
\hline & . & $\cdots$ & $\cdots$ & . & 0.5 & $\ldots$ & 1 & 3 & . & 4 \\
\hline & & & & & & & & & & \\
\hline & . & $\ldots$ & $\ldots$ & $\ldots$ & 0.5 & . & 1 & & . & 1 \\
\hline & . & . & $\ldots$ & $\ldots$ & 0.5 & . & 2 & 3 & . & 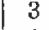 \\
\hline & . & . & $\cdots$ & $\cdots$ & 0.5 & $\cdots$ & 1 & $\cdots$ & . & \\
\hline & & & & & & & & & & \\
\hline & $\cdots$ & . & $\cdots$ & $\ldots$ & 0.5 & $\cdots$ & , & . & $\ldots$ & 0.5 \\
\hline & . & . & . & & 0.5 & . & . & $\cdots$ & . & 0.5 \\
\hline & . & $\ldots$ & . & $\ldots$ & 0.5 & $\cdots$ & & . & $\ldots$ & 0.5 \\
\hline & & & & & & & & & & \\
\hline & . & . & - & $\cdots$ & 2 & $\cdots$ & . & $\cdots$ & . & \\
\hline & - & . & & $\ldots$ & 0.5 & $\ldots$ & $\cdots$ & 1 & . & \\
\hline N. glory of sarni & . & & & . & 0.5 & & 1 & . & & \\
\hline
\end{tabular}

gentian violet, and safranin; and intermediate reactivity with temperature, but nearer to $N$. sarniensis var. corusca major.

Table A 12 shows the reaction-intensities in percentages of total starch gelatinized at definite intervals (minutes).

\section{Velocity-Reaction CuRtes.}

This section treats of the velocity-reaction curves of the starches of Nerine sarniensis var. corusca major, $N$. curvifolia var. fothergilli major, and $N$. glory of sarnia, showing the quantitative differences in the behavior toward different reagents at definite time-intervals. (Charts D 232 to D 252.)

Among the conspicnous features of these charts are:

(1) The closeness and correspondence of the curves of all three starches, excepting in the reactions with potassium sulphocyanate in which there appears to be a marked disproportionately low reactivity of $N$. sarniensis var. corusca major, in comparison with $N$. curvifolia var. fothergilli major, the departure becoming more and more marked during the course of the experiment. It is of importance to note that the reactions of the former and the hybrid are practically absolutely identical. With a slightly stronger solution of the reagent or a longer period of study it is probable that this discrepancy would become markedly less. The extremely rapid or slow reactions of all three starches with pyrogallic acid, sulphuric acid, potassium hydroxide, potassium iodide, sodium sulphide, cobalt nitrate, copper nitrate, cupric chloride, barium chloride, and mercuric chloride yield curves that are wholly or practically valueless for satisfactory differential study.

(2) The curve of $N$. sarniensis var. corusca major is higher than the curve of the other parent $N$. curvifolia var. fothergilli major in the reactions with chromic acid, nitric acid, potassium sulphocyanate, potassium sulphide, sodium hydroxide, calcium nitrate, uranium nitrate, and strontium nitrate; and lower in reactions with chloral hydrate, hydrochloric acid, sodium salicylate, but in several the differences are slight.

(3) The curves of the hybrid bear varying relations to those of the parents. There are marked tendencies to sameness to the pollen parent and to both parents; little tendency to the seed parent; none to be the highest of the three curves; and a very marked tendency to bo the lowest with equal inclination to each parent.

(4) An early period of high resistance followed by a rapid to moderate gelatinization is noted only in the experiments with chromic acid and nitric acid, especially in the first, and in the latter only in $N$. curvifolia var. fothergilli major.

(5) The earliest period during the 60 minutes that is best for the differentiation of the three starches is for chloral hydrate, potassium sulphide, sodium salicylate, and strontium nitrate at the end of 5 minutes; for nitric acid and hydrochloric acid at 15 minutes; for chromic acid at 30 minutes; and for potassium sulphocyanate, sodium hydroxide, calicum nitrate, and' uranium nitrate at 60 minutes. With the very slow reactions, including those with pyrogallic acid, sulphuric acid, potassium iodide, sodium sulphide, cobalt nitrate, copper nitrate, cupric chloride, barium chloride, and mercuric chloride, if any differentiation is possible, the longer the period of the reaction the better.

\section{ReACTION-INTENSITIES OF THE HrbRID.}

This section treats of the reaction-intensities of the hybrid as regards sameness, intermediateness, excess, and deficit in relation to the parent. (Table A 12 and Charts D 232 to D 252.) 
The reactivities of the hybrid are the same as those of the seed parent in the polarization reaction; the same as the pollen parent in the reactions with safranin, potassium sulphocyanate, sodium hydroxide, sodium sulphide, calcium nitrate, and uranium nitrate; the same as both parents with pyrogallic acid, potassium hydroxide, potassium iodide, cobalt nitrate, copper nitrate, cupric chloride, barium chloride, and mercuric chloride, in all of which the reactions are too slow for differentiation; intermediate in the temperature reaction, being closer to the seed parent; highest in none; and lowest with iodine, gentian violet, chloral hydrate, chromic acid, nitric acid, sulphuric acid, hydrochloric acid, potassium sulphide, sodium salicylate, and strontium nitrate (in five being closer to the seed parent, in four closer to the pollen parent, and in one as close to one as to the other parent).

The following is a summary of the reaction-intensities of the hybrid as regards sameness, intermediateness, excess, and deficit in relation to the parents: Same or practically the same as the seed parent, 1 ; the pollen parent, 6 ; both parents, 8 ; intermediate, 1 ; highest, 0 ; lowest, 10.

The tendency to lower curves than in either of the parents, the more marked influence of the pollen parent, the almost entire absence of intermediateness, and the entire absence of curves higher than those of the parents are quite conspicuous.

\section{Composite CuRves of the Reaction-intensities.}

This section treats of the composite curves of the reactions-intensities, showing the differentiation of the starches of Nerine sarniensis var. corusca major, N. curvifolia var. fothergilli major, and $N$. glory of sarnia. (Chart E 12.)

Among the most conspicuous features of this chart are :

(1) The very close correspondence in the rises and falls of all three curves, indicating a very close botanical relationship between the parents and but little botanical character variations in the hybrid from parental characters.

(2) In the curve of $N$. sarniensis var. corusca major in comparison with $N$. curvifolia var. fothergilli major the higher reactions with polarization, potassium sulphocyanate, sodium hydroxide, sodium salicylate, uranium nitrate, and strontium nitrate, and the same or practically the same with chloral hydrate, chromic acid, pyrogallic acid, nitric acid, sulphuric acid, potassium hydroxide, potassium iodide, potassium sulphide, sodium sulphide, sodium salicylate, cobalt nitrate, copper nitrate, cupric chloride, barium chloride, and mercuric chloride. In only the reactions with temperature, hydrochloric acid, potassium sulphocyanate, and strontium nitrate are there important differentiations.

(3) In $N$. sarniensis var. corusca major the very high reactions with polarization, sulphuric acid, potassium hydroxide, and sodium salicylate; the high reactions with iodine, chloral hydrate, hydrochloric acid, and strontium nitrate; the moderate reactions with gentian violet, safranin, chromic acid, and nitric acid; the low reactions with temperature, potassium sulphocyanate, and potassium sulphide; and the very low reactions with pyrogallic acid, potassium iodide, sodium hydroxide, sodium sul- phide, calcium nitrate, uranium nitrate, cobalt nitrate, copper nitrate, cupric chloride, barium chloride, and mercuric chloride.

(4) In $N$. curvifolia var. fothergilli major the very high reactions with polarization, nitric acid, hydrochloric acid, potassium hydroxide, and sodium salicylate; the high reactions with iodine and chloral hydrate; the moderate reactions with gentian violet, safranin, chromic acid, nitric acid, and strontium nitrate; the low reactions with temperature and potassium sulphide; and the very low reactions with pyrogallic acid, potassium iodide, potassium sulphocyanate, sodium hydroxide, sodium sulphide, calcium nitrate, uranium nitrate, cobalt nitrate, copper nitrate, cupric chloride, barium chloride, and mercuric chloride.

(5) In the hybrid $N$. glory of sarnia the very high reactions with polarization, sulphuric acid, potassium hydroxide, and sodium salicylate; the high reactions with hydrochloric acid; the moderate reactions with iodine, chloral hydrate, chromic acid, and strontium nitrate; the low reactions with gentian violet, safranin, temperature, nitric acid, and potassium sulphide; the very low reactions with pyrogallic acid, potassium iodide, potassium sulphocyanate, sodium hydroxide, sodium sulphide, calcium nitrate, uranium nitrate, cobalt nitrate, copper nitrate, cupric chloride, barium chloride, and mercuric chloride.

The following is a summary of reaction-intensities:

\begin{tabular}{l|c|c|c|c|c}
\hline & $\begin{array}{c}\text { Very } \\
\text { high. }\end{array}$ & High. & $\begin{array}{c}\text { Mod- } \\
\text { erate. }\end{array}$ & Low. & $\begin{array}{l}\text { Very } \\
\text { low. }\end{array}$ \\
\hline N. sarn. var. cor. maj......... & 3 & 3 & 6 & 3 & 11 \\
N. curv. var. foth. maj........ & 5 & 2 & 5 & 2 & 12 \\
N. glory of garnia............ & 4 & 1 & 4 & 4 & 12 \\
\hline
\end{tabular}

Notes on the Quantitative Reactions of THE NERINeS WITH THE Various Chemical Reagents.

(Charts D 253 to D 258.)

The most conspicuous features are:

(1) The three composite-curve charts are strikingly alike, showing very clearly the generic type of curve; and the curves run together quite closely, indicating nearly related members of the genus. The most marked differences exhibited by the five parents are seen in the reactions with chloral hydrate, nitric acid, hydrochloric acid, potassium sulphocyanate, potassium sulphide, and strontium nitrate. In the other reactions such differences as may exist are either of minor importance or possibly or probably fall within the limits of error of experiment, at least not within the limits of convincing differentiation.

(2) Comparisons of the curves of the five starches presented by each reagent show in the case of each reagent a correspondence in the type of curve, allowances being made for slight modifications due to variations in the rate of gelatinization and for small errors of estimation of percentages. Thus, comparing, for instance, the charts of the five reagents above noted, or better the special charts (D 253 to D 258) which give the curves of all five starches with each of the reagents, it will be observed that each chart has certain individualities by which it can be distinguished from the others. The charts for 
nitric acid and strontium nitrate are very much alike, the most distinct difference being noted in the curves during the first five minutes, yet, while there is a very close correspondence in the courses of the curves, there are curious alterations in the relative positions, as for instance, while the curve of $N$. curvifolia var. fothergilli major is the lowest and the curve of $N$. bowdeni intermediate in the nitric-acid reactions, the curve of the former is next to the lowest and that of the latter the lowest in the strontium-nirate reactions, showing that there are inherent important differences in the relations of these reagents to the starch molecules. Similar differences are very strikingly presented by certain starches of other genera which show more or less marked differences in the actions of these two reagents.

(3) Notable variations are shown in the degree of separation of the curves of the five starches in each of the charts. In the chart for hydrochloric acid all of the curves run closely together, those of $N$. crispa and $N$. elegans being identical, and those of the other three being almost identical. In the reactions with chloral hydrate the curves of $N$. curvifolia var. fothergilli major, $N$. elegans, and $N$. sarniensis var. corusca major are very nearly the same, but those of $N$. crispa and $N$. bowdeni are well separated from the former and from each other. In the reactions with nitric acid, potassium sulphocyanate, and potassium sulphide all the curves are fairly to well separated.

(4) In each chart the several curves bear the same position-relationship, there being no crossing of curves, so that if a given curve is the highest at the 5-minute interval it will not fall below another, although there may be dispersion or approximation of the curves during the progress of gelatinization-in the latter case they may become identical.

(5) The order of position of the five curves varies in the different reactions, as follows, in each case beginning with the highest and proceeding in order to the lowest:

Chloral hydrate: N. curv. var. foth. maj., N. elegans, N. sarn. var. cor. maj., N. crispa, N. bowdeni.

Nitric acid: N. elegans, N. crispa, N. bowdeni, N. garn. var. cor. maj., N. curv. var. foth. maj.

Hydrochloric acid: N. crispa, N. elegans, N. curv. var. foth. maj., N. bowdeni, N. sarn. var. cor. maj.

Potassium sulphocyanate: N. bowdeni, N. crispa, N. elegans, N. sarn. var. cor. maj., N. curv. var. foth. maj.

Potassium sulphide: N. crispa, N. sarn. var. cor. maj., N. curv. var. foth. maj., N. bowdeni, N. elegans.

Strontium nitrate: N. elegans, N. crispa, N. sarn. var. cor. maj., N. curv. var, foth. maj., N. bowdeni.

The variations in relative positions are quite remarkable and are expressions of definite physico-chemical peculiarities of the starch molecules in relation to the reagents. It will be observed that $N$. curvifolia var. fothergilli major is the highest in the reactions with chloral hydrate, but the lowest with nitric acid and potassium sulphocyanate; $N$. elegans is highest with nitric acid and strontium nitrate, but the lowest with potassium sulphide; $N$. bowdeni is the highest with potassium sulphocyanate, but the lowest with chloral hydrate and strontium nitrate, etc. It is of interest to note that while the charts for nitric acid and strontium nitrate bear a very close resemblance, as previously stated, the order of curves is not the same in both.
(6) In comparing the chart for hydrochloric acid with the abscissæ for hydrochloric acid of the compositecurve charts ( $\mathrm{E} 10, \mathrm{E} 11$, and $\mathrm{E} 12$ ) it will be seen that in the latter the differences between the parents is seemingly much exaggerated. This latter is owing to the very slow gelatinization after 15 minutes, rendering the curves of $N$. bowdeni and $N$. sarniensis var. corusca major disproportionately low. Both curves should perhaps be brought up as high as the 20-minute abscissa. The error is, however, of no essential importance, inasmuch as it does not give rise to error in the order of reactivity or essentially modify the generic type of curve.

(7) The hybrids in all three sets exhibit the same fundamental peculiarities in relation to their respective parents, in so far as each hybrid may in some reactions be intermediate, higher, lower, or the same as one or the other parent or both parents, as the case may be. It can not be foretold from the reactions of the parents with any given reagent what the reaction of the hybrid is likely to be. The hybrids tend to follow one parent closer than the other, in some reactions one parent and in others the other, there not being in any one of the three sets a universal sexual prepotency. In the first set the hybrids bear, on the whole, a closer relationship to the seed parent, but in the second and third sets to the pollen parents. In the first and second sets, in each of which there are two hybrids, the hybrids exhibit differences between each other in some reactions as marked as, or more marked than, the differences between the parents, but commonly the hybrids tend to be closely alike, especially when the parents are close, but there is no rule. As regards the latter, for instance, in the chloral-hydrate reactions of the first set (Chart D 190), the parents are well separated and likewise the two hybrids; in the second set (Chart D 211), the parents are well separated, but both hybrids are the same and also the same as one parent; and in the third set (Chart D 232) the parents are the same, but the hybrid is well separated from the parents, and so on with other reactions.

(8) No more striking feature seems to be presented than that of the shifting parental relationships of the two hybrids of each of the first two sets in the several reactions, as referred to in Section 6 and fully tabulated in Chapter V.

\section{Comparisons of the Starches of Narcissus poeticts ornatus, N. Poeticus poetarum, $N$. POETICUS HERRICK, AND N. POETICUS DANTE.}

In histologic characteristics, polariscopic figures, reactions with selenite, qualitative reactions with iodine, and qualitative reactions with various chemical reagents all four starches show properties in common in varying degrees of development together with certain individualities which collectively in each case serve to be characteristic. The starch of Narcissus poeticus poetarum in comparison with that of $N$. poeticus ornatus has a larger number of compound grains, more aggregates that are formed of a single primary grain inclosed in a secondary deposit, more irregularity of the grains, less distinctness of the hilum, more extensive fissuration but less branching, and lamellation not so distinct or so coarse; the polorization figure is less often well defined and the lines are more apt to be bisected and bent and less often form 
a cross; with selenite the quadrants are not so well defined and are more irregular in shape and size, the colors are not so pure, and there are fewer grains having a greenish tinge; with iodine the raw grains become more bluish and of a somewhat deeper tint, while the gelatinized grains and grain residues color less but the solution more. In the qualitative reactions with the various chemical reagents there are various differences. The starch of the hybrid $N$. poeticus herrick is in form, characters of the hilum, and characters of the lamellæ closer to $N$. poeticus ornatus than to the other parent, but in size the reverse. In polariscopic figure and appearances with selenite it is closer to $N$. poeticus ornatus; but in degree of polarization, the reverse. In the qualitative iodine reactions it is closer to $N$. poeticus poetarum. In the qualitative reactions with chloral hydrate, chromic acid, pyrogallic acid, nitric acid, and sulphuric acid it is closer to $N$. poeticus poetarum. The starch of the hybrid $N$. poeticus dante is in form closer to $N$. poeticus than to the other parent, but in the characters of the hilum, in lamellæ, and in size it is closer to the other parent $N$. poeticus poetarum. In the polariscopic figure and reactions with selenite it is closer to $N$. poeticus poetarum. In the qualitative iodine reactions it is closer to $N$. poeticus poetarum. In the qualitative reactions with chloral hydrate, chromic acid, pyrogallic acid, nitric acid, and sulphuric acid it shows a closer relationship to $N$. poeticus poetarum. The starch of the hybrid $N$. poeticus dante is more rounded than that of the other hybrid, and it does not show as close a relationship to $N$. poeticus ornatus. In character and eccentricity of the hilum it shows as close a relationship to $N$. poeticus poetarum as does that of the other hybrid to the other parent, and in the characters of the lamellæ the same holds true. In size it is larger than in the other hybrid, and therefore not so close to $N$. poeticus poetarum, yet it is closer to it than to the other parent. In polariscopic figure and appearances with selenite both hybrids bear the same relationship to the parents, and in the iodinequalitative reactions there are no differences between the hybrids. In the qualitative chemical reactions the starch of the hybrid $N$. poeticus dante bears a closer relationship than the starch of the other hybrid $N$. poeticus herrick to $N$. poeticus poetarum in the chloral-hydrate reaction, but not so close a relationship to this parent in the reactions with chromic acid, pyrogallic acid, nitric acid, and sulphuric acid.

Reaction-intensities Expressed by Light, Color, and Tempera-

Polarization:

N. poet. ornatus, low to very high, value 50 .

$\mathrm{N}$. poet. poetarum, low to very high, lower than in N. poet. ornatus, value 40 .

N. poet. herrick, low to very high, somewhat lower than in N. poet. ornatus, value 47 .

$\mathrm{N}$. poet. dante, low to very high, somewhat lower than in N. poet. Iodine: ornatus, value 47

N. poet. ornatus, light to moderate, value 40 .

N. poet. poetarum, moderate, somewhat higher than in $N$. poet. ornatus, value 45 .

N. poet. herrick, moderate, the same as in $\mathrm{N}$. poet. poetarum, value 45.

N. poet. dante, moderate, the same as in N. poet. poetarum, value 45 .
Gentian violet:

N. poet. ornatus, light to moderate, value 30 .

N. poet. poetarum, light to moderate, somewhat deeper than in N. poet. ornatus, value 35 .

N. poet. herrick, light to moderate, lighter than in either parent, value 25.

N. poet. dante, light to moderate, the same as in N. poet. poetarum, value 35 .

Safranin:

N. poet. ornatus, moderate, value 45 .

N. poet. poetarum, moderate, somewhat deeper than in N. poet. ornatus, value $\mathbf{5 0}$.

N. poet. herrick, light to moderate, lighter than in either parent, value 40.

N. poet. dante, moderate, the same as in $N$. poet. poetarum, value 50 .

Temperature:

N. poet. ornatus, in majority at 73 to $74^{\circ}$, in all at 77 to $78^{\circ}$, mean $77.5^{\circ}$.

N. poet. poetarum, in majority at 67 to $69^{\circ}$, in all at 71 to $73^{\circ}$, mean $72^{\circ}$.

N. poet. herrick, in majority at 69 to $71^{\circ}$, in all at 76 to $78^{\circ}$, mean $77^{\circ}$.

N. poet. dante, in majority at 71.2 to $73.1^{\circ}$, in all at 74 to $76^{\circ}$, mean $75^{\circ}$.

$N$. poeticus ornatus exhibits a higher reactivity than the other parent in the polarization reactions, and lower reactivities in those with iodine, gentian violet, safranin, and temperature. The hybrid $N$. poeticus herrick is higher than $N$. poeticus and lower than $N$. poeticus poetarum in the temperature reactions; the same as the latter parent in the iodine reaction; intermediate in polarization reaction; and the lowest in the reactions with gentian violet and safranin. The hybrid $N$. poeticus dante has the same or practically the same reactivity as $N$. poeticus ornatus in no reaction; the same or practically the same reactivity as $N$. poeticus poetarum in the reactions with iodine, gentian violet, and safranin; and intermediate in the polarization and temperature reactions. The two hybrids are alike in the polarization and iodine reactions, but $N$. poeticus herrick has lower reactivities than the other hybrid in the reactions with gentian violet, safranin, and temperature.

TABLE A 13.

\begin{tabular}{|c|c|c|c|c|c|c|c|c|c|}
\hline & $\underset{\sim-1}{\text { 日 }}$ & $\underset{\sim}{\mathbb{a}}$ & $\underset{\infty}{\text { 旦 }}$ & 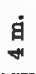 & है & $\begin{array}{l}\text { 日 } \\
19\end{array}$ & $\begin{array}{l}\text { 日 } \\
\text { 용 }\end{array}$ & $\underset{10}{10}$ & $\begin{array}{l}\text { घ́ } \\
8\end{array}$ \\
\hline \multicolumn{10}{|l|}{ Chloral hydrate: } \\
\hline N. poet. ornatus . . . . . . . & $\cdots$ & $\cdots$ & $\cdots$ & $\cdots$ & 0.5 & 6 & 24 & 28 & 34 \\
\hline N. poet. poetarum........ & . & . & - & . & 0.5 & 6 & 9 & 11 & 17 \\
\hline N. poet. herrick........... & . & $\cdots$ & . & $\cdots$ & 4 & 6 & 10 & 12 & 14 \\
\hline N. poet. dante. . . & . & . & . & . & 7 & 10 & 12 & 16 & 16 \\
\hline \multicolumn{10}{|l|}{ Chromic acid: } \\
\hline N. poet. ornatus. . . . . & $\cdots$ & . & . & . & 7 & 65 & 80 & 95 & 98 \\
\hline N. poet. poetarum. & - & $\cdots$ & . & $\cdots$ & 3 & 22 & 65 & 75 & 85 \\
\hline N. poet. herrick... & $\cdots$ & . & . & . & 5 & 42 & 70 & 82 & 90 \\
\hline N. poet. dante. . . . . . & . & . & . & . & 5 & 34 & 67 & 80 & 88 \\
\hline \multicolumn{10}{|l|}{ Pyrogallic acid: } \\
\hline N. poet. ornatus. . . & $\cdots$ & . & - & $\cdots$ & 2 & 20 & 68 & 81 & 88 \\
\hline N. poet. poetarum..... & - & . & $\cdots$ & . & 1 & 16 & 70 & 84 & 93 \\
\hline N. poet. herrick. . . . . . . & . & . & . & . & 2 & 19 & 69 & 83 & 91 \\
\hline N. poet. dante. .......... & . & . & . & . & 1 & 37 & 75 & 88 & 94 \\
\hline \multicolumn{10}{|l|}{ Nitric acid: } \\
\hline N. poet. ornatus... & . & $\cdots$ & . & $\cdots$ & 6 & 20 & 39 & 65 & 70 \\
\hline N. poet. poetarum. & . & . & . & . & 10 & 40 & 53 & 60 & 63 \\
\hline N. poet. herrick... & . & $\ldots$ & . & . & 30 & 56 & 69 & 76 & 78 \\
\hline N. poet. dante. . & . & . & . & . & 19 & 65 & 72 & 78 & 80 \\
\hline \multicolumn{10}{|l|}{ Sulphuric acid: } \\
\hline N. poet. ornatus ... & $\cdots$ & 93 & $\cdots$ & $\cdots$ & 99 & . & $\cdots$ & $\cdots$ & - \\
\hline N. poet. poetarum. . & $\cdots$ & 79 & $\cdots$ & $\cdots$ & 99 & . & . & . & - \\
\hline N. poet. herrick... & $\cdots$ & 98 & . & . & 99 & . & . & . & . \\
\hline N. poet. dante. ....... & $\cdots$ & 95 & . & . & 99 & $\cdots$ & . & . & . \\
\hline
\end{tabular}


Table A 13 shows the reaction-intensities in percentages of total starch gelatinized at definite intervals (minutes).

\section{Velocity-reaction CuRves.}

This section treats of the volocity-reaction curves of the starches of Narcissus poeticus ornatus, $N$. poeticus poetarum, $N$. poeticus herrick, and $N$. poeticus dante, showing the quantitative differences in the behavior toward different reagents at different time-intervals. (Charts D 259 to D 264.)

Conspicuous among the features of these charts are the following:

(1) In the five charts there is generally a manifest tendency in each chart for all four curves to keep together, the only places where there is leaning toward a well-marked separation are in the charts for chromic acid and nitric acid at the 15-minute interval. In the sulphuric-acid reaction gelatinization proceeds with such rapidity that there is not, except in one instance, what can be accepted as an entirely satisfactory differentiation of any one starch from any other, this instance being the starch of $N$. poeticus poetarum, which reacted with distinctly less rapidity than the other three (which react with identical intensity) during the first three minutes.

(2) The four curves bear varying relations to each other in the different reactions.

(3) The curve of $N$. poeticus ornatus is the highest of the four and well separated from the other three in the reactions with chloral hydrate and chromic acid; the lowest at first and intermediate finally with nitric acid; and practically the same, but with a lower tendency than in the other three, with pyrogallic acid, although in this reaction the curves of $N$. poeticus ornatus, $N$. poeticus -poetarum, and $N$. poeticus herrick are practically the same. There is an obvious tendency for the curves of $N$. poeticus poetarum, $N$. poeticus herrick, and $N$. poeticus dante to keep close in the reactions with chloral hydrate and chromic acid.

(4) The curves of the two hybrids tend to run closely. In the reactions with chloral hydrate and sulphuric acid they are the same; with chromic acid very nearly the same; and with pyrogallic acid and nitric acid they are separated sufficiently for differential purposes. The curve of the hybrid $N$. poeticus herrick is higher than the curve of the other hybrid in the chromic-acid reaction, lower in the pyrogallic-acid reaction, and for the most part lower in the nitric-acid reaction.

(5) An early period of resistance is noted particularly in the reactions with chromic acid and pyrogallic acid, and is suggested in the curves of the nitric acid.

(6) The earliest period at which the curves are best separated and hence the best for differential purposes is at 3 minutes in the reaction with sulphuric acid; at 5 minutes in those with chromic acid, pyrogallic acid, and nitric acid; and at 60 minutes in that with chloral hydrate.

\section{REACTION-INTENSITIES OF THE HYBRIDS.}

This section treats of the reaction-intensities of the hybrids as regards sameness, intermediateness, excess and deficit in relation to the parents. (Table A 13, Charts D 259 to D 264.)
The reactivities of the hybrid $N$. poeticus herrick are the same as those of the seed parent in none of the reactions; the same as those of the pollen parent with iodine, chloral hydrate, and pyrogallic acid; the same as both parents in none; intermediate with polarization, temperature, and chromic acid (in two nearer the seed parent and in one nearer the pollen parent); highest with nitric acid and sulphuric acid (in one as near to one as to the other parent and in one nearer the pollen parent); and lowest with gentian violet and safranin, being in both nearer the seed parent.

The reactivities of the hybrid $N$. poeticus dante are the same as those of the seed parent in the sulphuricacid reaction; the same as those of the pollen parent in the reactions with iodine, gentian violet, safranin, and chloral hydrate; the same as those of both parents in no reaction; intermediate in the reactions with polarization, temperature, chromic acid, and nitric acid (in two being closer to the seed parent, in one nearer the pollen parent, and in one mid-intermediate); highest with pyrogallic acid, being as near one as the other parent; and lowest in none.

Following is a summary of the reaction-intensities:

\begin{tabular}{|c|c|c|}
\hline & $\begin{array}{l}\text { N. poeticus } \\
\text { herrick. }\end{array}$ & $\begin{array}{l}\text { N. poeticus } \\
\text { dante. }\end{array}$ \\
\hline 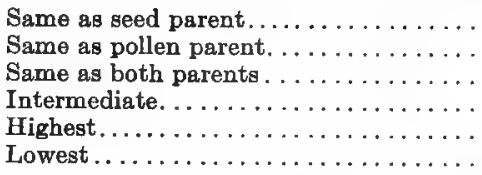 & $\begin{array}{l}0 \\
3 \\
0 \\
3 \\
2 \\
2\end{array}$ & $\begin{array}{l}1 \\
4 \\
0 \\
4 \\
1 \\
0\end{array}$ \\
\hline
\end{tabular}

The varying relationships of the two hybrids to the parents in the individual reactions is quite marked. Thus, in the polarization reactions both are intermediate and nearer the seed parent; in the iodine reactions both are the same as the pollen parent; in the gentian violet reaction one is lower than either parent and nearer the seed parent, but the other is the same as the pollen parent, etc.

\section{Composite Curves of Reaction-intensities.}

This section deals with the composite curves of the reaction-intensities showing the differentiation of the starches of Narcissus poeticus ornatus, $N$. poeticus poetarum, $N$. poeticus herrick, and $N$. poeticus dante. (Chart E 13.)

The most conspicuous features of this chart are:

(1) The marked closeness of all four curves and the very close correspondence in the rises and falls, showing agreement with a given species-type.

(2) In N. poeticus ornatus as compared with N.poeticus poetarum the higher reactions with polarization, chloral hydrate, chromio acid, nitric acid, and sulphuric acid; the same or practically the same reactions with pyrogallic acid; and the lower reactions with iodine, safranin, gentian violet, and temperature.

(3) In $N$. poeticus ornatus the very high reaction with sulphuric acid; the high reaction with chromic acid; the moderate reactions with polarization, iodine, and safranin; the low reactions with gentian violet, temperature, pyrogallic acid, and nitric acid; and the very low reaction with chloral hydrate. 
(4) In $N$. poeticus poetarum the very high reaction with sulphuric acid; the absence of any high reaction; the moderate reactions with polarization, iodine, safranin, temperature, and pyrogallic acid; the low reactions with gentian violet, chromic acid, and nitric acid; and the very low reaction with chloral hydrate.

(5) In the hybrid $N$. poeticus herrick the very high reactions with sulphuric acid; the absence of any high reaction; the moderate reactions with polarization, iodine, safranin, chromic acid, pyrogallic acid; the low reactions with gentian violet, temperature, and nitric acid; and the very low reaction with chloral hydrate.

(6) In the hybrid $N$. poeticus dante the very high sulphuric-acid reaction; the absence of any high reaction; the moderate reactions with polarization, iodine, safranin, chromic acid, and pyrogallic acid; the low reactions with gentian violet, temperature, and nitric acid; and the very low reaction with chloral hydrate.

The following is a summary of the reaction-intensities (10 reactions) :

\begin{tabular}{|c|c|c|c|c|c|}
\hline & $\begin{array}{l}\text { Very } \\
\text { high. }\end{array}$ & High. & $\begin{array}{l}\text { Mod- } \\
\text { erate. }\end{array}$ & Low. & $\begin{array}{l}\text { Very } \\
\text { low. }\end{array}$ \\
\hline $\begin{array}{l}\text { N. poet. ornatus } \ldots \ldots \ldots \ldots \ldots \\
\text { N. poet. poetarum } \ldots \ldots \ldots \ldots \ldots \\
\text { N. poet. herrick. } \ldots \ldots \ldots \ldots \ldots \\
\text { N. poet. dante. } \ldots \ldots \ldots \ldots \ldots\end{array}$ & $\begin{array}{l}1 \\
1 \\
1 \\
1\end{array}$ & $\begin{array}{l}1 \\
0 \\
0 \\
0\end{array}$ & $\begin{array}{l}3 \\
5 \\
5 \\
5\end{array}$ & $\begin{array}{l}4 \\
3 \\
3 \\
3\end{array}$ & $\begin{array}{l}1 \\
1 \\
1 \\
1\end{array}$ \\
\hline
\end{tabular}

14. Comparisons of the Starches of Narcissus TAZETTA GRAND MONARQUE, $N$. POETICUS ORNATUS, AND N. POETAZ TRIUMPH.

In histologic characteristics, polariscopic figures, reactions with selenite, reactions with iodine, and qualitative reactions with the various chemical reagents it will be noted that the starches of the parents and hybrid exhibit not only properties in common in varying degrees of development but also occasional individualities which collectively are in each case distinctive. In histologic properties the starches of the parents differ in welldefined respects. In the polariscopic figures and reactions with selenite there are no important differences. In the qualitative reactions with iodine, the raw grains of Narcissus tazetta grand monarque are colored less in comparison with those of the other parent, while after heating in water fewer grains are moderately colored and the solution is more deeply colored. In the qualitative reactions with chloral hydrate, chromic acid, pyrogallic acid, nitric acid, and sulphuric acid, there are in each case similarities and certain definite differences. The starch of the hybrid in comparison with the starches of the parents shows more irregularities in form than in either parent, and it is, on the whole, more closely related to $N$. tazetta grand monarque than to the other parent. In the character of the lamellæ, and in the size and proportions of different kinds of grains, the relationship is closer to $N$. tazetta grand monarque; in character of the hilum it is closer to the other parent, and in the eccentricity of the hilum it is the same as the parents. In the polariscopic figures, appearances with selenite, and iodine reactions it is closer to $N$. poeticus ornatus. In the qualitative reactions with the chemical reagent it is in all closer, on the whole, to $N$. tazetta grand monarque.

Reaction-intensities Expressed by Light, Color, and TemperaPolarization: ture Reactions.

N. taz. grand mon., low to very high, value 50 .

N. poet. ornatus, low to very high, same as N. taretta grand monarque, value 50 .

N. poetaz triumph, low to very high, same as both parente, value 50.
Iodine:

N. taz. grand mon., light to moderate, value 45.

N. poet. ornatus, light to moderate, less than $\mathbf{N}$. tagetta grand monarque, value 40.

N. poetaz triumph, light to moderate, the same as $N$. poeticus ornatus, value 40.

Gentian violet:

N. taz. grand mon., light to moderate, value 40.

$N$. poet. ornatus, light to moderate, less than $N$. tazetta grand monarque, value 35 .

N. poetaz triumph, light to moderate, the same as $N$. tazetta grand monarque, value 40 .

Safranin:

N. taz. grand mon., moderate, value 45.

N. poet. ornatus, moderate, the same as N. tazetta grand monarque, value 45 .

N. poetaz triumph, light to moderate, less than in either parent, value 40 .

Temperature:

N. taz. grand mon., in majority at 73 to $75^{\circ}$, in all at 76 to $77^{\circ}$, mean $76.5^{\circ}$.

N. poet. ornatus, in majority at 73 to $74^{\circ}$, in all at 77 to $78^{\circ}$, mean $77.5^{\circ}$.

N. poetaz triumph, in majority at 73 to $75^{\circ}$, in all at 76 to $77^{\circ}$, mean $76.5^{\circ}$.

The reactivity of $N$. tazetta grand monarque is the same or practically the same as that of the other parent in the polarization and safranin reactions; higher in the temperature reaction, and lower in the iodine and gentian-violet reactions. The reactivity of the hybrid is the same or practically the same as those of both parents in the polarization reaction; the same or practically the same as the reactivity of $N$. tazetta grand monarque in the gentian-violet and temperature reactions; the same or practically the same as that of the other parent in the iodine reaction; and the lowest of the three in the safranin reaction. In none of the five reactions is there intermediateness. In some respects the hybrid is closer to one parent and in other respects to the other.

Table A 14 shows the reaction-intensities in percentages of total starch gelatinized at definite intervals minutes ).

\section{Velootty-REadion CuRves.}

This section treats of the velocity-reaction curves of the starches of Narcissus tazetta grand monarque, $N$. poeticus ornatus, and $N$. poetaz triumph, showing quantitative differences in the behavior toward different reagents at definite time-intervals. (Charts D 265 to D 286.)

The most conspicuous features of this group of curves are:

(1) The closeness generally of all three curves in all of the reactions, with a tendency throughout, with the exception of that with sulphuric acid, to a moderate to low or very low reaction value. The curves of two or all three starches, excepting the reactions with the sulphuric acid, cobalt nitrate, barium chloride, and mercuric chloride, are satisfactorily separated, commonly well separated, for differentiation in reactivities. In the reactions with pyrogallic acid, hydrochloric acid, potassium hydroxide, potassium iodide, potassium sulphocyanate, potassium sulphide, sodium hydroxide, sodium sulphide, sodium salicylate, calcium nitrate, uranium nitrate, copper nitrate, and cupric chloride two of the curves tend to closeness and separation from the third, which two may be the curve of the hybrid and that of one or the other parent, or the curves of the parents. In some of the reactions the three curves do not closely correspond in course, as in the reactions with chloral hydrate, chromic acid, pyrogallic acid, nitric acid, potassium iodide, uranium nitrate, cobalt nitrate, and strontium nitrate; the departure of one from the course of the others may be in the curve of the hybrid or either parent, more often in the curve of $N$. tazetta grand monarque.

(2) The lower reactivity of $N$. tazetta grand monarque than of the other parent in the reactions with 
Table A 14.

\begin{tabular}{|c|c|c|c|c|c|c|c|c|c|}
\hline & 旦 & 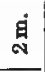 & 离 & 早 & 星 & $\underset{7}{8}$ & 昌 & 旦 & 圆 \\
\hline \multicolumn{10}{|l|}{ Chloral hydrate: } \\
\hline N. tazetta g. mon & $\cdots$ & . & & $\cdots$ & 5 & 24 & 32 & 36 & 40 \\
\hline N. poeticus ornat. & $\cdots$ & . & $\cdots$ & . & 0.5 & 6 & 24 & 28 & 34 \\
\hline N. poetaz triumph & . & . & $\cdots$ & $\cdots$ & 4 & 28 & 50 & 53 & 56 \\
\hline \multicolumn{10}{|l|}{ Chromic acid: } \\
\hline N. tazetta g. mon & $\cdots$ & $\cdots$ & $\cdots$ & $\cdots$ & 5 & 25 & 75 & 90 & 98 \\
\hline N. poeticus ornat. & . & $\cdots$ & $\cdots$ & $\cdots$ & 7 & 65 & 80 & 85 & 98 \\
\hline N. poetaz triumph. & $\cdots$ & . & $\cdots$ & $\cdots$ & 13 & 75 & 90 & 97 & 99 \\
\hline Pyrogallic acid: & & & & & & & & & \\
\hline N. tazetta g. mon & $\cdots$ & $\cdots$ & $\cdots$ & $\cdots$ & 1 & 20 & 23 & 47 & 78 \\
\hline N. poeticus ornat. & $\cdots$ & $\cdots$ & $\cdots$ & $\cdots$ & 2 & 20 & 68 & 81 & 88 \\
\hline N. poetaz triumph & $\cdots$ & $\cdots$ & $\cdots$ & $\cdots$ & 3 & 25 & 75 & 86 & 95 \\
\hline Nitric acid: & & & & & & & & & \\
\hline N. tazetta g. mon & $\cdots$ & $\cdots$ & $\cdots$ & $\cdots$ & 2 & 14 & 26 & 31 & 42 \\
\hline N. poeticus ornat & $\cdots$ & $\cdots$ & $\cdots$ & $\cdots$ & 6 & 20 & 39 & 65 & 70 \\
\hline N. poetaz triump & $\cdots$ & $\cdots$ & $\cdots$ & $\cdots$ & 10 & 60 & 74 & 86 & 88 \\
\hline Sulphuric acid: & & & & & & & & & \\
\hline N. tazetta g. mon & $\cdots$ & 86 & $\cdots$ & $\cdots$ & 99 & & $\cdots$ & $\cdots$ & *. \\
\hline N. poeticus ornat. & $\cdots$ & 93 & . & $\cdots$ & 99 & . & $\cdots$ & . & . \\
\hline N. poetaz triumph & . & 98 & $\cdots$ & $\cdots$ & 99 & . & $\cdots$ & $\cdots$ & .. \\
\hline Hydrochloric acid: & & & & & & & & & \\
\hline N. tazetta g. mon & $\cdots$ & $\cdots$ & $\cdots$ & $\cdots$ & 73 & 90 & 95 & 97 & 98 \\
\hline N. poeticus ornat & $\cdots$ & $\cdots$ & $\cdots$ & $\cdots$ & 88 & 95 & 97 & 98 & 99 \\
\hline $\begin{array}{l}\text { N. poetaz triumph. } \\
\text { Potassium hydroxide: }\end{array}$ & $\cdots$ & $\cdots$ & $\cdots$ & $\cdots$ & 90 & 98 & 99 & $\cdots$ & $\cdots$ \\
\hline N. tazetta g. mon. & $\cdots$ & .. & $\cdots$ & $\cdots$ & 16 & 32 & 38 & 42 & 46 \\
\hline N. poeticus ornat & . & . & . & $\cdots$ & 19 & 36 & 43 & 48 & 53 \\
\hline N. poetaz triumph & $\cdots$ & . & . & $\cdots$ & 35 & 64 & 75 & 86 & 91 \\
\hline Potarsium iodide: & & & & & & & & & \\
\hline N. tazetta g. mon & $\cdots$ & $\cdots$ & $\cdots$ & $\cdots$ & 3 & 17 & 55 & 69 & 75 \\
\hline N. poeticus ornat. & $\cdots$ & $\cdots$ & $\cdots$ & $\cdots$ & 5 & 51 & 68 & 77 & 80 \\
\hline N. poetaz triumph & $\cdots$ & $\cdots$ & $\cdots$ & $\cdots$ & 10 & 57 & 75 & 85 & 90 \\
\hline Potassium sulphocyan & & & & & & & & & \\
\hline N. tazetta g. mon & $\cdots$ & $\cdots$ & $\cdots$ & $\cdots$ & 39 & 62 & 76 & 89 & 94 \\
\hline N. pooticus ornat. & $\cdots$ & $\cdots$ & $\cdots$ & * & 45 & 70 & 80 & 90 & 97 \\
\hline N. poetaz triumph. & $\cdots$ & $\cdots$ & $\cdots$ & $\cdots$ & 67 & 83 & 92 & 95 & 98 \\
\hline Potassium sulphide: & & & & & & & & & \\
\hline N. tazetta g. mon. & $\cdots$ & $\cdots$ & $\cdots$ & $\cdots$ & $\cdots$ & 0.5 & 1 & 2 & 2 \\
\hline N. poeticus ornat. & $\cdots$ & $\cdots$ & $\cdots$ & $\cdots$ & $\cdots$ & 1 & 2 & 4 & 4 \\
\hline N. poetaz triumph & $\cdots$ & $\cdots$ & $\cdots$ & $\cdots$ & 5 & $\theta$ & 11 & 13 & 14 \\
\hline Sodium hydroxide: & & & & & & & & & \\
\hline N. tazetta g. mon & $\cdots$ & $\cdots$ & $\cdots$ & $\cdots$ & 5 & 43 & 50 & 73 & 78 \\
\hline N. poeticus ornat. & $\cdots$ & $\cdots$ & $\cdots$ & $\cdots$ & 18 & 49 & 62 & 75 & 80 \\
\hline N. poetaz triumph. & $\cdots$ & $\cdots$ & $\cdots$ & . & 31 & 65 & 85 & 90 & 92 \\
\hline Sodium sulphide: & & & & & & & & & \\
\hline N. tazetta g. mon & $\cdots$ & $\cdots$ & . & $\cdots$ & 2 & 7 & 28 & 40 & 50 \\
\hline N. poeticus of & . & $\cdots$ & $\cdots$ & $\cdots$ & $\mathbf{3}$ & 12 & 33 & 53 & 56 \\
\hline N. poetaz triumph. & . & $\cdots$ & $\cdots$ & $\cdots$ & 18 & 60 & 75 & 80 & 85 \\
\hline Sodium salicylate: & & & & & & & & & \\
\hline N. tazetta g. mon & $\cdots$ & $\cdots$ & $\cdots$ & $\cdots$ & 39 & 82 & 99 & $\cdots$ & $\cdots$ \\
\hline N. poeticus ornat. . & $\cdots$ & $\cdots$ & $\cdots$ & $\cdots$ & 50 & 92 & 99 & $\cdots$ & $\cdots$ \\
\hline N. poetaz triumph & $\cdots$ & $\cdots$ & $\cdots$ & $\cdots$ & 55 & 99 & $\cdots$ & $\cdots$ & $\cdots$ \\
\hline Calcium nitrate: & & & & & & & & & \\
\hline N. tazetta g. mon & $\cdots$ & $\cdots$ & $\cdots$ & $\cdots$ & 3 & 5 & 14 & 39 & 42 \\
\hline N. poeticus orna & $\cdots$ & $\cdots$ & $\cdots$ & $\cdots$ & 3 & 9 & 19 & 43 & 53 \\
\hline N. poetaz triumph. & $\cdots$ & . & $\cdots$ & - & 9 & 47 & 56 & 65 & 72 \\
\hline Uranium nitrate: & & & & & & & & & \\
\hline N. tazetta g. mon & $\cdots$ & $\cdots$ & $\cdots$ & $\cdots$ & 0.5 & 3 & 4 & 5 & 5 \\
\hline N. poeticus ornat. . & $\cdots$ & $\cdots$ & $\cdots$ & $\cdots$ & 1 & 5 & 7 & 10 & 12 \\
\hline N. poetaz triumph. . . . . . . & . & $\cdots$ & $\cdots$ & $\cdots$ & 5 & 14 & 20 & 25 & 25 \\
\hline Strontium nitrate: & & & & & & & & & \\
\hline N. tazetta g. mon. & $\cdots$ & $\cdots$ & $\cdots$ & . & 1 & 8 & 33 & 53 & 60 \\
\hline N. poeticus ornat. & . & . & $\cdots$ & . & 10 & 42 & 55 & 63 & 66 \\
\hline N. poetaz triumph. & . & $\cdots$ & $\cdots$ & . & 25 & 67 & 75 & 81 & 83 \\
\hline Cobalt nitrate: & & & & & & & & & \\
\hline N. tazetta g. mon & $\cdots$ & $\cdots$ & $\cdots$ & $\cdots$ & 0.5 & 1 & 2 & 2 & 2 \\
\hline N. poeticus ornat. & . & $\cdots$ & $\cdots$ & . & 0.5 & 1 & 3 & 3 & 3 \\
\hline N. poetaz triumph........ & .. & $\cdots$ & $\cdots$ & . & $\mathbf{1}$ & 3 & 5 & 6 & 6 \\
\hline Copper nitrate: & & & & & & & & & \\
\hline N. tazetta g. mon & $\cdots$ & * & $\cdots$ & $\cdots$ & 2 & 4 & 6 & 7 & $\theta$ \\
\hline oeticus ornat & $\cdots$ & . & $\cdots$ & $\cdots$ & 2 & 8 & 9 & 10 & 15 \\
\hline N. poetaz triumph. . & . & . & . & . & 10 & 25 & 36 & -. & 38 \\
\hline Cupric chloride: & & & & & & & & & \\
\hline N. tazetta g. mon ..... & $\cdots$ & . & $\cdots$ & $\cdots$ & 0.5 & 1 & 2 & 4 & 5 \\
\hline N. poeticus ornat. . & . & . & . & . & 1 & 2 & 4 & $\mathbf{5}$ & 6 \\
\hline N. poetaz triumph..... & - & $\cdots$ & . & $\cdots$ & 5 & 10. & 12 & 16 & 19 \\
\hline Barium chloride: & & & & & & & & & \\
\hline N. tazetta g. $\operatorname{mon} \ldots \ldots \ldots$ & $\cdots$ & $\cdots$ & $\cdots$ & $\cdots$ & $\cdots$ & $\cdots$ & $\cdots$ & $\cdots$ & \\
\hline N. poeticus ornat. & $\cdots$ & $\cdots$ & $\cdots$ & * & $\cdots$ & $\cdots$ & - & $\cdots$ & $?$ \\
\hline N. poetaz triumph........ & $\cdots$ & $\cdots$ & $\cdots$ & $\cdots$ & . & . & 1 & $\cdots$ & 1 \\
\hline Mercuric chloride: & & & & & & & & & \\
\hline N. tazetta g. mon. & $\cdots$ & -. & - & 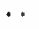 & - & $\cdots$ & 2 & 3 & 3 \\
\hline N. poeticus ornat. . & $\cdots$ & - & $\cdots$ & $\cdots$ & - & 3 & 4 & $\because:$ & 7 \\
\hline N. poetaz triumph......... & & $\cdots$ & $\cdots$ & $\cdots$ & 4 & 5 & 10 & 11 & 12 \\
\hline
\end{tabular}

chromic acid, pyrogallic acid, nitric acid, hydrochloric acid, potassium hydroxide, potassium iodide, potassium sulphocyanate, sodium hydroxide, sodium sulphide, sodium salicylate, calcium nitrate, uranium nitrate, strontium nitrate, and copper nitrate, and the same or practically the same reactivity with sulphuric acid, potassium sulphide, cobalt nitrate, cupric chloride, barium chloride, and mercuric chloride.

(3) The highest position of the hybrid curve of all three curves in all of the 21 reactions, excepting the barium chloride, in which latter owing to extremely slow reactions all three curves are absolutely or practically the same. In many reactions the hybrid curve is more separated from the parental curves than the latter are separated from each other, and in most instances the nearer parental curve is that of $N$. poeticus ornatus. There is in no instance a tendency either to intermediateness or to the lowest reactivity.

(4) An early period of comparative resistance followed by comparative rapid reaction is frequently noticed, sometimes in the case of one, two, or three of the starches. This is seen in all three starches in the reactions with chloral hydrate, chromic acid, pyrogallic acid, nitric acid, potassium iodide, and calcium nitrate; in the two parental starches with sodium sulphide and strontium nitrate; and in $N$. tazetta grand monarque with sodium hydroxide. In several, this resistant period is prolonged to 15 to 30 minutes.

(5) The earliest period during the 60 minutes at which the three curves are best separated for differentiation varies with the different reagents. Approximately, within the 5-minute interval in the reactions with sulphuric acid, sodium hydroxide, and sodium salicylate reactions; at the 15-minute interval with chromic acid, hydrochloric acid, potassium hydroxide, potassium sulphocyanate, sodium sulphide, calcium nitrate, and strontium nitrate; at the 30 -minute interval with chloral hydrate, pyrogallic acid, nitric acid, potassium iodide, and copper nitrate; and at the 60-minute interval with potassium sulphide, uranium nitrate, cobalt nitrate, copper nitrate, barium chloride, and mercuric chloride.

\section{ReAction-INTENSTIIES OF THE HYBRID.}

This section deals with the reaction-intensities of the hybrid as regards sameness, intermediateness, excess, and deficit in relation to the parents. (Table A 14 and Charts D 265 to D 286.)

The hybrid has the same reactivity as the seed parent in the reactions with gentian violet and safranin; the same as the pollen parent with polarization and iodine; the same as both parents with barium chloride, in which the reactions are too slow for differentiation; intermediate in none; highest with chloral hydrate, chromic acid, pyrogallic acid, nitric acid, sulphuric acid, hydrochloric acid, potassium hydroxide, potassium iodide, potassium sulphocyanate, potassium sulphide, sodium hydroxide, sodium sulphide, sodium salicylate, calcium nitrate, uranium nitrate, strontium nitrate, cobalt nitrate, copper nitrate, cupric chloride, and mercuric chloride (in 2 being closer to the seed parent, in 15 nearer the pollen parent, and in 3 as near one as the other parent); and lowest in the safranin reaction, as near one as the other parent.

The following is a summary of the reaction-intensities: Same as seed parent, 2; same as pollen parent, 2 ; same as both parents, 1 ; intermediate, 0 ; highest, 20 ; lowest, 1.

The most remarkable feature of these data is the almost universal higher reactivity of the hybrid in all of the chemical reactions, the only exception being with 
barium chloride in which the reactions are almost absolutely nil, yet even here there is at least the suggestion of highest reactivity. The inclination to the properties of the pollen parent are also strikingly manifested.

\section{Composite Curves of the Reaction-intensities.}

This section treats of the composite curves of the reaction-intensities, showing the differentiation of the starches of Narcissus lazetta grand monarque, $N$. poeticus ornatus, and $N$. poetaz triumph. (Chart E 14.)

The most conspicuous features of this chart are:

(1) The close correspondence in the courses of all three curves, and more particularly of the parental curves which not only tend almost invariably to marked closeness but also with few exceptions to keep below the hybrid curve.

(2) The curve of $N$. tazetta grand monarque tends usually to be lower than the curve of the other parent. It is distinctly lower in the reactions with chromic acid, pyrogallic acid, nitric acid, and hydrochloric acid; slightly lower or nearly the same with potassium hydroxide, potassium sulphocyanate, potassium sulphide, sodium hydroxide, sodium sulphide, sodium salicylate, calcium nitrate, uranium nitrate, strontium nitrate, cobalt nitrate, copper nitrate, cupric chloride, barium chloride, and mercuric chloride; higher with iodine, gentian violet, temperature, and chloral hydrate; and the same or practically the same with polarization, safranin, and sulphuric acid.

(3) In $N$. tazetta grand monarque the very high reaction with sulphuric acid; the high reactions with hydrochloric acid and sodium salicylate; the moderate reactions with polarization, iodine, gentian violet, safranin, chromic acid, and potassium sulphocyanate; the low reactions with temperature, pyrogallic acid, potassium iodide, sodium hydroxide, sodium sulphide, and strontium nitrate; and the very low reactions with chloral hydrate, nitric acid, potassium hydroxide, potassium sulphide, calcium nitrate, uranium nitrate, cobalt nitrate, copper nitrate, cupric chloride, barium chloride, and mercuric chloride.

(4) In $N$. poeticus ornatus the very high reactions with sulphuric acid and hydrochloric acid; the high reactions with chromic acid and sodium salicylate; the moderate reactions with polarization, safranin, and potassium sulphocyanate; the low reactions with gentian violet, temperature, pyrogallic acid, nitric acid, potassium hydroxide, potassium iodide, sodium hydroxide, sodium sulphide, calcium nitrate, strontium nitrate, and the very low reactions with chloral hydrate, potassium sulphide, uranium nitrate, cobalt nitrate, copper nitrate, cupric chloride, barium chloride, and mercuric chloride.

(5) In the hybrid the very high reactions with sulphuric acid, hydrochloric acid, and sodium salicylate; the high reactions with chromic acid and potassium sulphocyanate; the moderate reactions with polarization, iodine, gentian violet, safranin, pyrogallic acid, potassium hydroxide, potassium iodide, and sodium hydroxide; the low reactions with temperature, chloral hydrate, nitric acid, sodium sulphide, calcium nitrate, and strontium nitrate; and the very low reactions with potassium sulphide, uranium nitrate, cobalt nitrate, copper nitrate, cupric chloride, barium chloride, and mercuric chloride. The following is a summary of the reaction-intensities:

\begin{tabular}{|l|c|c|c|c|c}
\hline & $\begin{array}{c}\text { Very } \\
\text { high. }\end{array}$ & High. & $\begin{array}{r}\text { Mod- } \\
\text { erate. }\end{array}$ & Low. & $\begin{array}{l}\text { Very } \\
\text { low. }\end{array}$ \\
\hline N. tazetta grand monarque.... & 1 & 2 & 6 & 6 & 11 \\
N. poeticus ornatus.......... & 2 & 2 & 4 & 10 & 8 \\
N. poetaz triumph........... & 3 & 2 & 8 & 6 & 7 \\
\hline
\end{tabular}

15. Comparisons of the Starches of Narcissus gLORIA MUNDI, N. POETICUS oritatus, AND N. FIERX CROSS.

In histologic characteristics, polariscopic figures, reactions with selenite, reactions with iodine, and qualitative reactions with the various chemical reagents the starches of the parents and hybrid possess properties in common in varying degrees of development together with occasional individualities which collectively in each starch are distinctive. In histologic properties the parental starches differ in both minor and major respects. The starch of $N$. poeticus ornatus in comparison with that of the other parents shows in the polarization figure more distinctness and better definition, and other differences; and with selenite the quadrants are more often well defined, less irregular in shape, the colors not so often pure, and fewer grains have a greenish tiuge. In the qualitative iodine reactions no qualitative differences were recorded. In the qualitative reactions with chloral hydrate, chromic acid, pyrogallic acid, nitric acid, and sulphuric acid there are in each case characteristics in common and also individualities. The starch of the hybrid in comparison with the starches of the parents shows a closer relationship to that of $N$. gloria mundi in the form of the grains, character of the hilum, character and arrangement of the lamellæ, and in size; but it is closer to the other parent in the eccentricity of the hilum. In the polarization figures and in the reactions with selenite the relationship is closer to $N$. poeticus ornatus. In the iodine qualitative reactions differences between hybrid and parents, and between the latter were noted. In the qualitative reactions with the chemical reagents the hybrid shows certain resemblances to one parent and others to the other, but it is, on the whole, much more closely related to $N$. gloria mundi than to $N$. poeticus ornatus.

Reaction-intensities Expressed by Light, Color, and TemperaPolarization: ture Reactions.

N. gloria mundi, low to very high, usually moderate to moderately high, value 60 .

N. poeticus ornat., low to very high, lower than in N. gloria mundi, value 50.

$\mathrm{N}$. fiery cross, low to very high, the same as in N. poeticus ornatus, Iodine:

N. gloria mundi, moderate, value 60 .

N. poeticus ornat, moderate, much less than in N. gloria mundi, value 40.

$\mathrm{N}$. fiery cross, moderate, the same as $\mathrm{N}$. gloria mundi, value 60 .

Gentian violet:

N. gloria mundi, light to moderate, value 40.

$\mathrm{N}$. poeticus ornat., light to moderate, much less than in $\mathrm{N}$. poeticus mundi, value 30 .

$\mathrm{N}$. fiery cross, light to moderate, intermediate between the parents, Safranin: value 35 .

N. gloria mundi, moderate, value 40 . $\mathrm{N}$. poeticus ornat., moderate, higher than in $\mathrm{N}$. gloria mundi,
value 45 .

$\mathrm{N}$. fiery cross, moderate, the same as in N. gloria mundi, value 40. 
Temperature:

N. gloria mundi, in majority at 71 to $72.8^{\circ}$, in all at 74 to $75^{\circ}$, mean $74.5^{\circ}$.

N. poeticus ornat., in majority at 73 to $74^{\circ}$, in all at 77 to $78^{\circ}$, mean $77.5^{\circ}$.

$\mathrm{N}$. fiery cross, in majority at 71 to $72^{\circ}$, in all at 73.5 to $74.5^{\circ}$, mean $74^{\circ}$.

The reactivity of $N$. gloria mundi is higher than that of the other parent in the reactions with polarization, iodine, gentian violet, and temperature; and lower in the safranin reaction. The reactivity of the hybrid is the same or practically the same as that of $N$. gloria mundi in the iodine and safranin reactions, and slightly higher in the temperature reaction; the same or practically the same as that of the other parent in the polarization reaction; and mid-intermediate in the gentian violet reaction.

Table A 15 shows the reaction-intensities in percentages of total starch gelatinized at definite intervals (minutes) :

TABLe A 15.

\begin{tabular}{|c|c|c|c|c|c|c|c|c|c|}
\hline & $\underset{-}{\text { 旦 }}$ & $\begin{array}{l}\text { a } \\
\text { N }\end{array}$ & 良 & घ่ & 埕 & $\begin{array}{l}\text { g } \\
\stackrel{g}{n}\end{array}$ & 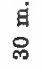 & $\begin{array}{l}\text { 白 } \\
\stackrel{4}{+}\end{array}$ & $\begin{array}{l}\dot{a} \\
\dot{8}\end{array}$ \\
\hline Chloral hydrate & & & & & & & & & \\
\hline $\mathrm{N}$ gloria mur & . & . & .. & . & 0.5 & 8 & 28 & 33 & 35 \\
\hline oeticus ornat & . & .. & .. & $\ldots$ & 0.5 & 6 & 24 & 28 & 34 \\
\hline N. fiery cross . & .. & . & .. & $\cdots$ & 0.5 & 3 & 5 & $\theta$ & 13 \\
\hline Chromic acid: & & & & & & & & & \\
\hline N. gloria mundi. & . & . & . & $\cdots$ & 2 & 25 & 65 & 82 & 90 \\
\hline N. poeticus ornatus. & . & . & .. & . & 7 & 65 & 80 & 95 & 98 \\
\hline N. fiery cross ... & . & .. & .. & .. & 3 & 12 & 60 & 85 & 95 \\
\hline Pyrogallic acid: & & & & & & & & & \\
\hline N. gloria mundi. & .. & . & .. & $\cdots$ & 1 & 18 & 65 & 78 & 91 \\
\hline N. poeticus ornatus. & . & . & $\therefore$ & . & 2 & 20 & 68 & 81 & 88 \\
\hline N. fiery cross. & . & . & .. & .. & 3 & 23 & 70 & 86 & 92 \\
\hline Nitric acid: & & & & & & & & & \\
\hline N. gloria mundi & . & . & .. & . & 8 & 23 & 47 & 55 & 61 \\
\hline N. poeticus ornatus... & . & . & .. & . & 6 & 20 & 39 & 65 & 70 \\
\hline N. fiery cross. & . & . & .. & . & 5 & 12 & 30 & 54 & 60 \\
\hline Sulphuric acid: & & & & & & & & & \\
\hline N. gloria mundi. . & . & 99 & . & . & $\cdots$ & . & . & $\cdots$ & \\
\hline N. poeticus ornatus..... & . & 93 & . . & .. & . & . & . & . & . \\
\hline N. fiery cross .......... & . & 97 & . & . & . & . & .. & . & \\
\hline
\end{tabular}

\section{VELOCITY-REACTION CURVES.}

This section treats of the velocity-reaction curves of the starches of Narcissus gloria mundi, $N$. poeticus ornatus, and $N$. fiery cross, showing quantitative differences in the behavior toward different reagents at definite time-intervals. (Charts D 287 to D 292.)

The most conspicuous features of these five charts are :

(1) The closeness of all three curves in all of the reactions, with the exception of that with chromic acid at the 15-minute interval, at which time the three curves are well separated; and also the tendency, with the exception that with sulphuric acid, for the reactions to be of moderate to low or very low intensity. In the sulphuric-acid reaction gelatinization proceeds so quickly that the curves are the same or practically the same, and in that with pyrogallic acid the curves are quite close, yet sufficiently separated and uniform in their courses to indicate clearly the reaction-intensity relationships.

(2) The relations of the parental curves to each other and to the hybrid vary in the reactions, and moreover vary during the progress of the reactions.
(3) The curve of $N$. gloria mundi is the highest of the three in the reaction with chloral hydrate; the highest during most of those with nitric acid and then intermediate; intermediate during most of those with chromic acid, otherwise the lowest; and lowest in those with pyrogallic acid.

(4) The hybrid curve tends to lowness or highness in relation to the parental curves, it being the highest of the three in the pyrogallic-acid reaction; the lowest in those with chloral hydrate and nitric acid; and lowest throughout nearly the whole 60-minute period in those with chromic acid, and finally intermediate but close to N. gloria mundi.

(5) An early period of comparative resistance is evident in one or more of the starches in all of the reactions, with the exception of the quick reaction with sulphuric acid, but in that with nitric acid it is seen only in the relation of the hybrid.

(6) The earliest period at which the curves are best separated for differential purposes is questionable. The sulphuric-acid reaction is so rapid that any differentiation must be made at the very beginning of the reaction. In the chromic-acid reaction it is probably at 15 minutes; in those with chloral hydrate and nitric acid probably at 30 minutes; and in that with pyrogallic acid probably at 45 or 60 minutes.

\section{REACTION-INTENSITIES OF THE HYBRID.}

This section treats of the reaction-intensities of the hybrid as regards sameness, intermediateness, excess, and deficit in relation to the parents. (Table A 15 and Charts D 287 to D 292.)

The reactivities of the hybrid are the same as those of the seed parent in the iodine reaction; the same as those of the pollen parent in the polarization and safranin reactions; the same as those of both parents in no reaction; intermediate in those with gentian violet and sulphuric acid, in both being mid-intermediate; highest in those with temperature and pyrogallic acid (in one closer to the seed parent and in the other closer to the pollen parent) ; and lowest in those with chloral hydrate, chromic acid, and nitric acid (in one being closer to the seed parent, in one closer to the pollen parent, and in one being as close to one as to the other parent).

The following is a summary of the reaction-intensities: Same as seed parent, 1 ; same as pollen parent, 2 ; same as both parents, 0 ; intermediate, 2 ; highest, 2 ; lowest, 3 .

The parents seem to have about equal influence on the properties of the starch of the hybrid.

\section{Composite Cunve of the Reaction-intensteies.}

This section treats of the composite curves of the reaction-intensities, showing the differentiation of the starches of Narcissus gloria mundi, $N$. poeticus ornatus, and $N$. fiery cross. (Chart E 15.)

The most conspicuous features of this chart are:

(1) The close correspondence of all three curves in their courses.

(2) In $N$. gloria mundi compared with the other parent the higher reactions with polarization, iodine, gentian violet, and temperature; the lower with chromic acid and nitric acid; and the same or practically the same with pyrogallic acid and nitric acid. 
(3) In N. gloria mundi the very high sulphuric-acid reactions; the high polarization and iodine reactions; the moderate with gentian violet, safranin, chromic acid, and pyrogallic acid; the low with temperature and nitric acid; and the very low with chloral hydrate.

(4) In $N$. poeticus ornatus the very high sulphuricacid reaction; the high with chromic acid; the moderate with polarization, iodine, and safranin; the low with gentian violet, temperature, pyrogallic acid, and nitric acid; and the very low with chloral hydrate.

(5) In the hybrid the very high sulphuric-acid reaction; the high iodine reaction; the moderate reactions with polarization, safranin, chromic acid, and pyrogallic acid; the low with gentian violet, temperature, and nitric acid; and the very low with chloral hydrate.

The following is a summary of the reaction-intensities (10 reactions):

\begin{tabular}{|c|c|c|c|c|c|}
\hline & $\begin{array}{l}\text { Very } \\
\text { high. }\end{array}$ & High. & $\begin{array}{l}\text { Mod- } \\
\text { erate. }\end{array}$ & Low. & $\begin{array}{l}\text { Very } \\
\text { low. }\end{array}$ \\
\hline 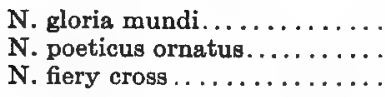 & $\begin{array}{l}1 \\
1 \\
1\end{array}$ & $\begin{array}{l}2 \\
1 \\
1\end{array}$ & $\begin{array}{l}4 \\
3 \\
4\end{array}$ & $\begin{array}{l}2 \\
4 \\
3\end{array}$ & $\begin{array}{l}1 \\
1 \\
1\end{array}$ \\
\hline
\end{tabular}

\section{Comparisons of the Starches of Narcissts telamoniUs plenus, N. poeticus ornatus, N. DOUBLOON.}

In histologic .characteristics, polariscopic figures, reactions with selenite, reactions with iodine, and qualitative reactions with the various chemical reagents the starches of the parents and hybrid exhibit not only properties in common in varying degrees of development but also certain individualities which collectively in each case are distinctive of the starch. In histologic properties the parental starches differ in certain well-defined respects. In $N$. poeticus ornatus the polariscopic figure is not so distinct or so well defined as in the other parent; and with selenite the quadrants are not so well defined and are more irregular in form, the colors are more often pure, and there are more grains with a greenish tinge. With iodine the raw grains of $N$. poeticus ornatus color less, and after boiling the grain-residues are more deeply colored and the solution less deeply colored than in $N$. telamonius plenus. In the qualitative reactions with chloral hydrate, chromic acid, pyrogallic acid, nitric acid, and sulphuric acid there are in each case rather striking differences. The starch of the hybrid in comparison with the starches of the parents shows in form a closer relationship to the starch of $N$. telamonius plenus than to that of the other parent, and the same relationship is true of the character of the hilum and the character of the lamellæ; in size of the grains the relationship is reversed; while in eccentricity of the hilum there is, on the whole, no appreciable difference between the three starches. In the polarization figure and reactions with selenite the relationship is closer to $N$. poeticus ornatus. In the qualitative iodine reactions the resemblances are closer to $N$. telamonius plenus. In the qualitative reactions with chloral hydrate, pyrogallic acid, and nitric acid the relationship is closer to $N$. telamonius plenus, while in those with the chromic acid and sul- phuric acid the relationship is reversed. In these reactions the three starches can be differentiated quite readily. The influences of each parent on the properties of the starch of the hybrid are manifest.

Reaction-intensities Expressed by Light, Color, and Temperature Reactions.

Polarization:

N. telamonius plen., low to very high, value 45 .

$\mathrm{N}$. poeticus ornat., low to very high, higher than in N. telamoniug plenus, value 50.

N. doubloon, low to very high, the same as in N. telamonius plenus, Iodine: value 45.

N. telamonius plen., moderate, value 45.

N. poeticus ornat., moderate, less than in N. telamonius plenus, value 40.

N. doubloon, moderate, the same es in N. telamonius plenus, value 45 .

Gentian violet:

N. telamonicus plen., light to moderate, value 40 .

N. poeticus ornat., light to moderate, less than in N. telamonius plenus, value 30 .

N. doubloon, light to moderate, less than in N. telamonius plenus. value 33 .

Safranin:

N. telamonius plen., moderate, value 50 .

N. poeticus ornat., moderate, less than in N. telamonius plenus, value 45.

N. doubloon, moderate, the same as in N. poeticus ornatus, value 451. Temperature:

N. telamonius plen., in majority at 70 to $72^{\circ}$, in all at 73 to $75^{\circ}$, mean $74^{\circ}$.

N. poeticus ornat., in majority at 73 to $74^{\circ}$, in all at 77 to $78^{\circ}$, mean $77.5^{\circ}$.

N. doubloon, in majority at 71.2 to $73^{\circ}$, in all at 75 to $77^{\circ}$, mean $76^{\circ}$.

The reactivity of $N$. telamonius plenus is lower than that of the other parent in the polarization reaction; and higher with iodine, gentian voilet, safranin, and temperature. The reactivity of the hybrid is the same or practically the same as that of $N$. telamonius plenus in the polarization and iodine reactions; the same or practically the same as that of the other parent in the safranin reaction; and intermediate in the gentian violet and temperature, both being closer to $N$. poeticus ornatus.

Table A 16 shows the reaction-intensities in percentages of total starch gelatinized at definite intervals (minutes) :

Table A 16.

\begin{tabular}{|c|c|c|c|c|c|c|c|c|c|}
\hline & $\underset{n \rightarrow-1}{\dot{g}}$ & $\underset{\text { N }}{\text { 日 }}$ & $\begin{array}{l}\text { g日 } \\
\infty\end{array}$ & $\begin{array}{l}\text { 日 } \\
\text { + }\end{array}$ & ฉ & $\underset{\sim}{\operatorname{\theta g}}$ & 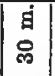 & $\begin{array}{l}\text { घ } \\
23\end{array}$ & $\begin{array}{l}\text { घ } \\
8\end{array}$ \\
\hline Chlo & & & & & & & & & \\
\hline & . & .. & .. & . & 2 & 11 & 20 & 22 & 24 \\
\hline & 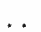 & . & .. & .. & 0.5 & 6 & 24 & 28 & 34 \\
\hline N. d & . & . & . & .. & 6 & 13 & 38 & 50 & \\
\hline Chromi & & & & & & & & & \\
\hline N. $t$ & . & & .. & . & 0.5 & 26 & 77 & 95 & 99 \\
\hline & . & & .. & .. & 7 & 65 & 80 & 95 & 98 \\
\hline & .. & . & .. & .. & 2 & 10 & 76 & 90 & 98 \\
\hline Pyrogallic & & & & & & & & & \\
\hline N. telamonius $\mathrm{pl}$ & . & .. & .. & $\cdots$ & 2 & 33 & 73 & 84 & \\
\hline N. & . & $\ldots$ & $\cdots$ & .. & 2 & 20 & 68 & 81 & 88 \\
\hline & .. & $\ldots$ & .. & $\ldots$ & 5 & 35 & 67 & 80 & 87 \\
\hline Nitric ac & & & & & & & & & \\
\hline N. $t$ & . & $\ldots$ & .. & .. & 14 & 65 & 75 & 80 & 85 \\
\hline $\mathrm{N}$ & . & $\ldots$ & $\cdots$ & .. & 6 & 20 & 39 & 65 & 70 \\
\hline N. doubloon... & .. & .. & . & . & 27 & 60 & 72 & 75 & 81 \\
\hline Sulphuric acid: & & & & & & & & & \\
\hline N. $t$ & .. & 99 & . & .. & . & . & .. & $\cdots$ & .. \\
\hline N. poeticus & & 93 & . & . & .. & $\cdots$ & . & .. & . \\
\hline N. doubloon. & & 97 & & & & & & $\ldots$ & \\
\hline
\end{tabular}




\section{VELOCITY-REACTION CURVES.}

This section treats with velocity-reaction curves of the starches of Narcissus telamonius plenus, $N$. poeticus ornatus, and $N$. doubloon, showing quantitative differences in the behavior toward different reagents at definite time-intervals. (Charts D 293 to D 298.)

The most conspicuous features of these charts are:

(1) The tendency in three of the charts to wellmarked separation of one of the three curves from the other two, to closeness of the curves in the reaction with pyrogallic acid, and to identity in the sulphuric-acid reaction. In the chloral-hydrate reaction the parental curves are in close correspondence in their courses, the hybrid curve departing; but in the charts for chromic acid and nitric acid the curves of $N$. telamonius plenus and the hybrid tend to closeness and the curve of $N$. poeticus ornatus to departure. With the exception of the very high reactivity with sulphuric acid, and the very low reactivity with chloral hydrate the reactions tend to be moderate to low.

(2) The relations of the parental curves to each other and to the hybrid vary in the four reactions.

(3) The curve of $N$. telamonius plenus is higher than the curve of the other parent throughout the whole, or the larger part, of the 60 minutes in the reactions with chloral hydrate, pyrogallic acid, and nitric acid, but is distinctly the lower in the reaction with chromic acid.

(4) The hybrid curves are very variable in their parental relationships. In the chloral-hydrate reaction the hybrid curve is distinctly the highest of the three curves; in that with chromic acid the lowest; in that with pyrogallic acid at first somewhat the highest and then passing on to be the lowest, although in this reaction all three curves tend to marked closeness; and in that with nitric acid it is at first the highest and then intermediate, but much closer to $N$. telamonius plemus than to the other parent. The relationship is, on the whole, rather closer to $N$. telamonius plenus.

(5) An early period of comparative resistance followed by a comparatively rapid reaction is noted with chromic acid and pyrogallic acid, not at all with nitric acid, and to a slight degree with chloral hydrate.

(6) The earliest period at which the curves are best separated for differential purposes is within or at 5 minutes in the reactions with sulphuric acid and nitric acid; at 15 minutes in those with chromic acid and pyrogallic acid; and either at 30 or 60 minutes in that with chloral hydrate-at the first $N$. telamonius plenus would be intermediate in position, while at the latter it would be lowest.

\section{Reaction-Intensities of the Hybid.}

This section treats of the reaction-intensities of the hybrid as regards sameness, intermediateness, excess, and deficit in relation to the parents. (Table A16 and Charts D 293 to D 298.)

The reactivities of the hybrid are the same as those of the seed parent in the polarization and iodine reactions; the same as those of the pollen parent in the safranin reaction; the same as those of both parents in that with pyrogallic acid; intermediate in those with gentian violet, temperature, nitric acid, and sulphuric acid (in two being closer to the seed parent and in two closer to the pollen parent); highest in none; and lowest in those with chloral hydrate and chromic acid (in one being as close to one as to the other parent, and in the other closer to the seed parent).

The following is a summary of the reaction-intensities (10 reactions): Same as seed parent, 2; same as pollen parent, 1 ; same as both parents, 1 ; intermediate, 4 ; highest, 0 ; lowest, 2.

The seed parent, $N$. poeticus ornatus, seems to be the more potent in influencing the characters of the starch of the hybrid.

\section{Composite Curves of the Reaction-intensities.}

This section treats of the composite curves of the reaction-intensities, showing the differentiation of the starches of Narcissus telamonius plenus, $N$. poeticus ornatus, and $N$. doubloon. (Chart E 16.)

The most conspicuous features of the chart are:

(1) The close correspondence of all three curves in their courses, especially of the parental curves.

(2) In N. telamonius plenus in comparison with the other parent the higher reactions with iodine, gentian violet, safranin, temperature, and nitric acid; the lower reactions with polarization and chloral hydrate; and the same or practically the same reactions with chromic acid, pyrogallic acid, and sulphuric acid.

(3) In $N$. telamonius plenus the very high reaction with sulphuric acid; the high reaction with chromic acid; the moderate reactions with polarization, iodine, gentian violet, safranin, and pyrogallic acid; the low reactions with temperature and nitric acid; and the very low reaction with chloral hydrate.

(4) In $N$. poeticus ornatus the very high reaction with sulphuric acid; the high reaction with chromic acid; the moderate reactions with polarization, iodine, and safranin; the low reactions with gentian violet, temperature, pyrogallic acid, and nitric acid; and the very low reaction with chloral hydrate.

(5) In the hybrid the very high reaction with sulphuric acid; the absence of any high reaction; the moderate reactions with polarization, iodine, safranin, and chromic acid; the low reactions with gentian violet, temperature, chloral hydrate, pyrogallic acid, and nitric acid; and the absence of any very low reaction.

The following is a summary of the reaction-intensities (10 reactions):

\begin{tabular}{l|c|c|c|c|c}
\hline & $\begin{array}{c}\text { Very } \\
\text { high. }\end{array}$ & High. & $\begin{array}{c}\text { Mod- } \\
\text { erate }\end{array}$ & Low. & $\begin{array}{l}\text { Very } \\
\text { low. }\end{array}$ \\
\hline $\begin{array}{l}\text { N. telamonius plenus ........... } \\
\text { N. poeticus ornatus......... }\end{array}$ & 1 & 1 & 5 & 2 & 1 \\
N. doubloon............... & 1 & 0 & 4 & 5 & 1 \\
\hline
\end{tabular}

\section{Comparisons of the Starches of Narcissus} PRINCESS MARY, N. POETICUS POETARUM, AND N. CRESSET.

In histologic characteristics, polariscopic figures, reactions with selenite, reactions with iodine, and qualitative reactions with various chemical reagents the starches of the parents and hybrids possess properties in common in varying degrees of development and individualities which collectively are in each case distinctive. In histo- 
logic properties the starches of the parents differ in certain well-defined respects. The starch of Narcissus poeticus poetarum in comparison with that of the other parent shows in the polarization figure less definition and some differences in the characters of the lines; and in the selenite reaction less clean-cut quadrants, more irregularity of shape, more often purity of colors, and more grains with a greenish tinge. With iodine no qualitative differences were recorded. In the qualitative reactions with the chemical reagents there are well-defined differences which for the most part are related to variations in the histologic peculiarities of the grains of the two plants. The starch of the hybrid in comparison with the starches of the parents contains a larger percentage of aggregates and compound grains than in either parent; it is more like the starch of $N$. princess mary as regards the absence of clearness of distinction between the primary and secondary starch deposits; but it is, on the whole, in closer relationship to the starch of $N$. poeticus poetarum. In the character and eccentricity of the hilum and size of the grains the relationship is closer to $N$. princess mary, but in the character of the lamellæ it is nearer the other parent. In character of the polariscopic figure, and in the reactions with selenite, the relationship is closer to $N$. princess mary. In the qualitative iodine reaction it is closer to $N$. poeticus poetarum. In all of the qualitative reactions with the chemical reagents (including chloral hydrate, chromic acid, pyrogallic acid, nitric acid, and sulphuric acid) characteristics of each of the parents are evident and also certain individualities not observed in the parents, but the resemblances of the hybrid, as a whole, are closer to $N$. princess mary than to $N$. poeticus poetarum.

Reaction-intensities Expressed by Light, Color, and TemperaPolarization:

N. princess mary, low to high, value 35 .

$\mathrm{N}$. poeticus poetar., low to high, higher than in N. princess mary, value 40 .

$\mathrm{N}$. cresset, low to high, same as in N. poeticus poetarum, value 40 Iodine:

N. princess mary, light to moderate, value 42.

N. poeticus poetar., light to moderate, slightly higher than in N. princess mary, value 45 .

$\mathrm{N}$. cresset, light to moderate, the same as in $\mathrm{N}$. poeticus poetarum, value 45.

Gentian violet:

N. princess mary, light to moderate, value 37 .

N. poeticus poetar., light to moderate, slightly lighter than in N. princess mary, value 35 .

$N$. cresset, light to moderate, the same as in N. princess mary, Safranin: value 37.

N. princess mary, moderate, value 50.

$\mathrm{N}$. poeticus poetar, moderate, the same as in $\mathrm{N}$. princess mąry, value 50 .

N. cresset, moderate, the same as in both parents, value 50 .

Temperature:

$\mathrm{N}$. princess mary, in majority at 70 to $72^{\circ}$, in all at 74 to $76^{\circ}$, mean $75^{\circ}$.

N. poeticus poetar., in majority at 67 to $69^{\circ}$, in all at 71 to $73^{\circ}$, mean $72^{\circ}$.

$\mathrm{N}$ : cresset, in majority at 71 to $73^{\circ}$, in all at 74.5 to $76^{\circ}$, mean $75.7^{\circ}$.

The reactivity of $N$. princess mary is the same or practically the same as that of the other parent in the safranin reaction; higher in the gentian-violet reaction; and lower in the polarization, iodine, and temperature reactions. The reactivity of the hybrid is the same or practically the same as that of $N$. princess mary with gentian violet; the same or practically the same as that of the other parent in the polarization and iodine reactions; the same as that of both parents with safranin; and the lowest of the three with temperature, but nearer N. princess mary.

Table A 17 shows the reaction-intensities in percentages of total starch gelatinized at definite intervals (minutes) :

TABLE A 17.

\begin{tabular}{|c|c|c|c|c|c|c|c|c|c|}
\hline & $\underset{\sigma}{\dot{g}}$ & $\underset{\text { N }}{\text { 日 }}$ & 百 & 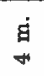 & $\begin{array}{l}\text { d } \\
\text { to }\end{array}$ & $\begin{array}{l}\text { 日் } \\
\stackrel{2}{2}\end{array}$ & 曋 & 星 & $\begin{array}{l}\text { a } \\
8\end{array}$ \\
\hline Chloral hydrate: & & & & & & & & & \\
\hline N. princess mary... & . & . & . & . & 2 & 5 & 6 & 8 & 15 \\
\hline N. poeticus poetar. & $\cdots$ & . & .. & . & 0.5 & 6 & 9 & 11 & 17 \\
\hline N. cresset........ & . & .. & .. & .. & 2 & 3 & 7 & 18 & 22 \\
\hline Chromic acid: & & & & & & & & & \\
\hline N. princess mary.. & $\cdots$ & . & . & . & 2 & 25 & 70 & 90 & 98 \\
\hline N. poeticus poetar. & . & . & . & .. & 3 & 22 & 65 & 75 & 85 \\
\hline N. cresset....... & .. & .. & . & . & 2 & 15 & 70 & 93 & 96 \\
\hline Pyrogallic acid: & & & & & & & & & \\
\hline N. princess mary. & . & $\cdots$ & . & . & $\mathbf{3}$ & 40 & 77 & 87 & 95 \\
\hline N. poeticus poetar. & .. & .. & . & . & 1 & 16 & 70 & 84 & 93 \\
\hline N. cresset...... & $\cdots$ & .. & $\cdots$ & . & 3 & 16 & 69 & 74 & 81 \\
\hline Nitric acid: & & & & & & & & & \\
\hline N. princess mary & - & . & . & . & 13 & 55 & 68 & 75 & 79 \\
\hline N. poeticus poetar. . & .. & . & . & .. & 10 & 40 & 53 & 60 & 63 \\
\hline N. cresset......... & . & . & . & . & 22 & 67 & 75 & 77 & 80 \\
\hline Sulphuric acid: & & & & & & & & & \\
\hline N. princess mary. & . & 95 & . & . & & $\cdots$ & . & & $\cdots$ \\
\hline N. poeticus poeta & . & 79 & . & . & 99 & . & * & . & . \\
\hline N. cresset.... & . & 98 & . & . & & . & & & \\
\hline
\end{tabular}

Velocity-Reaction Curves.

This section deals with the velocity-reaction curves of the starches of Narcissus princess mary, $N$. poeticus poetarum, and $N$. cresset, showing quantitative differences in the behavior toward different reagents at definite time-intervals. (Charts D 299 to D 304.)

The most conspicuous features of these charts are:

(1) The closeness of all three curves in all of the charts (with the exception of the very quick sulphuricacid reaction in which there is no differentiation) and the moderate to low or very low reactivities. In the sulphuric-acid reaction gelatinization proceeds so rapidly that there is differentiation only before the end of about 3 minutes, at the end of 2 minutes the reactions of $N$. princess mary and the hybrid are practically absolutely the same, but the reaction of the other parent is distinctly less. In the reaction with chloral hydrate there is unimportant separation of the curves, but in the other three reactions there are varying degrees of separation.

(2) The relationships of the parental curves to each other and to the curve of the hybrid vary in the different reactions and during the progress of the reactions.

(3) The curve of $N$. princess mary is the highest in the reaction with pyrogallic acid; lowest with chloral hydrate; intermediate. with nitric acid; and practically the same as that of the hybrid and higher than the curve of the other parent with chromic acid.

(4) The hybrid curve is the highest of the three in the reactions with chloral hydrate and nitric acid; it tends to be the lowest with pyrogallic acid; and it inclines to be the lowest at first and the highest later with chromic acid. It is more closely related to the curve of $N$. princess mary in the reaction with chloral hydrate; to the curve of the other parent with nitric acid; and first 
to one parent and then to the other with chromic acid and pyrogallic acid, the parental relationships being reversed in these two reactions.

(5) An early period of resistance followed by a comparatively rapid reaction is seen in the reactions with chromic acid and pyrogallic acid-in all three starches in the first and in the two starches in the second.

(6) The earliest period at which the three curves are best separated for differential purposes is in the sulphuric-acid reaction within the 5-minute period; in that with pyrogallic acid at 45 minutes; and in that with chloral hydrate at 60 minutes.

\section{Reaction-intensities OF the HrbRid.}

This section deals with the reaction-intensities of the hybrid as regards sameness, intermediateness, excess, and deficit in relation to the parents. (Table A 17 and Charts D 299 to D 304.)

The reactivities of the hybrid are the same as those of the seed parent in the reactions with gentian violet and chromic acid; the same as those of the pollen parent in those with polarization, iodine, and safranin; the same as those of both parents in none; intermediate in none; highest in those with chloral hydrate, nitric acid, and sulphuric acid, in all three being closer to the seed parent; and lowest in those with temperature and pyrogallic acid, in both being closer to the seed parent.

The following is a summary of the reaction-intensities (10 reactions): Same as seed parent, 2; same as pollen parent, 3 ; same as both parents, 0 ; intermediate, 0 ; highest, 3 ; lowest, 2 .

The seed parent, $N$. princess mary, has from these data exercised a far more potent influence than $N$. poeticus poetarum on the properties of the starch of the hybrid.

\section{Composite Curves of the Reaction-intensities.}

This section treats of the composite curves of the reaction-intensities, showing the differentiation of the starches of Narcissus princess mary, $N$. poeticus poetarum, and N. cresset. (Chart E 17.)

The most conspicuous features of this chart are:

(1) The very close correspondence in the curves, both as to nearness and course.

(2) In $N$. princess mary in comparison with the other parent the higher reactions with gentian violet, chromic acid, and nitric acid; the lower reactions with polarization and iodine; and the same or practically the same reactions with chloral hydrate, pyrogallic acid, and sulphuric acid.

(3) In N. princess mary the very high sulphuricacid reaction; the absence of any high reaction; the moderate reactions with iodine, safranin, chromic acid, and pyrogallic acid; the low reactions with polarization, gentian violet, temperature, and nitric acid; and the very low reaction with chloral hydrate.

(4) In $N$. poeticus poetarum the very high reaction with sulphuric acid; the absence of any high reaction; the moderate reactions with polarization, iodine, safranin, temperature, and pyrogallic acid; the low reactions with gentian violet, chromic acid, and nitric acid; and the very low reaction with chloral hydrate.

(5) In the hybrid the very high reaction with sulphuric acid; the absence of any high reaction; the mod- erate reactions with polarization, iodine, safranin, and chromic acid; the low reactions with gentian violet, temperature, pyrogallic acid, and nitric acid; and the very low reaction with chloral hydrate.

The following is a summary of the reaction-intensities (10 reactions):

\begin{tabular}{l|c|c|c|c|c}
\hline & $\begin{array}{c}\text { Very } \\
\text { high. }\end{array}$ & High. & $\begin{array}{c}\text { Mod- } \\
\text { erate. }\end{array}$ & Low. & $\begin{array}{c}\text { Very } \\
\text { low. }\end{array}$ \\
\hline N. princess mary ............ & 1 & 0 & 4 & 4 & 1 \\
N. poeticus poetarum........ & 1 & 0 & 5 & 3 & 1 \\
N. cresset ................ & 1 & 0 & 4 & 4 & 1 \\
\hline
\end{tabular}

18. Comparisons of the Starches of Narcissus abscissus, $N$. poeticus poetardar, and N. Will SCARLET.

In histologic characteristics, polariscopic figures, reactions with selenite, reactions with iodine, and qualitative reactions with the various chemical reagents the starches of the parents and hybrid exhibit properties in common in varying degrees of development, which collectively in each case are distinctive, although all three starches are very much alike. In histologic properties the starches of the parents differ very little, and the same is also true of the polariscopic figures and reactions with selenite. In the iodine reactions no qualitative differences were recorded. In the qualitative reactions with chloral hydrate, chromic acid, pyrogallic acid, nitric acid, and sulphuric acid there are properties in common and also individualities. The starch of the hybrid in comparison with the starches of the parents shows a closer relationship to Narcissus abscissus in the form of the grains, the character of the hilum, the character of the lamellæ, and the size of the larger grains; but closer to the other parent in the size of the smaller grains. The eccentricity of the hilum is about the same in all three starches, and in the hybrid the lamellæ are more distinct than in the parents, and the hilum is not so deeply and extensively fissured. In the polarization figures and reactions with selenite the relationship is closer to $N$. abscissus. In the qualitative iodine reactions it is closer to $N$. poeticus poetarum. In all of the qualitative reactions with the chemical reagents peculiarities of both parents are observed, but the resemblances are, on the whole, closer to $N$. abscissus. Such differences as have been recorded are only of a minor character.

Reaction-intensities Expressed by Light, Color, and TemperaPolarization: ture Reactions.

N. abscissus, low to high, value 43.

N. poeticus poetar., low to high, somewhat less than in N. abscissus, value 40 .

N. will scarlet, low to high, the same as in N. abscissus, value 43 . Iodine:

N. abscissus, light to moderate; value 40 .

$\mathrm{N}$. poeticus poetar., light to moderate, somewhat less than in $\mathrm{N}$. abscissus, value 45 .

$N$. will scarlet, light to moderate, the same as in $N$. poeticus poetarum, value 45 .

Gentian violet:

N. abscissus, light to moderate, value 33 .

$\mathrm{N}$. poeticus poetar., light to moderate, somewhat more than in N. abscissus, value 35 .

N. will scarlet, light to moderate, higher than in either parent, value 37 . 
Safranin:

N. abscissus, moderate, value 47.

N. poeticus poetar., moderate, somewhat more than in N. abscissus, value 50.

N. will scarlet, moderate, higher than in either parent, value 53.

Temperature:

N. abscissus, in majority at 69.5 to $71^{\circ}$, in all at 73 to $74^{\circ}$, mean $73.5^{\circ}$.

N. poeticus poetar., in majority at 69 to $71^{\circ}$, in all at 71 to $73^{\circ}$, mean $72^{\circ}$.

N. will scarlet, in majority at 69.8 to $71.9^{\circ}$, in all at 72 to $74^{\circ}$, mean $73^{\circ}$

The reactivity of $N$. abscissus is the same or practically the same as that of the other parent in not a single reaction; higher in the polarization reaction; and lower in those with iodine, gentian violet, safranin, and temperature. The reactivity of the hybrid is the same or practically the same as that of $N$. abscissus in the polarization reaction; the same or practically the same as that of the other parent in the iodine reaction; and the highest of the three in the reactions with gentian violet and safranin; and intermediate but close to the seed parent in the temperature reaction.

Table A 18 shows the reaction-intensities in percentages of total starch gelatinized at definite intervals (minutes) :

Table A 18.

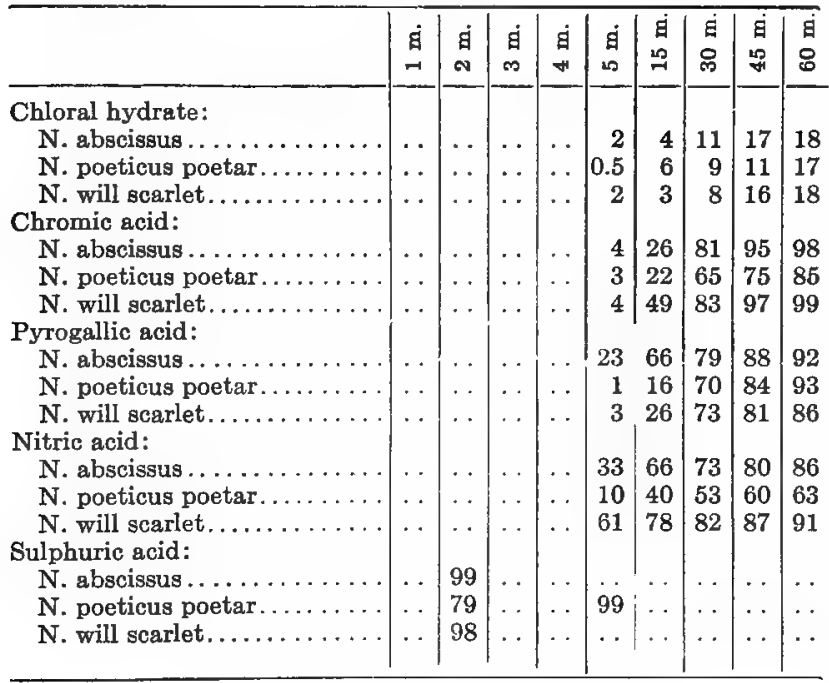

Velocity-Reaction CURVES.

This section treats of the velocity-reaction curves of the starches of Narcissus abscissus, $N$. poeticus poetarum, and $N$. will scarlet, showing qualitative differences in the behavior toward different reagents at definite timeintervals. (Charts D 305 to D 310.)

The most conspicuous features of these charts are:

(1) The close correspondence of all three curves (excepting in the pyrogallic-acid reaction, in which there is a disproportionate separation of the curve of $N$. $a b$ scissus from the other curves); and also the tendency for the reactions, excepting that with sulphuric acid, to be of moderate to low or very low intensity. The sulphuric-acid reaction is so very rapid that there is no differentiation to be seen in the charts, although, as will be seen from the preceding table, the reactivity of $N$. poeticus poetarum is less at first than that of either of the other starches. In the chloral-hydrate reaction the differences are of a very minor character, not sufficient for satisfactory differentiation.

(2) The relations of the parental curves to each other and to the hybrid vary in the reactions, and in the pyrogallic-acid reaction they vary during their course.

(3) The curve of $N$. abscissus is higher than that of the other parent in the reactions with chromic acid, pyrogallic acid, and nitric acid, in the two latter being quite well separated. A higher reactivity of $N$. abscissus is also indicated in the records of the reactions with chloral hydrate and sulphuric acid.

(4) The curve of the hybrid is the highest of the three in the reactions with chromic acid and nitric acid, and intermediate during the first part and lowest during the latter part of that with pyrogallic acid, although in this reaction there are but small differences between the hybrid and $N$. poeticus poetarum.

(5) An early period of resistance followed by comparatively rapid gelatinization is noted in all three starches in the reaction with chromic acid, in two with pyrogallic acid, and in one with nitric acid. The reaction with sulphuric acid is too rapid and with chloral hydrate too slow for a manifestation of this peculiarity.

(6) The earliest period at which the curves are best separated for differential purposes varies in the different reactions. This period is approximately in the reactions with sulphuric acid and pyrogallic acid within the 5-minute interval; in those with chromic acid and pyrogallic acid at the 15-minute interval; and in the chloral-hydrate reaction at probably 30 to 45 minutes, although at any time the differences in this reaction may fall wholly within the limits of error of experiment.

\section{REACTION-INTENSITIES OF THE HYBRID.}

This section treats of the reaction-intensities of the hybrid as regards sameness, intermediateness, excess, and deficit in relation to the parents. (Table A 18 and Charts D 305 to D 310.)

The reactivities of the hybrid are the same as those of the seed parent in the polarization and sulphuric acid; the same as those of the pollen parent in the iodine reaction; the same as both parents in that with chloral hydrate; intermediate in those with temperature and pyrogallic acid (in one being closer to one parent and in the other closer to the other parent) ; highest in those with gentian violet, safranin, chromic acid, and nitric acid (in three being closer to the pollen parent, and in one closer to the seed parent); and lowest in none.

The following is a summary of the reaction-intensities (10 reactions): Same as seed parent, 2; same as pollen parent, 1 ; same as both parents, 1 ; intermediate, 2 ; highest, 4 ; lowest, 0 .

The seed parent has probably slightly more influence than the pollen parent in determining the properties of the hybrid. The tendency of the hybrid to highness is evident, this being more marked than to intermediateness.

Composite CuRves of the Reaction-intensities.

This section treats of the composite curves of the reaction-intensities, showing the differentiation of the starches of Narcissus abscissus, $N$. poeticus poetarum, and $N$. will scarlet. (Chart E 18.)

The most conspicuous features of this chart are:

(1) The close correspondence of the three curves both as to closeness and course, the only tendency even 
to a moderate separation being in the reactions with chromic acid and nitric acid.

(2) In $N$. abscissus in comparison with the other parent the higher reactions with polarization, chromic acid, and nitric acid; the lower reactions with iodine, gentian violet, safranin, and temperature; and the same or practically the same reactions with chloral hydrate, pyrogallic acid, and sulphuric acid.

(3) In $N$. abscissus the very high reaction with sulphuric acid; the high reaction with chromic acid; the moderate reactions with polarization, iodine, safranin, and pyrogallic acid; the low reactions with gentian violet, temperature, and nitric acid; and the very low reaction with chloral hydrate.

(4) In $N$. poeticus poetarum the very high sulphuricacid reaction; the absence of a high reaction; the moderate reactions with polarization, iodine, safranin, temperature, and pyrogallic acid; the low reactions with gentian violet, chromic acid, and nitric acid; and the very low reaction with chloral hydrate.

(5) In the hybrid the very high reaction with sulphuric acid; the absence of a high reaction; the moderate reactions with polarization, iodine, safranin, chromic acid, and nitric acid; the low reactions with gentian violet, temperature, and pyrogallic acid; and the very low reaction with chloral hydrate.

The following is a summary of the reaction-intensities (10 reactions):

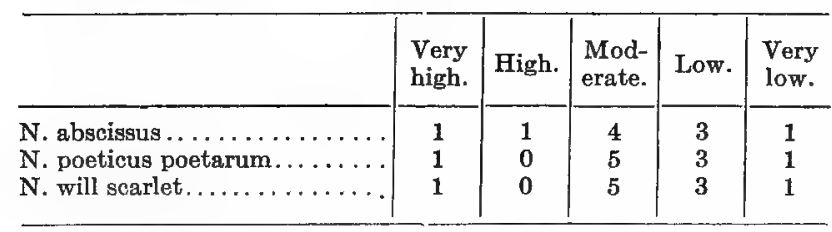

\section{Comparisons of the Starches of Narcissus ALBICANS, N. ABSCISSUS, AND N. BICOLOR APRICOT.}

In histologic characteristics, polariscopic figures, reactions with selenite, qualitative reactions with iodine, and qualitative reactions with the various chemical reagents the starches of the parents and hybrid exhibit properties in common in varying degrees of development together with certain individualities which collectively in each case are distinctive of the starch. In histologic properties there are certain well-defined differences between the starches of the parents. In Narcissus abscissus compared with the other parent the polariscopic figure is not so well defined, and there are minor differences in the lines; and with selenite the quadrants are not so clean-cut and are more irregular, the colors are more often pure, and more grains have a greenish tinge. In the iodine reactions no qualitative difference was recorded. In the qualitative reactions with chloral hydrate, chromic acid, pyrogallic acid, nitric acid, and sulphuric acid there are both properties in common and differences which are quite definite. The starch of the hybrid has fewer compound grains than in either parent, and in form generally shows a closer relationship to $N$. albicans than to $N$. abscissus. While the eccentricity of the hilum is about the same in all three starches, the charaoter of the hilum is somewhat closer to that of
$N$. abscissus. In the character of the lamellæ and in the size of the grains the relationship is closer to $N$. albicans. In the character of the polariscopic figure and the appearances with selenite the relationship is much closer to $N$. albicans. In the qualitative iodine reactions the raw grains show a closer relationship to $N$. albicans, but after heating the relationship is closer to the other parent. In the qualitative chemical reactions peculiarities of both parents are observed. With chloral hydrate the reactions, on the whole, more closely resemble those of $N$. albicans; but in those with chromic acid, pyrogallic acid, nitric acid, and sulphuric acid they resemble more closely those of the other parent. There are also certain individualities in the way of accentuation in the hybrid. Reaction-intensities Expressed by Light, Color, and Tempera-
ture Reactions.

Polarization:

N. albicans, low to high, value 37 .

N. abscissus, low to high, higher than in N. albicans, value 43 .

N. bicolor apricot, low to high, the same as in N. albicans, value 37 . Iodine:

N. albicans, moderate, value 55.

N. abscissus, light to moderate, much less than in $\mathrm{N}$. albicans, value 40 .

$\mathrm{N}$. bicolor apricot, moderate, intermediate between the parents, but much closer to $\mathrm{N}$. albicans, value 53 .

Gentian violet:

N. albicans, light to moderate, value 40.

$\mathrm{N}$. abscissus, light to moderate, lighter than in N. albicans, value 33 .

N. bicolor apricot, light to moderate, the same as N. albicans, value 40.

Safranin:

N. albicans, moderate, value 50 .

N. abscissus, moderate, less than in N. albicans, value 47 .

$\mathrm{N}$. bicolor apricot, moderate, the same as $\mathrm{N}$. albicans, value 50. Temperature:

N. albieans, in majority at 70.2 to $72^{\circ}$, in all at 73 to $75^{\circ}$, mean $74^{\circ}$.

N. abscissus, in majority at 69.5 to $71^{\circ}$, in all at 73 to $74^{\circ}$, mean $73.5^{\circ}$.

N. bicolor apricot, in majority at 71 to $72.5^{\circ}$, in all at 74 to $76^{\circ}$, mean $75^{\circ}$.

The reactivity of $N$. albicans is higher than that of the other parent in the reactions with iodine, gentian violet, and safranin; and lower in those with polarization and temperature. The reactivity of the hybrid is the same or practically the same as that of $N$. albicans with polarization, gentian violet, and safranin; intermediate

Table A 19.

\begin{tabular}{|c|c|c|c|c|c|c|c|c|c|}
\hline & 皇 & ล & 泉 & 昌 & $\underset{\text { a }}{\dot{9}}$ & $\begin{array}{l}9 \\
\square\end{array}$ & $\begin{array}{l}\text { 日 } \\
\text { 요 }\end{array}$ & $\begin{array}{l}\text { 豆 } \\
\text { Lी }\end{array}$ & $\begin{array}{l}\text { 永 } \\
8\end{array}$ \\
\hline Chloral hydrate: & & & & & & & & & \\
\hline & . . & $\ldots$ & & . & $0.5 \mathrm{t}^{\mathrm{n}}$ & 14 & 31 & 40 & 43 \\
\hline N. abscissus & & . & .. & . & 2 & 4 & 11 & 17 & 18 \\
\hline N. bicolor apricot & . & . & . & $\cdots$ & 3 & 5 & 9 & 15 & 21 \\
\hline Chromic acid: & & & & & & & & & \\
\hline N. albicans. . & . & . & . & $\cdots$ & 11 & 75 & 98 & 99 & \\
\hline N. ak & . & $\cdots$ & . & .. & 4 & 26 & 81 & 95 & 98 \\
\hline N. bicolor apricot & . & . & . & .. & 61 & 30 & 86 & 97 & 99 \\
\hline Pyrogallic acid: & & & & & & & & & \\
\hline N. albica: & . & . & . & - & 25 & 78 & 91 & 95 & 97 \\
\hline N. abscissus. & . & . & .. & . & 23 & 66 & 79 & 88 & 92 \\
\hline N. bicolor apr & $\cdots$ & . & .. & .. & 10 & 39 & 73 & 85 & 90 \\
\hline Nitric acid: & & & & & & & & & \\
\hline N. albicans... & .. & . & . & .. & 33 & 78 & 82 & 86 & 86 \\
\hline N. abscissus..... & . & .. & . & .. & 33 & 66 & 73 & 80 & 86 \\
\hline N. bicolor apricot & .. & . & . & .. & 16 & 56 & 68 & 76 & 80 \\
\hline Sulphuric acid: & & & & & & & & & \\
\hline N. albicans... & .. & 99 & . & . & . & . & $\cdots$ & & . \\
\hline N. abscissus ..... & . & 99 & $\cdots$ & - & ’ & . & . & - & .. \\
\hline & & & & & & & & & \\
\hline
\end{tabular}


but nearer $N$. albicans with iodine; and the lowest of the three, but nearer $N$. albicans, with temperature.

Table A 19 shows the reaction-intensities in percentages of total starch gelatinized at definite intervals (minutes).

\section{Velocity-REaCtion CURVES.}

This section treats of the velocity-reaction curves of the starches of Narcissus albicans, $N$. abscissus, and $N$. bicolor apricot, showing the quantitative differences in the behavior toward different reagents at definite timeintervals. (Charts D 311 to D 316.)

The most conspicuous features of these charts are:

(1) The close correspondence of the curves in their courses in all of the reactions (with the exception of the very rapid sulphuric-acid reaction, in which there is no differentiation) and the tendency mostly to a moderate or low reactivity.

(2) The relationships of the parental curves to each other and to the curve of the hybrid (excepting the quick sulphuric-acid reaction) vary in the different reactions and during their progress.

(3) The curve of $N$. albicans is distinctly higher than that of the other parent in reactions with the chloral hydrate, pyrogallic acid, chromic acid, and nitric acid, the degree of separation varying as stated.

(4) The hybrid curve is the same or practically the same as that of $N$.abscissus in the reactions with chloral hydrate and chromic acid, being fairly well separated from the curve of the other parent; and it is lowest in the reactions with pyrogallic acid and nitric acid, it being in both closer to $N$. abscissus.

(5) A tendency to an early period of resistance followed by comparatively high reactivity is indicated only in a minor degree, and almost solely that with chromic acid.

(6) The earliest period at which the three curves are best separated for differential purposes is in the reaction with sulphuric acid at the very beginning; with pyrogallic acid, chromic acid, and nitric acid at 15 minutes; and with chloral hydrate at 30 minutes or later.

\section{Reaction-Intensities OF THE Hybrids.}

This section deals with the reaction-intensities of the hybrids as regards sameness, intermediateness, excess, and deficit in relation to the parents. (Table A 19 and Charts D 311 to D 316.)

The reactivities of the hybrid are the same as those of the seed parent in the reactions with gentian violet and safranin; the same as those of the pollen parent with polarization and chloral hydrate; the same as those of both parents with sulphuric acid, in which the reactions occur too rapidly for differentiation; intermediate in those with iodine and chromic acid, in both being closer to those of the seed parent; highest in none; and the lowest in those with temperature, pyrogallic acid, and nitric acid, in one being closer to the seed parent and in two closer to the pollen parent.

The following is a summary of the reaction-intensities (10 reactions) : Same as seed parent, 3 ; same as pollent parent, 4 ; same as both parents, 1 ; intermediate, 2 ; highest, 0 ; lowest, 3 .

The seed parent seems to be much more potent in influencing the characters of the starch of the hybrid.
Composite Curves of the Reaction-intensities.

This section treats of the composite curves of the reaction-intensities, showing the differentiation of the starches of Narcissus albicans, $N$. abscissus, and $N$. bicolor apricot. (Chart E 19.)

The most conspicuous features of this chart are:

(1) The close correspondence of the curves both as to nearness and course.

(2) In $N$. albicans in comparison with the other parent the higher reactions with iodine, gentian violet, safranin, chloral hydrate, chromic acid, and pyrogallic acid; the lower reactions with polarization and temperature; and the same reactions with nitric acid and sulphuric acid.

(3) In $N$. albicans the very high sulphuric-acid reaction; the high reactions with chromic acid and pyrogallic acid, the moderate reactions with iodine, gentian violet, and safranin; the low reactions with polarization, temperature, and nitric acid; and the very low reaction with chloral hydrate.

(4) In $N$. abscissus the very high sulphuric-acid reaction; the high chromic-acid reaction; the moderate reactions with polarization, iodine, safranin, and pyrogallic acid; the low reactions with gentian violet, temperature, and nitric acid; and the very low reaction with chloral hydrate.

(5) In the hybrid the very high reaction with sulphuric acid; the high reaction with chromic acid; the moderate reactions with iodine, gentian violet, safranin, and pyrogallic acid; the low reactions with polarization, temperature, and nitric acid; and the very low reaction with chloral hydrate. The following is a summary of the reaction-intensities (10 reactions):

\begin{tabular}{|c|c|c|c|c|c|}
\hline & $\begin{array}{l}\text { Very } \\
\text { high. }\end{array}$ & High. & $\begin{array}{l}\text { Mod- } \\
\text { erate. }\end{array}$ & Low. & $\begin{array}{l}\text { Very } \\
\text { low. }\end{array}$ \\
\hline $\begin{array}{l}\text { N. albicans } \ldots \ldots \ldots \ldots \ldots \ldots \\
\text { N. abscissus } \ldots \ldots \ldots \ldots \ldots \ldots \\
\text { N. bicolor apricot } \ldots \ldots \ldots \ldots\end{array}$ & $\begin{array}{l}1 \\
1 \\
1\end{array}$ & $\begin{array}{l}2 \\
1 \\
1\end{array}$ & $\begin{array}{l}3 \\
4 \\
4\end{array}$ & $\begin{array}{l}3 \\
3 \\
3\end{array}$ & $\begin{array}{l}1 \\
1 \\
1\end{array}$ \\
\hline
\end{tabular}

20. Comparisons of the Starches of Narcissus EMPRESS, $N$. aLbiCaNs, AND $N$. MADAME DE GRAAFF.

In histologic characteristics, polariscopic figures, reactions with selenite, reactions with iodine, and qualitative reactions with various chemical reagents the starches of the parents and hybrid have properties in common in varying degrees of development together with certain individualities which collectively are in each case distinctive of the starch. The differences are, as a whole, of rather a minor character. In histologic properties the parental starches differ particularly in the number of aggregates, compound and composite grains, irregularity, and conspicuous forms, especially as regards the last. The nearly round and short elliptical grains seen in Narcissus albicans are not present in $N$. empress. There are minor differences in the hilum and lamellæ, and the grains are smaller in $N$. abscissus. In the polarization figures and reactions with selenite there are minor differences. In the reactions with iodine no qualitative differences were recorded. In the reactions with chloral hydrate, chromic acid, pyrogallic acid, nitric acid, and 
sulphuric acid, there are differences of minor characters. The starch of the hybrid has more isolated and more simple grains than either parent, and in form it is more closely related, on the whole, to $N$. empress than to $N$. albicans; moreover, some characteristios of the former are accentuated. The hilum is less fissured than in either parent, and in both character and eccentricity of the hilum it is in closer relationship to $N$. albicans. In the character and number of the lamellæ the relationship is closer to $N$. albicans, but in size the relationship is closer to $N$. empress. In the character of the polariscopic figure and appearance with selenite the relationship is closer to $N$. empress. In the qualitative iodine reactions the raw grains behave more like those of $N$. empress, while after the grains are boiled there are no differences noted in the three starches. In the qualitative reactions with the chemical reagents peculiarities of both parents are evident. In the reactions with chloral hydrate, chromic acid, nitric acid, and sulphuric acid the relationship is, on the whole, closer to $N$. empress; but in the pyrogallic-acid reaction the relationship is closer to the other parent.

Reactiowintensities Expressed by Light, Color, and Tempera-

Polarization: ture Reactions.

N. empress, low to high, value 42.

N. albicans, low to high, lower than in N. empress, value 37.

N. madame de graaff, low to high, the same as in N. albicans, Iodine: value 37.

N. empress, moderate, value 50.

N. albicans, moderate, higher than in N. empress, value 55 .

N. madame de graaff, moderate, the same as in N. empress, value 50. Gentian violet:

N. empress, light to moderate, value 43.

N. albicans, light to moderate, somewhat less than in N. empress, value 40 .

N. madame de graaff, light to moderate, the same as in N. empress, Safranin: value 43.

N. empress, moderate, value 53 .

N. albicans, moderate, somewhat less than in N. empress, value 50 . N. madame de graaff, moderate, the same as in N. empress, value 53. Temperature:

N. empress, in majority at 70 to $71^{\circ}$, in all at 73 to $74^{\circ}$, mean $73.5^{\circ}$. $\mathrm{N}$. albicans, in majority at 70.2 to $72^{\circ}$, in all at 73 to $75^{\circ}$, mean $74^{\circ}$. N. madame de graaff, in majority at 70 to $72^{\circ}$, in all at 73.5 to $75^{\circ}$, mean $74.25^{\circ}$

The reactivity of $N$. empress is higher than that of the other parent in the reactions with polarization, gentian violet, safranin, and temperature; and lower in the iodine reaction. The reactivity of the hybrid is the same or practically the same as that of $N$. empress in the reactions with iodine, gentian violet, and safranin, and the same or practically the same as that of the other parent in the polarization, iodine, and temperature reactions. In no reaction is there intermediateness of the hybrid.

Table A 20 shows the reaction-intensities in percentage of total starch gelatinized at definite time-intervals.

\section{Velocity-Reaction CuRves.}

This section treats of the velocity-reaction curves of the starches of Narcissus empress, $N$. albicans, and $N$. madame de graaff, showing the quantitative differences in the behavior toward different reagents at definite time-intervals. (Charts D 317 to D. 322.)
TABLE A 20.

\begin{tabular}{|c|c|c|c|c|c|c|c|c|c|}
\hline & - & ๙ & $\begin{array}{l}\text { 日 } \\
\infty\end{array}$ & $\begin{array}{l}\text { g } \\
+\end{array}$ & 自 & $\begin{array}{c}\dot{घ} \\
\mathscr{2}\end{array}$ & 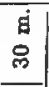 & $\begin{array}{l}\text { 日 } \\
\text { s }\end{array}$ & $\begin{array}{l}\dot{8} \\
8\end{array}$ \\
\hline Chloral hydrate: & & & & & & & & & \\
\hline & $\ldots$ & $\ldots$ & $\ldots$ & .. & 0.5 & 5 & 16 & 23 & \\
\hline N. a & $\cdots$ & $\ldots$ & . & .. & 0.5 & 14 & 31 & 40 & 43 \\
\hline N. madame de & & .. & .. & .. & 4 & 20 & 35 & 43 & 48 \\
\hline Chromic & & & & & & & & & \\
\hline N. em & $\cdots$ & . & . & . & 2 & 45 & 92 & 98 & 99 \\
\hline N. a & . . & .. & .. & . & 11 & 75 & 98 & 99 & \\
\hline N. $\mathrm{n}$ & $\cdots$ & .. & . & . & 1 & 33 & 77 & 91 & 98 \\
\hline Pyrogallic a & & & & & & & & & \\
\hline N. $e$ & $\cdots$ & & . & . & 3 & 13 & 50 & 61 & 78 \\
\hline & . & . & . & .. & 25 & 78 & 91 & 95 & 97 \\
\hline N. $\mathrm{r}$ & & .. & . . & .. & 1 & 32 & 56 & 68 & 79 \\
\hline Nitric acid & & & & & & & & & \\
\hline N. & . & . & . . & . & 12 & 52 & 58 & 65 & 70 \\
\hline & $\cdots$ & . & . & . & 33 & 78 & 82 & 86 & 86 \\
\hline & .. & . & .. & .. & 10 & 29 & 49 & 55 & 65 \\
\hline Sulphuri & & & & & & & & & \\
\hline N. empress. . & .. & 95 & . & $\cdots$ & $\cdots$ & -. & $\cdots$ & . & . \\
\hline & . & 99 & $\cdots$ & $\ldots$ & $\ldots$ & . & $\cdots$ & $\cdots$ & . \\
\hline N. madame de graafi & . & 98 & . & . & $\cdots$ & & $\cdots$ & & \\
\hline
\end{tabular}

The most conspicuous features of these charts are:

(1) The close correspondence in the courses of the three curves in all of the reactions (with the exception of the sulphuric-acid reaction, in which reaction is so rapid that there is no differentiation), and the tendency mostly to moderate to low reactivity.

(2) The varying relations of the parental curves to each other and the hybrid in the different reactions, excepting the sulphuric-acid reaction during the progress of the reactions.

(3) The curve of $N$. empress is distinctly lower than that of the other parent in the reactions with chloral hydrate, chromic acid, pyrogallic acid, and nitric acid, especially in that with pyrogallic acid.

(4) The hybrid curve is the highest of the three in the chloral-hydrate reaction; lowest with chromic acid and nitric acid; and intermediate with pyrogallic acid. In the reactions with chromic acid and nitric acid it is more closely related to $N$. empress, while in those with chloral hydrate and pyrogallic acid more closely related to $N$. albicans.

(5) A tendency to an early period of resistance fol lowed by a comparatively rapid reactivity is noticed in the reactions with chromic acid and pyrogallic acid-in all three starches in the former and in two in the latter. There are also suggestions of early resistance in the other two reactions.

(6) The earliest period at which the three curves are best separated for differential purposes is in the sulphuric-acid reaction at the very beginning of the reactions; in those with chromic acid, pyrogallic acid, nitric acid, and chloral hydrate at 15 minutes.

\section{REACTION-INTENSITIES OF THE HyBRID.}

This section treats of the reaction-intensities of the hybrid as regards sameness, intermediateness, excess, and deficit in relation to the parents. (Table A 20 and Charts D $31 \%$ to D 322.)

The reactivities of the hybrid are the same as those of the seed parent in the reactions with iodine, gentian violet, and safranin; the same as those of the pollen parent in the polarization reaction; the same as those of both parents in' none; intermediate with pyrogallic 
acid, and closer to that of the seed parent; highest with chloral hydrate, and nearer that of the pollen parent; and lowest with temperature, chromic acid, and nitric acid, in being closer to that of the seed parent and in three being closer to those of the pollen parent.

The following is a summary of the reaction-intensities (10 reactions): Same as seed parent, 4 ; same as pollen parent, 2; same as both parents, 0 ; intermediate, 1 ; highest, 1 ; lowest, 2.

The seed parent seems to be far more potent in determining the characters of the starch of the hybrid. The tendency to sameness or inclination of the hybrid to the seed parent is quite marked.

\section{Composite Curves of the Reaction-intensities.}

This section treats of the composite curves of the reaction-intensities, showing the differentiation of the starches of Narcissus empress, $N$. albicans, and $N$. madame de graaff. (Chart E 20.)

The most conspicuous features of this chart are:

(1) The close correspondence in the curves both as to course and nearness, the only well-marked tendency to departure being in the well-marked separation of the three curves in the chromic-acid reaction and of the parental curve in the pyrogallic-acid reaction.

(2) In $N$. empress in comparison with the other parent the higher reactions with polarization, gentian violet, and safranin; the lower reactions with iodine, chloral hydrate, chromic acid, pyrogallic acid, and nitric acid; and the same or practically the same reactions with temperature and sulphuric acid.

(3) In $N$.empress the very high reaction with sulphuric acid; the high reaction with chromic acid; the moderate reactions with polarization, iodine, gentian violet, and safranin; the low reactions with temperature, pyrogallic acid, and nitric acid; and the very low reaction with chloral hydrate.

(4) In $N$. albicans the very high reactions with sulphuric acid; the high reactions with chromic acid and pyrogallic acid; the moderate reactions with iodine, gentian violet, and safranin; the low reactions with polarization, temperature, and nitric acid; and the very low reaction with chloral hydrate.

(5) In the hybrid the very high sulphuric-acid reaction; the absence of a high reaction; the moderate reactions with iodine, gentian violet, safranin, and chromic acid; the low reactions with polarization, temperature, pyrogallic acid, and nitric acid; and the very low reaction with chloral hydrate. The following is a summary of the reaction-intensites (10 reactions) :

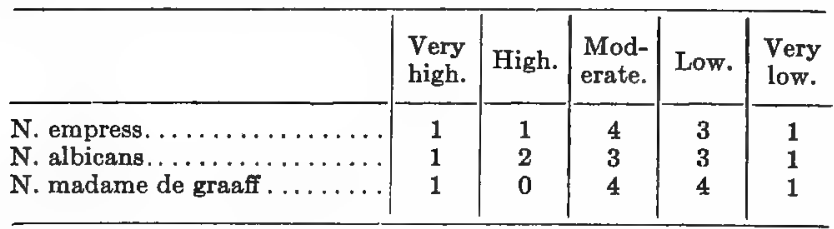

21. Comparisons of the Starches of Narcissus WEARDALE PERFECTION, N. MADAME DE GRAAFF, AND N. PYRAMUS.

In histologic characteristics, polariscopic figures, reactions with selenite, reactions with iodine, and qualitative reactions with the various chemical reagents the starches of the parents and hybrid have properties in common in varying degrees of development together with certain individualities which collectively in each case is distinctive of the starch. The differences are, however, for the most part of a very minor character. In histologic properties the parental starches differ in that in Narcissus madame de graaff in comparison with the other parent the relative number of compound grains and number of grains having both primary and secondary starch deposits are more numerous, there are more irregularities, and there is a larger number of forms. The hilum is not so often fissured or so deeply, and somewhat less eccentric; the lamellæ are somewhat less distinct and not so coarse; and the grains are, on the whole, larger. In the polariscopic figure there is less distinctness and definition and other differences, and in the selenite reaction the quadrants are less clean-cut and more of ten irregular, and the colors somewhat more pure, and there are more grains with a greenish tinge. In the qualitative iodine reactions the capsules color a red or reddish violet instead of nearly a reddish violet as in $N$. weardale perfection. In the reactions with chloral hydrate, chromic acid, pyrogallic acid, nitric acid, and sulphuric acid there are many differences, chiefly of minor importance, but which collectively distinguish one starch from the other. The starch of the hybrid shows in form, character, and eccentricity of the hilum, and character of the lamellæ a closer relationship to $N$. madame de graaff than to the other parent, but in size the opposite. In the polarization figure and appearances with selenite the relationship is closer to $N$. madame de graaff, but in the qualitative iodine reactions the relationship is reversed. In the reactions with the chemical reagents variable relationships, and hence the influences of one or the other or both parents, are recorded, and in some instances parental characteristics are exaggerated in the hybrid; but in all of the five reactions the relationships are, on the whole, closer to $N$. weardale perfection than to $N$. madame de graaff.

Reaotion-intensities Expressed by Light, Color, and TemperaPolarization: ture Reactions.

N. weardale perfect., low to high, value 37 .

N. madame de graaff, low to high, the same as in N. weardale perfection, value 37

$\mathrm{N}$. pyramus, low to high, higher than in either parent, value 42 .

Iodine:

N. weardale perfect., moderate, value 55 .

N. madame de graaff, moderate, less than in N. weardale perfection, value 50 .

$\mathrm{N}$. pyramus, moderate, the same as in $\mathrm{N}$. weardale perfection, value 55 .

Gentian violet:

N. weardale perfect., light to moderate, value 30 .

$\mathrm{N}$. madame de graaff, light to moderate, much more than in $\mathrm{N}$. weardale perfection, value 43 .

$\mathrm{N}$. pyramus, light to moderate, little less than in $\mathrm{N}$. weardale perfection, value 40 .

Safranin:

N. weardale perfect., light to moderate, value 40 .

N. madame de graaff, moderate, much more than in N. weardale perfection, value 53.

$\mathrm{N}$. pyramus, moderate, little less than in $\mathrm{N}$. weardale perfection, value 50.

Temperature:

N. weardale perfect., in majority at 68 to $69^{\circ}$, in all at 72 to $74^{\circ}$, mean $73^{\circ}$.

N. madame de graaff, in majority at 70 to $72^{\circ}$, in all at 73.5 to $75^{\circ}$, 'mean $74.25^{\circ}$.

N. pyramus, in majority at 73 to $74^{\circ}$, in all at 76 to $77^{\circ}$, mean $76^{\circ}$. 
The reactivity of $N$. weardale perfection is the same or practically the same as that of the other parent in the polarization reaction; higher in the iodine and temperature reactions; and lower in the gentian-violet and safranin reactions. The reactivity of the hybrid is the same or practically the same as that of $N$. weardale perfection in the iodine reaction; intermediate between those of the parents with gentian violet and safranin; lowest of the three in the temperature reaction; and the highest of the three in the polarization reaction.

Table A 21 shows the reaction-intensities in percentages of total starch gelatinized at definite intervals (minutes) :

TABLE A 21.

\begin{tabular}{|c|c|c|c|c|c|c|c|c|c|}
\hline & $\underset{-}{\text { 早 }}$ & 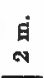 & $\underset{\infty}{\dot{B}}$ & $\underset{H}{\dot{q}}$ & घ & $\underset{\sim}{\stackrel{8}{g}}$ & $\begin{array}{l}\dot{g} \\
\text { 애 }\end{array}$ & $\begin{array}{l}8 \\
8 \\
2\end{array}$ & $\begin{array}{l}8 \\
8 \\
8\end{array}$ \\
\hline \multicolumn{10}{|l|}{ Chloral hydrate: } \\
\hline N. weardale perfect......... & . & $\cdots$ & $\cdots$ & $\cdots$ & 6 & 9 & 21 & 28 & 33 \\
\hline N. madame de graaff ....... & . & $\cdots$ & . & . & 4 & 20 & 35 & 43 & 48 \\
\hline N. pyramus . ............. & . & . & $\ldots$ & $\ldots$ & 2 & 5 & 19 & 21 & 23 \\
\hline \multicolumn{10}{|l|}{ Chromic acid: } \\
\hline N. weardale perfect......... & . & $\cdots$ & $\cdots$ & . & 5 & 40 & 91 & 99 & 99 \\
\hline N. madame de graaff... & $\cdots$ & . & . & . & 1 & 33 & 77 & 91 & 98 \\
\hline N. pyramus ......... & . & . & . & $\cdots$ & 7 & 64 & 95 & 99 & 99 \\
\hline \multicolumn{10}{|l|}{ Pyrogallic acid: } \\
\hline N. weardale perfect......... & $\cdots$ & $\cdots$ & $\cdots$ & $\cdots$ & $\mathbf{3}$ & 37 & 79 & 86 & 91 \\
\hline N. madame de graaff ....... & . & . & $\cdots$ & . & 1 & 32 & 56 & 68 & 79 \\
\hline N. pyramus . ....... & . & . & $\cdots$ & $\cdots$ & 10 & 50 & 80 & 88 & 91 \\
\hline \multicolumn{10}{|l|}{ Nitric acid: } \\
\hline N. weardale perfect.... & . & $\cdots$ & $\cdots$ & $\cdots$ & 11 & 48 & 57 & 66 & 69 \\
\hline N. madame de graaff .... & . & $\cdots$ & $\cdots$ & $\cdots$ & 10 & 29 & 49 & 58 & 65 \\
\hline N. pyramus. . . . . . . . & . & . & $\cdots$ & . & 18 & 54 & 63 & 70 & 75 \\
\hline \multicolumn{10}{|l|}{ Sulphuric acid: } \\
\hline N. weardale perfect... & $\cdots$ & 98 & $\cdots$ & $\cdots$ & $\cdots$ & $\cdots$ & $\cdots$ & $\cdots$ & $\cdots$ \\
\hline N. madame de graaff . & $\cdots$ & 98 & $\cdots$ & $\cdots$ & $\cdots$ & $\cdots$ & $\cdots$ & . & $\cdots$ \\
\hline N. pyramus.............. & $\cdots$ & 99 & $\cdots$ & $\cdots$ & $\cdots$ & $\cdots$ & $\cdots$ & $\cdots$ & $\cdots$ \\
\hline
\end{tabular}

Velocity-reaction CuRves.

This section treats of the velocity-reaction curves of the starches of Narcissus weardale perfection, N. madame de graaff, and $N$. pyramus, showing the quantitative differences in the behavior toward different reagents at definite time-intervals. (Charts D 323 to D 328.)

The most conspicuous features of these charts are:

(1) The close correspondence of the curves in each of the reactions during their progress (the curves of the sulphuric-acid reaction are identical, owing to the extremely rapid reaction), and the tendency of the reactions to be moderate to low.

(2) The varying relations of the parental curves to each other and the hybrid in the different reactions and (excepting with sulphuric acid) during the progress of the reactions.

(3) The curve of $N$. weardale perfection is lower than the curve of the other parent in the chloral-hydrate reaction; higher in those of chromic acid, pyrogallic acid, and nitric acid; and the same in that of sulphuric acid. In all except the latter they are sufficiently well separated for positive differentiation.

(4) The curve of the hybrid is the lowest of the three in the reaction with chloral hydrate; and the highest with chromic acid, pyrogallic acid, and nitric acid. The relationship is closer to $N$. weardale perfection in the chloral-hydrate reaction; and to this parent at first and to the other parent later in the reactions with chromic acid, pyrogallic acid, and nitric acid. On the whole, however, the relationship is distinctly closer to $N$. weardale perfection.

(5) A tendency to an early period of resistance followed by comparatively rapid reactivity is noted in the reactions with chromic acid and pyrogallic acid, with suggested resistance in the chloral hydrate reaction.

(6) The earliest period at which the three curves are best separated for differential purposes is in the sulphuric-acid reaction at the very beginning of the reaction; in the reactions with chromic acid, pyrogallic acid, and nitric acid at 15 minutes; and in the chloral-hydrate reaction at 60 minutes, or probably quite as good at 15 minutes.

\section{Reaction-Intensities of the HybRid.}

This section treats of the reaction-intensities of the hybrid as regards sameness, intermediateness, excess, and deficit in relation to the parents. (Table $A 21$ and Charts D 323 to D 328.)

The reactivities of the hybrid are the same as those of the seed parent in the iodine reaction; the same as those of the pollen parent in none; the same as those of both parents in the sulphuric-acid reaction, in which the reactions occur too rapidly for differentiation; intermediate in the reactions with gentian violet and safranin, in both being closer to those of the pollen parent; highest in the reactions with polarization, chromic acid, pyrogallic acid, and nitric acid, in one being as close to one as to the other parent, and in three closer to the seed parent; and lowest with temperature and chloral hydrate, in both being closer to the pollen parent.

The following is a summary of the reaction-intensities (10 reactions): Same as seed parent, 1; same as pollen parent, 0 ; same as both parents, 1 ; intermediate, 2 ; highest, 4 ; lowest, 2.

The seed parent exercises a distinctly more marked influence than the other parent in determining the characters of the starch of the hybrid. The almost entire absence of sameness to one or the other parent and the tendency, on the other hand, to highest and lowest reactivities are conspicuous features of the reactions of the hybrid.

\section{Composite CuRves of Reaction-intensities.}

This section treats of the composite curves of the reaction-intensities, showing the differentiation of the starches of Narcissus weardale perfection, $N$. madame de graaff, and $N$. pyramus. (Chant E 21.)

The most conspicuous features of this chart are:

(1) The close correspondence of all three curves both as to course and nearness, the only well-marked tendency to departure being in the chromic-acid reaction in which all three curves tend to be well separated.

(2) In $N$. weardale perfection in comparison with the other parent the higher reactions with iodine, temperature, chromic acid, pyrogallic acid, and nitric acid; the lower reactions with gentian violet, safranin, and chloral hydrate; and the same or practically the same reactions with polarization and sulphuric acid.

(3) In $N$. weardale perfection the very high sulphuric-acid reaction; the high chromic-acid reaction; the moderate reactions with iodine, safranin, and pyrogallic acid; the low reactions with polarization, gentian 
violet, temperature, and nitric acid; and the very low reaction with chloral hydrate.

(4) In $N$. madame de graaff the very high reaction with sulphuric acid; the absence of a high reaction; the moderate reactions with iodine, gentian violet, safranin, and chromic acid; the low reactions with polarization, temperature, pyrogallic acid, and nitric acid; and the very low reaction with chloral hydrate.

(5) In the hybrid the very high reaction with sulphuric acid; the high reaction with chromic acid; the moderate reactions with polarization, iodine, gentian, violet, safranin, and pyrogallic acid; the low reactions with temperature and nitric acid; and the very low reaction with chloral hydrate.

The following is a summary of the reaction-intensities (10 reactions):

\begin{tabular}{l|c|c|c|c|c}
\hline & $\begin{array}{c}\text { Very } \\
\text { high. }\end{array}$ & High. & $\begin{array}{c}\text { Mod- } \\
\text { erate. }\end{array}$ & Low. & $\begin{array}{c}\text { Very } \\
\text { low. }\end{array}$ \\
\hline N. weardale perfection......... & 1 & 1 & 3 & 4 & 1 \\
N. madame de graaff ........ & 1 & 0 & 4 & 4 & 1 \\
N. pyramus............... & 1 & 1 & 5 & 2 & 1 \\
\hline
\end{tabular}

\section{Comparisons of the Starches of Narcissus MONARCH, N. MADAME DE GRAAFF, AND N. LORD ROBERTS.}

In histologic characteristics, polariscopic figures, reactions with selenite, reaction with iodine, and reactions with the various chemical reagents the starches of the parents and hybrid have properties in common in varying degrees of development, the sum of which in case of each starch is distinctive of the starch. Such differences, as recorded, are of a minor character. The starch of $N$. madame de graaff, in comparison with that of the other parent, shows more aggregates and fewer compound grains, and the latter grains contain a larger number of components; there are more simple grains having both primary and secondary starch formation; and there is more irregularity and a greater variety of form. There is less fissuration of the hilum and more eccentricity. The lamellæ are more often visible, somewhat more distinct, and not so coarse. The grains are, on the whole, smaller. The polariscopic figure is more distinct and there are other minor differences; and with selenite the quadrants are more often clear-cut and less irregular in form. No qualitative differences were recorded in the iodine reactions. In the qualitative reactions with chloral hydrate, chromic acid, pyrogallic acid, nitric acid, and sulphuric acid there are various minor differences which collectively serve to differentiate the starches. The starch of the hybrid has more aggregates and compound grains than either parent and the grains are in form closer related to those of $N$. monarch than to those of the other parent. In the character and eccentricity of the hilum the relationship is closer to $N$. monarch; but in the character of the lamellæ and in the size of the grains to $N$. madame de graaff. In the polariscopic figure and reactions with selenite the relationship is closer to $N$. madame de graaff. In the qualitative reactions with iodine no differences were recorded in the three starches. In the qualitative reactions with chloral hydrate, chromic acid, pyrogallic acid, nitric acid, and sulphuric acid characteristics of both parents are manifest, certain reactions resembling in certain respects those of one parent and other reactions those of the other. The relationship is closer to $N$. monarch in the reactions with chloral hydrate and sulphuric acid; but closer to $N$. madame de graaff in those with chromic acid, pyrogallic acid, and nitric acid. The characters throughout indicate a close relationship of all three starches.

\section{Reaction-intensities Expressed by Light, Color, and Tempera} Polarization:

N. monarch, low to high, value 40 .

N. madame de graaff, low to high, somewhat lower than in $N$. monarch, value 37 .

N. lord roberts, low to high, the same as in N. madame de graaff, Iodine: value 37 .

N. monarch, moderate, value 50 .

N. madame de graaff, moderate, the same as in N. monarch, value 50.

N. lord roberts, moderate, the same as in the parent, value 50. Gentian violet:

N. monarch, moderate, value 45 .

N. madame de graaff, moderate, slightly less than in N. monarch, value 43.

N. lord roberts, moderate, the same as in N. monarch, value 45 . Safranin:

N. monarch, moderate, value 50.

N. madame de graaff, moderate, slightly more than in N. monarch, value 53.

N. lord roberts, moderate, the same as in N. monarch, value 50 .

Temperature:

N. monarch, in majority at 67 to $68.5^{\circ}$, in all at 72 to $73^{\circ}$, mean $72.5^{\circ}$.

N. madame de graaff, in majority at 70 to $72^{\circ}$, in all at 73.5 to $75^{\circ}$, mean $74.25^{\circ}$.

$\mathrm{N}$. lord roberts, in majority at 68 to $69.4^{\circ}$, in all at 73 to $74.5^{\circ}$, mean $73.75^{\circ}$.

The reactivity of $N$. monarch is higher than that of the other parent in the reactions with polarization, gentian violet, and temperature; the same or practically the same with iodine; and lower with safranin. The reactivity of the hybrid is the same or practically the same as those of the parents in the reaction with iodine; the same or practically the same as that of $N$. monarch with gentian violet and safranin; the same or practically the same as that of the other parent with polarization;

TABLE A 22.

\begin{tabular}{|c|c|c|c|c|c|c|c|c|c|}
\hline & घ & $\underset{\text { a }}{\text { G }}$ & 宣 & 五 & $\infty$ & $\mid \begin{array}{l}a \\
\text { a } \\
\text { 10 }\end{array}$ & $\begin{array}{l}\dot{\theta} \\
\text { 吕 }\end{array}$ & $\mid \begin{array}{l}\text { 日 } \\
\text { us }\end{array}$ & 昌 \\
\hline \multicolumn{10}{|l|}{ Chloral hydrate: } \\
\hline N. monarch. & $\cdots$ & $\cdots$ & .. & & 2 & 10 & 18 & 20 & \\
\hline & & & $\cdots$ & $\cdots$ & 4 & 20 & 35 & 43 & 48 \\
\hline & . & $\therefore$ & $\ldots$ & .. & & 11 & 20 & 27 & \\
\hline \multicolumn{10}{|l|}{ Chromic acid: } \\
\hline N. monarch & . & .. & $\cdots$ & .. & 33 & 71 & 95 & 99 & 99 \\
\hline & . & $\cdots$ & $\cdots$ & . & 1 & 33 & 77 & 91 & 98 \\
\hline & .. & . & $\cdots$ & . & 1 & 15 & 50 & 72 & 88 \\
\hline \multicolumn{10}{|l|}{ Pyrogallic acid: } \\
\hline & . & . & . & $\cdots$ & 7 & 56 & 72 & 82 & 86 \\
\hline & .. & . & .. & . & 1 & 32 & 56 & 68 & 79 \\
\hline N. lord rob & .. & $\cdots$ & $\cdots$ & .. & 2 & 36 & 63 & 73 & 33 \\
\hline \multicolumn{10}{|l|}{ Nitric acid: } \\
\hline N. monarch & . & $\cdots$ & $\cdots$ & . & 20 & 64 & 72 & 78 & \\
\hline N. m & $\cdots$ & $\cdots$ & $\cdots$ & .. & 10 & 29 & 49 & 58 & 65 \\
\hline $\begin{array}{l}\text { N. lord robert } \\
\text { Sulphuric acid: }\end{array}$ & \multicolumn{9}{|c|}{ Sulphuric acid: } \\
\hline N. monarch. & . & 96 & . & . & $\cdots$ & .. & $\cdots$ & . & \\
\hline N. $\mathrm{mad}$ & $\cdots$ & 98 & . & $\cdots$ & $\cdots$ & $\cdots$ & $\cdots$ & $\cdots$ & $\cdots$ \\
\hline N. Iord robert & & 95 & & & & & & & \\
\hline
\end{tabular}


and intermediate with temperature, but closer to $N$. madame de graaff.

Table A 22 shows the reaction-intensities of the starches expressed by the percentage of total starch gelatinized at definite time-intervals.

Velocity-Reaction CuRves.

This section treats of the velocity-reaction curves of the starches of Narcissus monarch, N. madame de graaff, and $N$. lord roberts, showing the quantitative differences in the behavior toward different reagents at definite time-intervals. (Charts D 329 to D 334.)

The most conspicuous features of these charts are:

(1) The correspondence in the courses of the three curves in all of the reactions (excepting the sulphuricacid reaction in which gelatinization is too rapid for differentiation), and the tendency to moderate to low reactivity. Inclination to separation of the curves is comparatively well marked in the pyrogallic acid.

(2) The varying relations of the parental curves to each other and to the curve of the hybrid in all of the reactions (excepting in that with sulphuric acid) during their progress.

(3) The curve of $N$. monarch is distinctly lower than that of the other parent in the reactions with chloral hydrate and pyrogallic acid; distinctly higher with chromic acid and nitric acid; and the same with iodine and sulphuric acid.

(4) The curve of the hybrid is intermediate in the reactions with chloral hydrate, pyrogallic acid, and nitric acid, but close to $N$. monarch with chloral hydrate and nitric acid, and to the other parent with pyrogallic acid; and the lowest of the three and well separated from the parental curves in the chromic-acid reaction.

(5) A tendency to an early period of resistance followed by comparatively high reactivity is evident, especially in the three starches in the pyrogallic-acid reaction and in two starches in the chromic-acid reaction, with a suggestion of resistance in the reactions with chloral hydrate and nitric acid.

(6) The earliest period at which the three curves are best separated for differential purposes is in the reaction with sulphuric acid at the very beginning; in those with chromic acid, pyrogallic acid, and nitric acid probably at 15 minutes; and with chloral hydrate at 60 minutes.

\section{Reaction-intensities of the Hrbaid.}

This section treats of the reaction-intensities of the hybrid as regards sameness, intermediateness, excess, and deficit in relation to the parents. (Table A 22 and Charts D 329 to D 334.)

The reactivities of the hybrid are the same as those of the seed parent in the reactions with gentian violet, safranin, and sulphuric acid; the same as those of the pollen parent in the polarization reaction; the same as those of both parents in the iodine reaction; intermediate in the reactions with temperature, chloral hydrate, pyrogallic acid, and nitric acid, being closer to the seed parent in two and to the pollen parent in two; highest in none; and lowest in the chromic-acid reaction.

The following is a summary of the reaction-intensities (10 reactions): Same as seed parent, 3 ; same as pollen parent, 1 ; same as both parents, 1 ; intermediate, 4 ; highest, 0 ; lowest, 1.
The parents appear to share about equally the determination of the properties of the starch of the hybrid. There is obviously a tendency to intermediateness, this being recorded in nearly half of the reactions.

\section{Composite Curves of Reaction-intensites.}

This section treats of the composite curves of the reaction-intensities, showing the differentiation of the starches of Narcissus monarch, $N$. madame de graaff, and $N$. lord roberts. (Chart E 22.)

The most conspicuous features of this chart are:

(1) The very close correspondence in all three curves in nearness and during their course, excepting in the chromic-acid reaction, in which the curve of $N$. monarch is well separated from the curves of the other parent and the hybrid.

(2) In $N$. monarch in comparison with the other parent the higher reaction with polarization, gentian violet, temperature, chromic acid, pyrogallic acid, and nitric acid; the lower with chloral hydrate; and the same with iodine and sulphuric acid.

(3) In $N$. monarch the very high sulphuric-acid reaction; the high chromic-acid reaction; the moderate reactions with polarization, iodine, gentian violet, safranin, and temperature; the low reactions with pyrogallic and nitric acids; and the very low reaction with chloral hydrate.

(4) In $N$. madame de graaff the very high sulphuricacid reaction; the absence of a high reaction; the moderate reactions with iodine, gentian violet, safranin, and chromic acid; the low reactions with polarization, temperature, pyrogallic acid, and nitric acid; and the very low reaction with chloral hydrate.

The following is a summary of the reaction-intensities (10 reactions) :

\begin{tabular}{l|c|c|c|c|c}
\hline & $\begin{array}{c}\text { Very } \\
\text { high. }\end{array}$ & High. & $\begin{array}{c}\text { Mod- } \\
\text { erate. }\end{array}$ & Low. & $\begin{array}{c}\text { Very } \\
\text { low. }\end{array}$ \\
\hline N. monarch. $\ldots \ldots \ldots \ldots \ldots \ldots \ldots$ & 1 & 1 & 5 & 2 & 1 \\
N. madame de gragff $\ldots \ldots \ldots \ldots$ & 1 & 0 & 4 & 4 & 1 \\
N. lord roberts............ & 1 & 0 & 4 & 4 & 1 \\
\hline
\end{tabular}

23. Comparisons of the Starches of Narcissus LEEDSII MINNIE HUME, N. TRIANDRUS ALBUS, AND N. AGNES HARVEY.

In histologic characteristics, polariscopic figures, reactions with selenite, reactions with iodine, and qualitative reaction with the various chemical reagents the starches of the parents and hybrid exhibit properties in common in varying degrees of development, which collectively are in each case distinctive. The differences are, on the whole, of a minor character, indicating close relationships of the three starches. In histologic properties in Narcissus triandrus albus in comparison with the other parent there are found a larger proportion of compound grains but fewer aggregates, somewhat fewer grains with primary and secondary deposits, and the grains are less irregular; the hilum is more often more deeply and more extensively fissured; the lamellæ are less often distinct and not so fine; and the grains are, as a whole, smaller than in $N$. leedsii minnie hume. 
The polariscopic figure is better defined and there are some differences in the lines. With selenite the quadrants are more often clean-cut and more regular in shape, the colors more often pure, and there are more grains having a greenish tinge. In the qualitative iodine reactions the capsules are more reddish than those of $N$. leedsii minnie hume. In the reactions with the chemical reagents there are various differences of a minor character which collectively differentiate each starch. The starch of the hybrid contains fewer compound grains and aggregates than either parent, and the relationship is, on the whole, closer to $N$. leedsii minnie hume than to the other parent. In the character of the hilum and character of the lamellæ the relationship is closer to $N$. leedsii minnie hume, while in size to $N$. triandrus albus. In the polariscopic figure and appearances with selenite the resemblances are closer to $N$. leedsii minnie hume, and the same is true of the qualitative iodine reactions. In the qualitative reactions with the chemical reagents the influences of both parents are manifest, and there are also individualities of a minor character of the hybrid. In all of these reactions the characters are, as a whole, more closely associated with those of $N$. leedsii minnie hume.

Reaction-intensities Expressed by Light, Color, and Tempera-

Polarization:

N. leedsii min. hume, low to very high, value 45 .

N. triandrus albus, low to high, higher than in N. leedsii minnie hume, value 50 .

$\mathrm{N}$. agnes harvey, low to high, the same as in $\mathbf{N}$. leedaii minnie

Iodine: hume, value 45 .

N. leedsii min. hume, moderate deep, value 60 .

N. triandrus albus, deep, deeper than in N. leedsii minnie hume, value 65 .

$\mathrm{N}$. agnes harvey, deep, the same as in N. leedsii minnie hume, value 60 .

Gentian violet:

N. leedsii min. hume, light to moderate, value 38 .

N. triandrus albus, light to moderate, lighter than in N. leedsii minnie hume, value 35 .

N. agnes harvey, light to moderate, the same as in N. leedsii

Safranin: minnie hume, value 38

N. leedsii min. hume, light to moderate, value 40.

N. triandrus albus, light to moderate, the same as in N. leedsii minnie hume; value 40.

N. agnes harvey, light to moderate, the same as in the parents, value 40.

Temperature:

N. leedsii min. hume, in majority at 70 to $71.2^{\circ}$, in all at 74.5 to $76^{\circ}$, mean $75.25^{\circ}$.

N. triandrus albus, in majority at 70 to $71^{\circ}$, in all at 73 to $75^{\circ}$, mean $74^{\circ}$.

N. agnes harvey, in majority at 70 to $71.8^{\circ}$, in all at 73.8 to $75^{\circ}$, mean $74.4^{\circ}$.

The reactivity of $N$. leedsii minnie hume is lower than that of the other parent in the polarization, iodine, and temperature reactions; the same or practically the same in the safranin reaction; and higher in the gentianviolet reaction. The reactivity of the hybrid is the same or practically the same as that of $N$. leedsii minnie hume in the polarization, iodine, and gentian-violet reactions; the same or practically the same as those of both parents in the safranin reaction; and intermediate in the temperature reaction, but closer to $N$. triandrus albus. All three starches are in these reactions either the same or practically the same or very nearly alike.
Table A 23 shows the reaction-intensities in percentages of total starch gelatinized at definite intervals (minutes) :

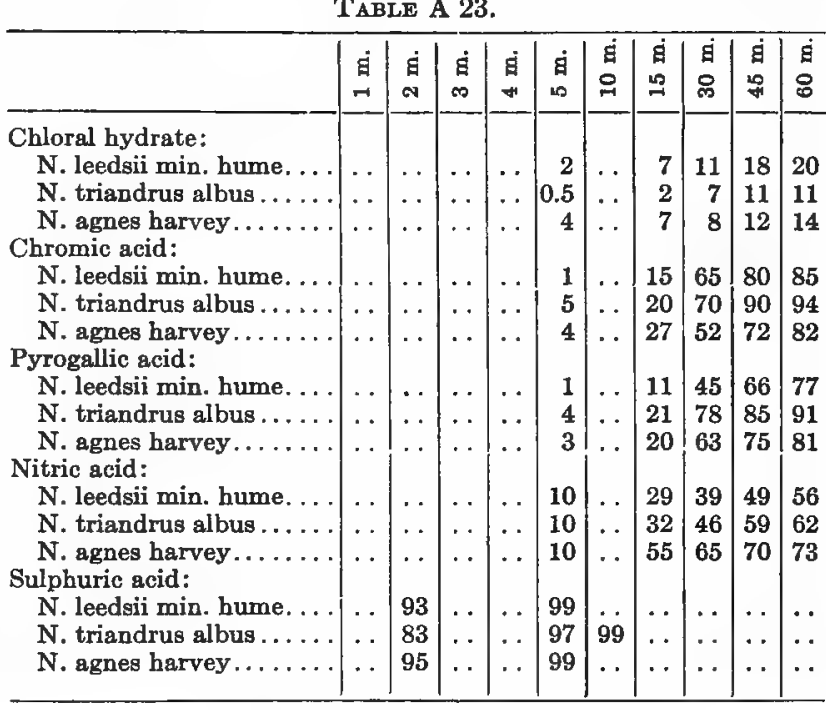

\section{Velocity-REaction CURVES.}

This section deals with the velocity-reaction curves of the starches of Narcissus leedsii minnie hume, $N$. triandrus albus, and $N$. agnes harvey, showing the quantitative differences in the behavior toward different reagents at definite time-intervals. (Charts D 335 to D 340.)

The most conspicuous features of these charts are:

(1) The close correspondence of all three starches in all of the reactions (with the exception of the sulphuric-acid reaction, which is too rapid for differentiation), and the tendency (with this exception) to a moderate, low, or very low reactivity.

(2) The varying relations of the parental curves to each other and to the curve of the hybrid in the different reactions (excepting the very rapid sulphuric-acid reaction) and during their progress.

(3) The curve of $N$. leedsii minnie hume is lower than that of the other parent in the reactions with chromic acid, pyrogallic acid, and nitric acid; and higher with chloral hydrate.

(4) The hybrid curve is the lowest of the three in the chromic-acid reaction; intermediate in the reactions with chloral hydrate and pyrogallic acid, but in the latter practically identical with that of $N$. triandrus albus; and highest with nitric acid.

(5) A tendency to a period of early resistance followed by a comparatively rapid reactivity is seen in all three starches in the chromic-acid and pyrogallicacid reactions.

(6) The earliest period at which the three curves are best separated for differential purposes is in the sulphuric-acid reaction at the very beginning of the reaction; in the reactions with chromic acid, pyrogallic acid, and nitric acid at 30 to $\mathbf{4 5}$ minutes, and with chromic acid at 60 minutes.

\section{Reaction-INTENSITIES OF THE HYBRID.}

This section treats of the reaction-intensities of the hybrid as regards sameness, intermediateness, excess, and 
deficit in relation to the parents. (Tables A 23 and Charts D 335 to D. 340.)

The reactivities of the hybrid are the same as those of the seed parent in the reactions with polarization, iodine, gentian violet, and sulphuric acid; the same as those of the pollen parent in none; the same as those of both parents in the safranin reaction; intermediate in those with temperature, chloral hydrate, and pyrogallic acid, in two being closer to those of the pollen parent and in one as close to one as the other parent: highest in the nitric-acid reaction, and closer to the pollen parent; and lowest in the chromic-acid reaction, being closer to the seed parent.

The following is a summary of the reaction-intensities (10 reactions) : Same as seed parent, 4 ; same as pollen parent, 0 ; same as both parents, 1 ; intermediate, 3 ; highest, 1; lowest, 1.

From the foregoing data it seems that the seed parent exercises a distinctly greater influence than the pollen parent on the characters of the starch of the hybrid. The most marked tendencies in the reactions are to sameness as the seed parent and to intermediateness.

\section{Composite CuRves of Reaction-intensities.}

This section treats of the composite curves of the reaction-intensities, showing the differentiation of the starches of Narcissus leedsii minnie hume, $N$. triandrus albus, and N. agnes harvey. (Chart E 23.)

The most conspicuous features of this chart are:

(1) The very close correspondence of all three curves in course and closeness throughout the chart.

(2) In $N$. leedsii minnie hume in comparison with the other parent the higher gentian-violet and chloralhydrate reactions; the lower reactions with polarization, iodine, temperature, chromic acid, pyrogallic, and nitric acid; and the same or practically the same in the reactions with safranin and sulphuric acid.

(3) In N. leedsii minnie hume the very high sulphuric-acid reaction; the high iodine reaction; the moderate polarization and safranin reactions; the low reactions with gentian violet, temperature, chromic acid, pyrogallic acid, and nitric acid; the very low reaction with chloral hydrate.

(4) In $N$. triandrus albus the very high sulphuricacid reaction; the high iodine reaction; the moderate reactions with polarization, safranin, chromic acid, and pyrogallic acid; the low reactions with gentian violet, temperature, and nitric acid; and the very low reaction with chloral hydrate.

(5) In the hybrid the very high sulphuric-acid reaction; the high iodine reaction; the moderate polarization and safranin reactions; the low gentian-violet, temperature, chromic-acid, pyrogallic-acid, and nitric-acid reactions; and the very low chloral hydrate reaction.

The following is a summary of the reaction-intensities (10 reactions):

\begin{tabular}{l|c|c|c|c|c}
\hline & $\begin{array}{c}\text { Very } \\
\text { high. }\end{array}$ & High. & $\begin{array}{c}\text { Mod- } \\
\text { erate. }\end{array}$ & Low. & $\begin{array}{l}\text { Very } \\
\text { low. }\end{array}$ \\
\hline N. leedsii minnie hume........ & 1 & 1 & 2 & $\mathbf{5}$ & $\mathbf{1}$ \\
N. triandrus albus........... & 1 & 1 & 4 & 3 & 1 \\
N. agnes harvey............. & 1 & 1 & 2 & $\mathbf{5}$ & 1 \\
\hline
\end{tabular}

24. Comparisons of the Starches of Narcisgus emperor, N. triandrus albUs, and N. J. T. BENNETT POE.

In histologic characteristics, polariscopic figures, reactions with selenite, reactions with iodine and qualitative reactions with the various chemical reagents, the starches of the parents and hybrid exhibit properties in common in varying degrees of development which collectively in case of each starch are distinctive. The differences are of a minor character. In histologic properties in Narcissus triandrus albus in comparison with the other parent there are more compound grains and aggregates, together with various other peculiarities, and there are various other differences in hilum, lamellæ, and size. The polariscopic figure is not so distinct but more often well defined, and there are other minor differences. With selenite the quadrants are more often clean-cut, the colors less often pure, and fewer grains with a greenish tinge. In the qualitative reactions with iodine no distinctive differences were recorded. In the qualitative reactions with chloral hydrate, chromic acid, pyrogallic acid, nitric acid, and sulphuric acid both methods of gelatinization common to both starches occur, and also methods observed in $N$. triandrus albus that are not seen or seen only in modified form in $N$. emperor. The starch of the hybrid contains fewer compound grains and aggregates than either parent, and shows, on the whole, a closer relationship to $N$. emperor than to the other parent. In character and eccentricity of the hilum and in size the relationship is closer to $N$. emperor; but in the character of the lamellæ closer to $N$. triandrus albus. In the character of the polarization figure and in the reactions with selenite the relationship is closer to $N$. triandrus albus. In the qualitative reactions with iodine the raw grains are more closely related to those of $N$. emperor, but the gelatinized grains show no differences from those of both parents. In the qualitative reactions with the chemical reagents the influences of both parents are manifest; in the chloral hydrate and sulphuric acid the resemblances are closer to $N$. emperor, while in the chromic acid, pyrogallic acid, and nitric acid the hybrid is closer to $N$. triandrus albus.

Reactiow-intensities Expressed by Light, Color, and TemperaPolarization:

N. emperor, low to high, value 60 .

$\mathrm{N}$. triandrus albus, low to high, lower than in N. emperor, value 50.

N. j. t. bennett poe, low to high, the same as in N. triandrus albus, value 60 .

Iodine:

N. emperor, moderate to deep, value 60.

N. triandrus albus, moderately deep, deeper than in N. emperor, value 65.

N. j. t. bennett poe, moderate to deep, the same as in N. emperor, value 60.

Gentian violet:

N. emperor, moderate, value 45 .

N. triandrus albus, light to moderate, lighter than in N. emperor, value 35 .

N. j. t. bennett poe, moderate, deeper than in either parent, value 50 , Safranin:

N. emperor, moderate, value 50 .

N. triandrus albus, light to moderate, lighter than in N. emperor, value 40.

N. j. t. bennett poe, moderate, deeper than in either parent, value 55. 
Temperature:

N. emperor, in majority at 69 to $71^{\circ}$, in all at 74 to $75.5^{\circ}$, mean $74.53^{\circ}$.

N. triandrus albus, in majority at 70 to $71^{\circ}$, in all at 73 to $75^{\circ}$, mean $74^{\circ}$.

N. j. t. bennett poe, in majority at 64 to $64.8^{\circ}$, in all at 69 to $71^{\circ}$, mean $70^{\circ}$.

The reactivity of $N$. emperor is higher than that of the other parent in the polarization, gentian violet, and safranin reaction; and lower in the iodine and temperature reactions. The reactivity of the hybrid is the same or practically the same as that of $N$. emperor in the polarization and iodine reactions; and the highest of the three in the gentian violet, safranin, and temperature reactions. There is no instance of intermediateness, and in certain respects the starch of the hybrid is nearer to one parent and in others to the other parent.

Table A 24 shows the reaction-intensities in percentages of total starch gelatinized at definite intervals (minutes):

Tabie A 24.

\begin{tabular}{|c|c|c|c|c|c|c|c|c|c|c|}
\hline & $\underset{-1}{\text { 早 }}$ & 囟 & $\underset{m}{\dot{B}}$ & $\underset{+1}{\dot{9}}$ & ${ }_{20}^{8}$ & $\begin{array}{l}\text { 日 } \\
\text { 울 }\end{array}$ & $\begin{array}{l}\text { 日 } \\
20\end{array}$ & 皃 & $\begin{array}{l}\text { 递 } \\
\stackrel{20}{+}\end{array}$ & $\begin{array}{l}\text { ㅁ } \\
8\end{array}$ \\
\hline \multicolumn{11}{|l|}{ Chloral hydrate: } \\
\hline N. emperor . .... & $\cdots$ & $\cdots$ & $\cdots$ & & 2 & $\cdots$ & 6 & 18 & 23 & 28 \\
\hline N. triandrus albus. & . & $\cdots$ & . & . & 0.5 & $\ldots$ & 2 & 7 & 11 & 11 \\
\hline N. j. t. bennett poe . . . . & . & $\cdots$ & . & . . & 4 & .. & 8 & 20 & 24 & 28 \\
\hline \multicolumn{11}{|l|}{ Chromic acid: } \\
\hline N. emperor . .. . & $\cdots$ & $\cdots$ & $\cdots$ & $\cdots$ & 3 & $\cdots$ & 39 & 75 & 94 & 97 \\
\hline N. triandrus albus... & . & . & $\cdots$ & . & 5 & . & 20 & 70 & 90 & 94 \\
\hline N. j. t. bennett poe ..... & . & . & . & . & 3 & .. & 51 & 87 & 95 & 99 \\
\hline \multicolumn{11}{|l|}{ Pyrogallic acid: } \\
\hline N. emperor . . . . & . & . & $\cdots$ & . & $\mathbf{5}$ & $\cdots$ & 20 & 74 & 82 & 93 \\
\hline N. triandrus albus.. & . & . & . & . & 4 & . & 21 & 78 & 85 & 91 \\
\hline N. j. t. bennett poe . & . & . & . & . & 20 & $\cdots$ & 60 & 85 & 95 & 98 \\
\hline \multicolumn{11}{|l|}{ Nitric acid: } \\
\hline N. emperor . . . . . . & $\ldots$ & . & $\cdots$ & . & 10 & . & 51 & 62 & 65 & 67 \\
\hline N. triandrus albus. . & . & . & . & . & 10 & $\cdots$ & 32 & 46 & 59 & 62 \\
\hline N. j. t. bennett poe & . & $\ldots$ & $\cdots$ & . & 15 & . & 57 & 63 & 69 & 72 \\
\hline \multicolumn{11}{|l|}{ Sulphuric acid: } \\
\hline N. emperor . . . . . & . & 94 & . & . & 99 & $\ddot{x}$ & $\cdots$ & $\cdots$ & $\cdots$ & . \\
\hline N. triandrus albus. & . & 83 & . & . & 97 & 99 & . & . & . & . \\
\hline N. j. t. bennett poe . . . . & . & 99 & . & . & . & . & . & . & . & .. \\
\hline
\end{tabular}

Velocity-REaCtion CuRtes.

This section treats of the velocity-reaction curves of the starches of Narcissus emperor, $N$. triandrus albus, and $N . j . t$. bennett poe, showing the quantitative differences in the behavior toward different reagents at definite time-intervals. (Charts D 341 to D 346.)

The most conspicuous features of these charts are:

(1) The correspondence in the three curves in all of the reactions, and the general tendency to a high to moderate reactivity.

(2) The varying relationships of the parental curves to each other and to the curve of the hybrid in the different reactions.

(3) The curve of $N$. emperor is practically the same as that of the other parent in the pyrogallic-acid reaction and higher in the reactions with chloral hydrate, chromic acid, pyrogallic acid, and sulphuric acid, the most marked difference being noted in the pyrogallicacid reaction and the least in the quick sulphuric-acid reaction.

(4) The curve of the hybrid is the same as that of $N$. emperor in the very rapid sulphuric-acid reaction; practically the same in that with chloral hydrate; nearly the same in that with pyrogallic acid; intermediate in none; and the highest of the three in those with chromic acid and pyrogallic acid. In all of the reactions the hybrid shows a higher reactivity than either parent.

(5) A tendency to an early period of resistance followed by a comparatively rapid reactivity is seen in all three starches in the reaction with chromic acid, and in the two parental starches in that with pyrogallic acid. The earliest period at which the three curves are best separated for differential purpose is in the sulphuric-acid reaction at the beginning; in those with chloral hydrate, chromic acid, pyrogallic acid, and nitric acid at 15 minutes.

\section{Reaction-InTENsities of THE Hybrid.}

This section treats of the reaction-intensities of the hybrid as regards sameness, intermediateness, excess, and deficit in relation to the parents. (Table A 24 and Charts D 341 to D 346.)

The reactivities of the hybrid are the same as those of the seed parent in the polarization and iodine reactions; the same as those of the pollen parent in none; the same as those of both parents in none; intermediate in none; highest in those with gentian violet, safranin, temperature, chloral hydrate, chromic acid, pyrogallic acid, nitric acid, and sulphuric acid (in six being closer to those of the seed parent, and in two closer to those of the pollen parent).

The following is a summary of the reaction-intensities (10 reactions): Same as seed parent, 2 ; same as pollen parent, 0 ; same as both parents, 0 ; intermediate, 0 ; highest, 8 ; lowest, 0 .

The seed parent seems to have almost entirely controlled the development of the properties of the hybrid, inasmuch as in 10 out of the 12 reactions there is sameness or nearness in relation to this parent. Another equally striking feature is the almost universal tendency for the reactivity of the hybrid to exceed parental extremes.

\section{Composite Curves of Reaction-intensities.}

This section treats of the composite curves of the reaction-intensities, showing the differentiation of the starches of Narcissus emperor, $N$. triandrus albus, and N. j.t. bennett poe. (Chart E 24.)

The most conspicuous features of this chart are:

(1) The close correspondence in the courses and closeness of the curves throughout the chart.

(2) In $N$. emperor in comparison with $N$. triandrus albus the higher reactions with polarization, gentian violet, safranin, chloral hydrate, and chromic acid; the lower reactions with iodine and nitric acid; and the same or practically the same reactions with temperature, pyrogallic acid, and sulphuric acid.

(3) In $N$. emperor the very high reaction with sulphuric acid; the high reactions with polarization and iodine; the moderate reactions with gentian violet, safranin, chromic acid, and pyrogallic acid; the low reactions with temperature and nitric acid; and the very low reaction with chloral hydrate.

(4) In $N$. triandrus albus the very high reaction with sulphuric acid; the high reaction with iodine; the moderate reactions with polarization, safranin, chromic acid, and pyrogallic acid; the low reactions with gentian 
violet, temperature, and nitric acid; and the very low reaction with chloral hydrate.

(5) In the hybrid the very high sulphuric-acid reaction; the high reactions with polarization, iodine, chromic acid, and pyrogallic acid; the moderate reactions with gentian violet, safranin, and temperature; the low reaction with nitric acid; and the very low reaction with chloral hydrate.

The following is a summary of the reaction-intensities (10 reactions) :

\begin{tabular}{l|c|c|c|c|c}
\hline & $\begin{array}{c}\text { Very } \\
\text { high. }\end{array}$ & High. & $\begin{array}{c}\text { Mod- } \\
\text { erate. }\end{array}$ & Low. & $\begin{array}{c}\text { Very } \\
\text { low. }\end{array}$ \\
\hline N. emperor ................ & 1 & 2 & 4 & 2 & 1 \\
N. triandrus albus........... & 1 & 1 & 4 & 3 & 1 \\
N. j. t. bennett poe.......... & 1 & 4 & 3 & 1 & 1 \\
\hline
\end{tabular}

Notes of the Narcissi.

The starches of the narcissi belong according to the foregoing data to the moderate to very low reaction group-average value low. The reaction-intensities, including the ten reactions (polarization, iodine, gentian violet, safranin, temperature, chloral hydrate, chromic acid, pyrogallic acid, nitric acid, and sulphuric acid), which were studied in all the sets, show that nearly 70 per cent are moderate or low (nearly equally divided), and about 10 per cent very low. From the records of Set 2 and Chart E 14, where 26 reactions are recorded, there are about 50 per cent of the reactions that are moderate or low and about 30 per cent very low. The comparatively lower reactivities shown by the latter are owing to the fact that the additional reagents represented include a relatively large number that are among the least reactive with starches in general.

The curves of the composite charts (Charts E 13 to E 24 inclusive) show a close general correspondence in the courses, indicating clearly in comparison with charts of other genera a definite type of Narcissus curve. The closeness of the parental and hybrid curves varies in the different charts. The sulphuric-acid reactions reach completion so rapidly that differentiation of the starches can be made only, if at all, at the very onset of the reaction. With the other agents there is closeness, or even marked closeness, inclination to separation of the curves being most marked in the reactions with chromic acid and pyrogallic acid, especially in the former. The two parental curves bear varying relations to each other, not only in the different sets but also in each set, sometimes the seed parent and sometimes the pollen parent showing the higher reactivity, and sometimes both are the same or practically the same.

The hybrids bear varying relationships to the parents, not only in the different sets but also in each set, each being in one reaction the same or practically the same as one parent or the other or both, and in another intermediate or developed in excess or deficit. Even the offspring of the same cross may show differences in the same reaction, as, for instance, the hybrids $N$. poeticus herrick and $N$. poeticus dante. The varying relationships of the hybrids are indicated grossly in the following recapitulation:
Summaries of Reaction-intensities of the Various Hybrids (10 Reactions Each, Except in One; 146 in $A l l)$ :

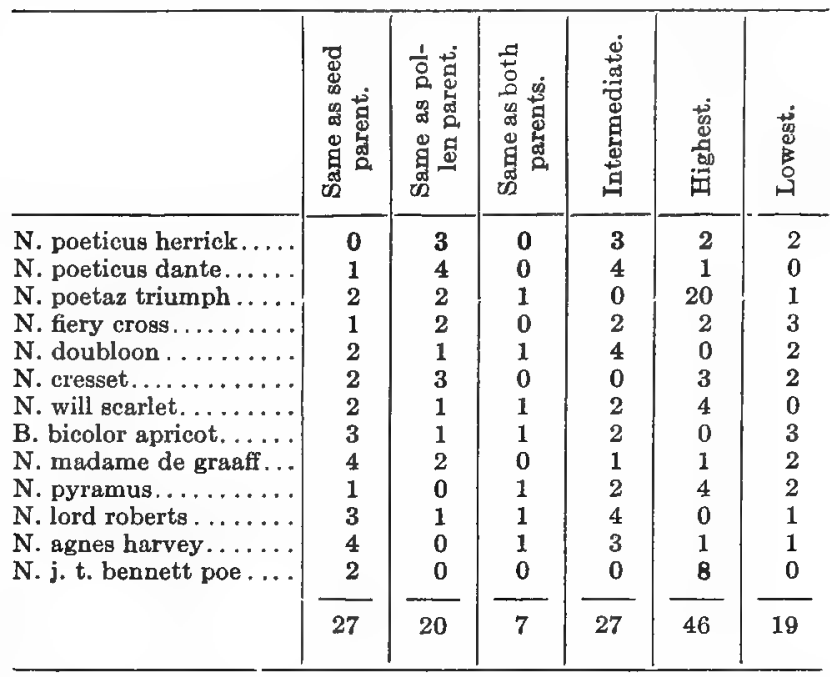

A corresponding shifting of relationship of the parents to each other and of the hybrid to the parents was recorded in the histologic characteristics, polariscopic figures, reactions with selenite, qualitative reactions with iodine, and qualitative reactions with the various chemical reagents. Among these will be found not only properties which are nearer to or identical with one or the other parent or the same as in both parents, or developed in excess or deficit, but also properties that are peculiar to the hybrid.

\section{Comparisons of the Starches of Lilidum martagon albUM, L. macUlatum, aNd L. MARHAN.}

In histologic characteristics, polariscopic figures, reactions with selenite, qualitative reactions with iodine, and qualitative reactions with the various chemical reagents all three starches exhibit properties in common in various degrees of development, the sum of which in each case is distinctive. The starch of Lilium maculatum in comparison with that of $L$. martagon album contains a less number of aggregates and compound grains, the grains are somewhat more irregular, and there is a form of irregularity that is peculiar. The hilum is more distinct, much more often fissured, and somewhat more eccentric. The lamellæ are less fine, more distinct, and less numerous. In size the grains are on the whole broader, absolutely and proportionately, in breadth to length. In the polariscopic, selenite, and qualitative iodine reactions there are various differences. In the qualitative reactions with chloral hydrate, chromic acid, potassium hydroxide, cobalt nitrate, and cupric chloride there are numerous differences, some of which are quite striking. The starch of the hybrid shows in form a closer relationship to that of $L$. maculatum. The hilum is more often fissured and occupied by a cavity than in either parent, and in character and eccentricity is in closer relationship to L. martagon album. The lamellæ are as distinct and fine as in L. martagon album, but in general characteristics and arrangement are the same as in both parents. In size the relationship 
is closer to $L$. martagon album. In the polariscopic, selenite, and qualitative iodine reactions the relationships are closer to $L$. maculatum. Here and there are data of development of the hybrid beyond parental extremes, as in the degree of irregularity of the grains, the appearance of secondary lamellæ, fissuration of and the cavities in the hilum, and in the bending and bisection of the lines of the polariscopic figure. In the qualitative reactions with the chemical reagents the resemblances are in the chloral-hydrate reactions closer to $L$. martagon album; but in those with chromic acid, potassium hydroxide, cobalt nitrate, and cupric chloride they are closer, on the whole, to those of $L$. maculatum. In some of these reactions the greater influence of one or the other parent is quite conspicuous.

\section{Reaction-intensities Expressed by Light, Color, and Tempera-} Polarization:

L. martagon album, low to high, value 65 .

L. maculatum, low to high, much lower than in L. martagon album, value 50 .

L. marhan, low to high, the same as in L. maculatum, value 50 . Iodine:

L. martagon album, moderate, value 65 .

L. maculatum, moderate, less than in L. martagon album, value 55 .

L. marhan, moderate, intermediate between the parents, value 58 . Gentian violet:

L. martagon album, moderate, value $\mathbf{5 5}$.

L. maculatum, moderate, less than in L. martagon album, value 45.

L. marhan, moderate, less than in either parent, value 43.

Safranin:

L. martagon album, moderate, value 50 .

L. maculatum, moderate, less than in L. martagon album, value 45 .

L. marhan, moderate, less than in either parent, value 43 .

Temperature:

L. martagon album, in majority at 59 to $61^{\circ}$, in all at 62 to $64^{\circ}$, mean $63^{\circ}$.

L. maculatum, in majority at 57 to $58^{\circ}$, in all at 60 to $62^{\circ}$, mean $61^{\circ}$

L. marhan, in majority at 56 to $58^{\circ}$, in all at 59 to $60^{\circ}$, mean $59.5^{\circ}$.

The reactivity of $L$. martagon album is higher than that of the other parent in the reactions with polarization, iodine, gentian violet, and safranin; and lower in that with temperature. The reactivity of the hybrid is the same or practically the same as that of $L$. macu latum in the polarization reaction; intermediate between those of the parents in the iodine reaction; lowest of the three in those with gentian violet and safranin; and the highest of the three in that with temperature. The reactions of the hybrid are closer throughout all five reactions to those of $L$. maculatum than to those of the other parent.

Table A 25 shows the reaction-intensities in percentages of total starch gelatinized at definite intervals (seconds and minutes).

\section{VeLOCITY-REaCTION CURVES.}

This section treats of the velocity-reaction curves of the starches of Lilium martagon album, L. maculatum, and $L$. marhan, showing the quantitative differences in the behavior toward different reagents at definite timeintervals. (Charts D 347 to D 353.)

These starches are generally so sensitive to the reagents used that only five of the reactions give satisfactory data for the construction of charts. In many of the reactions, notwithstanding the speed of gelatinization, more or less marked differences are recorded, yet little reliance should be placed on the figures unless they are confirmed by repeated experiment. In some instances the reactions of all three starches during the first minute are practically or absolutely alike, as in those with nitric acid, sulphuric acid, hydrochloric acid, potassium iodide, potassium sulphocyanate, potassium sulphide, sodium hydroxide, and sodium sulphide. In others there are such differences as to suggest that
TABLE A 25.

\begin{tabular}{|c|c|c|c|c|c|c|c|c|c|c|c|}
\hline & $\begin{array}{l}\mathbf{1} \\
\stackrel{10}{0}\end{array}$ & $\mid \begin{array}{l}\dot{\infty} \\
\dot{m}\end{array}$ & 百 & $\underset{\sim}{\text { 目 }}$ & $\begin{array}{l}\text { 自 } \\
\text { o }\end{array}$ & $\frac{\dot{\theta}}{+}$ & $\mid \begin{array}{l}\mid j \\
\infty\end{array}$ & $\mid \begin{array}{l}\text { g } \\
0 \\
0\end{array}$ & & \begin{tabular}{l|l} 
国 & \\
&
\end{tabular} & \begin{tabular}{l|l} 
日 \\
3 \\
3
\end{tabular} \\
\hline
\end{tabular}

Chloral hydrate:

L. martagon album

L. maculatum . ......

L. marhan ........... Chromic acid:

L. martagon album .

L. maculatum..........

L. marhan ...

Pyrogallic acid:

L. martagon album ....

L. maculatum.

L. marhan
Nitric acid:

L. martagon album ...

L. maculatum.........

L. marhan .............

Sulphuric acid:

L. martagon album ....

L. maculatum ..........

L. marhan ......

Hydrochloric acid:

L. martagon album .....

L. maculatum.

I. marhan ......

Potassium hydroxide:

L. martagon album .

L. maculatum. .........

L. marhan ..

Potassium iodide:

L. martagon album

L. maculatum. .........

L. marhan ...........

Potassium sulphocyanate:

L. martagon album ....

L. maculatum.........

L. marhan ............

Potassium sulphide:

L. martagon album .....

L. maculatum. .........

L. marhan ........... 10

Sodium hydroxide:

L. martagon album.....

L. maculatum. ........

L. marhan ............. 100

Sodium sulphide:

L. martagon album . . .

L. maculatum.........

L. marhan ............

Sodium salicylate:

L. martagon album . . .

L. maculatum. .........

L. marhan ... .

Calcium nitrate:

L. martagon album .

L. maculatum...........

L. marhan ... .

Uranium nitrate:

L. martagon album ....

L. maculatum.

L. marhan ......
Strontium nitrate:

L. martagon album

L. maculatum...........

L. marhan..

Cobalt nitrate:

L. martagon album

L. maculatum. ..........

L. marhan ..

Copper nitrate:

L. martagon album .....

L. maculatum..........

L. marhan ....

Cupric chloride:

L. martagon album .

L. maculatum...........

L. marhan ..

Barium chloride:

L. martagon album .....

L. maculatum

L. marhan .......

L. martagon album

L. maculatum. ............

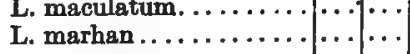

\begin{tabular}{c|c}
99 & $\ldots$ \\
99 & $\ldots$ \\
99 & $\ldots$ \\
& \\
$\cdots$ & $\cdots$ \\
99 & $\cdots$ \\
08 & $\ldots$ \\
00 & $\cdots$ \\
\hline
\end{tabular}

99.

$100 . \cdots$

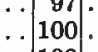

95.

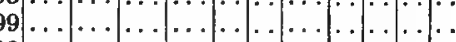

99

00.

99

98

99.

$\begin{array}{llllll} & \cdots & \cdots & \cdots & \cdots\end{array}$

\begin{tabular}{llll}
53 & . & 84 & 99 \\
\hline
\end{tabular}

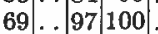

33 . . 90099

85
95

$95 . \cdots .99$

$66 . . . .99$

$93 . \ldots 100$

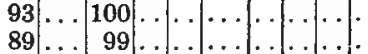

73 $\ldots 99 . . .6 . . . . .$.

95100

81 ...

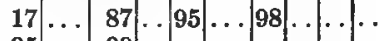

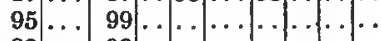

83 .

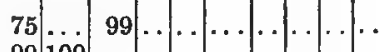

99100

$98 \quad 99$

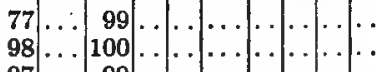

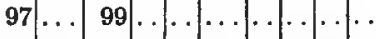

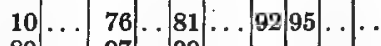

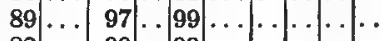

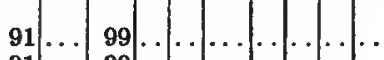

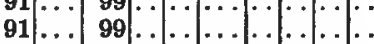

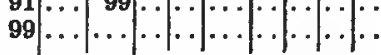


with reagents of suitable concentration there would be shown marked differentiation. Attention has been directed to greater resemblance generally of the hybrid to $L$. maculatum than to the other parent in histologic and certain qualitative peculiarities, and also in the reaction-intensities expressed by light, color, and temperature reactions, and it is of interest in this connection to note that in the reactions with calcium nitrate, uranium nitrate, cobalt nitrate, copper nitrate, cupric chloride, and barium chloride the figures show very definitely the same parental relationship, while in that with strontium nitrate the hybrid figure approximates mid-intermediateness, and in that with mercuric chloride a reactivity higher than in either parent. In the remaining reactions, all of which being less rapid, with chloral hydrate the reaction of the hybrid is practically mid-intermediate; with chromic acid and pyrogallic acid the reactions are closer to $L$. maculatum; and with sodium salicylate the reaction is at the end of 3 minutes distinctly lower than those of the parents and at 5 minutes mid-intermediate. Referring to the charts, it will be seen that in all five reactions the curve of $L$. martagon album is the lowest of the three; that the hybrid curve is practically the same as the curve of $L$. maculatum in the reactions with chromic acid, pyrogallic acid, and barium chloride; that the hybrid curve is intermediate in the chloral-hydrate reaction, but on the whole closer to $L$. maculatum; and that the hybrid curve is lower at first than that of either parent, and then intermediate, in the sodium salicylate reaction.

\section{Reaction-Intensities OF THE HybRID.}

This section treats of the reaction-intensities of the hybrid as regards sameness, intermediateness, excess, and deficit in relation to the parents. (Table A 25 and Charts D 347 to D 353.)

The reactivity of the hybrid is the same as that of the seed parent in none of the reactions; the same as those of the pollen parent in the reactions with polarization, chromic acid, pyrogallic acid, copper nitrate, and cupric chloride; the same as those of both parents with nitric acid, sulphuric acid, hydrochloric acid, potassium hydroxide, potassium iodide, potassium sulphocyanate, potassium sulphide, sodium hydroxide, and sodium sulphide, in all of which the reactions occur too rapidly for differentiation; intermediate with iodine, chloral hydrate, uranium nitrate, strontium nitrate, cobalt nitrate, and barium chloride (in four being closer to the seed parent, and in four closer to the pollen parent); highest with mercuric chloride, and as near one as the other parent; and lowest with gentian violet, safranin, temperature, sodium salicylate, and calcium nitrate (in three being closer to the pollen parent and in two closer to the seed parent).

The following is a summary of the reaction-intensities: Same as seed parent, 0; same as pollen parent, 5; same as both parents, 9 ; intermediate, 6 ; highest, 1 ; lowest, 5 .

The pollen parent has obviously exercised a much more potent influence than the other parent on the properties of the starch of the hybrid. The most conspicuous features of these reactions, apart from the many instances of sameness to both parents, are sameness to the pollen parent, intermediateness, and lowest reactivities.

\section{Composite Corves of Reaction-intensities.}

This section treats of the composite curves of the reaction-intensities, showing the differentiation of the starches of Lilium martagon album, $L$. maculatum, and L. marhan. (Chart E 25.)
The most conspicuous features of this chart are:

(1) The close correspondence of all three curves throughout, the curves keeping close together excepting in the barium-chloride reaction. In most of the charts there is either little or no differentiation of the three starches, as in the reactions with nitric acid, sulphuric acid, hydrochloric acid, potassium hydroxide, potassium iodide, potassium sulphocyanate, potassium sulphide, sodium hydroxide, and sodium sulphide. In all other reactions the curves of the hybrid and $L$. maculatum run very closely together, excepting in the reactions with sodium salicylate, calcium nitrate, uranium nitrate, strontium nitrate, in which the curves of the hybrid and $L$. martagon album are the same and below that of the other parent; in the cobalt-nitrate reaction, where the curve is intermediate, and in that of mercuric chloride, in which the curves of the parents are the same and the curve of the hybrid distinctly higher.

(2) In $L$. martagon album in comparison with the other parent the higher reactions with polarization, iodine, gentian violet, safranin; the lower reactions with temperature, chloral hydrate, chromic acid, pyrogallic acid, sodium salicylate, calcium nitrate, uranium nitrate, strontium nitrate, cobalt nitrate, copper nitrate, cupric chloride, and barium chloride; and the same or practically the same reactions with nitric acid, sulphuric acid, hydrochloric acid, potassium hydroxide, potassium iodide, potassium sulphocyanate, potassium sulphide, sodium hydroxide, sodium sulphide, and mercuric chloride.

(3) In L. martagon album, the very high reactions with chromic acid, pyrogallic acid, nitric acid, sulphuric acid, hydrochloric acid, potassium hydroxide, potassinm iodide, potassium sulphocyanate, potassium sulphide, sodium hydroxide, sodium sulphide, sodium salicylate, calcium nitrate, uranium nitrate, strontium nitrate, cobalt nitrate, copper nitrate, cupric chloride, and mercuric chloride; the high reactions with polarization, iodine, chloral hydrate, and barium chloride; the moderate reactions with gentian violet, safranin, and temperature.

(4) In $L$. maculatum, the very high reactions with chloral hydrate, chromic acid, pyrogallic acid, nitric acid, sulphuric acid, hydrochloric acid, potassium hydroxide, potassium iodide, potassium sulphocyanate, potassium sulphide, sodium hydroxide, sodium sulphide, sodium salicylate, calcium nitrate, uranium nitrate, strontium nitrate, cobalt nitrate, copper nitrate, cupric chloride, barium chloride, and mercuric chloride; the high reactions with temperature; and the moderate reactions with polarization, iodine, gentian violet, and safranin.

(5) In the hybrid, the very high reactions with chloral hydrate, chromic acid, pyrogallic acid, nitric acid, sulphuric acid, hydrochloric acid, potassium hydroxide, potassium iodide, potassium sulphocyanate, potassium sulphide, sodium hydroxide, sodium sulphide, sodium salicylate, calcium nitrate, uranium nitrate, strontium nitrate, cobalt nitrate, copper nitrate, cupric chloride, barium chloride, and mercuric chloride; the high reaction with temperature; the moderate reactions with polarization, iodine, gentian violet, and safranin.

The following is a summary of the reaction-intensities:

\begin{tabular}{|c|c|c|c|c|c|}
\hline & $\begin{array}{l}\text { Very } \\
\text { high. }\end{array}$ & High. & $\begin{array}{l}\text { Mod- } \\
\text { erate. }\end{array}$ & Low. & $\begin{array}{l}\text { Very } \\
\text { low. }\end{array}$ \\
\hline L. martagon album...$\ldots \ldots$ & 19 & 4 & 3 & 0 & 0 \\
\hline L. maculatum. ............ & 21 & 1 & 4 & 0 & 0 \\
\hline 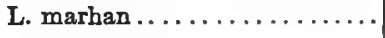 & 21 & 1 & 4 & $\mathbf{0}$ & 0 \\
\hline
\end{tabular}


26. Comparisons of the Starches of Liliom MARTAGON, L. MACULATUM, AND L. DALHANSONI.

In histologic characteristics, polariscopic figures, reactions with selenite, qualitative reactions with iodine and with the various chemical reagents all three starches exhibit properties in common in various degrees of development, the sum of which in each case is characteristic. The starch of $L$. maculatum in comparison with that of $L$. martagon contains no aggregates and few compound grains; the grains are more regular; broad forms are more numerous; and a larger number of grains are flattened. The hilum is more distinct, more often fissured, and less eccentric. The lamella are less fine, more distinct, and less numerous. In size there is more broadness. In the polariscopic, selenite, iodine, and aniline reactions there are various differences. In the qualitative reactions with chloral hydrate, chromic acid, potassium hydroxide, cobalt nitrate, and cupric chloride there are many differences which collectively are distinctive. The starch of the hybrid shows an absence of compound grains that were found in the starches of both parents; there is greater regularity of the grains than in either parent; and the starch shows, on the whole, a closer relationship to that of $L$. martagon. The hilum in character and eccentricity is more closely related to $L$. maculatum. The lamellæ in character and arrangement are more like those of $L$. martagon, but in number closer to the other parent. In size the larger grains are not so large as the corresponding grains in both parents, but their dimensions and also the common sizes are claser to those of $L$. martagon. In the polariscopic, selenite, iodine, and aniline reactions the relationships are closer to $L$. martagon. In the qualitative reactions with the chemical reagents closer resemblances to one or the other parent or in common to both parents are recorded. In the chloral-hydrate reactions the relationship is closer to L. maculatum, while in those with chromic acid, potassium hydroxide, cobalt nitrate, and cupric chloride the relationships are closer to $L$. martagon.

Reaction-intensities Expressed by Light, Color, and Temperature Reactions.

Polarization:

L. martagon, low to high, value 60 .

L. maculatum, low to high, lower than in L. martagon, value 50 .

L. dalhansoni, low to high, the same as in L. martagon, value 60 . Iodine:

L. martagon, moderate, value 60.

L. maculatum, moderate, less than in L. martagon, value 55.

L. dalhansoni, moderate to deep, higher than in either parent, value 65 .

Gentian violet:

L. martagon, moderate to moderately deep, value $\mathbf{5 5 .}$

L. maculatum, moderate, less than in L. martagon, value 45 .

L. dalhansoni, moderate, the same as in L. martagon, value 55. Safranin:

L. martagon, moderate, value 55 .

L. maculatum, moderate, less than in L. martagon, value 45.

L. dalhansoni, moderate, the same as in L. martagon, value 65 . Temperature:

L. martagon, in majority at 62 to $64^{\circ}$, in all at 66.5 to $68.3^{\circ}$, mean $67.4^{\circ}$.

L. maculatum, in majority at 57 to $58^{\circ}$, in all at 60 to $62^{\circ}$, mean $61^{\circ}$.

L. dalhansoni, in majority at 59 to $60.2^{\circ}$, in all at 63 to $64^{\circ}$, mean $63.9^{\circ}$.

The reactivity of $L$. martagon is higher than that of the other parent in the reactions with polarization, iodine, gentian violet, and safranin; and lower in those with temperature. The reactivity of the hybrid is the same or practically the same as that of $L$. martagon in the reactions with polarization, gentian violet, and safranin; the highest of the three in that with iodine; and intermediate in that with temperature. With the exception of the temperature reaction, the relationship of the hybrid is much closer to $L$. martagon than to the other parent.

Table A 26 shows the reaction-intensities in percentages of total starch gelatinized at definite intervals (seconds and minutes).

\section{Velocity-Reaction CuRves.}

This section treats of the velocity-reaction curves of the starches of Lilium martagon, L. maculatum, and L. dalhansoni, showing the quantitative differences in the behavior toward different reagents at definite time-intervals. (Charts D 354 to D 360.)

Most of the reactions occur with such rapidity that the data do not lend themselves to the making of charts. Gelatinization is complete within 15 to 30 seconds in the reactions with nitric acid, sulphuric acid, hydrochloric acid, potassium hydroxide, potassium iodide, potassium sulphocyanate, potassium sulphide, sodium hydroxide, and sodium sulphide. In certain other reactions, even though they proceed with speed, there are more or less distinctive differences, as, for instance, in the reactions with calcium nitrate, uranium nitrate, strontium nitrate, copper nitrate, cupric chloride, and mercuric chloride, in all of which gelatinization is almost if not complete within 3 minutes. In all of these reactions, excepting those with uranium nitrate, strontium nitrate, and cupric chloride the hybrid reactions are very distinctly closer to those of $L$. maculatum than to those of the other parent; in those with uranium nitrate and cupric chloride the hybrid is approximately mid-intermediate; and in those with strontium nitrate the same as $L$. martagon. In histologic and qualitative peculiarities, and in the polarization, iodine, and aniline reactions the hybrid shows in general a closer relationship to L. martagon; but occasionally closer to the other parent, or intermediate as in the temperature reaction. Referring to the charts, it will be seen that in all of them the curves of $L$. maculatum and the hybrid are almost exactly the same, and higher than the curve of the other parent; and that the hybrid curves tend to be slightly lower than those of $L$. maculatum. The relatively greater resistance of the starch of $L$. martagon is exhibited particularly in the curves for chromic acid, pyrogallic acid, and barium chloride.

\section{Reaction-intensities OF THE Hybids.}

This section treats of the reaction-intensities of the hybrids as regards sameness, intermediateness, excess, and deficit in relation to the parents. (Table A 26 and Charts D 354 to D 360.)

The reactivities of the hybrid are the same as those of the seed parent in the reactions with polarization, gentian voilet, and strontium nitrate; the same as those of the pollen parent with chloral hydrate; the same as those of both parents with nitric acid, sulphuric acid, hydrochloric acid, potassium hydroxide, potassium iodide, potassium sulphocyanate, potassium sulphide, sodium hydroxide, and sodium sulphide, in all of which the reactions occur too quickly for differentiation; intermediate with temperature, chromic acid, pyrogallic acid, calcium nitrate, uranium nitrate, cobalt nitrate, copper nitrate, cupric choride, and barium chloride (in seven closer to those of the pollen parent, in one closer to that of the seed parent, and in one mid-intermediate) ; highest with iodine and sodium salicylate (in one being closer to the seed parent, and in one closer to the pollen parent); and lowest with mercuric chloride, and closer to the pollen parent.

The following is a summary of the reaction-intensities: Same as seed parent, 4; same as pollen parent, 1; same as both parents, 9 ; intermediate, 9 ; highest, 2; lowest, 1. 
TABLE 26 A.

\begin{tabular}{|c|c|c|c|c|c|c|c|c|c|c|c|c|}
\hline & $\stackrel{\dot{m}}{2}$ & $\stackrel{\infty}{8}$ & 自 & ฉ & $\begin{array}{l}\text { 日 } \\
0\end{array}$ & $\begin{array}{l}\text { 早 } \\
+1\end{array}$ & 鬲 & $\begin{array}{l}\text { 目 } \\
\text { - }\end{array}$ & $\begin{array}{l}1 \\
0 \\
0\end{array}$ & $\begin{array}{l}\text { 日 } \\
8\end{array}$ & & 8 \\
\hline $\begin{array}{l}\text { hloral hydrate: } \\
\text { L. martagon. }\end{array}$ & & & & & & & & & & & & \\
\hline L. $\mathrm{n}$ & & & & & & & 82 & & $\begin{array}{l}30 \\
97\end{array}$ & 99 & & \\
\hline L. dalhan & & & & & $\ldots$ & & 80 & & & 100 & & \\
\hline Chromic acid: & & & & & & & & & & & & \\
\hline L. mar & & & & & & & 82 & & 89 & 99 & & \\
\hline L. m & & & & & 99 & & 99 & & & $\cdots$ & & \\
\hline L. dalha & & & & & & & 95 & & 99 & & & \\
\hline Pyrogallic acid: & & & & & & & & & & & & \\
\hline L. marta & & & & & & & 77 & & 89 & & 95 & \\
\hline L. $n$ & & & & & - & & $\begin{array}{l}99 \\
09\end{array}$ & & & & & \\
\hline L.d & & & & & & & 95 & & 99 & & & \\
\hline Nitric acid: & & & & & & & & & & & & \\
\hline & $\begin{array}{l}97 \\
99\end{array}$ & & & & & & & & $\cdots$ & & & \\
\hline L. d & 99 & & & & & & & & .. & & & \\
\hline Sulphu & & & & & & & & & & & & \\
\hline L. $n$ & & & 99 & & & & & & & & & \\
\hline L. $\mathrm{I}$ & & & 99 & & & & & & . & & & \\
\hline L. $\mathrm{d}$ & & & 99 & & & & & & . & & & \\
\hline Hydrochl & & & & & & & & & & & & \\
\hline L. m & 97 & & & & & & & & $\cdot$ & & & \\
\hline L. & 100 & & & & & & $\cdot$ & & . & & & \\
\hline L. dg & 99 & & & & & & . & & .. & . & & \\
\hline Potassium hydroxide: & & & & & & & & & & & & \\
\hline L. $m$ & 99 & & & & & & • & & 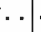 & & & \\
\hline L. I & 100 & & & & & & . & & . & & & \\
\hline L. d & 99 & & & & & & . & $\cdots$ & . & . & & \\
\hline Potassiu & & & & & & & & & & & & \\
\hline L. I & & 94 & & $\cdots$ & 98 & & 99 & & $\cdots$ & & & \\
\hline & & 100 & & & & & .. & & . & & & \\
\hline L. d & ... & 100 & & & & . & . & & . & & & \\
\hline ghocyanate: & & & & & & & & & & & & \\
\hline L. $\mathrm{m}$ & & 95 & & & & & & & & & & \\
\hline L. $\mathbf{n}$ & .. & 99 & & & & & . & & $\cdots$ & & & \\
\hline L. dal & $\cdots$ & 96 & & .. & & & .. & .. & . . & & & \\
\hline Potassium & & & & & & & & & & & & \\
\hline L. I & 95 & & & & & & . & & . & & & \\
\hline L. $n$ & 100 & . & & & & & . . & & . & & & \\
\hline L. de & 99 & $\ldots$ & & $\ldots$ & & & .. & .. & .. & . & & \\
\hline Sodium & & & & & & & & & & & & \\
\hline L. 1 & $\cdots$ & 97 & & & & & . & & & $\cdot$ & & \\
\hline & $\ldots$ & $100 \mid$ & & & & & & & & & & \\
\hline L. dalh & $\ldots$ & 99 & & & & & .. & & . & & & \\
\hline Sodium sulphide: & & & & & & & & & & & & \\
\hline L. $\mathrm{n}$ & $\cdots$ & 98 & & & & & $\cdots$ & & & & & \\
\hline L. $\mathrm{n}$ & $\ldots$ & 99 & & & & & . & & . & .. & & \\
\hline L. d & $\ldots$ & 98 & & & & & . & & . & ... & & \\
\hline Sodium & & & & & & & & & & & & \\
\hline & & . & & & 60 & & 84 & 98 & & & & \\
\hline & .. & .. & & & 69 & & 97 & 100 & . & & & \\
\hline L. $\mathrm{d}$ & . & .. & & $\ldots$ & 82 & & 99 & $\ldots$ & & . & & \\
\hline Calcium nitrate: & & & & & & & & & & & & \\
\hline L. I & & . & 10 & & 90 & & 97 & 99 & & & & \\
\hline L. I & & & 95 & & 99 & & & 1 & & & & \\
\hline L. dall & & & 84 & & 98 & & 99 & & & & & \\
\hline Uranium & & & & & & & & & & & & \\
\hline L. & & & 60 & & 97 & & 99 & & & & . & \\
\hline & & & 93 & & 100 & & & & & & & \\
\hline L. O & & .. & 72 & & 98 & & 99 & & & & . & \\
\hline Stronti & & & & & & & & & & & & \\
\hline & & & 77 & & 99 & & & & & & & \\
\hline & & & $\{95\}$ & 100 & & & $\therefore$ & & & & & \\
\hline & & 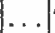 & 78 & 99 & 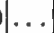 & & $\ldots$ & & & & & \\
\hline Cobalt nitrate & & & & & & & & & & & & \\
\hline & & . & 3 & & 66 & & 84 & & 95 & & & \\
\hline & & & 95 & & 99 & & .. & & & & & \\
\hline & & & 58 & & 95 & & 98 & & & & & \\
\hline Coppe & & & & & & & & & & & & \\
\hline L. $\mathbf{r}$ & & & 69 & & 98 & & 99 & & & & & \\
\hline & & & 99 & 100 & & & & & & & & \\
\hline & & & 95 & & 99 & & & & & & & \\
\hline Cupri & & & & & & & & & & & & \\
\hline L. & & & $60 \mid$ & & 95 & & 96 & & 99 & & & \\
\hline & & & 98 & & 100 & & & & & & & \\
\hline & & & 78 & & 99 & & . & & & & & \\
\hline Bariur & & & & & & & & & & & & \\
\hline & & . & 2 & & 52 & & 76 & & 86 & 89 & 90 & \\
\hline & & & 89 & & 97 & & 99 & & $\ldots$ & & & \\
\hline & & & 16 & & 89 & & 97 & & 99 & & & \\
\hline Mercuric chloride: & & & & & & & & & & & & \\
\hline & & & 80 & & 97 & & 98 & & & & & \\
\hline L. $\mathrm{x}$ (a) & & & 91 & & 99 & & & & . & & & \\
\hline & & & 95 & & 99 & & & & & & & \\
\hline
\end{tabular}

From the foregoing data the pollen parent has been by far the more potent in its influences on determining the properties of the starch of the hybrid. The tendency to intermediateness is quite manifest.

\section{Composite Curves of Reaction-intensities.}

This section treats of the composite curves of the reaction-intensities, showing the differentiation of the starches of Lilium martagon, $L$. maculatum, and $L$. dalhansoni. (Chart E 26.)

The most conspicuous features of this chart are:

(1) The close correspondence in the three curves excepting in the reactions with chromic acid, pyrogallic acid, and barium chloride, in which there occurs in each instance a marked drop in the curve of $L$. martagon, while the curves of $L$. maculatum and the hybrid tend to keep the same or quite close together. In a large number of reactions there is no differentiation between the three starches, as in those with chloral hydrate, nitric acid, sulphuric acid, hydrochloric acid, potassium hydroxide, potassium iodide, potassium sulphocyanate, potassium sulphide, sodium hydroxide, sodium sulphide, and uranium nitrate; and in other instances there is a tendency for the hybrid curve to be the same as that of one or the other parent, or occasionally above both or intermediate. In part the hybrid curve is more distinctly related to the curve of $\dot{L}$. maculatum than to that of the other parent, and in part the reverse.

(2) In $L$. martagon in comparison with the other parent, the high reactions with polarization, iodine, gentian violet and safranin; the same or practically the same with chloral hydrate, nitric acid, sulphuric acid, hydrochloric acid, potassium hydroxide, potassium iodide, potassium sulphocyanate, potassium sulphide, sodium hydroxide, sodium sulphide, calcium nitrate, uranium nitrate, and mercuric chloride; and the lower with temperature, chromic acid, pyrogallic acid, sodium salicylate, strontium nitrate, cobalt nitrate, copper nitrate, cupric chloride, and barium chloride.

(3) In $L$. martagon the very high reactions with chloral hydrate, nitric acid, sulphuric acid, hydrochloric acid, potassium hydroxide, potassium iodide, potassium sulphocyanate, potassium sulphide, sodium hydroxide, sodium sulphide, sodium salicylate, calcium nitrate, uranium nitrate, strontium nitrate, cobalt nitrate, copper nitrate, cupric chloride, and mercuric chloride; the high reactions with polarization, iodine, chromic acid, pyrogallic acid, and barium chloride; and the moderate reactions with gentian violet, safranin, and temperature.

(4) In $L$. macutatum the very high reactions with chloral hydrate, chromic acid, pyrogallic acid, nitric acid, sulphuric acid, hydrochloric acid, potassium hydroxide, potassium iodide, potassium sulphocyanate, potassium sulphide, sodium hydroxide, sodium sulphide, sodium salicylate, calcium nitrate, uranium nitrate, strontium nitrate, cobalt nitrate, copper nitrate, barium chloride, and mercuric chloride; the high temperature reaction; the moderate reactions with polarization, iodine, gentian violet, and safranin.

(5) In the hybrid, the very high reactions with chloral hydrate, chromic acid, pyrogallic acid, nitric acid, sulphuric acid, hydrochloric acid, potassium hydroxide, potassium iodide, potassium sulphocyanate, potassium sulphide, sodium hydroxide, sodium sulphide, sodium salicylate, calcium nitrate, uranium nitrate, strontium nitrate, cobalt nitrate, copper nitrate, cupric chloride, barium chloride, and mercuric chloride; the high reactions with polarization and iodine; and the moderate reactions with gentian violet, safranin, and temperature. 
Following is a summary of the reaction-intensities:

\begin{tabular}{|c|c|c|c|c|c|}
\hline & $\begin{array}{l}\text { Very } \\
\text { high. }\end{array}$ & High. & $\begin{array}{l}\text { Mod- } \\
\text { erate. }\end{array}$ & Low. & $\begin{array}{l}\text { Very } \\
\text { low. }\end{array}$ \\
\hline 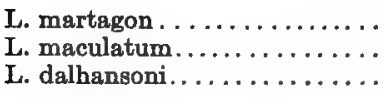 & $\begin{array}{l}18 \\
21 \\
21\end{array}$ & $\begin{array}{l}5 \\
1 \\
2\end{array}$ & $\begin{array}{l}3 \\
4 \\
3\end{array}$ & $\begin{array}{l}0 \\
0 \\
0\end{array}$ & $\begin{array}{l}0 \\
0 \\
0\end{array}$ \\
\hline
\end{tabular}

27. Comparisons of the Starches of Liliom TENUIFOLIUM, L. MARTAGON ALBDM, AND L. GOLDEN GLEAM.

In'the histologic characteristics, polariscopic figures, reactions with selenite, qualitative reactions with iodine, and qualitative reactions with the chemical reagents all three starches exhibit properties in common in various degrees of development, the sum of which in each case is characteristic. The starch of Lilium martagon album in comparison with that of $L$. tenuifolium contains very few compound grains and aggregates; there is less irregularity and variety in the forms, and the protuberances are less rounded; and a less number of grains are flattened. The hilum is not so distinct; less often occupied by a cavity; somewhat more fissured; and less eccentric. The lamellæ have the same characteristics and arrangement as in the other parent, but they are less numerous. The size is somewhat larger. In the polariscopic, selenite, and qualitative iodine reactions various differences are noted. In the qualitative reactions with chloral hydrate, chromic acid, potassium hydroxide, cobalt nitrate, and cupric chloride the differences are sufficient for easy differentiation. The starch of the hybrid shows in comparison with the starches of the parents fewer compound grains than in either parent, and there is an absence of aggregates; and the grains are more irregular than in either parent. The hilum is as distinct as in L. tenuifolium and more distinct than in the other parent; and it is fissured more often and the eccentricity is less than in either parent. The lamellæ are less distinct and less fine than in either parent. The size is about the same as in $L$. tenuifolium and slightly less than in the other parent. In the polariscopic, selenite, and qualitative iodine reactions there are leanings to one or the other parent, but the relationship is on the whole closer to $L$. tenuifolium. In the qualitative chemical reactions certain reactions lean to one parent and certain others to the other parent, but with chloral hydrate the relationship is closer to $L$. martagon album, and in those with chromic acid, potassium hydroxide, cobalt nitrate, and cupric chloride closer to L. tenuifolium.

Reaction-intensities Expressed by Light, Color, and TemperaPolarization: ture Reactions.

L. tenuifolium, low to high, value 50 .

L. martagon album, low to high, much higher than in L. tenuifolium, value 65 .

L. golden gleam, low to high, lower than in either parent, value 45 . Iodine:

L. tenuifolium, moderate, value 55 .

L. martagon album, moderate, much higher than in L. tenuifolium, value 65.

L. golden gleam, moderate, less than in either parent, value 50. Gentian violet:

L. tenuifolium, moderate, value 60 .

L. martagon album, moderate, less than in L. tenuifolium, value 55 .

L. golden gleam, moderate, less than in either parent, value 50 . Safranin:

L. tenuifolium, moderate, value 55 .

L. martagon album, moderate, less than in L. tenuifolium, value 50 .

L. golden gleam, moderate, less than in either parent, value 48 .

Temperature:

L. tenuifolium, in majority at 52 to $53^{\circ}$, in all at 55.6 to $56^{\circ}$, mean $55.8^{\circ}$.

L. martagon album, in majority at 59 to $61^{\circ}$, in all at 62 to $64^{\circ}$, mean $63^{\circ}$.

L. golden gleam, in majority at 53 to $54.4^{\circ}$, in all at 57 to $58.7^{\circ}$, mean $57.8^{\circ}$
Table A 27.

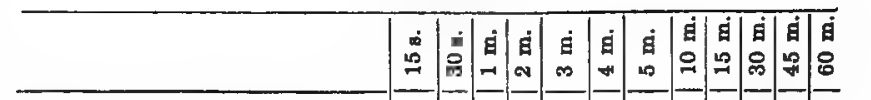

Chloral hydrate:

L. tenuifoliuma....

L. martagon album

L. golden gleam

L. tenuifoliun

L. martagon album.

L. golden gleam .

Pyrogallic acid:

L. tenuifolium ......

L. martagon album .

L. golden gleam

Nitric acid:

L. tenuifolium.

L. martagon album .

L. golden gleam

Sulphuric acid:

L. tenuifolium .

L. martagon album.

L. golden gleam .

Hydrochloric acid:

L. tenuifolium......

L. martagon album .

L. golden gleam....

Potassium hydroxide:

L. tenuifolium

L. martagon album ........ 99

L. golden gleam......... 100 .

Potassium iodide:

L. tenuifolium

L. martagon album ............

L. golden gleam.

Potassium sulphocyanate:

L. tenuifolium......

L martagon album ....................

L. golden gleam ........... 99 .

Potassium sulphide:

L. tenuifolium.... . .

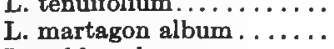

L. golden gleam ............

Sodium hydroxide:

L. tenuifolium...

L. martagon album ..........

L. golden gleam . ........ 100

Sodium sulphide:

L. tenuifolium

L. martagon album.

L. golden gleam. .

Sodium salicylate:

L. tenuifolium ....

L. martagon album . . .........

L. golden gleam

Calcium nitrate:

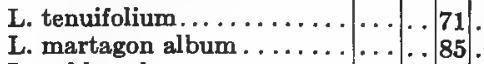

L. golden gleam. ............ 91 .

Uranium nitrate:

L. tenuifolium...

L. martagon album ............ 66

L. golden gleam ............ 88

Strontium nitrate:

L. tenuifolium ...............966.

L. martagon album........... 73

L. golden gleam ............. Cobalt nitrate:

L. tenuifolium $\ldots \ldots \ldots \ldots \ldots$
L. martagon album ........

L. golden gleam . ............ 75

Copper nitrate:

L. tenuifolium............. 90.

L. martagon album........... 75

L. golden gleam .............

Cupric chloride:

L. tenuifolium .............. 70 .

L. martagon album ............ 77

L. golden gleam ............. 82

Barium chloride:

L. teriuifolium.............. 66.

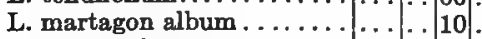

L. golden gleam............ 82 .

Mercuric chloride:

L. tenuifolium....

L. martagon album

L. golden gleam.... .

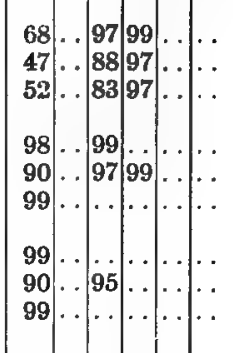

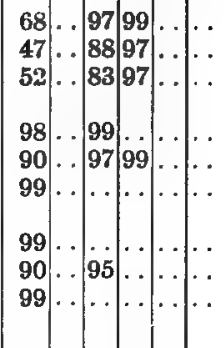

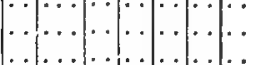

$\cdots \cdots$

.

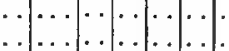

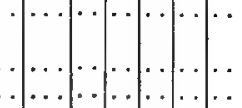

$\begin{array}{lllllll}\cdots & \cdots & \cdots & \cdots & \cdots & \cdots \\ \cdots & & & \end{array}$

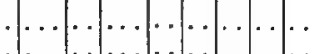

$\begin{array}{lllllll}\cdots & \cdots & \cdots & \cdots & \cdots & \cdots & \cdots\end{array}$

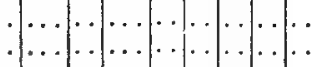

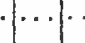

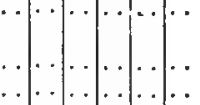

...

...

$53 .$.

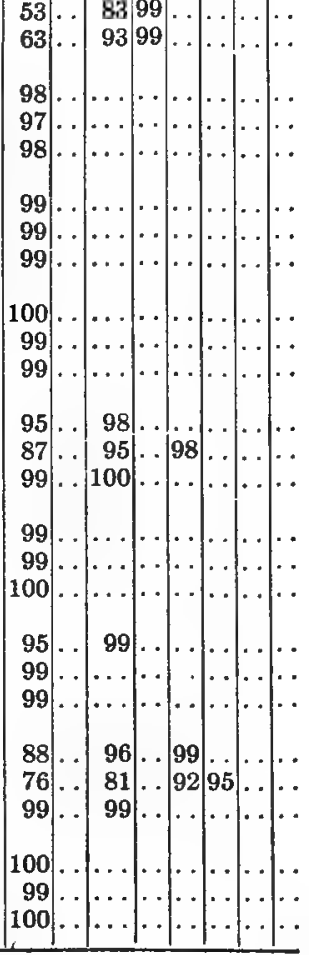


The reactivity of $L$. tenuifolium is lower than that of the other parent in the polarization and iodine reactions; and higher in the gentian violet, safranin, and temperature reactions. The reactivity of the hybrid is the lowest of the three in the reactions with polarization, iodine, gentian violet, and safranin; and intermediate with temperature. In the polarization, iodine, and temperature reactions the hybrid is closer to $L$. tenuifolium, and in those with gentian violet, safranin, and temperature closer to $L$. martagon album.

Table A 27 shows the reaction-intensities in percentages of total starch gelatinized at definite intervals (seconds and minutes).

\section{VELOCITY-REACTION CURVES.}

This section treats of the velocity-reaction curves of the starches of Lilium tenuifolium, L. martagon album, and $L$. golden gleam, showing the quantitative differences in the behavior toward different reagents at definite time-intervals. (Charts D 361 to D 366.)

These starches generally react so rapidly with the various reagents that there are few instances where the data are of value in presentation in the form of charts. In the reactions with nitric acid, sulphuric acid, hydrochloric acid, potassium hydroxide, potassium iodide, potassium sulphocyanate, potassium sulphide, sodium hydroxide, and sodium sulphide complete or nearly complete gelatinization occurs of all three starches within 15 to 30 seconds. In other reactions, notwithstanding the rapidity, more or less differentiation is evident, as with calcium nitrate, uranium nitrate, strontium nitrate, cobalt nitrate, copper nitrate, cupric chloride, and mercuric chloride, in which gelatinization is almost if not wholly completed in 3 minutes. Differences in these cases are quite noticeable at the end of 1 minute, $L$. tenuifolium has a lower reactivity than the other parent in the calcium-nitrate and cupric-chloride reactions, and a higher reactivity in the others, and the hybrid shows reactivities as high or higher than either parent. Not much importance is to be attached to these figures, although they are very suggestive, owing to the difficulties of obtaining accurate records. Referring to the charts, it will be noted that all three curves in each chart tend to closeness; that the hybrid curve is almost exactly the same as the curve of $L$. martagon album in the chloralhydrate reaction, but like that of the other parent in the chromic-acid and pyrogallic-acid reactions; that the parental curves are practically exactly the same in the sodium-salicylate reaction, but the hybrid curve definitely higher; that the hybrid curves are the highest in three out of the four reactions, namely, in those of chromic acid, sodium salicylate, and barium chloride; and that the parental curves differ somewhat in their relative positions, the curve of $L$. tenuifolium being higher than that of the other parent in the reactions with chIoral hydrate, chromic acid, and barium chloride, but the same in the reactions with sodium salicylate.

\section{Reaction-Intensities of the HyBRID.}

This section treats of the reaction-intensities of the hybrid as regards sameness, intermediateness, excess, and deficit in relation to the parents. (Table A $2 \%$ and Charts D. 361 to D 366.)

The reactivities of the hybrid are the same as those of the seed parent in the reactions with chromic acid, pyrogallic acid, potassium sulphocyanate, and mercuric chloride; the same as those of the pollen parent with chloral hydrate, potassium sulphide, sodium hydroxide, and sodium sulphide; the same as those of both parents with nitric acid, sulphuric acid, hydrochloric acid, potassium hydroxide, and potassium iodide, in all of which the reactions occur too rapidly for differentiation; intermediate with temperature and strontium nitrate, in both of which the reactions are closer to those of the seed parent; highest with sodium salicylate, calcium nitrate, uranium nitrate, cobalt nitrate, copper nitrate, cupric chloride, and barium chloride (in four being closer to the reactions of the seed parent, in two to those of the pollen parent, and in one as close to one as to the other parent); and lowest with polarization, iodine, gentian violet, and safranin (in two nearer the seed parent, and in two nearer the pollen parent).

The following is a summary of the reaction-intensities: Same as seed parent, 4 ; same as pollen parent, 4; same as both parents, 5 ; intermediate, 2 ; highest, 7; lowest, 4 .

These data indicate that the seed parent had a more marked influence than the pollen parent in determining the properties of the hybrid. The tendency to highest or lowest reactivity of the hybrid is quite marked, this being evident in nearly half of the reactions.

\section{Composite Curves of Reaction-Intensities.}

This section treats of the composite curves of the reaction-intensities, showing the differentiation of the starches of Lilium tenuifotium, L. martagon album, and L. golden gleam. (Chart E 26.)

The most conspicuous features of this chart are:

(1) The closeness of all three curves, the only point of important departure being in the barium-chloride reaction, in which there is a marked drop of the curve of $L$. martagon album from the curves of the other parent and the hybrid. Throughout a large part of the chart there is little or absolutely no differentiation of the curves, as in the reactions with nitric acid, sulphuric acid, hydrochloric acid, potassium hydroxide, potassium iodide, potassium sulphocyanate, potassium sulphide, sodium hydroxide, sodium sulphide, sodium salicylate, calcium nitrate, uranium nitrate, strontium nitrate, cobalt nitrate, copper nitrate, cupric chloride, and mercuric chloride. In the remaining 9 reactions the parental curves are well separated, and the hybrid curve tends usually to be close to or identical with that of $L$. tenuifolium rather than with that of the other parent.

(2) In $L$. tenuifolium, in comparison with the other parent, the lower reactions with polarization and iodine; the higher reactions with gentian violet, safranin, temperature, chloral hydrate, chromic acid, pyrogallic acid, cobalt nitrate, and barium chloride; and the same or practically the same reactions with nitric acid, sulphuric acid, hydrochloric acid, potassium hydroxide, potassium iodide, potassium sulphocyanate, potassium sulphide, sodium hydroxide, sodium sulphide, sodium salicylate, calcium nitrate, uranium nitrate, strontium nitrate, copper nitrate, cupric chloride, and mercuric chloride.

(3) In $L$. tenuifolium the very high reactions with chloral hydrate, chromic acid, pyrogallic acid, nitric acid, sulphuric acid, hydrochloric acid, potassium hydroxide, potassium iodide, potassium sulphocyanate, potassium sulphide, sodium hydroxide, sodium sulphide, sodium salicylate, calcium nitrate, uranium nitrate, strontium nitrate, cobalt nitrate, copper nitrate, cupric chloride, and mercuric chloride; the high reactions with gentian violet, temperature, and barium chloride; and the moderate reactions with polarization, iodine, and safranin.

(4) In $L$. martagon album the very high reactions with chromic acid, pyrogallic acid, nitric acid, sulphuric acid, hydrochloric acid, potassium hydroxide, potassium iodide, potassium sulphocyanate, potassium sulphide, sodium hydroxide, sodium salicylate, calcium nitrate, uranium nitrate, strontium nitrate, cobalt nitrate, cop- 
per nitrate, cupric chloride, and mercuric chloride; the high reactions with polarization, iodine, chloral hydrate, and barium chloride; and the moderate reactions with gentian violet, safranin, and temperature.

(5) In the hybrid the very high reactions with chromic acid, pyrogallic acid, nitric acid, sulphuric acid, hydrochloric acid, potassium hydroxide, potassium iodide, potassium sulphocyanate, potassium sulphide, sodium hydroxide, sodium sulphide, sodium salicylate, calcium nitrate, uranium nitrate, strontium nitrate, cobalt nitrate, copper nitrate, cupric chloride, barium chloride, and mercuric chloride; the high reactions with temperature and chloral hydrate; and the moderate reactions with polarization, iodine, gentian violet, and safranin.

Following is a summary of the reaction-intensities:

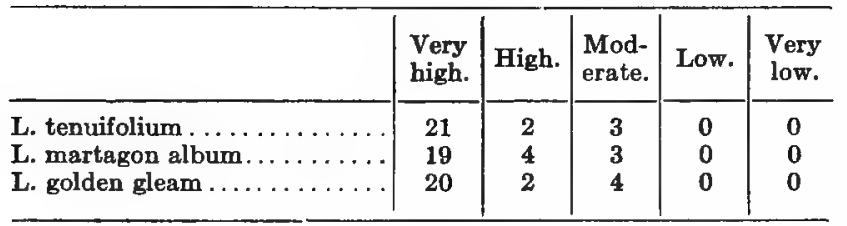

\section{Comparisons of the Starches of Lilium} CHaLCEDONICUM, L. CANDIDUM, and L. Testaceum.

In the histologic characteristics, polariscopic figures, reactions with selenite and qualitative reactions with iodine and with various chemical reagents all three starches possess properties in common in various degrees of development, the sum of which in each case is characteristic of the starch. The starch of Lilium candidum in comparison with that of $L$. chalcedonicum contains a larger proportion of grains that are regular in form, and there is a more marked tendency for the proximal end to be narrower than the distal end of the grain. The hilum is more often fissured and the eccentricity is less. The lamellæ are more distinct; broad, refractive lamellæ are more numerous; and there is often present a band of three or four broad lamellæ in the distal third of the grain; and the number is somewhat less. The sizes of corresponding types of grains are less. In the polariscopic, selenite, and qualitative iodine reactions there are numerous differences. In the qualitative reactions with chloral hydrate, chromic acid, potassium hydroxde, cobalt nitrate, and cupric chloride various differences are recorded, several of which are quite distinctive of one or the other parent. The starch of the hybrid in comparison with the starches of the parents is less regular in form than in either parent, and there is a kind of irregularity that is peculiar to the hybrid; and the grains tend to be less pointed at the proximal end than in $L$. chalcedonicum, but somewhat more pointed than in L. candidum. The hilum is in character closer to that of $L$. chalcedonicum, but in degree of eccentricity closer to that of $L$. candidum. The lamellæ are less distinct, less numerous, and finer than in either parent. The sizes of corresponding types of grains are closer to those of $L$. candidum and on the whole smaller than in the other parent. In the qualitative chemical reactions the hybrid leans to $L$. chalcedonicum, which reactions may be modified through the influence of the other parent.

Reaction-intensities Expressed by Light, Color, and Tempera-
ture Reactions. Polarization:

L. chalcedonicum, low to high, value 60 .

L. candidum, low to high, higher than in L. chalcedonicum, value 65 .

L. testaceum, low to high, the same as in $\mathrm{L}$. chalcedonicum, value 60 .
Iodine:

L. chalcedonicum, moderate, value 55 .

L. candidum, moderate, deeper than in L. chalcedonicum, value 65.

L. testaceum, moderate, less than in either parent, value 50.

Gentian violet:

L. chalcedonicum, moderate, value 60 .

L. candidum, moderate to very deep, much deeper than in $L$. chalcedonicum, value 80 .

L. testaceum, moderate to very deep, the same as in L. candidum, value 80.

Safranin:

L. chalcedonicum, moderate, value 65.

L. candidum, moderate to very deep, much deeper than in L. chalcedonicum, value 80 .

L. testaceum, moderate to very deep, the same as in L. candidum, value 80 .

Temperature:

L. chalcedonicum, in majority at 59.2 to $61^{\circ}$, in all at 63 to $64^{\circ}$, mean $63.5^{\circ}$.

L. candidum, in majority at 57 to $58.7^{\circ}$, in all at 60 to $62^{\circ}$, mean $61^{\circ}$.

L. testaceum, in majority at 61.2 to $63^{\circ}$, in all at 63.5 to $67^{\circ}$, mean $65.25^{\circ}$.

The reactivity of $L$. chalcedonicum is lower than that of the other parent in all five reactions. The reactivity of the hybrid is the same or practically the same as that of $\dot{L}$. chalcedonicum in the polarization reaction; the same or practically the same as that of the other parent in the gentian-violet and safranin reactions; and the lowest of the three in the iodine and temperature reactions. The hybrid in the polarization, iodine, and temperature reactions is closer to $L$. chalcedonicum than to the other parent, but in the gentian-violet and safranin reactions the reverse.

Table A 28 shows the reaction-intensities in percentages of total starch gelatinized at definite intervals (seconds and minutes).

\section{Velocity-Reaction CuRves.}

This section treats of the velocity-reaction curves of the starches of Lilium chalcedonicum, $L$. candidum, and L. testaceum, showing the quantitative differences in the behavior toward different reagents at definite time-intervals. (Charts D 367 to D 37\%.)

These starches react for the most part with such rapidity that but few data are of a character satisfactory for chart formation. However, even among the most rapid reacting reagents more or less marked differences are sometimes noted, as, for instance, in the reactions with nitric acid, sulphuric acid, hydrochloric acid, potassium hydroxide, potassium iodide, potassium sulphocyanate, potassium sulphide, sodium hydroxide, and sodium sulphide. Excepting those with hydrochloric acid and potassium hydroxide, there are varying degrees of lower reactivity of $L$. candidum than of the other parent and the hybrid. In other reactions that are less rapid, in which appróximately corresponding percentages of gelatinization occur in about 3 minutes (as in the reactions with calcium nitrate, uraniuma nitrate, strontium nitrate, copper nitrate, cupric chloride, and mercuric chloride), with uranium nitrate and strontium nitrate the reactivity of L. candidum is at the end of the first minute distinctly the lowest of the three; with calcium nitrate, cupric chloride, and mercuric chloride about the same as $L$. candidum and distinctly lower than in $L$. chalcedonicum; and with copper nitrate all three are alike. In all six charts the curves are from close to very close together. In all of the reactions the curves of $L$. chalcedonicum are higher than those of the other parent, the separation being well marked in all, especially with chloral hydrate and pyrogallic acid, which are distinctly the less rapid of the six. The hybrid is nearly the same as that of $L$. chalcedonicum in the reactions with chromic acid, sodium salicylate, and barium chloride; nearly the same as that of $L$. candidum with cobalt nitrate; distinctly intermediate with pyrogallic acid; and the highest of the 
TABle A 28.

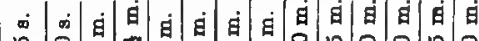

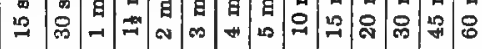

Chloral hydrate:

L. chalcedonic

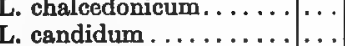

L. testaceum .

Chromio acid:

L. chalcedonicurn

L. candidum .... ...

L. testaceum.

Pyrogallic acid:

L. chalcedonicum

L. candidum ...........

L. testaceum. .

Nitric acid:

L. chalcedonicum

L. candidum . ............

L. testaceum.

Sulphuric acid:

L. chalcedonicum

L. candidum .

L. testaceum.

Hydrochloric acid:

L. chalcedonicum .

L. candidum ..............

L. testaceum.

Potassium hydroxide:

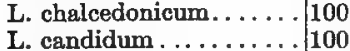

L. candidum ........... 100

Potassium iodide:

L. chalcedonicum.......... . 99

L. candidum ............. 95

L. testaceum...........

Potassium sulphocyanate:

L. chalcedonicum.

L. candidum ...........

L. testaceum.

Potassium sulphide:

L. chalcedonicum

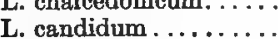

L. testaceum .............

Sodium hydroxide:

L. ehalcedonicum.......

L. candidum ...........

L. testaceum..........

Sodium sulphide:

L. chalcedonicum......

L. candidum ...........

L. testaceum.....

Sodium salicylate:

L. chalcedonicum . . . . . .

L. candidum ..........

L. testaceum.........

Calcium nitrate:

L. chalcedonicum.

L. candidum ...........

L. testaceum....

Uranium nitrate:

L. chalcedonicum .

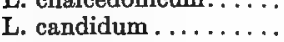

L. testaceum... .

Strontium nitrate:

L. chalcedonicum

L. candidum ....

L tegtaceum

Cobalt nitrate:

L. chalcedonicum.

L. testaceum................

Copper nitrate:

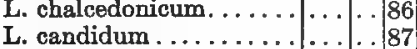

L. candidum ..............

L. testaceum.

L. chalcedonicum.

L. candidum .................

L testaceum ............. 10

Barium chloride:

L. chalcedonicum

L. candidum.................

L. testaceum.

Mercuric chloride:

L. chalcedonicum . .

....... 92

$\ldots \ldots \ldots \ldots 76$

L. testaceum............

$54 . .90 . .97 . .$.

. $34 . .74 .6889295$

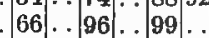

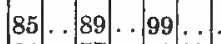

$\therefore 77 \quad 0395 \quad . .99$

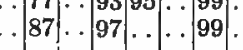

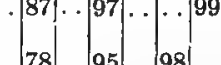

\begin{tabular}{l|l|l|l|l|l|l|l}
53 &. & 86 &. & 90 & 93 & 95
\end{tabular}

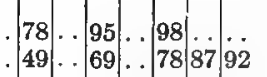

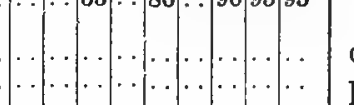
potassium iodide, potassium sulphide, and sodium hydroxide; the same as those of the pollen parent with gentian violet, safranin, and cupric chloride; the same as those of both parents with potassium hydroxide and copper nitrate; intermediate with chromic acid, pyrogallic acid, sulphuric acid, hydrochloric acid, calcium nitrate, cobalt nitrate, and barium chloride (in five being nearer the seed parent, in one nearer the pollen parent, and in one as near to one as to the other parent); highest with temperature, potassium sulphocyanate, sodium sulphide, sodium salicylate, uranium nitrate, and strontium nitrate (in all six being closer to the seed parent) ; and lowest with iodine, chloral hydrate, nitric acid, and mercuric chloride (in two being nearer the seed parent, in one nearer the pollen parent, and in one as close to one as to the other parent).

The following is a summary of the reaction-intensities: Same as seed parent, 4; same as pollen parent, 3; same as both parents, 2 ; intermediate, 7 ; highest, 6 ; lowest, 4.

The seed parent in comparison with the pollen parent has had a very potent influence in determining the properties of the starch of the hybrid. While there is a distinct tendency to intermediateness, there is an equal tendency to sameness as regards one or the other parent, and a decidedly greater tendency to highest and lowest reactivities of the hybrid.

\section{Composite Curves of Reaction-intensities.}

This section treats of the composite curves of the reaction-intensities, showing the differentiation of the starches of Lilium chalcedonicum, $L$. candidum, and $L$. testaceum. (Chart E 28.)

The most conspicuous features of this chart are:

(1) The close correspondence of all three curves, with the exception of those in the reactions with chloral hydrate and pyrogallic acid. It seems, judging from this and other records, that the reactions with chloral hydrate, chromic acid, and pyrogallic acid have a distinct tendency to be aberrant. This is seen in the reactions with chromic acid and pyrogallic acid of $L$. martagon in Chart E 26 ; with chloral hydrate and pyrogallic acid of $L$. candidum, and in the pyrogallic-acid reaction of the hybrid in this chart; and in the chromic-acid and pyrogallic-acid reactions of the hybrid, L. burbanki, in Chart E 29. In most of the charts there is little or no differentiation of the three starches, as in the reactions with nitric acid, sulphuric acid, hydrochloric acid, potassium hydroxide, potassium iodide, potassium sulphocyanate, potassium sulphide, sodium hydroxide, sodium sulphide, sodium salicylate, calcium nitrate, uranium nitrate, strontium nitrate, copper nitrate, cupric chloride, and mercuric chloride. The curves of the hybrid and L. candidum tend to be more closely related than the curves of the hybrid and the other parent, or the curves of the parents. 
(2) In $L$. chalcedonicum in comparison with that of the other parent, the lower reactions with polarization, iodine, gentian violet, safranin, and temperature; the higher reactions with chloral hydrate, chromic acid, pyrogallic acid, cobalt nitrate, cupric chloride, and barium chloride; and the same or practically the same with nitric acid, sulphuric acid, hydrochloric acid, potassium hydroxide, potassium iodide, potassium sulphocyanate, potassium sulphide, sodium hydroxide, sodium sulphide, sodium salicylate, calcium nitrate, uranium nitrate, strontium nitrate, copper nitrate, and mercuric chloride.

(3) In L. chalcedonicum the very high reactions with chromic acid, pyrogallic acid, nitric acid, sulphuric acid, hydrochloric acid, potassium hydroxide, potassium iodide, potassium sulphocyanate, potassium sulphide, sodium hydroxide, sodium sulphide, sodium salicylate, calcium nitrate, uranium nitrate, strontium nitrate, cobalt nitrate, copper nitrate, cupric chloride, barium chloride, and mercuric chloride; the high reactions with polarization, gentian violet, safranin, and chloral hydrate; and the moderate reactions with iodine and temperature.

(4) In $L$. candidum the very high reactions with gentian violet, safranin, chromic acid, nitric acid, sulphuric acid, hydrochloric acid, potassium hydroxide, potassium iodide, potassium sulphocyanate, potassium sulphide, sodium hydroxide, sodium sulphide, sodium salicylate, calcium nitrate, uranium nitrate, strontium nitrate, cobalt nitrate, copper nitrate, cupric chloride, and mercuric chloride; the high reactions with polarization, iodine, temperature, and barium chloride; and the moderate reactions with chloral hydrate and pyrogallic acid.

(5) In the hybrid, the very high reactions with chloral hydrate, chromic acid, nitric acid, sulphuric acid, hydrochloric acid, potassium hydroxide, potassium iodide, potassium sulphocyanate, potassium sulphide, sodium hydroxide, sodium sulphide, sodium salicylate, calcium nitrate, uranium nitrate, strontium nitrate, cobalt nitrate, copper nitrate, cupric chloride, and mercuric chloride; the high reactions with polarization and barium chloride; and the moderate reactions with iodine, temperature, and pyrogallic acid.

Following is a summary of the reaction-intensities:

\begin{tabular}{|c|c|c|c|c|c|}
\hline & $\begin{array}{l}\text { Very } \\
\text { high. }\end{array}$ & High. & $\begin{array}{l}\text { Mod- } \\
\text { erate. }\end{array}$ & Low. & $\begin{array}{l}\text { Very } \\
\text { low. }\end{array}$ \\
\hline 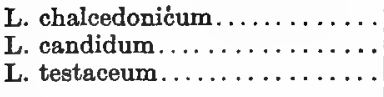 & $\begin{array}{l}20 \\
20 \\
21\end{array}$ & $\begin{array}{l}4 \\
4 \\
2\end{array}$ & $\begin{array}{l}\mathbf{2} \\
\mathbf{2} \\
\mathbf{3}\end{array}$ & $\begin{array}{l}0 \\
0 \\
0\end{array}$ & $\begin{array}{l}\mathbf{0} \\
\mathbf{0} \\
\mathbf{0}\end{array}$ \\
\hline
\end{tabular}

29. Comparisons of the Starches of Lilium PARDALINUM, L. PARRYI, AND L. BURBANKI.

In the histologic characteristics, polariscopic figures, reactions with selenite, qualitative reactions with iodine; and qualitative reactions with the various chemical reagents all three starches exhibit properties in common in varying degrees of development, the sum of which in each case being characteristic of the starch. The starch of $L$. parryi in comparison with that of $L$. pardalinum contains less numbers of compound grains and aggregates, and the grains are less irregular. The hilum is slightly less eccentric. The lamellæ are less distinct, and less numerous, and there is an absence of a broad refractive lamella that is found in $L$. pardalinum. The sizes of the corresponding forms of the grains are distinctly less. In the polariscopic, selenite, and qualitative iodine reactions there are some apparently minor differences. In the qualitative reactions with chloral hydrate, chromic acid, potassium hydroxide, cobalt nitrate, and cupric chloride various differences are recorded which seem to be of minor importance. The starch of the hybrid in comparison with the starches of the parents shows an absence of compound grains that are found in both parents; and the grains are more regular in form than in either parent. The hilum is less distinct, less often fissured, and less eccentric than in either parent. The lamellæ are in general characters like those of the parents, but they are less numerous. The sizes of the corresponding forms of grains are about mid-intermediate between those of the parents. In the polariscopic and selenite reactions the relationship of the hybrid is closer to $L$. parryi, but in the qualitative reactions closer to $L$. pardalinum. In the qualitative reactions with the chemical reagents in the reactions with chloral hydrate, chromic acid, potassium hydroxide, cobalt nitrate, and cupric chloride the relationship of the hybrid is closer to $L$. pardalium, but there are many instances of closeness to the peculiarities of $L$. parryi, especially in the chloral-hydrate and chromic-acid reactions. The influences of $L$. parryi are quite obvious, although, as a whole, superseded by those of the other parent.

\section{Reaction-intensities Expressed by Light, Color, and Tempera- ture Reactions.} Polarization:

L. pardalinum, low to high, value 55 .

L. parryi, low to high, lower than in L. pardalinum, value 50.

L. burbanki, low to high, the same as in L. parryi, value 50 .

Iodine:

L. pardalinum, light to moderate, value 40 .

L. parryi, moderate, much higher than in L. pardalinum, value $\mathbf{5 5}$.

L. burbanki, light to moderate, the same as in L. pardalinum, value 40 .

Gentian violet:

L. pardalinum, moderate to deep, value 65.

L. parryi, light to moderate, very much less than in L. pardalinum, value 40 .

L. burbanki, moderate, more than in L. parryi, value 45 .

Safranin:

L. pardalinum, moderate to deep, value 65 .

L. parryi, light to moderate, very much less than in L. pardalinum, value 35 .

L. burbanki, light to moderate, more than in L. parryi, value 40 . Temperature:

L. pardalinum, in majority at 58 to $60.5^{\circ}$, in all at 61 to $63^{\circ}$, mean $62^{\circ}$.

L. parryi, in majority at 47 to $48.5^{\circ}$, in all at 51 to $52^{\circ}$, mean $51.5^{\circ}$.

L. burbanki, in majority at 64 to $66^{\circ}$, in all at 67 to $68.5^{\circ}$, mean 67.75'.

The reactivity of $L$. pardalinum is higher than that of the other parent in the polarization, gentian-violet, and safranin reactions; and lower in the iodine and temperature reactions. The reactivity of the hybrid is the same or practically the same as that of $L$. pardalinum in the iodine reaction; the same or practically the same as that of $L$. parryi in the polarization reaction; lowest of the three in the temperature reaction; and intermediate in the gentian-violet and safranin reactions. The hybrid in the iodine and temperature reactions is closer to $L$. pardalinum than to $L$. parryi, but in the polarization, gentian violet, and safranin reactions closer to the latter parent.

Table A 29 shows the reaction-intensities in percentages of total starch gelatinized at definite intervals (seconds and minutes).

\section{VELOCITY-REACTION CURVES.}

This section treats of the velocity-reaction curves of the starches of Lilium pardalinum, L. parryi, and L. burbanki, showing the quantitative differences in the behavior toward different reagents at definite time-intervals. (Charts D 373 to D 378.)

These starches in common with the other lily starches are generally very sensitive to gelatinizing agents, but 
Table A 29.

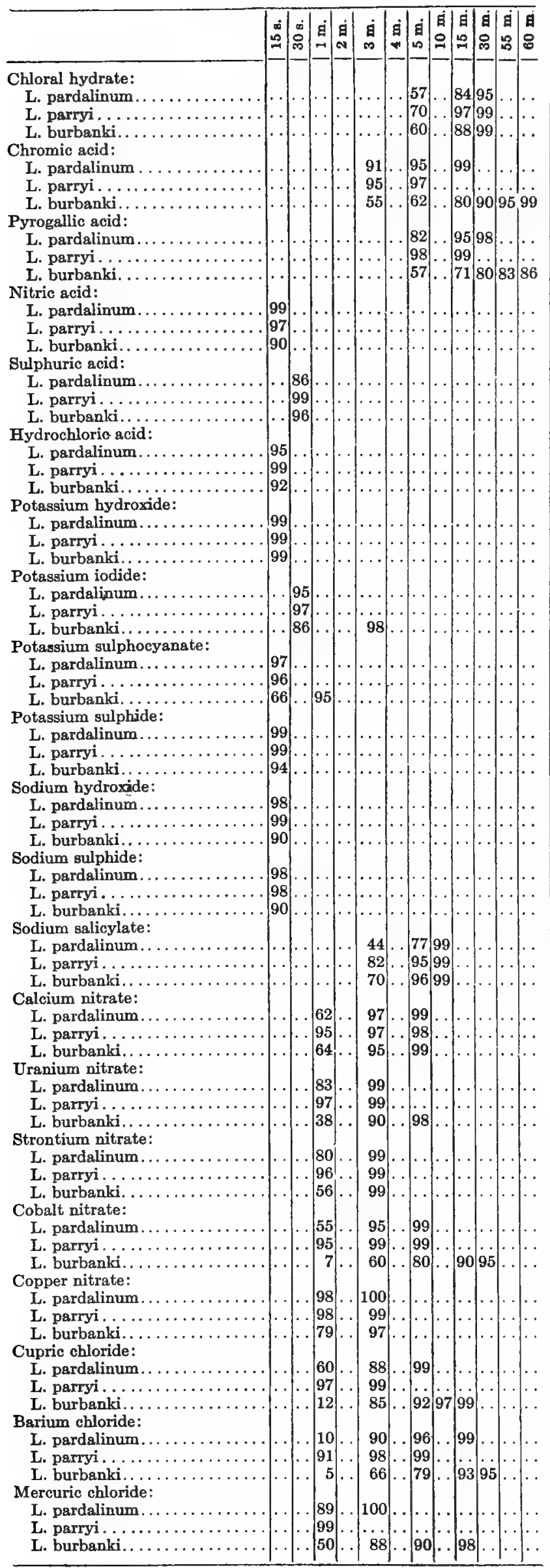

there is, on the whole, distinctly less sensitivity than of any of the four preceding groups, particularly as regards the hybrid. As a rule, however, the data are not of much usefulness excepting in very few instances for chart making. Gelatinization is nearly or practically complete in 15 to 30 seconds in the reactions with nitric acid, sulphuric acid, hydrochloric acid, potassium hydroxide, potassium iodide, potassium sulphocyanate, potassium sulphide, sodium hydroxide, and sodium sulphide. In the reactions with nitric acid, hydrochloric acid, potassium iodide, potassium sulphocyanate, sodium hydroxide, and sodium sulphide there are distinct indications of lower reactivity of the hybrid than of the parents. Gelatinization goes on very rapidly in all three starches during the first 1 to 3 minutes in the other reactions, so that in nearly all (excepting those with chloral hydrate, chromic acid, sodium salicylate, and cupric chloride) at least 90 per cent of the total starch is broken down within this period. In occasional instances the hybrid is comparatively resistant, as in the reactions with chromic acid, uranium nitrate, strontium nitrate, cobalt nitrate, copper nitrate, cupric chloride, barium chloride, and mercuric chloride, in some of which the resistance is quite marked or only noticeable during the first minute. There are also suggestions of differences in the parents, $L$. pardalinum showing generally a marked tendency to greater resistance than L. parryi. In these reactions the hybrid is generally distinctly closer to $L$. pardalinum than to the other parent, this being in accord with the findings in the histologic and quantitative peculiarities, and in the light, color, and temperature reactions. Referring to the charts, it will be seen that all three curves in each reaction tend to be from close to very close, the parental curves running together in five out of the six reactions, and the hybrid with the curves of $L$. parryi in the sodium-salicylate reactions. In all six charts the curves of $L$. parryi are higher than the curves of $L$. parryi in the reactions with chromic acid, cobalt nitrate, barium chloride, and mercuric chloride, keeping very close together, yet showing quite definite differences in the reactions. The hybrid curve is intermediate in the chloral-hydrate reaction; distinctly the lowest in those with chromic acid, pyrogallic acid, cobalt nitrate, barium chloride, and mercuric chloride; and nearly the same as L. parryi (at first intermediate) with sodium salicylate. There is in general a tendency to less reactivity of the hybrid than of the parents.

\section{Reaction-Intensities of the Hybrid.}

This section treats of the reaction-intensities of the hybrid as regards sameness, intermediateness, excess, and deficit in relation to the parents. (Table A 29 and Charts D 373 to D 378.)

The reactivities of the hybrid are the same as those of the seed parent in the iodine and calcium-nitrate reactions; the same as those of the pollen parent in the polarization reaction; the same as those of both parents in the potassium hydroxide reaction, in which the reactions occur too rapidly for differentiation; intermediate in the reactions with gentian violet, safranin, chloral hydrate, sulphuric acid, sodium salicylate, and barium chloride (in four being closer to those of the pollen parent, and in two closer to those of the seed parent); highest in none; and lowest in those with temperature, chromic acid, pyrogallic acid, nitric acid, hydrochloric acid, potassium iodide, potassium sulphocyanate, potassium sulphide, sodium hydroxide, sodium sulphide, uranium nitrate, strontium nitrate, cobalt nitrate, copper nitrate, cupric chloride, and mercuric chloride (in nine being 
closer to those of the seed parent, and in seven being as close to one as to the other parent). The following is a summary of the reaction-intensities: Same as seed parent, 2 ; same as pollen parent, 1 ; same as both parents, 1 ; intermediate, 6 ; highest, 0 ; lowest, 16.

The seed parent has according to these data to a far greater degree than the other parent influenced the properties of the starch of the hybrid. The tendency to lowest reactivity of the hybrid is even more conspicuous than the leanings to the seed parent. Intermediateness is fairly well marked.

\section{Composite Corves of the Reaction-intensities.}

This section treats of the composite curves of the reaction-intensities, showing the differentiation of the starches of Lilium pardalinum, $L$. parryi, and $L$. burbanki. (Chart E 29.)

The most conspicuous features of this chart are:

(1) The generally very close correspondence of all three curves, the most noticeable variations in the case of the parents being in the reactions with gentian violet and safranin; and of the hybrid with chromic acid, pyrogallic acid, cobalt nitrate, barium chloride, and mercuric chloride. There is no satisfactory differentiation of the three starches in the reactions with nitric acid, sulphuric acid, hydrochloric acid, potassium hydroxide, potassium iodide, potassium sulphocyanate, potassium sulphide, sodium hydroxide, and sodium sulphide; there is no differentiation of the parents in the copper-nitrate reaction, and not a very marked differentiation in those with calcium nitrate, uranium nitrate, strontium nitrate, cobalt nitrate, cupric chloride, barium chloride, and mercuric chloride. The hybrid curve tends to be somewhat erratic, and inclining to keep low and even below the parental curves, this being especially noticeable in the reactions with temperature, chromic acid, pyrogallic acid, uranium nitrate, cobalt nitrate, copper nitrate, cupric chloride, barium chloride, and mercuric chloride. With weaker reagents where the reactions occur with great rapidity, as in the nine reactions from nitric acid on to sodium sulphide, inclusive, this tendency would doubtless be made even more conspicuous. On the whole, the hybrid curve is much more closely related to the curve of $L$. pardalinum than to that of $L$. parryi.

(2) In L. pardalinum, in comparison with the other parent, the higher reactions with polarization, gentian violet, and safranin; the lower with iodine, temperature, chloral hydrate, chromic acid, pyrogallic acid, sodium salicylate, calcium nitrate, uranium nitrate, strontium nitrate, cobalt nitrate, cupric chloride, barium chloride, and mercuric chloride; and the same or practically the same reactions as those of the other parent with nitric acid, sulphuric acid, hydrochloric acid, potassium hydroxide, potassium sulphocyanate, potassium sulphide, sodium hydroxide, sodium sulphide, and copper nitrate.

(3) In $L$. pardalinum the very high reactions with chromic acid, pyrogallic acid, nitric acid, sulphuric acid, hydrochloric acid, potassium hydroxide, potassium iodide, potassium sulphocyanate, potassium sulphide, sodium hydroxide, sodium sulphide, sodium salicylate, calcium nitrate, uranium nitrate, strontium nitrate, cobalt nitrate, copper nitrate, cupric chloride, barium chloride, and mercuric chloride; the high reactions with gentian violet, safranin, temperature, and chloral hydrate; the moderate reactions with polarization and iodine.

(4) In $L$. parryi the very high reactions with temperature, chloral hydrate, chromic acid, pyrogallic acid, nitric acid, sulphuric acid, hydrochloric acid, potassium hydroxide, potassium iodide, potassium sulphocyanate, potassium sulphide, sodium hydroxide, sodium sulphide, sodium salicylate, calcium nitrate, uranium nitrate, strontium nitrate, cobalt nitrate, copper nitrate, cupric chloride, barium chloride, and mercuric chloride, reactions; the absence of a high reaction; the moderate reactions with polarization, iodine, and gentian violet; and the low reaction with safranin.

(5) In the hybrid the very high reactions with nitric acid, sulphuric acid, hydrochloric acid, potassium hydroxide, potassium iodide, potassium sulphocyanate, potassium sulphide, sodium hydroxide, sodium sulphide, sodium salicylate, calcium nitrate, strontium nitrate, copper nitrate, cupric chloride, and mercuric chloride; the high reactions with chloral hydrate, chromic acid, cobalt nitrate, and barium chloride; the moderate reactions with polarization, gentian violet, safranin, and temperature; and the low reactions with iodine and pyrogallic acid. ties:

The following is a summary of the reaction-intensi-

\begin{tabular}{|c|c|c|c|c|c|}
\hline & $\begin{array}{l}\text { Very } \\
\text { high. }\end{array}$ & High. & $\begin{array}{l}\text { Mod- } \\
\text { erate. }\end{array}$ & Low. & $\begin{array}{l}\text { Very } \\
\text { low. }\end{array}$ \\
\hline $\begin{array}{l}\text { L. pardalinum } \ldots \ldots \ldots \ldots \ldots \ldots \\
\text { L. parryi } \ldots \ldots \ldots \ldots \ldots \ldots \ldots \\
\text { L. burbanki......... }\end{array}$ & $\begin{array}{l}20 \\
22 \\
16\end{array}$ & $\begin{array}{l}4 \\
0 \\
4\end{array}$ & $\begin{array}{l}\mathbf{2} \\
\mathbf{3} \\
\mathbf{4}\end{array}$ & $\begin{array}{l}0 \\
1 \\
2\end{array}$ & $\begin{array}{l}\mathbf{0} \\
\mathbf{0} \\
\mathbf{0}\end{array}$ \\
\hline
\end{tabular}

\section{Notes ON THE LILIES.}

The starches of the various species of lilies belong to the quick-reacting group and they are universally so rapidly gelatinized by nitric acid, sulphuric acid, hydrochloric acid, potassium hydroxide, potassium iodide, potassium sulphocyanate, potassium sulphide, sodium hydroxide, and sodium sulphide that satisfactory differentiation is not possible, excepting with reagents of different concentration from those used in this research. Even with most of the other chemical reagents, they often react so rapidly that convincing differential data are not obtainable with the concentrations employed. The only reagents in the concentrations used that are really useful are chloral hydrate, chromic acid, pyrogallic acid, sodium salicylate, cobalt nitrate, and barium chloride. But in the reactions with polarization, iodine, gentian violet, safranin, and temperature conclusive data were usually recorded.

The hybrids tend in each case to be more closely related in the sum total of their characters to one or the other parent, and with far less inclination to intermediateness than to identical development or to excessive or deficient development beyond parental extremes. The tendency to exceed parental extremes is particularly well marked in the curve of $L$. burbanki, where there is shown a very distinct inclination to be below the lower of the parental curves. In the first and fourth groups, the hybrids are more closely related on the whole to the pollen parents; and in the second, third, and fifth groups to the seed parents. The general relationship of the 
hybrids to their respective parents in their quantitative reactions are exhibited in the following summary, the figures being, however, of an absolutely tentative character, because many of the reactions recorded as sameness are so only because the concentrations of the reagents were not adapted to elicit differences of a positive character.

Following is a summary of the reaction-intensities:

\begin{tabular}{|c|c|c|c|c|c|c|}
\hline & 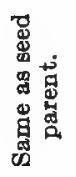 & 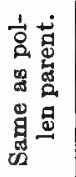 & 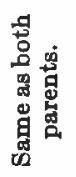 & 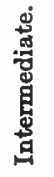 & 悹 & 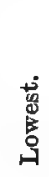 \\
\hline 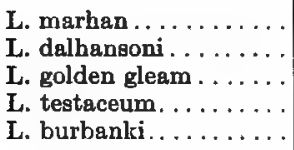 & $\begin{array}{l}0 \\
4 \\
4 \\
4 \\
2\end{array}$ & $\begin{array}{l}5 \\
1 \\
4 \\
3 \\
1\end{array}$ & $\begin{array}{l}9 \\
9 \\
5 \\
2 \\
1\end{array}$ & $\begin{array}{l}6 \\
9 \\
2 \\
7 \\
6\end{array}$ & $\begin{array}{l}1 \\
2 \\
7 \\
6 \\
0\end{array}$ & $\begin{array}{r}5 \\
1 \\
4 \\
4 \\
16\end{array}$ \\
\hline
\end{tabular}

The general picture presented by the five charts is that of a definite generic type, the curves bearing close relationships in their courses; but with a tendency to variability in the reactions with chloral hydrate, chromic acid, and pyrogallic acid, this latter indicating a marked molecular instability in relation to these special reagents. There is not the least evidence of subgeneric grouping such as was found in certain other genera studied, this being in accord with the findings in the preceding research in which it was stated upon the basis of that preliminary work that the division of Lilium into the six subgenera noted is probably botanically artificial.

The curves of Lilium martagon and its horticultural variety $L$. martagon album very closely coincide, the curve of the former inclining, where satisfactory differences can be made out, to be somewhat lower than that of the former, as in the reactions with polarization, iodine, chromic acid, pyrogallic acid, cobalt nitrate, and barium chloride; and rarely higher, as with safranin and chloral hydrate, the latter being the only one that is important.

It is of interest to note that in the fourth group $L$. chalcedonicum (subgenus Martagon) is crossed with L. candidum (subgenus Eulirion), yielding $L$. testaceum, which latter is classed in the subgenus Martagon and in the same subdivision of the subgenus as $L$. chalcedonicum. In this research the hybrid shows in the sum total of its characters a closer relationship, as a whole, to $L$. chalcedonicum than to the other parent. Thus, in the form of the grain, general characters of the hilum, characters and arrangements of the lamellæ, polariscopic figure, appearances with selenite, qualitative reactions with iodine, qualitative reactions with the various chemical reagents, and quantitative reactions in the polarization, iodine, chloral-hydrate, and chromicacid reactions it is closer to $L$. chalcedonicum; but in eccentricity of the hilum, size of the grains, and quantitative reactions with gentian violet, safranin, pyrogallic acid, cobalt nitrate, cupric chloride, and barium chloride it is distinctly much closer to the other parent. Curiously, while the foregoing data, as a whole, indicate a much closer relationship of the hybrid to $L$. chalcedonicum, the composite curves indicate the contrary, but this contradiction may be explained upon the basis of inadequate analysis with the chemical reagents, because of the great rapidity of many of the reactions. From the foregoing, qualitative data may be more important in the recognition and differentiation of starches than quantitative data, although theoretically one should expect them to go hand in hand.

\section{Comparisons of the Starches of Iris iberica, I. TROJANA, AND I. ISMALI.}

In the histologic characteristics, polariscopic figures, reactions with selenite, reactions with iodine, and qualitative reactions with various chemical reagents, the starches of the parents and hybrid exhibit properties in common in varying degrees of development, the sum of which in each case is characteristic of the starch. The starch of Iris iberica in comparison with that of I. trojana contains few aggregates, and more compound grains of more types; the grains are more irregular; and flattening of the distal end of elongated elliptical grains is more common. The hilum is more distinct and more frequently fissured. The lamellæ are coarser and more distinct; more apt to be irregular, especially between the hilum and the distal margin, following in their course the curvature of the notch in the distal margin; and the number is larger. The common sizes are largerlonger and broader or longer and of the same width than in the other parent. In the polariscopic, selenite, and qualitative iodine reactions there are a number of differences of an apparently minor character. In the qualitative reactions with chloral hydrate, hydrochloric acid, potassium iodide, sodium hydroxide, and sodium salicylate there are various differences, probably for the most part unimportant. The starch of the hybrid in comparison with the starches of the parents contains a less number of aggregates than in either parent; more compound grains than in I. iberica but less than in I. trojana; and the grains are much more irregular than in $I$. iberica and more irregular than in $I$. trojana. The hilum in character is more closely related to $I$. iberica, but in eccentricity to the other parent. The lamellæ are in character, arrangement, and number more closely related to I. iberica. The size is less than in either parent, but closer to $I$. iberica. In the degree of polarization and qualitative iodine reactions the relationship is closer to $I$. iberica, but in the qualitative polarization and selenite reactions closed to the other parent. In the qualitative chemical reactions there are leanings here and there to one or the other parent, but on the whole the relationships are much closer to 1 . iberica. It is of interest to note that a feature of $I$. iberica may be accentuated in the reactions of the hybrid.

Reaction-intensities Expressed by Light, Color, and TemperaPolarizatiọn:

I. iberica, low to high, value 50 .

I. trojana, low to moderately high, lower than in I. iberica, value 45 .

I. ismali, low to moderately high, lower than in either parent,

Iodine: value 40 .

I. iberica, light to moderate, value 40 .

I. trojana, moderate, deeper than in I. iberica, value 50 .

I. ismali, light to moderate, the same as in I. iberica, value 40 .

Gentian violet:

I. iberica, light to moderate, value 40.

I. trojana, moderate, deeper than in I. iberica, value 50.

I. ismali, light to moderate, the same as in $I$. iberica, value 40 . 
Safranin:

I. iberica, moderate, value 45 .

I. trojana, moderate, deeper than in I. iberica, value 50 .

I. ismali, moderate, the same as in I. iberica, value 45 . Temperature:

I. iberica, in the majority at 69 to $70^{\circ}$, in all at 71 to $72.5^{\circ}$, mean $71.75^{\circ}$.

I. trojana, in the majority at 70 to $71.5^{\circ}$, in all at 73.2 to $75^{\circ}$, mean $72.1^{\circ}$

I. ismali, in the majority at 69 to $71^{\circ}$, in all at 72 to $74^{\circ}$, mean $73^{\circ}$.

The reactivity of $I$. iberica is higher than that of the other parent in the polarization and temperature experiments, and lower in iodine, gentian-violet, and safranin reactions. The reactivity of the hybrid is the same or practically the same as that of $I$. iberica in the iodine, gentian-violet, and safranin reactions; the lowest of the three in the polarization reaction; and intermediate between those of the parents in the temperature reaction. The hybrid is nearer to $I$. iberica in the iodine, gentianviolet, and safranin reactions, nearer to the other parent in the polarization reactions, and intermediate in the temperature reaction.

Table A 30 shows the reaction-intensities in percentages of total starch gelatinized at definite intervals (minutes).

\section{Veloctty-Reaction Curves.}

This section treats of the velocity-reaction curves of the starches of Iris iberica, I. trojana, and I. ismali, showing the quantitative differences in the behavior toward different reagents at definite time-intervals. (Charts D 379 to D 399.)

The most conspicuous features of this group of curves are:

(1) The closeness of all three curves, indicating not only a corresponding relationship of the parents, but also very little modification of parental peculiarities in the hybrid. As regards the latter, the tendency of the curve is to follow closely that of one or the other parent or be of some degree of intermediateness. The only instances where there seems to be a notable inclination for separation of the curves are in the reactions with chloral hydrate, hydrochloric acid, sodium sulphide, calcium nitrate, and mercuric chloride; and with the exception of the last the hybrid curve is between the parental curves and distinctly closer to the curve of one or the other parent.

(2) The lower reactivity of $I$. iberica in comparison with the other parent with all of the chemical reagents (excepting in the very rapid sulphuric-acid and the very slow cobalt-nitrate and barium-chloride reactions, where the parental curves are practically absolutely the same), the absence of differentiation doubtless being due to the extreme slowness of gelatinization.

(3) The variable position of the hybrid curve in relation to the parental curves in the various reactions, with a very definite tendency to intermediateness or lowness. In some of the reactions one of the three starches may at first be comparatively slow in reacting, followed by a comparatively rapid reaction, so that the relations of the curves are changed. This is seen in the pyrogallicacid, strontium-nitrate, and copper-nitrate reactions, in which the hybrid curve is the lowest at the end of 5 minutes and subsequently intermediate; in the calciumnitrate reactions, where the curve of I. trojana is the lowest at 5 minutes and then the highest and well separated from the other curves; and in uranium-nitrate reaction where the parental curves change their relative positions after 5 minutes. The sulphuric-acid chart shows no differentiation, but the figures at the end of 2 minutes indicate the order of reactivity as follows: I. trojana, I. ismali, and $I$. iberica, making the hybrid intermediate. The
TABle A 30.

\begin{tabular}{|c|c|c|c|c|c|c|c|c|c|c|}
\hline & $\begin{array}{l}\text { 日 } \\
\text { 一 }\end{array}$ & $\underset{\text { ก }}{\text { ก }}$ & $\begin{array}{l}\text { 日 } \\
\text { ๓ }\end{array}$ & 五 & 官 & 몽 & $\begin{array}{l}\dot{g} \\
\stackrel{0}{\circ}\end{array}$ & $\begin{array}{l}\text { 永 } \\
\text { 品 }\end{array}$ & $\begin{array}{l}\text { घ } \\
\text { Н }\end{array}$ & $\begin{array}{l}\text { 1 } \\
8\end{array}$ \\
\hline Chloral hydrate: & & & & & & & & & & \\
\hline & & & & . & 6 & & 39 & 50 & & \\
\hline & $\cdots$ & & . & . & 18 & .. & 51 & 77 & 88 & \\
\hline & & & & . & & $\cdots$ & 42 & 76 & 36 & \\
\hline Chror & & & & & & & & & & \\
\hline & $\cdots$ & $\cdots$ & $\cdots$ & . & 6 & $\cdots$ & 70 & 90 & 97 & \\
\hline & & $\cdot$ & $\cdots$ & $\cdots$ & $\begin{array}{r}29 \\
0\end{array}$ & $\cdots$ & 0 & 98 & 68 & \\
\hline Pyrog & & & & & & & & 182 & 98 & \\
\hline & $\cdots$ & - & $\cdots$ & . & 22 & $\cdots$ & 72 & 81 & 86 & \\
\hline & & & .. & $\ldots$ & 28 & .. & 77 & 84 & 93 & 96 \\
\hline I. i & .. & . & . & .. & 16 & $\ldots$ & 75 & 81 & 92 & \\
\hline Nitri & & & & & & & & & & \\
\hline & $\cdots$ & $\cdots$ & . & $\cdots$ & 58 & .. & 73 & 77 & 81 & \\
\hline & & & & & 70 & .. & 82 & 86 & 90 & \\
\hline $\begin{array}{l}\text { I. } \mathbf{j} \\
\text { ulph }\end{array}$ & & - & $\cdots$ & $\cdots$ & 58 & . & 5 & 82 & 89 & 92 \\
\hline I. $\mathrm{i}$ & $\ldots$ & 85 & & .. & 99 & $\ldots$ & & $\cdots$ & & \\
\hline & & & & $\ldots$ & 99 & .. & .. & .. & .. & \\
\hline I. $\mathrm{i}$ & $\ldots$ & & & . & 97 & $\ldots$ & & .. & $\ldots$ & \\
\hline Hydr & & & & & & & & & & \\
\hline & $\cdots$ & .. & .. & .. & 53 & .. & 63 & 72 & 81 & 86 \\
\hline & $\cdots$ & . & $\cdots$ & $\cdots$ & 72 & . & 83 & 88 & $\cdots$ & 90 \\
\hline & & . & .. & . & 64 & . & 82 & 87 & . & \\
\hline Potas & & & & & & & & & & \\
\hline & & $\cdot$ & . & & $\begin{array}{l}82 \\
84\end{array}$ & & $\begin{array}{l}85 \\
92\end{array}$ & 89 & 93 & $\begin{array}{l}95 \\
96\end{array}$ \\
\hline & & & & 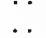 & 77 & $\cdots$ & $\begin{array}{l}92 \\
81\end{array}$ & $\begin{array}{l}80 \\
84\end{array}$ & 88 & $\begin{array}{l}90 \\
93\end{array}$ \\
\hline Potas & & & & & & & & & & \\
\hline & $\cdots$ & $\cdots$ & . & . & 52 & . & 68 & 78 & 86 & 89 \\
\hline & .. & .. & .. & .. & 58 & .. & 83 & 92 & 93 & 94 \\
\hline & & $\cdots$ & $\cdots$ & . & 65 & $\ldots$ & 85 & 89 & 91 & 83 \\
\hline sulphocyanate: & & & & & & & & & & \\
\hline & $\cdots$ & 84 & & . & 90 & . & 97 & $\cdots$ & $\cdots$ & $\cdots$ \\
\hline & & & $\cdots$ & $\ldots$ & 95 & . . & 98 & $\cdots$ & . & \\
\hline $\begin{array}{r}\text { I. i } \\
\text { PotaE }\end{array}$ & .. & & $\cdots$ & $\cdots$ & 93 & .. & 97 & .. & $\because$ & \\
\hline & $\ldots$ & $\cdots$ & & & 4 & & 5 & 6 & 7 & \\
\hline & $\ldots$ & $\ldots$ & . & $\ldots$ & 6 & .. & 11 & 16 & 20 & 23 \\
\hline & $\ldots$ & .. & . & .. & 5 & $\ldots$ & 10 & 13 & .. & 13 \\
\hline Sodiu & & & & & & & & & & \\
\hline & $\ldots$ & 59 & . & . & 80 & .. & 88 & 95 & 97 & 97 \\
\hline & $\ldots$ & 75 & $\ldots$ & $\ldots$ & 87 & .. & 91 & 95 & 97 & 97 \\
\hline & $\ldots$ & 60 & & & 82 & & 94 & 96 & 98 & 98 \\
\hline odiu & & & & & & & & & & \\
\hline & .. & $\cdots$ & $\cdots$ & $\cdots$ & 14 & $\cdots$ & 34 & 47 & 55 & 58 \\
\hline & .. & $\ldots$ & . & & 39 & .. & 58 & 67 & 72 & 77 \\
\hline & . & $\cdots$ & $\ldots$ & $\ldots$ & 17 & .. & 35 & 53 & 69 & 75 \\
\hline Sodi & & & & & & & & & & \\
\hline & . & $\ldots$ & . & . & 55 & 89 & 99 & $\cdots$ & . &. \\
\hline & . & $\cdots$ & $\cdots$ & & 77 & 99 & .. & $\ldots$ & .. & \\
\hline & $\cdots$ & $\ldots$ & $\ldots$ & & 75 & 99 & . & . & .. & \\
\hline Valci & & & & & & & & 45 & & \\
\hline & $\because$ & $\cdots$ & . & & $\begin{array}{r}13 \\
7\end{array}$ & & $\begin{array}{l}30 \\
66\end{array}$ & 71 & $\mid \begin{array}{l}07 \\
75\end{array}$ & $\begin{array}{l}100 \\
79\end{array}$ \\
\hline & & $\cdots$ & & & 19 & & 32 & 48 & 54 & 62 \\
\hline Uran & & & & & & & & & & \\
\hline & $\ldots$ & . & $\ldots$ & & 10 & & 20 & 22 & 25 & 29 \\
\hline & $\ldots$ & $\ldots$ & $\ldots$ & & $\mathbf{5}$ & .. & 25 & 32 & 40 & 45 \\
\hline & $\ldots$ & .. & $\ldots$ & $\cdots$ & 19 & $\ldots$ & 32 & 48 & 54 & 62 \\
\hline Stro & & & & & & & & & & \\
\hline & $\ldots$ & .. & $\cdots$ & & 12 & . & 48 & 67 & 78 & 80 \\
\hline & . & .. & . & & 1 & .. & 69 & 80 & 86 & 88 \\
\hline & & $\ldots$ & . & & 10 & .. & 50 & 68 & 80 & 86 \\
\hline & & & & & & & & & & \\
\hline & & .. & . & & 2 & .. & 4 & 6 & & \\
\hline & & $\ldots$ & . & & 1 & & 3 & 8 & 9 & \\
\hline & & $\ldots$ & $\ldots$ & . & 0.5 & .. & 2 & .. & & 3 \\
\hline & & & & & & & & & & \\
\hline & & .. & $\ldots$ & & 12 & .. & 19 & 50 & 54 & 61 \\
\hline & & .. & . & & 16 & .. & 25 & 70 & 76 & 81 \\
\hline & & .. & $\ldots$ & . & 4 & $\ldots$ & 22 & 54 & 60 & 63 \\
\hline & & & & & & & & & & \\
\hline & & . & $\ldots$ & . & 10 & . & 42 & 61 & 64 & 70 \\
\hline & & $\ldots$ & $\ldots$ & $\ldots$ & 15 & .. & 50 & 70 & 77 & 81 \\
\hline & & & $\cdots$ & $\ldots$ & 5 & & 22 & 51 & 61 & 68 \\
\hline Bariv & & & & & & & & & & \\
\hline & & . & . & & 1 & & 5 & 9 & 10 & 11 \\
\hline & & & & & 1 & & B & 7 & 9 & 11 \\
\hline & & $\ldots$ & $\ldots$ & . & 0.5 & & 1 & 2 & 3 & $\mathbf{5}$ \\
\hline & & & & & & & & & & \\
\hline & & . & 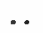 & $\cdots$ & 3 & .. & 11 & 15 & 22 & 52 \\
\hline & & . & . & & 6 & . & 18 & 32 & 40 & 46 \\
\hline & & & & & & & & 8 & 9 & 12 \\
\hline
\end{tabular}


hybrid and $I$. trojana curves are practically absolutely the same and above the $I$. iberica curve in the reactions with sodium salicylate; almost identical with the parental curves in the reaction with potassium sulphocyanate; at first intermediate and then the highest of the three in the reactions with sodium hydroxide, although there are but little differences; and the highestand then intermediate in the reactions with potassium iodide, tending to be close to the curve of $I$. trojana. The hybrid curve is lower than the parental curves in the reactions with potassium hydroxide, cupric chloride, cobalt nitrate, barium chloride, and mercuric chloride, although the cobalt-nitrate and barium-chloride curves are very little different from the parental curves; and the highest throughout the 60 minutes in the uranium-nitrate reaction.

(4) In very few reactions is there a marked period of early resistance followed by a comparatively rapid gelatinization. A brief period of early resistance of all three starches is suggested by the curves of the strontiumnitrate reaction, and of one or the other parent or the hybrid in the reactions with chloral hydrate, chromic acid, calcium nitrate, uranium nitrate, and copper nitrate, especially in the last.

(5) The earliest period during the 60 minutes at which the three curves are best separated to differentiate the starches varies with the different reagents. Approximately, this period occurs within 5 minutes in the reactions with pyrogallic acid, sulphuric acid, hydrochloric acid, potassium iodide, potassium sulphocyanate, sodium hydroxide, sodium salicylate, uranium nitrate, and copper nitrate; at 15 minutes with chromic acid, potassium hydroxide, calcium nitrate, strontium nitrate, and cupric chloride; at the end of 30 minutes with chloral hydrate, nitric acid, potassium sulphide, and sodium sulphide; and at the end of 60 minutes with cobalt nitrate, barium chloride, and mercuric chloride (with the last perhaps at the end of 30 to 45 minutes).

\section{Reaction-INTENSITIES OF THE HybRID.}

This section treats of the reaction-intensities of the hybrid as regards sameness, intermediateness, excess, and deficit in relation to the parents. (Table A 30 and Charts D 379 to D 399.)

The reactivities of the hybrid are the same as those of the seed parent in the iodine, gentian violet, and safranin reactions; the same as those of the pollen parent with potassium iodide and sodium hydroxide; the same as those of both parents with potassium sulphocyanate and sodium hydroxide; intermediate with temperature, chloral hydrate, chromic acid, pyrogallic acid, nitric acid, sulphuric acid, hydrochloric acid, potassium sulphide, sodium sulphide, calcium nitrate, strontium nitrate, and copper nitrate (in four being closer to the seed parent, in two being closer to the pollen parent, and in six being mid-intermediate) ; the highest with uranium nitrate, and nearer that of the pollen parent; and the lowest with polarization, potassium hydroxide, cobalt nitrate, cupric chloride, barium chloride, and mercuric chloride (in three being closer to the seed parent, in one closer to the pollen parent, and in two being as close to one as to the other parent).

The following is a summary of reaction-intensities: Same as seed parent, 3 ; same as pollen parent, 2 ; same as both parents, 2 ; intermediate, 12 ; highest, 1 ; lowest, 6 .

It seems from the foregoing data that the seed parent has exercised much more influence than the pollen parent on the characters of the starch of the hybrid. Apart from this the most conspicuous features are the marked tendency to intermediateness and a tendency to lowness of the hybrid.

\section{Composite Curves of Reaction-Intensitres.}

This section treats of the composite curves of the reaction-intensities, showing the differentiation of the starches of Iris iberica, I. trojana, and I. ismali. (Chart E 30.)

The most conspicuous features of this chart are:

(1) The closeness of all three curves, the parental curves running so closely together as to suggest very closely related species (I. iberica is, however, relegated to Oncocylus and I. trojana, to Apagon, well-separated subgenera of the rhizomatous series). (The groupings of the Irids by different botanists are by no means the same, and it is recognized as being questionable if the classification of the entire genus must not be reconstructed.)

(2) The curve of $I$. iberica tends, with the exception of the polarization and temperature reactions, to be below that of 1 . trojana; but the differences are usually slight, and most marked in those with iodine, gentian violet, temperature, chloral hydrate, chromic acid, potassium sulphocyanate, sodium sulphide, sodium salicylate, calcium nitrate, uranium nitrate, copper nitrate, cupric chloride, and mercuric chloride.

(3) The curve of the hybrid wavers in its parental relationships, sometimes being closer to one parent and at others to the other, with for the most part a tendency to sameness or intermediateness, occasionally above or below parental extremes.

(4) In $I$. iberica, the very high reactions with sulphuric acid, potassium sulphocyanate, and sodium salicylate; the high reactions with chromic acid and sodium hydroxide; the moderate reactions with polarization, iodine, gentian violet, safranin, temperature, pyrogallic acid, and potassium hydroxide; the low reactions with chloral hydrate, nitric acid, hydrochloric acid, sodium sulphide, calcium nitrate, strontium nitrate, copper nitrate, and cupric chloride; and the very low reactions with potassium sulphide, uranium nitrate, cobalt nitrate, barium chloride, and mercuric chloride.

(5) In $I$. trojana, the very high reactions with sulphuric acid, potassium sulphocyanate, and sodium salicylate; the high reactions with chromic acid and sodium hydroxide; the moderate reactions with polarization, iodine, gentian violet, safranin, chloral hydrate, pyrogallic acid, nitric acid, hydrochloric acid, potassium hydroxide, and potassium iodide; the low reactions with temperature, sodium sulphide, calcium nitrate, strontium nitrate, copper nitrate, and cupric chloride; and the very low reactions with potassium sulphide, uranium nitrate, cobalt nitrate, barium chloride, and mercuric chloride.

(6) In the hybrid, the very high reactions with sulphuric acid, potassium sulphocyanate, and sodium salicylate; the high reactions with chromic acid and sodium hydroxide; the moderate reactions with polarization, iodine, gentian violet, chloral hydrate, pyrogallic acid, nitric acid, potassium hydroxide, and potassium iodide; the low reactions with temperature, hydrochloric acid, sodium sulphide, calcium nitrate, uranium nitrate, strontium nitrate, copper nitrate, and cupric chloride; and the very low reactions with potassium sulphide, cobalt nitrate, barium chloride, and mercuric chloride.

Following is a summary of the reaction-intensities:

\begin{tabular}{l|c|c|c|c|c}
\hline & $\begin{array}{l}\text { Very } \\
\text { high. }\end{array}$ & High. & $\begin{array}{c}\text { Mod- } \\
\text { erate. }\end{array}$ & Low. & $\begin{array}{l}\text { Very } \\
\text { low. }\end{array}$ \\
\hline I. iberica. $\ldots \ldots \ldots \ldots \ldots \ldots \ldots \ldots$ & 3 & 2 & 7 & 9 & $\mathbf{5}$ \\
I. trojana $\ldots \ldots \ldots \ldots \ldots \ldots \ldots \ldots$ & 3 & 2 & 10 & 6 & 5 \\
I. ismali $\ldots \ldots \ldots \ldots \ldots \ldots \ldots$ & 3 & 2 & 9 & 8 & 4 \\
\hline
\end{tabular}


31. Comparisons of the Starches of Iris Iberica, I. Cengialti, and I. doraK.

In histologic characteristics, polariscopic figures, reactions with selenite, reactions with iodine, and qualitative reactions with various chemical reagents, the starches of the parents and hybrid exhibit properties in common in varying degrees of development, the sum of which in each case is characteristic of the starch. The three starches are very much alike, and notwithstanding the very close resemblances of the parental starches the hybrid starch shows clearly evidence of biparental inheritance. The starch of Iris iberica in comparison with that of $I$. cengialti contains more compound grains and aggregates, and there are two types of compound grains in the former that are not present in the latter; the grains are not quite so regular in form; and elongated elliptical grains are more common, but ovoid forms less common. The hilum is more distinct, less often fissured, and more eccentric. The lamellæ are less distinct, not quite so coarse, and more numerous. The size is somewhat less, with variations in ratio of length to width that are interesting. In the polariscopic, selenite, and qualitative reactions there are various differences. In the qualitative reactions with chloral hydrate, hydrochloric acid, potassium iodide, sodium hydroxide, and sodium salicylate, there are many differences and individualities, several of the latter being quite striking. The starch of the hybrid in comparison with the parental starches contains more compound grains and aggregates than in either parent, and the compounds are of the two types found in I. iberica, but not in the other parent; the grains are less regular than in either parent. The relationship is on the whole distinctly closer to $I$. iberica. The hilum in character is closer to $I$. iberica, but in eccentricity to the other parent. The lamellæ in character are closer to $I$. cengialti, but in number to $I$. iberica. The size is somewhat less than in either parent, and, on the whole, closer to $I$. cengialti. In the polariscopic, selenite, and qualitative iodine reactions there are leanings here and there toward one or the other parent, but, on the whole, the relationship is much closer to $I$. iberica. In the qualitative chemical reactions the latter statement holds with equal force.

\section{Reaction-intensities Expressed by Light, Color, and Tempera-}

Polarization:

I. iberica, low to high, value 50.

I. cengialti, moderately high to high, higher than in I. iberica, value 60 .

I. dorak, low to high, the same as in I. iberica, value $\mathbf{5 0}$.

Iodine:

I. iberica, light to moderate, value 40.

I. cengialti, moderate, deeper than in I. iberica, value 45.

I. dorak, light to moderate, the same as in I. iberica, value 40.

Gentian violet:

I. iberica, light to moderate, value 40 .

I. cengialti, moderate, deeper than in I. iberica, value 45 .

I. dorak, moderate, deeper than in either parent, value 50.

Safranin:

I. iberica, moderate, value 45 .

I. cengialti, moderate, deeper than in I. iberica, value 50 .

I. dorak, moderate, the same as in I. cengialti, value 50. Temperature:

I. iberica, in the majority at 69 to $70^{\circ}$, in all at 71 to $72.5^{\circ}$, mean $71.5^{\circ}$.

I. cengialti, in the majority at 70 to $72^{\circ}$ mean, in all at 74 to $76^{\circ}$, mean $75^{\circ}$.

1. dorak, in the majority at 68 to $70^{\circ}$, in all at 70 to $72^{\circ}$, mean $71.5^{\circ}$.

The reactivity of $I$. iberica is lower than that of the other parent in the polarization, iodine, gentian violet, and safranin reactions, and higher in the temperature reaction. The reactivity of the hybrid is the same or practically the same as that of $I$. iberica in the reactions with polarization and iodine; the same or practically the
TABLE A 31.

\begin{tabular}{|c|c|c|c|c|c|c|c|c|c|c|}
\hline & $\stackrel{\dot{g}}{-}$ & $\underset{\sim}{\mathbb{E}}$ & $\underset{\infty}{\dot{g}}$ & $\dot{\mathbf{g}}$ & 皇 & $\begin{array}{l}\text { a } \\
\text { 요 }\end{array}$ & $\begin{array}{l}\text { 五 } \\
\stackrel{20}{\rightarrow}\end{array}$ & $\begin{array}{l}\text { 白 } \\
\text { 品 }\end{array}$ & $\begin{array}{l}\text { 昌 } \\
\text { w }\end{array}$ & 8 \\
\hline Chloral hydrate: & & & & & & & & & & \\
\hline & . & .. & . & $\ldots$ & 6 & .. & 39 & 50 & 60 & \\
\hline & . & .. & .. & .. & 10 & .. & 34 & 52 & 62 & \\
\hline I. d & $\ldots$ & .. & .. & $\ldots$ & 6 & .. & 17 & 33 & 44 & \\
\hline Chrom & & & & & & & & & & \\
\hline 1. ib & $\ldots$ & $\cdots$ & .. & & 6 & .. & 70 & 80 & 97 & \\
\hline I. c & .. & .. & . & . & 10 & .. & 63 & 80 & 85 & 9 \\
\hline I. dor & . & . & $\ldots$ & $\ldots$ & 29 & .. & 86 & 95 & 97 & \\
\hline Pyrogall & & & & & & & & & & \\
\hline I. it & . & . . & . & $\cdots$ & 22 & . & 72 & 81 & 86 & \\
\hline I. $\mathrm{c}$ & $\ldots$ & .. & & . & 4 & .. & 45 & 71 & 78 & 8 \\
\hline I. dor & $\ldots$ & .. & .. & $\ldots$ & 20 & $\ldots$ & 70 & 85 & 91 & 96 \\
\hline Nitric & & & & & & & & & & \\
\hline & . & $\ldots$ & . & $\ldots$ & 58 & .. & 73 & 77 & 81 & 8 \\
\hline & . & $\ldots$ & $\ldots$ & . & 12 & $\ldots$ & 66 & 73 & 83 & 9 \\
\hline I. d & $\ldots$ & $\ldots$ & $\ldots$ & $\ldots$ & 65 & .. & 78 & 81 & 84 & 38 \\
\hline Sulphu & & & & & & & & & & \\
\hline I. i & . & 85 & .. & . & 99 & . & $\cdots$ & . & & \\
\hline I. $\mathrm{c}$ & . & 89 & . & . & 99 & . & .. & $\cdots$ & . & \\
\hline I. $d$ & . & 92 & .. & . & 99 & .. & . & .. & & \\
\hline Hydr & & & & & & & & & & \\
\hline I. i & . & . & $\ldots$ & . & 53 & .. & 63 & 72 & 81 & 86 \\
\hline & . & . & . & . & 60 & . & 82 & 90 & . & 92 \\
\hline I. d & $\cdots$ & . & $\cdots$ & $\ldots$ & 60 & .. & 82 & 92 & . & \\
\hline Potas & & 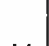 & $\therefore$ & & & & & & & \\
\hline & $\because$ & $\cdots$ & $\begin{array}{l}\cdots \\
\cdots\end{array}$ & . & $\begin{array}{l}82 \\
75\end{array}$ & $\therefore$ & $\begin{array}{l}85 \\
85\end{array}$ & $\begin{array}{l}89 \\
90\end{array}$ & $\begin{array}{l}93 \\
93\end{array}$ & 94 \\
\hline & $\ldots$ & $\cdots$ & $\cdots$ & . & 66 & . & 80 & 86 & 00 & \\
\hline Potas & & & & & & & & & & \\
\hline & . & .. & $\ldots$ & . & 52 & .. & 68 & 78 & 86 & \\
\hline & $\ldots$ & $\ldots$ & $\ldots$ & .. & 50 & .. & 82 & 86 & 91 & 93 \\
\hline I. d & $\ldots$ & $\ldots$ & $\ldots$ & $\ldots$ & 75 & . & 89 & 93 & 84 & \\
\hline Potas & & & & & & & & & & \\
\hline I. $\mathrm{i}$ & . & 84 & . & . & $\theta 0$ & . & 97 & & . & \\
\hline & $\ldots$ & 81 & .. & .. & 91 & $\ldots$ & 95 & 98 & . & \\
\hline I. $\mathrm{d}$ & $\cdots$ & 77 & $\cdots$ & . & 90 & . & 95 & $\ldots$ & . . & \\
\hline Pota & & & & & & & & & & \\
\hline & & . & $\ldots$ & .. & 4 & . & 5 & 6 & 7 & \\
\hline & $\ldots$ & $\ldots$ & $\ldots$ & $\ldots$ & 3 & .. & 4 & 5 & 10 & 10 \\
\hline & $\ldots$ & . & . & $\ldots$ & 4 & $\ldots$ & 6 & 8 & 9 & 12 \\
\hline Sodiu & & & & & & & & & & \\
\hline & .. & 59 & . . & . & 80 & .. & 88 & 95 & 97 & 97 \\
\hline & . & 50 & . . & . . & 74 & .. & 89 & 95 & 95 & 96 \\
\hline & $\ldots$ & 65 & . . & .. & 80 & .. & 90 & 95 & 95 & \\
\hline Sodiu & & & & & & & & & & \\
\hline & . & . & $\cdots$ & .. & 14 & . & 34 & 47 & 65 & \\
\hline & $\ldots$ & . & $\ldots$ & .. & 6 & .. & 48 & 60 & 66 & 66 \\
\hline & . & . & . & . & 27 & . & 47 & 60 & 66 & \\
\hline Sodiv & & & & & & & & & & \\
\hline & .. & . & $\ldots$ & .. & 55 & 89 & 99 & & . & \\
\hline & $\ldots$ & .. & $\ldots$ & .. & 55 & 95 & 99 & .. & .. & \\
\hline & $\ldots$ & $\ldots$ & $\ldots$ & $\ldots$ & 47 & 90 & 99 & $\ldots$ & .. & \\
\hline Calcix & & & & & & & & & & \\
\hline & $\cdots$ & . & $\cdots$ & .. & 13 & . & 30 & 45 & 54 & \\
\hline & $\ldots$ & .. & .. & .. & 6 & .. & 11 & 59 & 63 & 68 \\
\hline & . & .. & . & $\ldots$ & 14 & . & 28 & 48 & 60 & \\
\hline Uran & & & & & & & & & & \\
\hline & . & & $\cdots$ & .. & 10 & . & 20 & 22 & 25 & \\
\hline & $\ldots$ & . & . & . & 2 & .. & 10 & 20 & 33 & 36 \\
\hline & . & $\ldots$ & & $\ldots$ & 5 & & 18 & 32 & 39 & \\
\hline Stron & & & & & & & & & & \\
\hline & $\cdots$ & .. & $\ldots$ & . . & 12 & . . & 48 & 67 & 78 & \\
\hline & & & . & .. & 12 & .. & 58 & 71 & 78 & \\
\hline & & . & $\ldots$ & $\ldots$ & 20 & $\ldots$ & 55 & 65 & 72 & \\
\hline Cobs & & & & & & & & & & \\
\hline & . & . & . & & 2 & & 4 & 6 & 7 & \\
\hline & . & .. & . & $\ldots$ & 1 & .. & 2 & 5 & & \\
\hline & & .. & $\ldots$ & $\ldots$ & 0.5 & .. & 3 & 4 & 5 & \\
\hline & & & & & & & & & & \\
\hline & . & .. & $\ldots$ & .. & 12 & $\ldots$ & 19 & 50 & 54 & \\
\hline & . & $\ldots$ & $\ldots$ & .. & 10 & .. & 30 & 50 & 57 & \\
\hline & & $\ldots$ & $\ldots$ & $\ldots$ & 20 & $\ldots$ & 28 & 50 & 55 & \\
\hline Cupri & & & & & & & & & & \\
\hline & . & .. & $\ldots$ & . & 10 & .. & 42 & 61 & 64 & \\
\hline & & . & . & .. & 2 & .. & 15 & 55 & 62 & \\
\hline & & . & $\ldots$ & .. & 15 & .. & 56 & 64 & 66 & \\
\hline Bari & & & & & & & & & & \\
\hline & & . & . . & $\ldots$ & 1 & .. & 5 & 9 & 10 & \\
\hline & & .. & . & .. & 0.5 & .. & 1 & 2 & 3 & \\
\hline & & .. & $\ldots$ & .. & 1 & .. & 5 & 6 & 8 & \\
\hline Mercy & & & & & & & & & & \\
\hline & - & .. & . . & & $\mathbf{3}$ & .. & 11 & 15 & 22 & \\
\hline & .. & $\ldots$ & .. & $\ldots$ & 0.5 & .. & 2 & 3 & 9 & 1 \\
\hline I. do & & . & . & . & 6 & . & 11 & 17 & 21 & \\
\hline
\end{tabular}


same as that of the other parent in the safranin reaction; and the highest of the three in the temperature reaction. The hybrid is nearer $I$. iberica than to I. cengialti in the polarization, iodine, and temperature reactions, but nearer the other parent in the gentian violet and safranin reactions.

Table A 31 shows the reaction-intensities in percentages of total starch gelatinized at definite intervals (minutes).

\section{Velocity-Reaction CuRves.}

This section treats of the velocity-reaction curves of the starches of Iris iberica, I. cengialti, and I. dorak, showing the quantitative differences in the behavior toward different reagents at definite time-intervals. (Charts D 400 to D 420.)

The most conspicuous features of this group of curves are :

(1) The closeness of all three curves, occasionally almost identical, indicating corresponding relationships of the parents and little modification of parental peculiarities in the hybrid. The hybrid curve relative to the parental curves shows marked variability in so far as it sometimes follows one or the other parent closely, or is the highest or the lowest or tends to intermediateness, as the case may be. The hybrid curve inclines to differ as much from the parental curves as the latter do from each other. The tendency to separation of the parental curves is more marked in this group than in the previous group, and with the exception of the reactions with sulphuric acid, potassium sulphocyanate, potassium sulphide, sodium hydroxide, sodium salicylate, strontium nitrate, cobalt nitrate, copper nitrate, and barium chloride there is more or less marked separation, with a tendency generally for two of the three curves to keep close, sometimes the two parental curves and at others one parental curve with the hybrid curve. In some of the reactions noted there is definite although unimportant separation, as in those with sodium salicylate, strontium nitrate, copper nitrate, and barium chloride.

(2) The sameness or marked closeness of the parental curves in the reactions with chloral hydrate and chromic acid; the sameness or marked closeness of all three curves with sulphuric acid, potassium sulphocyanate, potassium sulphide, sodium hydroxide, sodium salicylate, strontium nitrate, cobalt nitrate, and copper nitrate; the sameness or marked closeness of the hybrid curve with one or the other parental curve with pyrogallic acid, nitric acid, hydrochloric acid, calcium nitrate, and mercuric chloride.

(3) The varying positions of the hybrid curves in relation to the parental curves in the different reactions, and the marked tendency for the hybrid curves to be higher or lower than the parental curves with almost not the least tendency to intermediateness.

(4) In a few instances there is evidence of a comparatively marked early resistance of one or two or all three starches, as the case may be, as in I. iberica in the chloral-hydrate and $I$. iberica and $I$. cengialti in the chromic-acid reactions; in I. cengialti in those with pyrogallic acid, nitric acid, sodium sulphide, copper nitrate, and cupric chloride. This peculiarity, in so far as the parents are concerned, is therefore almost confined to I. cengialti, and it is not observed in the hybrid unless perhaps in the uranium nitrate reaction.

(5) The earliest period during the 60 minutes at which the three curves are best separated to differentiate the starches varies with the different reagents. Approximately, this period occurs within 5 minutes in most of the reactions, including the reactions with pyrogallic acid, nitric acid, sulphuric acid, potassium hydroxide, potassium sulphocyanate, sodium hydroxide, sodium sulphide, sodium salicylate, calcium nitrate, uranium nitrate, and copper nitrate; at the end of 15 minutes with chloral hydrate, chromic acid, hydrochloric acid, potassium iodide, strontium nitrate, and cupric chloride; and at the end of 60 minutes with potassium sulphide, cobalt nitrate, barium chloride, and mercuric chloride. In some of these cases there is little or no practical differentiation at these respective periods.

\section{Reaction-Intensities of the Hybrid.}

This section treats of the reaction-intensities of the hybrid as regards sameness, intermediateness, excess, and deficit in relation to the parents. (Table A 31 and Charts D 400 to D 420 .)

The reactivities of the hybrid are the same as those of the seed parent in the reactions with polarization, iodine, sodium hydroxide, barium chloride, and mercuric chloride; the same as those of the pollen parent in those with safranin, hydrochloric acid, and potassium sulphide; the same as those of both parents in the cobalt-nitrate reaction; intermediate in that with calcium nitrate, and closer to the seed parent; highest in those with gentian violet, temperature, chromic acid, pyrogallic acid, nitric acid, sulphuric acid, potassium iodide, sodium sulphide, uranium nitrate, strontium nitrate, copper nitrate, and cupric chloride (in six being closer to the seed parent, in five closer to the pollen parent, and in one as close to one as to the other parent) ; and lowest with chloral hydrate, potassium hydroxide, potassium sulphocyanate, and sodium salicylate (in one being closer to the seed parent, in two closer to the pollen parent, and in one as close to one as to the other parent).

The following is a summary of the reaction-intensities: Same as seed parent, 5 ; same as pollen parent, 3 ; same as both parents, 2; intermediate, 1; highest, 11; lowest, 4.

The seed parent has apparently influenced to a more marked extent than the pollen parent the properties of the starch of the hybrid. The sameness to the seed parent coupled with the tendency to closeness to the seed parent in the reactions in which the hybrid is in excess of the parents is quite marked. The tendency to the highest or lowest reactivity of the hybrid is quite conspicuous, this being noted in more than half of the reactions.

\section{Composite Curves of Reaction-intensities.}

This section treats of the composite curves of the reaction-intensities, showing the differentiation of the starches of Iris iberica, I. cengialti, and I. dorak. (Chart E 31.)

The most conspicuous features of this chart are:

(1) The marked closeness of all three curves throughout, there being no tendency in any reaction for a marked departure of any one curve from the other two. The curves are so close as to suggest either very closely related species or mere varieties, the latter rather than the former. The species are, however, classed in different subgenera: I. iberica in Oncacyclus, and I. cengialti in Pogoniris and Regelia. I. cengialti is regarded as being probably a dwarf variety of $I$. pallida, which it closely resembles. For the most part the differences in the curves fall within or close to the limits of error of experiment, so that little or nothing of importance can be gained from a critical comparison. At some points one parental curve is higher than the other; and the hybrid curve courses with one or the other or both parental curves, here and there running above or below both.

(2) In $I$. iberica, the very high reactions with sulphuric acid, potassium sulphocyanate, and sodium salicylate; the high reactions with chromic acid and sodium 
hydroxide; the moderate reactions with polarization, iodine, gentian violet, safranin, temperature, pyrogallic acid, and potassium hydroxide; the low reactions with chloral hydrate, nitric acid, hydrochloric acid, sodium sulphide, calcium nitrate, strontium nitrate, copper nitrate, and cupric chloride; and the very low reactions with potassium sulphide, uranium nitrate, cobalt nitrate, barium chloride, and mercuric chloride.

(3) In $I$. cengialti, the very high reactions with sulphuric acid, potassium sulphocyanate, and sodium salicylate; the high reactions with polarization, chromic acid, and sodium hydroxide; the moderate reactions with iodine, gentian violet, safranin, hydrochloric acid, potassium hydroxide, and potassium iodide; the low reactions with temperature, chloral hydrate, pyrogallic acid, nitric acid, sodium sulphide, strontium nitrate, copper nitrate, and cupric chloride; and the very low reactions with potassium sulphide, uranium nitrate, cobalt nitrate, barium chloride, and mercuric chloride.

(4) In the hybrid, the very high reactions with sulphuric acid, potassium sulphocyanate, and sodium salicylate; the high reactions with chromic acid and sodium hydroxide; the moderate reactions with polarization, iodine, gentian violet, safranin, temperature, pyrogallic acid, nitric acid, hydrochloric acid, potassium hydroxide, and potassium iodide; the low reactions with chloral hydrate, sodium sulphide, calcium nitrate, strontium nitrate, copper nitrate, and cupric chloride; and the very low reactions with potassium sulphide, uranium nitrate, cobalt nitrate, barium chloride, and mercuric chloride.

Following is a summary of the reaction-intensities:

\begin{tabular}{|c|c|c|c|c|c|}
\hline & $\begin{array}{l}\text { Very } \\
\text { high. }\end{array}$ & High. & $\begin{array}{l}\text { Mod- } \\
\text { erate. }\end{array}$ & Low. & $\begin{array}{l}\text { Very } \\
\text { low. }\end{array}$ \\
\hline $\begin{array}{l}\text { I. iberica } \ldots \ldots \ldots \ldots \ldots \ldots \ldots \\
\text { I. cengialti } \ldots \ldots \ldots \ldots \ldots \ldots \ldots \\
\text { I. dorak } \ldots \ldots \ldots \ldots \ldots \ldots \ldots\end{array}$ & $\begin{array}{l}3 \\
3 \\
3\end{array}$ & $\begin{array}{l}\mathbf{2} \\
\mathbf{3} \\
\mathbf{2}\end{array}$ & $\begin{array}{r}7 \\
6 \\
10\end{array}$ & $\begin{array}{l}9 \\
9 \\
6\end{array}$ & $\begin{array}{l}\mathbf{5} \\
\mathbf{5} \\
\mathbf{5}\end{array}$ \\
\hline
\end{tabular}

32. Comparisons of the Starches of Iris cenGIALTI, I. PALIIDA QUEEN OF MAY, AND I. MRS. ALAN GREY.

In histologic characteristics, polariscopic figures, reactions with selenite and iodine, and with various chemical reagents the starches of the parents and hybrid exhibit properties in common in varying degrees of development, the sum of which in each case is characteristic of the starch. Inasmuch as one of the parents is probably merely a dwarf form of the other, but little difference is to be expected between either parents or parents and hybrid. The starch of $I$. cengialti in comparison with that of $I$. pallida queen of may contains fewer compound grains and aggregates; the grains are less irregular, more rounded, but not so slender. The hilum when not fissured is more distinct; more often, more deeply and more extensively fissured; and the eccentricity is groater. The lamellæ are usually not so distinct, coarser, and exhibit a notch corresponding to a notch in the distal margin that was not noted in $I$. pallida queen of may. The size of the grains is somewhat larger. In the polariscopic, selenite, and qualitative iodine reactions many differences are recorded. In the qualitative reactions with chloral hydrate, hydrochloric acid, potassium iodide, sodium hydroxide, and sodium salicylate various differences are noted, some of them quite individual and distinctive. The starch of the hybrid in comparison with the starches of the parents contains compound grains and aggregates in about the same numbers and of the same types as in I. pallida queen of may; the grains are more regular than in either parent. In certain respects the form is closer to that of $I$. cengialti, but in most features closer to that of the other parent. The hilum is in character closer to $I$. pallida queen of may, but the eccentricity is greater than in either parent, yet closer to this parent. The lamellæ are less distinct than in either parent, but they are in their general characters closer on the whole to I. cengialti. The size is less than in either parent, but closer to I. pallida queen of may. The polariscopic and selenite reactions are closer to those of $I$. pallida queen of may, but the qualitative iodine reactions are closer to those of the other parent. In the qualitative reactions with the chemical reagents the hybrid is very much more closely related to $I$. pallida queen of may.

Reaction-intensities Expressed by Light, Color, and Tempera-

\section{Polarization:} ture Reactions.

I. cengialti, moderately high to high, value 60 .

I. pallida queen of may, low to high, lower than in I. cengialti, value 50.

I. mrs. alan grey, low to high, lower than in either parent, value 45 . Iodine:

I. cengialti, moderate, value 45.

I. pallida queen of may, moderate, less than in I. cengialti, value 35 .

I. mrs. alan grey, moderate, deeper than in either parent, value 50. Gentian violet:

I. cengialti, moderate, value 45 .

I. pallida queen of may, moderate, slightly deeper than in I. cengialti, value 48 .

I. mrs. alan grey, light to moderate, less than in either parent, value 40 .

Safranin:

I. cengialti, moderate, value 50 .

I. pallida queen of may, moderate, slightly deeper than in I. cengialti, value 52.

I. mrs. alan grey, moderate, less than in either parent, value $\mathbf{4 5}$. Temperature:

I. cengialti, in the majority at 70 to $72^{\circ}$, in all at 74 to $76^{\circ}$, mean $75^{\circ}$.

I. pallida queen of may, in the majority at 71 to $73^{\circ}$, in all at 75 to $75.8^{\circ}$, mean $75.4^{\circ}$.

I. mre. alan grey, in the majority at 69 to $70^{\circ}$, in all at 73 to $74.5^{\circ}$, mean $73.75^{\circ}$.

The reactivity of 1 . cengialti is higher than that of the other parent in the reactions with polarization, iodine, and temperature; and lower with gentian violet and safranin. With the exception of the first two the differences are small, and in the case of temperature probably within the limits of error. The reactivity of the hybrid is the lowest of the three in the polarization, gentian-violet, safranin, and temperature reactions, and the highest of the three in the iodine reactions. The hybrid is closer to 1 . cengialti than to that of the other parent in the iodine, gentian-violet, safranin, and temperature reactions, but the reverse in polarization reactions.

Table A 32 shows the reaction-intensities in percentages of total starch gelatinized at definite intervals (minutes).

\section{Velocity-Reaction CuRves.}

This section treats of the velocity-reaction curves of the starches of Iris cengialti, I. pallida queen of may, and $I$. mrs. alan grey, showing the quantitative differences in the behavior toward different reagents at definite timeintervals. (Charts D 421 to D 441.)

The most conspicuous features of this group of charts are:

(1) The closeness of all three curves, with the exception of the chloral-hydrate reaction, in which the curves markedly diverge after the first 5 minutes. Excepting the reactions with nitric acid, sulphuric acid, potassium sulphide, cobalt nitrate, and barium chloride, there is sufficient separation of the curves, one or more, to permit of more or less satisfactory differentiation. It is of particular interest to note that the parental curves tend to a more marked closeness than does the 
Table A 32.

\begin{tabular}{|c|c|c|c|c|c|c|c|c|c|c|}
\hline & $\underset{-}{\mathrm{a}}$ & $\underset{\text { 早 }}{\dot{H}}$ & 旦 & $\underset{+}{\text { 百 }}$ & $\underset{n}{\mathrm{~g}}$ & $\begin{array}{l}\text { g } \\
\stackrel{9}{9}\end{array}$ & $\begin{array}{l}\text { 白 } \\
20\end{array}$ & $\begin{array}{l}\text { 白 } \\
\text { 豼 }\end{array}$ & $\begin{array}{l}\text { 自 } \\
\stackrel{\operatorname{mg}}{+}\end{array}$ & $\begin{array}{l}\text { 日 } \\
8\end{array}$ \\
\hline Chloral hydrate: & & & & & & & & & & \\
\hline I. cengialti... & $\ldots$ & $\cdots$ & $\cdots$ & $\cdots$ & 10 & & 34 & 52 & 62 & 66 \\
\hline I. pallida queen of may. . & . & $\ldots$ & . & $\ldots$ & 10 & . & 55 & 72 & 83 & 84 \\
\hline I. mrs. alan grey....... & . & . & . & . & 14 & . & 72 & 95 & 99 & .. \\
\hline Chromic acid: & & & & & & & & & & \\
\hline I. cengialti. . & $\ldots$ & & $\ldots$ & . & 10 & & 63 & 90 & 95 & 99 \\
\hline I. pallida queen of may. . & $\ldots$ & . & . & . & 5 & $\cdots$ & 40 & 81 & 95 & 98 \\
\hline I. mrs. alan grey ....... & . . & . & & . & 6 & . & 57 & 86 & 95 & 98 \\
\hline Pyrogallic acid: & & & & & & & & & & \\
\hline I. cengialti. . . . . . . . & . & $\cdots$ & $\cdots$ & . & 4 & . & 45 & 71 & 78 & 84 \\
\hline I. pallida queen of may. . & . & . & $\cdots$ & $\cdots$ & 4 & . & 30 & 67 & 84 & 92 \\
\hline I. mrs. alan grey........ & $\cdots$ & . & . & $\cdots$ & $\mathbf{5}$ & $\cdots$ & 16 & 56 & 66 & 78 \\
\hline Nitric acid: & & & & & & & & & & \\
\hline I. cengialti $\ldots \ldots \ldots \ldots$ & . & . & $\cdots$ & . & 12 & . & 66 & 73 & 83 & 90 \\
\hline I. pallida queen of may. . & $\ldots$ & $\cdots$ & . & . & 9 & . & 62 & 70 & 79 & 81 \\
\hline I. mrs. alan grey ........ & . . & . & $\cdots$ & $\ldots$ & .10 & . & 63 & 71 & 80 & 83 \\
\hline Sulphuric acid: & & & & & & & & & & \\
\hline I. cengialti. . . . . . . . & . & 89 & $\cdots$ & . & 99 & $\cdots$ & & . & . & . \\
\hline I. pallida queen of may. . & . & 89 & . & . & 99 & . & . & . & .. & .. \\
\hline I. mrs. alan grey........ & . & 93 & $\ldots$ & $\ldots$ & 99 & . & .. & .. & . & . \\
\hline Hydrochloric acid: & & & & & & & & & & \\
\hline 1. ceagialti. . . . . . . & . & $\cdots$ & $\cdots$ & $\cdots$ & 60 & . & 82 & 90 & .. & 92 \\
\hline I. pallida queen of may. . & $\cdots$ & . & . & . & 64 & . & 80 & 84 & & 86 \\
\hline I. mrs alan grey ........ & $\cdots$ & $\cdots$ & . & $\cdots$ & 20 & . & 62 & 75 & 86 & 86 \\
\hline Potassium hydroxide: & & & & & & & & & & \\
\hline I. cengialti. . . . & $\cdots$ & $\cdots$ & . & $\cdots$ & 75 & . & 85 & 90 & 93 & 94 \\
\hline I. pallida queen of may. . & $\cdots$ & $\cdots$ & . & $\cdots$ & 72 & $\cdots$ & 86 & 90 & 91 & 93 \\
\hline I. mrs. alan grey .... & $\cdots$ & $\cdots$ & $\cdots$ & $\cdots$ & 66 & $\cdots$ & 73 & 81 & 88 & 90 \\
\hline Potassium iodide: & & & & & & & & & & 93 \\
\hline $\begin{array}{l}\text { I. cengialti.... } \\
\text { I. pallida quee }\end{array}$ & $\cdots$ & $\cdots$ & $\cdots$ & $\cdots$ & $\begin{array}{l}50 \\
30\end{array}$ & & $\begin{array}{l}82 \\
75\end{array}$ & $\begin{array}{l}86 \\
83\end{array}$ & $\begin{array}{l}91 \\
88\end{array}$ & $\begin{array}{l}93 \\
90\end{array}$ \\
\hline $\begin{array}{l}\text { I. pallida queen of may . } \\
\text { I. mrs. alan grey........ }\end{array}$ & $\because$ & $\cdots$ & ’ & $\because$ & 30 & . & $\begin{array}{l}70 \\
53\end{array}$ & $\begin{array}{l}83 \\
77\end{array}$ & $\begin{array}{l}88 \\
81\end{array}$ & $\begin{array}{l}90 \\
83\end{array}$ \\
\hline Potassium sulphocyanate: & $\cdots$ & $\cdots$ & $\cdots$ & $\cdots$ & & & & & & \\
\hline I. cengialti. . . . . . . . & $\ldots$ & 81 & . & $\ldots$ & $\cdots$ & . & 91 & 95 & 98 & . \\
\hline I. pallida queen of may. . & $\ldots$ & 75 & . & . & $\therefore$ & . & 89 & 95 & 96 & . \\
\hline I. mrs. alan grey . . . . . & $\cdots$ & 66 & . & . & $\ldots$ & . & 77 & 90 & 93 & . \\
\hline Potassium sulphide: & & & & & & & & & & \\
\hline I. cengialti $\ldots \ldots \ldots \ldots \ldots$ & . & . & $\cdots$ & $\cdots$ & 3 & $\cdots$ & 4 & 5 & 10 & 10 \\
\hline 1. pallida queen of may. . & . & . & . & . & 2 & . & 6 & 10 & . & 10 \\
\hline I. mrs. alan grey ........ & $\ldots$ & $\cdots$ & $\cdots$ & $\cdots$ & 1 & $\cdots$ & 2 & 6 & $\ldots$ & 6 \\
\hline Sodium hydroxide: & & & & & & & & & & \\
\hline I. cengialti & - & 50 & $\cdots$ & . & 74 & $\cdots$ & 89 & 95 & 95 & 96 \\
\hline I. pallida queen & $\cdots$ & 58 & . & . & 75 & . & 90 & 92 & 95 & 95 \\
\hline I. mrs. alan grey. . . . . . & $\ldots$ & 45 & . & . & 64 & . & 75 & 90 & 93 & 94 \\
\hline Sodium sulphide: & & & & & & & & & & \\
\hline I. cengialti. . . . . . . . & $\cdots$ & $\cdots$ & . & . & 6 & $\cdots$ & 48 & 60 & 66 & 66 \\
\hline I. pallida queen of may. . & . & . & . & . & 12 & . & 50 & 53 & 59 & 62 \\
\hline I. mrs. alan grey........ & $\cdots$ & . & . & $\cdots$ & 7 & . & 20 & 31 & 40 & 52 \\
\hline Sodium salicylate: & & & & & & & & & & \\
\hline I. cengialti . . . . & $\cdots$ & . & $\ldots$ & . & 55 & 95 & 99 & $\ldots$ & . & $\cdots$ \\
\hline I. pallida queen of may. . & . & . & $\ldots$ & . & 80 & 99 & $\ldots$ & $\ldots$ & $\therefore$ & . \\
\hline I. mrs. alan grey ........ & . & . & $\ldots$ & $\ldots$ & 97 & 99 & . & . & $\ldots$ & . \\
\hline Calcium nitrate: & & & & & & & & & & \\
\hline I. cengialti. . . . . & $\ldots$ & . & $\ldots$ & $\ldots$ & 6 & . & 41 & 59 & 63 & 68 \\
\hline I. pallida queen of may. . & $\cdots$ & . & $\cdots$ & . & 7 & . & 45 & 50 & 56 & 60 \\
\hline I. mrs. alan grey ........ & . & . & . & . & 10 & . & 26 & 38 & 48 & 50 \\
\hline Uranium nitrate: & & & & & & & & & & \\
\hline I. cengialti.... . & $\ldots$ & $\ldots$ & . & $\ldots$ & 2 & $\ldots$ & 10 & 20 & 33 & 36 \\
\hline I. pallida queen of may. & . & $\ldots$ & . & . & 4 & . & 9 & 18 & 25 & 29 \\
\hline I. mrs. alan grey . . . . . . & . & . & . & $\ldots$ & 2 & . & 7 & 12 & 19 & 24 \\
\hline Strontium nitrate: & & & & & & & & & & \\
\hline I. cengialti .......... & $\cdots$ & . & $\ldots$ & . & 12 & .. & 58 & 71 & 78 & 86 \\
\hline I. pallida queen of may. . & . & . & . & $\cdots$ & 10 & . & 46 & 54 & 63 & 68 \\
\hline I. mrs. alan grey........ & $\cdots$ & $\cdots$ & . & . & 8 & . & 23 & 43 & 50 & 55 \\
\hline Cobalt nitrate: & & & & & & & & & & \\
\hline I. cengialti. . & $\ldots$ & $\ldots$ & $\cdots$ & $\cdots$ & 1 & $\ldots$ & 2 & 5 & 6 & 7 \\
\hline I. pallida queen of may. . & $\ldots$ & $\ldots$ & . & . & 0.5 & $\cdots$ & 1 & 2 & $\ldots$ & 3 \\
\hline I. mrs. alan grey........ & . & . & . & . & 0.5 & $\ldots$ & . & 1 & 2 & 3 \\
\hline Copper nitrate: & & & & & & & & & & \\
\hline I. cengialti $\ldots \ldots \ldots \ldots$ & . & . & $\therefore$ & . & 10 & . & 30 & 50 & 57 & 60 \\
\hline I. pallida queen of may. . & $\ldots$ & . & $\ldots$ & $\cdots$ & 12 & .. & 25 & 36 & 48 & 51 \\
\hline I. mrs. alan grey........ & . & . & $\cdots$ & . & 5 & .. & 12 & 20 & 30 & 31 \\
\hline Cupric chloride: & & & & & & & & & & \\
\hline I. cengialti. . & $\cdots$ & $\cdots$ & . & $\ldots$ & 2 & $\cdots$ & 15 & 55 & 62 & 68 \\
\hline I. pallida queen of may. & $\ldots$ & $\cdots$ & $\cdots$ & . & 6 & . & 19 & 48 & 60 & 63 \\
\hline I. mrs. alan grey........ & $\ldots$ & . & $\ldots$ & .. & 3 & . & 7 & 42 & 44 & 48 \\
\hline Barium chloride: & & & & & & & & & & \\
\hline I. cengialti. . . . . . . & . & .. & $\ldots$ & . & 0.5 & . & 1 & 2 & 3 & 5 \\
\hline I. pallida queen of may. . & . & . & . & . & 0.5 & . & 2 & 3 & 4 & 5 \\
\hline I. mars. alan grey ........ & $\cdots$ & . & . & . & 1 & . & 2 & . & 4 & 5 \\
\hline Mercuric chloride: & & & & & & & & & & \\
\hline I. cengialti. . . . . & $\cdots$ & $\cdots$ & $\ldots$ & $\ldots$ & 0.5 & $\cdots$ & 2 & 3 & 9 & 12 \\
\hline I. pallid & . & $\cdots$ & . & $\cdots$ & 0.5 & $\ldots$ & 5 & 9 & 10 & 14 \\
\hline I. mrs. alan grey. & & . & $\cdots$ & .. & 0.5 & & 1 & 2 & 4 & 4 \\
\hline
\end{tabular}

curve of the hybrid to either parent or to intermediateness. In fact, there is an inclination for the parental curves to be paired in their course and for the hybrid curve to be distinctly above or below the parental curves. In the chromic-acid reactions there is well-marked intermediateness of the hybrid, and in those with potassium, iodine, sodium sulphide, and cupric chloride a transient intermediateness during the first 5 minutes; but in this group, with the exception of the potassium iodide reaction, the differences in the curves of the three starches are slight and fall within the limits of error of experiment.

(2) The lower reactivity of $I$. cengialti in comparison with the other parent in the reactions with chloral hydrate and sodium salicylate; the higher reactivities in those with chromic acid, pyrogallic acid, potassium iodide, uranium nitrate, strontium nitrate, and copper nitrate; the same or nearly the same reactivities with hydrochloric acid, potassium hydroxide, potassium sulphocyanate, sodium hydroxide, sodium sulphide, calcium nitrate, cupric chloride, and mercuric chloride; and the same reactivities also with nitric acid, sulphuric acid, potassium sulphide, cobalt nitrate, and barium chloride, in which the reactivities of all three starches are the same or practically the same.

(3) The curves of the hybrid bear varying relations to the parental curves. The absence of sameness in any. instance to the seed parent, the almost entire absence of intermediateness of the curve, and the very marked tendency to the curve being the highest or lowest of the three are very striking. This low tendency is a most interesting peculiarity considering the very close relationship of the parents, and it recalls the same but even more marked peculiarity of the hybrids of the wellseparated parents-A maryllis belladonna and Brunsvigia josephince.

(4) In a few reactions there is evidence of an early period of resistance, and this may be noticeable in regard to one or more of three starches in any reaction. This resistance is seen in all three starches in the reactions with chloral hydrate, chromic acid, pyrogallic acid, nitric acid, strontium nitrate, and cupric chloride; with $I$. cengialti in the sodium-sulphide reaction; with both parents in that with calcium nitrate; and with the hybrid in that with cupric chloride particularly.

(5) The earliest period during the 60 minutes at which the three curves are best separated to differentiate the starches varies with the different reagents. Approximately, this period occurs within 5 minutes in the reactions with nitric acid, sulphuric acid, potassium hydroxide, potassium iodide, potassium sulphocyanate, sodium hydroxide, and sodium salicylate reactions; at $\mathbf{1 5} \mathrm{min}-$ utes with chloral hydrate, chromic acid, pyrogallic acid, hydrochloric acid, sodium sulphide, calcium nitrate, and strontium nitrate; at 30 minutes with copper nitrate and cupric chloride; and at 60 minutes with potassium sulphide, uranium nitrate, cobalt nitrate, barium chloride, and mercuric chloride. In a number of cases the assignment is very questionable, so that the classification must be looked upon as having merely a tentative valué.

\section{REACTION-INTENSITIES OF THE HyBRID.}

This section treats of the reaction-intensities of the hybrid as regards sameness, intermediateness, excess, and deficit in relation to the parents. (Table A 32 and Charts D 421 to D 441.)

The reactivities of the hybrid are the same as those of the seed parent in no reaction; the same as those of the pollen parent in that with cobalt nitrate; the same as those of both parents in those with nitric acid, sulphuric acid, and barium chloride, in all of which the 
progress of gelatinization is too fast or too slow for differentiation; intermediate with chromic acid, and closer to that of the seed parent; highest with iodine, temperature, chloral hydrate, and sodium salicylate (in one being nearer the seed parent, and in three nearer the pollen parent); and lowest with polarization, gentian violet, safranin, pyrogallic acid, hydrochloric acid, potassium hydroxide, potassium iodide, potassium sulphocyanate, potassium sulphide, sodium hydroxide, sodium sulphide, calcium nitrate, uranium nitrate, strontium nitrate, copper nitrate, cupric chloride, and mercuric chloride (in five being closer to the seed parent, in nine closer to the pollen parent, and in three being as close to one as to the other parent).

The following is a summary of the reaction-intensities: Same as seed parent, 0 ; same as pollen parent, 1 ; same as both parents, 3 ; intermediate, 1 ; highest, 3 ; lowest, 17.

Three features stand out most conspicuously: the more marked influence of the pollen parent on the properties of the starch of the hybrid, the remarkably strong tendency for the curve of the hybrid to be above or below the curves of the parents, especially to be below, and the almost entire absence of intermediateness.

Composite Curve of the Reaction-intensities.

This section treats of the composite curve of the reaction-intensities, showing the differentiation of the starches of Iris cengialti, I. pallida queen of may, and I. mrs. alan grey. (Chart E 32.)

The most conspicuous features of this chart are:

(1) The closeness of all three curves, excepting in the reactions with chloral hydrate, calcium nitrate, uranium nitrate, strontium nitrate, copper nitrate, and cupric chloride, in all of which, excepting the first, the separation is within comparatively narrow limits, and in all the separation is due in a large measure or solely to the hybrid curve going above or falling below the parental values, a tendency that was also recorded in the histologic and qualitative peculiarities and the reaction-intensities expressed by light, color, and temperature reactions of this summary.

(2) The curve of Iris cengialti tends to be higher than that of $I$. pallida queen of may in the reactions with polarization, iodine, temperature, nitric acid, sulphuric acid, potassium iodide, calcium nitrate, uranium nitrate, strontium nitrate, copper nitrate, and cupric chloride; lower with gentian violet, safranin, chloral hydrate, and pyrogallic acid; and the same or practically the same with chromic acid, sulphuric acid, potassium hydroxide, potassium sulphocyanate, potassium sulphide, sodium hydroxide, sodium sulphide, cobalt nitrate, barium chloride, and mercuric chloride. In several of the reactions where the curves differ they are so close as to be probably within the limits of error of experiment, as in the reactions with temperature, pyrogallic acid, nitric acid, hydrochloric acid, potassium iodide, calcium nitrate, uranium nitrate, copper nitrate, and cupric chloride. Charts D 421 to D 441 are to be taken with these data in determining differences in reactivity, but the differences will doubtless be found to hold excepting for slight variations.

(3) The curve of the hybrid is variable in its relations to the parental curves, commonly exhibiting either an inclination to be the same as the curve of one or both parents or to be above or below, but not to intermediateness. In Chart D 442 in the chromic-acid reactions there was definite intermediateness up to the 45 -minute record, and there were also transient intermediate tendencies in other reactions (see preceding section); but these are not apparent in this chart, owing to inherent defects of construction.
(4) In $I$. cengialti, the very high reactions with sulphuric acid, potassium sulphocyanate, and sodium salicylate; the high reactions with polarization, chromic acid, and sodium hydroxide; the moderate reactions with iodine, gentian violet, safranin, hydrochloric acid, potassium hydroxide, and potassium iodide; the low reactions with temperature, chloral hydrate, pyrogallic acid, nitric acid, sodium sulphide, strontium nitrate, copper nitrate, and cupric chloride; and the very low reactions with potassium sulphide, uranium nitrate, cobalt nitrate, barium chloride, and mercuric chloride.

(5) In 1 . pallida queen of may the very high reactions with sulphuric acid and sodium salicylate; the high reactions with polarization, chromic acid, potassium sulphocyanate, and sodium hydroxide; the moderate reactions with iodine, gentian violet, safranin, nitric acid, hydrochloric acid, potassium hydroxide, and potassium iodide; the low reactions with temperature, chloral hydrate, pyrogallic acid, sodium sulphide, calcium nitrate, strontium nitrate, copper nitrate, and cupric chloride; and the very low reactions with potassium sulphide, uranium nitrate, cobalt nitrate, barium chloride, and mercuric chloride.

(6) In the hybrid, the very high reactions with sulphuric acid and sodium salicylate; the high reactions with chloral hydrate, chromic acid, potassium sulphocyanate, and sodium hydroxide reactions; the moderate reactions with polarization, iodine, gentian violet, safranin, and potassium hydroxide; the low reactions with temperature, pyrogallic acid, nitric acid, hydrochloric acid, potassium iodide, sodium sulphide, calcium nitrate, and strontium nitrate; and the very low reactions with potassium sulphide, uranium nitrate, cobalt nitrate, copper nitrate, cupric chloride, barium chloride, and mercuric chloride.

Following is a summary of the reaction-intensities:

\begin{tabular}{l|c|c|c|c|c}
\hline & $\begin{array}{c}\text { Very } \\
\text { high. }\end{array}$ & High. & $\begin{array}{c}\text { Mod- } \\
\text { erate. }\end{array}$ & Low. & $\begin{array}{c}\text { Very } \\
\text { low. }\end{array}$ \\
\hline I. cengialti................ & 3 & 2 & 7 & 9 & 5 \\
I. pallida queen of may ....... & 2 & 4 & 7 & 8 & 5 \\
I. mrs. alan grey........... & 2 & 4 & 5 & 8 & 7 \\
\hline
\end{tabular}

33. Comparisons of the Starches of Iris PERsica var. PURPURea, I. Sindjarensis, AND I. PURSIND.

In histologic characteristics, polariscopic figures, reactions with selenite, reactions with iodine, and qualitative reactions with the various chemical reagents all three starches exhibit properties in common in varying degrees of development, the sum of which in case of each starch is distinctive of the starch. The starch of Iris sindjarensis in comparison with that of $I$. persica var. purpurea contains many more compound grains, all of the same types but in different proportions; and the grains are much more regular in form. The hilum is not so often or so deeply and extensively fissured; there is an absence of a single fissure in compound grains which passes through all of the hila, as was noted in the other parent; and eccentricity is usually greater. The lamellæ are not so coarse and are more regular, and the number is larger. The size is smaller. In the polariscopic, selenite, and qualitative iodine reactions there are various differences. In the qualitative reactions with chloral hydrate, hydrochloric acid, potassium iodide, sodium hydroxide, sodium salicylate, and mercuric chloride there are also many differences which on the whole definitely individualize each parent. The starch of the hybrid in comparison with the starches of the parents contains a less number 
of compound grains than in either parent; irregularity is intermediate; and, on the whole, the resemblances are distinctly closer to $I$. persica var. purpurea. The hilum in character is closer to $I$. persica var. purpurea, but in eccentricity closer to $I$. sindjarensis. The lamellæ in character and number are closer to $I$. persica var. purpurea. The size is closer to $I$. sindjarensis. In the polariscopic and selenite reactions the relationship is closer to $I$. persica var. purpurea, but in the qualitative iodine reactions closer to $I$. sindjarensis. In the qualitative reactions with the chemical reagents the leanings to one or the other parent are numerous and marked, but on the whole much more to I. persica var. purpurea than to the other parent; moreover, a feature that is characteristic of one parent may be accentuated in the hybrid, this being noted especially in the reactions with sodium hydroxide and sodium salicylate.

Reaction-intensities Expressed by Light, Color, and Temperature Reactions.

Polarization:

I. per. v. pur., moderately high to very high, value 70 .

I. sindjarensis, moderately high to very high, higher than in I. persica var. purpurea, value 75 .

I. pursind, moderately high to high, lower than in either parent,

Iodine: value 65 .

I. per. v. pur., moderate, value 55.

I. sindjarensis, moderate, less than in I. persica var. purpurea, value 50 .

I. pursind, moderate, the same as in I. sindjarensis, value 50. Gentian violet:

I. per. v. pur., moderate, value 45

I. sindjarensis, moderate, less than in I. persica var. purpurea, value 43.

I. pursind, light to moderate, less than in either parent, value 40 . Safranin:

I. per. v. pur., moderate, value 50 .

I. sindjarensis, moderate, less than in I. pereica var. purpurea, value 47.

I. pursind, moderate, less than in either parent, value 45.

Temperature:

I. per. v. pur., in the majority at 64 to $66^{\circ}$, in all at 68 to $70^{\circ}$, mean $69^{\circ}$.

I. sindjarensis, in the majority at 63.5 to $65^{\circ}$, in all at 66 to $67^{\circ}$, mean $66.5^{\circ}$.

I. puraind, in the majority at 64.5 to $66^{\circ}$, in all at 68 to $70^{\circ}$, mean $69^{\circ}$.

The reactivity of $I$. persica var. purpurea is higher than that of the other parent in the iodine, gentian violet, and safranin reactions, and lower in the polarization and temperature reactions. The reactivity of the hybrid is the same or practically the same as that of $I$. persica var. purpurea in the temperature reaction; the same or practically the same as that of $I$. sindjarensis in the iodine reaction; and the lowest of the three in the polarization, gentian violet, and safranin reactions. The hybrid is closer to $I$. persica var. purpurea than to the other parent in the polarization and temperature reactions; and the reverse in the iodine, gentian violet, and safranin reactions.

Table A 33 shows the reaction-intensities in percentages of total starch gelatinized at definite intervals (minutes).

\section{Velocity-reaction CURVes.}

This section treats of the velocity-reaction curves of the starches of Iris persica var. purpurea, I. sindjarensis, and $I$. pursind, showing the quantitative differences in the behavior toward different reagents at different timeintervals. (Charts D 442 to D 462.) are :

The most conspicuous features of this group of curves

(1) The marked closeness of all three curves throughout the various reactions, the only reaction in which there is a marked tendency to continually increasing differentiation during the 60 minutes being
TABLE A 33.

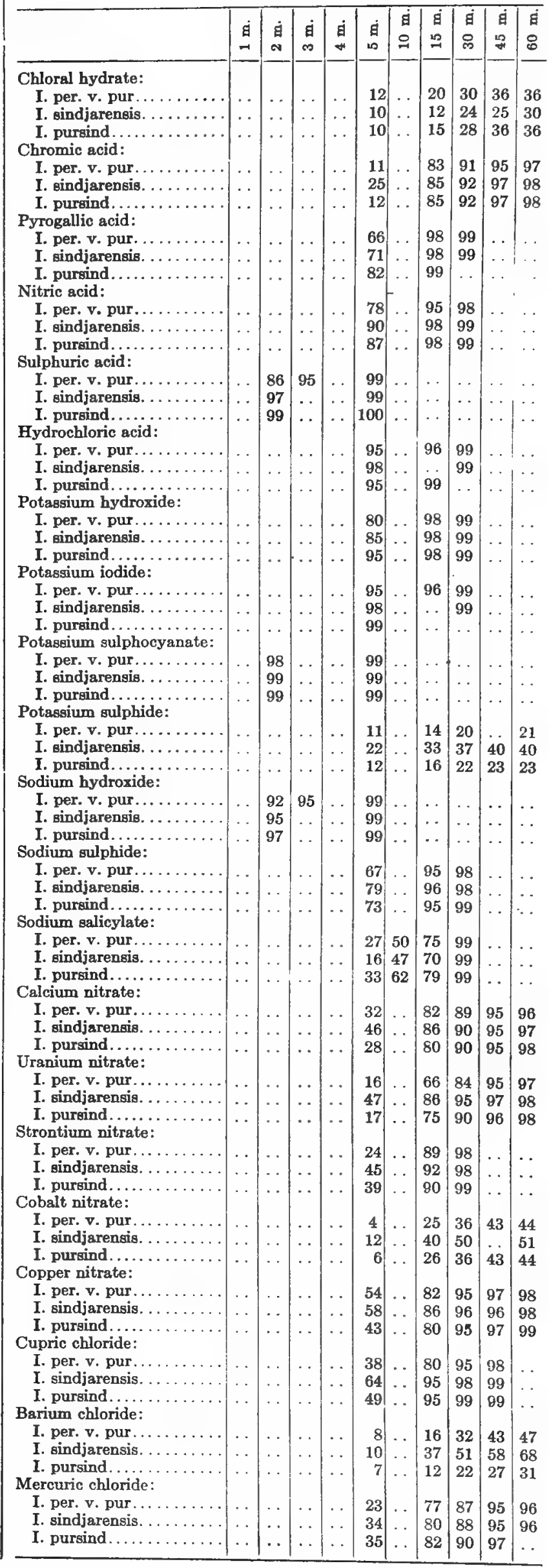


in that with barium chloride. In all other instances the most marked differentiation is noted early in the reactions, with an inclination for the differences to become less during the progress of the reactions. In many instances the curves are so close as not to permit of satisfactory differentiation, unless it be within the first 5 minutes, as in the reactions with chromic acid, pyrogallic acid, nitric acid, sulphuric acid, hydrochloric acid, potassium hydroxide, potassium iodide, sodium sulphide, calcium nitrate, strontium nitrate, copper nitrate, cupric chloride, and mercuric chloride; in others there may be as good or better differentiation at a later period, as in the reactions with chloral hydrate, potassium sulphide, sodium salicylate, uranium nitrate, cobalt nitrate, and barium chloride. Gelatinization occurs with such speed in the reactions with potassium sulphocyanate and sodium hydroxide as to render satisfactory differentiation impossible.

(2) The higher reactivity of $I$. persica var. purpurea than of the other parent in the reactions with chloral hydrate, sodium salicylate, and calcium nitrate; the lower reactivity with chromic acid, nitric acid, sulphuric acid, potassium sulphide, sodium sulphide, uranium nitrate, calcium nitrate, strontium nitrate, cobalt nitrate, cupric chloride, barium chloride, and mercuric chloride; and the same or practically the same reactivity with pyrogallic acid, hydrochloric acid, potassium hydroxide, potassium iodide, potassium sulphocyanate, sodium hydroxide, and cupric chloride. In some of the reactions where the curve is higher or lower the differences are unimportant and probably fall within the limits of error of experiment.

(3) The variable position of the hybrid curve in relation to one or both parental/ curves. There is a distinct tendency to intermediateness, and one also equally strong for the curve of the hybrid to be above or below the parental curves.

(4) There is an entire absence of any marked tendency to a period of early resistance followed by rapid reaction. There are mere suggestions of such resistance as, for instance, in I. persica var. purpurea and the hybrid in the chromic-acid and uranium-nitrate reactions; and of $I$. sindjarensis in the sodium-salicylate reaction.

(5) The earliest period during the 60 minutes at which the three curves are best separated to differentiate the starches varies with the different reagents. Approximately, this period occurs within 5 minutes in the reactions with chromic acid, pyrogallic acid, nitric acid, sulphuric acid, hydrochloric acid, potassium hydroxide, potassium iodide, potassium suphocyanate, sodium hydroxide, sodium sulphide, sodium salicylate, calcium nitrate, strontium nitrate, copper nitrate, cupric chloride, and mercuric chloride; at 15 minutes with chloral hydrate, potassium sulphide, uranium nitrate, and cobalt nitrate; and at 60 minutes with barium chloride.

\section{Reaction-INTENSities OF THE Hybrid.}

This section treats of the reaction-intensities of the hybrid as regards sameness, intermediateness, excess, and deficit in relation to the parents. (Table A 33 and Charts D 442 to D 462.)

The reactivities of the hybrid are the same as those of the seed parent with temperature, potassium sulphide, and cobalt nitrate; the same as those of the pollen parent with iodine and sulphuric acid; the same as those of both parents in the reactions with chromic acid, hydrochloric acid, potassium iodide, potassium sulphocyanate, and sodium hydroxide; intermediate with chloral hydrate, nitric acid, sodium sulphide, uranium nitrate, and strontium nitrate (in one being closer to the seed parent, in two closer to the pollen parent, and in two mid-intermediate); highest with pyrogallic acid, potassium hydroxide, sodium salicylate, cupric chloride, and mercuric chloride (in two being closer to the seed parent, in two closer to the pollen parent, and in one as close to one as to the other parent); and lowest with the polarization, gentian violet, safranin, calcium nitrate, copper nitrate, and barium chloride (in four being closer to the seed parent, and in two closer to the pollen parent).

The following is a summary of the reaction-intensities: Same as seed parent, 3; same as pollen parent, 2; same as both parents, 5; intermediate, 5; highest, 5; lowest, 6.

The influences of the seed and pollen parents seem to be about equal, slightly in favor of the former. Intermediateness is recorded in about one-fifth of the reactions, and highness and lowness in about two-fifths.

\section{Composite Corves of Reaction-Intensities.}

This section treats of the composite curves of the reaction-intensities, showing the differentiation of the starches of Iris persica var. purpura, I. sindjarensis, and I. pursind. (Chart $\mathrm{E} 33$.)

The most conspicuous features of this chart are:

(1) The marked closeness of all three curves throughout, the most noticeable differences being in the reactions with polarization, iodine, gentian violet, safranin, temperature, potassium hydroxide, uranium nitrate, cupric chloride, and barium chloride. In all other reactions (17 out of 26 ) the curves are nearly or practically identical, their closeness indicating very closely related parental species, or more likely varieties.

(2) The curve of $I$. persica var. purpurea tends to be lower than that of the other parent in the reactions with polarization, temperature, sulphuric acid, potassium sulphide, uranium nitrate, cupric chloride, and barium chloride; higher with iodine, gentian violet, and safranin ; and the same or practically the same with chloral hydrate, chromic acid, pyrogallic acid, nitric acid, hydrochloric acid, potassium hydroxide, potassium iodide, potassium sulphocyanate, sodium hydroxide, sodium sulphide, sodium salicylate, calcium nitrate, strontium nitrate, cobalt nitrate, copper nitrate, and mercuric chloride.

(3) The curve of the hybrid follows very closely the curves of the parents, it being closer to or identical with the curve of one or the other, or identical with both.

(4) In $I$. persica var. purpurea the very high reactions with pyrogallic acid, nitric acid, sulphuric acid, hydrochloric acid, potassium hydroxide, potassium iodide, potassium sulphocyanate, sodium hydroxide, sodium sulphide reactions; the high reactions with polarization, chromic acid, sodium salicylate, calcium nitrate, uranium nitrate, strontium nitrate, copper nitrate, cupric chloride, and meruric chloride; the moderate reactions with iodine, gentian violet, safranin, temperature; and the very low reactions with chloral hydrate, potassium sulphide, cobalt nitrate, and barium chloride. 
(5) In $I$. sindjarensis the very high reactions with pyrogallic acid, nitric acid, sulphuric acid, hydrochloric acid, potassium hydroxide, potassium iodide, potassium sulphocyanate, sodium hydroxide, sodium sulphide, and cupric chloride; the high reactions with polarization, chromic acid, sodium salicylate, calcium nitrate, uranium nitrate, strontium nitrate, copper nitrate, and mercuric chloride; the moderate reactions with iodine, gentian violet, safranin, and temperature; the low reactions with cobalt nitrate and barium chloride reactions; and the very low reactions with chloral hydrate and potassium sulphide.

(6) In the hybrid the very high reactions with pyrogallic acid, nitric acid, sulphuric acid, hydrochloric acid, potassium hydroxide, potassium iodide, potassium sulphocyanate, sodium hydroxide, and sodium sulphide; the high reactions with polarization, chromic acid, sodium salicylate, calcium nitrate, uranium nitrate, strontium nitrate, copper nitrate, cupric chloride, and mercuric chloride; the moderate reactions with iodine, gentian violet, safranin, and temperature; and the very low reactions with chloral hydrate, potassium sulphide, cobalt nitrate, and barium chloride.

Following is a summary of the reaction-intensities:

\begin{tabular}{|c|c|c|c|c|c|}
\hline & $\begin{array}{l}\text { Very } \\
\text { high. }\end{array}$ & High & $\begin{array}{l}\text { Mod- } \\
\text { erate. }\end{array}$ & Low. & $\begin{array}{l}\text { Very } \\
\text { low. }\end{array}$ \\
\hline $\begin{array}{l}\text { I. persica var. purpurea } \ldots \ldots \ldots \\
\text { I. sindjarensis. } \ldots \ldots \ldots \ldots \ldots \ldots \\
\text { I. pursind } \ldots \ldots \ldots \ldots \ldots \ldots \ldots\end{array}$ & $\begin{array}{r}9 \\
10 \\
9\end{array}$ & $\begin{array}{l}9 \\
8 \\
9\end{array}$ & $\begin{array}{l}4 \\
4 \\
4\end{array}$ & $\begin{array}{l}0 \\
2 \\
0\end{array}$ & $\begin{array}{l}4 \\
2 \\
4\end{array}$ \\
\hline
\end{tabular}

Notes ON THE IRISES. are:

Among the very striking features of the four charts

The closeness of all three curves in each chart and the wavering relationship of the hybrid curve to one or the other or both parental curves, occasionally going above or below parental extremes in Charts $\mathrm{E} 30, \mathrm{E} 31$, and $\mathrm{E} 33$, and frequently (15 out of 26 reactions) in Chart E 32; the close correspondence of the curves of the three sets of rhizomatous irids (Charts E $30, \mathrm{E} 31$, and $E 32)$; and the very definite differentiation of the curves of the rhizomatous and tuberous series.

In the first set the cross is between members of the subgenera Ococyclus and Apagon; in the second set, between members of the subgenera Ococyclus and Pogoniris and Regelia; in the third set, between members of the subgenus Pogoniris and Regelia; and in the fourth set, between members of the subgenus Juno. In the three sets of rhizomatous irids the curves are so nearly alike as to suggest that the subgeneric division of Hasselbring referred to in Part II is botanically largely artificial, and that the primary division into rhizomatous and tuberous groups is well founded in expressing fundamental botanical differentiation. Although only one set of tuberous irises was studied in detail in this research, cursory investigations were made with other members of this series (including $I$. histrio Reichb., $I$. tingitiana Boiss and Reut., I. reticulata M. Bieb., I. alata Poïr., and I. caucasica Hoffm.; the first three belonging to the subgenus Xiphion and the last two to the subgenus Juno), in all of which the reactions were in close correspondence with those of this set. In the previous research with irid starches it was found that the members of the rhizo- matous series have in comparison with those of the tuberous series, besides different histologic properties, a lower degree of polarization, lower reactivities with iodine, higher reactivities with gentian violet and safranin, and distinctly higher temperatures of gelatinization. Owing to improper strengths of the reagents, evidence was not recorded that is satisfactory to differentiate the starches then studied; but there was clear evidence of grouping of the two series, the members of the rhizomatous series having, as a whole, higher reactivities with chloral hydrate and chromic acid, and lower reactivities with ferric chloride and Purdy's solution. These results are in accord with those of the present research, there being in the rhizomatous series mean lower reactivities with polarization and iodine, higher reactivities with gentian violet and safranin, higher temperature of gelatinization, higher reactivity with chloral hydrate, the same or a tendency to a higher reactivity with chromic acid, and a lower reactivity with potassium hydroxide.

The types of curves of the rhizomatous and tuberous irids, respectively, differ chiefly in the relative lowness of the rhizomatous curve in the reactions with pyrogallic acid, nitric acid, hydrochloric acid, potassium hydroxide, potassium iodide, sodium hydroxide, sodium sulphide, calcium nitrate, uranium nitrate, copper nitrate, cupric chloride, and mercuric chloride, and the highness in those with chloral hydrate and sodium salicylate. Probably among the irids will be found some species or hybrid that will, as in case of the crinums, bridge the two series.

Owing to the almost invariable closeness of the three curves in each set, opportunity is rarely afforded for a satisfactory study of the relationships of the hybrid to one or the other or both parents. It will be seen by the following summary, the figures of which are to be taken as having only tentative values, that the different hybrids vary in their parental relationships, especially in their intermediate, highest, and lowest records.

The following is a summary of the reaction-intensities of the hybrids as regards sameness, intermediateness, excess, and deficit in relation to the parents:

\begin{tabular}{|c|c|c|c|c|c|c|}
\hline & 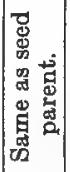 & 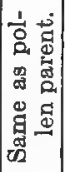 & 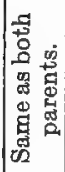 & 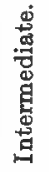 & 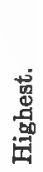 & 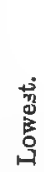 \\
\hline 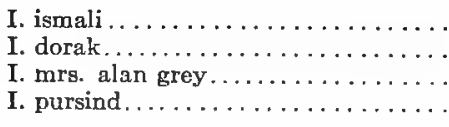 & $\begin{array}{l}3 \\
5 \\
0 \\
3\end{array}$ & $\begin{array}{l}2 \\
3 \\
1 \\
2\end{array}$ & $\begin{array}{l}2 \\
2 \\
3 \\
5\end{array}$ & $\begin{array}{r}12 \\
1 \\
1 \\
5\end{array}$ & $\begin{array}{r}1 \\
11 \\
3 \\
5\end{array}$ & $\begin{array}{r}6 \\
4 \\
17 \\
6\end{array}$ \\
\hline
\end{tabular}

The differences in the reactive-intensities of the rhizomatous and tuberous series are indicated in the following table:

\begin{tabular}{c|c|c|c|c|c}
\hline & $\begin{array}{c}\text { Very } \\
\text { high. }\end{array}$ & High. & $\begin{array}{l}\text { Mod- } \\
\text { erate. }\end{array}$ & Low. & $\begin{array}{l}\text { Very } \\
\text { low. }\end{array}$ \\
\hline $\begin{array}{l}\text { Rhizomatous series: } \\
\text { I. iberica-trojana-ismali..... }\end{array}$ & 3 & 2 & & & \\
$\begin{array}{c}\text { I. iberica-cengialti-dorak.... } \\
\text { I. cengialti-pallida-mra. }\end{array}$ & 3 & 3.1 & 8.7 & 7.7 & 4.7 \\
$\begin{array}{c}\text { Tuberous series: } \\
\text { I. persica-sindjarensis-pursind }\end{array}$ & $\mathbf{2 . 3}$ & 3.3 & 9.3 & 8.3 & 5 \\
\hline
\end{tabular}




\section{Comparisons of the Starches of Gladiolus CARDINALIS, G. TRISTIS, AND G. COLVILLEI.}

In histologic characteristics, polariscopic figures, reactions with selenite, qualitative reactions with iodine, and qualitative reactions with chemical reagents the parents and the hybrid exhibit properties in common in varying degrees of development and also individualities which collectively are in each case distinctive, although the starches show characters in general that are closely akin. The starch of Gladiolus tristis in comparison with that of $G$. cardinalis exhibits as prominent differences certain peculiarities of the aggregates and an absence of a type of compound grain that is found, and the presence of another type of compound grain that is not found in G. cardinalis; and sharply defined pressure facets are more common. The hilum is less distinct; an irregular cavity at the hilum is often larger and more irregular; fissuration is more common; and eccentricity is greater. The lamellæ are less distinct and numerous. The size of the grains is less. In the polariscopic, selenite, and qualitative iodine reactions there are many differences which seemingly are of a minor character, yet which collectively are quite diagnostic. In the qualitative reactions with chloral hydrate, hydrochloric acid, potassium iodide, sodium hydroxide, and sodium salicylate there are many differences, mostly minor, some individualizing one or the other parent. The starch of the hybrid in comparison with the starches of the parents contains certain compound grains similar to a type found only in G. cardinalis and also a linear type of aggregate that is found only in G. tristis. There are many minor differences, but the grains are on the whole more closely related to those of $G$. cardinalis. The hilum exhibits more numerous clefts and the fissuration is more varied than in either parent; eccentricity is about the same as in $G$. tristis and greater than in $G$. cardinalis; but in general characters the hilum is more like that of $G$. cardinatis. The lamellæ in character are mid-intermediate, but the number is in excess of the numbers in the parents. The size is closer to that of $G$. tristis. In the polariseopic, selenite, and qualitative iodine reactions there are leanings to one or the other parent, but the relationship is on the whole much closer to $G$. cardinalis. In the qualitative chemical reactions there are corresponding leanings and relationships.

Reaction-intensities Expressed by Light, Color, and TemperaPolarization:

G. cardinalis, high to very high, much higher than in G. tristis, value 85 .

G. tristis, moderate to high, value 65 .

G. colvillei, high to very high, not quite so high as in G. cardinalis, value 80 .

Iodine:

G. cardinalis, moderate to deep, the same as in G. tristis, value 60 .

G. tristis, moderate to deep, value 60 .

G. colvillei, moderate to deep, lighter than in either parent, value 55

Gentian violet:

G. cardinalis, moderate, higher than in G. tristis, value 50 .

G. tristis, light to moderate, value 40 .

G. colvillei, moderate, intermediate between the parents, value 47 . Safranin:

G. cardinalis, moderate, deeper than in G. tristis, value 53 .

G. tristis, light to moderate, value 45 .

G. colvillei, moderate, the same as in G. cardinalis, value 53. Temperature:

G. cardinalis, majority at 83 to $84.5^{\circ}$, all at 84 to $86^{\circ}$, mean $85^{\circ}$.

G. tristis, majority at 76 to $78^{\circ}$, all at 78 to $79^{\circ}$, mean $78.5^{\circ}$.

G. colvillei, majority at 78 to $80^{\circ}$, all at 82 to $83^{\circ}$, mean $82.5^{\circ}$.

The reactivities of $G$. cardinalis are higher than those of $G$. tristis in the polarization, gentian violet, and safranin; lower in the temperature reaction; and the same in that with iodine. The reactivities of the hybrid are in-
TABLE A 34.

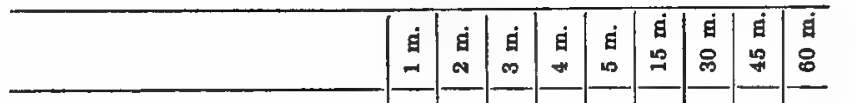

Chloral hydrate:

G. cardinalis.............

G. tristis...............

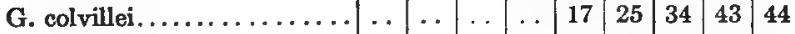

Chromic hydrate:

G. cardinalis.

G. tristis.

G. colvillei.

Pyrogallic acid:

G. cardinalis.

G. tristis. .

G. colvillei

Nitric acid:

G. cardinali

G. tristis. .

G. colvillei.

Sulphuric acid:

G. cardinalis

G. tristis.

G. colvillei

Hydrochloric acid:

G. cardinalis

G. tristis.

Potassium hydroxide:

G. cardinalis..........

G. tristis. .

\begin{tabular}{ll|l|l|l}
4 & 20 & 75 & 90 & 96
\end{tabular}

$\begin{array}{lllllll}3 & 60 & 95 & 98 & 99\end{array}$

\begin{tabular}{ll|l|l|l}
4 & 30 & 82 & 93 & 98
\end{tabular}

G. colvillei .

Potassium iodide:

G. cardinalis.

G. tristis. .

G. colvillei

Potassium sulphocyanate:

G. cardinalis

G. tristis. .

Potassium sulphide:

G. cardinalis...

G. tristis. .
G. colvillei.

Sodium hydroxide:

G. cardinalis

G. tristis. ....

G. colvillei

Sodium sulphide:

G. cardinalis

G. tristis.

G. colvillei....

Sodium salicylate:

G. cardinalis

G. tristis. .

G. colvillei...

Calcium nitrate:

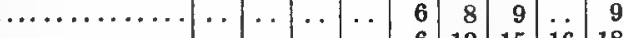

G. colvillei.................

Uranium nitrate:

G. cardinalis

G. colvillei................

Strontium nitrate:

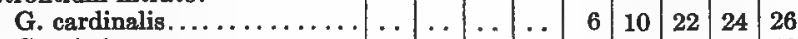

G. tristis................

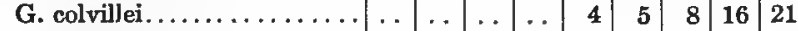

Cobalt nitrate:

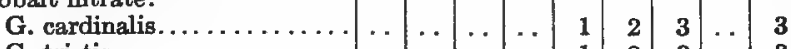

G. tristis.............................

G. colvillei.............

Copper nitrate:

G. cardinalis............. .

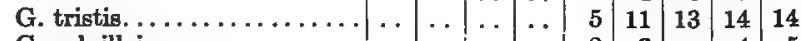

G. colvillei...............

Cupric chloride:

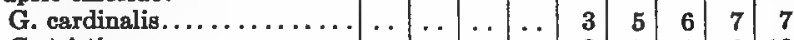

G. tristis................

G. colvillei...............

Barium chloride:

G. cardinalis.

$\begin{array}{lllllllllllll}\ldots & \ldots & \ldots & \ldots & \ldots & \ldots & \ldots & 1 & 2 & 3 & \ldots & 3\end{array}$

G. colvillei..............

Mercuric chloride:

G. cardinalis

G. tristis. .

G. colvillei............. 
termediate in the polarization, gentian-violet, and temperature reactions; lowest in the iodine reaction; and the same as that of $G$. cardinalis but higher than that of $G$. tristis in the safranin reaction. The hybrid is on the whole distinctly closer to G. cardinalis than to G. tristis.

Table A 34 shows the reaction-intensities in percentages of total starch gelatinized at definite intervals (minutes).

\section{Velocttr-Reaction CURVes.}

This section treats of the velocity-reaction curves of the starches of Gladiolus cardinalis, $G$. tristis, and $G$. colvillei, showing the quantitative differences in the behavior toward different reagents at definite time-intervals. (Charts D 463 to D 483 .)

Among the conspicuous features of these charts are:

(1) The higher reactivity of $G$. tristis in relation to the other parent and the hybrid throughout.

(2) The differences recorded between the reactions of the starches of the two parents with the various reagents, the curves varying very markedly in the extent of separation. Thus, the curves are very close throughout the whole or nearly the whole 60-minute period in the reactions with chloral hydrate, nitric acid, sulphuric acid, potassium hydroxide, potassium sulphide, sodium salicylate, calcium nitrate, uranium nitrate, cobalt nitrate, copper nitrate, cupric chloride, barium chloride, and mercuric chloride; they are well separated to widely separated in those with chromic acid, pyrogallic acid, hydrochloric acid, potassium iodide, potassium sulphocyanate, sodium hydroxide, sodium sulphide, and strontium nitrate.

(3) The almost universal tendency for the curve of G. cardinatis to be closer to the curve of the hybrid than to $G$. tristis. In only the reactions with chloral hydrate, sulphuric acid, potassium hydroxide, and sodium salicylate is the curve of $G$. cardinalis definitely closer to that of $G$. tristis. In the potassium-sulphide reactions gelatinization proceeded so slowly that such differences as were recorded fall within the limits of error of experiment. In the experiments with calcium nitrate, strontium nitrate, copper nitrate, and cupric chloride the $G$. cardinalis curve is practically intermediate.

(4) The curves of the hybrid bear varying relations to the parental curves, with a manifest tendency to sameness to the curves of $G$. cardinalis, and to intermediateness and to the lowest position, and almost invariably definitely toward the seed parent.

(5) An early period of resistance followed by a moderate to rapid gelatinization is noted in the chromic acid chart. In other charts the corresponding period is one of comparatively rapid gelatinization, as in the reactions with chloral hydrate, sulphuric acid, sodium salicylate, while in others gelatinization proceeds with marked slowness, yet steadily from the outstart, as instanced particularly in the reactions with potassium sulphide, uranium nitrate, cobalt nitrate, and in other very slow reactions. There are some gradations between these sets.

(6) The earliest period of the 60 minutes at which the three curves are best separated for differential purposes varies with the different reagents, and in some instances owing to the extremely slow reactions satisfactory differentiation is impossible. Approximately this period occurs at the end of 5 minutes in the reactions with chloral hydrate, sulphuric acid, and sodium salicylate; at 15 minutes with chromic acid, pyrogallic acid, hydrochloric acid, and potassium sulphocyanate; at 30 minutes with strontium nitrate; and at 60 minutes with nitric acid, potassium hydroxide, potassium iodide, potassium sulphide, sodium hydroxide, sodium sulphide, calcium nitrate, uranium nitrate, cobalt nitrate, copper nitrate, cupric chloride, barium chloride, and mercuric chloride. In a number of the reactions of the latter groups the differences are trivial and within the limits of error of experiment.

\section{Reaction-INTEnsities of THE HybRid.}

This section treats of the reaction-intensities of the hybrid as regards sameness, intermediateness, excess, and deficit in relation to the parents. (Table A 34 and Charts D 463 to D 483.)

The reactivities of the hybrid are the same as those of the pollen parent in none of the reactions; the same as those of the seed parent in the reactions with safranin, chromic acid, nitric acid, uranium nitrate, cupric chloride, barium chloride, and mercuric chloride; the same as those of both parents in that with cobalt nitrate, wherein the gelatinization is extremely slow; intermediate in those with polarization, gentian violet, temperature, and pyrogallic acid (in all four being closer to the seed parent); highest in none; and lowest with iodine, chloral hydrate, sulphuric acid, hydrochloric acid, potassium hydroxide, potassium iodide, potassium sulphocyanate, potassium sulphide, sodium hydroxide, sodium sulphide, sodium salicylate, calcium nitrate, strontium nitrate, and copper nitrate (in 12 being closer to the seed parent, and in 2 as close to one as to the other parent).

The following is a summary of the reaction-intensities: Same as seed parent, 7 ; same as pollen parent, 0 ; same as both parents, 1 ; intermediate, 4 ; highest, 0 ; lowest, 14.

The most striking features of the foregoing data are the absence of a single reaction in which there was sameness or even inclination more to the pollen than to the seed parent; the slight tendency to intermediateness; and the very strongly marked tendency for the curves of the hybrid to be below those of the parents.

\section{Composite Curves of the Reaction-Intensities.}

This section treats of the composite curves of the reaction-intensities, showing the differentiation of the starches of Gladiolus cardinatis, G. tristis, and G. colvillei. (Chart E 34.)

The most conspicuous features of this chart are:

(1) The varying relationship the curve of $G$. tristis bears to the curve of the other parent, sometimes above, below, or the same or practically the same. It is above in the reactions with temperature, chloral hydrate, pyrogallic acid, nitric acid, hydrochloric acid, potassium hydroxide, potassium iodide, potassium sulphocyanate, sodium hydroxide, sodium sulphide, sodium salicylate, calcium nitrate, uranium nitrate, strontium nitrate, and copper nitrate; below with polarization, gentian violet, and safranin; and the same or practically the same with iodine, chromic acid, sulphuric acid, potassium sulphide, cobalt nitrate, cupric chloride, barium chloride, and mercuric chloride. The other parent, G. cardinalis, is higher in only the polarization, gentian-violet, and safranin reactions.

(2) The varying degrees of separation of the parental curves, the most marked separation being noted in the reactions with polarization, temperature, pyrogallic acid, potassium iodide, potassium sulphocyanate, sodium hydroxide, sodium sulphide, and strontium nitrate.

(3) The marked tendency for the curve of the hybrid to be closer to the curve of $G$. cardinalis than to the other parent, and to be lowest of the three.

(4) In $G$. tristis the very high reactions with sulphuric acid; the high reactions with polarization, iodine, and sodium salicylate; the moderate with gentian violet, 
safranin, chromic acid, pyrogallic acid, and potassium sulphocyanate; the low with temperature, chloral hydrate, and hydrochloric acid, potassium iodide, sodium hydroxide, and sodium sulphide; and the very low reactions with nitric acid, potassium hydroxide, potassium sulphide, calcium nitrate, uranium nitrate, strontium nitrate, cobalt nitrate, copper nitrate, cupric chloride, barium chloride, and mercuric chloride.

(5) In $G$. cardinatis the very high reactions with polarization and sulphuric acid; the high reactions with iodine and sodium salicylate; the moderate reactions with gentian violet, safranin, and chromic acid; the low reactions with chloral hydrate and hydrochloric acid; and the very low reactions with temperature, pyrogallic acid, nitric acid, potassium hydroxide, potassium iodide, potassium sulphocyanate, potassium sulphide, sodium hydroxide, sodium sulphide, calcium nitrate, uranium nitrate, strontium nitrate, cobalt nitrate, copper nitrate, cupric chloride, barium chloride, and mercuric chloride.

(6) In the hybrid the very high reactions with polarization and sulphuric acid; the absence of any high reaction; the moderate reactions with iodine, gentian violet, safranin, chromic acid, and sodium salicylate; the low reaction with temperature; the very low reactions with chloral hydrate, pyrogallic acid, nitric acid, hydrochloric acid, potassium hydroxide, potassium iodide, potassium sulphocyanate, potassium sulphide, sodium hydroxide, sodium sulphide, calcium nitrate, uranium nitrate, strontium nitrate, cobalt nitrate, copper nitrate, cupric chloride, barium chloride, and mercuric chloride.

Following is a summary of the reaction-intensities:

\begin{tabular}{|c|c|c|c|c|c|}
\hline & $\begin{array}{l}\text { Very } \\
\text { high. }\end{array}$ & High. & $\begin{array}{l}\text { Mod- } \\
\text { erate. }\end{array}$ & Low. & $\begin{array}{l}\text { Very } \\
\text { low. }\end{array}$ \\
\hline $\begin{array}{l}\text { G. tristis. } \ldots \ldots \ldots \ldots \ldots \ldots \ldots \\
\text { G. cardinalis. } \ldots \ldots \ldots \ldots \ldots \ldots \ldots \\
\text { G. colvillei........ }\end{array}$ & $\begin{array}{l}1 \\
2 \\
2\end{array}$ & $\begin{array}{l}3 \\
2 \\
0\end{array}$ & $\begin{array}{l}5 \\
3 \\
5\end{array}$ & $\begin{array}{l}6 \\
2 \\
1\end{array}$ & $\begin{array}{l}11 \\
17 \\
18\end{array}$ \\
\hline
\end{tabular}

\section{Comparisons of the Starches of Tritonia pottsi, T. CROcosmia aUREa, AND T. Cronos- MEFLORA.}

In histologic characteristics, polariscopic figures, reactions with selenite, reactions with iodine, and qualitative reactions with the various chemical reagents the starches of the parents and hybrid exhibit properties in common in varying degrees of development and also certain individualities, which latter, although as a rule of a minor character, are in conjunction with the properties in common sufficient for differential purposes. The starch of Tritonia crocosmia aurea in comparison with that of $T$. pottsii shows among the most conspicuous differences in form a larger proportion of permanently isolated grains; more numerous compound grains of two components; less numerous grains with well-defined pressure facets; triangular grains more elongated; and varied proportions of other types of grains. The hilum is more refractive; a rounded or irregular cavity is more frequently found; more often fissured, and the clefts are as a rule deeper; there are some differences in the forms of fissuration; and eccentricity is slightly greater. The lamellæ are less distinct; a marginal band of refractive lamellæ is more frequently present; the numbers are about the same. The sizes differ but little. In the polariscopic, selenite, and qualitative iodine reactions there are numerous differences which are seemingly of a minor character. In the qualitative reactions with chloral hydrate, hydrochloric acid, potassium iodide, sodium hydroxide, and sodium salicylate many differences are recorded, some of which are individually quite distinctive. The starch of the hybrid in comparison with the parental starches is found to show markedly the influences of both parents; leaning to one or the other parent or sameness with both are very conspicuous. In form the differences are essentially in the varying proportions of different types of grains, the starch of the hybrid being closer to that of T. crocosmia aurea. The hilum in eccentricity is closer to that of $T$. crocosmia aurea, but in every other character closer to the other parent. The lamellæ and size differ but little from those of the parents, and in both respects the relationship is closer to $T$. pottsii. In the polariscopic, selenite, and qualitative iodine reactions, and in the reactions with the various chemical reagents there are leanings to one or the other parent, or sameness to both, but on the whole distinctly toward $T$. crocosmia aurea. Notwithstanding the closeness of all three starches it is quite remarkable how readily the variable parental leanings of the hybrid are detected.

\section{Reaction-intensities Eapressed by Light, Color, and Tempera- ture Reactions.}

Polarization:

T. pottsii, moderate to very high, value 70 .

T. crocosmia aurea, high to very high, higher than in $\mathrm{T}$. pottsii, value 75 .

T. crocosmæflora, moderate to very high, lower than in T. pottsii,

Iodine: value 67 .

T. pottsii, very light, value 10 .

T. crocosmia aurea, moderate, value 50 .

T. crocosmmflora, light, value 25 .

Gentian violet:

T. pottsii, light to moderate, value 40

T. crocosmia aurea, light to moderate, lighter than $T$. pottsii, value 35 .

T. crocosmæflora, light to moderate, the same as $T$. pottaii, Safranin: value 40 .

T. pottsii, light to moderate, value 40 .

T. crocosmia aurea, light to moderate, lower than $T$. pottsii, value 35 .

T. crocosmæflora, light to moderate, deeper than in the parents, value 45 .

\section{Temperature:}

T. pottsii, majority at 73 to $75^{\circ}$, all at 76 to $77.5^{\circ}$, mean $76.75^{\circ}$.

T. crocosmia aurea, majority at 78 to $80^{\circ}$, all at 80 to $82^{\circ}$, mean $81^{\circ}$.

T. crocosmaflora, majority at 74 to $76^{\circ}$, all at 76 to $78^{\circ}$, mean $77^{\circ}$.

The reactivity of $T$. pottsii is higher than that of $T$. crocosmia aurea in the polarization and iodine reactions, and higher in the gentian-violet, safranin, and temperature reactions. The reactivity of the hybrid is intermediate in the iodine reaction; the same as that of $T$. pottsii in the gentian-violet and temperature reactions; lowest of the three in the polarization reaction; and the highest of the three in the safranin reaction. The relationship throughout is closer to $T$. pottsii.

Table A 35 shows the reaction-intensities in percentages of total starch gelatinized at definite intervals (minutes).

\section{Velocity-reaction Curves.}

This section treats of the velocity-reaction curves of the starches of Tritonia pottsii, T. crocosmia aurea, and T. crocosmoflora, showing the quantitative differences in the behavior toward different reagents at definite timeintervals. (Charts D 484 to D 504.)

Among the most conspicuous features of these charts are the following:

(1) Excepting the sulphuric-acid and barium-chloride reactions in which the differences in reactivity are insignificant, the starches of the parents exhibit welldefined differences which are very variable in extent with the different reagents. With all of the reagents, excepting those noted and chloral hydrate, $T$. pottsii has the higher reactivity, but in the reactions with the latter it 
TABLE A 35.

\begin{tabular}{|c|c|c|c|c|c|c|c|c|c|}
\hline & $\underset{9}{-1}$ & $\underset{\mathrm{N}}{\dot{\mathrm{g}}}$ & $\underset{\infty}{\dot{E}}$ & $\stackrel{g}{*}$ & $\underset{n}{0}$ & $\begin{array}{l}\text { 日 } \\
\text { 号 } \\
\rightarrow\end{array}$ & ${ }_{0}^{\dot{B}}$ & $\begin{array}{l}\text { घี } \\
\text { 요 }\end{array}$ & $\begin{array}{l}8 \\
8\end{array}$ \\
\hline \multicolumn{10}{|l|}{ Chloral hydrate: } \\
\hline T. pottsii..... & $\cdots$ & $\cdots$ & $\cdots$ & $\cdots$ & 10 & 26 & 48 & 60 & 63 \\
\hline T. crocosmia aurea. . . . . . . & $\cdots$ & $\cdots$ & $\cdots$ & . & 15 & 40 & 52 & 62 & 66 \\
\hline T, crocosmmflora... & $\cdots$ & $\cdots$ & $\cdots$ & $\cdots$ & 8 & 20 & 28 & 29 & 30 \\
\hline \multicolumn{10}{|l|}{ Chromic acid: } \\
\hline T. pottsii. .... & $\cdots$ & $\cdots$ & $\ldots$ & $\cdots$ & 5 & 50 & 98 & 99 & \\
\hline T. crocosmia aurea. & . & $\cdots$ & . & . & 2 & 24 & 54 & 80 & 90 \\
\hline T. crocosmæflora... & $\cdots$ & . & . & . & 5 & 36 & 95 & 98 & 99 \\
\hline Pyrogallic acid: & & & & & & & & & \\
\hline T. pottsii........ & $\cdots$ & . & . & $\cdots$ & 13 & 43 & 78 & 91 & 96 \\
\hline T. crocosmia aurea. . . . . . . & $\cdots$ & . & . & $\cdots$ & 2 & 9 & 20 & 40 & 50 \\
\hline T. crocosmæflora. . . . . . . & $\cdots$ & . & . & $\cdots$ & 7 & 40 & 62 & 73 & 90 \\
\hline Nitric acid: & & & & & & & & & \\
\hline T. pottsii........ & $\cdots$ & $\cdots$ & $\cdots$ & $\cdots$ & 10 & 25 & 47 & $\because$ & 50 \\
\hline T. crocosmia au & $\cdots$ & $\cdots$ & . & $\cdots$ & 2 & 5 & 9 & 12 & 12 \\
\hline T. crocosmaflora & $\cdots$ & $\cdots$ & $\cdots$ & $\cdots$ & 12 & 32 & 62 & 68 & 70 \\
\hline $\begin{array}{l}\text { Sulphuric acid: } \\
\text { T. pottsii....... }\end{array}$ & $\therefore$ & & & & 99 & & & & \\
\hline $\begin{array}{l}\text { T. pottsii. . . . . } \\
\text { T. crocosmia aurea }\end{array}$ & $\begin{array}{l}\cdots \\
\cdots\end{array}$ & $\cdots$ & $\cdots$ & $\cdots$ & 95 & 99 & $\cdots$ & $\cdots$ & $\cdots$ \\
\hline $\begin{array}{l}\text { T. crocosmia aurea } \\
\text { T. crocosmeflora.. }\end{array}$ & $\ldots$ & $\cdots$ & $\ddot{*}$ & $\cdots$ & 99 & 99 & $\cdots$ & $\cdots$ & $\cdots$ \\
\hline Hydrochloric acid: & & & & & & $\cdots$ & & $\cdots$ & $\cdots$ \\
\hline T. pottsii....... & $\cdots$ & $\ldots$ & $\ldots$ & $\ldots$ & 80 & 92 & 95 & 97 & 99 \\
\hline T. crocosmia aurea. & $\cdots$ & . & . & . & 51 & 73 & 86 & 90 & 92 \\
\hline T. crocosmæflora... & $\cdots$ & . & . & . & 78 & 81 & 93 & 98 & 99 \\
\hline Potassium hydroxide: & & & & & & & & & \\
\hline T. pottsii........ & $\cdots$ & $\cdots$ & $\ldots$ & . & 9 & 15 & 28 & 33 & 39 \\
\hline T. crocosmia aurea & $\cdots$ & . & . & . & 2 & 5 & 9 & 14 & 20 \\
\hline T. crocosmæflora . . . . . . . & $\cdots$ & . & $\cdots$ & . & 7 & 12 & 17 & 23 & 33 \\
\hline Potassium iodide: & & & & & & & & & \\
\hline T. pottsii........... & $\cdots$ & $\cdots$ & $\cdots$ & $\cdots$ & 15 & 29 & 45 & 62 & 67 \\
\hline T. crocosmia aurea ........ & $\cdots$ & $\cdots$ & $\cdots$ & $\cdots$ & 9 & 12 & 18 & 22 & 27 \\
\hline T. crocosmeflora . . . . . . . & $\cdots$ & $\cdots$ & $\cdots$ & . & 10 & 20 & 39 & 50 & 61 \\
\hline Potassium sulphocyanate: & & & & & & & & & \\
\hline T. pottsii. $\ldots \ldots \ldots \ldots \ldots$ & . & $\ldots$ & $\ldots$ & $\cdots$ & 78 & 85 & 93 & 95 & 97 \\
\hline T. crocosmia aurea. & $\cdots$ & $\cdots$ & . & $\cdots$ & 33 & 57 & 75 & 82 & 86 \\
\hline T. crocosmmflora. . & $\cdots$ & . & $\ldots$ & . & 69 & 86 & 93 & 95 & 97 \\
\hline Potassium sulphide: & & & & & & & & & \\
\hline T. pottsii ........ & $\cdots$ & $\cdots$ & $\cdots$ & $\cdots$ & 5 & 7 & $\cdots$ & 8 & 8 \\
\hline T. crocosmia aur & $\cdots$ & $\cdots$ & $\cdots$ & $\cdots$ & 0.5 & 1 & . & 2 & 2 \\
\hline T. crocosmeflora. & $\cdots$ & $\cdots$ & $\cdots$ & $\cdots$ & 1 & 2 & . & $\cdots$ & 4 \\
\hline Sodium hydroxide: & & & & & & & & & \\
\hline T. pottsii. ...... & $\cdots$ & $\cdots$ & $\cdots$ & $\cdots$ & 62 & 77 & 81 & 84 & .87 \\
\hline T. crocosmia a & $\cdots$ & $\cdots$ & $\cdots$ & $\cdots$ & 16 & 33 & 50 & 56 & 58 \\
\hline T. crocosmeflora. & $\cdots$ & $\cdots$ & $\cdots$ & $\cdots$ & 60 & 71 & 77 & 89 & 91 \\
\hline Sodium sulphide: & & & & & & & & & \\
\hline T. pottsii $\ldots \ldots \ldots \ldots \ldots$ & $\cdots$ & $\cdots$ & $\cdots$ & $\cdots$ & 25 & 34 & 54 & 62 & 68 \\
\hline T. crocosmia aurea ......... & $\cdots$ & $\cdots$ & $\cdots$ & . & 4 & 13 & 22 & 27 & 29 \\
\hline T. crocosmæflora. & 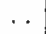 & . & $\cdots$ & . & 16 & 29 & 42 & 60 & 65 \\
\hline Sodium salicylate: & & & & & & & & & \\
\hline T. pottsii...... & . & $\cdots$ & . & $\cdots$ & 65 & 92 & 99 & $\cdots$ & \\
\hline T. crocosmia aurea & $\cdots$ & $\cdots$ & $\cdots$ & $\cdots$ & 11 & 60 & 95 & . & 95 \\
\hline T. crocosmæflora.. & $\cdots$ & $\cdots$ & $\cdots$ & $\cdots$ & 60 & 90 & 99 & . & $\ldots$ \\
\hline Calcium nitrate: & & & & & & & & & \\
\hline T. pottsii......... & . & $\cdots$ & $\cdots$ & $\cdots$ & 15 & 19 & 22 & 26 & 36 \\
\hline T. crocosmia aurea... & . & $\cdots$ & $\cdots$ & $\cdots$ & 3 & $\mathbf{5}$ & 10 & 14 & 14 \\
\hline T. crocosmeflora... & $\cdots$ & . & $\cdots$ & $\cdots$ & 6 & 11 & 16 & 23 & 31 \\
\hline Uranium nitrate: & & & & & & & & & \\
\hline T. pottsil. & . & $\cdots$ & $\cdots$ & $\cdots$ & 5 & 9 & 13 & 16 & 16 \\
\hline T. crocosmia aurea & . & . & $\cdots$ & . & 1 & 3 & 4 & 5 & 5 \\
\hline T. crocosmæflora... & $\cdots$ & $\cdots$ & . & $\cdots$ & 1 & 6 & 7 & $\cdots$ & 8 \\
\hline Strontium nitrate: & & & & & & & & & \\
\hline T. pottsii ......... & $\cdots$ & $\cdots$ & $\cdots$ & $\cdots$ & 10 & 24 & 38 & 41 & 50 \\
\hline T. crocosmia aurea. & - & $\cdots$ & $\cdots$ & . & 3 & 8 & 23 & 33 & 43 \\
\hline T. crocosmæflora . . . . . . . . & . & . & . & . & 12 & 26 & 43 & 51 & 60 \\
\hline Cobalt nitrate: & & & & & & & & & \\
\hline T. pottaii... & . & . & $\cdots$ & $\cdots$ & 7 & 11 & 15 & $\cdots$ & 15 \\
\hline T. crocosmia aurea & . & . & $\cdots$ & $\cdots$ & 1 & 2 & $\mathbf{3}$ & 4 & 4 \\
\hline T. crocosmeflora . . . . . . . & $\ldots$ & $\cdots$ & $\cdots$ & $\cdots$ & 1 & 2 & 3 & 4 & 4 \\
\hline Copper nitrate: & & & & & & & & & \\
\hline T. pottsii..... & $\cdots$ & 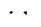 & $\cdots$ & . & 11 & 20 & 24 & 28 & 31 \\
\hline T. crocosmia aurea. & . & $\cdots$ & . & . & 5 & 6 & 7 & & 8 \\
\hline T. crocosmaflora . . . & $\cdots$ & . & . & $\cdots$ & 6 & 15 & 17 & 18 & 21 \\
\hline Cupric chloride: & & & & & & & & & \\
\hline T. pottsii . . . . . . . & . & $\cdots$ & . & $\cdots$ & 10 & 14 & 16 & . & 16 \\
\hline T. crocosmia aurea. & & . & $\cdots$ & . & 2 & $\mathbf{5}$ & 6 & 7 & 8 \\
\hline T. crocosmæflora...... & $\cdots$ & $\cdots$ & $\cdots$ & $\cdots$ & 10 & 11 & 12 & 15 & 15 \\
\hline Barium chloride: & & & & & & & & & \\
\hline T. pottsii. . & & $\cdots$ & $\cdots$ & . & 2 & $\cdots$ & 3 & & 3 \\
\hline T. crocosmia aurea. & $\cdots$ & $\cdots$ & $\cdots$ & $\because$ & & 1 & $\cdots$ & & 1 \\
\hline T. crocosmeflora... & $\ldots$ & .. & . & $\cdots$ & 0.5 & 1 & . & 2 & 2 \\
\hline Mercuric chloride: & & & & & & & & & \\
\hline T. pottsii....... & $\cdots$ & $\cdots$ & $\cdots$ & $\cdots$ & 6 & 9 & 12 & 13 & 15 \\
\hline T. crocosmia a & $\cdots$ & $\cdots$ & $\cdots$ & $\cdots$ & 1 & 2 & 3 & 4 & 4 \\
\hline T, crocosmeflora.... & $\cdots$ & $\cdots$ & $\cdots$ & $\cdots$ & 3 & 6 & 9 & 10 & 11 \\
\hline
\end{tabular}

has a somewhat lower reactivity. The differences are, on the whole, such as to suggest well-separated species.

(2) The curves of the hybrid bear varying relationships to the parental curves, tending for the most part to intermediateness and toward the curves of the seed parent.

(3) An early period of marked resistance is rarely observed, but to the contrary the opposite tendency is usually present, so that the percentage of starch gelatinized during the first 5 minutes is proportionately larger, commonly very much larger, than at any subsequent 5-minute interval. An early period of resistance is noticeable particularly in the reactions with chromic acid and pyrogallic acid, while a low degree of resistance is noted particularly in those with hydrochloric acid, potassium sulphocyanate, sodium hydroxide, sodium sulphide, and sodium salicylate ( $T$. pottsii and the hybrid).

(4) The earliest period during the 60 minutes at which the three curves are best separated, and hence the best time for the differentiation of the starches, is variable in relation to the different reagents. Approximately this period occurs at the end of 5 minutes in the reactions with potassium sulphocyanate, sodium sulphide, and sodium salicylate; at 15 minutes with chloral hydrate, chromic acid, pyrogallic acid, hydrochloric acid, potassium iodide, sodium hydroxide, calcium nitrate, uranium nitrate, copper nitrate, cupric chloride, and mercuric chloride; at 30 minutes with nitric acid, potassium hydroxide, strontium nitrate, and cobalt nitrate; and at 60 minutes with potassium sulphide.

\section{Reaction-intensities of the Hybrid.}

This section treats of the reaction-intensities of the hybrid as regards sameness, intermediateness, excess, and deficit in relation to the parent. (Table A 35 and Charts D 484 to D 504.)

The reactivities of the hybrid are the same as those of the seed parent in the gentian-violet and temperature reactions; the same as those of the pollen parent in the cobalt-nitrate reaction; the same as those of both parents in the sulphuric-acid and barium-chloride reactions; intermediate in those with iodine, chromic acid, pyrogallic acid, hydrochloric acid, potassium hydroxide, potassium iodide, potassium sulphocyanate, potassium sulphide, sodium hydroxide, sodium sulphide, sodium salicylate, calcium nitrate, uranium nitrate, copper nitrate, cupric chloride, and mercuric chloride (in 14 being closer to the seed parent and in 2 closer to the pollen parent) ; highest with safranin, nitric acid, and strontium nitrate (in 2 being closer to the seed parent and in the other to the pollen parent); and lowest with polarization and chloral hydrate, in both being closer to the seed parent.

The following is a summary of the reaction-intensities: Same as seed parent, 2; same as pollen parent, 1; same as both parents, 2 ; intermediate, 17 ; highest, 3 ; lowest, 2 .

The pollen parent seems to have had very little influence in determining the characters of the starch of the hybrid. The tendency to intermediateness of the hybrid is exceptionally well marked, and there is very little tendency for the hybrid curve to be higher or lower than the parental curves.

\section{Composite CuRves of Reaction-Intensities.}

This section treats of the composite curves of the reaction-intensities, showing the differentiation of the starches of Tritonia pottsii, $T$. crocosmia aurea, and T. crocosmoeflora. (Chart E 35.)

Among the conspicuous features of the chart are:

(1) The usually well-marked separation of the curves of the parents, together with an almost invariably 
higher position of the curve of Tritonia pottsii and the close correspondence of the two curves in the up-anddown variations. The only places at which the curve of $T$. pottsii is distinctly lower than that of $T$. crocosmia aurea are in the polarization, iodine, and chloral-hydrate reactions. The curve is the same or practically the same in the reactions with sulphuric acid, potassium sulphide, sodium salicylate, and barium chloride.

(2) In $T$. pottsii the very high reactions with sulphuric acid; the high reactions with polarization, chromic acid, hydrochloric acid, potassium sulphocyanate, and sodium salicylate; the moderate reactions with gentian violet, safranin, and pyrogallic acid; the low reactions with temperature, chloral hydrate, nitric acid, potassium iodide, sodium hydroxide, sodium sulphide, and strontium nitrate; and the very low reactions with iodine, potassium hydroxide, potassium sulphide, calcium nitrate, uranium nitrate, cobalt nitrate, copper nitrate, cupric chloride, barium chloride, and mercuric chloride.

(3) In $T$. crocosmia aurea the very high reaction with sulphuric acid; the high reactions with polarization and sodium salicylate; the moderate reactions with iodine, chromic acid, and hydrochloric acid; the low reactions with gentian violet, safranin, temperature, chloral hydrate, pyrogallic acid, potassium sulphocyanate, and sodium hydroxide; and the very low reactions with nitric acid, potassium hydroxide, potassium iodide, potassium sulphide, sodium sulphide, calcium nitrate, uranium nitrate, strontium nitrate, cobalt nitrate, copper nitrate, cupric chloride, barium chloride, and mercuric chloride.

(4) In the hybrid the very high reactions with sulphuric acid and sodium salicylate; the high reactions with polarization, chromic acid, hydrochloric acid, and potassium sulphocyanate; the moderate reactions with gentian violet, safranin, pyrogallic acid, and sodium hydroxide; the low reactions with iodine, temperature, nitric acid, potassium iodide, sodium sulphide, and strontium nitrate; and the very low reactions with chloral hydrate, potassium hydroxide, potassium sulphide, calcium nitrate, uranium nitrate, cobalt nitrate, copper nitrate, cupric chloride, barium chloride, and mercuric chloride.

Following is a summary of the reaction-intensities:

\begin{tabular}{|c|c|c|c|c|c|}
\hline & $\begin{array}{l}\text { Very } \\
\text { high. }\end{array}$ & High. & $\begin{array}{l}\text { Mod- } \\
\text { erate. }\end{array}$ & Low. & $\begin{array}{l}\text { Very } \\
\text { low. }\end{array}$ \\
\hline $\begin{array}{l}\text { T. pottsii } \ldots \ldots \ldots \ldots \ldots \\
\text { T. crocosmia aures } \ldots \ldots \ldots \ldots \\
\text { T. crocosmøflora.......... }\end{array}$ & $\begin{array}{l}1 \\
1 \\
2\end{array}$ & $\begin{array}{l}5 \\
2 \\
4\end{array}$ & $\begin{array}{l}3 \\
\mathbf{3} \\
\mathbf{4}\end{array}$ & $\begin{array}{l}7 \\
7 \\
6\end{array}$ & $\begin{array}{l}10 \\
13 \\
10\end{array}$ \\
\hline
\end{tabular}

\section{Comparisons of the Starches of Begonia SINGLE CRIMSON SCARLET, B. SOCOTRANA, AND B. MRS. HEAL.}

In the histologic characteristics, polariscopic figures, reactions with selenite and iodine, and qualitative reactions with the various chemical reagents the three starches have properties in common in various degrees of development and in each case certain individualities. The starch of Begonia socotrana in comparison with that of $B$. single crimson scarlet contains no compound grains or aggregates; the grains are not so often irregular, but where irregularity exists it is more marked; the grains are more elongated and the round type few. The hilum is somewhat less distinct and more often fissured, and a peculiar form of fissure is found ecentricity is greater. The lamellæ are somewhat more distinct and somewhat less regular, and there is an absence of a very coarse lamella near the hilum and also of one outlining the primary starch deposit in compound grains if the deposit consists of both primary and secondary lamellæ. Other- wise the character and arrangements are the same. The size is larger. In the polariscopic, selenite, and qualitative iodine reactions there are many differences. In the qualitative reactions with chloral hydrate, chromic acid, pyrogallic acid, nitric acid, and strontium nitrate there are also many differences, many quite striking and distinctive of one or the other parent. The starch of the hybrid in comparison with the starches of the parents exhibits but few individualities in form, and in this histological character it is in closer relationship to $B$. socotrana. The starch of the hybrid is closer to that of B. single crimson scarlet in the general characters of the hilum, but nearer the other parent in form, eccentricity of the hilum, size, and arrangement of the lamellæ (excepting when the grain consists of a primary and a secondary part, when the relationship is closer to the first parent). Certain irregularities of form are seen that are not present in either parent, and the lamellæ are more distinct and not so fine as they are in the parents. In the characters of the polariscopic figure and in the selenite reaction it is closer to $B$. single crimson scarlet. In the iodine reactions it is closer to $B$. single crimson scarlet. In the qualitative reactions with chloral hydrate, chromic acid, pyrogallic acid, nitric acid, and strontium nitrate the relationship is closer to $B$. single crimson scarlet. Some of the grains during gelatinization behave like those of one parent and others like those of the other, and some show associated peculiarities of both parents. The resemblances are, on the whole, more closely related to $B$. single crimson scarlet, as is also the case in the quantitative reactions.

Reaction-intensities Expressed by Light, Color, and TemperaPolarization:

B. sing. crim. scar., moderately high to high, value 60 .

B. socotrana, moderately high to high, the same as in B. single crimson scarlet, value 60 .

B. mrs. heal, moderately high to high, less than in either parent, Iodine: value $\mathbf{5 5}$.

B. sing. crim. scar., moderate, value 45 .

B. socotrana, light to moderate, much less than in $\mathbf{B}$. single crimson scarlet, value 30 .

B. mrs. heal, moderate, the same as in B. single crimson scarlet, value 45 .

Gentian violet:

B. sing. crim. scar., moderate, value 45 .

B. socotrana, light to moderate, much less than in B. single crimson Bcarlet, value 35 .

B. mrs. heal, moderate, same as in B. single crimson scarlet, Safranin: value 45.

B. singl crim. scar., moderate to deep, value 60 .

B. socotrana, moderate to deep, less than in B. single crimson scarlet, value 55 .

B. mrs. heal, moderate to deep, same as in B. single crimson searlet, value 60 .

Temperature:

B. sing. crim. scar., in the majority at 67 to $68.5^{\circ}$, in all at 70 to $72^{\circ}$, mean $71^{\circ}$.

B. socotrana, in the majority at 79 to $80^{\circ}$, in all at 81 to $81.8^{\circ}$, mean $81.4^{\circ}$.

B. mrs. heal, in the majority at 67 to $69^{\circ}$, in all at 71 to $72^{\circ}$, mean $71.5^{\circ}$.

The reactivity of $B$. single crimson scarlet is higher than that of the other parent in the iodine, gentian violet, safranin, and temperature reactions; and the same or practically the same in the polarization reaction. The reactivity of the hybrid is the same or practically the the same as that of $B$. single crimson scarlet in the reactions with iodine, gentian violet, safranin, and temperature; and is the lowest of the three in the polarization reaction. The hybrid is closer to $B$. single crimson scarlet than to the other parent in the reactions with iodine, gentian violet, safranin, and temperature, and is the same in relation to both parents in the polarization reaction. 
TABLe A 36.

—

Chloral hydrate:

B. sing. crim. scar.

B. socotrana.

B. mrs. heal . .

Chromic acid:

B. sing. crim. scar.

B. socotrana....

B. mrs. heal .

B. sing, crim, scar

B. socotrana..........

B. mrs. heal ...

Nitric acid:

B. sing. crim. scar. ... 100 .

8899

387995

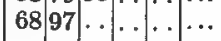

B. socotrana............

B. mrs. heal .... . . . 9599

Sulphuric acid:

B. sing. crim. sear. ... 9s 100 .

B. socotrana........... 9299 ...

B. mrs. heal ......... 90 99

Hydrochloride acid:

B. sing. crim. scar. . . . . 96 96 . 100 .

B. socotrana.....

B. mrs. heal .....

Potassiumhydroxide

B. sing. crim. scar. 100

$87 . \cdots 99$

B. socotrana..... 100

B. mrs. heal ...

Potassium iodide:

B. sing. crim. scar.

.........999

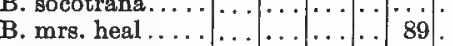

Potassium sulphocyanate:

B. sing. crim. scar. $\ldots . \ldots 99$

B. socotrana.......

B. mrs. heal .

Potassium sulphide:

B. sing. crim. scar. ... 100 ..

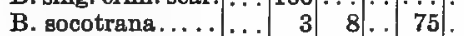

B. murs. heal ......

Sodium hydroxide:

B. sing. crim. sear.

B. socotrang ...

B. mrs. hesl .....

Sodium sulphide:

B. sing. crim. scar.

B. Bocotrana.....

Sodium salicylate:

B. sing. crim. scar.

B. socotrana.....

B. mrs. heal ....

Calcium nitrate:

B. sing. crim. scar. ...

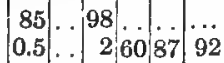

20 . 67 95

B. socotrana.........

\begin{tabular}{ll|l|l|l|l}
58 &. & 88 & 92 & 95 & 97
\end{tabular}

B. mrs. heal ....

Uranium nitrate:

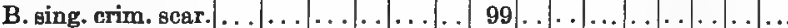

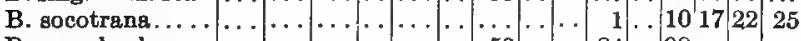

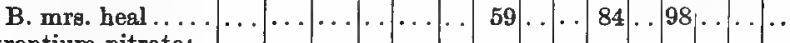

Strontium nitrate:

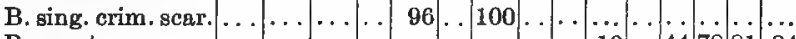

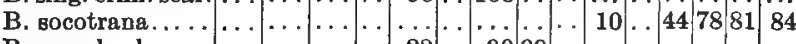

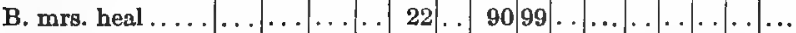

Cobalt nitrate:

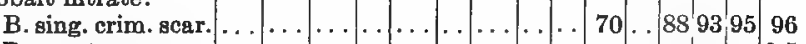

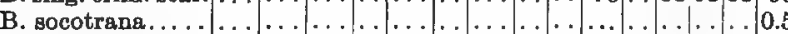

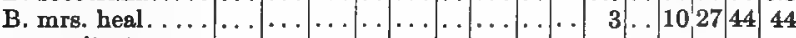

Copper nitrate:

B. sing. crim. scar.

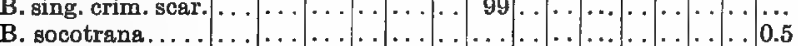

B. rars. heal ....

Cupric chloride:

B. sing, crim. scar.

B. socotrana..

B. mrs, heal ...

Barium chloride:

B. sing. crim. scar.

B. socotrana......

B. mrs. heal .....

Mercuric chloride:

B. sing. crim. scar.
B. socotrana.....

B. socotran

B. mrs. heal ..............
Table A 36 shows the reaction-intensities in percentages of total starch gelatinized at definite intervals (seconds and minutes).

\section{Velocity-Reaction Curves.}

This section treats of the velocity-reaction curves of the starches of Begonia single crimson scarlet, $B$. socotrana, and $B$. mrs. heal, showing quantitative differences in the behavior toward different reagents at definite timeintervals. (Charts D 505 to D 526.)

The most conspicuous features of this group of curves are:

(1) The extraordinary variation of the relations of the curves in the different charts : in some, all three curves being practically identical or close together; in others, two curves keeping close and the third well separated or even separated to the extreme; and in others, all three being well separated from one another. These peculiarities are due largely primarily to the remarkable variations in the reactivities of $B$. socotrana in relation to the different reagents (with one reagent being very reactive and with another the reverse); and secondarily to the almost uniformly very high reactivities of $B$. single crimson scarlet (18 very high, 2 high, and 1 low), together with the marked variations in the relationships of the hybrid to $B$. single crimson scarlet, the hybrid being in many reactions identical or practically identical with this parent and in others having varying degrees of intermediateness, but being much closer, as a rule, to this parent than to the other. Excepting the sulphuric-acid and potassium-hydrate charts, in which the reactions of all three starches are shown to occur with great rapidity, there is a tendency to a well-marked or even extreme separation of the parental curves, the starch of $B$. single crimson scarlet showing, with one exception (barium chloride), a very high to high reactivity, and that of $B$. socotrana, with seven exceptions (chloral hydrate, chromic acid, nitric acid, sulphuric acid, potassium hydroxide, potassium sulphide, and sodium salicylate) a low or usually very low reactivity.

(2) The higher reactivity of $B$. single crimson scarlet than of $B$. socotrana with chloral hydrate, chromic acid, pyrogallic acid, nitric acid, hydrochloric acid, potassium iodide, potassium sulphocyanate, potassium sulphide, sodium hydroxide, sodium sulphide, sodium salicylate, calcium nitrate, uranium nitrate, strontium nitrate, cobalt nitrate, copper nitrate, cupric chloride, barium chloride and mercuric chloride, and the same reactivities with sulphuric acid and potassium hydroxide. There are small differences in the reactivities of the parents with chloral hydrate, potassium sulphide, and sodium salicylate, and from large to very large differences in the other reactions noted, excepting the sulphuric-acid and potassium-hydroxide reactions, in which the two are the same.

(3) The tendency of the hybrid curves to be the same or nearly the same as the curves of $B$. single crimson scarlet, or be of some degree of intermediateness, usually closer to this parent, throughout the whole series of reactions. (See following subsection.)

(4) A period of early resistance followed by a comparative rapid reaction is conspicuous for its almost entire absence. Such a period is suggested in the reactions of the hybrid in the calcium-nitrate reaction, in $B$. single crimson scarlet in the barium-chloride reaction, and in $B$. socotrana in the chromic-acid reaction.

(5) The earliest period during the 60 minutes at which the three curves are best separated to differentiate the starches varies with the different reagents. With five exceptions this occurs in 5 minutes. The exceptions 
are chromic acid, barium chloride, and mercuric chloride in 15 minutes, pyrogallic acid in 30 minutes, and cobalt nitrate in 45 minutes.

\section{Reaction-intensities of the Hybid.}

This section treats of the reaction-intensities of the hybrid as regards sameness, intermediateness, excess, and deficit in relation to the parents. (Table A 36 and Charts D 515 to D 526.)

The reactivities of the hybrid are the same as those of the seed parent in the reactions with iodine, gentian violet, safranin, temperature, nitric acid, hydrochloric acid, potassium iodide, potassium sulphocyanate, and potassium sulphide; the same as those of the pollen parent in none; the same as those of both parents in the reactions with sulphuric acid and potassium hydroxide; intermediate with chloral hydrate, chromic acid, pyrogallic acid, sodium hydroxide, sodium sulphide, sodium salicylate, calcium nitrate, uranium nitrate, strontium nitrate, cobalt nitrate, copper nitrate, cupric chloride, barium chloride, and mercuric chloride (in all 14 being nearer the seed parent) ; highest in none; and lowest in the polarization reaction, in which it is as close to one as to the other parent.

The following is a summary of the reaction-intensities: Same as seed parent, 9 ; same as pollen parent, 0 ; same as both parents, 2 ; intermediate, 14 ; highest, 0 ; lowest, 1.

Sameness as the seed parent and intermediateness with a universal inclination to the seed parent are very conspicuous features of these data. In the two reactions wherein all three starches are the same the reactions occurred with such rapidity as not to permit of differentiation, and in the polarization reaction in which the hybrid shows the lowest reactivity of the three and is as closely related to one as to the other parent the crudity of the method of valuation of the reaction has not brought out differences that probably exist. The properties of the starch seem to have been determined primarily by the seed parent, the effect of the other parent being expressed in the lowering of reactive-intensities, varying in degree in the different reactions, but never so far as to the point of mid-intermediateness.

\section{Composite Curves of the Reaction-intensities.}

This section treats of the composite curves of the reaction-intensities, showing the differentiation of the starches of Begonia single crimson scarlet, $B$. socotrana, and B. mrs. heal. (Chart E 36.)

The most conspicuous features of this chart are:

(1) The generally close accord of the curves of $B$. single crimson scarlet and the hybrid and the extraordinarily erratic course of the curve of $B$. socotrana throughout most of the chart. The hybrid, which is a tuberous form, follows very closely, as a rule, the reactivities of the first parent, which is also tuberous, while the other parent, which is semituberous (bulbils), has a very different type of curve-far more different from that of the other parent than was recorded in the curves of the tender and hardy crinums and the rhizomatous and tuberous irises.

(2) The curve of $B$. single crimson scarlet is higher than the curve of $B$. socotrana throughout the chart (excepting in the reactions with polarization, sulphuric acid, and potassium hydroxide, in which they are alike), and in most instances it tends to be very much higher, the only reactions in which there is marked approximation being those with chloral hydrate, potassium sulphide, and sodium salicylate.

(3) In $B$. single crimson scarlet the very high reactions with chloral hydrate, chromic acid, nitric acid, sulphuric acid, hydrochloric acid, potassium hydroxide, potassium iodide, potassium sulphocyanate, potassium sulphide, sodium hydroxide, sodium sulphide, sodium salicylate, calcium nitrate, uranium nitrate, strontium nitrate, cobalt nitrate, copper nitrate, cupric chloride, and mercuric chloride; the high reactions with polarization, safranin, pyrogallic acid, and cobalt nitrate; the moderate reactions with iodine, gentian violet, and temperature; and the low reaction with barium chloride.

(4) In $B$. socotrana the very high reactions with chloral hydrate, sulphuric acid, potassium hydroxide, potassium sulphide, and sodium salicylate; the high reactions with polarization and nitric acid; the moderate reactions with safranin and chromic acid; the low reactions with iodine, gentian violet, temperature, sodium hydroxide, and strontium nitrate; and the very low reactions with pyrogallic acid, hydrochloric acid, potassium iodide, potassium sulphocyanate, sodium sulphide, calcium nitrate, uranium nitrate, cobalt nitrate, copper nitrate, cupric chloride, barium chloride, and mercuric chloride.

(5) In the hybrid the very high reactions with chloral hydrate, nitric acid, sulphuric acid, hydrochloric acid, potassium hydroxide, potassium iodide, potassium sulphocyanate, potassium sulphide, sodium hydroxide, sodium sulphide, sodium salicylate, calcium nitrate, uranium nitrate, strontium nitrate, copper nitrate, and cupric chloride; the high reactions with safranin and chromic acid; the moderate reactions with polarization, iodine, and gentian violet; the low reactions with temperature, pyrogallic acid, and mercuric chloride; and the very low reactions with cobalt nitrate and barium chloride.

Following is a summary of the reaction-intensities:

\begin{tabular}{|c|c|c|c|c|c|}
\hline & $\begin{array}{l}\text { Very } \\
\text { high. }\end{array}$ & High. & $\begin{array}{l}\text { Mod- } \\
\text { erate. }\end{array}$ & Low. & $\begin{array}{l}\text { Very } \\
\text { low. }\end{array}$ \\
\hline B. single crimson scarlet....... & 18 & 4 & 3 & 1 & $\mathbf{0}$ \\
\hline B. socotrana $\ldots \ldots \ldots \ldots \ldots \ldots$ & 5 & 2 & 2 & $\mathbf{5}$ & 12 \\
\hline B. mrs. heal . . . . . . . . . . & 16 & 2 & $\mathbf{3}$ & $\mathbf{3}$ & 2 \\
\hline
\end{tabular}

37. Comparisons of the Starches of Begonia DOUBLE Light ROSE, B. Socotrana, AND B. ENSTGN.

In histologic characteristics, polariscopic figures, reactions with selenite, reactions with iodine, and qualitative reactions with various chemical reagents all three starches have properties in common in varying degrees of development, the sum of which in each case is distinctive of the starch. The starch of Begonia socotrana in comparison with that of $B$. double light rose shows an absence of aggregates and has more numerous irregularities. The hilum is less distinct, somewhat more often fissured, and more eccentric. The lamellæ are not so distinct; more distinct at the distal than at the proximal end, instead of sometimes the reverse as in $B$. double light 
rose; and they are more numerous. The size is larger than in $B$. double light rose. In the polariscopic, selenite, and iodine reactions there are various differences which seem to be of a minor character, and the same is true of the reactions with chloral hydrate, chromic acid, pyrogallic acid, nitric acid, and strontium nitrate. The starch of the hybrid is closer to that of $B$. double light rose in the form of the grains, character of the hilum, character of the lamellæ, and size of the smaller grains, but nearer to $B$. socotrana in the eccentricity of the hilum and size of the larger grains. It is closer to $B$. double light rose in the appearance with selenite, but nearer the other parent in the polariscopic figures. It is closer to the first parent in the iodine reactions. In the qualitative reactions with chloral hydrate, chromic acid, pyrogallic acid, nitric acid, and strontium nitrate, while closer to $B$. double light rose, the influences of $B$. socotrana are quite manifest in each.

\section{Reaction-intensities Expressed by Light, Color, and Tempera- ture Reactions.}

Polarization:

B. doub. light rose, moderately high to high, value 70 .

B. socotrana, moderate to moderately high, less than in B. double light rose, value 60

B. ensign, moderate to high, intermediate between parents, value 67 . Iodine:

B. doub. light rose, moderate, value 45 .

B. socotrana, light to moderate, less than in B. double light rose, value 30 .

B. ensign, light to moderate, intermediate between the parents, value 40 .

Gentian violet:

B. doub. light rose, light to moderate, value 40.

B. socotrana, light to moderate, less than in B. double light rose, value 35 .

B. ensign, light to moderate, less than in either parent, value 30 . Safranin:

B. doub. light rose, moderate to deep, value 60 .

B. socotrana, moderate, less than in B. double light rose, value 55 .

B. ensign, moderate to deep, less than in either parent, value 50.

Temperature:

B. doub. light rose, in the majority at 60 to $61^{\circ}$, in all at 62 to $64^{\circ}$, mean $63^{\circ}$.

B. socotrana, in the majority at 79 to $80^{\circ}$, in all at 81 to $81.8^{\circ}$, mean $81.4^{\circ}$.

B. ensign, in the majority at 64 to $65.5^{\circ}$, in all at 66 to $68^{\circ}$, mean $67^{\circ}$.

The reactivity of $B$. double light rose is higher than that of the other parent in all five reactions. The reactivity of the hybrid is intermediate between those of the parents in the polarization, iodine, and temperature reactions, and is the lowest of the three with gentian violet and safranin. The hybrid is closer to $B$. double light rose than to $B$. socotrana in the polarization, iodine, and temperature reactions, and the reverse in those with gentian violet and safranin.

Table A 37 shows the reaction-intensities in percentages of total starch gelatinized at definite intervals (seconds and minutes).

\section{Velocity-REaction CURVes.}

This section treats of the velocity-reaction curves of the starches of Begonia double light rose, $B$. socotrana, and $B$. ensign, showing quantitative differences in the behavior toward different reagents at definite time-intervals. (Charts D 527 to D 532.)

The most conspicuous features of these five charts are:

The marked diversity of the relations of the three curves, all three running close in the choral-hydrate

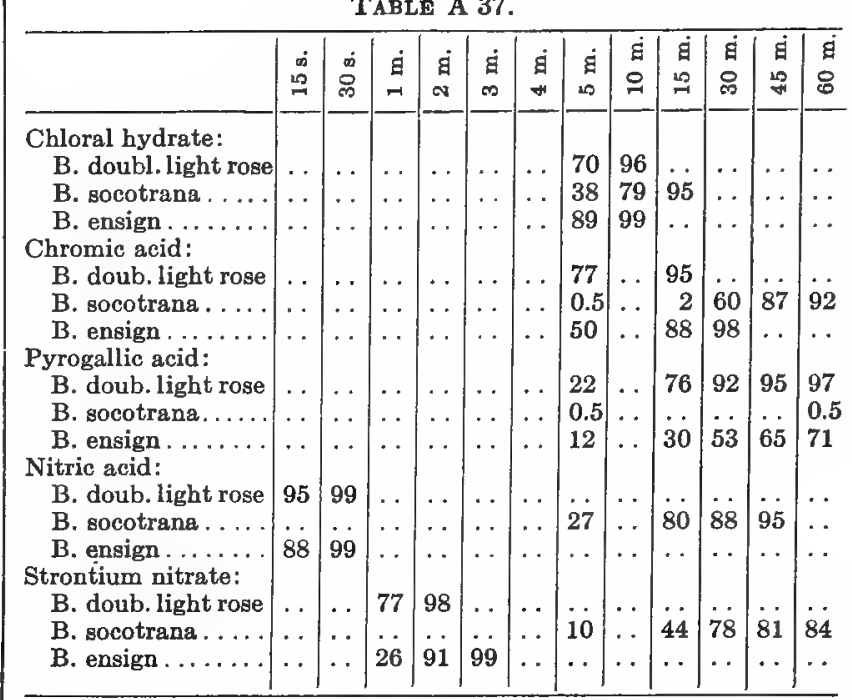

reactions, two being close and the other well separated in those with nitric acid and strontium nitrate, two being somewhat close and the other well separated in that with chromic acid, and all three being well separated in that with pyrogallic acid. The tendency in all for the hybrid and $B$. double light rose curves to be closely related, and to be higher-usually much higher-than the curves of $B$. socotrana. The tendency in all of the reactions to intermediateness, highest or lowest reactivity, with an inclination in 8 out of 10 reactions toward the reactivity of the seed parent. The short period of very high resistance of $B$. socotrana in the chromic-acid reaction.

\section{Reaction-Intensities of the Hybrid.}

This section treats of the reaction-intensities of the hybrid as regards sameness, intermediateness, excess, and deficit in relation to the parents. (Table A 37 and Charts D 527 to D 532.)

The reactivities of the hybrid are not the same as those of either or both parents in a single reaction; intermediate in the reactions with polarization, iodine, temperature, chromic acid, pyrogallic acid, nitric acid, and strontium nitrate, in all being closer to those of the seed parent; highest in that with chloral hydrate, being closer to that of the seed parent; and the lowest in those with gentian violet and safranin, in both being closer to the pollen parent.

The following is a summary of the reaction-intensities: Same as seed parent, 0 ; same as pollen parent, 0 ; same as both parents, 0 ; intermediate, 7 ; highest, 1 ; lowest, 2.

The following features of the hybrid are particularly conspicuous: The absence of any reaction that is the same as either or both parents; the marked tendency to intermediateness; the occasional tendency to the highest or lowest reactivity; and the markedly stronger influence of the seed parent on the properties of the starch.

\section{Composite Curves of Reaction-intensities.}

This section treats of the composite curves of the reaction-intensities, showing the differentiation of the 
starches of Begonia double light rose, $B$. socotrana, and B. ensign. (Chart E 3\%.)

The most conspicuous features of this chart are: The generally close correspondence in the courses of the three curves, although in some instances the curves are well separated. The higher position of the curve of $B$. double light rose in relation to that of $B$. socotrana throughout excepting in the nitric-acid reaction, in which the curves are the same. The varying relationship of the hybrid curve to the parental curves. It is intermediate in the reactions with polarization, iodine, temperature, chromic acid, and pyrogallic acid; lower than the parental curves in those with gentian violet and safranin; the same or nearly the same as that of $B$. double light rose in those with chloral hydrate and strontium nitrate; and the same as both parents in that with nitric acid.

\section{Comparisons of the Starches of Begonia} DOUBLE WHITE, B. SOCOTRANA, AND B. JULIUS.

In the histologic characteristics, polariscopic figures, reactions with selenite, reactions with iodine, and qualitative reactions with various chemical reagents all three starches have properties in common in varying degrees of development, together with individualities, which collectively in each case serve to be distinctive. The starch of Begonia socotrana in comparison with that of $B$. double white shows an absence of compounds and aggregates; more irregularity of the grains and some marked differences in the causes of the irregularities; grains often elongated; and comparatively few round and triangular forms. The hilum is less distinct, much less often fissured, shows an absence of certain forms of fissuration, and eccentricity is more. The lamellæ are finer but not so distinct, there is an absence of two lamellæ which are quite conspicuous in the other parent; they are more often not regular and show waviness; and they are slightly less numerous. In size the grains are somewhat larger and more slender. In the polariscopic, selenite and qualitative iodine reactions there are many differences. In the qualitative reactions with chloral hydrate, chromic acid, pyrogallic acid, nitric acid, and strontium nitrate the differences are numerous and some of them quite individualize the parent. The starch of the hybrid is more closely related to $B$. double white in form, character and arrangement of the lamellæ, and size of the grains; nearer to $B$. socotrana in the characters of the irregularities of the grains and in the character and eccentricity of the hilum; and it has fewer irregularities than either parent. In the polarization figures it resembles both parents equally. In the iodine reactions the heated grains more closely resemble those of $B$. double white, while the unheated grains more closely resemble those of $B$. socotrana. In the qualitative reactions with chloral hydrate, chromic acid, pyrogallic acid, nitric acid, and strontium nitrate peculiarities of both parents are manifest, but the reactions, as a whole, more closely resemble those of $B$. double white than of $B$. socotrana.

Reaction-intensities Expressed by Light, Color, and Tempera-

Polarization: ture Reactions.

B. double white, low to moderately high, value 55 .

B. socotrana, moderate to moderately high, higher than in B. double white, value 60 .

B. julius, moderate to moderately, the same as in B. double white, value 60 .
Iodine:

B. double white, light, value 25.

B. socotrana, light to moderate, deeper than in B. double white, value 30 .

B. julius, light to moderate, deeper than in either parent, value 40. Gentian violet:

B. double white, light to moderate, value 30 .

B. socotrana, light to moderate, deeper than in B. double white, value 35 .

B. julius, moderate to moderately deep, deeper than in either Safranin: parent, value 45.

B. double white, light to moderate, value 40 .

B. socotrana, moderate, much deeper than in B. double white, value 55 .

B. julius, moderately deep, deeper than in either parent, value 60 . Temperature:

B. double white, in the majority at 60 to $61.5^{\circ}$, in all at 65 to $66.5^{\circ}$, mean $62.75^{\circ}$.

B. socotrana, in the majority at 79 to $80^{\circ}$, in all at 81 to $81.8^{\circ}$, mean $81.4^{\circ}$.

B. julius, in the majority at 65 to $66^{\circ}$, in all at 67 to $69^{\circ}$, mean $68^{\circ}$

The reactivity of $B$. double white is lower than that of the other parent in the polarization, gentian-violet, and safranin reactions, and higher in the temperature reaction. The reactivity of the hybrid is the same or practically the same as that of $B$. socotrana in the polarization reactions; highest of the three in those with iodine, gentian violet, and safranin; and intermediate in that with temperature. The hybrid is closer to $B$. double white than to $B$. socotrana in the temperature reaction; and the reverse in those with polarization, iodine, gentian violet, and safranin.

Table A 39 shows the reaction-intensities in percentages of total starch gelatinized at definite intervals (seconds and minutes) :

Table A 38.

\begin{tabular}{|c|c|c|c|c|c|c|c|c|c|c|c|c|}
\hline & $\begin{array}{l}m \\
\ddot{n} \\
\ddot{n}\end{array}$ & $\begin{array}{l}\dot{m} \\
\text { 品 }\end{array}$ & g & $\underset{\sigma}{E}$ & $\begin{array}{l}\tilde{\varepsilon} \\
\infty\end{array}$ & $\dot{a}$ & 㝴 & $\begin{array}{l}\text { g } \\
\text { ᄋ }\end{array}$ & 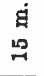 & 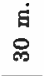 & 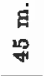 & $\begin{array}{l}\text { घี } \\
\text { ८े }\end{array}$ \\
\hline Chloral & & & & & & 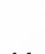 & 8 & 99 & & & 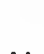 & \\
\hline & & & & & $\therefore$ & & 38 & 79 & 95 & & ․ & .. \\
\hline & & & $\ldots$ & & . . & .. & 90 & 99 & 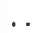 & .. & .. & .. \\
\hline Chron & & & & & & & & & & & & \\
\hline B. double white & & & . & & . & . & 97 & . & 99 & & & \\
\hline & & & . & & .. & .. & 0.5 & .. & 2 & 60 & 87 & 92 \\
\hline B. & & & $\ldots$ & & . & . . & 75 & .. & 95 & 99 & $\cdots$ & .. \\
\hline Pyrog & & & & & & & & & & & & \\
\hline B. & & & . & & .. & .. & 84 & . & 95 & 99 & $\cdots$ & \\
\hline B. 8 & & & . & & . . & . & 0.5 & . & & & & 0.5 \\
\hline & & & . & . & . & . & 20 & . & 75 & 90 & 92 & 95 \\
\hline Nitric & & & & & & & & & & & & \\
\hline B. & 100 & & . & & .. & .. & $\because$ & $\cdots$ & & & & $\cdots$ \\
\hline B. & & & . & & . & .. & 27 & .. & 80 & 88 & 95 & . \\
\hline & 99 & 100 & . & & . & . & .. & $\cdots$ & . & . & $\cdots$ & - \\
\hline Stront & & & & & & & & & & & & \\
\hline & & & 97 & 100 & . & . & & . & & & & \\
\hline & & & & & .. & .. & 10 & .. & 44 & 78 & 81 & 84 \\
\hline B. julius & & & 84 & 99 & . & . & . & - & $\cdots$ & . & $\cdots$ & * \\
\hline
\end{tabular}

Veloctty-Reaction CuRtes.

This section treats of the velocity-reaction curves of the starches of Begonia double white, B. socotrana, and $B$. julius, showing quantitative differences in the behavior toward different reagents at definite time-intervals. (Charts D 533 to D 538.)

These charts bear close resemblances to the corresponding charts in the preceding set, but the differences are sufficient to show that there are differences in parentage and offspring. There is a tendency in this set to a 
higher reactivity of the seed parent, which in turn tends to affect in the same direction the reactivities of the hybrid.

\section{Reaction-intensitiss OF the Hybrid.}

This section treats of the reaction-intensities of the hybrid as regards samencss, intermediateness, excess, and deficit in relation to the parents. (Table A 38 and Charts D 533 to D 538.)

The reactivities of the hybrid are the same as those of the seed parent in the nitric-acid reaction; the same as those of the pollen parent in the polarization reaction; the same as those of both parents in none; intermediate in the reactions with temperature, chromic acid, pyrogallic acid, and strontium nitrate, in all of which being closer to those of the seed parent; highest with iodine, gentian violet, safranin, and chloral hydrate (in three being closer to those of the pollen parent and in one closer to that of the seed parent) ; and lowest in none.

The following is a summary of the reaction-intensities: Same as the seed parent, 1; same as the pollen parent, 1 ; same as both parents, 0 ; intermediate, 4 ; highest, 4 ; lowest, 0 .

In these reactions the reactivities of the hybrid bear only a somewhat closer relationship to the seed parent, and there is a marked inclination to intermediateness and highest reactivity.

\section{Composite CURVES OF the REaCtion-Intensities.}

This section treats of the composite curves of the reaction-intensities showing the differentiation of the starches of Begonia double white, B. socotrana, and $B$. julius. (Chart E 38.)

The most conspicuous features of this chart are: The generally close correspondence in the courses of all three curves, although in three instances the curves are well separated. The lower position of the curve of $B$. double white in relation to that of the other parent in the reactions with polarization, iodine, gentian violet, and safranin; the higher position with temperature, chloral hydrate, chromic acid, pyrogallic acid, and strontium nitrate; and the same position with nitric acid. The varying relationship of the hybrid curve to the parental curves. It is the same as the curve of $B$. socotrana in the reaction with polarization; the same as that of $B$. double white with chloral hydrate and strontium nitrate; the same as both parents with nitric acid; the highest in the three with iodine, gentian violet, and safranin; and intermediate with temperature, chromic acid, and pyrogallic acid.

\section{Comparisons of the Starches of Begonia DOUBLE DEEP ROSE, B. SOCOTRANA, AND B. SUCCESS.}

In the histologic characteristics, polariscopic figures, reactions with selenite, reactions with iodine, and qualitative reactions with various reagents all three starches have properties in common in varying degrees of development, the sum of which in each case is distinctive. The starch of Begonia socotrana in comparison with that of $B$. double deep rose shows an absence of compound grains and aggregates; the grains are more regular, but such irregularities as occur are more obvious and striking; the grains are more elongated; and round and nearly round forms are very rare. The hilum is somewhat less rarely fissured; there is an individual form of fissuring; and there is more eccentricity. The lamellæ are finer and less distinct; several are present that are not seen in B. double deep rose; and they are much more numerous. The size is larger. The reactions with polarization, selenite, and iodine exhibit many differences. In the qualitative reactions with chloral hydrate, chromic acid, pyrogallic acid, nitric acid, and strontium nitrate the differences are numerous and some of them are quite striking and distinctly individualize the starch. The starch of the hybrid in comparison with the starches of the parents shows a closer relationship to the starch of $B$. double deep rose in the characters of the irregularities of the grains and in the characters of the hilum; more like the other parent in the form of the grains, eccentricity of the hilum, character and arrangement and number of the lamellæ, and size of the grains. It has, however, less irregularities in the grains than in either parent. It is nearer $B$. socotrana in the polarization figures and appearances with selenite, and nearer also in the iodine reactions. It shows peculiarities of both parents in the quantitative reactions with chloral hydrate, chromic acid, pyrogallic acid, nitric acid, and strontium nitrate, but is closer to $B$. double deep rose.

Reaction-intensities Expressed by Light, Color, and TemperaPolarization:

B. double deep rose, moderately low to high, value 50 .

B. socotrana, moderate to high, higher than in B. double deep rose, value 60 .

B. success, moderate to high, the same as in N. socotrana, value 60 . Iodine:

B. double deep rose, moderate, value 45 .

B. socotrans, light to moderate, much lighter than in B. double deep rose, value 30 .

B. success, light to moderate, the same as in B. socotrana, value 30 . Gentian violet:

B. double deep rose, light to moderate, value 40.

B. socotrana, light to moderate, less than in B. double deep rose, value 35 .

B. success, light to moderate, the same as in B. socotrana, value 35 . Safranin:

B. double deep rose, moderate to deep, value 60 .

B. socotrana, moderate, less than in B. double deep rose, value 55 .

B. success, moderate to deep, the same as in B. double deep rose, value 60 .

Temperature:

B. double deep rose, in majority at 64 to $65.5^{\circ}$, in all at 67 to $68.8^{\circ}$, mean $67.8^{\circ}$.

B. socotrana, in majority at 79 to $80^{\circ}$, in all at 81 to $81.8^{\circ}$, mean $81.4^{\circ}$.

B. success, in majority at 62 to $64^{\circ}$, in all at 68 to $69^{\circ}$, mean $68.5^{\circ}$.

The reactivity of $B$. double deep rose is lower than that of the other parent in the polarization reaction; and higher in those with iodine, gentian violet, safranin, and temperature. The reactivity of the hybrid is the same or practically the same as that of $B$. double deep rose in the reaction with safranin; the same or practically the same as those of $B$. socotrana with polarization, iodine, and gentian violet; and intermediate between those of the parents in that with temperature. The hybrid is closer to $B$. double deep rose than to $B$. socotrana in the safranin and temperature reactions, and the reverse in those with polarization, iodine, and safranin.

Table A 39 shows the reaction-intensities in percentages of total starch gelatinized at definite intervals ( onds and minutes): 
Table A 39.

\begin{tabular}{|c|c|c|c|c|c|c|c|c|c|c|c|c|}
\hline & $\stackrel{m}{2}$ & $\begin{array}{l}\dot{0} \\
\stackrel{\infty}{\infty}\end{array}$ & $\underset{-1}{\dot{a}}$ & a & $\dot{g}$ & 皇 & 目 & $\stackrel{9}{9}$ & $\begin{array}{l}\text { 日 } \\
\sim 9\end{array}$ & $\begin{array}{l}\text { 白 } \\
\text { 品 }\end{array}$ & $\begin{array}{l}\text { g } \\
\text { 20 }\end{array}$ & $\begin{array}{l}\dot{\text { g }} \\
\stackrel{8}{\circ}\end{array}$ \\
\hline $\begin{array}{l}\text { Chloral hydrate: } \\
\text { B.doubledeeprose }\end{array}$ & & . & $\cdots$ & & & & 9 & & & & & \\
\hline B. socotrana.... & . & . & . & . & . & . & 38 & 79 & 95 & & . & . \\
\hline B. success . . & $\ldots$ & .. & .. & & . & & 86 & 99 & & & & \\
\hline Chromic acid: & & & & & & & & & & & & \\
\hline B.doubledeeprose & $\cdots$ & .. & . & & . & $\cdots$ & 65 & & 95 & 99 & & \\
\hline B. socotrana..... & ... & . & .. & . & .. & .. & 0.5 & . & 2 & 60 & 87 & 92 \\
\hline B. success... & $\ldots$ & .. & .. & . & .. & . & 73 & .. & 95 & $\cdots$ & $\ldots$ & \\
\hline Pyrogallic acid: & & & & & & & & & & & & \\
\hline B. doubledeeprose & $\cdots$ & $\cdots$ & . & $\cdots$ & . & $\cdots$ & 25 & . & 77 & 88 & 95 & 96 \\
\hline B. socotrana..... & $\ldots$ & • & .. & . & $\cdots$ & . & 0.5 & .. & & & & 0.5 \\
\hline B. success. & .. & . & . & . & .. & . & 43 & .. & 87 & 92 & 96 & \\
\hline Nitric acid: & & & & & & & & & & & & \\
\hline B. double deeprose & 100 & . & . & . & $\cdots$ & . & & . & & & & \\
\hline B. socotrana ..... & & 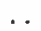 & . & . & .. & . & 27 & . & 80 & 88 & 95 & . \\
\hline B. success........ & 100 & $\ldots$ & .. & . & . & .. & $\cdots$ & .. & . & . & $\cdots$ & . \\
\hline $\begin{array}{l}\text { Strontium nitrate: } \\
\text { B doubledeeprose }\end{array}$ & & & 80 & 99 & & & & & & & & \\
\hline B. socotrana.... & & & & & & $\ldots$ & 10 & . & 44 & 78 & 81 & 84 \\
\hline B. succes & & . & 88 & 99 & . & 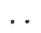 & & . & .. & & & \\
\hline
\end{tabular}

Velocity-REaction CURVes.

This section treats of the velocity-reaction curves of the starches of Begonia double deep rose, $B$. socotrana, and $B$. success, showing quantitative differences in the behavior toward different reagents at definite time-intervals. (Charts D 539 to D 544.)

These charts differ from those of the last set chiefly in the reversal of the relative positions of the curves of the seed parent and hybrid and the more marked closeness of these curves in the pyrogallic-acid reaction. The nitric-acid and strontium-nitrate curves are in the two sets in each case practically the same.

\section{Reaction-INTENSITIES OF THE HYBRID.}

This section treats of the reaction-intensities of the hybrid as regards sameness, intermediateness, excess, and deficit in relation to the parents. (Table A 39 and Charts D 539 to D 544.)

The reactivities of the hybrid are the same as those of the seed parent in the reactions with safranin and nitric acid; the same as those of the pollen parent with polarization, iodine, and gentian violet; the same as those of both parents in none; intermediate with temperature and chloral hydrate, in both being closer to those of the seed parent; highest with chromic acid, pyrogallic acid, and strontium nitrate, in all three being closer to those of the seed parent; and the lowest in none.

The following is a summary of the reaction-intensities: Same as seed parent, 2; same as pollen parent, 3; same as both parents, 0 ; intermediate, 2 ; highest, 3 ; lowest, 0 .

In these few reactions the tendencies seem to be about equal to sameness as one or the other parent, intermediateness and highest reactivity; but the influences of the seed parent in determining the properties of the starch of the hybrid distinctly dominate those of the other parent.

\section{Composite CuRtes of the Reaotion-Intensities.}

This section treats of the composite curves of the reaction-intensities, showing the differentiation of the starches of Begonia double deep rose, B. socotrana, and B. success. (Chart E 39.)
The most conspicuous features of this chart are:

(1) The generally close correspondence of all three curves, although in some instances the curves are well separated, as in the preceding sets.

(2) The higher position of the curve of $B$. double deep rose in the relation to the curve of the other parent in the reactions with iodine, gentian violet, safranin, temperature, chloral hydrate, chromic acid, pyrogallic acid, and strontium nitrate; the lower position with polarization; and the identical position with nitric acid.

(3) The varying position of the hybrid curve in relation to the parental curves. It is the same or practically the same as the curve of $B$. double deep rose in the reactions with safranin, temperature, chromic acid, pyrogallic acid, and strontium nitrate; the same as that of $B$. socotrana in those with polarization, iodine, and gentian violet; the same as the curves of both parents in that with nitric acid; and intermediate in that with chloral hydrate.

\section{Notes on the Begonias.}

The most conspicuous features of these records are observed in the very definite and commonly wide differences between the properties of the seed parents on the one hand and of Begonia socotrana, the pollen parent, on the other, representing two quite different groups of begonias. Histologically, the starches of the seed parents have characters in common which definitely group them from the starch of $B$. socotrana. Even far greater distinctions are seen in the records of the temperatures of gelatinization and of the quantitative reactions with hydrochloric acid, potassium iodide, potassium sulphocyanate, sodium hydroxide, sodium sulphide, calcium nitrate, uranium nitrate, strontium nitrate, copper nitrate, cupric chloride, and mercuric chloride. The very large differences in the temperature reactions of the two groups exceed any records thus far made of members of any genus. The least difference between members of the tuberous group and $B$. socotrana is $11.4^{\circ}$, the greatest $18.65^{\circ}$, and the average $14.85^{\circ}$. Such differences indicate corresponding marked physico-chemical peculiarities of the starch molecules and prepare one for finding similar diversities in the reactions with various chemical reagents. Comparisons of the data of the four seed parents indicate well-separated horticultural or subgeneric specimens. Inasmuch as $B$. socotrana is the pollen parent in each set, it is of exceptional interest to learn to what extent and in what directions the characters of the hybrids are influenced by this parent. Inasmuch as the seed parents exhibit among themselves distinctive peculiarities it is to be expected that the hybrid in any set will be definitely different from the hybrids of the other sets, and such has been found to be a fact. The hybrids show marked variability in their relations to their parents, each exhibiting characters that are either common to both parents or individually parental, and in varying. degrees of development, sometimes being like one parent or the other, or identical with both or having development beyond parental extremes in one direction or the other. While the inclination of the hybrid is, on the whole, very definitely toward, even at times exceeding, the development of the seed parent the influences of $B$. socotrana are themselves sometimes so potent that the seed parent seems to be without effect. 
The following is a summary of the reaction-intensities of the hybrid as regards sameness, intermediateness, excess, and deficit in relation to the parents:

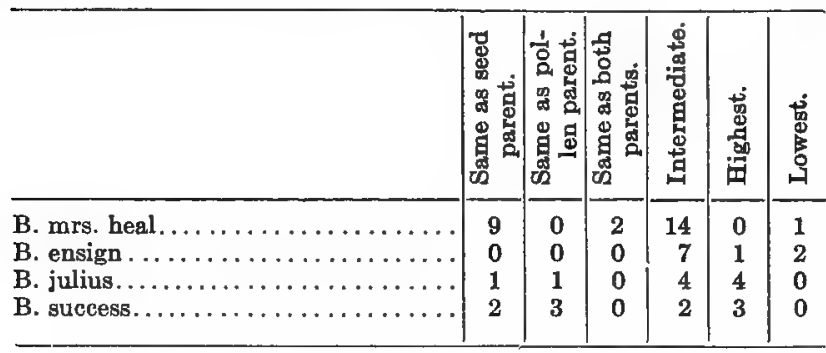

40. Comparisons of the Starches of Richardia albo-Macdlata, R. elliottiana, and R. Mrs. ROOSEVELT.

In the histologic characteristics, polariscopic figures, reactions with selenite, reactions with iodine and qualitative reactions with the various chemical reagents the starches of the parents while exhibiting certain properties in common also show certain minor peculiarities by which collectively they may be distinguished. The starch of Richardia elliottiana in comparison with that of $R$. albo-maculata is found to differ very little, chiefly in the proportions of different kinds of grains. The hilum is more often fissured, more frequently visible, and shows more often a tendency to eccentricity. The lamellæ are more numerous. The size on the whole tends to be slightly less. The polariscopic, selenite, and qualitative iodine reactions exhibit many slight differences. In the qualitative reactions with chloral hydrate, chromic acid, hydrochloric acid, potassium hydroxide, and sodium salicylate there are a number of points of differentiation, mostly apparently of a very minor character. The starch of the hybrid is in form, character of the hilium, lamellæ, size, polariscopic and selenite reactions, iodine reactions and qualitative chemical reactions slightly closer to $R$. albo-maculata than to the other parent, but such differences as are observed are it seems of a decidedly minor character. These starches are not well adapted for differential study not only because of their very close similarities in their properties, but also because of their small size and the differences in gelatinizability of the inner and outer parts, the former gelatinizing with comparative rapidity and the latter with comparative difficulty, excepting in the rapid reactions. On this account only few reactions were studied.

Reaction-intensities Expressed by Light, Color, and TemperaPolarization:

R. albo-maculata, moderate to high, value 70 .

R. elliottiana, moderate to high, lower than R. albo-maculata, value 65 .

R. mrs. roosevelt; moderate to high, between the parents, value 67 . Iodine:

R. albo-maculata, moderate, value 45.

R. elliottiana, moderate, less than R. albo-maculata, value 40 .

R. mrs. roosevelt, moderate, the same as R. albo-maculata, value 45 . Gentian violet:

R. albo-maculata, light, value 30 .

R. elliottiana, light, slightly deeper than in R. albo-maculata, value 33 .

R. mrs. roosevelt, light, deeper than in either parent, value 35 .
Safranin:

R. albo-maculata, light, value 33.

$\mathrm{R}$. elliottiana, light, slightly deeper than in R. albo-maculata, value 35 .

R. mrs. roosevelt, light, light to moderate, deeper than in the parents, value 38 .

Temperature:

R. albo-maculata, majority at 75 to $76^{\circ}$, all at 77 to $78.5^{\circ}$, mean $77.7^{\circ}$.

R. albo-maculata, majority at 75 to $76^{\circ}$, all at 77 to $78.5^{\circ}$, mean $77.7^{\circ}$.

R. elliottiana, majority at 74 to $75^{\circ}$, all at 76 to $77^{\circ}$, mean $76.5^{\circ}$.

R. mrs. roosevelt, majority at 74 to $76^{\circ}$, all at 76 to $78^{\circ}$, mean $77^{\circ}$.

The reactivities of $R$. albo-maculata are higher than those of the other parent in the polarization and iodine reactions, and lower in the gentian violet, safranin, and temperature reactions. The hybrid in the polarization and temperature reactions is intermediate in value; in the iodine reaction it is the same as in $R$. albo-maculata and higher than in $R$. elliottiana; and in the gentianviolet and safranin reactions the figures are closer to, but in excess of, those of $R$. elliottiana, and beyond the parental extremes.

Table 40 shows the reaction-intensities in percentages of total starch gelatinized at definite intervals (minutes) : TABLe A 40 .

\begin{tabular}{|c|c|c|c|c|c|c|c|c|c|c|}
\hline & $\underset{\square}{\mathrm{g}}$ & $\underset{\mathbf{N}}{\dot{\boldsymbol{d}}}$ & 百 & $\underset{*}{\text { 百 }}$ & $\begin{array}{l}\text { g } \\
\text { s }\end{array}$ & $\stackrel{\dot{E}}{\circ}$ & $\underset{\text { g }}{\dot{E}}$ & 㿟 & $\begin{array}{l}\text { g } \\
\text { is }\end{array}$ & 용 \\
\hline \multicolumn{11}{|l|}{ Chloral hydrate: } \\
\hline R. albo-maculata ....... & $\cdots$ & $\cdots$ & 95 & 99 & $\cdots$ & $\cdots$ & $\cdots$ & . & . & . \\
\hline R. elliottiana........ & . & $\cdots$ & 92 & $\cdots$ & 97 & $\cdots$ & $\cdots$ & & $\cdots$ & . \\
\hline R. mrs. roosevelt. . . . . . & . & . & 99 & . & . & . & . & . & . & . \\
\hline \multicolumn{11}{|l|}{ Chromic acid: } \\
\hline R. albo-maculata....... & . & $\cdots$ & $\cdots$ & . & 2 & . & 65 & 96 & 98 & 99 \\
\hline R. elliottiana......... & . & . & $\therefore$ & . & 3 & . & 68 & 97 & 99 & . \\
\hline R. mrs. roosevelt. . . . . . & $\cdots$ & $\cdots$ & . & $\cdots$ & 6 & $\cdots$ & 67 & 97 & 99 & . \\
\hline \multicolumn{11}{|l|}{ Pyrogallic acid: } \\
\hline R. albo-maculata ... & $\cdots$ & $\cdots$ & $\cdots$ & . & 4 & . & 5 & 9 & 10 & 11 \\
\hline R. elliottiana......... & . & . & . & . & 2 & . & 3 & 5 & 7 & 9 \\
\hline R. mrs. roosevelt . . . . . & . & . & . & . & 3 & . & 4 & 6 & 7 & 8 \\
\hline \multicolumn{11}{|l|}{ Nitric acid: } \\
\hline R. albo-maculata... & $\cdots$ & $\cdots$ & . & . & 6 & . & 22 & 28 & 40 & 48 \\
\hline R. elliottiana.......... & $\cdots$ & . & . & . & 4 & . & 16 & 20 & 30 & 36 \\
\hline R. mrs. roosevelt . . . . . . & . & . & . & . & 6 & $\cdots$ & 16 & 22 & 36 & 41 \\
\hline \multicolumn{11}{|l|}{ Sulphuric acid: } \\
\hline R. albo-maculata ...... & . & $\cdots$ & . & . & 97 & 99 & - & $\cdots$ & $\cdots$ & . \\
\hline R. elliottiana. . . . . . & . & $\cdots$ & . & . & 98 & 99 & . & . & . & . \\
\hline R. mrs. roosevelt . . & . & . & . & . & 97 & 99 & . & $\cdots$ & $\cdots$ & . \\
\hline \multicolumn{11}{|l|}{ Hydrochloric acid: } \\
\hline R. albo-maculata.. & $\cdots$ & $\cdots$ & $\cdots$ & $\ldots$ & 18 & $\ldots$ & 35 & 62 & 75 & 82 \\
\hline R. elliottiana...... & $\ldots$ & . & $\cdots$ & & 16 & . & 33 & 55 & 70 & 80 \\
\hline R. mrs, roosevelt. .. & . & . & . & . & 16 & .. & 29 & 32 & 61 & 78 \\
\hline \multicolumn{11}{|l|}{ Potassium hydroxide: } \\
\hline R. albo-maculata... & . & $\cdots$ & . & . & 3 & . & 8 & 10 & 13 & 21 \\
\hline R. elliottiana..... & . & .. & . & . & 8 & . & 13 & 14 & 17 & 23 \\
\hline R. mrs. roosevelt. . & . & $\cdots$ & . & . & 9 & . & 14 & 15 & 25 & 38 \\
\hline \multicolumn{11}{|l|}{ Sodium salicylate: } \\
\hline R. albo-maculata ... & . & $\ldots$ & 92 & . & 99 & . & $\therefore$ & $\cdots$ & $\cdots$ & . \\
\hline R. elliottiana...... & . & . & 91 & $\ldots$ & 99 & . & $\cdots$ & . & $\ldots$ & . \\
\hline R. mrs. roosevelt . . . . . & . & . & 94 & . & 99 & . & $\cdots$ & $\cdots$ & $\cdots$ & \\
\hline
\end{tabular}

Velocity-Reaction Curves.

This section treats of the velocity-reaction curves of the starches of Richardia albo-maculata, $R$. elliottiana, and $R$. mrs. roosevelt. (Charts D 545 to D 55\%.)

There are very few points of interest in the accompanying eight charts. The starches are so nearly alike that but little differences are shown in any of the charts. In the reactions with chloral hydrate, sulphuric acid, and sodium salicylate gelatinization occurs so rapidly that 
such differences as are recorded probably fall within the limits of error of experiment; in those with chromic acid and pyrogallic acid the differences are insignificant; and in those with nitric acid, hydrochloric acid, and potassium hydroxide the differences are not marked, yet sufficient for definite differential purposes. In the latter reactions it will be observed that the relations of the curves of the three starches differ in each-in the nitricacid reaction the starch of $R$. albo-maculata is the most reactive, $R$. elliottiana the least, and the hybrid intermediate; in the hydrochloric-acid reaction the order of reactivity is $R$. albo-maculata, $R$. elliottiana, and hybrid; and in the potassium-hydroxide reaction the order is hybrid, $R$. elliottiana, and $R$. albo-maculata. The greatest interest centers perhaps in the differences in reactivity toward the different reagents, there being represented in the eight charts almost the extremes of reactivities. In the chloral-hydrate, sulphuric-acid, and sodium-salicylate reactions within 5 minutes all three starches are gelatinized; with pyrogallic acid there is very little effect even at the end of 60 minutes; while with chromic acid, nitric acid, hydrochloric acid, and potassium hydroxide there are in-between gradations. It is also of interest to note the different courses of the curves with these four reagents.

\section{Reaction-INTENsities of the HrbRid.}

This section treats of the reaction-intensities of the hybrid as regards sameness, intermediateness, excess, and deficit in relation to the parents. (Table A 40 and Charts D 545 to D 552.)

The reactivities of the hybrid are the same as those of the seed parent in the iodine reaction; the same as those of the pollen parent in none; the same as those of both parents in the reactions with chromic acid, pyrogallic acid, sulphuric acid, and sodium salicylate; intermediate in the polarization, temperature, and nitric acid reactions, in all being mid-intermediate; highest with gentian violet, safranin, chloral hydrate, and potassium hydroxide; and the lowest with hydrochloric acid, it being closer to that of the pollen parent.

The following is a summary of the reaction-intensities: Same as seed parent, 1; same as pollen parent, 0 ; same as both parents, 4 ; intermediate, 3 ; highest, 4; lowest, 1.

It is interesting to note that while in one reaction there is sameness in relation to the seed parent, there is not in any reaction sameness to the pollen parent, although in 5 reactions out of the 13 the inclination is to the pollen parent and in only the one referred to is it to the seed parent. Tendencies to mid-intermediateness, to highest reactivity, and to sameness as both parents are quite apparent.

\section{Composite Curves of the Reaction-intensities.}

This section treats of the composite curves of the reaction-intensities, showing the differentiation of the starches of Richardia albo-maculata, R. elliottiana, and R. mrs. roosevelt. (Chart $\mathrm{E} 40$.)

The most conspicuous features of this chart are:

Marked closeness, almost identity, of all three curves. In fact, such differences as are shown are usually so small as to fall within the limits of error of record. It would perhaps be hazardous to reach a definite diagnosis of one from the other by these curves, yet if taken in connection with the curves showing the reaction-intensities at definite time-intervals differentiation appears to be satisfactory. From these curves one might naturally be led to the belief that we are dealing with varieties of a species and not with two recognized species (even though they might belong to a species subgroup) and a hybrid. From these investigations (which are inconclusive) the parents should be regarded as varieties of a given species. It is of interest to compare these curves with those of the hippeastrums, the parents of which are garden varieties that have come from closely related parentage. The marked excursions of the curves, showing wide variations in the reactive intensities with the different reagents, are very striking.

\section{Comparisons of the Starches of Musa} ARNOLDIANA, M. GILLETII, AND M. HYBRIDA.

In the histologic characteristics, polariscopic figures, reactions with selenite, reactions with iodine, and qualitative reactions with the various chemical reagents the starches of the parents have properties in common in varying degrees of development and also certain individualities, and the starch of the hybrid has properties like those of one or the other or both parents, and also certain individualities; but it is, on the whole, distinctly closer to Musa gilletii than to the other parent. The starch of $M$. gilletii in comparison with that of $M$. arnoldiana has only one of the two types seen in M. arnoldiana, but there are aggregates that are not found in the latter; and there are more numerous elongated forms. The hilum is somewhat more often fissured, and eccentricity is somewhat less in some of the forms. The lamellæ are more often distinct, not so fine, and less numerous. The size is slightly larger. In the polariscopic, selenite, and qualitative iodine reactions there are many differences which seem to be of a minor character. In the qualitative reactions with chloral hydrate, chromic acid, pyrogallic acid, sodium salicylate, and cobalt nitrate there are very many differences, many of which quite definitely individualize one or the other parent. The starch of the hybrid in comparison with the starches of the parents shows in almost every feature a closer relationship to the starch of the pollen parent. It contains the two types of compound grains found in $M$. arnoldiana and the aggregates of the other parent, and there is a type of compound grain that is peculiar to the hybrid. The hilum is more frequently fissured than in either parent. The lamellæ are in character and arrangement more like those of $M$. gilletii, but in number closer to $M$. arnoldiana. In size some of the grains exceed those of the parents. In the polariscopic, selenite, and qualitative iodine reactions there are many differences, but the inclinations of the hybrid are distinctly to $M$. gitletii. In the qualitative chemical reactions the leanings are very definitely to one or the other or both parents, with, on the whole, a distinctly closer relationship to $M$. gilletii, the pollen parent.

Reaction-intensities Expressed by Light, Color, and Tempera-
ture Reactions.

Polarization:

M. arnoldiana, low to high, value 40 .

M. gilletii, low to high, higher than in M. arnoldiana, value 45 .

M. hybrida, low to high, higher than in either parent, value 50. 
Iodine:

M. arnoldiana, moderate, value 55.

M. gilletii, moderate, somewhat less than in M. arnoldiana, value 50 .

M. hybrida, moderate, the same as in M. gilletii, value 50 .

Gentian violet:

M. arnoldiana, light to deep, value 50 .

M. gilletii, light to deep, somewhat less, value 45 .

M. hybrida, light to deep, the same as in M. gilletii, value 45.

Safranin:

M. arnoldiana, moderate to deep, value 60 .

M. gilletii, moderate to deep, less than in M. arnoldiana, value 50 .

M. hybrida, moderate to deep, the same as in M. gilletii, value 50 . Temperature:

M. arnoldiana, majority at 60 to $61^{\circ}$, all at 64.5 to $65.8^{\circ}$, mean $65^{\circ}$. M. gilletii, majority at 64 to $66.5^{\circ}$, all at 67.5 to $69^{\circ}$, mean $68.4^{\circ}$.

M. hybrida, majority at 65.2 to $67^{\circ}$, all at 69 to $70^{\circ}$, mean $69.75^{\circ}$.

In not one of the five reactions are the figures for the two parents the same. The polarization reaction of $M$. gilletii is higher, and those with iodine, safranin, gentian violet, and temperature are lower than those of the other parent. The hybrid has the same degree of reactivity as $M$. gilletii in the reactions with iodine, gentian violet, and safranin; higher reactivity than either parent in that with polarization; and a lower reactivity in that with temperature. In all of these reactions the hybrid is closer to $M$. gilletii than to the other parent. In no instance is there intermediateness, and in two records the reactions are in excess or deficit of the parental extremes.

Table A 41 shows the reaction-intensities in percentages of total starch gelatinized at definite intervals (seconds and minutes) :

Table A 41.

\begin{tabular}{|c|c|c|c|c|c|c|c|c|c|c|c|c|}
\hline & $\underset{\rightarrow-1}{20}$ & 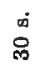 & $\underset{n-1}{g}$ & ฉ & 目 & घ & a & 뭉 & 里 & 임 & 帛 & 8 \\
\hline \multicolumn{13}{|l|}{ Chloral hydrate: } \\
\hline M. arnoldiana... & . & $\cdots$ & $\cdots$ & $\cdots$ & $\ldots$ & $\cdots$ & 55 & $\cdots$ & 90 & 99. & & \\
\hline M. & $\cdots$ & . & $\cdots$ & . & . & . & 30 & $\cdots$ & 69 & 78 & 88 & 95 \\
\hline & . . & . & . & . & $\ldots$ & . & 28 & . & 58 & 70 & 74 & 77 \\
\hline \multicolumn{13}{|l|}{ Chromic acid: } \\
\hline M. arnoldiana.. . & $\cdots$ & $\cdots$ & $\cdots$ & $\cdots$ & $\cdots$ & $\cdots$ & 95 & $\cdots$ & 100 & $\cdots$ & $\cdots$ & $\cdots$ \\
\hline $\mathbf{M}$. & $\cdots$ & $\cdots$ & . & $\cdots$ & $\cdots$ & $\cdots$ & 70 & . & 90 & 99 & $\cdots$ & $\cdots$ \\
\hline \multirow{2}{*}{\multicolumn{13}{|c|}{ Pyrogallic acid: }} \\
\hline & & & & & & & & & & & & \\
\hline M. arnoldiana.. & $\cdots$ & $\cdots$ & $\cdots$ & $\cdots$ & $\cdots$ & $\because$ & 86 & $\cdots$ & 95 & 99 & & \\
\hline M. $\mathrm{g}$ & $\cdots$ & - & . & $\cdots$ & $\cdots$ & - & 11 & . & 54 & 73 & 81 & 84 \\
\hline M. hybride & $\cdots$ & $\cdots$ & . & . & . & . & 14 & . & 55 & 73 & . & 79 \\
\hline \multicolumn{13}{|l|}{ Nitric acid: } \\
\hline M. ar & 98 & $\cdots$ & $\cdots$ & $\cdots$ & $\cdots$ & $\cdots$ & & $\cdots$ & & $\ddot{\therefore}$ & $\cdots$ & $\cdots$ \\
\hline M. & 67 & $\cdots$ & - & $\cdots$ & . & . & 90 & $\cdots$ & 93 & 96 & . & $\cdots$ \\
\hline M. hybrida. .... & 47 & - & $\cdots$ & . & . & . & 90 & $\cdots$ & 93 & 95 & . & . \\
\hline \multicolumn{13}{|l|}{ Sulphuric acid: } \\
\hline M. arnoldiana. . & $\cdots$ & 95 & $\because$ & $\cdots$ & $\cdots$ & $*$ & $\cdots$ & $\cdots$ & $\cdots$ & $\cdots$ & $\cdots$ & $\cdots$ \\
\hline & $\cdots$ & 75 & 96 & $\cdots$ & $\cdots$ & - & $\cdots$ & $\cdots$ & $\ldots$ & . & $\cdots$ & 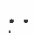 \\
\hline M. hybrida. . . . & . & 48 & 95 & . & $\cdots$ & $\cdots$ & $\cdots$ & $\cdots$ & $\cdots$ & $\cdots$ & $\cdots$ & $\cdots$ \\
\hline \multicolumn{13}{|l|}{ Hydrochloric acid: } \\
\hline M. arnoldiana.. & $\cdots$ & 99 & $\ddot{0}$ & $\cdots$ & $\cdots$ & $\cdots$ & $\cdots$ & $\cdots$ & & $\cdots$ & $\cdots$ & $\cdots$ \\
\hline M. & & 75 & 96 & $\cdots$ & $\ldots$ & & & . & $\ldots$ & $\ldots$ & $\cdots$ & . \\
\hline M. hybrida. ... . & . & 84 & 89 & . & 98 & . & 99 & . & $\cdots$ & $\cdots$ & . & . \\
\hline \multicolumn{13}{|l|}{$\begin{array}{l}\text { Potassium hydrox- } \\
\text { ide: }\end{array}$} \\
\hline M. arnoldiana.. & 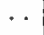 & 99 & $\ddot{0}$ & $\cdots$ & $\cdots$ & $\cdots$ & $\cdots$ & . & & & $\cdots$ & . \\
\hline M. & & 85 & 93 & $\cdots$ & $\cdots$ & $\cdots$ & $\cdots$ & . & $\cdots$ & $\cdots$ & $\cdots$ & . \\
\hline M. hybrida . .... & . & 91 & 95 & $\cdots$ & . & . & $\cdots$ & $\cdots$ & & $\cdots$ & . & . \\
\hline \multicolumn{13}{|l|}{ Potassium iodide: } \\
\hline M. arnoldiana.. & $\cdots$ & $\cdots$ & 98 & $\cdots$ & $\ddot{0}$ & $\cdots$ & $\ddot{0}$ & $\cdots$ & & $\cdots$ & $\cdots$ & . \\
\hline M. g & - & . & 75 & $\cdots$ & 85 & . & 87 & . & 96 & $\cdots$ & $\cdots$ & $\cdots$ \\
\hline M. hybrida. ... . & $\cdots$ & - & 62 & $\cdots$ & 78 & $\cdots$ & 84 & $\cdots$ & 95 & $\cdots$ & $\cdots$ & . \\
\hline \multicolumn{13}{|l|}{$\begin{array}{l}\text { Potassium aulpho- } \\
\text { cyanate: }\end{array}$} \\
\hline & . & 96 & 99 & $\cdots$ & $\cdots$ & . & & $\cdots$ & & & $\cdots$ & \\
\hline M. gille & . & 14 & 87 & . & 97 & - & $\ddot{x}$ & . & 99 & $\cdots$ & $\cdots$ & . \\
\hline M. hybr & . & 12 & 81 & - & $\cdots$ & . & 95 & . & 99 & & $\cdots$ & . \\
\hline
\end{tabular}

TABle A 41.-Continued.

\begin{tabular}{|c|c|c|c|c|c|c|c|c|c|c|c|c|}
\hline & $\begin{array}{l}\infty \\
12 \\
-1\end{array}$ & 施 & $\underset{-}{\dot{a}}$ & $\begin{array}{l}\text { घं } \\
\text { N }\end{array}$ & $\underset{\infty}{\text { 白 }}$ & $\begin{array}{l}\dot{g} \\
\vec{H}\end{array}$ & وص & $\begin{array}{l}\text { 日 } \\
0\end{array}$ & $\begin{array}{l}\text { व } \\
\stackrel{2}{-1}\end{array}$ & $\begin{array}{l}\text { घं } \\
\text { ᄋ }\end{array}$ & $\begin{array}{c}\dot{1} \\
40\end{array}$ & $\begin{array}{l}\text { 며 } \\
8\end{array}$ \\
\hline \multicolumn{13}{|l|}{ Potassiumsulphide: } \\
\hline M. arnoldiana.. . & . & 99 & 1 & . & . & . & & . & & . & $\cdots$ & . \\
\hline M. gilletii . . . . . . & . & 70 & $\ldots$ & . & . & . & 95 & $\cdots$ & 97 & . & . & . \\
\hline M. hybrida. .... & $\cdots$ & 64 & . & $\ldots$ & $\cdots$ & . & 92 & . & 95 & . & . & . \\
\hline \multicolumn{13}{|l|}{ Sodium hydroxide: } \\
\hline M. arnoldiana.. & . & 99 & . & $\cdots$ & . & . & & $\cdots$ & & $\cdots$ & $\cdots$ & . \\
\hline M. gilletii ..... . & . & 65 & 84 & . . & . & . & 95 & . & 98 & $\cdots$ & . & $\ldots$ \\
\hline M. hybrida. .... & $\ldots$ & 36 & 68 & $\ldots$ & $\ldots$ & . & 93 & . & 97 & . & . & . \\
\hline \multicolumn{13}{|l|}{ Sodium sulphide: } \\
\hline M. arnoldiana.. & . & 96 & 99 & . & . & . & . & $\cdots$ & $\cdots$ & & $\cdots$ & . \\
\hline M. gilletii . . . . . & . & 18 & 42 & - & . & . & 81 & $\cdots$ & 89 & 95 & $\cdots$ & .. \\
\hline M. hybrida. . . . . & . & 8 & 38 & $\cdots$ & . & . & 70 & . & 87 & 95 & . & . \\
\hline \multicolumn{13}{|l|}{ Sodium salicylate: } \\
\hline M. arnoldiana.. . & $\ldots$ & $\ldots$ & $\cdots$ & . & 75 & . & 95 & 99 & & $\ldots$ & $\cdots$ & $\cdots$ \\
\hline M. gilletii . . . . . & . & . & $\cdots$ & . & 74 & . & 85 & 95 & 99 & . & $\ldots$ & . \\
\hline M. hybrida. . . . & $\ldots$ & . & . & $\ldots$ & 62 & . & 73 & 90 & 98 & $\ldots$ & . & . \\
\hline \multicolumn{13}{|l|}{ Calcium nitrate: } \\
\hline M. arnoldiana.. . & $\ldots$ & . & 95 & . & $\ldots$ & . & 99 & . & & & - & $\cdots$ \\
\hline M. gilletii . .... & . & $\cdots$ & 10 & . & . & $\ldots$ & 80 & . & 86 & 90 & $\cdots$ & 93 \\
\hline M. hybrida. . . . & $\cdots$ & $\cdots$ & 8 & $\cdots$ & . & . & 58 & & 74 & 80 & 86 & 90 \\
\hline \multicolumn{13}{|l|}{ Uranium nitrate: } \\
\hline M. arnoldiana.. & $\cdots$ & . & 84 & $\cdots$ & 99 & $\cdots$ & $\cdots$ & . & & 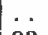 & $\therefore$ & $\because$ \\
\hline M. gilletii . .... . & . & . & 10 & . & 77 & . & 80 & $\cdots$ & 90 & 93 & 95 & 97 \\
\hline M. hybrida. . . . & . & . & 8 & . & 54 & . & 73 & . & 83 & 87 & 93 & 95 \\
\hline \multicolumn{13}{|l|}{ Strontium nitrate: } \\
\hline M. arnoldiana.. & . & . & 95 & . & 99 & . & $\ldots$ & $\cdots$ & $\cdots$ & . & $\cdots$ & $\cdots$ \\
\hline M. gilletii . .... . & . & . & 14 & $\cdots$ & 83 & . & 87 & . & 95 & 97 & . & $\cdots$ \\
\hline M. hybrida. .... & $\ldots$ & $\cdots$ & 15 & . & 72 & . & 76 & . & 92 & 95 & . & . \\
\hline \multicolumn{13}{|l|}{ Cobalt nitrate: } \\
\hline M. arnoldiana.. & $\ldots$ & . & $\ldots$ & $\ldots$ & & $\ldots$ & 98 & $\cdots$ & 99 & & & $\cdots$ \\
\hline M. gilletii . .... & . & . & $\ldots$ & . & . & $\ldots$ & 14 & .. & 28 & 38 & 48 & 52 \\
\hline M. hybrida. . . . & $\ldots$ & $\cdots$ & $\ldots$ & . & . & . & 10 & . & 21 & 30 & 40 & 44 \\
\hline \multicolumn{13}{|l|}{ Copper nitrate: } \\
\hline M. arnoldiana... & . & $\cdots$ & 99 & . & - & $\cdots$ & $\ldots$ & . & & . & . & .. \\
\hline M. gilletii..... & . & . & 16 & . & . & . & 72 & & 95 & 98 & . & $\cdots$ \\
\hline M. hybrida. . . . & . & . & 8 & . & . & . & 59 & . & 88 & 90 & . & 90 \\
\hline \multicolumn{13}{|l|}{ Cupric chloride: } \\
\hline M. arnoldiana... & $\ldots$ & . & 87 & . & 99 & $\ldots$ & $\ldots$ & . & & $\cdots$ & $\cdots$ & $\cdots$ \\
\hline M. gilletii..... & $\ldots$ & $\cdots$ & 10 & . & 55 & . & 60 & & 79 & 84 & 85 & 89 \\
\hline M. hybrida. . . . . & . & $\ldots$ & 5 & . & 50 & $\ldots$ & 55 & . & 70 & 80 & 82 & 85 \\
\hline \multicolumn{13}{|l|}{ Barium chloride: } \\
\hline M. arnoldiana.. & . & $\cdots$ & $\cdots$ & $\cdots$ & . & . & 74 & & 95 & 98 & $\cdots$ & \\
\hline M. gilletii . . . . . & . & . & .. & . & . & . & 5 & & 51 & 56 & . & 56 \\
\hline M. hybrida. . . . & . & . & . & . & $\ldots$ & . & 5 & & 26 & 40 & . & 42 \\
\hline \multicolumn{13}{|l|}{ Mercuric chloride; } \\
\hline M. arnoldiana.. & . & & 68 & . & 97 & $\cdots$ & 99 & . & & $\cdots$ & $\cdots$ & $\cdots$ \\
\hline M. gilletii . . . . . & . & $\cdots$ & 10 & . & 39 & . & 54 & . & 61 & 71 & 75 & 79 \\
\hline M. hybrida. & . & . & 3 & . & 31 & .. & 48 & . & 55 & 62 & 68 & 72 \\
\hline
\end{tabular}

Velocity-Reaction CuRves.

This section treats of the velocity-reaction curves of the starches of Musa arnoldiana, M. gilletii, and $M$. $h y$ brida, showing the quantitative differences in the behavior towards different reagents at definite time-intervals. (Charts D 553 to D 573.)

Among the conspicuous features of these charts are:

(1) The high to very high reactivity of the starch of Musa arnoldiana throughout all of the reactions, in only one of which is the reaction high. In not less than 11 reactions out of the 20 at least 95 per cent of the total starch was gelatinized within 2 minutes, and in the others with the exception of chloral hydrate, pyrogallic acid, and barium chloride a similar intensity of reaction occurred in 5 minutes or less. The maximum time (99 per cent in 30 minutes) was in the chloral-hydrate reactions. In many of the reactions not only was the reactivity of this starch greater than in case of the other parent and the hybrid; but sometimes also markedly higher.

(2) The marked tendency for the curves of $M$. gilletii and $M$. hybrida to run close together, and in many in- 
stances to be well separated from the curve of $M$. arnoldiana. The tendency for the hybrid reactions throughout (excepting those with nitric acid, sulphuric acid, and potassium hydroxide which are so rapid that no satisfactory differentiation can be made, and in that with pyrogallic acid, in which the curve is practically identical with that of the pollen parent), to be lower than that in either parent; and also to show a distinctly closer relationship to $M$. gilletii than to $M$. arnoldiana.

(3) The considerable differences in the interrelations of the three curves: Thus, in the reactions with chloral hydrate, chromic acid, sodium salicylate, calcium nitrate, uranium nitrate, strontium nitrate, and barium chloride the curves are quite evenly separated, the curve of $M$. gilletii in each chart being between the curves of $M$. arnoldiana and the hybrid. In the reactions with pyrogallic acid, nitric acid, potassium iodide, potassium sulphocyanate, potassium sulphide, sodium hydroxide, sodium sulphide, cobalt nitrate, copper nitrate, cupric chloride, and mercuric chloride there is an obvious pairing of the curves of $M$. gilletii and the hybrid, the curves being to more or less marked degrees separated from the curve of $M$. arnoldiana, and from each other, excepting in the latter in the pyrogallicacid reactions, where the curves of $M$. gitletii and the hybrid are practically identical. In the reactions with nitric acid, potassium iodide, and sodium hydroxide the only important differences are noted at the very beginning of gelatinization. In the other reactions, with the exceptions noted, while the curves tend in general to run closely, there are sufficient differences to permit of diagnosis.

(4) An early period of resistance is noted in very few of the reactions. In fact, there is generally a marked tendency for an immediate high to very high degree of reactivity which may be followed by a progressively lessening. An early period of resistance is seen in the reactions of chromic acid with $M$. hybrida, of pyrogallic acid, and, particularly, of barium chloride, with both $M$. gilletii and $M$. hybrida.

(5) The earliest period during the 60 minutes of observation at which the curves are best separated for the differentiation of the three starches is variable with the different reagents. In case of the very rapid reactions, including those with nitric acid, sulphuric acid, hydrochloric acid, potassium hydroxide, potassium iodide, potassium sulphocyanate, potassium sulphide, and sodium hydroxide, the period is noted within the first minute of the reactions; in those with chromic acid, pyrogallic acid, sodium sulphide, sodium salicylate, calcium nitrate, uranium nitrate, strontium nitrate, calcium nitrate, copper nitrate, cupric chloride, and mercuric chloride within 5 minutes; and in those with chloral hydrate and barium chloride within 15 minutes. From this data the best period for the differentiation of members of this genus would be, perhaps, on the whole, 5 minutes after the beginning of the reaction; or better, to use in most cases weaker reagents.

\section{Reaction-Intensities of THE Hybrid.}

This section treats of the reaction-intensities of the hybrid as regards sameness, intermediateness, excess, and deficit in relation to the parents. (Table A 41 and Charts D 553 to D 573.)
The reactivities of the hybrid are the same as those of the seed parent in no reaction; the same as those of the pollen parent in the reactions with iodine, gentian violet, safranin, and pyrogallic acid; the same as those of both parents in none; intermediate with hydrochloric acid, and potassium hydroxide, being closer to the pollen parent in one and mid-intermediate in the other; highest in none; and the lowest with polarization, temperature, chloral hydrate, chromic acid, nitric acid, sulphuric acid, potassium iodide, potassium sulphocyanate, potassium sulphide, sodium hydroxide, sodium sulphide, sodium salicylate, calcium nitrate, uranium nitrate, strontium nitrate, cobalt nitrate, copper nitrate, cupric chloride, barium chloride, and mercuric chloride, in all of which being closer to the pollen parent.

The following is a summary of the reaction-intensities: Same as seed parent, 0 ; same as pollen parent, 4; same as both parents, 0 ; intermediate, 2 ; highest, 0 ; lowest, 20 .

Lowest reactivity of the three starches and sameness and inclination to the pollen parent are two features that stand out with marked conspicuousness. The pollen parent seems to have been pre-eminent in determining the characters of the starch of the hybrid, inasmuch as in 25 of the 26 reactions this parent bears the closer relationship to the hybrid, while in the remaining reaction there is mid-intermediateness, but of doubtful valuation.

\section{Composite CuRves of the Reaction-intensities.}

This section treats of the composite curves of the reaction-intensities, showing the differentiation of the starches of Musa arnoldiana, $M$. gilletii, and $M$. hybrida. (Chart E 41.)

The most conspicuous features of the chart are: The general correspondence in the ups and downs of the curves, excepting in the case of $M$. arnoldiana in many reactions which occur so rapidly that differences are not satisfactorily demonstrated. The three curves from the polarization to the sulphuric acid reactions are in close accord, but from the latter on to the sodium-sulphide reaction the curve of $M$. arnoldiana shows practically no change, and from then on such alterations as are exhibited occur within the 5-minute limit, excepting in the barium-chloride reaction, in which the limit is extended to 15 minutes. With $M$. gilletii and $M$. hybrida, however, the variations from reagent to reagent are commonly well marked. With somewhat weaker reagents the curve of $M$. arnoldiana would in all probability correspond in its variations with the curves of $M$. gilletii and the hybrid. The curve of $M$. arnoldiana is the highest throughout, excepting in the polarization reaction, and in many instances it is much higher than the curve of $M$. gilletii and the hybrid. The curve of $M$. gilletii is higher than the curve of $M$. hybrida in the reaction with temperature, chloral hydrate, hydrochloric acid, potassium sulphocyanate, potassium sulphide, sodium hydroxide, sodium salicylate, uranium nitrate, and strontium nitrate; and the same or nearly the same in all other reactions, excepting with polarization, in which it is lower, the same, or nearly the same. The best reagents in the differentiation of these two starches are chloral hydrate, potassium sulphide, sodium hydroxide, sodium salicylate, uranium nitrate, and strontium nitrate. The very high reactions of $M$. arnoldiana with chromic acid, pyrogallio 
acid, nitric acid, sulphuric acid, hydrochloric acid, potassium hydroxide, potassium iodide, potassium sulphocyanate, potassium sulphide, sodium hydroxide, sodium sulphide, sodium salicylate, calcium nitrate, uranium nitrate, strontium nitrate, cobalt nitrate, copper nitrate, cupric chloride, barium chloride, and mercuric chloride; the high reactions with safranin and chloral hydrate; the moderate reactions with polarization, iodine, gentian violet, and temperature; and the absence of any low or very low reactivities. The very high reactivities of $M$. gilletii with sulphuric acid, hydrochloric acid, potassium hydroxide, potassium iodide, potassium sulphocyanate, potassium sulphide, sodium hydroxide, sodium salicylate, and strontium nitrate; the high reactions with chromic acid, nitric acid, sodium sulphide, and uranium nitrate; the moderate reactivities in the polarization, iodine, gentian violet, and safranin, temperature, chloral hydrate, calcium nitrate, and copper nitrate reactions; the low reactions with pyrogallic acid, cobalt nitrate, cupric chloride, barium chloride, and mercuric chloride; and the very low reaction with cobalt nitrate. The very high reactivities of $M$. hybrida with sulphuric acid and the other reagents noted under $M$. gilletii, excepting strontium nitrate; the high reactions with chromic acid, nitric acid, sodium sulphide, and strontium nitrate; the moderate reactions with polarization, iodine, gentian violet, safranin, temperature, calcium nitrate, uranium nitrate, and copper nitrate; the low reactions with chloral hydrate, pyrogallic acid, cupric chloride, and mercuric chloride; and the very low reactions with cobalt nitrate and barium chloride.

Following is a summary of the reaction-intensities:

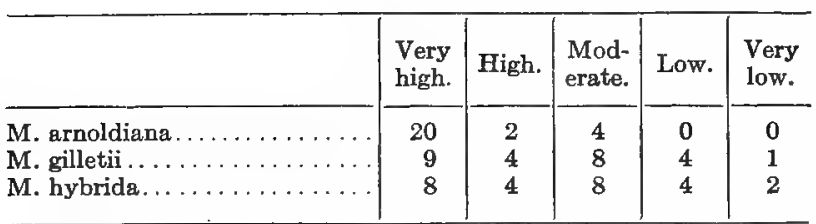

\section{Comparisons of the Starches of Phaius} gRANDIFOLIUS, P. WALLICHII, AND P. HYBRIDUS.

In the histologic characteristics, polariscopic figures, reactions with selenite, qualitative reactions with iodine, and qualitative reactions with the various chemical reagents, the parents and hybrid exhibit properties in common in varying degrees of development, and also certain individualities by which collectively they can be identified. The starch of Phaius wallichii in comparison with that of $P$. grandifolius shows larger proportions of aggregates and compound grains; more frequent irregúlarities, but given forms of irregularity vary in frequency; and the forms are of more varied types. The hilum is more often distinct, slightly more refractive, and rarely fissured; a longitudinal slit-like cavity at the hilum and a deflected oblique fissure are more frequently noted; eccentricity is more variable and less. The lamellæ exhibit some differences in distribution and form; secondary sets are more numerous; the number is about the same. The size of the larger grains is longer and less wide; that of the common-sized grains about the same. In the polariscopic, selenite, and qualitative iodine reactions there are various differences. In qualitative reactions with chloral hydrate, chromic acid, pyrogallic acid, hydrochloric acid, potassium hydroxide, potassium iodide, potassium sulphocyanate, potassium sulphide, sodium hydroxide, sodium sulphide, and sodium salicylate there are very many points of difference which seem to be wholly of a minor character. The starch of the hybrid in comparison with the starches of the parents contains larger proportions of aggregates and compound grains than in either parent; irregularities are less frequent; and there are more grains of a slender type than in $P$. grandifolius, but less than in $P$. wallichii. The hilum is more refractive and more frequently demonstrable than in either parent; a slit-like cavity at the hilum is as frequently apparent as in $P$. grandifolius, but less frequently than in $P$. wallichii; fissuration is slightly more varied and more frequent than in either parent; clefts in the form of a soaring-bird figure are seen, this form not being observed in the parents; eccentricity is the same as in $P$. wallichii. The lamellæ of the primary sets are coarser than in the parents; a refractive border at the proximal and lateral margins is less frequent, and it is of the same width as in P. grandifolius, but less broad as a rule than in $P$. wallichii. Secondary sets of lamellæ are somewhat more frequent, often larger and commonly located as in $P$. grandifolius; but less numerous and less varied in location than in $P$. wallichii; and the number is about the same as in the parents. The size is closer to that of $P$. grandifolius. In the polarization and selenite reactions there are many inclinations to one or the other parent, but on the whole to $P$. grandifolius; while in the qualitative iodine reactions the leanings are on the whole to $P$. wallichii. In the qualitative chemical reactions the peculiarities of one or the other or both parents are very well manifested, but in each the reactions are on the whole closer to those of $P$. grandifolius.

Reaction-intensities Expressed by Light, Color, and Temperature Reactions.

Polarization:

P. grandifolius, high to very high, value 85 .

$P$. wallichii, high, lower than in P. grandifolius, value 80 .

P. hybridus, high to very high, slightly higher than in P. grandifolius, value 87 . Iodine:

P. grandifolius, moderate, value 50 .

P. wallichii, moderate, lighter than in P. grandifolius, value 40 .

P. hybridus, moderate, intermediate between the parents, but nearer to $\mathrm{P}$. wallichii, value 43 .

Gentian violet:

P. grandifolius, moderate to deep, value 57 .

P. wallichii, light to moderate, lighter than in P. grandifolius, value 50 .

P. hybridus, moderate to deep, deeper than either parent, value 60 . Safranin:

P. grandifolius, moderate to deep, value 60

$\mathbf{P}$. wallichii, light to moderate, lighter than in $\mathbf{P}$. grandifolius, value 55 .

P. hybridus, moderately deep to deep, deeper than in either parent, value 65 .

Temperature:

P. grandifolius, in the majority at 65 to $66^{\circ}$, in all but rare grains at 68 to $69^{\circ}$, mean 68.5 .

P. wallichii, in the majority at 64 to $65^{\circ}$, in all but rare grains at 67 to $68^{\circ}$, mean $67.5^{\circ}$.

P. hybridus, in the majority at 64 to $66^{\circ}$, in all but rare grains at 66 to $68^{\circ}$, mean $67^{\circ}$.

In the reactions with polarization, iodine, gentian violet, and safranin $P$. grandifolius exhibits higher reactivities than the other parent, but in the temperature 
TABLE A 42.

\begin{tabular}{|c|c|c|c|c|c|c|c|c|c|c|}
\hline & घี & $\underset{g}{\text { ล }}$ & घं & $\begin{array}{l}\dot{\xi} \\
+1\end{array}$ & ถ⿱ & $\begin{array}{l}\text { g } \\
\stackrel{-}{O}\end{array}$ & 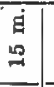 & $\begin{array}{l}\text { घं } \\
\text { Оे }\end{array}$ & 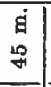 & $\begin{array}{l}\dot{8} \\
8\end{array}$ \\
\hline \multicolumn{11}{|l|}{ Chloral hydrate: } \\
\hline P. grandifolius. & $\cdots$ & $\cdots$ & 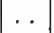 & $\cdots$ & 30 & . & 50 & 65 & 79 & 80 \\
\hline P. & & & . & & 27 & . & 48 & 61 & 67 & 67 \\
\hline P. b & .. & .. & . & . & 21 & . & 44 & 56 & 66 & \\
\hline \multicolumn{11}{|l|}{ Chromic acid: } \\
\hline P. $\mathrm{gr}$ & . & $\cdots$ & $\cdots$ & & 30 & . & 70 & 99 & & \\
\hline P. V & .. & . & .. & . & 67 & . & 97 & 99 & . & . \\
\hline P. $\mathrm{h}$ & $\cdots$ & . & . & . & 44 & . & 87 & 99 & . & $\cdots$ \\
\hline Pyrogal & & & & & & & & & & \\
\hline $\begin{array}{l}\text { P. gra } \\
\text { P. wal }\end{array}$ & $\because$ & . & $\because$ & . & $\begin{array}{r}6 \\
63\end{array}$ & . & $\begin{array}{l}34 \\
80\end{array}$ & $\begin{array}{l}50 \\
85\end{array}$ & $\begin{array}{l}58 \\
91\end{array}$ & $\begin{array}{l}68 \\
94\end{array}$ \\
\hline P. h & . & . & . & .. & 8 & . & 62 & 70 & 77 & 84 \\
\hline Nitric a & & & & & & & & & & \\
\hline P. g & 72 & . & . . & & 95 & 99 & & $\cdots$ & . & \\
\hline P. V & 90 & . & .. & . & 99 & . & - & & . & $\cdots$ \\
\hline P. h & 78 & . & . & .. & 99 & .. & . & . & . & .. \\
\hline Sulphur & & & & & & & & & & \\
\hline & 93 & 98 & 100 & & $\cdots$ & . & & . & .. & \\
\hline P. $v$ & 96 & 99 & 100 & .. & . & . & & . & . & \\
\hline P. 1 & 92 & 99 & 100 & .. & . & .. & . & .. & .. & \\
\hline Hydroch & & & & & & & & & & \\
\hline P. & 96 & 99 & $\cdots$ & . & - & . & 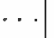 & . & . & \\
\hline P. & 99 & $\cdots$ & $\cdots$ & . & $\cdots$ & $\cdots$ & . & . & . & $\cdots$ \\
\hline P. b & 99 & .. & . & .. & .. & . & . & . & . & $\cdots$ \\
\hline Potassiu & & & & & & & & & & \\
\hline P. & 99 & . & $\cdots$ & . & . & . & . & . & . & \\
\hline P. & 100 & $\ldots$ & . & . . & $\ldots$ & . & & .. & .. & \\
\hline P. 1 & 99 & $\ldots$ & .. & . & . & . & . & . & . & \\
\hline Potass & & & & & & & & & & \\
\hline P. & - & $\cdots$ & $\cdots$ & . & 68 & . & 90 & 95 & 97 & 99 \\
\hline P. $v$ & .. & $\ldots$ & .. & .. & 90 & .. & 95 & 98 & 99 & \\
\hline P. h & . & .. & $\cdots$ & . & 82 & . & 92 & 95 & 98 & 99 \\
\hline hocyanate: & & & & & & & & & & \\
\hline P. & . & $\cdots$ & .. & .. & 97 & . & 99 & $\cdots$ & $\cdots$ & \\
\hline P. V & $\cdots$ & . & . & . & 99 & .. & & . & . & .. \\
\hline P. h & $\cdots$ & . & . & . & 97 & . & 99 & . & . & \\
\hline Potassi & & & & & & & & & & \\
\hline P. g & $\cdots$ & 99 & . & $\cdots$ & $\cdots$ & . & & . & . & \\
\hline P. & . & 99 & .. & . & & . & $\cdots$ & . & . & . \\
\hline P. & . & 95 & . . & .. & 99 & $\ldots$ & & . & $\ldots$ & . \\
\hline Sodium & & & & & & & & & & \\
\hline P. g & $\cdots$ & & 99 & .. & & . & & $\cdots$ & $\cdots$ & \\
\hline P. & . . & 92 & $\ldots$ & . & 97 & . & 99 & . & . & \\
\hline & $\ldots$ & 84 & $\ldots$ & $\ldots$ & 95 & . & 99 & $\ldots$ & $\ldots$ & \\
\hline Sodiux & & & & & & & & & & \\
\hline P. & . & 84 & $\ldots$ & . & 95 & . . & 99 & . & . & \\
\hline P. & . & 92 & . & . & 96 & . & 99 & . & $\cdots$ & . \\
\hline P. 1 & .. & 90 & . & . & 95 & . & 99 & . & . & \\
\hline Sodiur & & & & & & & & & & \\
\hline P. & . & .. & . & . & 39 & - & 84 & 99 & . & \\
\hline P. & . & . & . & .. & 54 & . & 97 & 99 & . & . \\
\hline P. 1 & . & . & $\ldots$ & .. & 54 & . . & 96 & 98 & .. & .. \\
\hline Calciv & & & & & & & & & & \\
\hline & $\cdots$ & . . & . . & .. & 72 & . & 91 & 97 & 99 & \\
\hline & .. & . & . & .. & 83 & . & 99 & & $\cdots$ & \\
\hline & .. & $\ldots$ & $\ldots$ & .. & 75 & . & 99 & . & .. & .. \\
\hline Urani & & & & & & & & & & \\
\hline P. & .. & . . & . & . & 65 & . & 90 & 95 & 98 & \\
\hline & .. & . & . & . & 90 & . & 98 & 99 & $\ldots$ & . \\
\hline & . & .. & . & . & 68 & . & 95 & 98 & $\cdots$ & \\
\hline Stront & & & & & & & & & & \\
\hline & & 84 & . & . & 95 & . & & & $\cdots$ & \\
\hline & .. & 91 & . & . & 99 & $\cdots$ & & $\cdots$ & .. & .. \\
\hline & .. & 83 & $\ldots$ & .. & 98 & $\ldots$ & 100 & & $\ldots$ & \\
\hline Jobalt & & & & & & & & & & \\
\hline & . & . & . & . & 9 & . . & 22 & 56 & 69 & 72 \\
\hline & .. & $\cdots$ & .. & & 48 & .. & 78 & 87 & 90 & 96 \\
\hline & $\cdots$ & .. & . & . . & 10 & . & 62 & 76 & 82 & 86 \\
\hline Copp & & & & & & & & & & \\
\hline $\mathbf{P}$ & . & .. & . & & 96 & . & 99 & $\ldots$ & $\cdots$ & $\cdots$ \\
\hline & . & . . & . & . & 99 & . & & & . & \\
\hline & . & . . & . . & . & 98 & . & 99 & . & $\cdots$ & \\
\hline Gupric & & & & & & & & & & \\
\hline & . & $\cdots$ & . & . & 51 & . . & 76 & 84 & 87 & \\
\hline & .. & .. & $\ldots$ & .. & 82 & . & 95 & 97 & 98 & 99 \\
\hline & . & . & . & .. & 65 & . & 82 & 92 & 95 & 96 \\
\hline Bariun & & & & & & & & & & \\
\hline P. & . . & . & & & 1 & . & 2 & 3 & & \\
\hline & & . & & & 2 & .. & 8 & 11 & 19 & 25 \\
\hline & .. & $\ldots$ & $\ldots$ & . & 1 & .. & 3 & 5 & 6 & 8 \\
\hline Merc & & & & & & & & & & \\
\hline P. & . & . & . & . & 55 & . . & 74 & 83 & 90 & 90 \\
\hline & . & . & . . & . . & 81 & . & 91 & 95 & 97 & 99 \\
\hline P. hybr & .. & . & . & . & 68 & . & 85 & 90 & 95 & 95 \\
\hline
\end{tabular}

reactions lower activity. The hybrid shows in the reactions with polarization, gentian violet, and safranin higher reactivities than either of the parents; with iodine intermediateness, but nearer to $P$. wallichii; and with temperature practically the same reactivity as that of $P$. wallichii.

Table A 42 shows the reaction-intensities in percentages of total starch gelatinized at definite intervals (minutes).

\section{VELOCITY-íREACTION CURVES.}

This section treats of the velocity-reaction curves of the starches of Phaius grandifolius, $P$. wallichii, and $P$. hybridus, showing the quantitative differences in the behavior toward different reagents at definite time-intervals. (Charts D 574 to D 594.)

Among the conspicuous features of these charts are: The correspondence in the courses and the closeness of all three curves in the several reactions. Owing to the very rapid reactions of the starches with nitric acid, sulphuric acid, hydrochloric acid, potassium hydroxide, potassium sulphocyanate, potassium sulphide, sodium hydroxide, sodium sulphide, strontium nitrate, and copper nitrate (10 out of the 21 chemical reagents), satisfactory studies of the curves can not be made. Omitting these, the curves tend to run very closely excepting in the reactions with pyrogallic acid and copper nitrate, in each of which there is well-marked separation. The curve of $P$. grandifolius is higher than that of the other parent in only the chloral-hydrate reaction, and definitely lower in those of the reactions with chromic acid, pyrogallic acid, potassium iodide, sodium salicylate, calcium nitrate, uranium nitrate, cobalt nitrate, cupric chloride, barium chloride, and mercuric chloride. The curves of the hybrid vary in the different reactions in their parental relationships. There is a marked tendency to intermediateness, and there is about an equal tendency to excess or deficit of reaction as there is to sameness to one or the other and both parents, and there is about equal inclination to one as to the other parent. In only two of the charts (pyrogallic acid and cobalt nitrate) is there evidence of an early period of resistance followed by a moderate to rapid gelatinization. In both only two of the starches ( $P$. grandifolius and $P$. hybridus) exhibit this feature, but neither to a marked degree. The earliest period of the experiments at which the curves are best separated for differential purposes is with chromic acid, potassium iodide, sodium salicylate, calcium nitrate, uranium nitrate, cupric chloride, and mercuric chloride at 5 minutes; pyrogallic acid and cobalt nitrate at $\mathbf{1 5}$ minutes; chloral hydrate at 45 minutes; and barium chloride at 60 minutes.

\section{REACTION-INTENSITIES OF THE HYBRID.}

This section treats of the reaction-intensities of the hybrid as regards sameness, intermediateness, excess, and deficit in relation to the parents. (Table A 42 and Charts D 574 to D 594.)

The reactivities of the hybrid are the same as those of the seed parent in the strontium-nitrate reaction; the same as those of the pollen parent in the reactions with temperature, sodium sulphide, and sodium salicylate; the same as those of both parents with sulphuric acid, hydrochloric acid, potassium hydroxide, potassium sulphocyanate, and copper nitrate, in most all being too fast for satisfactory differentiation; intermediate with iodine, chromic acid, pyrogallic acid, nitric acid, potassium iodide, calcium nitrate, uranium nitrate, cobalt nitrate, cupric chloride, barium chloride, and mercuric chloride (in 4 being closer to the seed parent, in 2 closer to the pollen parent, and in 4 being intermediate); 
highest with polarization, gentian violet, and safranin, in all closer to the seed parent; and lowest with chloral hydrate, potassium sulphide, and sodium hydroxide (in 2 being closer to the pollen parent, and in 1 as close to one as to the other parent).

The following is a summary of the reaction-intensities: Same as seed parent, 1; same as pollen parent, 3; same as both parents, 5 ; intermediate, 11 ; highest, 3 ; lowest, 3 .

In these reactions the parents seem to share about equally their influences in determining the characters of the starch of the hybrid. The tendency to intermediateness is quite marked, and in about one-half of these reactions there is mid-intermediateness. There is a stronger tendency to highest or lowest reactivity than to sameness to one or the other parent.

\section{Composite Corves of the ReaCtion-intensities.}

This section treats of the composite curves of the reaction-intensities, showing the differentiation of the starches of Phaius grandifolius, $P$. wallichii, and $P$. hybridus. (Chart E 42.) are:

Among the most conspicuous features of this chart

The very close correspondence in the rises and falls of the curves and in most of the reactions the closeness of the curves to one another, suggesting closely related members of the same genus. The curve of Phaius grandifolius is higher than the curve of the other parent $P$. wallichii in the reactions with polarization, iodine, gentian violet, safranin, chloral hydrate, and sodium hydroxide; lower with temperature, chromic acid, pyrogallic acid, potassium iodide, sodium salicylate, calcium nitrate, uranium nitrate, cobalt nitrate, cupric chloride, barium chloride, and mercuric chloride; and the same or practically the same with nitric acid, sulphuric acid, hydrochloric acid, potassium hydroxide, potassium sulphocyanate, potassium sulphide, sodium sulphide, strontium nitrate, and copper nitrate. In $P$. grandifolius the very high reactions with polarization, nitric acid, sulphuric acid, hydrochloric acid, potassium hydroxide, potassium sulphocyanate, potassium sulphide, sodium hydroxide, sodium sulphide, calcium nitrate, strontium nitrate, and copper nitrate; the high with safranin, chromic acid, potassium iodide, sodium salicylate, uranium nitrate; the moderate with iodine, gentian violet, temperature, cupric chloride, and mercuric chloride; the low with chloral hydrate, pyrogallic acid, and cobalt nitrate; and the very low with barium chloride. In $P$. wallichii the very high reactions with polarization, chromic acid, nitric acid, sulphuric acid, hydrochloric acid, potassium hydroxide, potassium iodide, potassium sulphocyanate, potassium sulphide, sodium hydroxide, sodium sulphide, sodium salicylate, calcium nitrate, uranium nitrate, strontium nitrate, copper nitrate, and cupric chloride; the high with safranin and mercuric chloride; the moderate with iodine, gentian violet, temperature, pyrogallic acid, and cobalt nitrate; the low with chloral hydrate; and the very low with barium chloride. In $P$. hybridus the very high reactions with polarization, nitric acid, hydrochloric acid, potassium hydroxide, potassium sulphocyanate, potassium sulphide, sodium hydroxide, sodium sulphide, sodium salicylate, calcium nitrate, uranium nitrate, strontium nitrate, and copper nitrate; the high with gentian violet, safranin, chromic acid, potassium iodide, cupric chloride, and mercuric chloride; the moderate with iodine and temperature; the low with chloral hydrate, pyrogallic acid, and cobalt nitrate; and the very low with barium chloride.
Following is a summary of the reaction-intensities:

\begin{tabular}{|c|c|c|c|c|c|}
\hline & $\begin{array}{l}\text { Very } \\
\text { high. }\end{array}$ & High. & $\begin{array}{l}\text { Mod- } \\
\text { erate. }\end{array}$ & Low. & $\begin{array}{l}\text { Very } \\
\text { low. }\end{array}$ \\
\hline P. grandifolius. . . . . . . . & 12 & 5 & 5 & 3 & 1 \\
\hline P. wallichii. . . . $\ldots \ldots \ldots$ & 17 & 2 & 5 & 1 & 1 \\
\hline P. hybridus . . . . . . . . . & 14 & 6 & 2 & 3 & 1 \\
\hline
\end{tabular}

43. Comparisons of the Starches of Miltonia VEXILLARIA, M. REZLII, AND M. BLEUANA.

In the histologic characteristics, polariscopic figures, reactions with selenite, qualitative reactions with iodine, and qualitative reactions with the various chemical reagents, all three starches exhibit properties in common in varying degrees of development together with individualities, the sum of which in each case is characteristic of the starch. The starch of Miltonia rozlii in comparison with that of $M$. vexillaria shows less numerous compound grains; more varied aggregates and a larger number of the mosaic type; irregularities more frequent and more pronounced (there are differences in the frequency of the appearance of given forms of irregularity) ; a somewhat abrupt flattening at the distal margin may be observed, which peculiarity is not seen in the other starch ; flattening is more frequent in grains with secondary lamellæ. The hilum is somewhat more frequently fissured, and when not fissured is less distinct; quito refractive hila rare; cavity directed longitudinally and clefts more frequent; fissure projected from the hilum generally deeper, more frequently branched and more common; eccentricity less. The lamellæ are less often demonstrable, and there are a number of variations in their distribution and grouping. The size is larger, with a marked tendency to broadness. In the polariscopic, selenite, and qualitative iodine reactions there are many differences. In the qualitative reactions with chloral hydrate, chromic acid, hydrochloric acid, potassium iodide, and sodium salicylate there are many similarities and dissimilarities, some of the latter being quite marked. The starch of the hybrid in comparison with the starches of the parents contains larger numbers of compound grains and aggregates; irregularities are slightly less than in $M$. vexillaria and considerably less than in $M$. roczlii; a lateral extension of secondary lamella is less frequently seen than in $M$. rozzlii. The hilum when fissured is more distinct and is more frequently refractive than in either parent and there are various modifications in the characters of the fissures and clefts; eccentricity is about the same as in $M$. roczlii and less than in $M$. vexillaria. The size is larger than in either parent. The hybrid starch is in form, character of the hilum, and characters of the lamellæ more closely related to $M$. vexitlaria; but in eccentricity of the hilum and size it is closer to $M$. rcozlii. In the polariscopic, selenite, and qualitative iodine reactions there are obvious leanings to one or the other parent, but the relationship is on the whole distinctly closer to $M$. vexillaria. In the qualitative chemical reactions, while the relationships are on the whole distinctly closer to $M$. vexillaria, the influences of $M$. roezlii on the hybrid starch are markedly manifest.

Reaction-intensities Expressed by Light, Color, and Tempera-

Polarization:

M. vexillaria, high to very high, value 85 .

M. rozlii, moderate to very high, lower than in M. vexillaria, value 75 .

M. bleuana, high to very high, higher than in either parent, Iodine: value 88

M. vezillaria, moderate, value 55 .

M. rcezlii, moderate, lighter than in M. vexillaria, value 50 .

M. bleuana, moderate, the same as in $\mathbf{M}$. vexillaria, value 55 . 
Gentian violet:

M. vexillaria, moderate, value 50 .

M. rcezlii, moderate to deep, deeper than in M. vexillaria, value 55 . M. bleuana, moderate to deep, lighter than in $M$. vexillaria, Safranin: value 47 .

M. vexillaria, moderate to moderately deep, value 55 .

M. rœzlii, moderate to deep, considerably deeper than in M. vexillaria, value 65 .

M. bleuana, moderate to moderately deep, the same as in M. vexillaria, value 55 .

Temperature:

M. vexillaria, in the majority at 70 to $71^{\circ}$, in all but rare grains at 73 to $74^{\circ}$, mean $73.5^{\circ}$.

M. rcezlii, in the majority at 74 to $76^{\circ}$, in all but rare grains at 76 to $77^{\circ}$, mean $76.5^{\circ}$

M. bleuana, in the majority at 69 to $71^{\circ}$, in all but rare grains at 72 to $74^{\circ}$, mean $73^{\circ}$.

$M$. vexillaria shows a higher reactivity than the other parent in the polarization, iodine, and temperature reactions, and a lower reactivity in the gentian-violet and safranin reactions. The hybrid has the highest reactivities of the three in the polarization and temperature reactions, the lowest reactivity in the gentian-violet reactions, and the same or practically the same reactivities as $M$. vexillaria in the iodine and safranin reactions. In all five reactions the hybrid is either the same as or closer to $M$. vexillaria.

Table A 43 shows the reaction-intensities in percentages of total starch gelatinized at definite intervals (minutes).

\section{Velocitr-Reaction CuRves.}

This section treats of the velocity-reaction curves of the starches of Miltonia vexillaria, $M$. rezlii, and $M$. bleuana, showing the quantitative differences in the behavior toward different reagents at definite time-intervals. (Charts D 595 to D 609.)

Among the conspicuous features of these charts are: The closeness and correspondence of the curves in each of the reactions. The reactions with nitric acid, sulphuric acid, hydrochloric acid, and potassium hydroxide occur with such rapidity that there is practically no differentiation. The curve of $M$. vexillaria is higher than the curve of the other parent in the reactions with chloral hydrate, chromic acid, pyrogallic acid, potassium iodide, potassium sulphocyanate, potassium sulphide, sodium hydroxide, sodium sulphide, sodium salicylate, calcium nitrate, uranium nitrate, strontium nitrate, copper nitrate, cupric chloride, and mercuric chloride; and lower with cobalt nitrate and barium chloride. The hybrid, while bearing varying relations to one or the other or both parents as regards sameness, intermediateness, excess, and deficit in reactivities, shows a remarkable inclination to an almost universally higher reactivity than either of the parents, and, moreover, a similar inclination to the seed parent; in only 2 of the 26 reactions is there a manifest leaning toward the pollen parent. An early period of high resistance followed by rapid to moderate gelatinization is entirely absent from this set of reactions. The earliest period during the 60 minutes that is best for the differentiation of the three starches is for chromic acid, potassium iodide, potassium sulphide, potassium sulphocyanate, sodium hydroxide, sodium sulphide, sodium salicylate, uranium nitrate, strontium nitrate, cobalt nitrate, copper nitrate, and cupric chloride at 5 minutes; calcium nitrate at 15 minutes; chloral hydrate, pyrogallic acid, barium chloride, and mercuric chloride at 30 minutes. The reactions with nitric acid, sulphuric acid, hydrochloric acid, and potassium hydroxide are too fast for differentiation of the starches.

\section{REACTION-INTENSITIES OF THE HYBRID.}

This section treats of the reaction-intensities of the hybrid as regards sameness, intermediateness, excess, and
TABLE A 43.

\begin{tabular}{|c|c|c|c|c|c|c|c|c|c|c|}
\hline & $\underline{-1}$ & ล & $\underset{\infty}{8}$ & 百 & מ & $\begin{array}{l}\text { 日 } \\
\text { 足 }\end{array}$ & 豆 & 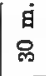 & $\begin{array}{r}8 \\
4 \\
4\end{array}$ & 8 \\
\hline $\begin{array}{l}\text { hloral hy } \\
\text { M. vexill }\end{array}$ & & & & & 67. & & 84 & 97 & 98 & \\
\hline & & . & & . & 60 & $\ldots$ & 71 & 82 & 84 & \\
\hline & & $\cdots$ & . & . & 62 & . & 81 & 95 & 97 & \\
\hline hror & & & & & & & & & & \\
\hline & $\cdots$ & $\cdots$ & - & . & 42 & $\cdots$ & 87 & 97 & 99 & \\
\hline & $\begin{array}{l}\cdots \\
\cdots\end{array}$ & $\because$ & $\because$ & $\cdots$ & $\begin{array}{l}37 \\
63\end{array}$ & $\cdots$ & 91 & & 96 & 98 \\
\hline Pyroge & & $\cdots$ & & & 05 & & & 90 & & \\
\hline & . & $\cdots$ & $\cdots$ & & 50 & . & 72 & 84 & 88 & 34 \\
\hline & & . & . & .. & 43 & $\ldots$ & 63 & 72 & 77 & \\
\hline & & . & $\cdots$ & $\cdots$ & 63 & . & 82 & 96 & 97 & 100 \\
\hline Jit & & & & & & & & & & \\
\hline & 88 & $\begin{array}{l}92 \\
93\end{array}$ & . & $\cdot$ & 97 & $\cdots$ & 99 & & & \\
\hline & 86 & $\begin{array}{l}95 \\
99\end{array}$ & $\cdots$ & & 85 & $\cdots$ & 9. & 99 & $\cdots$ & \\
\hline ulphu & & & & & & $\cdots$ & $\cdots$ & $\cdots$ & & $\cdots$ \\
\hline & 99 & & . & & & & $\ldots$ & $\cdots$ & & \\
\hline & 97 & 98 & . & 89 & 100 & . & . & . & . & 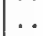 \\
\hline & 9 & $\cdots$ & . & .. & $\cdots$ & $\cdots$ & . & $\cdots$ & . & . \\
\hline Hyd & & & & & & & & & & \\
\hline & 97 & 99 & & . & & $\cdots$ & $\cdots$ & . & . & \\
\hline M. & 94 & 97 & . & .. & 99 & . & . & $\ldots$ & . . & \\
\hline & 99 & $\cdots$ & $\cdots$ & . & $\cdots$ & . & $\cdots$ & $\cdots$ & . & \\
\hline Potassiu & & & & & & & & & & \\
\hline & 98 & $\cdots$ & $\cdots$ & $\cdots$ & $\begin{array}{r}99 \\
\end{array}$ & & $\cdots$ & $\cdots$ & $\cdots$ & \\
\hline & & $\because$ & & & 100 & $\cdots$ & $\cdots$ & $\cdots$ & $\cdots$ & \\
\hline $\begin{array}{r}\text { M. b } \\
\text { Potass }\end{array}$ & & $\cdots$ & & & 100 & $\cdots$ & $\cdots$ & $\cdots$ & - & " \\
\hline & . & . & . & $\cdots$ & 84 & $\cdots$ & 97 & 99 & & \\
\hline & $\cdots$ & $\cdots$ & . & . & 75 & & 85 & 90 & & 95 \\
\hline & $\cdots$ & . & . & . & 92 & 95 & 98 & 99 & & \\
\hline e: & & & & & & & & & & \\
\hline & . & 95 & & . & 99 & $\cdots$ & & & . & $\cdots$ \\
\hline & $\cdots$ & 85 & $\cdots$ & $\cdots$ & 89 & $\cdots$ & 95 & 98 & . & . \\
\hline & $\cdots$ & & $\cdots$ & $\cdots$ & 99 & $\cdots$ & $\cdots$ & $\cdots$ & $\cdots$ & \\
\hline $\begin{array}{c}\text { Potass } \\
\text { M. }\end{array}$ & . & $\cdots$ & $\ldots$ & $\cdots$ & 83 & $\ldots$ & 87 & 90 & 92 & \\
\hline & . & . & $\ldots$ & .. & 72 & . & 84 & 85 & 87 & 88 \\
\hline & . & . & . & . & 96 & .. & 98 & 99 & - & \\
\hline Sodi & & & & & & & & & & \\
\hline & . & $\cdots$ & $\cdots$ & - & 95 & . & 99 & & & \\
\hline & $\cdots$ & $\cdots$ & $\cdots$ & . & 87 & . & 92 & 95 & . & 95 \\
\hline & $\cdots$ & $\cdots$ & $\cdots$ & $\cdots$ & 98 & $\cdots$ & 99 & $\cdots$ & . & . \\
\hline & & & & & & & & & & \\
\hline & $\cdots$ & $\cdots$ & $\because$ & $\cdots$ & $\begin{array}{l}79 \\
58\end{array}$ & $\cdots$ & $\begin{array}{l}89 \\
72\end{array}$ & 95 & $\begin{array}{l}96 \\
90\end{array}$ & \\
\hline & $\cdots$ & $\because$ & $\cdots$ & $\cdots$ & $\begin{array}{l}58 \\
95\end{array}$ & $\because$ & \begin{tabular}{|l|}
72 \\
99
\end{tabular} & $r 6$ & & 83 \\
\hline Sodi & & & & & & & & $\cdots$ & & \\
\hline & .. & . & . & . & 80 & . & 98 & . & $\cdots$ & \\
\hline & $\cdots$ & . & . & . & 78 & & 96 & . & . & $\cdot$ \\
\hline & $\cdots$ & $\cdots$ & . & $\cdots$ & 86 & 95 & 99 & $\cdots$ & . & \\
\hline Calc & & & & & & & & & & \\
\hline $\begin{array}{l}\mathrm{M} \\
\mathrm{M}\end{array}$ & 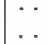 & $\cdots$ & $\cdots$ & $\cdots$ & $\begin{array}{l}84 \\
82\end{array}$ & $\cdots$ & $\begin{array}{l}95 \\
89\end{array}$ & $\begin{array}{l}96 \\
90\end{array}$ & 91 & $\begin{array}{l}98 \\
92\end{array}$ \\
\hline & . . & . & . & . & 97 & $\because$ & 99 & 00 & & \\
\hline Uranil & & & & & & & & & & \\
\hline & $\cdots$ & $\cdots$ & . & . & 83 & $\cdots$ & 90 & 95 & 96 & 98 \\
\hline & $\cdots$ & . & . & $\cdots$ & 77 & $\cdots$ & 87 & 95 & . & 96 \\
\hline & $\cdots$ & $\cdots$ & . & . & 95 & $\cdots$ & 99 & $\cdots$ & $\cdots$ & $\cdots$ \\
\hline $\begin{array}{l}\text { tro } \\
\text { M }\end{array}$ & & & & & & & & & & \\
\hline & $\cdots$ & .. & . & $\cdots$ & $\begin{array}{l}91 \\
86\end{array}$ & $\begin{array}{l}95 \\
\ldots\end{array}$ & 95 & 96 & 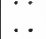 & \\
\hline & $\ldots$ & $\ldots$ & . & . & 99 & $\ldots$ & $\ldots$ & $\ldots$ & .. & \\
\hline Cob & & & & & & & & & & \\
\hline & . & . & . & $\cdots$ & 16 & $\cdots$ & 46 & 52 & 56 & 60 \\
\hline & $\cdots$ & . . & . & . & 48 & $\ldots$ & 56 & 62 & 64 & 70 \\
\hline & $\cdots$ & $\cdots$ & . & . & 67 & $\cdots$ & 81 & 89 & 90 & 91 \\
\hline opl & & & & & & & & & & \\
\hline & . & . & $\cdots$ & $\cdots$ & 84 & $\cdots$ & 95 & 96 & 97 & 89 \\
\hline & $\cdots$ & . & . & . & 73 & $\cdots$ & 83 & 90 & 95 & 95 \\
\hline & $\cdots$ & $\cdots$ & $\ldots$ & . & 98 & $\ldots$ & 99 & . & . & \\
\hline & & & & & & & & & & \\
\hline & $\cdots$ & $\cdots$ & $\cdots$ & $\cdots$ & 56 & $\cdots$ & 70 & 78 & 81 & 85 \\
\hline & $\cdots$ & $\cdots$ & . & . & 52 & $\cdots$ & 64 & 68 & 70 & 72 \\
\hline & $\cdots$ & $\cdots$ & $\cdots$ & $\cdots$ & 81 & $\cdots$ & 90 & 95 & 97 & 99 \\
\hline Bariu & & & & & & & & & & \\
\hline & . & $\cdots$ & $\cdots$ & $\cdots$ & 2 & . & 6 & 7 & 10 & 12 \\
\hline & • & $\ldots$ & . & . & 6 & . & 11 & 15 & 18 & 22 \\
\hline & $\cdots$ & $\cdots$ & $\cdots$ & $\ldots$ & 10 & $\ldots$ & 20 & 25 & 30 & 33 \\
\hline Mer & & & & & & & & & & \\
\hline & . & $\cdots$ & . & $\cdots$ & 43 & . & 60 & 75 & 80 & 85 \\
\hline & & . & $\cdots$ & . & 42 & . & 53 & 57 & 60 & 60 \\
\hline & & & $\cdots$ & . & 75 & & 90 & 97 & 98 & 98 \\
\hline
\end{tabular}


deficit in relation to the parents. (Table A 43 and Charts D 595 to D 609.)

The reactivities of the hybrid are the same as those of the seed parent in the reactions with iodine, safranin, and potassium sulphocyanate; the same as those of the pollen parent in none; the same as those of both parents in those with sulphuric acid, hydrochloric acid, and potassium hydroxide, in all of which gelatinization occurs very quickly ; intermediate, but nearer the seed parent, in that with chloral hydrate; highest with polarization, chromic acid, pyrogallic acid, nitric acid, potassium iodide, potassium sulphide, sodium hydroxide, sodium sulphide, sodium salicylate, calcium nitrate, uranium nitrate, strontium nitrate, cobalt nitrate, copper nitrate, cupric chloride, copper chloride, barium chloride, and mercuric chloride (in 14 being closer to the seed parent, in 2 closer to the pollen parent, and in 1 as close to one as to the other parent) ; and lowest with gentian violet and temperature, in both being closer to the seed parent-in the latter practically the same.

The following is a summary of the reaction-intensities: Same as seed parent, 3; same as pollen parent, 0 ; same as both parents, 3 ; intermediate, 1 ; highest, 17 ; lowest, 2.

Two very conspicuous features of these data are the very markedly dominating influence of the seed parent on the properties of the starch of the hybrid, and the equally marked tendency to reactivities of the hybrid, higher than those of the parents. In 20 out of the 26 reactions the seed parent is the same or closer to the hybrid, while in only 2 is there closeness to the pollen parent; and in 17 reactions the hybrid exceeds the reactivilies of the parents.

\section{Composite Curves of Reaction-intensities.}

This section treats of the composite curves of the reaction-intensities, showing the differentiation of the starches of Miltonia vexillaria, $M$. rozlii, and $M$. bleuana. (Chart E 43.)

The most conspicuous features of this chart are: The close correspondence in the rises and falls of all three curves excepting in the reactions with gentian violet, chloral hydrate, and calcium nitrate. In the gentianviolet reactions the curves of $M$. vexillaria and the hybrid fall, while the curve of $M$. rozlii rises; in the chloralhydrate reactions the curves of the former rise while the curve of the latter falls; and in the calcium-nitrate reactions the curve of $M$. rozlii appears aberrant by falling. $M$. vexillaria has higher reactivities than the other parent in the reactions with polarization, iodine, choral hydrate, pyrogallic acid, potassium iodide, potassium sulphocyanate, potassium sulphide, sodium hydroxide, calcium nitrate, strontium nitrate, copper nitrate, cupric chloride, and mercuric chloride; lower reactivities with gentian violet, safranin, temperature, cobalt nitrate, and barium chloride; and the same or practically the same reaction-intensities with chromic acid, nitric acid, sulphuric acid, hydrochloric acid, potassium hydroxide, sodium sulphide, sodium salicylate, and uranium nitrate. In $M$. vexillaria the very high reactions with polarization, nitric acid, sulphuric acid, hydrochloric acid, potassium hydroxide, potassium iodide, potassium sulphocyanate, sodium hydroxide, sodium salicylate, calcium nitrate, strontium nitrate, and copper nitrate; the high reactions with chloral hydrate, chromic acid, sodium sulphide, and uranium nitrate; the moderate reactions with iodine, gentian violet, safranin, pyrogallic acid, and potassium sulphide; the low reactions with temperature, cobalt nitrate, cupric chloride, and mercuric chloride; and the very low reactions with barium chloride. In $M$. razlii the very high reactions with nitric acid, sulphuric acid, hydrochloric acid, potassium hydroxide, potassium sulphocyanate, sodium salicylate, and strontium nitrate; the high reactions with polarization, safranin, chromic acid, sodium hydroxide, sodium sulphide, uranium nitrate, and copper nitrate; the moderate reactions with iodine, gentian violet, temperature, potassium iodide, and calcium nitrate; the low reactions with chloral hydrate, pyrogallic acid, potassium sulphide, cobalt nitrate, cupric chloride, and mercuric chloride; and the very low reactions with barium chloride. In M. bleuana the very high reactions with polarization, chromic acid, nitric acid, sulphuric acid, hydrochloric acid, potassium hydroxide, potassium iodide, potassium sulphocyanate, potassium sulphide, sodium hydroxide, sodium sulphide, sodium salicylate, calcium nitrate, uranium nitrate, strontium nitrate, and copper nitrate; the high reactions with chloral hydrate, pyrogallic acid, cupric chloride, and mercuric chloride; the moderate reactions with iodine, gentian violet, safranin, and cobalt nitrate; the low reaction with temperature; and the very low reaction with barium chloride.

Following is a summary of the reaction-intensities:

\begin{tabular}{|c|c|c|c|c|c|}
\hline & $\begin{array}{l}\text { Very } \\
\text { high. }\end{array}$ & High. & $\begin{array}{l}\text { Mod- } \\
\text { erate. }\end{array}$ & Low. & $\begin{array}{l}\text { Very } \\
\text { low. }\end{array}$ \\
\hline 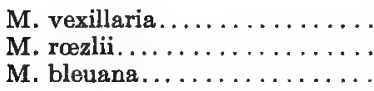 & $\begin{array}{r}12 \\
7 \\
16\end{array}$ & $\begin{array}{l}4 \\
7 \\
4\end{array}$ & $\begin{array}{l}5 \\
5 \\
4\end{array}$ & $\begin{array}{l}4 \\
6 \\
1\end{array}$ & $\begin{array}{l}1 \\
1 \\
1\end{array}$ \\
\hline
\end{tabular}

44. Comparison of the Starches of Crmbididm LOWIANUM, C. EBURNEUM, AND C. EBURNeLOWTANUM.

In the histologic characteristics, polariscopic figures, reactions with selenite, qualitative reactions with iodine, and qualitative reactions with the various chemical reagents all three starches exhibit properties in common in varying degrees of development together with certain individualities which collectively are in each case characteristic. The starch of Cymbidium lowinaum in comparison with that of $C$. eburneum has somewhat less numerous grains of the disaggregate type; pressure facets on separated grains are more numerous; the surfaces of disaggregates are more regular; large grains of the iso lated disaggregate type are more numerous and more varied in form; compactly arranged triplets and quadruplets are more common; components of doublets are more often of equal size; and mosaics of five to ten components are more rounded. The hilum has a cavity or cleft more often; it is more often fissured; there are various modifications of fissuring; eccentricity is less. The lamellæ are much less often demonstrable; there is an absence of a secondary set of lamellæ at right angle to the primary set; the number is probably less. The size is on the whole smaller, and differences are noted in the proportion of length to width. In the polariscopic, selenite, and qualitative iodine reactions various differences are recorded in the three starches, mostly apparently of a very minor character. In the qualitative reactions with chloral hydrate, chromic acid, nitric acid, potassium hydroxide, potassium iodide, potassium sulphocyanate, and sodium salicylate various points of difference have been demonstrated, but these seem to be of minor character. Throughout, with few exceptions, the hybrid is much closer to C. lowianum.

Reaction-intensities Expressed by Light, Color, and Temperature Reactions.

Polarization:

C. lowianum, high, value 80 .

C. eburneum, high, lower than in C. lowianum, value 75 .

C. eburn.-low., high, the same as in C. lowianum, value 80 . 
Iodine:

C. lowianum, moderate, value 50 .

C. eburneum, moderate, lighter than in C. lowianum, value 45.

C. eburn.-low., moderate, the same as in C. lowianum, value 50 .

Gentian violet:

C. lowianum, moderate to moderately deep, value 55 .

C. eburneum, light to moderately deep, slightly deeper than in C. lowianum, value 57

C. eburn.-low., light to moderately deep, the same as in C. lowianum, value 55

Safranin:

C. lowianum, moderate to moderately deep, value 52.

C. eburneum, moderate to moderately deep, slightly deeper than in C. lowianum, value 55 .

C. eburn.-low., moderate to moderately deep, the same as in C. lowianum, value 52 .

Temperature:

C. lowianum, in the majority at 58 to $60^{\circ}$, in all at 62 to $63^{\circ}$, mean $62.5^{\circ}$.

C. eburneum, in the majority at 58 to $59.5^{\circ}$, in all at 65 to $66.5^{\circ}$, mean $65.76^{\circ}$.

C. eburn.-low., in the majority at 61 to $63^{\circ}$, in all but rare grains at 67 to $68^{\circ}$, mean $67.5^{\circ}$.

C. lowianum exhibits a higher reactivity than the other parent in the polarization, iodine, and temperature reactions, and a lower reactivity in the gentian-violet and safranin reactions. The hybrid has the same reactivities as $C$. lowianum in the reactions with polarization, iodine, gentian violet, and safranin, but has a lower reactivity than either parent with temperature, in which it is nearer to $C$. eburneum.

Table A 44 shows the reaction-intensities in percentages of total starch gelatinized at definite intervals (seconds and minutes).

\section{VELOCITY-REACTION CURVES.}

This section treats of the velocity-reaction curres of the starches of Cymbidium lowianum, C. eburneum, and C. eburneo-lowianum, showing the quantitative difference in the behavior toward different reagents at definite timeintervals. (Charts D 616 to D 618.)

The reactions with the various reagents, with rare exceptions, occur with such rapidity that such differences as may have been noted are not conclusive, all three starches being gelatinized completely or practically completely within a minute or two, and often within 15 to 30 seconds. Where no differences are recorded between the reactions of the parents those of the hybrid may be distinctly different, as in the chloral-hydrate, pyrogallicacid, and barium-chloride reactions, especially in the last. For the reason stated, only the curves of these three reactions have been charted.

\section{Reaction-Intensities OF THE HYBRID.}

This section treats of the reaction-intensities of the hybrid as regards sameness, intermediateness, excess, and deficit in relation to the parents. (Table A 44 and Charts D 616 to D 618. .)

The reactivities of the hybrid are the same as those of the seed parent in the reactions with polarization, iodine, gentian violet, and safranin; the same as those of the pollen parent in none; the same as those of both parents with sulphuric acid, hydrochloric acid, potassium hydroxide, potassium iodide, potassium sulphocyanate, potassium sulphide, sodium hydroxide, sodium sulphide, and strontium nitrate, in all of which the reactions are too rapid for differentiation; intermediate or highest in none; and the lowest with temperature, chloral hydrate, chromic acid, pyrogallic acid, nitric acid, sodium salicylate, calcium nitrate, cobalt nitrate, copper nitrate, cupric chloride, barium chloride, and mercuric chloride (in 1 being closer to the pollen parent, and in 12 as close to one as to the other parent).

The following is a summary of the reaction-intensities: Same as seed parent, 4; same as pollen parent, 0 ;
TABLE A 44.

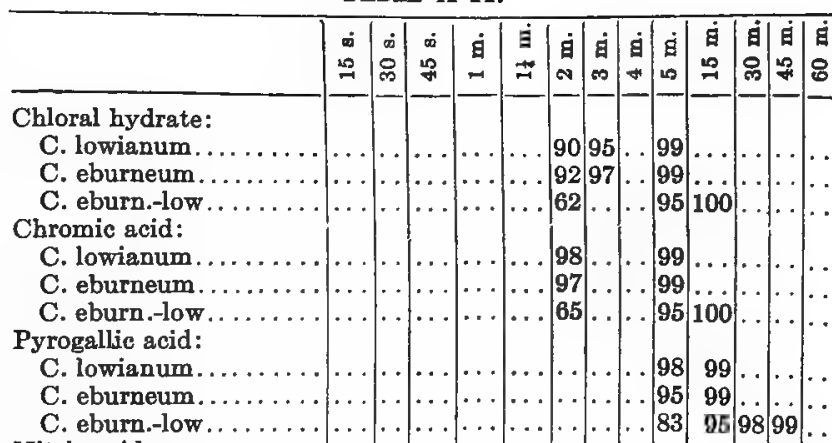

Nitric acid:

C. lowianum

C. eburneum.

Sulphuric acid:

C. lowianum.

C. eburneum.

C. eburn.-low

Hydrochloric acid:

C. lowianum

C. eburneum..................

C. eburn.-low.

Potassium hydroxide:

C. lowianum......... 100

C. eburneum......... 100

C. eburn.-low.

Potassium iodide:

C. lowianum.

C. eburn.-low.

Potassium sulphocyanate

C. lowianum.

C. eburneum. 95.

$83 \quad 959899$

Potassium sulphide:

C. lowianum. .

C. eburneum........... 100

C. eburn.-low

Sodium hydroxide:

C. lowianum.

... 100

C. eburneum......... 100

C. eburn.-low

Sodium sulphide:

C. lowianum

C. eburneum

C. eburn.-low

Sodium salicylate:

C. lowianum

C. eburneum

C. eburn.-low

Calcium nitrate:

C. lowianum.

C. eburneum

C. eburn

Uranium nitrate:

C. lowianum

C. eburneum.............

C. eburn.-low...

Strontium nitrate:

C. lowianum...

C. eburneum.

C. eburn.-low

Cobalt nitrate:

C. lowianum

C. eburneum.

Copper nitrate:

C. lowianum

C. eburneum.

C. eburn.-low.

Cupric chloride:

C. lowianum........... $95 .$.

C. eburneum

C. eburn.-low.
Barium chloride:

C. lowianum......... . . . . . .

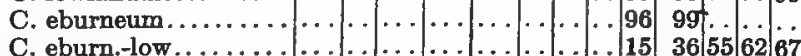

Mercuric chloride:

C. lowianum

C. eburneum

.........

C. eburn.-low......... ( 
same as both parents, 9 ; intermediate, 0 ; highest, 0 ; lowest, 13.

The most striking features of the foregoing data are in the hybrid the entire absence of sameness to the pollen parent, of intermediateness, and of highest reactivity; the frequent sameness of reactivity in relation to both parents ; and the large number of lowest reactivities, with almost universal closeness to one as to the other parent. The very high reactivities of all three starches makes differentiation in most instances impossible or unsatisfactory. The seed parent seems to have had on the whole a somewhat higher reactivity than the pollen parent in the reactions with polarization, iodine, gentian violet, and safranin, but in the chemical reactions the reactivities of the parents seem to be almost if not absolutely identical. It is all the more remarkable that with this parental identity the hybrid should show in any reaction a departure from the parental standard. With modified strengths of reagents undoubtedly parental differences would be brought out, and hybrid-parental differences markedly exaggerated.

\section{Composite Curves of the Rfaction-intensities.}

This section treats of the composite curves of the reaction-intensities, showing the differentiation of the starches of Cymbidium lowianum, C. eburneum, and $C$. eburneo-lowianum.

The most conspicuous features of this chart are: The marked closeness of all three curves throughout, excepting in the pyrogallic-acid and barium-chloride reactions, in the latter the hybrid curves exhibiting an exceptionally marked departure from the parental standard. The parental curves are the same or practically the same excepting in the reactions with polarization, iodine, gentian violet, safranin, and temperature, and among these the only important difference is noted in the temperature reactions, there being a difference of $3.25^{\circ}$ in the mean temperature of gelatinization. With weaker reagents more or less marked differences in the parents would be elicited in at least most of the reactions where they appear to be identical in the chart. The curve of C. lowianum is higher than the curve of the other parent in the polarization, iodine, and temperature reactions; lower with gentian violet and safranin; and the same or practically the same in all with the chemical reactions. In $C$. lowianum the very high reactivities in the reactions with polarization, chloral hydrate, chromic acid, pyrogallic acid, nitric acid, sulphuric acid, hydrochloric acid, potassium hydroxide, potassium iodide, potassium sulphocyanate, potassium sulphide, sodium hydroxide, sodium sulphide, sodium salicylate, calcium nitrate, uranium nitrate, strontium nitrate, cobalt nitrate, copper nitrate, cupric chloride, barium chloride, and mercuric chloride; the high reaction with temperature; and the moderate reactions with iodine, gentian violet, and safranin. In C. lowianum the very high reactions with chloral hydrate, chromic acid, pyrogallic acid, nitric acid, sulphuric acid, and hydrochloric acid, potassium hydroxide, potassium iodide, potassium sulphocyanate, potassium sulphide, sodium hydroxide, sodium sulphide, sodium salicylate, calcium nitrate, uranium nitrate, strontium nitrate, cobalt nitrate, copper nitrate, cupric chloride, barium chloride, and mercuric chloride; the high reaction with polarization; and the moderate reactions with iodine, gentian violet, safranin, and temperature. In the hybrid the very high reactions with polarization, chloral hydrate, chromic acid, pyrogallic acid, nitric acid, sulphuric acid, hydrochloric acid, potassium hydroxide, potassium iodide, potassium sulphocyanate, potassium sulphide, sodium hydroxide, sodium sulphide, sodium salicylate, calcium nitrate, uranium nitrate, strontium nitrate, cobalt nitrate, copper nitrate, cupric chloride, and mercuric chloride; the moderate reactions with iodine, gentian violet, safranin, and temperature; and the low reaction with barium chloride.

Following is a summary of the reaction-intensities:

\begin{tabular}{|c|c|c|c|c|c|}
\hline & $\begin{array}{l}\text { Very } \\
\text { high. }\end{array}$ & High. & $\begin{array}{l}\text { Mod- } \\
\text { erate. }\end{array}$ & Low. & $\begin{array}{l}\text { Very } \\
\text { low. }\end{array}$ \\
\hline C. lowianum.. & 22 & 1 & 3 & 0 & 0 \\
\hline C. eburneum........... & 21 & 1 & 4 & 0 & 0 \\
\hline C. eburn.-low.............. & 21 & 0 & 4 & 1 & 0 \\
\hline
\end{tabular}

\section{Comparisons of the Starches of Calanthe} ROSEA, C. VESTITA VAR. RUBRo-ocUlata, AND C. VEITCHII.

In the histologic characteristics, polariscopic figures, reactions with selenite, qualitative reactions with iodine, and qualitative reactions with the various chemical reagents all three starches exhibit properties in common in varying degrees of development and certain more or less well-defined individualities which collectively in each are distinctive. The hybrid Calanthe veitchii is in form, on the whole, much closer to $C$. rosea, but there are some forms that are the same as those found in and peculiar to C. vestita var. rubro-oculata. In hilum and lamellæ the starch is closer to C. rosea, but in size and proportions of length to width of the grains it is closer to C. vestita var. rubro-oculata. In polariscopic figures and reactions with selenite it is closer to C. vestita var. rubro-oculata. In the qualitative iodine reactions it is slightly closer to C. rosea. In the qualitative reactions with chloral hydrate, potassium hydroxide, and sodium salicylate it is closer to $C$. vestita var. rubro-oculata, while in the chromic-acid and hydrochloric-acid reactions it is closer to C. rosea.

Reaction-intensities Expressed by Light, Color, and Temperature Reactions.

Polarization:

C. rosea, low to very high, value 55 .

C. vest. v. rubro-oc., moderate to very high, much higher than C. rosea, value 70 .

C. veitchii, low to very high, intermediate between the parents, Iodine: value 60 .

C. rosea, light to moderate, value 40 .

C. vest. v. rubro-oc., moderate, deeper than C. rosea, value 50.

C. veitchii, moderate, intermediate between the parents, value 43 . Gentian violet:

C. rosea, moderate to moderately deep, value 55 .

C. vest. v. rubro-oc., moderate to deep, deeper than C. rosea, value 60 .

C. veitchii, moderate to moderately deep, intermediate between Safranin the parents, value 57 .

C. rosea, moderate to moderately deep, value 60 .

C. vest. v. rubeo-oc., moderate to moderately deep, deeper than C. rosea, value 65.

C. veitchii, moderate to moderately deep, the same as C. vestita var. rubro-oculata, value 65 .

Temperature:

C. rosea, in the majority at 74 to $76^{\circ}$, in all at 75 to $77^{\circ}$, mean $76^{\circ}$.

C. vest. v. rubro-oc., in the majority at 72 to $74^{\circ}$, in all at 74 to $75^{\circ}$ mean $74.5^{\circ}$.

C. veitehii, in the majority at 71 to $72^{\circ}$, in all at 73 to $74^{\circ}$, mean $72.5^{\circ}$.

C. rosea has lower reactivities than the other parent in the reactions with polarization, iodine, gentian violet, safranin, and temperature. The hybrid has an intermediate reactivity between the parents in the polarization, iodine, and gentian-violet reactions; the same reactivity as $C$. vestita var. rubro-oculata in the safranin reaction; and a higher reactivity than either parent in the temperature reaction. 
Table A 45 shows the reaction-intensities in percentages of total starch gelatinized at definite intervals (minutes) :

Table A 45.

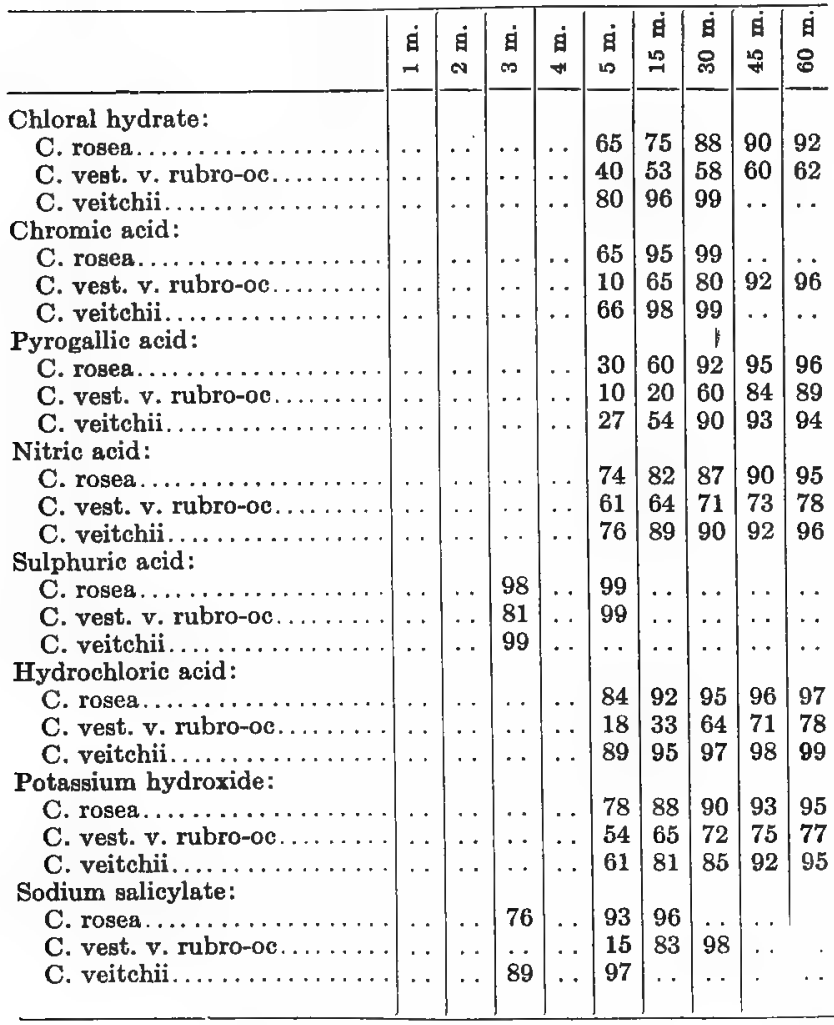

Velocity-REACTION CURtes.

This section treats of the velocity-reaction curves of the starches of Calanthe rosea, C. vestita var. rubrooculata, and $C$. veitchii, showing the quantitative differences in the behavior toward different reagents at definite time-intervals. (Charts D 619 to D 626.)

Among the conspicuous features of these charts are: The marked separation of all three curves in the reactions with chloral hydrate and potassium hydroxide; the practical identity of all three with sulphuric acid; the closeness of the curves of $C$. rosea and the hybrid curves with pyrogallic acid, chromic acid, hydrochloric acid, and sodium salicylate; and the lower curves of $C$. vestita var. rubro-oculata in all but the sulphuric-acid reactions (even in the latter there is a slightly lower reactivity, although not shown in the chart; see reactions in Table A 45). The curve of $C$. rosea is higher than the curve of the other parent. usually very much higher, in every chart, excepting that of sulphuric acid, in which the differences between the reactions of the parents are not presented, owing to the great rapidity of gelatinization. Even with this reagent differences are shown by the figures of the preceding tables, there being 98 per cent of the total starch of $C$. rosea and only 81 per cent of the total starch of $C$. vestita var. rubro-oculata gelatinized in 3 minutes. The curves of the hybrid $C$. veitchii tend in all of the experiments to be closer, and usually much closer, to the curves of $C$. rosea than to those of the other parent. An early period of comparatively high resist- ance followed by a rapid to moderate rapidity of gelatinization is noted in only the starch of $C$. vestita var. rubro-oculata, and in the reactions as above stated. The earliest period during the 60 minutes that is best for the differentiation of all three starches is for chromic acid, hydrochloric acid, potassium hydroxide, and sodium salicylate at 5 minutes, and for chloral hydrate, pyrogallic acid, and nitric acid at $\mathbf{1 5}$ minutes.

\section{Reaction-Intensities of THE Hybrid.}

This section treats of the reaction-intensities of the hybrid as regards sameness, intermediateness, excess, and deficit in relation to the parents. (Table $\mathbf{A} 45$ and Charts D 619 to D 626 .)

The reactivities of the hybrid are the same as those of the seed parent in the reactions with chromic acid and sulphuric acid; the same as those of the pollen parent with safranin; the same as those of both parents with polarization, iodine, gentian violet, pyrogallic acid, and potassium hydroxide (in 4 being closer to the seed parent and in 1 as close to one as to the other parent); highest with temperature, chloral hydrate, nitric acid, and sodium salicylate, in all being closer to those of the seed parent; and the lowest with hydrochloric acid.

The following is a summary of the reaction-intensities: Same as seed parent, 2; same as pollen parent, 1; same as both parents, 0 ; intermediate, 5 ; highest, 4 ; lowest, 1.

The most conspicuous features of these data are the pre-eminence of the seed parent in determining the properties of the starch of the hybrid, and the distinct tendency to intermediateness and to highest and lowest reactivities of the hybrid.

\section{Composite Curves of the Reaction-intensitirs.}

This section treats of the composite curves of the reaction-intensities, showing the differentiation of the starches of Calanthe rosea, C. vestita var. rubro-oculata, and C. veitchii. (Chart $\mathrm{E} 45$. .)

The most conspicuous features of this chart are: The close correspondence in the rises and falls of all three curves excepting in the chloral-hydrate reactions, where one of the curves diverges, the curve of $C$. vestita var. rubro-oculata falling instead of rising in harmony with the curves of the other parent and the hybrid. The curve of $C$. rosea is higher than the curve of the other parent in the reactions with chloral hydrate, chromic acid, pyrogallic acid, nitric acid, sulphuric acid, hydrochloric acid, and potassium hydroxide, and lower with polarization, iodine, gentian violet, safranin, and temperature. In $C$. rosea the very high reactions with chromic acid and sulphuric acid; the high reactions with safranin, pyrogallic acid, and hydrochloric acid; the moderate reactions with polarization, iodine, gentian violet, chloral hydrate, nitric acid, and potassium hydroxide; the low reaction with temperature. In $C$. vestita var. rubro-oculata the very high reaction with sulphuric acid; the high reactions with polarization, gentian violet, and safranin; the moderate reactions with iodine and chromic acid; the low reactions with temperature, chloral hydrate, pyrogallic acid, nitric acid, hydrochloric acid, and potassium hydroxide. In the hybrid C. veitchii the very high reactions with chloral hydrate, chromic acid, sulphuric acid, and hydrochloric acid; the 
high reactions with polarization and safranin; and the moderate reactions with iodine, gentian violet, temperature, pyrogallic acid, nitric acid, and potassium hydroxide.

Following is a summary of the reaction-intensities:

\begin{tabular}{|c|c|c|c|c|c|}
\hline & $\begin{array}{l}\text { Very } \\
\text { high. }\end{array}$ & High. & $\begin{array}{l}\text { Mod- } \\
\text { erate. }\end{array}$ & Low. & $\begin{array}{l}\text { Very } \\
\text { low. }\end{array}$ \\
\hline C. rosea ............... & 2 & 3 & 6 & 1 & 0 \\
\hline C. vest. v. rubro-oe $\ldots \ldots \ldots \ldots$ & 1 & 3 & 2 & 6 & 0 \\
\hline C. veitchii $\ldots \ldots \ldots \ldots \ldots \ldots$ & 4 & 2 & 6. & 0 & 0 \\
\hline
\end{tabular}

46. Comparisons of the Starches of Calanthe vESTITA VAR. RUBRO-OCDLATA, C. REGNIERI, AND C. BRYAN.

In the histologic characteristics, polariscopic figures, reactions with selenite, qualitative reactions with iodine, and qualitative reactions with the various chemical reagents the starches of parents and hybrid exhibit properties in common in varying degrees of development and in each case more or less marked individualities. The hybrid $C$. bryan is in form in the majority of the grains closer to $C$. regnieri, and in a minority of the grains closer to $C$. vestita var. rubro-oculata. In hilum and lamellæ it is closer to C. regnieri. In mean size the grains are larger than those of either parent but closer to $C$. regnieri, while in proportion of length to width they are closer to the other parent. In polariscopic figure and reactions with selenite it is closer to $C$. regnieri. In the qualitative reactions with iodine it is closer to C. regnieri. In the qualitative changes during heat gelatinization it is, during the first stages, closer to $C$. regnieri, but during the later stages closer to the other parent. In the qualitative reactions with chloral hydrate, chromic acid, nitric acid, and sodium salicylate it is closer to $C$. vestita var. rubro-oculata, but in those with hydrochloric acid and sodium salicylate it is closer to $C$. rignieri.

Reaction-intensities Expressed by Light, Color, and Tempera-

Polarization:

C. vest. v. rubro-oc., moderate to very high, value 70 .

C. regnieri, very low to very high, much lower than in C. vestita var. rubro-oculata, value 35 .

C. bryan, very low to very high, intermediate between the parents, value 45.

Iodine:

C. vest. v. rubro-oc., moderate, value 50 .

C. regnieri, moderately light, lighter than in C. vestita var. rubrooculata, value 35 .

C. bryan, moderate, intermediate between the parents, value 38 . Gentian violet:

C. vest. v. rubro-oc., moderate to deep, value 60.

C. regnieri, light to moderately deep, lighter than in C. vestita var. rubro-oculata, value 50 .

C. bryan, moderate to moderately deep, intermediate between Safranin: parents, value 53

C. vest. v, rubro-oc., moderate to moderately deep, value 65 .

C. regnieri, moderate to moderately deep, lighter than in C. vestita var. rubro-oculata, value 60 .

C. bryan, moderate to moderately deep, intermediate between the parents, value 63 .

Temperature:

C. vest. v. rubro-oc., in the majority at 72 to $74^{\circ}$, in all at 74 to $75^{\circ}$, mean $74.5^{\circ}$

C. regnieri, in the majority at 70 to $72^{\circ}$, in all but rare grains at 76 to $78^{\circ}$, mean $77^{\circ}$

C. bryan, in the majority at 72 to $74^{\circ}$, in all but rare grains at 76 to $77^{\circ}$, mean $76.5^{\circ}$.
C. vestita var. rubro-oculata exhibits a higher reactivity than the other parent in all five reactions, the difference being very marked in the polarization reactions; slight in those with temperature; and little in the others. The hybrid $C$. bryan has intermediate reactivities between the parents in all of the reactions, being generally somewhat closer to $C$. vestita var. rubro-oculata than to the other parent.

Table A 46 shows the reaction-intensities in percentages of total starch gelatinized at definite intervals (seconds and minutes) :

\begin{tabular}{|c|c|c|c|c|c|c|c|c|c|c|}
\hline & $\stackrel{\dot{m}}{\dot{0}}$ & घं & $\begin{array}{c}\mathrm{g} \\
\mathrm{N}\end{array}$ & $\underset{\infty}{\dot{1}}$ & घं & gi & $\underset{\sim}{g}$ & 星 & $\begin{array}{l}\dot{g} \\
\text { \& }\end{array}$ & $\begin{array}{l}\dot{q} \\
8 \\
8\end{array}$ \\
\hline \multicolumn{11}{|l|}{ Chloral hydrate: } \\
\hline C. vest. v. rubro-oc ..... & . & . & . & . & . & 40 & 53 & 58 & 60 & 62 \\
\hline C. regnieri. . . . . . . . & . & . & . & . & . & 67 & 95 & 99 & . & \\
\hline C. bryan .... & . & . & . & . & . & 61 & 75 & 89 & 91 & 94 \\
\hline Chromic acid: & & & & & & & & & & \\
\hline C. vest. v. rubro-oc ..... & $\cdots$ & $\ldots$ & $\cdots$ & $\cdots$ & $\ldots$ & 10 & 65 & 80 & 92 & 96 \\
\hline C. regnieri. . . . . . . . . & . & . & . & . & . & 75 & 90 & 99 & $\ldots$ & . \\
\hline 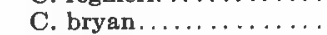 & . & . & . & $\cdots$ & . & 40 & 85 & 93 & 99 & $\cdots$ \\
\hline \multicolumn{11}{|l|}{ Pyrogallic acid: } \\
\hline C. vest. v. rubro & . & $\cdots$ & . & $\cdots$ & $\cdots$ & 10 & 20 & 60 & 84 & 89 \\
\hline C. regnieri.... & . & . & $\ldots$ & . & . & 25 & 66 & 93 & 96 & 98 \\
\hline C. bryan ..... & . & . & . & . & . & 15 & 33 & 80 & 85 & 92 \\
\hline \multicolumn{11}{|l|}{ Nitric acid: } \\
\hline C. vest. v. rubr & . & . & . & . & . & 61 & 64 & 71 & 73 & 78 \\
\hline C. regnieri. ... & . & . & . & $\cdots$ & $\cdots$ & 86 & 93 & 96 & & . \\
\hline C. bryan..... & . & . & . & . & . & 62 & 75 & 81 & 88 & 89 \\
\hline \multicolumn{11}{|l|}{ Sulphuric acid: } \\
\hline C. vest. v. rubro-oc & 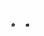 & . & . & 81 & . & 99 & . & $\cdots$ & $\cdots$ & \\
\hline C. regnieri. ....... & 99 & . & . & $\ldots$ & . & . & $\cdots$ & . & . & \\
\hline C. bryan. ...... & $\cdots$ & . & . & 97 & . & 99 & $\cdots$ & . & . & . \\
\hline \multicolumn{11}{|l|}{ Hydrochloric acid: } \\
\hline C. vest. v. rubro-oc & . & . & . & . & . & 18 & 33 & 64 & 71 & 78 \\
\hline C. regnieri. ... & . & . & . & . & . & 42 & 71 & 89 & 92 & 95 \\
\hline C. bryan.......... & . & . & . & . & . & 58 & 74 & 92 & 94 & 96 \\
\hline \multicolumn{11}{|l|}{ Potassium hydroxide: } \\
\hline C. vest. v. rubro-oc & . & . & . & . & . & 54 & 65 & 72 & 75 & 77 \\
\hline C. regnieri. ... & . & . & . & $\ldots$ & . & 77 & 80 & 85 & 90 & 93 \\
\hline C. bryan....... & . & . & . & . & . & 53 & 62 & 71 & 75 & 77 \\
\hline \multicolumn{11}{|l|}{ Sodium salicylate: } \\
\hline C. vest. v. rubro oc & $\cdots$ & . & $\cdots$ & $\cdots$ & . & 15 & 83 & 98 & . & $\cdots$ \\
\hline C. regnieri. ... & . & . & . & 96 & . & 99 & & $\cdots$ & . & \\
\hline C. bryan.......... & . & . & . & . & .. & 53 & 99 & $\ldots$ & . & . \\
\hline
\end{tabular}

\section{Veloctty-reaction CuRves.}

This section treats of the velocity-reaction curves of the starches of Calanthe vestita var. rubro-oculata, $C$. regnieri, and $C$. bryan, showing the quantitative differences in the behavior toward different reagents at definite time-intervals. (Charts D 627 to D 634.)

Among the most conspicuous features of these charts are: The generally close correspondence in the courses of all three curves. The well-marked separation of the parental curves, even in the sulphuric-acid reactions, which occur very quickly, there being as high a gelatinization of one parent in one-half a minute as in the other in 5 minutes. The curve of $C$. vestita var. rubrooculata is lower than the curve of the other parent in all of the 8 reactions. The curves of the hybrid show a very marked tendency to intermediateness, and when not mid-intermediate the inclination seems to be more marked toward the pollen parent. In other reactions, in one there is sameness, in relation to the seed parent and in another the hybrid reaction is the highest of the three and nearer the pollen parent. A tendency to an early 
period of high resistance followed by a rapid to moderate gelatinization is not noticeable excepting the reactions with chromic acid, pyrogallic acid, and sodium salicylate with $C$. vestita var. rubro-oculata, and in the pyrogallicacid reaction with the hybrid $C$. bryan. The earliest period during the 60 minutes at which it is best for the differentiation of the three starches seems, for chromic acid, sulphuric acid, hydrochloric acid, potassium hydroxide, and sodium salicylate, at 5 minutes; for pyrogallic acid at 10 minutes; and for chloral hydrate and nitric acid at 15 minutes.

\section{Reaction-Intensities of the Hybrid.}

This section treats of the reaction-intensities of the hybrid as regards sameness, intermediateness, excess, and deficit in relation to the parents. (Table A 46 and Charts D 627 to D 634.)

The reactivities of the hybrid are the same as those of the seed parent in the potassium-hydroxide reaction; the same as those of the pollen parent or both parents in none; intermediate in the polarization, iodine, gentian violet, safranin, temperature, chloral hydrate, chromic acid, pyrogallic acid, nitric acid, sulphuric acid, and sodium salicylate reactions (in 1 being closer to the seed parent, in 4 closer to the pollen parent, and in 5 being mid-intermediate); highest in the hydrochloricacid reaction, and closer to the pollen parent; and the lowest in none.

The following is a summary of the reaction-intensities: Same as seed parent, 1; same as pollen parent, 0 ; same as both parents, 0 ; intermediate, 11; highest, 1 ; lowest, 0 .

The pollen parent seems to have been more effective than the seed parent in determining the characters of the starch of the hybrid. Intermediateness is quite marked, and in about one-half of these reactions there is midintermediateness.

\section{Composite Curves of the Reaction-intensities.}

This section treats of the composite curves of the reaction-intensities, showing the differentiation of the starches of Calanthe vestita var. rubro-oculata, C. regnieri, and C.bryan. (Chart E 46.)

The most conspicuous features of this chart are: The very close correspondence in the rises and falls of all three curves excepting in the chloral-hydrate reactions, in which the curve of $C$. vestita var. rubro-oculata falls instead of rises in harmony with the curves of the other parent and the hybrid, as in the preceding set of Calanthe. The marked separation of the curves of the two parents in the reactions with polarization, chloral hydrate, chromic acid, pyrogallic acid, and nitric acid, and their closeness in the others. The tendency in general for the curve of the hybrid to have a position of some degree of intermediateness and with an apparent closer relationship to C. regnieri than to the other parent. The higher position of the curve of $C$. vestita var. rubrooculata than that of the other parent in the reactions with polarization, iodine, gentian violet, safranin, and temperature; and the lower positions with chloral hydrate, chromic acid, pyrogallic acid, nitric acid, sulphuric acid, hydrochloric acid, and potassium hydroxide. In
C. vestita var. rubro-oculata the very high reaction with sulphuric acid; the high reactions with polarization and safranin; the moderate reactions with iodine, gentian violet, and chromic acid; and the low reactions with temperature, chloral hydrate, pyrogallic acid, nitric acid, hydrochloric acid, and potassium hydroxide. In $C$. regnievi the very high reactions with chloral hydrate and sulphuric acid; the high reactions with safranin, chromic acid, pyrogallic acid, and nitric acid; the moderate reactions with gentian violet, hydrochloric acid, and potassium hydroxide; and the low reactions with polarization, iodine, and temperature. In the hybrid C. bryan the high reaction with sulphuric acid; the high reactions with safranin and chromic acid; the moderate reactions with polarization, gentian violet, chloral hydrate, chromic acid, pyrogallic acid, and hydrochloric acid; and the low reactions with iodine, temperature, nitric acid, and potassium hydroxide.

Following is a summary of the reaction-intensities (12 reactions) :

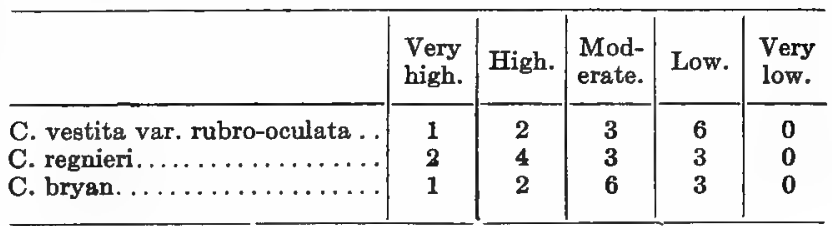

Notes on the CaLanthes.

In comparing the two composite-curve charts it will be observed that the curves correspond with sufficient closeness to indicate a common generic type. The three parents show marked closeness (or even a practical identity) in the reactions with iodine, gentian violet, safranin, temperature, sulphuric acid, and potassium hydroxide; but more or less marked differences in those with polarization, chloral hydrate, chromic acid, pyrogallic acid, nitric acid, and hydrochloric acid. The greatest interest in these charts doubtless centers in the differences in the relations of the hybrid curves to the parental curves, in the first set the hybrid curve tending in general to follow more closely the parent (seed parent) having the higher mean reactivity, and in the second set to follow more closely the parent (pollen parent) having the lower mean reactivity. In both sets $C$. vestita var. rubro-oculata is a parent, in one the pollen parent and in the other the seed parent, but in neither does the hybrid show as much closeness to it as to the other parent. The relations of the hybrid curves as regards sameness, intermediateness, and excess are quite different, as indicated in the summaries. Owing to peculiarities of the grains of Calanthe referred to in Part II, page 769 , the studies of the reactions with different reagents were limited to comparatively few of the reagents, and it is obvious for reasons stated that the data recorded must be accepted with reserve.

\section{Notes ON THE ORCHIDS.}

The composite curve charts of Phaius and Miltonia are very much alike, indicating closely related genera, and quite different from those of Cymbidium and Calanthe, which differ very markedly from each other and also from Phaius and Miltonia. 


\title{
- CHAPTER IV.
}

\section{GENERAL AND SPECIAL CONSIDERATIONS OF THE REACTION-INTENSITIES OF THE STARCHES OF PARENT-STOCKS AND HYBRID-STOCKS.}

\author{
(Charts A 1 to A 26, B 1 to B 42, C 1, D 1 to D 691, E I to E 46, and F 1 to F 14. Tables B 1 and B 2.)
}

The reaction-intensities of starches lend themselves admirably to presentation in the form of charts, which charts in turn are peculiarly well adapted for comparative purposes. It has been found advantageous, as stated in Chapter II, to render these data in three main and various special forms of charts, each serving to accentuate some special feature or features of the reactions. Of the three main forms, one presents the reactionintensities of different starches with each agent and reagent with reference especially to the specific properties of each agent and reagent, and to these peculiarities with reference to varietal, species, subgeneric, and generic groupings; another form exhibits in particular the progress of gelatinization of the starches of the parents and hybrid with different reagents in terms of percentage of starch gelatinized; and a third form gives a composite picture of the reaction-intensities of the starches of the parents and hybrid with all or some of the agents and reagents which serves in a special way to differentiate varieties, species, subgenera, and genera, and to exhibit the relations of parents and hybrids. These three forms of charts are included in the present chapter under the corresponding headings above given, and several special charts have been added which later receive adequate attention. The second and third forms have had more or less detailed comment in the preceding chapter, but additional remarks that are desirable or necessary will follow in the second and third sections of this chapter. The first form of chart will be taken up for consideration in the immediately following section. It has been found advantageous to present these charts in two series, A 1 to A 26 and B 1 to B 42, which series are complementary, but demand separate consideration. The first series gives the reaction-intensities of all or most of the starches, and the second series only those of selected starches, the reasons for the latter being stated in subsequent pages.

\section{Reaction-intensities of Starches with Each Agent and Reagent. \\ (Charts A 1 to A 26.)}

The reaction-intensities of different starches with different agents and reagents differ within wide extremes, owing in part to inherent peculiarities of the starch molecules and in part to peculiarities of the reagents as regards both chemical composition and concentration of solution. In some instances the starch molecules alone or largely determine the reaction, while in others both starch and reagent play important parts, as in chemical reactions generally. Thus, as will be stated fully later on, in the polarization reaction the starch molecule undergoes no change, the reaction being physical; hence it expresses peculiarities that are inherent to the molecule. In the gentian-violet and safranin reactions the organization of the molecule is either unaffected or affected to an undetectable degree, the reactions being presumably adsorption phenomena. In the iodine reaction there is probably a combination of the iodine and starch, but without apparent intermolecular disorganization. In the temperature and chemical-reagent reactions there is an intermolecular breaking down by a process of hydration, with which process there may be associated reactions that vary in character in accordance with peculiarities of the composition of the reagents. If the molecules of the starches from different sources are in the form of stereoisomers it follows, as a corollary, that they must act differently with different agents and reagents and that, inasmuch as the agents and reagents differ, each starch should show differences that are related to variation in the kind of agent and in the composition and concentration of the reagents. In other words, the reaction in each case is conditioned by the kind of starch and the kind of agent or reagent. Such is in fact what has been found experimentally, as the subsequent data show.

The most conspicuous features of these charts may be summed up as follows, consideration in detail being given under the corresponding headings:

The wide range of reaction-intensities, the extent of which varying with the different agents and reagents, and being most marked with the reagents.

The manifest tendency to grouping of the reaction-intensities of different starches in harmony in general with botanical groupings.

The individuality or specificity of each chart that is definitely related to the character of the agent or reagent, this characteristic being most obvious in the reactions in which the starch molecule is disorganized.

The specificities of the components of the reagents that are accountable for variations in the reaction-intensities and in the qualitative changes apart from those dependent upon differences in stereoisomeric forms of starch.

The variable relationships of the reaction-intensities in the different charts as regards sameness, intermediateness, excess and deficit of reactions of the hybrid starch in comparison with the parental starches.

Variations in the reaction-intensities of the starches as regards height, sum, and a rerage.

The average temperatures of gelatinization compared with the average reaction-intensities. 
Wide Range of Reaction-Intensities.

(Charts A 1 to A 26, )

In comparing the range of reaction-intensities it must be borne in mind that the values expressed in the polarization, iodine, gentian-violet, safranin, temperature, and chemical-reagent charts are not formulated upon the same basis of calibration. In the first four instances the values are grossly quantitative, and the abscissæ are founded upon crude and entirely arbitrary standards and do not likely represent values that are equivalent to those of the temperature or chemical-reagent records. The temperature values are based upon a scale that is different from those of the first group and from those of the chemical reagents. The calibrations in the first group, apart from the crudeness, are probably defective because the reaction-intensities of the starches studied do not extend, as in the case of those of the chemical reagents, between the extreme limits of the chart. The range in the temperature of gelatinization charts closely resembles in its limitations the ranges in the iodine, gentian-violet, and safranin charts.

In these charts the abscissæ-values, in comparison with the corresponding values in the chemical-reagent charts, are much too limited, but at present we have no data which enable us to state (in terms of light, color, and temperature reactions) the equivalent of a given reaction-intensity that is expressed in time-per cent of starch gelatinized. For instance, a difference of $2.5^{\circ}$ in the temperature of gelatinization which is represented by the space between two abscissæ appears small on the chart, yet this difference may have a differential value that is equal to several times this abscissæ-value in the chemical-reagent charts. These temperature differences would have been nearly equitably expressed in comparison with the chemical-reagent values had the temperature scale been between the extremes of say $50^{\circ}$ and $85^{\circ}$ instead of $40^{\circ}$ and $95^{\circ}$. A similar change could have been made to advantage in the scales of the other charts mentioned. Comparing cursorily these five charts (A 1 to A 5), it will be noted that notwithstanding the comparatively limited ranges of reaction-activities each may readily be distinguished from the others, with the exception of the gentian-violet and safranin charts, which are very much alike and which, while easily differentiated from the other charts, are distinguished from each other only and doubtfully by careful comparison (see also Chart B 2). In fact, the differences in the latter are unimportant because the crudeness of the method of valuation probably makes them fall within the limits of error or observation. Among the chemical-reagent charts the variations in reaction-intensities range in nearly all, from reactions which are complete within a few seconds to those in which so little as 2 per cent or less of the starch is gelatinized in 60 minutes. In exceptional charts (Charts A 10 and A 18, sulphuric acid and sodium salicylate) the extent of the variations is distinctly limited generally because of rapidity of gelatinization of the starches, in the former most of the reactions being shown to be complete within 5 minutes, and in the latter within $\mathbf{1 5}$ minutes.
Manifest Tendency to Groupings of ReactionINTENSITIES.

In both the preceding and present researches, particularly in the former because of the relatively large numbers of species and varieties included among many of the several genera, it has been found that the reactionintensities of the representatives of a genus tend to be confined usually within well-restricted limits, the maxima and minima reactions of members of the genus being in general wider apart as they are botanically farther separated, the greatest differences being noted when specimens are included which belong to well-defined generic subdivisions. Where the representatives of a genus are not so far separated as to fall into such subdivisions, the variations tend to be confined to a space on the charts that rarely exceeds 3 to 5 abscissæ (22 being the chart limit), frequently less; but where there are representatives that belong to different well-defined subgeneric divisions (for instance, subgenera, tender and hardy species, tuberous and rhizomatous forms, etc.) the variations are, on the whole, much more-extensive, equivalent usually to the space of 10 to 20 abscissæ or they may extend to practically the extremes of the chart. As extraordinary as it may seem, while such extreme variations may be found with one reagent, little or no difference may be found with another reagent; and with other reagents all intermediate values may be noted between these extremes. These facts are well illustrated in Begonia: No differences are noted in the reaction-intensities of these starches in Charts A 10 and A 12 (sulphuric-acid and potassium-hydroxide reactions), gelatinization in all being complete within less than a minute; while in a number of other charts (as in Chart A 9, the nitric-acid reactions) the same remarkably rapid reaction occurs in the starch of only one of the parents and in the hybrid, while the reaction of the other parental starch is remarkably slow.

The extent of generic differentiation varies in the different charts. Some differentiation is evident, for instance, in Charts A 6, A 15, A 18 (chloral-hydrate, potassium-sulphide, and sodium-salicylate reactions); there is better differentiation in Chart A 7 (chromicacid reactions); and still better differentiation in Chart A 8 (pyrogallic-acid reactions). The grouping of members of a genus and the differentiation of the genus upon the basis of reaction-intensities can be rendered satisfactory only when large numbers of members of each genus are studied; when the maximum, minimum, and average values are determined with a number of reagents; and when it is recognized that members of subgenera and of other generic divisions may exhibit in the sum of their reactions differences that may be as divergent as those of different genera. For instance, in Nerine, it will be seen that in 17 of the 26 charts the values of the 3 groups are within very restricted limits and constitute a group of close values; and, moreover, that while the maximum, minimum, and average values of the group may be about the same as the corresponding values of other generic groups, in certain reactions they will be found to be different, so that in the final summing up the genus stands very distinctly apart from the other genera. In the remaining 9 charts there are varying degrees of departure from this well-defined grouping, 
chiefly because of the comparative less reactivity of the first set of parents and hybrid than of the other sets. In Chart A 6 (chloral-hydrate reactions) there i: marked extension of the maximal and minimal limits of the reactions owing to the prolongation of 4 of the 11 lines, so that the group is nothing like so distinctly individualized as in the 17 charts referred to wherein the maxima and minima are close. In Charts A9, A11, A12, $A 14, A 15$, and $A 21$ (nitric acid, hydrochloric acid, potassium hydroxide, potassium sulphocyanate, potassium sulphide, and strontium nitrate) there is a wellmarked separation of the first from the second and third sets, the latter showing about the same, and the former distinctly higher reaction-intensities. Such peculiarities are found to be common among the other genera where a number of sets of parents and hybrids are included, from which it is obvious that where a genus is represented by a single such set the maximum, minimum, and mean reactive-intensities are to be taken merely tentatively as representing the generic standards.

This statement finds immediate application to a number of generic groups represented in these charts, including Amaryllis-brunsvigia (bigeneric), Gladiolus, Tritonia, Richardia, Musa, Phaius, Miltonia, and Cymbidium. The maximum, minimum, and average values differ not only in the case of different sets of parents and hybrids of the same genus, but also of the members of the same set with different reagents. Thus, in Nerine, in Charts A 8 and A 17 (pyrogallic-acid and sodium-sulphide reactions) and in certain other charts, the maxima, minima, and averages for all of the species and hybrids are practically absolutely the same, but in Charts A 11 and A 14 (hydrochloric-acid and potassium-sulphocyanate reactions) and in others, all three are different in all three sets of starches. Finally, generic grouping may seem ingly be set aside in some instances by wide differences in the reaction-intensities of one or more sets included in the genus group. This is well illustrated in Crinum, Iris, and Begonia in Chart A 9 (nitric-acid reactions). The species of Crinum studied in this research are divisible into two horticultural groups, which are distinguished as tender and hardy, the starch of the former being characterized by generally low reactivities and those of the latter by generally high reactivities, the differences being so marked that it is necessary to recognize in these starches two distinct subgeneric groups. Such differences are well shown in other charts, such as Charts A 8, $A 10, A 11$, and $A 12$, but there is an entire absence of such distinction in Charts A 6, A 7, A 15, A 19, A 22, A 23, A 25, and others. In fact, in several of the latter the differences are so slight as to suggest very closely related members of the genus. In Iris there is a very conspicuous example of subgeneric grouping: In Charts $\mathrm{A} 5, \mathrm{~A} 6, \mathrm{~A} \%, \mathrm{~A} 10$, and $\mathrm{A} 15$ the reaction-intensities of the members of all four sets are nearly the same or do not differ to a marked degree; but in A 8, A 9, A 11, A 12, A 13, A 14, A 16, A 17, A 18, A 19, A 20, A 21, A 22, A 23, A 24, A 25, and A 26 there is a well-marked grouping, the first three sets constituting one group and the last set another group.

With the exception of Charts A 6 and A 18 the first group is characterized by lower reaction-intensities, which with rare exceptions tend to be very close in all three sets, thus forming a very distinct group. While in Charts A 6 and A 18 the same grouping remains, there is a reversal of the reaction-intensities, the first group showing less reactivity than the second group. Even more interesting is Begonia: In Chart A 9 there is no obvious differentiation of any of the sets of members of a set, but in Chart A 6 there appears a very conspicuous differentiation in the comparative slowness of the $B$. socotrana reaction; and in all other charts, with four exceptions, the length of the line is accentuated in varying degree, thus markedly characterizing the sets of this group. This seemingly aberrant reaction-intensity of this exceptional species gives a peculiar generic picture, and means, as in the instances of Crinum and Iris, two generic types.

The correspondence of the grouping of the reactionintensities of starches in accordance in general with genera is usually quite evident, this being not only more marked with some than with other agents and reagents, as stated, but also more marked with some than with other groups. A given group may stand out very conspicuously in one chart, but not in another, or even not be differentiated from adjoining groups, yet be more or less distinctly differentiated from the same groups in other charts. For instance, in Chart A 10 (sulphuricacid reactions), taking the genera represented by Nerine, Narcissus, Lilium, Iris, Gladiolus, and Tritonia, it will be seen that with the exception of Gladiolus there is no differentiation of the rcaction-values that even suggests that the records are those pertaining to different genera; in fact, they are so nearly alike as to indicate that the several groups belong to a single genus. The Gladiolus reactions take place with comparative slowness, which distinctly differentiates this genus from the five other genera. In Chart A 11 (hydrochloric-acid reactions) Lilium stands very distinctly apart from the other five genera; Nerine and Narcissus are not differentiated from each other, but they differ from Lilium, Iris, Gladiolus, and Tritonia.

It will be seen that three of the four sets of Irids are practically alike and markedly different from the fourth set, showing what marked differences may be exhibited by members of subgenera or of similar divisions of genera. In Chart A 12 (potassium-hydroxide reactions) the picture is radically changed in a number of particulars: Lilium remains conspicuous as before; $\mathrm{Ne}$ rine and Narcissus are very definitely grouped, the lines of the former being very short and those of the latter quite long; Iris differs but little, as a whole, from the preceding chart; and in both Gladiolus and Tritonia the lines are prolonged and about the same, giving no differentiation between these two genera. In Chart A 13 (potassium-iodide reactions) the picture again differs: Lilium is about the same; the Nerine lines are very considerably prolonged and markedly exceed the length of the Narcissus lines which are slightly shortened in comparison with the length in the preceding chart, thus showing a marked reversal of the quantitative relationships. The Narcissus lines and those of the first three sets of Irids are about the same, whereas in the preceding chart the latter are, on the whole, distinctly shorter; and Gladiolus and Tritonia are about the same, but longer than the Narcissus and Iris lines, and shorter than the 
Nerine lines. In Chart A 15 (potassium-sulphide reactions) Lilium remains the same; Nerine and Narcissus are distinctly different, the lines of the former being much shorter than those of the latter; and the lines of Narcissus, Iris (all four groups), Gladiolus, and Tri tonia are all prolonged to about the same level, so that there are no generic differentiations of these four genera. In Chart A 18 (sodium-salicylate reactions) there is a noticeable absence of resemblance of the lines collectively to those of any of the preceding charts. Here, Nerine, Narcissus, Lilium, and Iris (the first three sets of the last) are, on the whole, very much alike. The third set of Iris, which in the other charts shows greater reactivity than the other three sets, now shows the opposite relationship ; and, moreover, while this set in the previous charts is markedly different from Gladiolus and Tritonia, here it is the same. Similar differences will be found in other generic groups, in other sets, and also with other reagents. These characteristics demonstrate conclusively that the starches of different generic groups and subgroups differ within wide limits in their molecular structures; that there are very definite generic and subgeneric peculiarities; and that these differences can satisfactorily be reduced to figures and charts.

\section{Individuality or Specificity of Each Chart.}

The individuality or specificity of each chart is very pronounced and is most striking in the reactions in which there occurs intermolecular disorganization of the starch. Inasmuch as the starches are the same in each of the charts (except in some instances as to number), and the agents and reagents are variable, this individuality is definitely associated with peculiarities of the latter. Taking the charts, as a whole, it will be seen that no two are alike, although in exceptional instances, and for very obvious reasons, they differ in only minor degrees and even within the limits of error of experiment; well-marked examples of the latter are found in the gentian-violet and safranin, and in the copper-nitrate and cupric-chloride charts. On the other hand, where in accordance with general laboratory experience no material differences should be expected, excepting such as would be dependent upon differences in the concentration of the reagents, as in the potassium and sodiumhydroxide charts, respectively, the individualization is not only very marked, but also in a measure entirely independent of differences in concentration.

As previously stated, these 26 charts fall naturally into two primary divisions in accordance with whether or not in the reactions there occurs intermolecular disorganization. In conformity with recognized principles of physical chemistry, comparatively limited variations should, as a rule, be expected when in the reactions the starch molecules remain wholly or apparently intact, as in the polarization, iodine, gentian-violet, and safranin reactions; but wide to extremely wide variations when the molecules are broken down, especially in cases of reagents which may have multiple active components taking part in the disintegrative processes. As previously stated, the polarization reaction is a light reaction in which the molecules are undisturbed; the gentian-violet and safranin reactions are, in all likelihood, adsorptive phenomena which, as far as known, do not involve dis- arrangement of the starch molecules; and the iodine reaction seems to be of a kind in which an unstable iodide of starch is formed, but without obvious intermolecular disorganization; the temperature reaction is one of hydration which, while causing intermolecular breaking down, does not give rise to a loss of typical starch properties; and the reactions with the various chemical reagents are primarily phenomena of hydration, such as are brought about by heat, but modified quantitatively and qualitatively by differences in the components of the reagents which take part in the reaction.

It is obvious that the polarization reactions stand entirely apart from all others; that the gentian-violet and safranin reactions constitute an isolated pair; that the iodine reactions stand by themselves; and that the temperature and chemical-reagent reactions form a welldefined group, the former representing one and the latter another subgroup. In the temperature reaction we have a typical manifestation of the simplest form of the process of gelatinization, while in the chemical-reagent subgroup there is this same type but which is more or less materially modified by various substances that have chemical relations to the starch molecule. A comparison of the temperature and chemical-reagent charts will show that the latter not only differ markedly from the former, but also as much or more from each other. It would seem to follow, as a corollary, that the more varied and widespread the chemical disturbances in the starch molecules the more varied the reactions and the better the differentiation of genera, species, parents, and hybrids.

The individuality of each of the chemical-reagent charts that is definitely associated with peculiarities of the reagent is due in part to concentration and in part to composition of the reagent. This salient point is elicited clearly when the data recorded in any two arbitrarily selected charts are compared. Thus, taking Charts A 6 and A 7 (chloral-hydrate and chromic-acid reactions) a first glance will indicate that the average length of the ordinate in the former is greater than in the latter and, hence, that the concentration (reactive-intensity of the reagent) is less than in the latter; but it will also be very apparent, upon comparing the lengths of the ordinates of any given set of parents and hybrid, or of any generic group in the two charts, that the differences are not such as are to be expected were the reactionintensities exhibited by those reagents dependent solely upon differences in concentration.

Should the differences in the reaction-intensities depend merely upon differences in concentration (as of the same reagent) it seems obvious that if with a given starch the reaction with one reagent is equal to the length of say 2 abscissæ, and with another reagent to the length of 3 abscissæ, a corresponding though not necessarily proportional relationship should be found in the reactions of the different starches. In fact, not only may there be an entire absence of such quantitative relationship, but also a reversal of reaction-intensities, the reagent of higher concentration being the stronger in some reactions but the weaker in others. Thus, in Chart A 6 (chloralhydrate reactions), in the Amaryllis-Brunsvigia-Brunsdonna set, it will be seen that the ordinates for Amaryllis and Brunsvigia extend to the abscissæ values 96 and 82, respectively, and that those for the hybrids extend to 
30 and 28 , respectively ; meaning that 96 and 82 per cent, respectively, of the total starch was gelatinized in 60 minutes and that 95 per cent of the starch of each hybrid was gelatinized in 30 and 28 minutes, respectively. Turning now to Chart A 7 (chromic-acid reactions), it will be noted that while there is considerable shortening of the Amaryllis and Brunsvigia lines the hybrid ordinates are virtually absolutely the same. Taking the Hippeastrum, Homanthus, and Crinum groups, it will be noticed that in Chart A 6 the average reactivity of the Hippeastrum group is slightly less than the reactivities of the Homanthus and Crinum groups, which are nearly alike; while in Chart $A \%$ the average reactivity of the first group is greater than in either of the other groups, and the reactivity of the Crinum group is somewhat less than that of Hippeastrum group. In Chart A 6 the average reactivity of Nerine is greater than in Chart A 7, the reverse of what was noted in Amaryllis-Brunsvigia, Hippeastrum, Hcemanthus, and Crinum. In Narcissus the same reversal is noted except in one parent and the two hybrids of the first set. In Chart $A Y$ there are, in comparison with the preceding, generally higher reactivities of Lilium, Iris, Gladiolus, Tritonia, Musa, Phaius, Miltonia, Cymbidium, and Calanthe; but the opposite with Begonia. Among the first generic groups there will be found many exceptions-that is, lower reactivities. For instance, the reaction of Lilium martagon instead of being shorter is longer; the reaction of $L$. chalcedonicum and $L$. candidum are shorter, but not the reaction of $L$. testaceum; and those of $L$. pardalinum and $L$. parryi are shortened, while the reactivity of $L$. burbanki is lengthened. Similar inequalities appear in other groups. Finally, in Begonia the reactions with a single exception instead of being shorter are longer, especially the reaction of $B$. socotrana.

The remarkable differences in the behavior of different reagents, irrespective of concentration of solution, are perhaps better presented in charts of reactions of very closely allied reagents, for instance, in Charts A 12 and A 16 (potassium-hydroxide and sodium-hydroxide reactions). The average reaction-intensity exhibited by the potassium-hydroxide chart is in some instances greater and in others less than by the sodium-hydroxide chart. The records are so pregnant with interest that each set or group may with ample justification be taken up separately. Beginning with the Amaryllis-brunsvigia set it will be seen that with potassium hydroxide the reactions with the four starches occur with such rapidity that gelatinization is practically or absolutely complete within 1 minute; with sodium hydroxide all four reactions differ to so marked a degree that each is at a glance differentiated from the others-in Amaryllis $9^{7}$ per cent of the starch is gelatinized in 3 minutes, in Brunsvigia 95 per cent in 15 minutes, in Brunsdonna sandera alba 65 per cent in 60 minutes, and in Brunsdonna sanderc 88 per cent in 60 minutes. The average reactivity of Hippeastrum with potassium hydroxide is 74 per cent, with sodium hydroxide 44 per cent, in 60 minutes; that of $H o$ manthus is about the same with both reagents, the chief difference being seen in the marked elongation of the $H$. puniceus ordinate in the sodium-hydroxide reaction. The Crinum ordinates differ in the two charts very little, the only noticeable differences being seen in the $C$. moorei,
C. Kircape, and $C$. powellii ordinates, mostly not at all marked. In Nerine there are wide differences, the potassium hydroxide ordinates being very markedly shorter than those of sodium hydroxide, the former indicating almost if not complete gelatinization of all of the starches in 3 minutes or less, and the latter an average gelatinization of about 15 per cent in 60 minutes. This wide difference in comparison with what was noted in Hippeastrum, Hamanthus, and Crinum is remarkable. Narcissus, like the last three genera, does not show very much difference with these reagents, the averages being 63 and 83 per cent, respectively, in 60 minutes, the shortening being due almost wholly to the greater reactivities of the parents. The starches of Lilium gelatinize with great rapidity with both reagents. The Iris ordinates are longer throughout in the potassiumhydroxide chart except in case of $I$. trojana, the ordinate remaining the same in the sodium-hydroxide chart notwithstanding that the ordinates of the other parent ( $I$. iberica) and the hybrid ( $I$. ismali) are materially shortened. In Gladiolus and Tritonia the ordinates are very nearly the same in the potassium hydroxide chart, but both are shortened in the sodium-hydroxide chart, Gladiolus somewhat less than Tritonia. In Begonia a striking difference is seen in the $B$. socotrana ordinates but very little differences in the others; thus, in the potassium-hydroxide reaction this starch is completely gelatinized in one-sixth of a second, while in the sodiumhydroxide reaction only 84 per cent is gelatinized in 60 minutes-a remarkable difference. Richardia was not studied with sodium hydroxide. Musa, Phaius, Miltonia, and $C y m b i d i u m$ all show shorter ordinates generally with potassium hydroxide than with sodium hydroxide, the most conspicuous variation being noticed in the sodium-hydroxide chart in the markedly disproportionate elongation of the $M$. roezlii ordinate.

Similar characteristics are found in Charts A 15 and A 17 (potassium-sulphide and sodium-sulphide reactions), given groups acting with greater reactivity with potassium sulphide than with sodium sulphide, with others the reverse, and members of the same group bearing varying quantitative relationships in the two reactions, etc. The Amaryllis-Brunsvigia group has in the potassium-sulphide reactions much shorter ordinates than in the sodium-sulphide reactions, Amaryllis belladonna and Brunsdonna sanderæ being alike, and $B$. sanderce alba between them and the ordinate of Brunsvigia josephina; while in the sodium-sulphide chart the Amaryllis belladonna and Brunsvigia josephince ordinates are almost exactly the same, and those of the hybrids longer than those of the parents, and nearly alike. The Hippeastrum and Homanthus ordinates are, on the whole, closely alike in both charts, but the Crinum ordinates show some noticeable differences. The Nerine group is particularly conspicuous because of the less length of all of the ordinates in the potassium-sulphide chart than in the sodium-sulphide chart; because of the marked difference between the lengths of those of the first group and those of the second and third groups in the potassium-sulphide charts; and because all three groups have almost exactly the same length of ordinates in the sodium-sulphide chart. Narcissus has, to the contrary, distinctly longer ordinates in the potassium-sulphide 
chart than in the sodium-sulphide chart. Iris is, like Nerine, conspicuous by the differences of the ordinates, but particularly in reversed ways. The Iris ordinates in the potassium-sulphide chart are distinctly longer than in the other chart and they are of about the same length (the opposite to what is seen in Nerine); and in the sodium-sulphide chart the ordinates of three of the groups are the same, while those of the fourth group are much shortened. More or less marked differences in the two charts are seen in the remaining generic groups, especially in members of Begonia, Musa, and Miltonia.

Another pair of reagents that yield reactions worthy of especial examination are represented in Charts A 23 and $\mathrm{A} 24$ (copper-nitrate and cupric-chloride reactions). These two charts are in the corresponding groups almost the same throughout, the chief differences being noted in Crinum powellii, Lilium burbanki, Iris sindjarensis, I. pursind, Begonia mrs. heal, Musa gilletii, Miltonia (both parents and hybrid), and Cymbidium eburneo-lowianum. These differences are in every case such as not to fall within the limits of error of experiment.

Any two or more of these charts can thus be compared with the certainty of finding results that conform to those referred to in the preceding pairs.

The one feature above all others that serves to individualize each chart is the variable relationships of the reaction-intensities of the members of each of the different sets of parents and hybrid and of groups of sets in the different charts. For instance, taking the AmaryllisBrunsvigia set it will be seen upon comparing the different charts that differences in the average reactionintensities of this set in comparison with the differences in other sets and groups of sets are nothing like so striking and characteristic as are the differences in the group itself in the various charts. In other words, while there is a general tendency for the average reactionintensity of this group to rise or fall with the averages of other groups in the different charts, the individual members of the group exhibit marked independence in the direction and extent of the changes. Thus, in this group in the charts of chloral hydrate, pyrogallic acid, potassium iodide, potassium sulphocyanate, sodium hydroxide, sodium salicylate, cobalt nitrate, copper nitrate, cupric chloride, and mercuric chloride the four ordinates are in couples, the parental couple being in the chloralhydrate reaction shorter than the hybrid couple, but in the other reactions the reverse. In the reactions of chromic acid, nitric acid, hydrochloric acid, potassium hydroxide, sodium salicylate, and barium chloride all four ordinates are the same or closely the same, there being neither the coupling so obvious in the previous set nor any marked departure of any from an average standard. In the reactions of potassium sulphide, calcium nitrate, strontium nitrate, and uranium nitrate (with the exception of potassium sulphide and strontium nitrate) no two of the four ordinates are alike with any reagent, and the relative lengths of the four ordinates vary in the different reactions, the order of length being:

Potassium sulphide: Brunsvigia, Brunsdonna sanderce alba, Amaryllis, and Brunsdonna sandero.

Calcium nitrate: Brunsdonna sanderce alba, B. sanderce, Brunsvigia (these two being the same), and Amaryllis.
Strontium nitrate: Brunsvigia, Brunsdonna sanderce alba, $B$. sanderoe (these two being the same), Amaryllis.

Uranium nitrate: Brunsdonna sanderce alba, Brunsdonna sanderœ, Brunsvigia, and Amaryllis.

Such variations will be treated quite fully in the following subsection:

\section{The Specificities of the Components of the REAGENTS.}

(Charts B 1 to B 42.)

Inasmuch as different starches behave differently, qualitatively and quantitatively, with a given reagent, and a given starch differently with different reagents, it follows, as a corollary, that certain peculiarities of the reactions are to be attached to the starches and certain others to the reagents-in other words, the characters of the reactions are conditioned, as before stated, by both starch and reagent. In this research the phenomena of gelatinization have been taken as the chief indices in the differentiation of starches and it has been shown that a considerable variety of reagents may be used.

The terms gelatinized starch and soluble starch are used synonymously, yet starch may be in a soluble form without being gelatinized or gelatinizable, for it has been shown that raw starch through the agency of acid can be converted into soluble starch without apparent antecedent change in the structure of the starch grain that can be detected in the reaction of the grains in polarized light; that such grains can be dissolved in hot water without the appearance of gelatinization; and that such grains in solid form or in solution yield the blue starch-reaction with iodine. (See preceding memoir,* page 105.) It is therefore obvious that the changes expressed by gelatinization and solubility are independent, although usually associated; and, as a consequence, that a gelatinizing reagent may give rise coincidently to such molecular alterations as will convert an insoluble into a soluble and gelatinized starch or into a soluble but ungelatinizable starch. In all of the experiments with these reagents the former change has been brought about; but accompanying alterations may occur, hence, the question naturally arises in conjunction with the use of different reagents as to the meanings of the differences in the two cases.

It is of importance to note that in all of these investigations the soluble non-gelatinizable form was prepared by the use of acids, inorganic or organic, non-volatile or volatile. On the other hand, as far as the voluminous records go, alkalies always give rise to soluble starch of the gelatinized form. This indicates clearly that the actions of the acids and alkalies may be inherently quite different. When the grains are heated in water, gelatinization occurs at a given temperature, varying within narrow limits, the mean temperature differing in starches from different sources. In accordance with the foregoing, heat and alkalies may be placed in one and acids in another category, but without the assumption that the actions of the several members of each class are precisely the same. Gelatinization is undoubtedly due to a hydration of the starch molecules, but the alteration from

\footnotetext{
* Carnegie Inst. Wash. Pub. No. 173 (1913).
} 
the insoluble to the soluble non-gelatinizable form is apparently not in any way related to water, inasmuch as it may be brought about in anhydrous starch by anhydrous acetic acid, and is therefore an anhydrous process unless water is derived in some obscure way by intramolecular disorganization. There is at all events no intermolecular disorganization such as occurs antecedent to and associated with obvious gelation.

The foregoing changes in the starch molecules in association with the more or less marked differences exhibited by a given starch in the reactions with different reagents indicate clearly that beneath and overshadowed by the conspicuous phenomena of gelation there lay processes or reactions that vary, within even wide limits, in relation to the components of the reagents. Moreover, raw starch presents certain very striking characteristics in its relations to water, entirely apart from the phenomena of hydration that is expressed by gelation. It has been found that raw starch is not only highly hygroscopic and clings tenaceously to water, but also that its behavior toward water is in certain respects different from that of hydrated starch, the percentage of water in the raw grains being influenced to a very limited degree and that of hydrated starch to a maximum degree, in the presence of water by changes in temperature. Airdried starches from different sources have been found to contain from 9.9 to 35 per cent of water; the figure varying with the kind of starch, impurities, and percentage of moisture in the air. Freshly prepared starch may contain as much as 45 per cent of water. Anhydrous starch is obtained by subjecting the starch to a temperature of $120^{\circ}$ or in vacuo at $100^{\circ}$. Starch that has been partially or completely dehydrated and then placed in water at room temperature takes up water very rapidly with the evolution of heat, the amount being in direct relationship to the degree of dehydration and the kind and amount of starch. A preparation consisting of 20 grams of air-dried potato starch in 20 grams of water showed an increase of temperature equal to $3^{\circ}$; and a similar preparation of anyhydrous starch, an increase of $13.8^{\circ}$. The formation of heat has been ascribed to an actual chemical combination of the starch and water (see preceding memoir, page 167 ), but it can satisfactorily and better be accounted for upon the basis of adsorption (which, however, is in fact a form of chemical union).

The level of aqueous saturation is maintained within very narrow limits, and it is very much more influenced by variations in external moisture than by changes in temperature that occur below the temperature of gelation; and it is reached before there is the least detectable change in the starch grain or starch molecule. This level is, however, not only materially higher in hydrated starch, but also variable within wide degrees and in direct relation to moisture and temperature, and it probably reaches its highest level at the baking temperature of bread (Katz, Zeitsch. physiol. Chem., 1915, XCV, 104). As the temperature falls, even though in the presence of an atmosphere saturated with moisture, there is some reversion of hydrated starch to raw or insoluble starch.

Starch grains do not either gelatinize or pass into solution in their normal state because apparently of the existence of some peculiar surface condition which, like an osmotic membrane, serves to prevent a further inflow of water after a certain level of partial saturation has been reached, and which likewise prevents an outflow of water as long as external conditions are unalteredin other words, maintains a state of physico-chemical equilibrium as regards water within and without the starch grain. That such a surface condition exists seems evident in the sudden dissipation of this level at the temperature of gelation and in the absence of this level in comminuted and otherwise injured grains in which the starch molecules of the interior of the grain are freely exposed to the water. The intracapsular starch thus exposed exhibits a similar but not identical surface condition, which is owing to differences in the intracapsular and capsular starches, as will be noted more particularly later. Therefore, in studying the phenomena of gelatinization and absorption of water both of these surface conditions must be considered, as must also be both forms of starch.

When raw starch in water is subjected to slowly rising temperature, at a certain temperature that varies for different starches and within narrow limits for each starch there occurs a loss of anisotropy (which indicates an intermolecular disorganization) that is immediately followed by a rapid taking up of water attended by swelling and gelatinization. This disappearance of anisotropy is taken to mean that immediately antecedent a modification or removal of the surface condition has occurred. This surface condition may likewise be affected by various gelatinizing reagents such as have been used in this research, and thus hydration of the starch grain permitted as in the case of gelation by heat; or there may be the opposite effect, as when there is present a sufficient quantity of alcohol, acetone, alcohol-ether, brine or other so-called dehydrating reagent. Analogous phenomena have been noted in the study of certain other colloids, from which it seems that heat and other gelatinizing agents are effective by affecting primarily the surface condition, thus giving rise to an alteration in the level of aqueous saturation. The underlying cause of this peculiar surface condition is at present problematical, but it seems that it is to be located directly or indirectly either in a hypothetical deposit on the surface of the grain by the cell-sap or in the modified form of the starch that constitutes the capsular part of the grain (the so-called starch cellulose). This part of the grain is the last to be deposited, and it differs from the inner part (or so-called starch granulose) especially in density, solubility in cold and hot water, digestibility, dextrin products of digestion, resistance to decomposing agents, and in both quantitative and qualitative color reactions with iodine. The degree of resistance varies in starches from different sources, and it is so marked in some instances in the initial stage of the reaction as to render gelatinization very slow for a period varying from 1 to 10 minutes, to be followed by gelatinization that varies in rapidity from slow to very rapid, as will be seen by an examination of Charts D 1 to D 691 that exhibit the velocities of gelatinization. Upon this assumption, any agent which affects the physico-chemical condition of the capsular part of the grain will modify the surface conditions or 
surface tension so that hydration may be augmented or inhibited.

As stated elsewhere (see preceding memoir, pages 95 and 96), while there can be no doubt of the essential part played by water in the swelling, gelatinization, pseudosolution, and true solution of starch, it seems that none of these phenomena is due to either hydrolysis (decomposition in which molecules of water are taken up and become an integral part of the molecules) or hydration in the strictly chemical sense (the formation of derivatives in which basic matter is substituted by hydrogen atoms of water, or the actual combination of water so that the molecules of water constitute intramolecular components of the derivatives). The terms hydrolysis and hydration are often used synonymously, but at times incorrectly, because while hydration may mean hydrolysis, it may on the other hand signify a union or impregnation with water which is an extramolecular and not an intramolecular phenomenon. According to the recent developments of physical chemistry, none of the processes concerned in the conversion of raw starch into the so-called soluble starch, of which starch-paste and pseudo-solution and true solution are simple modifications, is one of hydrolysis or hydration in the strictly chemical sense, but one of adsorption, that is, an extramolecular union with water that is of a physico-chemical character, such, for instance, as is observed in the deposition of moisture on glass and the taking up of water by hygroscopic substances in which there may be no true chemical union in the conventional meaning, but a mere surface combination or surface condensation. The combination is, of course, actually chemical, but it is not chemical in the customary sense any more than is the solution of sugar in water chemical, and thus in the form technically of a hydrate. Starch in common with other organic colloids is hygroscopic, and the so-called process of hydration or hydrolysis that is associated with swelling and gelatinization is explicable upon the basis of adsorption-that is, a physico-chemical affinity that is specific and selective, and supplemental to satisfied affinities according to the laws of stoichiometry. This, however, does not preclude the possibility or probability of the occasional occurrence, of reagent reactions that are strictly speaking those of hydration.

It seems clear from the foregoing that in the gelatinization of normal starch grains the first and essential step is the modification or dissipation of the surface condition that prevents an inflow of water after the normal point of partial saturation, or state of physicochemical equilibrium as regards water, has been reached. This barrier it seems is not mechanical but physicochemical, as is suggested by the fact that corresponding or analogous phenomena have been observed in the behavior of other colloids in vitro and in the living cells, where it seems to have been clearly demonstrated that they are manifestations of surface tension. Heat, when a certain temperature is reached, is assumed to give rise to a surface alteration or change in surface tension that causes a mass action of the molecules of water with a consequent inflow of water and attendant gelatinization, and it has been found that the addition of various substances to the water may lower or raise the temperature of gelatinization-in other words, aid or oppose the action of heat in altering the surface tension. The various gelatinizing reagents which are active at room temperature are undoubtedly effective by causing similar or identical alterations in surface tension, for evidence has been found that the ions do not form an adsorption union with the starch molecules but give rise to the surface alteration that leads to an adsorption union of molecules of water and starch; and it would seem to follow, in accordance with our knowledge of the behavior of other colloids with ions and molecules of different kinds, that this surface change, as well as subsequent phenomena, are modifiable in relation to the kinds and concentrations of ions and molecules taking part in the reactions. Hence, the phenomena of gelatinization brought about in distilled water by heat would likely be different in certain respects from those due to some chemical reagent, such as chromic acid; and those of any given reagent will differ from those of every other reagent. Such is in fact what has been found in this research

Samac (Studien über Pflanzenkolloide I. Die Lösungsquellung der Stärke bei Gegenwart von Kristalloiden. Dresden, 1912, S. 42) made studies with potato starch in which he used equimolecular solutions of various electrolytes and non-electrolytes in concentrations varying from 0.25 to 10 gram-molecules to the liter. Both cations and anions were found to be effective. Lithium, sodium, potassium, ammonium, magnesium, calcium, strontium, and barium chloride in weak solution raised the temperature of gelatinization; and with increasing increments of concentration there occurred with some a further elevation followed by a fall, but with others a fall, the effects being different according to the kind of cation present. Sulphate, oxalate, tartrate, acetate, chloride, bromide, nitrate, iodide, sulphocyanate, and carbonate of potassium, and also calcium nitrate, sodium sulphate, and ammonium sulphate, behaved differently in accordance with the kind of anion. With some, in any concentration, the temperature of gelatinization was raised; with others, with increasing increments of concentration a rise was followed by a fall; and with others there was a fall with any concentration. Sulphuric acid, hydrochloric acid, and acetic acid likewise caused varying effects. With sulphuric acid and hydrochloric acid increasing increments of concentration caused a rise followed by a fall, while under the same conditions acetic acid caused a fall. Both potassium hydroxide and ammonia in all concentrations caused a fall. Dextrose and glycerin, which are in any concentration without detectable gelatinizing action at room temperatures, caused with increasing increments of concentration a steady elevation of the temperature of gelatinization; and urea and chloral hydrate, under the same conditions, caused a steady lowering. Both acetic acid and potassium hydroxide in any concentration caused a fall ; but acetate of potassium in increasing increments of concentration caused a rise followed by a fall. These results are in harmony with those obtained by various investigators in swelling and precipitation experiments with proteins.

The starch molecule like the protein molecule has the property of acting as an acid or base to form salts, this being explicable upon the assumption that both starch and protein molecules are produced by a condensation 
of two different kinds of groups. The starch molecule behaves as an amphoteric electrolyte, acting as an acid or base in relation to the components of the reagents to form different salts, the reactions being attended by the splitting off of hydrogen or hydroxyl ions. All of the reagents used in this research to gelatinize starch are aqueous solutions of electrolytes or imperfect electrolytes, and hence each is partially ionized, the degree of ionization varying with the different reagents; moreover, there is a variety of elements and molecules, acid and base, that may enter into chemical combination with the starch molecules. Hence it follows that each solution is a complex that consists of molecules of water and solute, and of ions of water and of solute. Having now a starch molecule that may assume either acid or basic properties, and reagents that contain both water and various kinds of elements and molecules that may enter into chemical combination with the starch to form salts, it is obvious that the phenomena of gelatinization or swelling, quantitatively and qualitatively, may vary more or less markedly in accordance with the chemical reactions that occur coincidently with the adsorption of water. An examination of the list of reagents used in this research will show that there are well-defined classifications or groupings in accordance with peculiarities of the substances entering into the reagents as the solutes, as, for instance, organic acid, inorganic acids, potassium salts, sodium salts, hydroxides, sulphides, nitrates, chlorides, ete. Not only are variations to be expected in the reactions because of differences in the composition of these reagents, but also because of differences in the molecular arrangements of the starch molecules. If the starches from different plant sources exist in different stereoisomeric forms, it seems upon the basis of our knowledge of the peculiarities of stereoisomers in general that variations in the reactions that are due to this peculiarity may be as great or even greater than those due to differences in the reagents - that is, that variations in the reactions of different starches with a given reagent may be as marked or more marked than those in the case of a single starch with different reagents. This has been found to be a fact by the results of this research.

In the study of the phenomena of gelatinization that are definitely associated with peculiarities of the reagents the object has been to demonstrate differences in the behavior of different reagents without reference to the cause of these differences, except as they go to prove the existence of starch in stereoisomeric forms that are modified in specific relationship to the plant source. Obviously, there would be many advantages in a combined study of both gross phenomena of gelatinization and reactions that occur during and subsequent to gelatinization, and much is to be gained by the use of reagents in equimolecular solutions; but certain unavoidable conditions attending this research made it necessary to pursue the studies of the actions of reagents with reference to effect and without more than incidental reference to cause.

It will be recognized, from what has been stated, that the reactions are conditioned by both starch and reagent. Having a number of starches of presumably different stereoisomeric forms, there remained the selection of the kind and concentration of reagents that would elicit such differences in the reactions as would demonstrate clearly not only isomerism but an isomerism that is specific in relation to genera, species, varieties and hybrids. It was found advantageous, in formulating these solutions, to disregard entirely concentrations upon the gram-molecular basis and to determine experimentally the strengths of solution that seemed best adapted to give wide ranges of reaction with different starches under the same conditions of experiment. The marked variations in the behavior of different starches with a given reagent, and of different reagents with a given starch, are presented in striking form in Charts A 1 to A 26 ; but these features are brought out even better in certain respects in Charts $\mathrm{E} 1$ to $\mathrm{E} 46$, and very much better in most respects in Charts B 1 to B 42 . The first group of charts has been considered in a previous subsection of this chapter; the second group will be taken up in a subsequent subsection; and the third group will here be studied in only sufficient detail to meet requirements.

In the construction of the group of charts designated $B 1$ to $B 42$ the main purpose was to bring out certain extraordinary peculiarities in the reactions of selected pairs (occasionally more) of reagents with a number of starches which are taken tentatively to be representative of genera and of subgeneric divisions. In the selection of the reagents for comparison it seemed that characteristics peculiar to each of the several reagents could be presented particularly well if in one group of this series of charts the reactions of a given reagent are taken as the standard of comparison with the reactions of each of the other 25 agents and reagents; and if in a second group we compare the reactions of certain two or more agents or reagents, selected because of certain peculiarities, such as similarity or dissimilarity of agent and reagent, this plan was carried out. In the first series the reactions of nitric acid are taken as the standard; and in the second series the reactions of anilines, inorganic acids, hydroxides, sulphides, etc., various combinations of two or more agents and reagents were made.

To reiterate, there is in the polarization reactions no molecular alteration of the starch molecule; color reactions are present with gentian violet and safranin which are attributable to adsorption without detectable attendant molecular disorganization; in the iodine reactions there is in all probability a union of iodine and starch to form an unstable iodide of starch, but no intermolecular breaking down; in the temperature reactions intermolecular disorganization is associated with the adsorption of water, but without the loss of properties that characterize the starch molecule; and in the chemical-reagent reactions not only intermolecular disorganization occurs, but various associated reactions that depend upon the acid or base character and particular elements and molecules of the reagents. From this it would follow that these reactions fall into well-defined groups: the polarization, aniline, iodine, temperature, and chemical-reagent reactions, respectively.

When the reaction-intensities with polarization, gentian violet, safranin, iodine, and temperature are plotted out in curves, as in Chart B 1, and the chemical-reagent reaction-intensities are plotted out, as in Charts $\mathrm{B} 2$ to 
B 42, it will be apparent that there is a well-marked line of demarcation between these two groups; and also that when the five curves of Chart B 1 are compared differences are exhibited that are in harmony with the similarities and dissimilarities of the characters of the reaction-processes. The polarization curve stands in its peculiarities quite apart from the others, and it appears, on the whole, to be in its course without more than incidental relationship to the courses of the other curves; but the gentian-violet and safranin curves show almost throughout their courses, close correspondence in their variations with each other (see also Chart B 2), yet an absence of correspondence with the other three curves. Such differences as are recorded in these two curves are doubtless attributable to errors of experiment. When the crudity of the method of valuation of these reactions is considered, it is remarkable that the curves are so close, rather than that there are. some discrepancies. The iodine and temperature curves bear certain well-defined similarities, but they lack the close agreement seen in the two aniline curves; and they differ enough to indicate that the processes involved in the two reactions are not the same. The absence of conformity of the aniline and iodine curves, together with the agreement of the former, is convincing evidence that here also the processes of the two sets of reactions can not be the same. While the iodine and temperature curves show similarities (Chart B 3) they differ as much in general from each other as do the iodine and aniline curves.

It will be seen that the iodine curve remains at variable distances above the temperature curve, excepting in Lilium tenuifolium, $L$. chalcedonicum, $L$. pardalimum, Iris iberica, Tritonia pottsii, and Phaius grandifolius, where in 5 of the 6 it is below and in one the same. The iodine valuations are only approximate, yet the errors of observation are probably not sufficient to alter the curve in any essential respect, at least in so far as concerns general comparisons. On the other hand, the temperature valuations are approximately scientifically correct inasmuch as the errors of experiment fall within such very narrow limits as not to affect appreciably the position of the curve at any point. While certain variations in the quantitative differences between these curves, and at points the inversion and reversion of the curves, might suggest errors of valuation, they are in conformity with the findings shown in the other charts, as will be seen. Some of the variations of the iodine records are probably due to differences in the behavior of this reagent with the capsular and intracapsular parts of the grains. Nägeli found that iodine in weak solutions may penetrate the capsular part to the intracapsular part of the grains, coloring the latter but not the former. It would seem, therefore, that the iodine reactions of the raw starch grains, as here studied, are reactions essentially, and with weak solutions solely, of the intracapsular part of the grain, and that the differences in color values of the reactions are dependent in part upon the peculiarities of the intracapsular starch, and in part upon variations in the transmissive and reactive properties of the capsule. With a given strength of iodine solution, when the grains are gelatinized by heating, both intracapsular and capsular parts color, the former very much more than in the normal grain, and the latter a different color from the intracapsular partthe former blue, and the latter violet, old-rose, etc.

Heating the starch grains in water, and various reagents gelatinize starch, but the molecular processes involved can not, for reasons stated, be precisely the same. The qualitative gelatinization changes in different starches differ from each other; those caused by heat differ from those caused by chemical reagents; and those caused by one reagent differ from those caused by another. The quantitative differences are in all corresponding cases far more marked than the qualitative changes. In the gelatinization caused by heat the change in surface tension that gives rise to the inflow of water is due, in accordance with our knowledge in general of colloidal swelling, to ionic action. Both hydrogen and hydroxyl ions are present, but it seems that the hydrogen ion is the effective agent, and effective only at certain temperatures that vary with the kind of starch. With the chemical reagents there are not only hydrogen and hydroxyl ions present, but also they are in comparatively very high concentration; and, moreover, there are in the different solutions other kinds of ions and also molecules that vary in kind and concentration. In these reagents the ion concentration is without the aid of heat sufficient to bring about the alteration in surface tension that permits of hydration of the starch, and also there are components of the solutions that with the amphoteric starch molecule may form various chemical combinations and influence the processes of gelatinization, as previously stated. If these statements are justified, such should be indicated when, for instance, the temperature-reaction experiments are compared with those of chloral hydrate, pyrogallic acid, nitric acid, and other reagents.

In comparing the curves of Charts B 4, B 5, and B 6, it will be seen in each that the temperature-curve differs markedly from the reagent curve, although there are many suggestions of correspondence in the variations; but they differ quite as distinctly from each other as do the reagent-curves from each other. Moreover, not only are there marked quantitative differences, but these differences not infrequently take the form of inversion of the curves, so that while with one starch temperature reactivity may be higher than reagent activity, in another starch there may be the reverse. For instance, in the temperature chloral-hydrate chart (Chart B 4) it will be seen that, here and there, varying direct and inverse relationships in the up and down courses of the curves occur, the one curve keeps continually above the other with variable degrees of separation, and then the curves will cross or become inverted, and at varying distances recross, such crossing and recrossing occurring a number of times. Thus, the temperature curve is higher than the chloral-hydrate curve in Amaryllis belladonna, Hamanthus katherina, H. puniceus, Nerine bowdeni, $N$. sarniensis var. corusca major, Lilium martagon, $L$. tenuifolium, L. chalcedonicum, L. pardalinum, Iris trojana, Begonia single crimson scarlet, B. socotrana, and Miltonia bleuana. In Amaryllis belladonna the temperature curve is lower than the chloral-hydrate curve, but in Brunsvigia josephince the reverse. In the three Hlippeastrums the temperature curve is the higher; the 
difference between the two curves in each is nearly the same; both are higher in the second and third than in the first; and the curve in all three is lower than in Amaryllis and Brunsvigia. In Homanthus the curves are inverted, the temperature curve being the lower, and the distance between the curves is practically the same. In the Crinums the curves recross, the temperature curves being the higher, and the distances between the curves in the three species are quite different-in the two hardy species the distances are small but different, and in the tender species well marked, showing definite subgeneric division. In the three Nerines, in the first the temperature curve is the higher, and in the second and third the lower. In other words, Nerine crispa has a higher reactivity in the temperature than in the chloralhydrate reaction, while $N$. bowdeni and $N$. sarniensis var. corusca major exhibit the opposite peculiarity.

These remarkable inversions and reversions, both intergeneric and intrageneric, have been found to be common in the researches with the various reagents, as will be seen. In Narcissus the temperature curve is again the higher, and in Lilium inversion again occurs, the temperature curve in all four being the lower, the distance between the two curves being very marked in the first species, marked in the other three, and nearly the same in each. In Iris the temperature curve is the higher in the first, third, and fourth, and lower in the second; and the distance between the curves is different in each, it being greatest by far in the fourth. In both Gladiolus and Tritonia the temperature curve is the higher, and the difference between the two curves is small and practically the same in both genera. In Begonia inversion again occurs, in both the temperature curve being lower and very markedly lower than the chloralhydrate curve, the separation being greater in Begonia socotrana. In Phaius. crossing again occurs, and again in Miltonia, the separation in the former being distinct and in the latter marked. While the courses of these curves vary greatly, the variations are not more than in the temperature-pyrogallic acid and temperaturenitric-acid charts (Charts B 5 and B 6), or when the temperature curve is compared with that of any other of the reagents, or when the curves of almost any two reagents arbitrarily selected are compared.

Comparisons of the temperature-pyrogallic acid and temperature-chloral hydrate charts (B 5 and B 4) bring out many striking differences: The range of reaction intensities of pyrogallic acid is distinctly greater than with chloral hydrate; the temperature and pyrogallicacid curves show far less tendency than the temperature and chloral-hydrate curves to any relationship in their courses; the variations in the degrees of separation in the temperature and pyrogallic-acid curves bear no evident relationship to what was seen in the temperaturechloral hydrate chart; and the points of inversion and recrossing of the curves have no correspondence unless of apparently a purely accidental character. The temperature-chloral hydrate reactions with Amaryllis and Brunsvigia show only small differences between the two curves, the temperature curve being the lower in Amaryllis and the higher in Brunsvigia; and in the temperaturepyrogallic acid reactions the temperature curve is the lower in both, and there is extremely little or practically no separation in Amaryllis but marked separation in Brunsvigia. In the former, in Hippeastrum, the temperature curve is the higher, while in the latter it is the lower, and the manner of separation of the curves is very different. In the former, in Homanthus, the temperature curve is the lower; in the latter, in the first species it is the higher and in the second species the lower, and the differences in the degree of separation are very different. In the former, in Crinum, the temperature curve is the higher in all three species; in the latter, it is the lower in all three, and the separations of the curves wholly unlike. In the former, in Nerine, the temperature curve is the higher in one and the lower in two; in the latter, it is higher in all three; and while the chloral-hydrate curve is high in the former the pyrogallic-acid curve is very low, almost zero, in the latter. In both the former and the latter charts, in Lilium the temperature curve is the lower, and there are some differences in the separation of the curves. In Iris and throughout the remainder of the charts similar differences will be found. Comparing now the temperaturenitric acid chart (Chart B 6 ) with the foregoing, it will be seen that it presents a very different picture, and here also there are the vagrant variations in the degrees of separation of the curves and the vagrant inversions and reversions, but which do not bear more than accidental relationships to the variations observed heretofore. In other words, each chart presents evidence in support of certain well-defined principles regarding reactive intensities of different starches with different reagents, and is a specific and characteristic picture that is indicative of the particular reagent.

From the point of view of strictly fair comparisons of the temperature and chemical-reagent reactivities some fallacy is introduced, because these two groups of reactivities have not an identical basis of valuation, and therefore because the value expressed by the space between any two abscissæ in the temperature reactions may not have the equivalent values of reagent reactions. In constructing the temperature scale in this research advantage was taken of data obtained in the previous investigation, and the scale was made to include what was believed to be the lowest and highest temperatures of gelatinization of the kinds of starches that were likely to be studied, this scale being taken to be the equivalent in values of the scale of reaction-intensities with reagents that was made to extend between the extremes of highest and lowest possible reactivities. But it will be seen, upon examination of Charts B $4, B$, and B 6 , that the temperature reactions are limited in the starches examined between $55.8^{\circ}$ (Lilium tenuifolium) and $83^{\circ}$ (Homanthus katherince); whereas, in the chloral-hydrate reactions the values extend between 5 per cent of the total starch gelatinized in 60 minutes (Crinum zeylanicum) to 99 per cent in 10 minutes (Begonia single crimson scarlet), and in both the pyrogallic-acid and nitric-acid reactions the values vary practically from extreme to extreme of the scale.

The temperature scale as thus constructed represents a scale that has just about one-half the abscissæ values represented by the chemical-reagent scale. If now the former scale is modified so that the extremes represent the extreme temperatures recorded among the starches 
studied, the maximum and minimum temperatures will be as shown in Chart B 6 , in which the temperatures as plotted out by the standard scale are represented by the heavy continuous line, and those by the modified scale by the broken line. It will be seen that the effect of the new scale is not only to accentuate differences, but also to bring about some differences in the relative positions of the curves as regards inversion and reversion. The first noticeable difference of importance is seen in Hippeastrum, in which in all three starches with the old calibration the temperature curve is the higher, while with the new it is lower in two and higher in one, and with marked differences in the degree of separation of the two curves. In Homanthus with the former the temperature curve is the higher in both species, while with the latter the two curves are practically alike in the first species and the temperature curve is very much lower in the second species, and so on throughout the chart. It will be seen, however, that the important characteristics pointed out in the preceding charts are present with both forms of calibration-that is, independence in the variations of the two curves during their progress, with some tendency to concordance, inversions and reversions of the curves at points, and independence of the fluctuations of the curves of each reagent and of the points of inversion, recrossing and separation of the curves in each chart of that which is recorded in any other chart. The standard calibration adopted for the temperature experiments is preferable to the other because better adapted for future investigations and, therefore, also for comparisons of the results of the present research with those of subsequent studies.

The peculiarities elicited by these charts are extraordinary; they are harmonious in the demonstration of certain fundamental principles; and they positively indicate that they are conditioned by both kind of reagent and kind of starch. It is, consequently, well worth while to extend these studies by means of a group of charts in which a given reagent will be taken as a standard of comparison with each of the other reagents, and in addition to supplement this with another group in which each chart shall present the reactive-intensities of two selected reagents. To this end one group of charts, Charts B' 6 to B 30, inclusive, and another, B 31 to B 42, have been prepared. In the former the nitric-acid reactions are taken as the standard of comparison, these reactions being particularly well adapted for the purpose because of their wide range and their exceptional value in the differentiation of genera, subgeneric divisions, species, and hybrids. Much space would be required to go over all the first group of charts individually and in detail, and indeed this is not necessary if the plan adopted in comparing Charts B 1 and B' 6 is pursued. There are, however, several points to which, because of their broad application, especial reference should be made: First, the marked differences exhibited by the various agents and reagents in the range of activities, even when the latter are plotted out upon the same basis of valuation, as in the case of all of the chemical reagents; second, the independence of the curve of each agent and reagent of the curve of every other (in several instances, however, as in the anilines and copper salts, there are no important differences); third, the wide differences in values exhibited by different agents and reagents in the differentiation of genera, subgeneric divisions, species, etc.; fourth, the differentiation of certain genera, subgeneric divisions, and species by one reagent without differentiation by others; fifth, the differences in the manner of differentiation by different agents and reagents of genera, subgeneric divisions, and species; sixth, the repeated inversions and reversions of the two curves in almost every chart, and the entire independence of the points of crossing in one chart of those in another; seventh, the marked variations that occur in the degree of separation of the two curves in each chart, and in each chart compared with each other chart; and eighth, the suggestion at least of a tendency to some correspondence, varying in extent, throughout the series of curves in the up and down movements of the curves. Of not less or even of greater interest and value are the second group of charts (Charts B 31 to B 42, inclusive) which present the reaction-intensities of selected pairs of reagents, such as chromic acid and pyrogallic acid, sulphuric acid and hydrochloric acid, nitric acid and sulphuric acid, nitric acid and hydrochloric acid, potassium hydroxide and sodium hydroxide, potassium sulphide and sodium sulphide, etc. Probably in no other way can the data of the specificity of each agent and reagent and of each form of starch be more convincingly exhibited. These charts are worthy of careful study.

The differences shown in the reactions of chromic acid and pyrogallic acid (Chart B 31) are very striking and full of interest, and the chart is worthy of a carefully detailed study. Considered from a rather general aspect, it will be seen that the chromic-acid curve undergoes much less variation than that of pyrogallic acid; that in some parts of the chart the chromic-acid curve is higher, in other parts lower, and in other parts the same or practically the same as the pyrogallic-acid curve; that the two curves rise and fall for the most part at the same ordinates and at points to indicate generic and subgeneric dividing lines; that the quantitative differences between the curves vary within wide limits, not only in different genera but also among members of the same genus, especially among subgeneric representatives; and that inversions and reversions of the curves occur at a number of ordinates at which such deviations are consistent with plant differentiation.

Among the many peculiarities worthy of more than passing notice are the following: In Amaryllis and Brunsvigia chromic acid failed to bring out any differentiation at the end of the 30-minute period, at which time there was 99 per cent of the total starch of each gelatinized, although, as shown by our records during the earlier part of the experiments, the former showed distinctly less reactivity than the latter. Pyrogallic acid elicited, from the beginning and throughout the reaction, very definite differentiation; and it showed very much less reactivity than chromic acid with Amaryllis, but the same reactivity with Brunsvigia, 90 per cent of the former being gelatinized in 60 minutes and 98 per cent of the latter, in 30 minutes. The Hippeastrums show distinctly higher reactivities with chromic acid than with pyrogallic acid, and the quantitative differences exhibited by $H$. titan and $H$. ossultan are very markedly larger than those shown by $H$. doones. In Homanthus the 
reactivities with chromic acid are moderate and those with pyrogallic acid very low; while the corresponding reactivities with $H$. puniceus are high and very high, respectively. The chromic-acid reaction is as much higher than the pyrogallic-acid reaction in $H$. katherince as it is lower in $H$. puniceus. This interesting inversion of reactive intensities of the two starches with these reagents is consistent with well-separated characters of these species, as already pointed out. In Crinum the two hardy species are much more reactive to chromic acid than to pyrogallic acid, whereas the reverse relationship is seen in the reactions of the tender species; moreover, curves of the latter are inverted in comparison with the former. In Nerine the chromic-acid reactions are moderate, while those of pyrogallic acid are so very low as to be almost absolutely negligible, making a very marked difference between the reaction-intensities. In Narcissus the chromic-acid reaction is moderate and the pyrogallicacid reaction low, but without much difference between them. In Litium all of the reactions are high to very high, the chromic-acid reactions being the higher except in one species, in which both reactions are the same, although during the earlier part of the experiments chromic acid showed a somewhat higher reactive intensity than pyrogallic acid.

The degree of separation of the two curves in the other three specimens is not alike in any two. In Iris the chromic-acid reactions are high in all four starches, and the pyrogallic-acid reactions moderate in two, low in one, and very high in one. The distance between the curves is marked in all four, and in $I$. persica var. purpurea the curves are inverted-in other words, the first three starches are more sensitive to chromic acid than to pyrogallic acid, while in the last there is the reverse. Throughout this group of charts it will be seen that this form of Iris exhibits a number of peculiarities of reactivity which definitely differentiate it from the preceding three, which in turn seem to be closely related in their reactivities. Inversion and reversion of the curves of the irids corresponding to the foregoing will be found in Charts B 7, B 8, B 9, B 10, B 12, B 22, and B 36 . In Gladiolus and Tritonia the chromic-acid reactions are high and the pyrogallic-acid reactions moderate, the reactions of the two starches with each reagent being the same or practically the same, but the reaction-intensities with the two reagents being markedly different. In Begonia the chromic-acid and pyrogallic-acid reactions are distinctly higher in Begonia single crimson scarlet than in $B$. socotrana, and the difference between the two reactions is very much greater in the latter than in the former. In Phaius and Miltonia the chromic-acid reactions are much higher than the pyrogallic-acid reactions, but the amount of separation between the two curves is nearly the same.

Examining this chart (B 31) from the aspect of generic and subgeneric differentiation, it is essential to bear in mind that certain genera are represented by individuals that show such marked differences as to indicate that they belong to subgenera or some other form of subgeneric division, as in Homanthus, Crinum, Iris, and Begonia, and that on this account variations of their curves may be such as to appear to be opposed to recognized generic grouping. With this peculiarity in view, beginning with Amaryllis and Brunsvigia (closely related genera), it will be seen the positions of the two curves in each are very different-in Amaryllis the two curves are well separated, but in Brunsvigia they are the same. There is here a definite separation of the two genera. These genera are well separated from Hippeastrum, and the latter from the Hcrmanthus, by the marked differences in the curves. In the three forms of $\mathrm{Hip}$ peastrum the chromic-acid curve is higher or even much higher than in the preceding and succeeding genera, and it is in two well above and in one definitely above the pyrogallic-acid curve. The pictures presented by the curves in these three generic groups are so different that one could not possibly be confounded with another. In Homanthus there is a drop of the chromic-acid curve in $H$. katherino and $H$. puniceus; and a very marked drop of the pyrogallic-acid curve in the former, but a marked rise in the latter, giving rise to a well-defined separation of this genus from Hippeastrum and to inversion of the curves in $H$. puniceus with consequent separation of the two species. In Crinum the picture is again different, there being a rise of the chromic-acid curve accompanied by a rise of the pyrogallic-acid curve in two and a fall in one.

Inversion of the curres occurs in relation to $C$. zeylanicum, this feature of itself differentiating this tender species from the two hardy species. In Nerine the picture is again and markedly altered. Both curves fall, the chromic-acid curve to a moderate level and the pyrogallic-acid curve almost to zero, and with very little or practically no difference in the reactivities of the four starches with each of the reagents. In Narcissus, while the chromic-acid curve remains at practically the same level as in Nerine the pyrogallic-acid curve has risen almost to the level of moderate reactivity, thus causing some separation of the two curves and giving a generic combination of the two curves which differs from that found in any other part of the chart. In Lilium the picture is again changed and is again distinctive of the genus. And so on, as we pass to Iris, Gladiolus and Tritonia, Begonia, Phaius, and Miltonia, the curves rary in their positions and degree of separation in such manners as to differentiate or suggest, as the case may be, not only generic but subgeneric groups. The Gladiolus and Tritonia curves are practically identical, the explanation for which has been referred to repeatedly. The first three and the last of the Iris are well separated; but Begonia shows curves of the two starches which, while well separated, rather indicate well-separated species than representatives of subgenera, as in the case of many of the other charts.

While it is true that in a number of instances a genus is represented by only a single species and that, inasmuch as the reactivities of different species of a genus exhibit varying reactivities with the same reagents and thus suggest that the differences (in so far as they are applied to the differentiation of genera) may be merely casual, it will nevertheless be found perfectly clear by examination of the accompanying charts that the evidence in support of the generic and subgeneric differentiations and other relations here noted is cumulative and convincing. The very marked differences in the reactivities of subgeneric groups which are quite as great, on the whole, 
as those of different genera, represent probably the most remarkable feature of the chart, and they might naturally be regarded as being accidental were it not that corresponding peculiarities have been recorded in nearly all instances where the reactivities of two agents or reagents have been compared. A further consideration of this striking phenomenon will be taken up later.

The inorganic acids, here typified by nitric acid, sulphuric acid, and hydrochloric acid (Chart B 32) are of pecular interest because of their pre-eminently hydrionic character, and because in each, in accordance with ionic action in relation to the swelling of proteins, the active agent in bringing about the alteration in surface tension that initiates gelatinization is the anion. But that these ions alone are insufficient to account for differences in the phenomena of gelatinization due to these agents, that the cations in each acid play a part, and that the reactions are modified by both concentration and kind of ions, is rendered apparent by a study of the curves. The most conspicuous features of this chart are: The wide differences exhibited by the different kinds of starch, and the obvious generic and subgeneric groupings; the identity or practical identity of the reactions of two or all three of the acids with certain starches in contrast with the marked to very marked variations with others; and the tendency generally for the nitric-acid and the hydrochloric-acid curves to run closely together and, as a rule, well apart from the sulphuric-acid curve, with, however, occasional greater closeness of the hydrochloric and sulphuric-acid curves than of the nitric-acid and hydrochloric-acid curves. This separation of the curves, while in part unquestionably due to differences in concentration of the reagents, is also partly due to differences in the characters of the reactions dependent upon the cations. In Amaryllis and Brunsvigia all three reagents yield exceedingly rapid reactions, but in Brunsvigia the nitric-acid reaction is distinctly less rapid than the sulphuric-acid and hydrochloric-acid reactions, the last two being the same. In Crinum moorei, Lilium martagon, L. tenuifolium, L. chalcedonicum, L. pardalinum, and Begonia single crimson scarlet the reactions with all three reagents are very rapid, and are the same or practically the same. The sulphuric-acid and hydrochloricacid reactions are nearly the same or practically the same in Brunsvigia josephince, Crinum longifalium, Iris persica var. purpurea, Phaius grandifolius, and Miltonia bleuana. The nitric-acid and hydrochloric-acid reactions tend to be close to very close, and at the same time well separated from the sulphuric-acid reactions, in $H i p$ peastrum titan, $H$. ossultan, $H$. docones, Hcemanthus katherince, Crinum zeylanicum, Iris iberica, I. trojana, and $I$. cengialti; to be approximately mid-intermediate in Homanthus puniceus, Nerine crispa, $N$. bowdeni, $N$. sarniensis var. corusca major, Narcissus tazetta grand monarque, Gladiolus Tristis, and Tritonia pottsii.

Curiously, in only 1 of the 28 starches (Begonia socotrana) is the hydrochloric reaction lower than the reactions of the other two acids; and not only is the difference in the reaction-intensities very marked between this and the next closer or nitric-acid reaction, but the difference between the latter and the sulphuric-acid reaction is also very marked; and the three reactions form a group that is widely and remarkably different from the reactions observed in the other Begonias. It is of especial interest to note that in Homanthus, Crinum, and Iris, among which there are subgeneric representatives, the subgeneric differentiation is in each genus well marked. These extraordinary variations in the relations of the reactions of the three reagents are inexplicable upon the basis merely of differences in ionic and molecular concentration of the reagents; or upon differences in the starches that may be assumed to be due to varying proportions of components of a mechanical mixture; or upon differences in reaction owing to the amount or kind of impurities; but they are entirely explicable upon the basis of different stereoisomeric forms of starch that have specific and varying relationships to the kinds and concentrations of solutes in aqueous solution.

The potassium-hydroxide and sodium-hydroxide chart (Chart B 33) presents features which, while less extraordinary, are quite interesting and significant. These reagents, like the acids, bear very close relationships, but there are aqueous solutions that are pre-eminently cationic, and here, as in the acid chart, it will be seen that reaction-intensities vary within the extremes of the abscissæ and elicit very definitely but in modified forms the generic and subgeneric divisions that are brought out so strikingly by the acids. Moreover, it is perfectly obvious that here, as in preceding charts, while certain differences may justifiably be attributed to differences in the concentration of the reagents, other differences seem to be inseparable from the presence of stereoisomers and of components of the solute that form specific and variable kinds of products through chemical union with the raw-starch molecules and their derivatives. The concentration of the potassium-hydroxide solution is 1.5 grams to 110 c.c. of water, and of the sodiumhydroxide solution 0.5 gram to 100 c.c. of water. It will be seen that the curves tend for the most part to keep close together in their variations; that while generally the potassium-hydroxide curve is the higher it is in a number of instances somewhat or even markedly lower, and in other instances the same or practically the same as the sodium-hydroxide curve; and that the generic and subgeneric divisions that were demonstrated in the preceding charts are here also elicited but in modified forms. The two reactions are the same or practically the same in Hamanthus katherina, Crinum zeylanicum, Lilium martagon, $L$. tenuifolium, L. chalcedonicum, $L$. pardalinum, Iris trojana, and Begonia single crimson scarlet. The potassium-hydroxide reactions are higher in all of the remaining starches excepting Crinum longifolium, Narcissus tazetta grand monarque, Iris iberica, I. cengialti, I. persica var. purpurea, Gladiolus tristis, and Tritonia pottsii, in which group it is markedly to very markedly lower, chiefly the latter. The very marked differences in the reaction-intensities of the two reagents in Nerine and Begonia in comparison with the differences generally stand out very conspicuously.

One feature of especial interest is to be noted in the species of Crinum: C. moorei is more sensitive to potassium hydroxide than to sodium hydroxide; $C$. longifolium shows the reverse; and $C$. zeylanicum about equal reactivity with the two reagents. Another feature is to be found in species of Iris, the first three showing with sodium hydroxide the same sensitivity and the last a 
very much higher sensitivity than the former; while with potassium hydroxide there are three gradations of sensitivity. The reactions of Iris persica var. purpurea differentiate it from the first three members of this genus. Another feature is seen in the very striking differences in Begonia; in the first Begonia both reactions are very high and the same, while in the second the potassium-hydroxide reaction is similarly high and the sodium-hydroxide reaction is low and far separated from the former.

Potassium sulphide and sodium sulphide (Chart B 34) elicit reactions which as a whole are quite different from those recorded in the preceding charts, but are nevertheless in entire support of the fundamental peculiarities that have been found to be set forth by the reactions of each pair of reagents thus far studied - that is, an independence of each reagent in its reactions that is due to both concentration and kind of solute; an independence of the reactions of each starch that is dependent upon differences in stereoisomeric forms; and an independence of the course of each curve to such a degree that there may not only be most variable quantitative differences but also inversion, yet with a manifest tendency to conforming with the peculiarities of a prototype (say the nitric-acid curve). Probably the first feature that will attract attention is the very marked differences in the behaviors of Amaryllis and Brunsvigia with these closely related reagents, the former exhibiting a very high reactivity with potassium sulphide and a moderate reactivity with sodium sulphide, thus showing a very wide difference in reactivity, there being 97 per cent of the total starch of Amaryllis gelatinized in 3 minutes and only 91 per cent of the total starch of Brunsvigia in 60 minutes; whereas with sodium sulphide the reactivities of both starches are very nearly the same, 90 and 96 per cent, respectively, in 60 minutes being recorded, A maryllis throughout the course of the reaction showing only slightly less reactivity than Brunsvigia.

It will be noted that the two curves here are entirely different from those of the three preceding charts (Charts B 31, B 32, and B 33), which also so differ from each other that each chart is very definitely individualized. The reactions of the sulphides are the same or practically the same in Brunsvigia josephince, Hippeastrum titan, H. ossultan, Homanthus josephinc, Crinum zeylanicum, Lilium martagon, $L$. tenuifolium, $L$. chalcedonicum, $L$. pardalinum, and Begonia single crimson scarlet. The potassium-sulphide reactions are higher in A maryllis belladonna, Homanthus puniceus, Nerine crispa, N. bowdeni, N. sarniensis var. corusca major, Begonia socotrana, and Phaius grandifolius; and lower in Hippeastrum daones, Crinum moorei, C. longifolium, Nancissus tazetta grand monarque, Iris iberica, I. trojana, I. cengialti, $I$. persica var. purpurea, Gladiolus tristis, Tritonia pottsii, and Miltonia vexillaria. For the most part the curves are well separated, this feature being particularly accentuated in Amaryllis belladonna, Crinum moorei, Nerine crispa, Iris persica var. purpurea, and Begonia socotrana. Homanthus katherince and $H$. puniceus are not nearly so well differentiated as in the preceding charts; the hardy and tender Crinums are well differentiated, as in the previous pairs of reactions. The Irids show nearly the same reactivities with potassium sulphide, while three show nearly the same reactivities with sodium sulphide, but higher than with potassium sulphide, and one a very much higher reactivity than the first three with sodium sulphide and a corresponding difference in relation to potassium sulphide, showing a marked subgeneric subdivision such as was noted with other reagents. In Gladiolus and Tritonia the potassium-sulphide curves are well below the sodium-sulphide curves, the difference in each being about the same. In Begonia the differentiation of the two starches is very striking. In Phaius and Miltonia the generic differences are pronounced, not only in regard to the degree of separation of the curves, but also in respect to the inversion of the curves. The high reactivities shown in Amaryllis belladonna, Nerine crispa, and Begonia socotrana with potassium sulphide in comparison with the moderate to very low reactivities with the other reagent, together with the very opposite in Crinum moorei, Iris persica var. purpurea, and Miltonia bleuana, are striking manifestations of differences in the molecular constitution of starches from different plant sources.

The reaction-intensities of potassium iodide and potassium sulphocyanate (Chart B 35) present very much closer relationships than do those of any of the pairs of reagents thus far considered, yet here also are found the fundamental peculiarities that have characterized all of the comparisons brought out in the preceding charts. The reactivities of these reagents are the same in Hamanthus katherince, Crinum moorei, C. zeylanicum, C. longifolium, Lilium martagon, L. tenuifolium, L. chalcedonicum, L. pardalinum, and Begonia single crimson scarlet. The reactions of potassium iodide are higher than those of potassium sulphocyanate in Amaryllis belladonna and Brunsvigia josephince, and lower with all of the remaining starches, except the group noted. The curves show for the most part a marked concordance in their upand-down movements, but the degree of separation of the curves is quite variable and there are inversions only of Amaryllis and Brunsvigia.

A comparative examination of the curves of the reactions of sodium hydroxide and sodium salicylate (Chart B 36) brings out one very exceptional feature that is associated with the latter reagent, and various features that are in harmony with characteristics that are common to the other charts. The marked limitations of the reactions of sodium salicylate are most striking and peculiar to this reagent. In only two reactions (those with Crinum zeylanicum and Begonia single crimson scarlet) is there a departure from the narrow limits of the upper six abscissæ (a trifle more than one-fourth of the highest and lowest limits of reaction-intensities). This limitation greatly restricts the value of the reagent in the differentiation of starches from different plant sources, yet there are in some instances marked to very marked differentiation, especially of subgeneric groups. The differences in the reactions of the two species of Hamanthus are not of themselves sufficient to definitely indicate subgeneric division, but rather well-separated species; in Crinum the two hardy forms are well differentiated from the tender form; in Iris the first three stand definitely apart from the fourth; and in Begonia there are striking differences between the two starches. 
The independence of the variations in the courses of these two curves, together with the individuality of the salicylate curve when compared with curves of the reactions of the other reagents, suggests peculiar relationships of the salicylate with the starch molecule that are worthy of special study. While this reagent is, at least in the concentration used, of comparatively little value in the differentiation of genera, it is not only of marked usefulness in recognition of subgeneric groups, as stated, but also in the differentiation of species and hybrids (see Chart A 18, page 183); and it has proven of much value in the study of the qualitative reactions of different starches, as will be found by reference to data in Part II and to Tables $\mathrm{C} 1$ to $\mathrm{C} 17$ in subsequent pages. Lens (Seventh Inter. Congress Applied Chem., London, 1909 ; Jour. Soc. Chem. Ind., 1909, xxvII, 731) had already found that this reagent could be used in the microchemical differentiation of starches from different sources. He states that if a trace of rye starch, in a hanging drop of a solution of 1 part of sodium salicylate in 11 parts of water, is examined under a magnification of 200 , at the ordinary temperature, it will be found that after the lapse of an hour (more distinctly after 24 hours) most of the large granules have swollen and that only a small part resists the action of the salicylate and still shows the polarization cross between crossed nicols. In the case of wheat starch, only a few of the large granules become swollen; after 1 to 24 hours the outline of the unswollen wheat starch-granules is sharply defined, and the granules, unlike those of rye starch, do not become flattened (starch of any kind which has been altered by storage in a moist condition swells on treatment with the salicylate solution). Barley and millet starches swell to a small extent only. Only few of the grains of oat, maize, rice, potato, bean, pea, lentil, and arrowroot starches become swollen.

The calcium-nitrate and strontium-nitrate curves (Chart B 37) exhibit wide excursions, those of the latter being the more marked; and the fluctuations tend with few exceptions to correspond in their directions, although with more or less marked quantitative variations. Both generic and subgeneric differentiations are as conspicuous as in the preceding charts; but inversion of the curves does not occur at any point. The reactions of these reagents are the same or practically the same in Amaryllis belladonna, Hamanthus katherina, Crinum zeylanicum, Lilium chalcedonicum, L. pardalinum, and Begonia single crimson scarlet; and very nearly the same in Hippeastrum titan, L. martagon, and L. tenuifolium. Elsewhere the differences range within variable limits, the widest being in Brunsvigia josephince, Crinum moorei, $C$. longifolium, Nerine crispa, $N$. bowdeni, $N$. sarniensis var. corusca major, and Begonia socotrana.

The curves of the uranium-nitrate and cobalt-nitrate reactions (Chart B 38) bear in general close relationships to the curves of the preceding chart, the most noticeable differences being apparent in the generally higher reactivities of calcium nitrate and strontium nitrate, particularly the latter. The curves tend to be distinctly closer than with the latter reagents; no inversion of the curves occurs at any place; and generic and subgeneric differentiations, especially the latter, are with rare exceptions well marked.
The copper-nitrate and cupric-chloride curves (Chart B 39) are very similar to those of the two preceding charts, the reactions tending to be the same or somewhat greater than with uranium and cobalt nitrate, but as a whole distinctly lower than with calcium nitrate and strontium nitrate. Both generic and subgeneric distinctions are well marked.

Barium chloride and mercuric chloride in the concentrations used are the weakest of all of the reagents in the gelation of starch. Both curves (Chart B 40) are therefore lower, as a whole, than is found in the other charts, the barium-chloride curve being distinctly the lowest curve recorded. The fluctuations in this chart are in close correspondence with those of the immediately preceding charts. No inversion of the curves occurs except possibly in Hamanthus puniceus, where the difference in the reactions falls within the limits of error of experiment.

Reviewing these charts, as a whole, from both general and special aspects, it will be found that they may be divided primarily into two well-defined groups in accordance with the peculiarities of the curves: first, those showing the reactions with polarization, gentian violet, safranin, and iodine; second, those showing reactions with temperature and chemical reagents. This distinction is due in part to differences in the method of calibrating reaction-values and (in pant and chiefly) to differences in the inherent characters of the reactions. As before noted, and of fundamental importance at this juncture, the scale-values in the experiments with polarization, gentian violet, safranin, iodine, and temperature are different from those in the chemical reagent experiments; the polarization reaction is an optic phenomenon that is without associated molecular disturbance; the gentian-violet and safranin reactions are probably simple phenomena of adsorption, but without apparent molecular disturbance; the iodine reaction is probably a manifestation of chemical combination of the iodine with the starch to form a feeble union, but without a detectable appearance of intermolecular disorganization; the temperature reaction elicits an intermolecular disaggregation that is associated with hydration; and the chemical-reagent reactions are expressions of not only intermolecular breaking down and hydration, but also various quantitative and qualitative modifications in the starch molecules and their derivatives that depend upon differences in concentration and components of the reagents, the starch molecule because of its amphoteric properties combining with both acids and bases, and the gelatinization processes being more or less modified by some reagents by associated chemical changes. The polarization curve (Chart B 1) bears no well-defined relationship, except of an apparently accidental character, to any of the other curves. The gentian-violet and safranin curves (Chart B 2) are very much alike, and where differences are noted they are doubtless to be attributed to errors of experiment; and these curves stand apart from all other curves. The iodine and temperature curves (Chart 3) show in general a closeness which suggests that since in the temperature reaction there is intermolecular disorganization there is a more marked molecular change in the iodine reaction than is shown by the microscope in ordinary or polarized light. 
Inasmuch as the temperature valuations are quite exact (as exact as the determinations of the meltingpoints of crystalline substances), and as the iodine valuations are of a gross character, it seems probable that seeming deviations from what is judged to be the normal in the two charts may be due to errors of experiment; but some of these differences are explicable only upon the assumption of peculiarities of the molecules of the different starches, causing them to behave differently with different reagents, as was found in the study of the reactions with the chemical reagents. The temperature curve, while very much more limited in its excursions than the curves of most of the chemical reagents, bears in general a well-defined relationship in its fluctuations to the variations collectively of the latter. This relationship becomes more obvious when the temperature values are in a modified form to render them more consistent with the chemical reagent values, as shown in Chart B 6, in which the temperature and nitric-acid curves are figured, the former being exhibited in, one curve in accord with the standard calibration and in another with a modified valuation so formulated that these values, like the chemical reagent values, extend over the entire limits of chart between the highest and lowest abscissæ. When, however, the iodine values are similarly modified (Chart B 8) there is no more similarity, on the whole, between this modified form of curve and the nitric-acid curve than there is when the standard calibration is used-in fact, if anything, there is a greater lack of correspondence. Comparisons of this modified curve with curves of the reactions of other reagents are fully confirmative of these findings in support of inherent differences in the behavior of the starch molecules in these reactions. In a word, these facts indicate quite convincingly that the iodine, temperature, and nitric-acid reactions are in some way or ways fundamentally different and that there is an obscure relationship between the temperature and nitric-acid curves that does not exist between the iodine and nitric-acid curves. In these comparisons the nitric-acid curve has been taken as a prototype of the chemical-reagent curves. When the latter are individually compared with this prototype and with each other it will be found that, while no two are alike, all conform to this type in a manner that is comparable to the conformity of the members of a genus to a generic prototype. In other words, the variations shown by the different reagents are comparable to the variations exhibited by the members of a genus.

Sufficient reference has doubtless been made to the peculiarities of the reactions of the various reagents, individually and in couples, that are specific to each reagent in association with peculiarities of the various stereoisomeric forms of starch, yet it seems that additional statements may be made with profit in respect especially to certain reactions of well-defined natural groups of reagents, such as the inorganic acids, hydroxides, sulphides, nitrates, chlorides, potassium salts, sodium salts, copper salts, etc. The only organic acid used in this research is pyrogallic acid, to the solution of which was added a small amount of oxalic acid for the purpose of preservation. Chromic acid, while belonging to the inorganic group that comprises nitric, sulphuric, and hydrochloric acids, may for certain reasons be con- sidered with pyrogallic acid, and then with the other three acids. Chromic acid acts on the starch grain in a manner that is not only entirely individual and distinctive in comparison with the actions of the other acids, but also quite different from that of any other reagent. This acid causes the grain at first to be altered into a gelatinized capsule and a semi-liquid contents; the capsule then ruptures at some point and the contents flow out; and then both capsular part and escaped contents pass rapidly into solution. Pyrogallic acid brings about changes that belong to a fundamental type that is common to the other chemical reagents, but variously modifiable with each reagent. By comparing the chromic-acid and pyrogallic-acid curves (Chart B 31), and then these with the nitric-acid, sulphuric-acid, and hydrochloricacid curves (Chart B 32), it will be seen that the first two differ markedly from each other, that the chromicacid curve is not in closer relationship than the pyrogallic-acid curve to the curves of the group of inorganic acids, and that the pyrogallic-acid curve is more closely related than the sulphuric-acid curve to the nitric-acid and hydrochloric-acid curves. The sulphuric-acid curve in comparison with the nitric- and hydrochloric-acid curves appears to be vagrant, but this seeming discrepancy may be due, in a large measure at least, to the higher reactive-intensity of this reagent.

These five reagents undoubtedly have, because of their inherent chemical differences, different chemical relationships to the starch molecule and accordingly yield reactions that can not be identical qualitatively. Chromic acid and nitric acid apparently stand apart from the other acids because of their oxidizing properties, but it may be, as suggested by the investigations of Sacharow and of Grüiss (see previous memoir, pages 95, 146, and 186), that oxygen is essential in both the initial and final stages of the saccharification of starch. If this is so, the part played by oxygen in the actions of the other reagents is masked. However, chromic acid has been used commercially to liquefy starch and form dextrin and sugar because of its asserted oxidizing power. Nitric acid has been found similarly valuable to form oxalic acid from starch and other carbohydrates. Pyrogallic acid, on the other hand, is an active deoxidizer, taking up oxygen freely; and, moreover, this acid does not, as is well known, form true salts. Both sulphuric and hydrochloric acids have been employed by a large number of investigators to reduce starch to dextrin and sugar (see Publication No. 173, page 104). While our knowledge of the exact characters of the intermediate products of saccharification is very limited, it is justifiable, from what is known, to assume that the interactions of these various reagents with the starch molecule may be quite as varied as those which occur in the evolution of oxygen from peroxides, chlorates, and permanganates, respectively, and that they may differ even more than the processes of enzymes and acids, respectively, in the liquefaction, dextrinization, and saccharification of starch (see previous memoir, page 149).

Probably no two pairs of curves elicit more interest than those of potassium and sodium hydroxides and nitric and hydrochloric acids when the members of each pair and of the two pairs are compared. The first two reagents are pre-eminently cationic; the latter is pre-emi- 
nently anionic. It might naturally be expected that if one of the two reagents of either pair exhibits a higher reactivity than the other member of the pair with a given starch the same relationship in reaction-intensity should be found in the reactions with other starches, but it will be seen in each of these pairs of curves that there is not only an absence of consistent relationship in so far as one curve is always higher than the other, but also in other respects, so that there is more or less marked independence in the courses of the curves-independence quite as conspicuous as has been found in the comparisons of any pair of microscopic and macroscopic characters of the plants themselves. Thus, in Amaryllis belladonna with potassium hydroxide (Chart B 33) there is complete gelatinization in 1 minute, and with sodium hydroxide a not quite complete gelatinization in $3 \mathrm{~min}-$ utes; while in the Brunsvigia josephince reactions the records with the same reagents are 98 per cent in 1 minute and 95 per cent in 15 minutes, respectively. With the first starch the reagents exhibit but little difference, but with the second a marked difference, while in both the potassium hydroxide is the stronger in its actions. In other instances the values may be the same, or the curves may be more or less separated, or inverted so that the potassium hydroxide is the less effective.

Passing from starch to starch it will be seen that the separation of the curves observed in Brunsvigia is as well marked in Hippeastrum. In Homanthus katherince the reactions of both reagents are very slow, almost nil; but in $H$. puniceus there is a wide separation of thecurves, the potassium curve being high and the sodiumhydroxide curve low. In Crinum moorei the two reactions are very high and in C. zeylanicum very low. In C. longifolium both are very high, but not so high as in C. moorei. In C. moorei and C. zeylanicum there is in each little difference in the potassium and sodium curves, in the latter practically none; but in $C$. longifolium the curves are well separated. Subgeneric differentiation here, as in the case of the species of Hcomanthus, is quite marked. In Nerine the two curves are antipodal, the potassium-hydroxide curve being very high and the sodium-hydroxide curve very low, making the separation exceptionally wide. In Narcissus the curves of both reagents are low to very low, and the reactivities of the reagents are in inverse relationship to what has been heretofore noted, this starch being more responsive to the sodium than to the potassium salt. In Lilium the reactions with both reagents take place with such rapidity that there is not satisfactory differentiation. In Iris interesting differences in the curves are seen, and so on with the other starches. Similar peculiarities will be found in the comparisons of the curves of the pair of acids.

Comparing now the pairs of acid and base curves (Charts B 15 and B 33) it will be noticed that notwithstanding the opposite characters of the ions the curves of the two charts bear in general resemblances that conform closely to a common type of curve; that in each pair one of the two reagents tends to be the more active, or to have the same reactivity as the companion reagent throughout most of the chart; that in each pair of curves the quantitative relationships may be so altered that there may be not only very variable degrees of dif- ferences in the extent of separation of the curves, but also inversions and recrossings of the curves; and that in the two charts the ordinates at which sameness of reactivity-intensity of the reagents, higher reactivity of one reagent over the other, inversion, recrossing, etc., may have no correspondence. These facts demonstrate an individuality of each reagent and each form of starch.

It will also be seen that while the two pairs of curves are in general in their fluctuations in accord they may not correspond in the extent of the variations. This feature is conspicuous in Nerine, Narcissus, Iris, Gladiolus, Tritonia, and Begonia. Thus, in Nerine both of the acid curves fall, the hydrochloric-acid curve for the first two species (the values for the second and third being the same), and the nitric-acid curve for all three species, making about the same difference between the two curves for the first two species and a more marked difference for the third species. The picture here is entirely different from that of the potassium and sodiumhydroxide chart. In Narcissus the hydrochloric-acid curve is high and the nitric-acid curve very low; the potassium and sodium-hydroxide curves are both very low; the nitric-acid reaction is practically the same as that of potassium hydroxide, somewhat lower than that of sodium hydroxide, and markedly lower than that of hydrochloric acid. In Iris both acid curves fall to the level of moderate to low reactivity in the first three starches, and in all practically the same; but in the fourth starch both reactions are very high, the hydrochloric-acid reaction being distinctly higher than the nitric-acid reaction. With the base reagents both curves fall to the level of high to moderate reactivity in the first three starches, and rise to high reactivity in the fourth starch. The positions of the curves of the first three starches differ entirely from those of the acids, while those of the fourth starch are practically precisely the same as those of the acids. In Gladiolus and Trilonia both pairs of curves fall to the levels of low to very low reactivity, the nitric-acid curve falling to a lower level than the hydrochloric-acid curve; the hydroxide curves fall to an intermediate position, the sodium curve being lower than that of potassium. Begonia shows striking similarities and dissimilarities: In $B$. single crimson scarlet all four reagents act with great energy, gelatinization being complete in one minute or less. In $B$. socotrana both acid curves fall, one to the level of the line of demarcation of high to moderate activity, and the other to very low reactivity; whereas with the hydroxides the reaction with the potassium salt is very rapid and is over in less than a minute, while with the sodium salt it is very slow. Moroever, in the acid reactions, while most of the starches show a lower reactivity with nitric acid, $B$. socotrana shows a markedly lower reactivity; and in the potassium-sodium chart most of the starches show a higher reactivity to potassium than to sodium, the starch of $B$. socotrana also showing this character. In other words, this species is aberrant, as it were, in its reactions with the acids in comparison with the reactions of the other Begonias and most other starches, but in harmony in the potassium and sodium reactions. In both Phaius and Miltonia there is a reversal of the reaction-intensities of the two acids, but not of the hydroxides, as compared with $B$. 
socotrana. Additional comparisons of the data of these charts will bring out many interesting facts.

The potassium-sulphide and sodium-sulphide chart (Chart $B$ 34) bears in certain respects closer resemblances to the hydroxide chart (Chart B 33) than to the acid chart (Chart B 15), and in other respects the reverse, thus indicating that the alteration of the hydroxides into the sulphides has yielded reagents which give rise to reactions that suggest the presence of both active cations and anions, in contradistinction to the reactions of the hydroxides and acids which are pre-eminently cationic and anionic, respectively. These sulphide reactions vary in intensity in both directions to almost the extreme limits of the abscissæ, from the extremely high reactivities of potassium sulphide that are recorded in Litium, Begonia, and Phaius in which complete gelatinization occurs in 2 minutes or less, to the extremely low reactivities in Hippeastrum, Homanthus, Crinum, etc., where 5 per cent or less is gelatinized in 60 minutes. The deviations of these curves from the acid and base curves are much more marked than the variations of the curves themselves, and the quantitative differences between the curves tend to be more marked and erratic, and inversions to be more frequent, than in the acid and base curves. In Nerine there occurs in the sulphide curves, as in those of the hydroxide, an inversion, in both charts the potassium salt is the stronger. In Iris there is a marked separation of the curves, as was found to be the case with one exception in the hydroxide reactions; but in three of the starches there was no separation of the acid curves. In Begonia socotrana the curves are less like those of the bases than of the acids, while in Miltonia they stand apart from both base and acid curves. The wide separation of the sulphide curves in Amaryllis is very conspicuous in comparison with the small separation of the base curves and the absence of separation of the acid curves. Similar peculiarities will be found in the reactions of these three pairs of reagents with other starches.

The potassium-iodide and potassium-sulphocyanate reactions (Chart B 35) bear, on the whole, far closer resemblances to the hydroxide reactions than to the acid or sulphide reactions. In contradistinction to the sulphides these reagents contain acid radicals that are probably almost inert. Comparing this chart with the base chart (Chart B 33), the most noticeable differences will be found in the reactivities with Amaryllis, Brunsvigia, Hamanthus puniceus, Nerine, Iris, Begonia, Phaius, and Miltonia. Amaryllis and Brunsvigia each exhibits practically no difference in the potassium-iodide or potassium-sulphocyanate reactions, but $A$ maryllis and Brunsvigia are differentiated from each other by both reagents, both starches reacting more readily with potassium iodide than with the other reagent. In Hormanthus puniceus, while these reagents do not differ in their reactivities, potassium hydroxide yields a markedly different result from that of sodium hydroxide. In Nerine reactivity with the iodide is very low and with the sulphocyanate low; while in the hydroxide reactions those with potassium hydroxide are very high and those with sodium hydroxide very low. In Iris the potassium iodide reactions are very much lower in the first three Irids and somewhat lower in the fourth; while in the hydroxide reactions in two there are very marked differences, in one no difference, and in another a marked difference, the potassium reactions being the lower when difference exists. In Begonia the iodide and sulphocyanate reactions show very little difference, in $B$. single crimson scarlet both reagents acting with great intensity and in $B$. socotrana with great slowness, the iodide being practically inert; while in the hydroxide reactions both reagents act with great intensity with $B$. single crimson scarlet, potassium hydroxide acts with equal vigor, but sodium hydroxide with low intensity with $B$. socotrana. In Phaius and Miltonia both the iodide and the sulphocyanate show differences between these genera and between the members of each genus, the iodide being less active than the sulphocyanate. While in both Phaius and Miltonia marked differences exist between the reaction-intensities of the iodide and the sulphocyanate, there are comparatively small differences between the intensities of the hydroxides.

The curve of sodium salicylate (Chart B 36) stands alone, as before stated, and therefore is not comparable, as in the foregoing instances, with that of any other reagent.

Calcium nitrate and strontium nitrate (Chart B 37) exhibit differences that are most pronounced in Brunsvigia, Crinum, Nerine, and Miltonia. The calcium curve appears to correspond more particularly with the curves of potassium iodide, potassium sulphocyanate, and sodium hydroxide; while the strontium curve appears to be more closely related to the curves of uranium nitrate, copper nitrate, cupric chloride, and mercuric chloride. All of the latter curves appear to be very closely related to a common type, which suggests that the reactions, in so far as the latter depend upon the reagents, are due essentially to differences in the basic ions or cations.

Differentiation of Subgeneric Groups.-There is probably no feature of these charts more prominent or of greater value in proof of the worth of the gelatinization method in the differentiation of starches from different sources than the constancy and definiteness in similar and dissimilar directions of the differentiation of subgeneric representatives. Hamanthus katherino and $H$. puniceus are, from the standpoint of the systematist, at most well-separated species, but from the results of this research they are probably to be regarded as representatives of well-defined subgeneric groups. Had this marked subgeneric differentiation been indicated by the reactions of a single or an occasional reagent it might naturally be regarded as being accidental, but it is evident throughout the charts of the reactions of the 21 reagents, except the chloral-hydrate and sodium-salicylate reactions. The one species is as definitely and widely differentiated from the other as are genera in general, with the exception only of the closely related Gladiolus and Tritonia. While at the end of 60 minutes there is only slight and questionable differentiation in the chloralhydrate reactions, and in the sodium-salicylate reactions no differentiation, there are differences of importance shown during the progress of the reactions (Charts D 106 and $D$ 118). The hardy and tender Crinums are with every reagent markedly differentiated, but by some to a better degree than by others. The abscissæ of the two hardy Crinums are in all of the reactions above those 
of the tender Crinum, so that in every chart the curves of these three species are V-shaped, and the first segment of the $V$ is longer than the second, the difference in length varying with the different reagents. In Iris the first three specimens are definitely differentiated from the fourth in most of the charts by the distinctly lower reactivities of the former, the exceptions being in the reactions of chloral hydrate, chromic acid, sulphuric acid, potassium sulphocyanate, potassium sulphide, and sodium salicylate (in the chloral-hydrate and potassiumsulphide reactions those of the former are the higher). In other words, in only 4 of the 21 reactions is there not a definite separation of the first three from the fourth. In Begonia the differentiation is not only very marked, but also in certain respects extraordinary: $B$. socotrana is a very exceptional form of the genus, is semituberous, and is botanically quite different from the tuberous $B e-$ gonia single crimson scarlet. The starches of the two plants in histologic and polariscopic characters, qualitative reactions with various reagents, are alike in many respects and very dissimilar in others, so that each exhibits certain striking and distinctive characteristics (see Chapters III and V, and Part II, Chapter VIII). These peculiarities together with the remarkable differences in their reaction-intensities constitute one of the exceptionally interesting findings of this research.

The curves of the reactions of the four tuberous Begonias (Charts E 36, E 37, E 38, and E 39) tend to be as much in accord as should be expected in plants that have such a botanical relationship, but the curve of $B$. socotrana (Chart $\mathrm{E} 36$ ) appears definitely to be vagrant in nearly all of the reactions. The four hybrids incline, on the whole, to an obviously closer relationship to the tuberous parents than to $B$. socotrana. Examinations of the curves of the preceding charts (Charts B 11 et seq.) will show that: With chloral hydrate there is definite but not marked differentiation, 99 per cent of the total starch of $B$. single crimson scarlet being gelatinized in 10 minutes and 95 per cent of the starch of $B$. socotrana in 15 minutes. With chromic acid there is 98 per cent in 15 minutes and 92 per cent in 60 minutes, respectively, a wide difference. With pyrogallic acid, 95 per cent in 45 minutes and only 0.5 per cent, or almost nothing, in 60 minutes, giving a much wider difference than with the preceding reagent. With sulphuric acid a practically complete gelatinization occurs in both starches in a minute, while with hydrochloric and nitric acids with the starch of the first plant there is immediate gelatinization with both reagents; and with $B$. socotrana with the hydrochloric acid there is 45 per cent in 45 minutes, and with nitric acid only 12 per cent in 60 minutes. With potassium hydroxide there is an almost instantaneous gelatinization of both starches. With potassium iodide there is practically complete gelatinization of one in 30 seconds, while with the other there is almost no detectable effect, only about 1 per cent being gelatinized in 60 minutes-almost the absolute extremes of reaction-intensity. With potassium sulphocyanate peculiarities are elicited that are almost identical with those of the last reagents, the only difference being a somewhat larger percentage of starch of $B$. socotrana gelatinized in 60 minutes-here 18 per cent. With potassium sulphide the differences between the reactions of two starches is positive, complete gelatinization occurring in the starch of $B$. single crimson scarlet in 15 seconds and 99 per cent in the case of $B$. socotrana in 5 minutes. With nearly all of the remaining reagents (including sodium hydroxide, sodium sulphide, calcium nitrate, uranium nitrate, strontium nitrate, copper nitrate and cupric chloride) gelatinization of the starch of $B$. single crimson scarlet is with each reagent complete within 2 minutes, while with the starch of $B$. socotrana it varies from 0.5 per cent to 84 per cent in 60 minutes (with two reagents there was 84 per cent, with one 25 per cent, with one 9 per cent, with one 1 per cent, and with two 0.5 per cent). With sodium salicylate the figures for the first starch are 97 per cent in 3 minutes, and for the second 99 per cent in 10 minutes. With cobalt nitrate the figures for first are 66 per cent in 60 minutes (the lowest record for this starch with any of the reagents), and for the second 0.5 per cent in 60 minutes. With mercuric chloride the first starch shows a gelatinization of 96 per cent in 15 minutes, and the second 0.5 per cent in 60 minutes. The extraordinary differences exhibited by these starches are at present inexplicable, and they open a field of most interesting and promising research of the most fundamental character.

Inversion and Reversion of Reaction-intensities.The inversion and reversion of the reaction-intensities of different starches with different pairs of reagents is also a feature of exceptional interest and of pre-eminent importance in proof of the existence of starches from different plant sources being in stereoisomeric forms. It is obvious, as before stated, that if we were dealing with starches that differ from each other because merely of differences in density, reaction, impurities, percentage of water, or varying proportions of several modifications of starch in the form of mechanical mixtures, the two curves would be alike or one would always be above the other, the distance, however, varying in relationship to the rapidity of reaction, the slower the reaction the greater probably the tendency in general to separate. It has been repeatedly noted that inversion and reversion of the curves is not limited to the distinction of genera, although it is more apt to be associated with genera, and next in order with subgeneric groups, and next with species. In other words, if with any two reagents a member of a given genus will exhibit a greater reactivity with one than the other reagent the same peculiarity will probably be found with all other members of the genus unless there are definite subgeneric divisions of the genus, under which conditions the subgeneric divisions may be as distinctly differentiated as may be genera by inversion or reversion of the reaction-intensities.

Sometimes species of a genus which are not recognized as belonging to subgeneric groups may exhibit inversion or reversion in their reactivities in relation to the reactivities of the other species, as has been found, for instance, in Nerine. These inversions and reversions are, as a rule, not so apt to occur with reagents of a similar as of a dissimilar character. Moreover, the points at which inversions and reversions of the curves of any pair of reagents occur may be the same or different from those at which inversions and reversions of another pair occur-that is, two genera or representatives of two subgeneric divisions, or two species of a genus, may be 
distinctly differentiated by the inversion or reversion of the reactive-intensities of a given pair of reagents, but not by another pair. Thus, in the chloral-hydrate and nitric-acid reactions (Chart B 11) the first inversion seen occurs in the curves between Hippeastrum and IIamanthus, the three species Hæmanthus showing a higher reactivity with nitric acid than with chloral hydrate, while Homanthus katherince shows the reverse. But the differentiation here is not generic because the second species, Homanthus puniceus, exhibits a reversion in relation to the first species. In the chromic-acid and pyrogallic-acid reactions the reverse is noted in the behavior of these two species, $H$. katherina showing in common with Hippeastrum a higher reactivity with chromic acid, while $H$. puniceus shows the inversion. In other charts (as, for instance, in Chart B 32 and B 36) all species of Hippeastrum and Hemanthus show in common a higher reactivity with one of the two reagents; while in other charts there are various modifications. For instance, in Chart B 35 each Hippeastrum shows different reactivities with the two reagents, but the Hæmanthuses no difference.

Crossing of the curves occurs again between Nerine bowdeni and $N$. sarniensis corusca major, thus markedly differentiating the first from the last two species of this generic group. The same separation will be seen in Chart B 2 (gentian violet and safranin), while in Chart B 4 (chloral hydrate and temperature) and Chart 8 (nitric acid and iodine) the crossing occurs between $N$. crispa and $N$. bowdeni. The next crossing occurs between Iris and Gladiolus; the next between Tritonia and Begonia and the next between Begonia and Phaius-all representing generic lines of division. Comparing the locations of these points of inversion or reversion with those in the nitric-acid and chromic-acid chart (Chart B 12) it will be found that with two exceptions (between Iris and Gladiolus, and between Tritonia and Begonia) the points are entirely different. The first crossing here occurs between Brunsvigia and Hippeastrum; the second between Hæmanthus and Crinum; the third between Crinum moorei and C. zeylanicum; the fourth between C. zeylanicum and C. longifolium; the fifth between $\mathrm{Ne}$ rine sarniensis var. corusca major and Narcissus; the sixth between Narcissus and Lilium; the seventh between Lilium and Iris; the eighth between Iris cengialti and I. persica var. purpurea; the ninth between Iris and Gladiolus; and the tenth between Tritonia and Begonia. Some of these ten inversions and reversions occur between generic representatives, while others represent subgeneric dividing lines.

The different points of inversion and reversion of the curves shown in these charts (Charts B 1 to B 40) are exhibited collectively in Chart B 41, this presentation rendering further detailed statement in regard to each chart unnecessary. Even a superficial study of the varying points of crossing of the curves and of the totals of this chart brings out very interesting and significant comparisons. In confirmation of statements made in preceding pages, it will be found that in some of the charts (12 out of the 40) no crossing of the curves occurs at any part; that in most of the charts there are inversions and reversions, the number ranging from 3 to 10 ; that inversions and reversions are, on the whole, more common when the agents and reagents are of dissimilar character and when they exhibit wide and frequently varying ranges of reaction-intensities; and that the crossings of the curves are most apt to occur at points of separation of genera and subgeneric representatives, and in variable numbers with different reagents and different starches at such places. The closely related genera Amaryllis and Brunsvigia are distinguished by the inversion of the reactions in only a single instance (Chart B 4, temperature and chloral-hydrate reactions). Brunsvigia and Hippeastrum have a separation by 9 crossings, but the latter is separated from Hremanthus by only 3. Curiously, the two species of Homanthus are separated by 6 crossings, these variations of the curves suggesting subgeneric division of the species. Hamanthus is separated from Crinum by 8 crossings, and Crinum from Nerine by 7 ; but there are 9 between Crinum moorei and $C$. zeylanicum, and 11 between the latter and $C$. longifolium, markedly differentiating the two hardy forms from the tender form. The separation of Nerine from Crinum and from Narcissus is well marked, there being 7 crossings at the former point and 14 at the latter. Narcissus is separated from Lilium by 9 , and the latter from Iris by 15. The separation of the first three Irids from the fourth is evident by 8 . Gladiolus and Tritonia are separated by only 3 , but these two are separated from Iris by 12 and from Begonia by 11 . The remarkable differences exhibited by the tuberous and semituberous Begonias are here illustrated by the separation of the two by 15 crossings. Begonia is separated from Phaius by 7 , and Phaius from Miltonia by 8 .

Wide Differences in the Reactions with Different Pairs of Reagents.-Another feature of exceptional interest is the wide differences in the reactions of different pairs of starches with different reagents, as has been referred to repeatedly, and which is worthy of some special notice. This peculiarity is well exemplified, for instance, in Amaryllis and Brunsvigia. Little or, in some instances, no difference is observed in the reactions of these starches with chromic acid, sulphuric acid, hydrochloric acid, nitric acid, potassium hydroxide, potassium iodide, potassium sulphocyanate, sodium sulphide, cobalt nitrate and barium chloride; distinct but not marked differences are noted with chloral hydrate and sodium salicylate; and marked differences are recorded with pyrogallic acid, potassium sulphide, sodium hydroxide, calcium nitrate, uranium nitrate, strontium nitrate, copper nitrate, and cupric chloride. The reactions of Amaryllis are higher than those of Brunsvigia with chloral hydrate, nitric acid, hydrochloric acid, sulphuric acid, potassium sulphide, sodium hydroxide, sodium salicylate, calcium nitrate, uranium nitrate, strontium nitrate, cobalt nitrate, and cupric chloride; lower with pyrogallic acid, potassium hydroxide, potassium iodide, potassium sulphocyanate, barium chloride, and mercuric chloride; and the same with chromic acid and sodium sulphide. Even better illustrations are to be found with other pairs of starches, as, for instance, the two Begonias.

Limitation of Number of Gelatinizing Reagents, Etc. -The variety of the reagents used in this research to gelatinize starch, together with the amphoteric properties of the starch molecules, may give the impression 
that almost any kind of reagent in aqueous solution may react with starch in this way. In fact, however, it is rather surprising to find how few reagents outside of certain well-defined groups are effective. It is also to be noted that there are various substances which while in any concentration in aqueous solution may be practically or absolutely inactive as a gelatinizing agent at room temperature may aid or hinder the gelatinizing effect of heat, as is evident by their property of lowering or raising the temperature of gelatinization (page 146). As a corollary, there may be found two reagents, each of which when alone is active, that may be inactive when associated in solution, as, for instance, solutions of potassium hydroxide and nitric acid, both of which are active when in separate solution, but inactive in the form of potassium nitrate; and that a gelatinizing reagent may be rendered less active or even inert by the presence of another reagent, as, for instance, the presence of alcohol, glycerine, or sodium chloride in concentration.

In the selection of the reagents used in this research a very large number of most varied kinds, electrolytes and non-electrolytes, and in various concentrations, were tried, the number aggregating probably 200 ; but unfortunately only a partial list was preserved. One of the difficulties met with in making this selection and in determining the concentration was in the wide differences in the behavior of different starches that could not be foretold excepting to a very limited degree. That is, if a given reagent in any concentration was found to be useless when tested with a given starch it could not be set aside because it might be found to be not only active but even extremely active with another starch. It was also found that there are certain starches that have a high to very high reactivity; others low to very low reactivity, and others high to moderate reactivity with a given reagent in given concentration. Thus, with a given reagent while the starches of Litium tend to high to very high reactivity, those of Hippeastrum and Hiomanthus tend mostly to low or very low reactivity, and those of the Irids mostly to intermediate gradation or moderate reactivities. It was also found that certain reagents are with all starches very strong gelatinizers, while others, in any concentration, tend to be relatively feeble; and still others that represent intermediate gradations. The reactions with sulphuric acid and sodium salicylate are mostly high to very high; those of chromic acid mostly moderate to high; those of barium chloride mostly low to very low; those of pyrogallic and nitric acids widely variable with different starches, etc.

It is obvious, in so far as values of individual reagents are concerned, that it must be recognized that the most useful in the differentiation starches are those whose activities show the most marked differences with different starches-or, in other words, which show the widest and most numerous fluctuations of the reactionintensity curves, as is instanced in the records of pyrogallic acid and nitric acid; that the fast-reacting reagents are of especial value in the differentiation of the slow to very slow reacting starches; and that the slow-reacting reagents are similarly valuable in relation to the rapidly reacting starches. A selection of the reagents on this basis is manifestly necessary where starches of diverse character are to be studied. In the testing of the various reagents to determine their values it was found in practice desirable to make at the outstart very concentrated solutions, using in the case of acids and bases generally approximately 50 per, cent solutions, and of salts approximately saturated solutions, and then modify the concentrations in the direction the intensity of the reaction indicates. It was also found of advantage to use for the first test a form of starch that is classed among the readily gelatinized and readily obtainable, such as that of Lilium candidum, and then make the final tests with this starch and with others which are classed among those having mostly a high, moderate, low, and very low reactivity, respectively. In this way reagents were selected which in kind and concentration have served admirably, although by no means perfectly, in eliciting peculiarities of the various starches here studied.

The following very incomplete list of the reagents and their effects shown by the starch of Lilium candidum, may be of advantage to subsequent investigators:

\begin{tabular}{|c|c|c|}
\hline Reagent. & $\begin{array}{l}\text { Concentration of } \\
\text { aqueous solution. }\end{array}$ & $\begin{array}{l}\text { Percentage of starch } \\
\text { gelatinized. }\end{array}$ \\
\hline Pyrogallic acid & $\begin{array}{l}4 \text { gms. to } 35 \text { c.c., } \\
\text { with } 0.3 \text { gm. of } \\
\text { oxalic acid }\end{array}$ & 92 p. ct. in $60 \mathrm{~min}$. \\
\hline Tartaric acid. & Concentrated.... & No effect in $60 \mathrm{~min}$. \\
\hline Lactic acid. . & Do......... & Do. \\
\hline Tannic acid. & Do........... & Do. \\
\hline Citric acid.. & Do.. & Do. \\
\hline & Do......... & Do. \\
\hline Chromic acid & 2.5 gms. in 20 c.c. & 95 p. ct. in 60 min. \\
\hline Nitric acid... & 10 gms. in 35 c.c. & 99 p. ct. ir \\
\hline & in 27 c.c. & 97 p. ct. \\
\hline Hyd & 9 gms. in 10 c.c.. & 100 p. ct. in $15 \mathrm{sec}$. \\
\hline Tun & Concentrated.... & No effect in $60 \mathrm{~min}$. \\
\hline Phos & Do........ & Do. \\
\hline Phos & Do.......... & Do. \\
\hline Chlor & in 5 c.c. & $\begin{array}{l}\text { Do. } \\
100 \text { p. ct. }\end{array}$ \\
\hline & $0.75 \mathrm{gm}$. in 55 c.c. & 100 p. c \\
\hline Potas & Concentrated.... & \\
\hline Potassiu & & $\begin{array}{l}\text { in majority } \\
\text { 1.; no further } \\
60 \text { min. }\end{array}$ \\
\hline Potas & a 30 c.c. & 95 p. c \\
\hline & Conce & No ef \\
\hline & Do & $100 \mathrm{p} . \mathrm{c}$ \\
\hline Pota & Do & No effect in $60 \mathrm{~min}$. \\
\hline Potas & & \\
\hline & & $\begin{array}{l}\text { Almost complete in } \\
60 \mathrm{~min} \text {. }\end{array}$ \\
\hline ots & $1 \mathrm{gm}$ & 93 p. ct. in 1 \\
\hline & $5 \mathrm{gm}$ & 98 p. ct. in $60 \mathrm{sec}$. \\
\hline $\begin{array}{l}\text { sulphate } \\
\text { aganate. }\end{array}$ & $\begin{array}{r}\text { Concentrated.... } \\
\text { Do......... }\end{array}$ & $\begin{array}{l}\text { No effect in } 60 \mathrm{~min} \text {. } \\
\text { Do. }\end{array}$ \\
\hline Sodium hydroxide... & $0.5 \mathrm{gma}$ in 100 c.c. & 88 p. ct. in 15 sec. \\
\hline & $1 \mathrm{gm}$. in 45 c.c.. & 97 p. ct. in 30 sec. \\
\hline Sodin & $10 \mathrm{gms}$. in 10 c.c. & 95 p. ct. in 10 eec. \\
\hline & trated.. & Complete in $10 \mathrm{sec}$. \\
\hline & & No effect in $60 \mathrm{~min}$. \\
\hline & & \\
\hline $\mathrm{C}$ & $\begin{array}{l}\text { gms. in } 16 \text { c.c.. } \\
\text { Concentrated.... }\end{array}$ & Incomple \\
\hline & & No effect in $60 \mathrm{~min}$. \\
\hline & & $\begin{array}{l}100 \mathrm{p} \text {. ct. in less than } \\
30 \mathrm{~min} \text {. }\end{array}$ \\
\hline & $5 \mathrm{gms}$. in $7 \mathrm{c.c}$ & 98 p. ct. in 3 min. \\
\hline & & 100 p. ct. in 30 min. \\
\hline & & 96 p. ct. in 30 min. \\
\hline & Concentrated & $\begin{array}{l}\text { No effect in } 60 \mathrm{~min} \text {. } \\
100 \text { p. ct. in } 2 \mathrm{~min} \text {. }\end{array}$ \\
\hline Cobalt nitrate & $9 \mathrm{gms}$. in 15 c.c. . & 97 p. ct. in $15 \mathrm{~min}$. \\
\hline
\end{tabular}




\begin{tabular}{|c|c|c|}
\hline Reagent. & $\begin{array}{l}\text { Concentration of } \\
\text { aqueous solution. }\end{array}$ & $\begin{array}{l}\text { Percentage of starch } \\
\text { gelatinized. }\end{array}$ \\
\hline 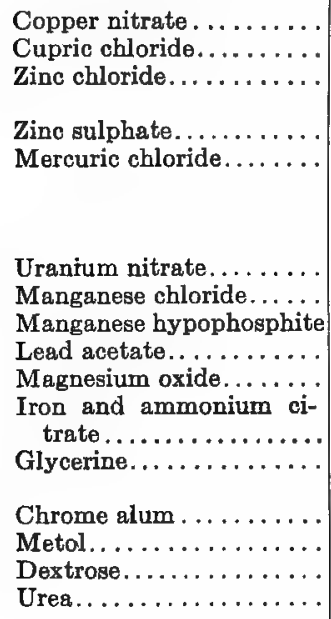 & 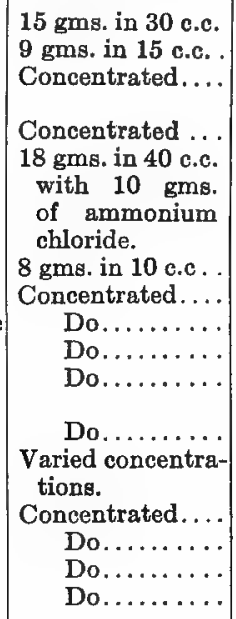 & $\begin{array}{l}98 \text { p. ct. in } 5 \mathrm{~min} . \\
\text { No effect in } 60 \mathrm{~min} . \\
\text { Do. } \\
\text { Do. } \\
\text { Do. } \\
\\
\text { Do. } \\
\text { Do. }\end{array}$ \\
\hline
\end{tabular}

Many interesting and unexpected peculiarities will be found upon examination of the foregoing table. For instance, potassium nitrate is inert with the starch of Lilium candidum, while potassium nitrite causes complete gelatinization in 1 minute; and while the former has been found to be inactive with this starch, it is recorded by other investigators as being active in relation to the starches of Triticum and $Z$ ea. This latter peculiarity is noted in the case of tannic acid. The sulphides of potassium and sodium are very active, but the sulphide of calcium is inactive. Strontium nitrate gelatinized 98 per cent of the starch in 3 minutes, while strontium bromide required 30 minutes for the same effect; but the corresponding potassium salts showed a reversal of reaction-intensities. Barium chloride is very active, but barium nitrate is inactive; and zinc chloride and zine sulphate show the same characteristics. Sodium hydroxide and hydrochloric acid when in separate solutions are very active, but sodium chloride is inactive, etc.

A detailed study of the specific properties of the ions and molecules of these reagents in their relations to the starch molecules in the phenomena of gelatinization, and also in the subsequent disintegration processes, is of prime importance, and not only in the elucidation of the chemistry of the starch molecule, but also in colloidal chemistry in general. Inasmuch, however, as the fundamental object of these gelatinization experiments has been the differentiation of starches from different sources by peculiarities of the quantitative and qualitative reactions, as this object has been attained without reference to the precise natures of the chemical reactions involved, and as detailed study of parts played by the different ions and molecules is therefore needless for the fulfilment of the purposes of the investigation and would lead us far beyond the limitations of space in this memoir, further study of this nature has been omitted.

Variable ReLationships of the Reaction-Intensities as Regards Sameness, Intermediateness, Etc.

That we are dealing in the starches from different plant sources with stereoisomers, and not merely with mechanical mixtures of varying proportions of several kinds of starch or with starches that differ because of varying impurities, etc., is evidenced by variations observed in the reaction-intensity relationships of the parental and hybrid starches with different reagents (see charts of both $A$ and $B$ series). Were there, for instance, merely mechanical mixtures of varying proportions representing the parental and hybrid starches, respectively, and a given reagent, it might be found that the reactivities are in the order of seed parent, pollen parent, and hybrid, and that if there were used other concentrations of the same reagent, while the reactionintensities would be increased or decreased, the order of reactivity would not be changed. Moreover, it would be expected that with all reagents the same order of reactivity would be found. It also seems clear, if impurities played any important part, that when closely related reagents, such as potassium and sodium hydroxide, are used, while some differences in mean reaction-intensity might be expected, there should not be a change in the order of reactivity. The opposite is shown by these charts. Thus, Charts A 6, A 7 , A 8 (chloral-hydrate, chromic-acid, and pyrogallic-acid reactions) of the $\mathrm{Ama}$ ryllis-Brunsvigia-Brunsdonna reactions show in the chloral-hydrate reactions that the order of reactivity is Brunsdonna sandero, $B$. sanderce alba, Amaryllis belladonna, and Brunsvigia josephince, the first two showing a markedly greater reactivity than the second two, and the reactions of the members of each pair being closely alike. In the chromic-acid reactions all four are alike, so that while there is marked differentiation with chloral hydrate there is none with chromic acid. In the pyrogallic-acid reactions there is somewhat better differentiation than in the chloral-hydrate reactions, and also an entire change in the order of reactivities, here the order being Brunsvigia josephina, A maryllis belladonna, Brunsdonna sanderoe alba, and $B$. sandero, the hybrids, as in the chloral-hydrate reactions, being nearly the same, but the parental starches well differentiated from each other; moreover, here the parental starches are more reactive, while in the chloral-hydrate reactions they are less reactive. Corresponding phenomena are observed in instances where the reagents are chemically very closely related, as in the cases of potassium and sodium hydroxide, potassium and sodium sulphide, and mineral acids, which would seem to eliminate the possibility of these changes being due to mechanical mixtures of different starches or to impurities. The Amaryllis set exhibits with potassium hydroxide no noticeable differences in the reactivities of the four starches, because probably of the great rapidity of gelatinization, and little or very little difference is found in the reactions with the nitric, sulphuric, and hydrochloric acids. But with sodium hydroxide and all of the other reagents, excepting chromic acid, one or more of the reactivities will be found at variance with the others; and, moreover, the relationships of order of reaction-intensity are of the most varied character. Thus, in the sodium hydroxide chart the order of reactivity is Amaryllis belladonna, Brunsvigia josephina, Brunsdonna sandero alba, and $B$. sandero, which order is entirely different from what is found in the chloral-hydrate and pyrogallic-acid charts. Comparing the potassium-sulphide and sodium-sulphide charts it is seen that in the former the order is A maryllis 
belladonna and Brunsdonna sandero (both the same), Brunsvigia josephina, and Brunsdonna sanderce alba; and in the sodium-sulphide chart, Brunsvigia josephine, Amaryllis belladonna, Brunsdonna sandera, and $B$. sanderce alba. Viewing the various charts of this set, all sorts of variations in the relative reaction-intensities of these four starches will be found: In some, such as in the charts for chromic acid, potassium hydroxide, and barium chloride, there are practically or absolutely no differences; the charts for nitric acid, sulphuric acid, and hydrochloric acid show some but not marked differences; the charts for chloral hydrate, potassium iodide, potassium sulphocyanate, and cobalt nitrate show welldefined pairing-in all three reactions the parents and the hybrids, respectively, are paired, in the chloralhydrate reaction the parental pair having the less reactivity, while in the potassium-sulphocyanate and cobaltnitrate reactions the greater reactivity. In other instances there may be a single pair, the other two starches differing from this pair and from each other, as in the reactions of pyrogallic acid, potassium sulphide, strontium nitrate, cupric chloride, and mercuric chloride; in other instances all four are unlike, as in the charts of sodium hydroxide, sodium sulphide, calcium nitrate, and so on.

Pairing when present may be confined to either the parents or the hybrids, or there may be pairing of both parents and both hybrids, and in one instance (potassium-sulphide chart) Amaryllis and Brunsdonna sanderce are paired, and show distinctly different reactionintensities from those of the other parent and the other hybrid, which two latter in turn differ markedly. In other words, if any given set of parents and offspring be taken and their reaction-intensities with the different reagents be compared, it will be found that there are not only very marked-differences in the average reactionintensities of the several members with the different reagents, but also most remarkable variations in the relative reaction-intensities with these reagents, so that while a given starch may show the highest reactivity of the set with one reagent it may show the least with another, and so on, each starch being capable of reacting in a way independently of the others, so that all possible combinations of varying relationships may occur. This means, of course, that in one reaction the hybrid may be the same as that of the seed parent, in another the same as that of the pollen parent, in another the same as the reactions of both parents, in another intermediate, in another in excess of those of either parents, etc. Each reagent, therefore, has the property of eliciting some definite parental phase. A somewhat detailed consideration of this important phenomenon will be taken up in Chapter V.

\section{Variations in the Reaction-INTENSities as RE- gards Height, SUM, aNd AVERAGe. \\ (Table B 1, Chart C 1.)}

The valuations of the reaction-intensities have been based, as has been repeatedly stated, on definite but arbitrary scales: Those of the reaction-intensities of the polarization, iodine, gentian-violet, and safranin reactions on a scale of 0 to 105 ; those of the temperatures of gelatinization on a scale of $40^{\circ}$ to $95^{\circ}$, and those of the reactions with the chemical reagents on a scale that shows in one segment the percentage of total starch gelatinized within 60 minutes, and in another the time of complete or practically complete gelatinization within the same period. Inasmuch as in all three sets the same abscissæ are used, and as the scale-values bear in all of the charts the same relationships, the figures of one scale always have a fixed value in relation to given figures of the other scales; hence, if the scale for the polarization reactions were adopted for valuation of all kinds of reactions the values in all cases would be comparable upon a common basis. For purposes of gross comparisons this scale has been divided arbitrarily into 5 parts which are intended to designate very high, high, moderate, low, and very low reactivity, respectively. Thus, any reaction that falls between 80 and 105 (or in the temperature scale 52.5 and $42.5^{\circ}$; or in the chemical reagent scale 25 and 0 minutes), both inclusive, is recorded as being very high; between 60 and less than 80 , etc., as being high, etc. Table B 1 gives, in connection with each starch, the numbers of the 26 reactions that fall under one or another of these divisions; the sum of the individual reactionintensity values of each starch; and the average of this sum, which latter is obtained by dividing by 26 . Such data constitute a very satisfactory basis for comparisons of the reaction-intensities of the different starches individually, generically, and so on, and they are rendered of additional value if they are also reduced to chart form. (Chart C1.)

The most conspicuous features of the table and chart are: The close correspondence in the numerical distribution of the reaction-intensities (very high, high, moderate, low, very low) of the several starches of each set of parents and hybrids and of each generic group, together with the close correspondence of the sum and the average values, except when the set or genus represented contains members of subgenera or subgeneric groups; and the varying values of the different generic groups.

It will be seen, for example, in Hippeastrum, in which generic group the parents are closely related, and where consequently there is but little deviation in the reactions of the hybrids from those of the parents, that the figures in each of the columns of the chart for all of the parents and hybrids are in close correspondence, and that the sums and averages of the reaction-intensities are also quite close. The range of these figures in the table for all the starches studied is limited by 2614 (sum) and 100 (average) in Cymbidium lowianum and 525 (sum) and 20 (average) in Homanthus katherino. In the first column (very high reactivities) the figures range from 2 to 4 ; in the second column, from 0 to 3 ; in the third column, from 3 to 5 ; in the fourth column, from 3 to 6 ; in the fifth column, from 11 to 14 ; in the sixth column, from 748 to 925 ; and in the last column, from 29 to 36 . These ranges will be found to be within very narrow limits when compared with the figures of the table, as a whole. Such correspondences are also well marked in Nerine, Narcissus, Litium, Gladiolus, Tritonia, Phairu, Miltonia, and Cymbidium. On the other hand, when the genus is represented by bigeneric parents or by members of subgenera or subgeneric groups, there may be more or less marked deviations from those found when the parents are monogeneric and not so far separated 
TABLE B 1-Summary of the Reaction-intensities and the Sum and the Average Reaction-values of the Starches of Parentand Hybrid-stocks:

\begin{tabular}{|c|c|c|c|c|c|c|c|c|}
\hline & $\begin{array}{l}\frac{d}{60} \\
\frac{6}{8} \\
\frac{8}{2}\end{array}$ & 姁 & 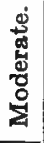 & 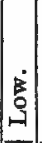 & 客 & 煦 & 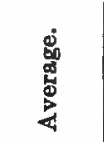 & 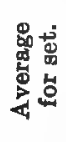 \\
\hline Amaryllis belladonna . . . . . . & 11 & 5 & 7 & 1 & 2 & 1973 & $76)_{70}$ & \\
\hline Brunsvigia josephinæ. . . . . . . & 8 & 5 & 7 & 5 & 1 & 1760 & $68\}^{72}$ & \\
\hline Brunsdonna sand. alba...... & 8 & 4 & 2 & 4 & 8 & 1362 & $52\}_{535}$ & 62.7 \\
\hline Brunsdonna sander $œ . . . . .$. & 8 & 4 & 2 & 4 & 8 & 1437 & $55\}^{53.5}$ & \\
\hline Hippeastrum titan......... & 2 & 2 & 4 & 4 & 14 & 748 & 29 & \\
\hline Hippeastrum cleonia........ & 2 & 3 & 3 & 6 & 12 & 821 & 32 & 30.3 \\
\hline Hippeastrum titan-cleonia... & 2 & 2 & 4 & 5 & 13 & 790 & 30 & 30.0 \\
\hline Hippeastrum ossultan....... & 4 & 0 & 4 & 5 & 13 & 838 & 32 & \\
\hline Hippeastrum pyrrha. . . . . . . & 3 & 1 & 4 & 5 & 13 & 822 & $31\}$ & 32.3 \\
\hline Hippeastrum ossult.-pyrh. ... & 5 & 0 & 5 & 3 & 13 & 880 & 34 & \\
\hline Hippeastrum døeones....... & 3 & 2 & 4 & 6 & 11 & 925 & 36 & \\
\hline Hippeastrum zephyr........ & 2 & 3 & 3 & 5 & 13 & 833 & 32 & 34 \\
\hline Hippeastrum dxon.-zeph ..... & 2 & 3 & 3 & 5 & 13 & 872 & 34 & \\
\hline Hæmanthus katherinæ....... & 1 & 3 & 3 & 1 & 18 & 525 & 20 & \\
\hline Hæmanthus magnificus . . . . . & 1 & 3 & 3 & 4 & 15 & 652 & 25 & 22 \\
\hline Hæmanthus andromeda..... & 2 & $\mathbf{0}$ & 5 & 1 & 18 & 535 & 21 & \\
\hline Hæmanthus katherinæ....... & 1 & 3 & 3 & 1 & 18 & 525 & 20 & \\
\hline Hæmanthus puniceus........ & 4 & 6 & 3 & 8 & 5 & 1264 & 48 & 30.3 \\
\hline Hæmanthus könig albert..... & 2 & 1 & 4 & 2 & 17 & 597 & 23 & 80.0 \\
\hline Crinum moorei............ & 12 & 3 & 4 & 6 & 1 & 1907 & 73 & \\
\hline Crinum zeylanicum......... & 1 & 3 & 3 & 2 & 17 & 594 & 23 & 39 \\
\hline Crinum hybridum j. c. h. .... & 1 & 2 & 2 & 4 & 17 & 550 & 21 & \\
\hline Crinum zeylanicum......... & 1 & 3 & 3 & 2 & 17 & 594 & 23 & \\
\hline Crinum longifolium......... & 9 & 5 & 2 & 9 & 1 & 1685 & 65 & 40 \\
\hline Crinum kircape. . . . . . . . & 1 & 5 & 0 & 7 & 13 & 841 & 32 & \\
\hline Crinum longifolium......... & 9 & 5 & 2 & 9 & 1 & 1685 & 65 & \\
\hline Crinum moorei........... & 12 & 3 & 4 & 6 & 1 & 1907 & 73 & 73.3 \\
\hline Crinum powellii........... & 15 & 5 & 3 & 3 & 0 & 2142 & 82 & \\
\hline Nerine crispa. . . . . . . . . . & 5 & 3 & 5 & 2 & 11 & 1093 & 42 & \\
\hline Nerine elegans............ & 7 & 2 & 3 & 3 & 11 & 1147 & 44 & \\
\hline Nerine dainty maid. . . ...... & 5 & 4 & 2 & 3 & 11 & 1144 & 44 & 44 \\
\hline Nerine queen of roses....... & 6 & 3 & 5 & 1 & 11 & 1199 & 46 & \\
\hline Nerine bowdeni............ & 3 & 3 & 6 & 3 & 11 & 1039 & 40 & \\
\hline Nerine aarn. var. cor. maj.... & 4 & 4 & 4 & 3 & 11 & 1015 & 39 & \\
\hline Nerine giantess............ & 4 & 4 & $\mathbf{5}$ & 2 & 11 & 1051 & 40 & 39 \\
\hline Nerine abundance......... & 4 & 3 & 5 & 2 & 12 & 957 & 37 & \\
\hline Nerine sarn. var. cor. maj... & 4 & 4 & 4 & 3 & 11 & 1015 & 39 & \\
\hline Nerine curv, var. foth. maj... & 5 & 2 & 5 & 2 & 12 & 1001 & $38\}$ & 37.2 \\
\hline Nerine glory of sarnia....... & 4 & 1 & 4 & 4 & 12 & 869 & 33 & \\
\hline Narcissus taz. grand mon.... & 1 & 2 & 6 & 6 & 11 & 864 & 33 & \\
\hline Narcissus poeticus ornatus... & 2 & 2 & 4 & 10 & 8 & 938 & $36\}$ & 37 \\
\hline Narcissus poetaz triumph... & 3 & 2 & 8 & 6 & 7 & 1088 & 42 & \\
\hline Lilium martagon album..... . & 19 & 4 & 3 & 0 & 0 & 2502 & 96 & \\
\hline Lilium maculatum......... & 21 & 1 & 4 & 0 & 0 & 2551 & 98 & 97 \\
\hline Lilium marhan. . . . . . . . . . & 21 & 1 & 4 & 0 & 0 & 2541 & 97 & \\
\hline Lilium martagon. . . . . . . . . & 18 & 5 & 3 & 0 & 0 & 2430 & 93 & \\
\hline Lilium maculatum......... & 21 & 1 & 4 & 0 & 0 & 2551 & $98\}$ & 97 \\
\hline Lilium dalhansoni. . . . . . . . . & 21 & 2 & 3 & 0 & 0 & 2580 & 99 & \\
\hline Lilium tenuifolium . . . . . . . . & 21 & 2 & 3 & 0 & 0 & 2567 & 99 & \\
\hline Lilium martagon album..... . & 19 & 4 & 3 & 0 & 0 & 2502 & 96 & 97 \\
\hline Lilium golden gleam. . . . . . . & 20 & 2 & 4 & 0 & 0 & 2529 & 97 & \\
\hline Lilium chalcedonicum....... & 20 & 4 & 2 & 0 & 0 & 2519 & 97 & \\
\hline Lilium candidum. ......... & 20 & 4 & 2 & 0 & 0 & 2460 & 94 & 95 \\
\hline Lilium testaceum.......... & 21 & 2 & 3 & 0 & 0 & 2482 & 95 & \\
\hline Lilium pardalinum . . . . . . . . & 20 & 4 & 2 & 0 & 0 & 2530 & 97 & \\
\hline Lilium parryi............ & 22 & 0 & 3 & 1 & 0 & 2556 & 98 & 94.6 \\
\hline Lilium burbanki........... & 16 & 4 & 4 & 2 & 0 & 2309 & 89 & \\
\hline Iris iberica . . . . . . . . . & 3 & 2 & 7 & 9 & 5 & 1139 & 44 & \\
\hline Iris trojana. . . . . . . . & 3 & 2 & 10 & 6 & 5 & 1282 & 49 & 46 \\
\hline Iris ismali. . . . . . . . . . & 3 & 2 & 9 & 8 & 4 & 1181 & 45 & \\
\hline Iris iberica . . . . . . . . . . & 3 & 2 & 7 & 9 & 5 & 1139 & 44 & \\
\hline Iris cengialti. . . . . . . & 3 & 3 & 6 & 9 & 5 & 1166 & 44 & 44 \\
\hline Iris dorak. . . . . . . . . . . & 3 & 2 & 10 & 6 & 5 & 1181 & 45 & \\
\hline Iris cengialti, . . . . . . . . . & 3 & 2 & 7 & 9 & 5 & 1166 & 44 & \\
\hline Iris pallida queen of may..... & 2 & 4 & 7 & 8 & 5 & 1100 & $42\}$ & 42 \\
\hline Iris mrs. alan grey ......... & 2 & 4 & 5 & 8 & 7 & 1085 & 41 & \\
\hline Iris persica var. purpurea.... . & 9 & 9 & 4 & 0 & 4 & 1853 & 71 & \\
\hline Iris sindjarensis........... & 10 & 8 & 4 & 2 & 2 & 1922 & 74 & 72 \\
\hline Iris pursind . .............. & 9 & 9 & 4 & 0 & 4 & 1858 & 71) & \\
\hline
\end{tabular}

TABLE B 1.-Continued.

\begin{tabular}{|c|c|c|c|c|c|c|c|c|}
\hline & $\begin{array}{l}\frac{d}{50} \\
.0 \\
0 \\
0 \\
0 \\
0\end{array}$ & 遒 & 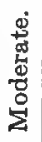 & $\begin{array}{l}8 \\
8 \\
9 \\
\end{array}$ & 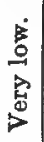 & 㫛 & 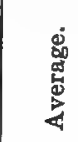 & 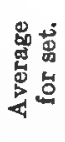 \\
\hline Gladiolus cardinalis. ......... & 2 & 2 & 3 & 2 & 17 & 695 & 27) & \\
\hline Gladiolus tristis............ & 1 & 3 & 5 & 6 & 11 & 877 & $34\}$ & 27 \\
\hline Gladiolus colvillei .......... & 2 & 0 & 5 & 1 & 18 & 604 & 23 & \\
\hline Tritonia pottsii............ & 1 & 5 & 3 & 7 & 10 & 964 & 37 & \\
\hline Tritonia crocosmia aurea..... & $\overline{1}$ & 2 & 3 & 7 & 13 & 741 & $28\}$ & 33.3 \\
\hline Tritonia crocosmgflora....... & 2 & 4 & 4 & 6 & 10 & 950 & 36 & \\
\hline Begonia sing. crim. scar ...... & 18 & 4 & 3 & 1 & 0 & 2369 & 91 & \\
\hline Begonia socotrana......... & 5 & 2 & 2 & 5 & 12 & 982 & 38 & 70 \\
\hline Begonia mrs. heal. . . . . . . . & 16 & 2 & 3 & 3 & 2 & 2117 & 81) & \\
\hline Musa arnoldiana........... & 20 & 2 & 4 & 0 & 0 & 2502 & 96 & \\
\hline Musa gilletii. .............. & 9 & 4 & 8 & 4 & 1 & 1811 & 69 & 77 \\
\hline Musa hybrida............. & 8 & 4 & 8 & 4 & 2 & 1728 & 66 & \\
\hline Phaius grandifolius......... & 12 & 5 & 5 & 3 & 1. & 2000 & 77 & \\
\hline Phaius wallichii........... & 17 & 2 & 5 & 1 & 1 & 2157 & 83 & 80 \\
\hline Phaius hybridus. . . . . . . . . & 14 & 6 & 2 & 3 & 1 & 2096 & 81 & \\
\hline Miltonia vexillaria........... & 12 & 4 & 5 & 4 & 1 & 1969 & 76 & \\
\hline Miltonia roezlii............ & 7 & 7 & 5 & 6 & $\overline{1}$ & 1755 & 67 & 77 \\
\hline Miltonia bleuana........... & 16 & 4 & 4 & 1 & 1 & 2258 & 87 & \\
\hline Cymbidium lowianum ....... & 22 & 1 & 3 & 0 & 0 & 2614 & 100 & \\
\hline Cymbidium eburneum. ...... & 21 & 1 & 4 & 0 & 0 & 2602 & 100 & 99 \\
\hline Cymbidium eburneo-lowianum & 21 & 0 & 4 & 1 & 0 & 2510 & 97) & \\
\hline
\end{tabular}

as to fall into subgeneric divisions, as in the case of the genera just referred to. In the Amaryllis-Brunsvigia set two closely related genera are represented and there is a tendency to higher reactivity of Amaryllis belladonna than of Brunsvigia josephina, differences being noted especially in the numbers of the very high and the low reactivities, and in the sums and averages. The hybrids show distinctly lower reactivities, as a whole, than those of either parent, and there is striking identity as regards the distribution of the reaction-intensities among the several divisions, but there are distinct though not marked differences in both sums and averages, so that while these two starches are not distinguishable from each other by differences in distribution of the reactionintensities they may be distinguished by the sums and averages of the reaction-intensities. In the Crinums there are subgeneric groups characterized by tender and hardy species, the former having a tendency to distinctly lower reactivities than the latter. Each of the hybrids tends to be more closely related in its reaction-intensities to either seed or pollen parent.

The differences in distribution in the highly reactive species and hybrids are conspicuous especially in the high number of very high reactivities and the low number of the very low reactivities, and for the reverse in the low reactive species and the hybrids. The sums and averages are markedly different in the two groups. In Homanthus, $H$. puniceus seems to be representative of a subgeneric group that differs from that of which the other two species belong. In Iris, the I. persica-sindjarensispersica var. purpurea set stands distinctly apart from the other three, exhibiting markedly higher reactivities. In Begonia, $B$. socotrana is evidently variant in relation to the other species, and is, as is well known, an exceptional form of this genus. In Musa there is a very wellmarked tendency for higher reactivities of one than of the other parent, which indicates that these species represent some form of generic subdivision. 
With these exceptions, the figures for the several members of each group and each genus tend to be distributed among the several divisions in case of each genus with remarkable uniformity, in some genera a conspicuously large number falling among the very high, or the very high and high reactions, or the very low, or the very low and low reactivities, and so on. Such differences, of themselves, are usually quite definite in making distinct groups which upon comparison will be found to agree remarkably with botanical classification. Thus Hippeastrum, Nerine, Gladiolus, and Tritonia are characterized particularly by the relatively large number of reactions that are very low (the number varying in the different genera) and the fairly uniform distribution of the remaining reactions among the other divisions, chiefly among the moderate and low. In Lilium, Phains, and Cymbidium the characterization is by the very large number of very high reactions and the fairly uniform distribution of the other reactions among the other divisions, especially generally among the high and moderate. In Amaryilis-Brunsvigia, Crinum, Hamanthus, Iris, Begonia, and Musa variations from these systems may be observed because of certain subgeneric peculiarities that have already been referred to.

These data indicate quite clearly that peculiarities in the distribution of these reaction-intensities are intimately related to generic and subgeneric divisions, and that when the distributions in the case of members of a set or of a genus may be alike or nearly alike there may be differences in the sums and averages that are more or less definitely distinctive. For instance, the distribution in Brunsdonna sanderce alba and $B$. sanderce is identical, but the sums and averages differ sufficiently to differentiate these hybrids. In Nerine, the distributions differ very little; in some cases the sums and averages are absolutely or practically identical, and in others they differ within small to very narrow limits. Under such conditions positive identification of different members of the group can not satisfactorily be made. Corresponding conditions are found in relation to intergeneric differentiation. Thus, the distributions in Hippeastrum and Nerine are closely the same, and were dependence placed upon this feature to distinguish genera it would naturally be concluded that the genera are alike; but upon a careful examination of the two sets of figures it will be found that in Hippeastrum there is a manifest tendency for a shifting of the reaction-intensities toward the very low reactivity end, and in Nerine in the same direction, but to a slightly less degree, so that in the final summing up the sums and averages in the former fall lower than in the latter-in Hippeastrum, ranging from 748 to 925 and 29 to 36 , respectively; and in Nerine from 869 to 1199 and 33 to 46 , respectively. In Gladiolus and Tritonia, very closely related genera, the distribution closely corresponds to the preceding groups in the several respects referred to. On the other hand, Lilium and Cymbidium, while in general very closely alike in distribution, sum, and average are very markedly different from all other groups. Phaius values bear a close resemblance to the figures of LiTium and Cymbidium. Iris in its first three sets stands apart from all other genera in the manner of distribution of the reactionintensities, yet the sums and averages are close to but somewhat less than in Nerine. In other words, different genera may or may not exhibit distinctive peculiarities in the distribution, sum, and average of the reaction-intensities. The value of such data seems to lay particularly in showing that members of a genus that are not so differentiated as to fall into subgeneric divisions tend to exhibit a method of distribution of the reaction-intensities according to a definite system, which system is composed of the averages of the number of very high, high, moderate, low, and very low reaction-intensities, of the average of the sum of the reaction-intensities, and of the average of the latter. For comparative purposes the system represented by Hippeastrum, I ris (first three sets), and Lilium may be taken because they show different types:

\begin{tabular}{|c|c|c|c|}
\hline & $\begin{array}{c}\text { Hippe- } \\
\text { astrum. }\end{array}$ & Iris. & Lilium. \\
\hline Very high ...... & 2.8 & 2.7 & 20 \\
High......... & 1.8 & 2.6 & 2.7 \\
Moderate...... & 3.7 & 7.6 & 3.1 \\
Low ........ & 5 & 8 & 0.2 \\
Very low ...... & 12.8 & 5.1 & 0 \\
Sum ......... & 836. & $1,160$. & $2,447$. \\
Average...... & 31. & 44. & 94. \\
\hline
\end{tabular}

If the figures for any given member of any one of the genera represented be compared with the figures for the genus, it will be found that those for the corresponding columns differ, if at all, only within narrow limits. Thus, in case of Hippeastrum the figure in the first column of this table and chart is 2.8 , while the figures for the nine starches represented in this genus vary between 2 and 5 ; in the last column the figure is 12.8 , while the range for all of these starches is from 11 to 14 . The sum is 836 , and the range from 748 to 925 . The average is 31 , and the range from 29 to 36 . And so on with Iris and Lilium. When, however, there are subgeneric groups there may be as many types as there are groups, as is well illustrated by instances referred to.

Obviously, the method of differentiating genera, subgeneric groups, species, hybrids, and varieties by such a system has its limitations, not because of the failure of the data per se, but because of the faultiness of the method of formulating the data. This is manifest, for instance, in Hippeastrum and Nerine, in which the data as tabulated indicate very closely related genera or even subgenera, yet these genera, although belonging to the same family, are well separated and are not confounded by the botanist. When, however, the data are presented in other forms, as in other tables and charts, the genera are as markedly differentiated from each other, and the members of each genus from each other, as they are by the data of the systematist. Finally, it is of interest to note that in summing up these averages intermediateness of the hybrid is not the rule, the tendency being more frequently for the hybrid values to exceed or fall below those of the parents than to be intermediate.

\section{Average Temperatures of Gelatinization Compared with the Average Reaction-intensities.}

\section{(Table B 2, Chart B .12)}

During the progress of the research it was found that the temperatures of gelatinization bore varying relationships to the average reaction-intensities, as a whole, of different members of certain sets, different sets, and dif- 
Table B 2.-Temperatures of Gelatimization.

\begin{tabular}{|c|c|c|c|c|c|c|}
\hline \multirow[b]{2}{*}{ Amaryllis belladonna. . . . . } & \multicolumn{2}{|c|}{$\begin{array}{c}\text { In majority } \\
\text { of the } \\
\text { grains. }\end{array}$} & \multicolumn{2}{|c|}{$\begin{array}{l}\text { In all or } \\
\text { practically } \\
\text { all of the } \\
\text { grains. }\end{array}$} & \multirow{2}{*}{$\begin{array}{c}\begin{array}{c}\text { Mean } \\
\text { of } \\
\text { latter. }\end{array} \\
72.7^{\circ}\end{array}$} & $\begin{array}{l}\text { Aver- } \\
\text { age } \\
\text { for } \\
\text { set. }\end{array}$ \\
\hline & 70 & to $71^{\circ}$ & $72.5 \mathrm{t}$ & $73^{\circ}$ & & \\
\hline Brunsvigia josephinæ. ...... & 65 & 66 & 70 & 72 & 71 & \\
\hline Brunsdonna sand. alba & 70 & 71 & 71.5 & 73 & 72.25 & 71.18 \\
\hline Brunsdonna sanderœ.. & 70 & 71.5 & 72 & 72.5 & 72.75 & \\
\hline Hippeastrum titan .... & 74 & 75 & 77 & 77.5 & 77.25 & \\
\hline Hippeastrum cleonia. ...... & 71 & 73 & 73 & 74 & 73.5 & 74.42 \\
\hline Hippeastrum titan-cleonia... & 72 & 74 & 73 & 74 & 73.5 & \\
\hline Hippenstrum ossultan ...... & 73 & 74 & 75 & 76 & 75.5 & \\
\hline Hippeastrum p.rr & 71 & 73 & 73 & 74 & 73.5 & 73.8 \\
\hline Hippeastrum ossu & 70 & 72 & 72 & 73 & 72.5 & \\
\hline Hippeastrum dæ0 & 72.5 & 74 & 74 & 75 & 74.5 & \\
\hline Hippeastrum zeph & 72 & 73 & 73 & 75 & 74 & 73.7 \\
\hline Hippeastrum dæon.-zep & 72 & 73 & 72 & 73 & 72.5 & \\
\hline Hæmanthus katherinæ.... . & 79 & 80 & 82 & 84 & 83 & \\
\hline Hremanthus magnificus. . . . . & 77 & 77.5 & 78 & 79 & 78.5 & 81 \\
\hline Hæmanthus andromeda.... & 75.5 & 80 & 81 & 82 & 81.5 & 01 \\
\hline Hæmanthus katherinæ . . . . . & 79 & 80 & 82 & 84 & 83 & \\
\hline Hrmanthus puniceus...... & 77 & 79 & 81 & 82.5 & 81.75 & 82.7 \\
\hline Hæmanthus könig albert.... & 80 & 82 & 82.5 & 84 & 83.25 & \\
\hline Crinuìin n & 68 & 70 & 70 & 71 & 70.5 & \\
\hline Crinum zeylanicum ....... & 77 & 78 & 79 & 80 & 79.5 & 77 \\
\hline Crinum hybridum j c. h. . & 78 & 80 & 80 & 82 & 81 & \\
\hline Crinum zeylanicum ....... & 77 & 78 & 79 & 80 & 79.5 & \\
\hline Crinum longifolium ... & 70 & 71 & 74 & 75 & 74.5 & 77.3 \\
\hline Crinum kircape . . . . . . & 75 & 76 & 77 & 79 & 78 & \\
\hline Crinum longifolium .... & 70 & 71 & 74 & 75 & 74.5 & \\
\hline Crinum moorei........ & 68 & 70 & 70 & 71 & 70.5 & 71.2 \\
\hline Crinum powellii.......... & 65 & 67 & 68 & 69 & 68.5 & 11.2 \\
\hline Nerine crispa........... & 64 & 65 & 70 & 71.5 & 70.7 & \\
\hline Ner & 68.5 & 70 & 75 & 76.9 & 75.9 & \\
\hline Nerine dai & 69 & 70.5 & 72.5 & 73.8 & 73.2 & 72.9 \\
\hline Nerine que & 68 & 69.1 & 71 & 72.8 & 71.9 & \\
\hline Nerine bow & 67.6 & 67.9 & 74 & 75 & 74.5 & \\
\hline Ner & 70 & 71 & 76 & 78.8 & 78.4 & \\
\hline Nerine giantes & 68.2 & 69.1 & 70.9 & 71 & 70.95 & \\
\hline Nerine abundan & 69 & 69.9 & 73.9 & 74.8 & 74.3 & \\
\hline Nerine sarn. var. cor. maj... & 70 & 71 & 76 & 78.8 & 78.4 & \\
\hline Nerine curv. var. foth. maj.. & 68.1 & 69 & 73.2 & 74.3 & 73.8 & 76.2 \\
\hline Nerine glory of sarnia ...... & 70 & 72 & 75.8 & 77 & 76.4 & \\
\hline Narcissus poeticus ornat. . . & 73 & 74 & 77 & 78 & 77.5 & \\
\hline Narcissus poeticus poetar. . & 67 & 69 & 71 & 73 & 72 & \\
\hline Narcissus poeticus herrick... & 69 & 71 & 76 & 78 & 77 & 75.3 \\
\hline Narcissus poeticus dante.... & 71.2 & 73.1 & 74 & 76 & 75 & \\
\hline ind mon... & 73 & 75 & 76 & 77 & 76.5 & \\
\hline Narcissus poeticus & 73 & 74 & 77 & 78 & 77.5 & 73.5 \\
\hline Narci & 73 & 75 & 76 & 77 & 76.5 & \\
\hline Nar & 71 & 72.8 & 74 & 75 & 74.5 & \\
\hline Narcissus poeticus ornatus. . & $7 \overline{3}$ & 74 & 77 & 78 & 77.5 & 75 \\
\hline $\mathrm{Nar}$ & 71 & 72 & 73.5 & 74.5 & 74 & \\
\hline Narcissus telamo & 70 & 72 & 73 & 75 & 74 & \\
\hline Narcissus poeticus ornatus. . & 73 & 74 & 77 & 78 & 77.5 & 75.8 \\
\hline Narcissus doubloon . . . . . . . & 71.2 & 73 & 75 & 77 & 76 & \\
\hline Narcissus princess mar & 70 & 72 & 74 & 76 & 75 & \\
\hline Narcissus poeticus poetar. . & 67 & 69 & 71 & 73 & 72 & 74.2 \\
\hline Narcissus cresset. . . . . . . . & 71 & 73 & 74.5 & 76 & 75.7 & \\
\hline $\mathrm{Nar}$ & 69.5 & 71 & 73 & 74 & 73.5 & \\
\hline poetar. . & 69 & 71 & 71 & 73 & 72 & 72.8 \\
\hline lat & 69.8 & 71.9 & 72 & 74 & 73 & \\
\hline & 70.2 & 72 & 73 & 75 & 74 & \\
\hline $\mathbf{N}$ & 69.5 & 71 & 73 & 74 & 73.5 & 74.2 \\
\hline t.... & 71 & 72.5 & 74 & 76 & 75 & \\
\hline $\mathrm{Na}$ & 70 & 71 & 73 & 74 & 73.5 & \\
\hline Narcissus albicans. & 70.2 & 72 & 73 & 75 & 74 & 73.9 \\
\hline nadame de graaff & 70 & 72 & 73.5 & 75 & 74.25 & \\
\hline eardale perfect. . & 68 & 69 & 72 & 74 & 73 & \\
\hline Narcissus madame de graaff & 70 & 72 & 73.5 & 75 & 74.25 & 74.4 \\
\hline Narcissus pyran & 73 & 74 & 76 & 77 & 76 & \\
\hline $\mathbf{N a}$ & 67 & 68.5 & 72 & 73 & 72.5 & \\
\hline e de graaff & 70 & 72 & 73.5 & 75 & 74.25 & 73.5 \\
\hline Ho & 68 & 69.4 & 73 & 74.5 & 73.75 & \\
\hline min. hu & 70 & 71.2 & 74.5 & 76 & 75.25 & \\
\hline Narcissus triandrus albu & 70 & 71 & 73 & 75 & 74 & 74.5 \\
\hline Narcissus agnes harvey.. & 70 & 71.8 & 73.8 & 75 & 74.4 & \\
\hline Narcissus emperor . ........ & 69 & 71 & 74 & 75.5 & 74.53 & \\
\hline Narcissus triandrus albus... & 70 & 71 & $7 \overline{3}$ & 75 & 74 & 72.8 \\
\hline Narcissus j. t. bennett poe. . & 64 & 64.8 & 69 & 71 & 70 & \\
\hline
\end{tabular}

TABLE B 2.-Continued.

\begin{tabular}{|c|c|c|c|c|c|c|}
\hline \multirow[b]{2}{*}{ Lilium martagon album . . . . } & \multicolumn{2}{|c|}{$\begin{array}{c}\text { In majority } \\
\text { of the } \\
\text { grains. }\end{array}$} & \multicolumn{2}{|c|}{$\begin{array}{l}\text { In all or } \\
\text { practically } \\
\text { all of the } \\
\text { grains. }\end{array}$} & \multirow{3}{*}{$\begin{array}{c}\begin{array}{c}\text { Mean } \\
\text { of } \\
\text { latter. }\end{array} \\
63 \\
61\end{array}$} & \multirow{2}{*}{$\begin{array}{c}\text { Aver- } \\
\text { age } \\
\text { for } \\
\text { set. }\end{array}$} \\
\hline & 59 & 61 & 62 & 64 & & \\
\hline Lilium maculatum . . . . . . . & 57 & 58 & 60 & 62 & & 61.2 \\
\hline Lilium marhan .......... & 56 & 58 & 59 & 60 & 59.5 & \\
\hline Lilium martagon. . . . . . . . . & 62 & 64 & 66.5 & 68.3 & 67.4 & \\
\hline Lilium maculatum . . . . . . . & 57 & 58 & 60 & 62 & 61 & 64.1 \\
\hline Lilium dalhansoni......... & 59 & 60.2 & 63 & 64 & 63.8 & \\
\hline Lilium tenuifolium....... & 52 & 53 & 55.6 & 56 & 55.8 & \\
\hline Lilium martagon album . . . & 59 & 61 & 62 & 64 & 63 & 58.8 \\
\hline Lilium golden gleam . . . . . . & 53 & 54.4 & 57 & 58.7 & 57.8 & \\
\hline Lilium chalcedonicum ...... & 59.2 & 61 & 63 & 64 & 63.5 & \\
\hline Lilium candidum . . . . . . . & 57 & 58.7 & 60 & 62 & 61 & 63.2 \\
\hline Lilium testaceum ......... & 61.2 & 63 & 63.5 & 67 & 65.25 & \\
\hline Lilium pardalinum........ & 58 & 60.5 & 61 & 63 & 62 & \\
\hline Lilium parryi. . . . . . . . . & 47 & 48.5 & 51 & 52 & 51.6 & 60.4 \\
\hline Lilium burbanki. . . . . . . . & 64 & 66 & 67 & 68.5 & 67.75 & \\
\hline Iris iberica. . . . . . . . . . . & 69 & 70 & 71 & 72.5 & 71.75 & \\
\hline Iris trojana . . . . . . . . . & 70 & 71.5 & 73.2 & 75 & 74.1 & 72.0 \\
\hline Iris ismali. . . . . . . . . . & 69 & 71 & 72 & 74 & 73 & \\
\hline Iris iberica. . . . . . . . . & 69 & 70 & 71 & 72.5 & 71.75 & \\
\hline Iris cengialti . . . . . . . . & 70 & 72 & 74 & 76 & 75 & 72.6 \\
\hline Iris dorak . . . . . . . & 68 & 70 & 70 & 72 & 71 & \\
\hline Iris cengialti $\ldots \ldots \ldots \ldots \ldots$ & 70 & 72 & 74 & 76 & 75 & \\
\hline Iris pallida queen of may ... & 71 & 73 & 75 & 75.8 & 75.4 & 74.5 \\
\hline Iris mrs. alan grey . . . . . . . . & 69 & 70 & 73 & 74.5 & 73.75 & \\
\hline Iris persica var. purpurea. . . & 64 & 66 & 68 & 70 & 69 & \\
\hline Iris sindjarensis. . . . . . . . & 63.5 & 65 & 66 & 67 & 66.5 & 68.2 \\
\hline Iris pursind $\ldots \ldots \ldots \ldots \ldots \ldots$ & 64.5 & 66 & 68 & 70 & 69 & \\
\hline Gladiolus cardinalis . . . . . . & 83 & 84.5 & 84 & 86 & 85 & \\
\hline Gladiolus tristis........... & 76 & 78 & 78 & 79 & 78.5 & 82 \\
\hline Gladiolus colvillei......... & 78 & 80 & 82 & 83 & 82.5 & \\
\hline Tritonia pottsii.......... & 73 & 75 & 76 & 77.5 & 76.75 & \\
\hline Tritonia crocosmia aurea... & 78 & 80 & 80 & 82 & 81 & 78.2 \\
\hline Tritonia crocosmæflora ..... & 74 & 76 & 76 & 78 & 77 & \\
\hline Begonia sing. crim. scar. . . & 67 & 68.5 & 70 & 72 & 71 & \\
\hline Begonia socotrana. . . . . . . & 79 & 80 & 81 & 81.8 & 81.4 & 74.9 \\
\hline Begonia mrs, heal. . . . . . . . . & 67 & 69 & 71 & 72 & 71.5 & \\
\hline Begonia doub. light rose.... & 60 & 61 & 62 & 64 & 63 & \\
\hline Begonia socotrana. . . . . . . & 79 & 80 & 81 & 81.8 & 81.4 & 70.5 \\
\hline Begonia ensign . ......... & 64 & 65.5 & 66 & 68 & 67 & \\
\hline egonia double white...... & 60 & 61.5 & 65 & 66.5 & 62.75 & \\
\hline egonia socotrana . . . . . . & 79 & 80 & 81 & 81,8 & 81.4 & 71.7 \\
\hline Begonia julius.... . . . . . . & 65 & 66 & 67 & 69 & 68 & \\
\hline Begonia doub. deep rose. ... & 64 & 65.5 & 67 & 68.8 & 67.8 & \\
\hline Begonia socotrana....... & 79 & 80 & 81 & 81.8 & 81.4 & 72.6 \\
\hline Begonia success........... & 62 & 64 & 68 & 69 & 68.5 & \\
\hline Richardia albo-maculata... & 75 & 76 & 77 & 78.5 & 77.7 & \\
\hline Richardia elliottiana. . . . . . & 74 & $7 \overline{5}$ & 76 & 77 & 76.5 & 77.1 \\
\hline Richardia mrs. roosevelt... . & 74 & 76 & 76 & 78 & 77 & \\
\hline usa arnoldiana $\ldots \ldots \ldots \ldots$ & 60 & 61 & 64.5 & 65.8 & 65 & \\
\hline usa gilletii . . . . . . . & 64 & 66.5 & 67.5 & 69 & 68.4 & 67.7 \\
\hline Musa hybrida. ........... & 65.2 & 67 & 69 & 70 & 69.75 & \\
\hline haius grandifolius......... & 65 & 66 & 68 & 69 & 68.5 & \\
\hline Phaius wallichii....... & 64 & 65 & 67 & 68 & 67.5 & 67.7 \\
\hline haius hybridus ..... & 64 & 66 & 66 & 68 & 67 & \\
\hline Miltonia vexillaria . . . & 70 & 71 & 73 & 74 & 73.5 & \\
\hline Miltonia roezlii . . . . . . & 74 & 76 & 76 & 77 & 76.5 & 74.7 \\
\hline Iiltonia bleuana . . . . . . . & 69 & 71 & 72 & 74 & 73 & \\
\hline ymbidium lowianum ..... & 58 & 60 & 62 & 63 & 62.5 & \\
\hline $\begin{array}{l}\text { ymbidium eburneum....... } \\
\text { Symbidium eburneo-lowia- }\end{array}$ & 58 & 59.5 & 65 & 66.5 & 65.75 & 65.2 \\
\hline num $\ldots \ldots \ldots \ldots \ldots$ & 61 & 63 & 67 & 68 & 67.5 & \\
\hline Jalanthe rosea . . . . . . . . & 74 & 76 & 75 & 77 & 76 & \\
\hline Calanthe vest. var, rub.-oc.. & 72 & 74 & 74 & 75 & 74.5 & 74.3 \\
\hline Galanthe veitchii......... & 71 & 72 & 73 & 74 & 72.5 & \\
\hline Calanthe vest. var. rub.-oc.. & 72 & 74 & 74 & 75 & 74.5 & \\
\hline Galanthe regnieri......... & 70 & 72 & 76 & 78 & 77 & 76 \\
\hline Valanthe bryan........... & 72 & 74 & 76 & 77 & 76.5 & \\
\hline
\end{tabular}

Average mean temperature of gelatinization of the seed parentstocks............................. $72.07^{\circ}$

Average mean temperature of gelatinization of the pollenparent stocks............................ $73.08^{\circ}$ Average mean temperature of gelatinization of the hybridstocks.......................... $72.63^{\circ}$ 
ferent genera, the reaction being in some instances higher, or lower, or the same, or about the same, as the average reaction-intensity. In comparing the data of different genera, species, or hybrids, it was usually found that the two tend to fall and rise together-in other words, that if in one set the average mean temperature of gelatinization and the average reaction-intensity is at a given standard and if in the next set the temperature is higher, the average reaction-intensity will be higher, although the quantitative relationship between the two may vary; but.one may rise and the other fall, and so on. The varying relationships of these two sets of reactions will be seen by comparing the records in Table B 2 and Chart B 42. Strictly equivalent values in the two cases are not given because the scales are different and arbitrary. The range of temperature reactions are included between $51.5^{\circ}$ (Lilium parryi) and $83.25^{\circ}$ (Hcemanthus könig albert), representing a range of only about three-fifths of the scale, while in the reaction-intensities, as a whole, the entire scale is included; hence, it follows that strictly comparative values of the excursions of the temperature curve should be amplified two-fifths. This fault, however, does not interfere with the gross comparisons sought. Taking the two averages for the Amaryllis-brunsvigia-brunsdonna group as a startingpoint, it will be observed that there is a well-marked separation of the two curves and that the temperature curve is the lower. Both curves fall in Hippeastrum, the temperature curve less than the other, and there is an inversion of the positions of the two curves, the temperature curve now being the higher. In Homanthus both curves are still lower, both being close in the first set but well separated and again reversed in the second set, the temperature curve now being the lower as in Amaryllisbrunsvigia-brunsdonna. This last crossing is due to peculiarities, several times referred to, of Hamanthus puniceus. In Crinum both curves rise and undergo a marked separation in the last set, the temperature curve remaining in all three sets lower and changed to a less degree than the other curve. In Nerine both curves fall and approximate. In Narcissus the reaction-intensity curve remains at the same level as in the last set of Nerine, but the temperature curve rises to a point slightly above the reaction-intensity curve. In all of the following generic groups the temperature curve falls below the other curve, the degree being very variable, and the range of variability far in excess of what can be accounted for by error of calibration above referred to.

These average differences do not begin to bring out or even indicate the extent and kind of these rariations that are found when the data for members of different sets are compared. For instance, in Amaryllis-brunsvigia-brunsdonna the temperatures of gelatinization are nearly the same, the maximum difference being only $1.75^{\circ}$, but the reaction-intensities vary between 76 and 52, the temperatures for Amaryllis and Brunsdonna sanderce being practically absolutely the same, while the reaction-intensity averages are 76 and 55, respectivelya wide difference. In other words, there may be no difference in the temperature of gelatinization, but a wide difference in reaction-intensities. In the Crinum longifolium-moorei-powellii set, $C$. powellii has the lowest temperature of gelatinization, but the highest average reaction-intensity. In Iris, in the first three sets the temperatures are uniformly higher than in the fourth set, but the relative reaction-intensities are the opposite, they being very much lower in the first three sets than in the last set, and the difference is proportionately far more marked than in the temperatures of gelatinization. In Begonia, in $B$. socotrana the temperature of gelatinization is very much higher than in the other members of the genus represented, but the reaction-intensity is very decidedly lower. On the other hand, in Hippeastrum the temperatures of gelatinization and average reaction-intensities are in both cases very closely alike. In Homanthus katherina the temperature of gelatinization is distinctly higher than in $H$. magnificus, but with the average reaction-intensity, although there is a tendency, on the whole, for a starch that has a high temperature of gelatinization to have a corresponding reaction-intensity.

In comparing the data of this table it is worthy of note that while there may be evidence in some reaction of a grouping of genera and of subgeneric divisions there may not be in others. For instance, the temperature of gelatinization of the members of two genera may be close, as in the case of Hippeastrum and Nerine, but the sum and average reaction-intensities may be distinctly different; or the temperatures may more or less distinctly individualize the genus, as in the case of Lilium; or they may individualize subgeneric groups, as in Iris, in which the first three sets and the last set stand distinctly apart from each other. While it may not be possible positively to recognize a genus upon the basis of temperature of gelatinization and average reaction-intensitiy, it is at least possible to state that it may be this or that genus or positively that it can not be a certain genus. For instance, having the data for Hippeastrum and Nerine, it could perhaps not be stated conclusively which is which, although there is evident differentiation; but neither could possibly be confounded with Amaryllis-brunsvigia, Lilium, Iris, Musa, Phaius, Miltonia, or Cymbidium; nor could Lilium be mistaken for Iris or for any other genus with the exception, possibly, of Cymbidium. Lilium and Cymbidium are very widely separated genera, one belonging to Liliaceæ and the other to Orchidaceæ, and there should be a wide difference in the sum-total of their reactivities, but the reason why they are not here so differentiated is owing to their great sensitivity to the chemical reagents. So far as the temperature of gelatinization is concerned, it is well established that starches obtained from very remote plant sources may have the same temperature of gelatinization, which peculiarity applies also to every reagent, both of which being in accord with what is to be expected of stereoisomers. On the other hand, they may exhibit differences, which vary in degree with different reagents. Hence, it follows that the starches are to be distinguished from each other by the collective peculiarities of each starch compared with those of other starches.

\section{Velocity-REactions With Different REAGents. \\ (Charts D 1 to D 691.)}

In the preceding section it was shown, among various conspicuous phenomena, that different starches exhibit a wide range of reaction-intensities with a given agent or 
reagent; that the reactions of a given starch may vary with different agents and reagents within wide limits; that there is a manifest tendency to groupings of reaction-intensities of different starches that are, on the whole, very closely in harmony with the plant groupings of the systematist; that the most variable relationships exist between the starches in their reaction-intensities, as regards sameness, intermediateness, excess and deficit of reaction-intensity development of the hybrid in relation to the reactions of the parents; and that the differences in the reactions are conditioned by differences of the starch molecule, by the characters of the agents, and by molecular constitution and concentration of the reagents. The comparative studies of the reactions with the chemical reagents have as their sole basis values that are expressed in terms of percentage of starch gelatinized in 60 minutes or less. There was no note regarding differences that were recorded in the comparative percentages of the entire number of grains and total starch gelatinized at definite time-intervals, and only the most casual references were made to peculiarities observed in the progress of curves of the reactions from period to period; yet both of these features are found to be of great importance, alone and in conjunction with the findings presented in the foregoing sections, in the determination of generic, species, varietal, parental, and hybrid peculiarities of starches. The reaction-intensities of different starches with different reagents recorded in Part II, Chapter I, include the percentages of both the entire grains and total starch gelatinized at definite time-intervals. The data of the total starch gelatinized have been tabulated in Section 3 of each of the Comparisons of the Starches of the Parent- and Hybrid-Stocks in Chapter III, and they are here presented with few unimportant exceptions in the form of Charts D 1 to D 634 which admirably exhibit both intensity and progress of the reactions, and render comparisons of the behavior of both starches and reagents very satisfactory. Additional charts (Charts D 635 to D 691) have been introduced to show the relationships between the percentages of entire grains and total starch gelatinized at given time-intervals. There will also be found among Narcissus, Lilium, and Begonia a few charts that show differences between these percentages, and a few additional charts to bring out certain generic peculiarities.

These charts are so very numerous and the curves so exceedingly varied that detailed descriptions and comparisons are rendered impracticable because of necessary limitations of space, although it will be perfectly manifest, after even a superficial survey, that the results of such a study would prove of great value in many directions; yet very much that is of more than mere passing interest, value and suggestiveness can be brought out by even casual examination.

\section{Percentage of Total Starch Geuatinized at Definite Time-Intervals. \\ (Charts D 1 to D 634.)}

The curves of total starch gelatinized vary widely and the number and forms of types recognized are purely arbitrary. In some instances the curve is nearly or absolutely rectilinear, but in most cases it is circumlinear and varied, but suggestive usually of an ellipse, hyperbola or parabola or some modification of one of the three. The rectilinear curves are presented in the form of three types or what may tentatively be regarded as three modifications or forms of a single type:

(a) A form that is characterized by an immediate, very rapid and continually rapid rise of the curve at an angle approximating about $1^{\circ}$ to $2^{\circ}$ with the vertical, thus representing a complete or practically complete gelatinization in 1 or 2 minutes. This curve should probably be circumlinear inasmuch as it is likely that during equal increments of time larger increments of the starch are gelatinized during the earlier than later periods of the reactions, but the time-intervals here are too short for such determinations. This belief is supported by the fact that when the reactions of the same starch but with a weakened reagent are somewhat less rapid, as when complete gelatinization occurs at the end of 5 minutes, this variation is noted and the circumlinear character of the curve is quite marked, the increments of gelatinized starch falling very rapidly and disproportionately after the first minute. This form of curve is illustrated in the Amaryllis-Brunsvigia-Brunsdonna group in the reactions with nitric acid, sulphuric acid, hydrochloric acid, and potassium hydroxide (Charts D 4 , D 5, D 6, and D \%). It will be seen that in some of the reactions the line is straight and in others curved.

(b) Another form of the rectilinear type presents a curve that is almost if not entirely rectilinear, but having an inclination that rarely is less than an angle of $80^{\circ}$ with the vertical, which is equivalent to a maximum of approximately 15 per cent of the total starch gelatinized in 60 minutes. This form of curve is associated usually with weak gelatinizing reagents and exceptionally resistant starches. It will very frequently be found in the study of these charts that while a given starch may show such a curve with one reagent, a curve of the first form or of an entirely different type may be exhibited with another reagent. Such a curve is well typified in the reactions of Brunsdonna sanderce alba with sodium sulphide, cobalt nitrate, cupric chloride, barium chloride, and mercuric chloride (Charts D 12, D 17, D 19, D 20, D 21).

(c) A third form of the rectilinear curve links in its varied positions the first and third forms, and were it not that the first two forms are very common and the third form relatively rare, there would be no good reason for the recognition of three forms. This form is illustrated in the reactions of Brunsvigia josephine with mercuric chloride (Chart D 21), of Crinum kircape with sodium sulphide (Chart D 159), and of Nerine bowdeni with uranium nitrate (Chart D 225).

The circumlinear type of curves is divisible into three forms :

(a) One form shows that gelatinization begins and proceeds rapidly, there being progressively or practically progressively decreasing increments of starch gelatinized with additional increments of time. This form is illustrated in the reactions of Amaryllis belladonna with sodium sulphide (Chart D 12). This form of curve is very common, perhaps the most common of all. An examination of this series of charts (Charts D 1 to D 634) will elicit most varied and modified gradations in both directions from what may properly be regarded as a true hyperbolic form. 
(b) Another form is an inversion of the latter, gelatinization proceeding very slowly at first and then increasing with additional increments of time. Such curves are illustrated in the reactions of Brunsdonna sanderce alba with uranium nitrate (Chart D 15), of Hippeastrum pyrrha with nitric acid (Chart D 46), of Crinum kircape with strontium nitrate (Chart D 163), and of Nerine sarniensis var. corusca major and $N$. giantess with potassium sulphocyanate (Chart D 219). In this form there is a tendency to a continuously increasing increment of starch gelatinized with increasing increments of time.

(c) A third form, and one that is frequently observed, shows reactions that begin relatively or absolutely slowly, followed by progressively increasing reaction, and this in turn by progressively decreasing reaction, with additional increments of time, thus giving a curve that approximates the form of the letter $f$. Such a curve is typified in the reactions of all four starches of the Amaryllis-Brunsvigia-Brunsdonna group with chloral hydrate (Chart D 1), and in one or more of these starches with chromic acid, pyrogallic acid, potassium iodide, calcium nitrate, and copper nitrate (Charts D 2, D 8, D 14, and D 18). This curve is a modification of the first form of the circumlinear type, the modification being brought about chiefly by a relatively marked early resistance of the grains to the reagent. The duration of the period and the degree of resistance are very variable. In some instances there is merely a suggestion of resistance; and in others resistance is very marked in both degree and duration; and in others various intermediate gradations and variations. Thus, in the reactions of Amaryllis belladonna and Brunsvigia josephince with cobalt nitrate (Chart D 17) there is only slight evidence of this early resistance, while in the Brunsdonna sanderce alba and $B$. sandero reactions the resistance is very marked (Chart D 2), in the latter instance there being only 3 and 1 per cent respectively of the total starch gelatinized in 5 minutes; while 77 and 79 per cent, respectively, was gelatinized during the succeeding 10 minutes. In the chromic-acid reactions of the Nerine crispa-elegansdainty maid-queen of roses group this period lasts in all four starches for 15 minutes, followed by a rapid gelatinization, giving a well-marked $f$ form of curve. While all four starches may show this resistance with one reagent, one or all may not with others, and the degree and duration of the resistance, may either or both be quite variable. Thus, in the chloral-hydrate reactions, two of the starches show slight early resistance, and two not any (Chart D 190); in the potassium-sulphocyanate reactions all four show a resistant period, two for 5 minutes, and so on. The inclination of this form of curve is very variable, in some instances, being less than $30^{\circ}$ (Chart D 2); in others, about $50^{\circ}$ (Chart D 1), in others about $80^{\circ}$ (Chart D 18); and in others, between or beyond these extremes, the less the angle the less rapid, as a whole, is the process of gelatinization.

Curves are not infrequently found which do not pursue a uniform rectilinear or curvilinear course, so that they are not classifiable among the forms stated. In other words, they appear to be at times erratic in their courses. For instance, in the reactions of Brunsdonna sanderce with sodium sulphide (Chart D 12) the curve during the first 15 minutes appears like a segment of the $f$ form, but between the 15-minute and 45-minute intervals the curve drops instead of rises. In the sodiumhydroxide reactions with Brunsdonna sanderce alba (Chart D 11), it seems from the courses of the curves of the other starches shown in the chart that the curve should have risen decidedly more by the end of the 15minute interval, impinging at perhaps the 30 per cent abscissa instead of at the 16 . In some instances these seeming or actual aberrations in the progress of gelatinization may be due to errors of experiment that are attributable to errors of estimation or to variations in attendant conditions; but in most and probably in nearly all instances they are owing to peculiarities, molecular or physical, of the starch grains, as is indicated by the occurrence of identical or practically identical records when experiments have been repeated, even under varying incidental conditions.

The curves of gelatinization of the starches constituting a parental-hybrid group tend usually to divergence in their courses during the early part of the reactions, and when a definite position-relationship (highest, intermediate, same or lowest) is once established it is commonly retained throughout the courses of the curves, but the degree of separation may be very variable, usually increasing for a variable period and then decreasing or increasing, more frequently decreasing. In some instances there is little or no difference between two or more of the curves of the group during an early period of the experiment, the length of which period being variable, this period being followed by variable degree of divergence; and in other instances, while divergence may be marked during the early and mid-periods of experiment, there may be sameness during the final period, and so on. Crossing of curves is occasionally observed, but recrossing is very rare. Such peculiarities as are here indicated are illustrated in large part by the $A$ maryllisBrunsvigia-Brunsdonna reactions (Charts D 1 to D 21). In most of these charts (excepting those in which gelatinization is very rapid or very slow) there occurs primarily divergence and secondarily convergence. In Chart D 21 there is practically divergence from beginning to end of reaction. Charts belonging to the divergent type are common, for instance, among the Crinum zeylanicum-longifolium-kircape group (Charts D 148 to D 168).

Different starches may exhibit with a given reagent the same or different curves. Thus the chloral-hydrate reactions with different starches show varying differences in regard to both type and form of type and in the degree of inclination of the curves. This feature is shown by both the individuals of the groups of parental and hybrid starches and by the different generic groups, as seen, for instance, by an examination of the reactions of the four starches as presented in Chart D 1, and by the reactions of various generic representatives shown in Charts D 22, D 85, D 127, D 190, D 265, D 361, D 379, D 463, D 484, D 505, D 545, D 574, D 595, D 616, and D 619 . Similar variations will be found in the reactions of other reagents, these differences being usually more conspicuous in the case of reagents that act usually with moderate activity than with those which act commonly with either much or little intensity. 
A given starch may exhibit like or unlike reactions with different reggents, and the curves vary as much as do those of different starches with the same reagent, so that there may be most varied forms of the different types. This feature will be found to be well exhibited when the curves of the reactions of any given starch of any one of the generic groups are compared, for instance, the curves of Amaryllis belladonna (Chart D 1 to D 21). The curve in the chloral-hydrate reaction is of the $f$ form, having an inclination of about $50^{\circ}$, so that the upper end is at the termination of the 60-minute interval. The curve of the chromic-acid reaction is of the $f$ form, but it terminates at the end of the 30-minute interval, giving it an inclination of about $30^{\circ}$, which indicates a very much more rapid gelatinization. It will be seen, however, that during the first 5 minutes the percentage gelatinized in both reactions is practically the sarne (12 and 10 per cent, respectively), that the gain in the chromic-acid reaction occurs during the next 10 minutes; and that the quantities gelatinized during the interval between 15 and 30 minutes are the same in both reactions. The pyrogallic-acid and chloral-hydrate curves bear a close resemblance; but the former is lower throughout, especially at the end of the 5-minute interval, indicating a more marked early resistance to this reagent than to chloral hydrate. From this point onward to the end of 60 minutes the curves run very closely parallel.

In 11 of the 21 experiments with different reagents the curves belong to the form of circumlinear type that is characterized by progressively decreasing increments of starch gelatinized during additional incremonts of time. These curves vary markedly in character. In some the increment of starch gelatinized during the first 5 minutes is very disproportionate to the quantities subsequently broken down, as is noted particularly in the reactions of potassium sulphide, sodium hydroxide, calcium nitrate, and strontium nitrate (Charts D 10, D 11, D 14, and D16), in each of which about 98 per cent of the total starch was gelatinized in 5 minutes. In the sodiumsulphite reactions the increments of gelatinized starch are $66,14,4,3$, and 2 per cent. In the other reactions of this group, including those of potassium iodide, sodium salicylate, uranium nitrate, copper nitrate, and cupric chloride (Charts D 8, D 13, D 15, D 18, and D 19), the curves exhibit various modifications in comparison with the foregoing. In the mercuric-chloride reactions the curve is of a modified $f$ form, tending, in fact, like the accompanying Brunsvigia josephince curve, to be rectilinear, but at an angle of about $18^{\circ}$ as compared with about $26^{\circ}$ for the latter. In the reactions of nitric acid, sulphuric acid, hydrochloric acid, and potassium hydroxide (Charts D 4, D 5, D 6, and D \%), the curve is rectilinear and almost vertical, while in the barium-chloride reactions (Chart D 20) it is rectilinear and almost, horizonlal.

Starches of members of a genus tend, as a rule, in their reactions with each reagent to yield curves that are of or incline to the same type and type form, except when there are subgeneric representatives or widely separated species, in which case it may be found that there is or is not relationship in the characters of the curves, and this peculiarity may also apply to the curves of hybrids in relation to those of its parents. For instance, taking the chloral-hydrate reactions: of the starches of Lilium (Charts D 347, D 354, D 367, and D 373) the concordance of both type and type-form is obvious; of the starches of Nerine (Charts D 190, D 211, and D 232), the curves of the five parental starches are of the $f$ form, but vary in their courses sufficiently for easy differentiation; of the starches of Crinum moorei, C. longifolium and $C$. powellii compared with those of $C$. zeylanicum, where we have subgeneric or the equivalent of subgeneric representatives (Charts D 127, D 148, and D 169), the curves of the first three conform to a given type-form, while the curve of the latter is of an entirely different type; of the starches of Begonia, where similarly wellseparated starches are represented by those of the seed parent on the one hand and by the starch of B. socotrana (pollen parent) on the other (Charts D 463, D 52\%, D 533, and D 539), the curves are closely similar; of the starches of Amaryllis and Brunsvigia, where two recognized genera are represented, the curves are much alike (Chart D 1). Varieties that are offspring of closely related parental stock, as in Hippeastrum (Charts $\mathrm{D} 22, \mathrm{D} 43$, and D 64), tend to show marked closeness in the curves and this may also be seen not only in closely related species, as in Phaius (Chart D 574) and Iris (Chart D 421), but also in closely related genera, as in Gladiolus and Tritonia (Charts D 463 and D' 484). The curves of hybrids show, as will be pointed out particularly hereafter, the most varied relationships to the parental curves, varying between identity and great dissimilarity.

Taking the reactions of all of the parental starches with any given reagent and comparing them with those of other rcagents, it becomes apparent that those of each reagent represent a group in which there are both similarities and dissimilarities; and that the different groups as such exhibit similarities and dissimilarities, the reactions collectively of each group being quite as or even more distinct from those of another group as are those of members of the same group; that the more closely related the starches the more marked the tendency generally to closeness of the curves, yet sometimes distantly or wholly unrelated starches may exhibit almost if not identical curves with a given reagent. In a word, the peculiarities of these reactions are of such characters as should logically be expected if we are dealing with stereoisomeric forms of starch.

The starches of the hybrid and parents usually take on within a brief period after the beginning of gelatinization definite relationships, which may be the same or different in the reactions with different reagents. That is, if shortly after the beginning of the reaction the positions of the three curves should be in the order of intensity of reactivity, seed parent, pollen parent, and hybrid (highest, intermediate, and lowest), this relationship usually tends to be continued during the entire period of gelatinization, but with varying degrees of separation of the curves. The hybrid curve may bear any relationship to one or the other or both parental curves - that is, be higher or lower than either, or intermediate, or the same as one or the other or both. Rarely the parental curves cross (Chart D 169), or the hybrid curve crosses one or the other parental curve (Chart D 89). The hybrid curves tend usually to follow closely the parental curves, but they may differ as much or more from the parental 
curves as do the latter from each other (Charts D 241, $\mathrm{D} 277$, and D 343). When there are two hybrids of the same parentage, the curves may differ quite as much or more from each other, as the parental curves differ from each other. (Charts D 1 to D 21.)

\section{Percentages of Total Staroh and Entire Number of Grains Gelatinized at Definite TIME-INTERVALS.}

(Charts D 635 to D 688; also D 261, D 268, D 290, D 296, D 302, D 308, D 314, D 320, D 326, D 332, D 338, D 344, D 350, D 351 , D 357, D 365, D 366, D 508, D 530, D 536, D 542.)

The curves of the percentages of total starch and the entire number of grains completely gelatinized tend in general to correspond in their courses; but both may differ in varying ways, relatively and absolutely, in accordance with the kind of starch and the reagent, excepting, of course, when the reactions are too fast or too slow for definite differentiation.

When starch is gelatinized it passes into an imperfect or pseudo-solution, and the grains, like solid particles or masses of other substances passing into solution, show differences in solubility of both grains in their entirety and parts of individual grains. Some grains may undergo complete gelatinization, while others do not exhibit any obvious change; and other grains show very variable proportions that have undergone a breaking down. These peculiarities have been observed in all kinds of starch with the same reagent. They are constant for the same starch with the same reagent; variable with the same starch with different reagents; and variable with different starches with the same reagent. The behavior of each starch with the different reagents is, as a whole, so characteristic and specific as to be diagnostic. These several points will be found to be well illustrated if there be taken a number of starches that are representative of different generic and subgeneric divisions, plotting in curves the data of the reactions of one of the starches with one reagent, and supplementing this group with curves of the reactions of a few arbitrarily selected starches with several reagents. Thus, taking the pyrogallic-acid reactions (Charts D 635 to D 649), it will be found that the curves of the percentages of total starch and the entire number of grains completely gelatinized differ widely; that the two curves of each starch tend in general to correspondence in their courses; that the degree of correspondence varies from marked closeness to an almost lack of any likeness; and that the degree of separation of the curves varies in the different starches and also during the progress of the reactions. It is obvious that the farther the separation of the curves the smaller relatively the percentage of the entire number of grains completely gelatinized, and the higher relatively the proportion of the total starch gelatinized in the partially gelatinized grains.

In some of the starches it will be seen that during the progress of the reactions the increasing height of the curve of the percentage of total starch gelatinized is almost if not directly proportional to the increase in percentage of the entire number of grains completely gelatinized-in other words, the total per cent gelatinized is not appreciably or but little contributed to by the amount of gelatinization in grains that have undergone only varying degrees of partial disorganization; in others, there will be found the reverse, the major portion of the percentage of total starch gelatinized being yielded by grains that have been only in part, but to varying degrees, broken down; in others, there are various gradations between the former. These peculiarities are constant with each starch with each reagent, except in very rare instances, indicating thereby that they are in part expressions of inherent constitutional properties of starch molecules that differ in accordance with the plant source. In reactions that are completed within 2 to 5 minutes or so, or which are so slow that a very small percentage of the starch is gelatinized by the end of 60 minutes, the differences between the two percentages may be so small as to be undetectable, or if detectable of little or no value in demonstrating this peculiarity. This is found, for instance, in Lilium tenuifolium (Chart D 644), 99 per cent of the total starch is gelatinized in 5 minutes, 93 of this 99 per cent being contributed by grains completely gelatinized and the remaining 6 per cent of grains being only partially gelatinized, and 1 per cent unaffected. Additional instances are found, but in the opposite direction, in the reactions of Homanthus katherince (Chart D 639), Iris iberica (Chart D 684), and Richardia albo-maculata (Chart D 652).

Taking, in turn for comparative purposes, several selected charts of this series, and beginning with those of Lilium tenuifolium (Chart D 644) and Homanthus katherince (Chart D 639), which represent opposite extremes of reaction-intensities, and wherein the two percentage curves in each are almost identical, variations in the courses of these curves will be found that are coupled with variations in the degree of separation of the curves during the progress of reactions, each chart being in one or both respects different from the other charts, and therefore characteristic of starch plus reagent. In Cymbidium lowianum (Chart D 657) the reactions occur rapidly, gelatinization being practically complete in 15 minutes, 98 per cent of the total starch being gelatinized in 5 minutes, of which quantity 87 was made up of the starch of completely gelatinized grains; while in Richardia albo-maculata only 11 per cent of the total starch was gelatinized in 60 minutes, of which quantity 6 per cent was made up of the starch of grains completely gelatinized. In some of the other charts gelatinization is shown to proceed with fair to moderate activity, but during the earlier part of the 60-minute period the proportion of gelatinized starch contributed by grains that are entirely broken down is decidedly less than that by the partially gelatinized grains. This peculiarity is well illustrated, for instance, in Iris iberica (Chart D 646), Iris trojana (Chart D 647), and Phaius grandifolius (Chart D 655). In Iris iberica, at the end of 5-minute period, 20 per cent of the total starch was gelatinized, of which quantity only 2 per cent was contributed by grains that were entirely gelatinized; at 15 minutes the figures are 62 and 30 , respectively; at 30 minutes, 81 and 42 , respectively; at 45 minutes, 86 and 53 , respectively; and at 60 minutes, 54 and 90, respectively. Similar data are recorded in the other two charts, the proportions in each varying at the different periodsat the end of 60 minutes, in Iris iberica, 54: 70, in I. trojana, 63:96, and in Phaius grandifolius, 28:67, of the gelatinized starch was contributed by the grains that 
were entirely gelatinized. In Narcissus tazetta grand monarque, during the first 15 minutes less than 0.5 per cent of the grains, but 20 per cent of the total starch, were gelatinized, and during the progress of the reaction both curves rise, but the curve of the percentage of total starch rises somewhat more rapidly than the other. In certain of the charts this progressive separation is seen, as in Amaryllis belladonna (Chart D 635) and Tritonia pottsii (Chart D 651); in others, there is for a time separation, this being followed by approximation, as in Hippeastrum titan (Chart D 636) and Homanthus puniceus (Chart D 640); and in others, there is an early marked separation followed in time by approximate parallelism, as in Gladiolus tristis (Chart D 650) and Calanthe rosea (Chart D 658), and so on with various differences.

While no two charts are identical some are quite similar, yet readily differentiated. Such similarity is apt to be found in very closely related varieties and speciesfor instance, in Hippeastrum titan, $H$. ossultan, and $H$. docones (Charts D 636, D 637, and D 638), and in Iris (Charts D 646, D 647, and D 648). Those of the several species of Litium differ markedly (Charts D 643, D 644, and D 645). Those of widely separated species, such as Homanthus katherince and $H$. puniceus, are decidedly different from each other, which species for reasons as stated, probably represent subgeneric groups. The same peculiarities are true in Iris, those of $I$. iberica (Chart D 646), I. trojana (Chart D 647) and I. cengialti (Chart D 648) having a close general resemblance, and markedly contrasted with the curves of the apparently distantly related $I$. persica var. purpurea (Chart D 649), which curves are quite different from the former. Gladiolus and Tritonia (Charts D 650 and D 651), while representing closely related genera and exhibiting at the end of the 60-minute period the same percentages of both total starch and entire number of grains completely gelatinized, nevertheless present differences in the courses of the curves that are quite definitely distinctive.

In some of the charts it will be seen that there is an early period of resistance of the starch to gelatinization. This is manifest in some instances in the percentage of completely gelatinized grains, but not in the percentage of total starch gelatinized, as in Iris iberica and I. trojana (Charts D 646 and D 647), and in Litium chalcedonicum . (Chart D 645); in others, it may be the reverse, as in Narcissus tazetta grand monarque (Chart D 642) ; and in others, in both percentages, as in Amaryllis belladonna (Chart D.635) and Hippeastrum titan (Chart D 636). In other charts both curves may begin at once to rise rapidly, but the percentage curve of total starch rises more rapidly than the other, as in Hcomanthus puniceus (Chart D 640), L. martagon (Chart D 643), Musa arnoldiana (Chart D 654), and Miltonia vexillaria (Chart D 656). In the different starches these changes go on with varying rapidity and relationships, so that by the end of the 5-minute period not only may the two curves of any given starch be well separated but their courses may be quite different. Thus, the figures for the percentages of total starch and number of grains completely gelatinized in 5 minutes in the above four species are 33 and 65,30 and $7 \%, 30$ and 86 , and 27 and 50 , respectively. It is to be noted that while in the four cases the percentages of the entire number of grains com- pletely gelatinized are the same or nearly the same, the percentages of total starch are in all distinctly different. This is of diagnostic importance because it indicates inherent individual peculiarities of the several starches. The preceding groups of charts indicate to what degree the reactions of different starches with a given reagent may differ in the percentages of both total starch and entire number of grains completely gelatinized, and also the tendencies in general to similarities of the pair of curves of closely related starches and to dissimilarities of distantly or unrelated starches.

When similarities are observed, as in the very closely related Hippeastrums, such peculiarity is to be expected in the reactions of the same starches with other reagents. For instance, in the reactions with chloral hydrate (Charts D 659, D 660, and D 661) the three pairs of curves are closely alike, the type of curve is the same as is seen in the pyrogallic-acid reactions (Charts D 636, D 637, and D 638), but the positions of the curves in the two reactions are different, owing to the distinctly lower reactivities of these starches with chloral hydrate. When, however, the reactions of the starches of well-separated or unrelated species are studied it is found that there may be the widest variations in the relationships of the two curves, not only with different agents but also with the same reagent, even to the extent that the percentage of total starch gelatinized will give a type of curve entirely different from that of the percentage of grains completely gelatinized. Thus, examining the pyrogallicacid reactions of the various starches (Charts D 635 to D 658), it will be found that there is with few exceptions a well-marked tendency to separation of the two curves, and that in some instances the two curves are not of the same type, as in Lilium chalcedonicum (Chart D 645) and Iris trojana (Chart D 647). In contrast with this, in the chloral-hydrate reactions (Charts D. 659 to $\mathrm{D} 667$ ) both curves tend to marked closeness in course and hence to the same type. Comparisons of the pyrogallic-acid and chloral-hydrate reactions of the same starch bring out many interesting points. For instance, in Amaryllis belladonna (Charts D 635 and D 662) in the pyrogallic-acid reaction the two curves become widely separated during their progress, the percentage of completely gelatinized grains ceases to increase after 30 minutes, but the quantity of gelatinized starch is materially being added to by the grains that are undergoing partial gelatinization; while in the chloral-hydrate reaction the curves keep very close throughout. The most marked difference between the reactions of the two reagents is seen in the curves of the percentage of the entire number of grains completely gelatinized, which differ greatly, while the total percentage curves differ comparatively very little. In Homanthus puniceus (Charts D 640 and D 664) the pyrogallic-acid and chloral-hydrate curves are of different types; and the curves of both pairs of percentages tend to closeness, more particularly the chloral-hydrate curves. In Narcissus tazetta grand monarque (Charts D 642 and D 665) both pairs are again different, not only from those of the preceding charts, but also from each other, and as markedly in the latter as in the former case. Here the types of the pairs of curves are distinctly different, and while the two curves in the pyrogallic-acid reaction tend to progressive separation, those of the chloral-hydrate reaction tend to 
continued closeness. In Iris iberica (Charts D 646 and D 666) there is a difference in the type of the two curves in the pyrogallic-acid reaction, but not in the chloralhydrate reaction, and in the former the curves tend to marked separations, but in the latter to marked closeness. In Phaius grandifolius (Charts D 655 and D 667) the same peculiarities are observed. Similar pairs of charts of the curves of other starches with these and other reagents exhibit corresponding characteristics. It is of importance to recognize that the differences between the two curves may be as marked in the reactions of the same starch with different reagents as it is in the case of different starches with the same reagent. Indications of these differences have had incidental reference in the immediately preceding statements, and they may be sufficiently accentuated by reference to a single generic group of reactions, as, for instance, the reactions of Iris iberica with different reagents (Charts D 668 to D 688), that which is found here being taken as a rough index or suggestion of the records of the other starches.

\section{Composite Reaction-intensity Corves with Different Agents and Reagents. (Charts E 1 to E 46, and D 1 to D 691.)}

In the construction of the composite reaction-intensity curves the abscissæe are, in the polarization, iodine, gentian-violet, and safranin reactions in terms of gross quantitative light and color values based on an arbitrary scale of 105 in divisions of twentieths; in the temperatures of gelatinization, in the centigrade scale in divisions of $2.5^{\circ}$; and in the reactions with the chemical reagents on a duplex scale, the upper portion giving the time of complete or practically complete gelatinization ( 95 per cent or more of the total starch), and the lower portion of the scale the percentage of total starch gelatinized when complete or practically complete gelatinization has occurred within not less than an hour. The ordinates represent the agents and reagents used in the reactions. The reaction-intensity of each agent and reagent is marked upon its ordinate and upon the proper abscissa, and then a line is continued from ordinate to ordinate, making an irregular curve. This form of chart is especially useful in the differentiation and recognition of varieties, species, subgenera and genera, and in comparisons of the peculiarities of parents and hybrids. The method of construction is, however, faulty, and the curves are at times misleading because differences that have been recorded antecedent to the record used in the chart may be of very different significance, on which account there will be found here and there what appear to be discrepancies from what should be expected upon the basis of the data of the systematist; but as previously stated, each of these different kinds of charts brings out in a particular way certain features, and it is of primary importance to note that there are presented in Charts D 1 to D 691 data of the progress of the reactions that are of essential importance in connection with understanding and proper interpretation of these composite charts. In a word, the composite charts exhibit in a gross and by no means accurate way comparative reaction-intensities. For instance, the reaction-intensities of two or more starches may be shown to be 95 per cent of the total starch gelatinized in 30 minutes, or pre- cisely the same, whereas the records for the preceding periods may or may not have shown any differences. This is illustrated in the uranium-nitrate reactions of Amaryllis belladonna, Phaius grandifolius, and Miltonia vexillaria (Chart D 689), wherein at the end of the 5-minute period the figure for both A maryllis and Phaius is the same or 65 per cent; and that of Miltonia 83; and at 15 minutes, and thence onward, they are practically exactly the same for all three. Then again, the curves of gelatinization of any given starch may undergo a complete change in its relationships to other curves during its progress. This is well shown in the cobaltnitrate reactions with the same starches (Chart D 690). At the end of the 5-minute period the order of reactivity is Miltonia, Amaryllis, and Phaius; at 15 minutes, Amaryllis, Miltonia, and Phaius; and at the end of the 30, 45, and 60 minute intervals, Amaryllis, Phaius, and Miltonia.

In making the composite charts the records of these species at the end of 60 minutes are taken, and quite a different impression is given of relative reaction-intensities than if the records had been used at the 5- or 15minute periods. Another source of fallacy is to be found in the tendency in most of the reactions for convergence or divergence of the curves, this being apparent not only in the charts of the reactions of the starches of parents and hybrid, but also when the curves of arbitrarily selected starches are compared. This latter is set forth in the pyrogallic-acid reactions of the Amaryllis, Phains, and Miltonia starches (Chart D 691). Here it will be noted that while the Miltonia curve is highest, that of A maryllis lowest, and that of Phaius intermediate, at the end of the 5-minute period the figures are 50,6, and 5 per cent, respectively; at the end of the 15-minute period 34,40 , and 72 per cent, respectively; at the end of the 30-minute period 50, 75 , and 84 per cent, respectively; and at the end of 60 minutes 94,90 , and 67 per cent, respectively. In a word, at the end of the 5-minute period there was no practical difference between Amaryllis and Phaius, but a wide difference between them and Miltonia; and during the progress of the reactions, while gelatinization in Phaius tends to keep about parallel in intensity with that in Miltonia, that in Amaryllis tends to approach more and more closely the intensity of reaction in Miltonia, so that by the end of the hour the figures for Miltonia and Amaryllis are very nearly the same (94 and 90 per cent, respectively) while the figure for Phaius is only 67 per cent. Notwithstanding the grossness of this method of charting and the manifest tendency to introduce fallacies, it will be apparent by even a cursory survey of these charts from the aspect of taxonomy that they are not without rery considerable value, and that by necessary modifications in the plan of charting we shall arrive at a positive means by 'which plants can be identified and classified by the physicochemical peculiarities of their starches and other complex metabolites, in other words, by a strictly scientific method.

In Publication 173 similar charts were presented. In their formulation the number of reactions was less, the reagents somewhat different from those used in the present research, and the values expressed were in terms of complete or practically complete gelatinization time. Attempts were made in the present investigation to lessen 
the sources of fallacy by increasing the number and changing the concentration of the reagents and modifying the standard of values in accordance with the abscissæ here used. Notwithstanding the crudities of the methods adopted and the fallacies introduced in the formulation of the composite charts in the former memoir the following was rendered apparent: That the reactions of members of a genus constitute a well-defined group, the mean of the character-values constituting a distinct generic type, this type tending to be similar to the types of very closely related genera and dissimilar to the types of distantly related or unrelated genera; that the reactions of different species of a genus yield curves that tend to be closely in conformity with the generic type of curve, but when there are representatives of subgenera or similar generic subdivisions there may be departures or aberrations from this generic type so that there may be as many subgeneric or group types as there are subgenera or subgeneric groups; that the reactions of varieties of a species yield curves that very closely correspond with those of the species; and that the generic, subgeneric, and species differentiations are in general in close accord with established botanical data. The results of the present research are in harmony with those of the preceding investigation, but some unexpected variations have been found, especially in the extent of certain generic and subgeneric differentiations which will be referred to here with sufficient detail.

Taking up first those genera which are best represented by species and varieties, but in which there are not included subgeneric or similar generic group representatives, such as Hippeastrum (Charts E 2, E 3, and E 4), Nerine (Charts E 10, E 11, and E 12), Narcissus (Charts E 13 to E 24, inclusive), and Lilium (Charts E 25 to E 29, inclusive), it will be apparent upon even superficial examination that the starches of the varieties or species, or of both varieties and species, of each genus have curves that are in general very similar in form and that the type form of the curve in each genus is different from that of any other, and so markedly so that the curves of the members of one genus could not be confounded with those of another any more than could the plants themselves. It will also be noted that when the starches are from very closely related plants, as in the Hippeastrums, the curves are very closely alike, while in Nerine and Narcissus, respectively, where there are instances of both botanical closeness and separation, the variations from the mean or the generic type of curve tend to be more and more marked as the representatives of the genus are botanically farther separated. The curves of Litium, while yielding a generic type very different from the Hippeastrum, Nerine, and Narcissus types, are of little usefulness in the differentiation of the various membiers of the genus represented because of the very rapid gelatinization of the starches with nearly all of the reagents. In order to satisfactorily differentiate these starches reagents of such modified strengths must be used as will render gelatinization very much less rapid, and probably additional reagents may be necessary. In other genera studied, where there are only the two parental and the hybrid representatives of the genus, as in Gladiolus (Chart E 34), Tritonia (Chart E 35), Richardia (Chart E 40), Musa (Chart E 41), Phaius (Chart E 42), Miltonia (Chart E 43),
Cymbidium (Chart E 44), corresponding peculiarities will be found, although in Giladiolus and Tritonia, closely related genera, the curves are so much alike as to indicate different species rather than different genera. There is also much resemblance between the Amaryllis and Phaius charts which represent very widely separated genera, but this singular peculiarity will be referred to particularly later on. In the A maryllis-Brunsvigia reactions (Chart E 1 ), where there is bigeneric representation, the curves are quite different.

When genera are represented by subgenera or subgeneric groups, as in Hæamanthus (Chart E 6), Crinum (Charts E \%, E 8, and E 9), Iris (Charts E 30, E 31, $\mathrm{E} 32$, and E 33), and Begonia (Chart E 36), the curves of the subgeneric representatives may differ not only markedly but to even a much more marked degree than the curves of different genera generally of the same family-a most curious and as yet inexplicable phenomenon. In Hamanthus the curve of H. puniceus is so variant in comparison with those of $H$. katherina, $H$. magnificus, and both hybrids that it seems that this species must be separated botanically sufficiently far from the other two to be regarded as belonging to a different subgenus, although this differentiation may not have been recognized by the systematist. In Crinum the curves of the representatives of the hardy and tender forms ( $C$. moorei and C. longifolium, hardy; C. zeylanicum, tender) differ so markedly as to suggest members of different genera. In Iris, in the first three sets (Charts $\mathrm{E} 30$, $\mathrm{E} 31$, and $\mathrm{E} 32$ ), the reactions of rhyzomatous forms are represented, and it will be seen that all of the curves conform closely to a common type; but in the fourth set (Chart E 33) the reactions are of tuberous forms, all three curves conform with great closeness to a common type, and they all differ materially from the rhyzomatous type, and in fact so different are they that they would certainly not in the present stages of the investigation be recognized as belonging to the same genus. In $\mathrm{Be}$ gonia there is found an even more remarkable instance of subgeneric differentiation in the curves of the tuberous and semituberous forms, the former being represented by four garden varieties and the latter by $B$. socotrana, a very exceptional and isolated species of the genus. Comparing the curves of these charts (Charts E 36 to E 39) it will be seen that the curves of the tuberous forms are in close conformity to a common type, while the curve of $B$. socotrana is so very unlike the curves of the former in a large number of the reactions with the chemical reagents as to suggest anything but generic relationship to the tuberous forms. Unfortunately, the number of reactions of the latter were with a single exception very limited, but the curve of the reactions of $B$. single crimson scarlet (Chart E 36) can with perfect safety be taken as very closely typifying the eurves of the others.

The Amaryltis and Phaius curves (Charts E 1 and E 42), while representing wholly unrelated and widely separated genera, give the impression of curves of closely related genera or even of species of a genus; in fact, the resemblance is much closer than that of related genera here represented, as, for instance, of A maryllis and Brunsvigia (Chart E 1), of Phaius and Miltonia (Charts E 42 and $\mathrm{E} 43$ ), or of Phaius and Cymbidium (Charts E 42 and $\mathrm{E} 44)$. While there is some resemblance between 
Phaius and Miltonia, there is exceedingly little between Phaius and Cymbidium. Obviously, from what is manifest by the curves generally of these charts, this resemblance must be seeming rather than actual, and due to faultiness in the methods of experiment and charting. That the Amaryllis and Phaius starches differ far more than is indicated by the composite curves is shown by the records of the velocity reactions (Charts D 1 to D 21, and D 574 to D 594), and it is obvious that in the construction of composite charts the recognition of such differences is essential to even an approximately accurate presentation of the reaction peculiarities of any starch. It will probably be found that taxonomic differences of much value will be brought out by differences in the ratios of the reaction-intensities of different pairs or combinations of certain pairs of reagents, and there undoubtedly yet remain many reagents that can be employed to advantage in these studies, it being not improbable that the differences in reactions of a very few reagents may be specific in the differentiation of certain genera, as has been found, for instance, in the tests for proteins, all proteins responding to certain of the protein tests, but some only to certain tests to which others do not respond. Similar restricted methods of differentiation are by no means rare even to the systematist. Then again, in comparing these curves it will be seen that no less than 7 of the 21 reagents have, apparently at least, proved useless because of the energy with which they cause gelatinization. Modifications of the strengths of these alone, or in conjunction with the other reagents, may elicit generic differences of such a character as to indicate the wide separation of these genera.

These composite charts were studied individually in Chapter III, Section 6, of the comparisons of the reactions of the members of each set of parent- and hybrid-stocks, and two or more of them were considered comparatively whenever there were two or more sets belonging to the same genus. The main object in these studies was to bring out the relations of the hybrids in their reactions, individually and collectively, to one or the other or both parents. If now these charts are studied collectively, with especial reference to the relationships of the hybrid curves to the parental curves, much data of comparative interest will be elicited that is likely to be missed otherwise. When the parental curves run very closely together, the hybrid curve tends to similar closeness; but when the parental curves tend to separation, and especially with variance in their courses, the hybrid curve may tend to follow the curve of one or the other parent, to be intermediate, or to be more or less distinctly independent of both parental curves. Intermediateness is much more of an exception than a rule, and therefore except in few instances is far from being a criterion of a hybrid. (See also Tables $\mathrm{F}$ and H.) In Hippeastrum (Charts E 2 to E 4), Narcissus (Charts $\mathrm{E} 13$ to $\mathrm{E} 24$ ), Iris (Charts $\mathrm{E} 30$ to $\mathrm{E} 33$ ), and Richardia (Chart E 40) the parental curves tend in each group and genus to marked closeness in their positions and courses, and the hybrid curves similarly tend to closeness to the parental curves, but varying from reaction to reaction in their parental relationships. When the parents are well separated species, as in Hcomanthus (Chart E 5), Crinum (Chart E 9), Nerine (Charts E 10 to E 12), Narcissus (Chart E 14), etc., and the parental curves are generally well separated and somewhat variant in their courses, though on the whole conforming to generic types, the hybrid curves tend to equal or greater degrees of variance. And when the parents are representatives of different genera, as in the Amaryllis-Brunsvigia group (Chart $\mathrm{E} 1$ ), or of subgenera or subgeneric groups, as in Homanthus (Chart $\mathrm{E}$ 6), Crinum (Charts $\mathrm{E}$ ' and E 8), and Begonia (Chart E 36) - where the parental curves are not only well separated but tend to more or less markedly different courses-the hybrid curves show their greatest variabilities in their relations to the parental curves, in some instances tending to have in general marked closeness to the curves of one parent, in others to have a position of intermediateness which is usually closer to one of the parents than to the other, and in others to have a more or less wide departure from both parental curves. When there are two hybrids of the same parentage, as in Amaryllis-Brunsvigia (Chart E 1), Nerine (Charts $\mathrm{E} 10$ and $\mathrm{E} 11$ ) and Narcissus (Chart E 13), the hybrids of each pair of parents tend to differ less from each other, as a rule, than the parents differ from each other; unless, as in case of Amaryllis-Brunsvigia, the parents are so far separated as to give well separated curves, in which case the curves of the hybrids may not only be quite at variance with the parental curves, but also be distinctly better separated from each other, and show even more marked differences from the parental curves than the latter show in relation to each other.

In a number of sets of parent- and hybrid-stocks studied a given parent is found to be the seed parent in one set and the pollen parent in another, or the seed parent or the pollen parent in both sets, but with an associated parent that is different in each of the two setsas in Homanthus (H. katherince, which is the seed parent in two sets, the pollen parents being different); Crinum (C. moorei, C. zeylanicum, and C. longifolium, which are differently paired in the three sets); Nerine ( $N$. sarniensis corusca major) ; Narcissus (N. poeticus ornatus, $N$. poeticus poetarum, $N$. abscissus, $N$. albicans, $N$. madame de graaff, and $N$. triandrus albus); Lilium (L. martagon album and L. maculatum) ; Iris (I. iberica and $I$. cengialti); and Calanthe (C. vestita var. rubrooculata). In connection therewith many interesting features have been recorded in the histologic and polariscopic properties and in the reactions with heat and various chemical reagents which show most varying trangmissibilities in both kind and degree of parental characters to the hybrid, but a detailed review is not necessary and is prohibited by want of space in an already too voluminous report. The most important of such data will be found presented for the most part and in succinct form in Chapter III, and in detail in Part II, Chapter I, under the appropriate headings.

\section{Series of Charts.}

The various charts of the reaction-intensities are referred to particularly or incidentally with frequency throughout Part I, and it was found in the final arrangement of the report that it was desirable chiefly for convenience of reference to bring all of them together in one section. In addition to these a series, $\mathrm{F} 1$ to $\mathrm{F} 14$, is included, but which belongs in the next chapter, in several of which certain reaction-intensities are also recorded. 
Chart A 1.-Polarization Reactions.

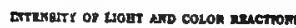

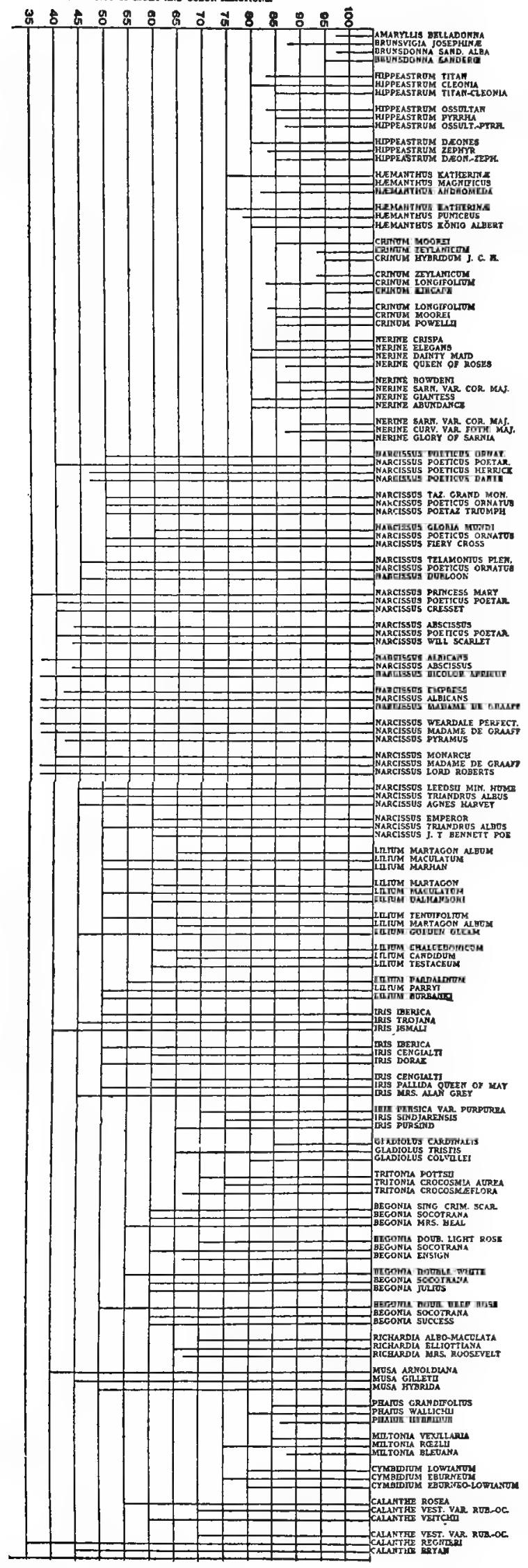

Chant A 2.-Iodine Reactions.

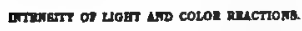

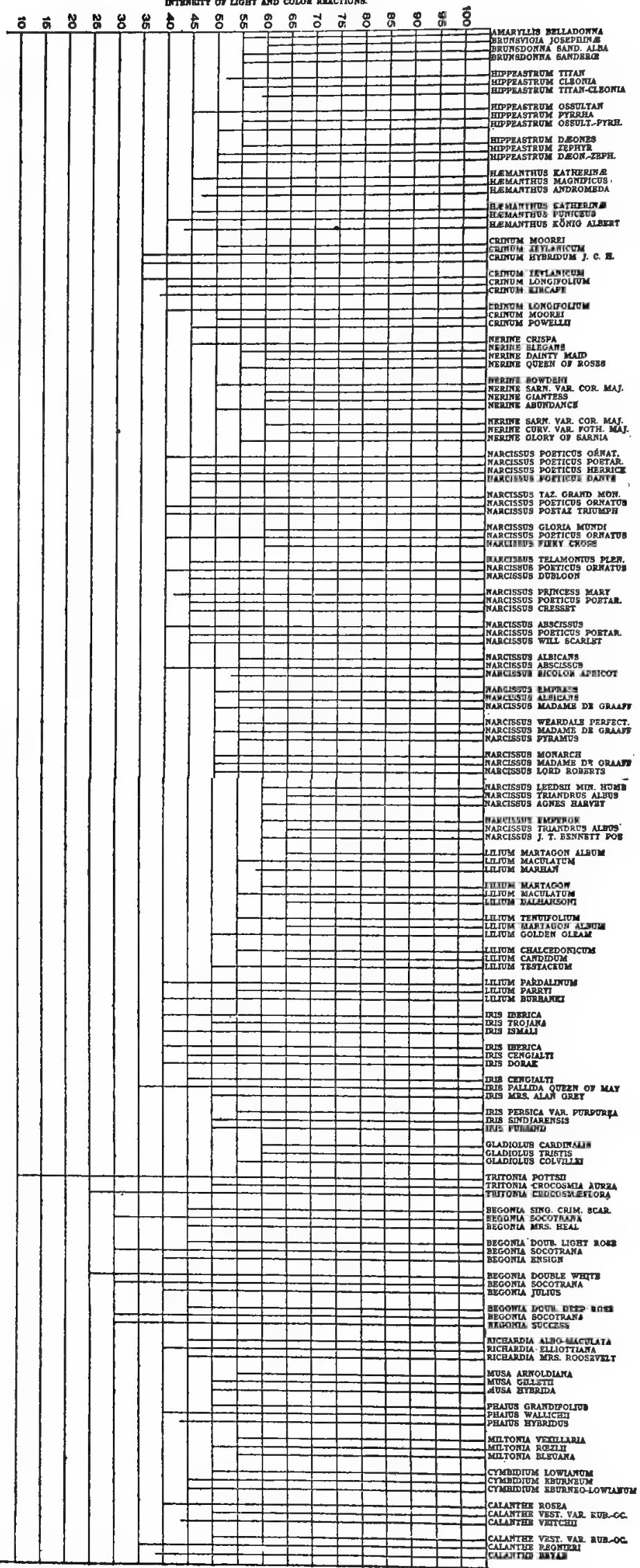


Chart A 3.-Gentian-violet Reactions.

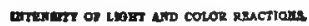

ตั

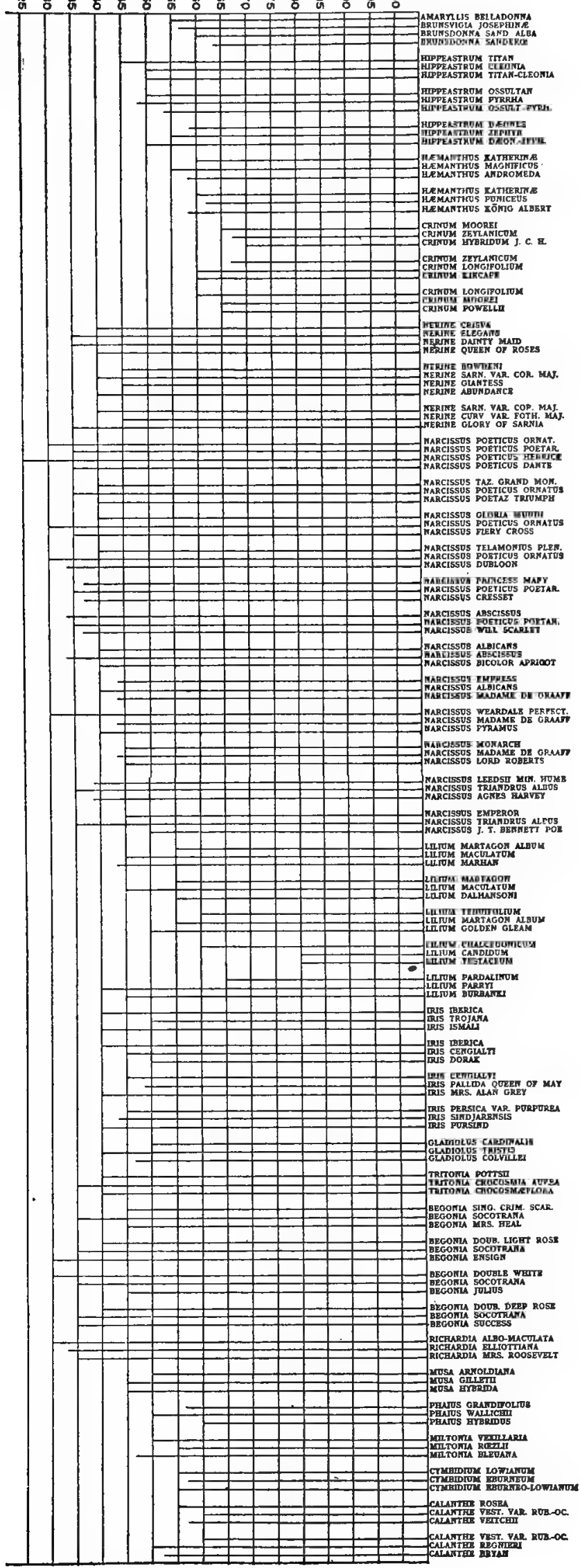

Chart A 4.-Safranin Reactions.

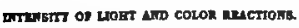

\&

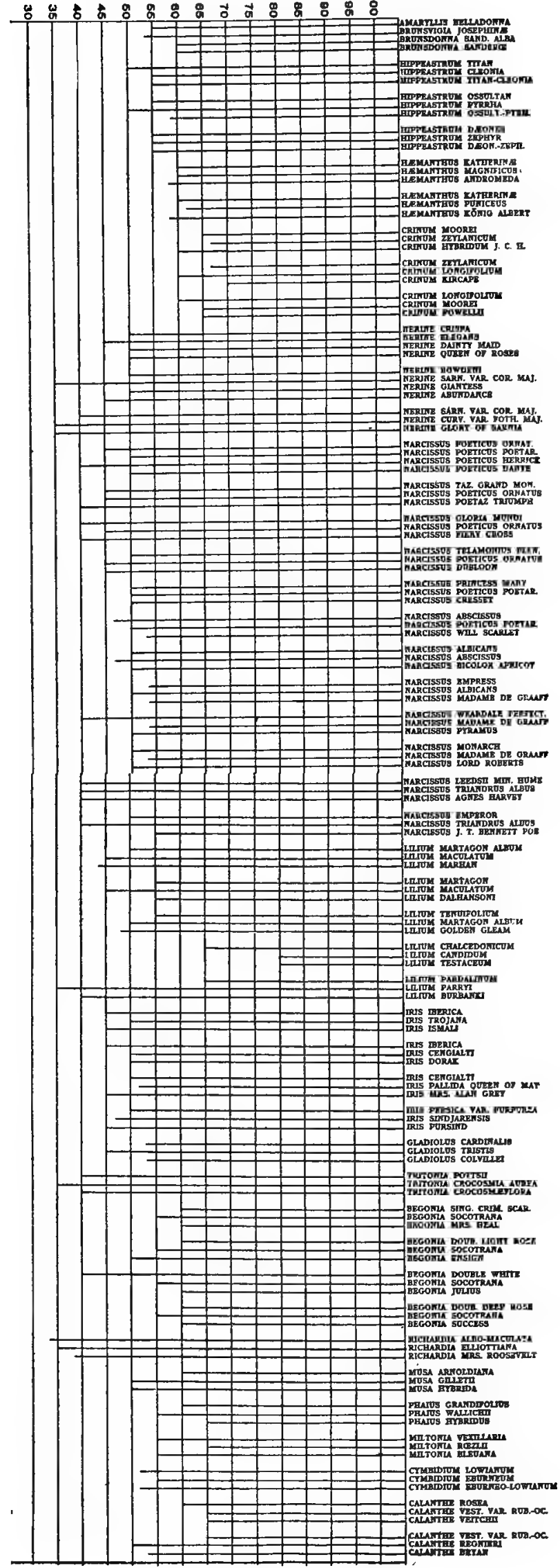


Chart A 5.-Temperature of Gelatinization Reactions.

:

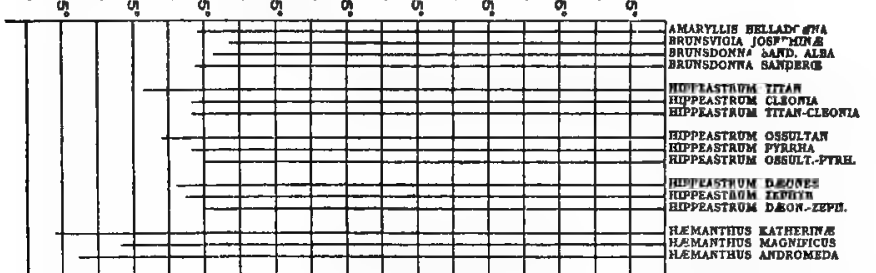

Chart A 6.-Chloral-hydrate Reactions.

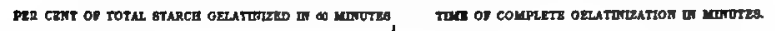

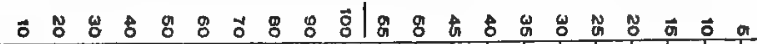

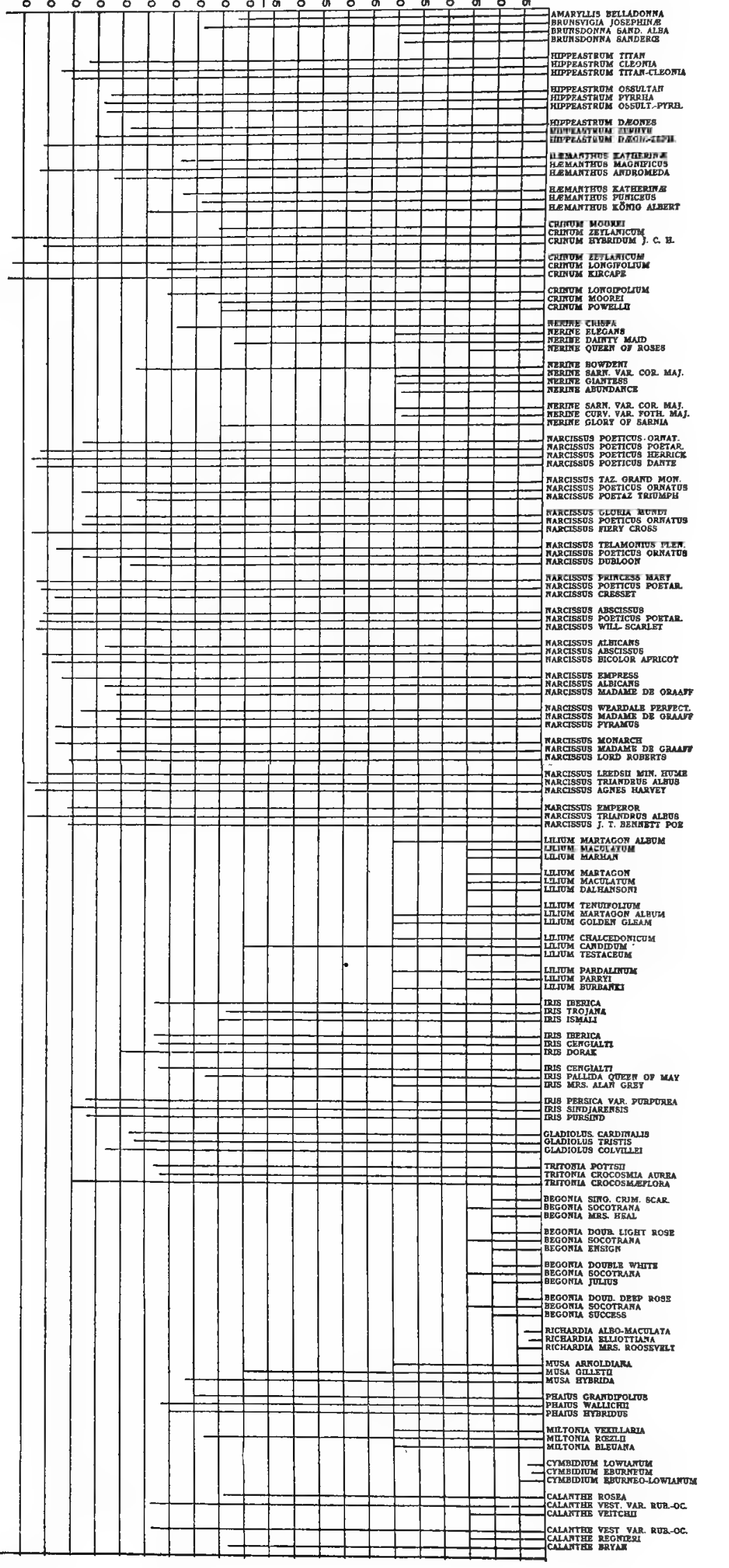


ChART A 7.-Chromic-acid Reactions.

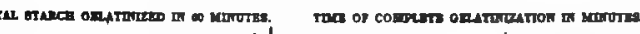

Б
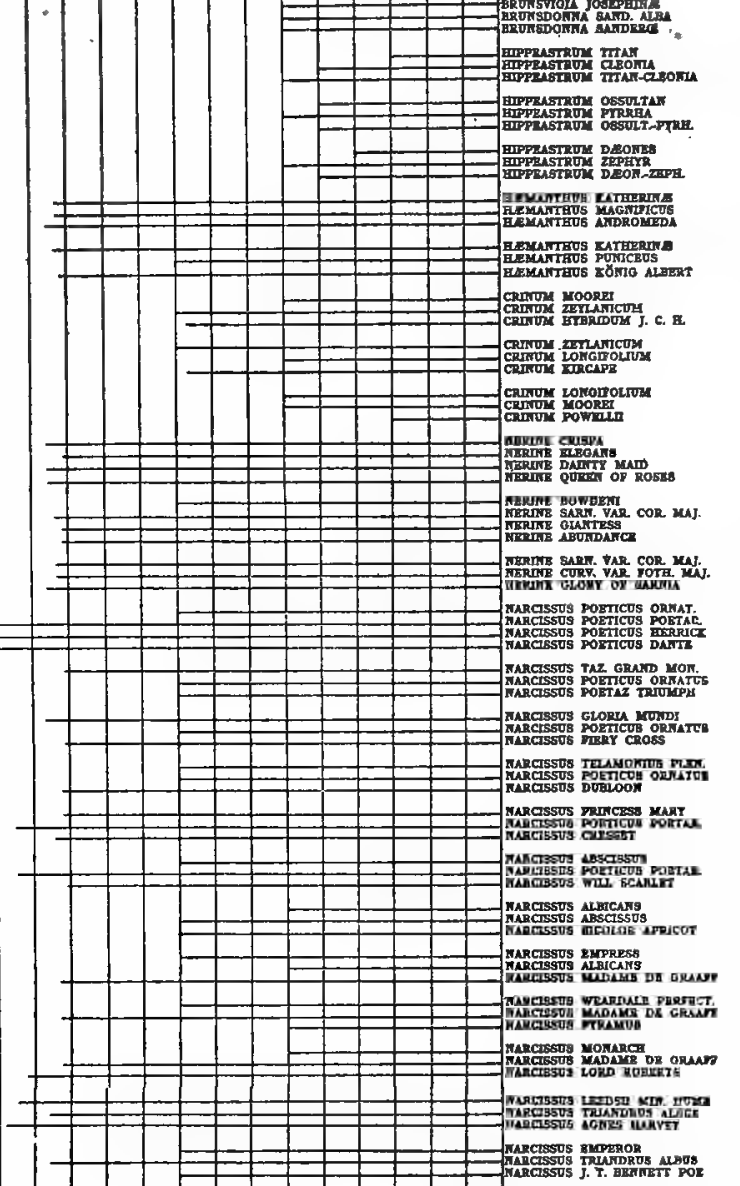

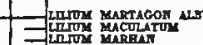

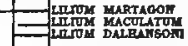

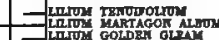

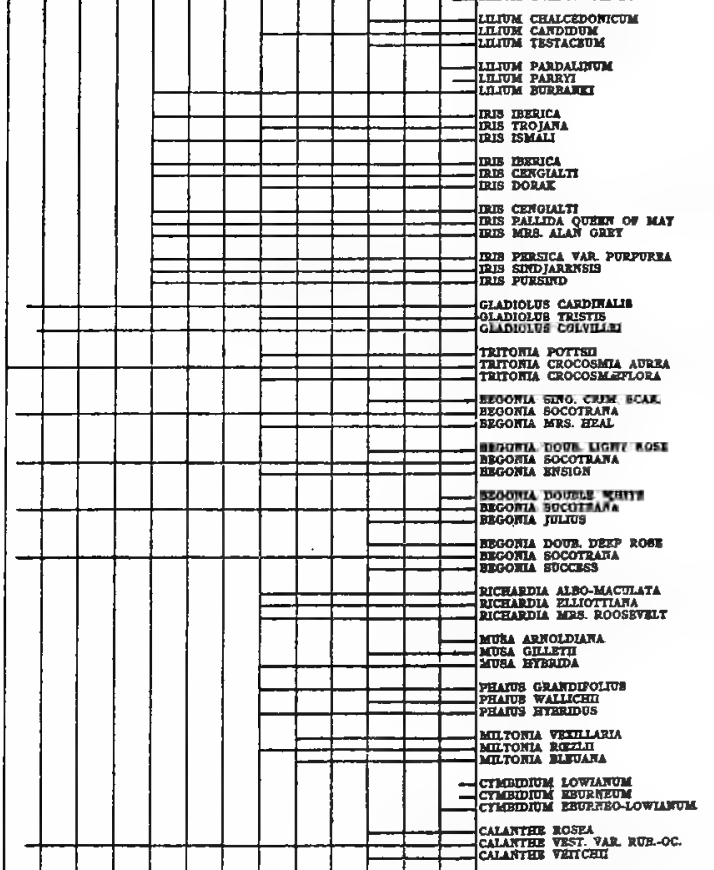

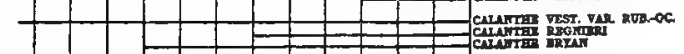

Chart A 8.-Pyrogallic-acid Reactions.

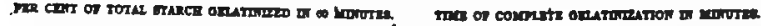

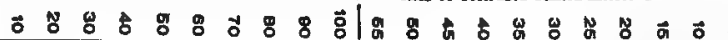

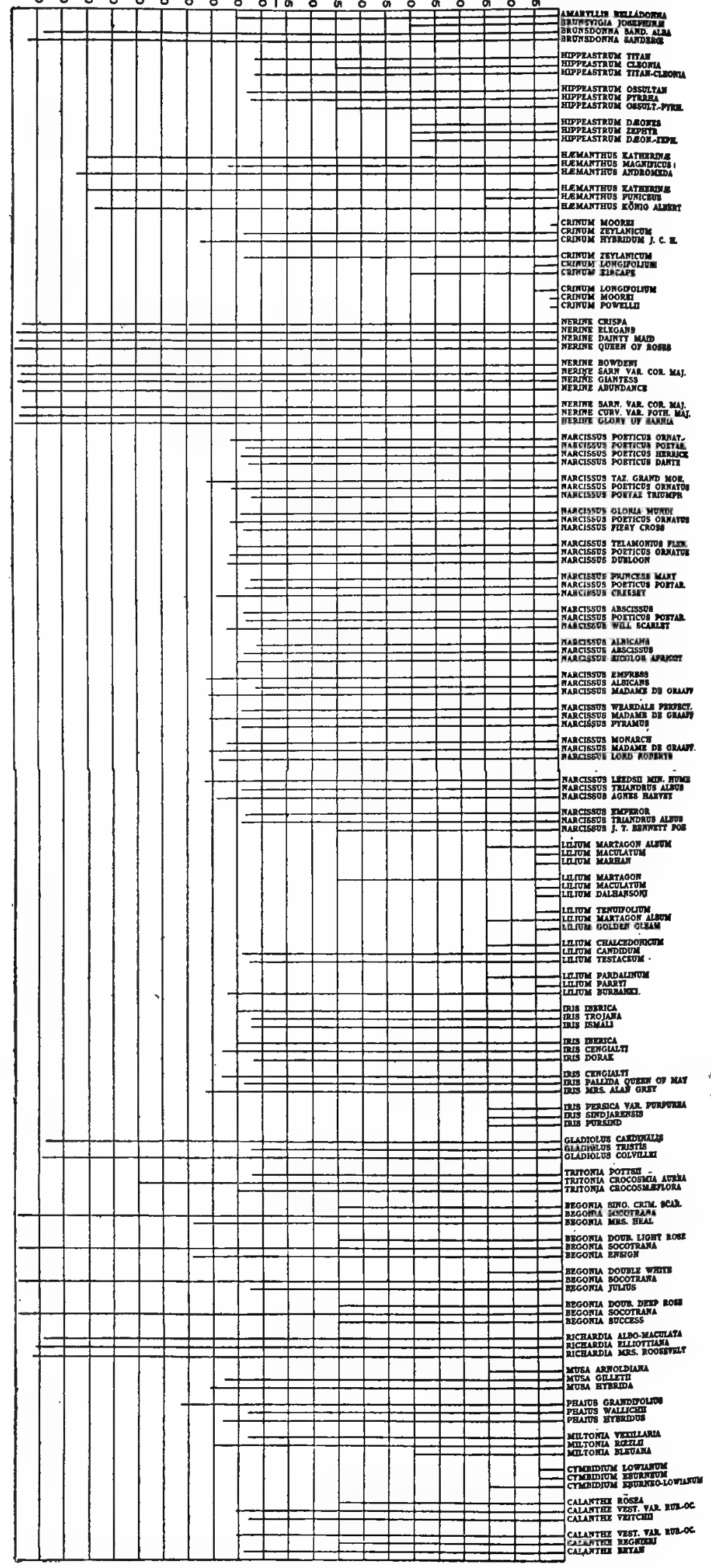


Chart A 9.-Nitric-acid Reactions.

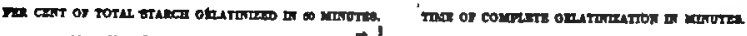

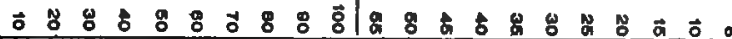

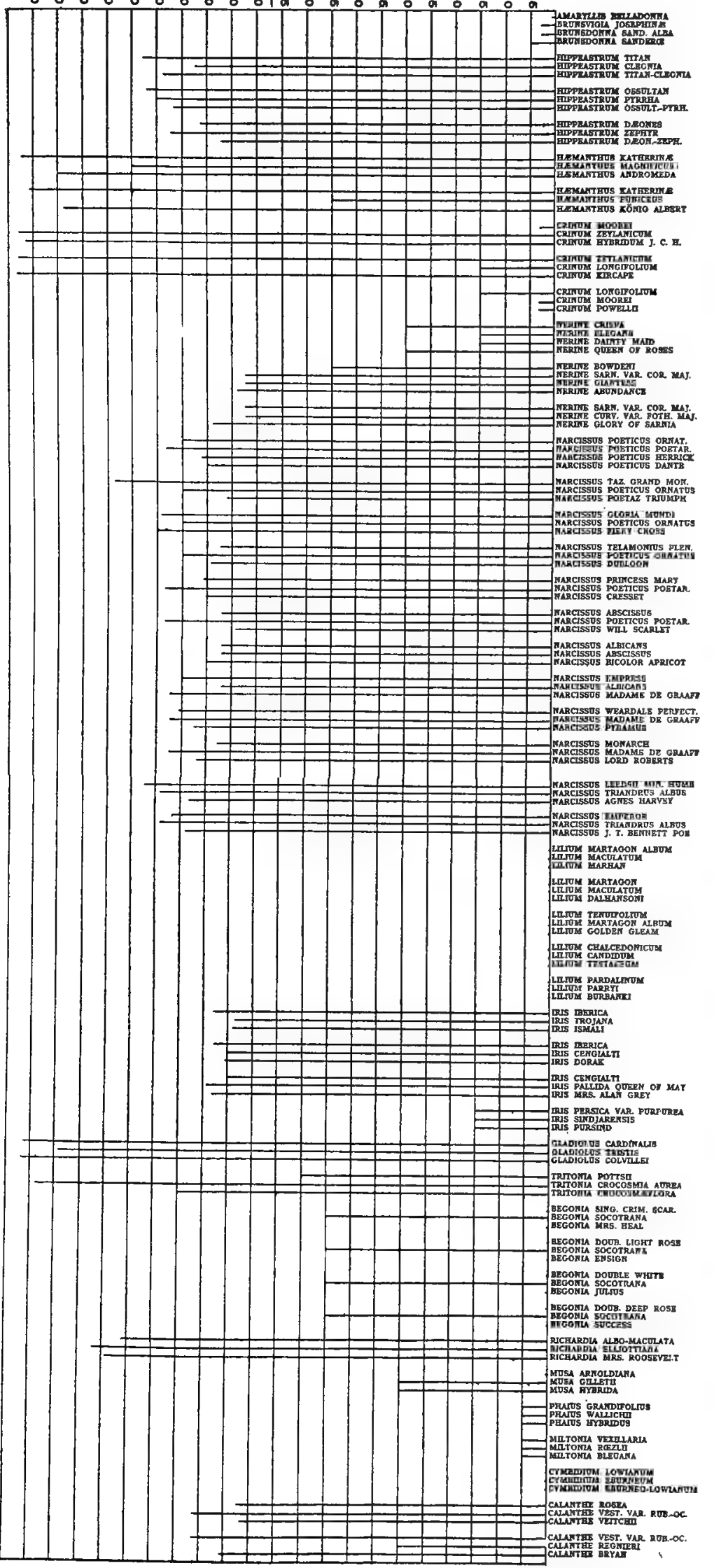

Chart A 10.-Sulphuric-acid Reactions. rew corro

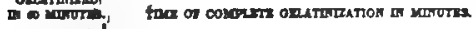

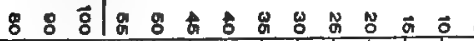

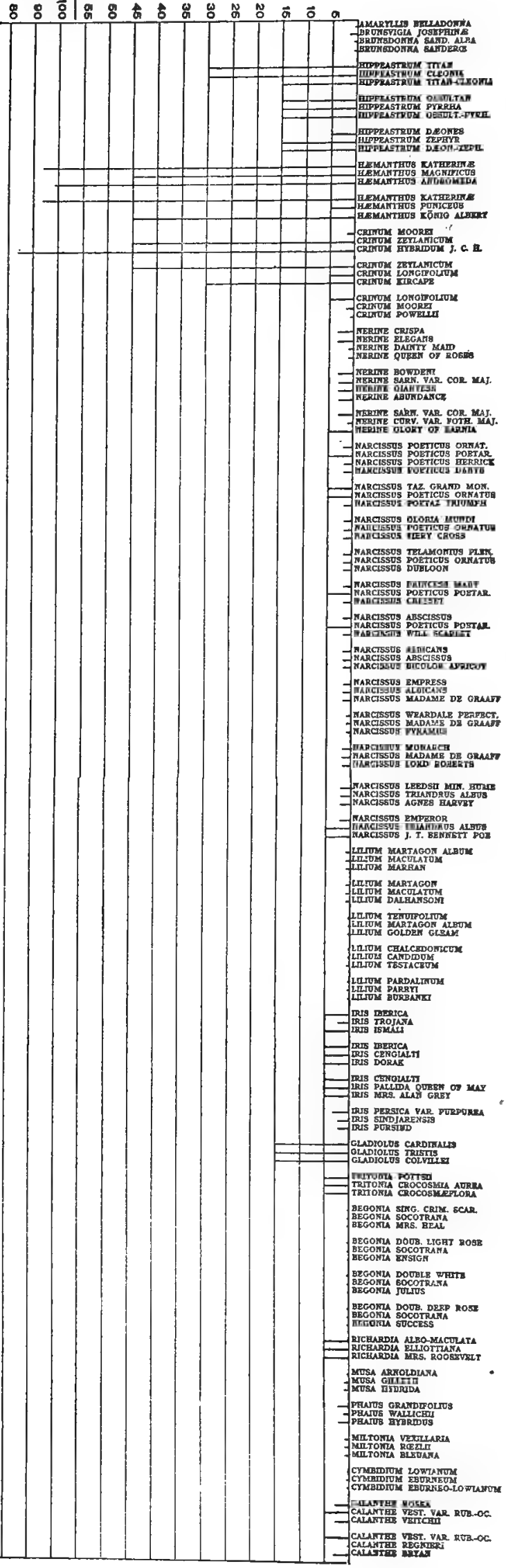


Chart A 11.-Hydrochloric-acid Reactions.

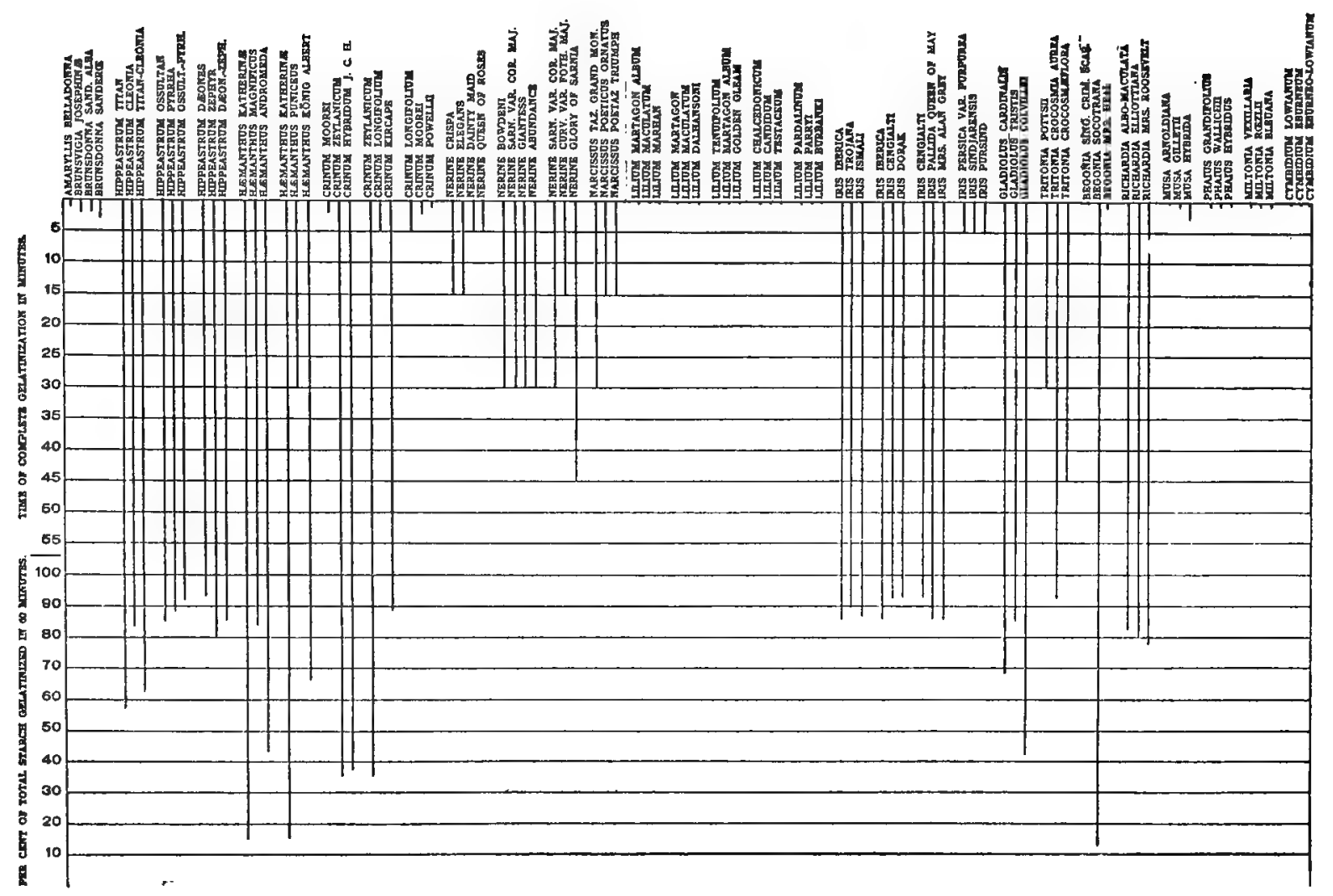

Chart A 12.-Potassium-hydroxide Reactions.

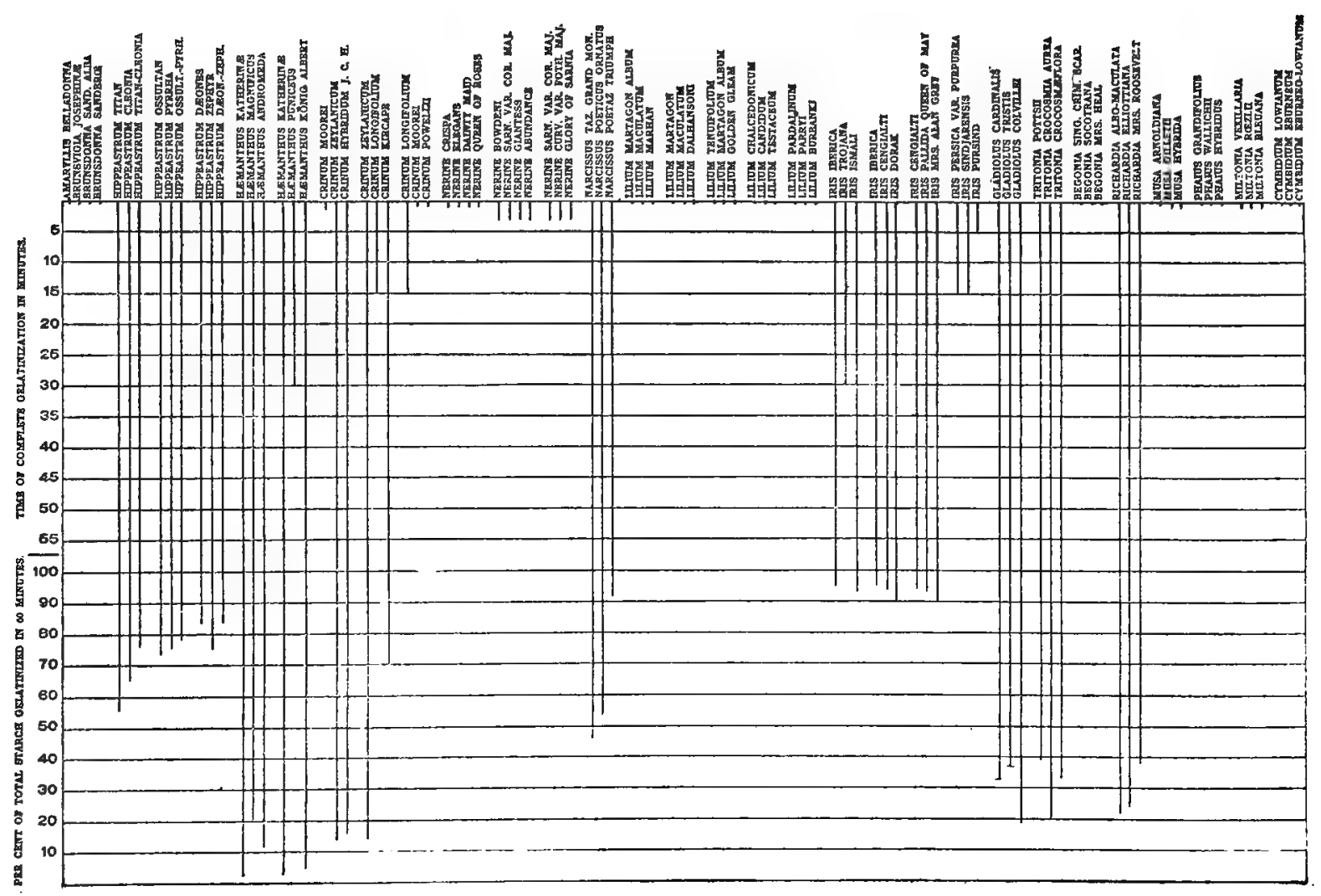


Chart A 13.-Potassium-iodide Reactions.

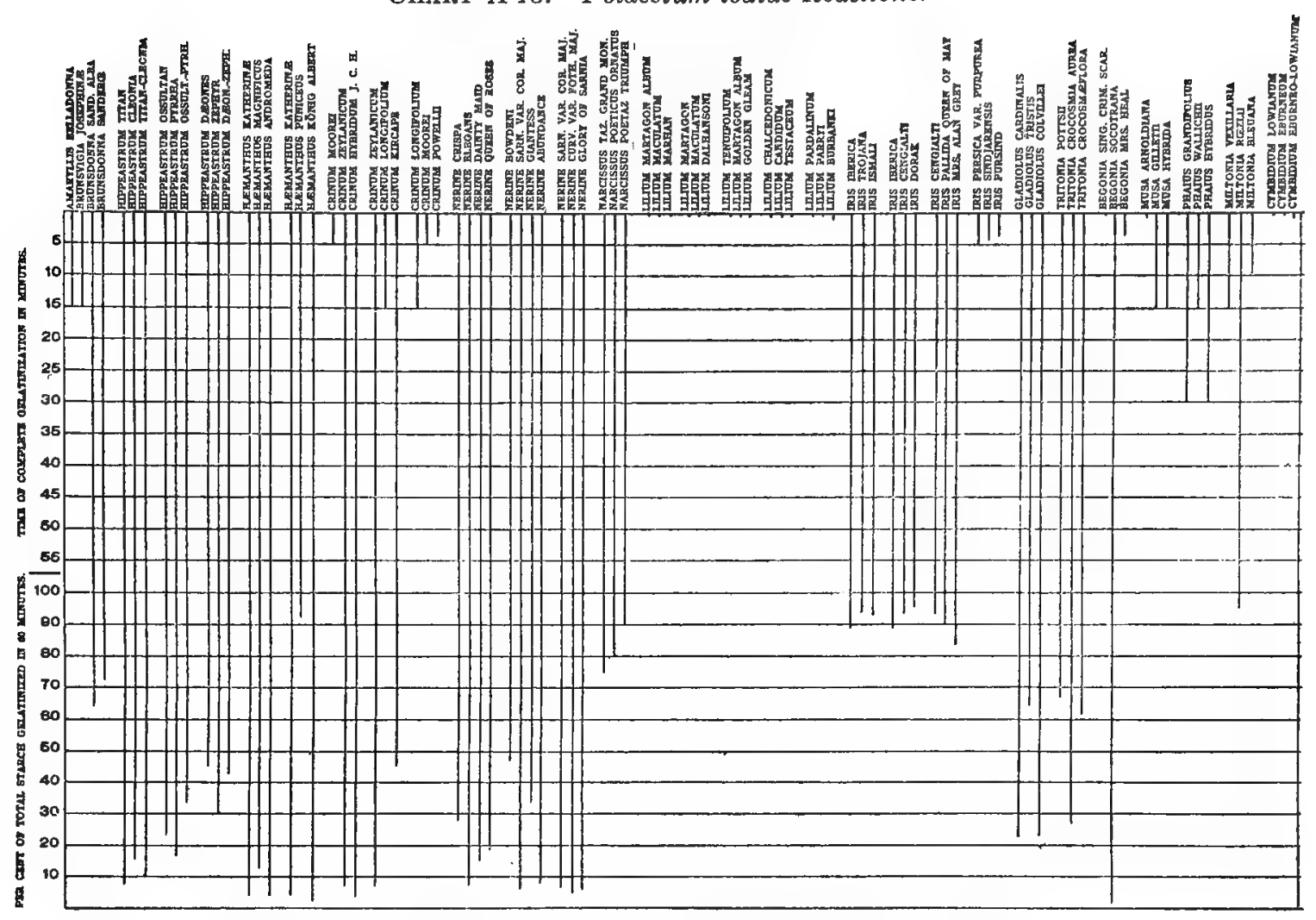

Chart A 14.-Potassium-sulphocyanate Reactions.

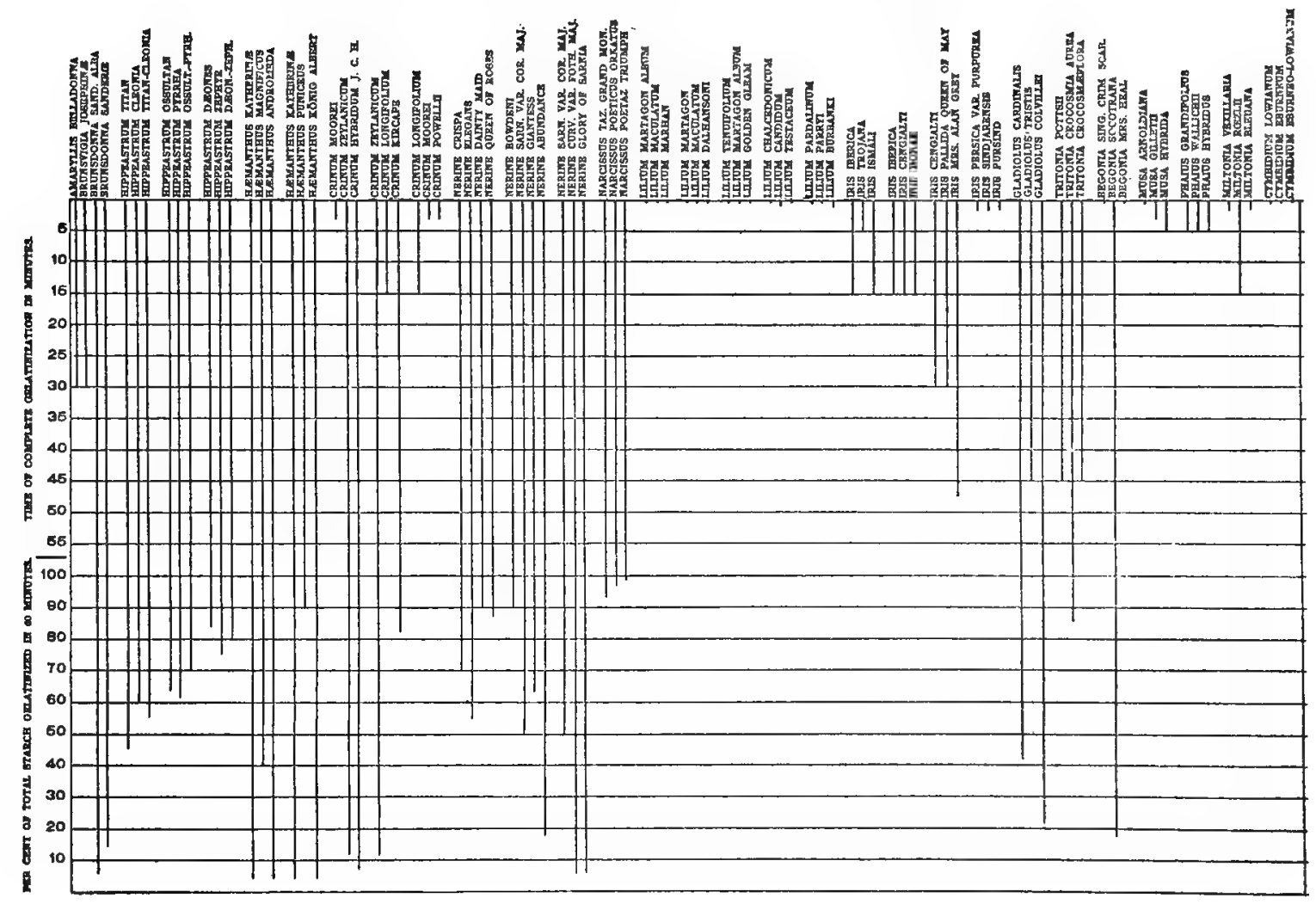


Chart A 15.-Potassium-sulphide Reactions.

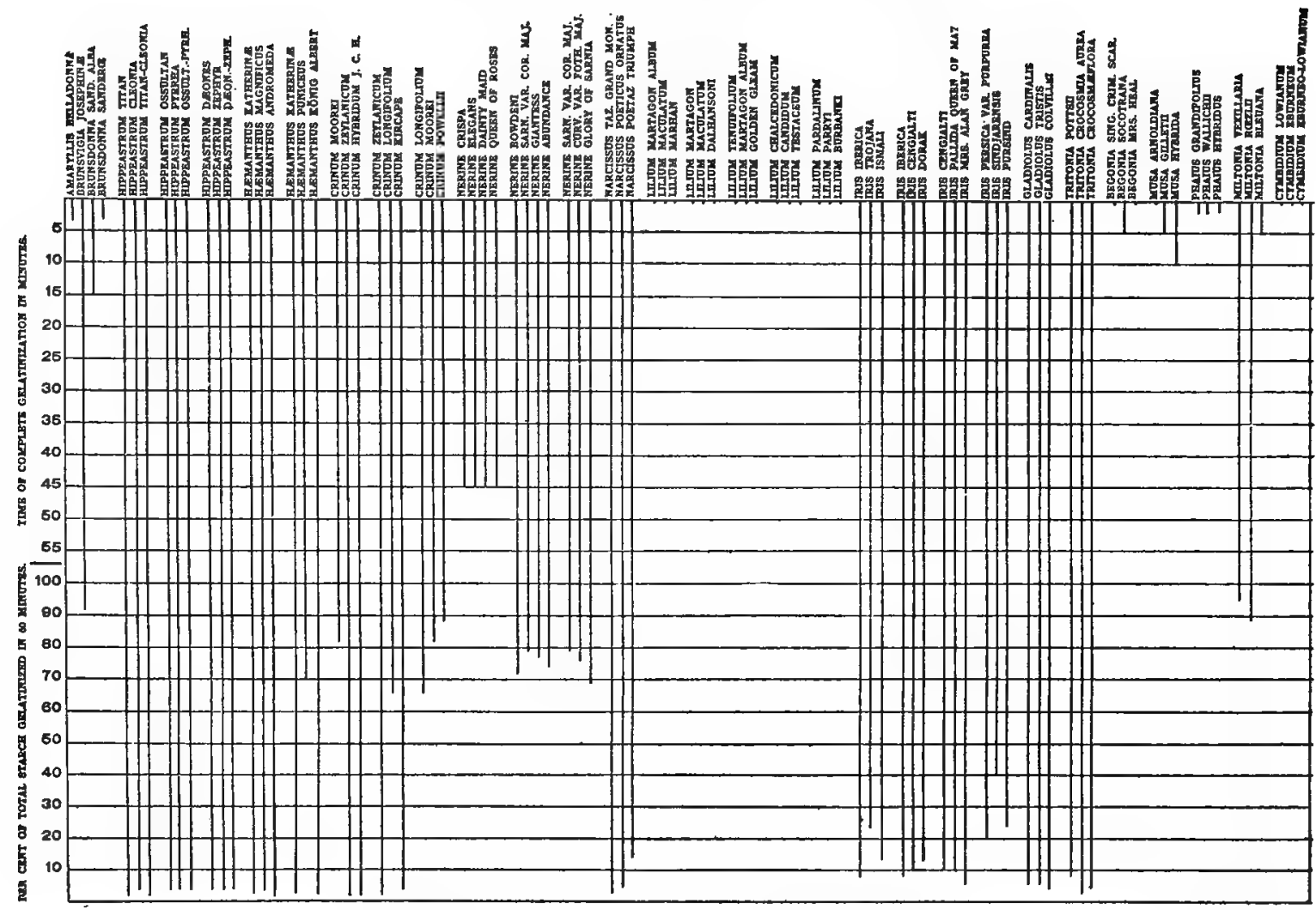

Chart A 16.-Sodium-hydroxide Reactions.

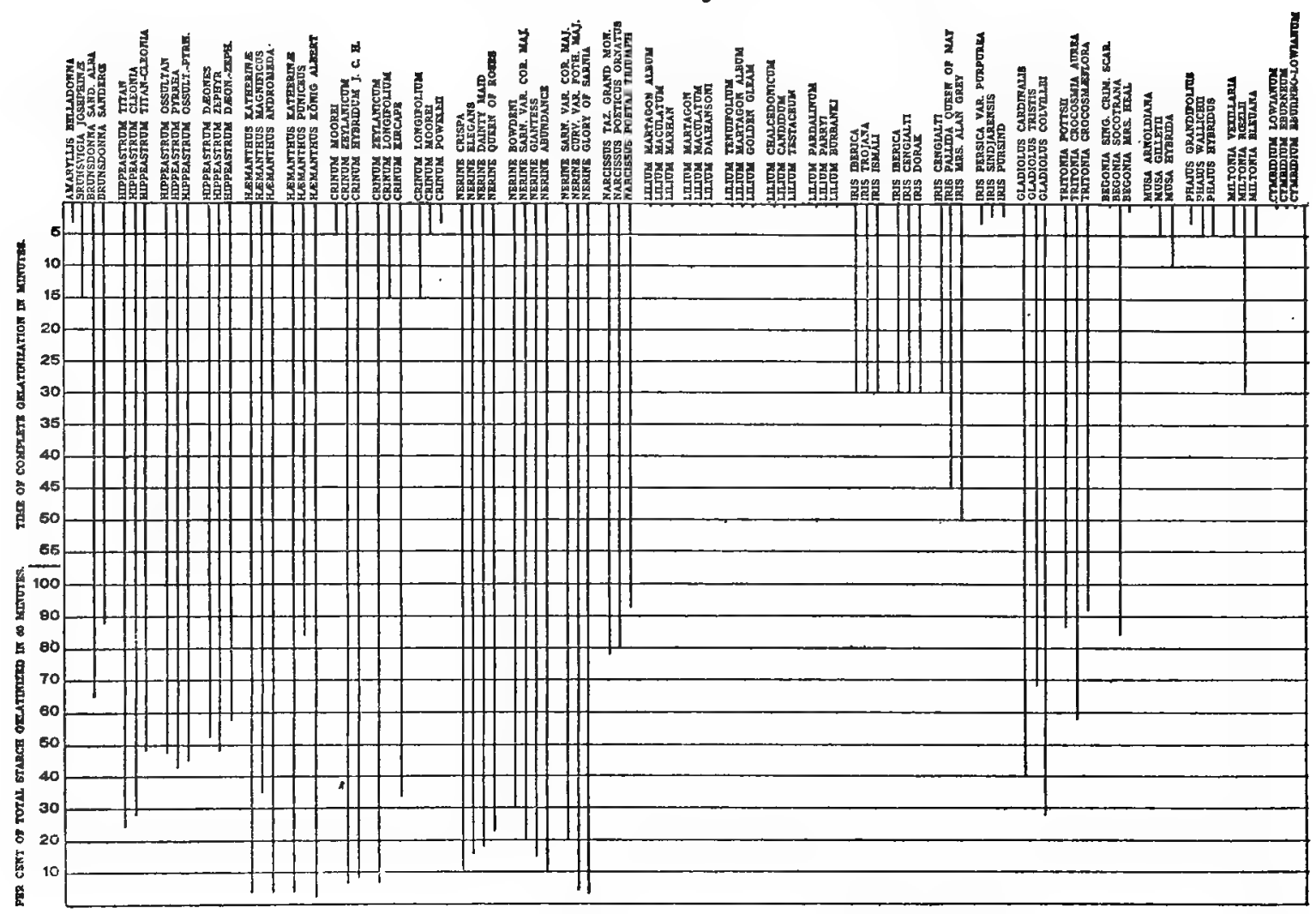


Chart A 17.-Sodium-sulphide Reactions.

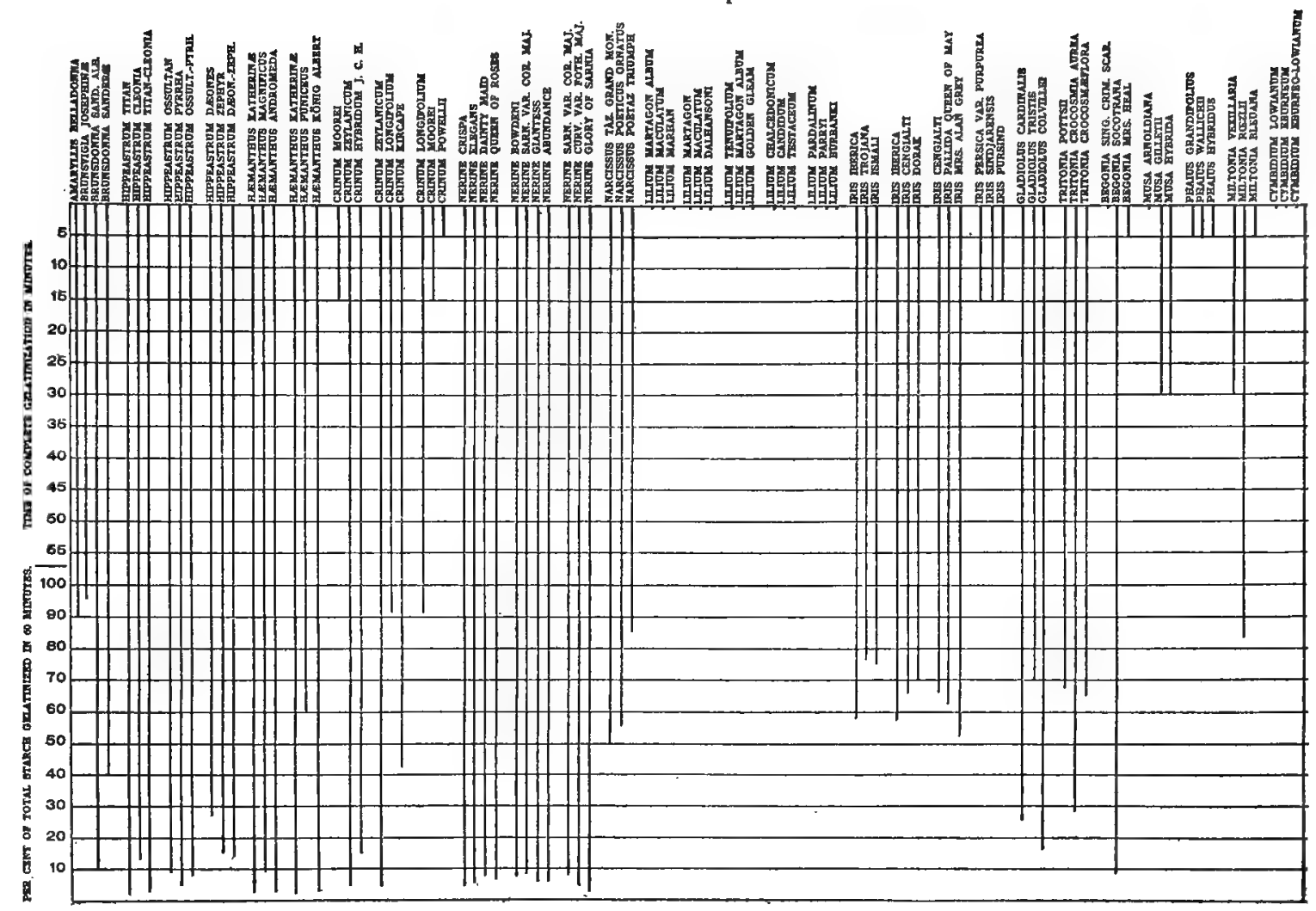

Chart A 18.-Sodium-salicylate Reactions.

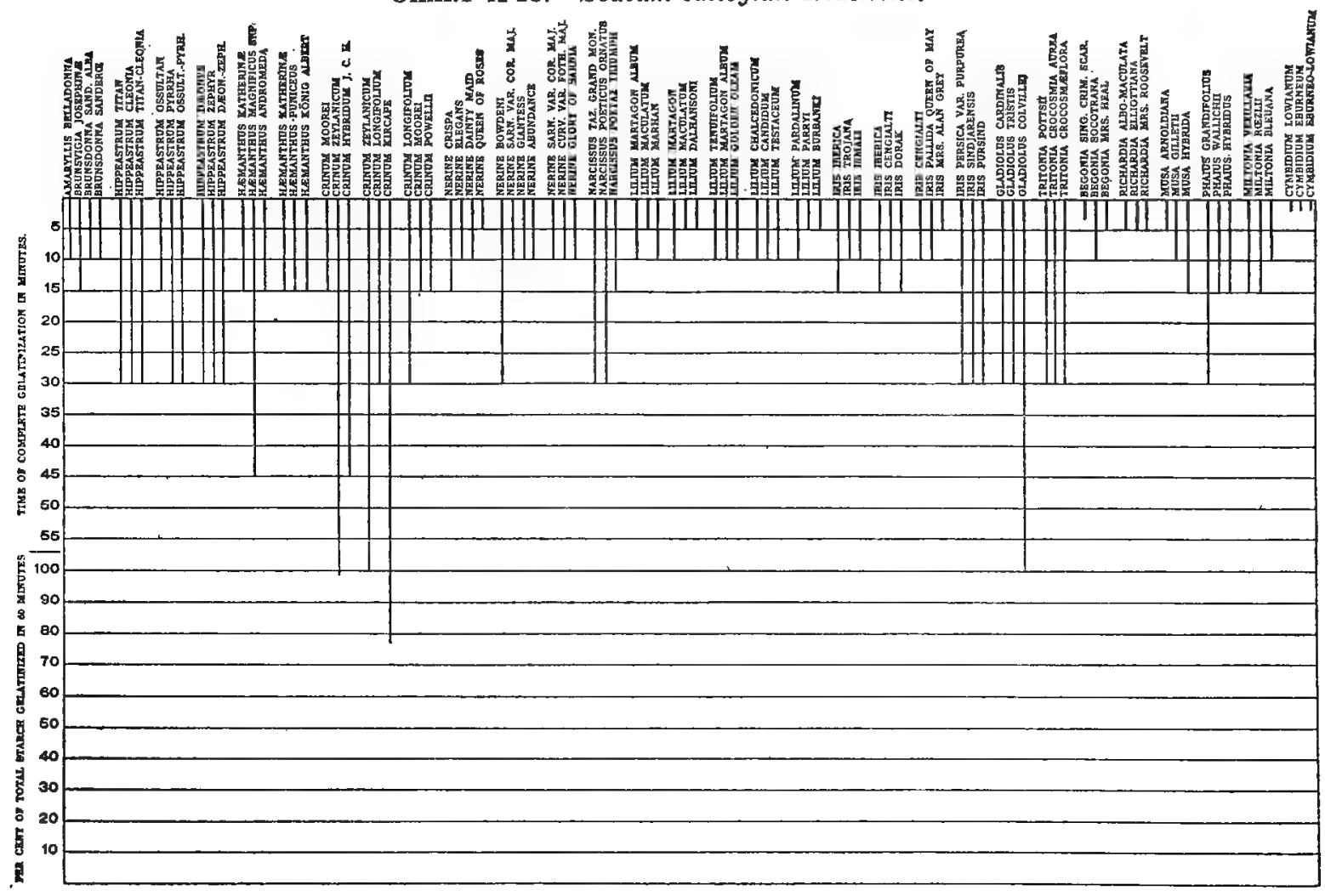


Chart A 19.-Calcium-nitrate Reactions.

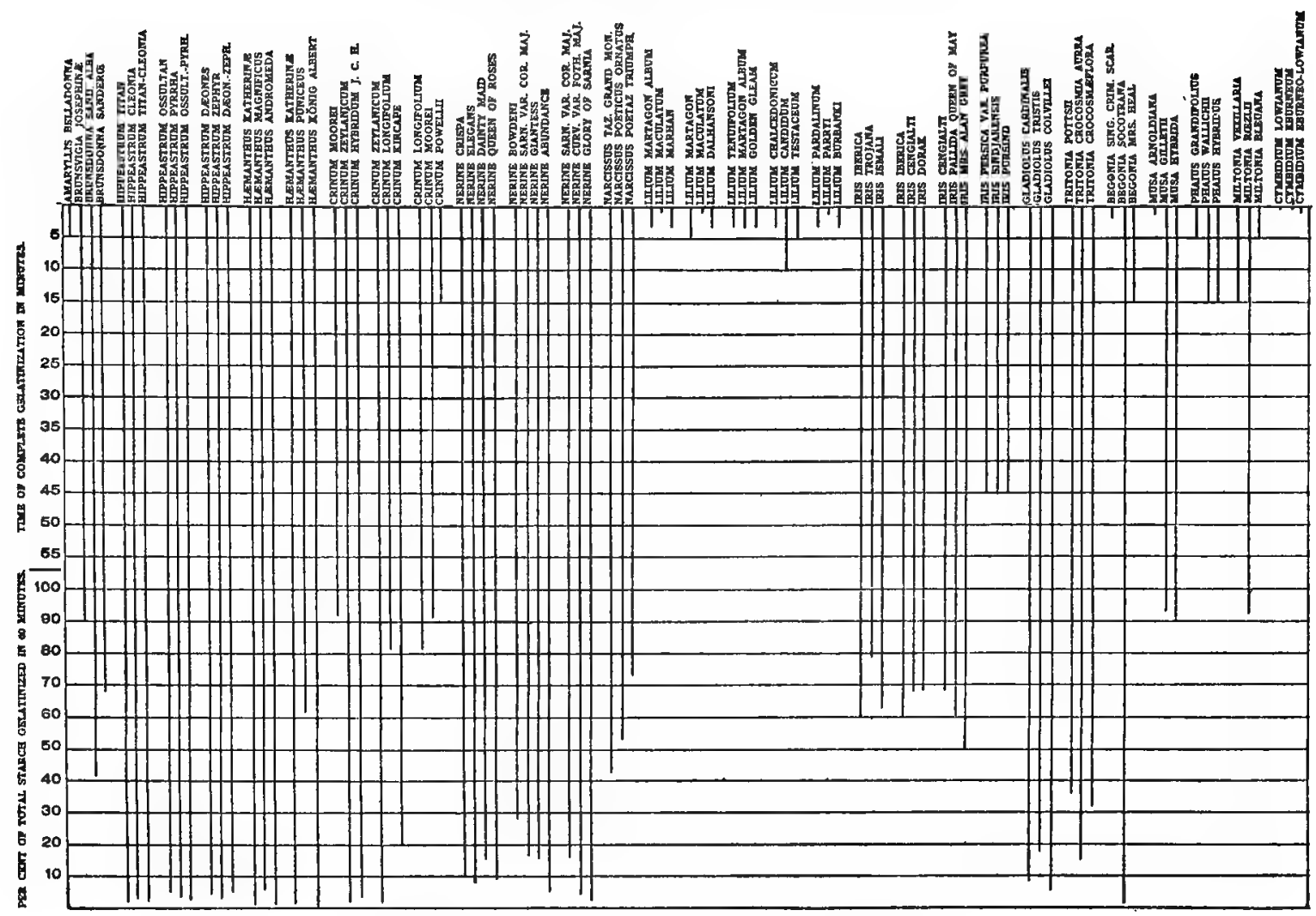

Chart A 20.-Uranium-nitrate Reactions.

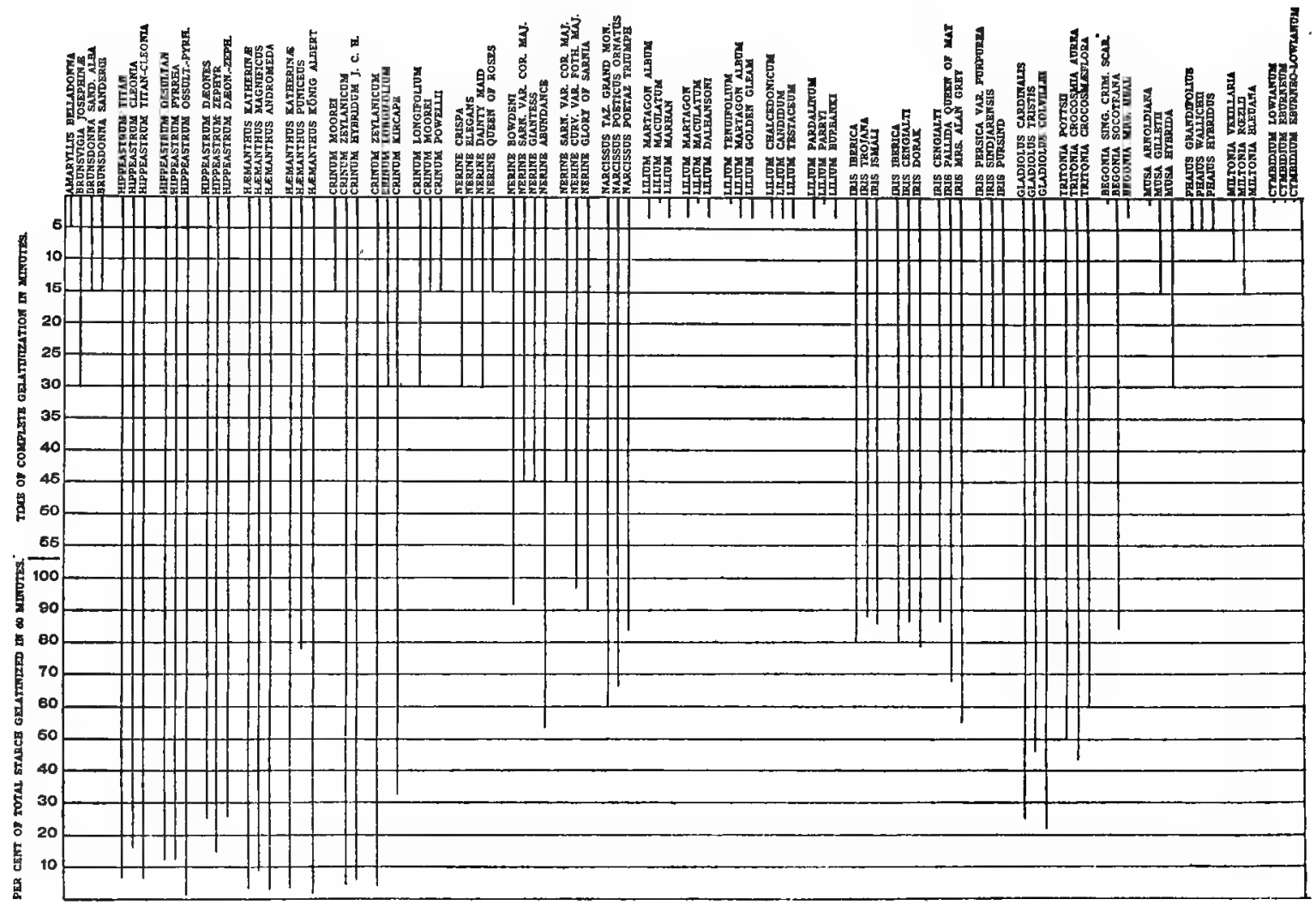


Chart A 21.-Strontium-nitrate Reactions.

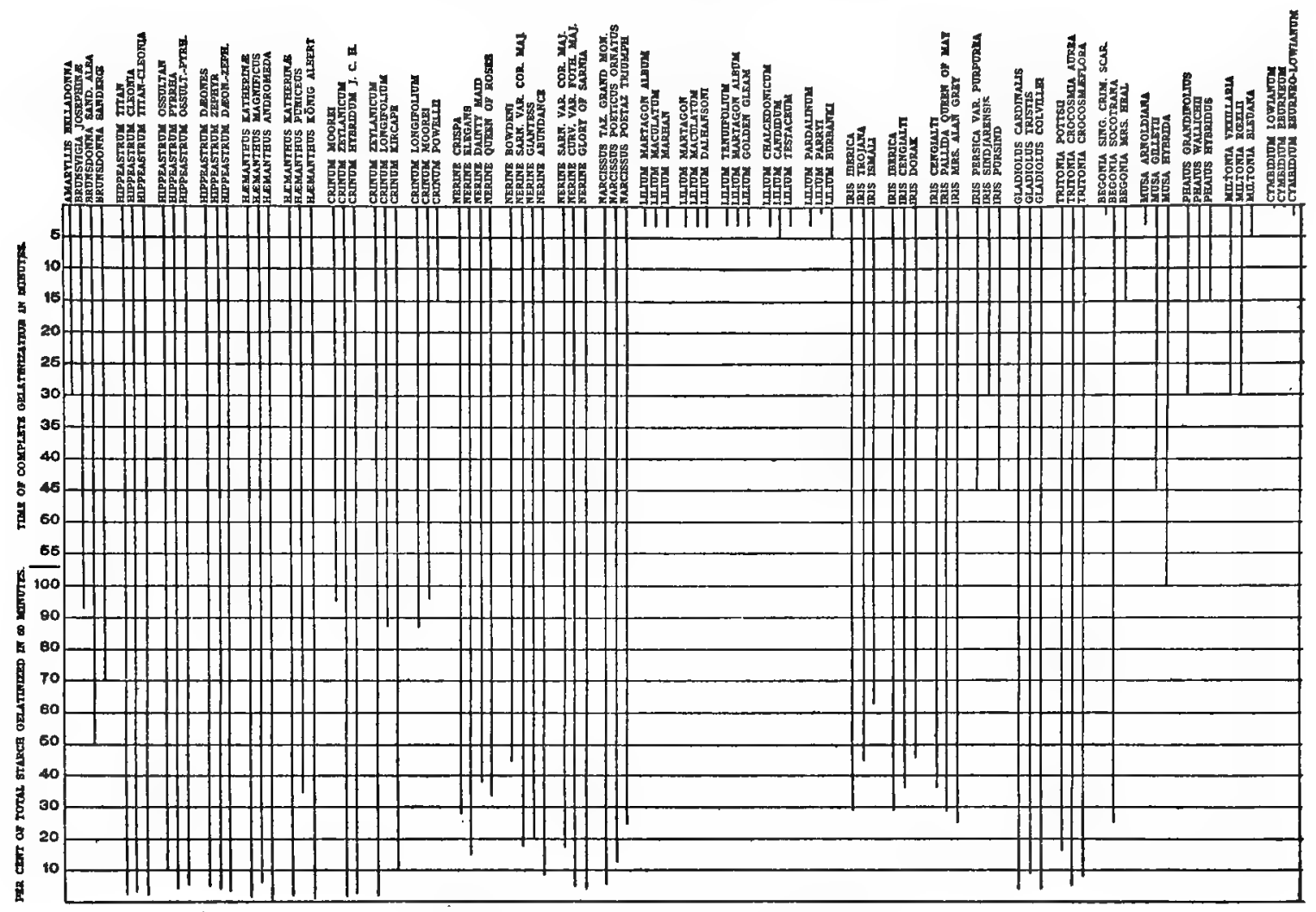

Chart A 22.-Cobalt-nitrate Reactions.

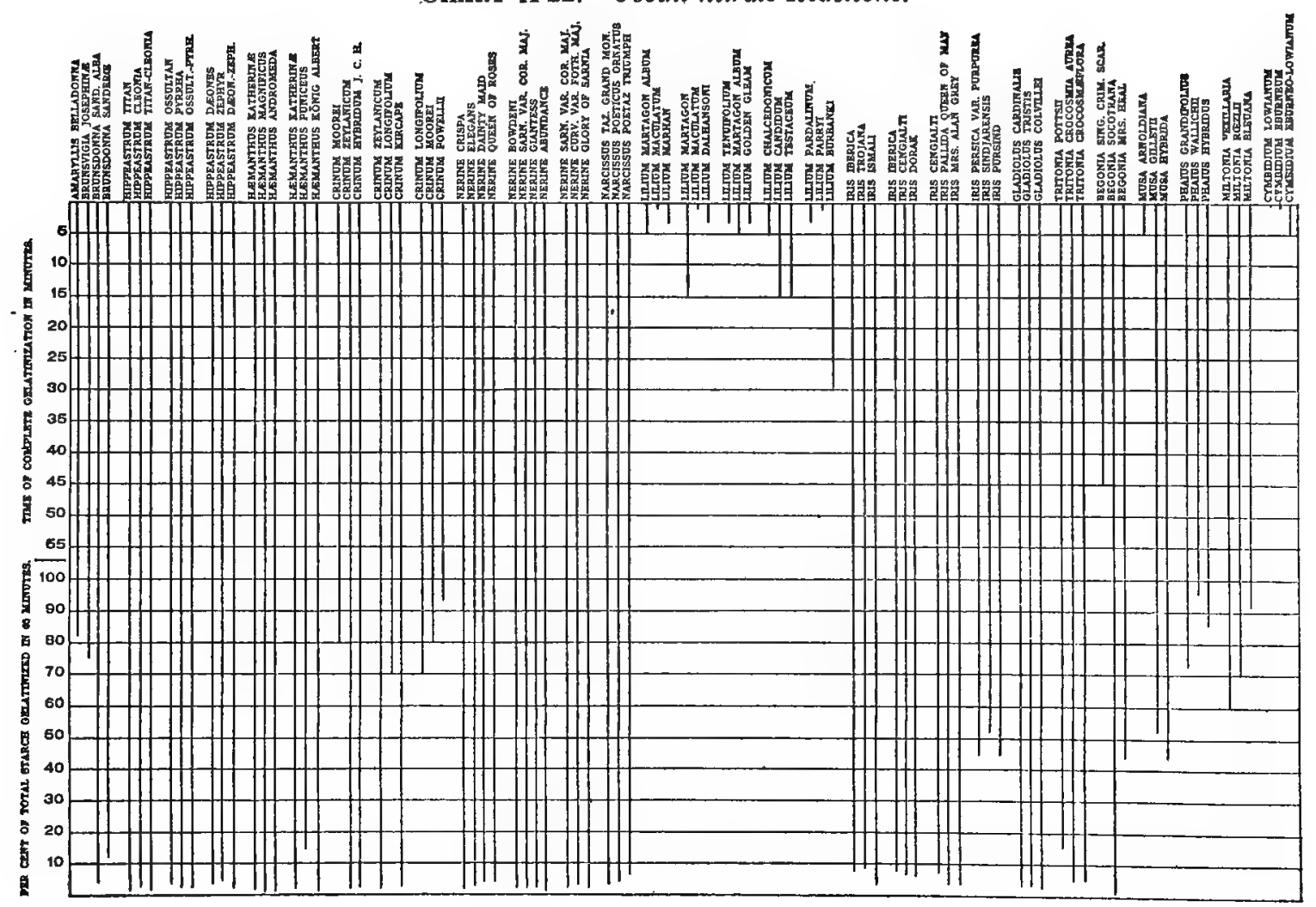


Chart A 23.-Copper-nitrate Reactions.

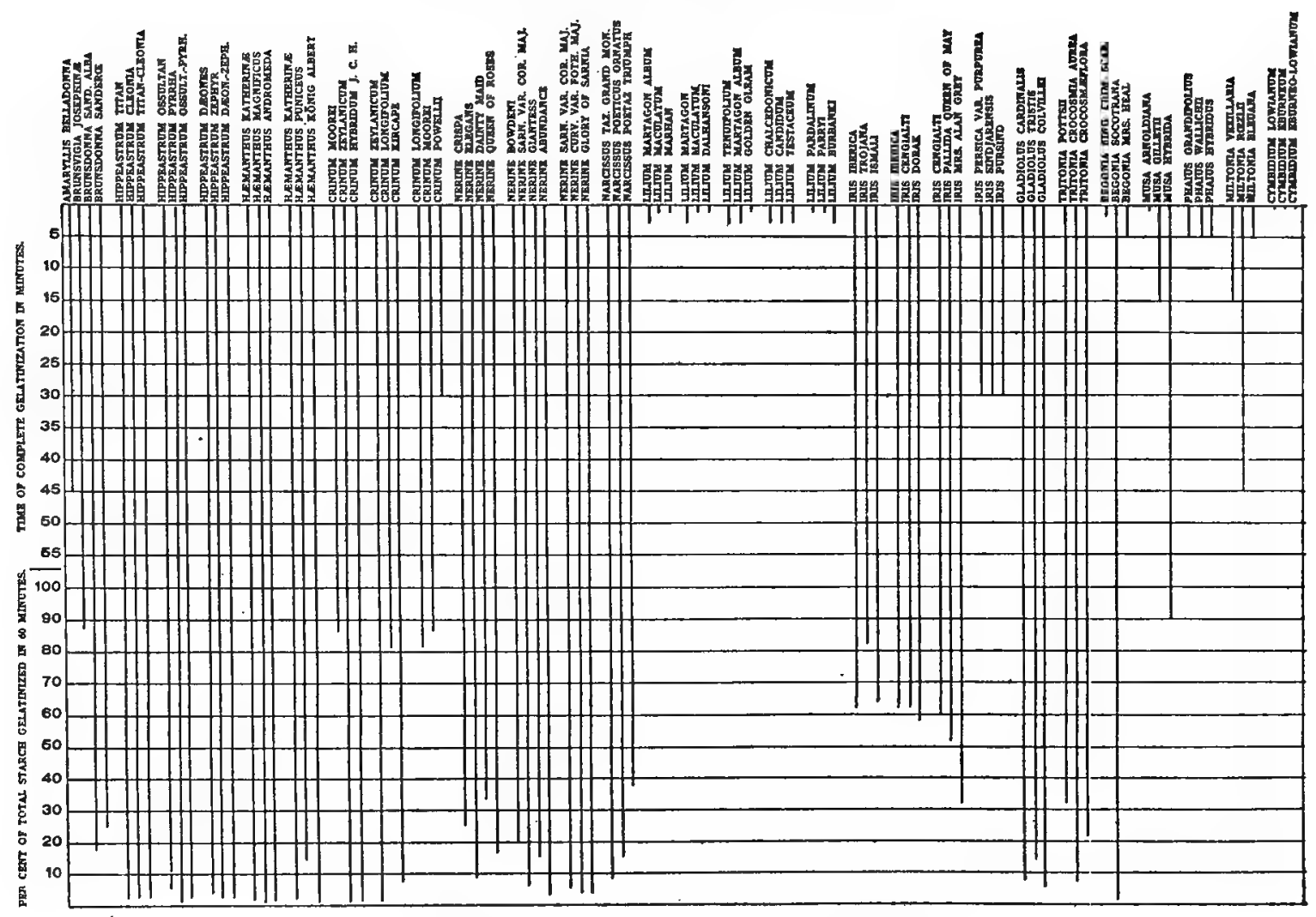

ChAR'T A 24.-Cupric-chloride Reactions.

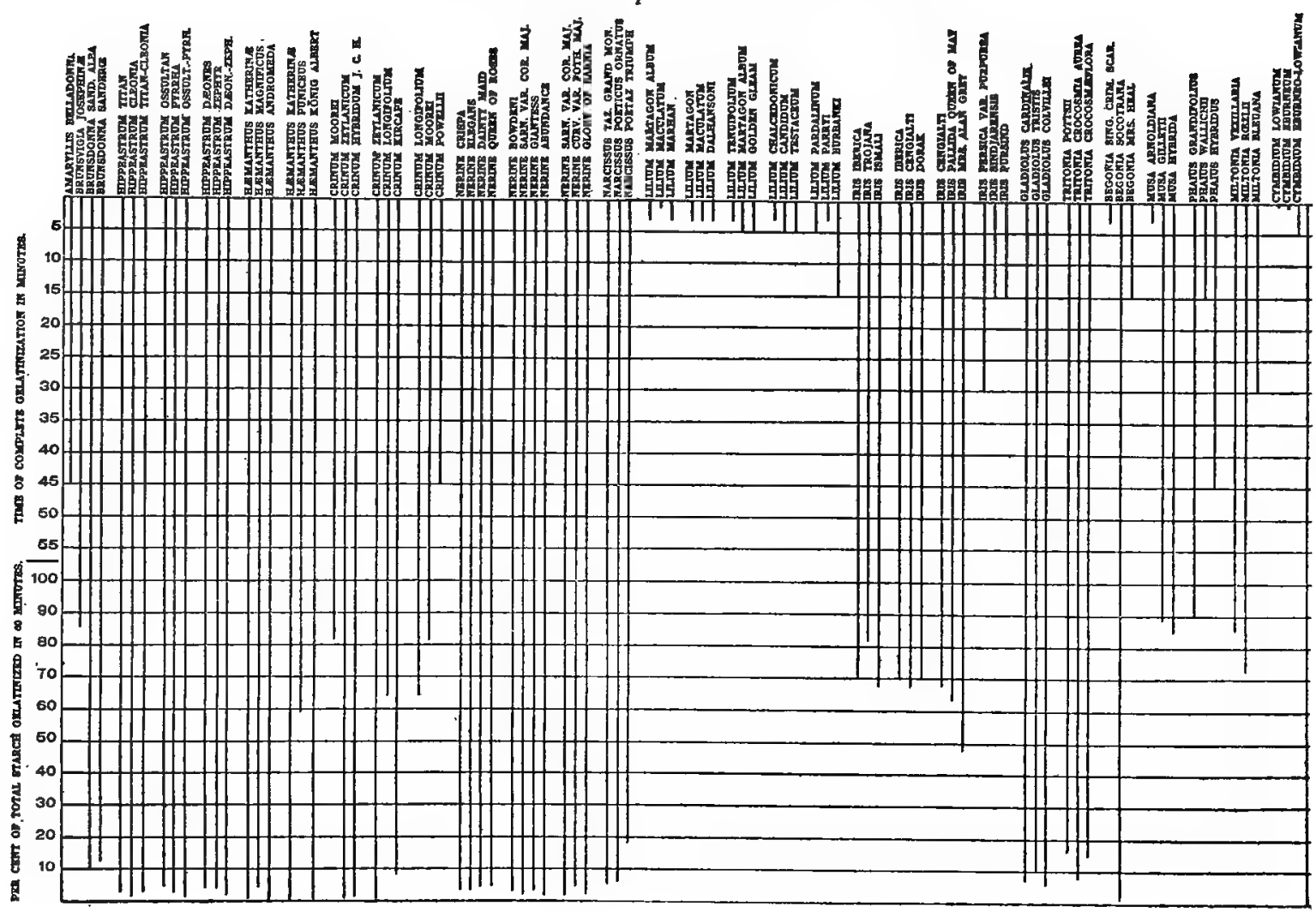


Chart A 25.-Barium-chloride Reactions.

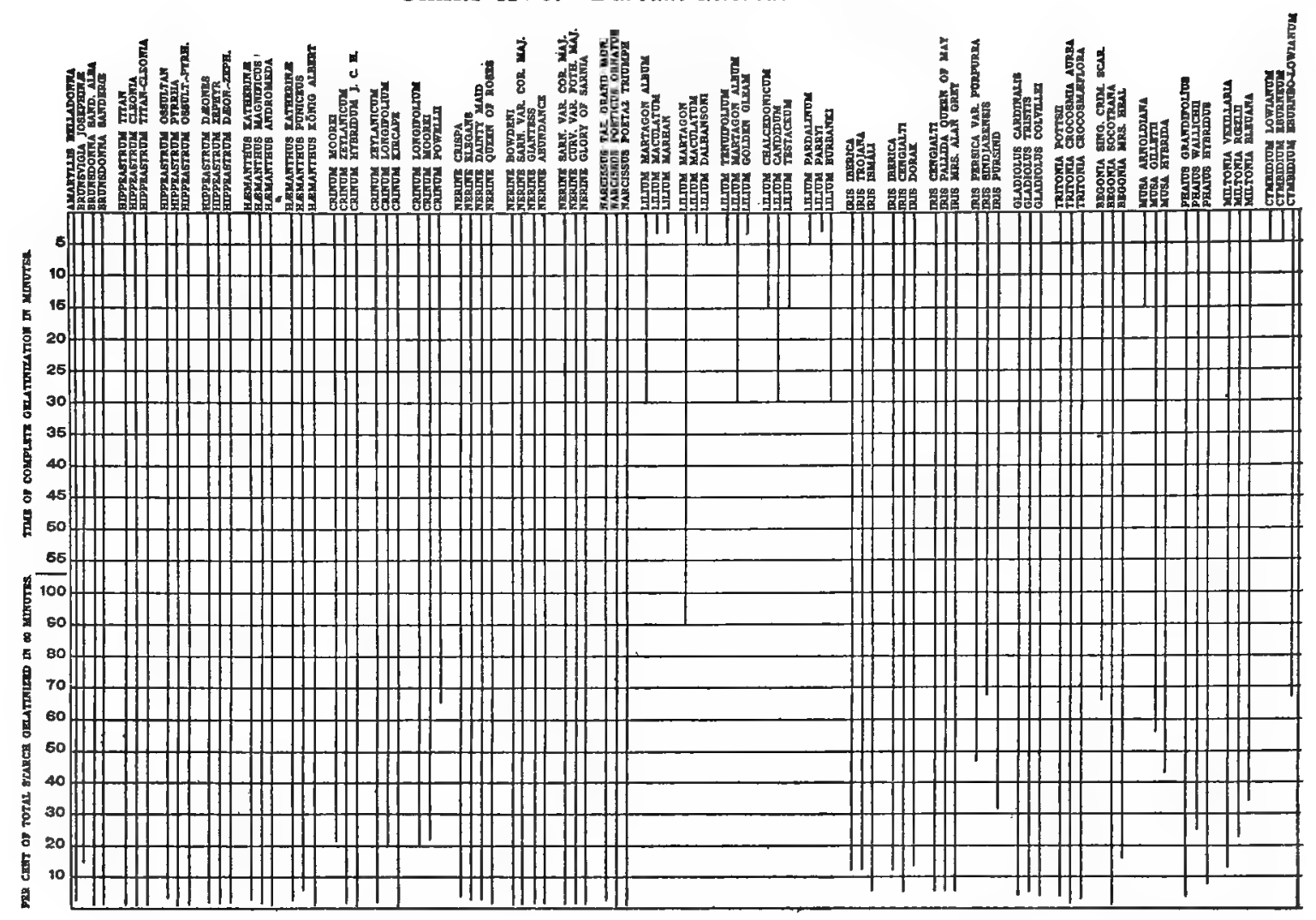

Chart A 26.-Mercuric-chloride Reactions.

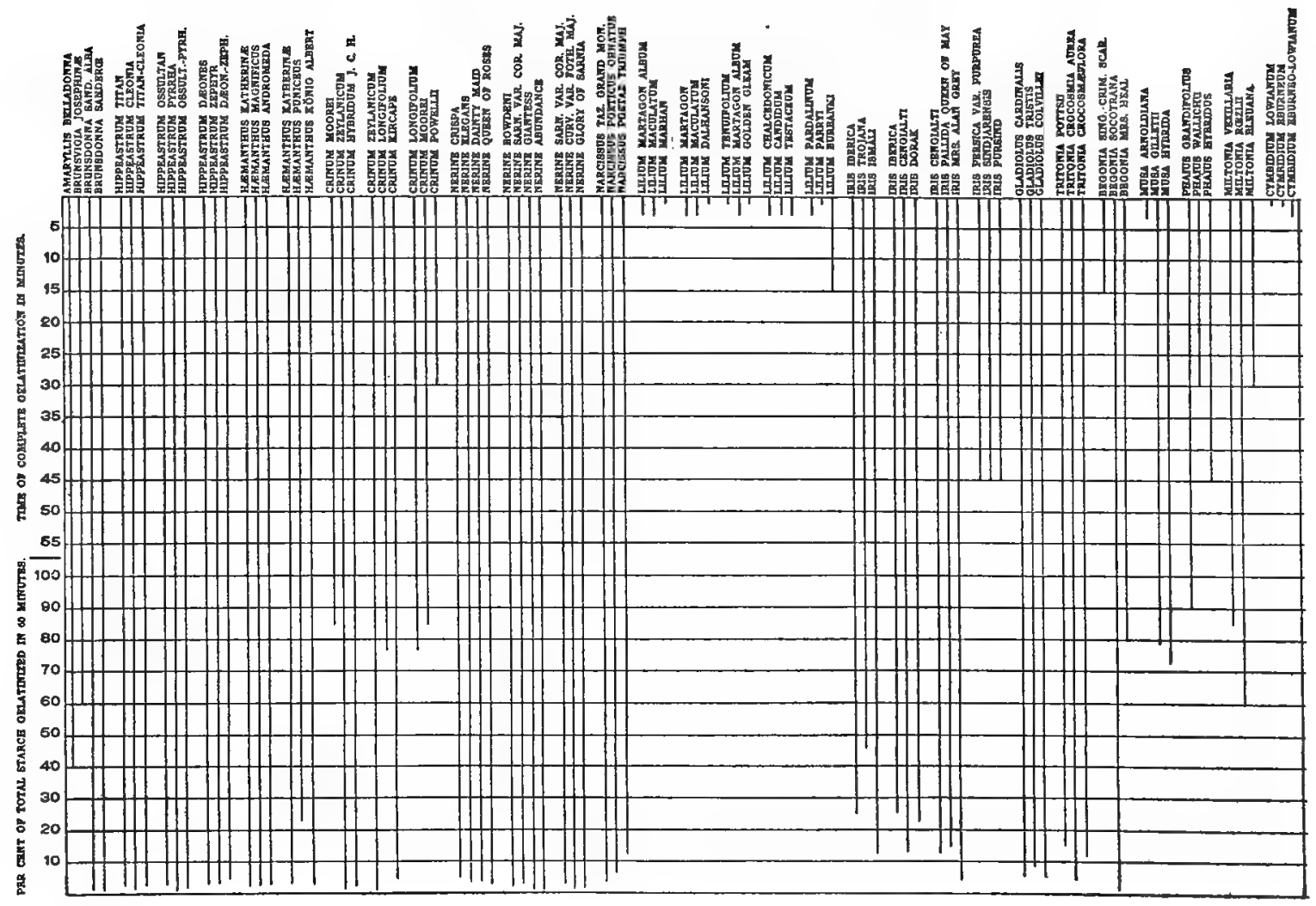


ChART B 1.-Polarization (-), Iodine (-), Gentian-violet (....), Safranin $(\ldots .$.$) , and Temperature$ $($ (......) Reactions.

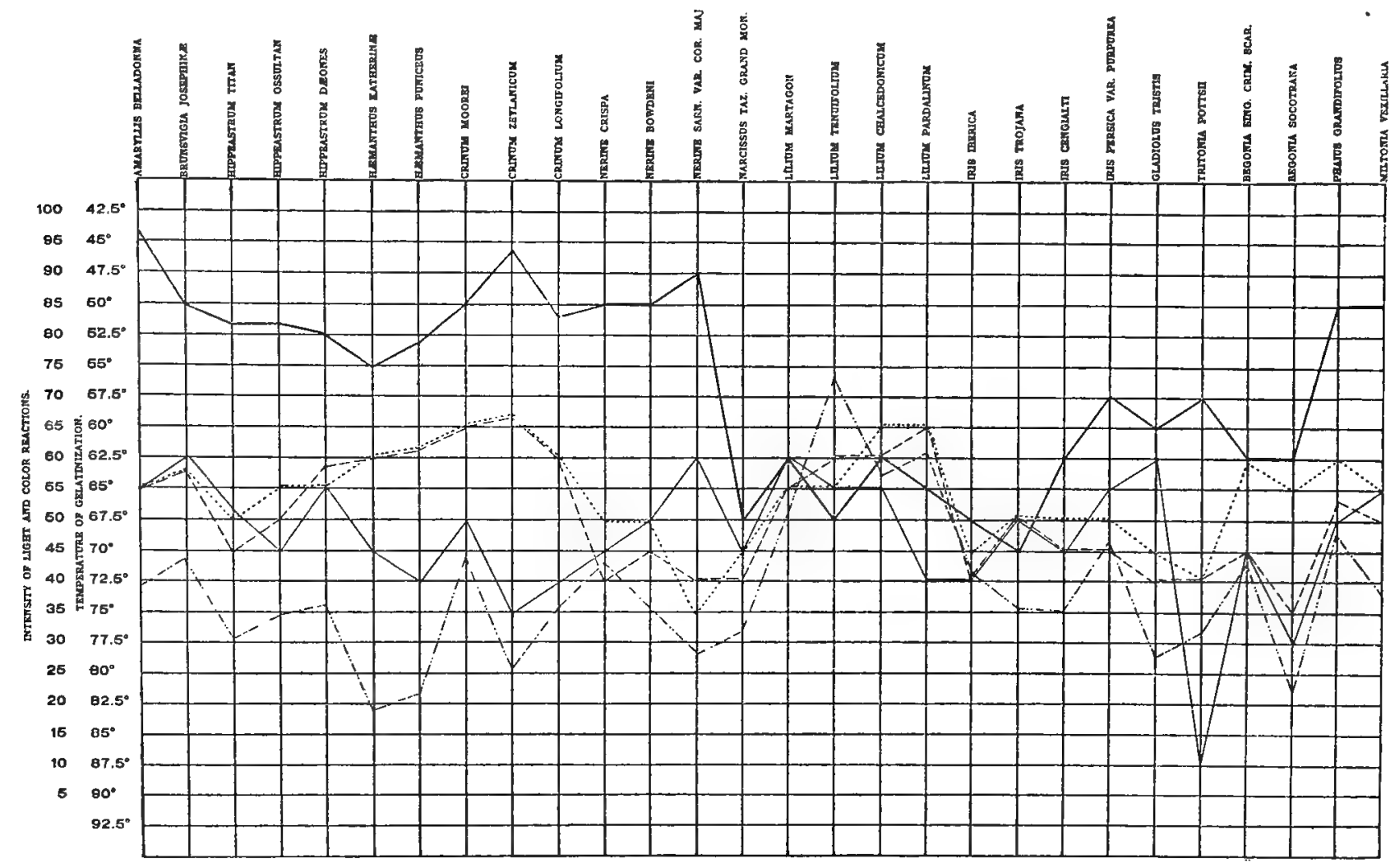

Chart B 2.-Gentian-violet $(\longrightarrow)$ and Safranin $(\longrightarrow$ Reactions.

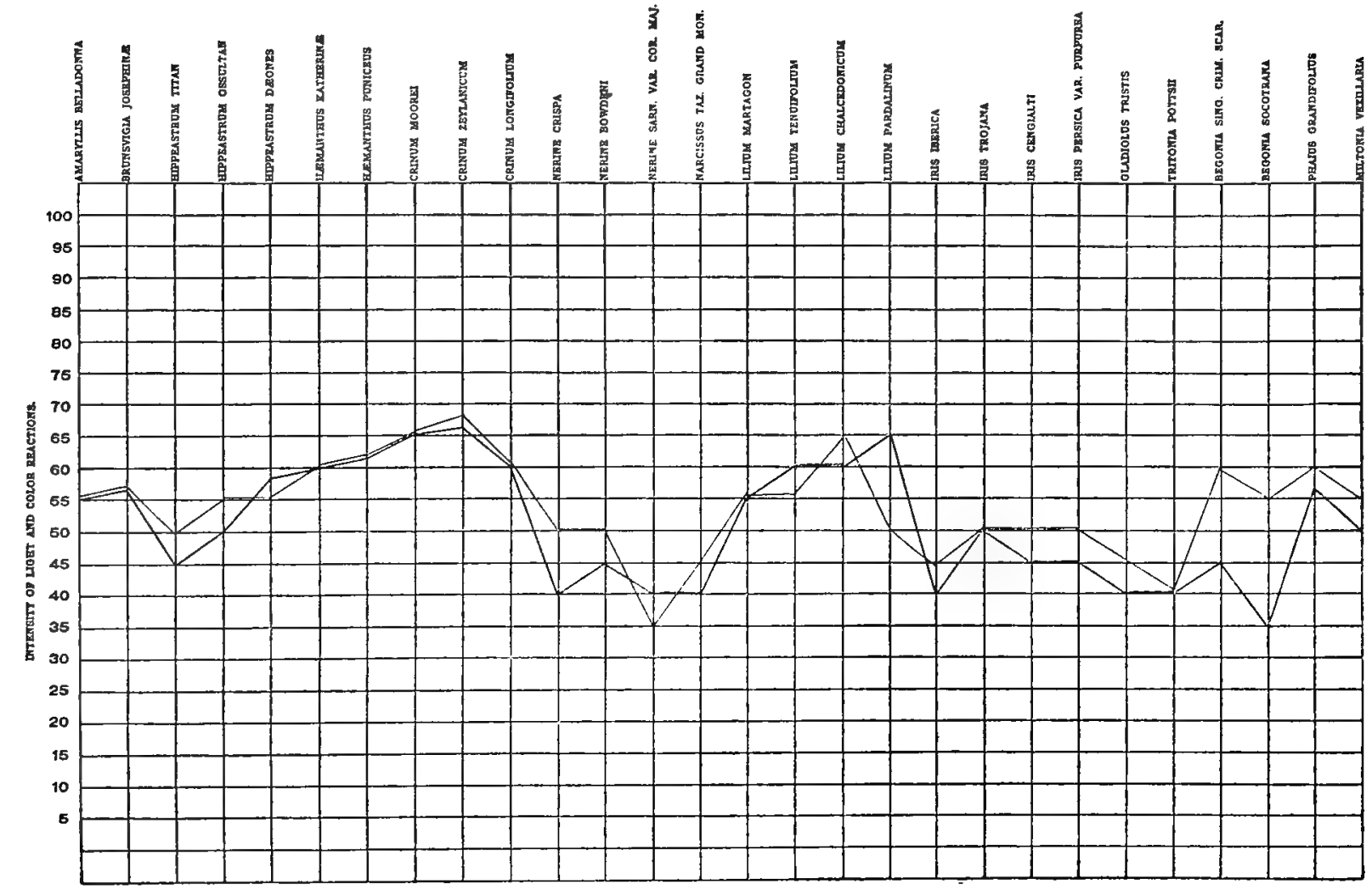


Chart B 3.-Temperature $(-$ ) and Iodine $(-$ Reactions.

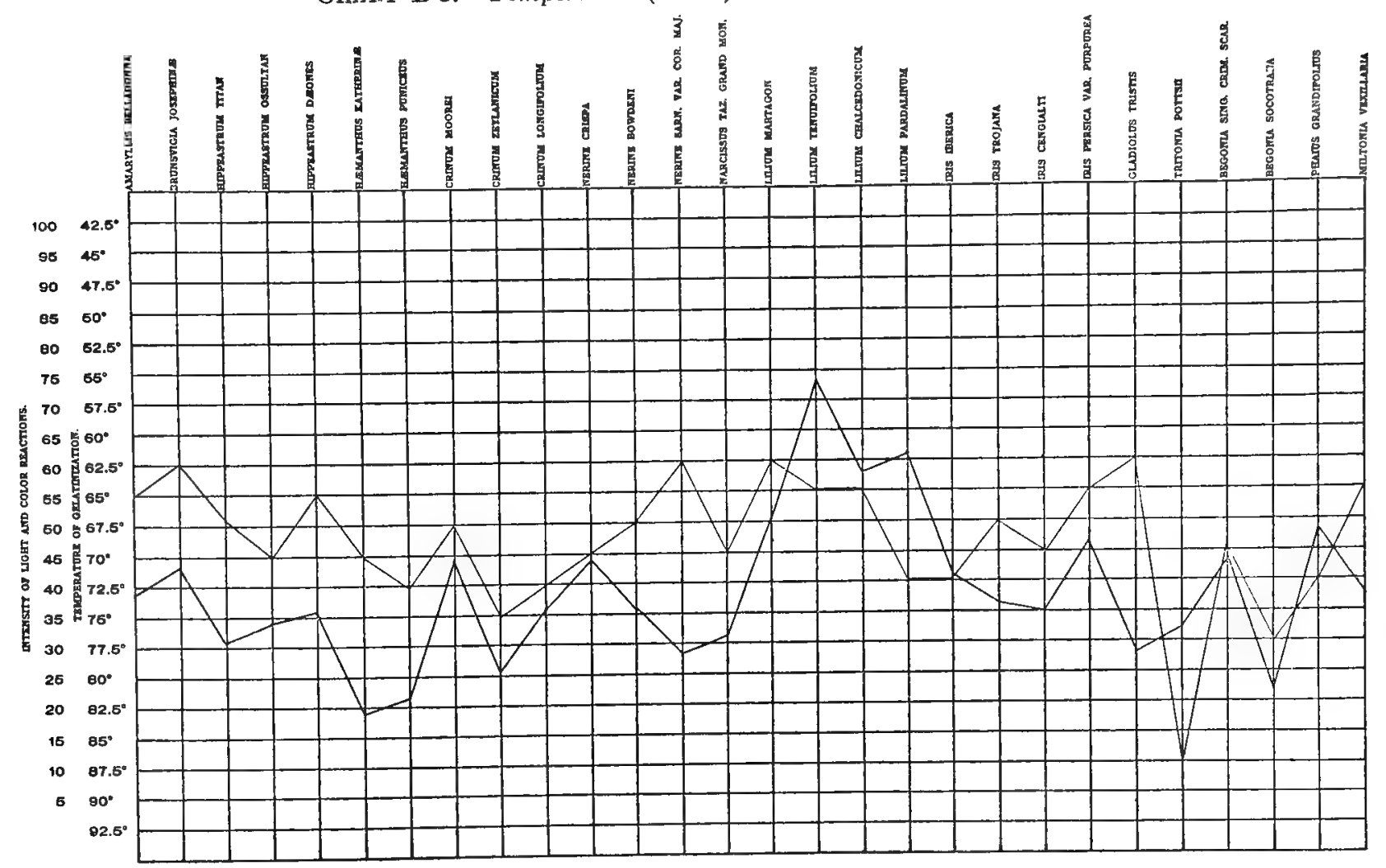

CHART B 4.-Temperature (-) and Chloral-hydrate $(-)$ Reactions.

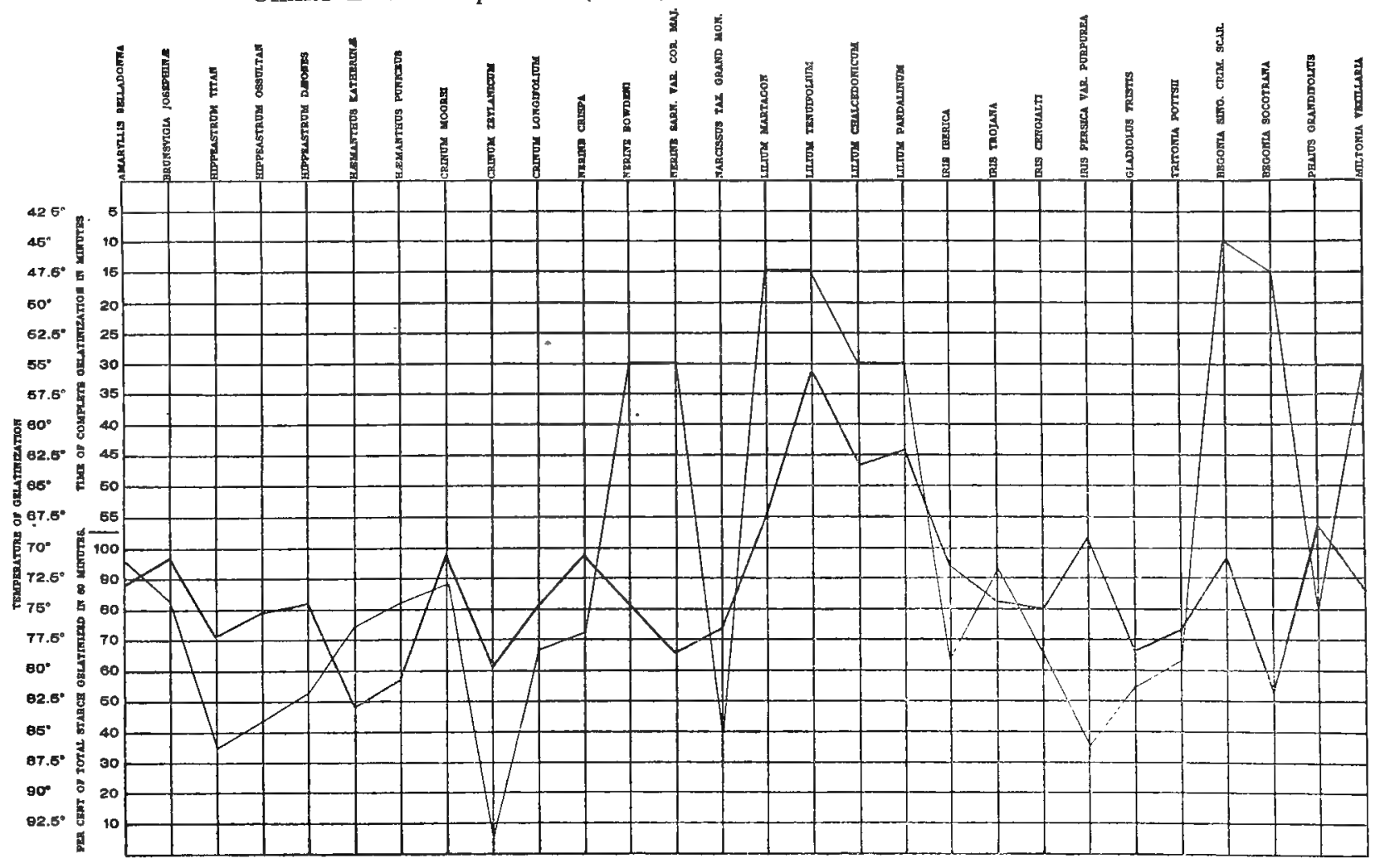


Chart B 5.-Temperature (-) and Pyrogallic-acid $(-)$ Reactions.

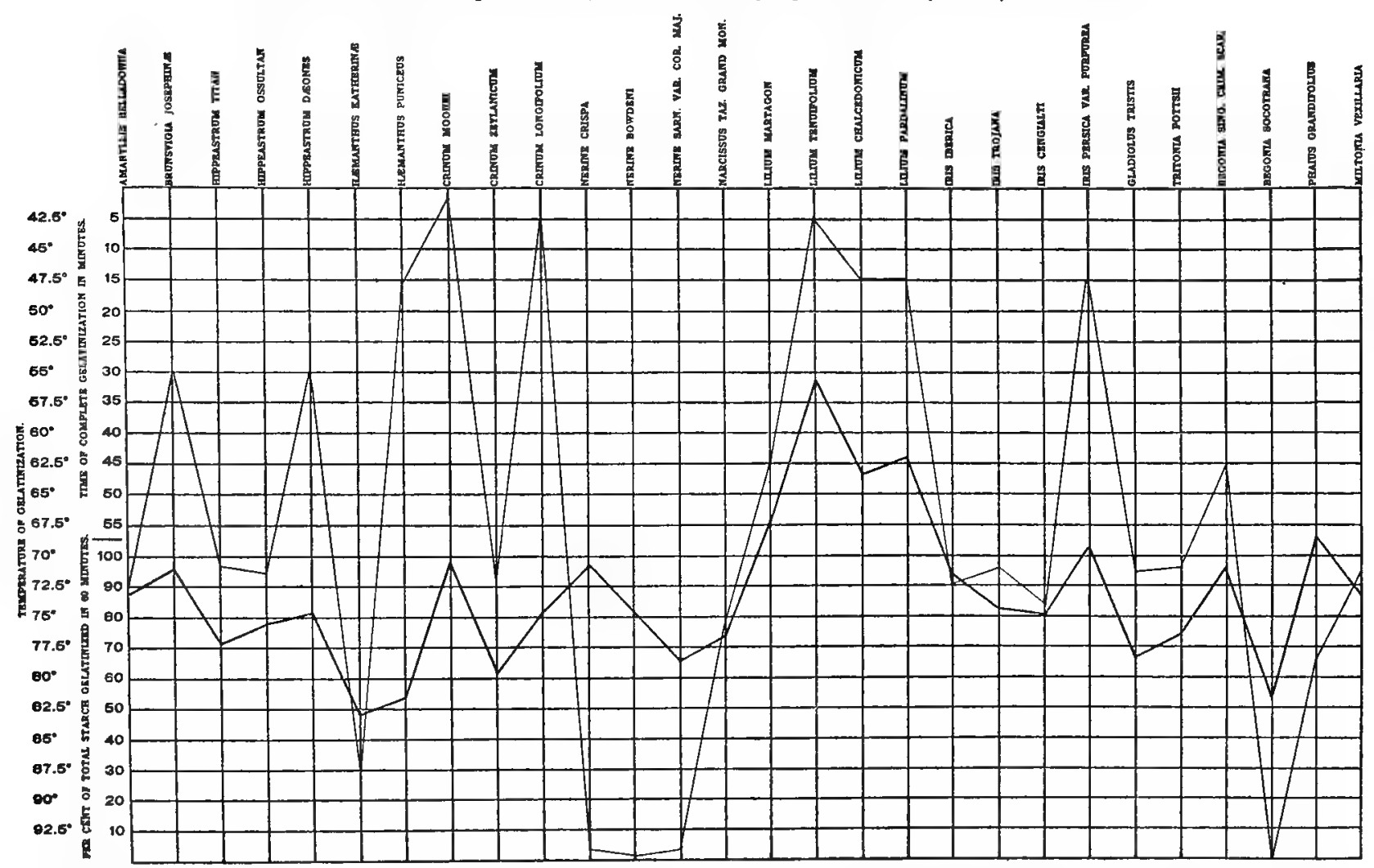

ChART B 6.-Temperature (standard ——and new --.- calibrations) and Nitric-acid $(\longrightarrow$ Reactions.

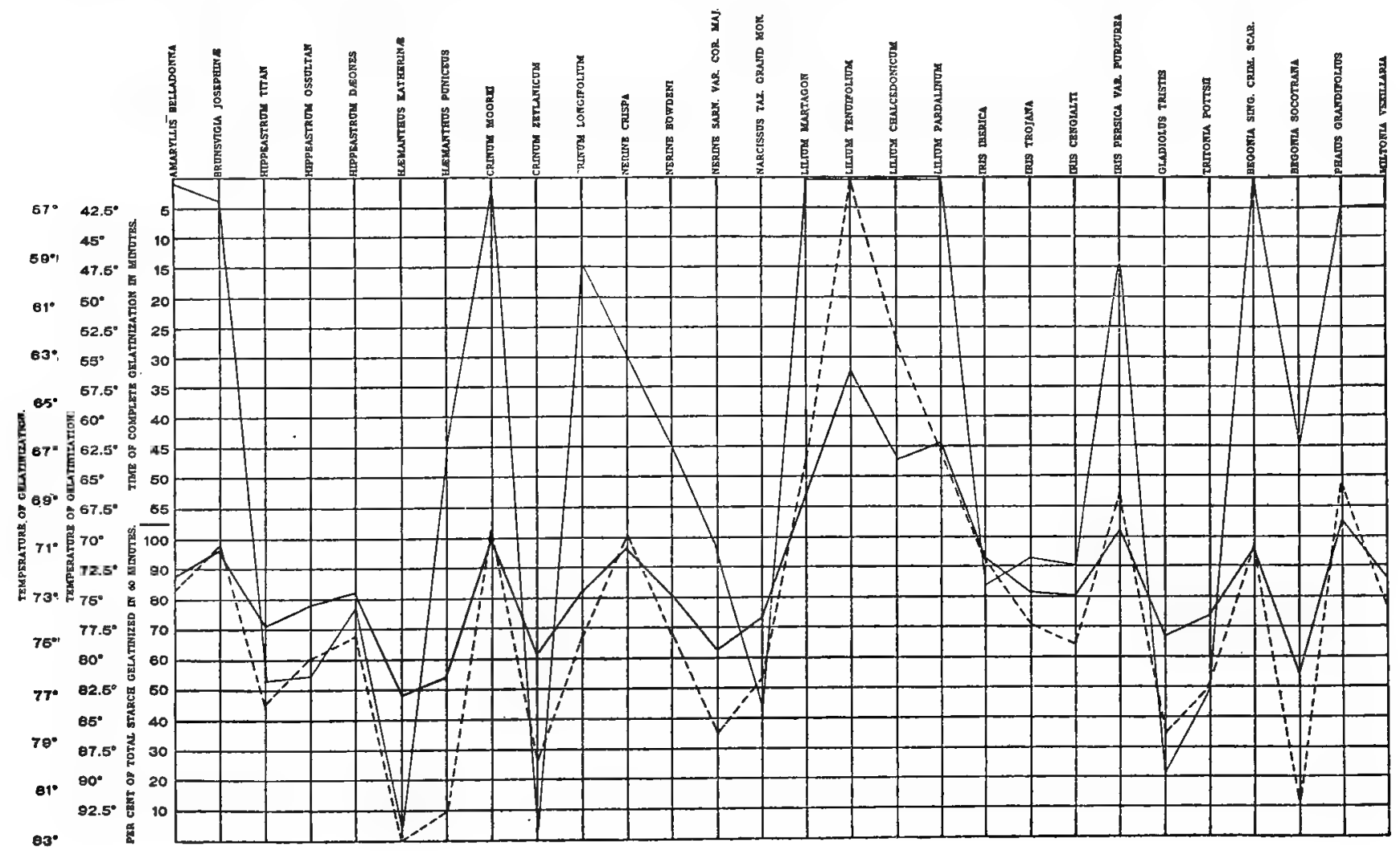


Chart B 7.-Nitric-acid $(\longrightarrow$ ) and Polarization $(\longrightarrow)$ Reactions.

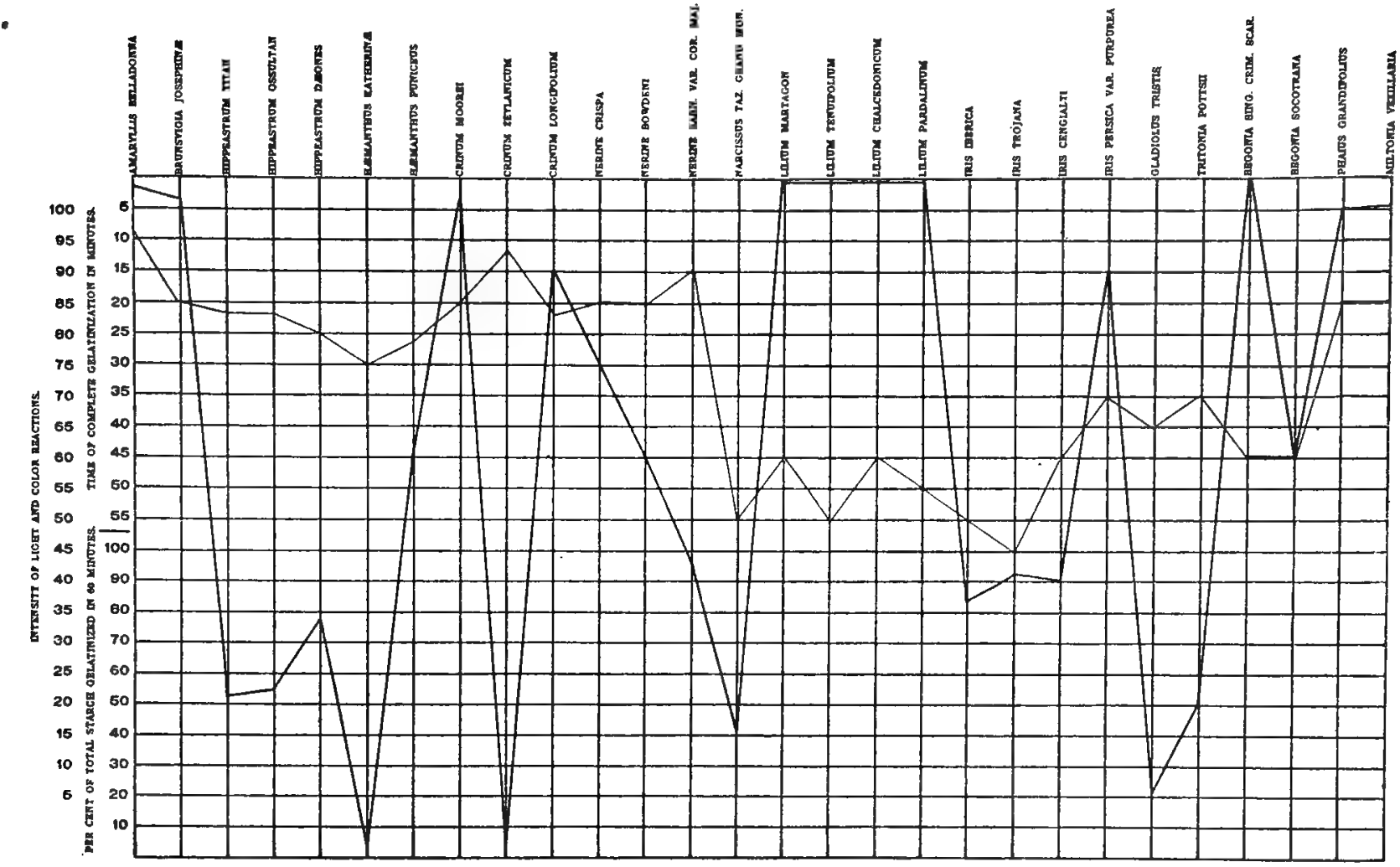

Chart B 8.-Nitric-acid (—) and Iodine (standard - and new -.... calibrations) Reactions.

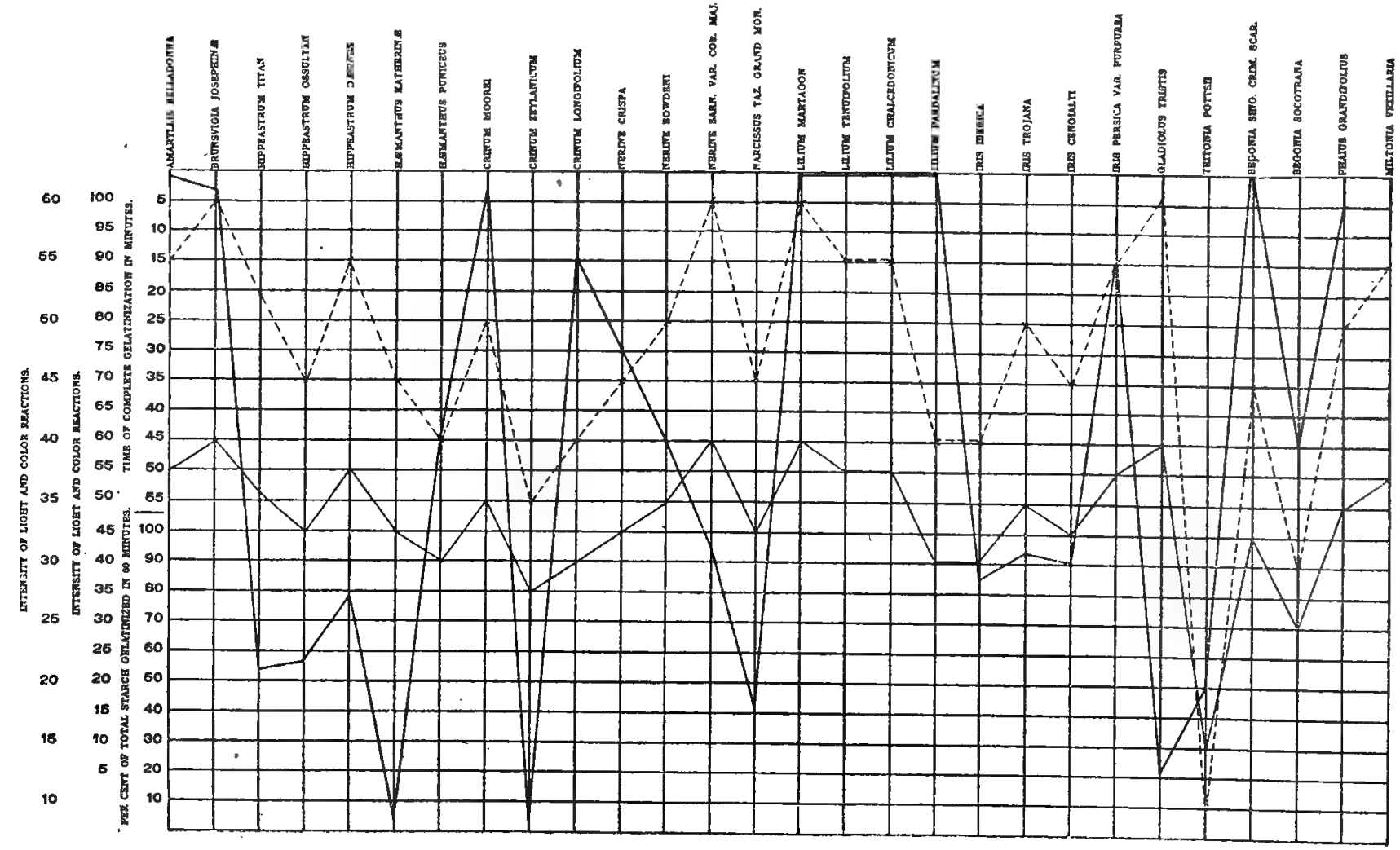


Chart B 9.-Nitric-acid (-) and Gentian-violet $(\longrightarrow)$ Reactions.

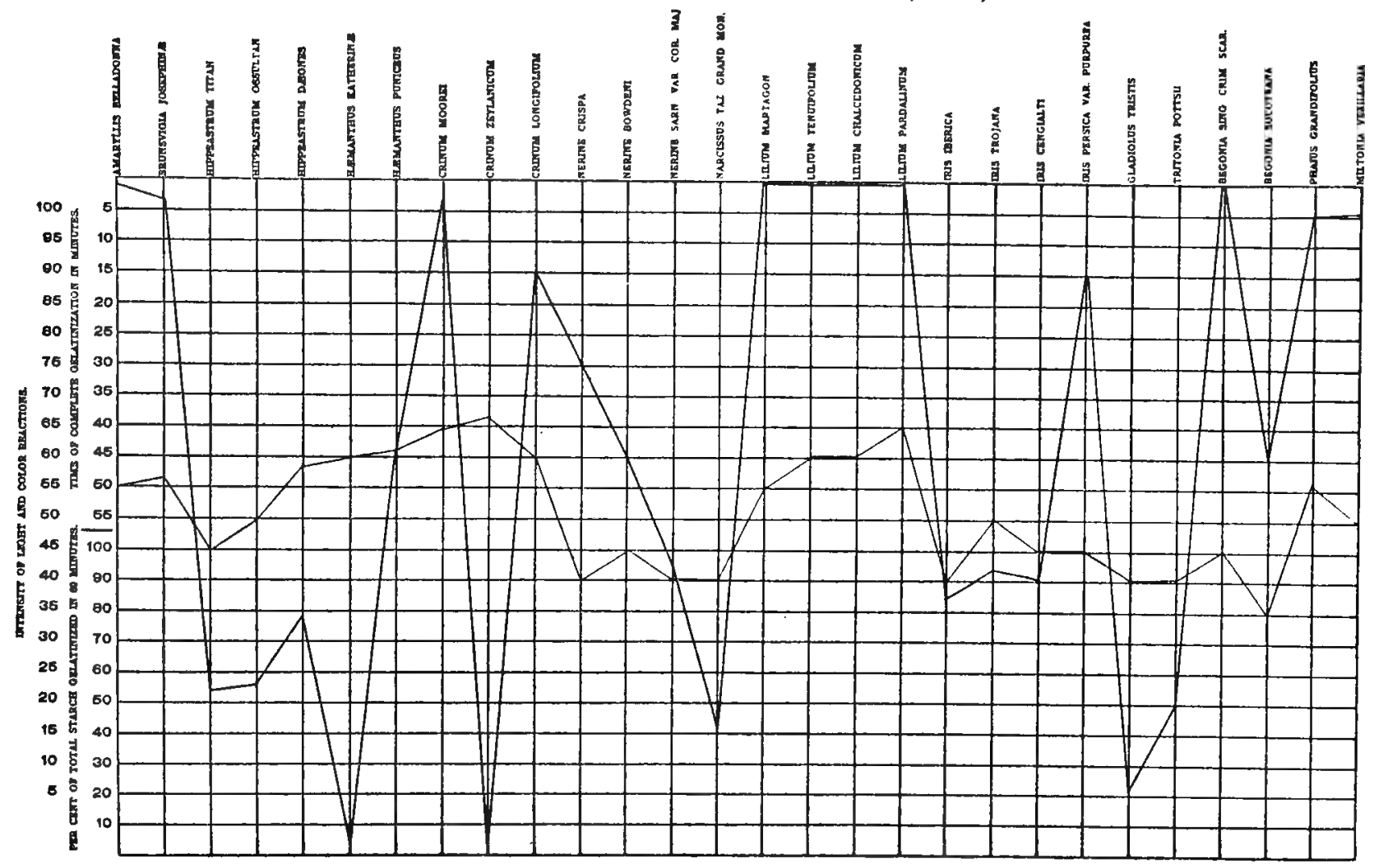

Chart B 10.-Nitric-acid (-) and Safranin (-) Reactions.
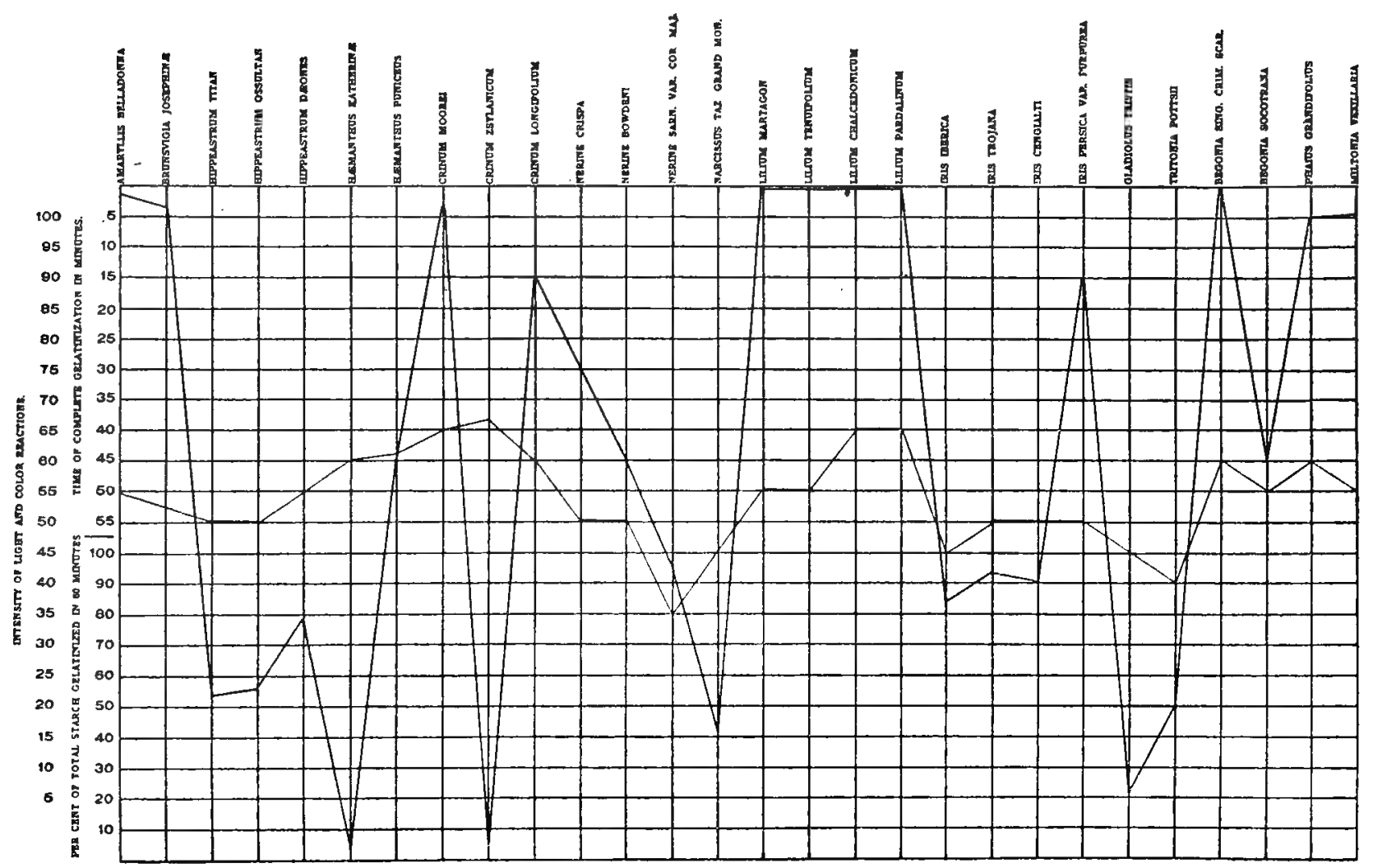
Chart B 11.-Nitric-acid $(\longrightarrow$ ) and Chloral-hydrate $(\longrightarrow$ Reactions.

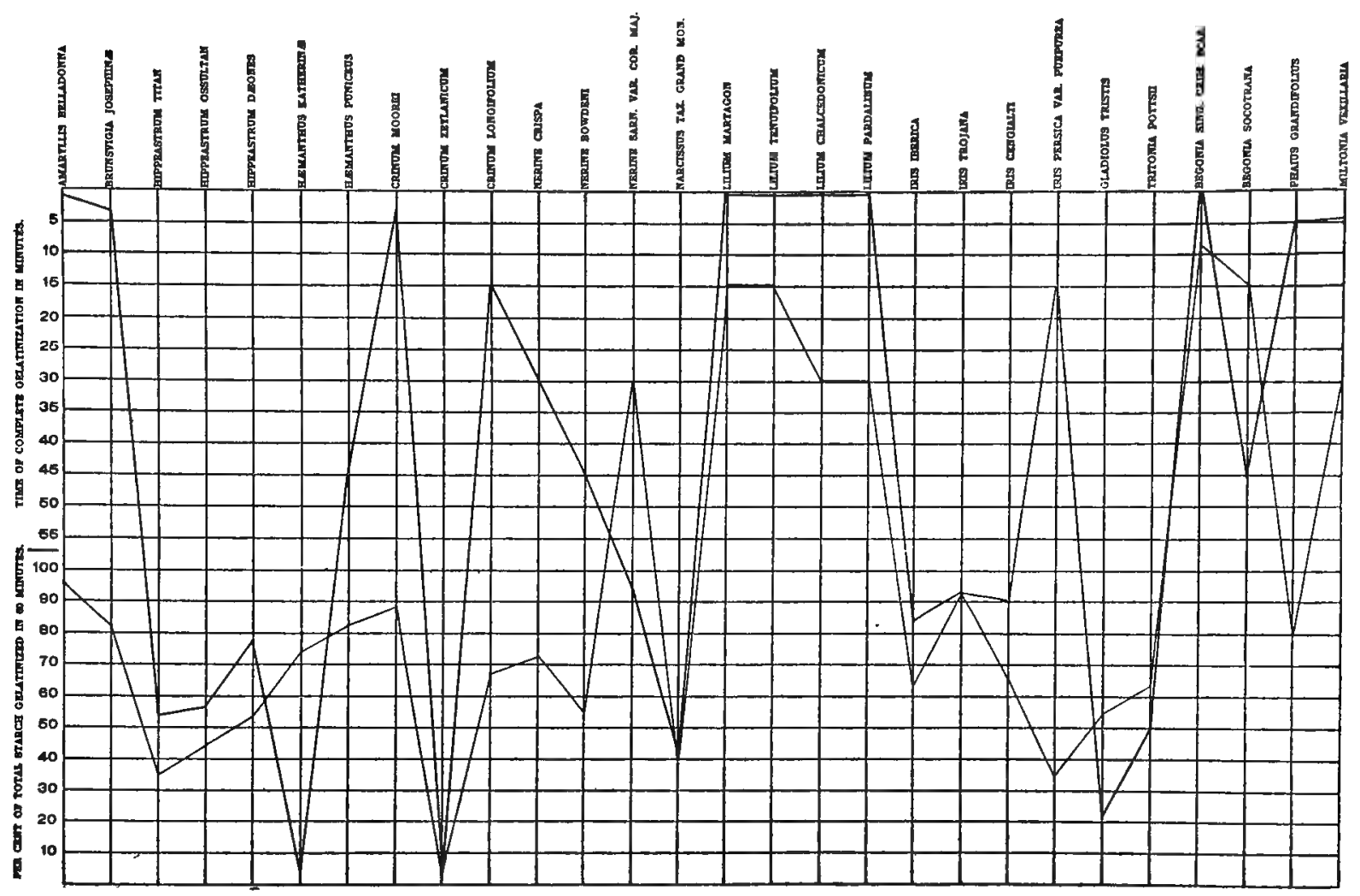

Chart B 12.-Nitric-acid $(\longrightarrow)$ and Chromic-acid $(\longrightarrow$ Reactions.

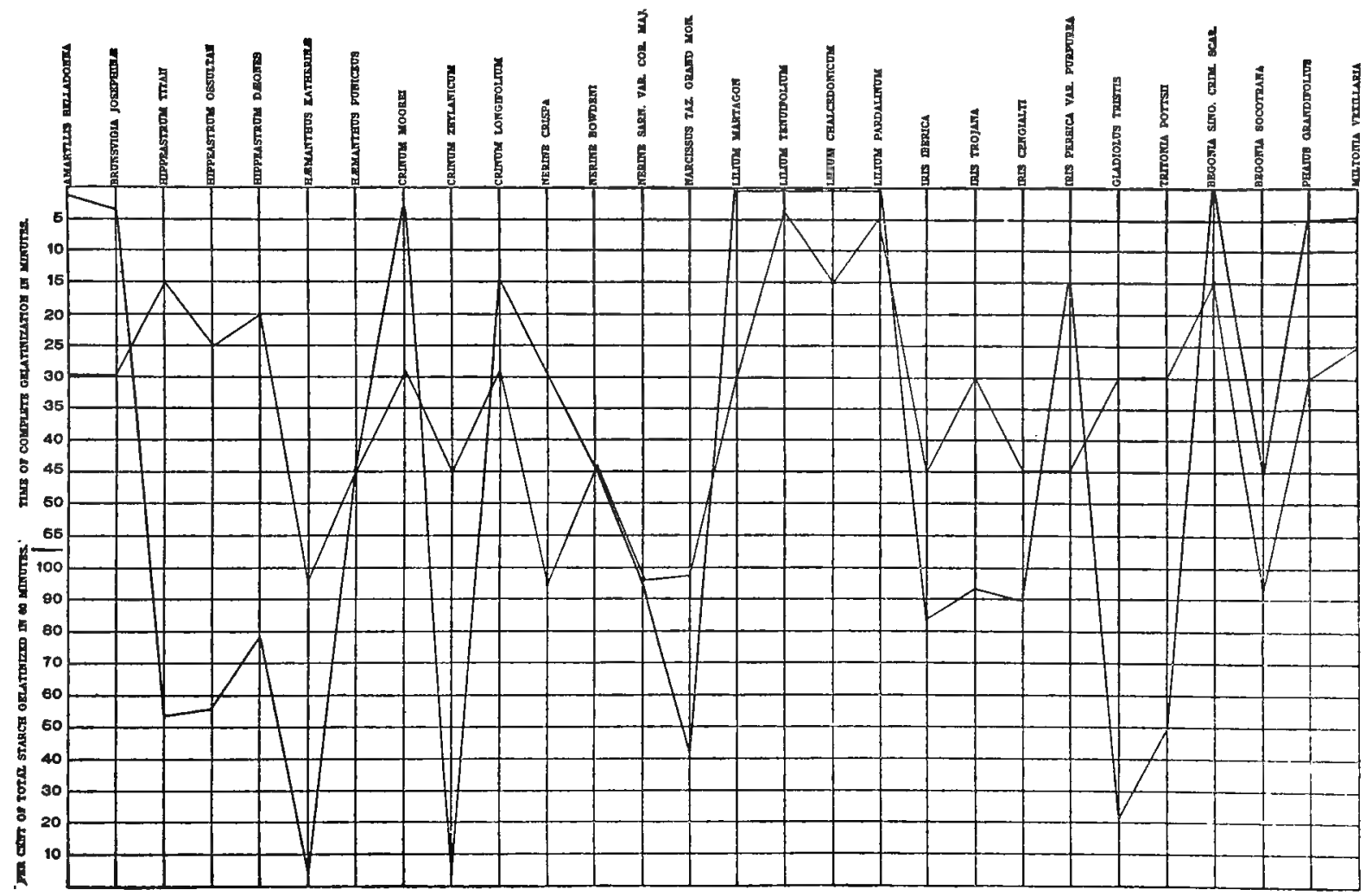


Chart B 13.-Nitric-acid (-) and Pyrogallic-acid $(\longrightarrow$ Reactions.

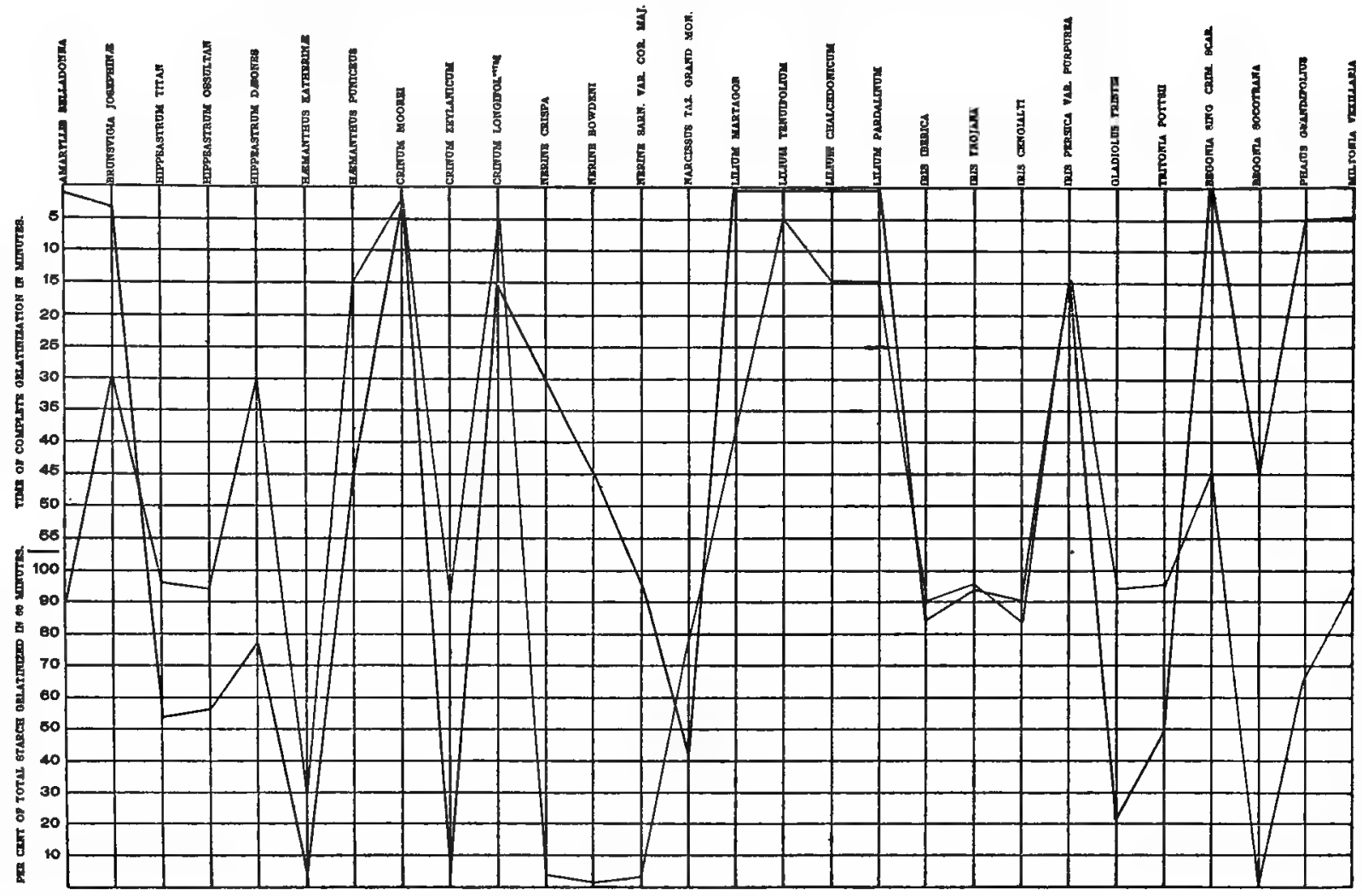

CHART B 14.-Nitric-acid (-) and Sulphuric-acid (-) Reactions.

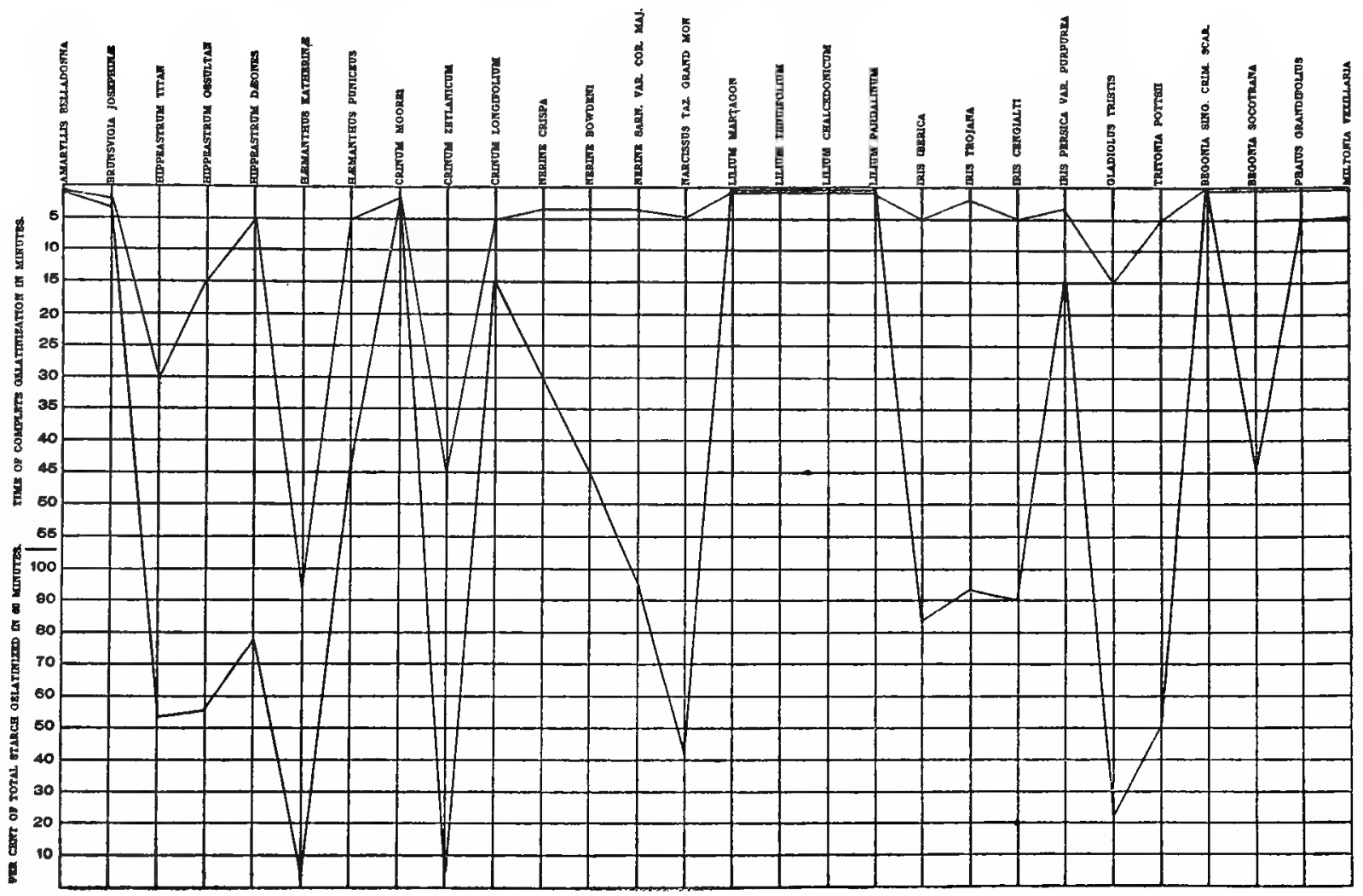


Chart B 15.-Nitric-acid (-) and Hydrochloric-acid $(-)$ Reactions.

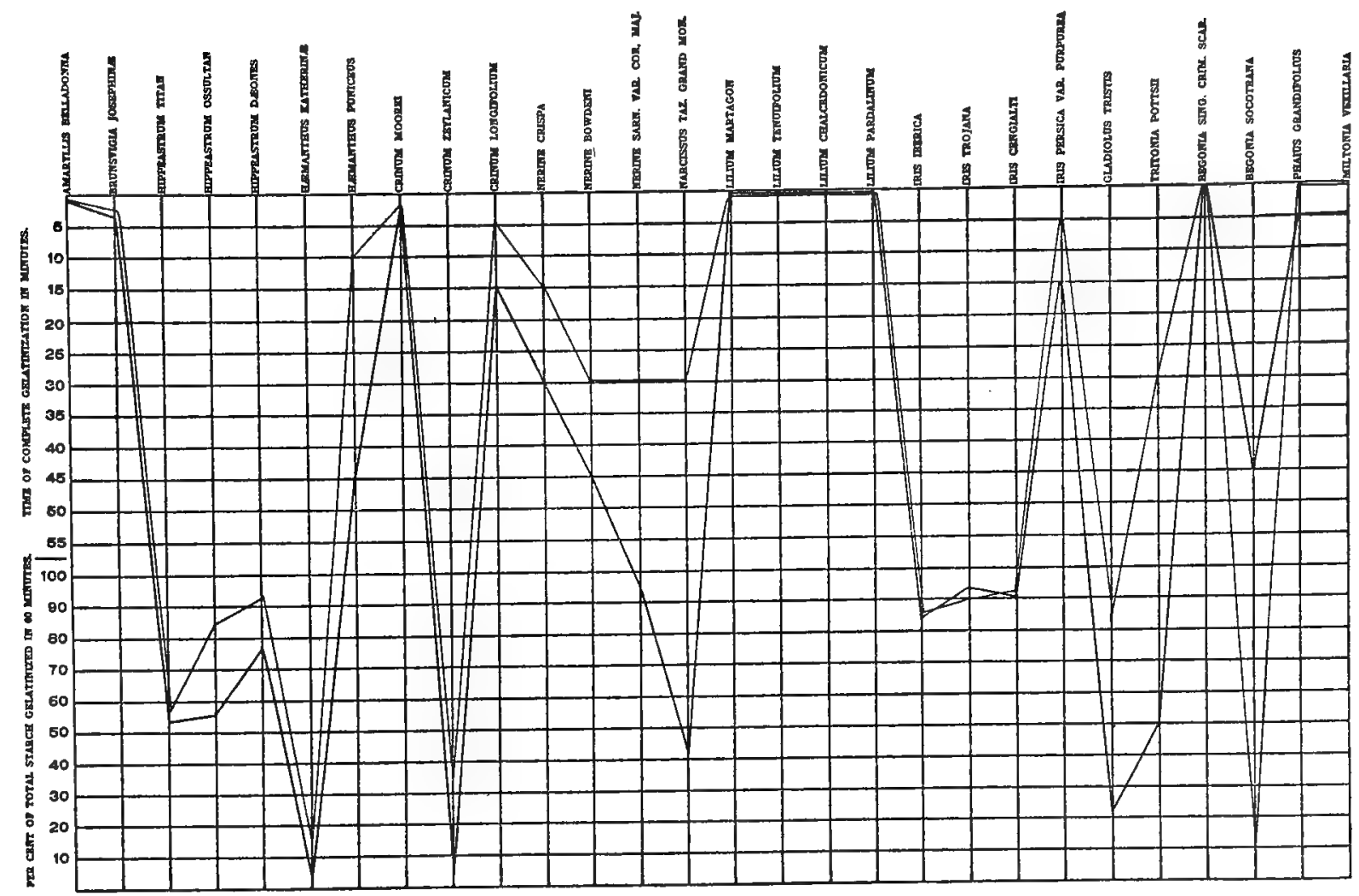

Chart B 16.-Nitric-acid (-) and Potassium-hydroxide $(-)$ Reactions.

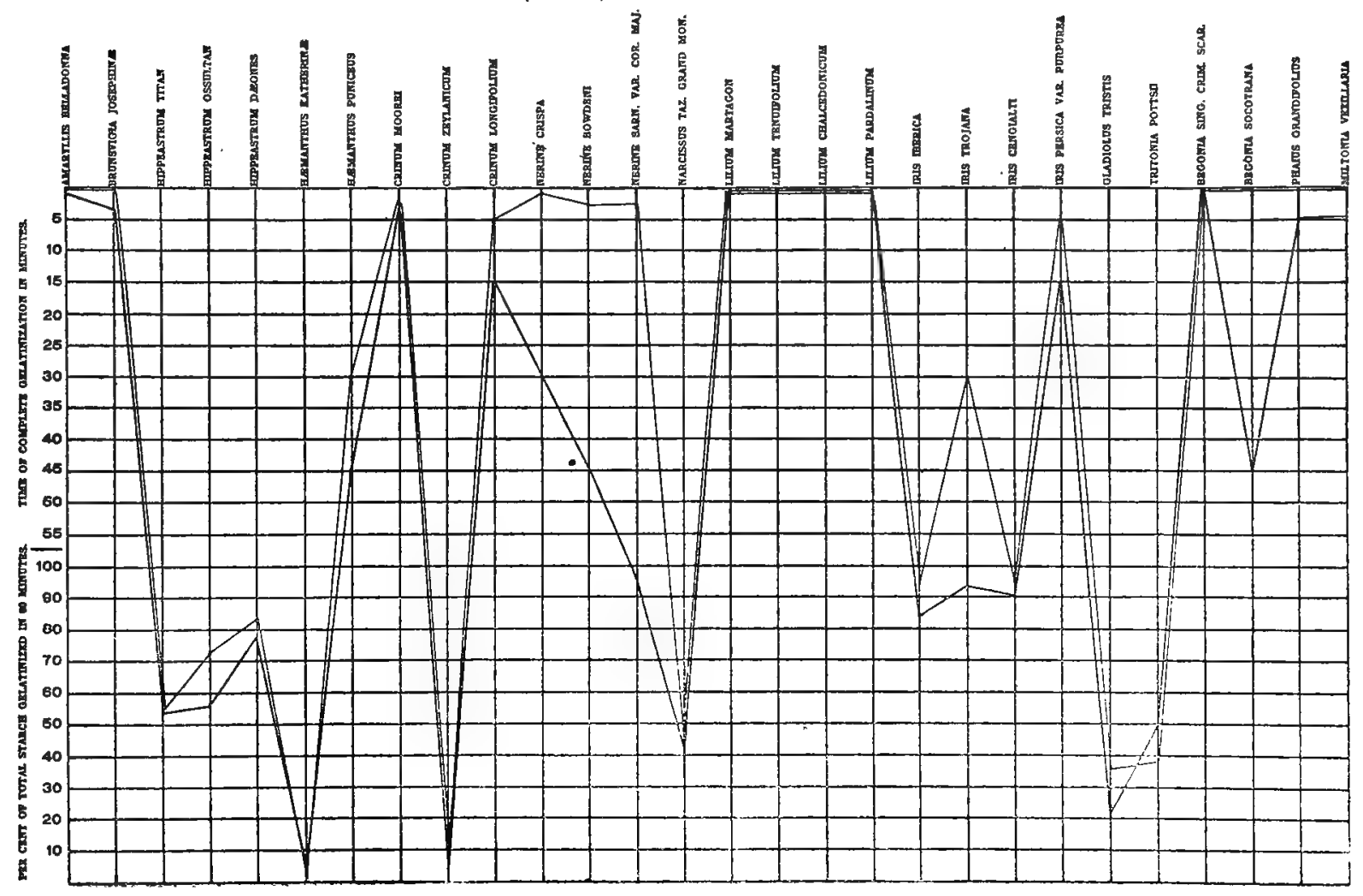


Chart B 17.-Nitric-acid (-) and Potassium-iodide $(-)$ Reactions.

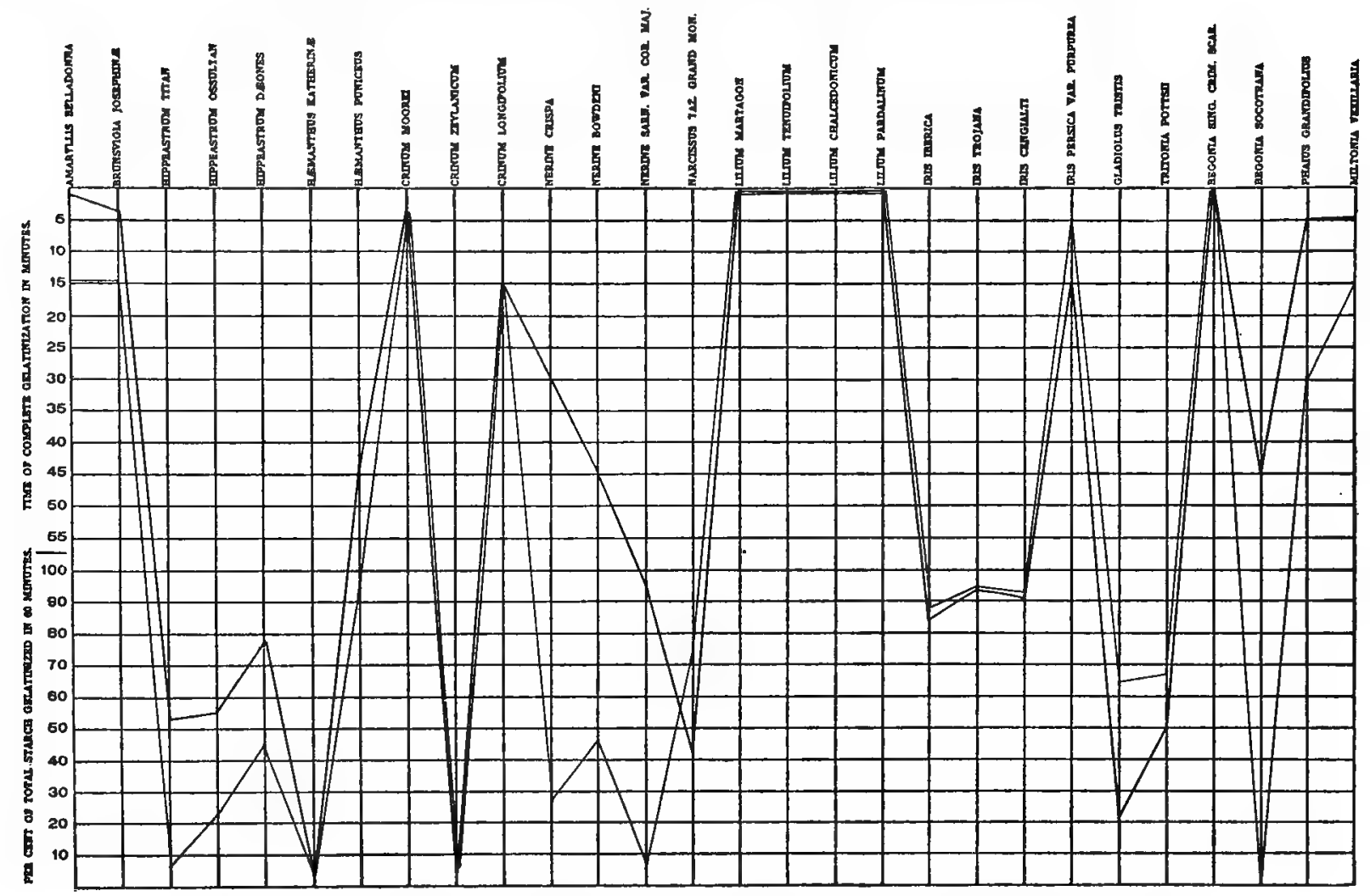

Chart B 18.-Nitric-acid $(\longrightarrow$ ) and Potassium-sulphocyanate $(\longrightarrow$ Reactions.

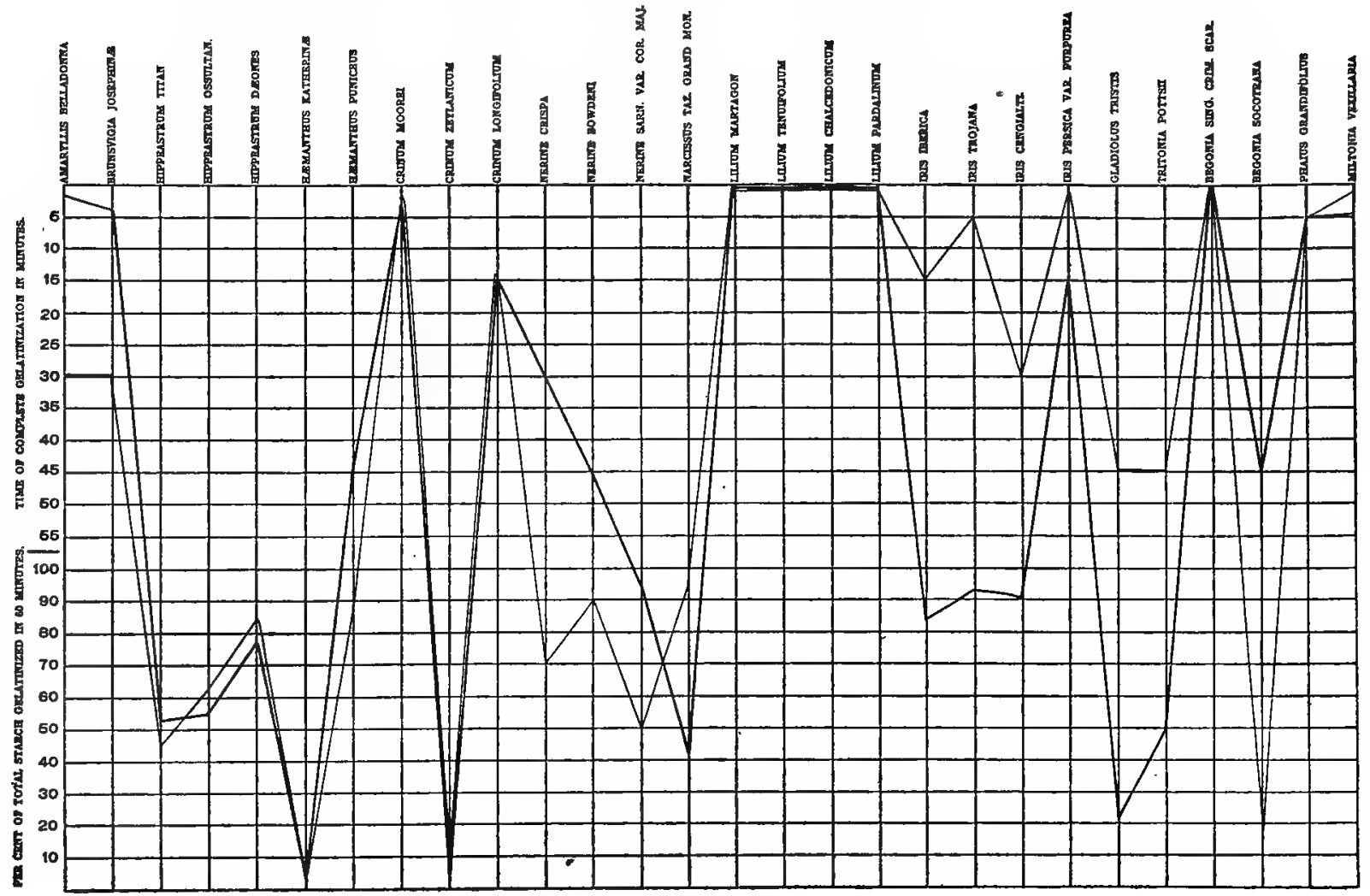


197

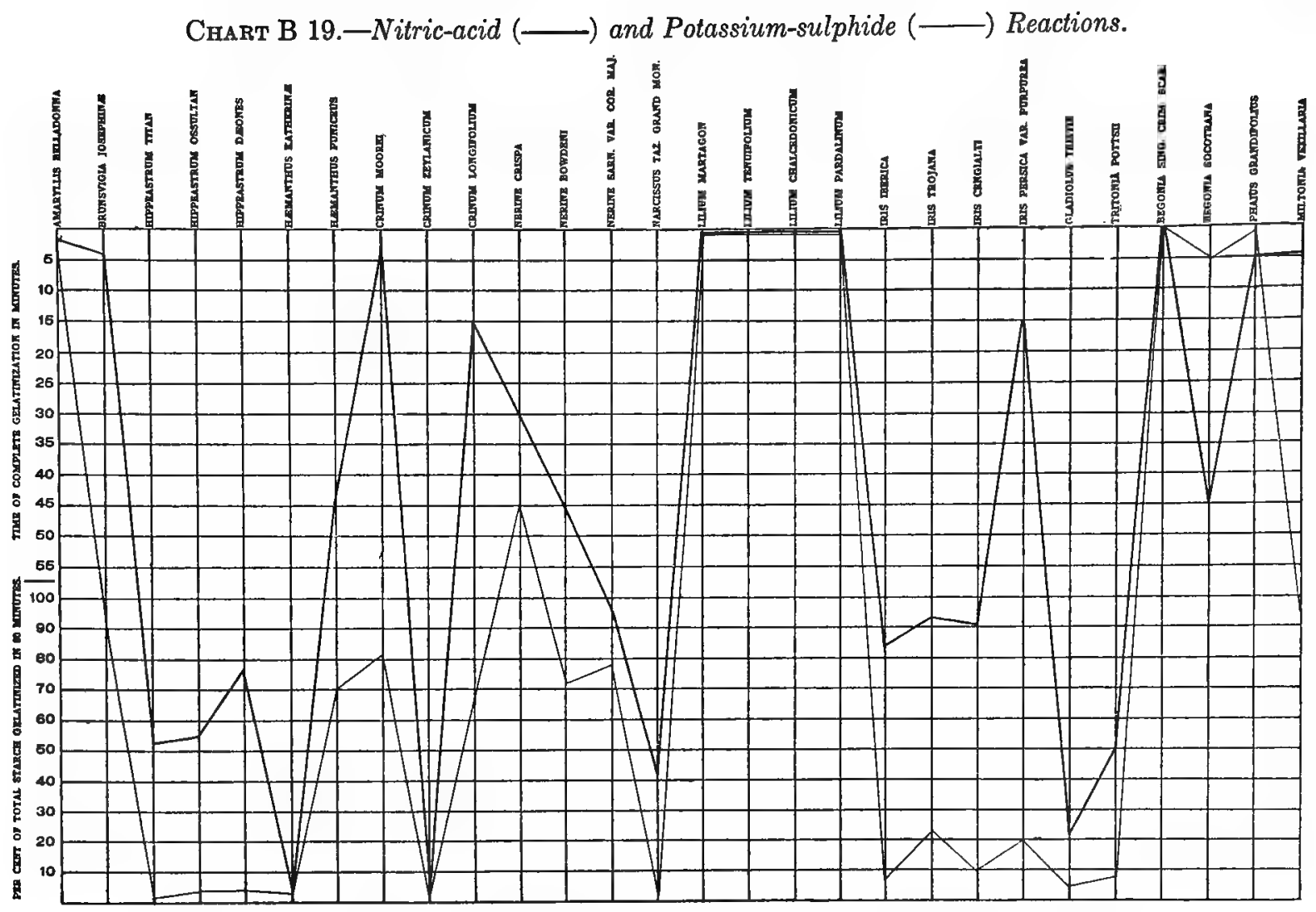

Chart B 20.-Nitric-acid (-) and Sodium-hydroxide (-) Reactions.

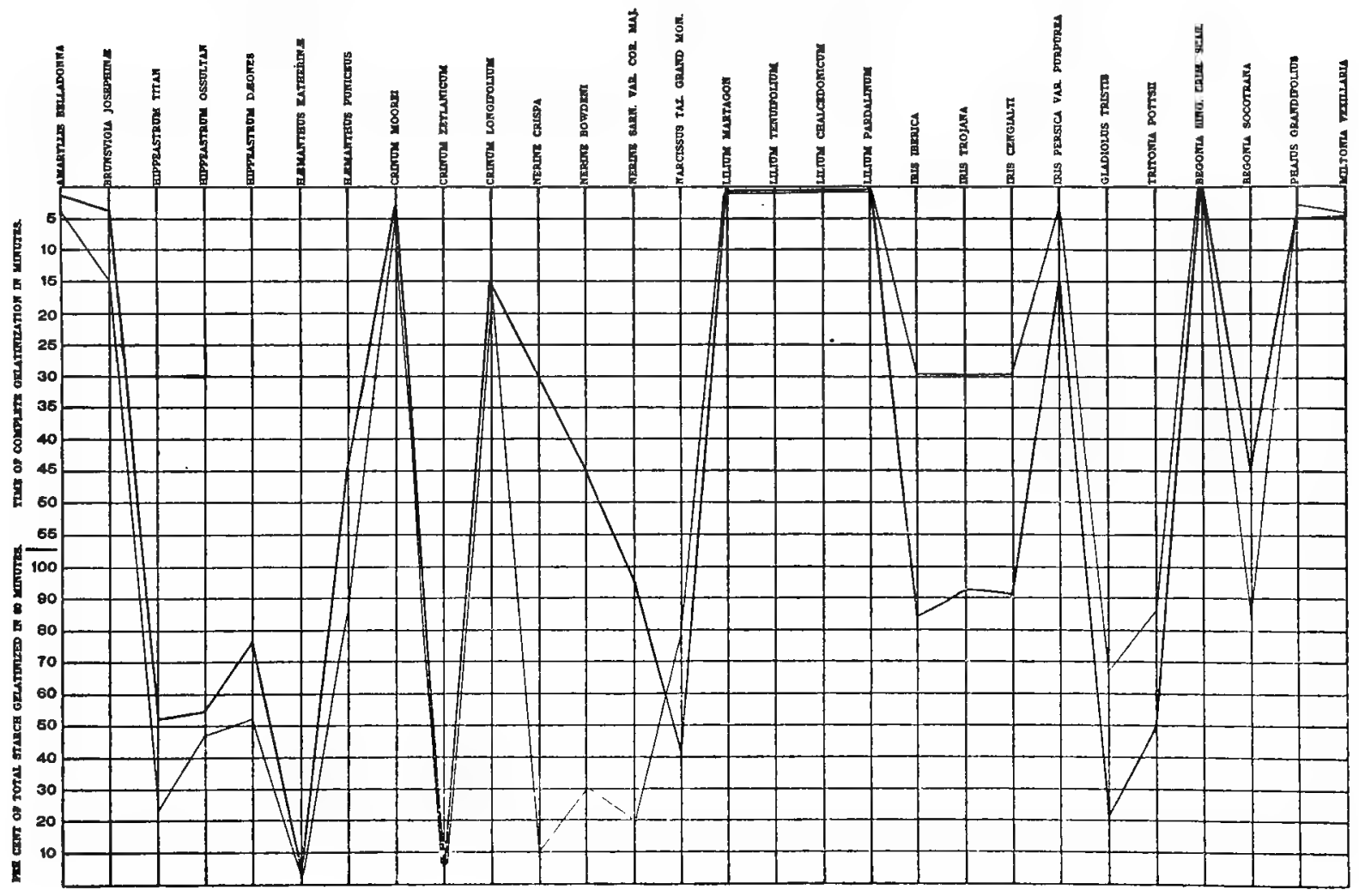


ChART B 21.-Nitric-acid (-) and Sodium-sulphide $(\longrightarrow)$ Reactions.

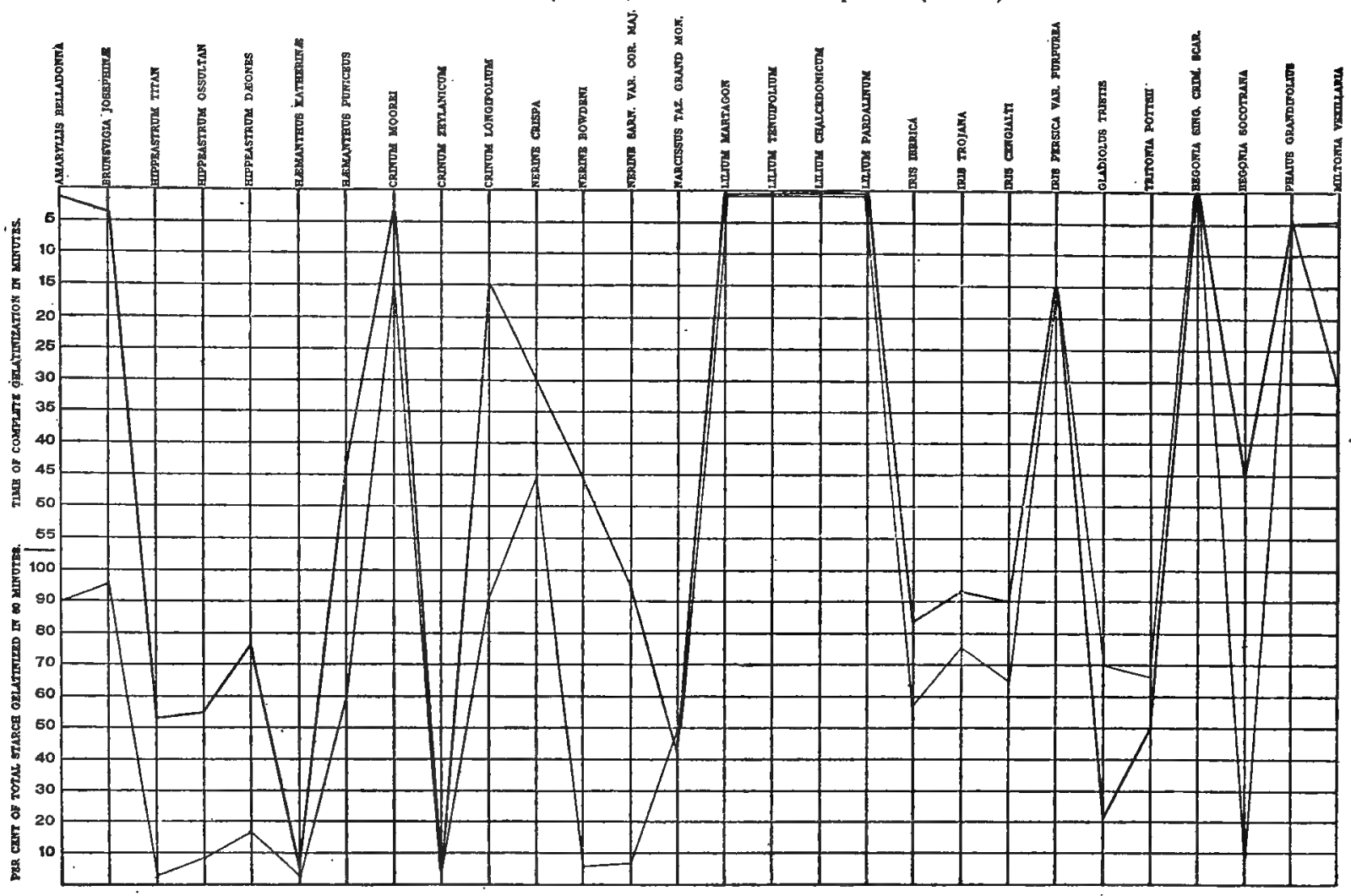

Chart B 22.-Nitric-acid (-) and Sodium-salicylate $(-)$ Reactions.

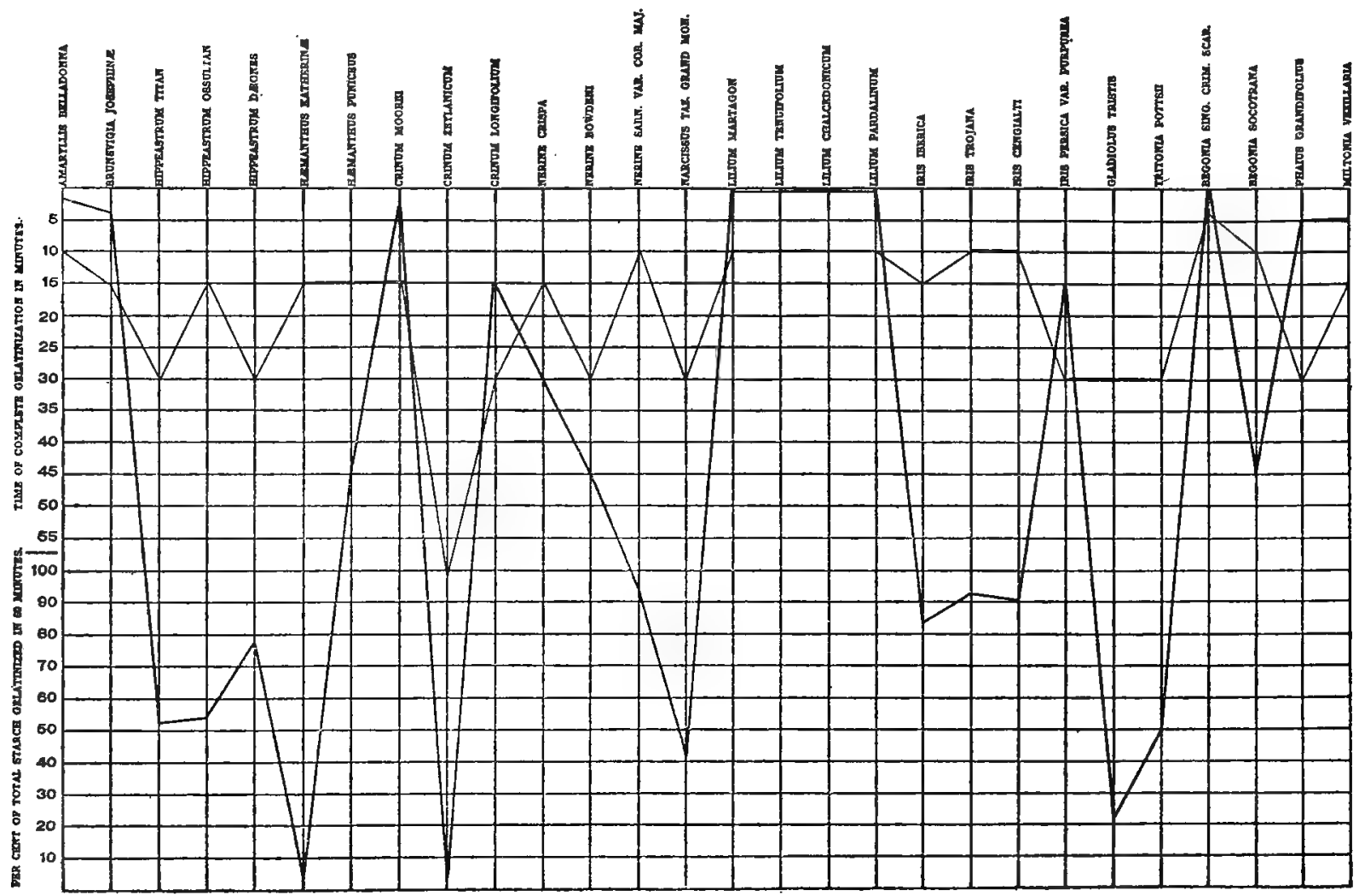


199

Chart B 23.-Nitric-acid $(\longrightarrow$ ) and Calcium-nitrate $(\longrightarrow)$ Reactions.

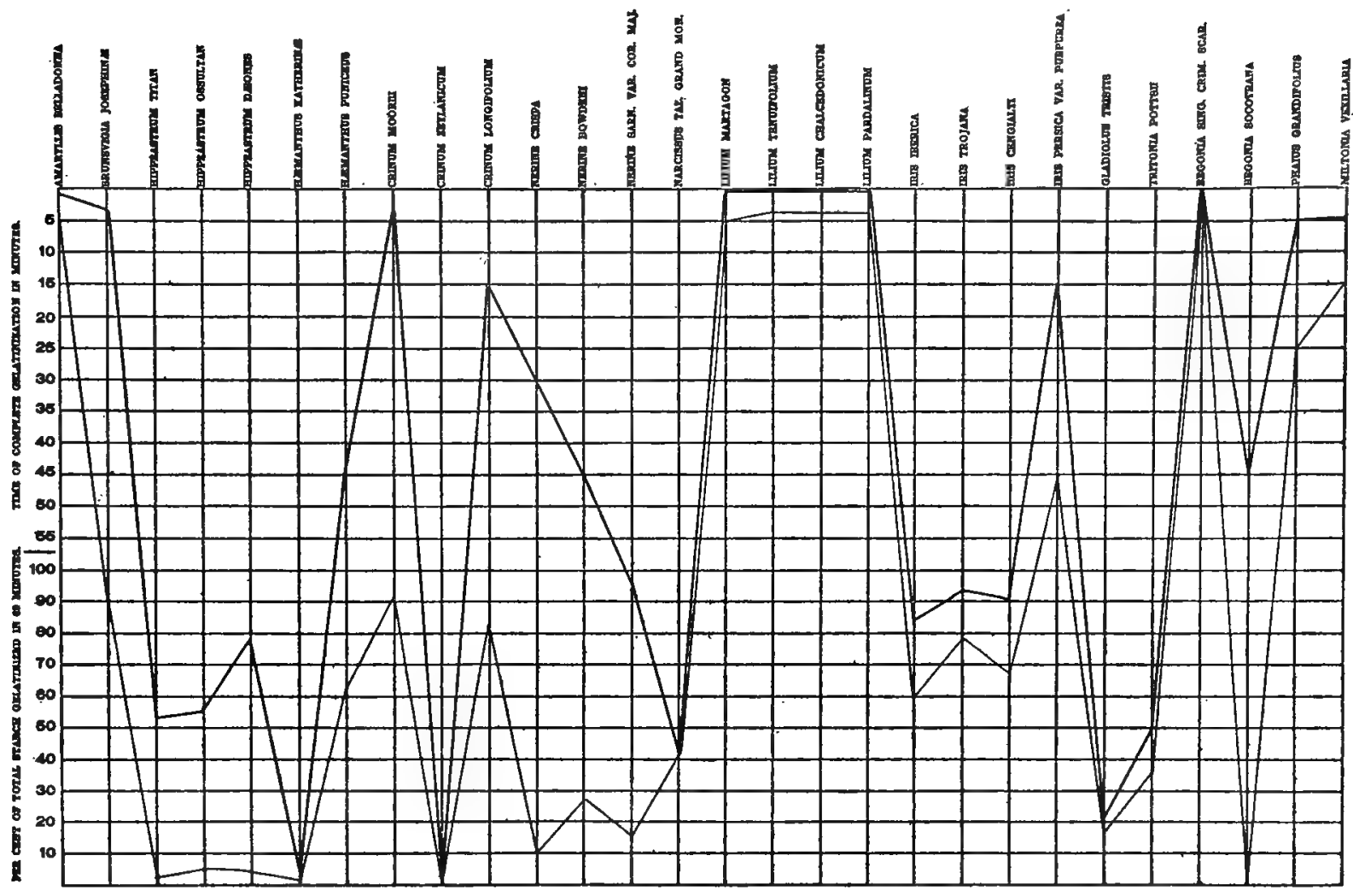

Chart B 24.-Nitric-acid $(-$ ) and Uranium-nitrate $(-)$ Reactions.

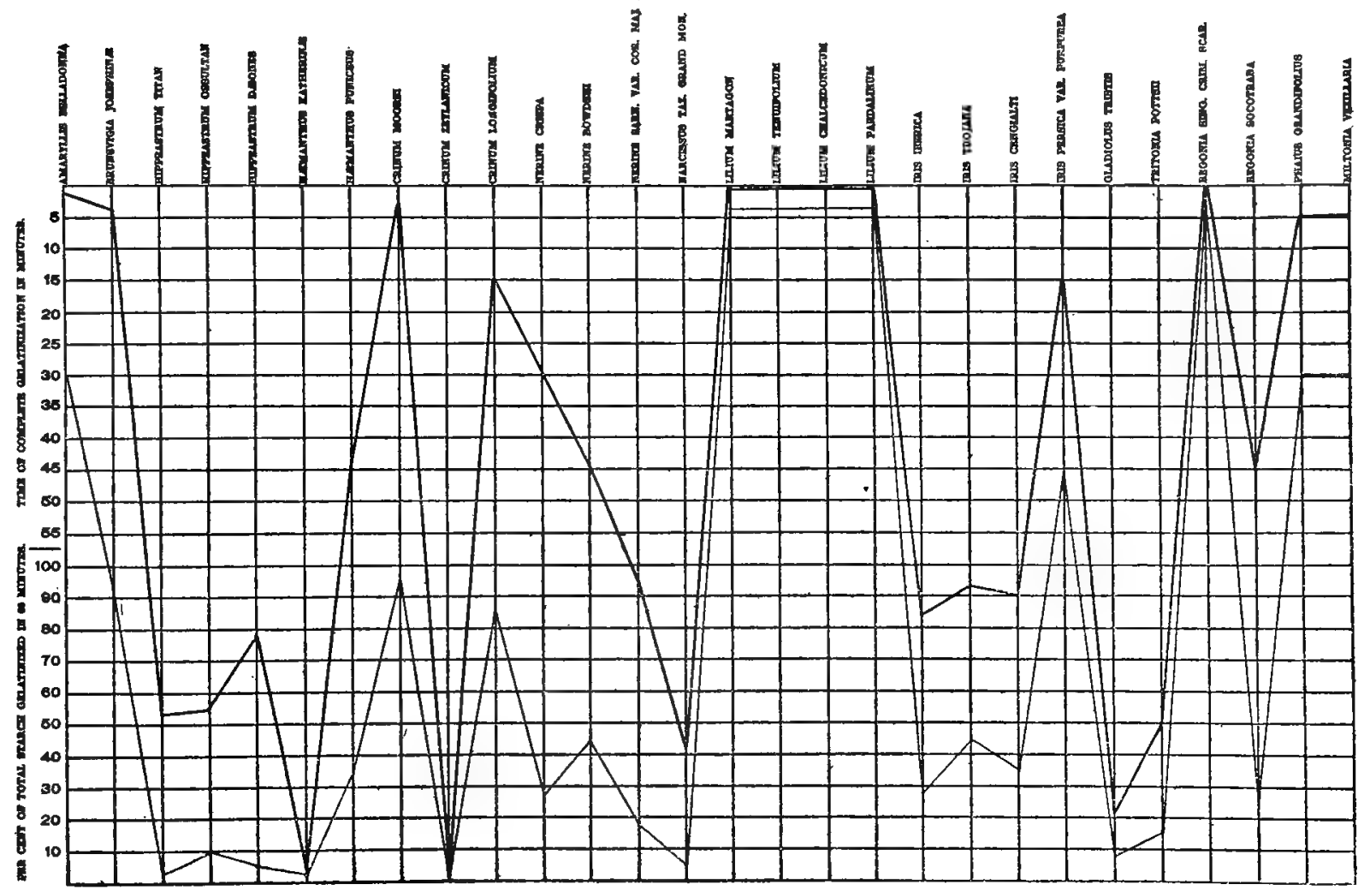


200

Chart B 25.-Nitric-acid (-) and Strontium-nitrate (-) Reactions.

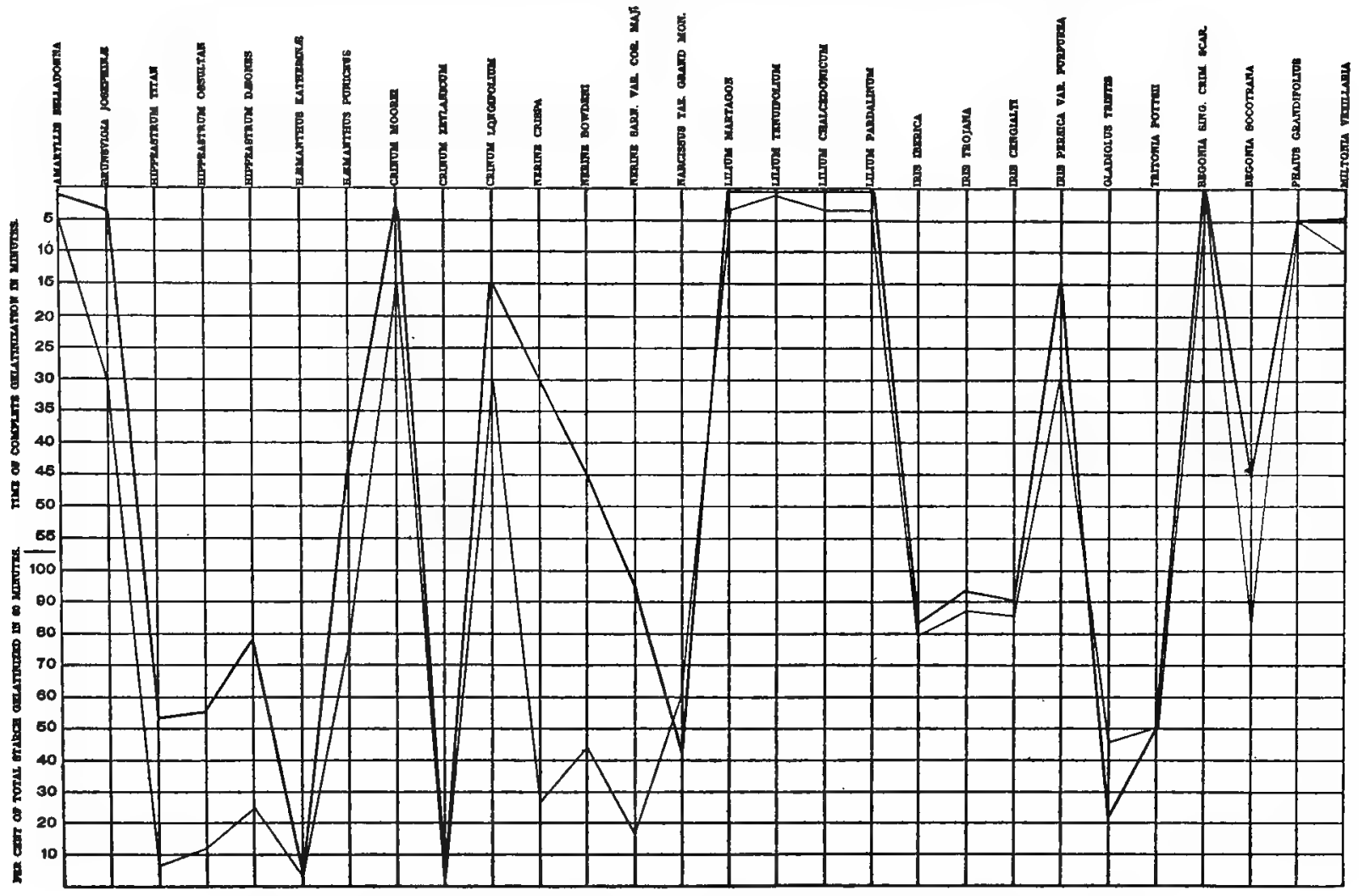

Chart B 26.-Nitric-acid (-) and Cobalt-nitrate $(-)$ Reactions.

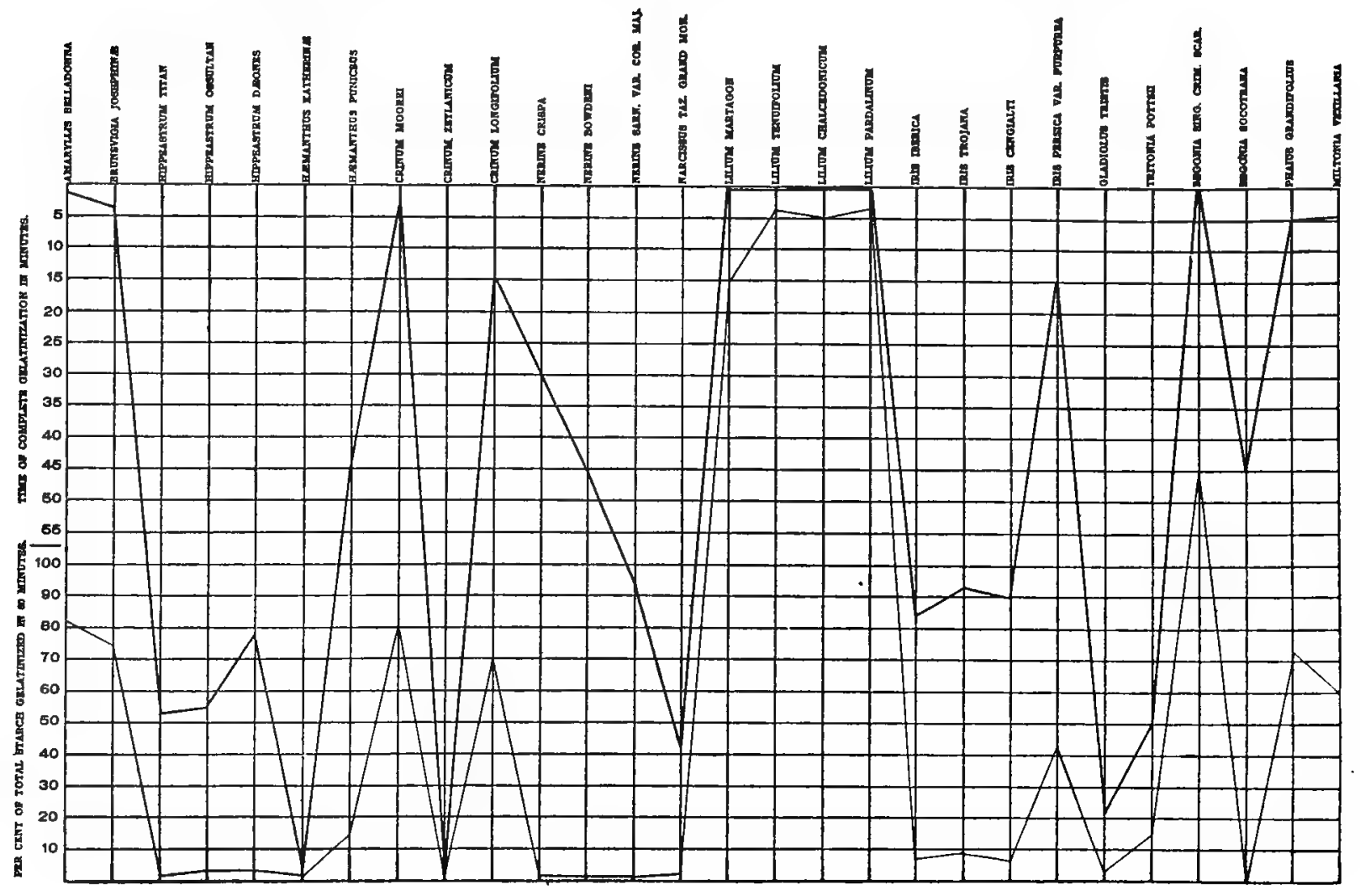


201

Chart B 27.-Nitric-acid (-) and Copper-nitrate $(\longrightarrow)$ Reactions.

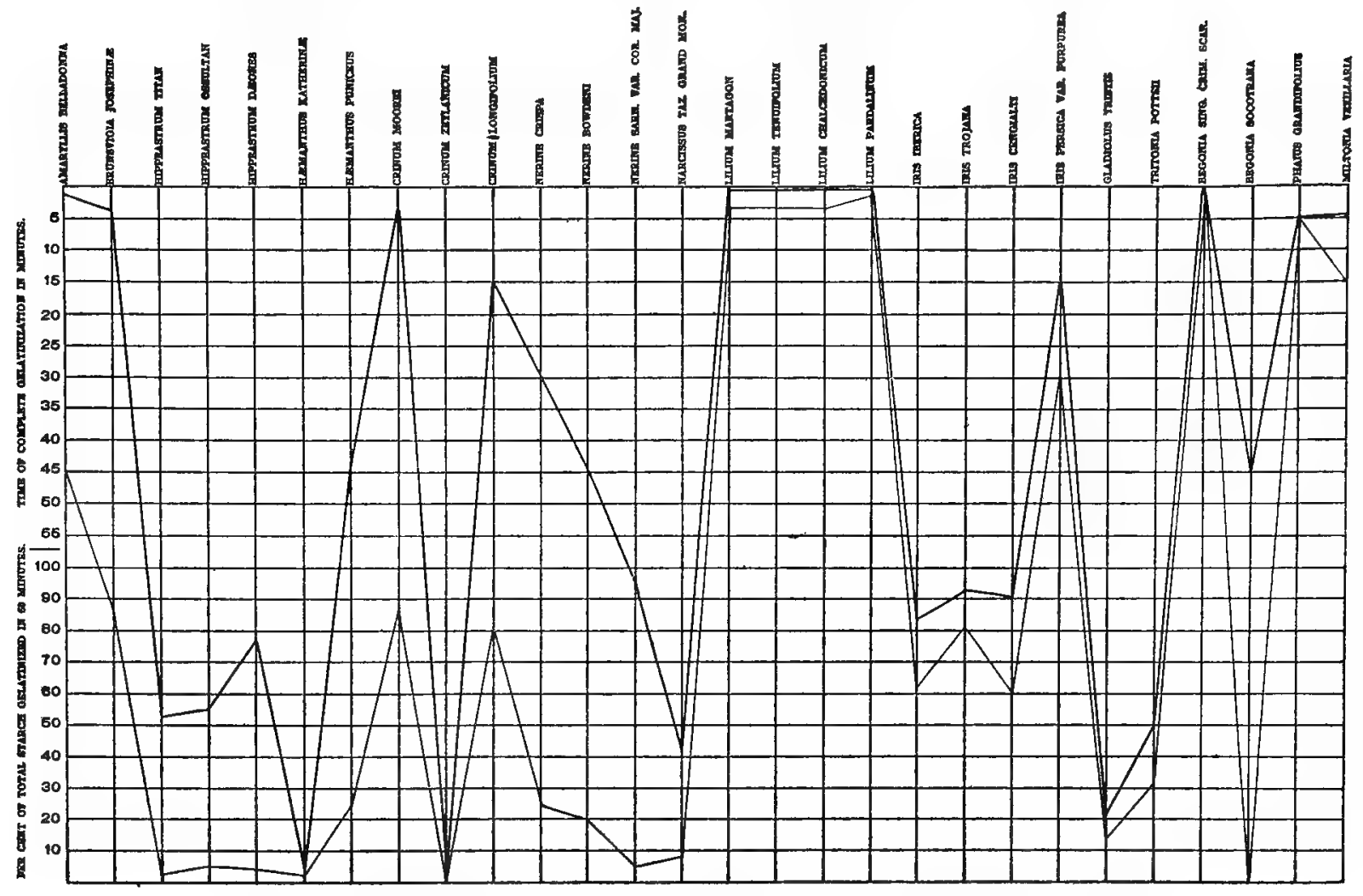

Chart B 28.-Nitric-acid (-) and Cuprio-chloride $(-)$ Reactions.

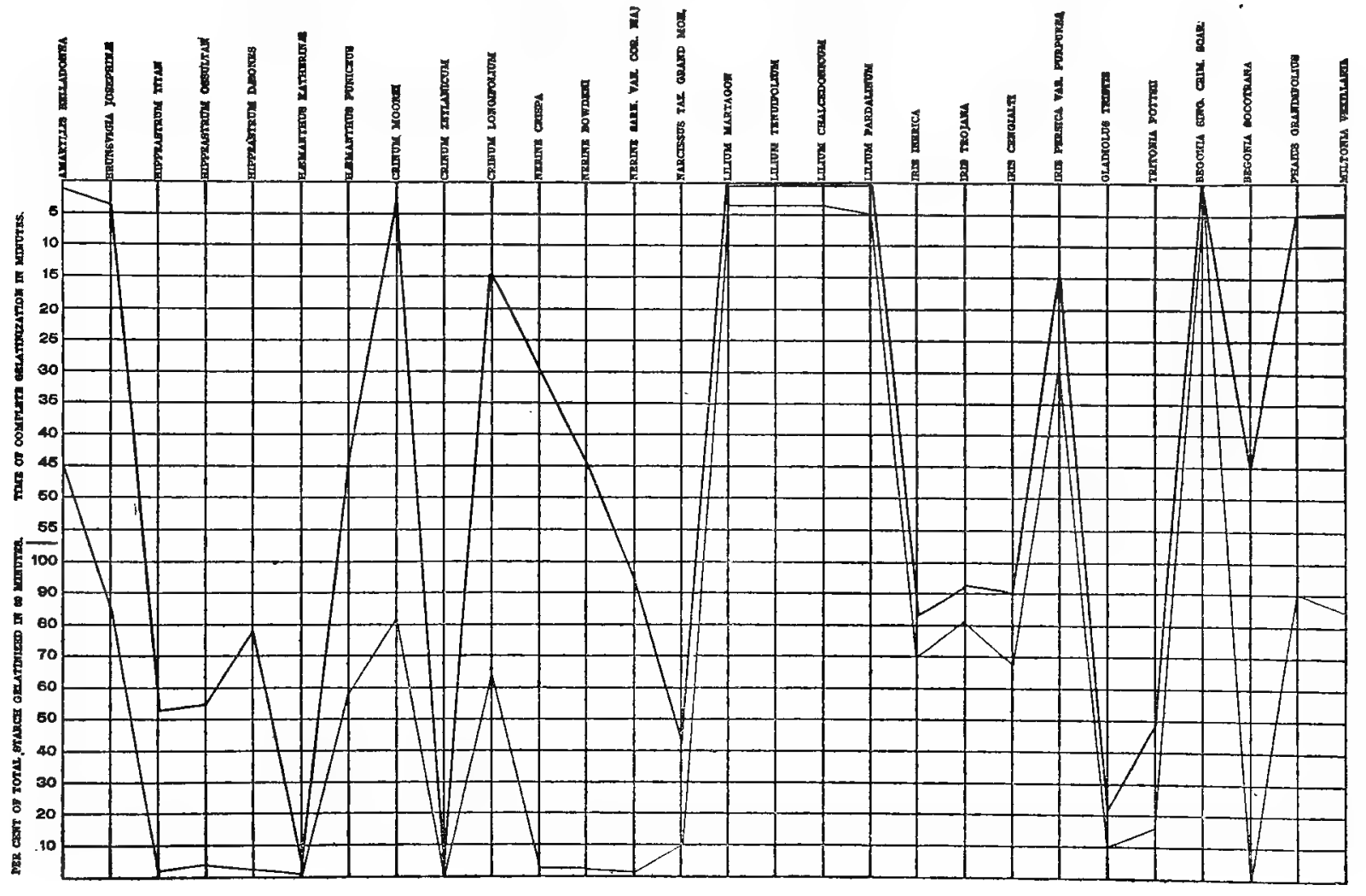


Chart B 29.-Nitric-acid (-) and Barium-chloride (-) Reactions.

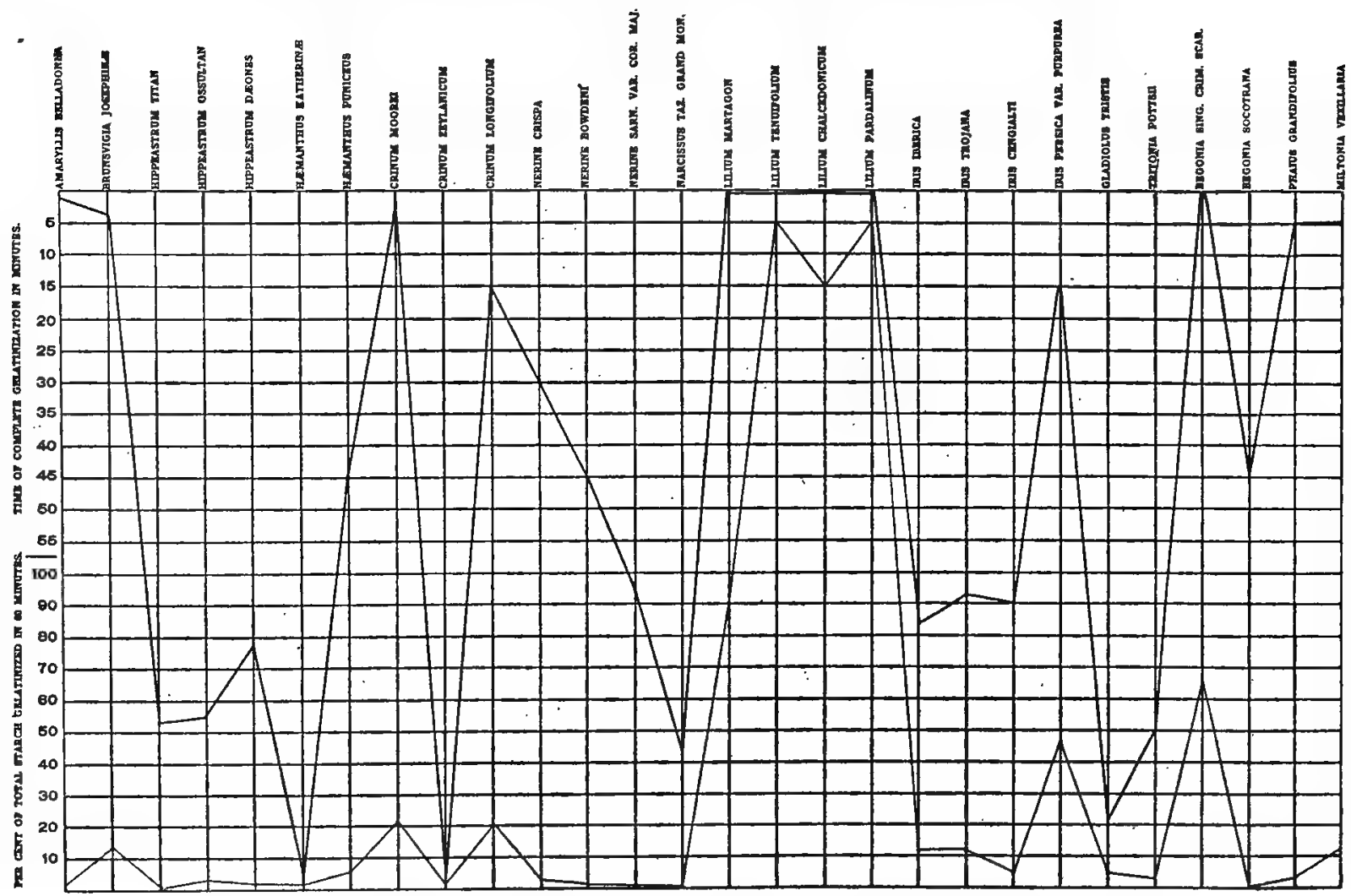

Chart B 30.-Nitric-acid $(-$ ) and Mercuric-chloride $(\longrightarrow)$ Reactions.

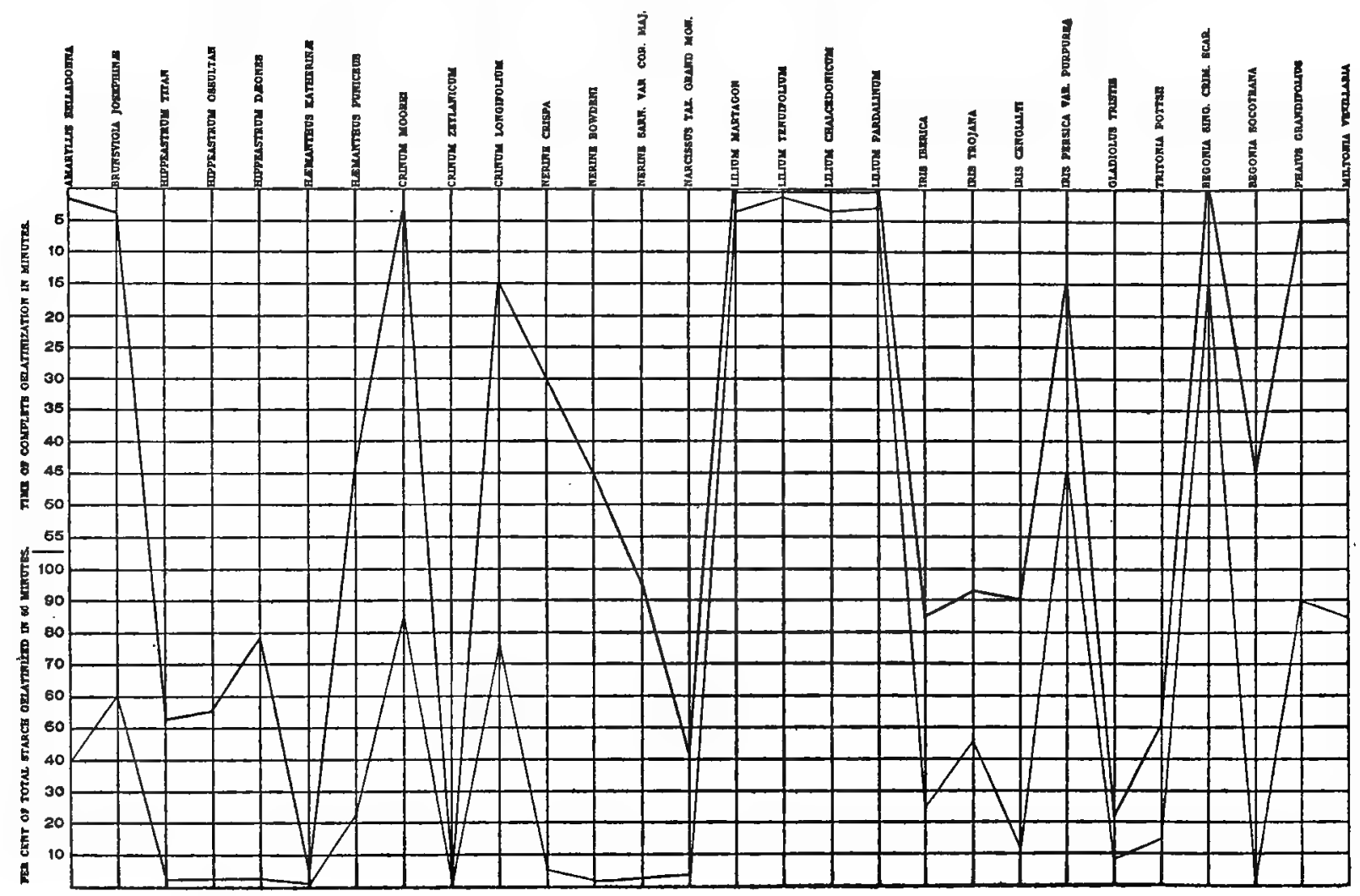


Chart B 31.-Chromic-acid (-) and Pyrogallic-acid (-) Reactions.

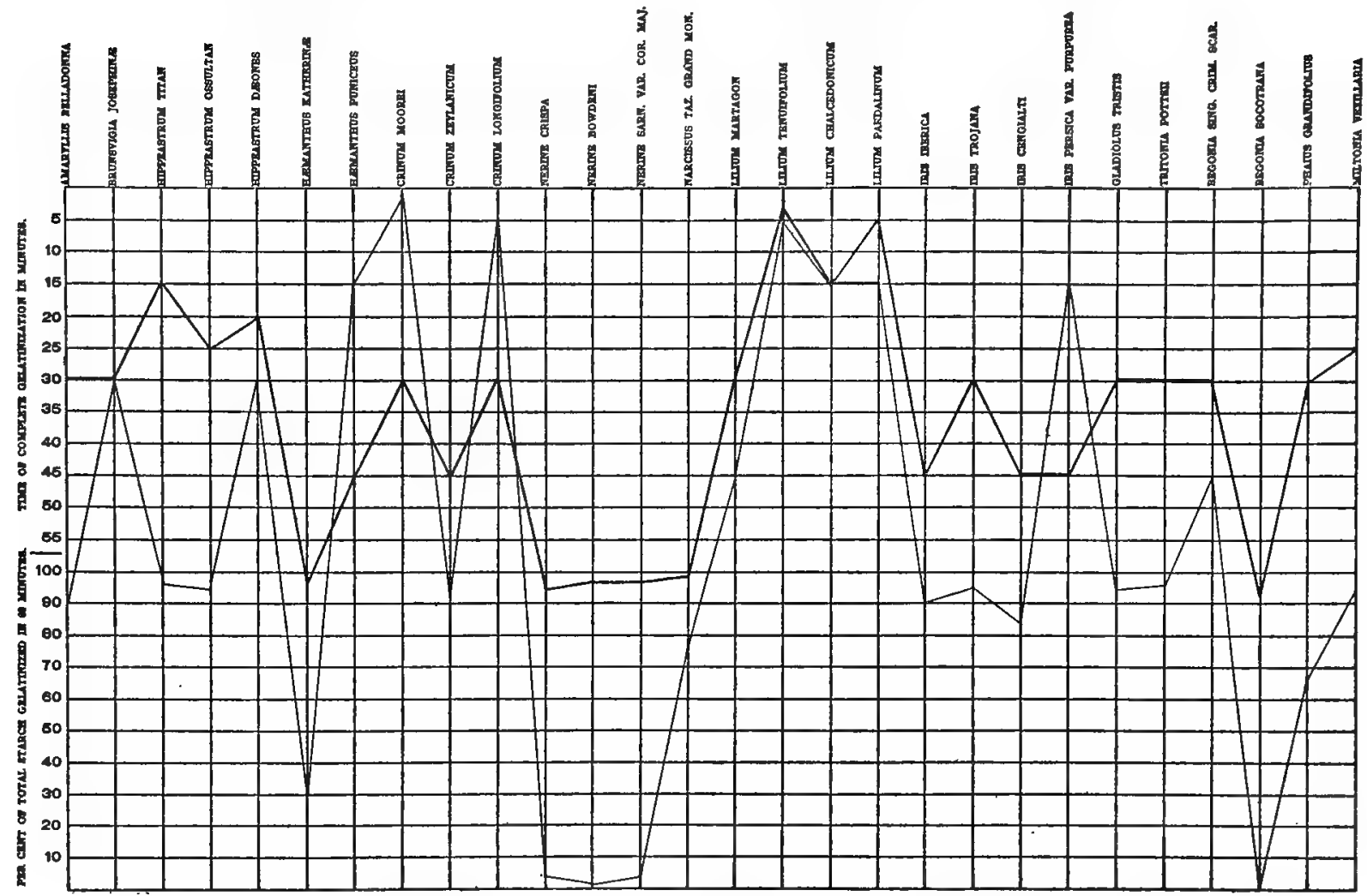

ChART B 32.-Nitric-acid (—), Sulphuric-acid (—), and Hydrochloric-acid (....) Reactions.

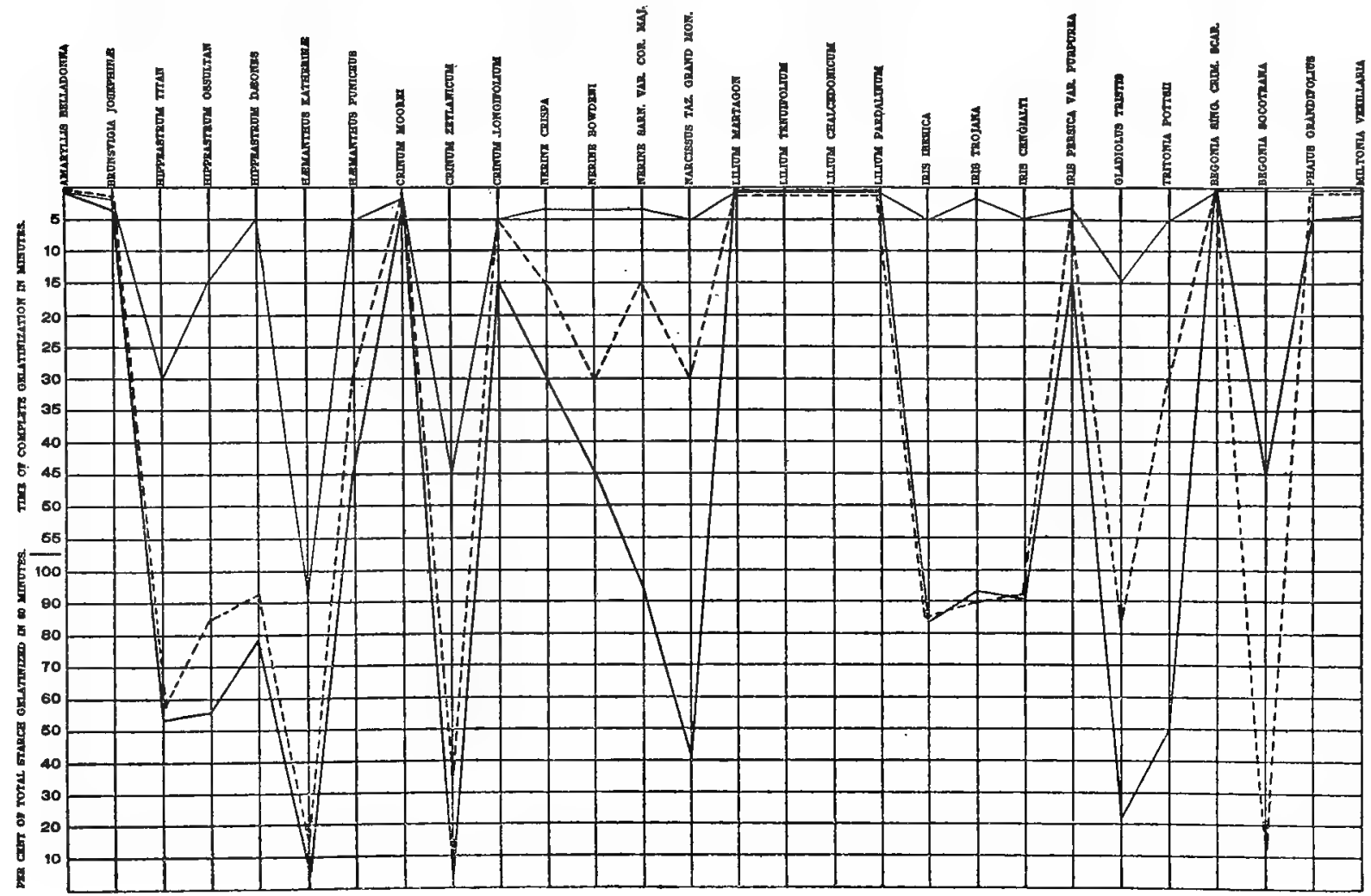


Chart B 33.-Potassium-hydroxide (-) and Sodium-hydroxide $(\longrightarrow)$ Reactions.

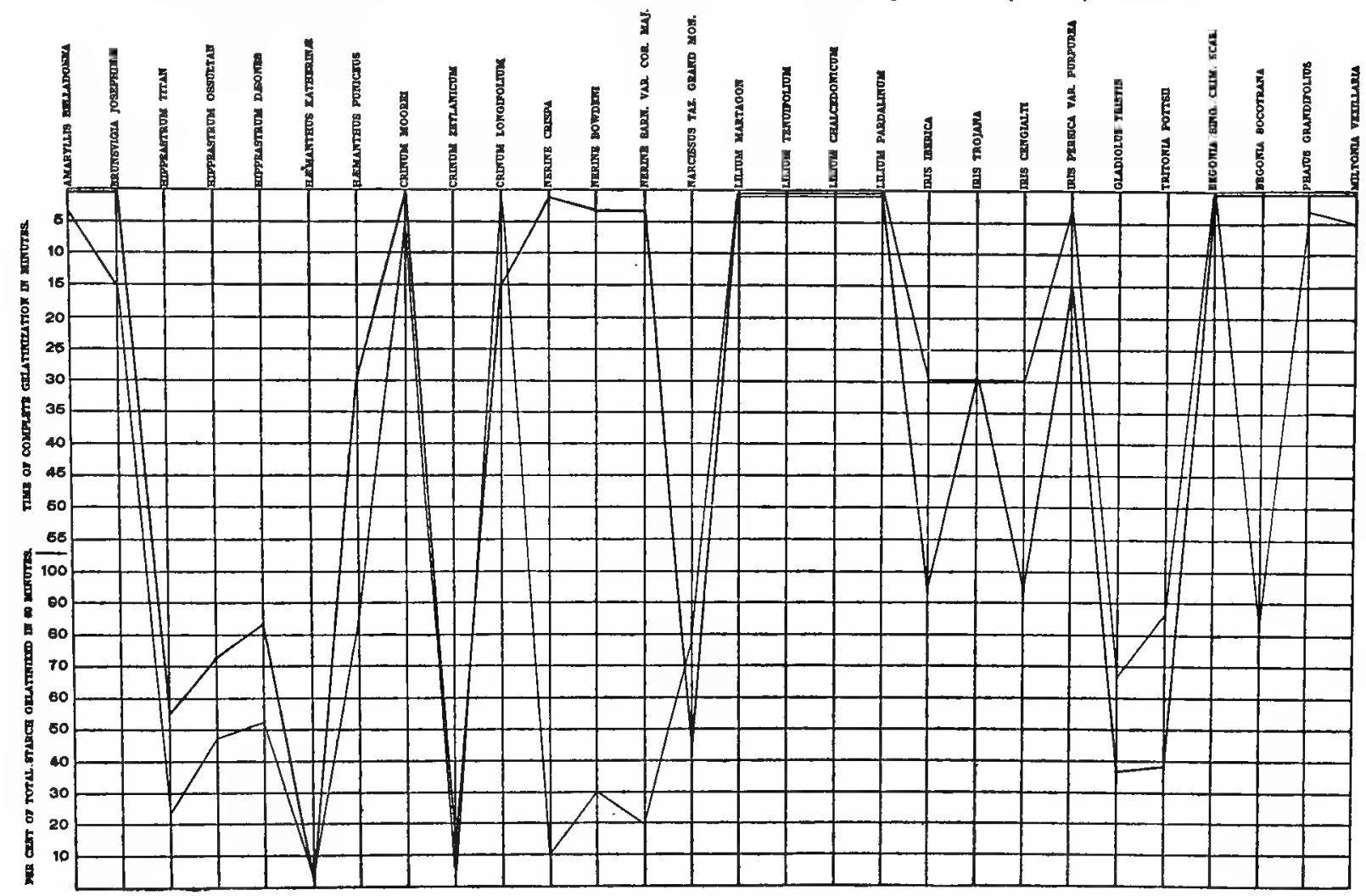

Chart B 34.-Potassium-sulphide $(\longrightarrow$ ) and Sodium-sulphide $(-)$ Reactions.

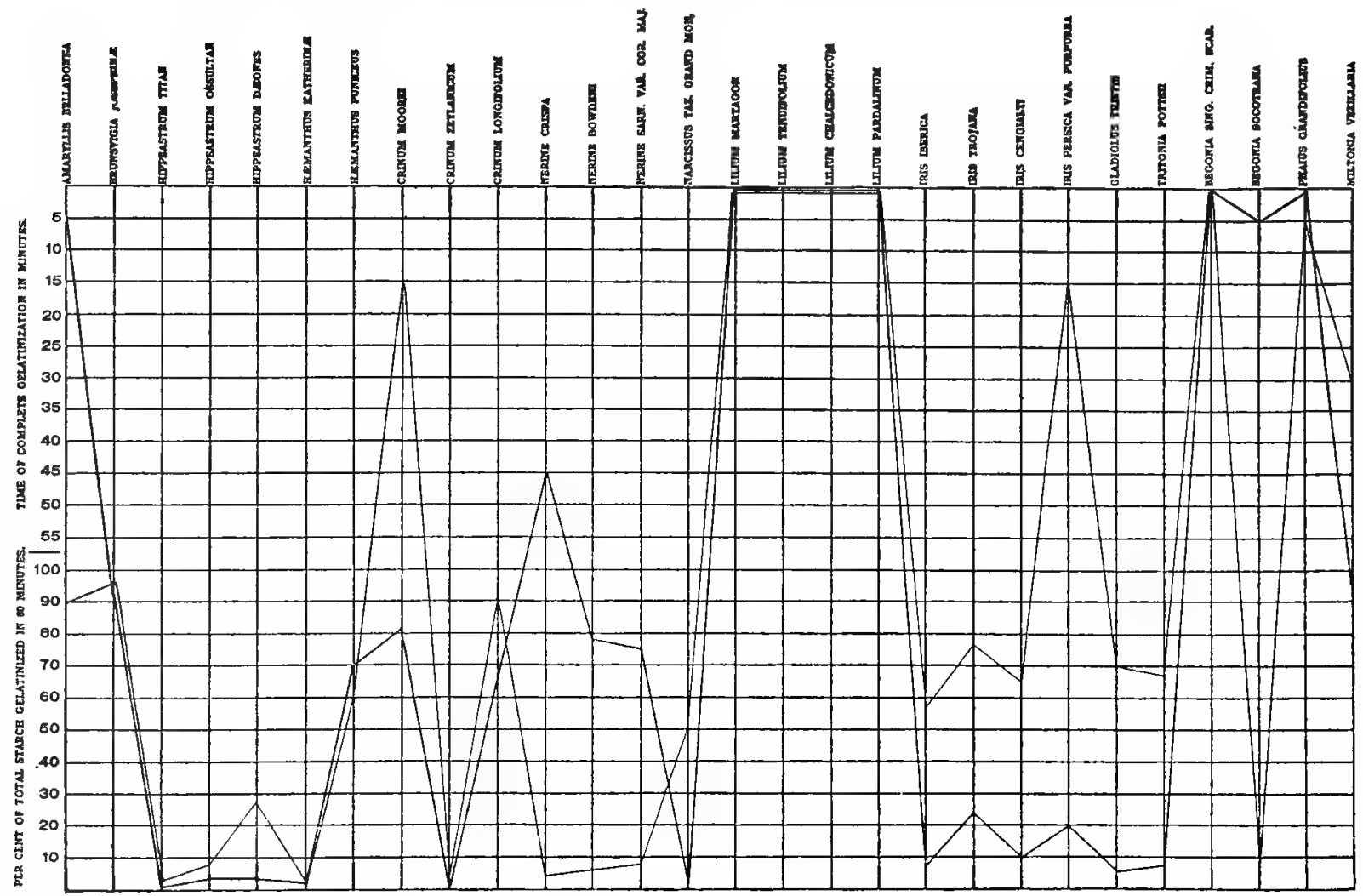


Chart B 35.-Potassium-iodide (-) and Potassium-sulphocyanate $(\longrightarrow)$ Reactions.

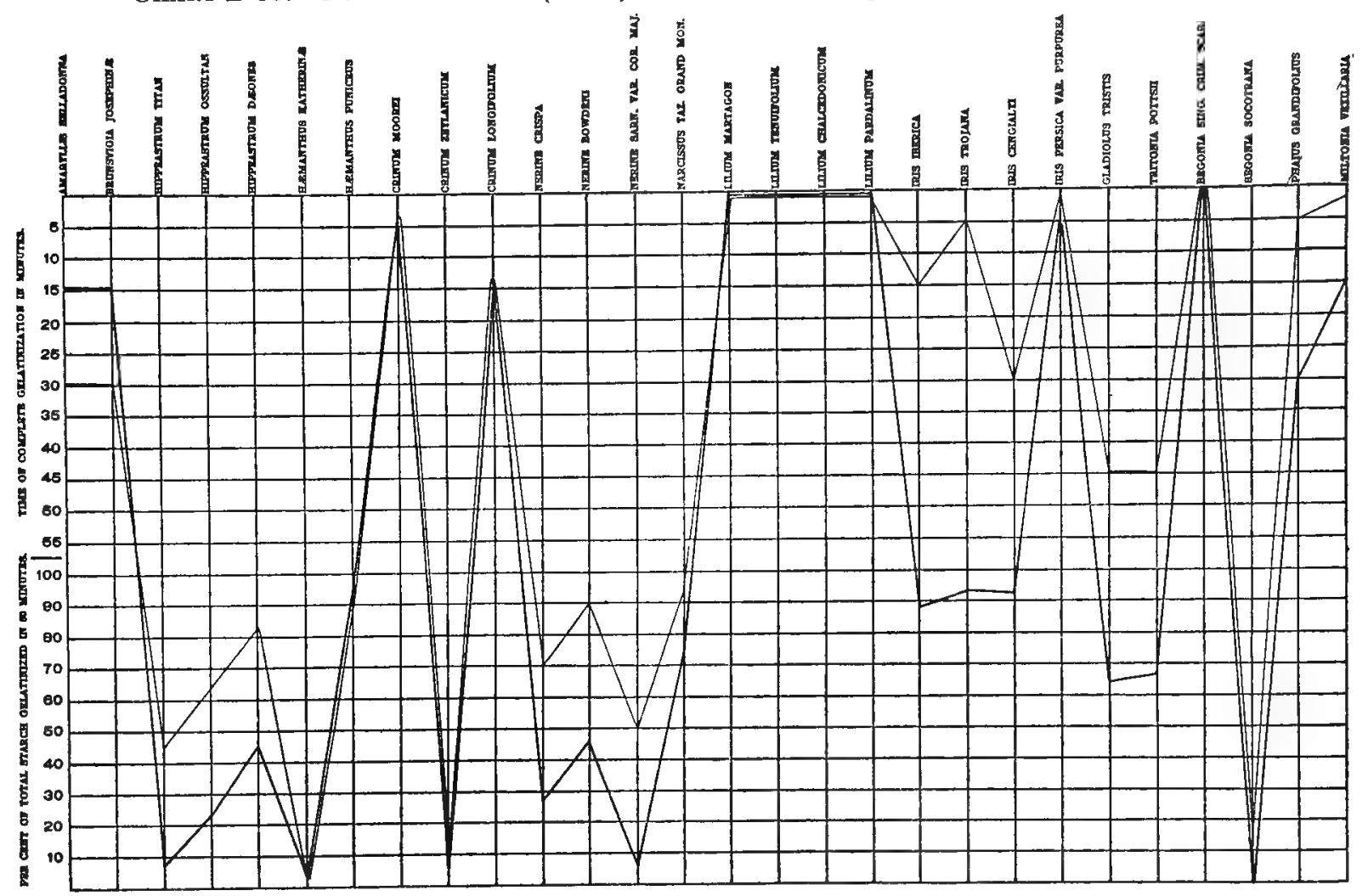

Chart B 36.-Sodium-hydroxide $(-)$ and Sodium-salicylate $(-)$ Reactions.

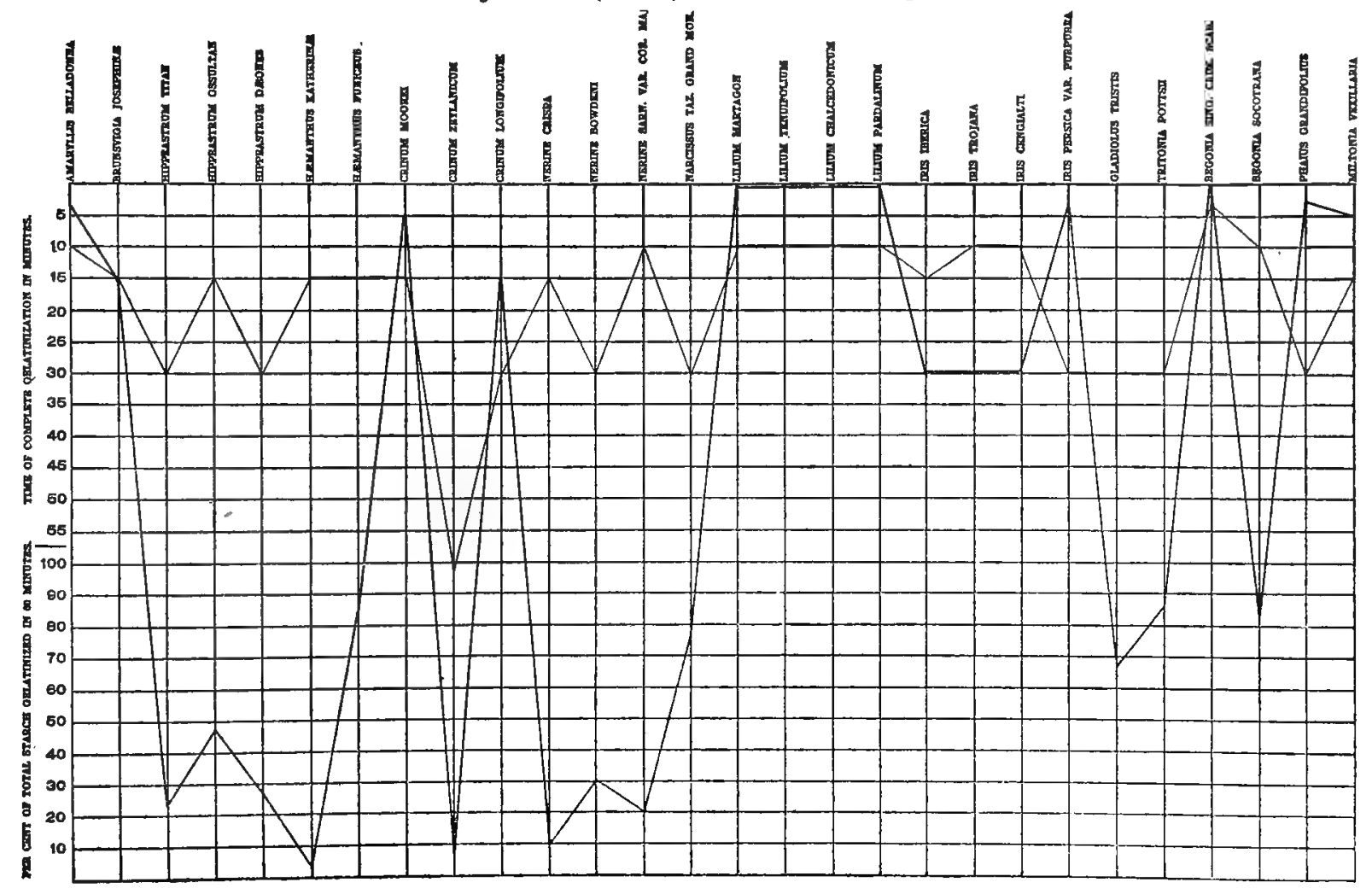


ChART B 37.-Calcium-nitrate $(\longrightarrow)$ and Strontium-nitrate $(\longrightarrow)$ Reactions.

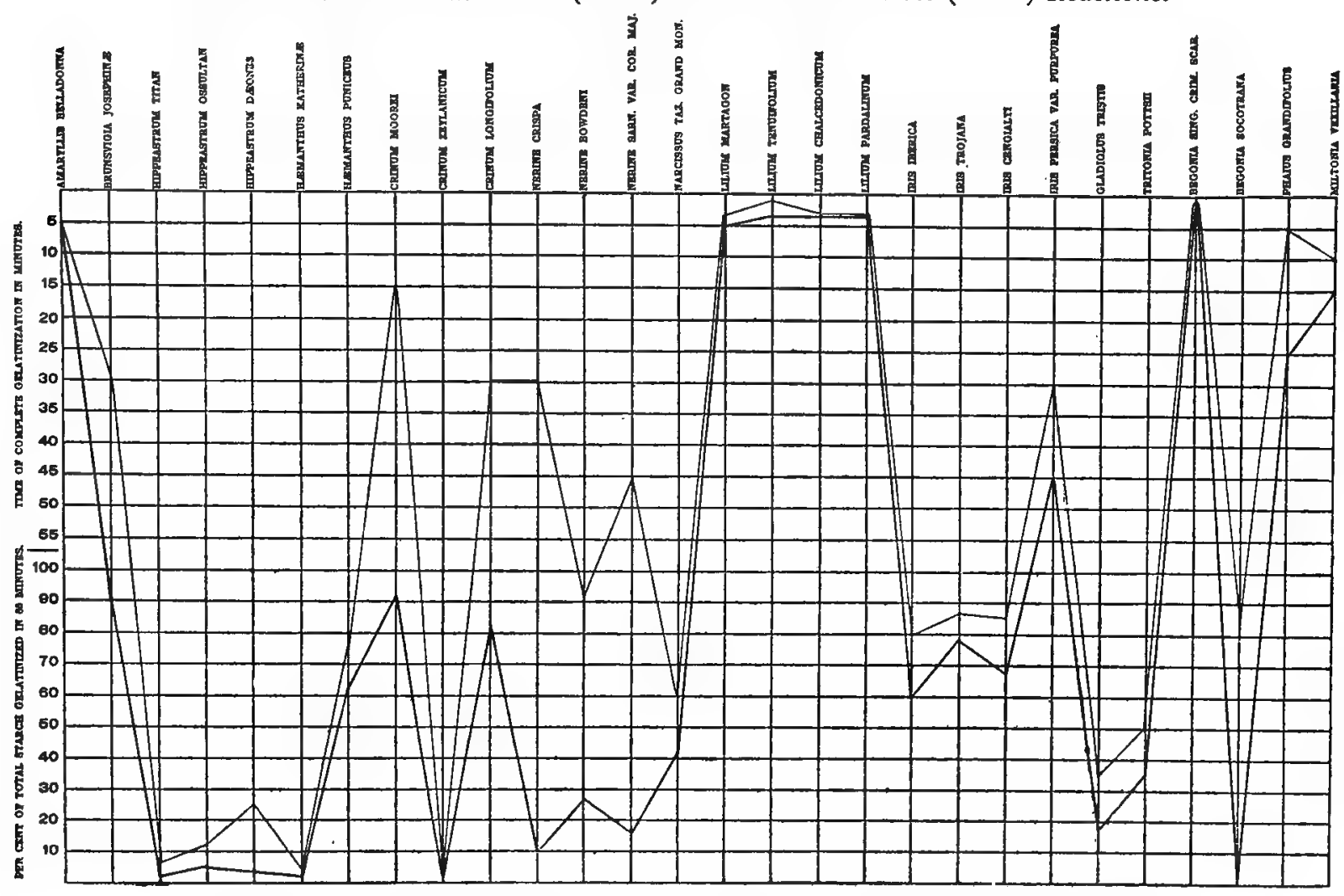

Chakt B 38.-Uranium-nitrate $(\longrightarrow)$ and Coball-nitrate $(-)$ Reactions.

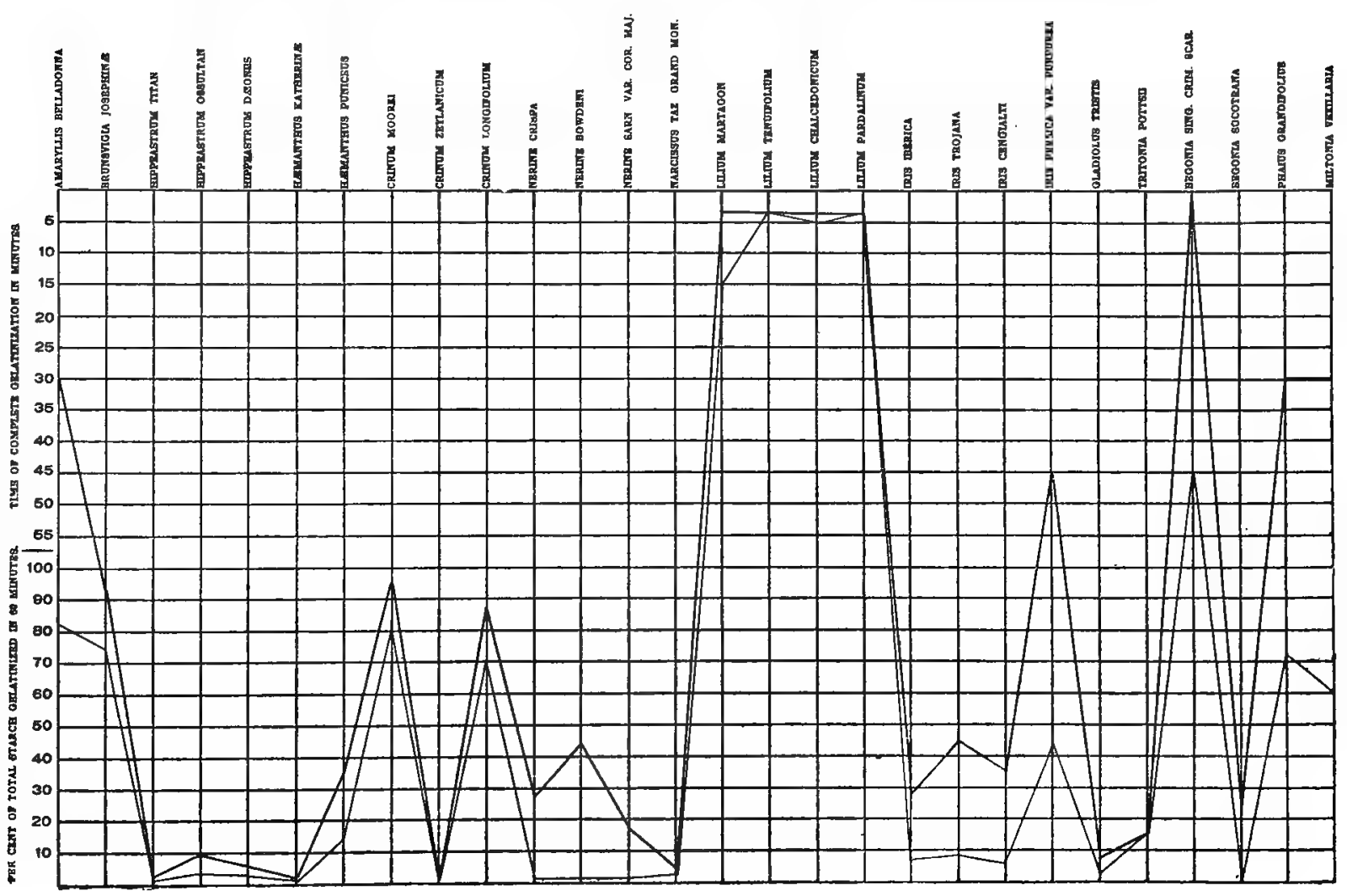


Chart B 39.-Copper-nitrate $(\longrightarrow$ ) and Cupric-chloride $(\longrightarrow)$ Reactions.

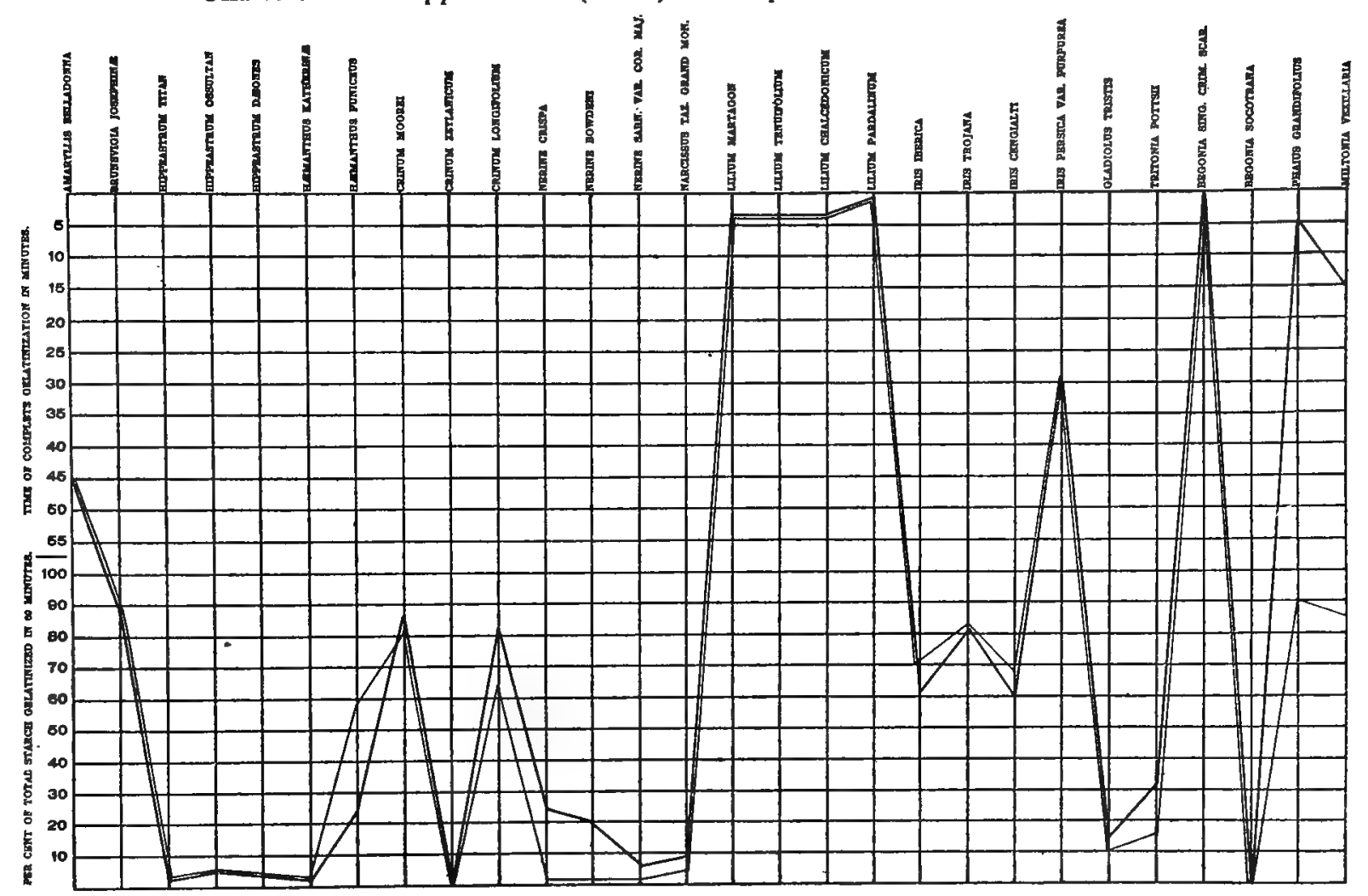

Chant B 40.-Barium-chloride $(\longrightarrow$ ) and Mercuric-chloride $(\longrightarrow$ Reactions.

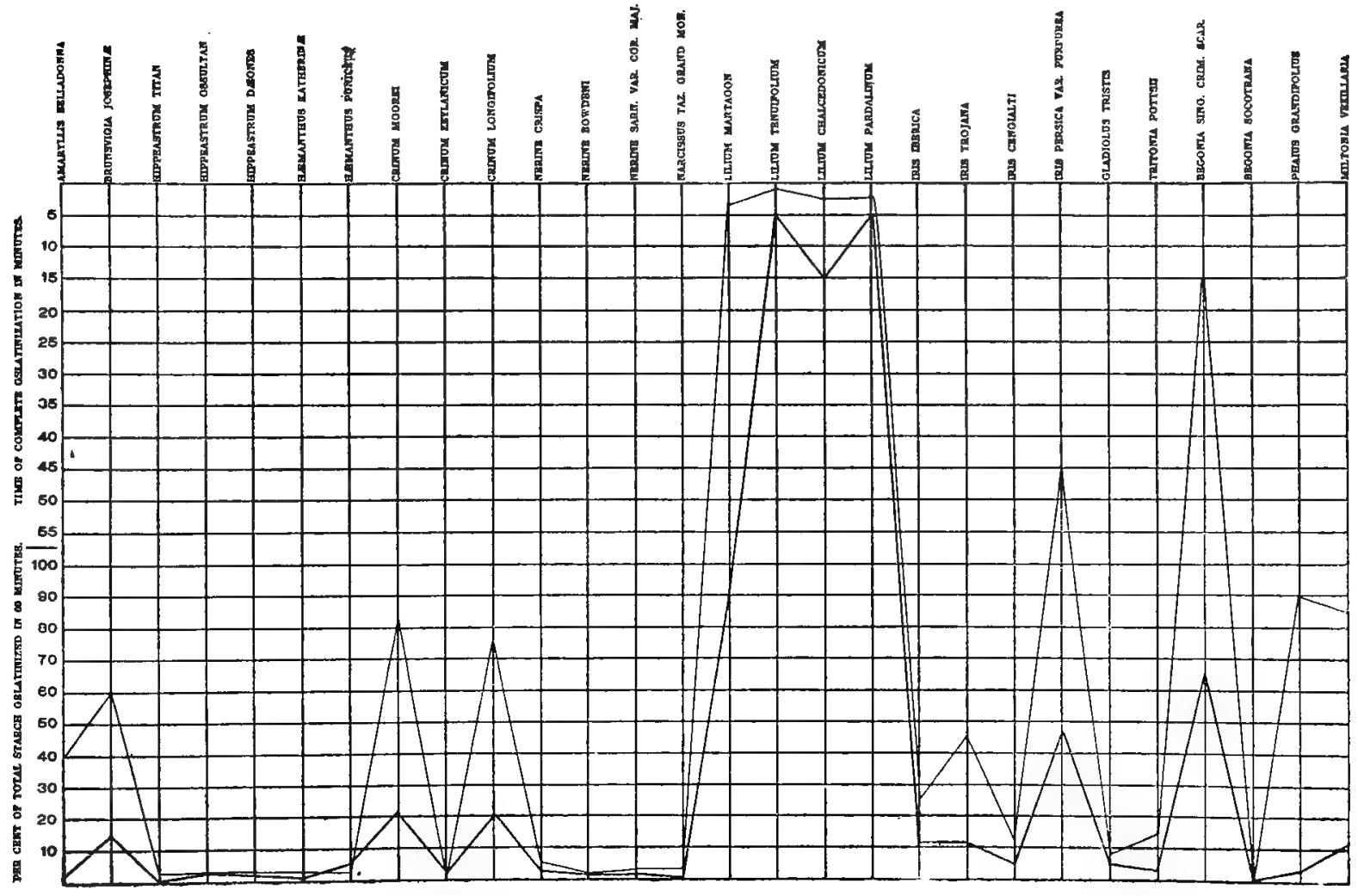


Chart B 41.-Points of Inversion and Recrossing of the Curves.

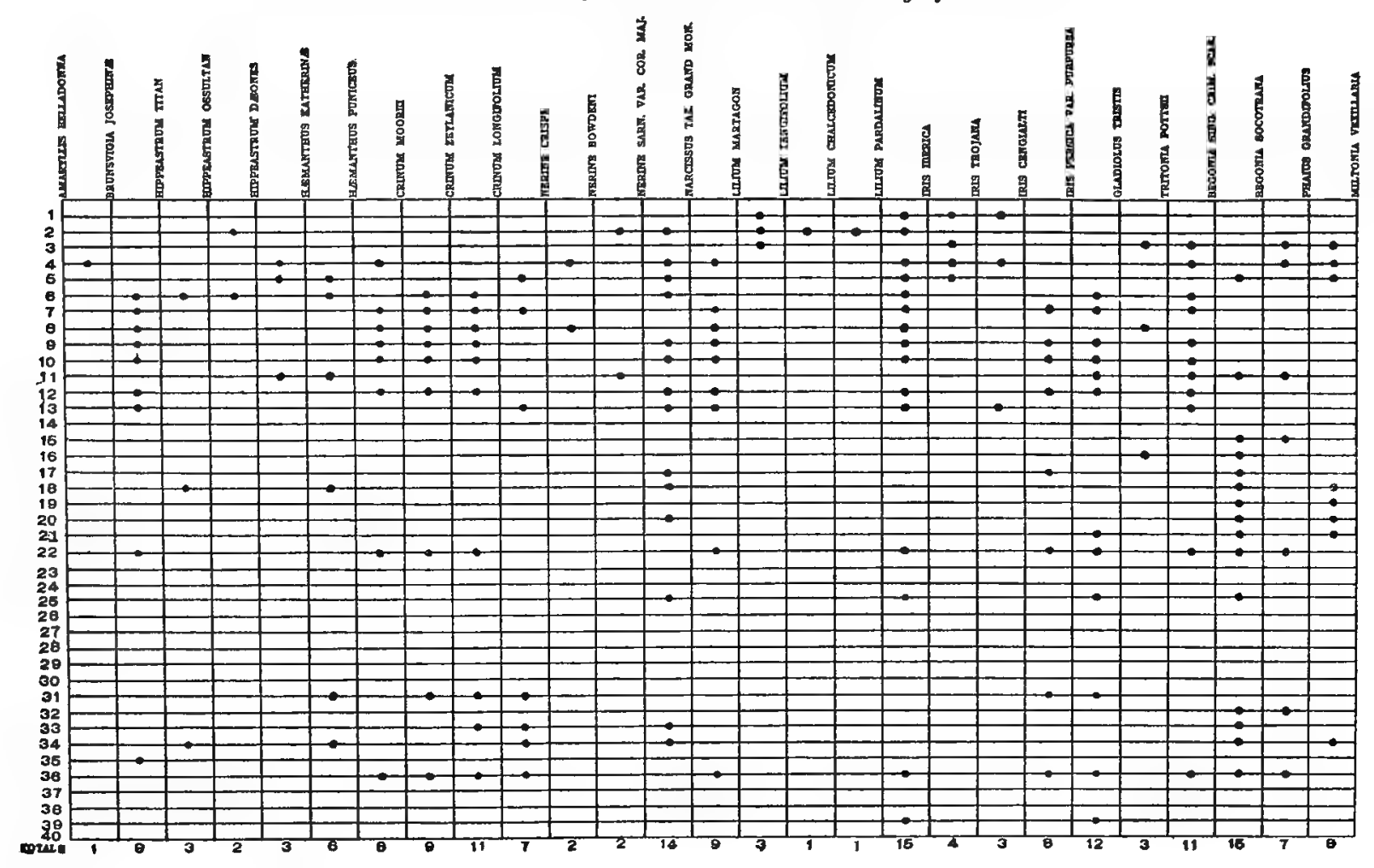

CHART B 42.-Average Reaction-Intensities (-) and Temperatures of Gelatinization (-).

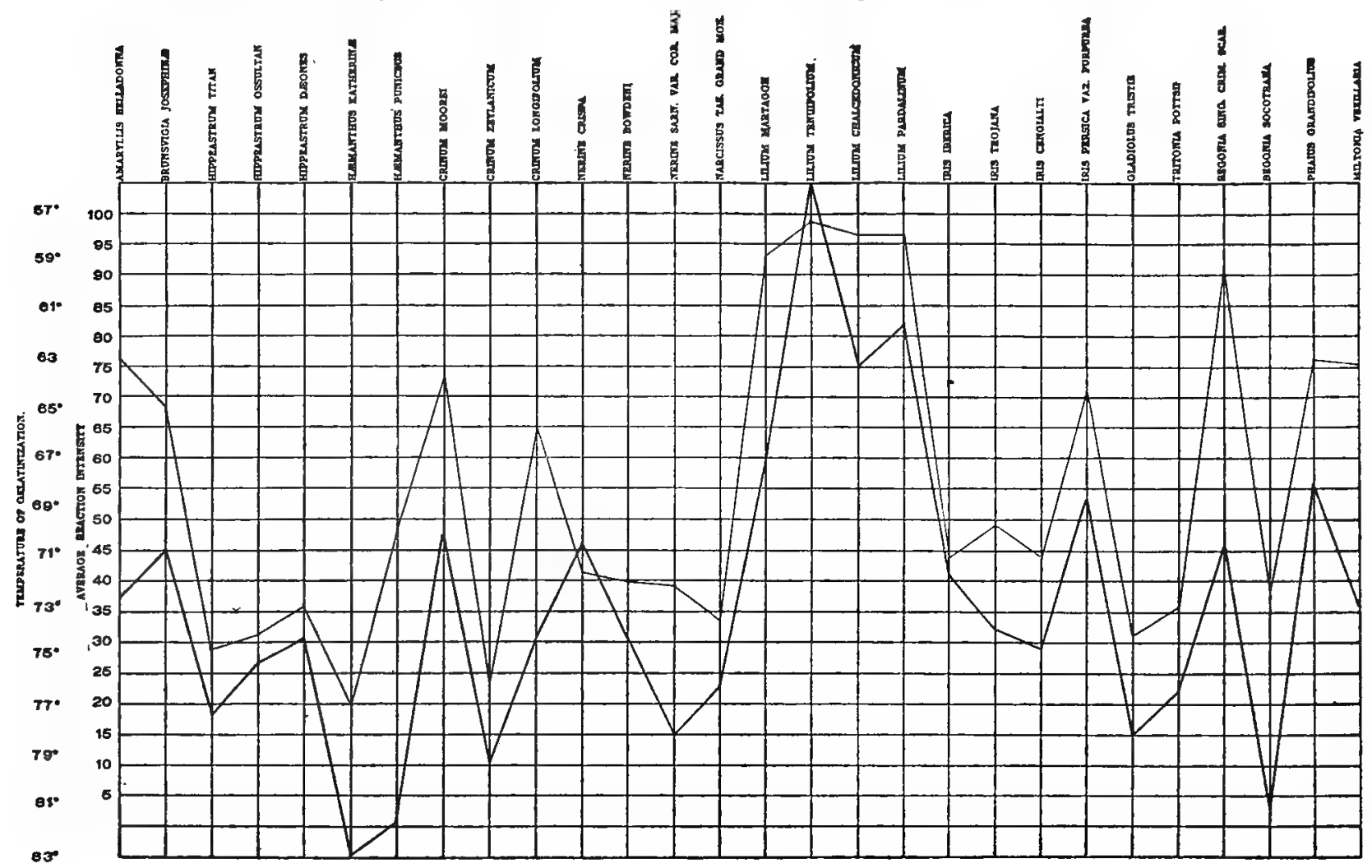


Chart C 1.-Height, Sum, and Average of Reaction-Intensities of Starches of Hybrid-Stocks and Parent-Stocks.

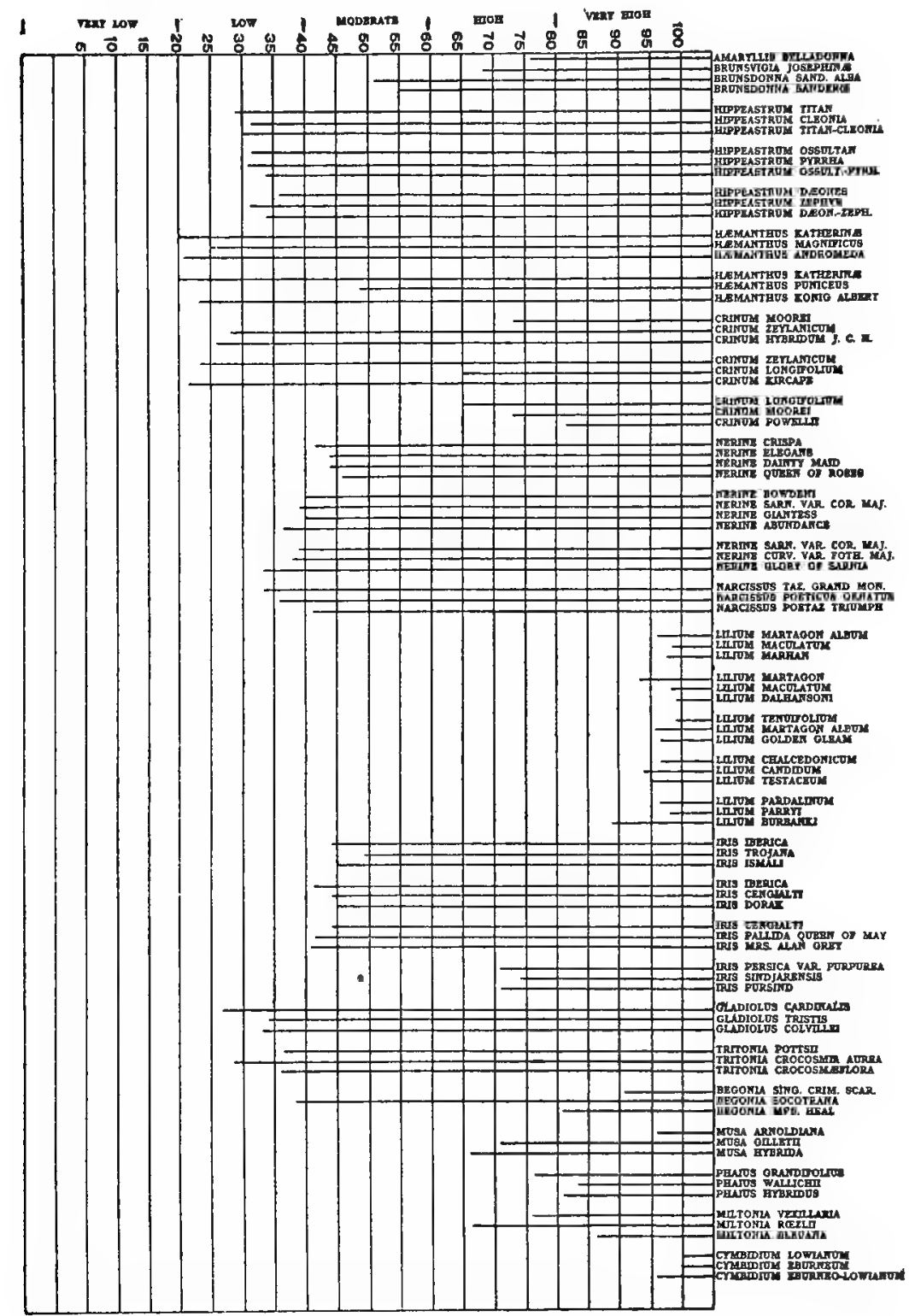




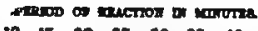

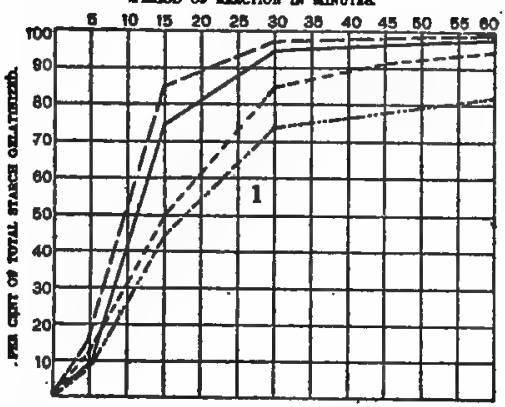

Monod on paction In moths

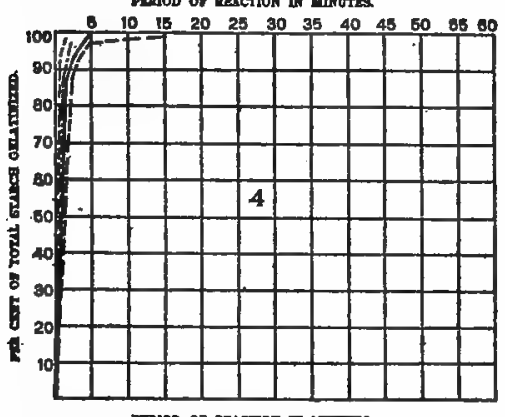

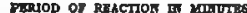

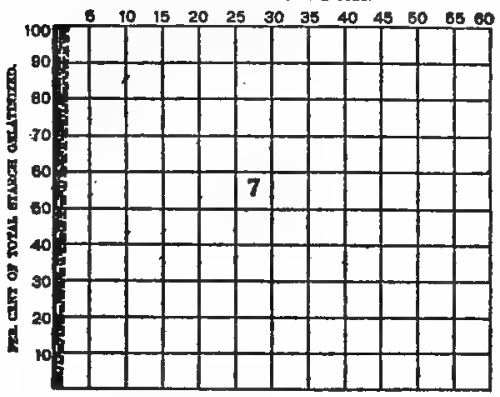

morod os mection in ymatres

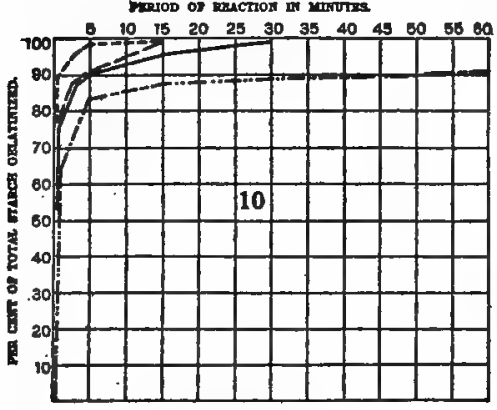

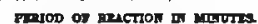

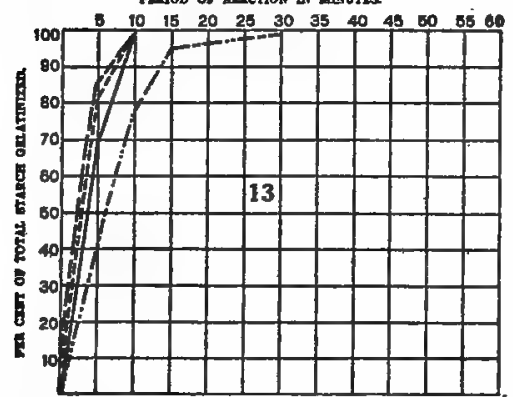

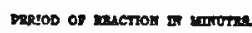

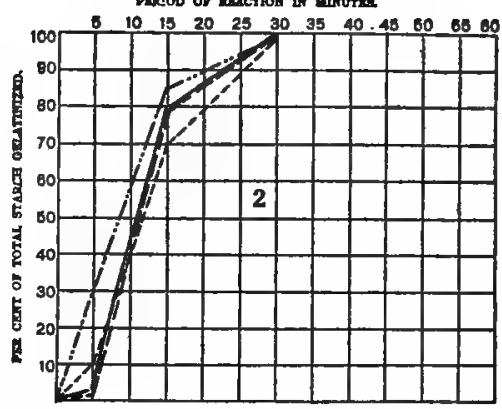

pRriob of Reuctor an warotes

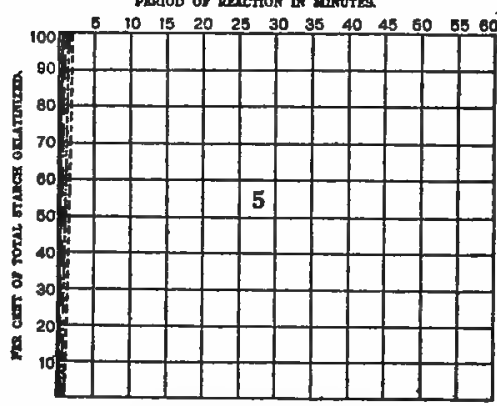

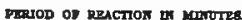

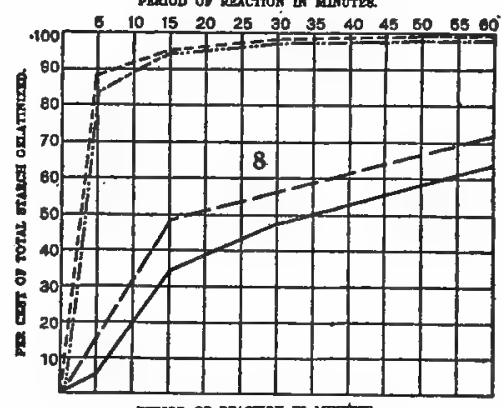

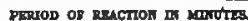

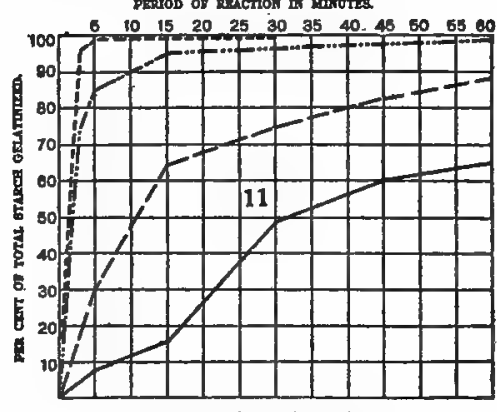

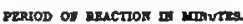

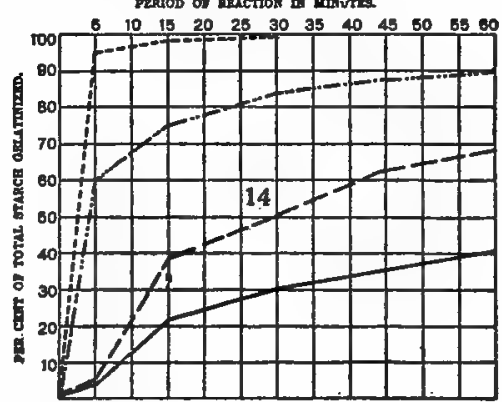

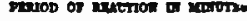

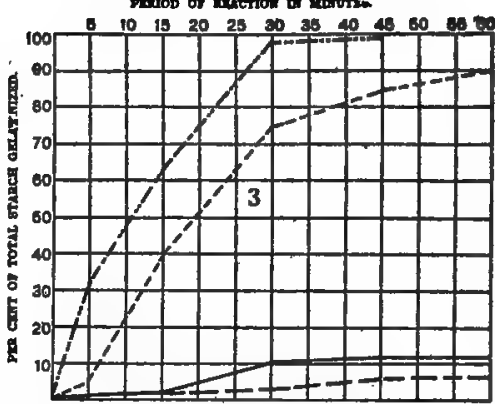

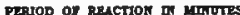

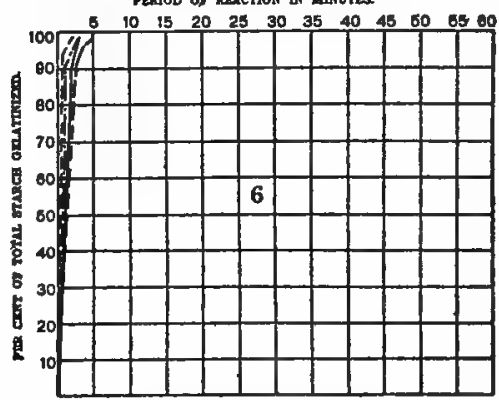

marod of Elsction, in morota

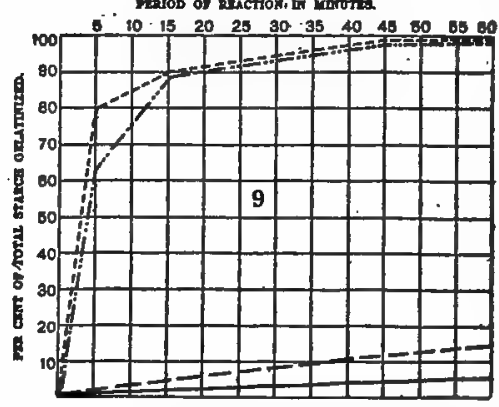

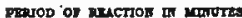

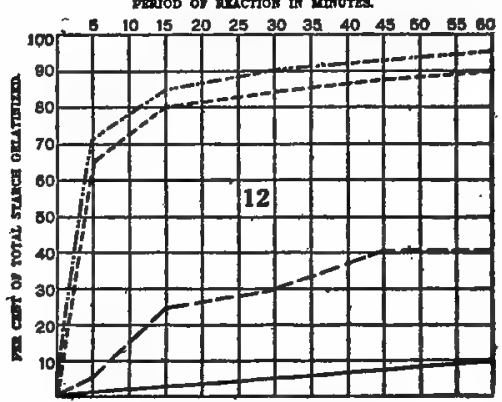

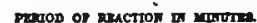

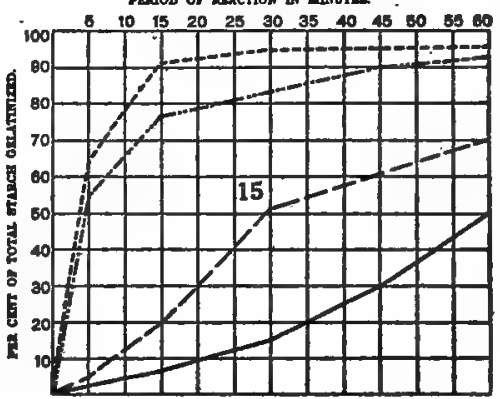

Charts D 1 to D 15.-Velocity-Reactions of Starches of Amaryllis belladonna (.....), Brunsoigia josephince (-.....), Brunsdonna sanderce alba (-), and Brunsdonna sanderce (- $\longrightarrow$ ).

1. With Choral Hydrate.

2. With Chromic Acid.

3. With Pyrogallic Acid

4. With Nitric Acid.

5. With Sulphuric Acid.
6. With Hydrochloric Acid.

7. With Potassium Hydroxide.

8. With Potasgium Iodide.

9. With Potaesium Sulphocyanate.

10. With Potassium Sulphide.
11. With Sodium Hydroxide.

12. With Sodium Sulphide.

13. With Sodium Salicylate.

14. With Calcium Nitrate.
15. With Uranium Nitrate. 

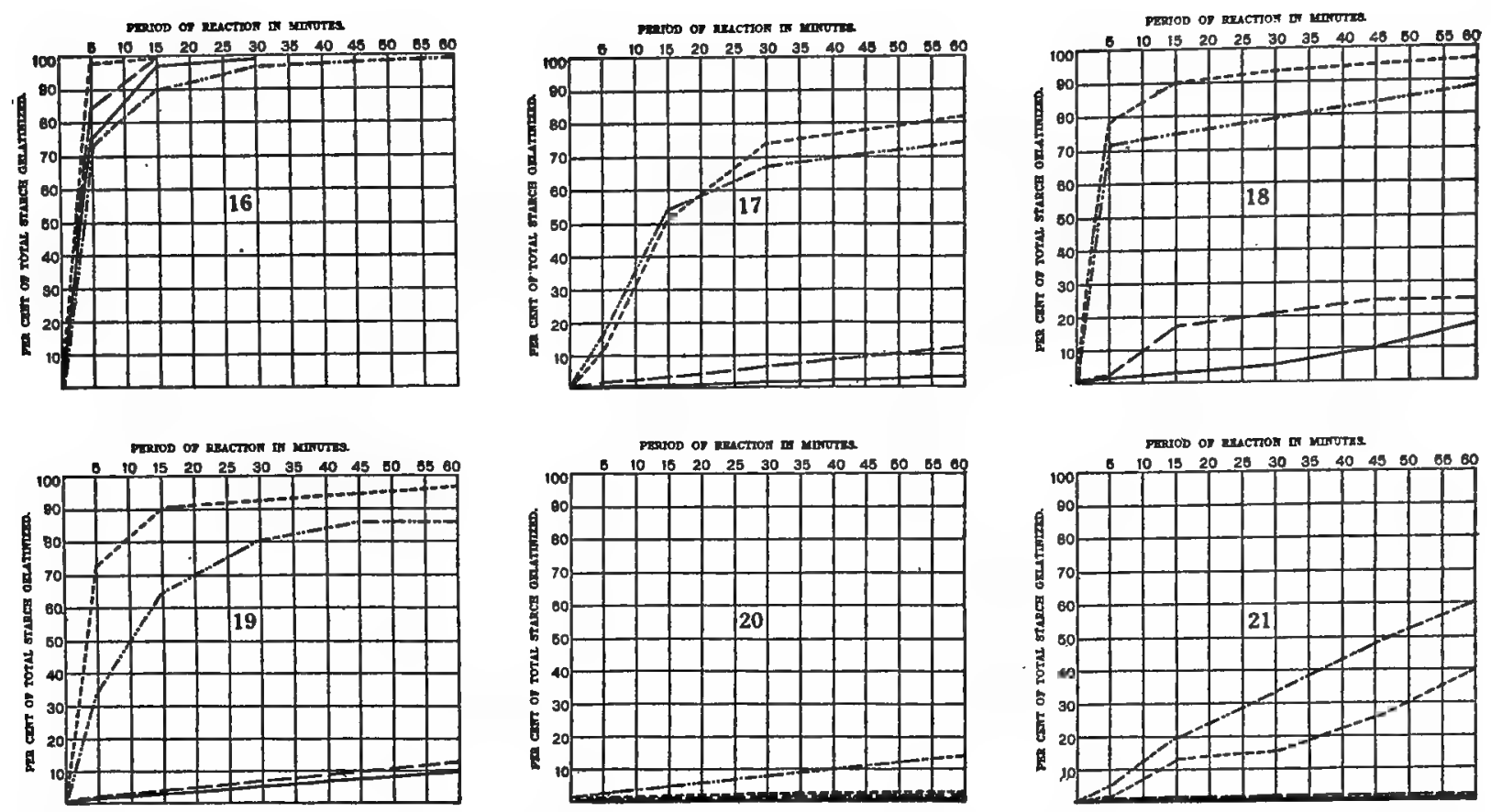

Charts D 16 to D 21.-Velocity-Reactions of Starches of Amaryllis belladonna (--.--), Brunsvigia josephince ( (......), Brunsdonna sanderœ alba (—), and Brunsdonna sanderæ (- $\longrightarrow$ ).
16. With Strontium Nitrate.
18. With Copper Nitrate.
20. With Barium Chloride.
17. With Cobalt Nitrate.
19. With Cupric Chloride.
21. With Mercuric Chloride.
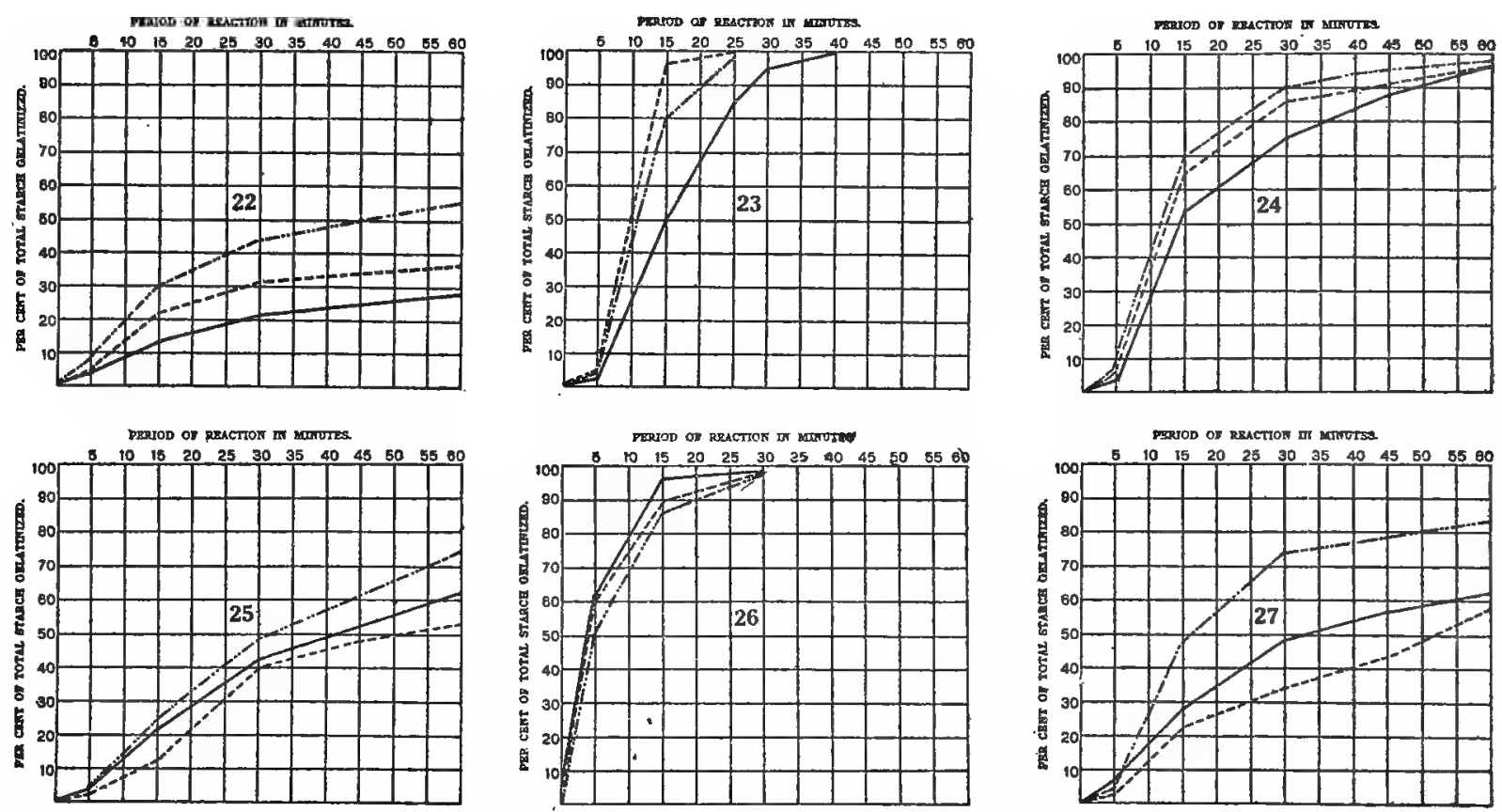

Charts D 22 то D 27.-Velocity-Reactions of Starches of Hippeastrum titan (....), H. cleonia (...... ), and H. titan-cleonia (-). 


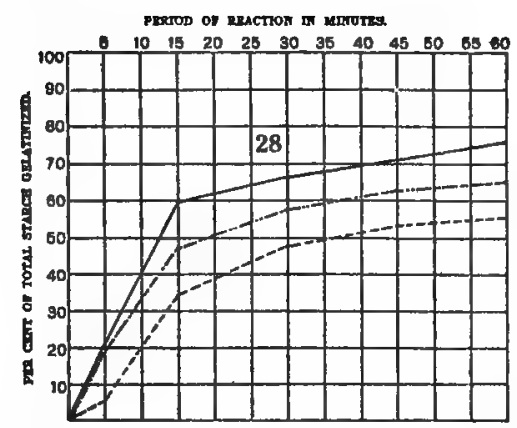

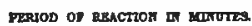

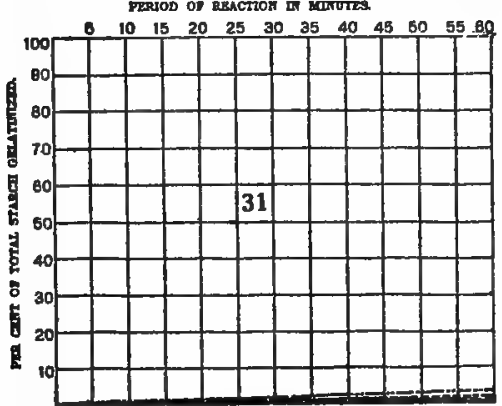

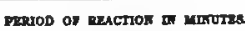

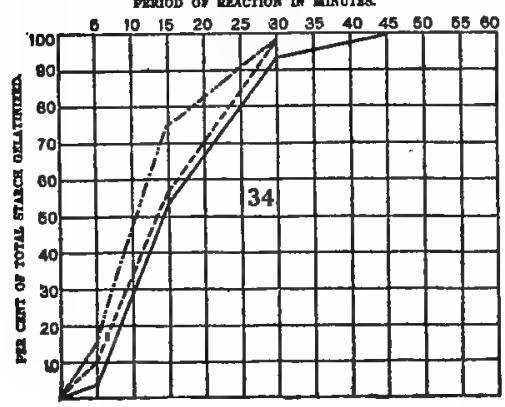

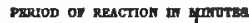

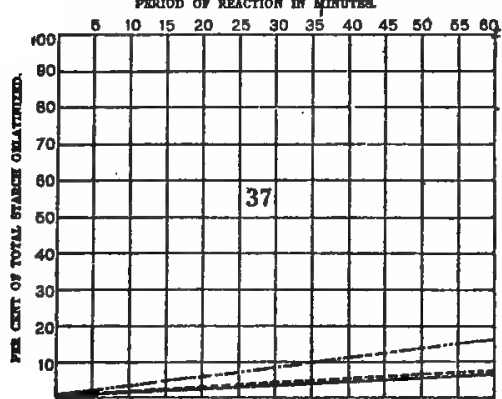

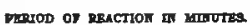

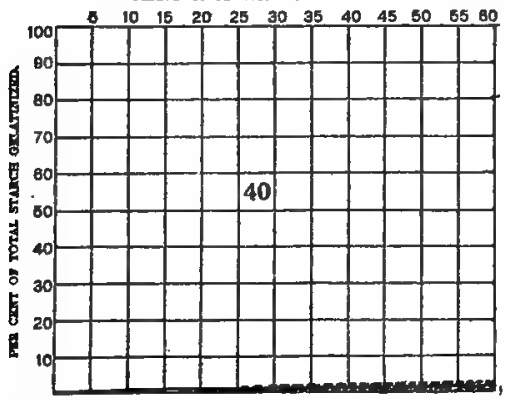

panod an kenction in wowrm

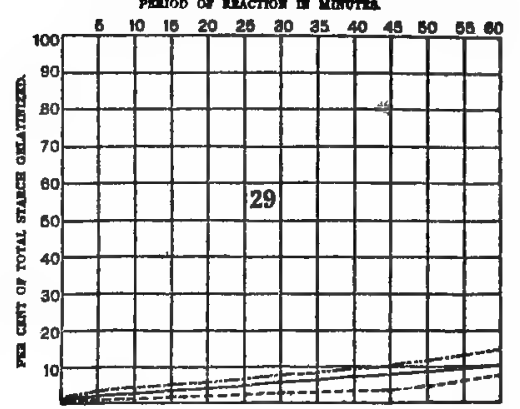

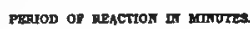

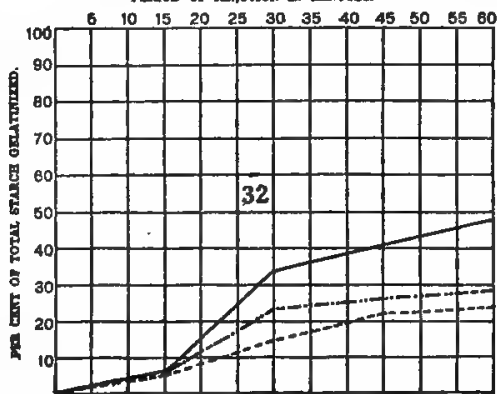

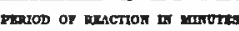

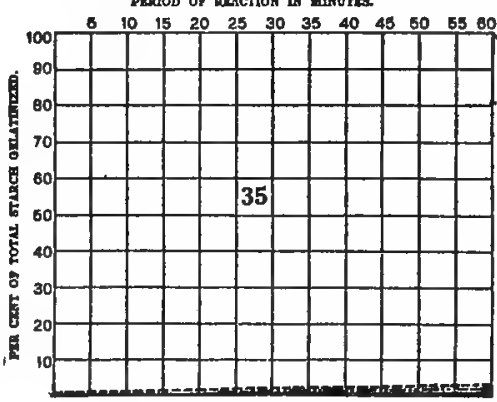

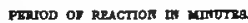

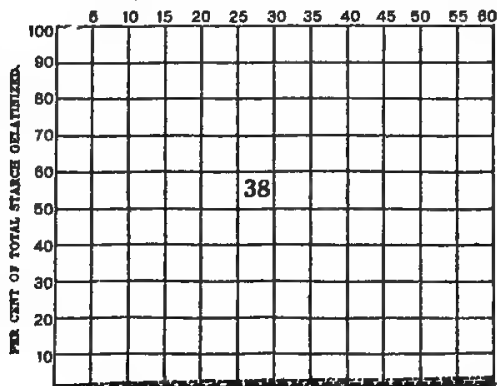

PERTOD of REACTON or MerTUES

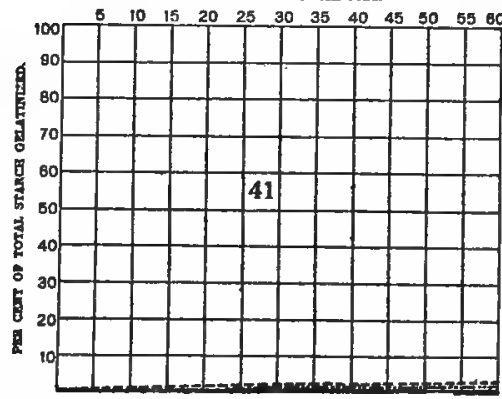

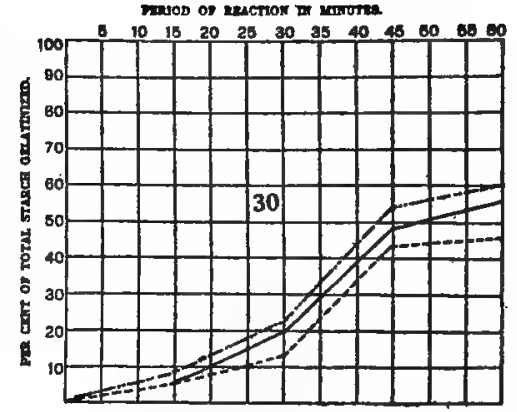

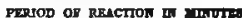

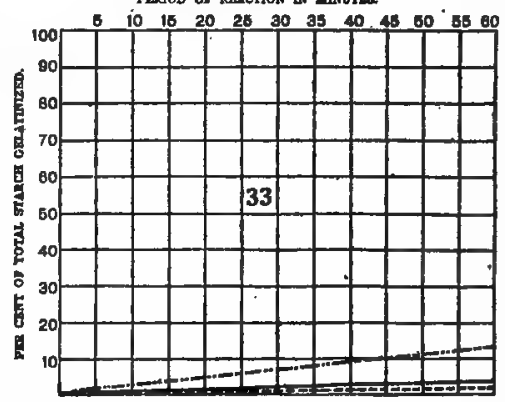

praion of mectron an urrorm

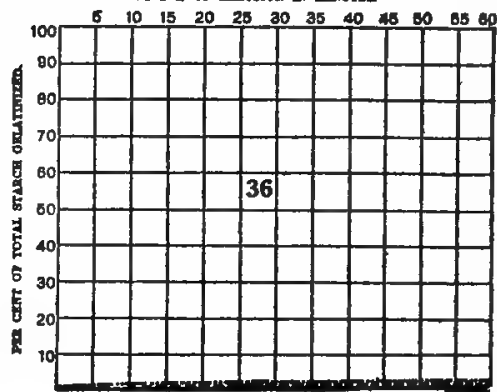

Marod os mectros an unsures

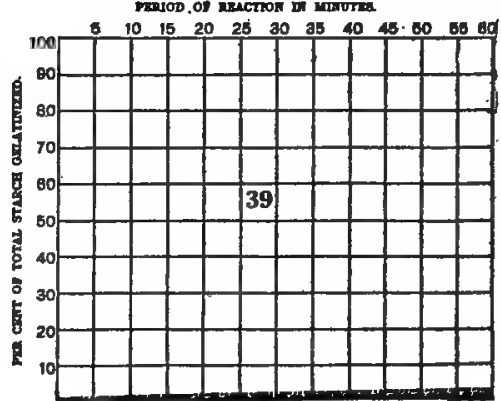

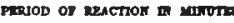

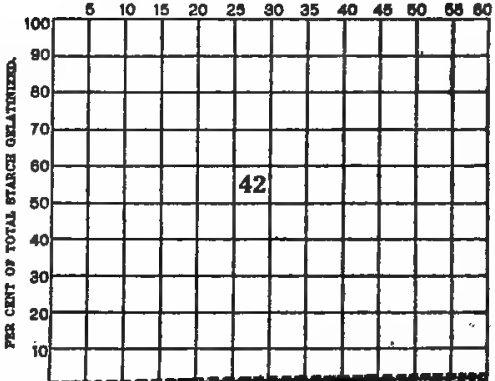

Charts D 28 то D 42.-Velocity-Reactions of Starches of Hippeastrum titan (-...-), H. cleonia ( .......), and

28. With Potassium Hydroxide.

29. With Potassium Iodide.

30. With Potassium Sulphocyanate.

31. With Potassium Sulphide.

32. With Sodium Hydroxide.

\section{H. titan-cleonia (-).}

33. With Sodium Sulphide.

34. With Sodium Salicylate.

35. With Calcium Nitrate.

36. With Uranium Nitrate.

37. With Strontium Nitrate.
38. With Cobalt Nitrate.

39. With Copper Nitrate.

40. With Cupric Chloride.

41. With Barium Chloride. 


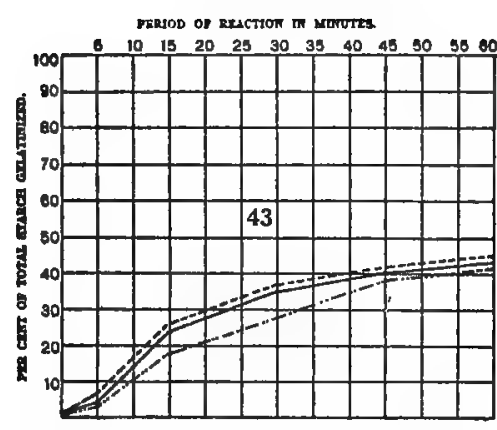

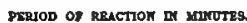

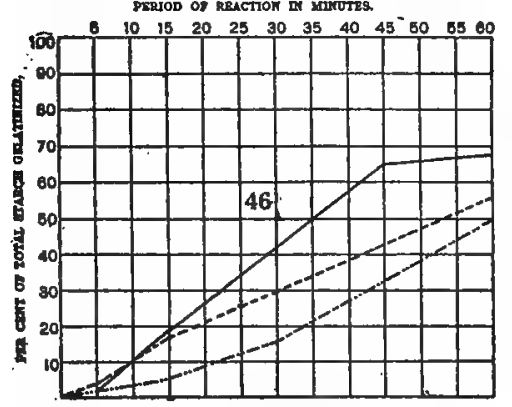

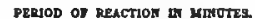

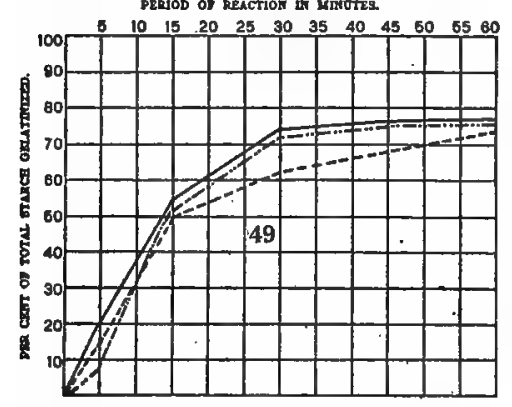

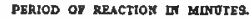

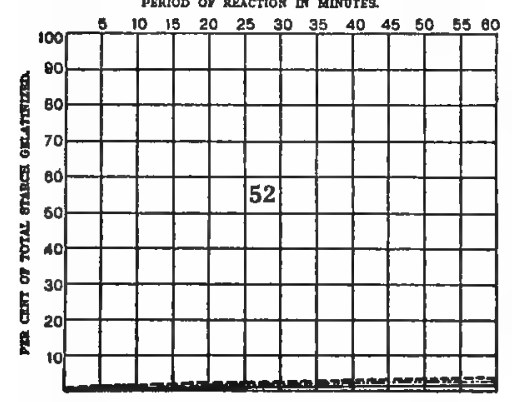

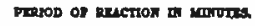

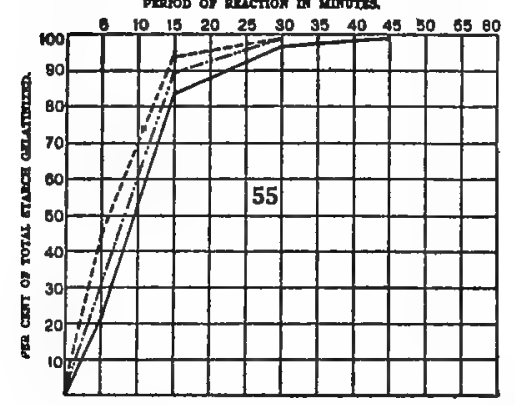

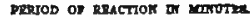

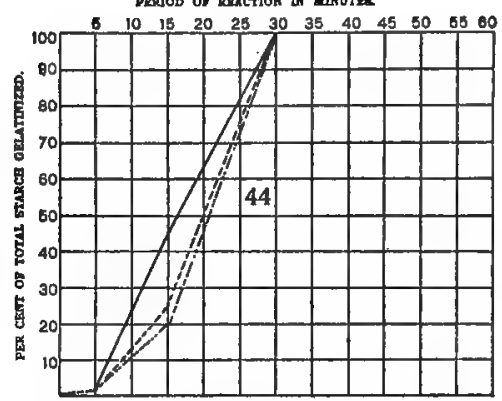

PERrod Op ABaction IN MRNOTES

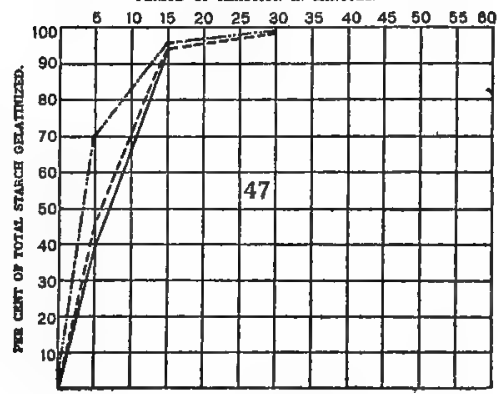

PERTOD OF RECCTIOK W MHTOTSS

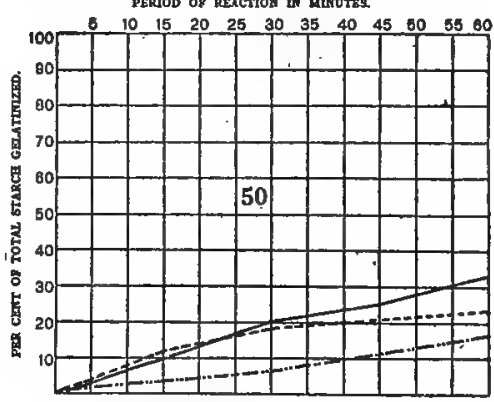

PEROD OF RERCTION In METUTRs.

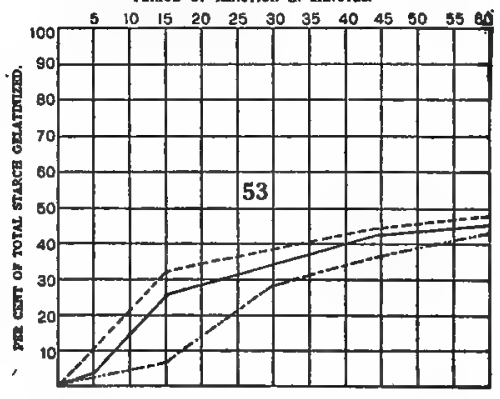

PEROD OP RZACTION IN MATSUTES

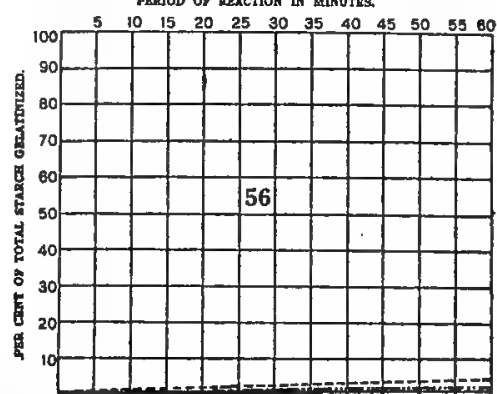

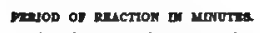

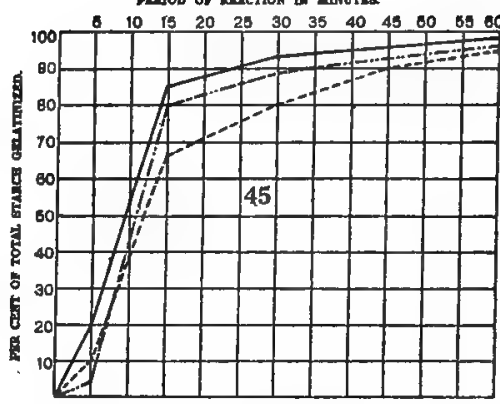

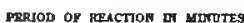

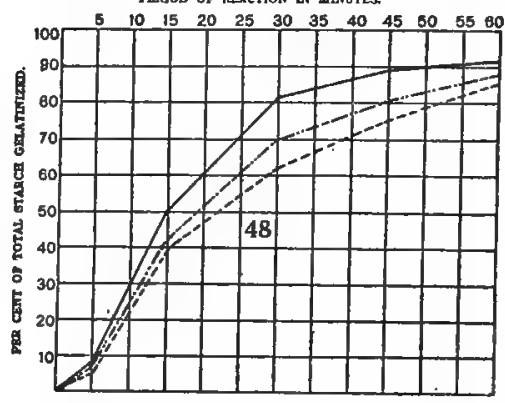

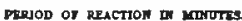

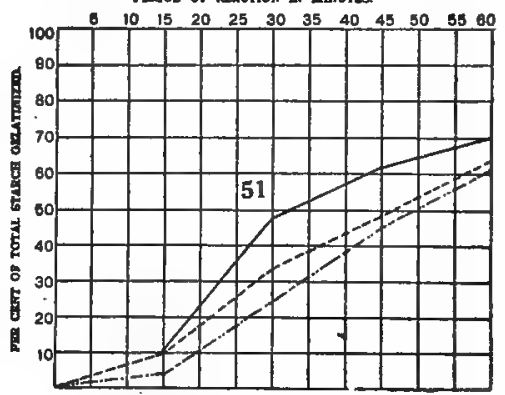

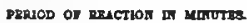

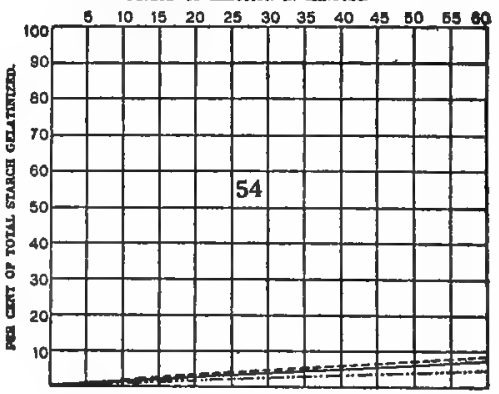

PENOD OI RRACTION IN MENOTRS

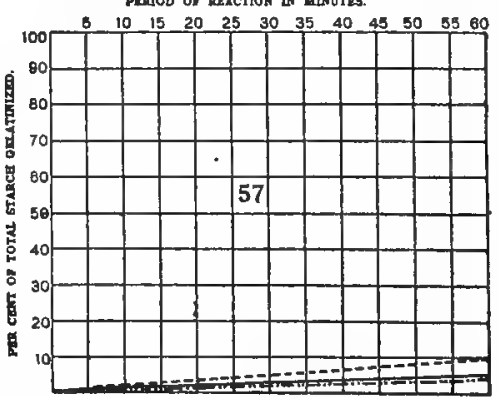

CharTs D 43 to D 57.-Velocity-Reactions of Starches of Hippeastrum ossultan ( -...), H. pyrrha ( ......), and H. ossultan-pyrrha (-).

43. With Chloral Hydrate. 44. With Chromic Acid. 45. With Pyrogallic Acid

46. With Nitric Acid

47. With Sulphuric Acid.
48. With Hydrochloric Acid.

49. With Potassium Hydroxide.

50. With Potassium Iodide.

51. With Potassium Sulphocyanate.

52. With Potaseium Sulphide.
53. With Sodium Hydroxide.

54. With Sodium Sulphide.

55. With Sodium Salicylate.

57. With Uranium Nitrate. 
merob of reaction Is hapteses

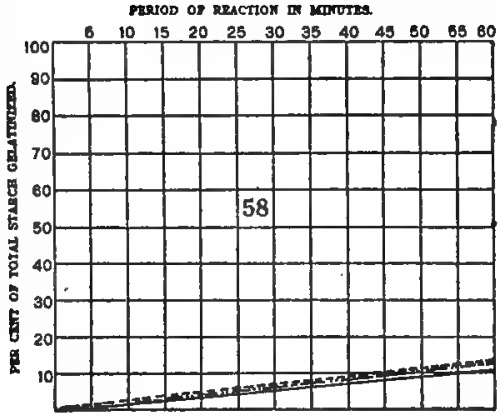

DRRIOD OF RRACTION DI MTNTTE

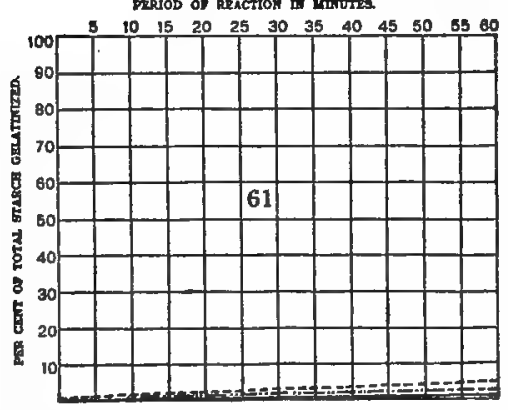

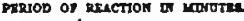
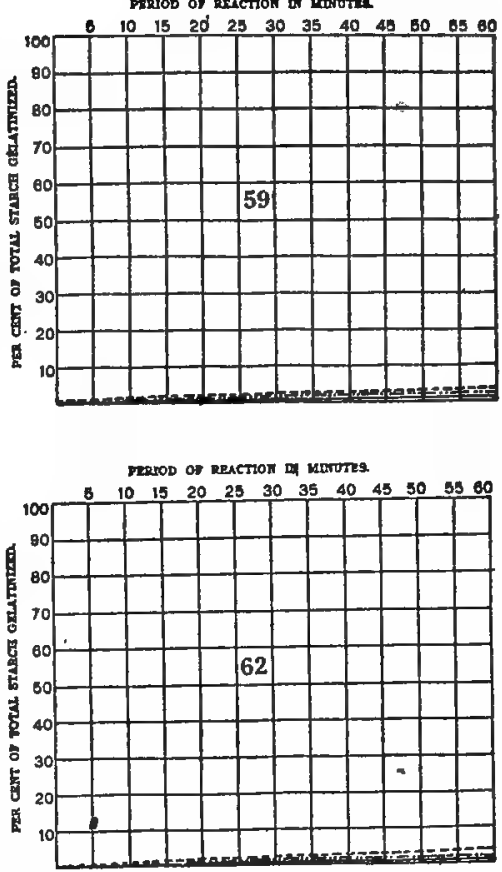

maros of ancroin a kerotse

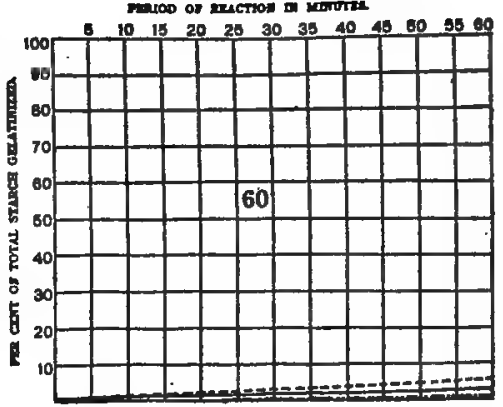

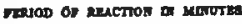

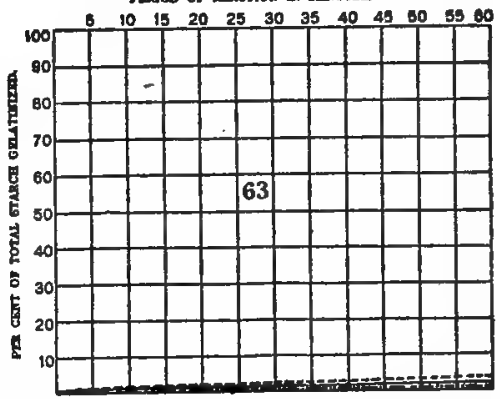

Charts D 58 ro D 63.-Velocity-Reactions of Starches of Hippeastrum ossultan ( .....), H. pyrrha (.......), and H. ossultan-pyrrha (-).

60. With Copper Nitrate.

62. With Barium Chloride

58. With Strontium Nitrate

69. With Cobalt Nitrate.

61. With Cupric Chloride.

63. With Merourio Chloride.
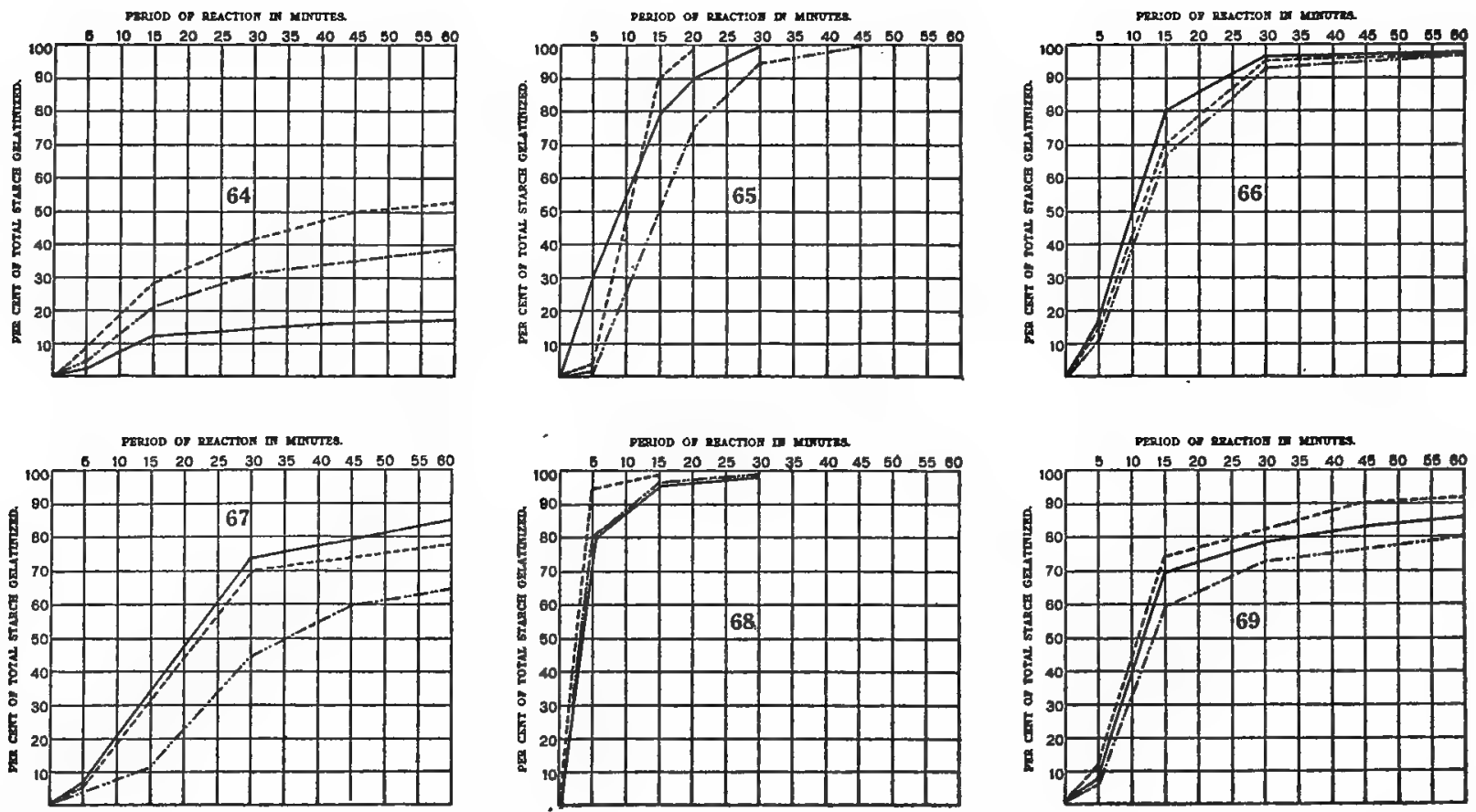

Charts D 64 to D 69.-Velocity-Reactions of Starches of Hippeastrum dwones (....), H. zephyr (-......), and $H$. doones-zephyr (-).
64. With Chloral Hydrato.
66. With Pyrogallic Acid.
67. With Nitric Acid.
68. With Sulphuric Acid.
69. With Hydrachlorio Acid. 
Prouod of maction in ywotre.

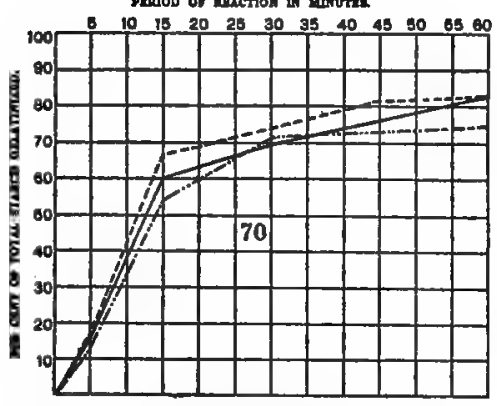

pentod of Rection on Merotra

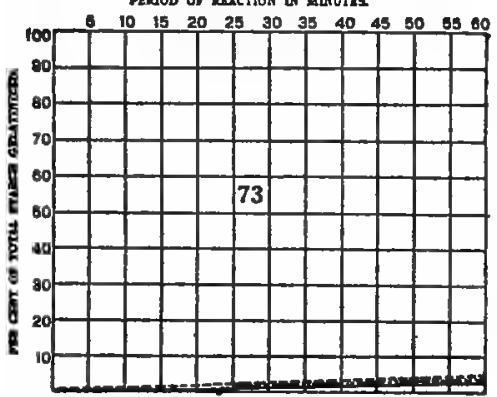

prouob os pectron in morotes.

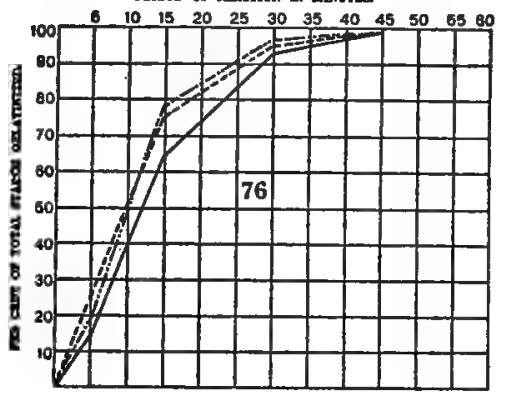

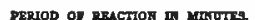

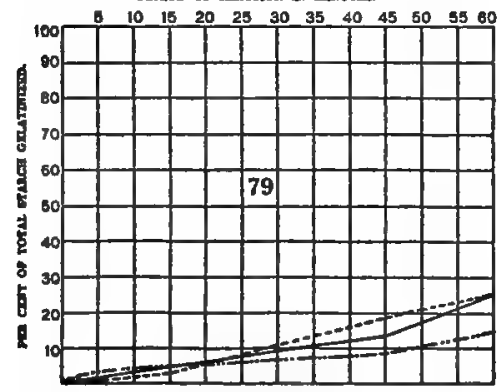

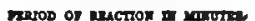

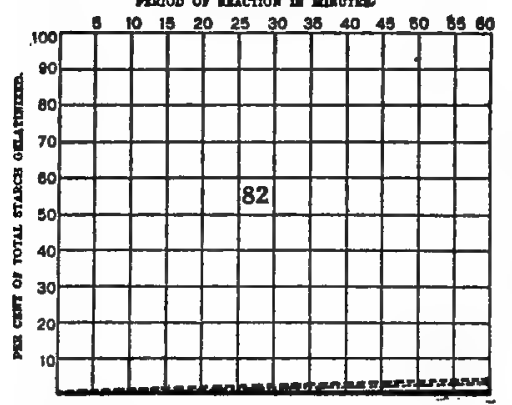

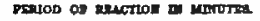

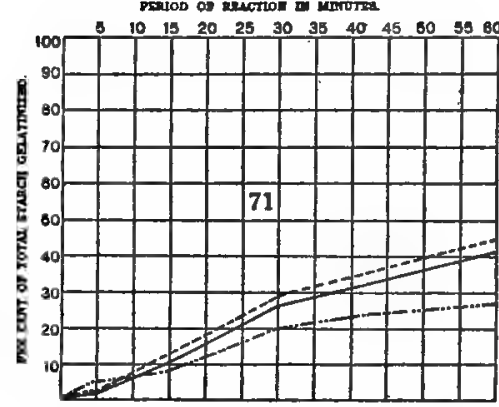

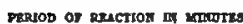

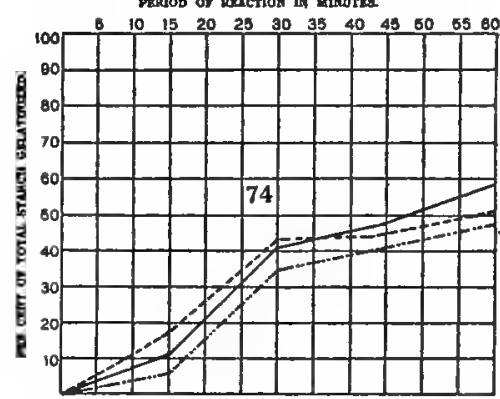

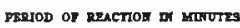

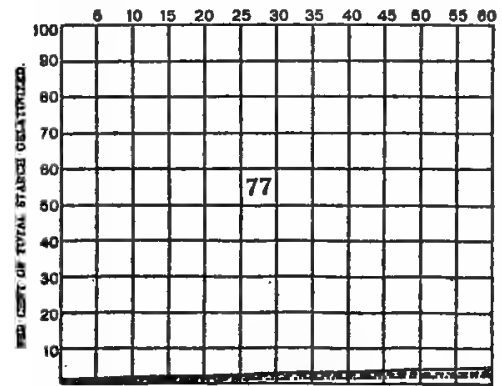

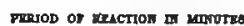

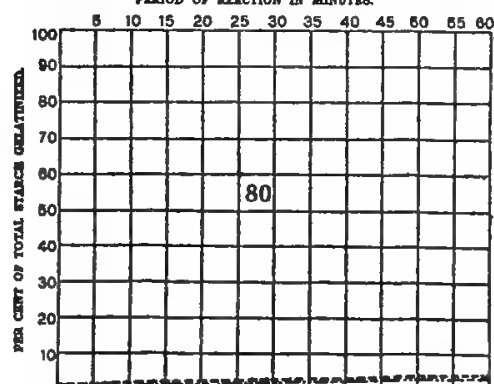

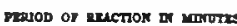

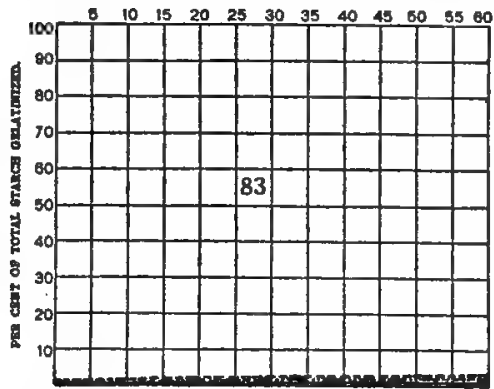

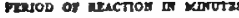

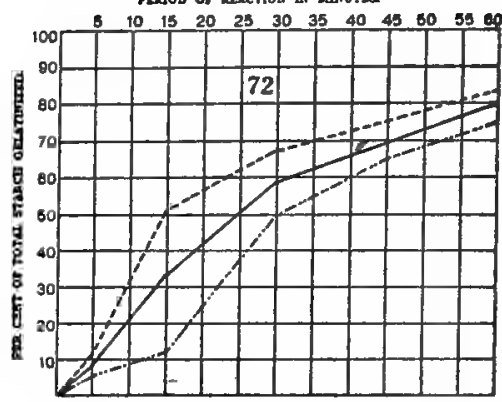

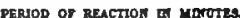

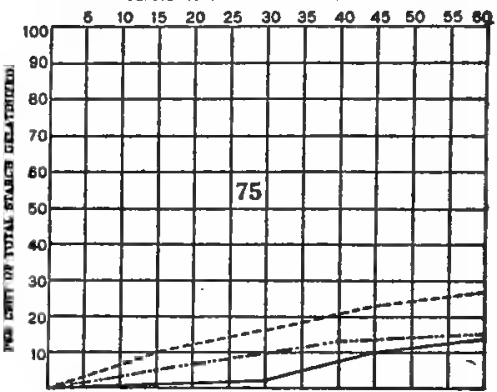

presod of enctioni us menutes

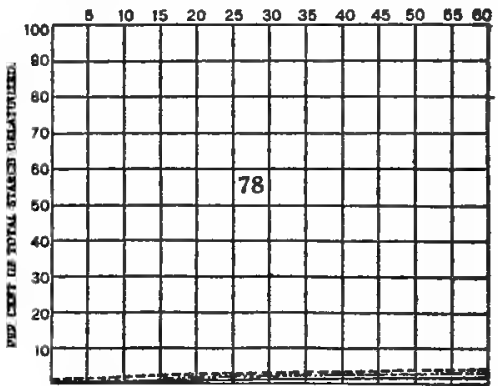

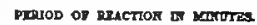

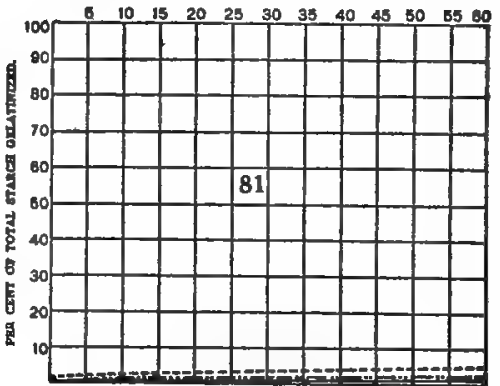

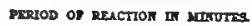

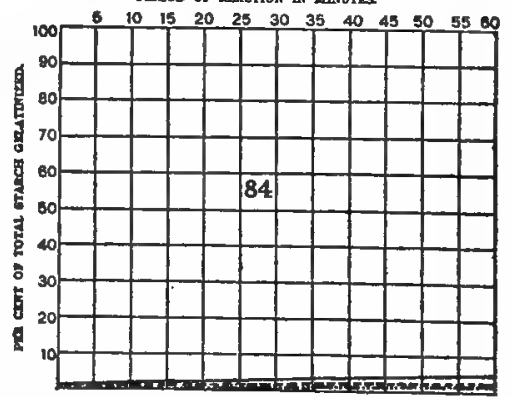

Charts D 70 то D 84.-Velocity-Reactions of Starches of Hippeastrum dceones (-...), H. zephyr ( ......), and $H$. daones-zephyr (-).

70. With Potassium Hydroxide.

71. With Potassium Iodide.

72. With Potareium Sulphocyanate.

73. With Potssaium Sulphide.

74. With Sodium Hydroxide.
75. With Sodium Sulphide.

76. With Sodium Salicylate.

77. With Calcium Nitrate.

78. With Uranium Nitrate.

79. With Strontium Nitrate.
80. With Cobalt Nitrate.

81. With Copper Nitrate.

83. With Cupric Chloride

84. With Mercuric Chloride. 


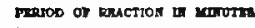

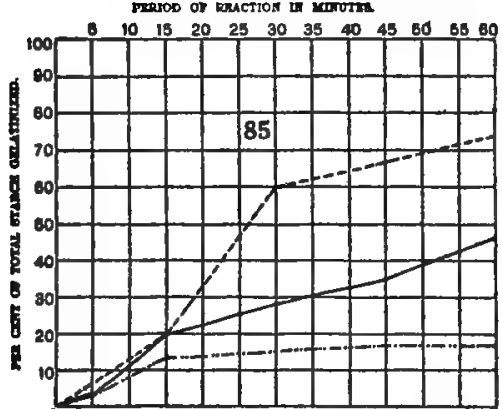

meriod oy reaction an umoteg.

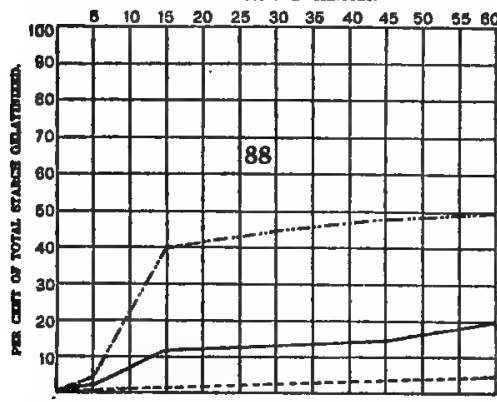

PRRIOD OF REACTION If MTNUTES

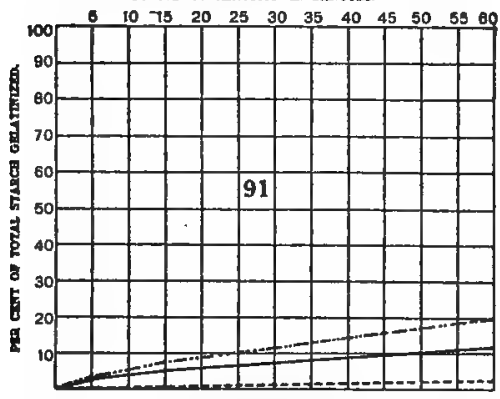

PENOD on DESction IT METTES

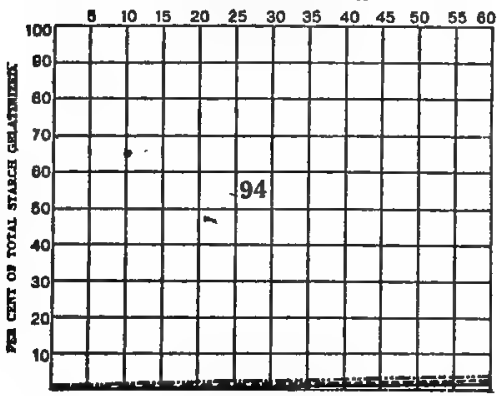

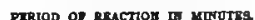

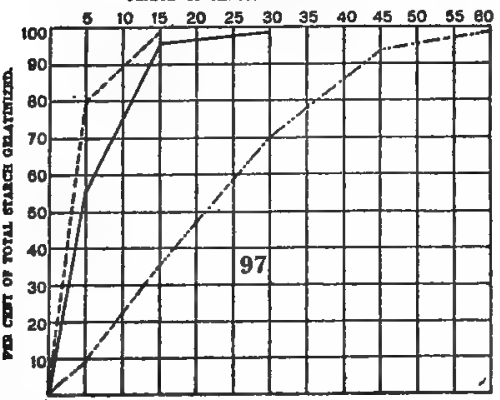

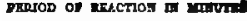

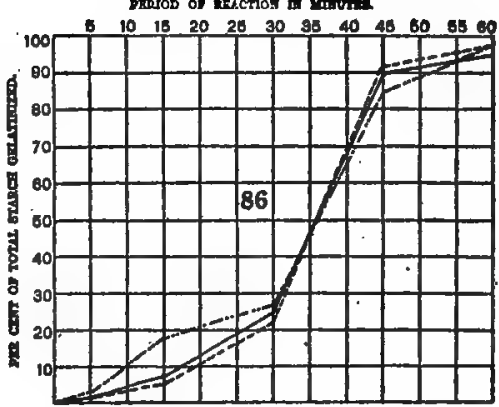

MERiod of anction IN MDNoTEs

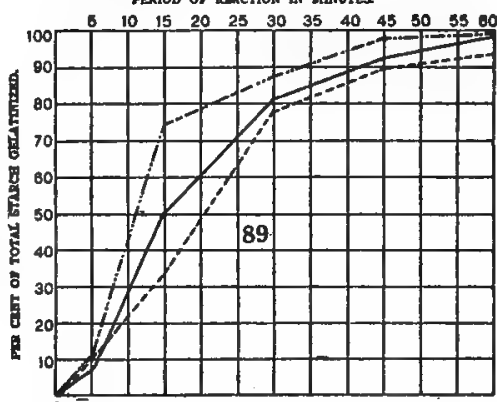

Frerod of genction as Mmotzs

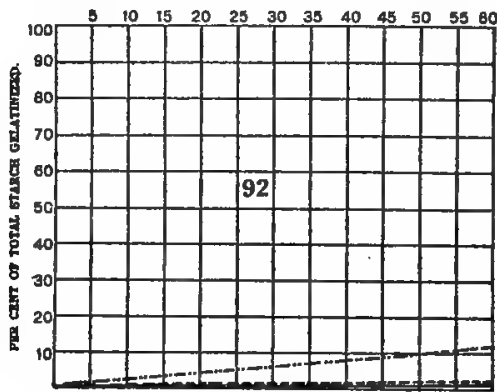

PERIOD of REACtION LT HESTRES

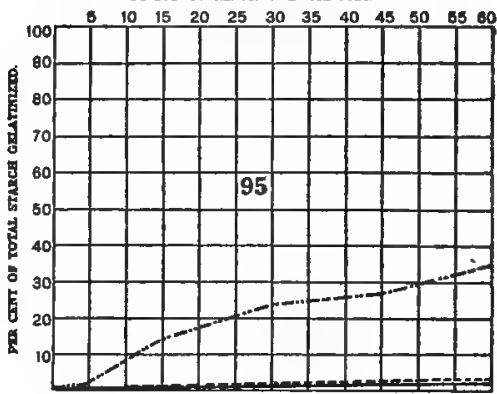

(10)

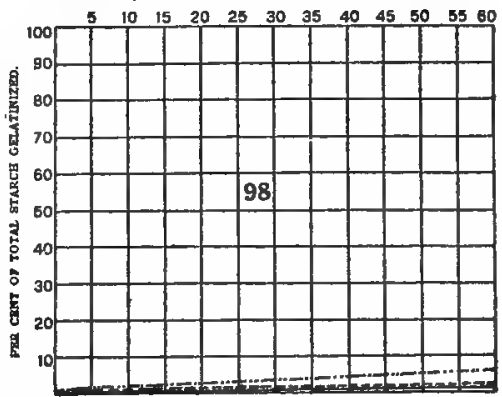

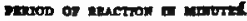

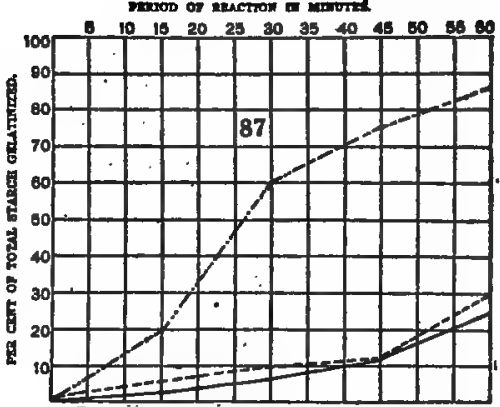

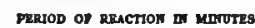

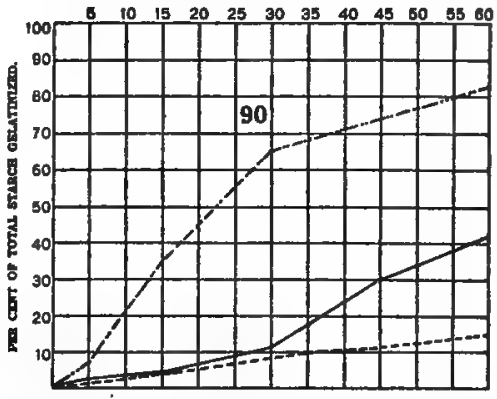

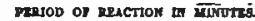

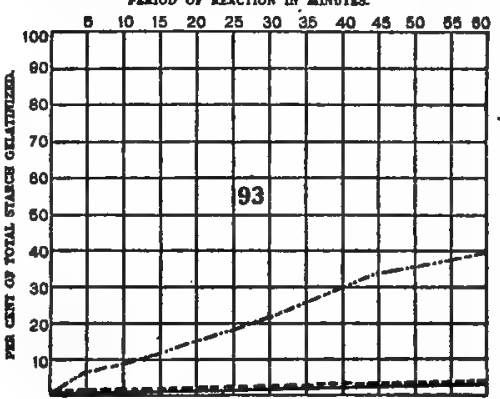

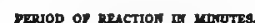

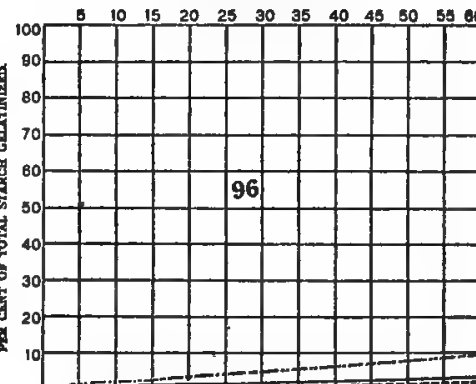

ERLoD of enector mortes

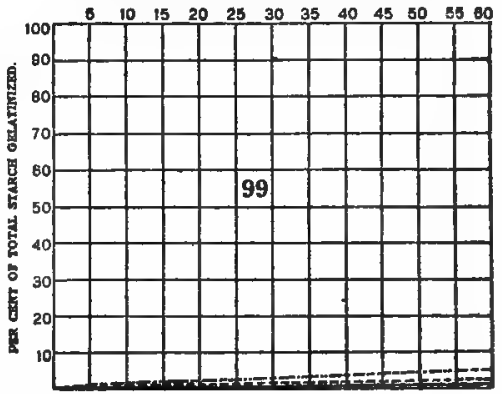

Charts D 85 to D 99.-Velocity-Reactions of Starches of Hœmanthus katherince (.....), H. magnificus ( .......), and $H$. andromeda (—).

85. With Chloral Hydrate.

86. With Chromic Acid

87. With Pyrogallic Acid.

88. With Nitric Acid.

89. With Sulphuric Acid.
90. With Hydrochloric Acid.

91. Wi th Potasium Hydroxide.

92. With Potassium Iodide.

03. With Potaseium Sulphocyanate.

94. With Potarium Sulphide.
95. With Sodium Hydroxide.

96. With Sodium Sulphide.

97. With Sodium Salicylate.

98. With Calcium Nitrate. 

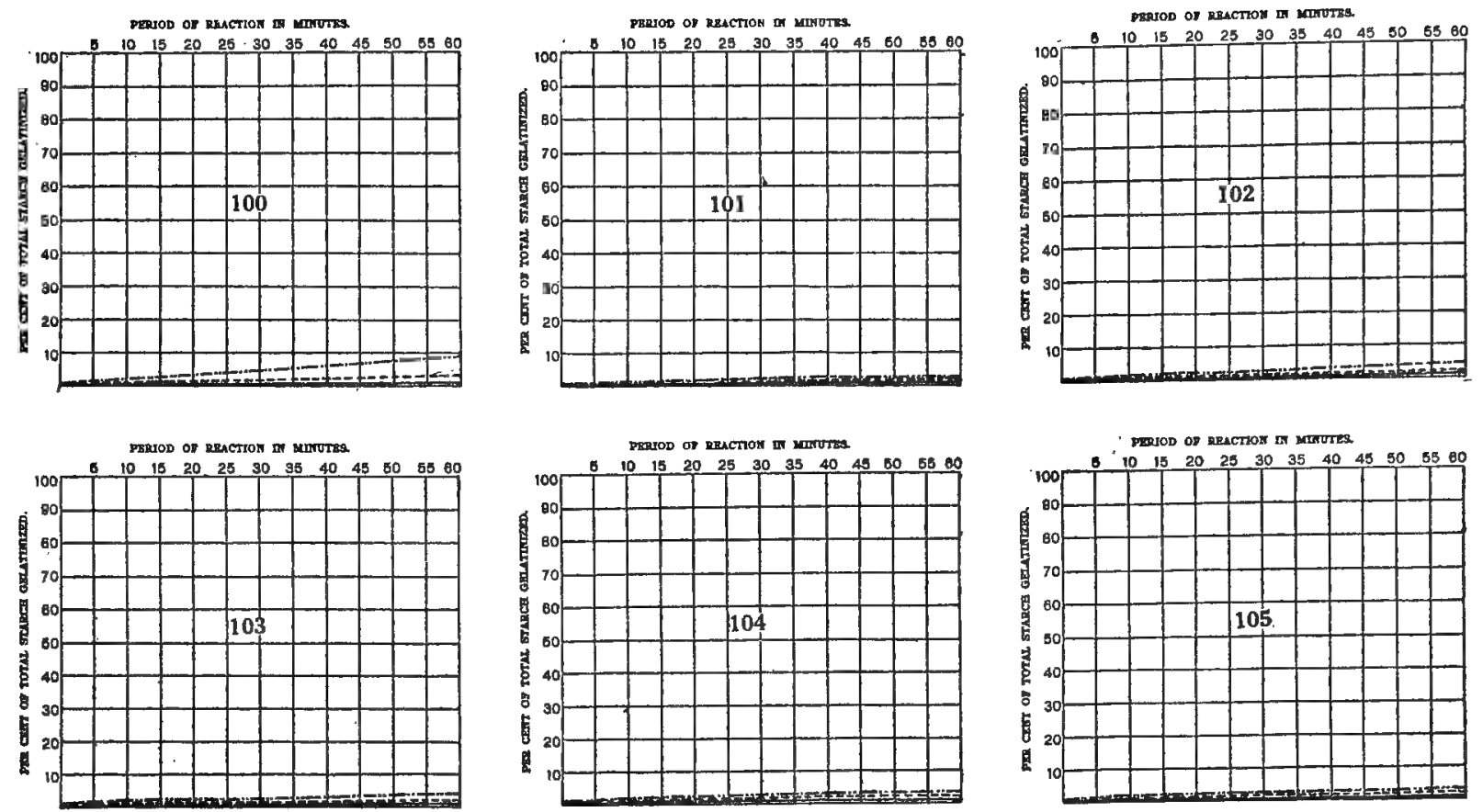

Charts D 100 to D 105.-Velocity-Reactions of Starches of Homanthus katherina ( -...-), H. magnificus ( -......), and H. andromeda (-).

100. With Strontium Nitrate.

102. With Copper Nitrate.

104. With Barium Chloride. 101. With Cobalt Nitrate.
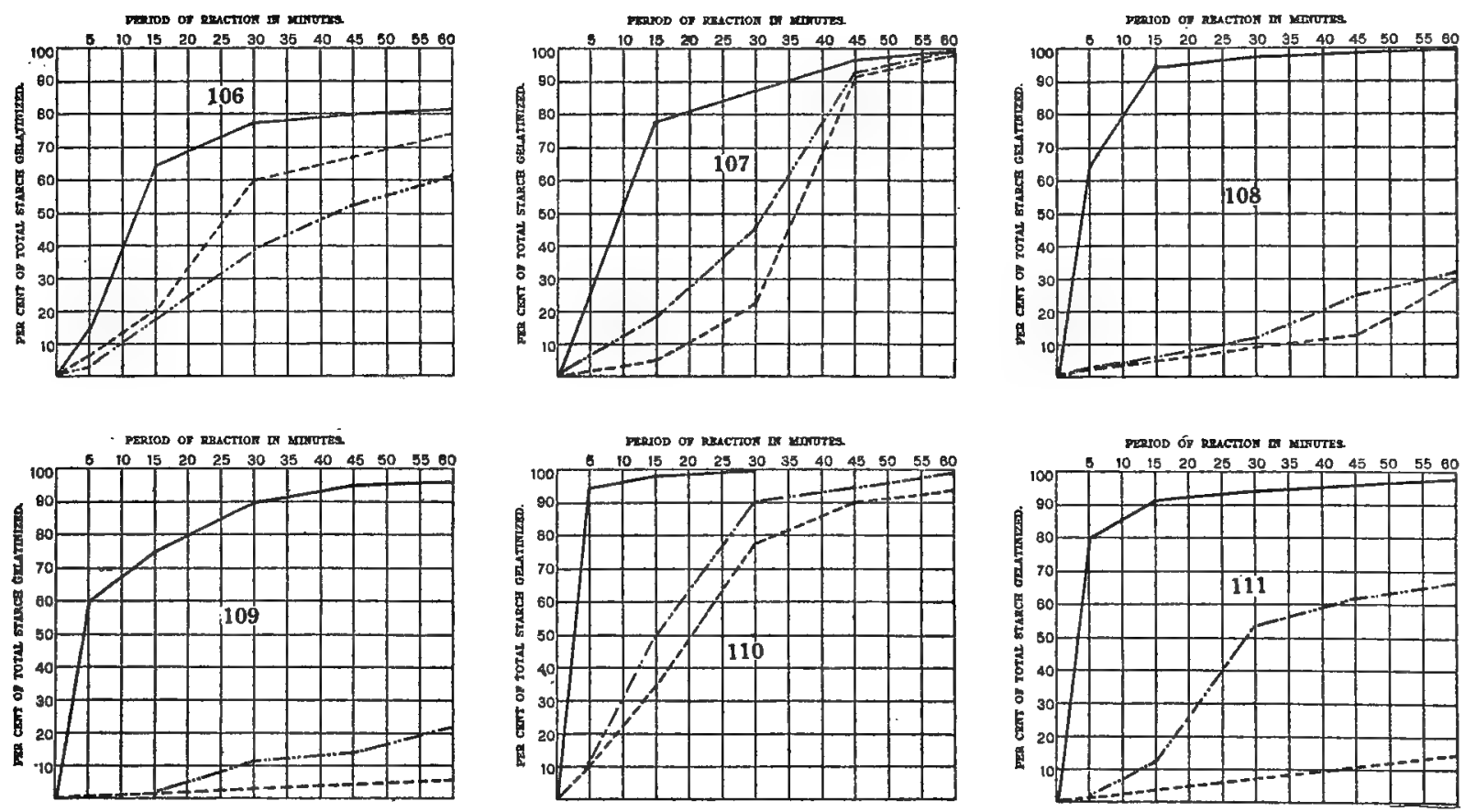

CharTs D 106 to D 111.-Velocity-Reactions of Starches of Homanthus katherince ( - ...-), Hamanthus puniceus (-......), and Homanthus könig albert (-). 
mos of acherion th mortes.

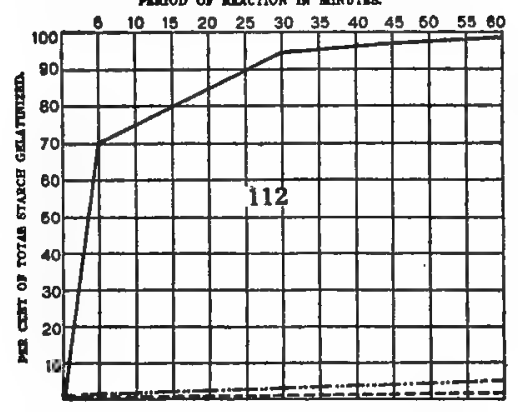

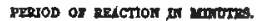

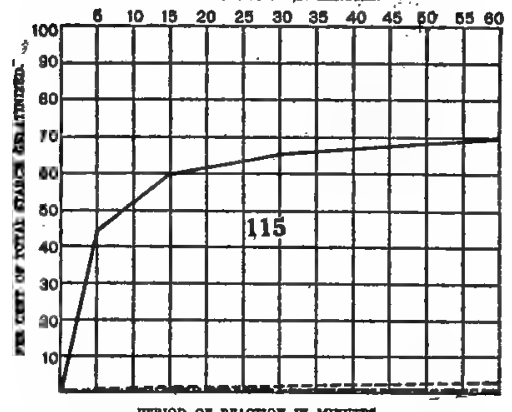

in

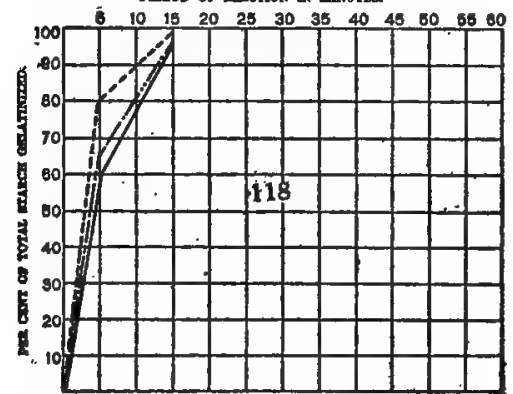

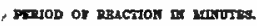

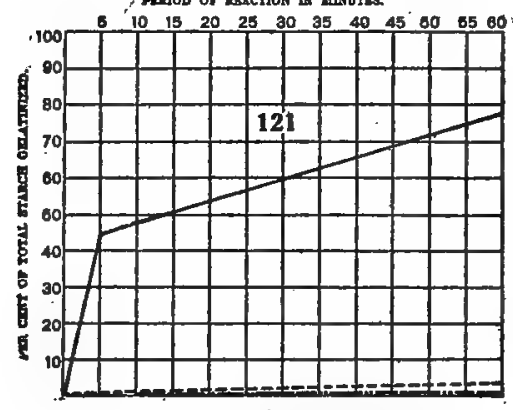

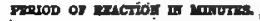

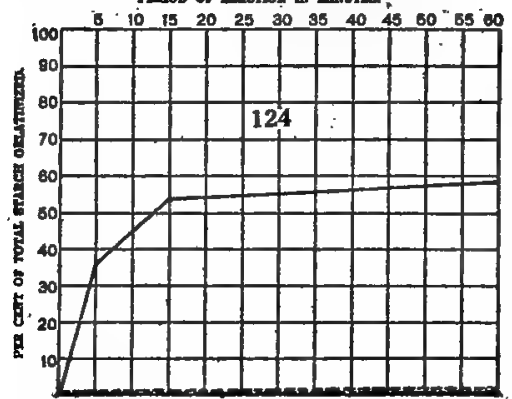

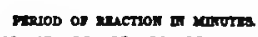

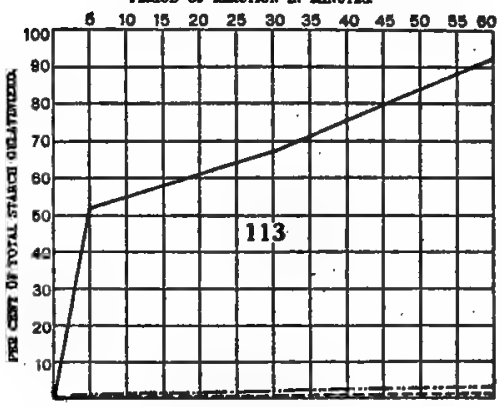

pranob of ezection no morotsa

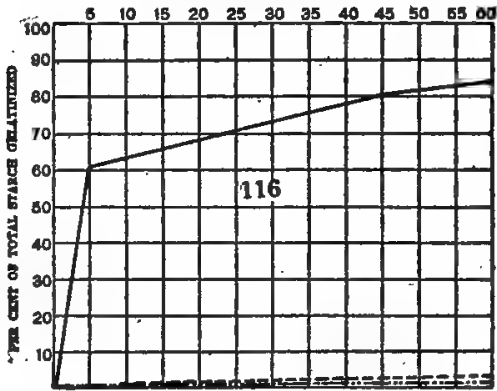

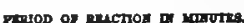

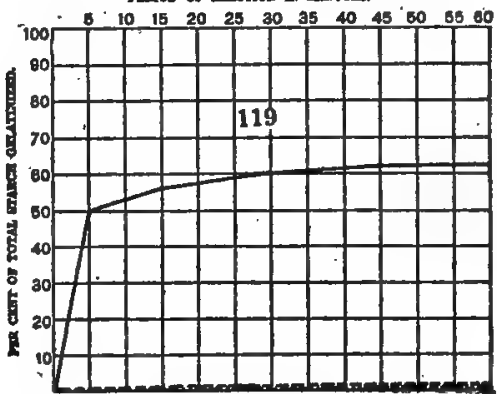

pretos of mecrion an verotres

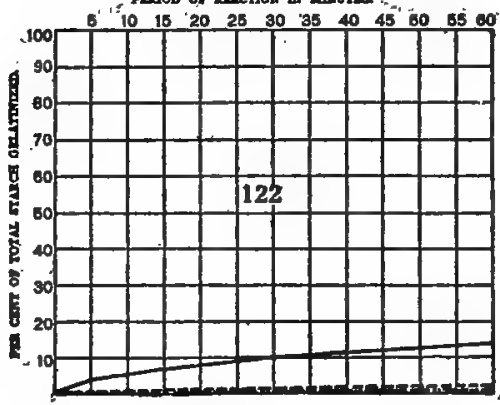

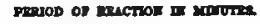

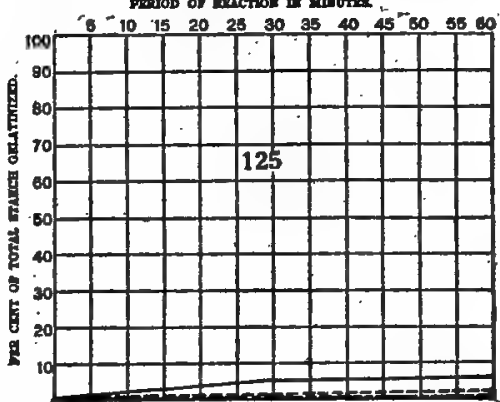

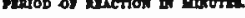

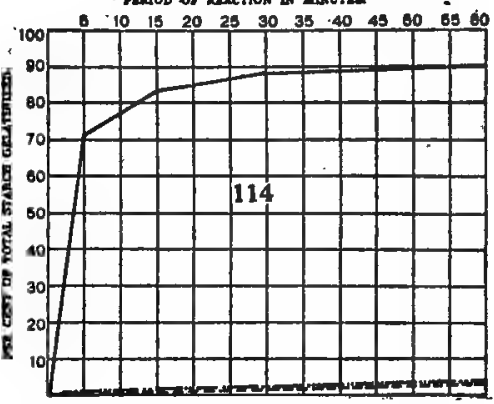

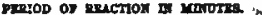

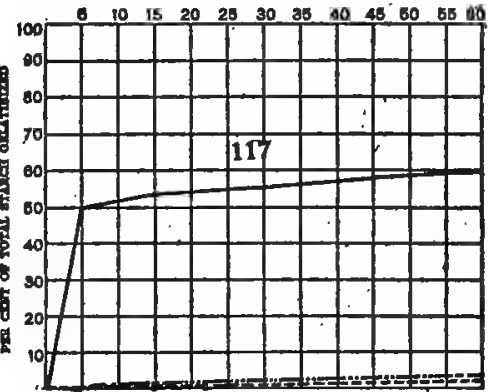

mod of puction on martres

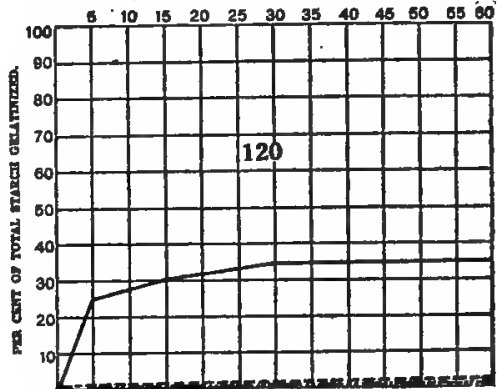

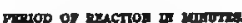

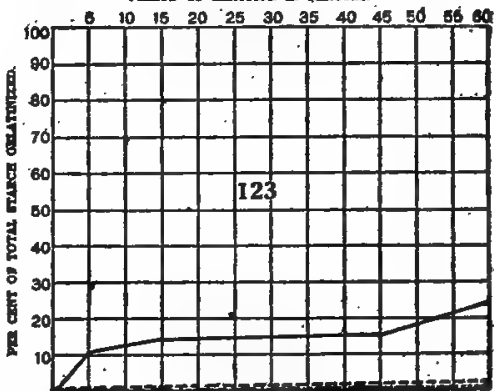

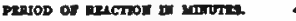

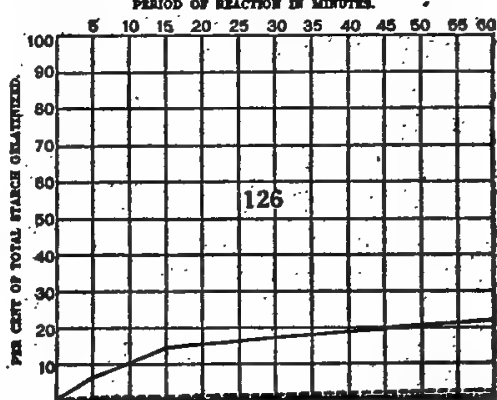

ChaRTs D 112 то D 126.-Velocity-Reactions of Starches of Hæmanthus katherinæ (-...-), Homanthus puniceus ( -......), and Hamanthus könig albert (-).

$\begin{array}{lll}\text { 112. With Potassium Hydroxide. } & \text { 117. With Sodium Sulphide. } & \text { 122. With Cobalt Nitrate. } \\ \text { 113. With Potassium Iodide. } & 118 . \text { With Sodium Salicylate. } & \text { 123. With Copper Nitrate. } \\ \text { 114. With Potassium Sulphocyanate. } & 119 . \text { With Calcium Nitrate. } & \text { 124. With Cupric Choride. } \\ \text { 115. With Potassium Sulphide. } & \text { 120. With Uranium Nitrate. } & \text { 125. With Barium Chloride. } \\ \text { 116. With Sodium Bydroxide. } & \text { 121. With Strontium Nitrate. } & \text { 126. With Mercuric Chloride. }\end{array}$




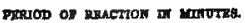

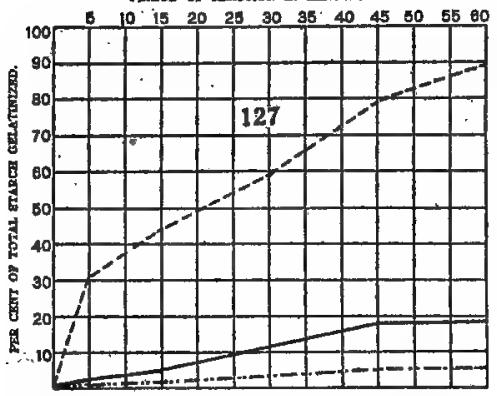

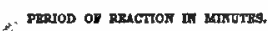

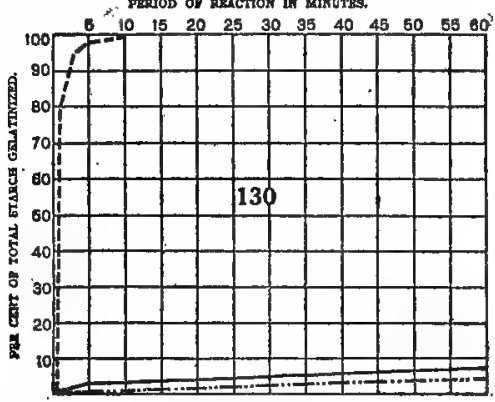

PERTOD Of RRACTION WA MESTITS

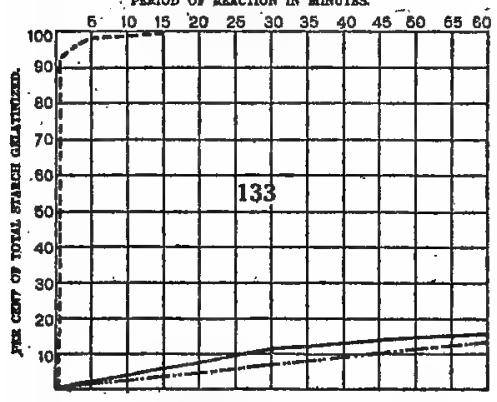

PRRTOD OY RRACTRON DN MERUTBS

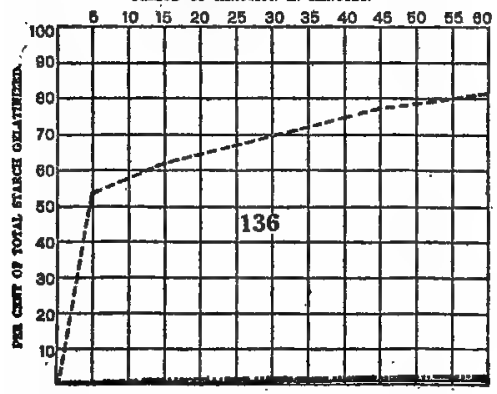

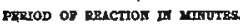

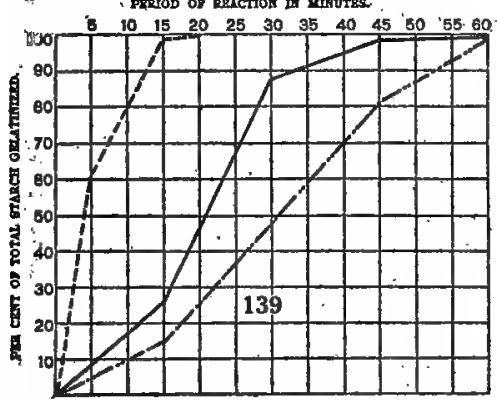

Charts D 127 to D 141.-Velocity-Reactions of Starches of Crinum moorei

127. With Chloral Hydrate. 128. With Chromic Acid.

129. With Pyrogallie Acid.

130. With Nitric Acid.

131. With Sulphurio Aoid.
Fgrod of meaction af Mersts.

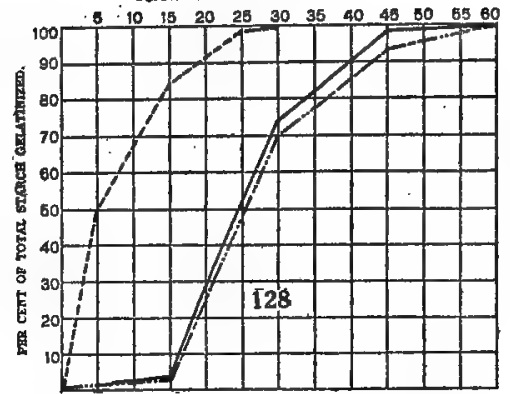

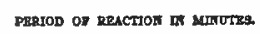

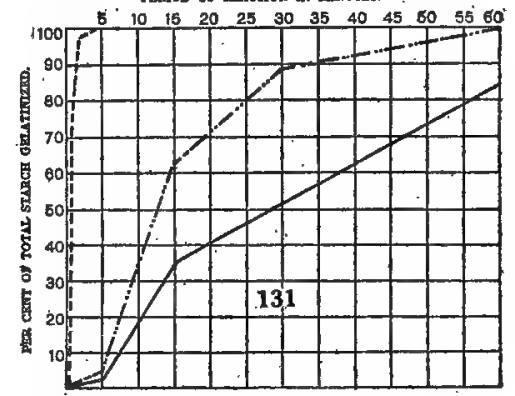

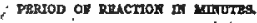

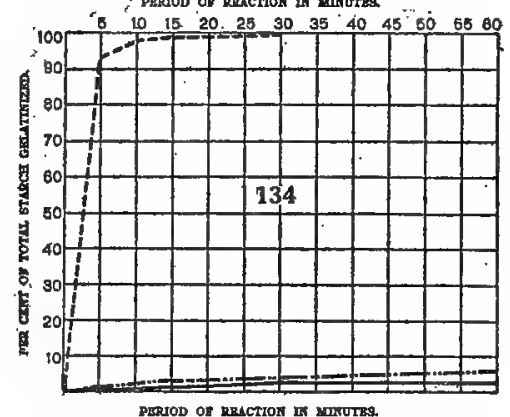

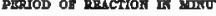

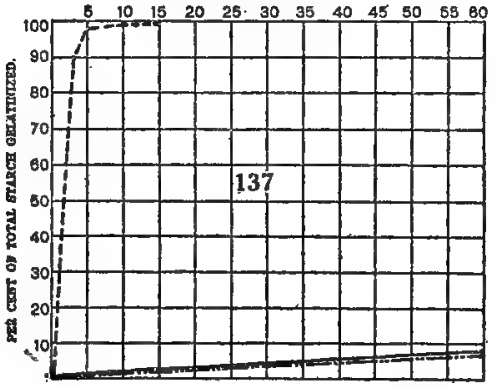

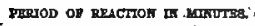

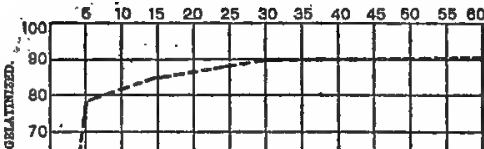

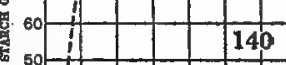

40

:

20

要

and Crinum hybridum j.c.h. (-)

132. With Hydrochloric Acid.

133. With Potassium Hydroxide.

134. With Potassium Iodide.

135. With Potagsium Sulphocyanste.

136. With Potassium Sulphide.

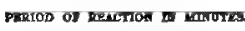

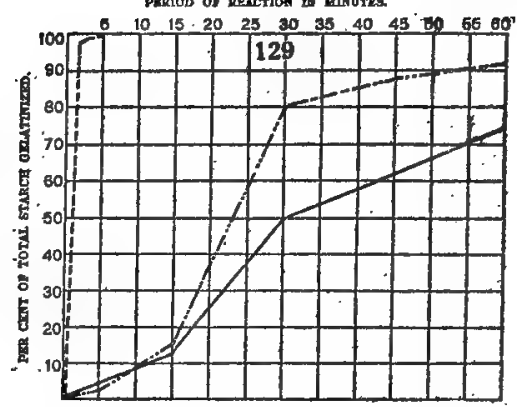

PERTOD OP REACTIOR II MLTUTES

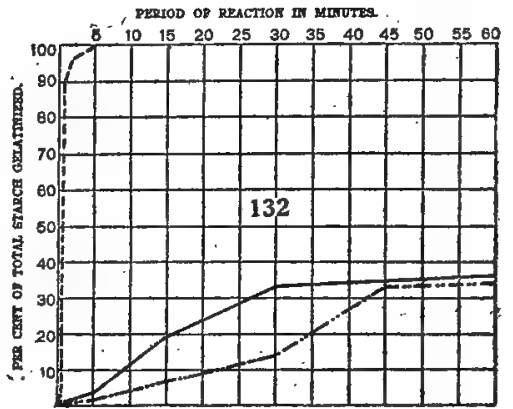

PERTOD OP DRACTIOF IN MUNUTE

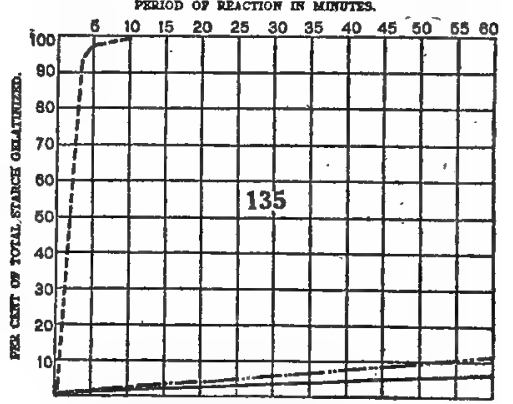

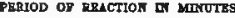

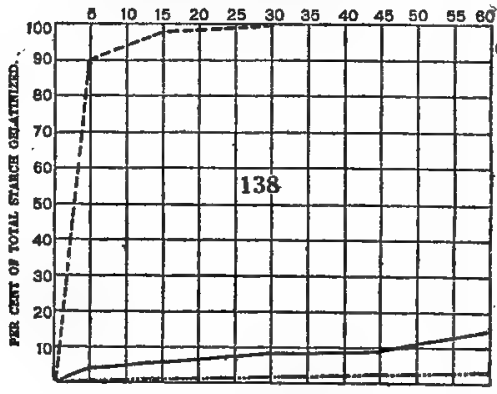

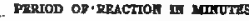

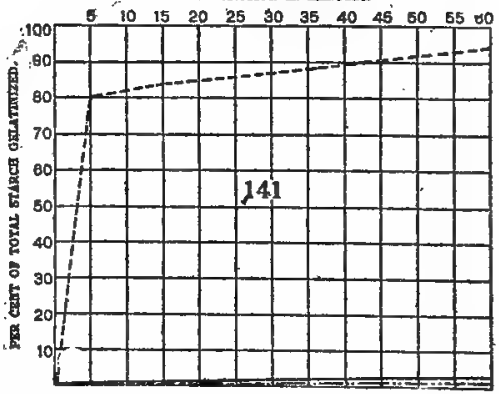

4 (and

( ...-), Crinum zeylanicum ( ......),

137. With Sodium Hydroxide.

138. With Sodium Sulobide.

139. With Sodium Salicylate.

140. With Caloium Nitrate. 

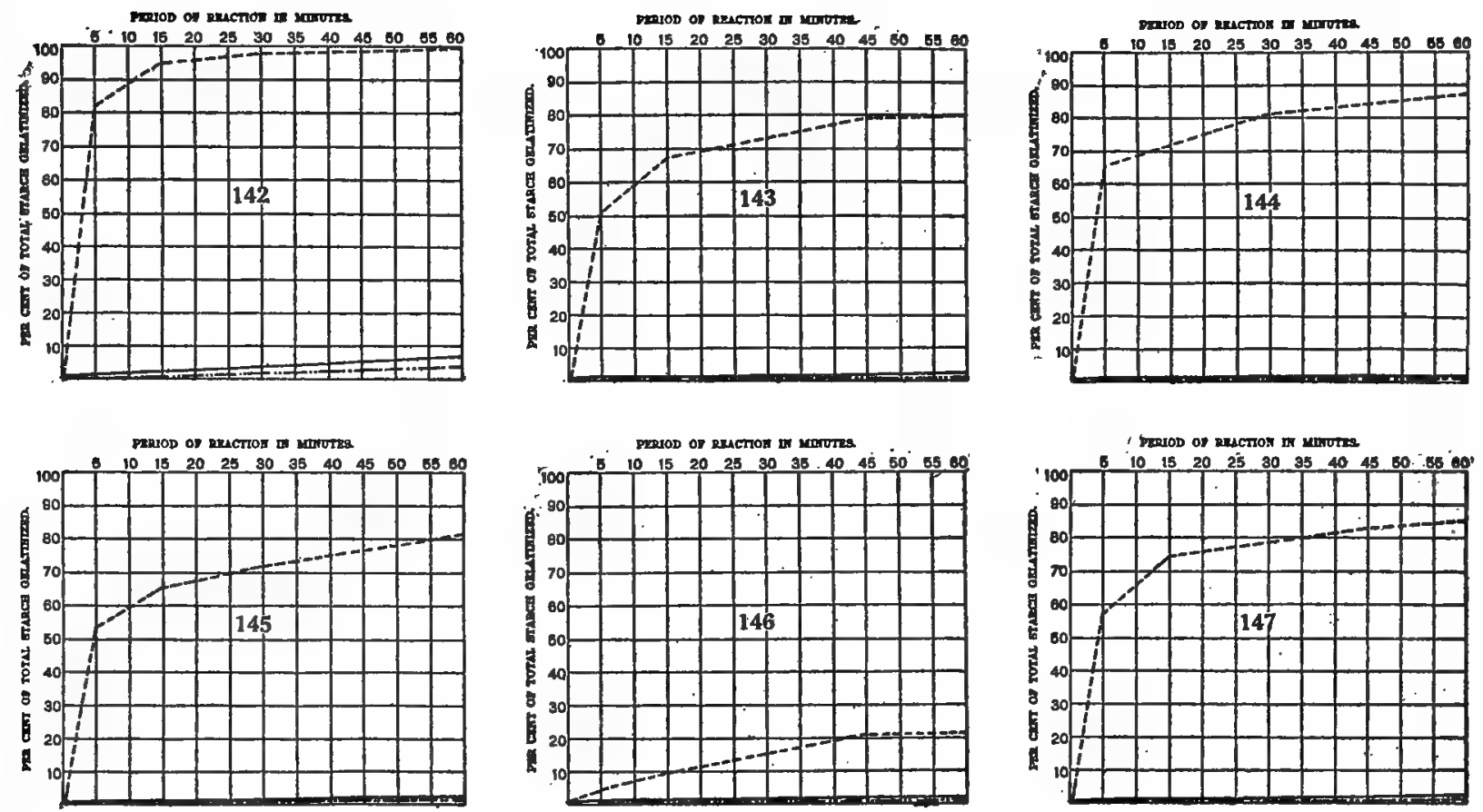

Charts D 142 to D 147.-Velocity-Reactions of Starches of Crinum moorei (.....), Crinum zeylanicum ( ......-), and Crinum hybridum j.c.h. (—).

142. With Strontium Nitrate. 143. With Cobalt Nitrate.

144. With Copper Nitrate.

146. With Barium Chloride.

147. With Mercurio Chloride.
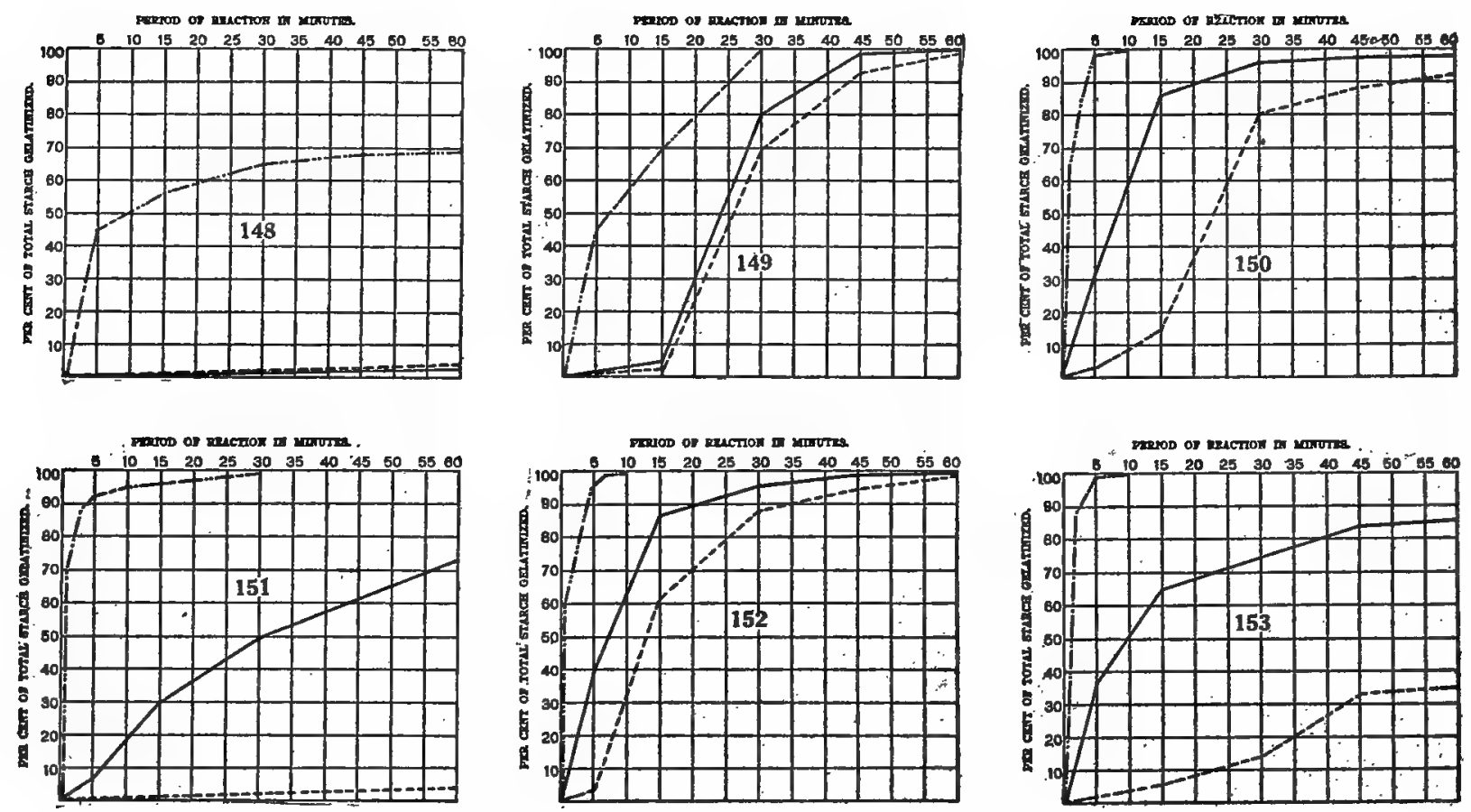

Charts D 148 то D 153.-Velocity-Reactions of Starches of Crinum zeylanicum (-..--), Crinum longifolium ( .......-), and Crinum kircape (-). 


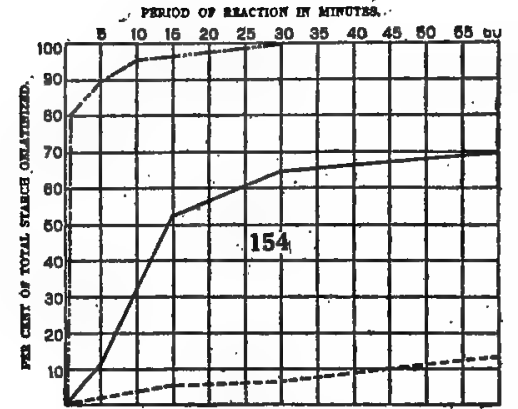

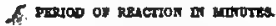

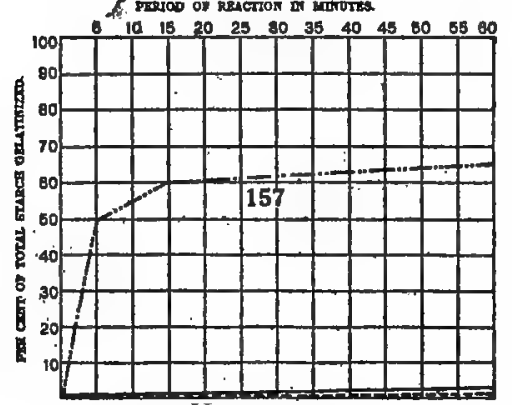

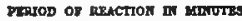

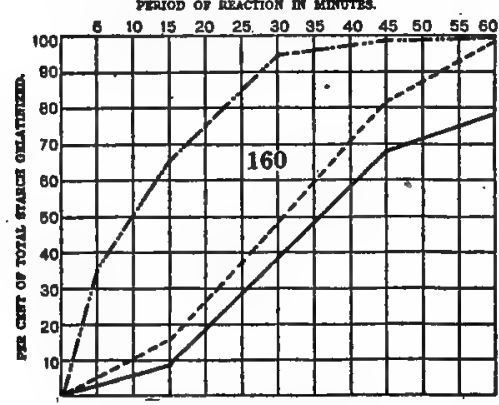

PERYOD Of REACTOX IT MHNOTE

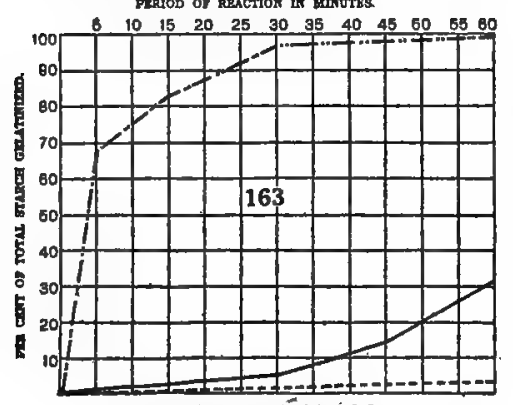

Patrod of ReActros If xutures.

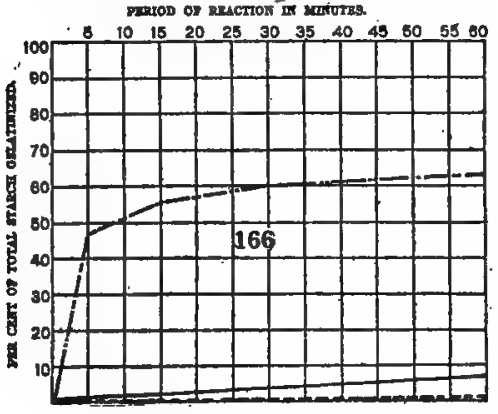

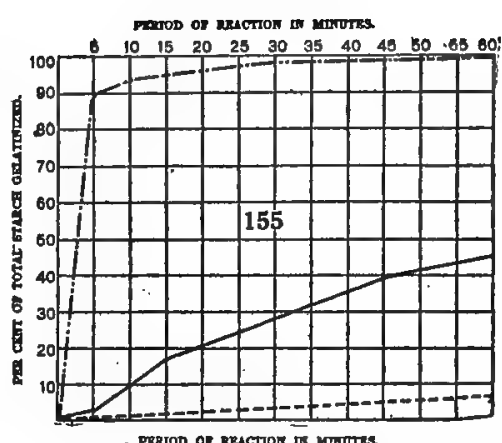

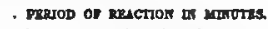

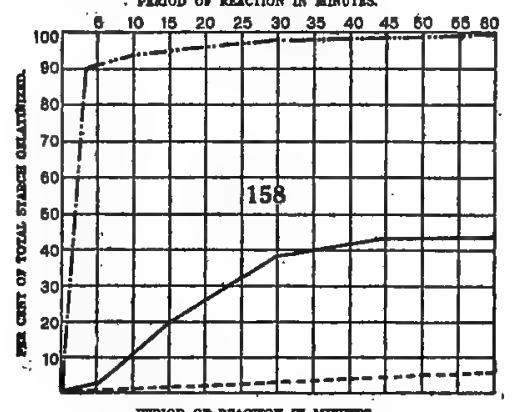

FRRTOD OT RRACTIOE ET MEROTIS

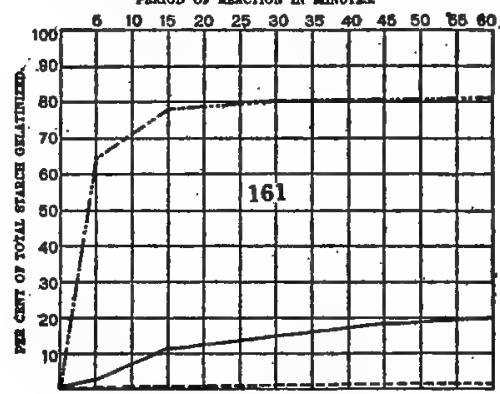

PRRIOD OY REACTION II MANUTES

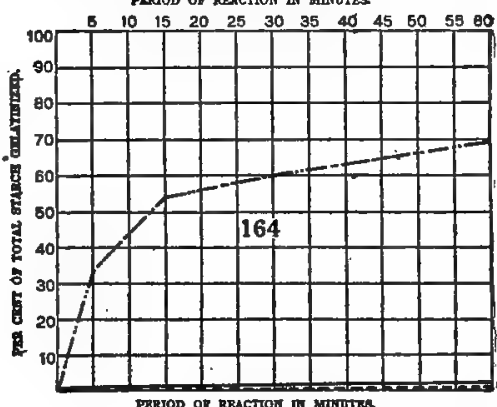

PREOD OF REACTIOA II MANUTES

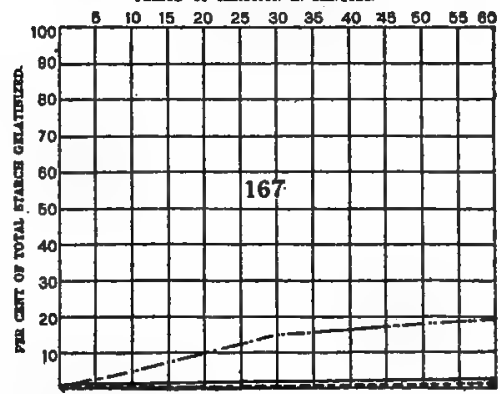

ronos as

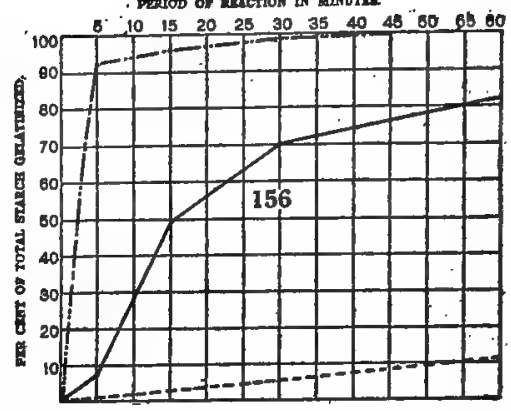

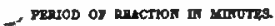

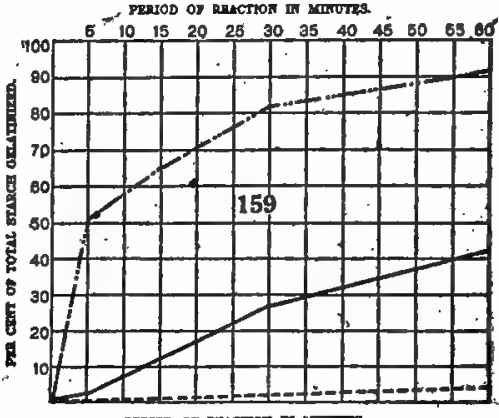

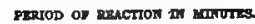

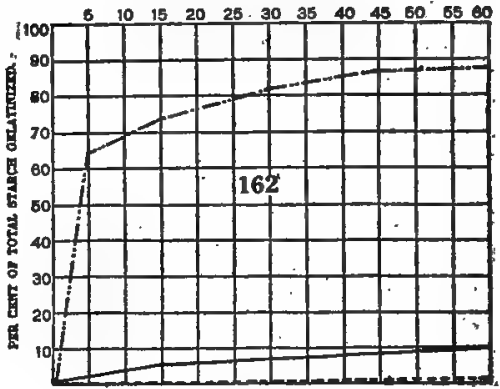

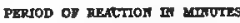

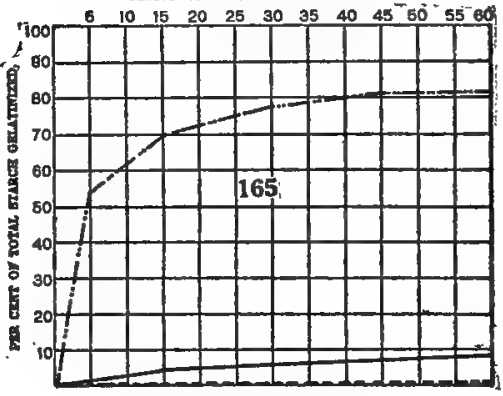

presod oy aracrion an umores

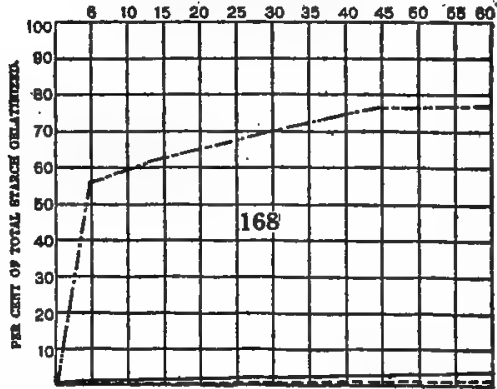

CHars D 154 то D 168.-Velocity-Reactions of Starches of Crinum zeylanicum (.....), Crinum longifolium (-.....), and Crinum kircape (-).

154. With Potagsium Hydroxide.

155. With Potassium Iodide.

156. With Potagsium Sulphocyanate.

158. With Sotassium Sulphide.
159. With Sodium Sulphide.

160. With Sodium Salicylate.

161. With Calcium Nitrate.

163. With Uranium Nitrate.
164. With Cobalt Nitrate.

165. With Copper Nitrate.

166. With Cupric Chloride.

167. With Barium Chloride.

168. With Mercuric Chloride. 


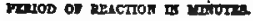

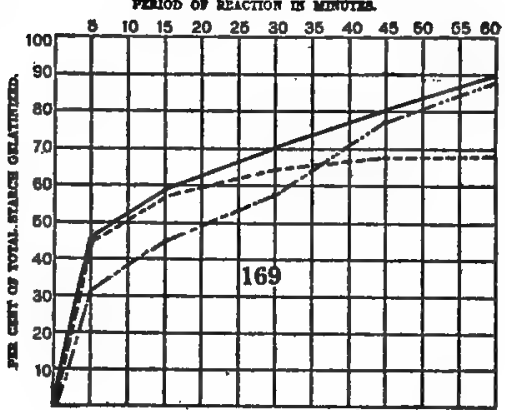

PERTOD of ReAcTion IE METURE

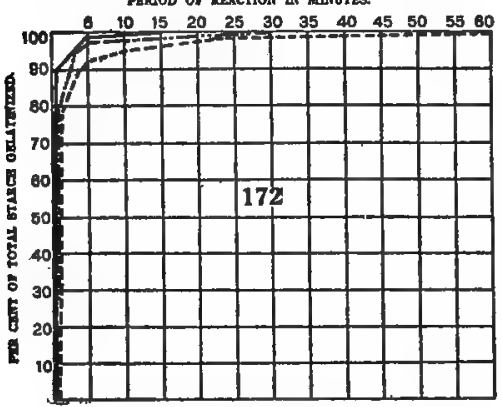

PERTOD OF RRACTION II MRETER

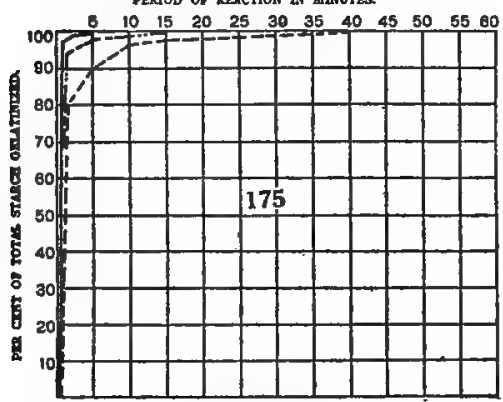

prarod of mectios w ansortes

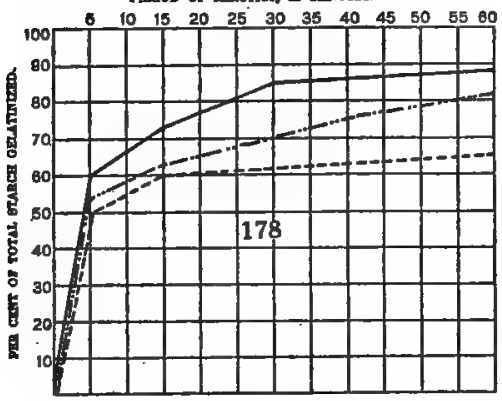

PERToD of ERACTION EN METUTEs

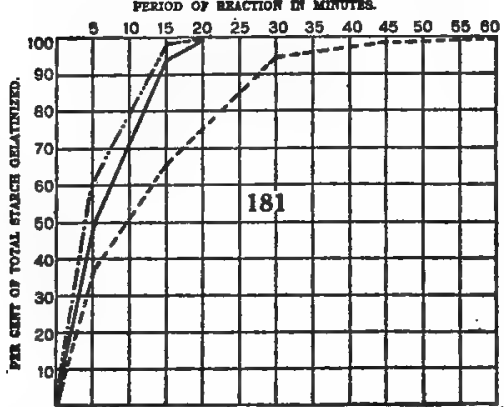

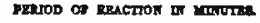

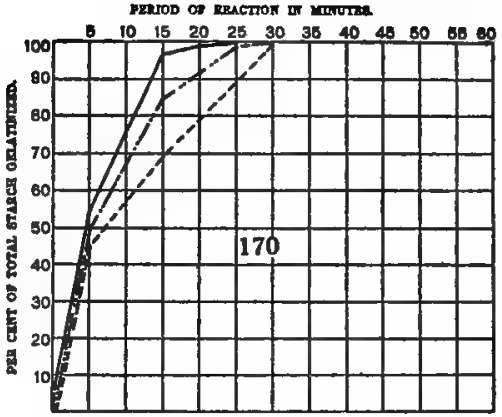

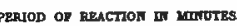

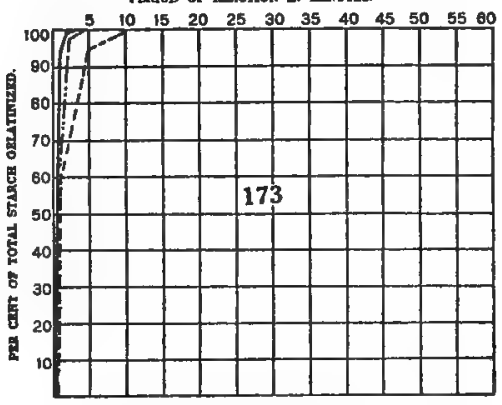

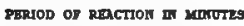

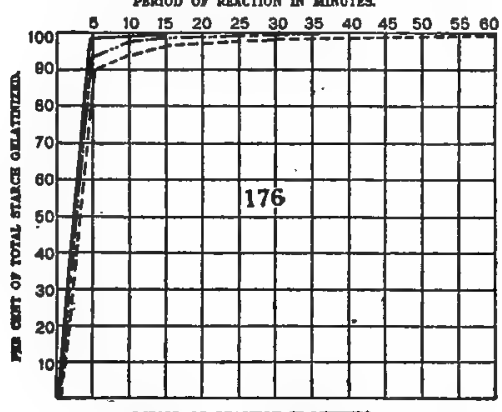

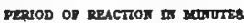

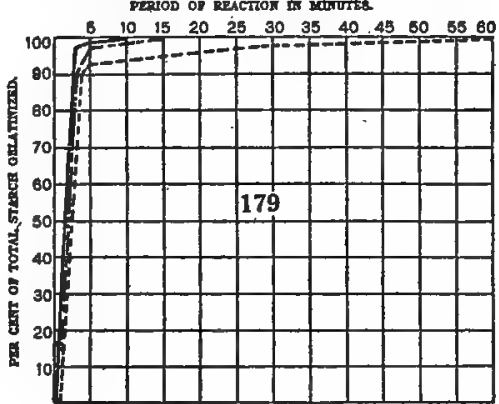

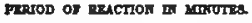

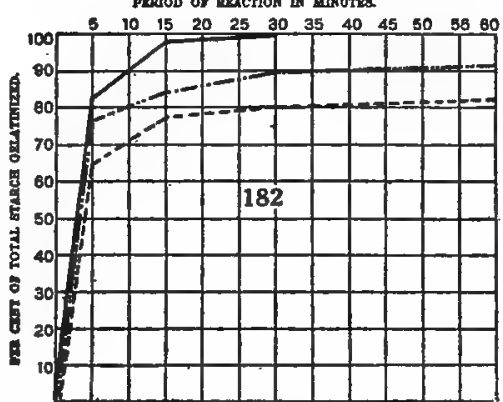

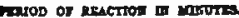

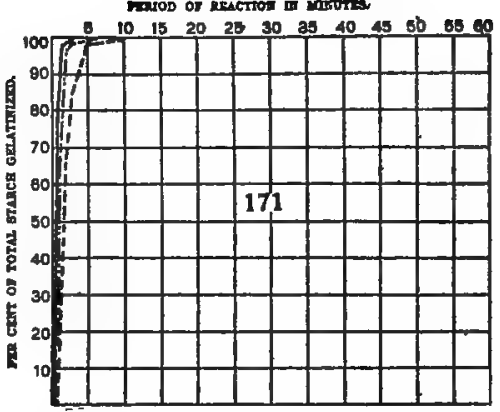

Mexiod of Rractor m smotres

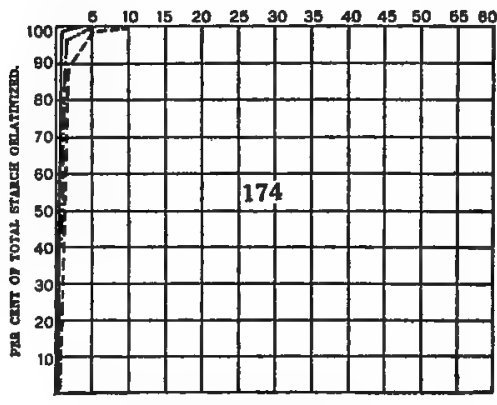

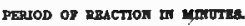

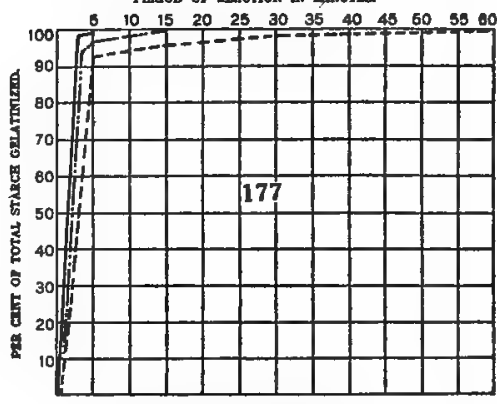

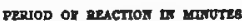

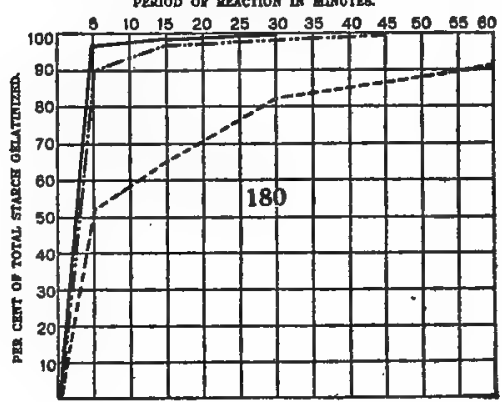

PBarod of ReAction w manutes

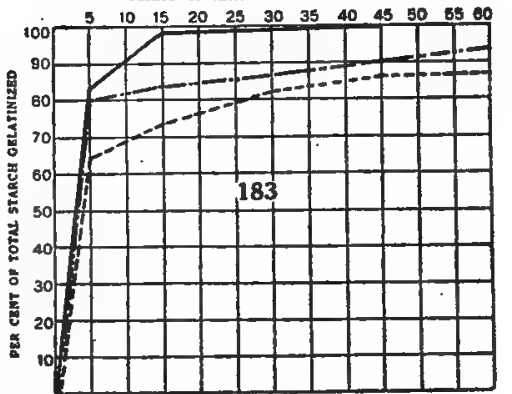

Charts D 169 to D 183.-Velocity-Reactions of Starches of Crinum longifolium (.....), Crinum moorei (-......), and Crinum powellii ( $\longrightarrow$ ).

169. With Chloral Hydrate.

170. With Chromic Acid.

171. With Pyrogallic Acid

173. With Nitric Acid.
174. With Hydrochloric Acid.

175. With Potassium Hydroxide.

176. With Potassium Iodide.

177. With Potgsgium Sulphocyanato

178. With Potassium Hydroxide.
179. With Sodium Hydroxide.

180. With Sodium Sulphide.

181. With Sodium Salicylate.

182. With Calcium Nitrate. 

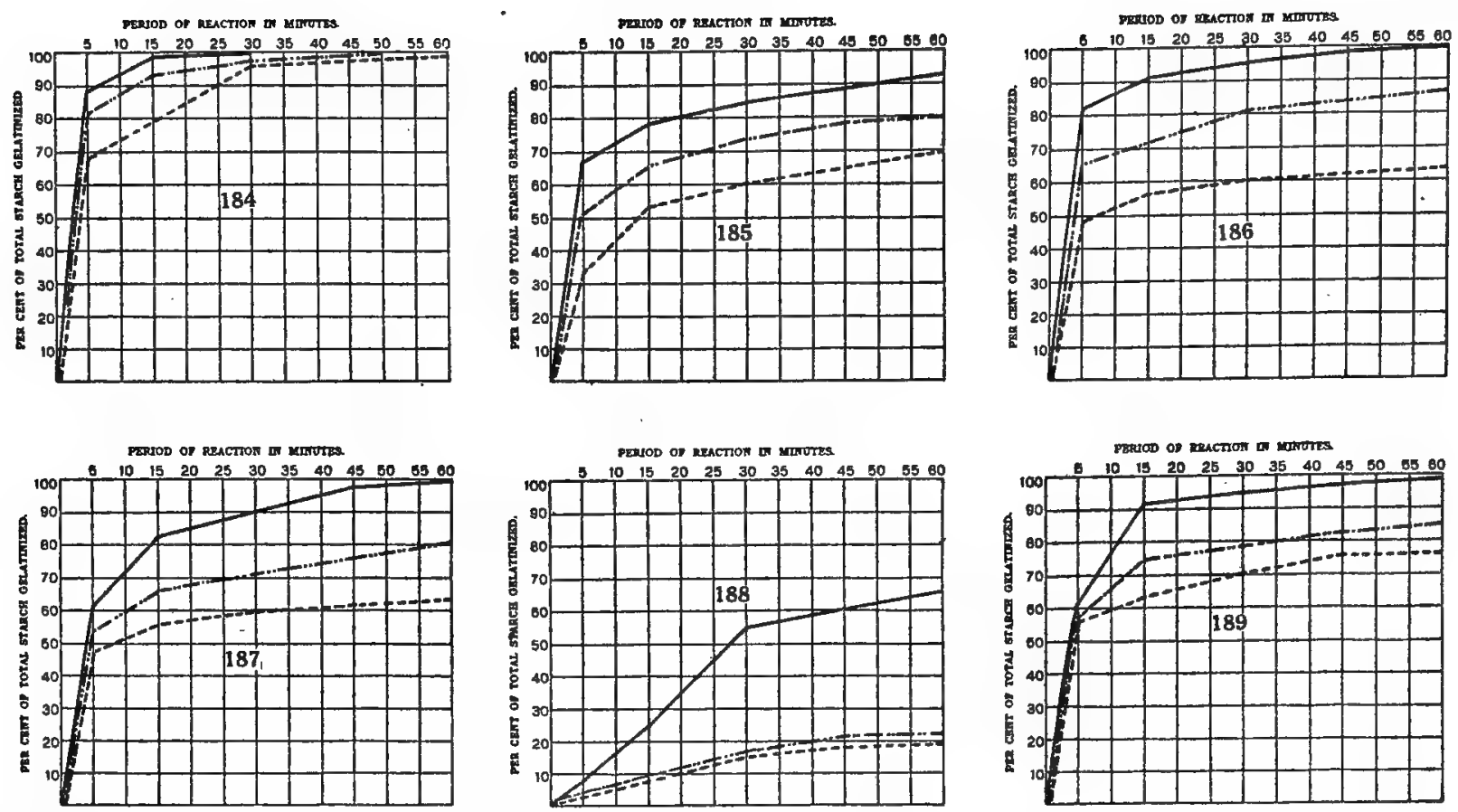

Charts D 184 to D 189.-Velocity-Reactions of Starches of Crinum longifolium ( -...-), Crinum moorei (-.....-), and Crinum powellii (-).

184. With Strontium Nitrate. 185. With Cobalt Nitrate.
186. With Copper Nitrate.

187. With Cupric Chloride
188. With Barium Chloride

189. With Mercuric Chloride.
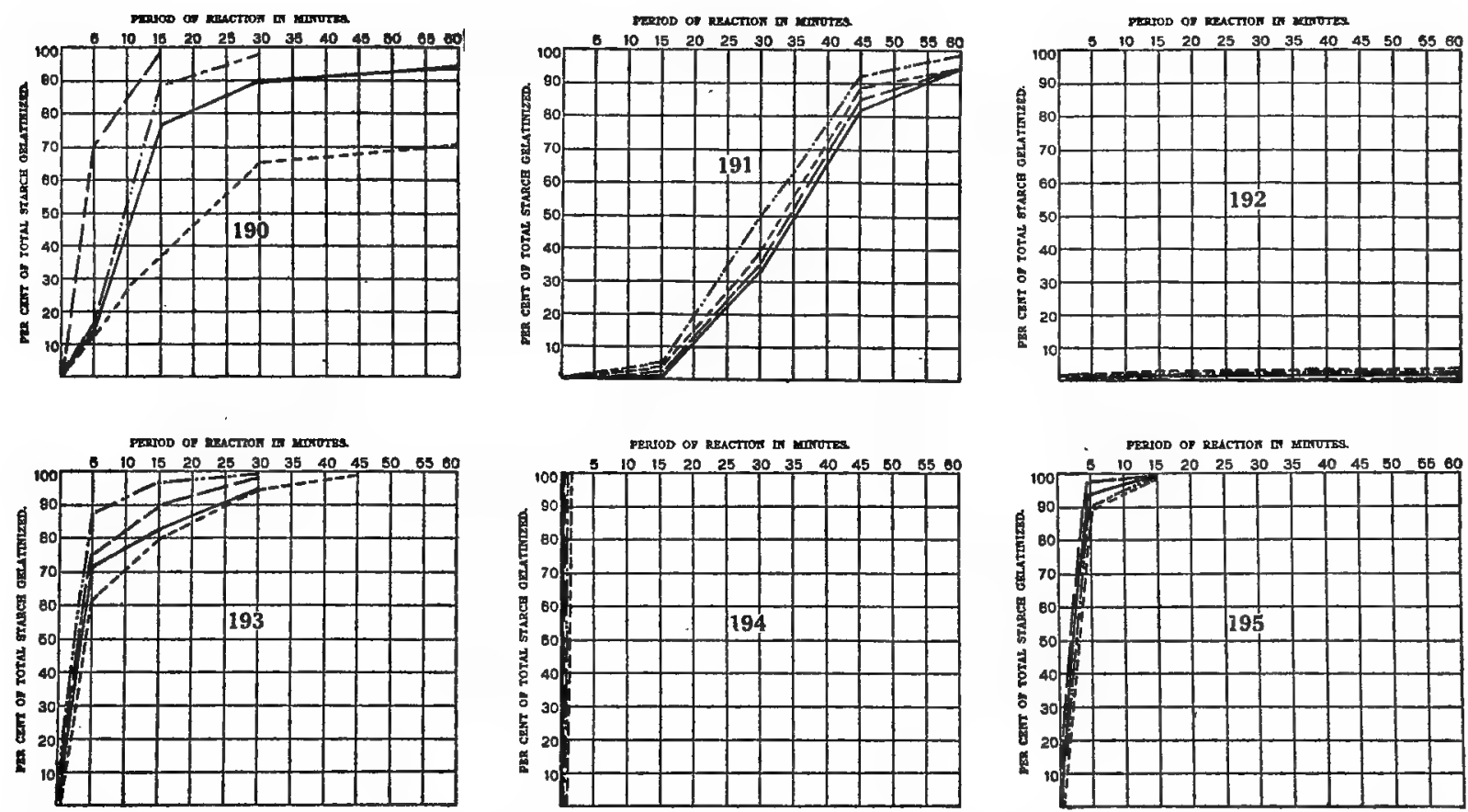

Charts D 190 to D 195.-Velocity-Reactions of Starches of Nerine crispa ( .....), Nerine elegans ( ... _...), Nerine dainty maid (

190. With Chloral Hydrate.

191. With Chromio Acid
192. With Pyrogallic Aoid.

193. With Nitrio Aoid.
194. With Sulphurio Acid.

195. With Hydrochlorio Acid. 

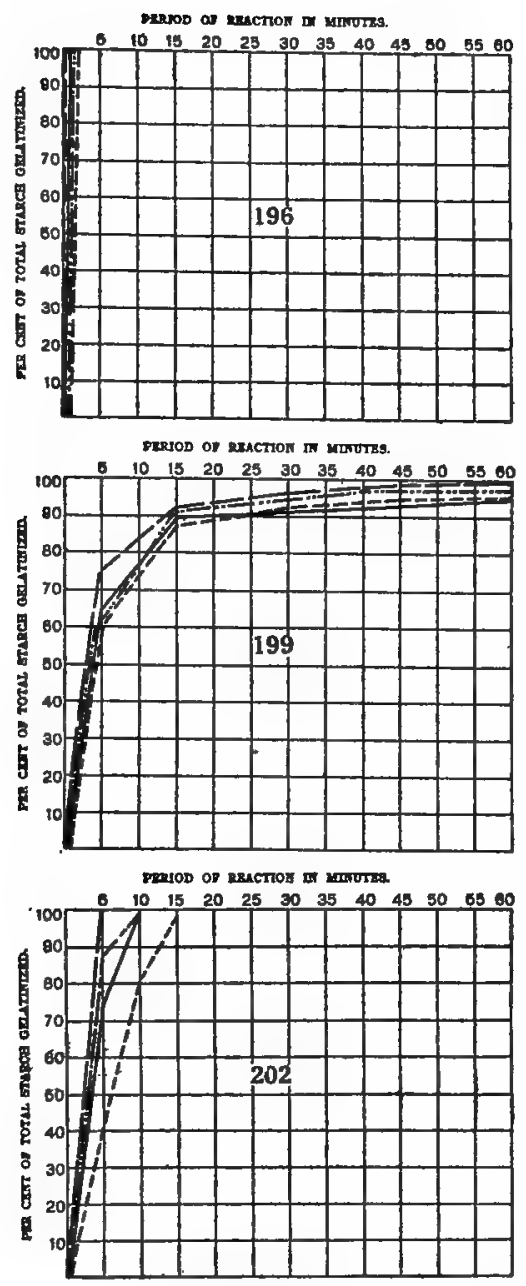

PERIOD OF REACTIOH II MITUTES.

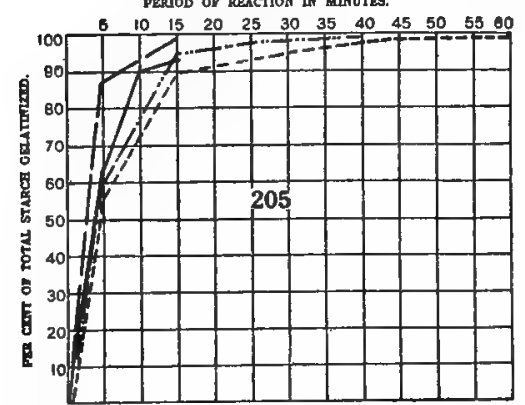

maios of gection al movote.

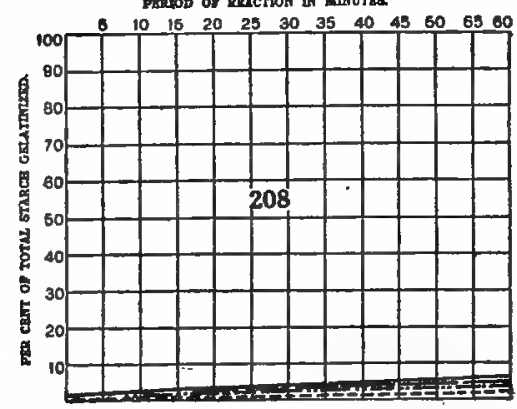

mono of Reactios a Menotes

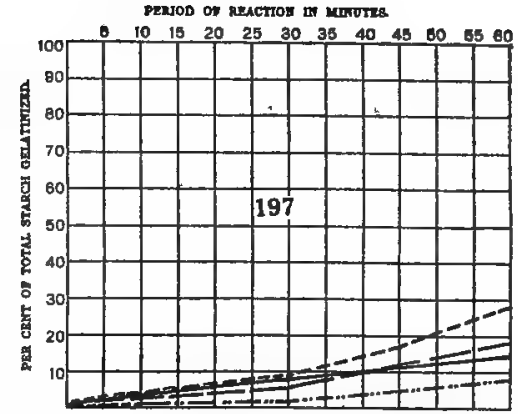

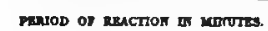

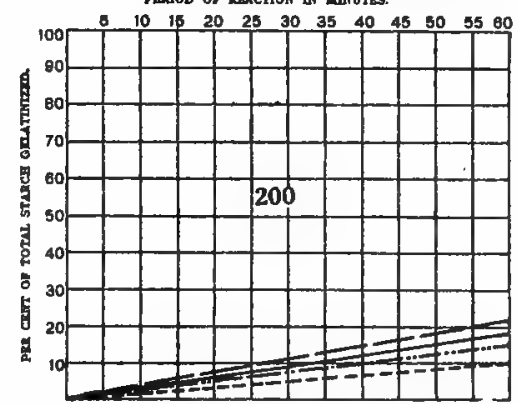

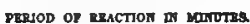

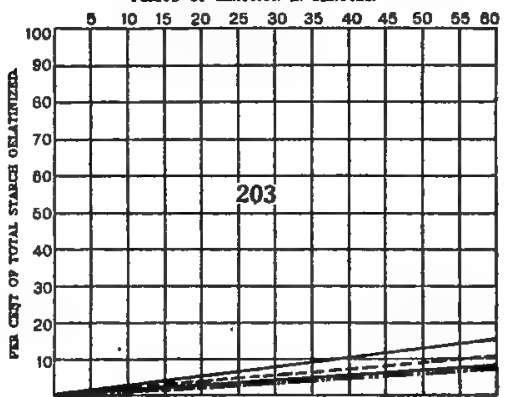

prsarod of REAction II MLNUTEs.

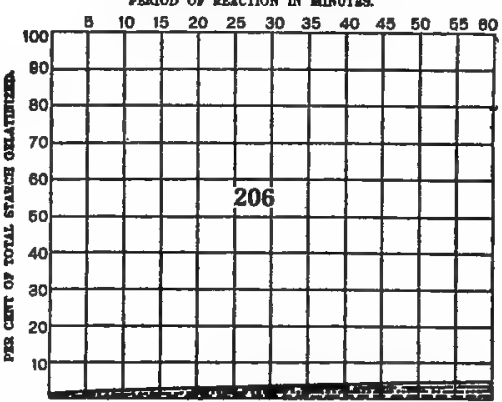

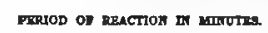

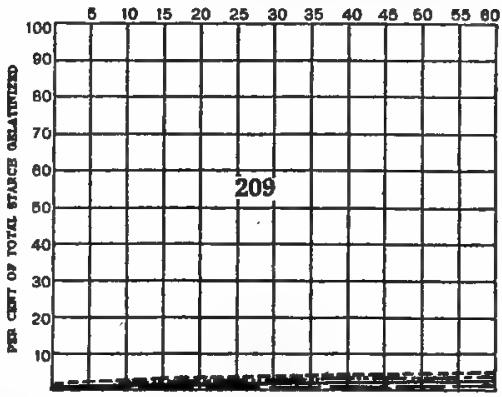

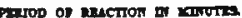

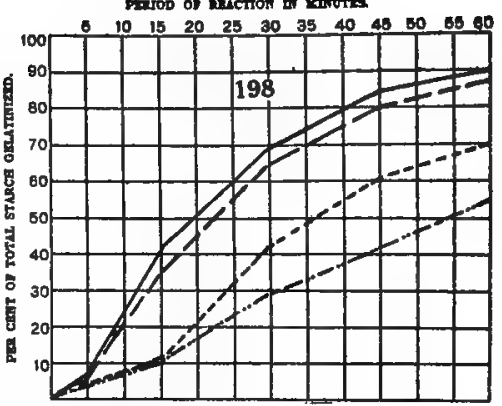

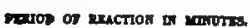

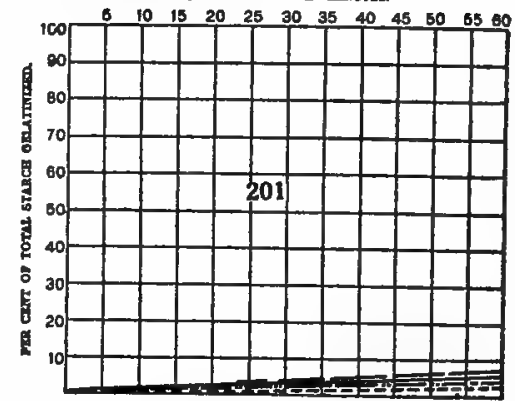

Premos of kenctiox at merotrs.

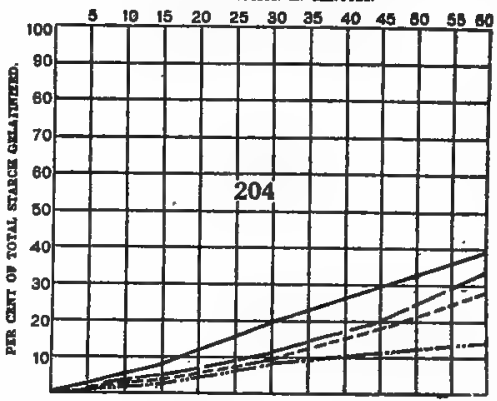

provod of pencros an umuma

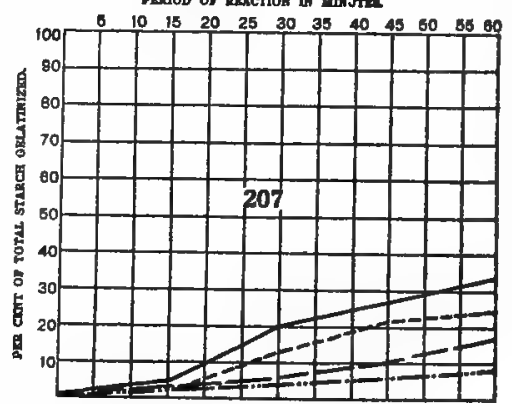

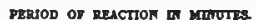

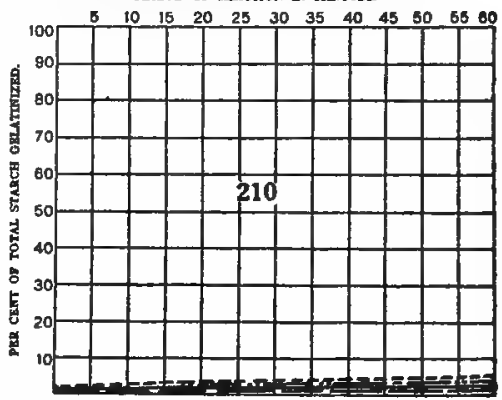

Charts D 196 to D 210.-Velocity-Reactions of Starches of Nerine crispa ( -...-), Nerine elegans ( ......-), Nerine

dainty maid

196. With Potassium Hydroxide.

197. With Potassium Iodide.

198. With Potassium Sulphocyanate.

199. With Potassium Sulphide.

200. With Sodium Hydroxide.
), and Nerine queen of roses (

201. With Sodium Sulphide.

202. With Sodium Salicylate.

203. With Calcium Nitrate.

205. With Uranium Nitrate.
206. With Cobalt Nitrate.

207. With Copper Nitrate.

208. With Cupric Chloride.

210. With Mercurio Chloride. 

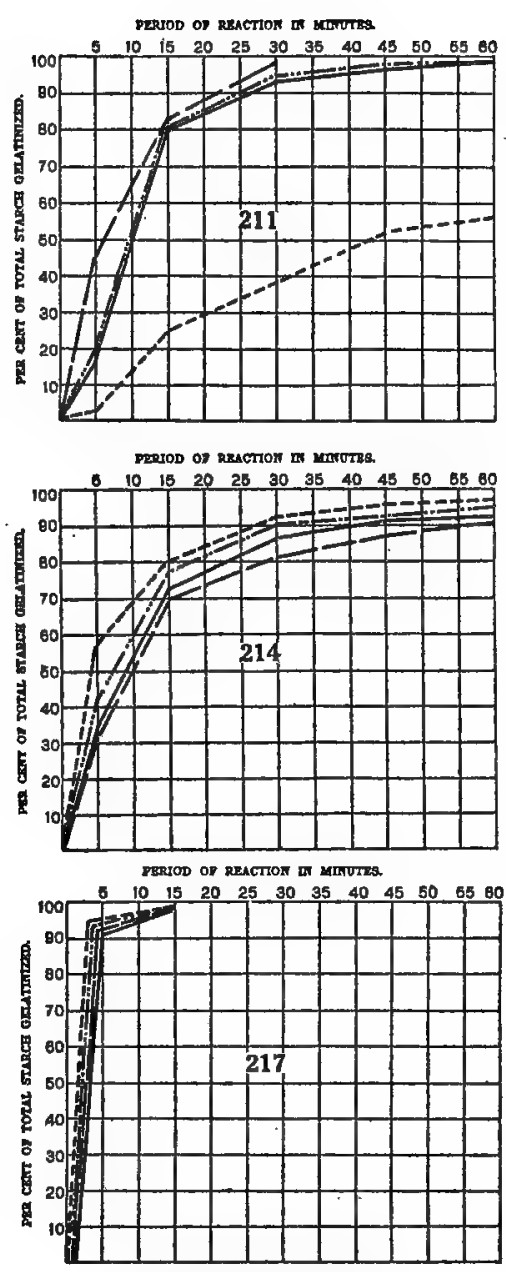

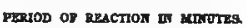

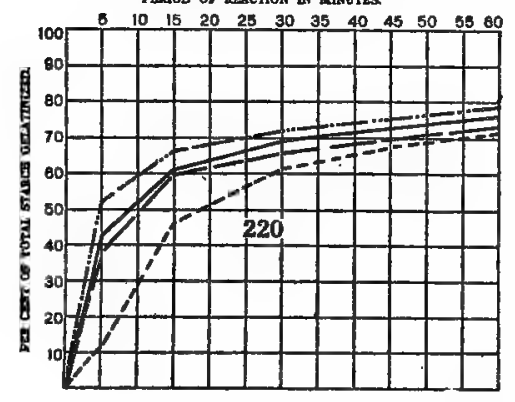

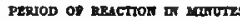

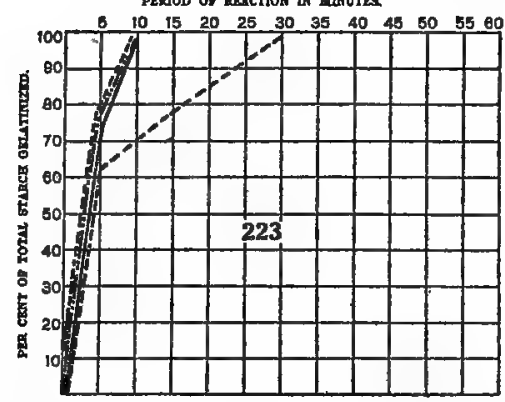

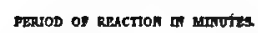

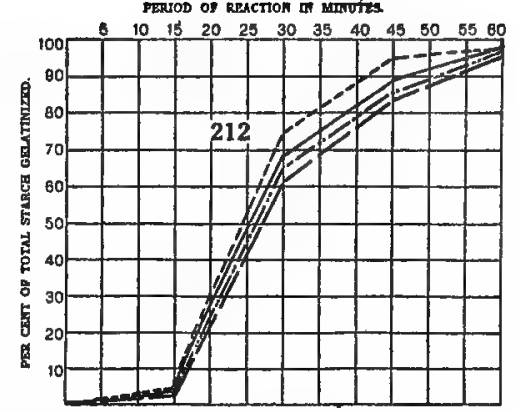

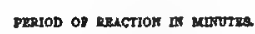

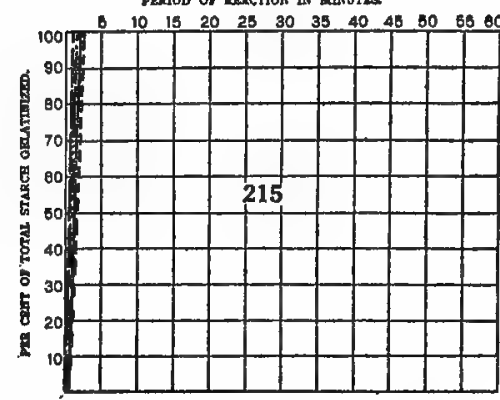

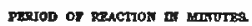

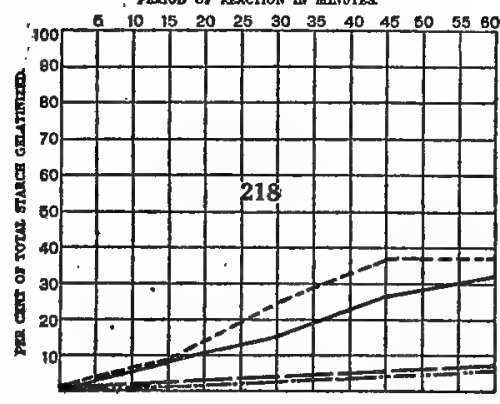

PReiod of RECCTon IT METVTres

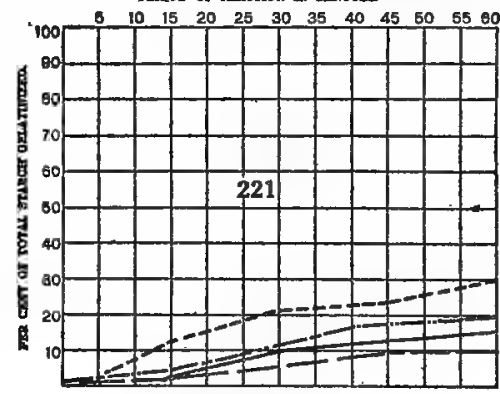

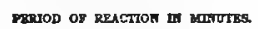

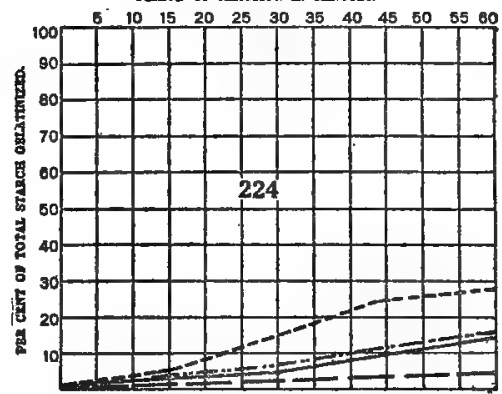

merod of ersection an Matrotes

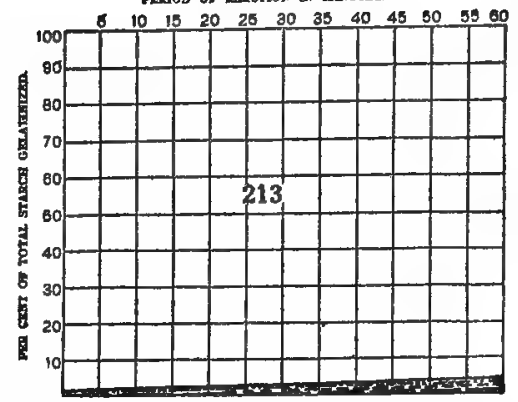

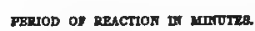

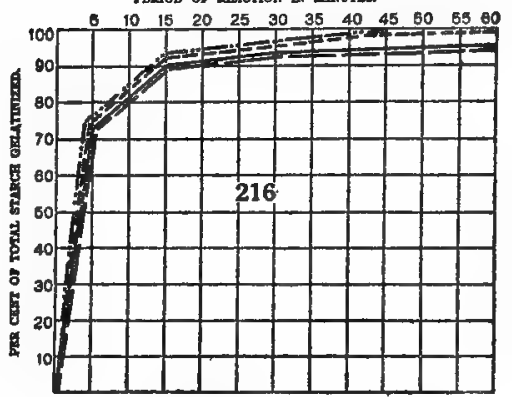

Parrod or kectron as vesutras

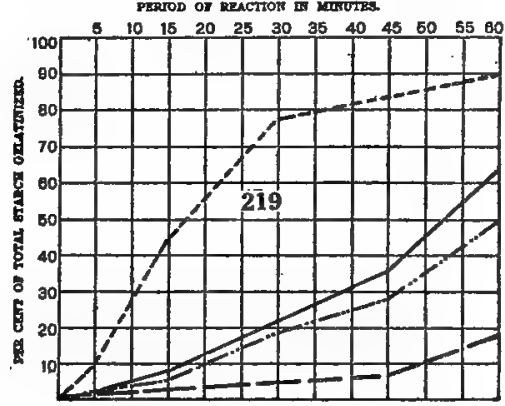

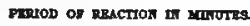

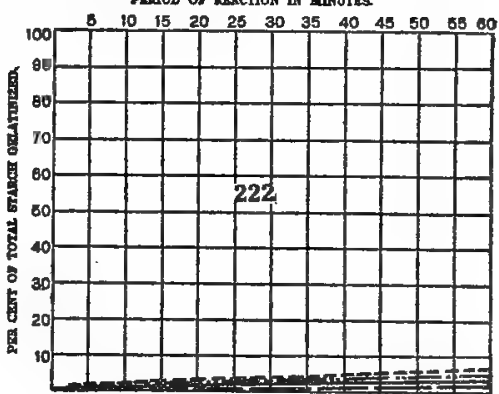

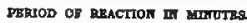

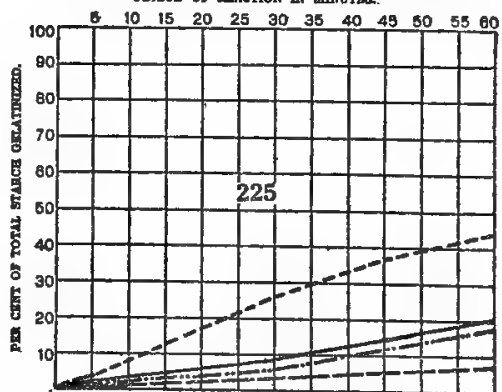

Charts D 211 to D 225.-Velocity-Reactions of Starches of Nerine bowdeni (.....), Nerine sarniensis var. corusca major ( .......), Nerine giantess (-), and Nerine abundance (- - -

211. With Chloral Hydrate.

212. With Chromic Acid.

213. With Pyrogallic Acid.

215. With Sulphusic Acid.
216. With Hydrochloric Acid.

217. With Potassium Hydrozide

219. With Potassium Sulphoc

219. With Potassium Sulphocyanate.
220. With Potassium Sulphide.
221. With Sodium Hydroxide.

222. With Sodium Sulphide.

223. With Sodium Salicylate.

225. With Uranium Nitrate. 


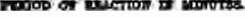

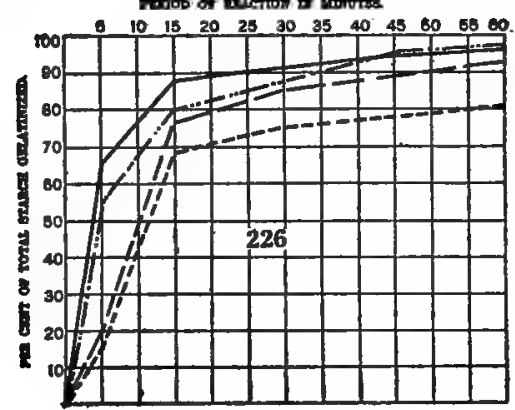

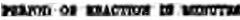

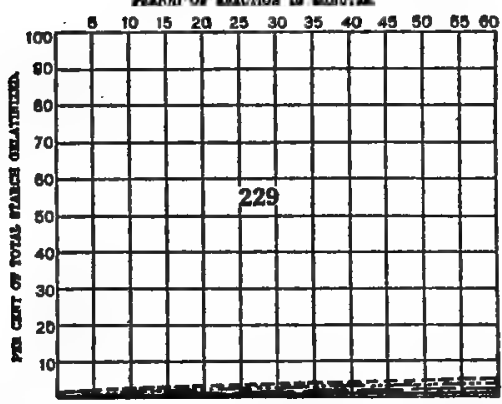

mono of ancton to more

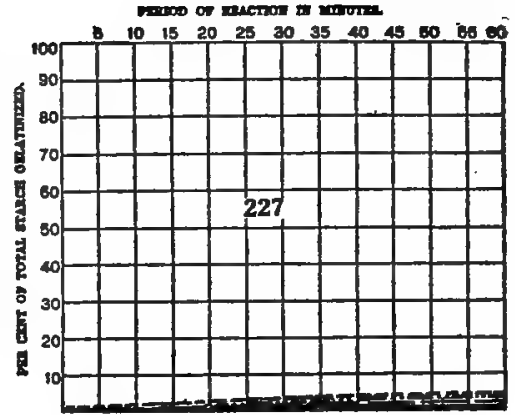

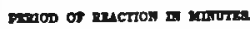

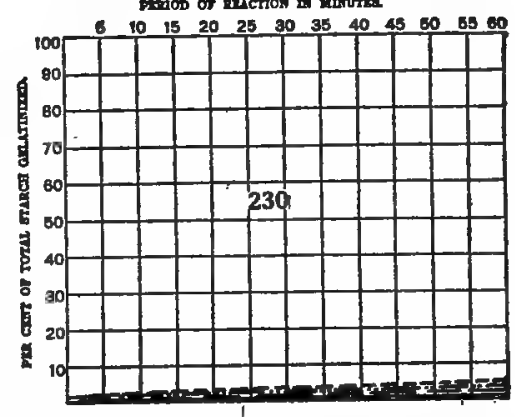

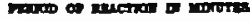

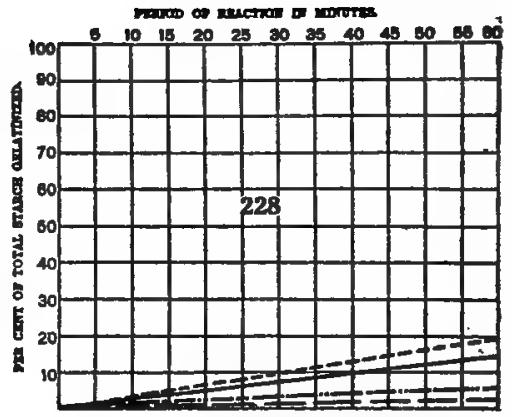

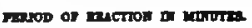

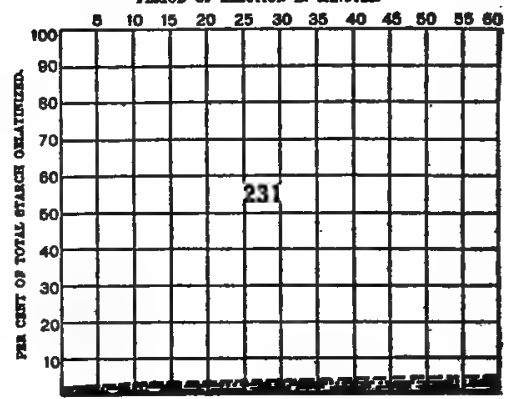

Charts D 226 to D 231.-Velocity-Reactions of Starches of Nerine bowdeni (-.---), Nerine sarniensis var. corusca major ( .......), Nerine giantess (-), and Nerine abundance (- -$)$ ).

226. With Strontium Nitrato.

228. With Copper Nitrate.

230. With Barium Chloride.

227. With Cobalt Nitrate.

229. With Cupric Chloride.
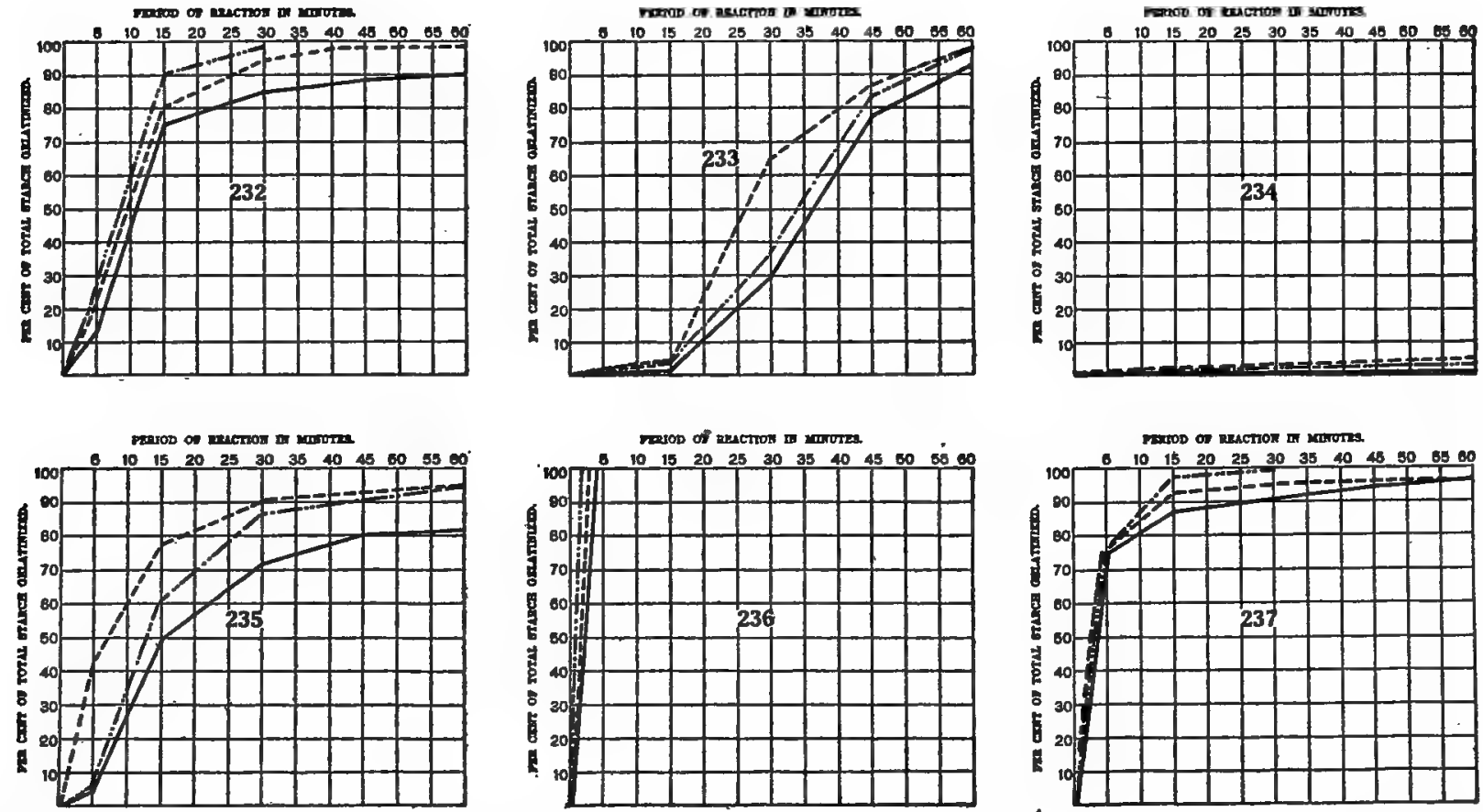

Charts D 232 to D 237.-Velocity-Reactions of Starches of Nerine sarniensis var. corusca major (--.--), Nerine curviflora var. fothergilii major (....... ), and Nerine glory of sarnia (-).

232. With Choral Hydrate. 233. With Chromio Acid.
234. With Pyrogallic Aoid.

235. With Nitric Acid.
236. With Sulphuric Acid.

237. With Hydrochlorio Aoid. 


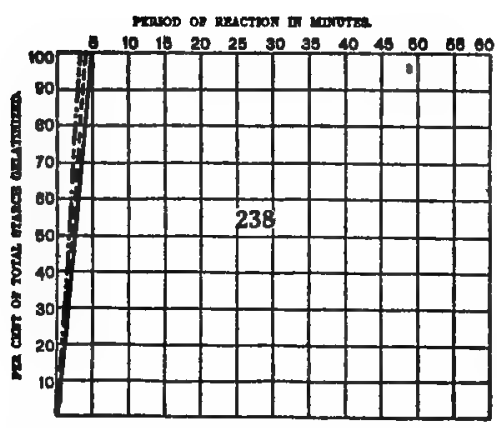

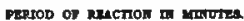

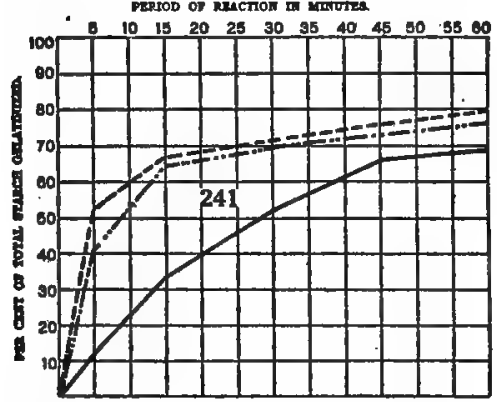

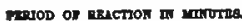

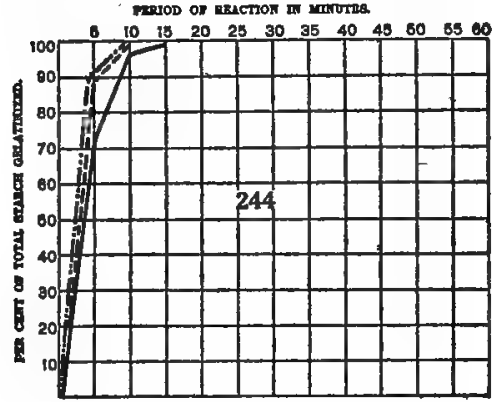

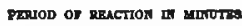

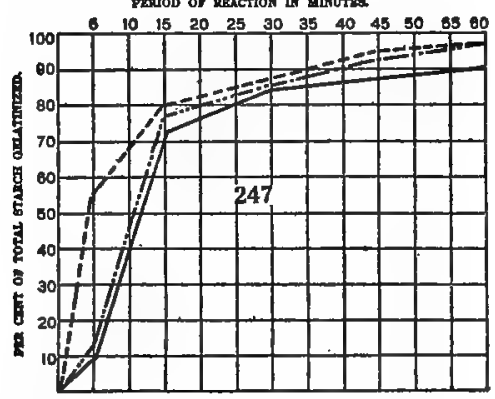

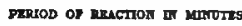

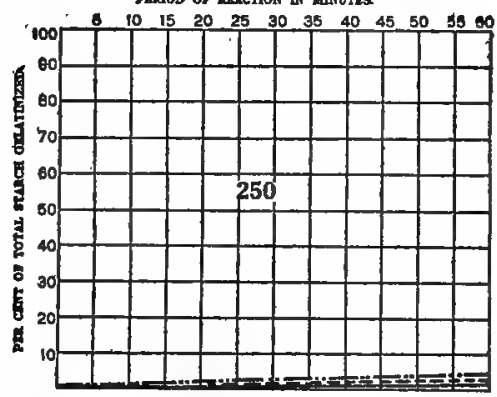

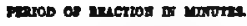

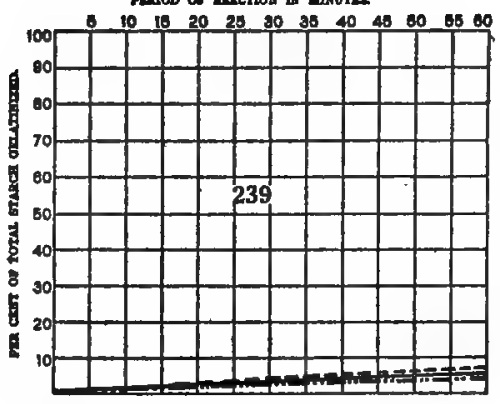

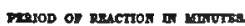

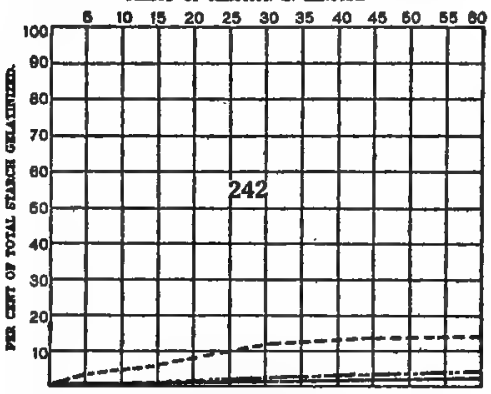

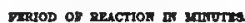

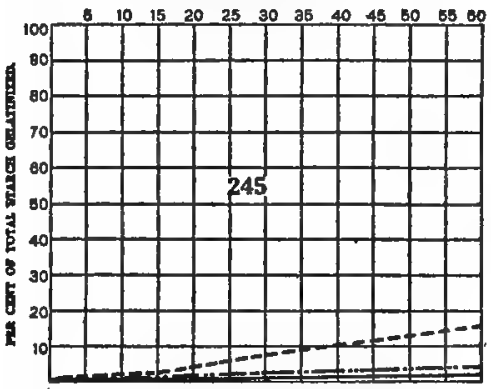

persod or aruction in unvores.

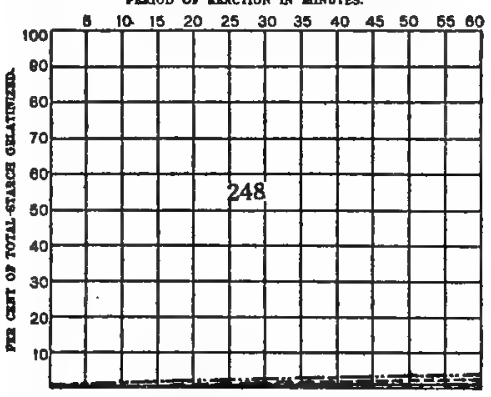

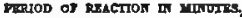

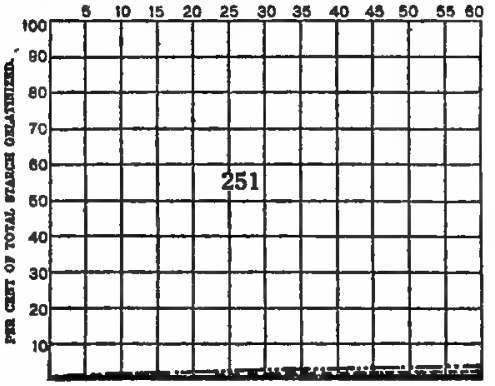

(1)

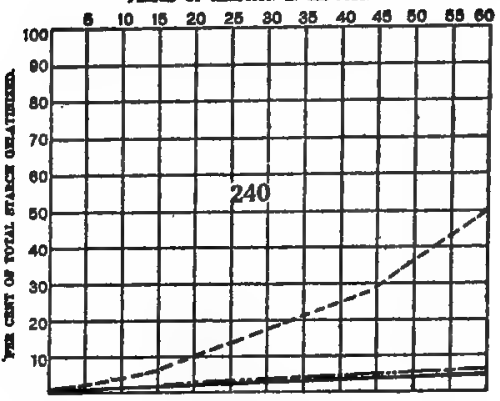

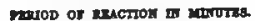

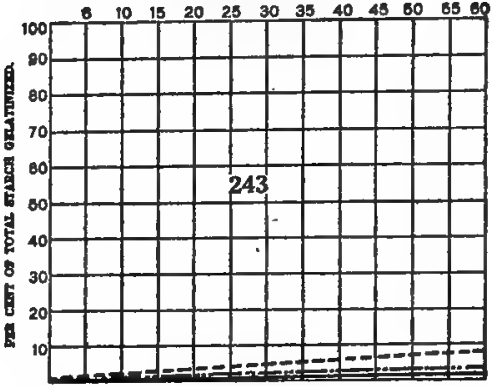

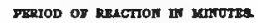

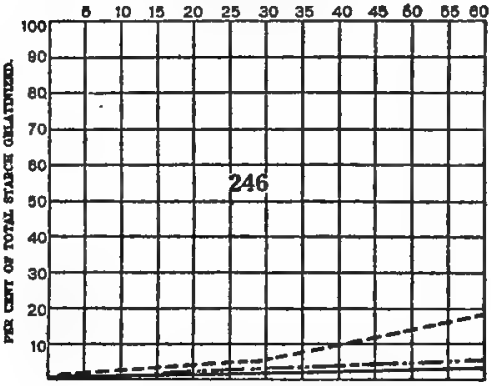

PBeriod of Eraction in knvotes

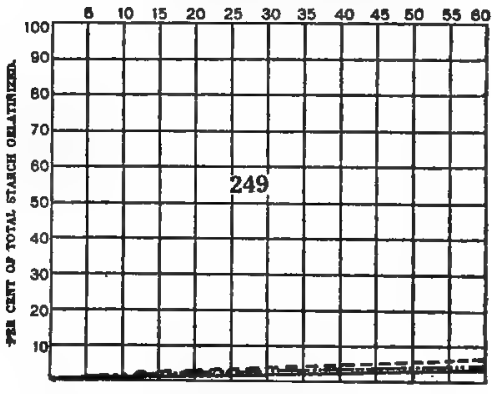

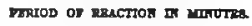

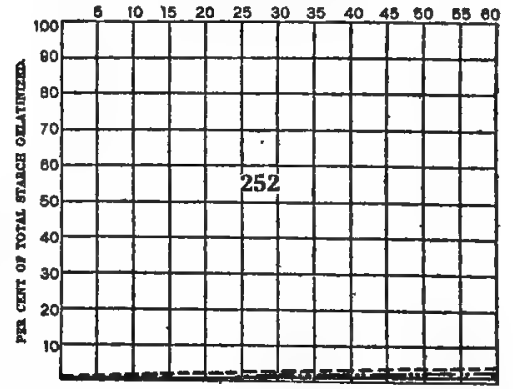

Charts D 238 to D 252.-Velocity-Reactions of Starches of Nerine sarniensis var. corusca major (.....), Nerine curviflora var. fothergilii major (.......), and Nerine glory of sarnia (-).

238. With Potassium Hydroride.

239. With Potassium Iodide.

240. With Potassium Sulphocyanate.

241. With Potagsium Sulphide.

242. With Sodium Bydroxide.
243. With Sodium Sulphide.

244. With Sodium Salicylate.

245. With Calcium Nitrate.

246. With Uranium Nitrate.

247. With Strontium Nitrate.
248. With Cobalt Nitrate.

249. With Copper Nitrate.

251. With Barium Chloride.

252. With Mercuric Chloride. 

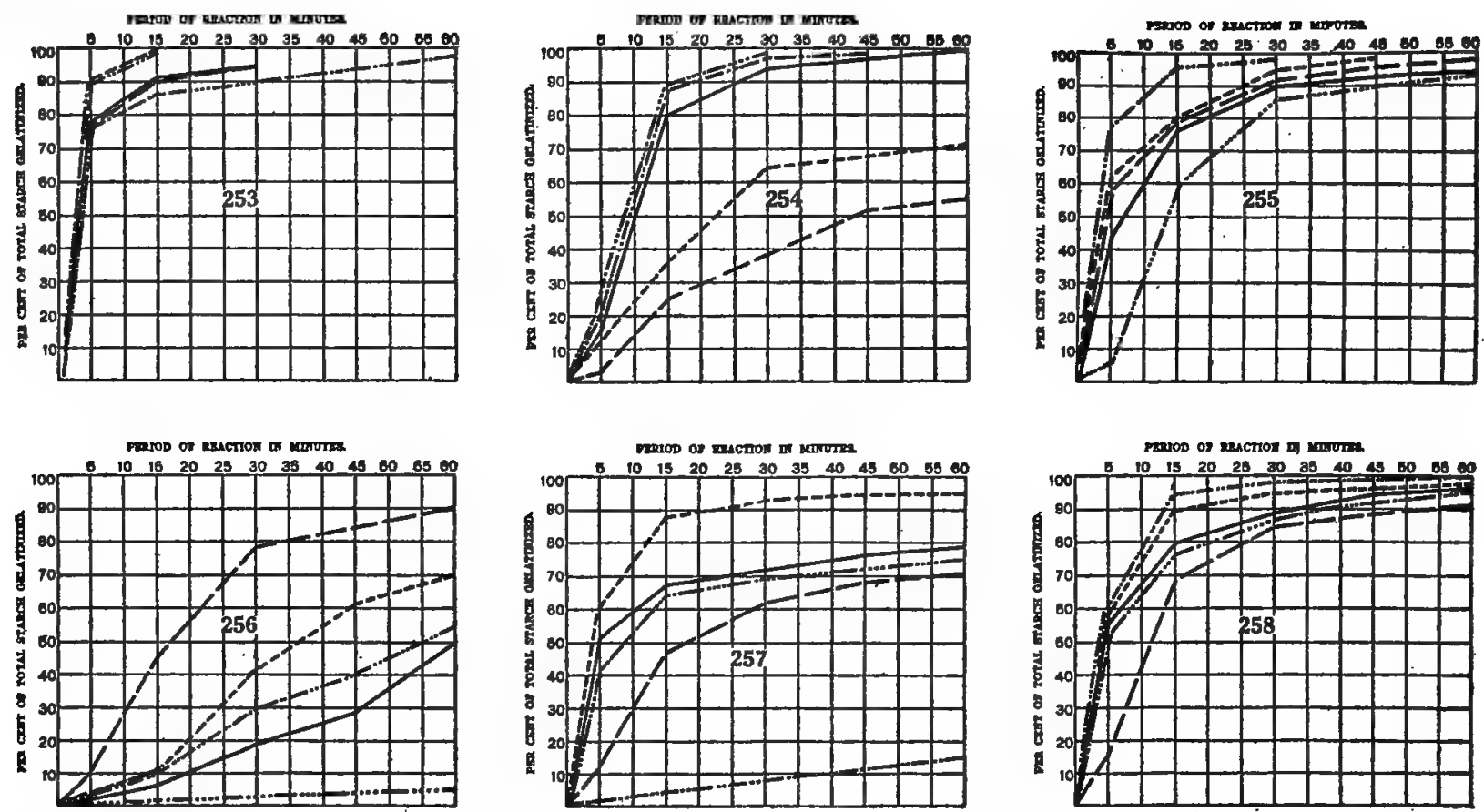

Charts D 253 то D 258.-Velocity-Reactions of Starches of Nerine curvifolia var. fothergilli major (-.........), N. elegans (-...-), N. sarniensis var. corusca major (-), N. crispa (----), and N. bowdeni (-- - ).

253. With Hydrochloric Acid. 254. With Chloral Hydrate.
255. With Nitrio Acid.

256. With Potaseium Sulphooy anate.
257. With Potaseium Sulphide.

258. With Strontium Nitrate.
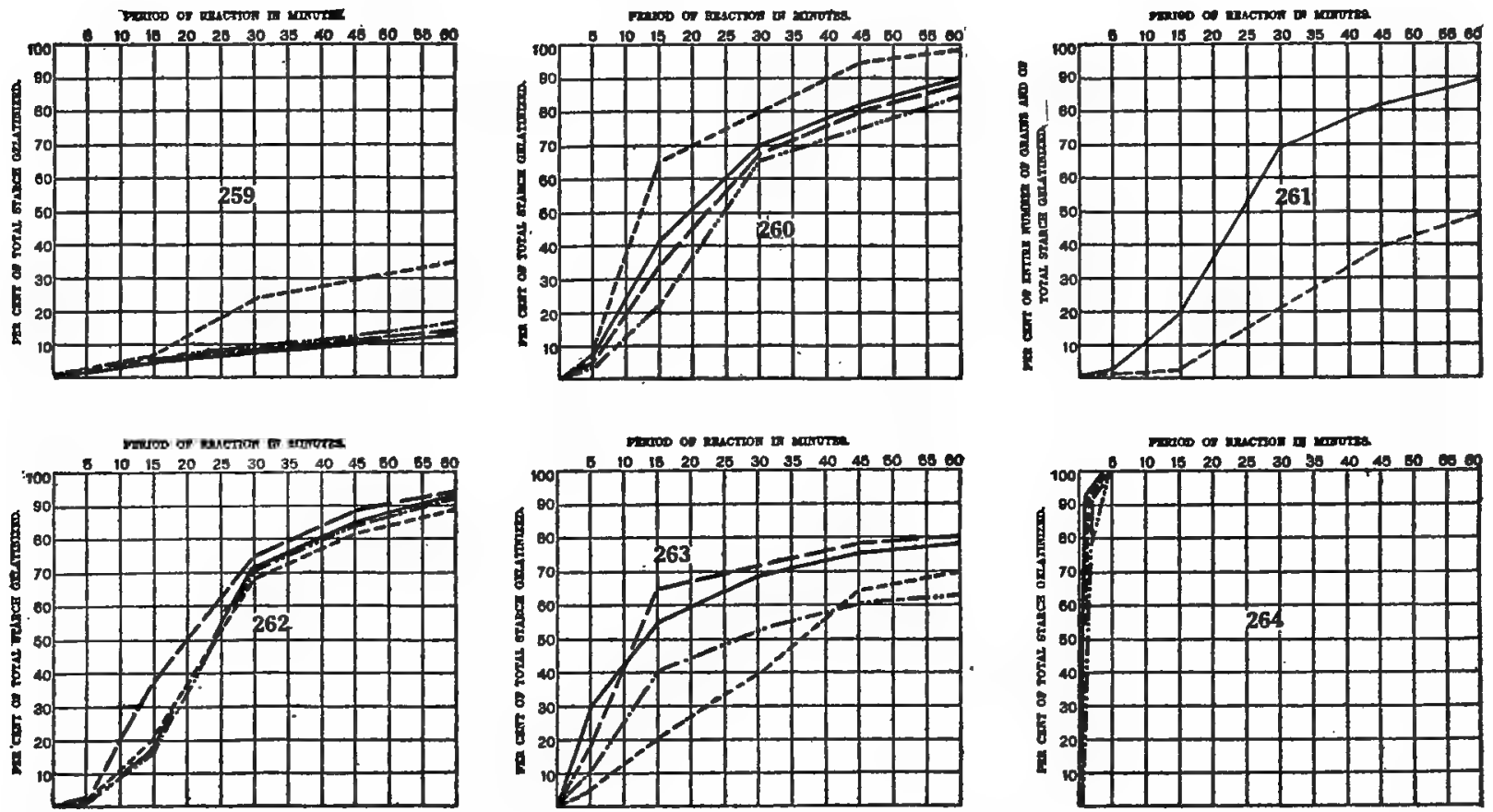

Charts D 259, D 260, D 262 to D 264.-Velocity-Reactions of Starches of Narcissus poeticus ornatus (-...-), $N$. poeticus poetarum (.......), N. poeticus herrick (—), and N. poeticus dante (- -
259. With Chloral Hydrate.
262. With Pyrogallio Acid.
263. With Nitrio Acid.
260. With Chromic Acid.
64. With Sulphuric Acid.

Chart D 261.-Velocity-Reactions of Pyrogallic Acid with the Starch of Narcissus poeticus ornatus. Percentage of entire number of grains (.....) and of total starch $(-)$ gelatinized. 


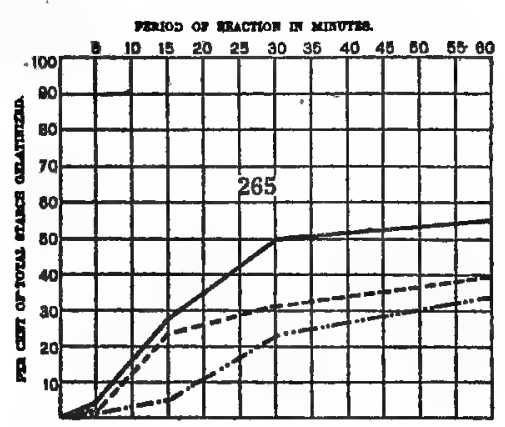

pearod oz enecrion of monutre.

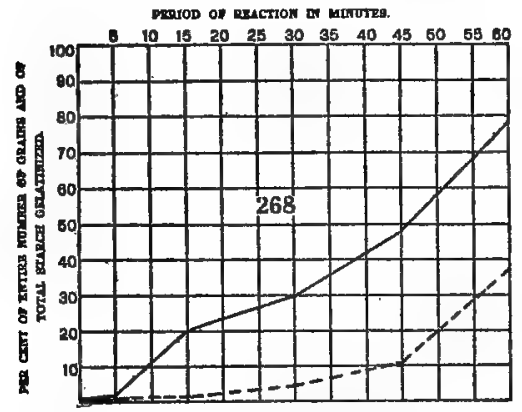

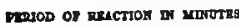

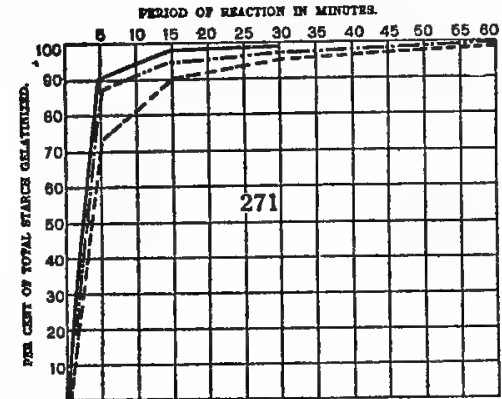

men precror

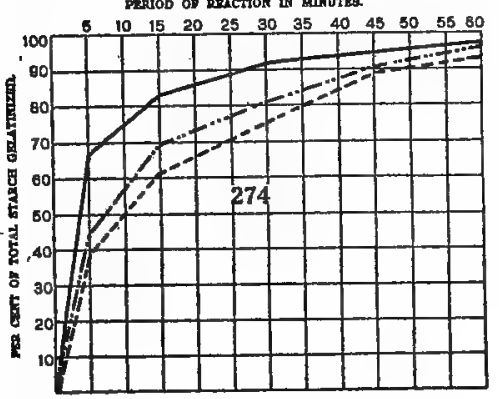

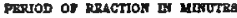

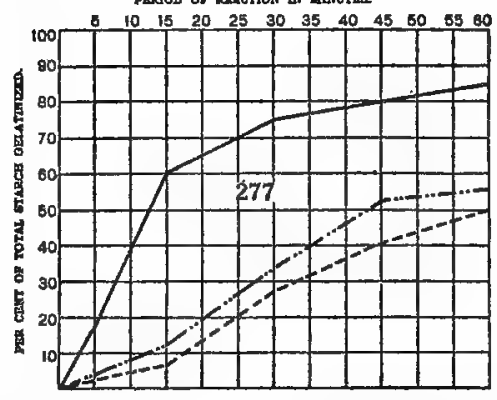

neatob of practios of mantse

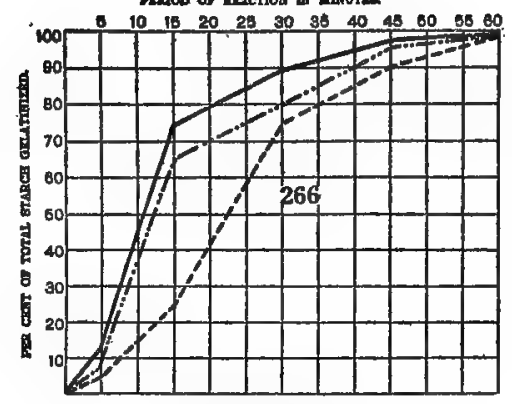

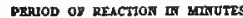

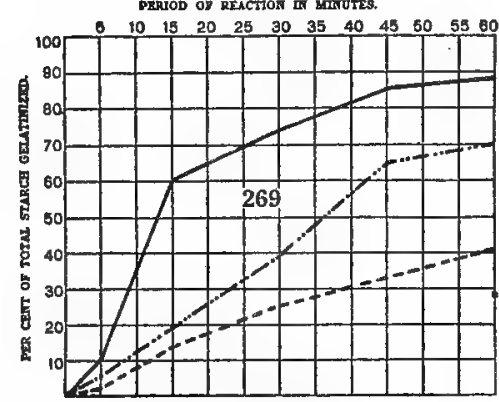

preen of Resction as Mnevtse

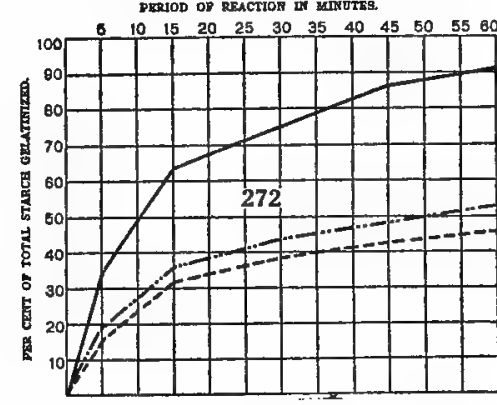

PERTOD of Renctror w MITUTES.

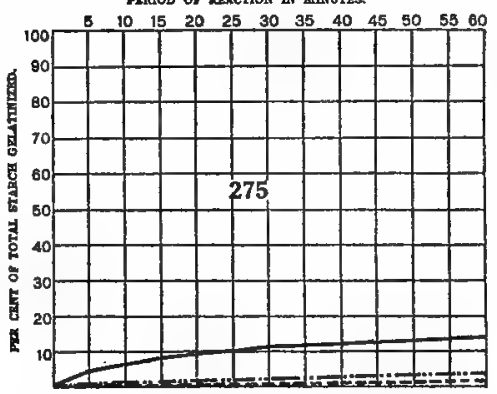

PRPUOD OF REACTION DA MINUTES

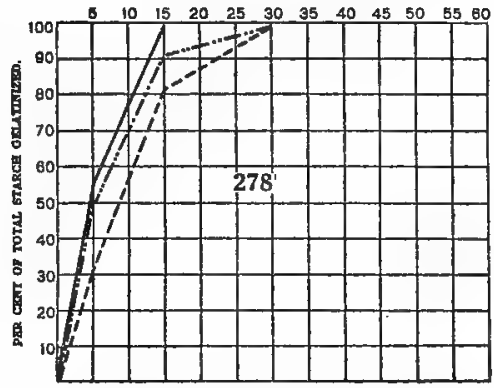

perop of pectrox of wintres

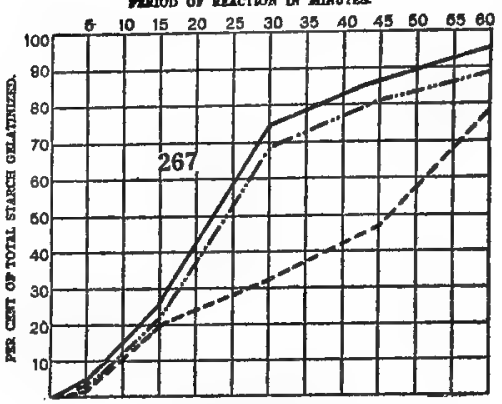

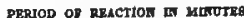

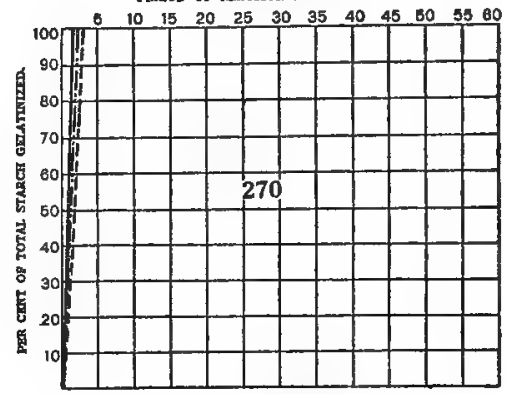

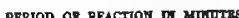

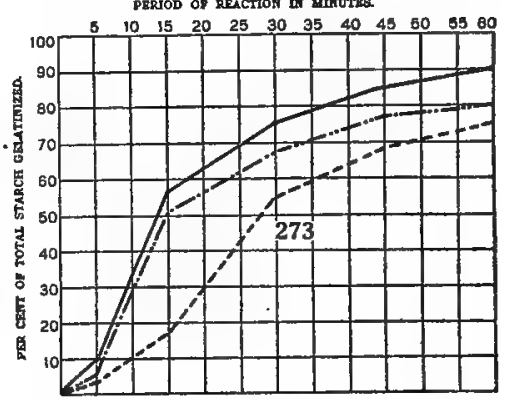

PERTOD Of RRACTION IN MERUTES

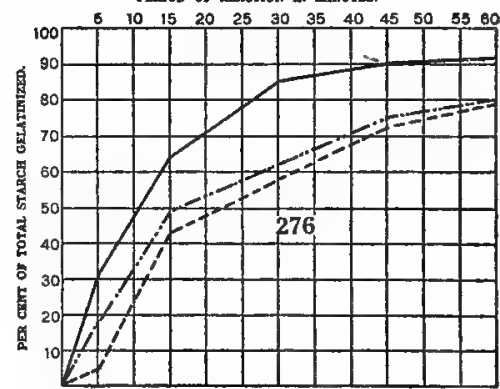

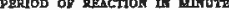

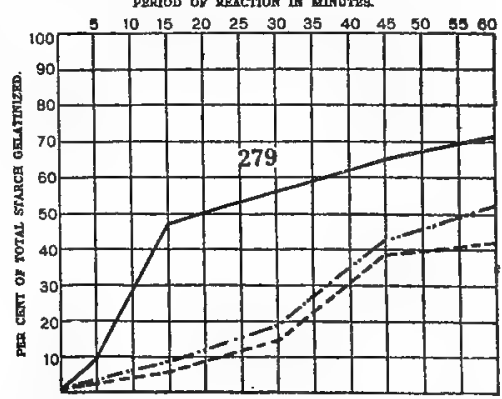

Charts D 265 то D 267, D 269 то D 279.-Velocity-Reactions of Starches of Narcissus tazetta grand monarque (-..-), Narcissus poeticus ornatus (-.....-), and Narcissus poetaz triumph (-).
205. With Chloral Hydrate.
266. With Chromic Acid.
267. With Pyrogallic Acid
269. With Nitric Acid.
270. With Sulphuric Acid.
271. With Hydrochloric Acid.
272. With Potassium Hydroxide.
273. With Potessium Iodide.
275. With Potassium Sulphocyansto.
276. With Sodium Hydroxide.
278. With Sodium Sulphide.
279. With Calcin

CharT D 268.-Velocity-Reactions of Pyrogallic Acid with the Starch of Narcissus tazetta grand monarque. Percentage of entire number of grains (....) and of total starch (_) gelatinized. 

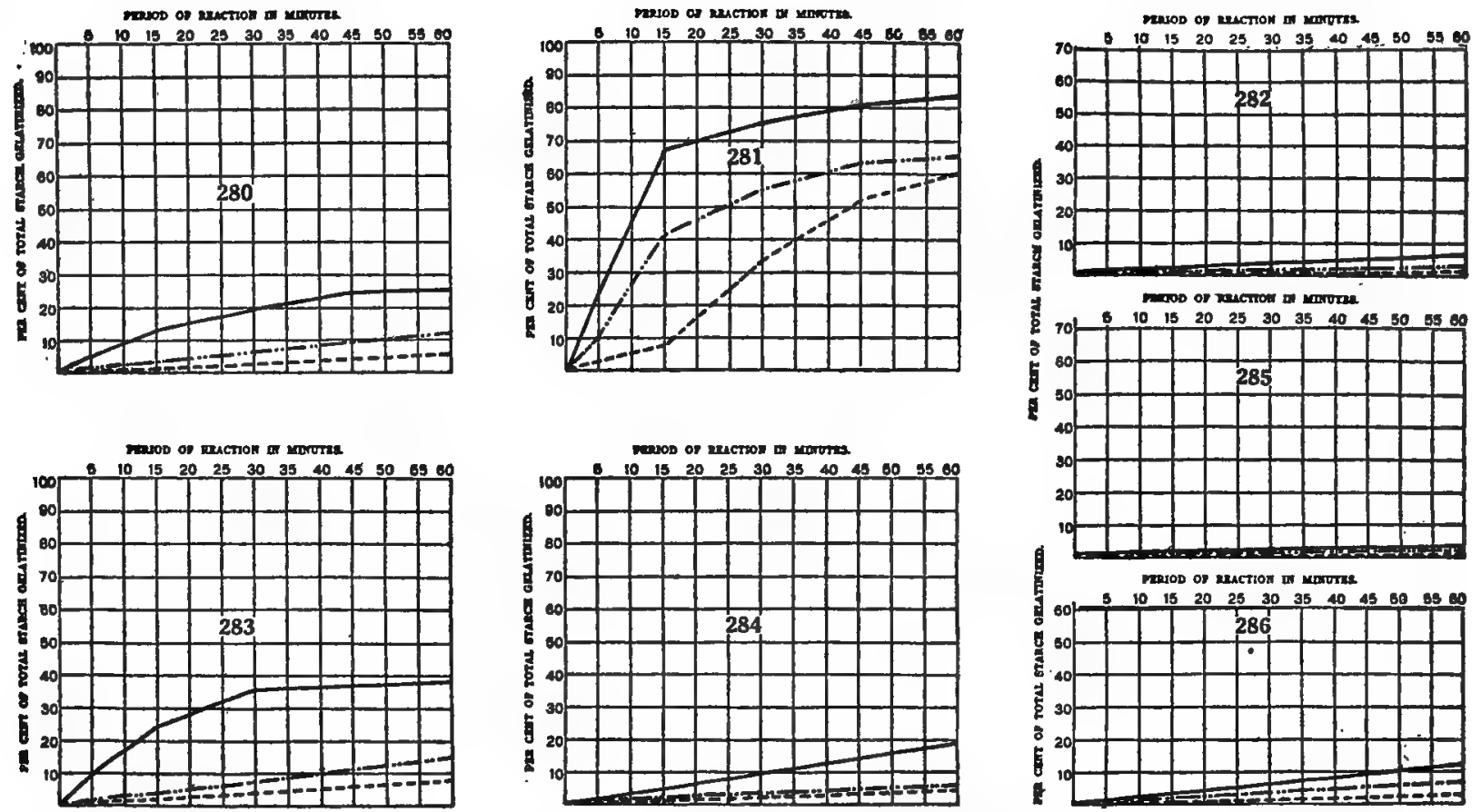

Charts D 280 то D 286.-Velocity-Reactions of Starches of Narcissus tazetta grand monarque (-..--), Narcissus poeticus ornatus (....), and Narcissus poetaz triumph (-). 280. With Uranium Nitrate.
281. With Strontium Nitrate.

282. With Cobalt Nitrate.

283. With Copper Nitrate.

284. With Cuprio Chloride.
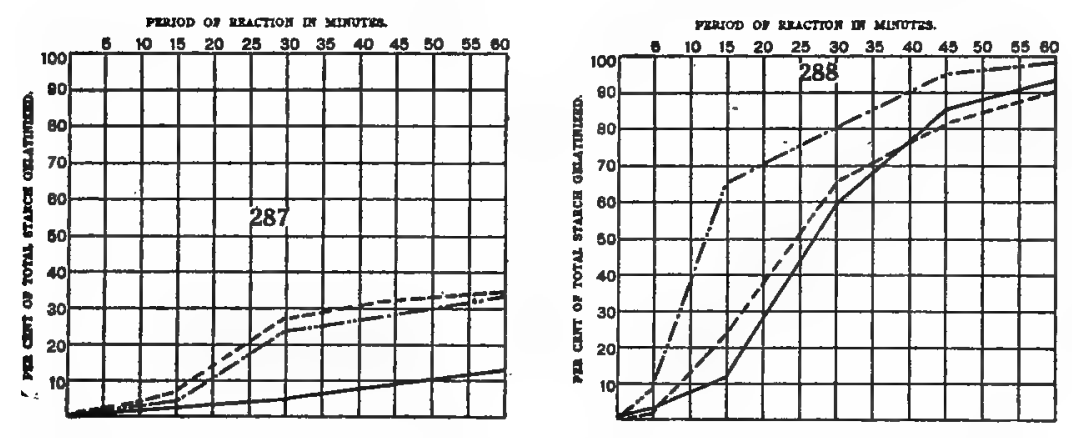

285. With Barium Chloride.

286. With Mercuric Chloride.
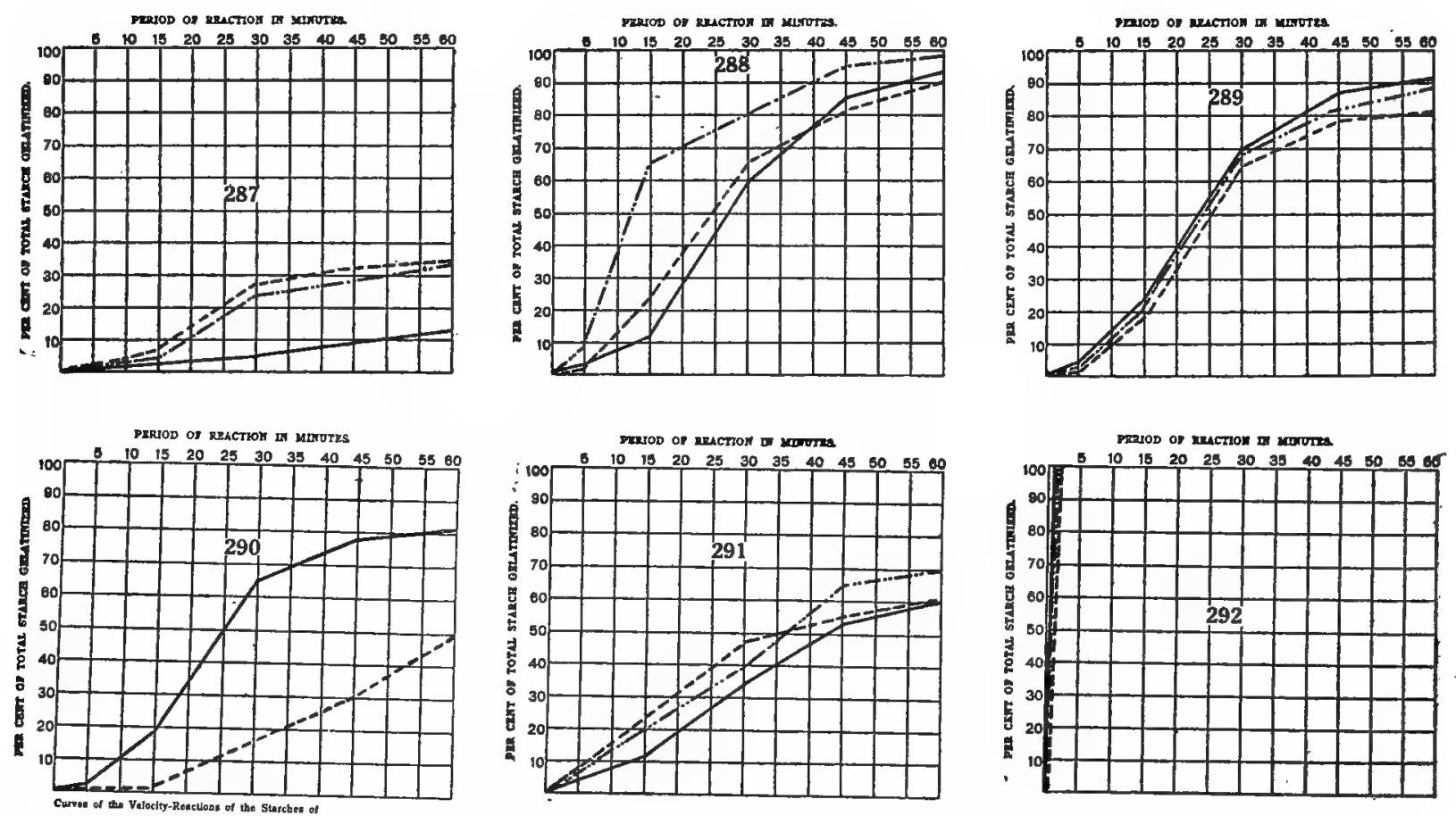

Charts D 287 to D 289 and D 291, D 292.-Velocity-Reactions of Starches of Narcissus gloria mundi (..-.-), Narcissus poeticus ornatus ( ......-), and Narcissus fiery cross (-).
287. With Chloral Hydrate.
288. With Chromic Acid.
289. With Pyrogallic Acid.
291. With Nitric Acid.

Chart D 290.-Velocity-Reactions of Pyrogallic Acid with the Starch of Narcissus gloria mundi. Percentage of entire number of grains (....) and of total starch (_- gelatinized. 

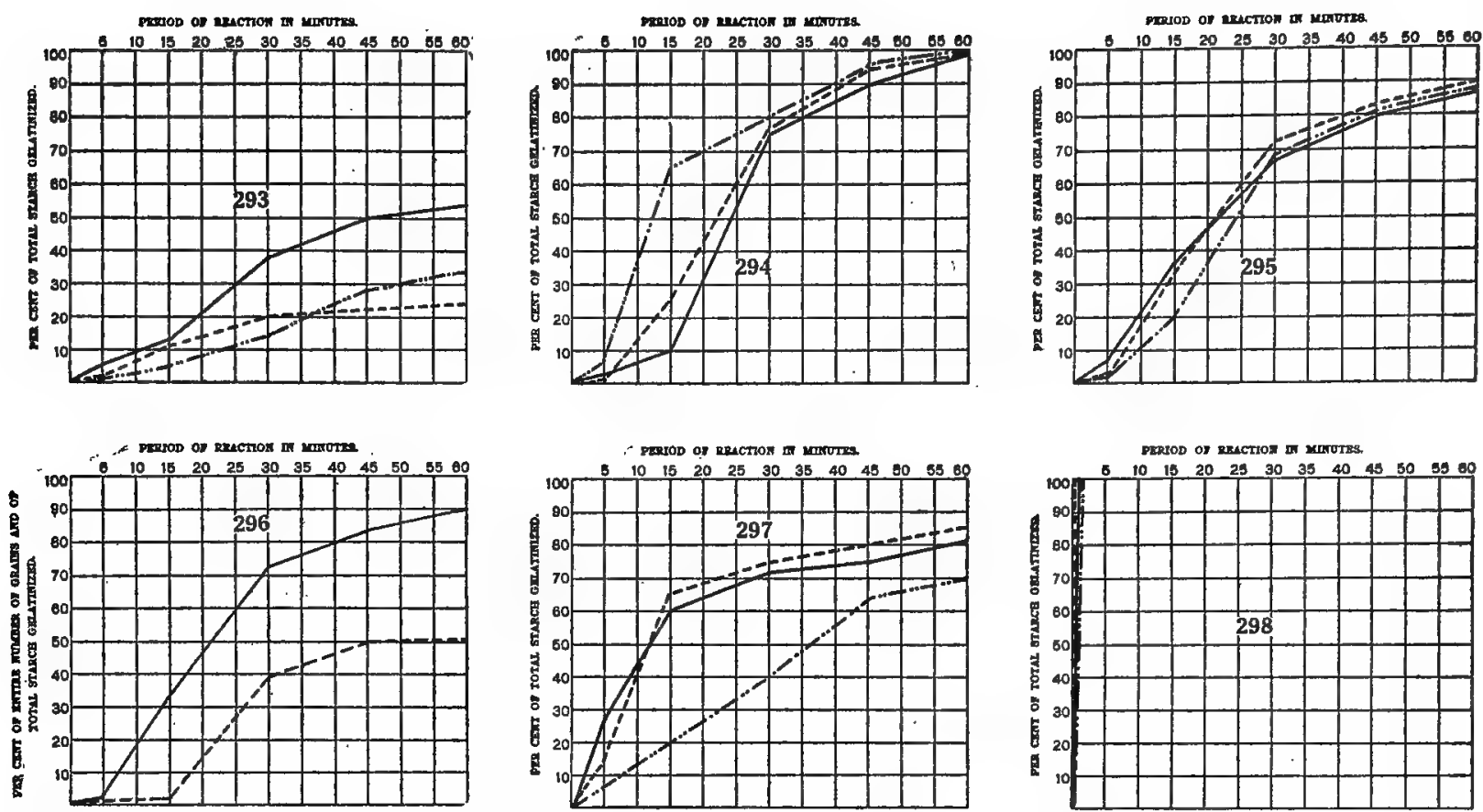

Charts D 293 то D 295, D 297, D 298.-Velocity-Reactions of Starches of Narcissus telamonius plenus (-.--), Narcissus poeticus ornatus (-.....-), and Narcissus doubloon (-).
293. With Chloral Hydrate.
294. With Chromic Acid.
295. With Pyrogallio Acid.
297. With Nitric Acid.

Chart D 296.-Velocity-Reactions of Pyrogallic Acid with the Starch of Narcissus telamonius plenus. Percentage of entire number of grains ( ....) and of total starch (-) gelatinized.
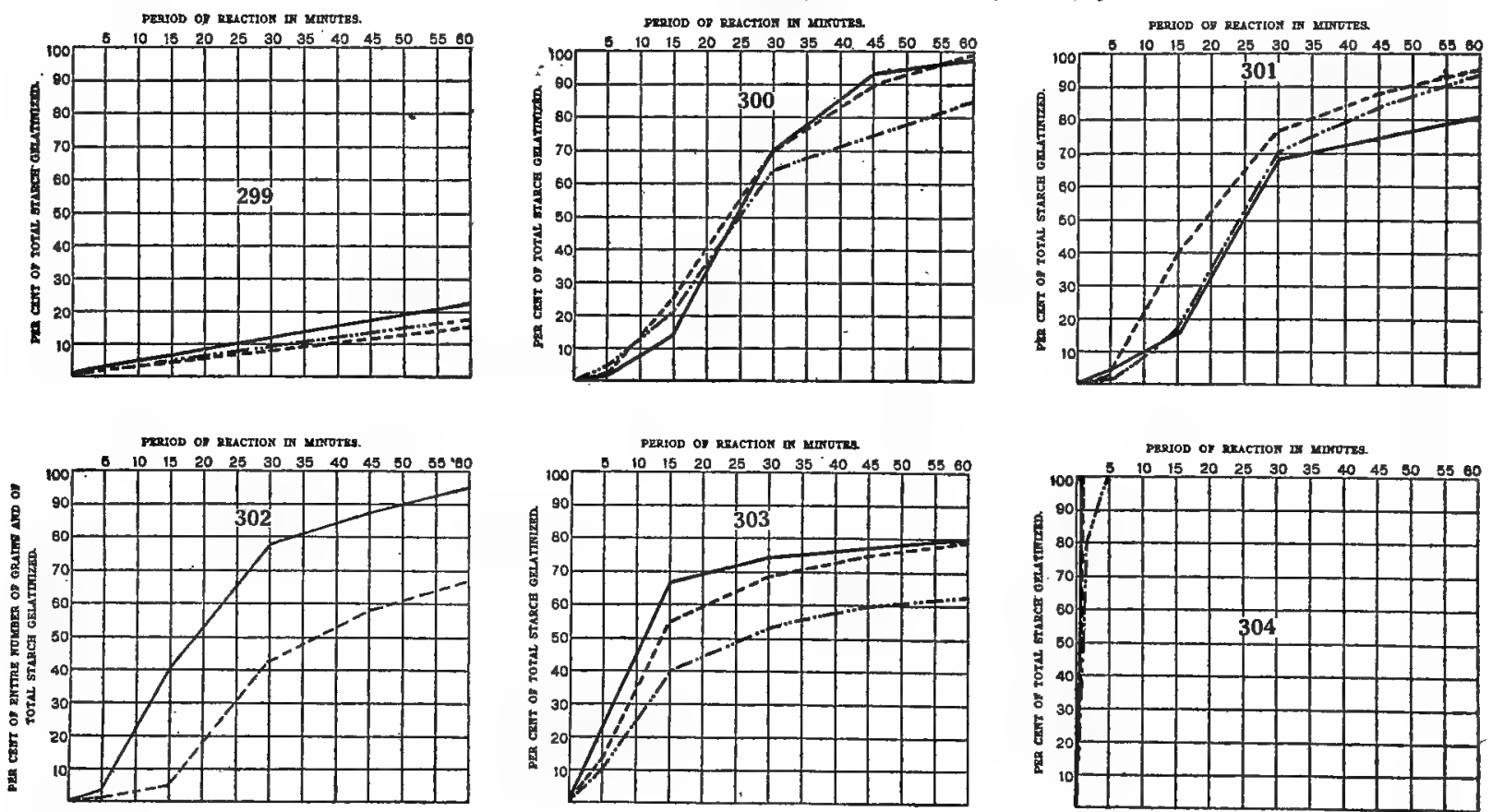

Charts D 299 to D 301, D 303, D 304.-Velocity-Reactions of Starches of Narcissus princess mary (....-), Narcissus poeticus poetarum (.......), and Narcissus cresset (-).

299. With Chloral Hydrate.

301. With Pyrogallic Acid.

303. With Nitric Acid.

Chart D 302.-Velocity-Reactions of Pyrogallic Acid with the Starch of Narcissus princess mary. Percentage of entire number of grains (....) and of total starch (-) gelatinized. 

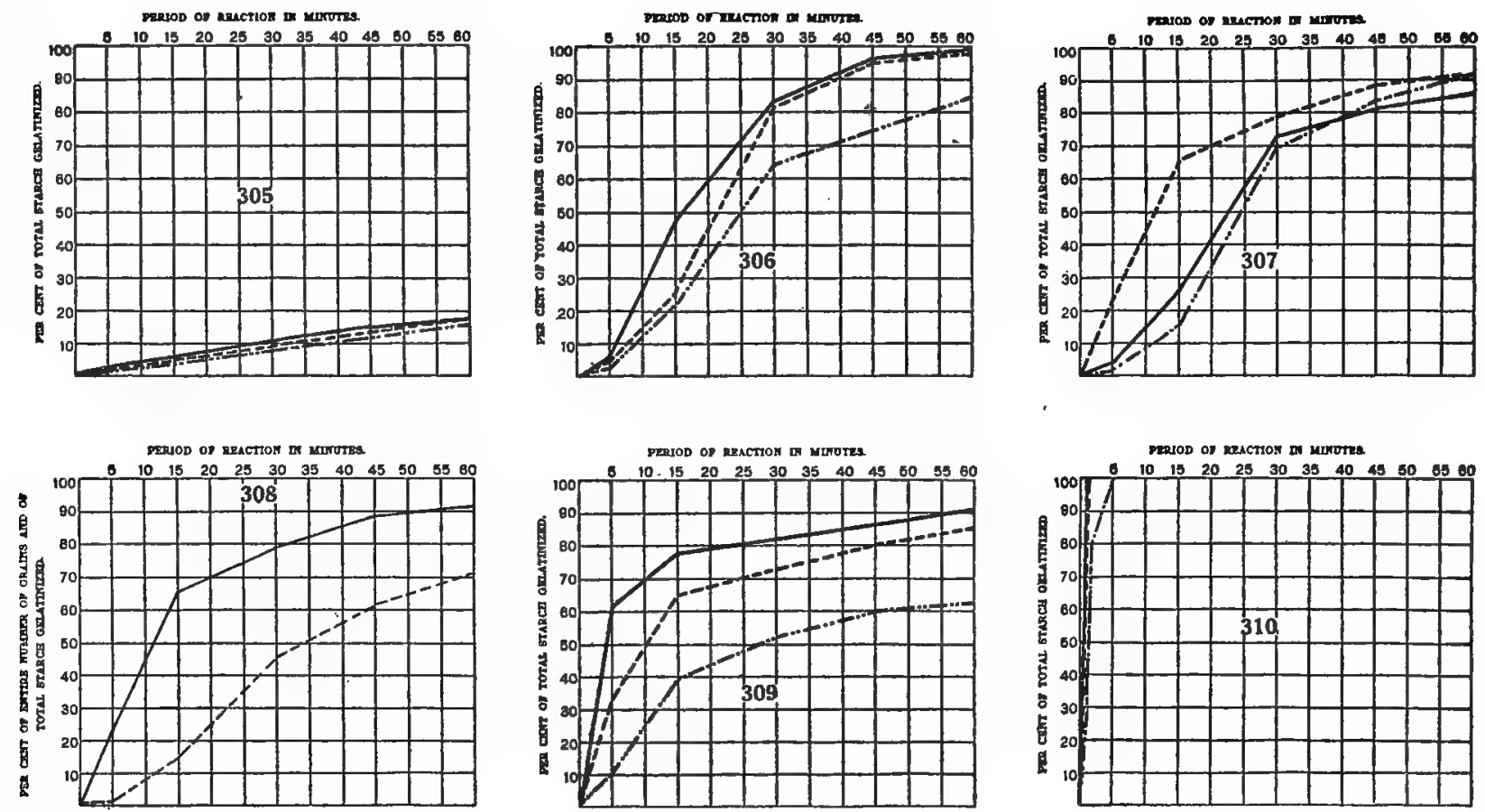

Charts D 305 to D 307, D 309, D 310.-Velocity-Reactions of Starches of Narcissus abscissus ( --.--), Narcissus poeticus poetarum ( -......), and Narcissus will scarlet (-). 305. With Chloral Hydrate.
306. With Chromic Acid.

307. With Pyrogallic Acid.

309. With Nitric Acid

310. With Sulphuric Acid.

Chart D 308.-Velocity-Reactions of Pyrogallic Acid with the Starch of Narcissus abscissus. Percentage of entire number of grains (----) and of total starch (-) gelatinized.
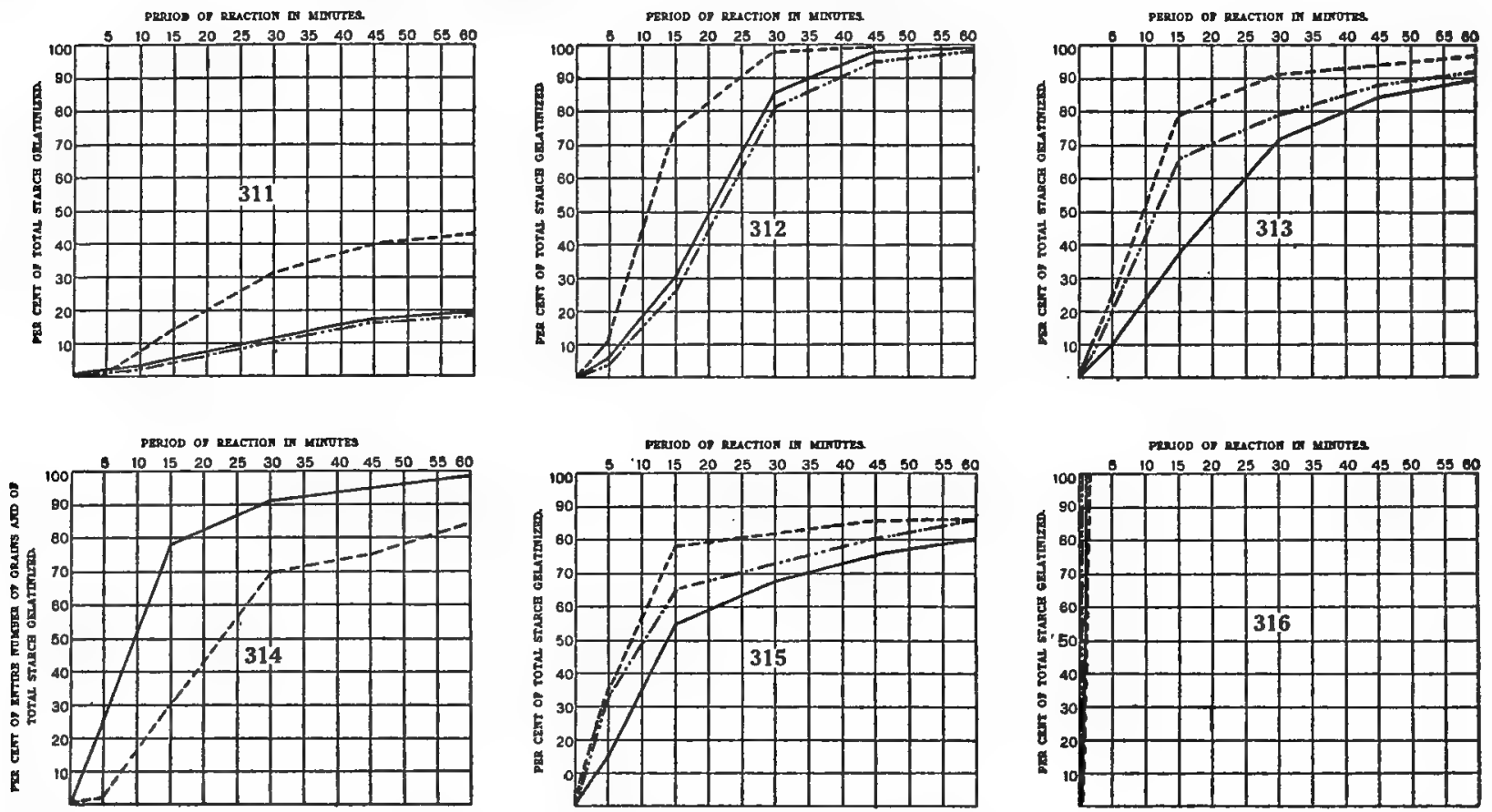

Charts D 311 тo D 313, D 315, D 316.-Velocity-Reactions of Starches of Narcissus albicans (....-.), Narcissus abscissus ( .......), and Narcissus bicolor apricot (- - ).

311. With Chloral Hydrate.
312. With Chromic Acid.

313. With Pyrogallic Acid.

315. With Nitric Acid.

316. With Sulphuric Aeid.

Chart D 314.-Velocity-Reaction of Pyrogallic Acid with the starch of Narcissus albicans. Percentage of entire number of grains (.....) and of total starch (-) gelatinized. 

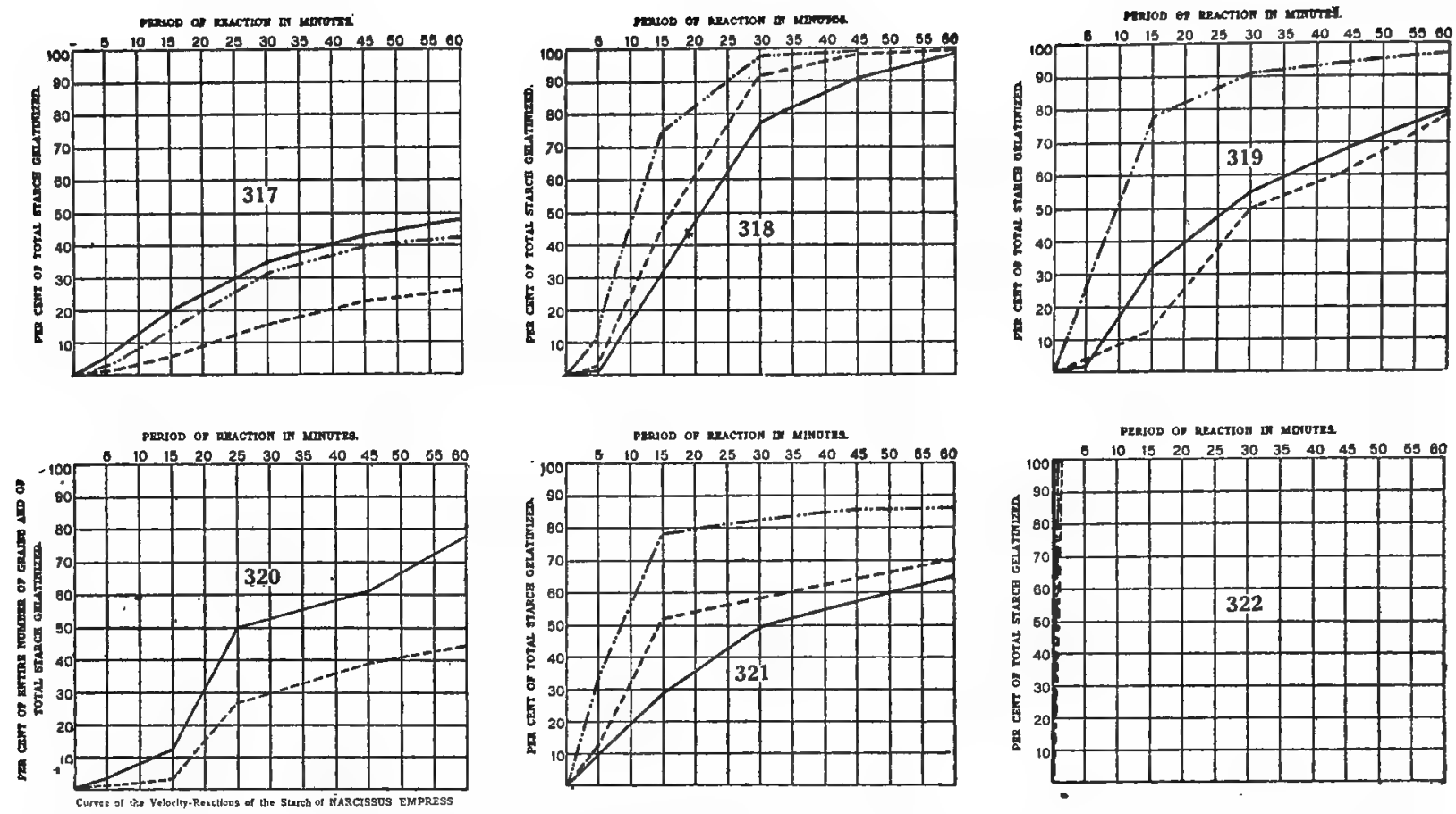

Charts D 317 to D 319, D 321, D 322.-Velocity-Reactions of Starches of Narcissus empress (-..--), Narcissus albicans ( ....... ), and Narcissus madame de graaff (-).

317. With Chloral Hydrate.
318. With Chromic Acid.

319. With Pyrogallio Acid.

321. With Nitrio Acid.

322. With Sulphuric Acid.

Chart D 320.-Velocity-Reactions of Pyrogallic Acid with the Starch of Narcissus empress. Percentage of entire number of grains (.....) and of total starch (-) gelatinized.
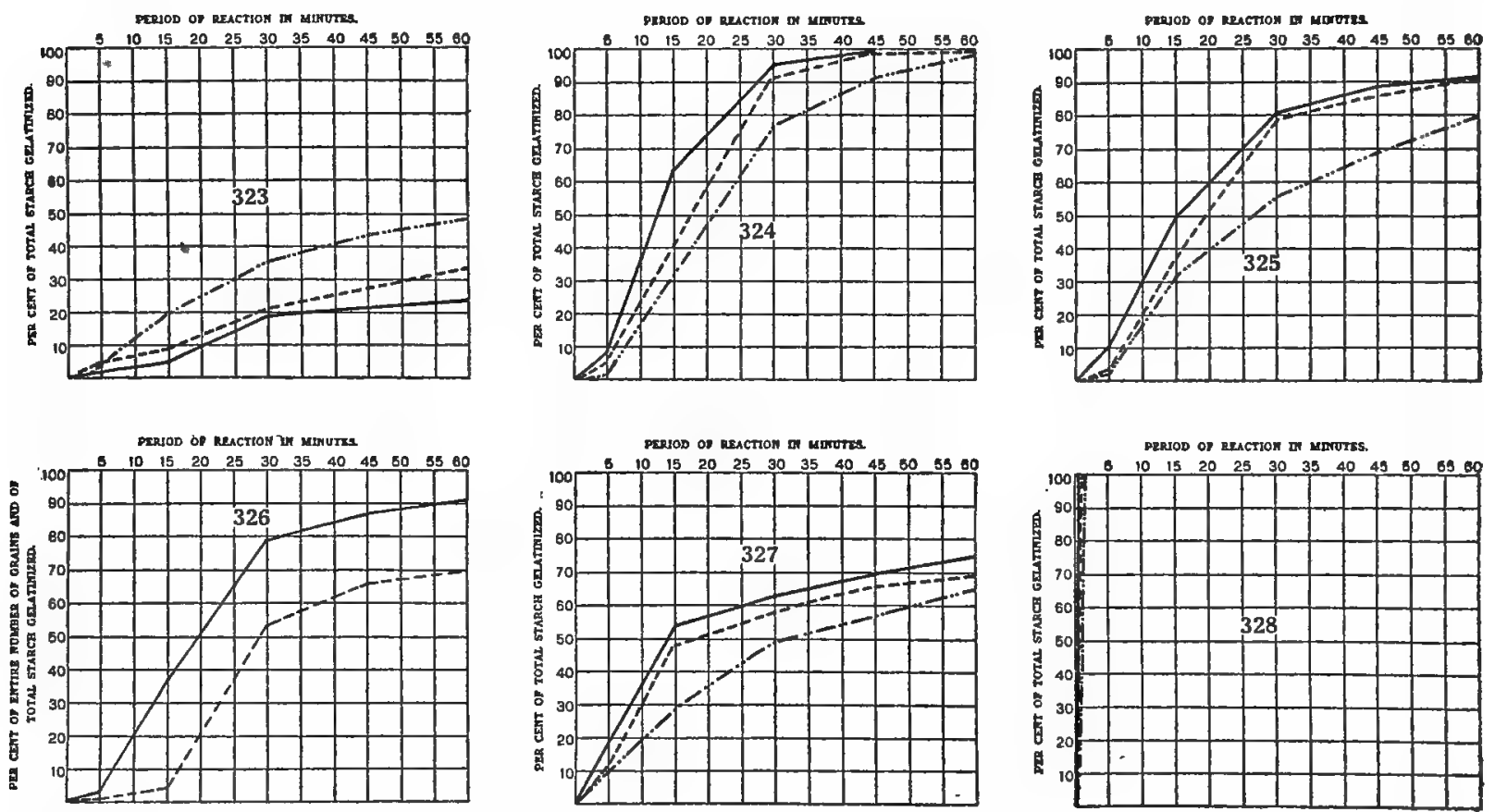

Charts D 323 to D 325, D 327, D 328.-Velocity-Reactions of Starches of Narcissus weardale perfection (....-), Narcissus madame de graaff (-.....), and Narcissus pyramus (-

$\begin{array}{ll}\text { 323. With Chloral Hydrate. } & \text { 325. With Pyrogallic Acid. With Nitric Acid, } \\ \text { 324. With Chromic Acid. } & \text { 328. With Sulphuic Acid }\end{array}$

328. With Sulphuric Acid.

Cenart D 326.-Velocity-Reactions of Pyrogallic Acid with the Starch of Narcissus weardale perfection. Percentage of entire number of grains (.....) and of total starch (- $(-)$ gelatinized. 

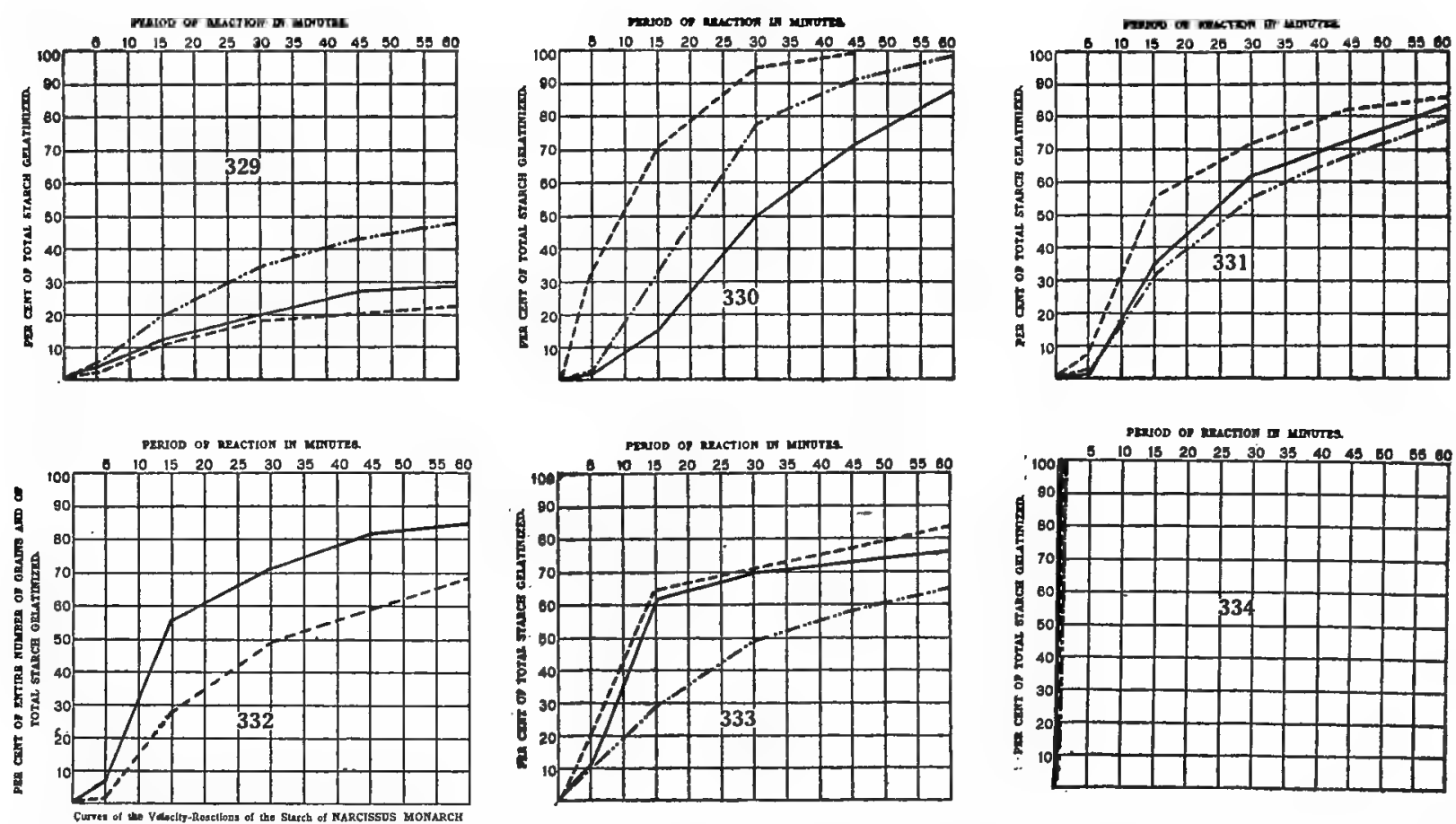

Charts D 329 to D 331, D 333, D 334.-Velocity-Reactions of Starches of Narcissus monarch ( .....), Narcissus madame de graaff ( .......), and Narcissus lord roberts (-).

329. With Chloral Hydrate.

331. With Pyrogallic Acid.

333. With Nitric Acid.

334. With Sulphuric Acid.

Chart D 332.-Velocity-Reactions of Pyrogallic Acid with the Starch of Narcissus monarch. Percentage of entire number of grains (....) and of total starch (-) gelatinized.
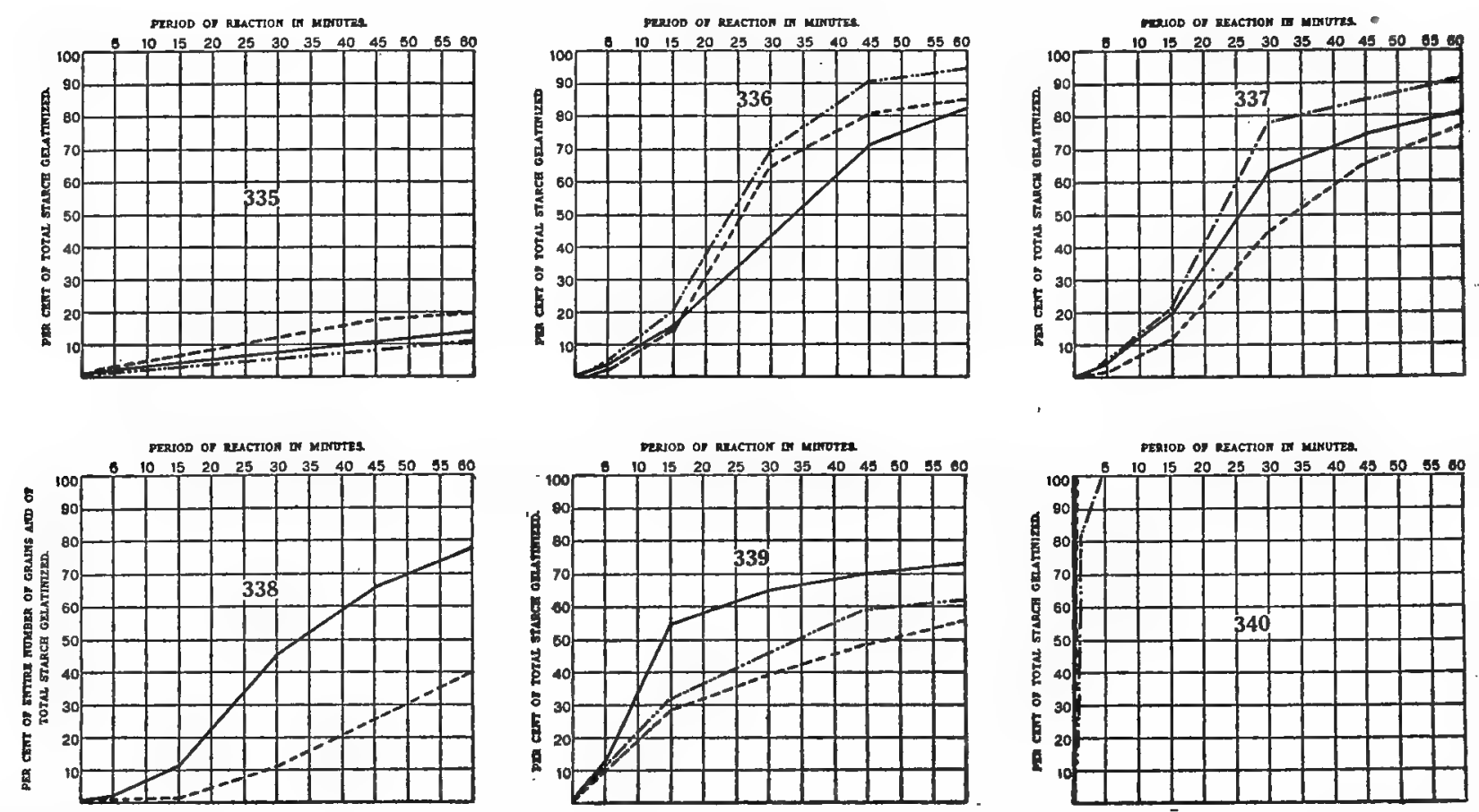

Charts D 335 to D 337, D 339, D 340.-Velocity-Reactions of Starches of Narcissus leedsii minnie hume (.....), Narcissus triandrus albus (.....) and Narcissus agnes harvey (-).

335. With Sulphuric Acid.

335. With Chlphuric Acid.

337. With Chromic Acid.

339. With Nitric Acid.

Chart D 338.-Velocity-Reactions of Pyrogallic Acid with the Starch of Narcissus leedsii minnie hume. Percentage of entire number of grains (....) and of total starch (- $(-)$ gelatinized. 

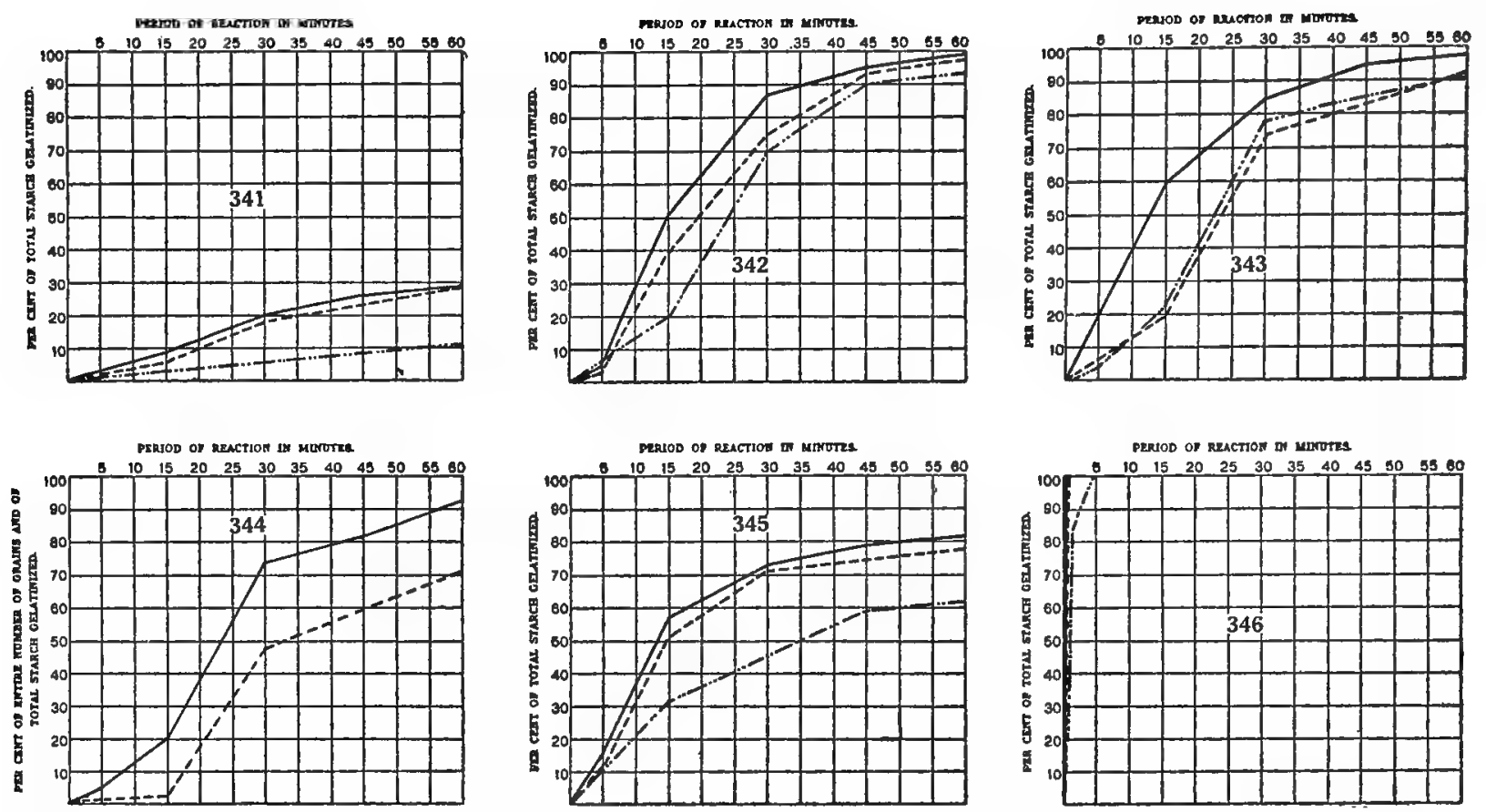

Charts D 341 to D 343, D 345, D 346.-Velocity-Reactions of Starches of Narcissus emperor (-.... ), Narcissus triandrus albus ( ......-), and Narcissus $j . t$. bennett poe (- - ).

$\begin{array}{lll}\text { 341. With Chloral Hydrate. } & \text { 343. With Pyrogallio Acid. } & \text { 345. With Nitric Acid. } \\ \text { 342. With Chromic Acid. } & \text { 346. With Sulphuric Acid. }\end{array}$

$\begin{array}{lll}\text { 341. With Chloral Hydrate. } & \text { 343. With Pyrogallio Acid. } & \text { 345. With Nitric Acid. } \\ \text { 342. With Chromic Acid. } & \text { 346. With Sulphuric Acid. }\end{array}$

$\begin{array}{lll}\text { 341. With Chloral Eydrate. } & \text { 343. With Pyrogallio Acid. } & \text { 345. With Nitric Acid. } \\ \text { 342. With Chromic Acid. } & \text { 346. With Sulphuric Acid. }\end{array}$

Chart D 344.-Velocity-Reactions of Pyrogallic Acid with the Starch of Narcissus emperor. Percentage of entire number of grains (-..-) and of total starch (-) gelatinized.
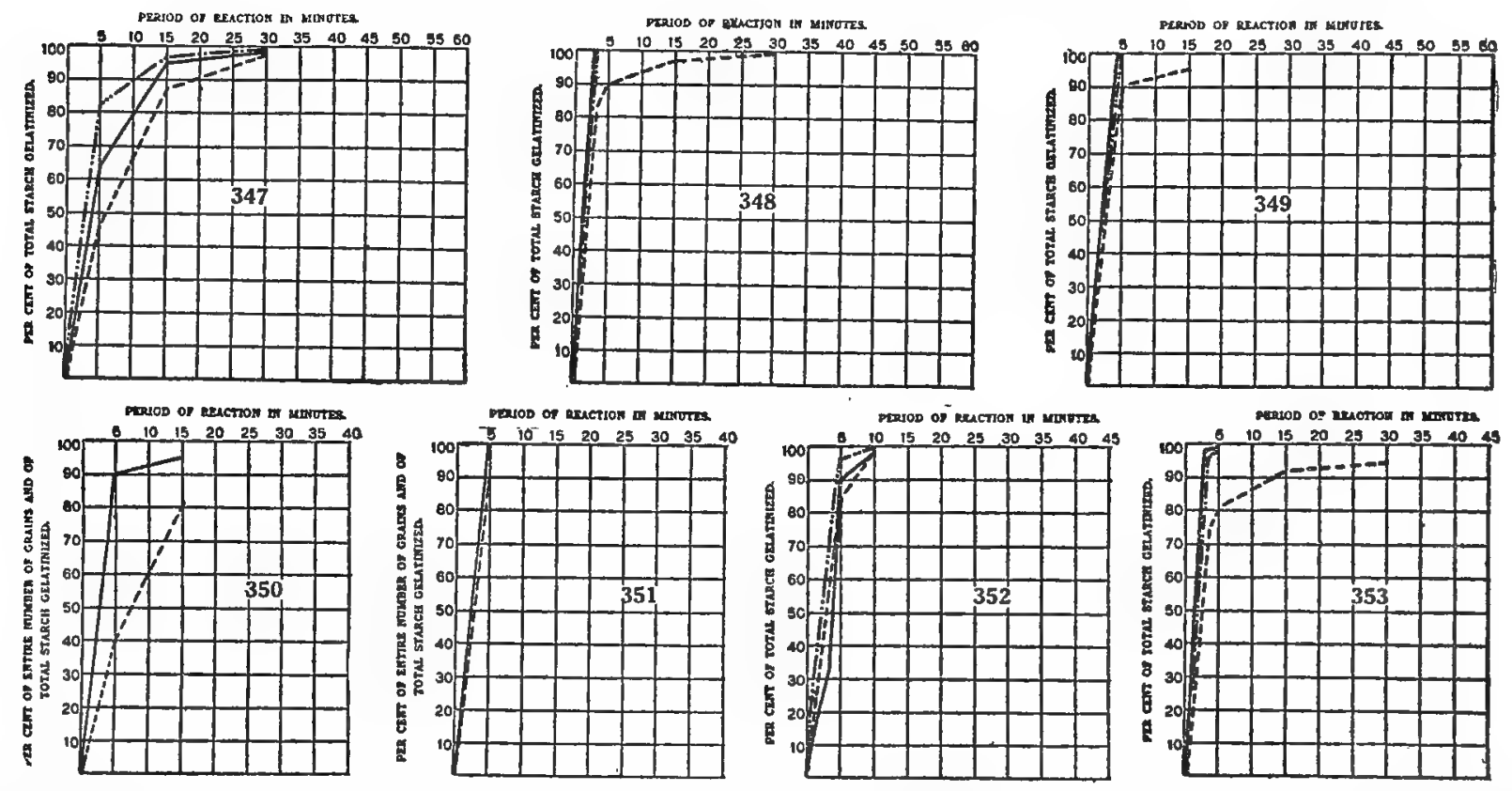

Charts D 347 to D 349, D 352, D 353.-Velocity-Reactions of Starches of Lilium martagon album (......), Lilium maculatum ( -.....-), and Lilium marhan (-).

347. With Chloral Hydrate.
348. With Chromic Acid.

349. With Pyrogallic Acid.

352. With Sodium Salicylate.

353. With Barium Chloride.

Charts D 350 And D 351.-Velocity-Reactions of Pyrogallic Acid with the Starches of Lilium martagon album and L. maculatum. Percentage of entire number of grains (....) and of total starch (- ( ) gelatinized. 

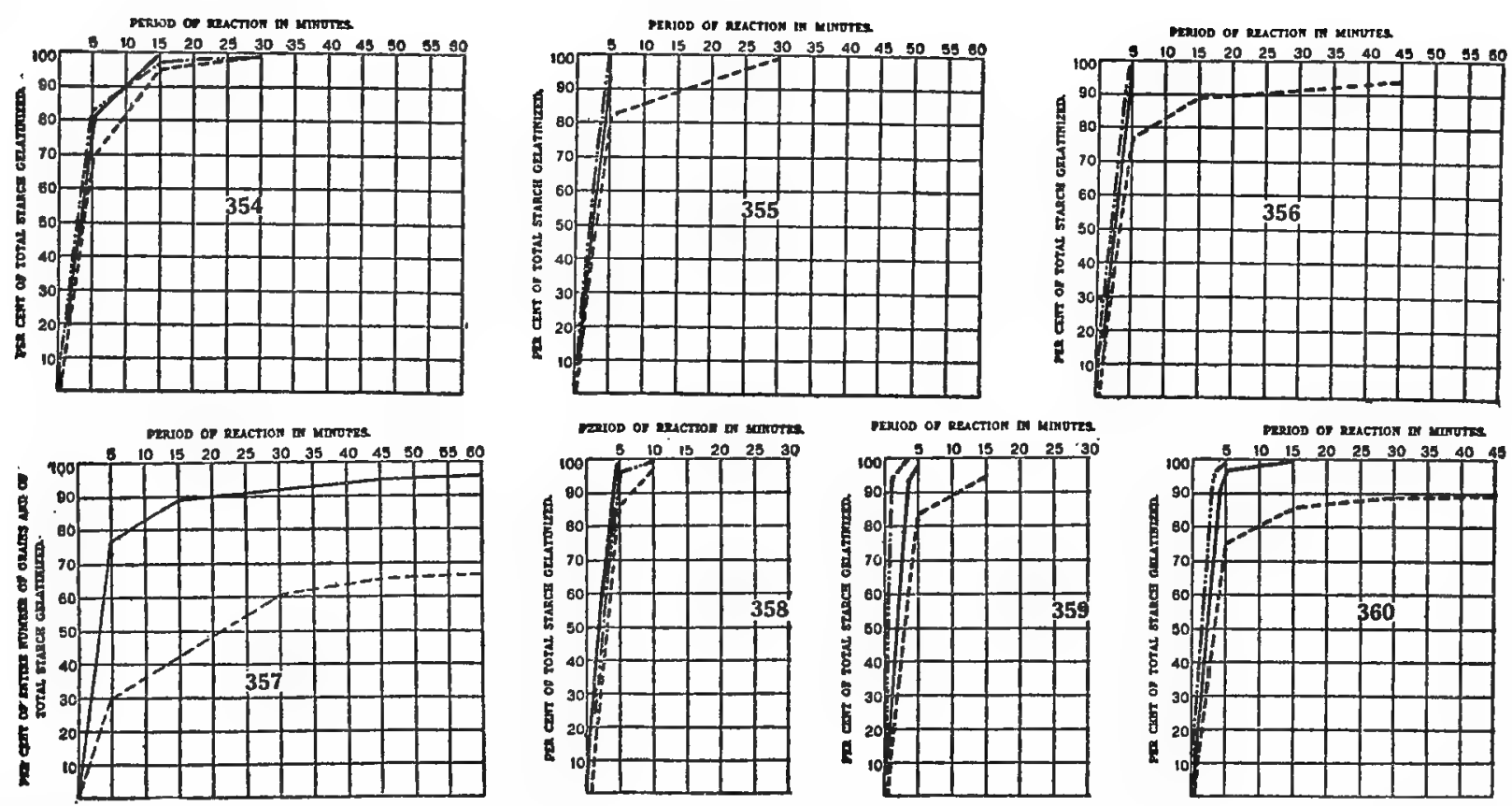

Charts D 354 то D 356, D 358 то D 360.-Velocity-Reactions of Starches of Lilium martagon (..... ), Lilium maculatum ( .......), and Lilium dalhansoni (-).

354. With Chloral Hydrate. 355. With Chromic Acid.

356. With Pyrogallio Acid.
358. With Sodium Salicylate.

359. With Cobalt Nitrate.

Chart D 357.-Velocity-Reactions of Pyrogallic Acid with the Starch of Lilium martagon. Percentage of entire number of grains (-..--) and of total starch (-) gelatinized.
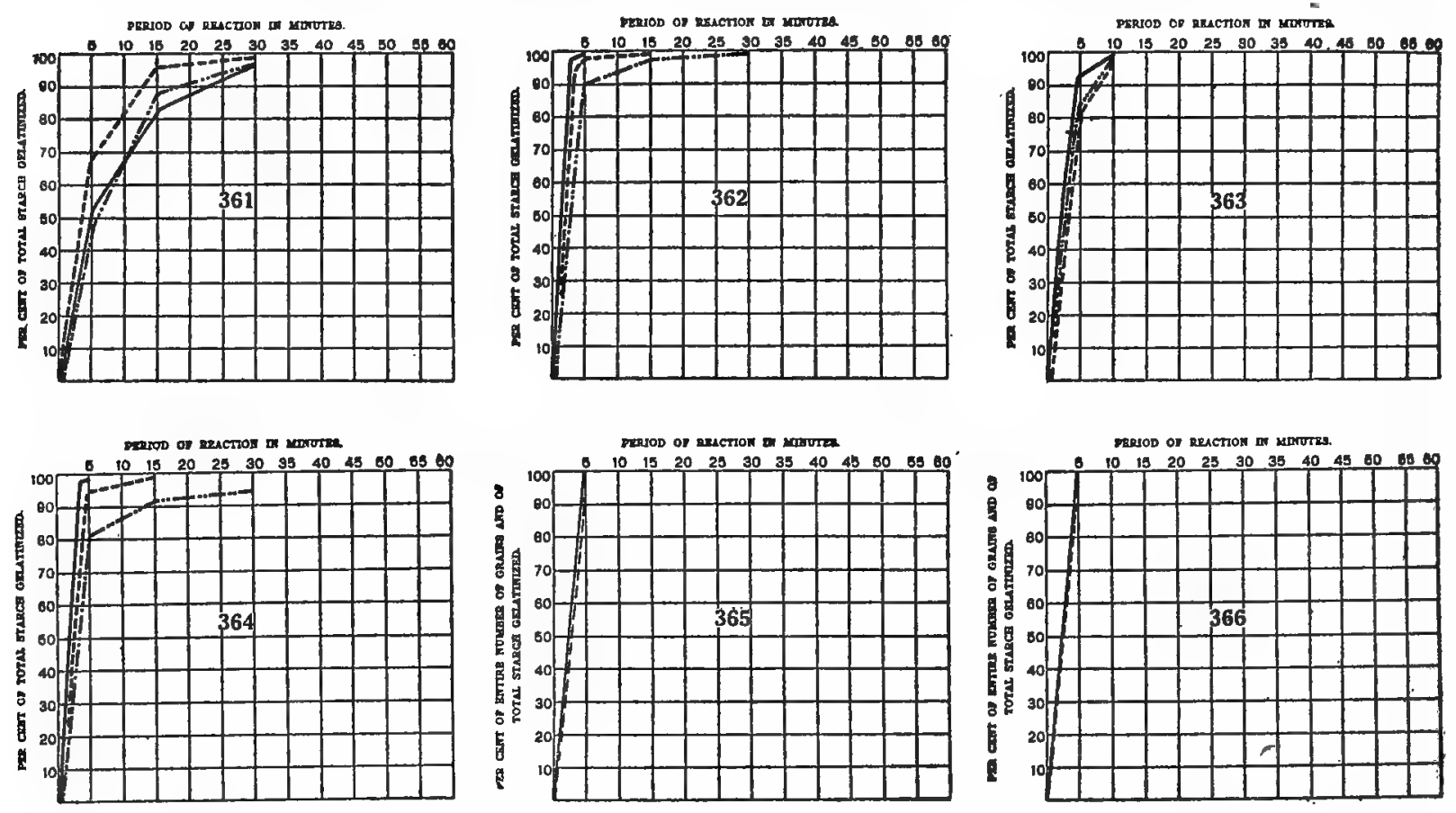

Charts D 361 тo D 364.-Velocity-Reactions of the Starches of Lilium tenuifolium (.....), Lilium martagon album (-.....), and Lilium golden gleam (-).

$\begin{array}{ll}\text { 361. With Chloral Hydrate. } & \text { 363. With Sodium Salicylate. } \\ \text { 362. With Chromic Acid. } & \text { 364. With Barium Chloride. }\end{array}$

Chants D 365 and D 366.-Velocity-Reactions of Pyrogallic Acid with the Starches of Lilium tenuifolium and L. golden gleam. Percentage of entire number of grains (_...) and of total starch (_- gelatinized. 

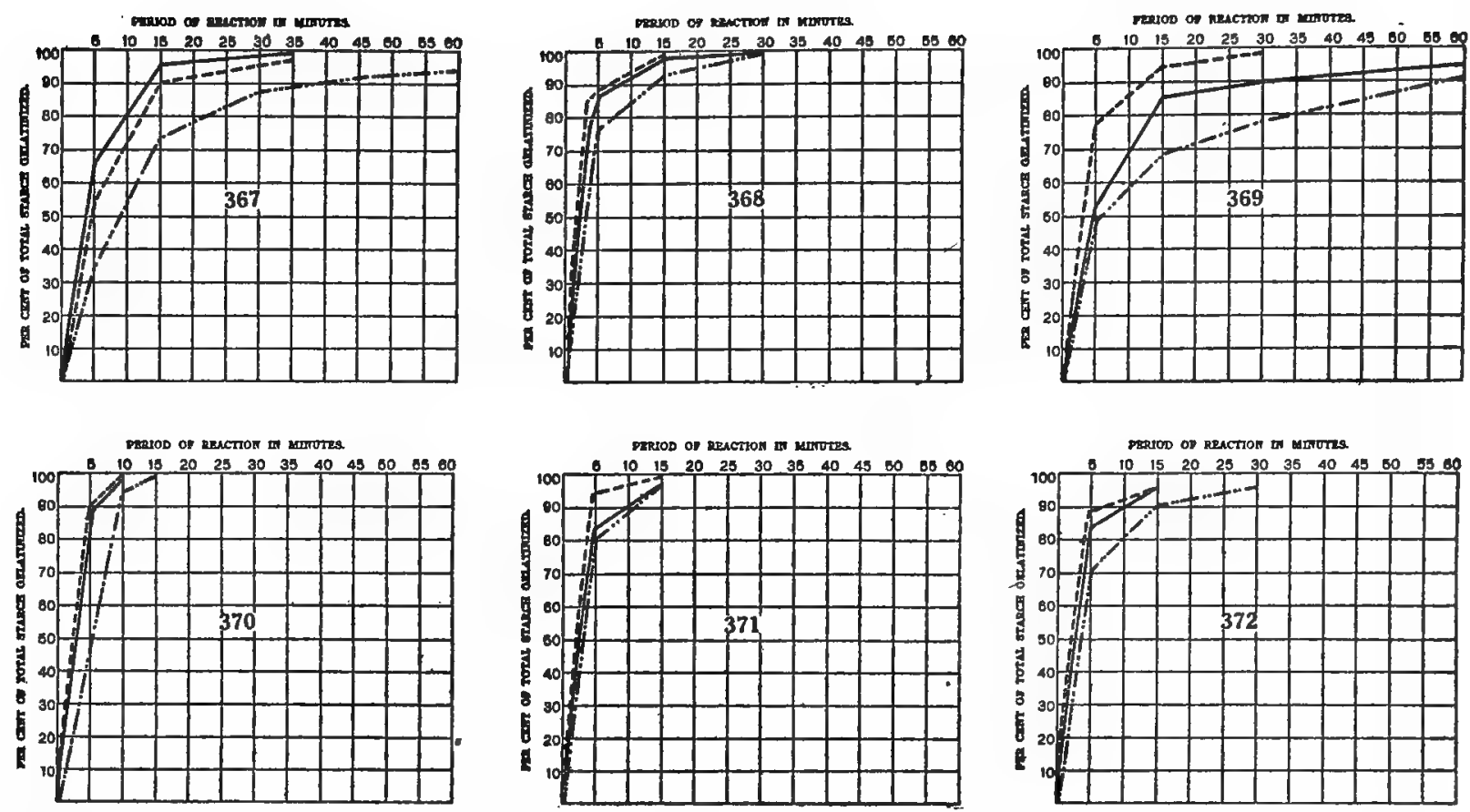

Charts D 367 то D 372.-Velocity-Reactions of Starches of Lilium chalcedonicum (--.--), Lilium candidum (...-), and Lilium testaceum (- - .

367. With Chloral Hydrate. 368. With Chromio Aoid.

369. With Pyrogallic Acid. 370. With Sodium Balicylate.
371. With Cobalt Nitrate.

372. With Barium Chloride.
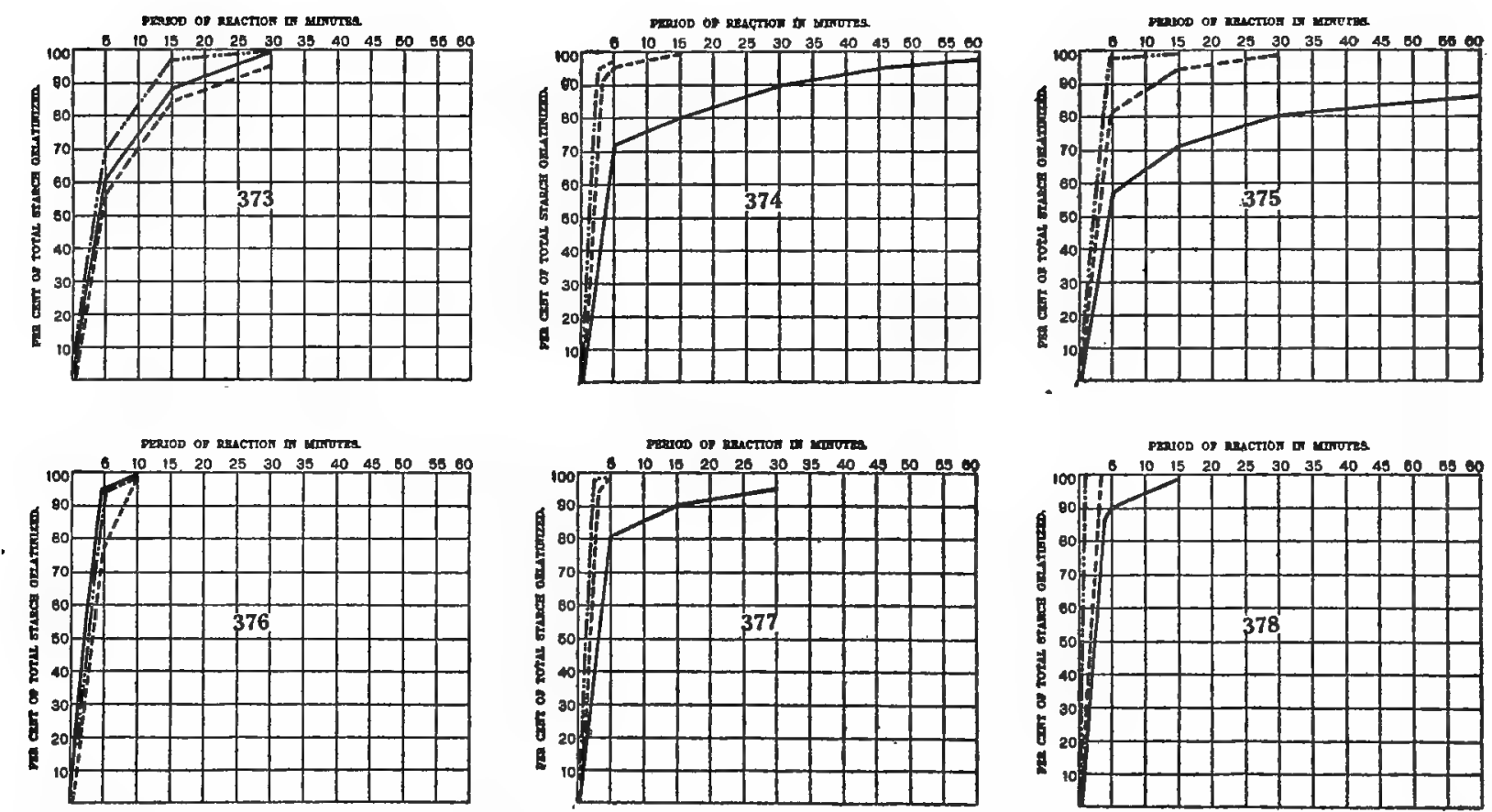

Charts D 373 то D 378.--Velocity-Reactions of Starches of Lilium pardalinum (-...-), Lilium parryi (-.....-), and Litium burbanki (-).

375. With Pyrogallic Acid. 376. With Sodium Salicylate.
377. With Cobslt Nitrate.

378. With Mercurio Chloride.
373. With Chloral Hydrate 374. With Chromio Acid. 


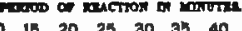

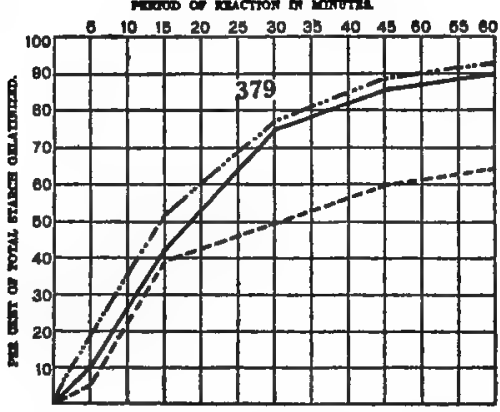

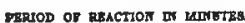

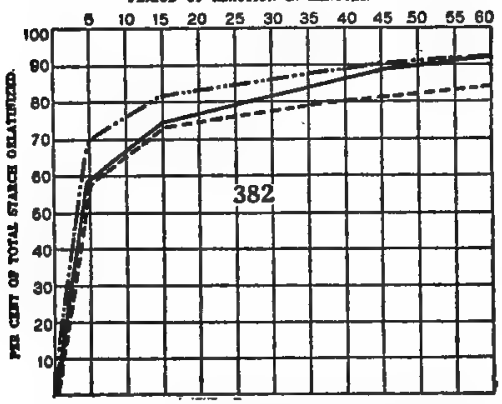

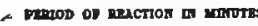

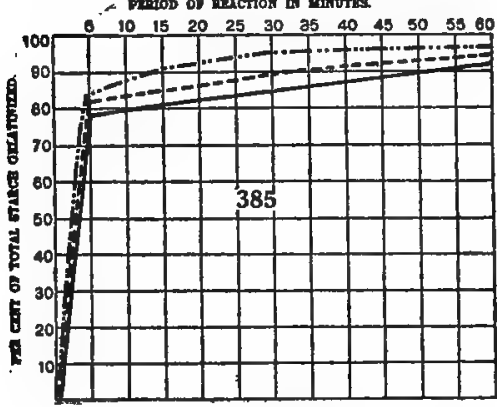

PERIOD OP RRACTION IN WUNUTES

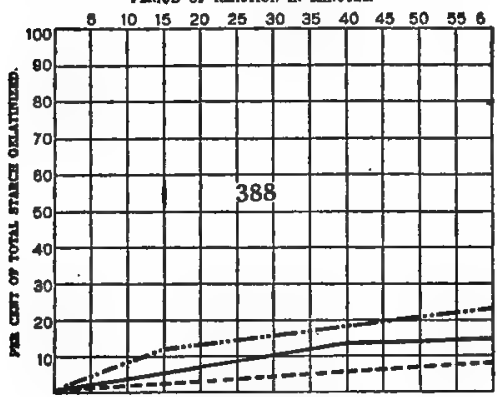

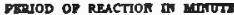

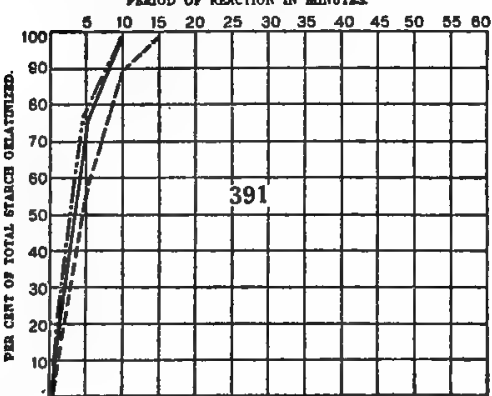

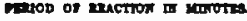

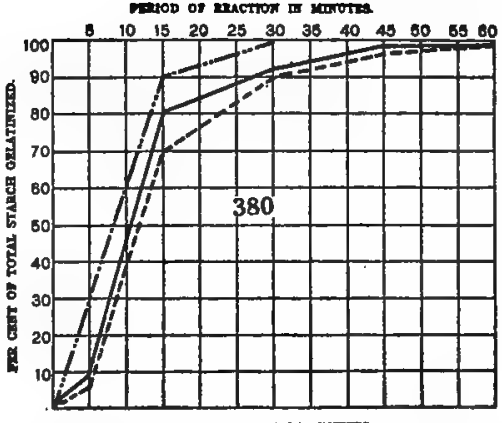

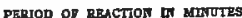

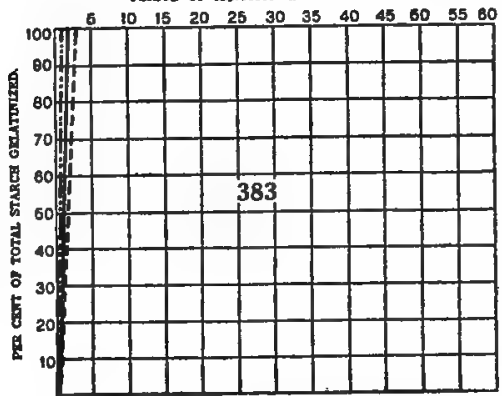

PERTOD OF REACTION DF MHOTES

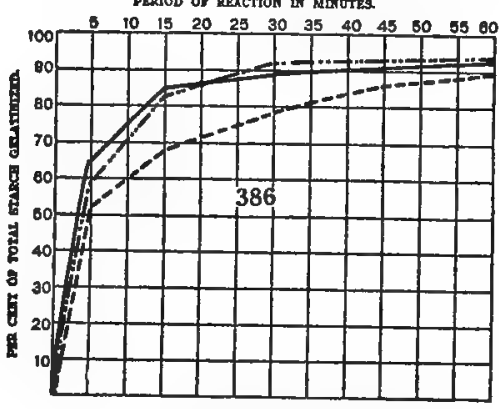

PERLOD of REACTIOA IN MRTOTES.

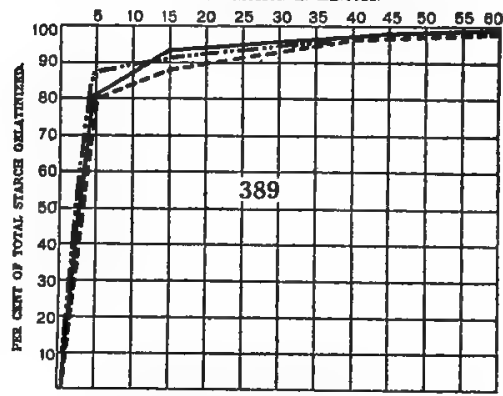

PERTOD OF REACTION IN MCTUTE

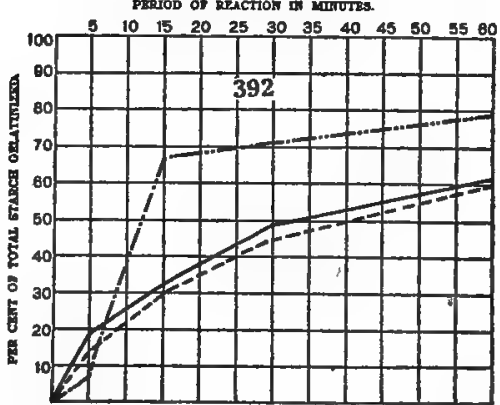

Furos of mecton a merom.

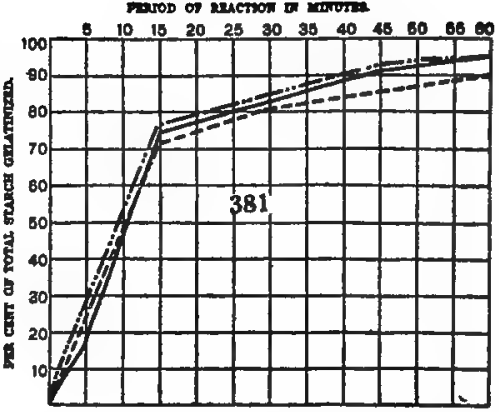

PRDTOD OP REACTIOA IA MLTUTES

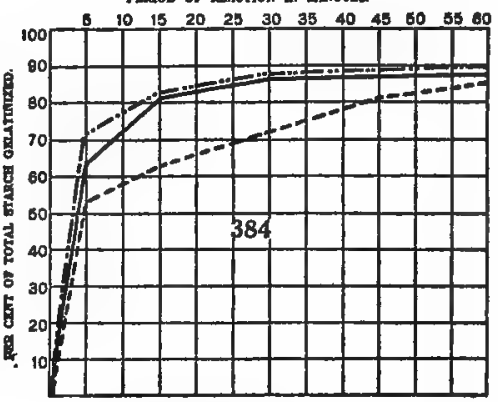

PERIOD Of REAction m MLTOTES.

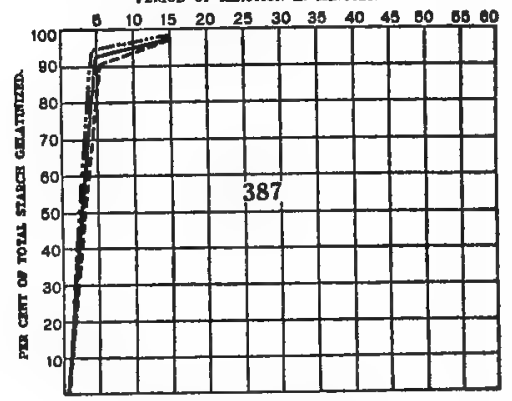

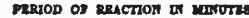
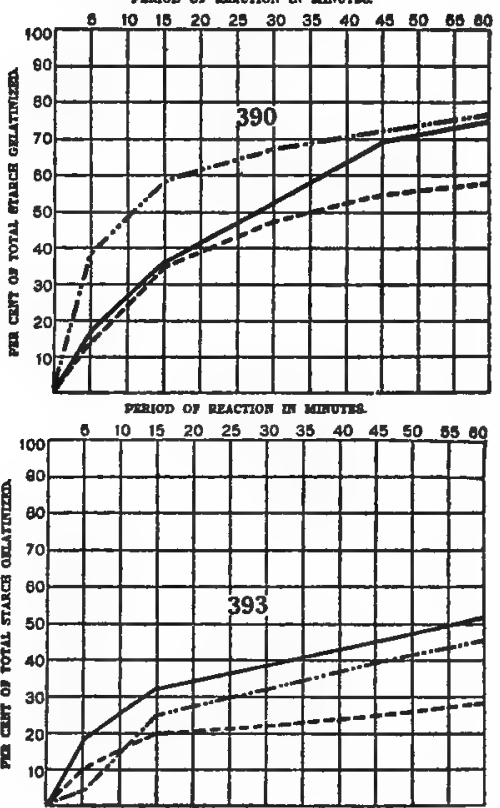

Charts D 379 to D 393.-Velocity-Reactions of Starches of Iris iberica (-....), Iris trojana (......-), and Iris ismali (-).

379. With Chloral Hydrate. 380. With Chromic Acid. 381. With Pyrogallic Acid 382. With Nitric Acid. 383. With Sulphuric Acid.
384. With Hydrochloric Acid.

385. With Potassium Hydroxide.

386. With Potassium Iodide.

387. W:th Potassium Sulphocyanate.

388. With Potassium Sulphide.
389. With Sodium Hydroxide

390. With Sodium Sulphide.

391. With Sodium Salicylate.

392. With Calcium Nitrate.

393. With Uranium Nitrate. 

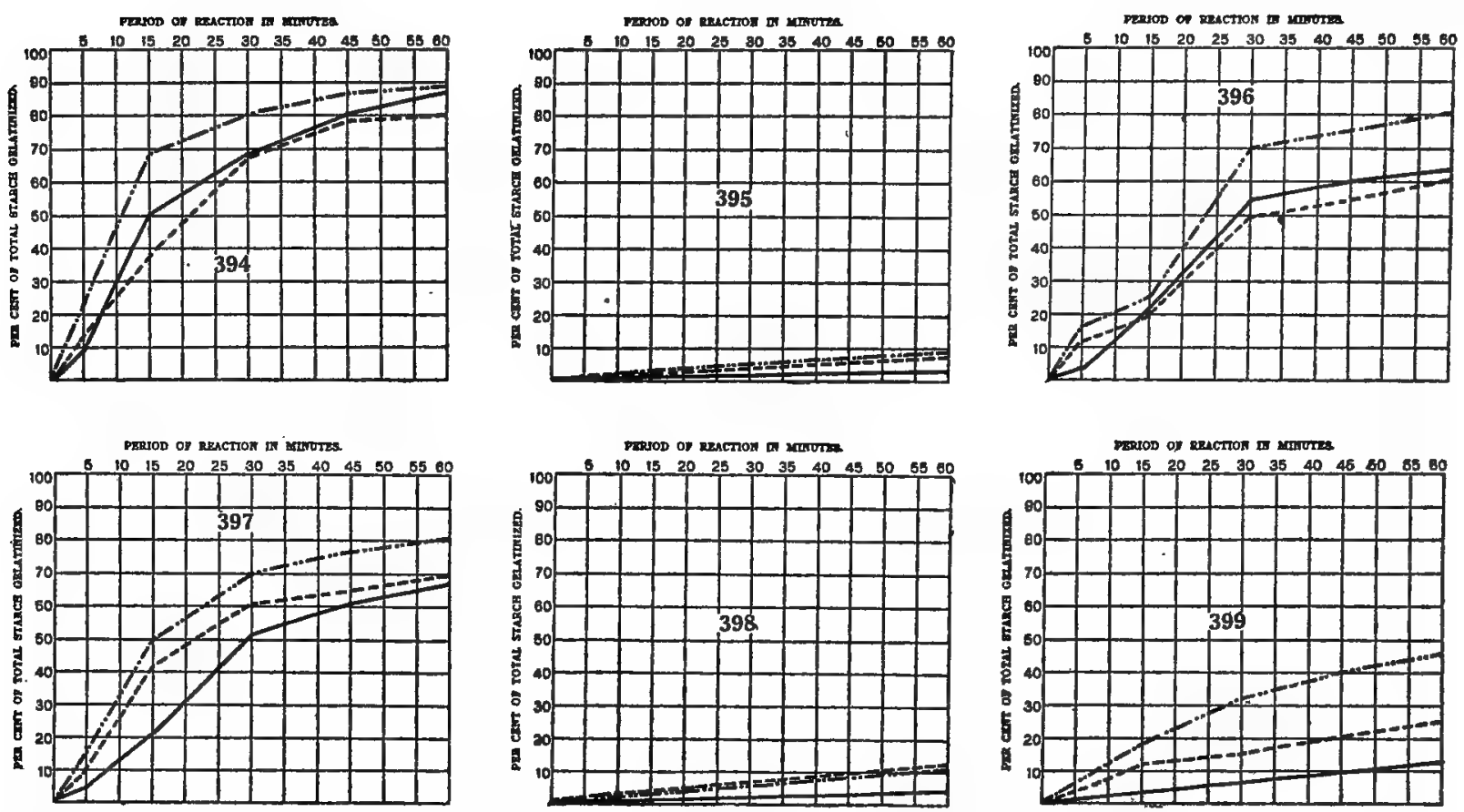

Charts D 394 to D 399.-Velocity-Reactions of Starches of Iris iberica Iris ismali (-).

394. With Strontium Nitrate.

396. With Copper Nitrate. 395. With Cobalt Nitrate.
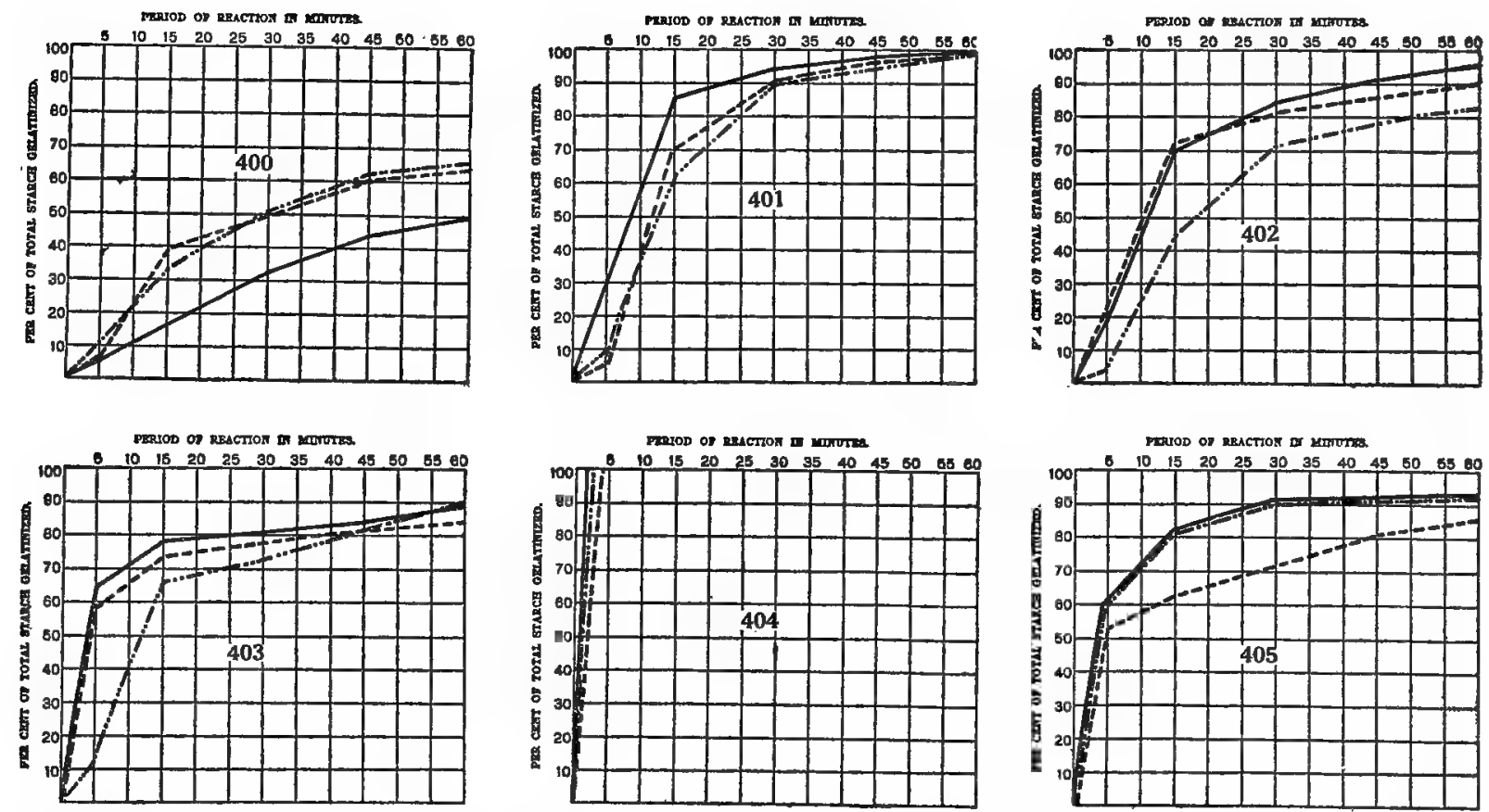

Charts D 400 то D 405.-Velocity-Reactions of Starches of Iris iberica (....), Iris cengialti (-......), and Iris dorak (-). 
Pariod os exction is umona.

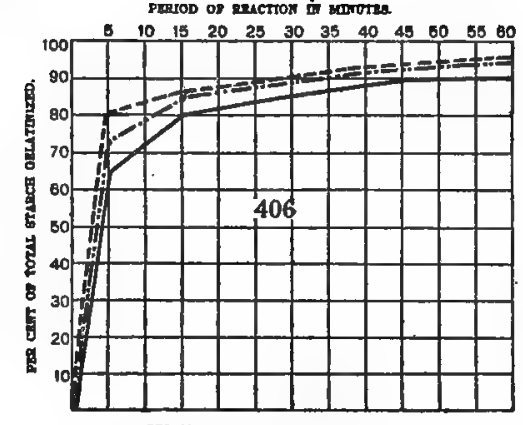

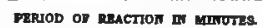
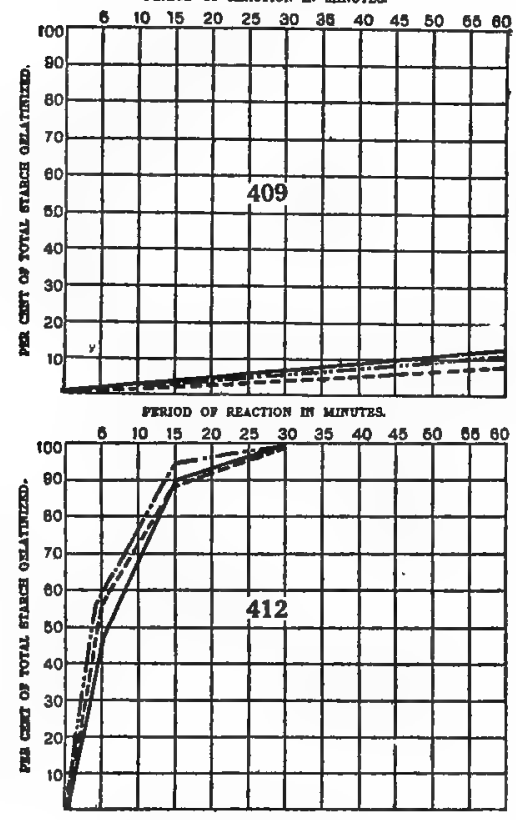

Rractios In MTNOTES

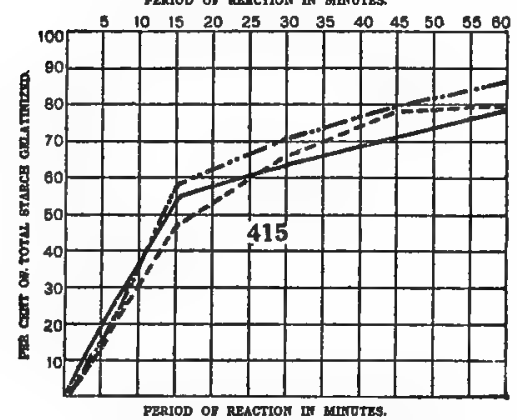

gerion of anection

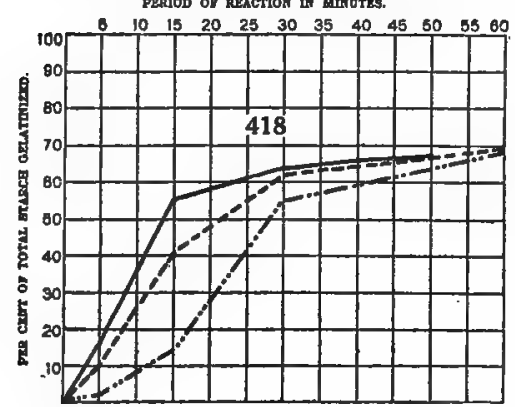

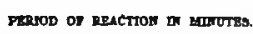

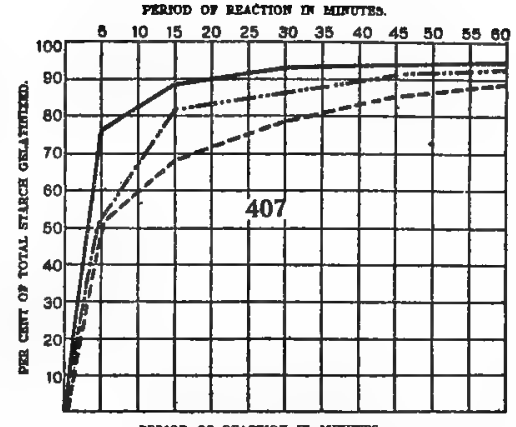

PERTO OF RRACTIO
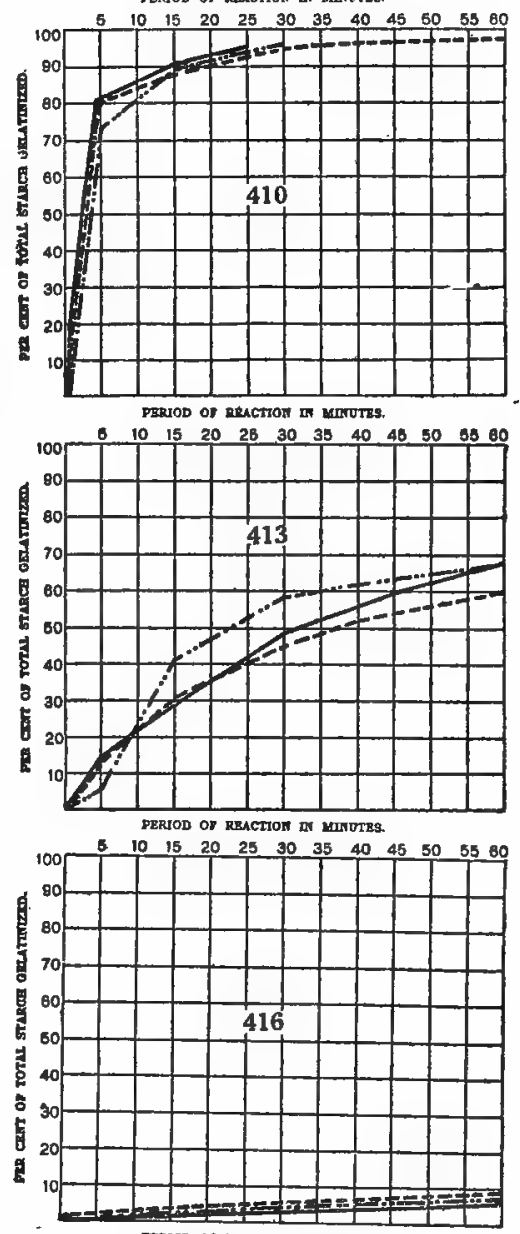

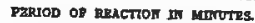

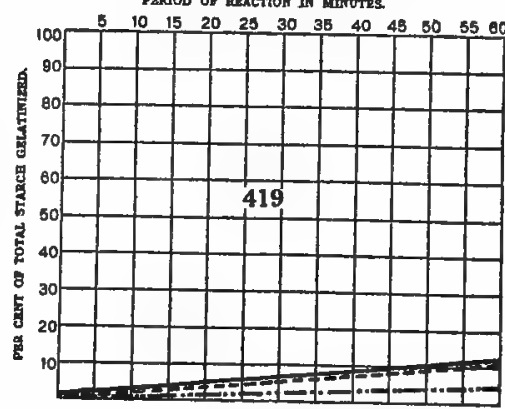

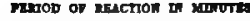

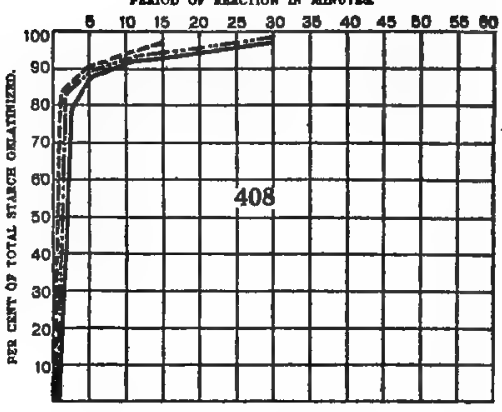

paruod of Rection $m$ undm

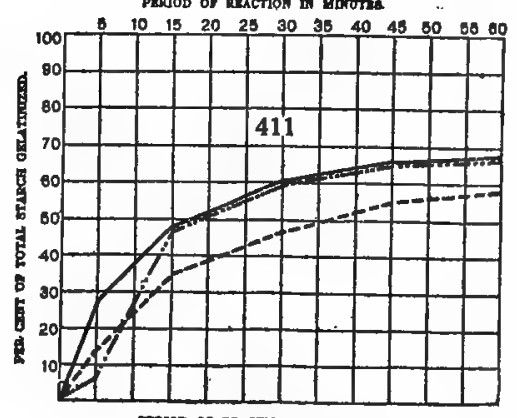

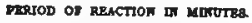

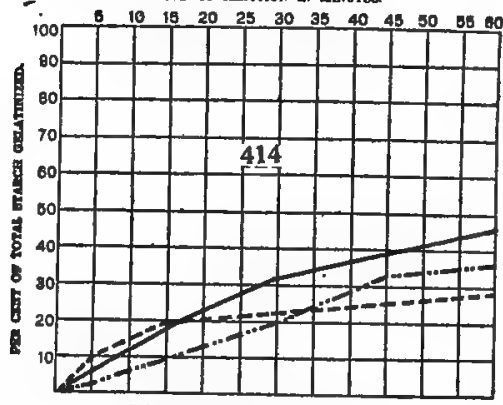

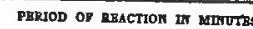

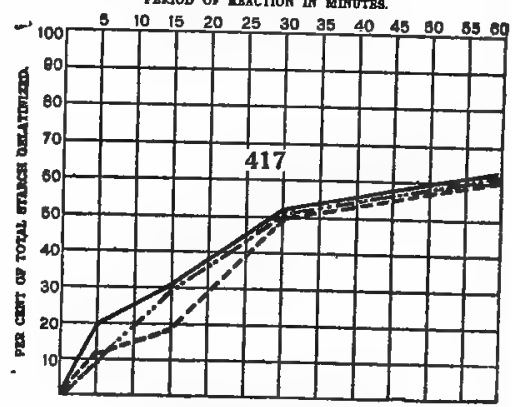

PERTOD OF RRACTTON ER Mathotes.

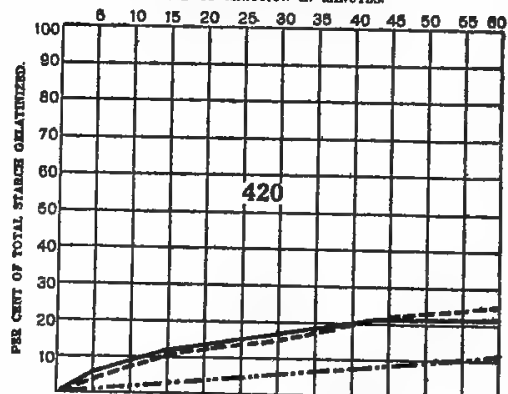

Charts D 406 то D 420.-Velocity-Reactions of Starches of Iris iberica (.....), Iris cengialti (-......), and Iris dorak (-).

406. With Potassium Hydroxide. 407. With Potassium Iodide. 408. With Potassium Sulphooyanate. . 409. With Potassium Sulphide. 410. With Sodium Hydroxide.
411. With Sodium Sulphide.

412. With Sodium Salicylate.

413. With Calcium Nitrate.

414. With Uranium Nitrate.
415. With Strontium Nitrate.
416. With Cobalt Nitrate.

417. With Copper Nitrate.

418. With Cupric Chloride.

420." With Mercuric Chloride. 


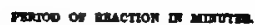

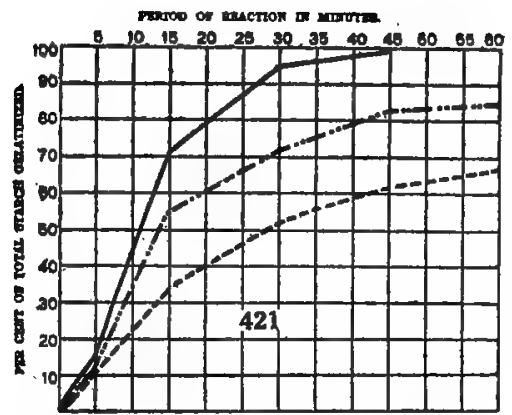

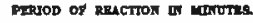

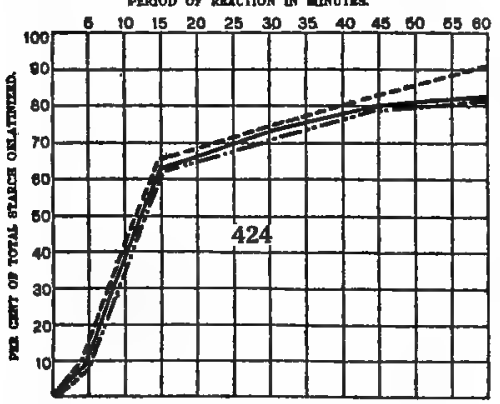

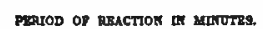

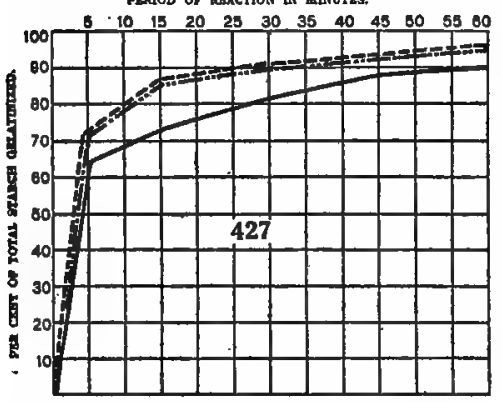

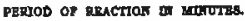

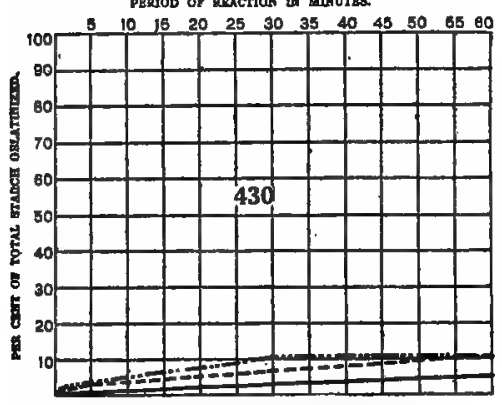

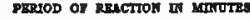

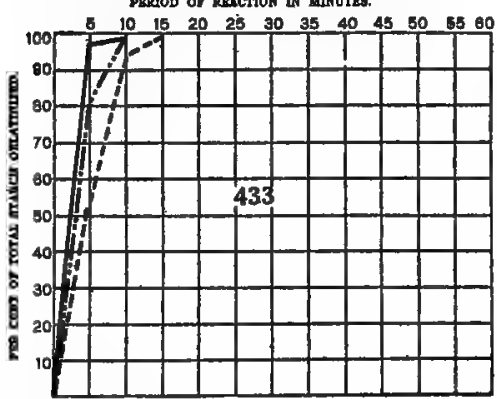

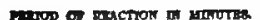

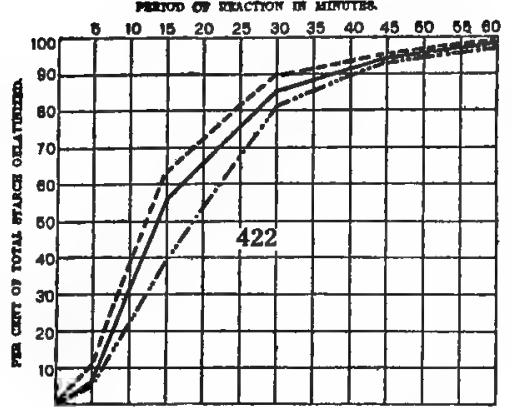

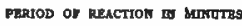

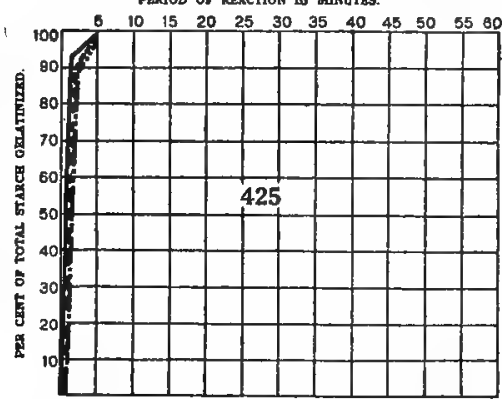

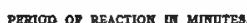
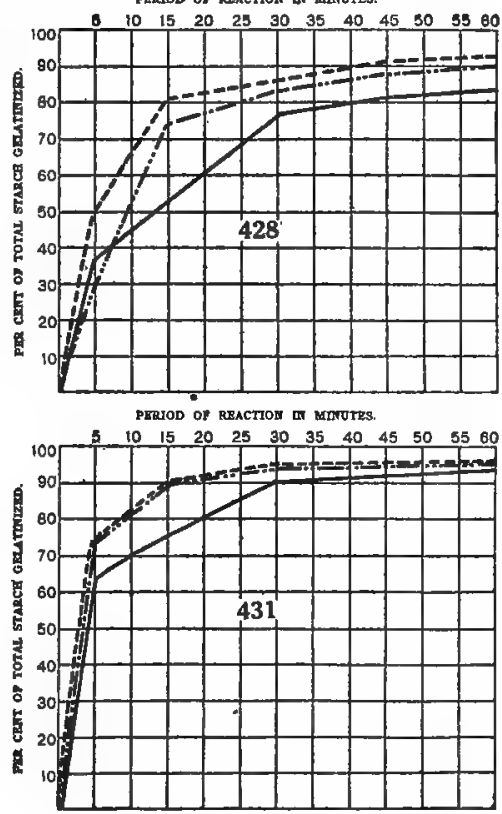

PERSOD OB PRACTIOS II MTSUTES

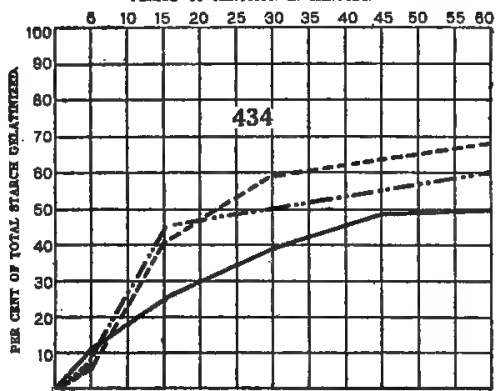

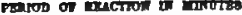

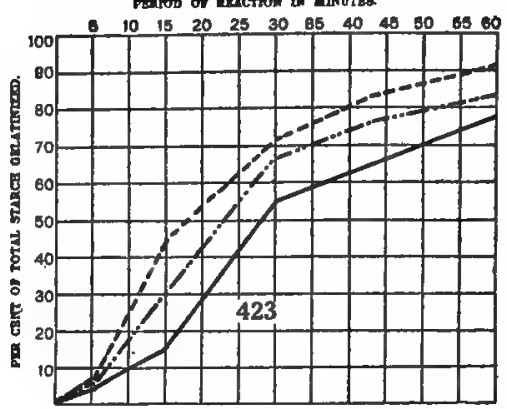

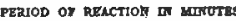

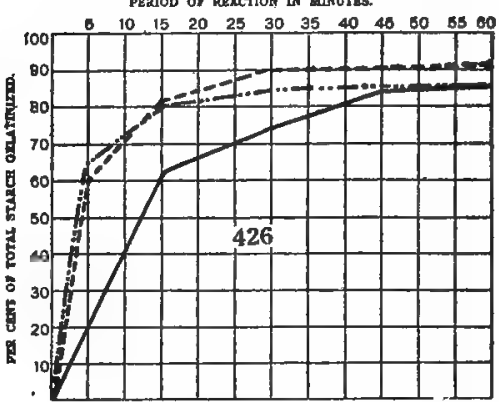

Propos on anection

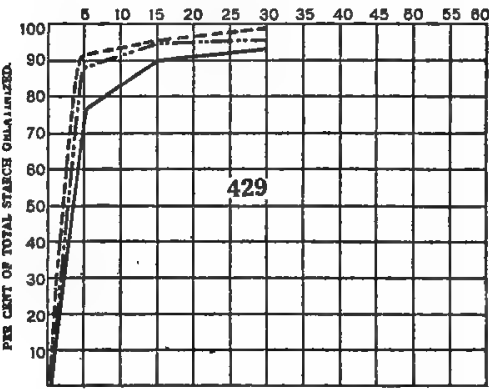

Den of

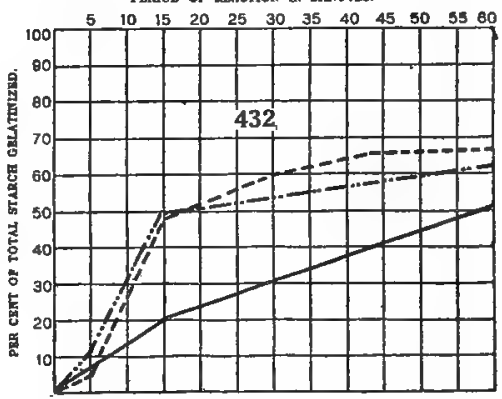

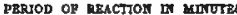

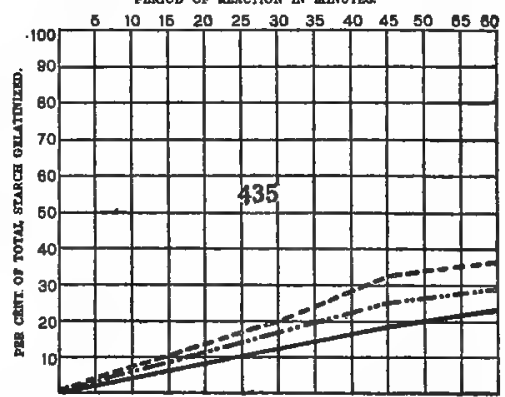

Charts D 421 то D 435.-Velocity-Reactions of Starches of Iris cengialti (....-), Iris pallida queen of may (.......), and Iris mrs. alan grey (-).

421. With Chloral Hydrate. 422. With Chromic Acid. 423. With Pyrogallic Acid. 424. With Nitric Acid.
426. With Hydrochloric Acid.

427. With Potassium Hydrozide.

428. With Potassium Iodide.

429. With Potassium Sulphocyanate. 430. With Potassium Sulphide.
431. With Sodium Hydroxide

432. With Sodium Sulphide.

433. With Sodium Salicylate

434. With Calcium Nitrate.

435. With Uranium Nitrate. 


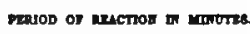

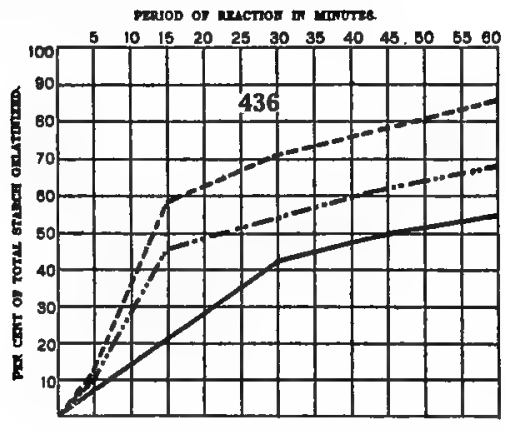

PERTOD of REACTIO II MUTUTR:

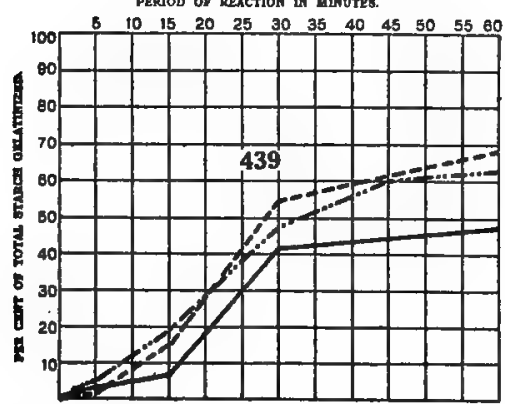

praros of maction in montes

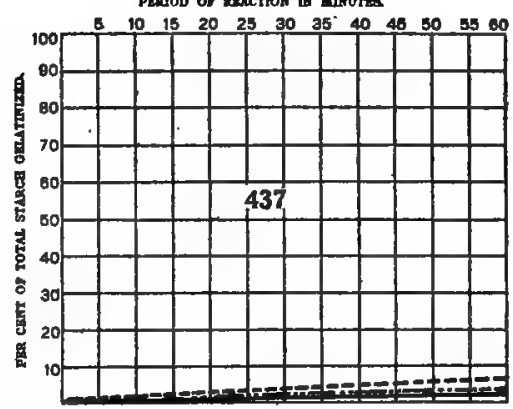

PERTOD of ARACTON 4 MLTUTES

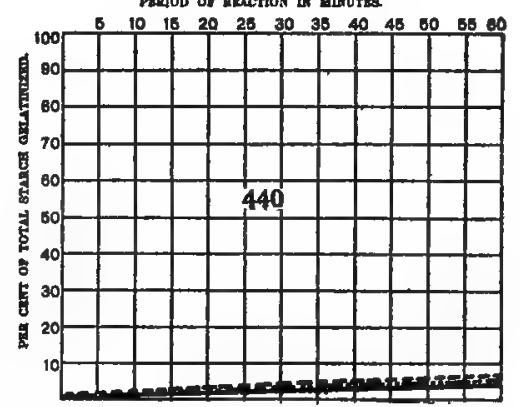

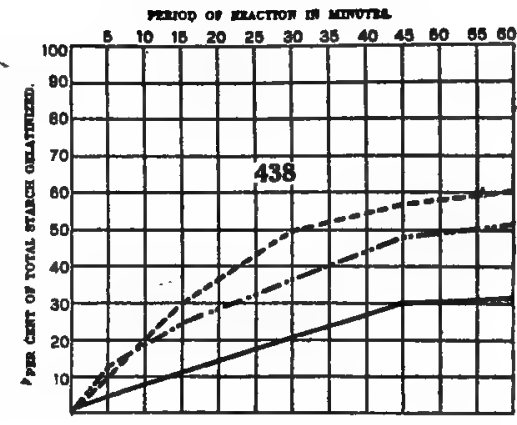

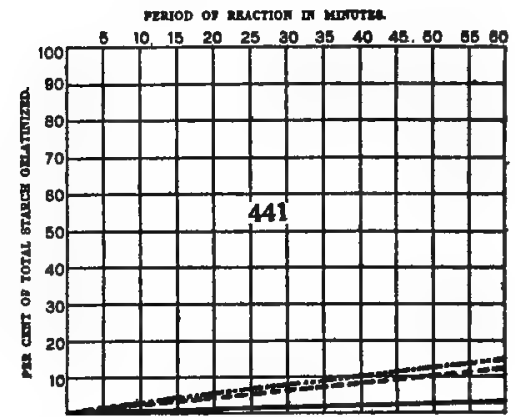

Charts D 436 to D 441.-Velocity-Reactions of Starches of Iris cengialti (....-), Iris pallida queen of may (.....), and Iris mrs. alan grey (-).

438. With Copper Nitrate.

436. With Strontium Nitrate.

440. With Barium Chloride. 437. With Cobalt Nitrate.

PERIOD of REACTION IA MDVTE:

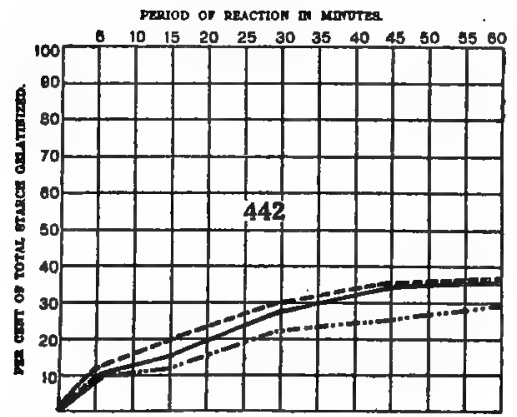

PERYod of Eucton as knotes
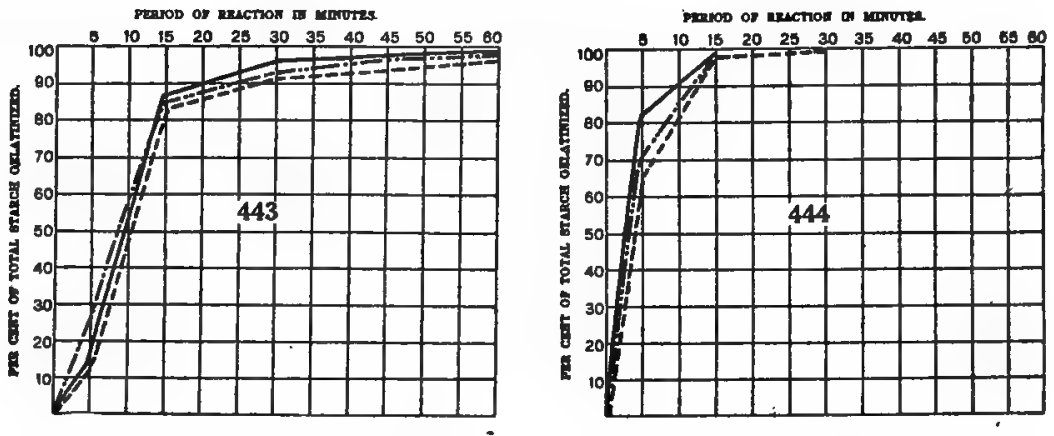

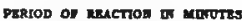
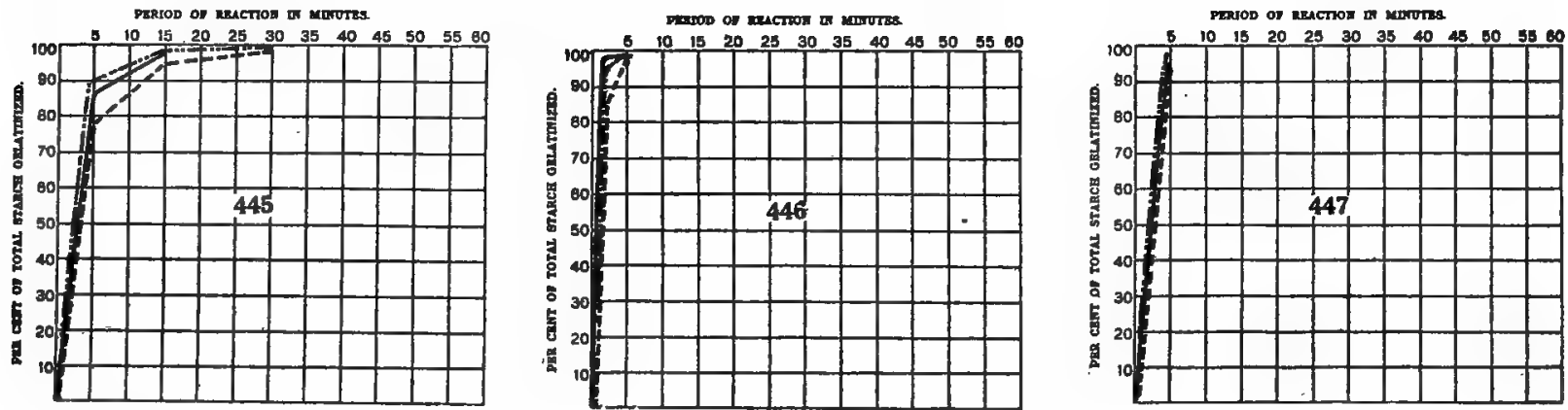

Charts D 442 to D 447.-Velocity-Reactions of Starches of Iris persica var. purpurea (.....), Iris sindjarensis $(\ldots \ldots \ldots)$, and Iris pursind (-).

442. With Chloral Hydrate. 443. With Chromic Acid.
444. With Pyrogallic Acid 445. With Nitric Acid.
446. With Sulphuric Acid.

447. With Hydrochloric Acid. 


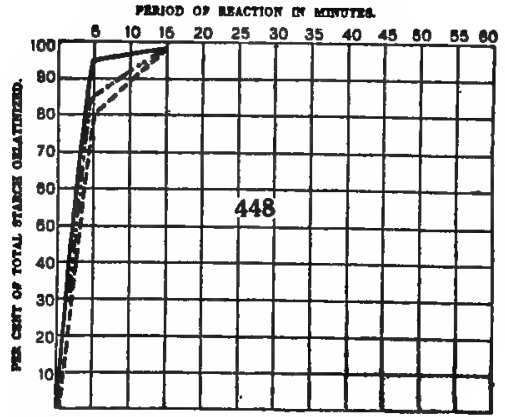

DENOD or Rriction in Merteres

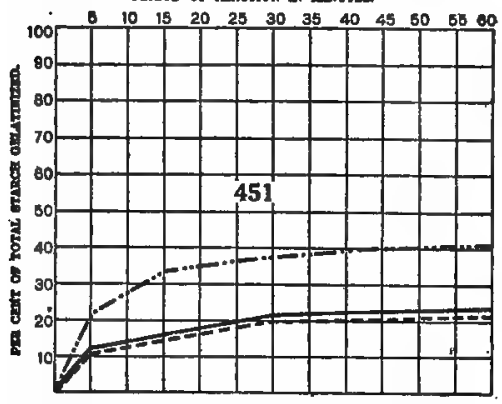

PBEROD OF REACTION DA MDTUTES.

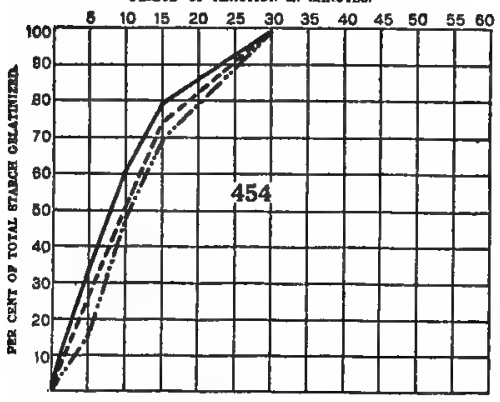

PERIOD OF REACTIOR IN MENTTES.

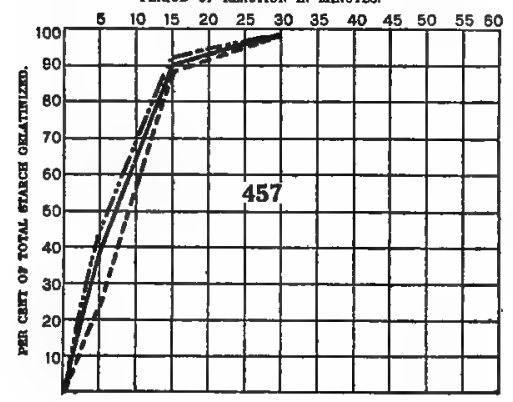

PBNOD OF REACTION IN MPNUTES.

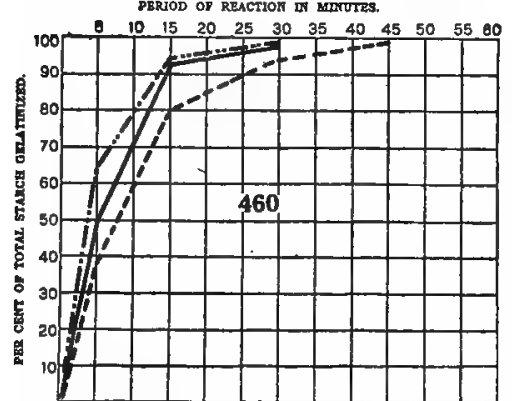

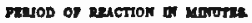

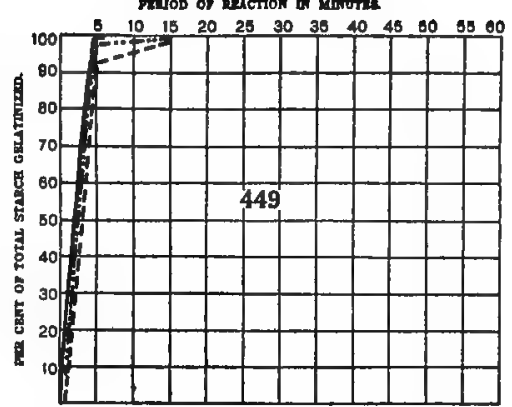

Dxerod of REAction ar vanutses

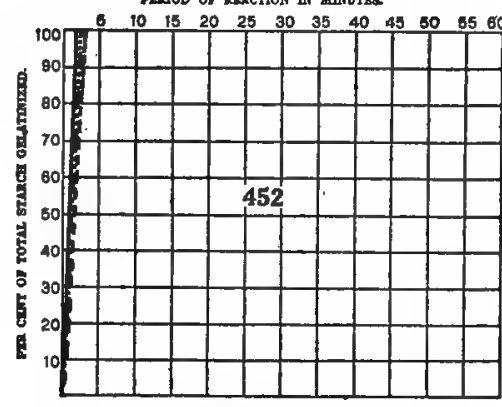

prerod or xenctron an nerutra.

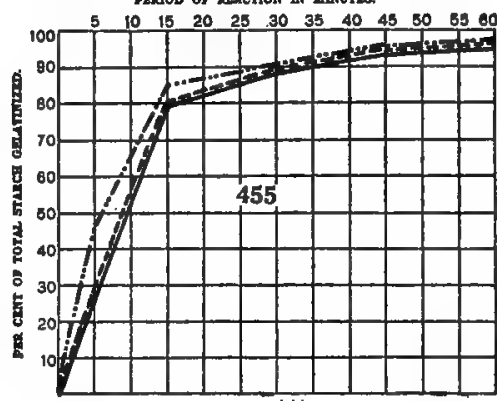

PERIOD OF REACTION TH MLTUTER

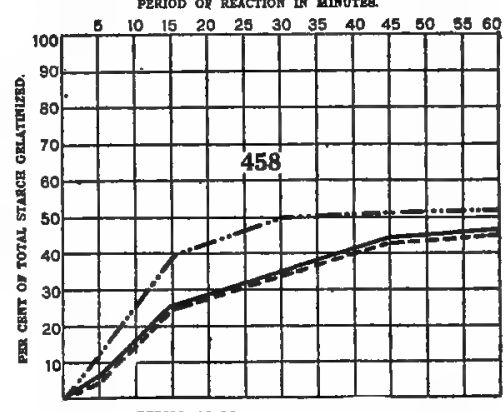

PRProd of penction on ararotes

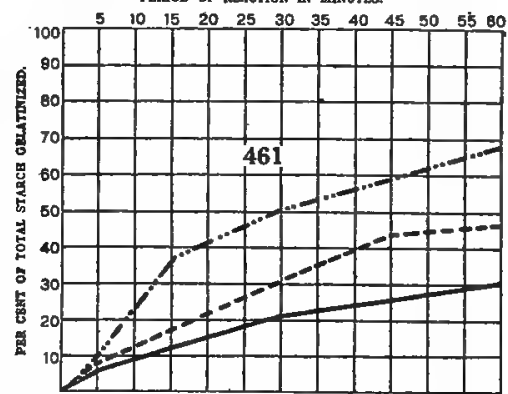

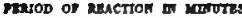

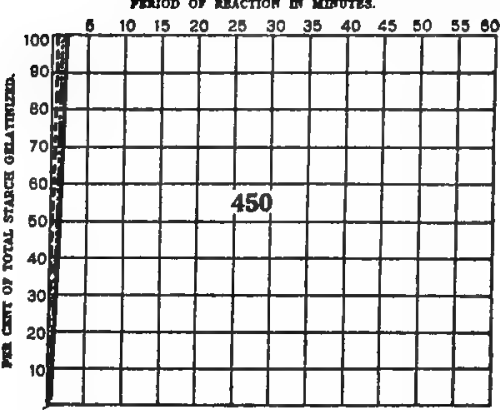

persod or xractior as umvires.

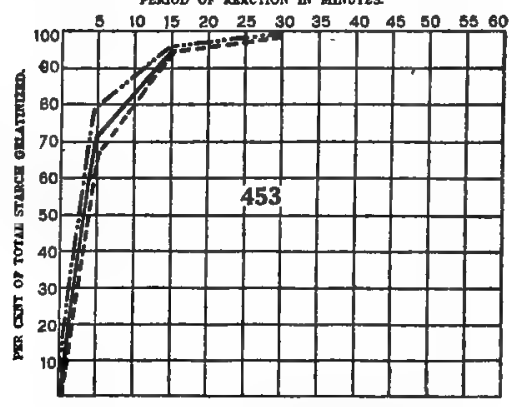

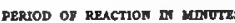

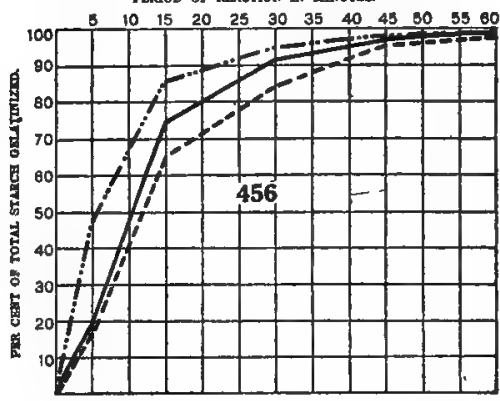

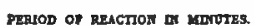

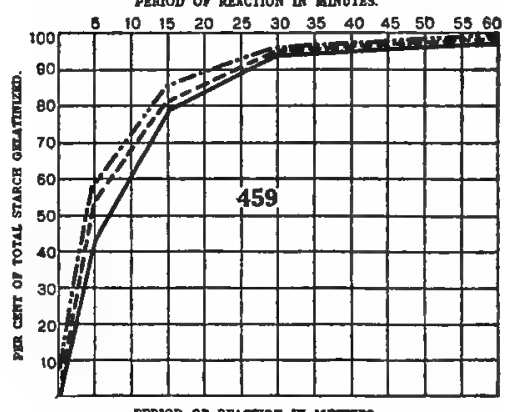

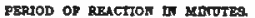

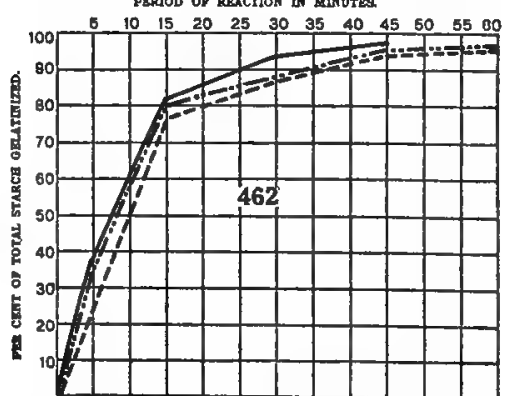

Charts D 448 to D 462.-Velocity-Reactions of Starches of Iris persica var. purpurea (-.-.-), Iris sindjarensi

448. With Potasgium Hydroxide.

449. With Potassium Iodide.

450. With Potassium Sulphocyanste.

451. With Potassium Sulphide. $(\ldots-\ldots)$, and Iris pursind (-).

453. With Sodium Sulphide.

454. With Sodium Salicylate.

455. With Calcium Nitrate.

457. With Strontium Nitrate.
458. With Cobalt Nitrate.

459. With Copper Nitrate.

460. With Cupric Chloride.

462. With Mercuric Chloride. 
arod of necrion to worves

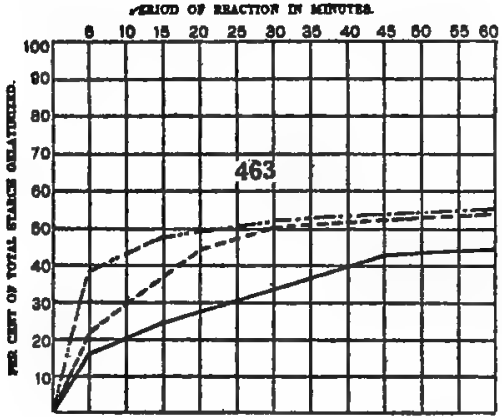

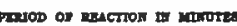

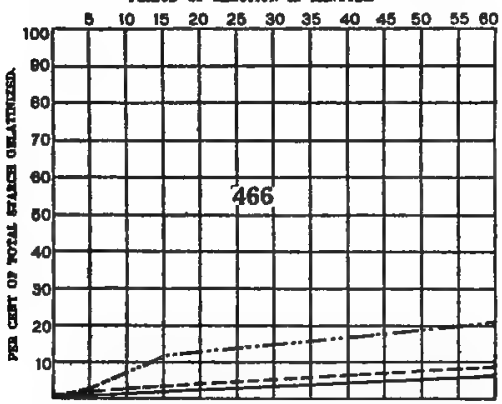

pespod of praction an Mandes.

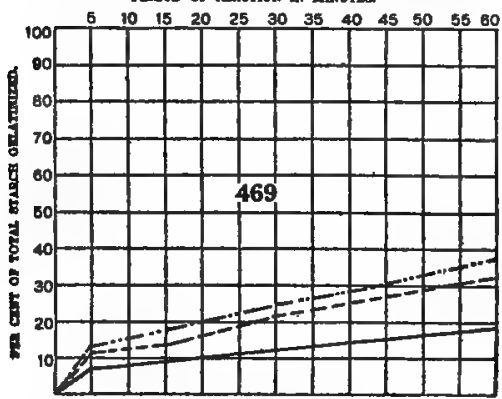

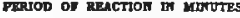

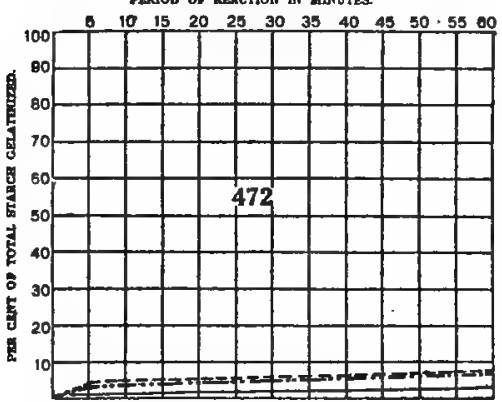

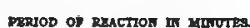

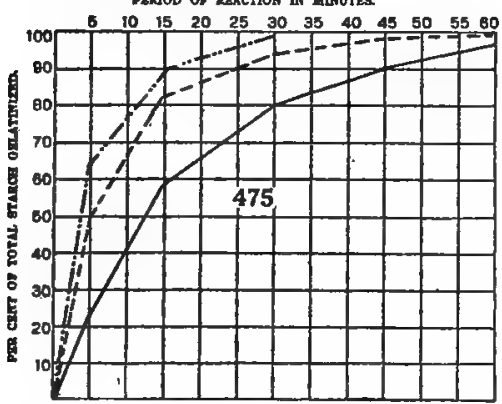

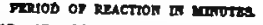

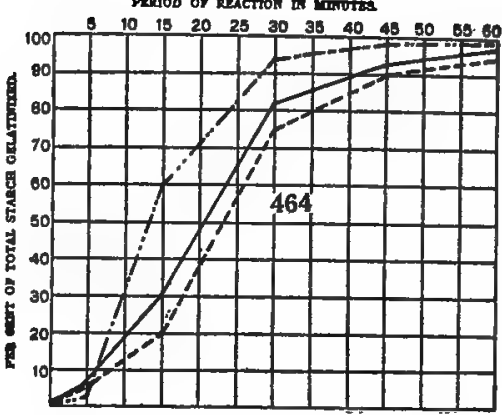

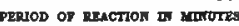

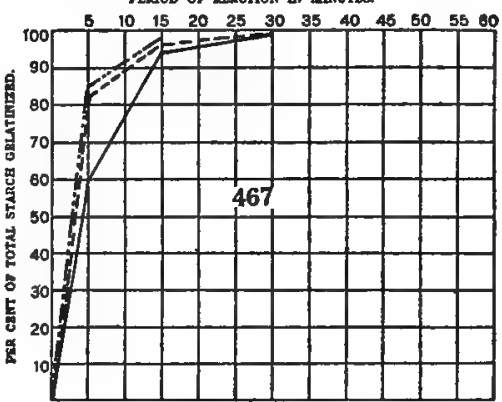

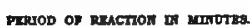

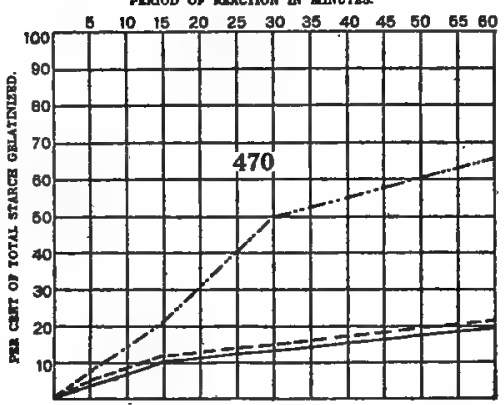

PEgrod on peaction

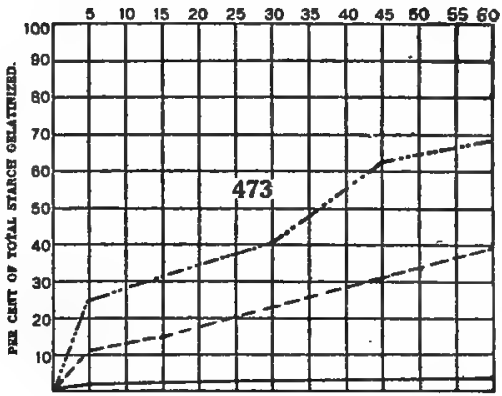

PERJOD OF RRACT.VN

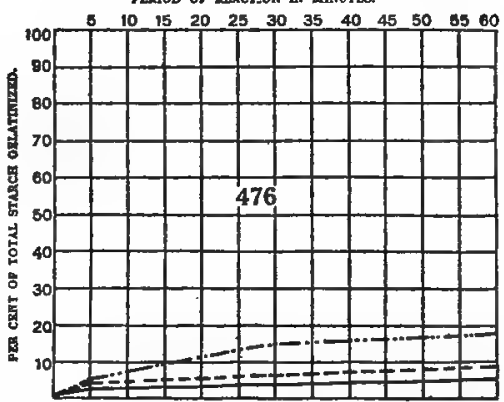

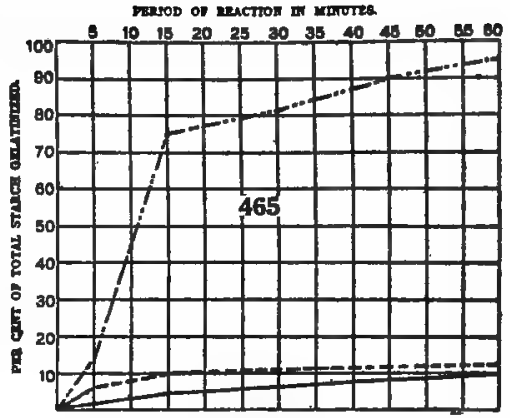

prevos of naction a merves

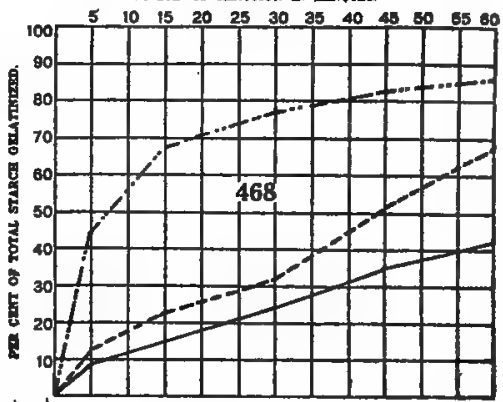

period of gractios $\mathrm{m}$

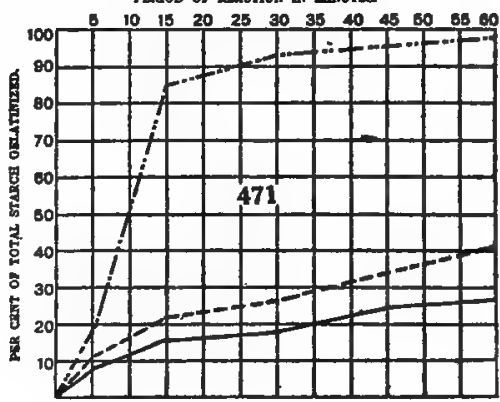

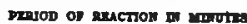

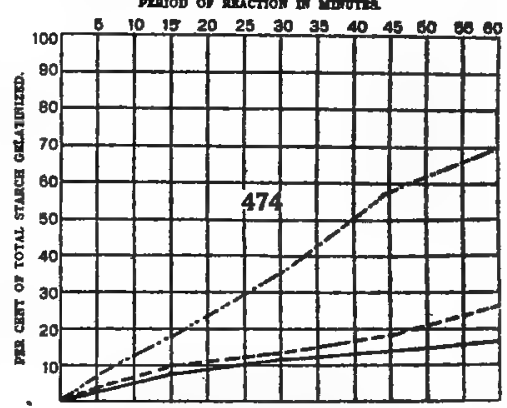

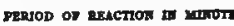

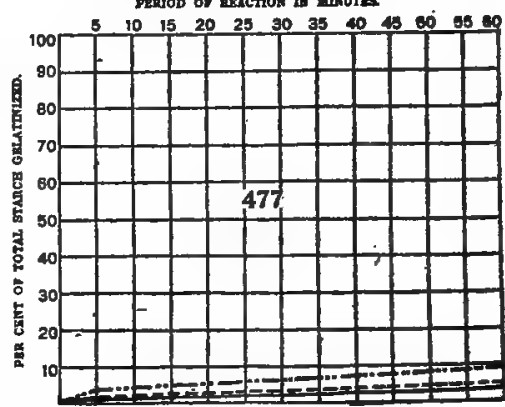

Charts D 463 тo D 477.-Velocity-Reactions of Starches of Gladiolus cardinalis (....), Gladiolus tristis (-......), and Gladiolus colvillei (-).

463. With Chloral Hydrate. 464. With Chromic Acid.

465. With Pyrogallic Acid.

466. With Nitric Acid.
468. With Hydrochloric Acid.

469. With Potassium Hydroxide.

470. With Potassium Iodide.

471. With Potsasium Sulphocyanate.

472. With Potassium Sulphide.
473. With Sodium Hydroxide.

474. With Sodium Sulphide.

475. With Sodium Salieylate.

477. With Uranium Nitrate 

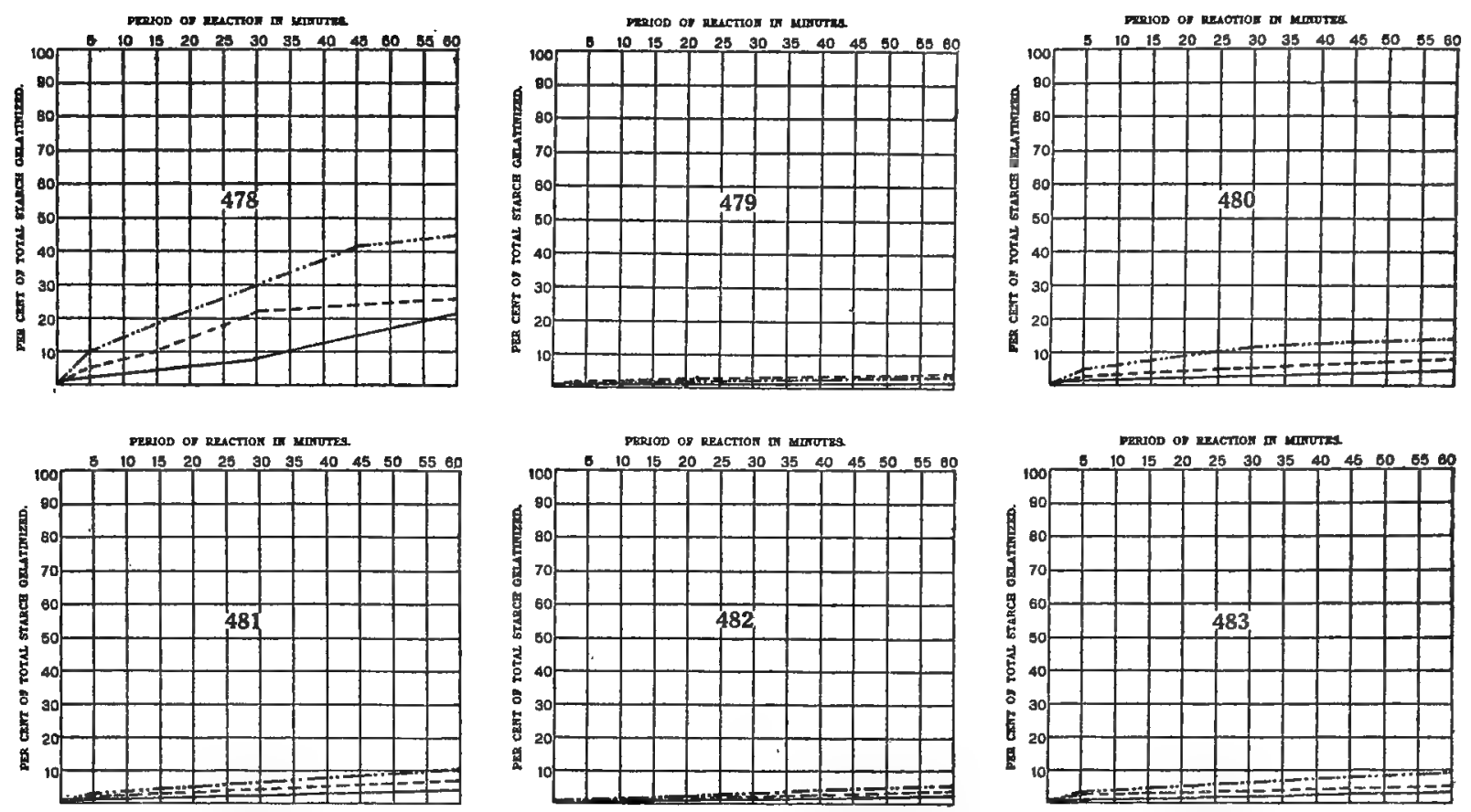

Charts D 478 to D 483.--Velocity-Reactions of Starches of Gladiolus cardinalis (-...-), Gladiolus tristis (-......), and Gladiolus colvillei (-).

478. With Strontium Nitrate.

480. With Copper Nitrate.

482. With Barium Chloride. 479. With Cobalt Nitrate.
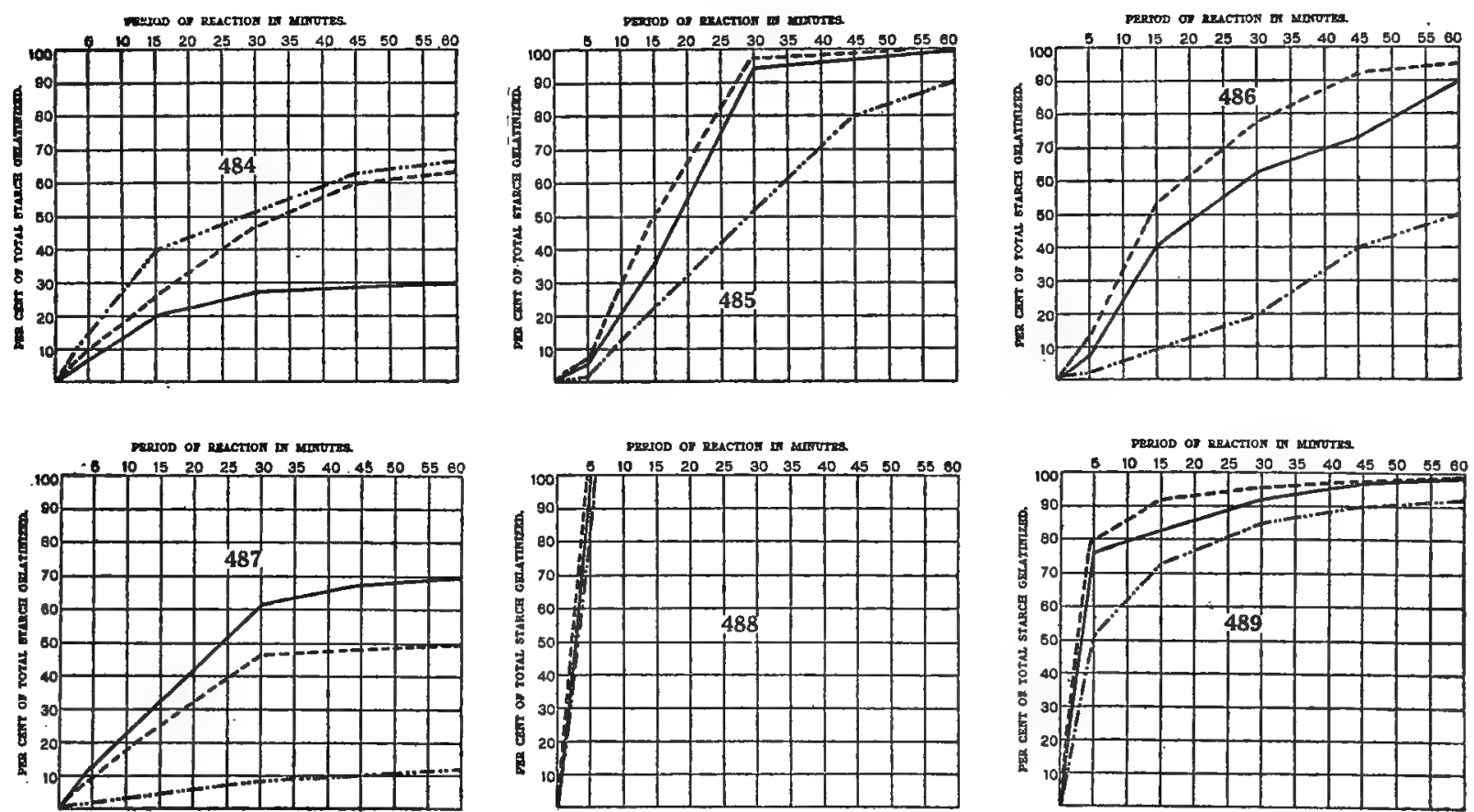

Charts D 484 тo D 489.-Velocity-Reactions of Starches of Tritonia pottsii (-..--), Tritonia crocosmia aurea (-......), and Tritonia crocosmaeflora (-
484. With Chloral Hydrate.
486. With Pyrogallic Acid.
488. With Sulphuric Acid
485. With Chromic Acid.
487. With Nitrie Acid.
489. With Hydrochloric Acid. 
mesod of enction a worme

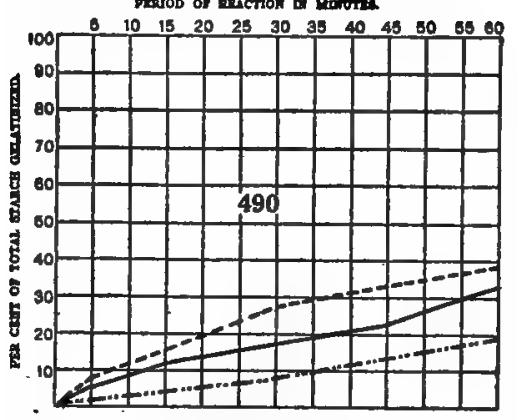

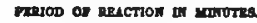

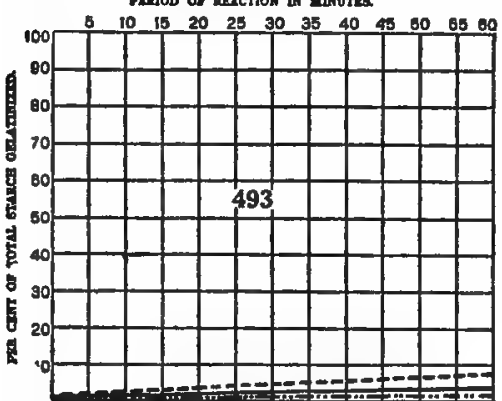

Thop or reaction of umotras

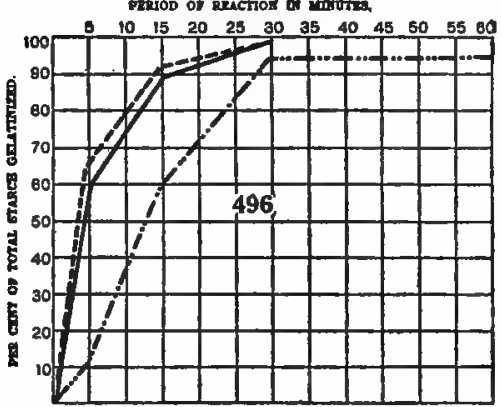

PENOD of REACTON EN UENOTES.

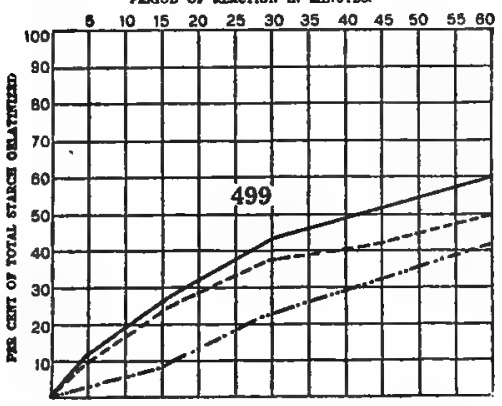

perod of Resction If satotes

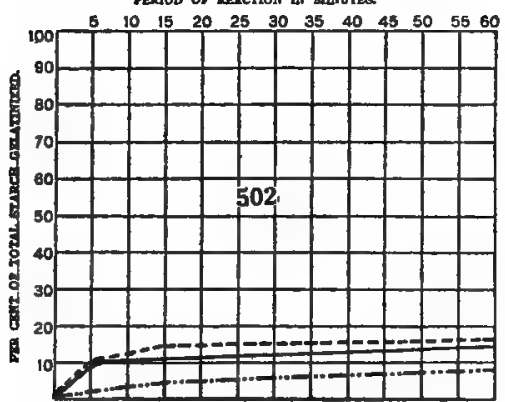

mano os muction a kerotes

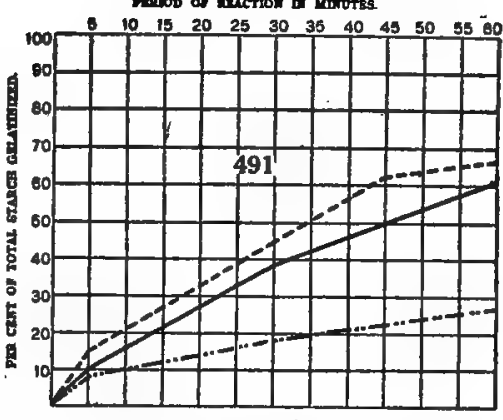

pretod of section an morotess

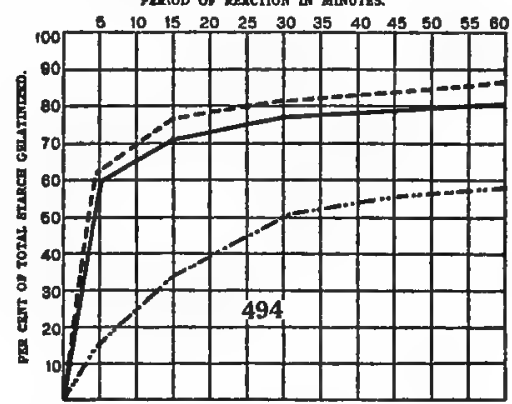

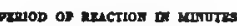

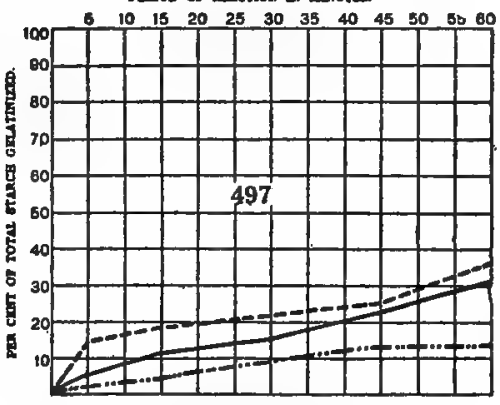

PERSOD OF REACTION IN MLNOTRS

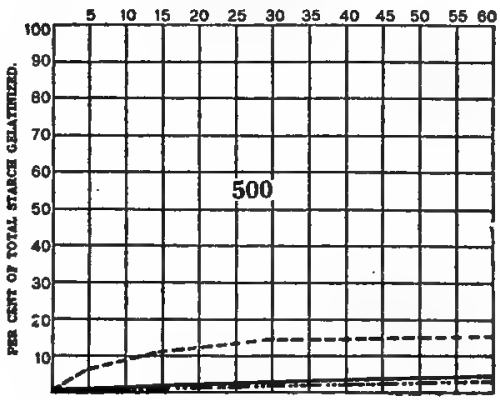

MRIOD OP RHACTION ET MR⿴囗十⺝

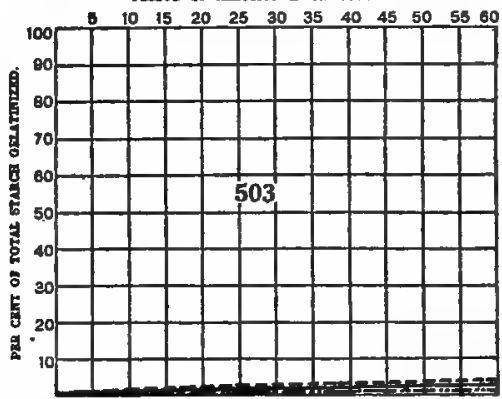

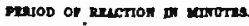

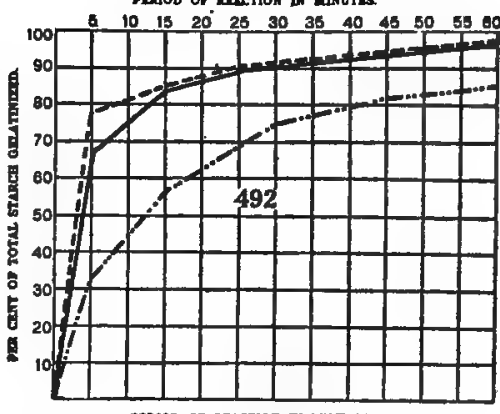

PEarod on REuction or marotes

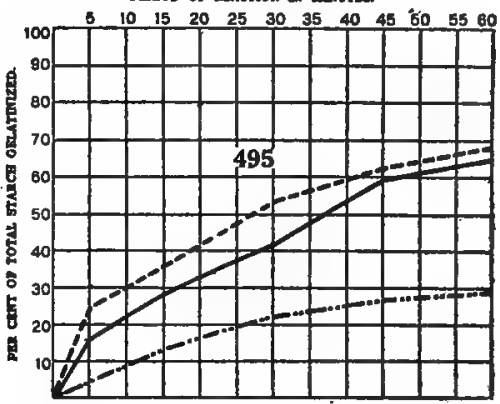

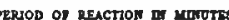

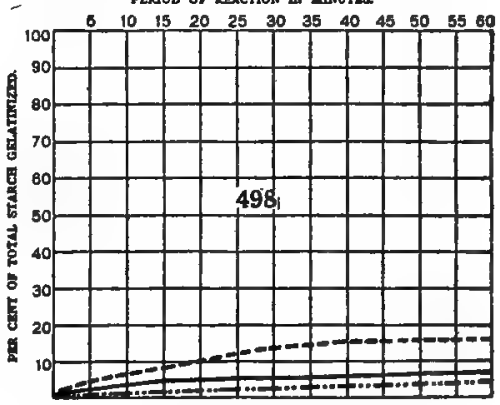

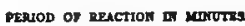

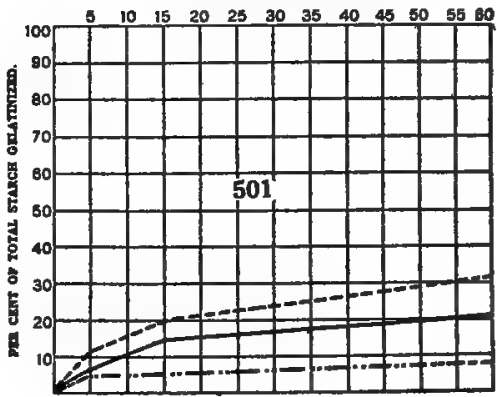

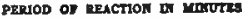

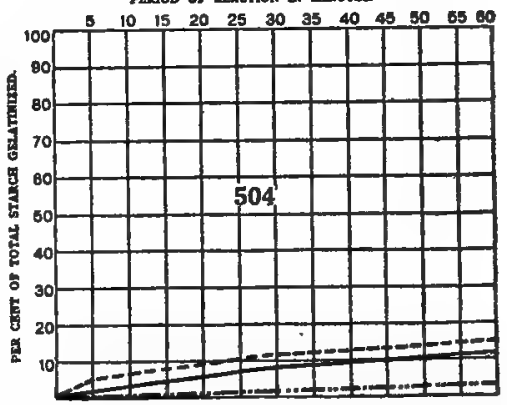

Charts D 490 to D 504.-Velocity-Reactions of Starches of Tritonia pottsii (-..-.), Tritonia crocosmia aurea (......), and Tritonia crocosmoeflora (-).

490. With Potagsium Hydroxide. 491. With Potassium Iodide. 492. With Potasaium Sulphocyanate. 493. With Potassium Sulphide. 494. With Bodium Bydroride.
495. With Sodium Sulphide.

496. With Sodium Salicylate.

497. With Calcium Nitrate.

499. With Uranium Nitrate
500. With Cobalt Nitrate.

501. With Copper Nitrate.

502. With Cupric Chloride.

504. With Mercuric Chloride. 

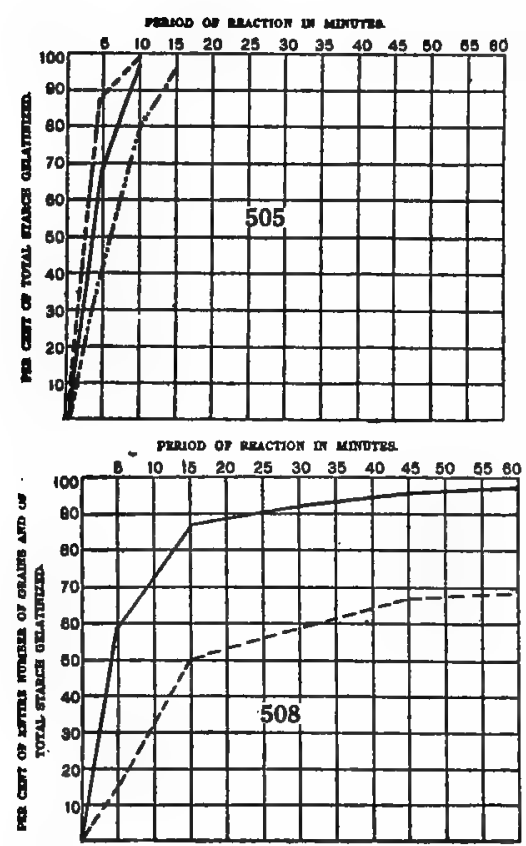

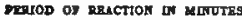

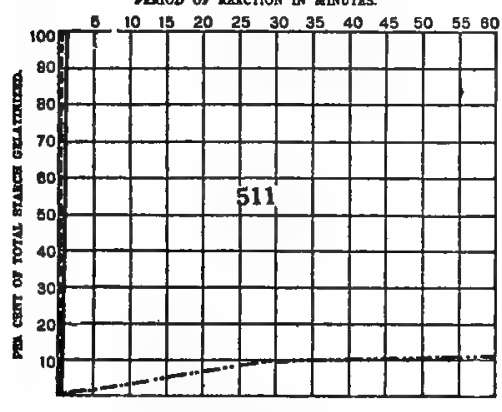

parad os peiction as mortrat

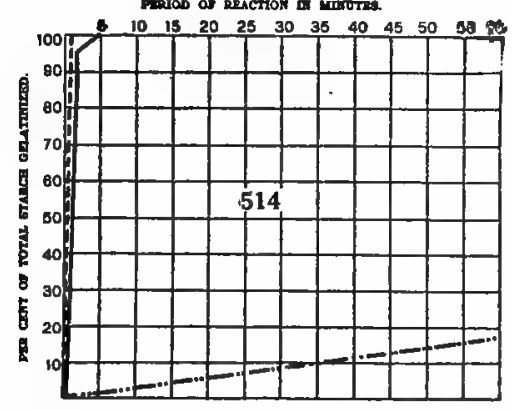

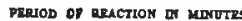

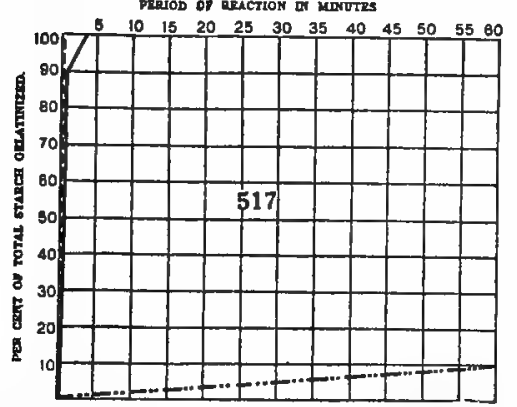

mas of anchou in arome.

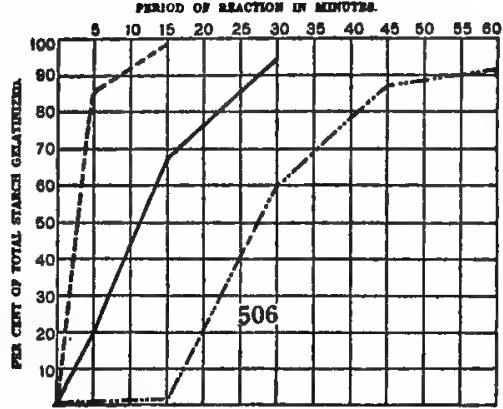

percod of graction as servizs.

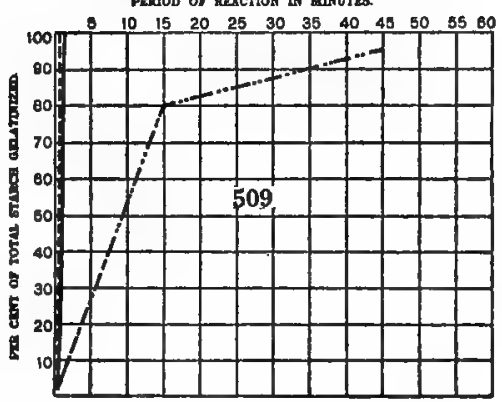

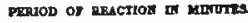

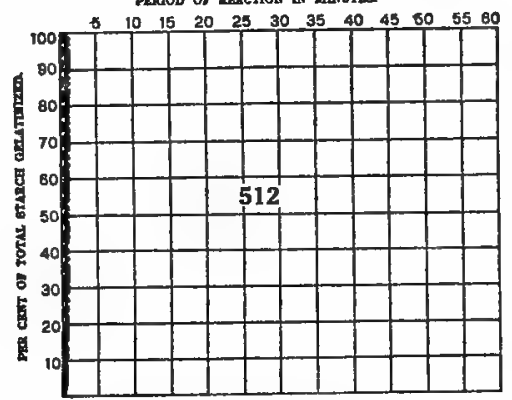

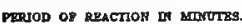

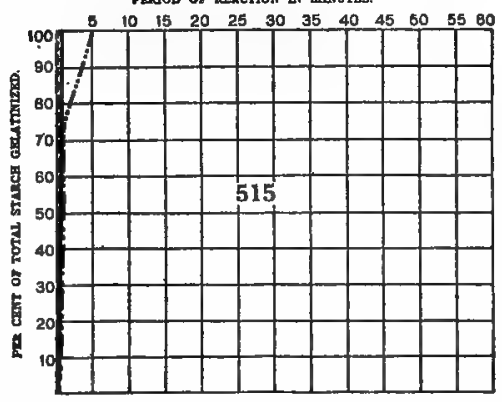

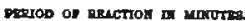

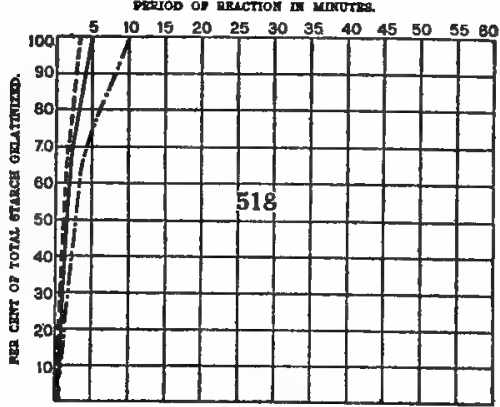

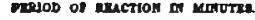

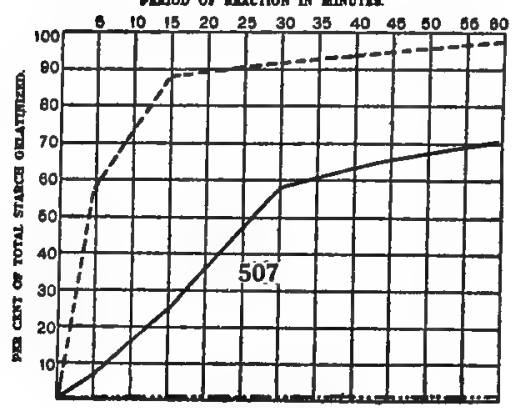

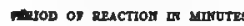

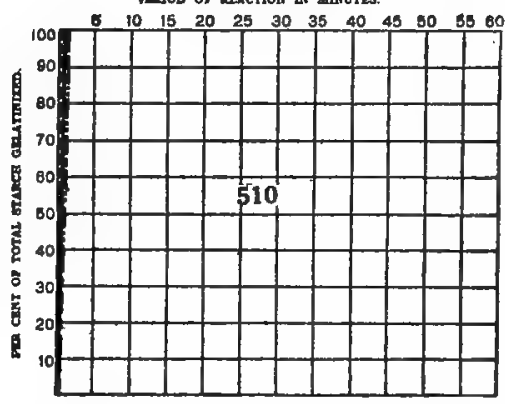

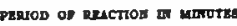

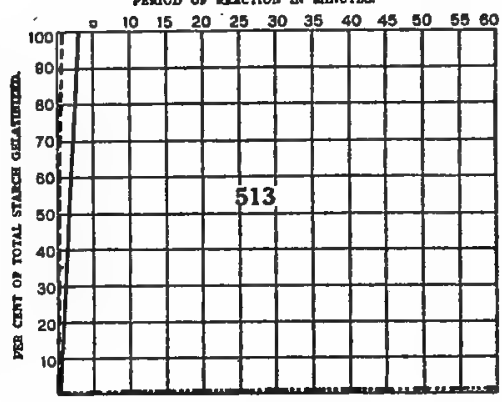

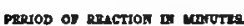

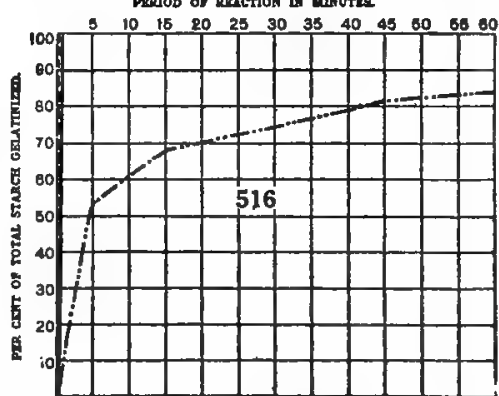

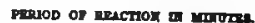

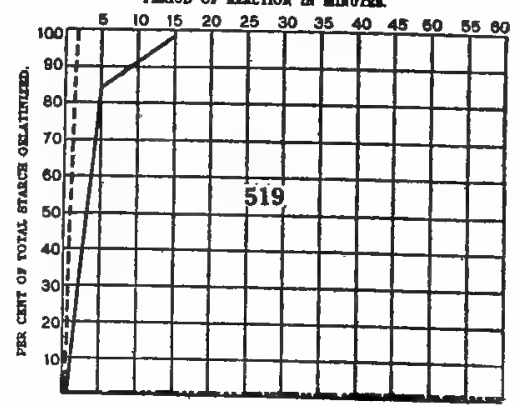

Charts D 505 то D 507, D 509 то D 519.-Velocity-Reactions of Starches of Begonia single crimson scarlet (-....), Begonia socotrana (-.....-), and Begonia mrs. heal (-).
505. With Chloral Hydrate.
506. With Chromic Acid.
507. With Pyrogallic Acid.
509. With Nitric Acid.
511. With Hydrochloric Acid
512. With Potassium Hydroxide.
513. With Potassium Iodide.
514. With Potassium Sulphocyanate.
516. With Sodium Hydroxide.
517. With Sodium Sulphide.
519. With Calcium Nitrate.

CharT D 508.-Velocity-Reactions of Pyrogallic Acid with the Starch of Begonia single crimson scarlet. Percentage of entire number of grains (....-) and total starch (-) gelatinized. 

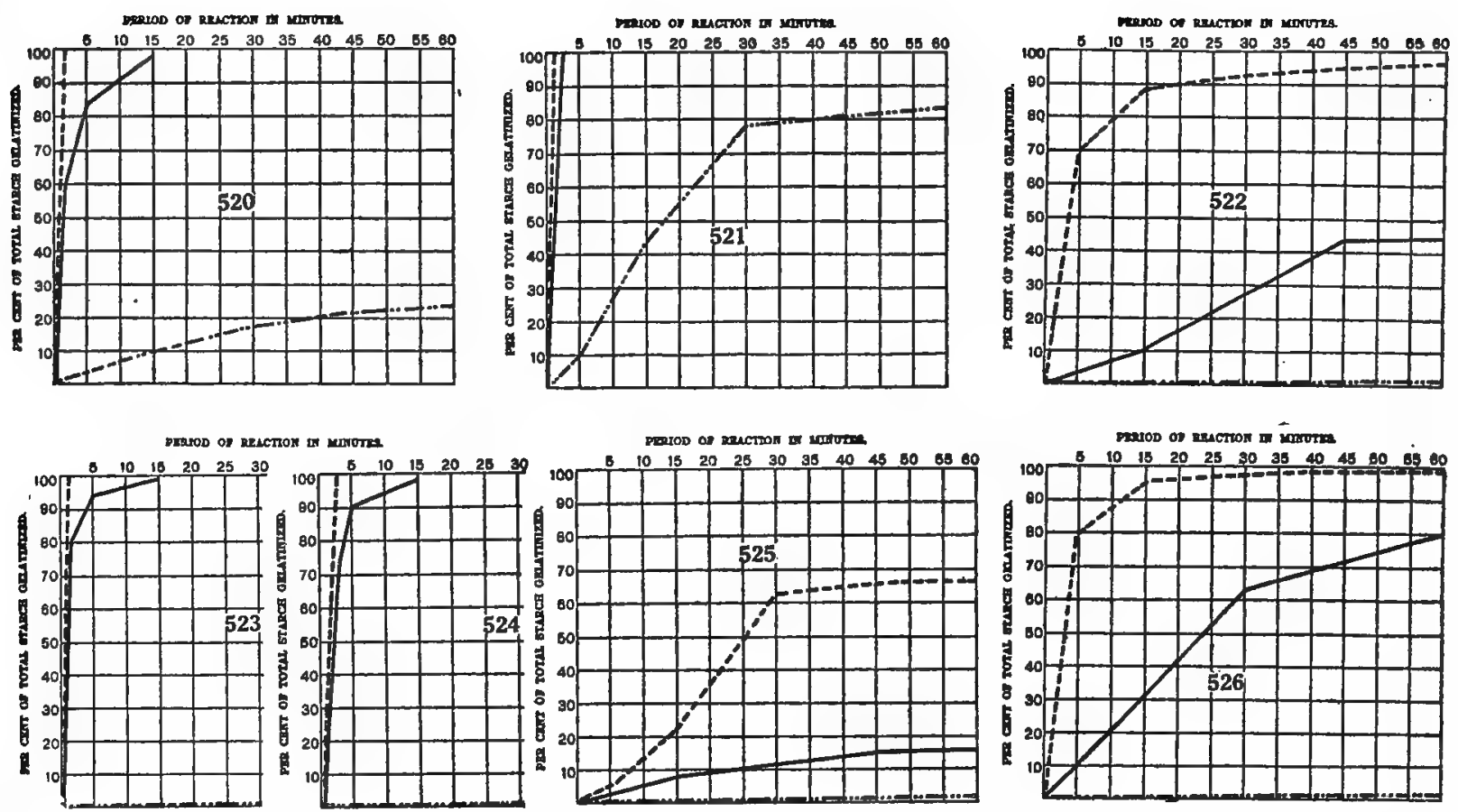

Charts D 520 to D 526.-Velocity-Reactions of Starches of Begonia single crimson scarlet (-..--), Begonia socotrana ( ......-), and Begonia mrs. heal (-).

520. With Uranium Nitrate.
521. With Strontium Nitrate.

522. With Cobalt Nitrate.
523. With Copper Nitrate.

524. With Cupric Chloride.

526. With Mercurio Chloride.
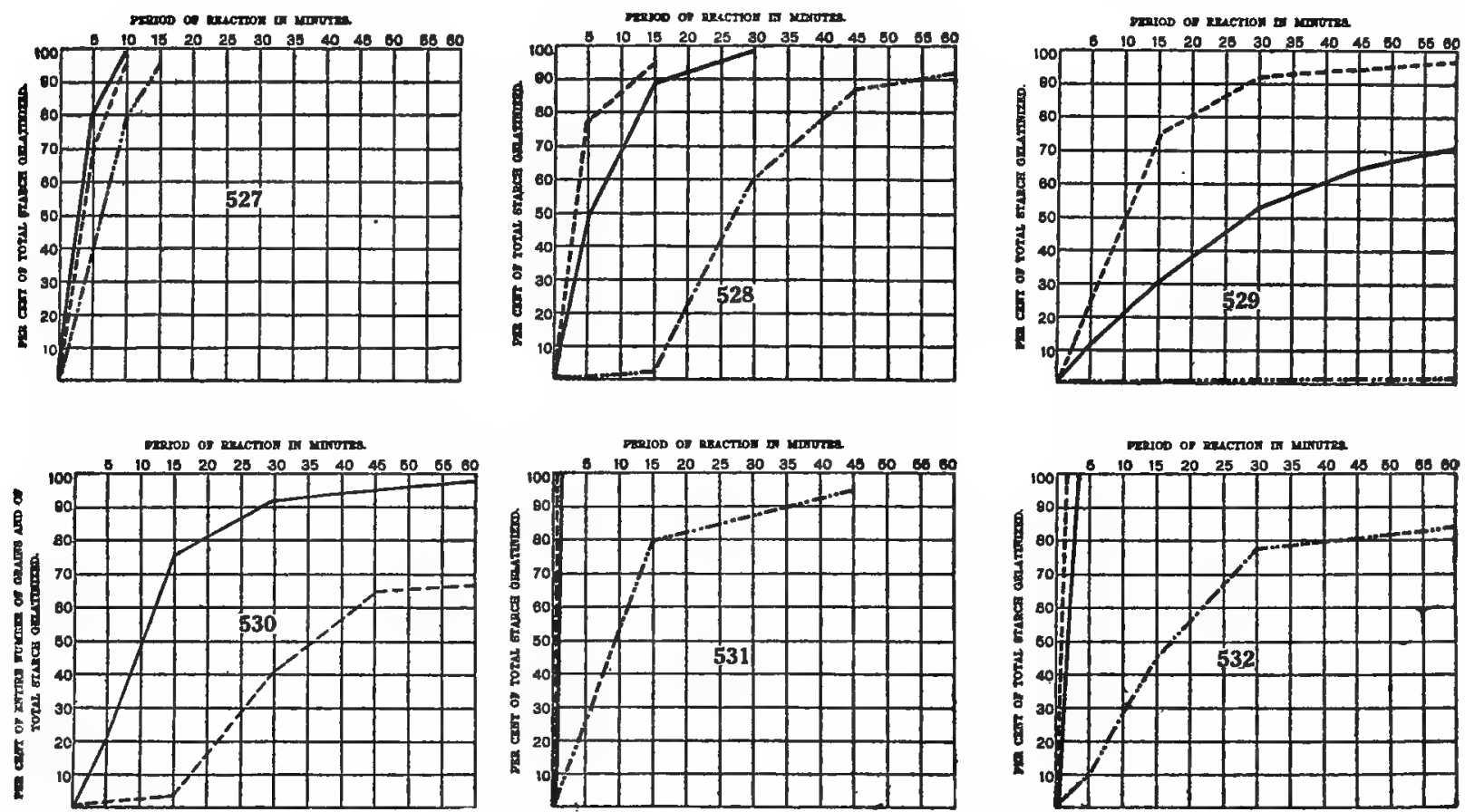

Charts D 527 to D 529, D 531, D 532.-Velocity-Reactions of the Starches of Begonia double light rose (.....), Begonia socotrana (.......), and Begonia ensign

$\begin{array}{ll}\text { 527. With Chloral Hydrate. } & \text { 529. With Pyrogallic Acid. } \\ \text { 528. With Chromic Acid. } & \text { 532. With Nitric Acid. }\end{array}$

$\begin{array}{ll}\text { 527. With Chloral Hydrate. } & \text { 529. With Pyrogallic Acid. } \\ \text { 528. With Chromic Acid. } & \text { 532. With Nitric Acid. }\end{array}$

$\begin{array}{ll}\text { 527. With Chloral Hydrate. } & \text { 529. With Pyrogallic Acid. } \\ \text { 528. With Chromic Acid. } & \text { 532. With Nitric Acid. }\end{array}$

Chart D 530.-Velocity-Reactions of Pyrogallic Acid with the Starch of Begonia double light rose. Percentage of entire number of grains (.....) and of total starch (-) gelatinized. 

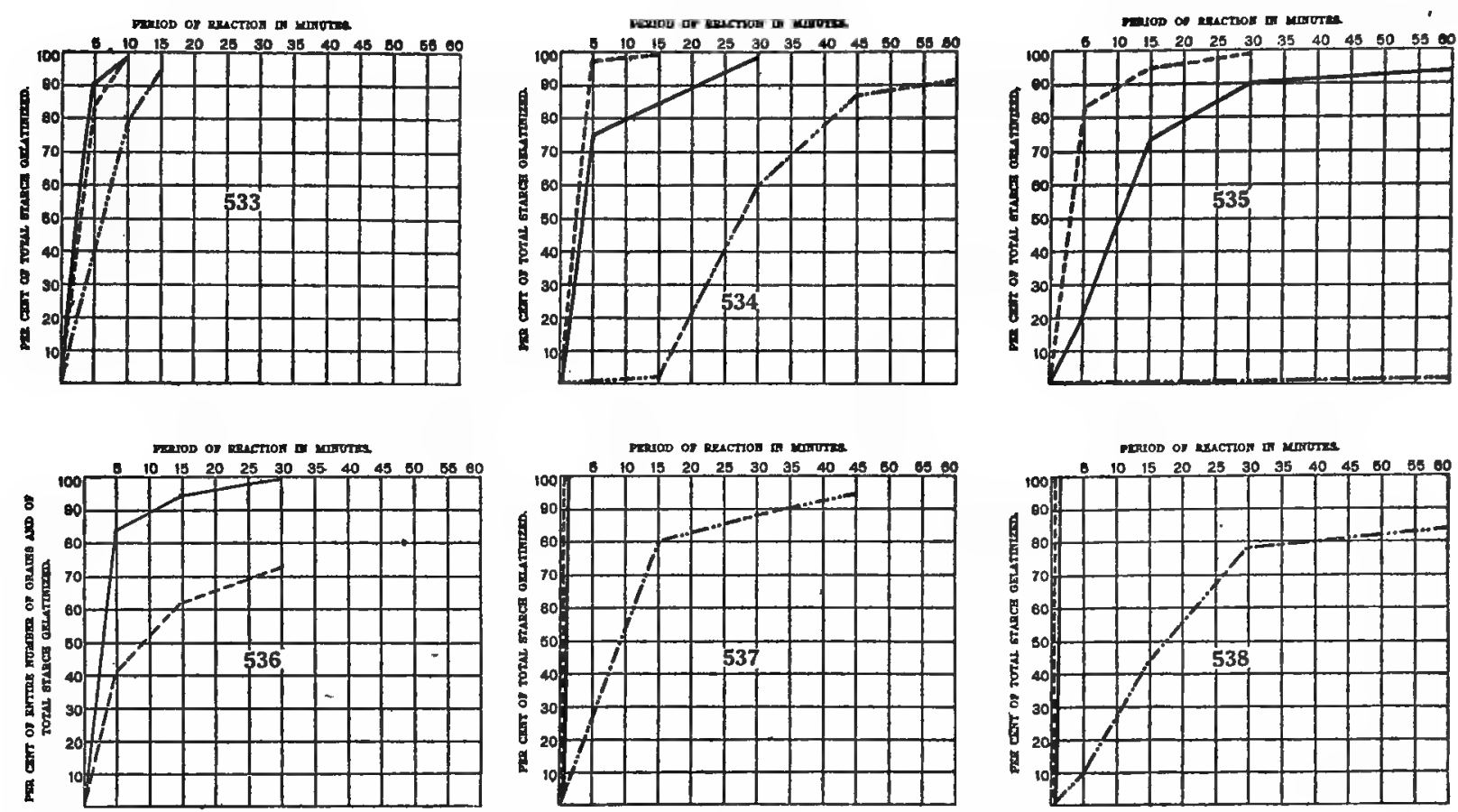

Charts D 533 to D 535, D 537, D 538.--Velocity-Reactions of Starches of Begonia double white (-----), Begonia socotrana (......), and Begonia julius (-).

533. With Chloral Hydrate. 534. With Chromic Acid.

535. With Pyrogallio Acid.

537. With Nitric Acid.

538. With Strontium Nitrate.

Chart D 536.-Velocity-Reactions of Pyrogallic Acid with the Starch of Begonia double white. Percentage of entire number of grains (...-) and total starch (-) gelatinized.
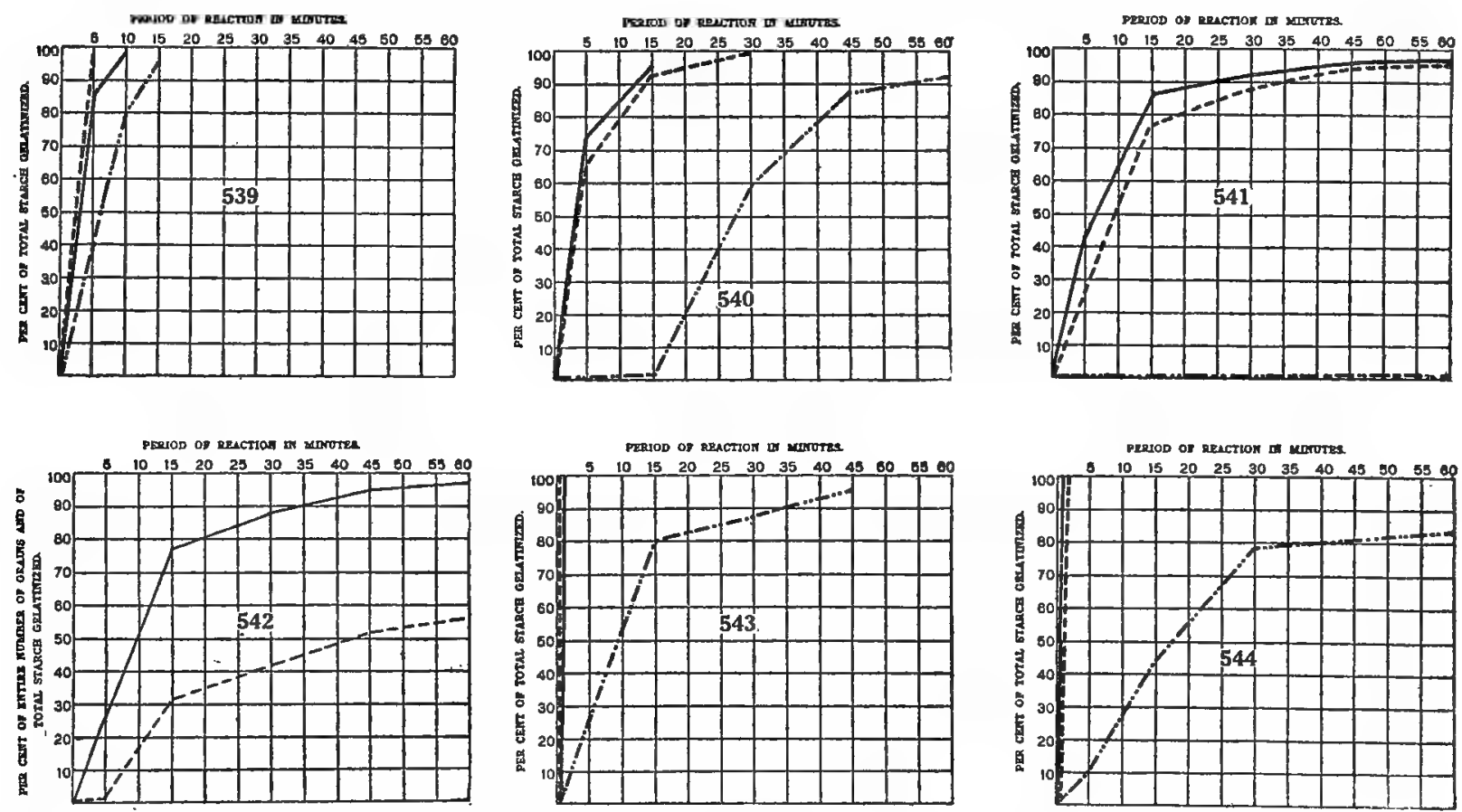

Charts D 539 to D 541, D 543, D 544.-Velocity-Reactions of Starches of Begonia double deep rose (--.--), Begonia socotrana (.......), and Begonia success (539. With Chloral Hydrate.
540. With Chromic Acid.

541. With Pyrogallic Acid.

543. With Nitric Acid.

544. With Strontium Nitrate.

Chart D 542.-Velocity-Reactions of Pyrogallic Acid with the Starch of Begonia double deep rose. Percentage of entire number of grains (....) and total starch (- $(-)$ gelatinized. 
mono of encrion

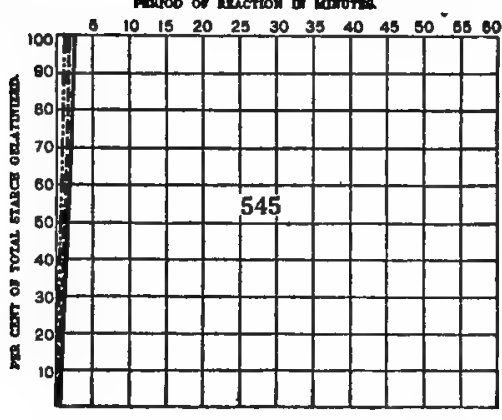

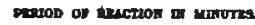
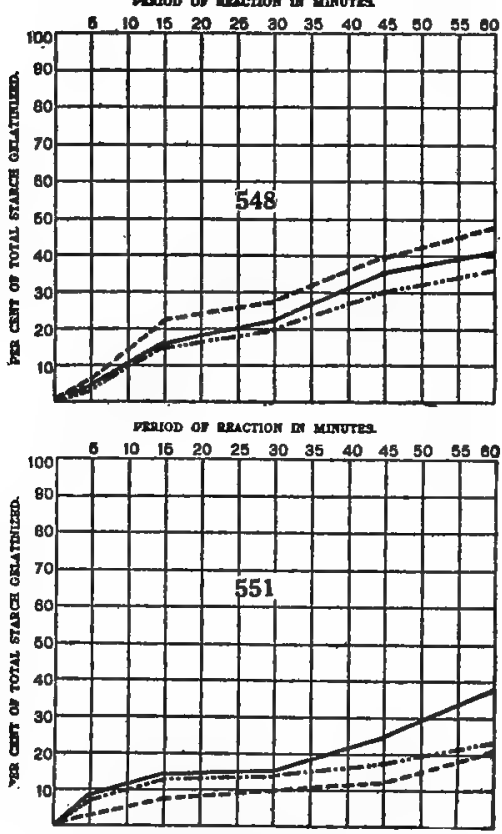

PERTOD OP REACtion AS MENUTRE

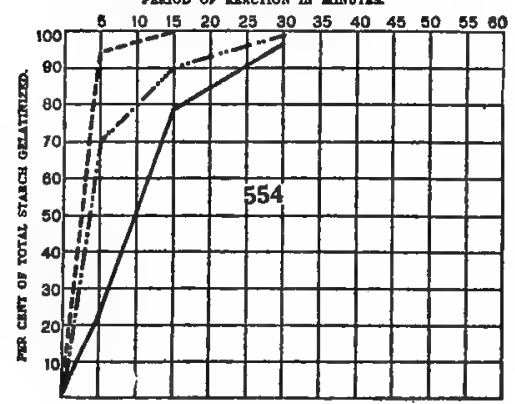

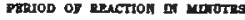

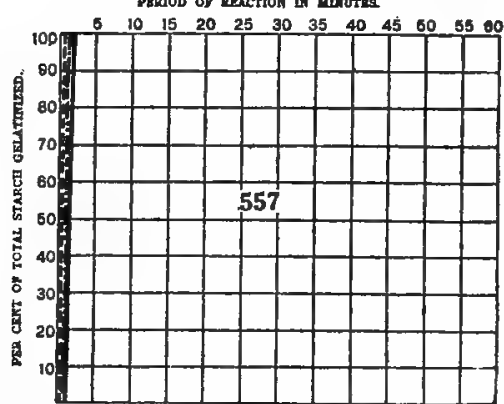

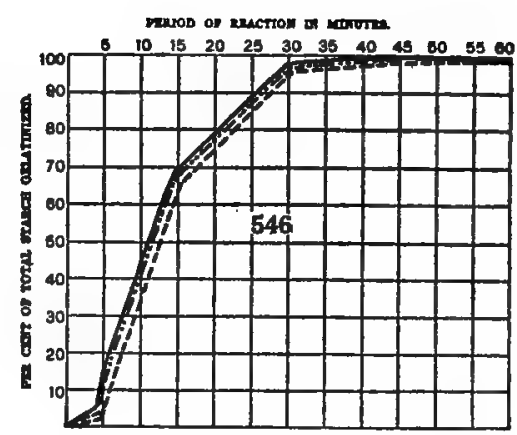

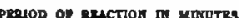

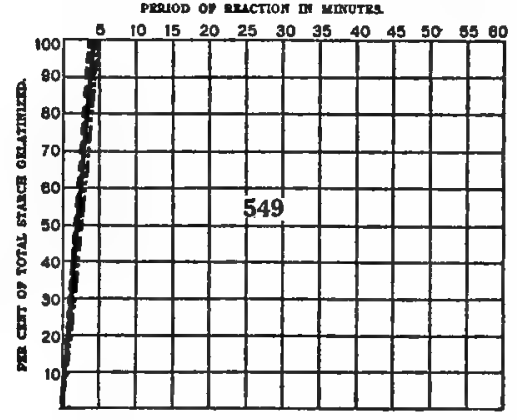

peruod of menction an worutes

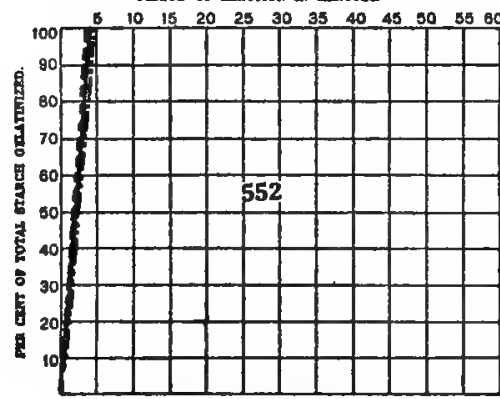

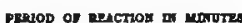

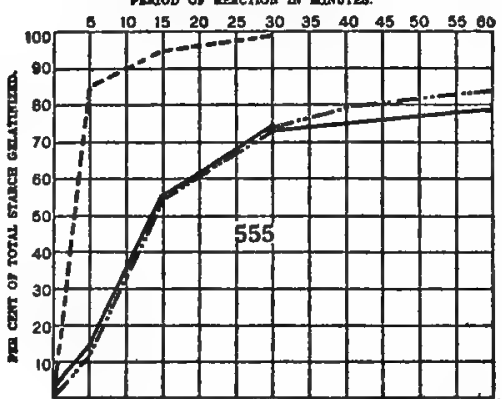

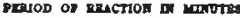

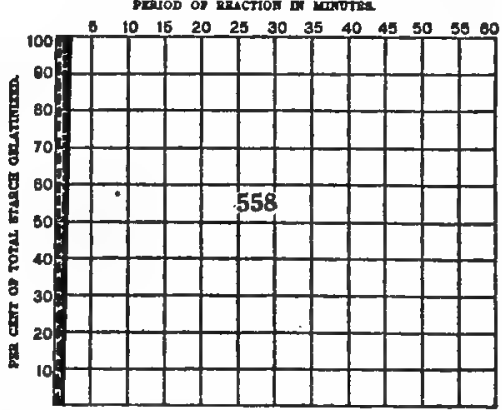

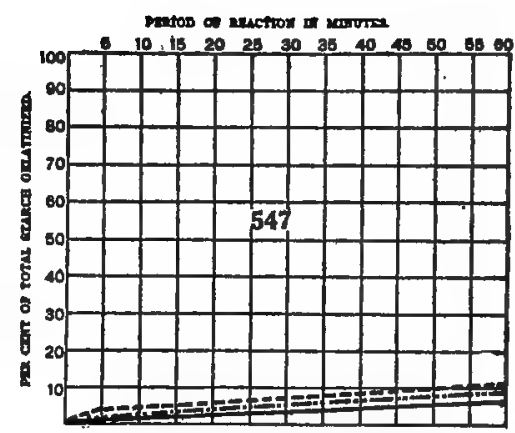

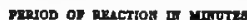

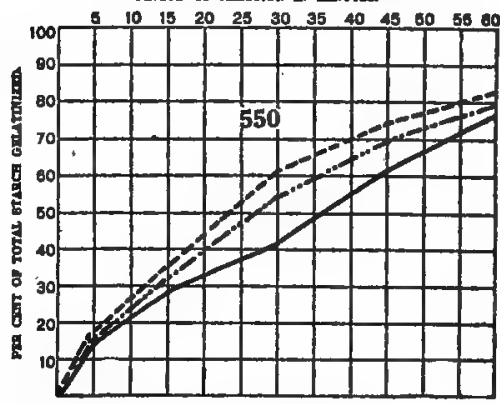

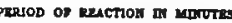

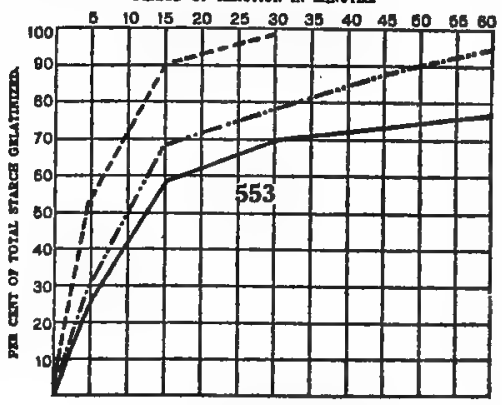

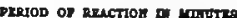

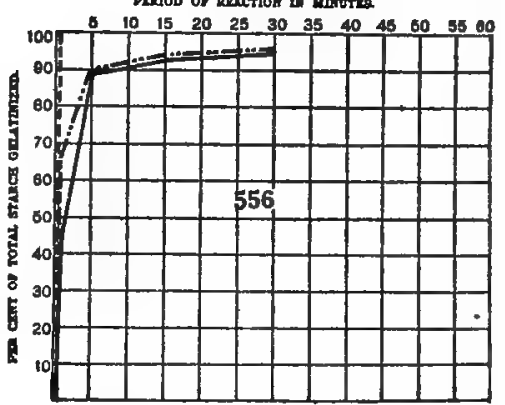

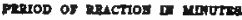

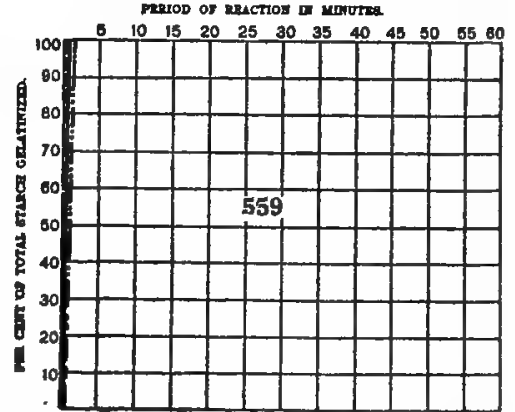

Charts D 545 to D 559.-Velocity-Reactions of Starches of Richardia albo-maculata ( .....), Richardia elliottiana

545. With Chloral Hydrate.

546. With Chromic Acid.

547. With Pyrogallic Acid.

548. With Nitric Acid.

549. With Sulphuric Acid.

( ......), and Richardia mrs. roosevelt (-).

\begin{abstract}
550. With Hydrochloric Acid.
55. With Potassium Hydroxide.

552. With Sodium Salicylat
\end{abstract}

554. With Chromic Acid.
555. With Pyrogallic Acid.

556. With Nitric Acid.

557. With Sulphuric Acid.

558. With Hydrochloric Acid

559. With Potassium Hydroride. 

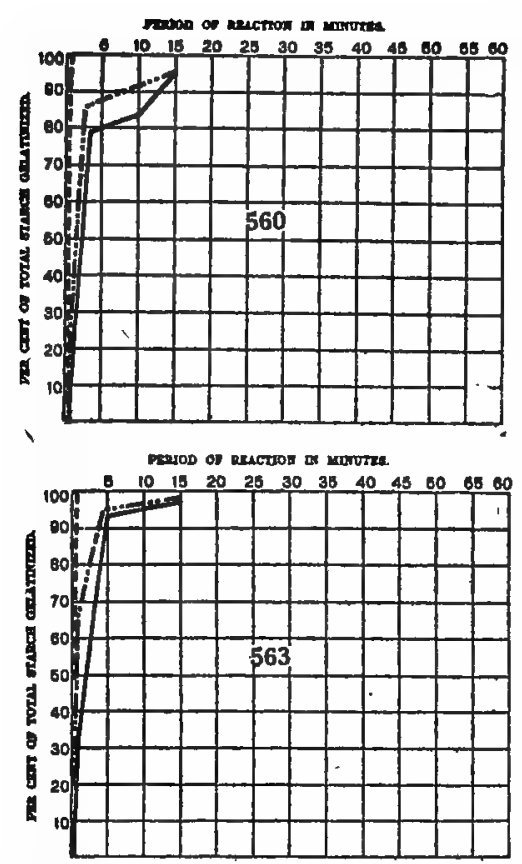

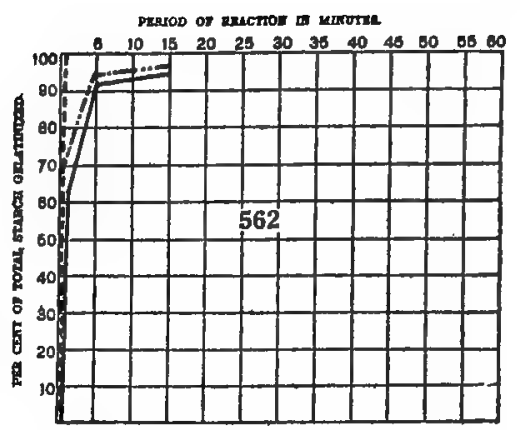

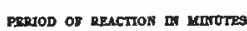
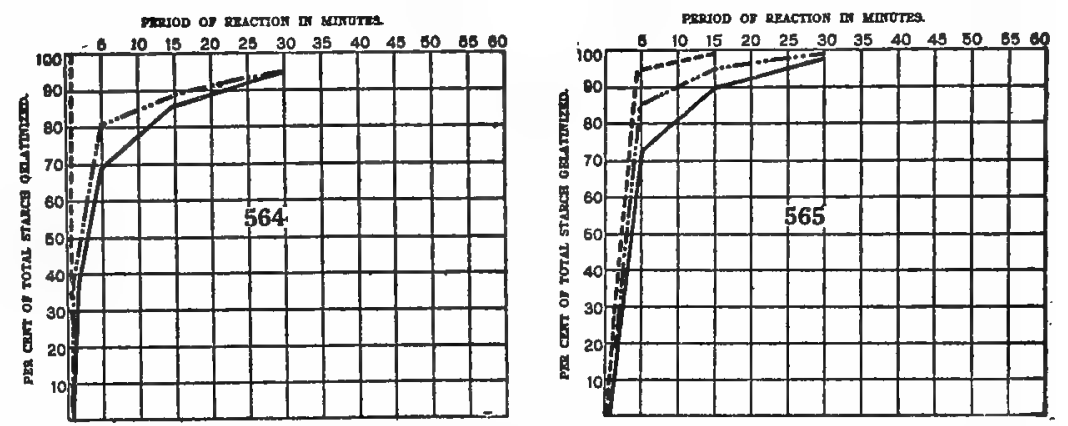

Charts D 560 to D 565.-Velocity-Reactions of Starches of Richardia albo-maculata ( .....), Richardia elliottiana ( -......), and Richardia mrs. roosevelt (-).
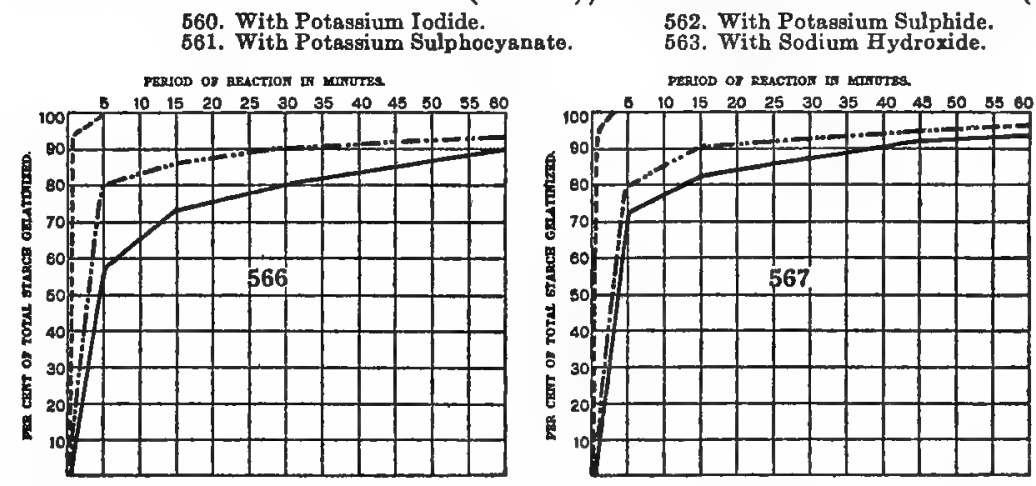

564. With Sodium Sulphide.

562. With Potagium Sulphide. With Sodium Hydroxide. 565. With Sodium Salicylate.

$\begin{array}{lllllllllll}10 & 15 & 20 & 25 & 30 & 35 & 40 & 45 & 50 & 55 & 00\end{array}$
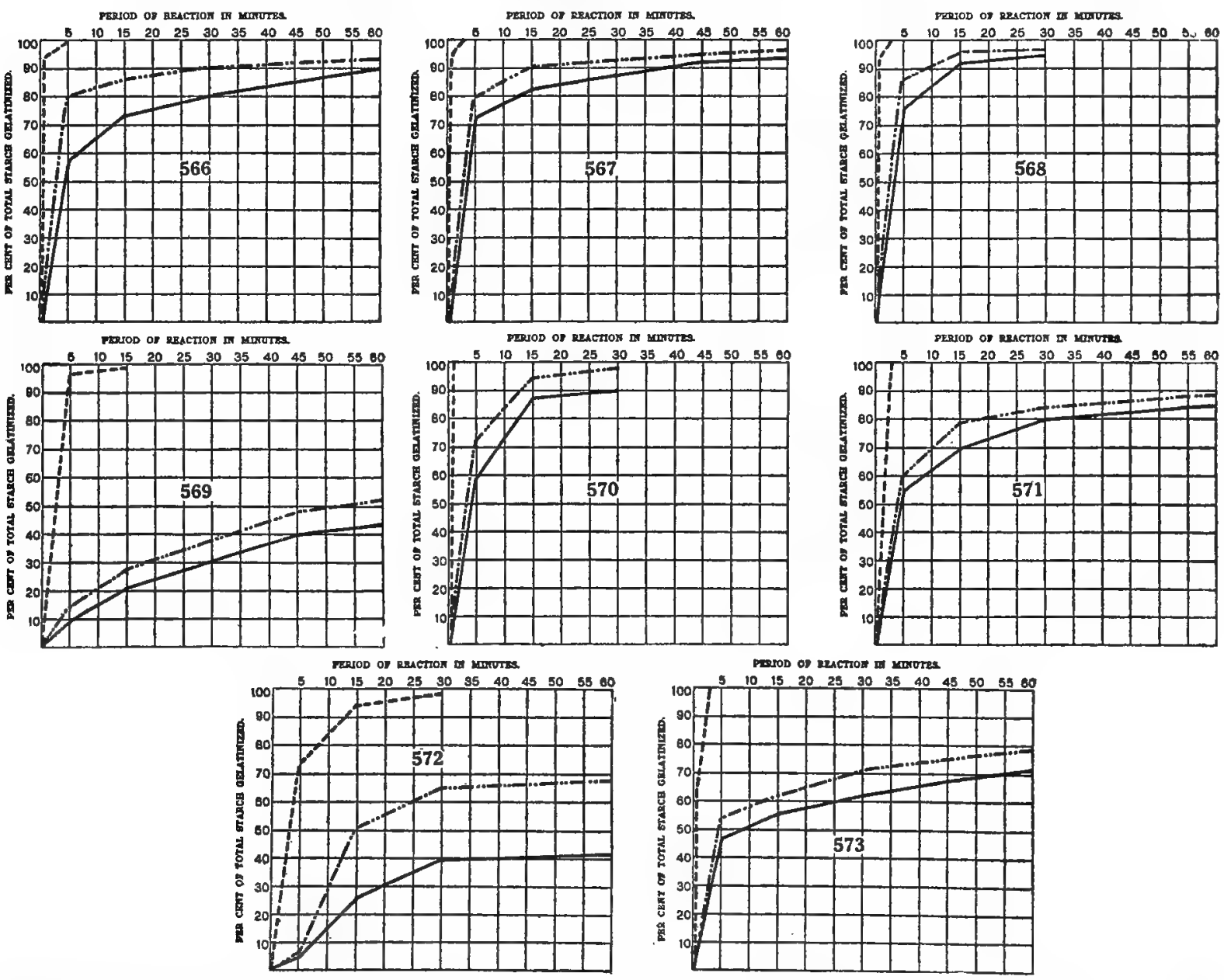

Charts D 566 to D 573.-Velocity-Reactions of Starches of Musa arnoldiana ( -....), Musa gilletti ( .......), and Musa hybrida (-). 


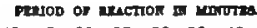

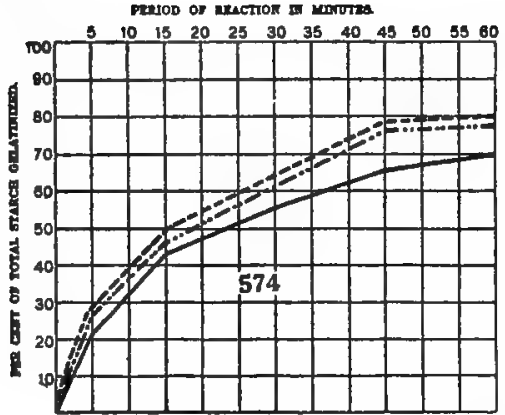

PERIOD O) penctron D MDVTS

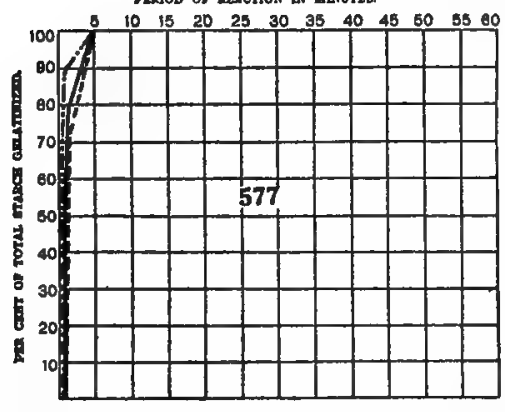

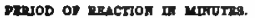

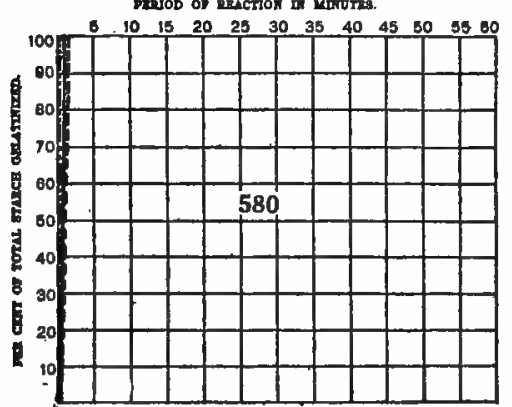

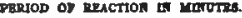

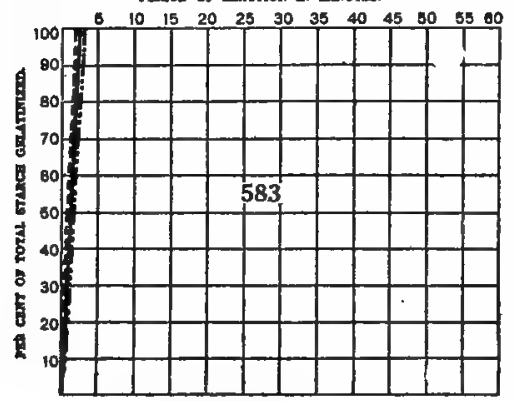

prefos of genction ar Mesctres.

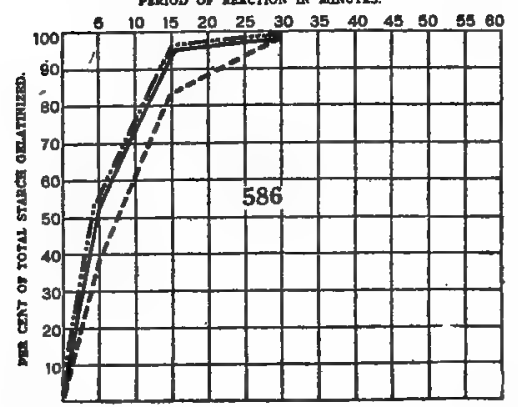

Pmiod of Eectros if mortse

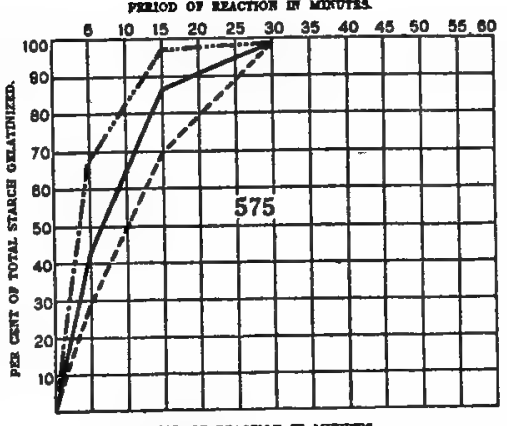

Pretod of exachon If warures

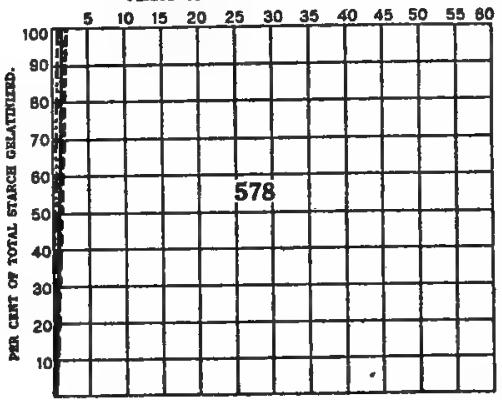

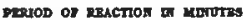

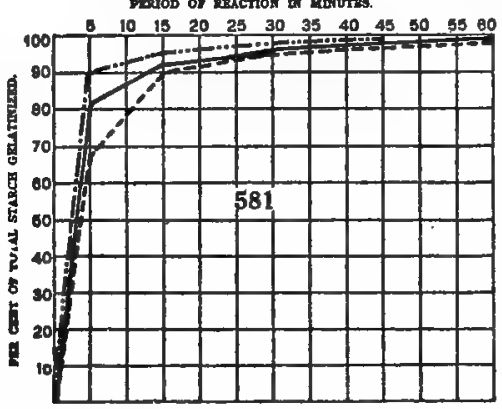

PRUOD Ob RPACTION WN MLTOTES

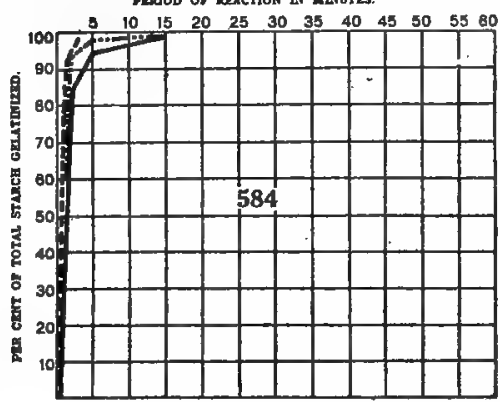

prefos of menctiox un matres

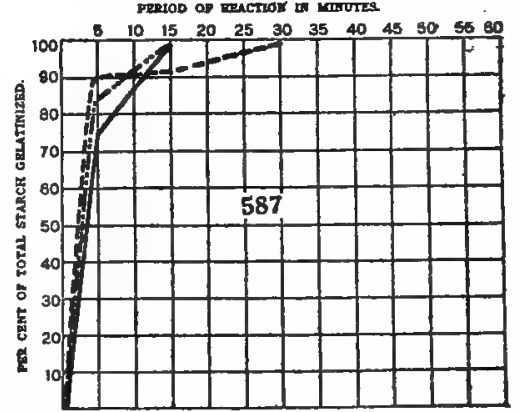

marod or anctor

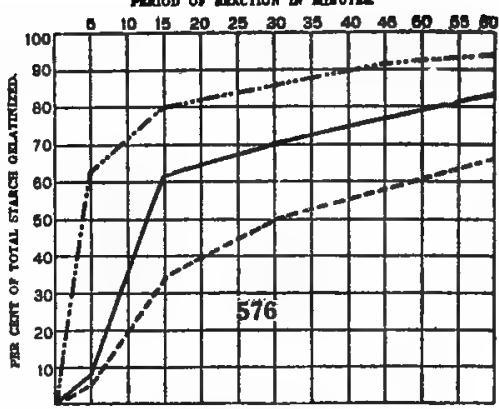

mos of penctox of usprotes.

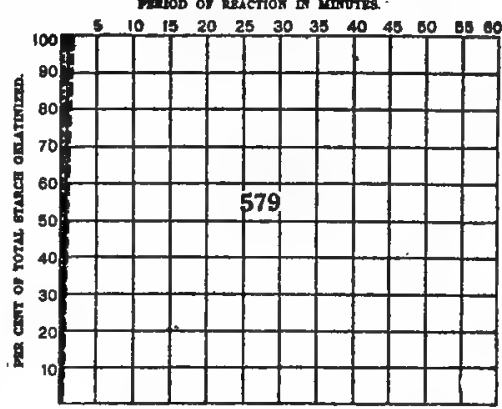

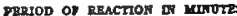

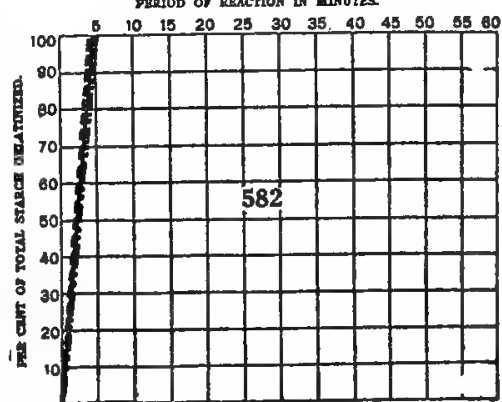

muod of enction on Mervte:

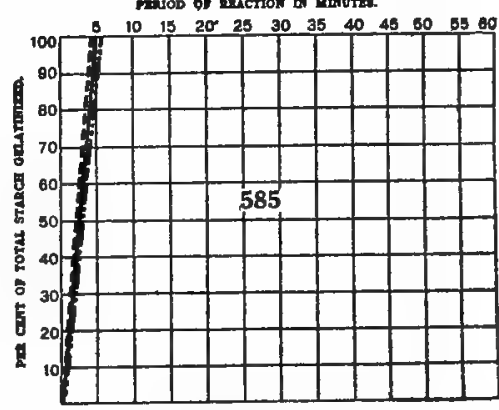

pesiod os penction a morotea.

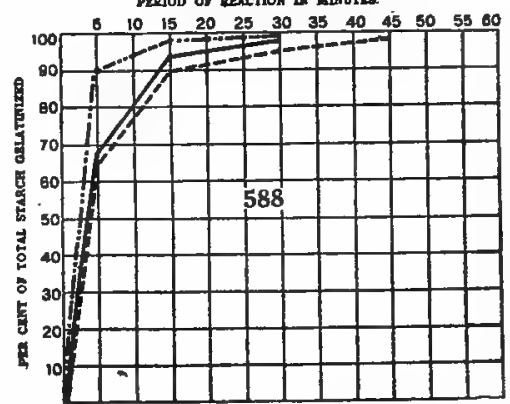

Charts D 574 to D 588.-Velocity-Reactions of Starches of Phaius grandifolius ( -...-), Phaius wallichii (-......),

and Phaius hybridus (-).

579. With Hydrochloric Acid.

580. With Potssssium Hydroxide.

581. With Potrasium Iodide.

582. With Potassium Sulphocyanate.

683. With Potassium Sulphide.
584. With Sodium Hydroxide. 685. With Sodium Sulphide.

586. With Sodium Salicylate.

588. With Calcium Nitrate.
576. With Pyrogallic Acid.

578. With Sulphuric Acid. 

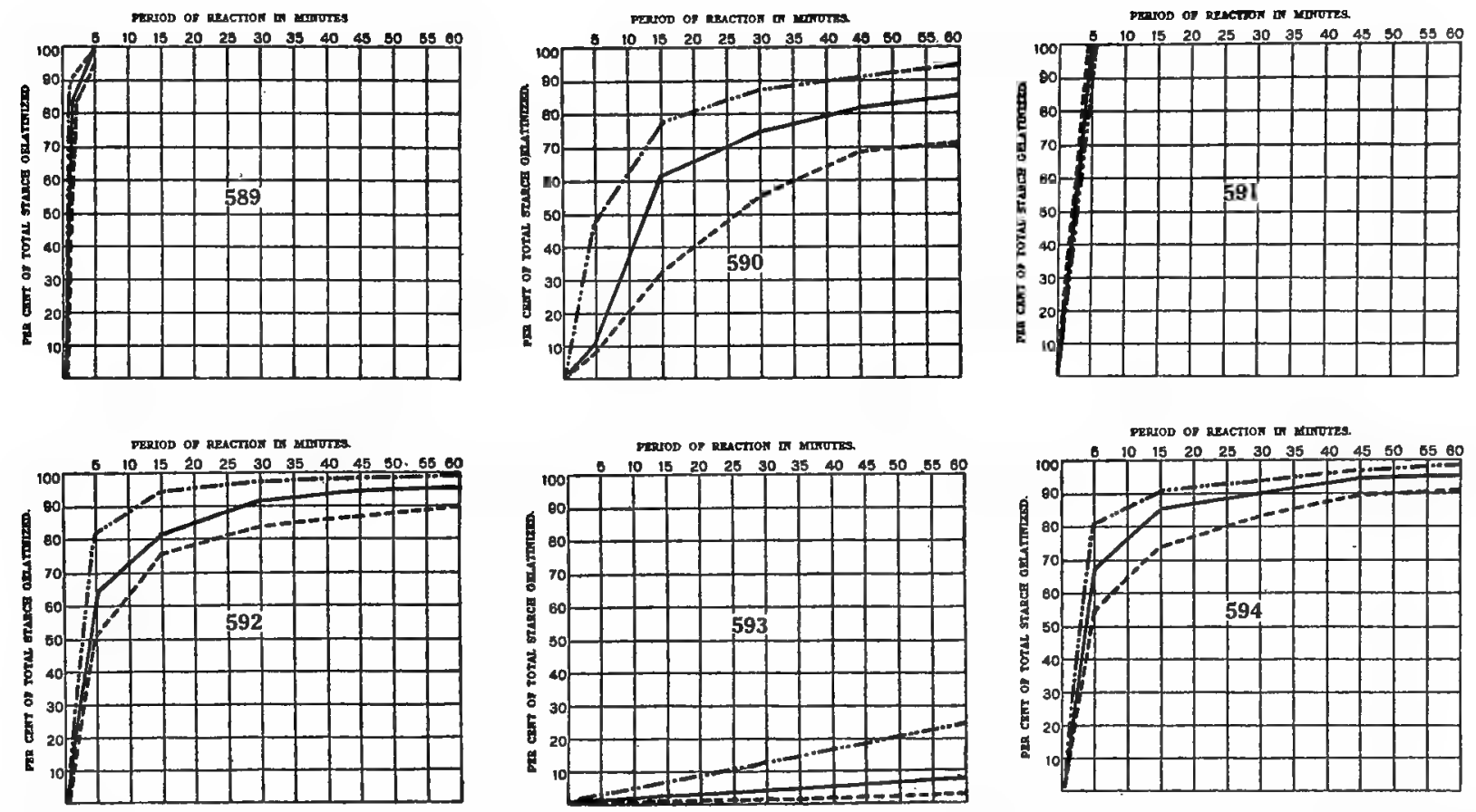

Charts D 589 то D 594.-Velocity-Reactions of Starches of Phaius grandifolius (-...-), Phaius wallichii ( .......), and Phaius hybridus (-).

589. With Strontium Nitrate. 590. With Cobalt Nitrate.
591. With Copper Nitrate. 592. With Cupric Chloride.
593. With Barium Chloride. 594. With Mercurio Chloride. 


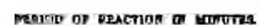

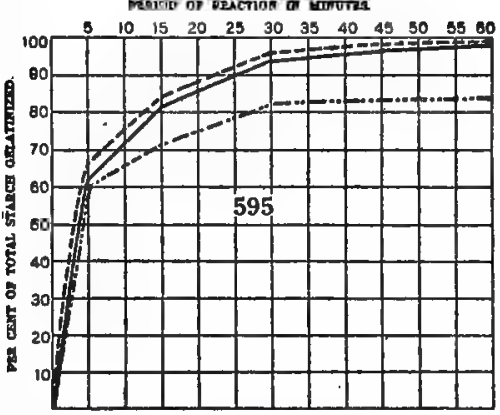

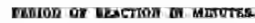

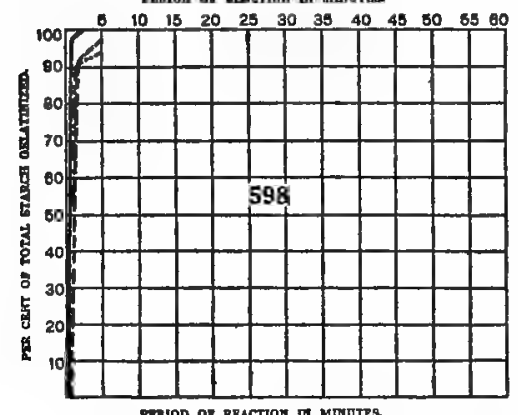

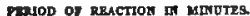

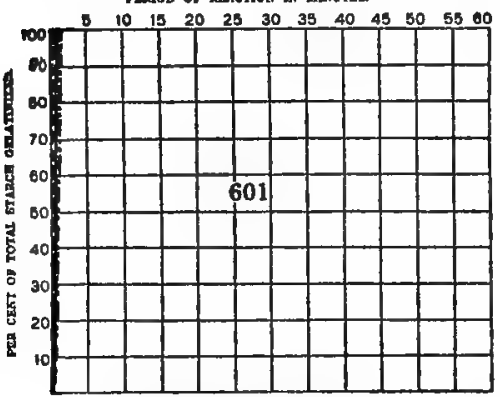

PTIOD OP REACTION IA Marotes

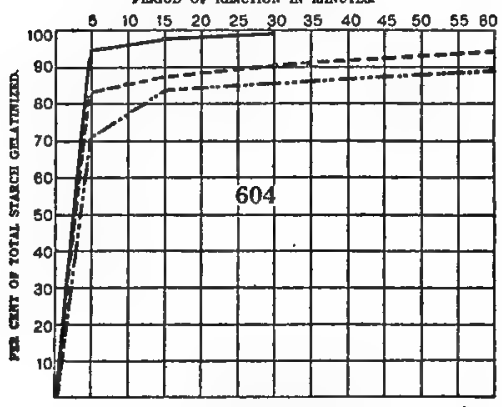

(2)

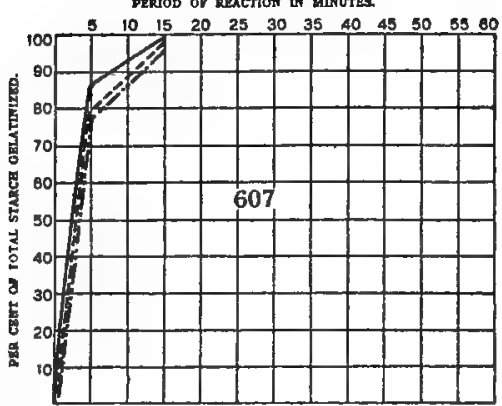

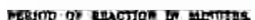

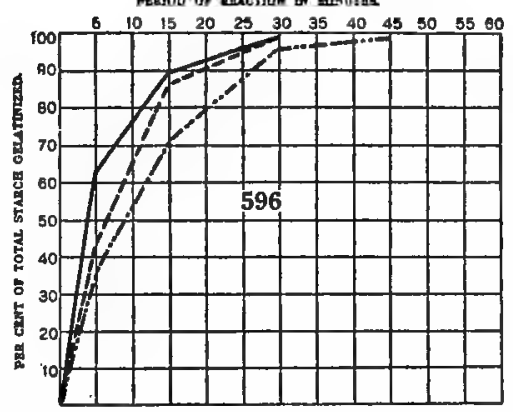

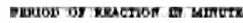

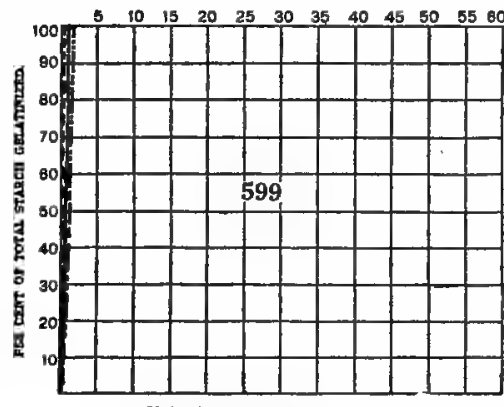

PERIOD OT REACTION II MURUTES

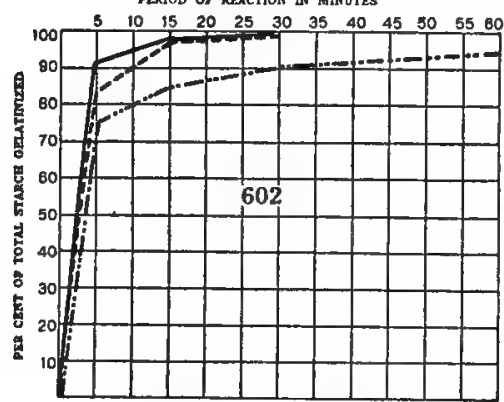

PERrod OF PFACTIOA II MDNOTES

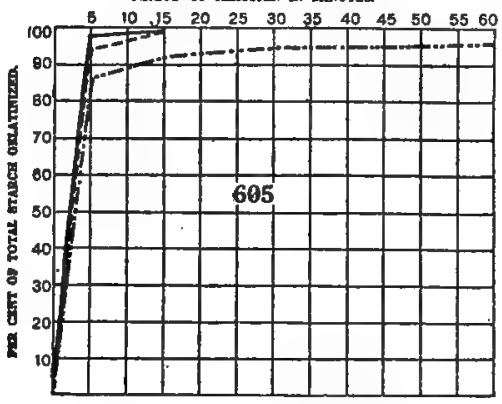

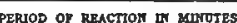

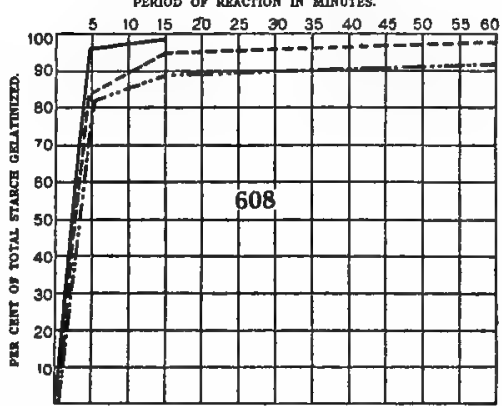

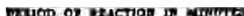

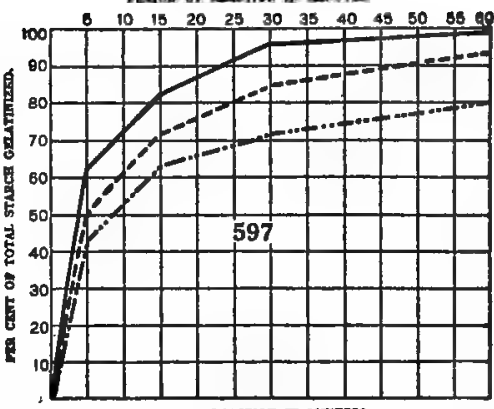

10

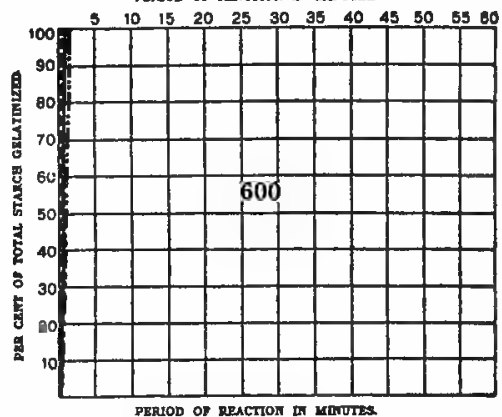

PERLOD OP REACTION पT KENUTES

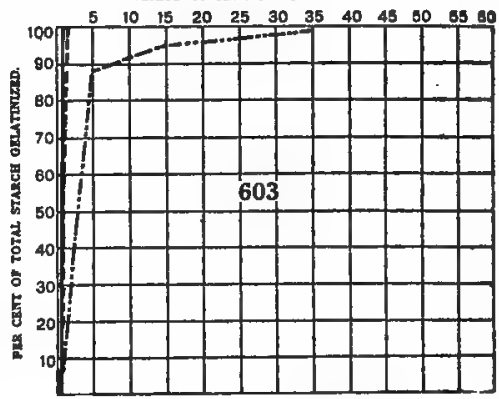

prerod of menction at untures

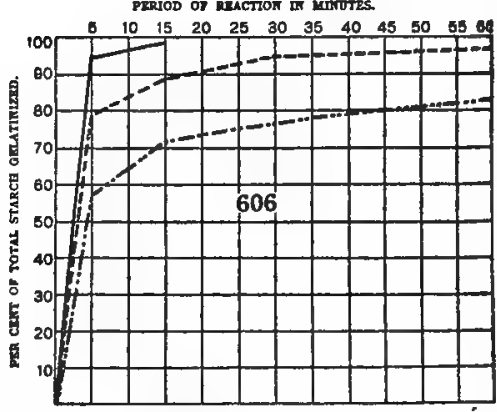

(1)

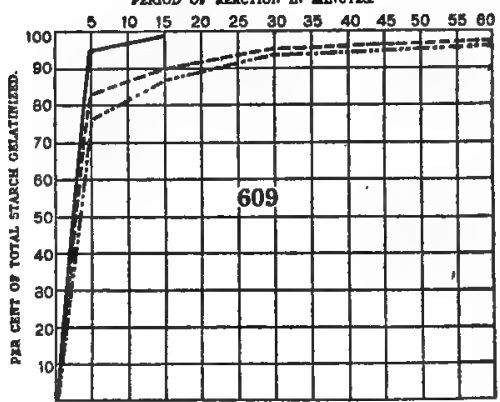

Charts D 595 to D 609.-Velocity-Reactions of Starches of Miltonia vexillaria ( ...-.), Miltonia razlii (....... -), and Miltonia bleuana (-).

600. With Hydrochloric Acid.

601. With Potassium Hydroxide

602. With Potassium Iodide.

603. With Potasoium Sulphocyanste. 604. With Potassium Bulphide.
605. With Sodium Hydroxide.

606. With Sodium Sulphide.

606. With Sodium Sulphide.

607. With Sodium Salicylate.

608. With Caloium Nitrate.
609 . With Uranium Nitrate. 

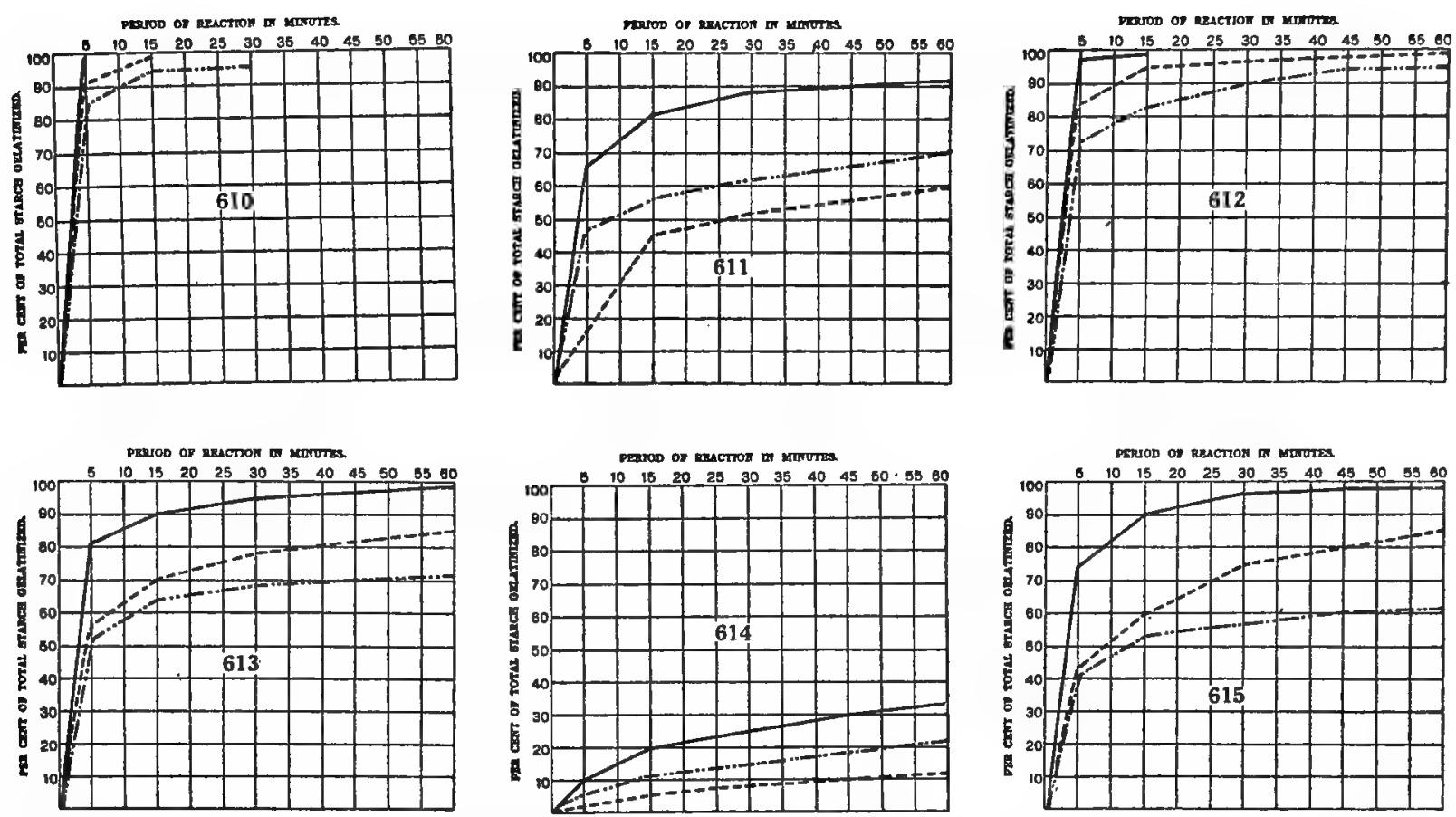

Charts D 610 to D 615.-Velocity-Reactions of Starches of Miltonia vexillaria (--.--), Miltonia rozlii ( -......), and Miltonia bleuana (-).

610. With Strontium Nitrate. 611. With Cobalt Nitrate.

612. With Copper Nitrate.

614. With Barium Chloride.

615. With Mercuric Chloride.
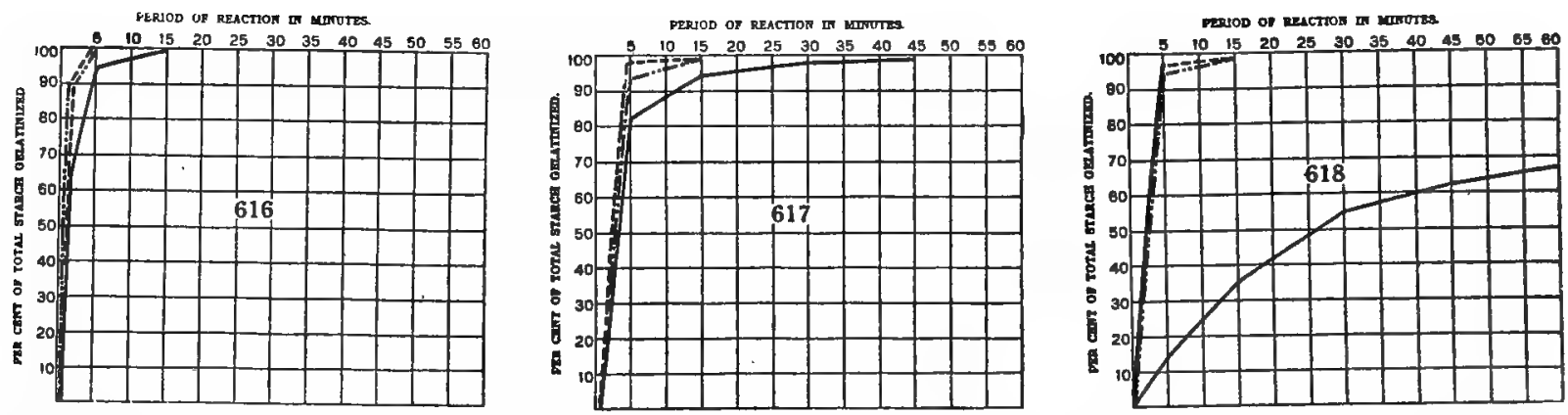

CharTs D 616 to D 618.-Velocity-Reactions of the Starches of Cymbidium lowianum (....-), Cymbidium eburneum (-.....), and Cymbidium eburneo-lowianum ( -....). 

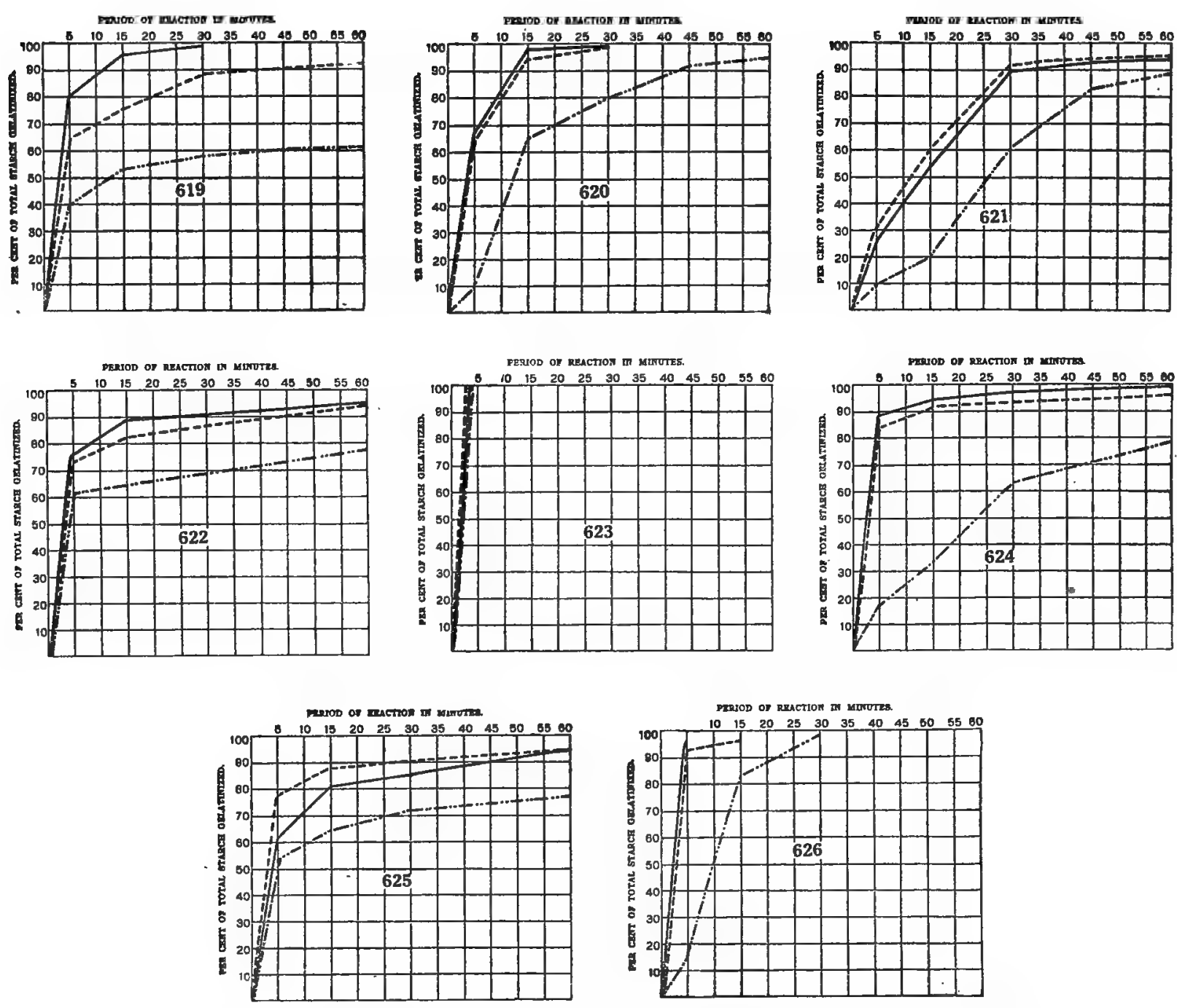

Charts D 619 to D 626.-Velocity-Reactions of Starches of Calanthe rosea (--.-.), Calanthe vestita var. rubrooculata (......-), and Calanthe veitchii (-).

619. With Chlorsl Hydrate. 620. With Chromic Acid.
o21. With Pyrogallio Acid.
622. With Nitric Acid.

623. With Sulphuric Acid.

624. With Hydrochloric Acid.
625. With Potasaium Hydroxide 626. With Sodium Salicylate. 

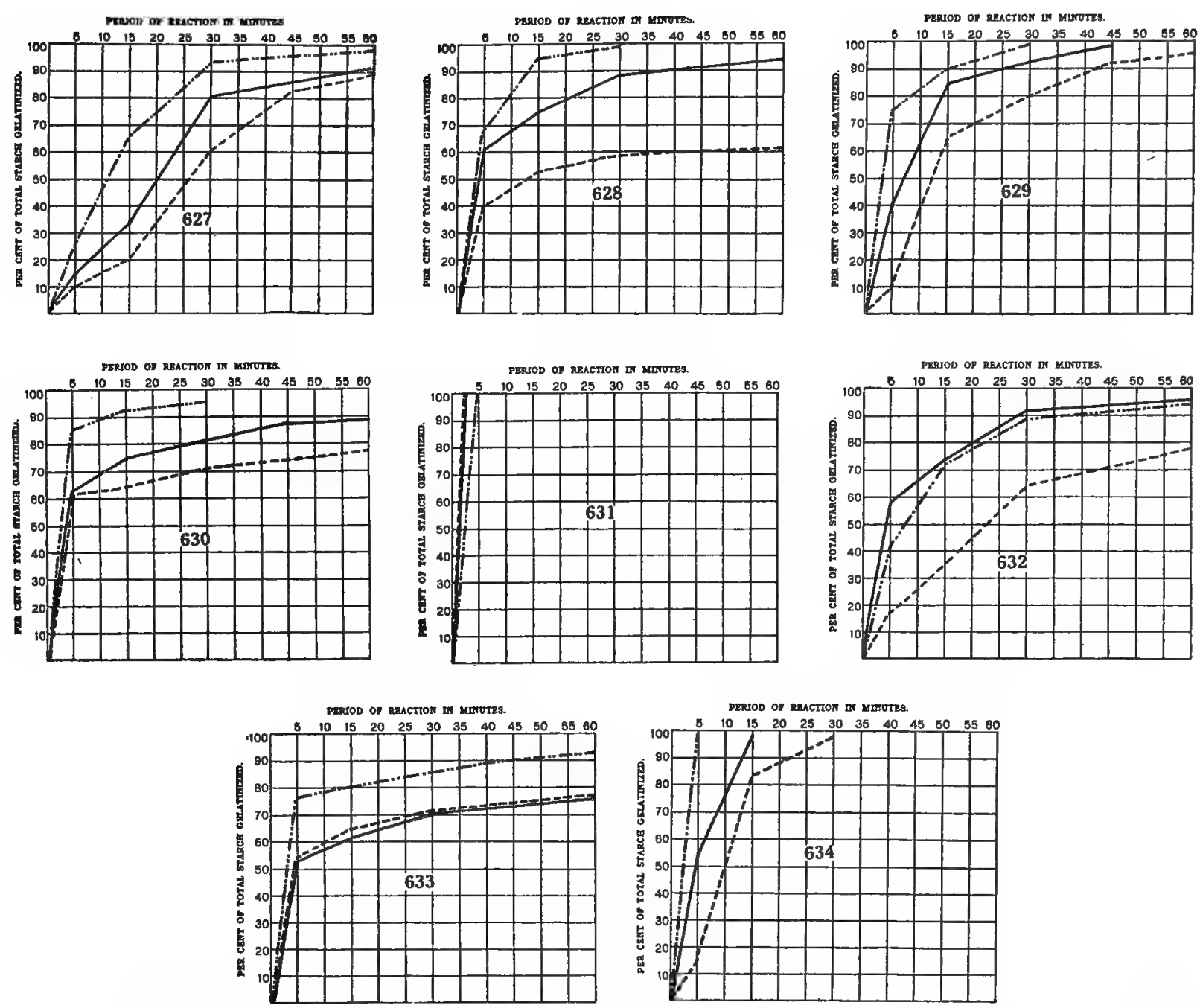

Charts D 627 to D 634.-Velocity-Reactions of Starches of Calanthe vestita var. rubro-oculata (.....), Calanthe regnieri (.......), and Calanthe bryan (—).

627. With Pyrogallic Acid.
628. With Chloral Hydrate.

629. With Chromic Adid.
630. With Nitric Acid.

631. With Sulphuric Acid.

632. With Hydrochloric Acid.
633. With Potgssium Hydroxide.

634. Wi th Sodium Salicylate. 


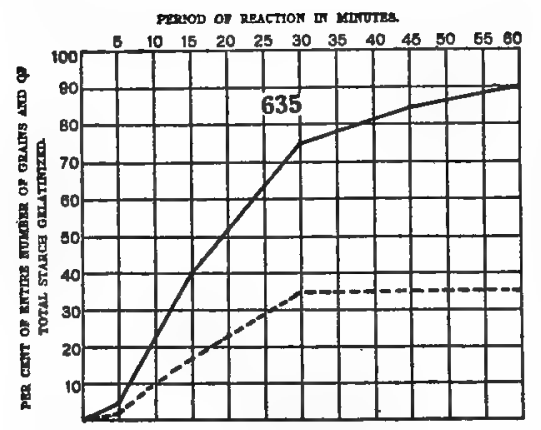

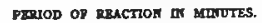

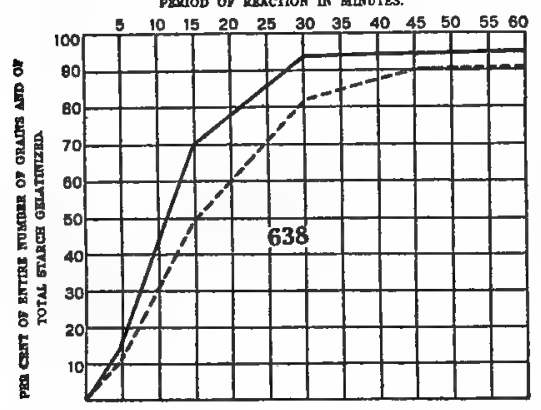

PRRTOD OF REACTON Wn MQNOTES.

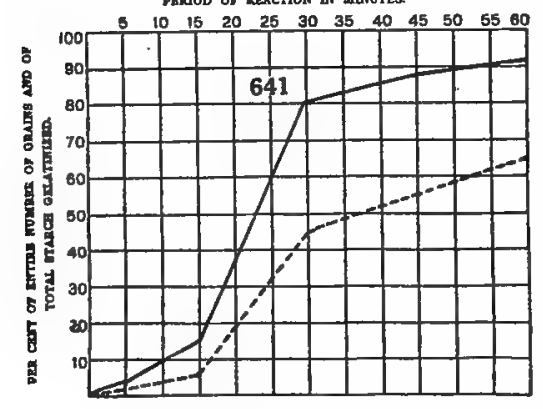

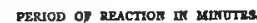

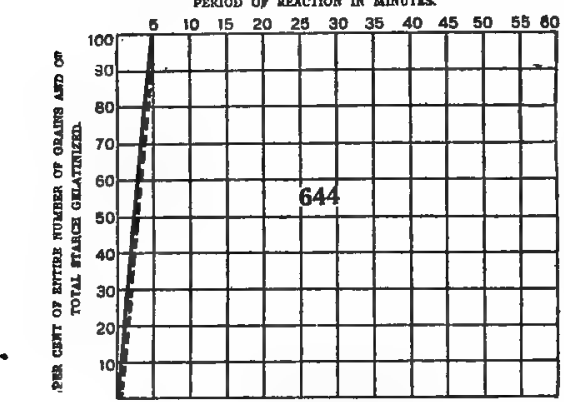

NERTOD OI RBACTION IT MINTTES

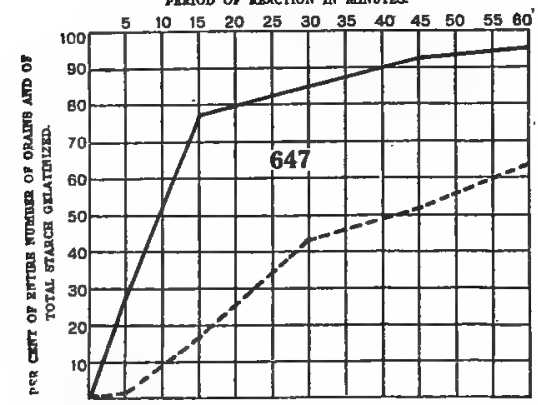

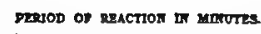

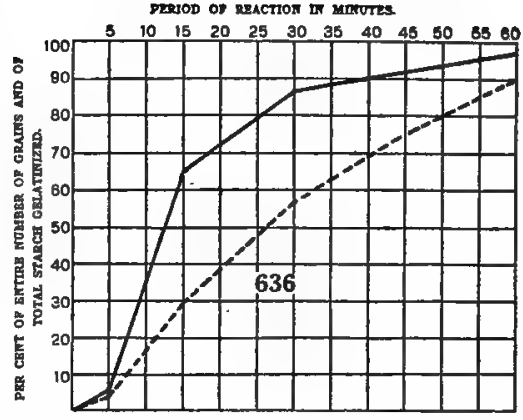

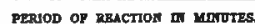

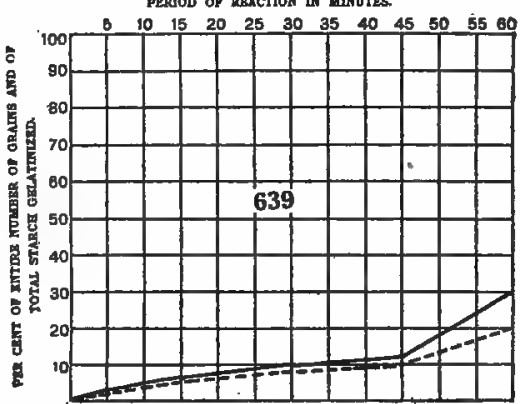

PERTod of HeActios as MUNUTBE

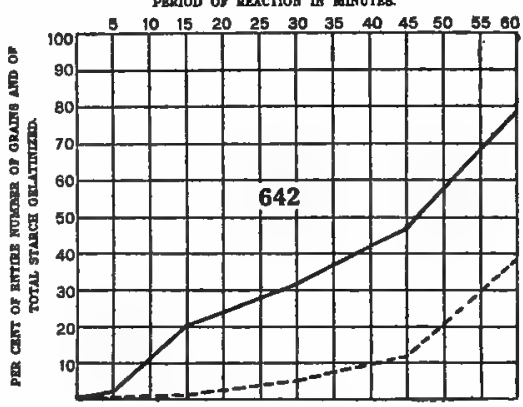

PERIOD OI REACTION IS YTNOTES.

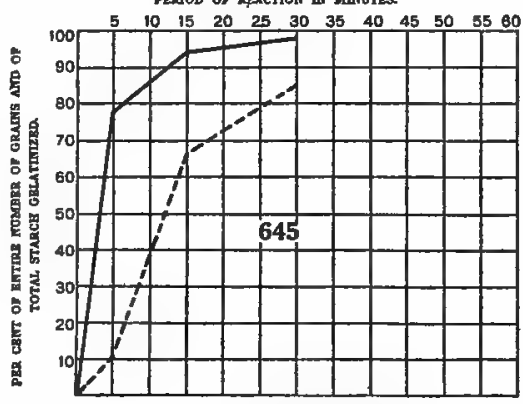

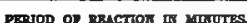

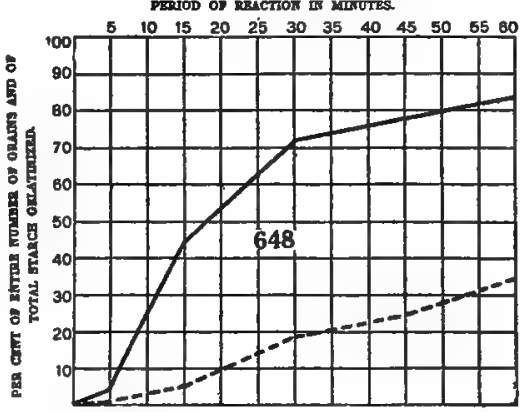

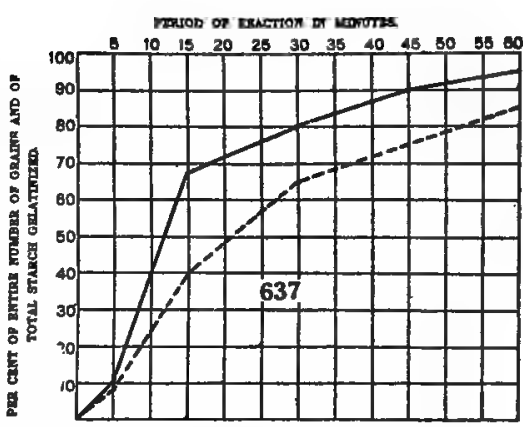

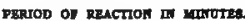

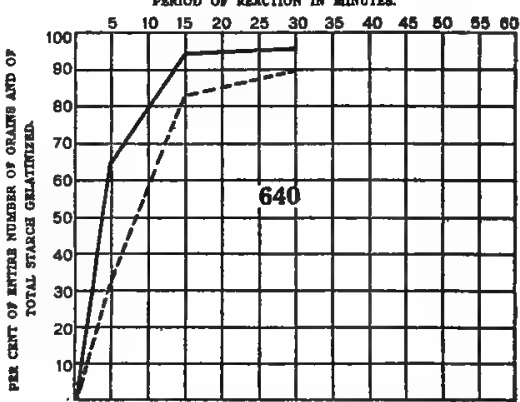

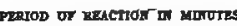

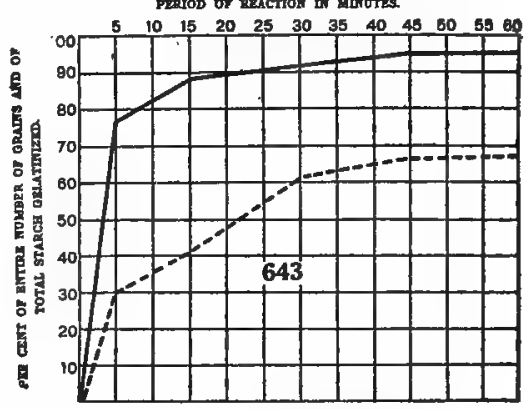

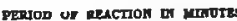

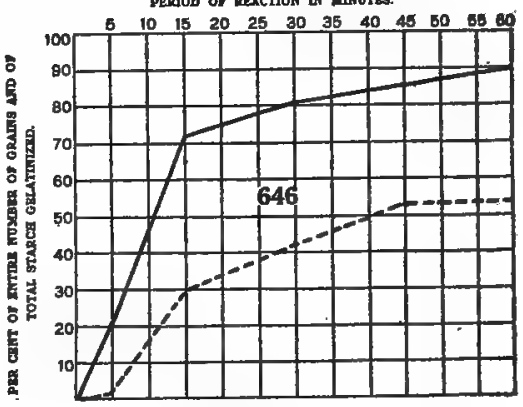

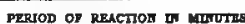

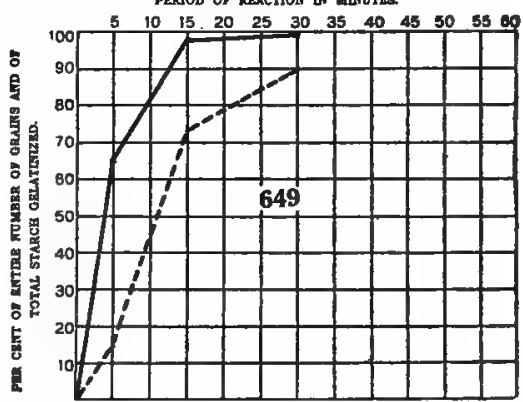

Charts D 635 то D 649.-Velocity-reactions of pyrogallic acid with various starches, showing the percentage of the entire number of grains (..-) and of the total starch (-) gelatinized.

635. With Amaryllis belladonna. 636. With Hippeastrum titan.

637. With Hippeastrum ossultan.

638. With Hippeastrum dasones.

639. With $\mathrm{H}$ mmanthus katherinæ.
640. With Homanthus puniceus.

641. With Crinum zeylanicum.

642. With Narcisaus taz. grand mon.

644 . With
645. With Lilium chalcedonicum.

646. With Iris iberics.

647. With Iris trojana.

648. With Iris cengialti.

649. With Iris persica var. purpurea. 

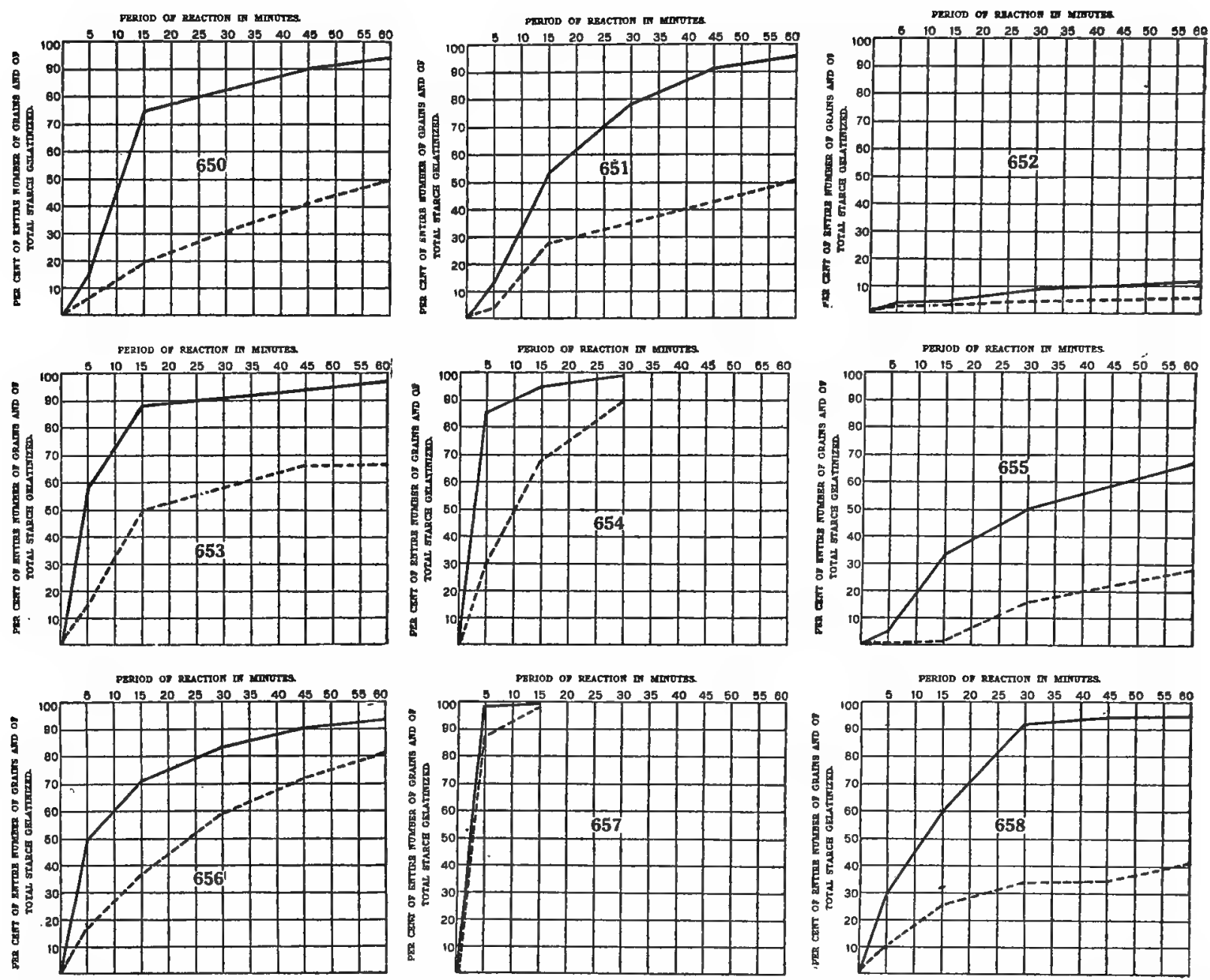

ChARTs D 650 то D 658.-Velocity-reactions of pyrogallic acid with various starches, showing the percentage of the entire number of grains (....), and of the total starch (-) gelatinized.

650. With Gladiolus tristis.

651. With Tritonis pottsil.

652. With Richardia albo-maculata.

See also Charts:

261. Narcissus poeticus ornatus. 290. Narcissua gloria mundi.

296. Narciasus telamonius plenus.

308. Narcissus abscissus.

314. Narcissus albicans.
653. With Begonia sing. crim. Bcar. 655. With Musa arnoldiana.

320. Narcissus empress. 326. Narciasus weardale perfection. 344. Narcissus emperor. 350. Lilium martagon album. 351. Lilium maculatum.
656. With Miltonia vexillaria. 657. With Cymbidium lowianum. 658. With Calanthe rosea.

357. Lilium martagon.

530. Begonia double light rose.

535. Begonia double white.

542. Begonia double deep rose. 

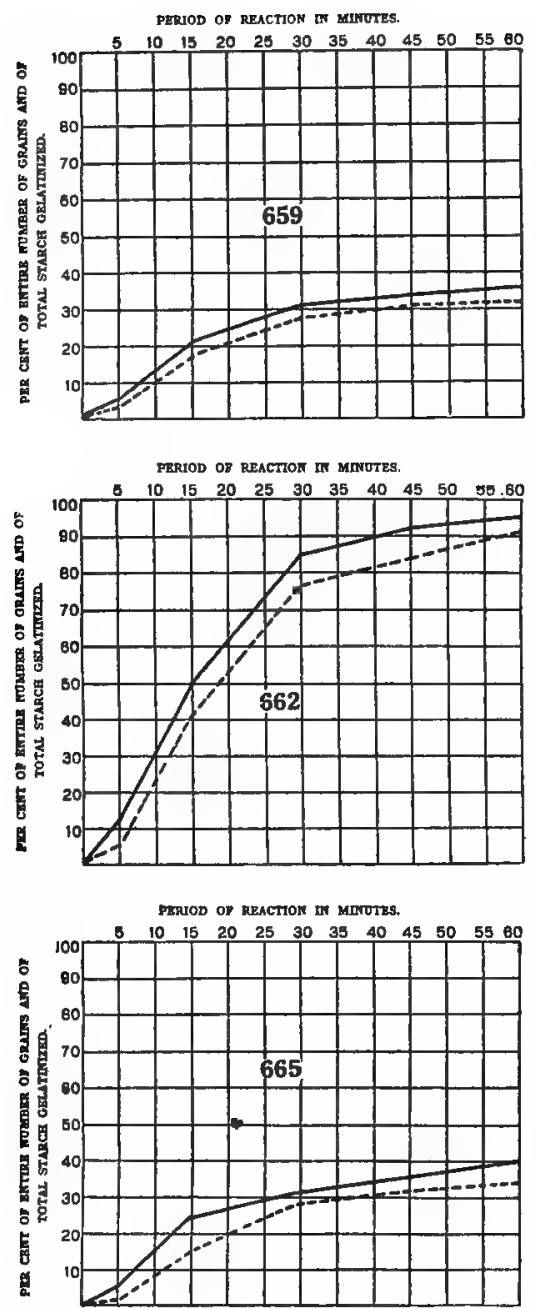

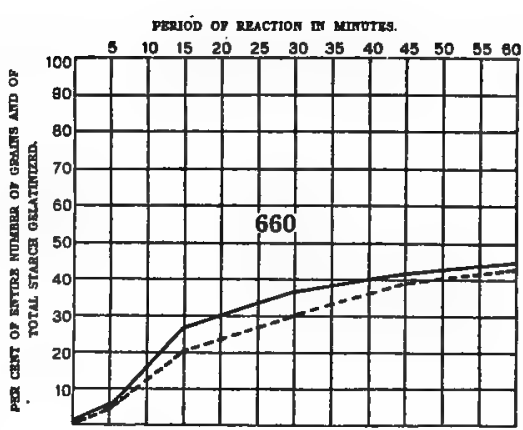

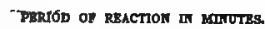

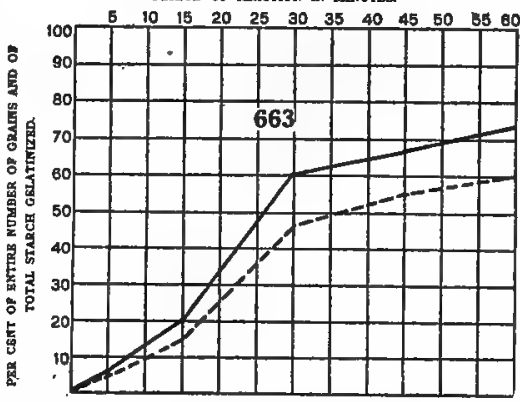

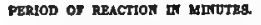

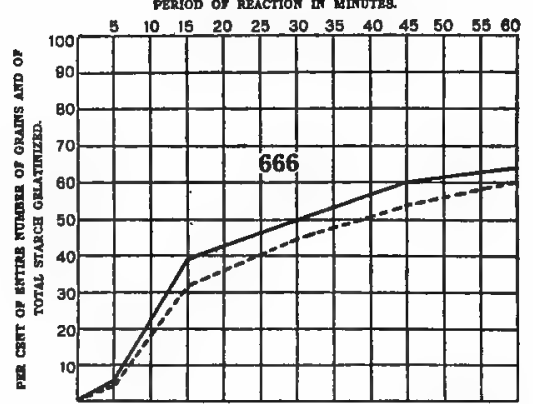

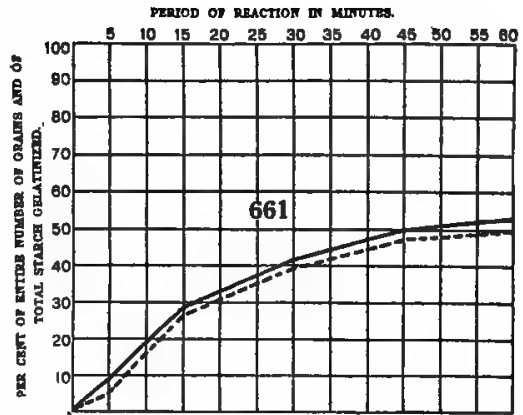

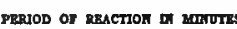

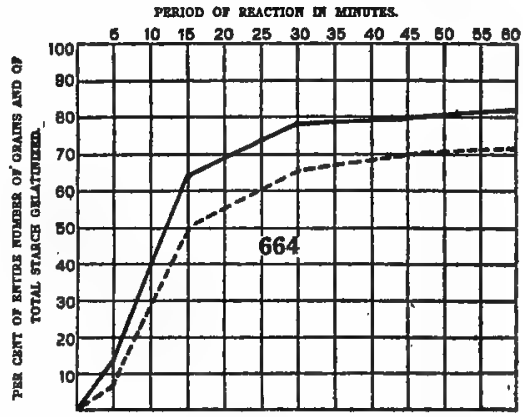

PERIOD OI REACTION IR MATUTES.

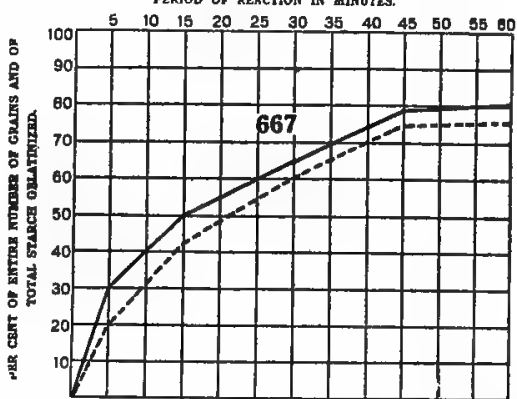

ChaRTs D 659 To D 667.-Velocity-Reactions of chloral hydrate with various starches, showing the percentage of entire number of grains ( -..--) and total starch (-) gelatinized.

659. With Hippeastrum titan. 660. With Hippeastrum ossultan 681. With Hippeastrum dæones.
662. With Amaryllis belladonna. 663. With Hrmanthus katherina. 664. With Hæmanthus puniceus.
665. With Narcissue taz. grand mon.

666. With Iris iberica.

667. With Phaius grandifolius. 

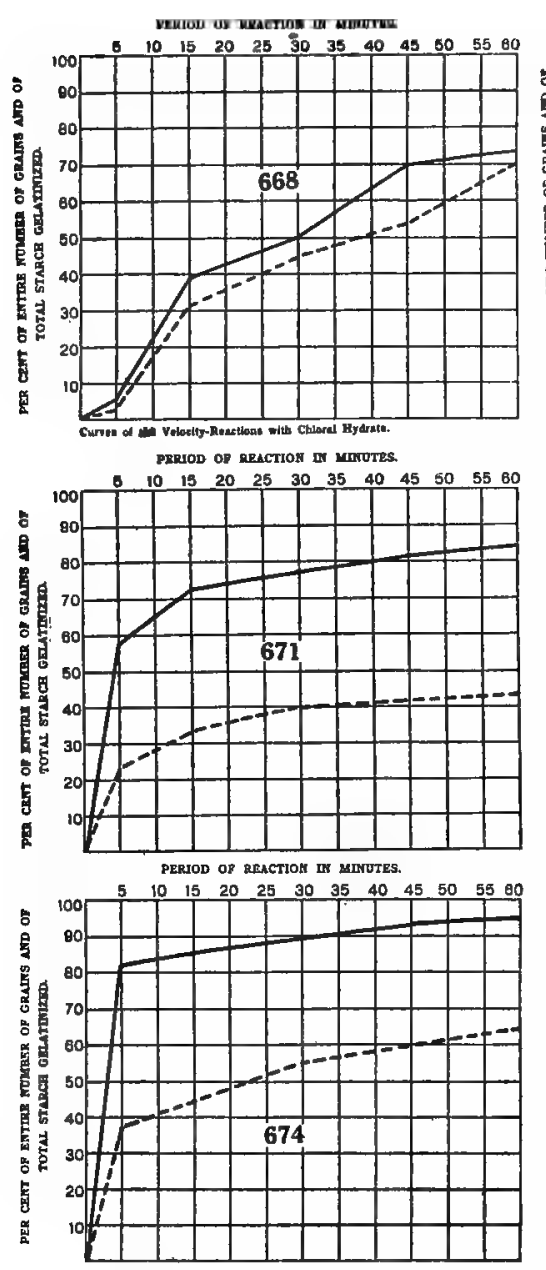

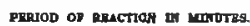

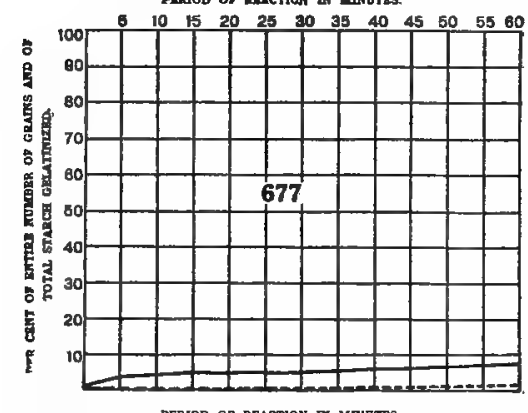

PERIOD OF REACTION IA MINUTES

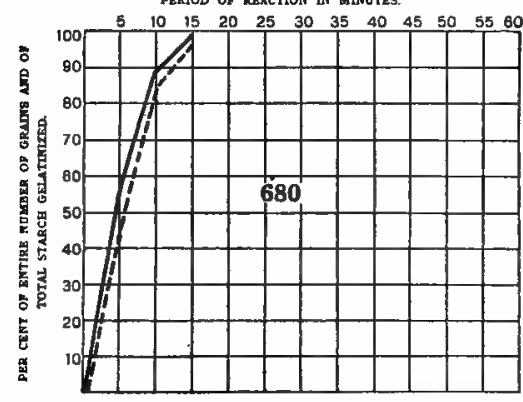

PESTOD ON DBACTION M MINUTES.

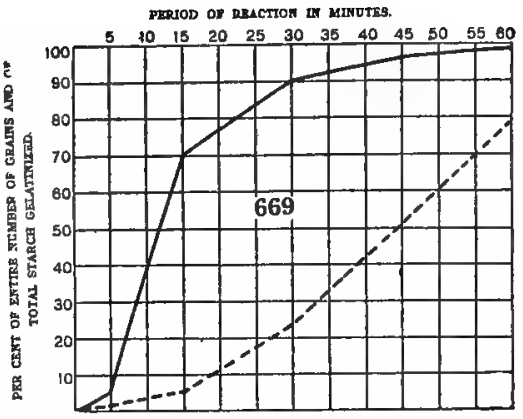

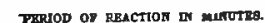

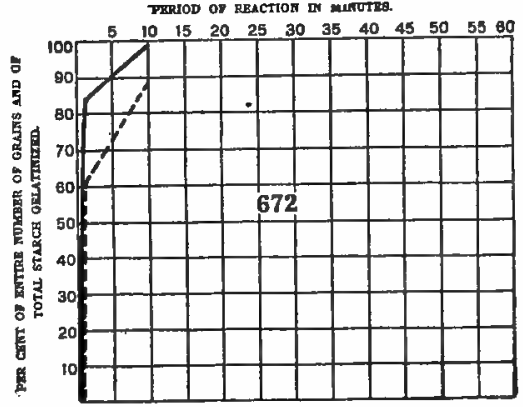

PERTOD OP RRACTION IN MENTTES

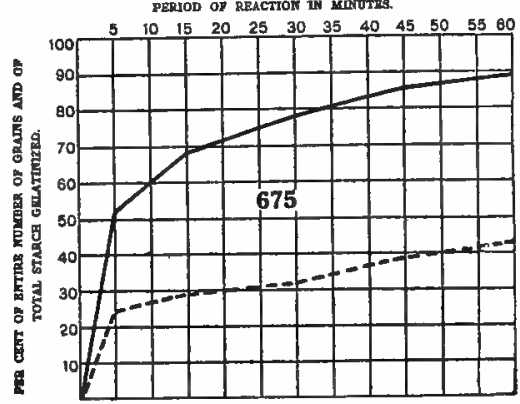

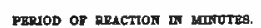

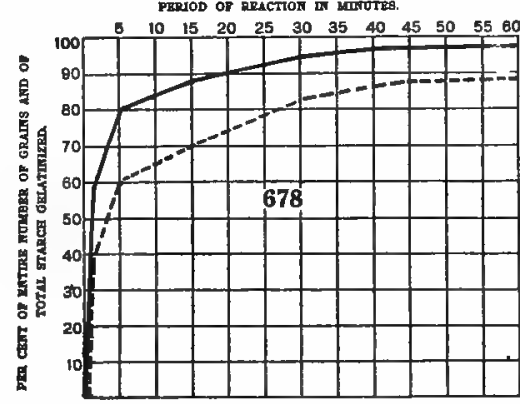

PERTOD OR PRACTOO IT MUNUTBS.

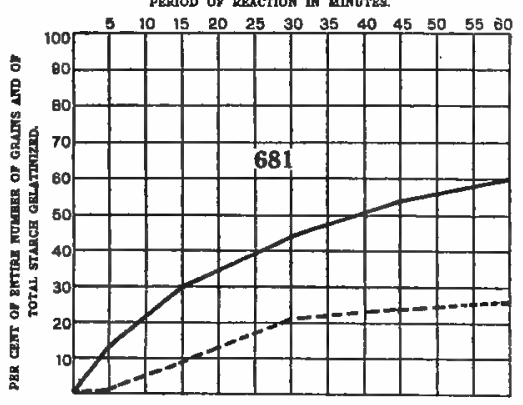

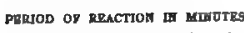

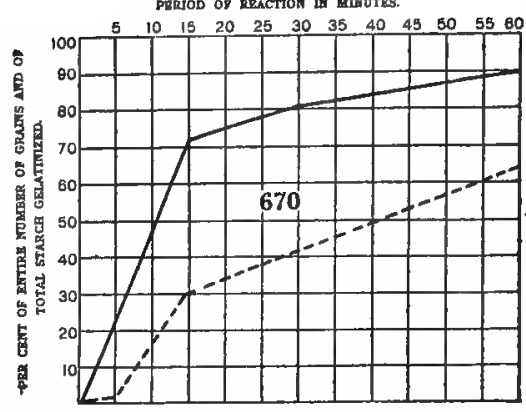

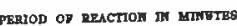

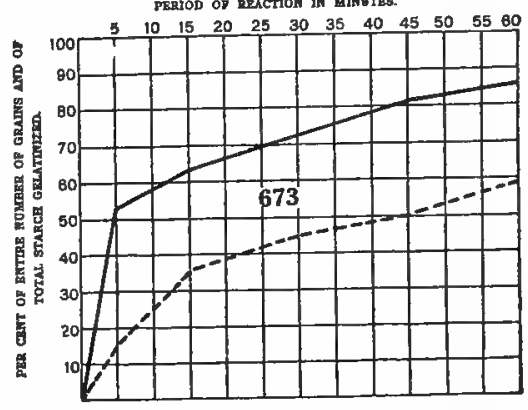

PRRIOD OE RRACTIOA IN MLINOTES.

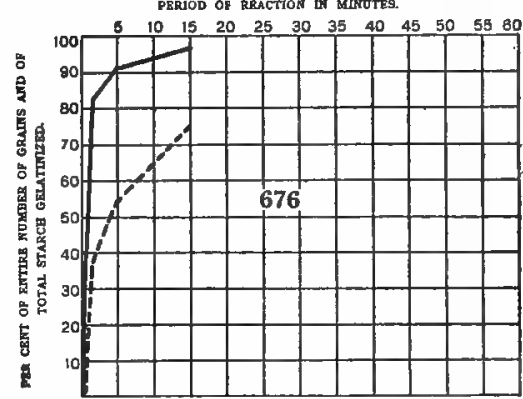

PERTOD OF REACTION DN MTNOTES.

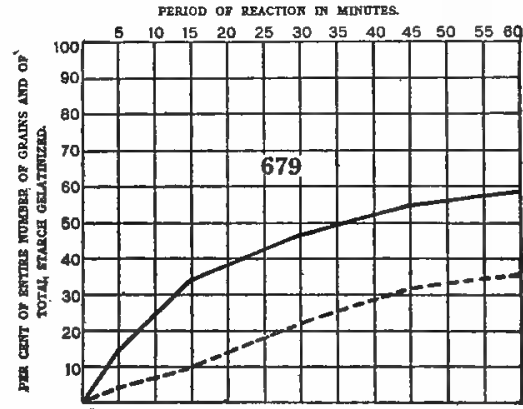

PRDIOD OF REAC

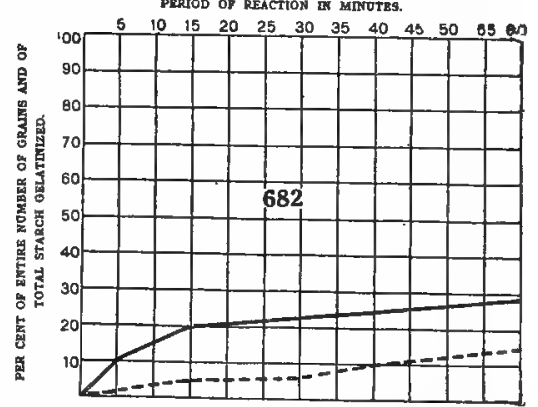

ChaRTS 668 to D 682.-Velocity-Reactions of Starch of Iris iberica with various reagents, showing the percentage of the entire number of grains (-..-) and of the total starch (- gelatinized. 668. With Chloral Hydrate. 669. With Chromic Acid.

670. With Pyrogallic Acid.

671. With Nitric Acid.
672. With Sulphuric Acid. 673. With Hydrochloric Acid. 674. With Potassium Hydroxide. 675. With Potassium Iodide. 676. With Potassium Sulphocyanate 677. With Potassium Sulphide.
678. With Sodium Hydroxide.

679. With Sodium Sulphide.

680. With Sodium Salicylate.

682. With Calcium Nitrate. 


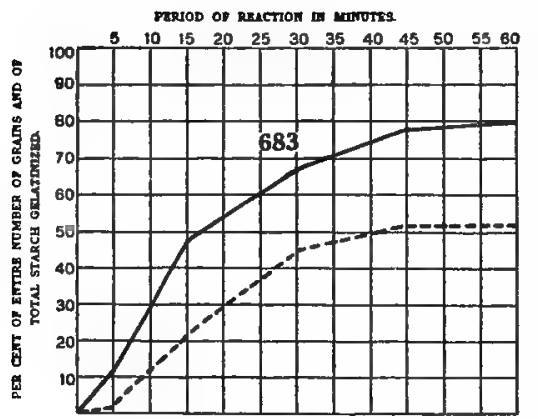

PERTOD OP RRACrios LN MANUTBS.

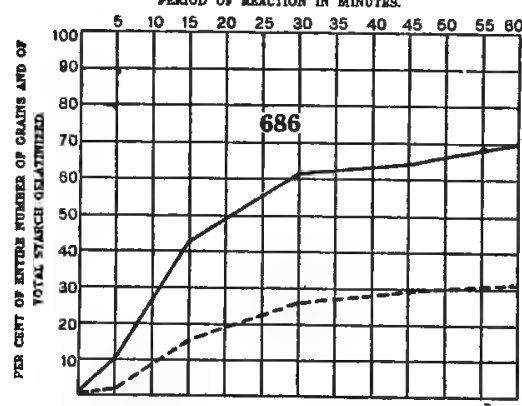

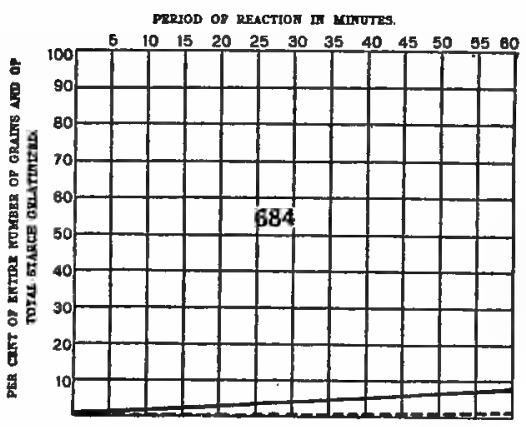

Prerod op penction ux merotres.

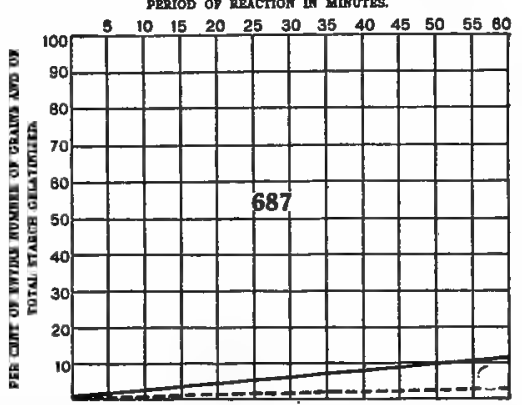

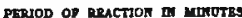

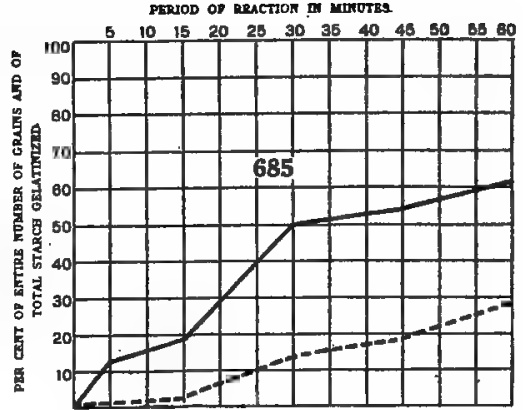

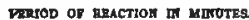

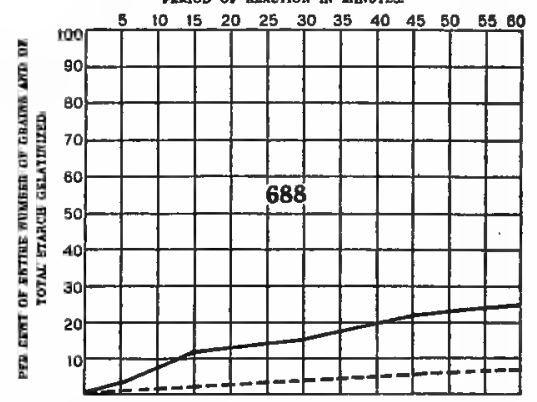

Charts D 683 to D 688.-Velocity-Reactions of Starch of Iris iberica with various reagents, showing the percentage of entire number of grains (-..-.), and total starch 683. With Strontium Nitrate. 684. With Cobalt Nitrate.

685. With Copper Nitrate. 686. With Cupric Chloride.
-) gelatinized.

687. With Barium Chloride.

688. With Mercuric Chloride.
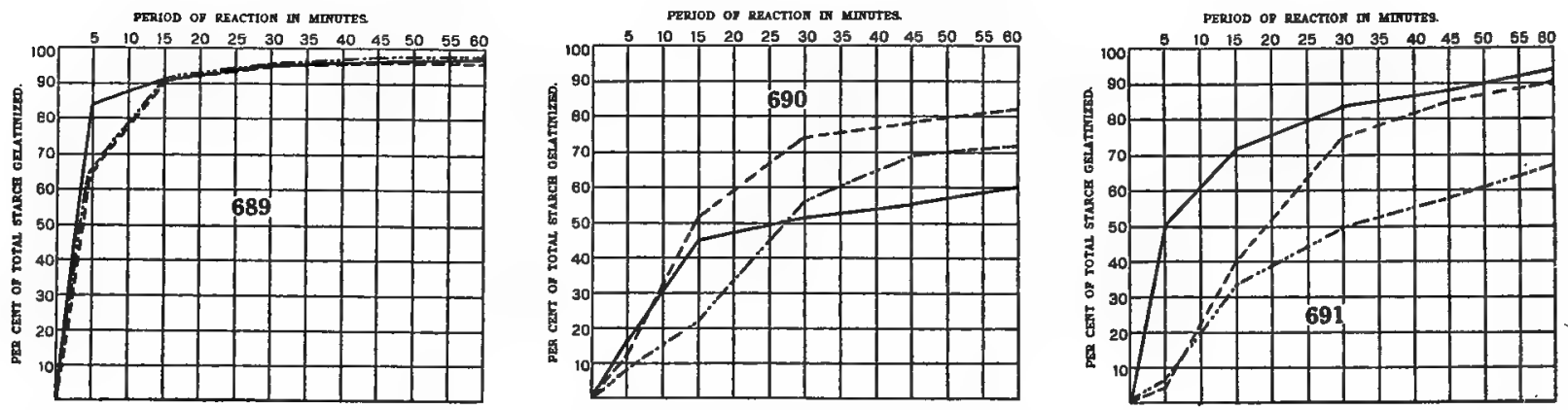

Charts D 689 to D 691.-Velocity-Reactions of Starches of Amaryllis belladonna (-----), Phaius grandifolius (-.....), and Miltonia vexillaria (_-). 


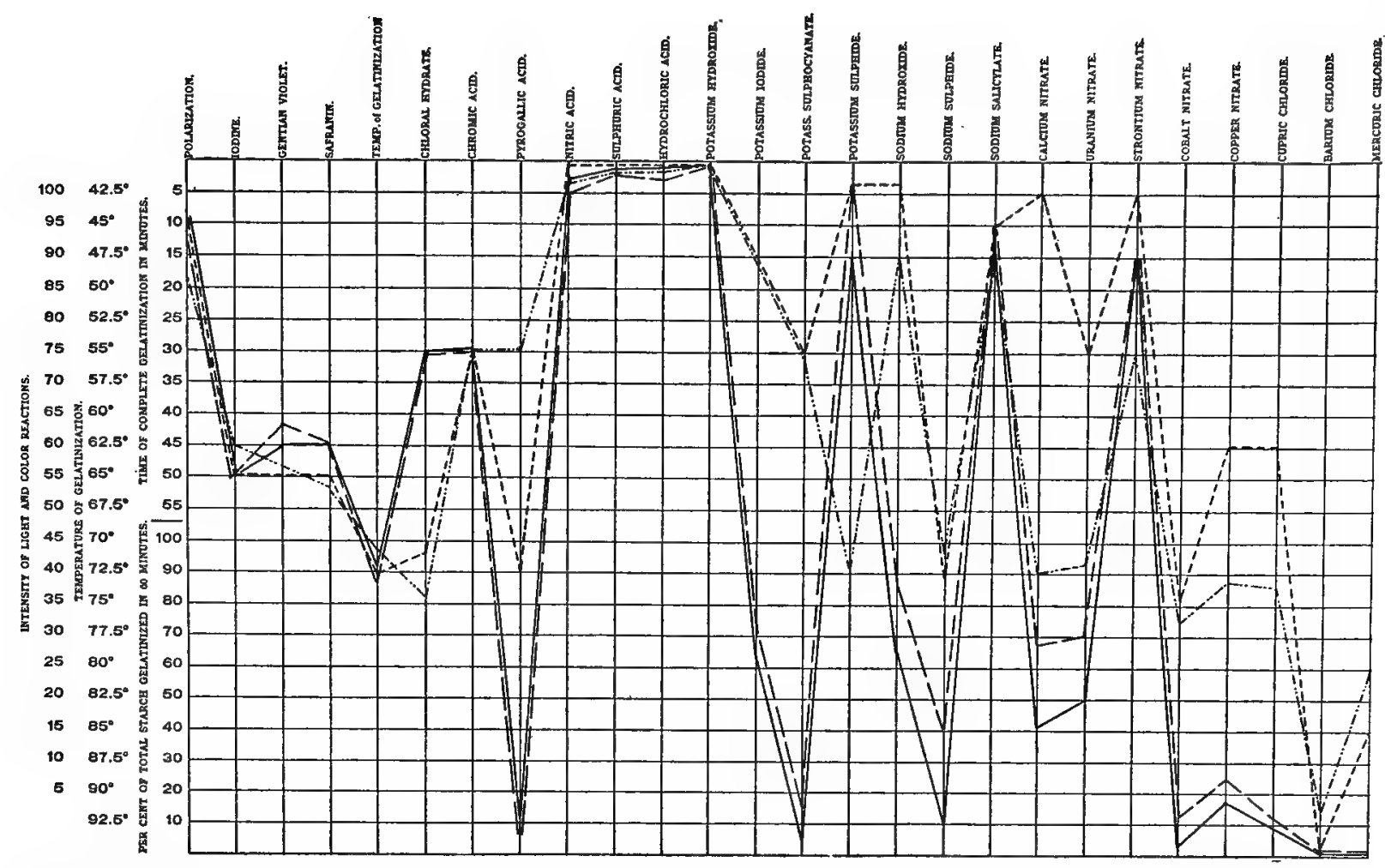

Chart E 1.-Composite Curves of the Starches of Amaryllis belladonna (-..--), Brunsvigia josephince (-.....-), Brunsdonna sanderce alba (-), and Brunsdonna sanderce (- - -

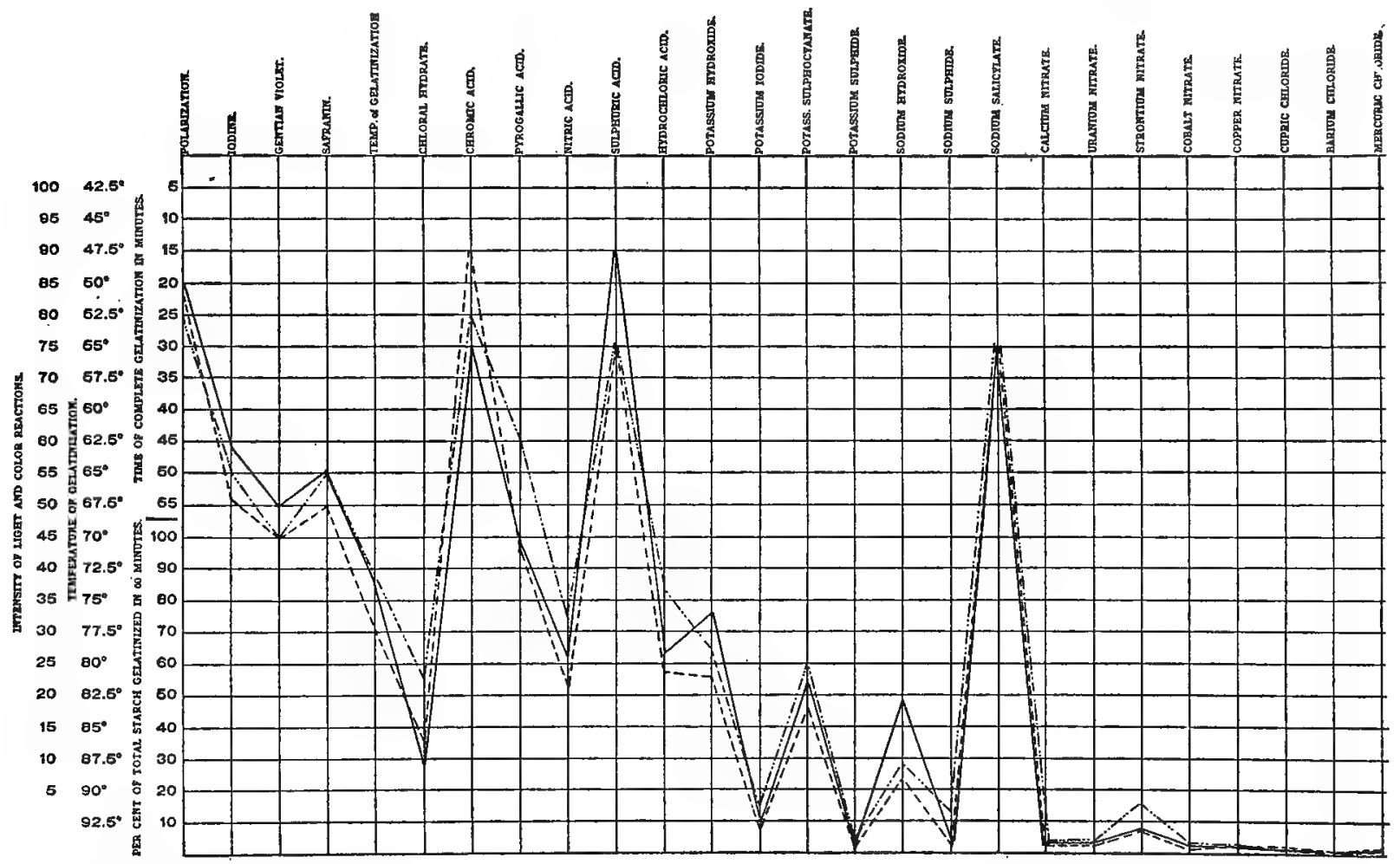

CHART E 2.-Composite Curves of the Starches of Hippeastrum titan (-..-), Hippeastrum cleonia (-.....-), and Hippeastrum titan-cleonia (-). 


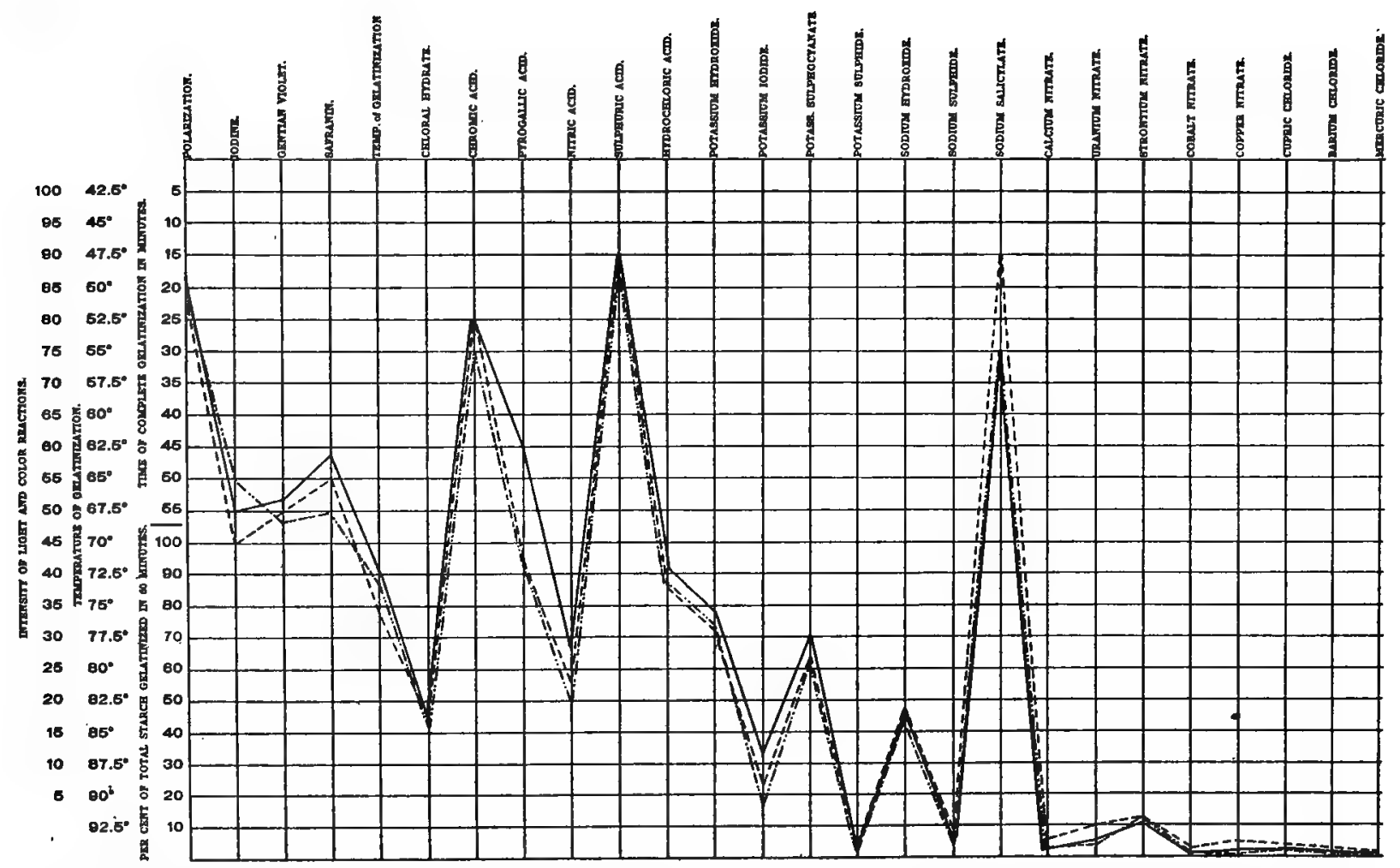

Chart E 3.-Composite Curves of the Starches of Hippeastrum ossultan (--..-), Hippeastrum pyrrha (-......), and Hippeastrum ossultan-pyrrha (-).

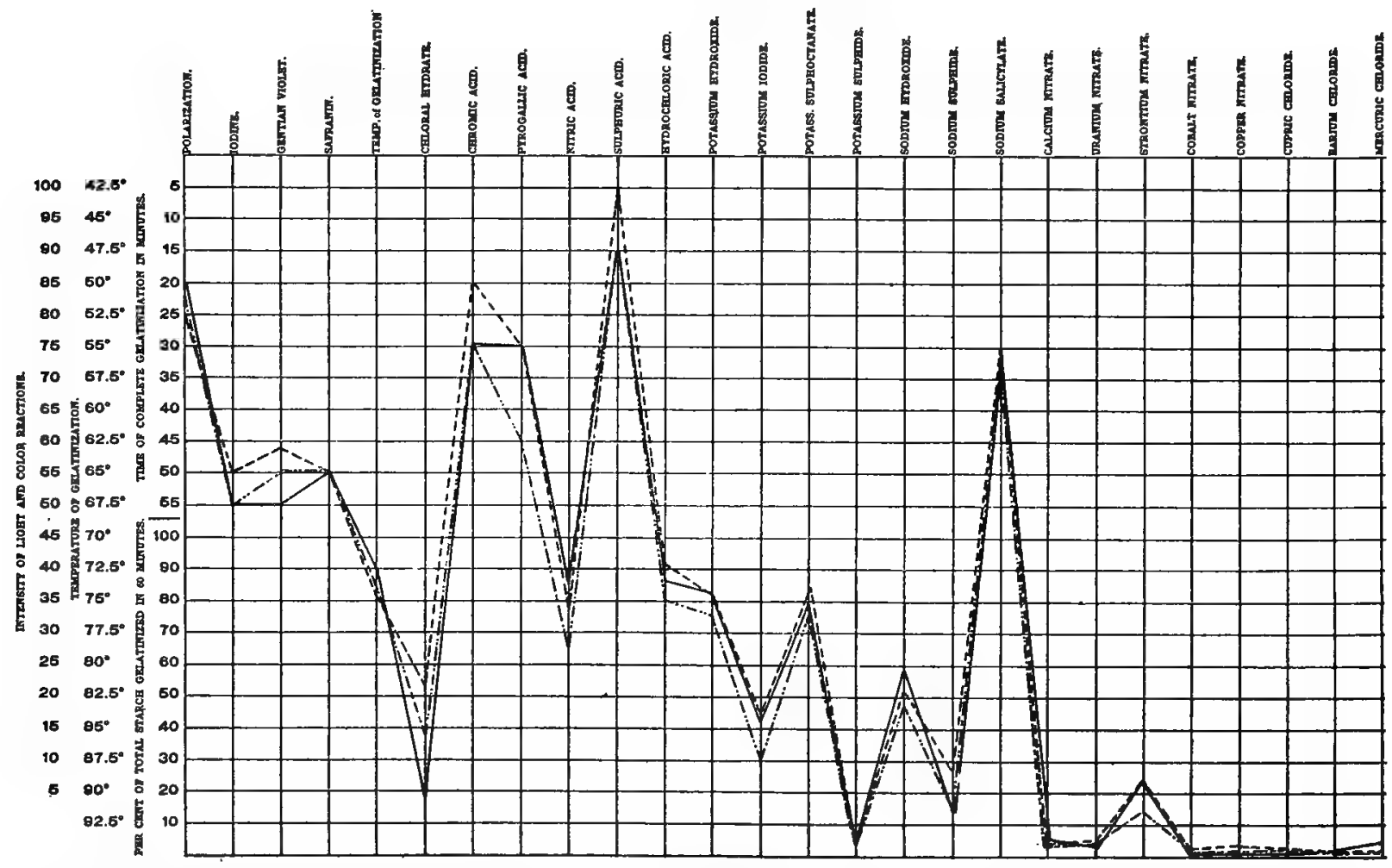

Chart E 4.-Composite Curves of the Starches of Hippeastrum doeones (-...), Hippeastrum zephyr (-.....), and Hippeastrum doones-zephyr (-). 


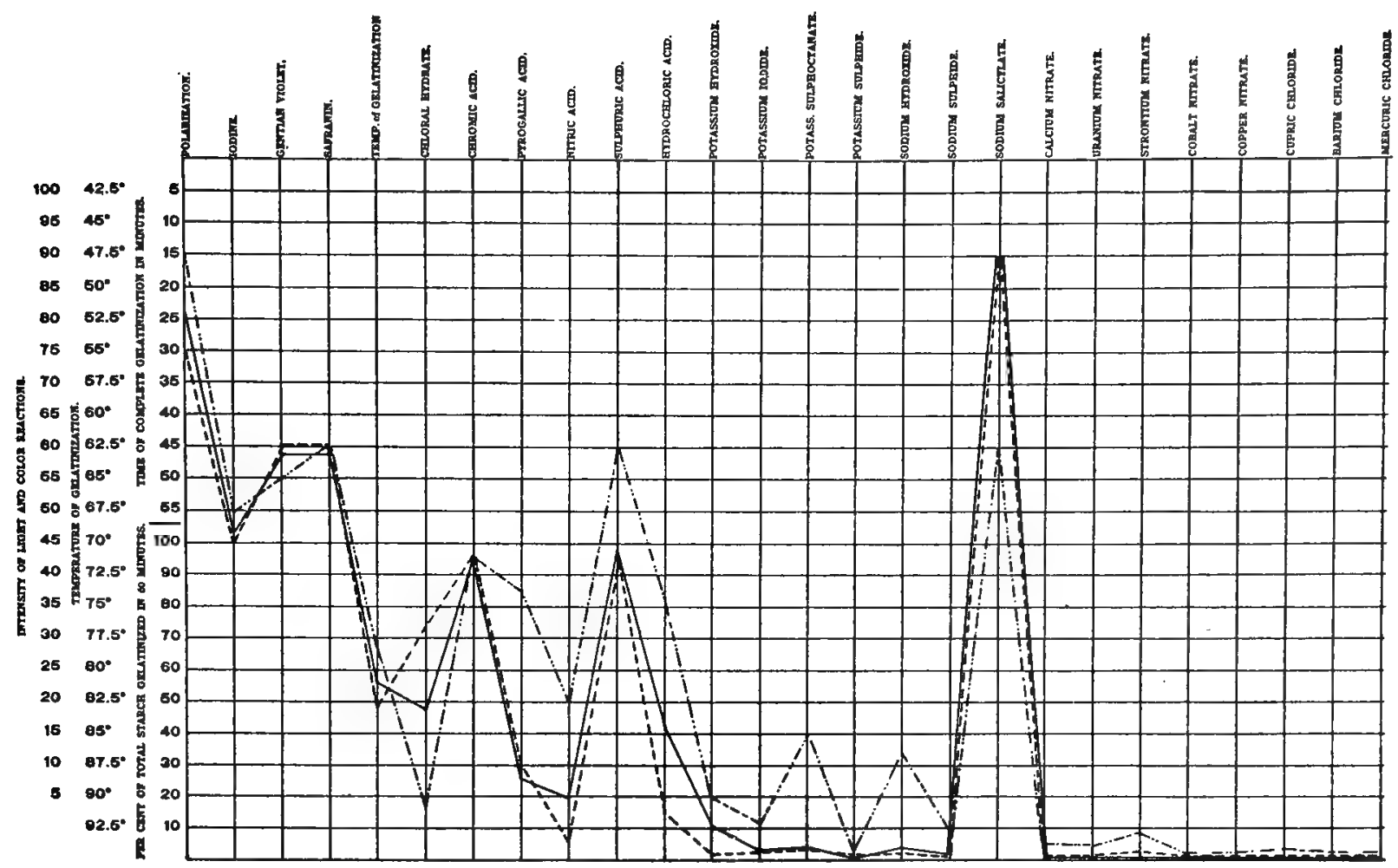

Chart E 5.-Composite Curves of the Starches of Homanthus katherina (.....), Homanthus magnificus (-......), and Homanthus andromeda (-).

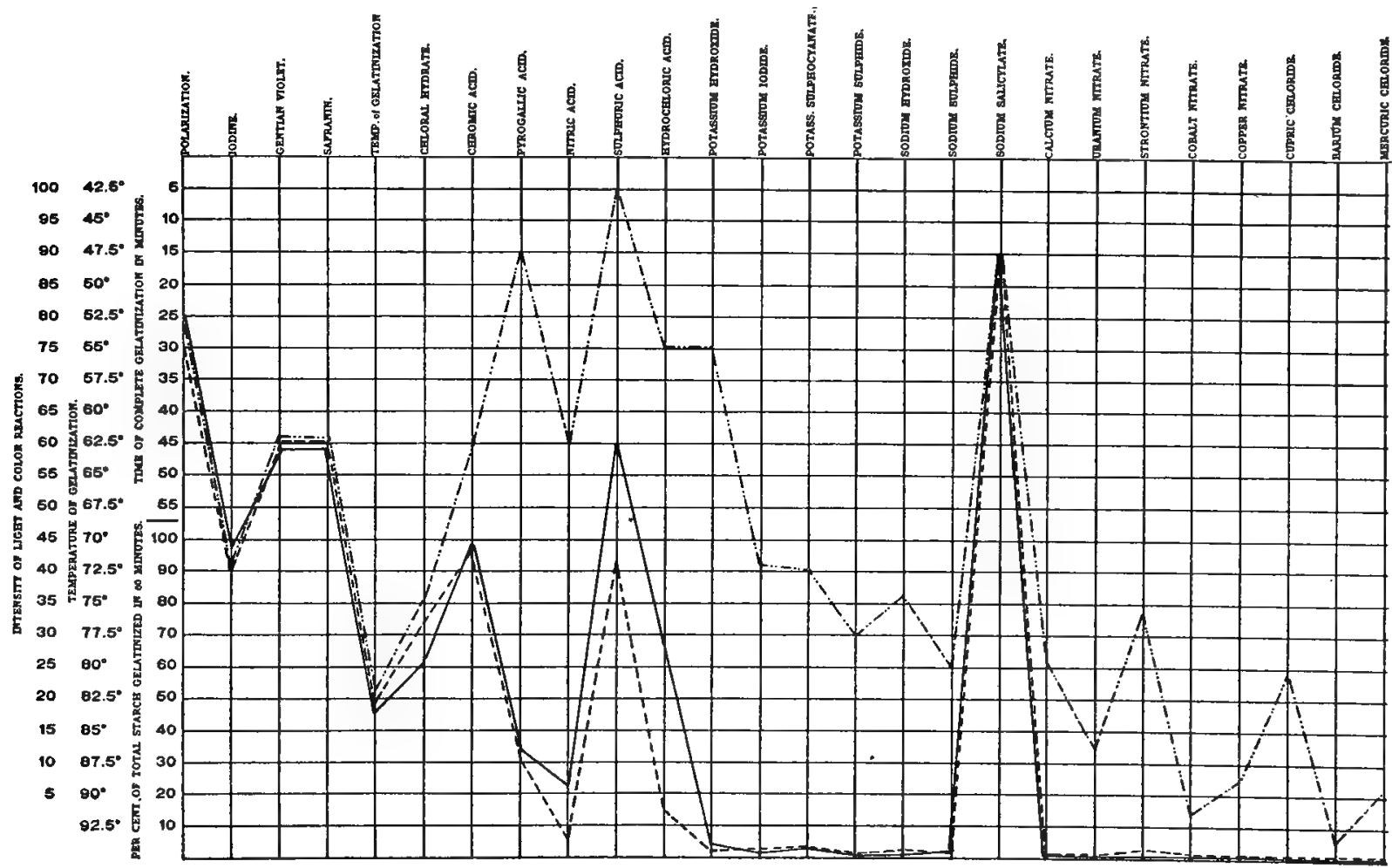

Chart E 6.-Composite Curves of the Starches of Homanthus katherinae (-..--), Hamanthus puniceus (-.....-), and Homanthus könig albert (-). 
266

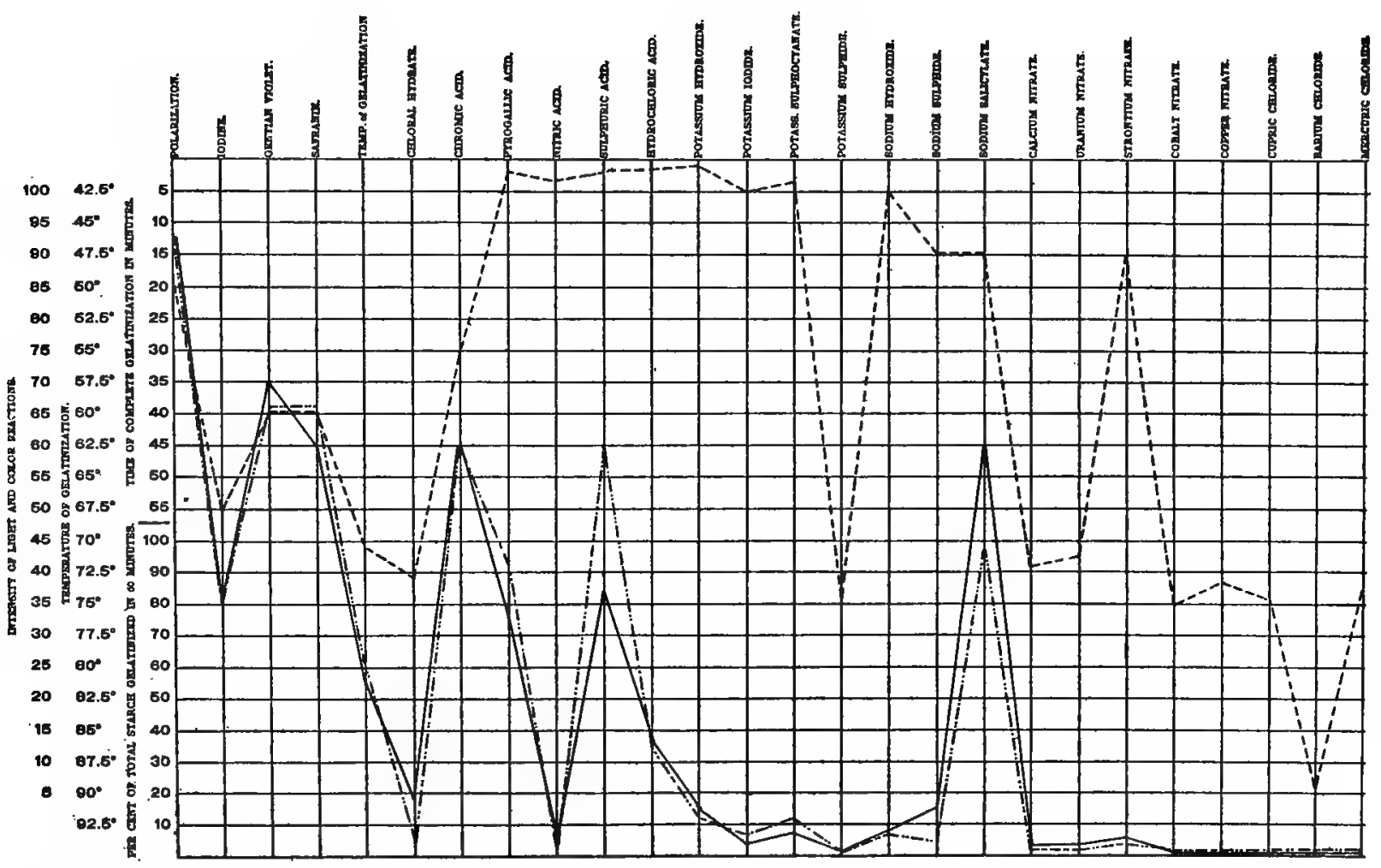

Chart E 7.-Composite Curves of the Starches of Crinum moorei (_...-), Crinum zeylanicum (_......), and Crinum hybridum j.c.h. (-).

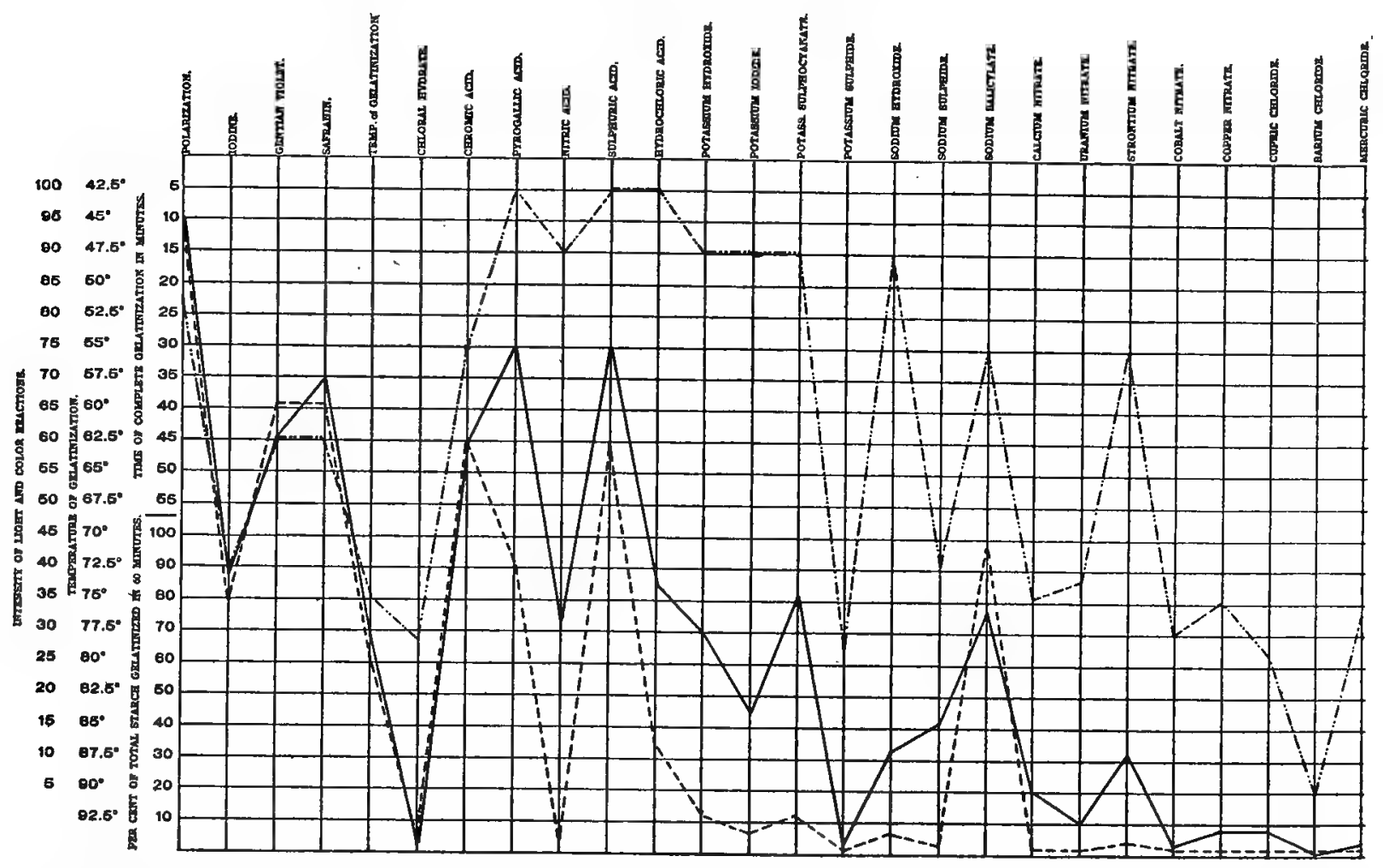

Chart E 8.-Composite Curves of the Starches of Crinum zeylanicum (....), Crinum longifolium (-.....), and Crinum kircape (-). 


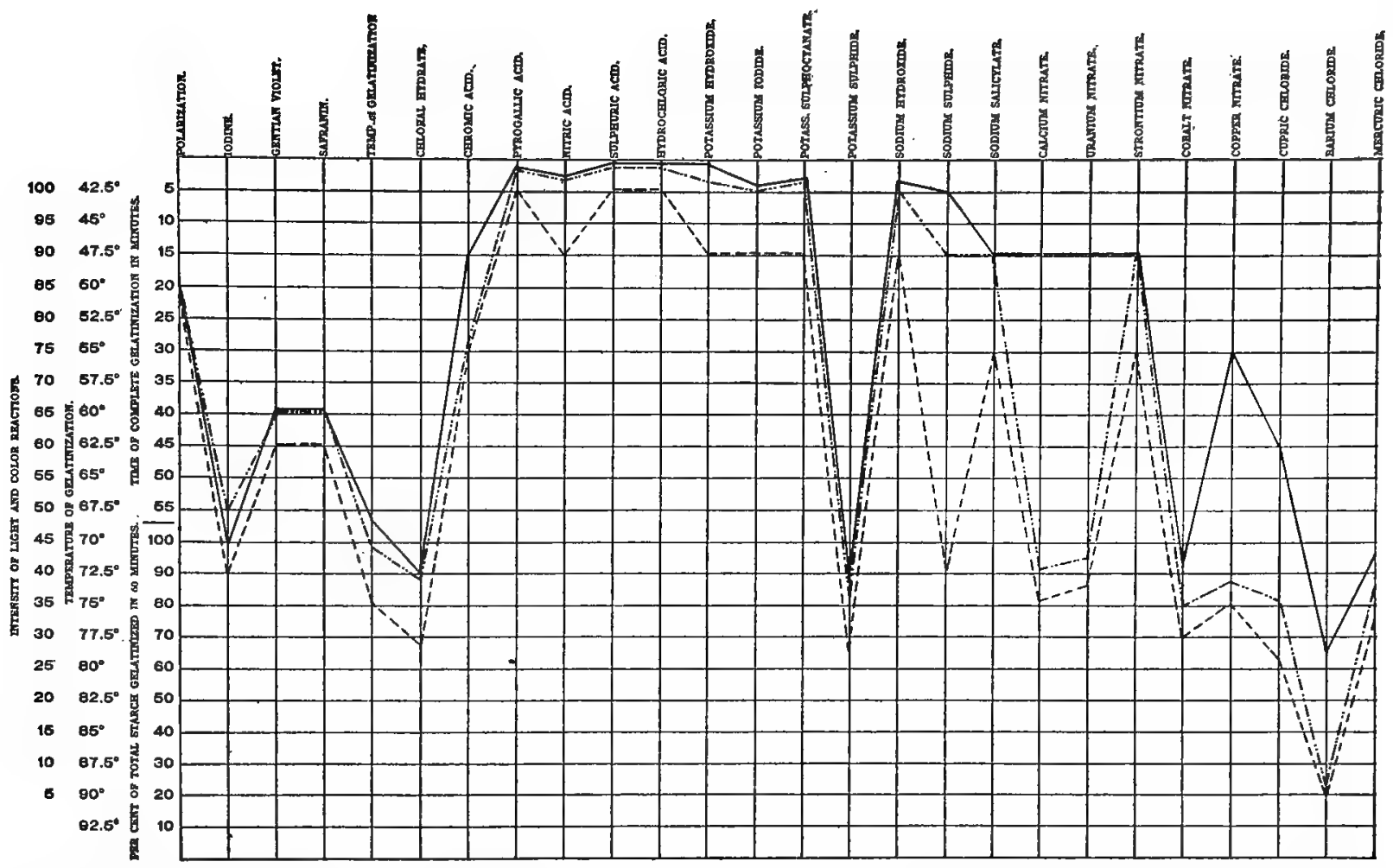

Chart E 9.-Composite Curves of the Starches of Crinum longifolium (...-), Crinum moorei (_......), and Crinum powellii (-).

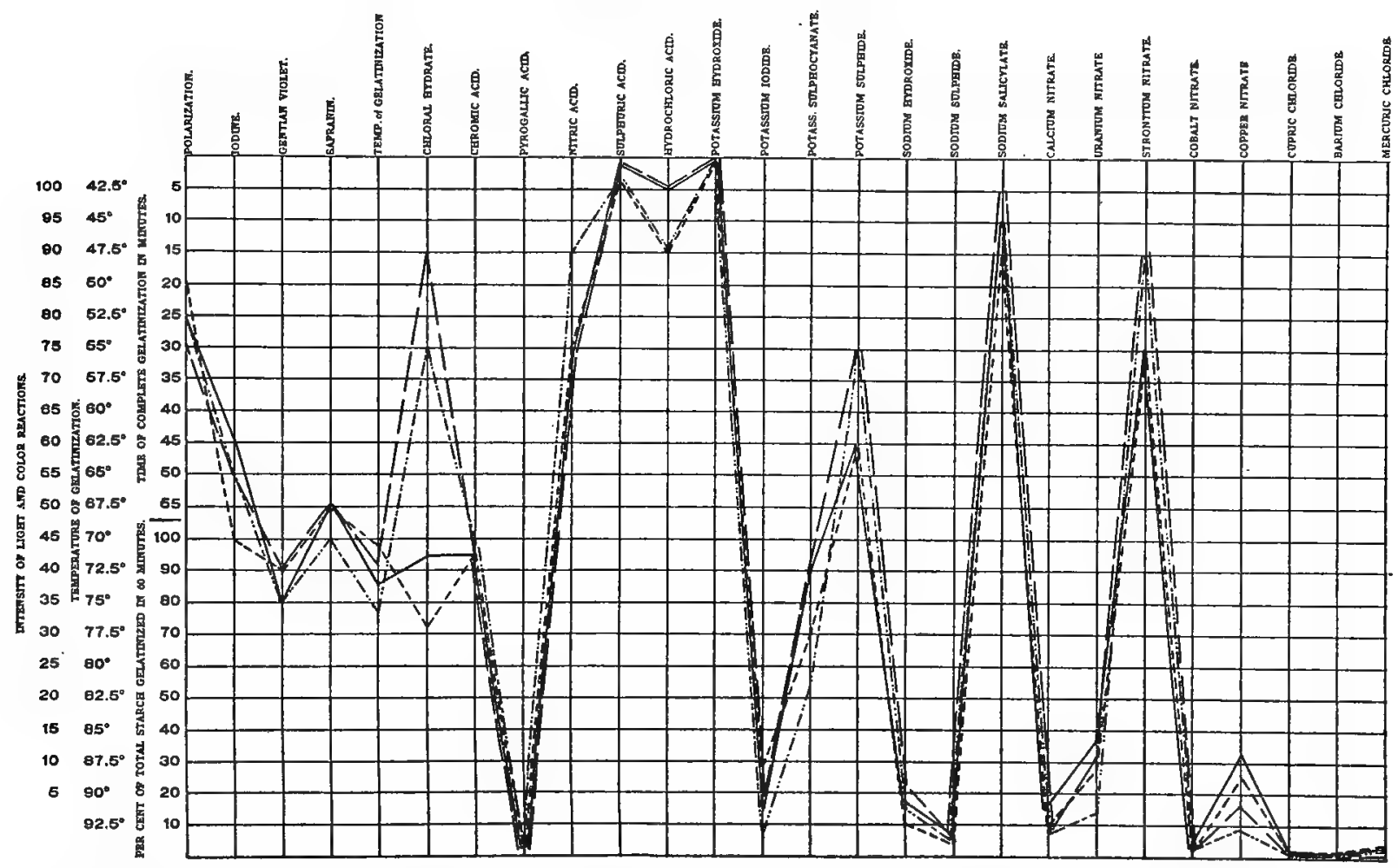

Chart E 10.-Composite Curves of the Starches of Nerine crispa (-...-), Nerine elegans (.......), Nerine dainty maid (-), and Nerine queen of roses (- - - 


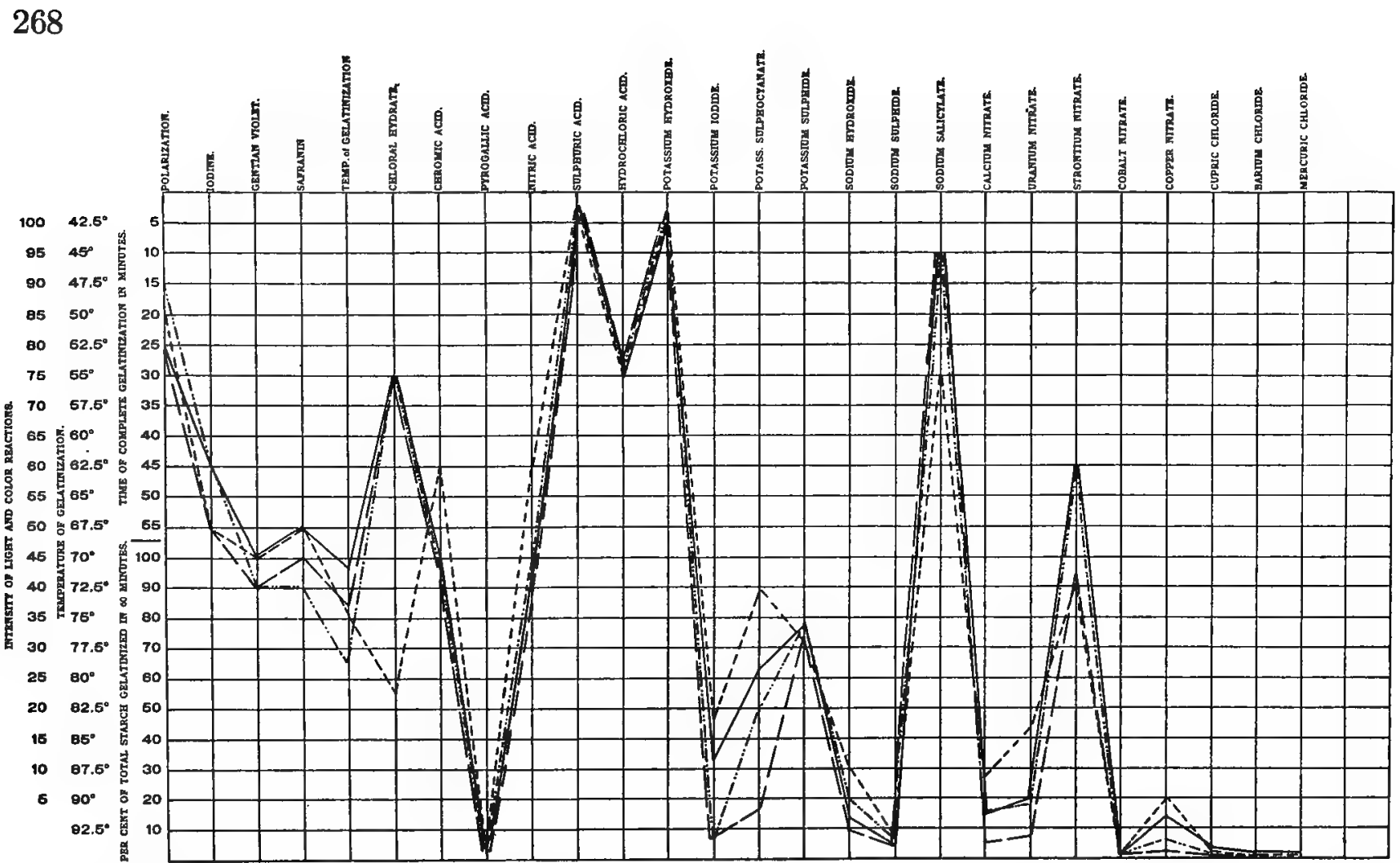

Chart E 11.-Composite Curves of the Starches of Nerine bowdeni (-----), Nerine sarniensis var. corusca major ( -.....), Nerine giantess (-), and Nerine abundance (- $\longrightarrow$ ).

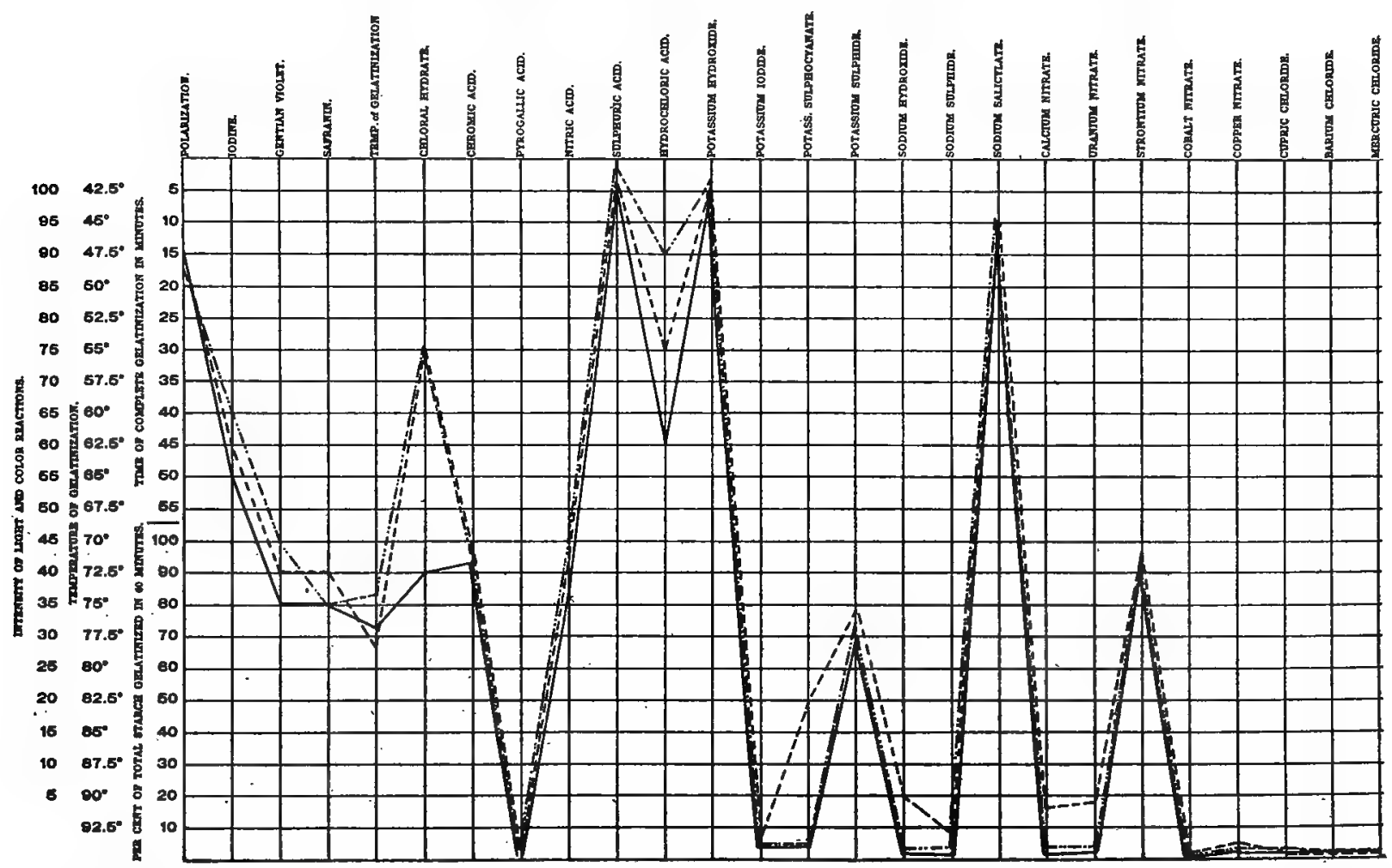

Chart E 12.-Composite Curves of the Starches of Nerine sarniensis var. corusca major (-----), Nerine curviflora var. fothergilii major (......-), and Nerine glory of sarnia (- - ). 


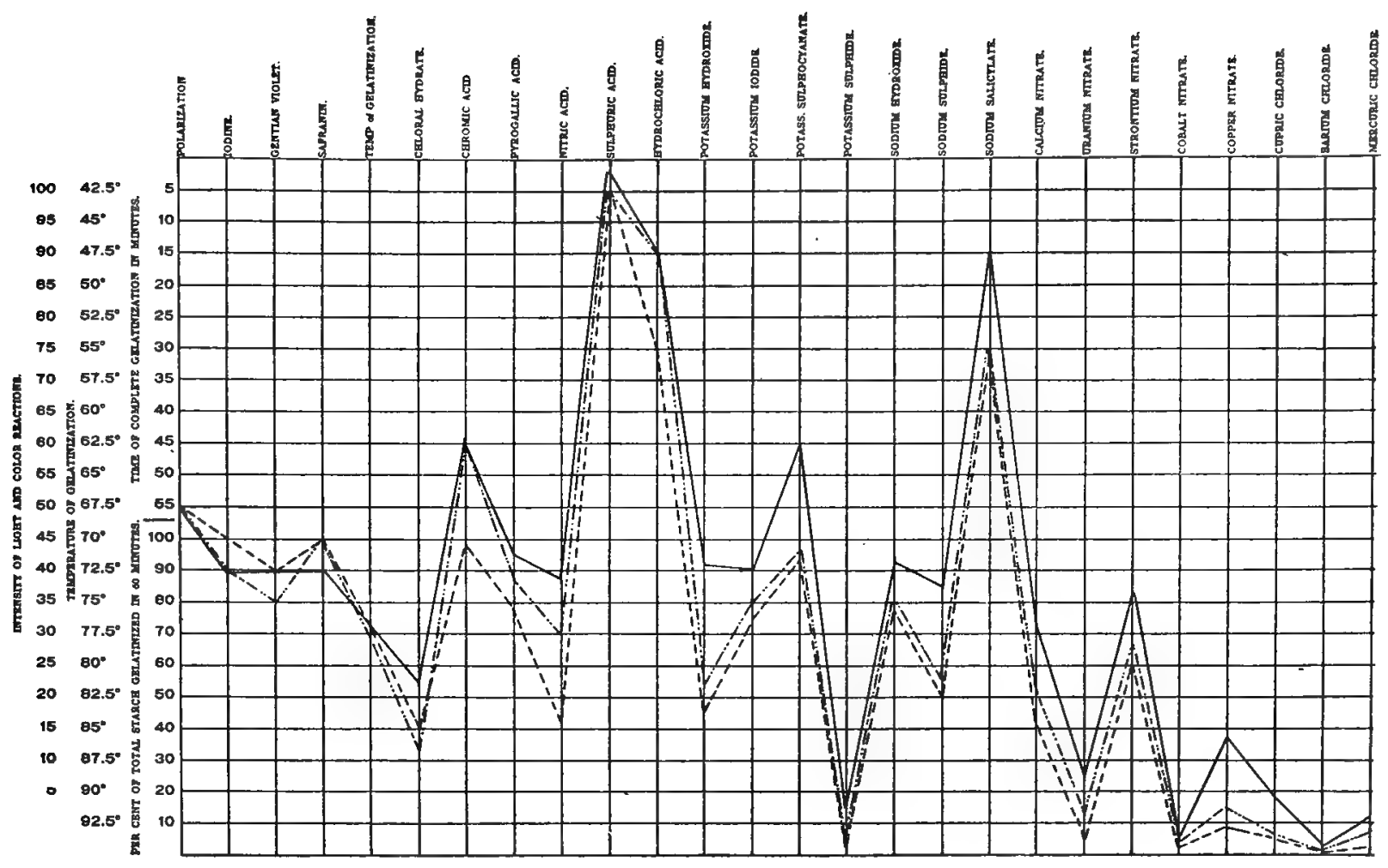

Chart E 14.-Composite Curves of the Starches of Narcissus tazetta grand monarque (-...-.), Narcissus poeticus ornatus ( .......), and Narcissus poetaz triumph (_- $)$.

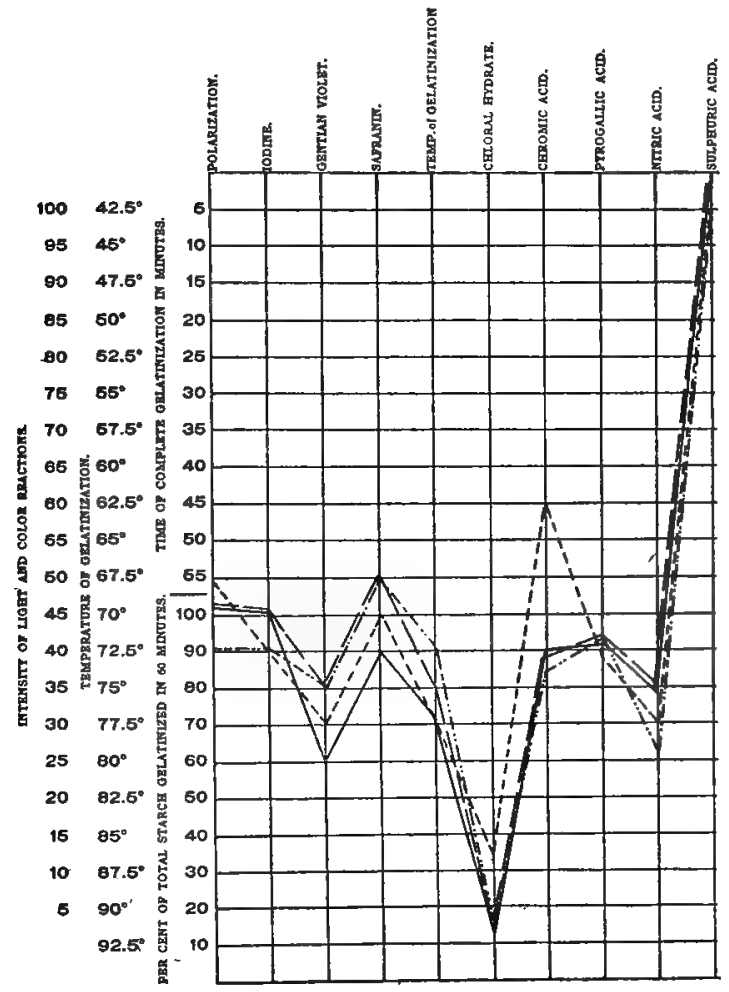

Chart E 13.-Composite Curves of the Starches of Narcissus poeticus ornatus (-...-), Narcissus poeticus poetarum ( .......), Narcissus poeticus herrick (- - $)$, and Narcissus poeticus dante (- - $)$.

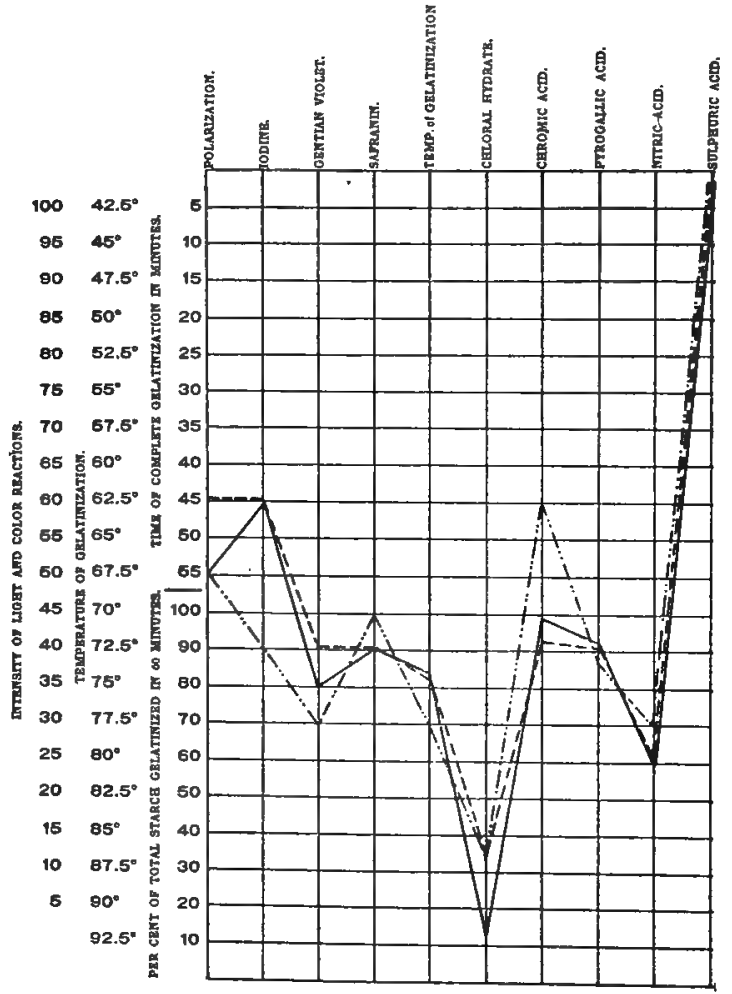

Chart E 15.-Composite Curves of the Starches of Narcissus gloria mundi (...-), Narcissus poeticus ronatus (......), and Narcissus fiery cross (- $\longrightarrow$ ). 


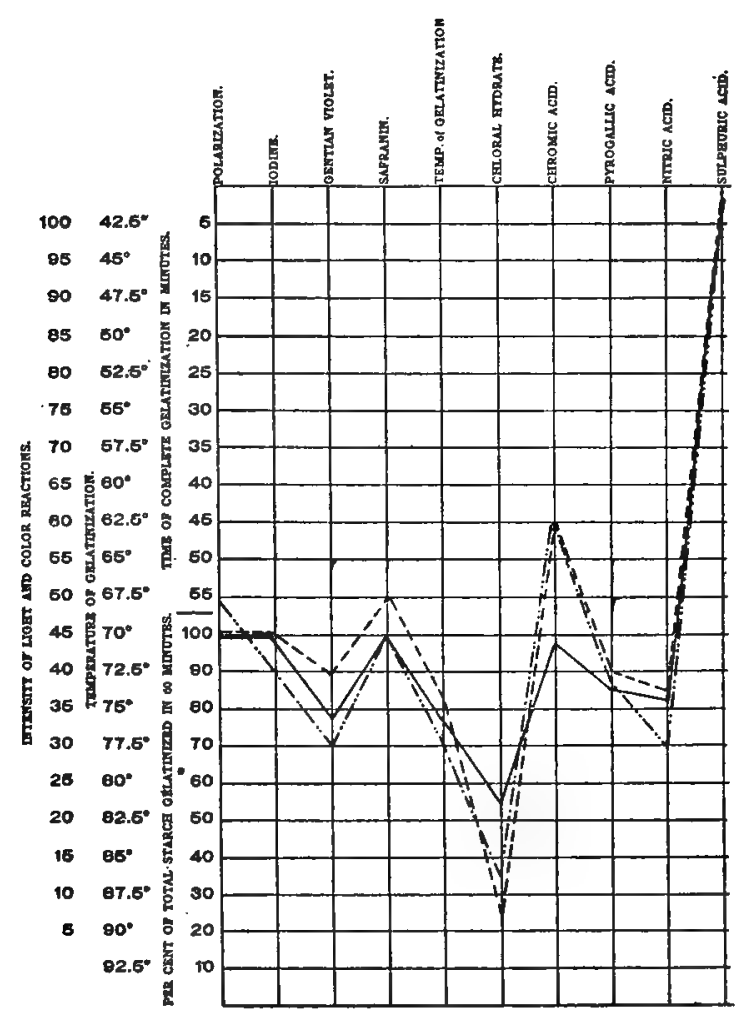

Chart E 16.-Composite Curves of the Starches of Narcissus telamonius plenus (-.--), Narcissus poeticus ornatus ( -......), and Narcissus doubloon (-).

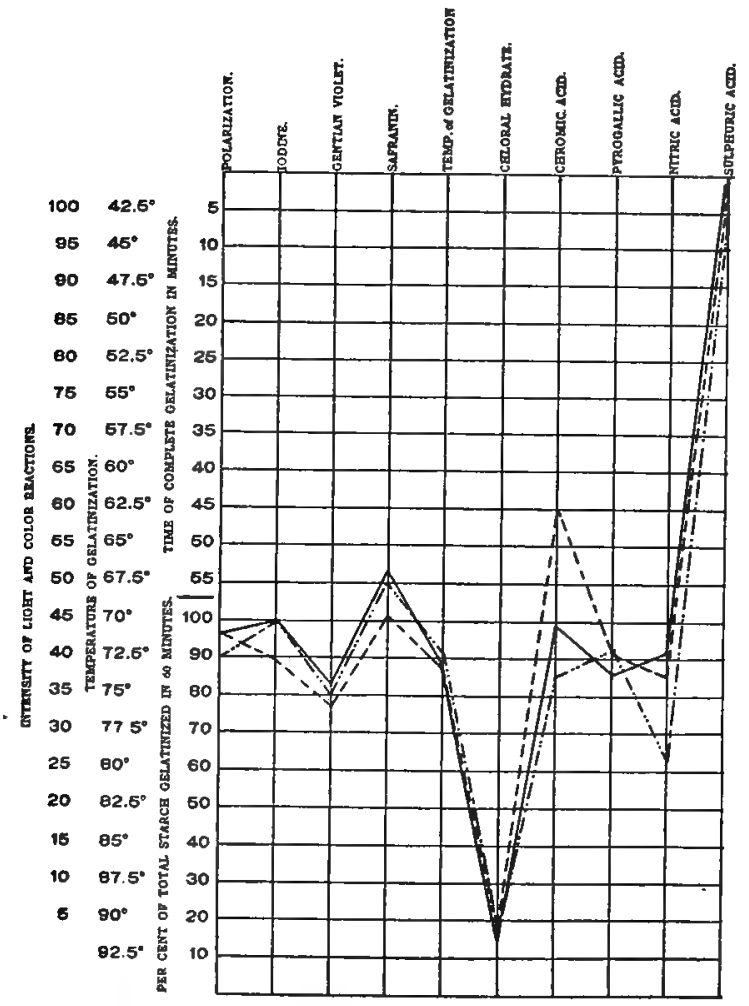

Chart E 18.-Composite Curves of the Starches of Narcissus abscissus (.....), Narcissus poeticus poetarum (.......), and Narcissus will scarlet (-).

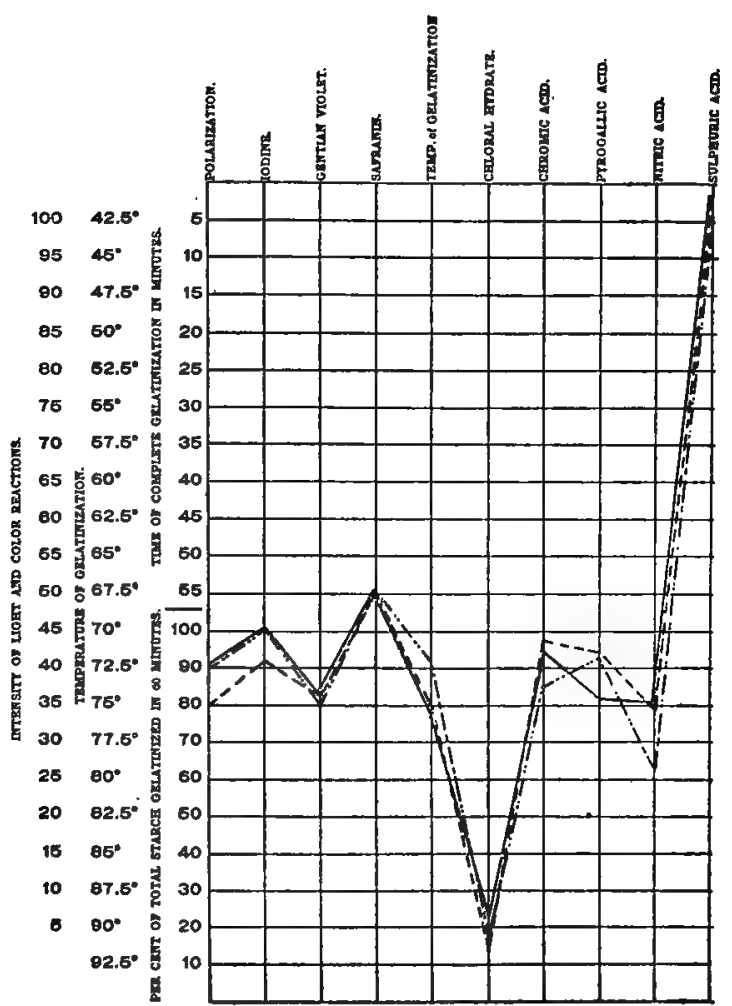

CHART E 17.-Compósite Curves of the Starches of Narcissus princess mary (--.--), Narcissus poeticus poetarum (......), and Narcissus cresset (-).

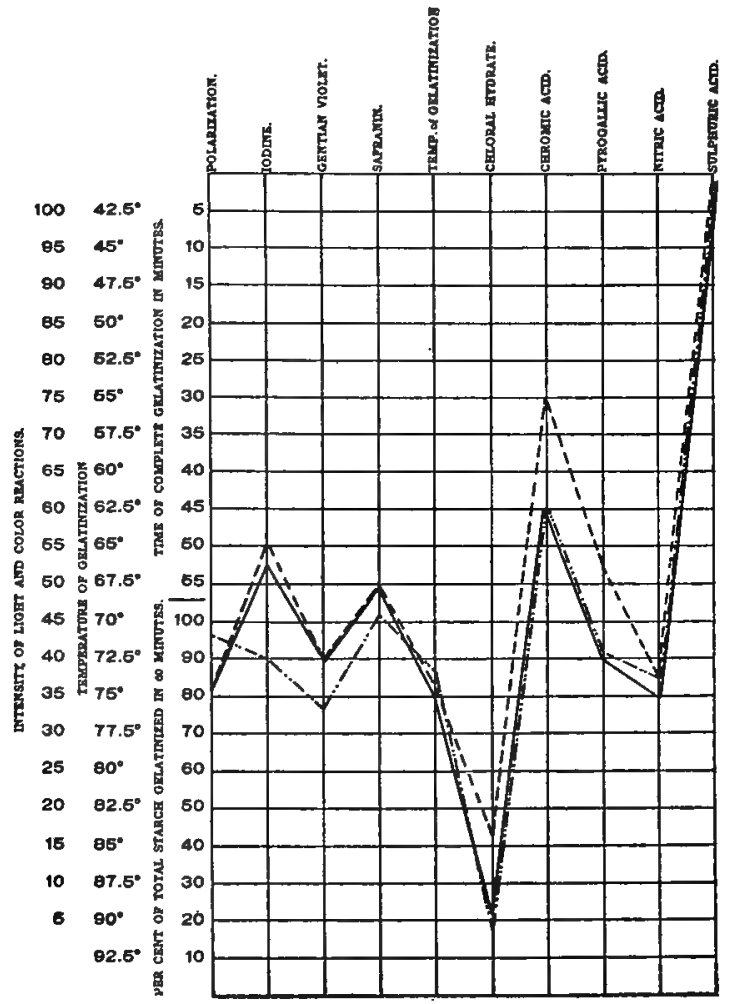

Chart E 19.-Composite Curves of the Starches of Narcissus albicans (...-) ), Narcissus abscissus (......-), and Narcissus bicolor apricot (—). 


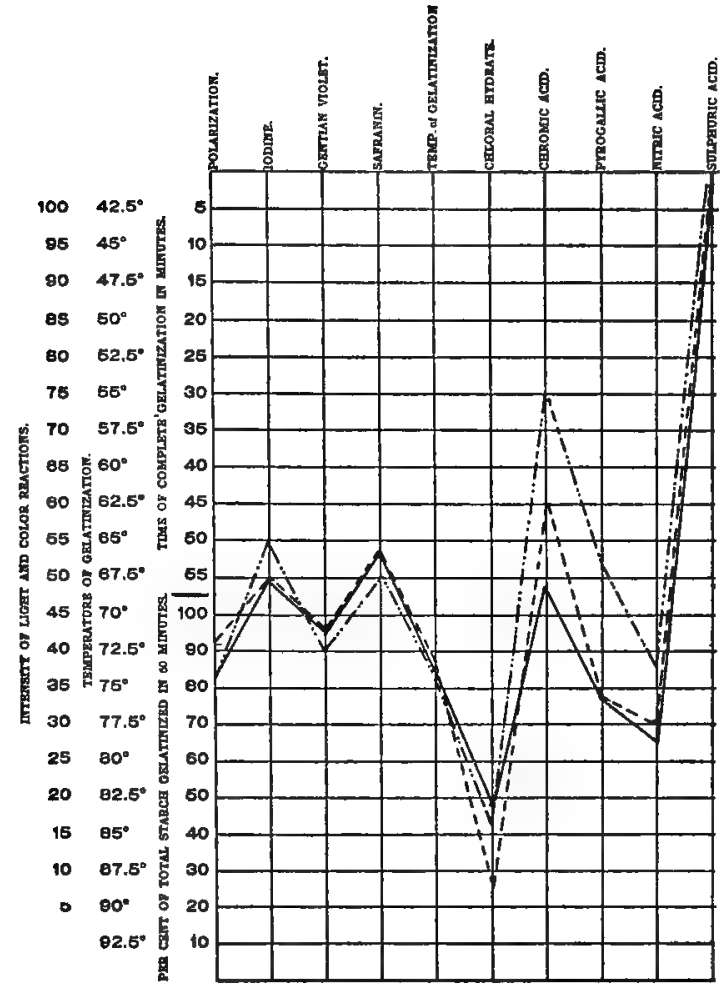

Chart E 20.-Composite Curves of the Starches of Narcissus empress (-.-.-), Narcissus albicans (.......), and Narcissus madame de graaff (-).

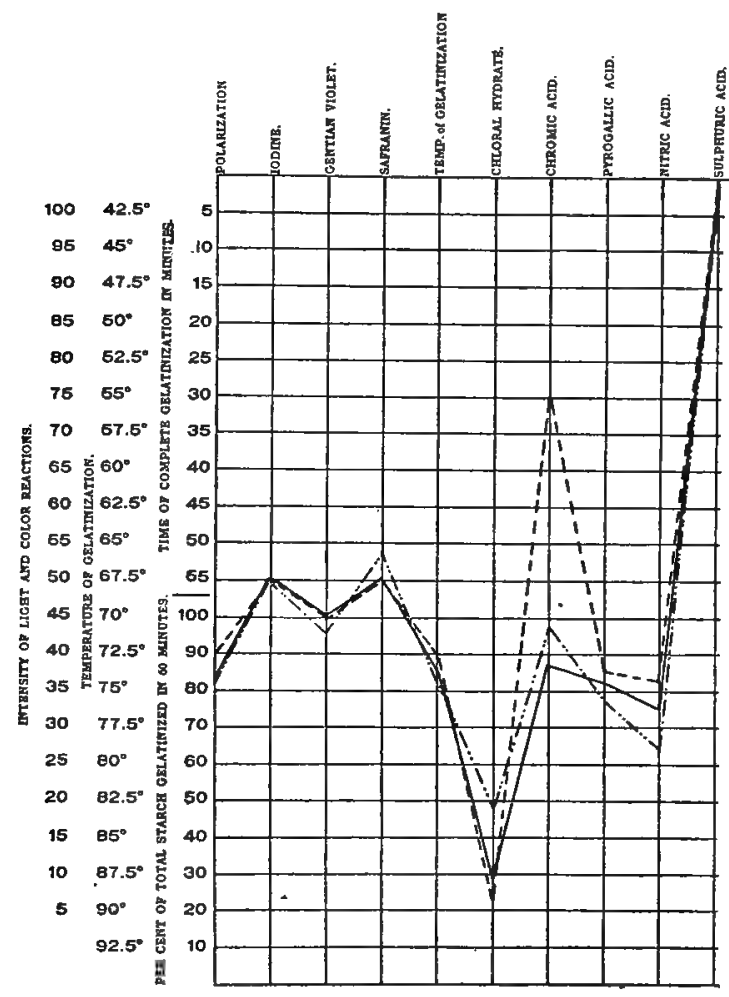

Chart E 22.-Composite Curves of the Starches of Narcissus monarch (.....), Narcissus madame de graaff (.......), and Narcissus lord roberts (-).

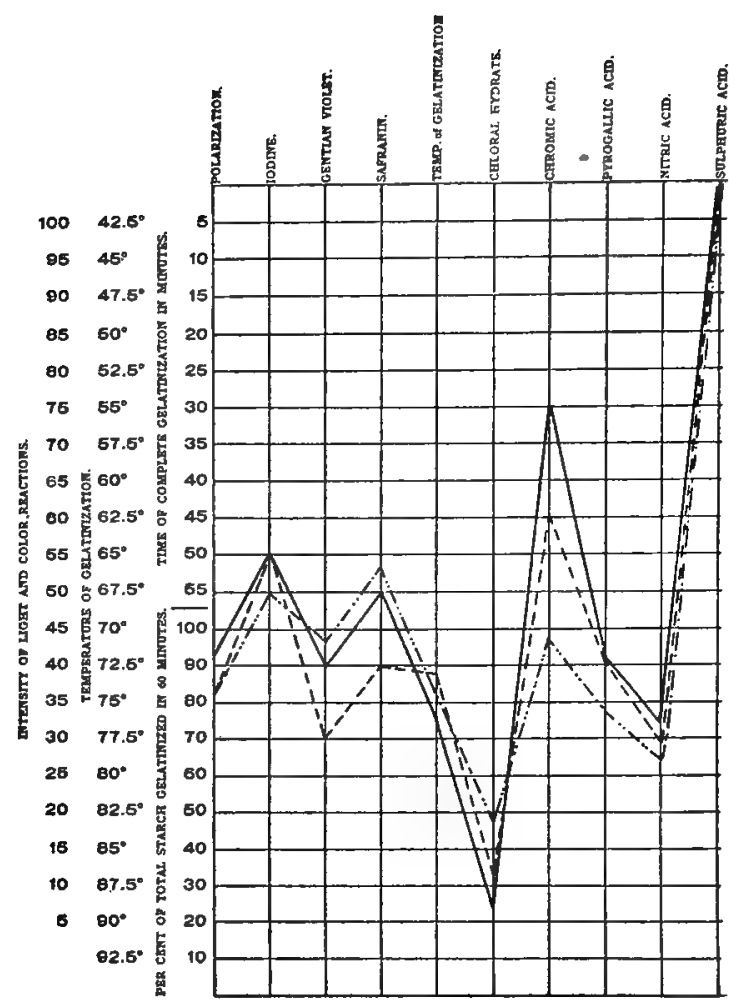

Chart E 21.-Composite Curves of the Starches of Narcissus weardale perfection (-.--), Narcissus madame de graaff (-......), and Narcissus pyramus (—).

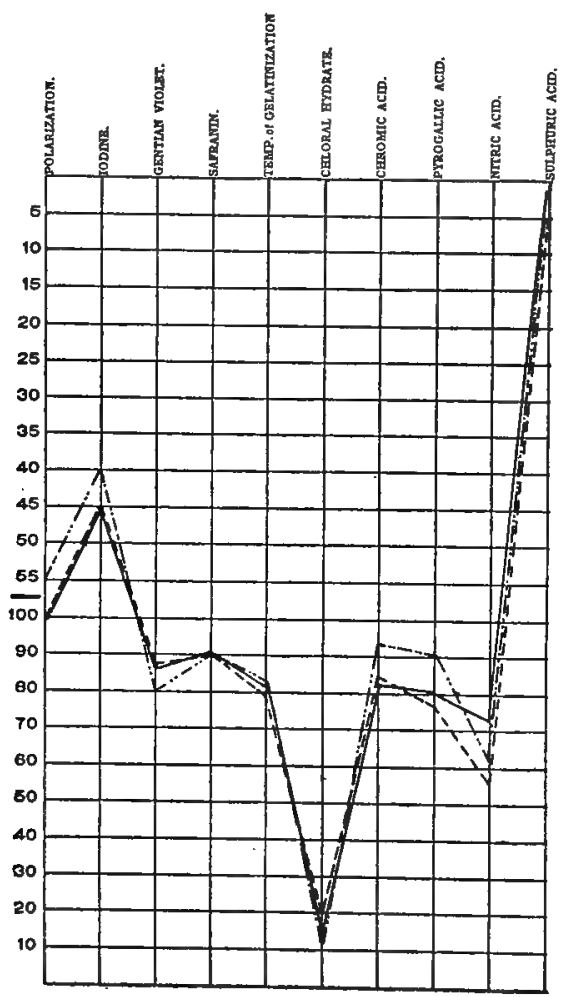

Chart E 23.-Composite Curves of the Starches of Narcissus leedsii minnie hume (...-), Narcissus triandrus albus (......), and Narcissus agnes harvey (-). 


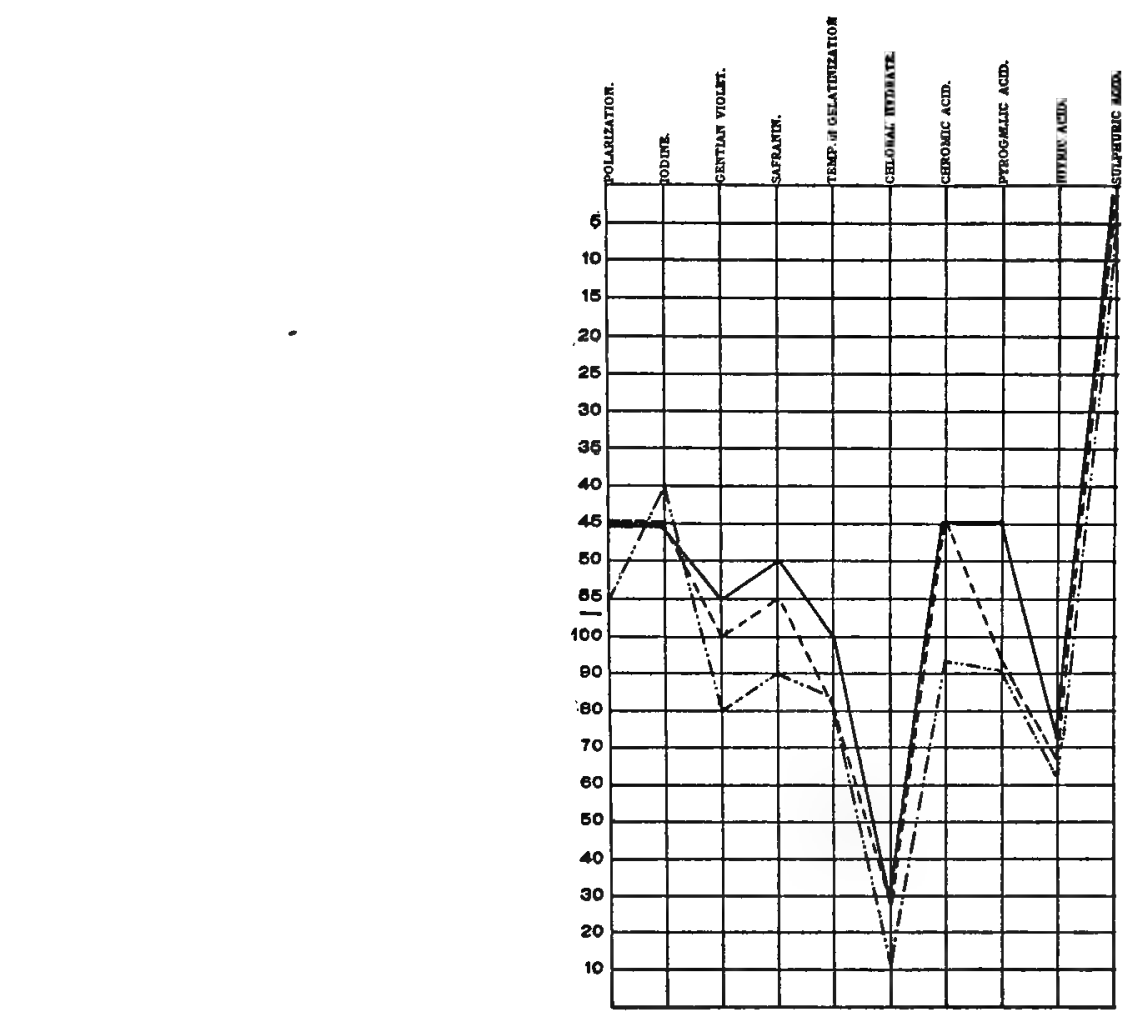

Chart E 24.-Composite Curves of the

Starches of Narcissus emperor (....-),

Narcissus triandrus albus (......-), and

Narcissus $j$. t. bennet poe (-).

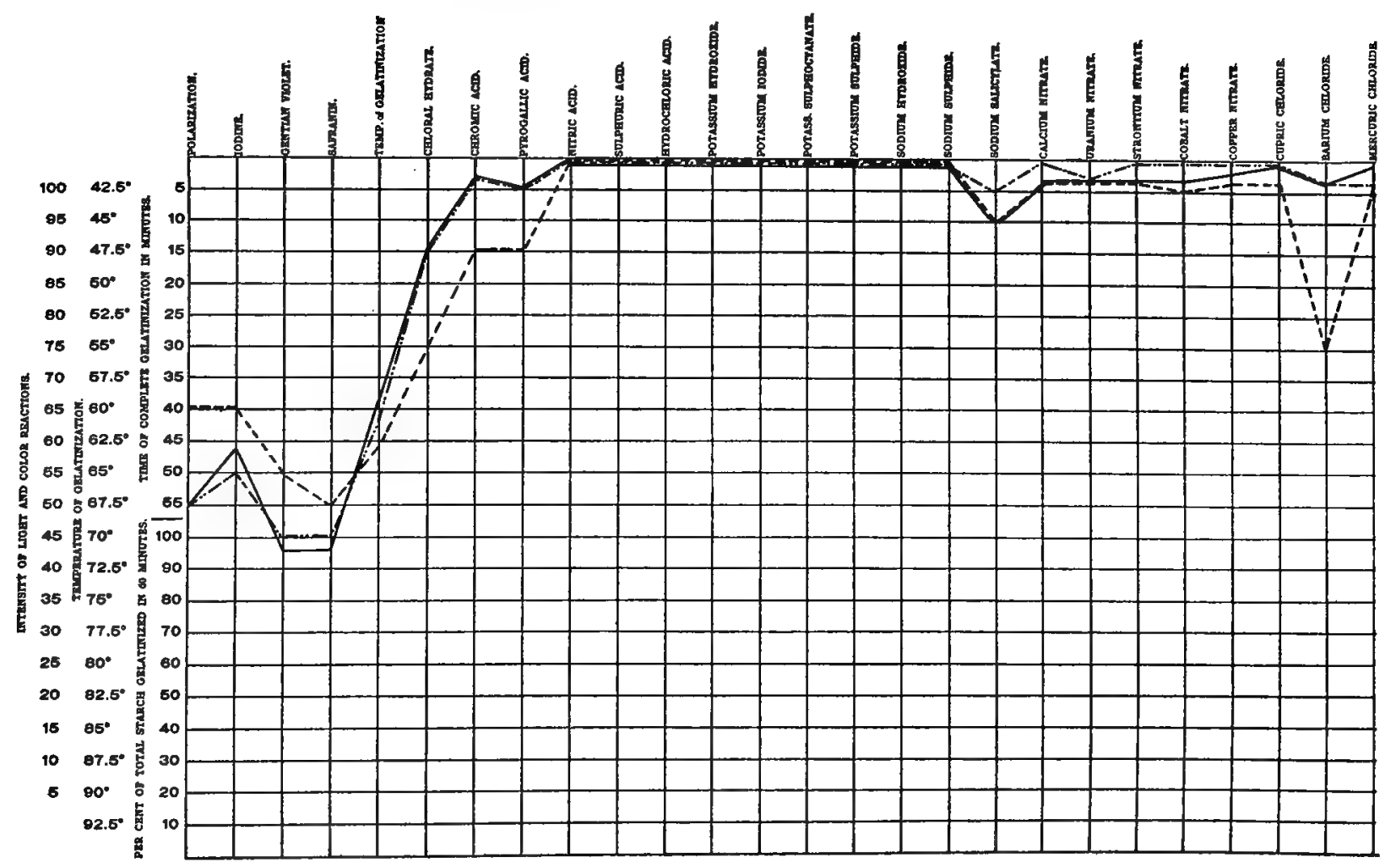

CharT E 25.-Composite Curves of the Starches of Lilium martagon album (...--), Lilium maculatum (......-), and Lilium marhan (-). 


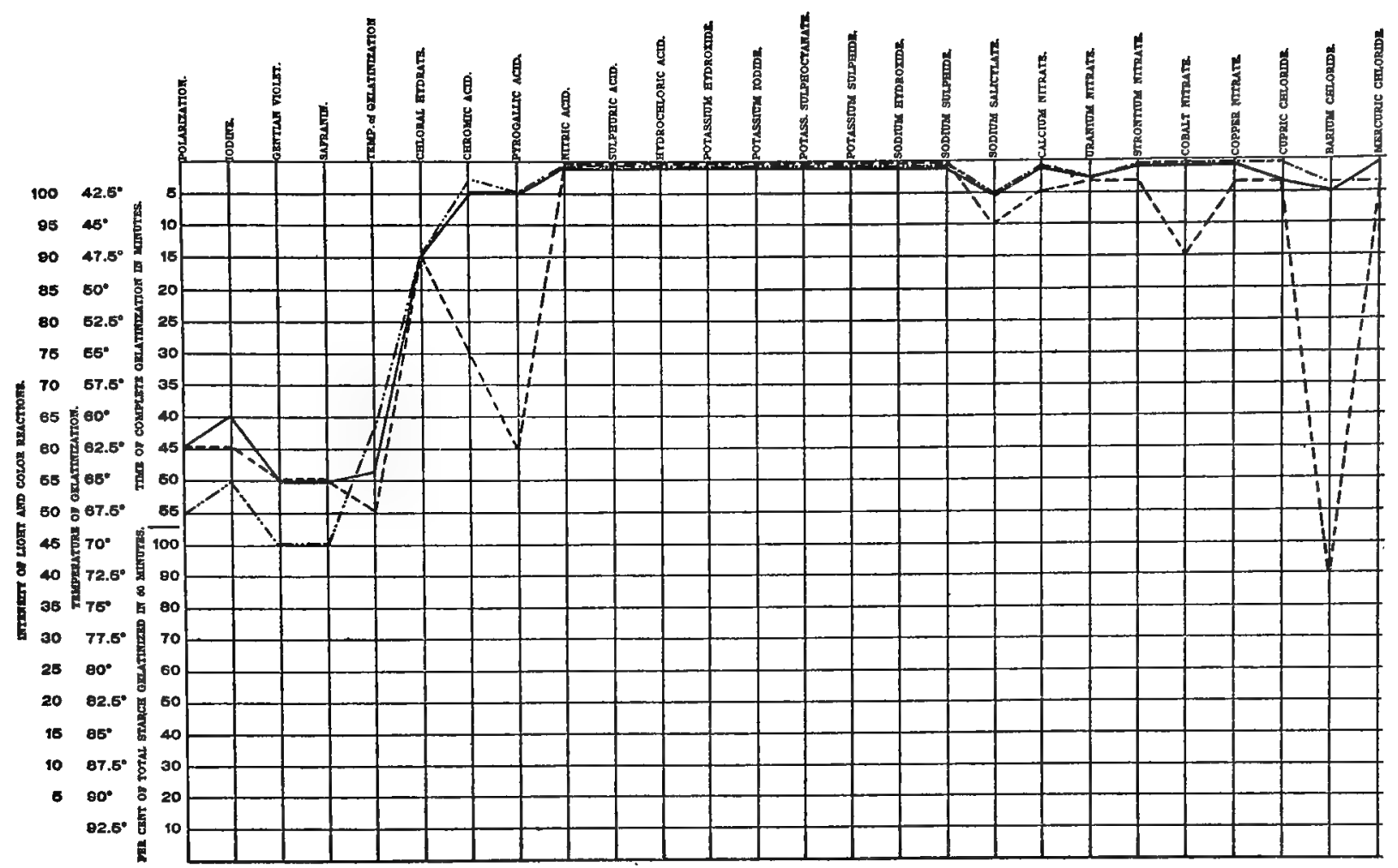

Chart E 26.-Composite Curves of the Starches of Lilium martagon (-...- ), Lilium maculatum (....... ), and Lilium dalhansoni (-).

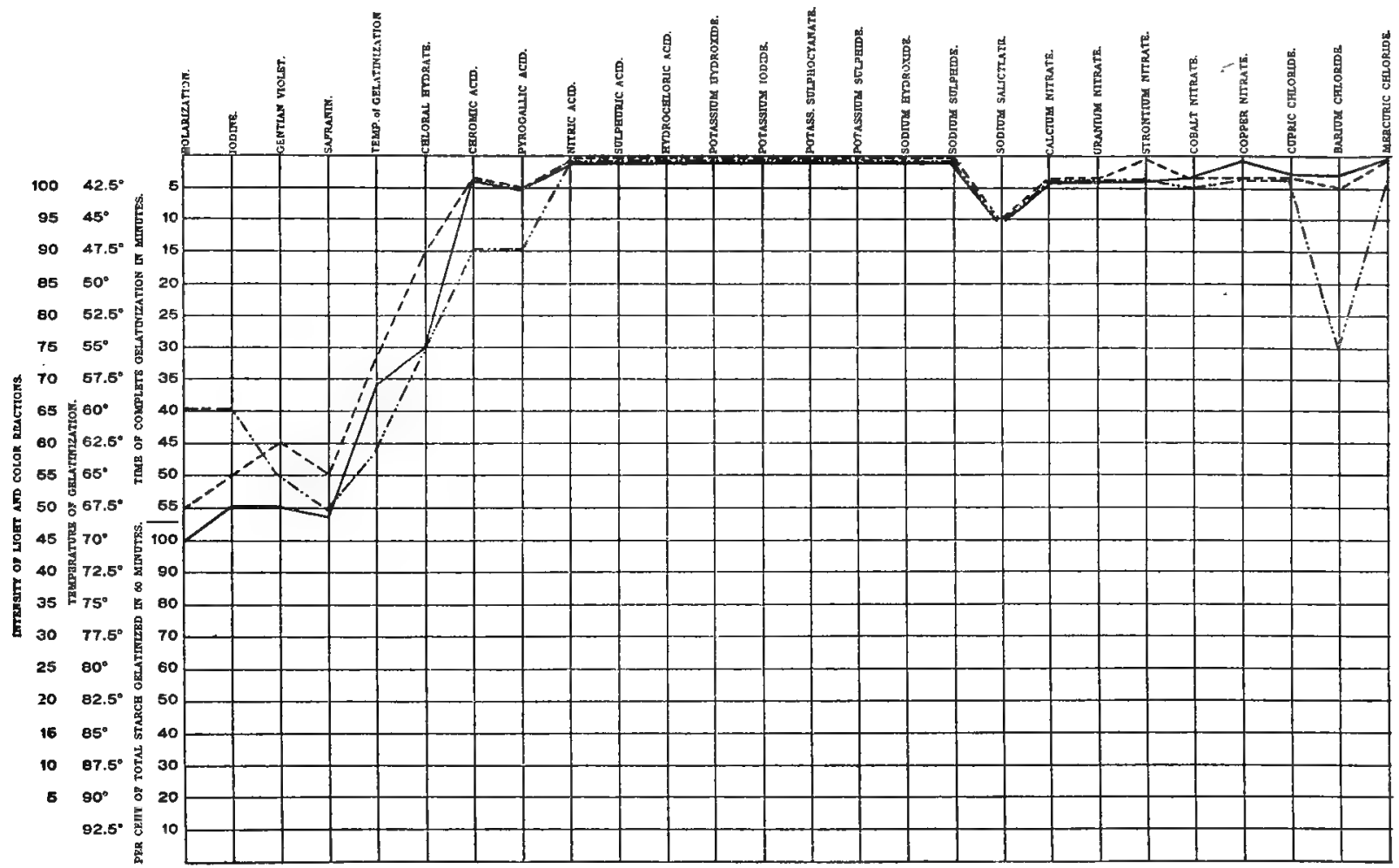

Chart E 27.-Composite Curves of the Starches of Lilium tenuifolium (....), Lilium martagon album ( ......-), and Lilium golden gleam (-). 


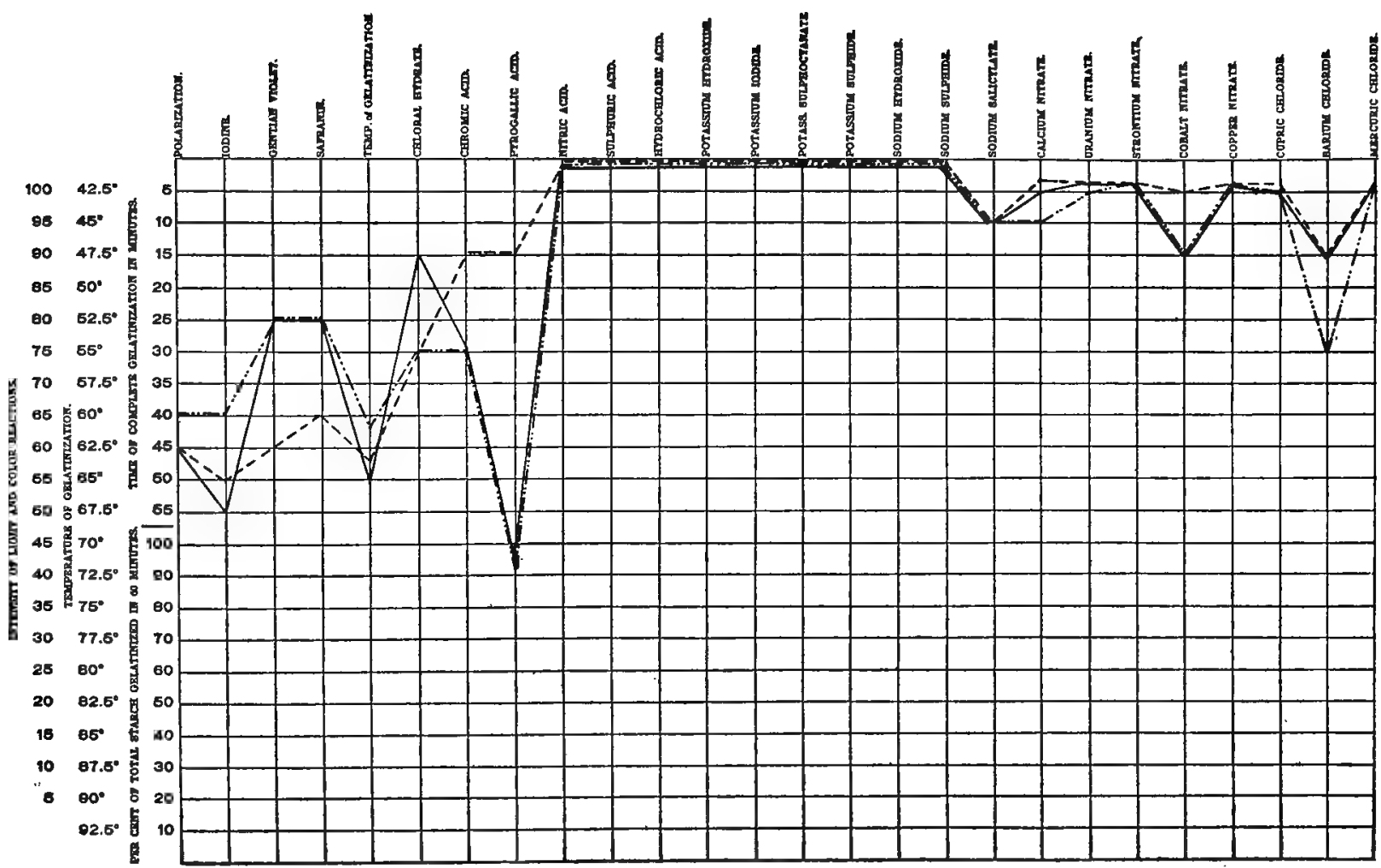

Chart E 28.-Composite Curves of the Starches of Lilium chalcedonicum (-...-), Lilium candidum (-.....-), and Lilium testaceum (-).

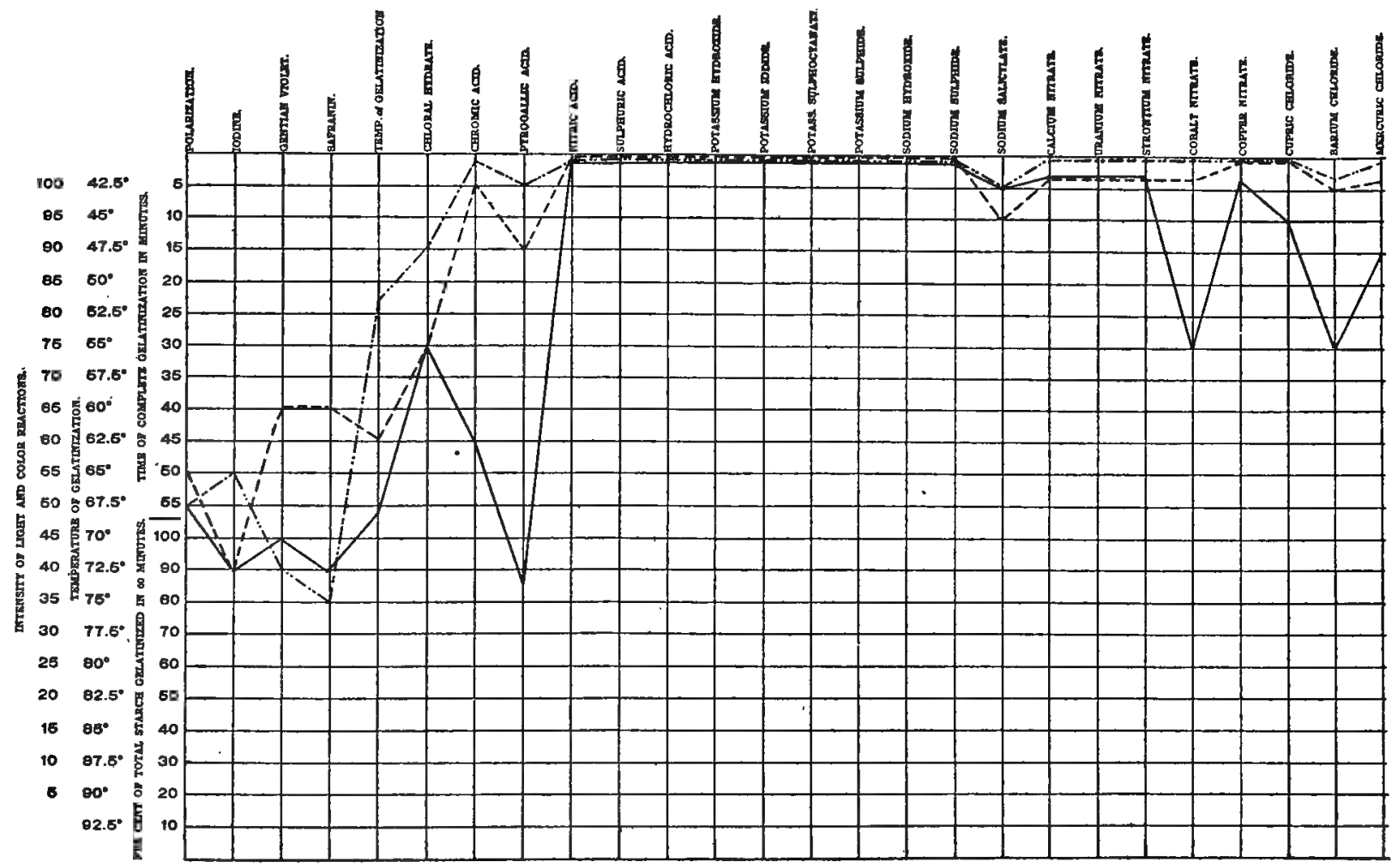

Chart E 29.-Composite Curves of the Starches of Lilium pardalinum (....), Lilium parryi ( ...... -), and Lilium burbanki (-). 


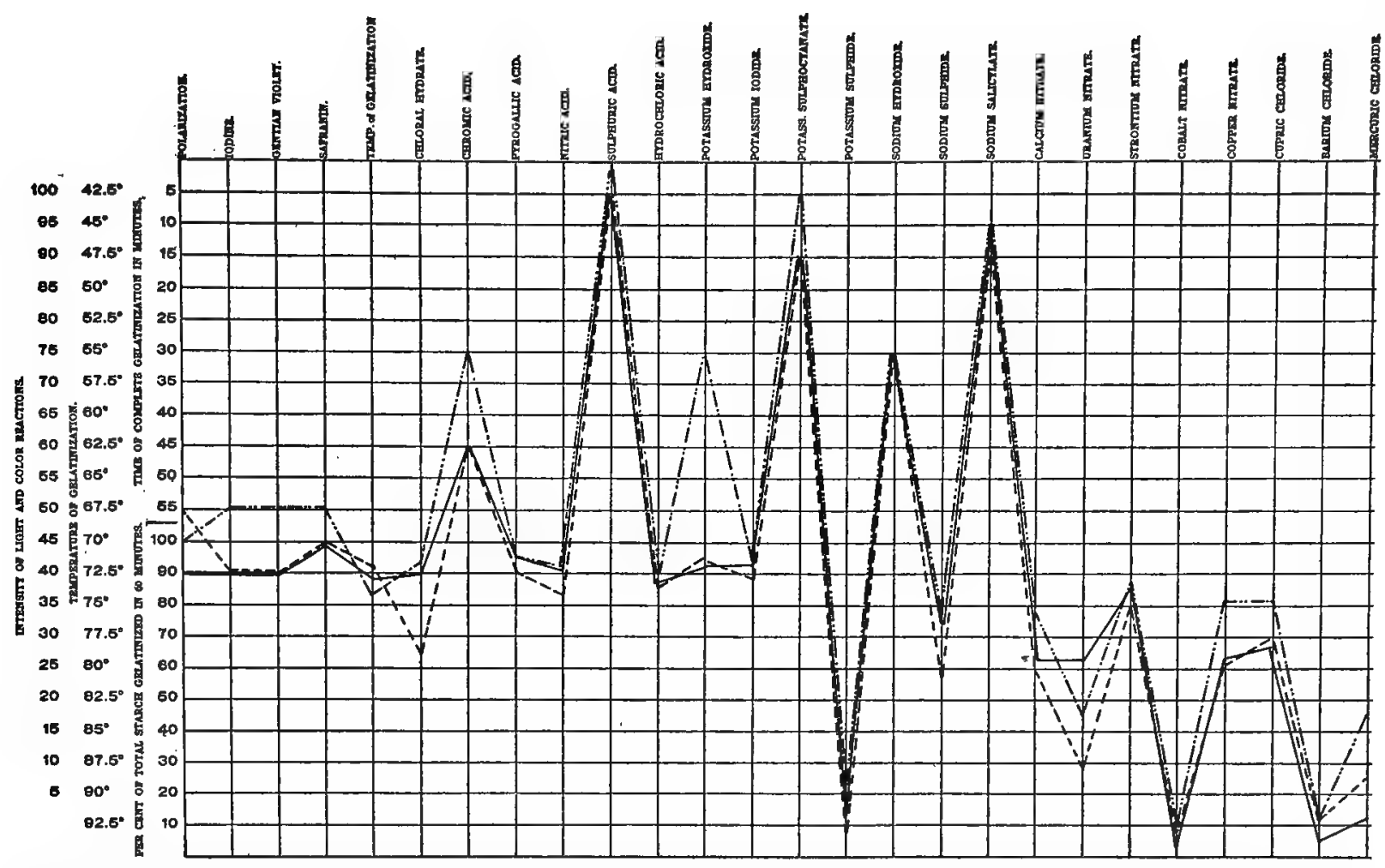

Сhart E 30.-Composite Curves of the Starches of Iris iberica ( ....), Iris trojana ( .......), and Iris ismali (-).

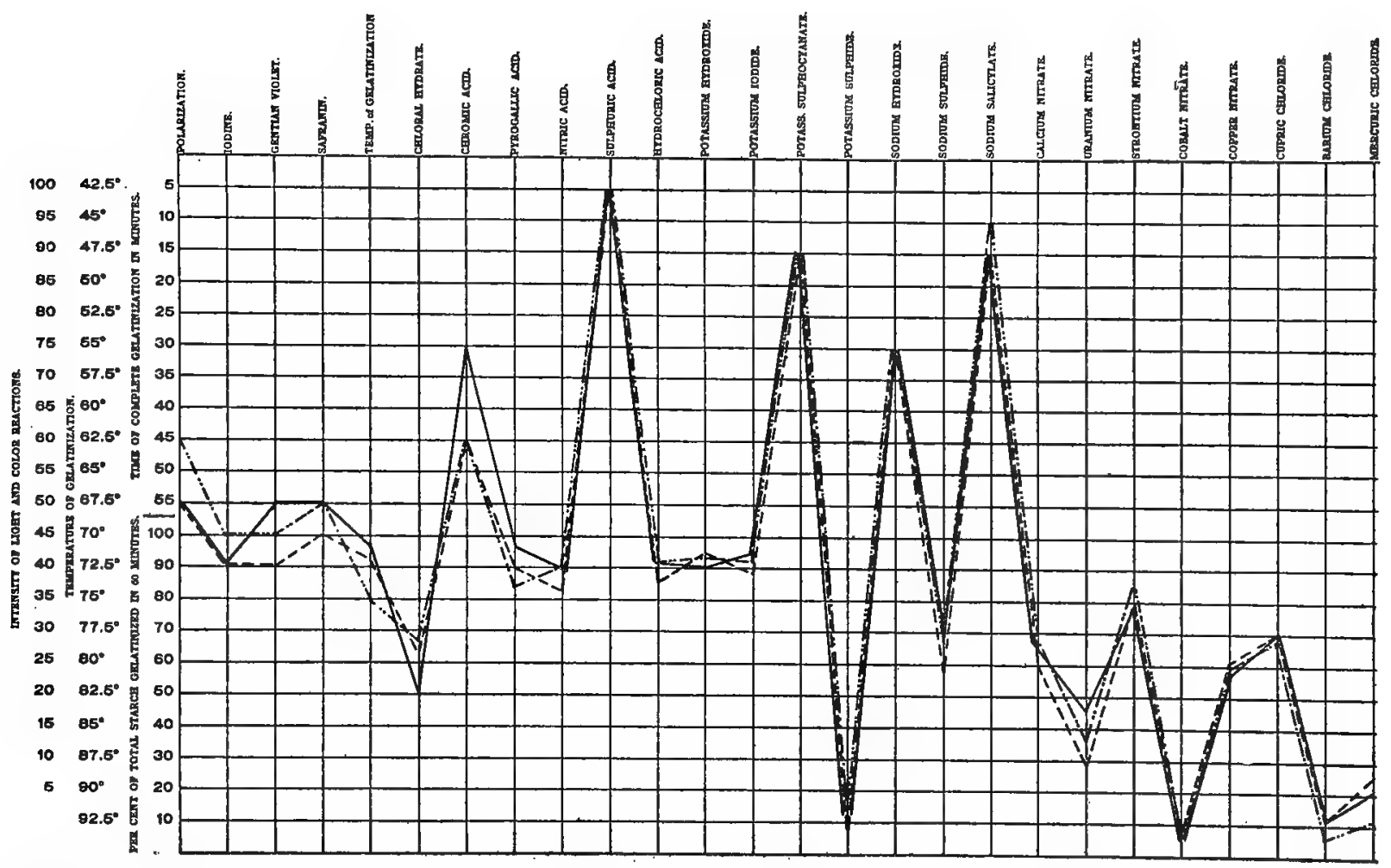

Chart E 31.-Composite Curves of the Starches of Iris iberica ( .... ), Iris cengialti ( .......), and Iris dorak ( $\longrightarrow$ ). 


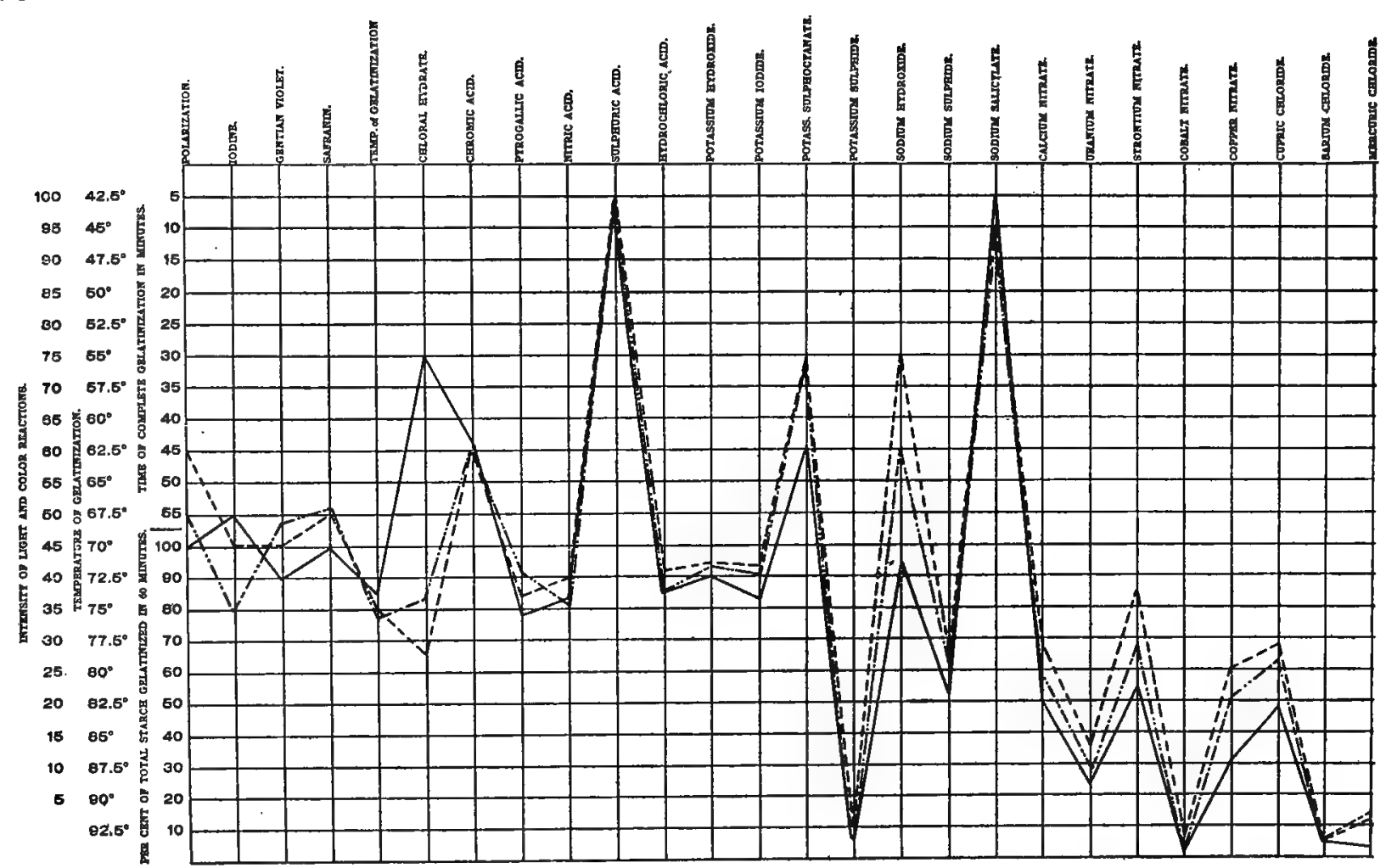

Снart E 32.-Composite Curves of the Starches of Iris cengialti ( ....), Iris pallida queen of may ( .......), and Iris mrs. alan grey (-).

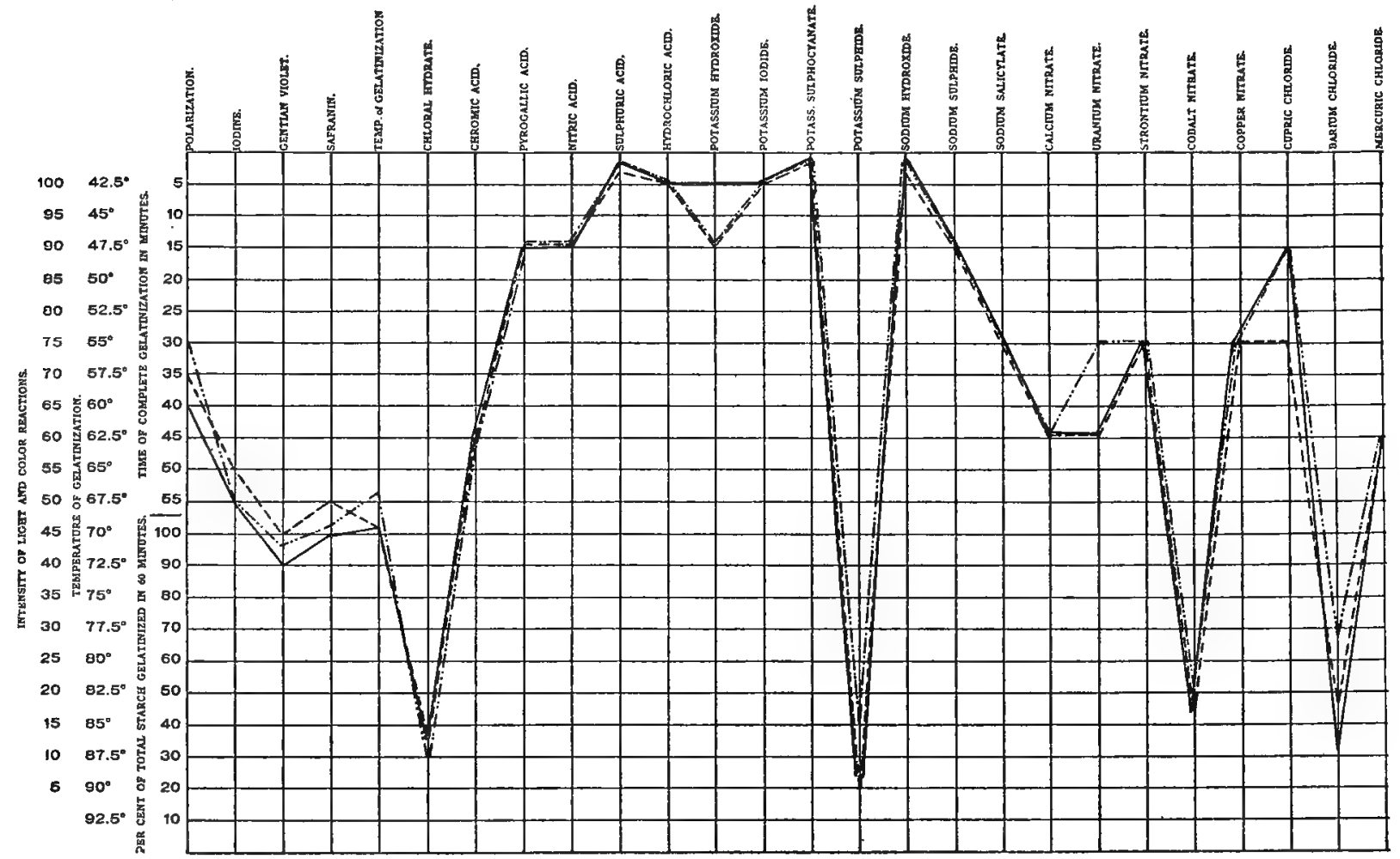

Chart E 33.-Composite Curves of the Starches of Iris persica var. purpurea (.....), Iris sindjarensis ( .......), and Iris pursind (-). 


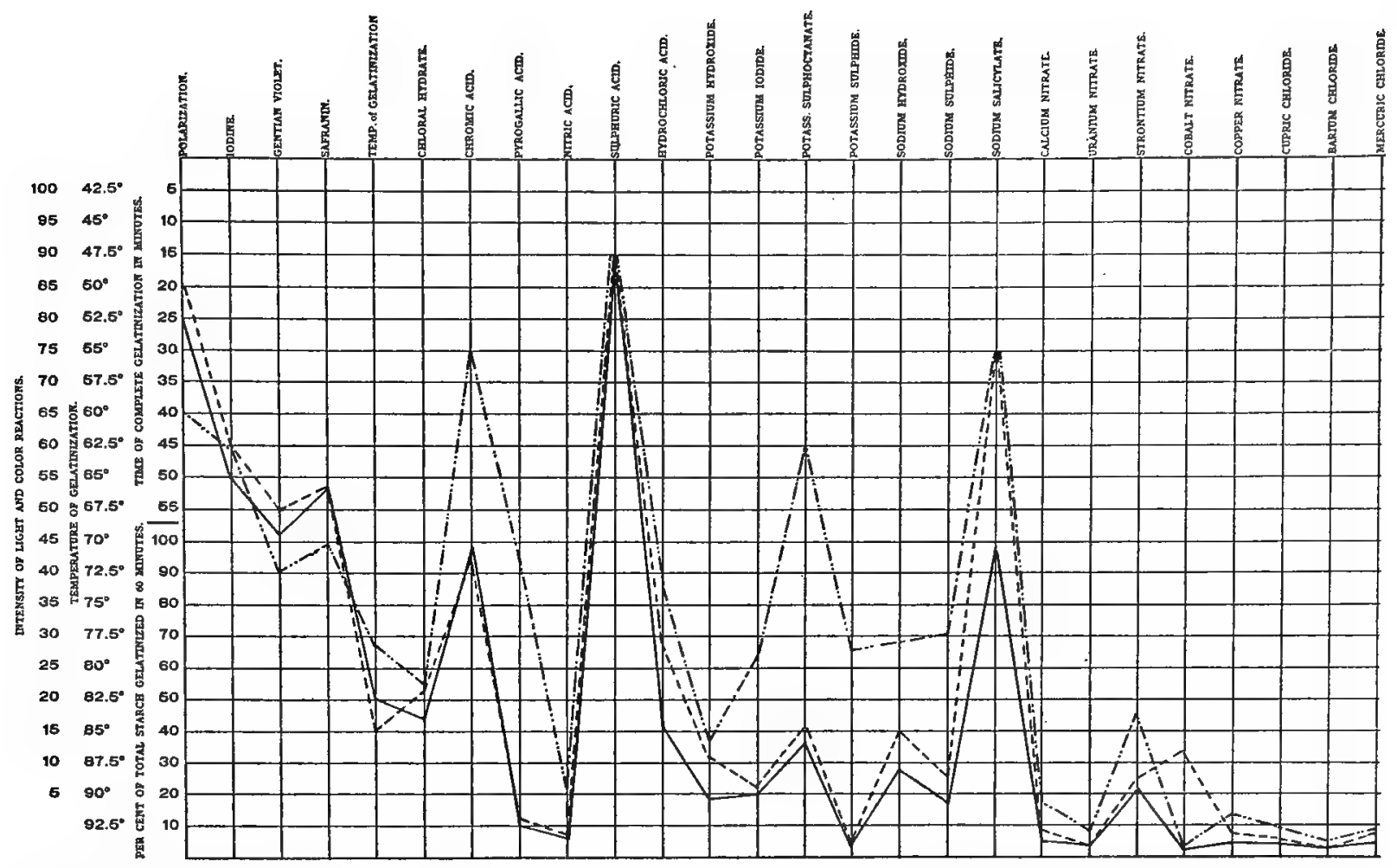

CharT E 34.-Composite Curves of the Starches of Gladiolus cardinalis (-...), Gladiolus tristis (-.....), and Gladiolus colvillei (-).

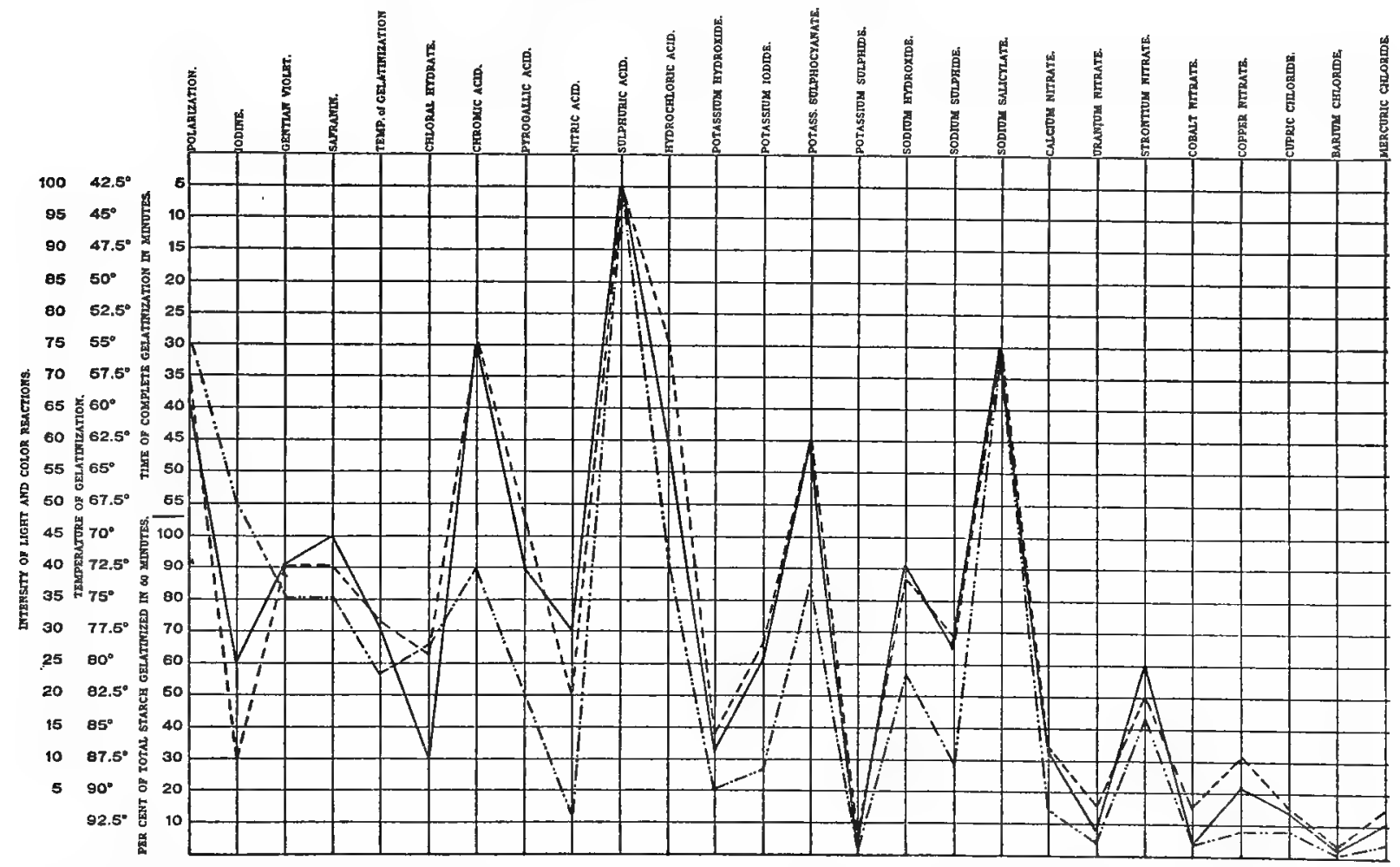

Chart E 35.-Composite Curves of the Starches of Tritonia pottsii (--..-), Tritonia crocosmia aurea (-......), and Tritonia crocosmaflora (-). 


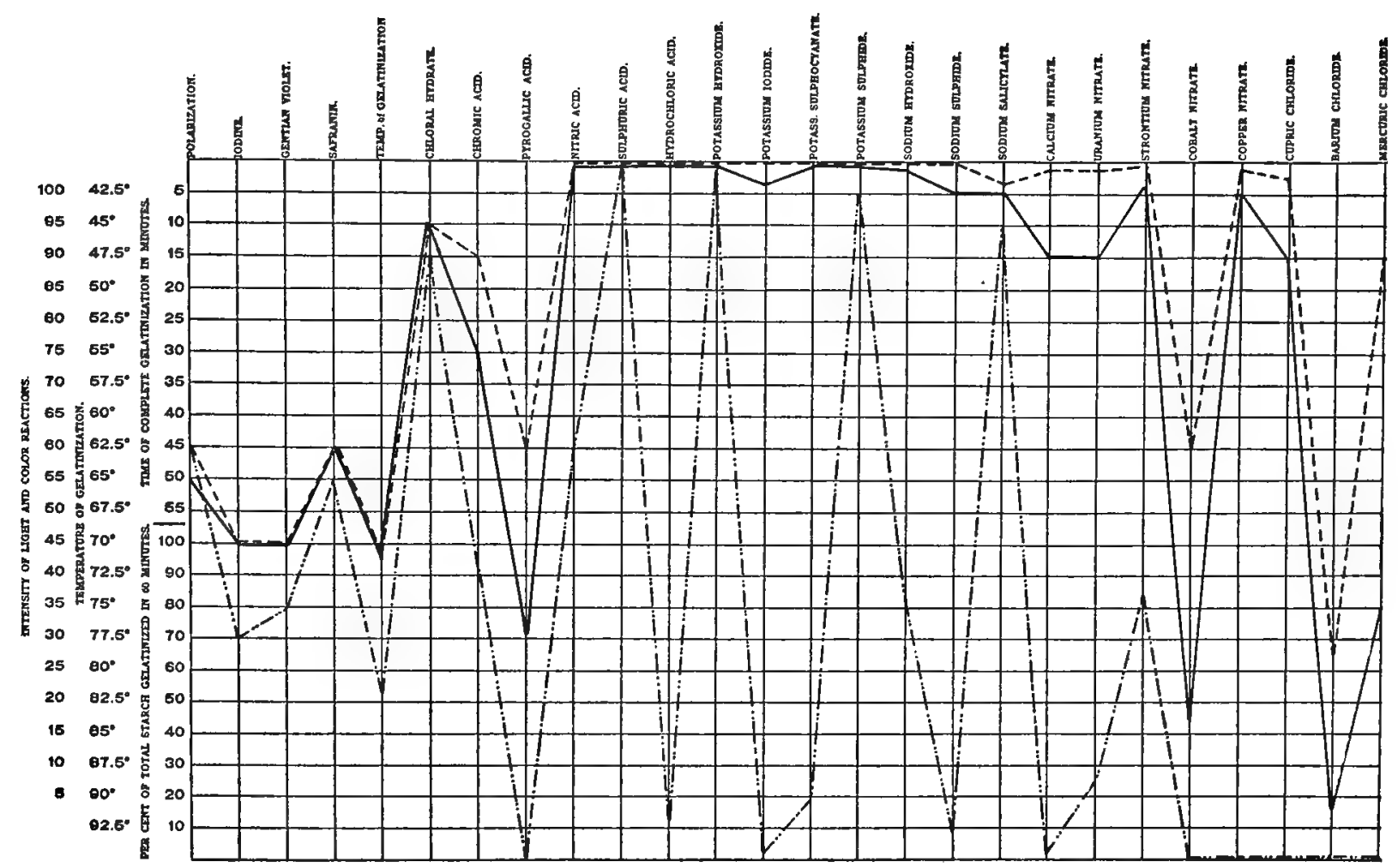

Chart E 36.-Composite Curves of the Starches of Begonia single crimson scarlet (-..--), Begonia socotrana (......), and Begonia mrs. heal (-).

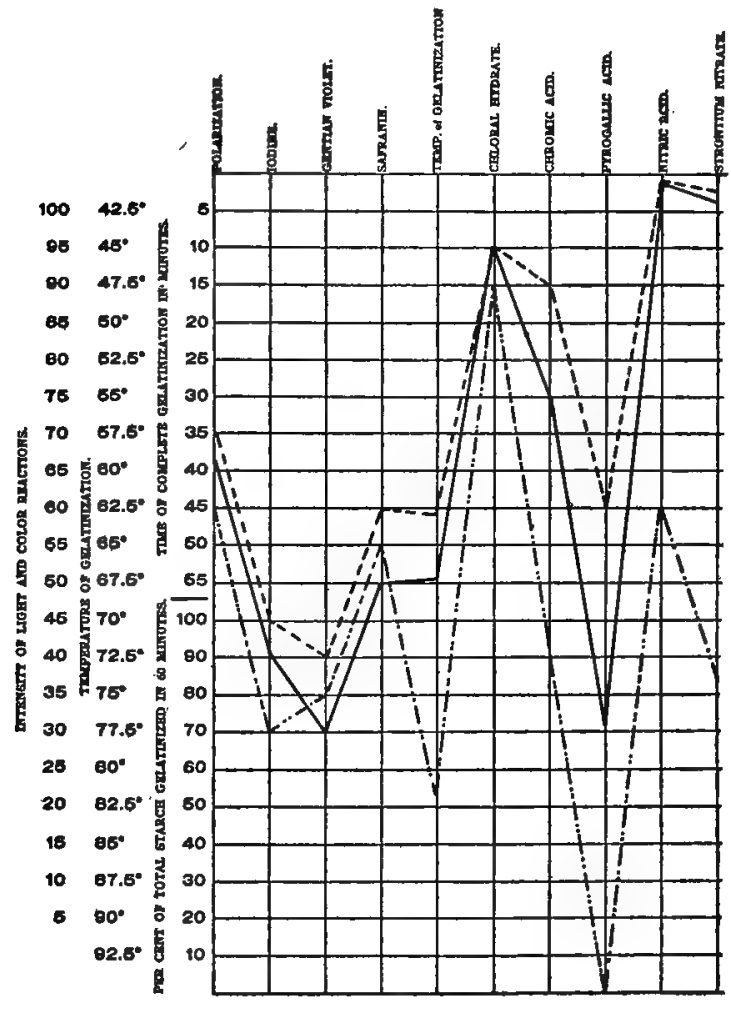

Chart E 37.-Composite Curves of the Starches of Begonia double light rose (-.-.-), Begonia socotrana ( (.......), and Begonia ensign (-

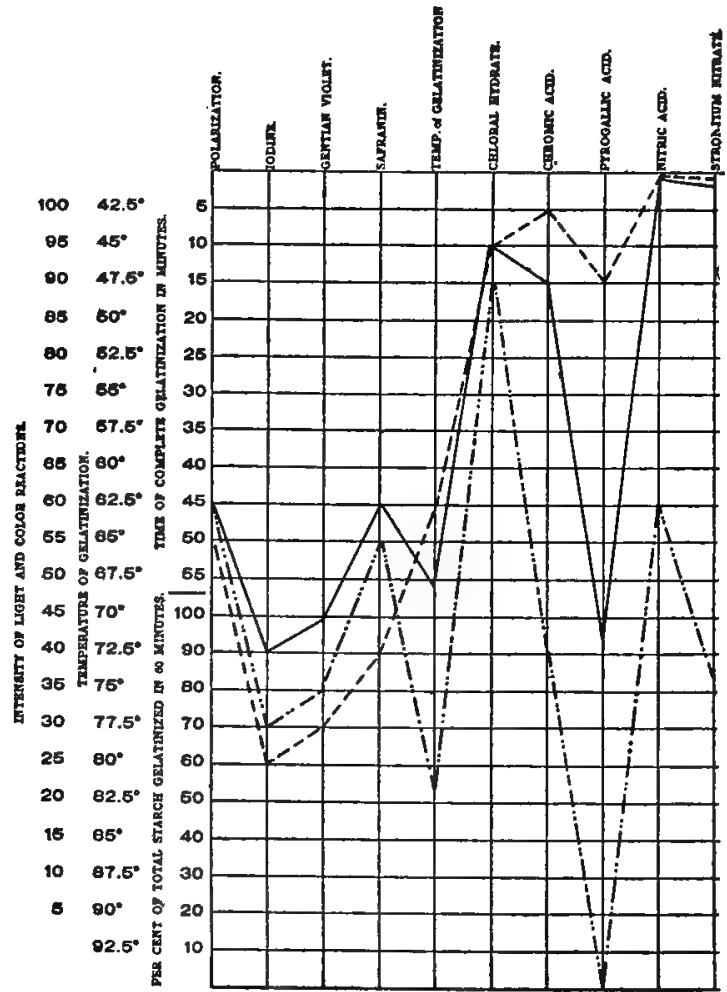

Chart E 38.-Composite Curves of the Starches of Begonia double white (..--.), Begonia socotrana $(-.-.-)$, and Begonia julius (-). 


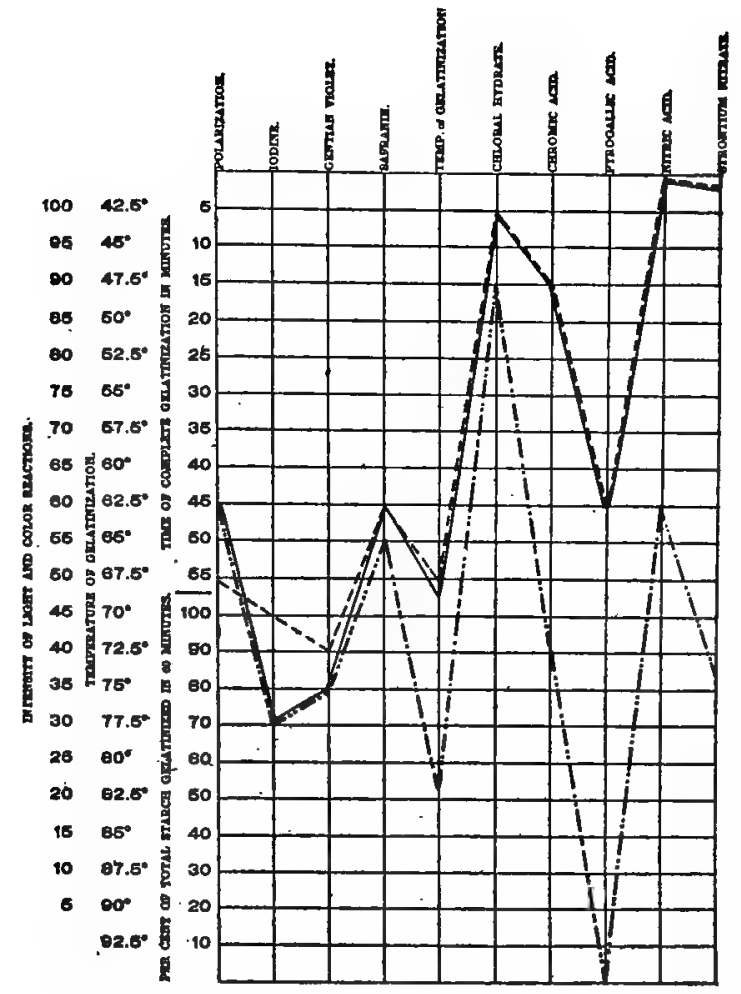

Chart E 39.-Composite Curves of the Starches of Begonia double deep rose (...-.), Begonia socotrana (.......), and Begonia success (-).

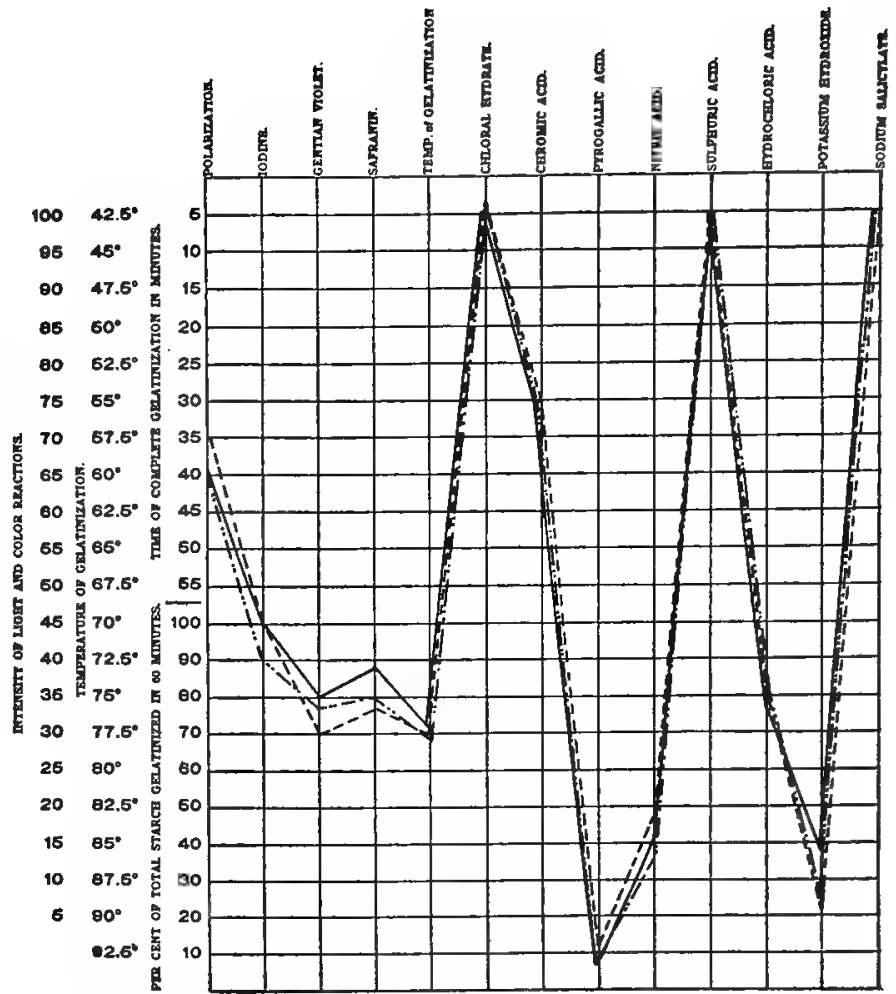

Chart E 40.-Composite Curves of the Starches of Richardia albo-maculata (-...), Richardia elliottiana (......), and Richardia mrs. roosevelt (-).

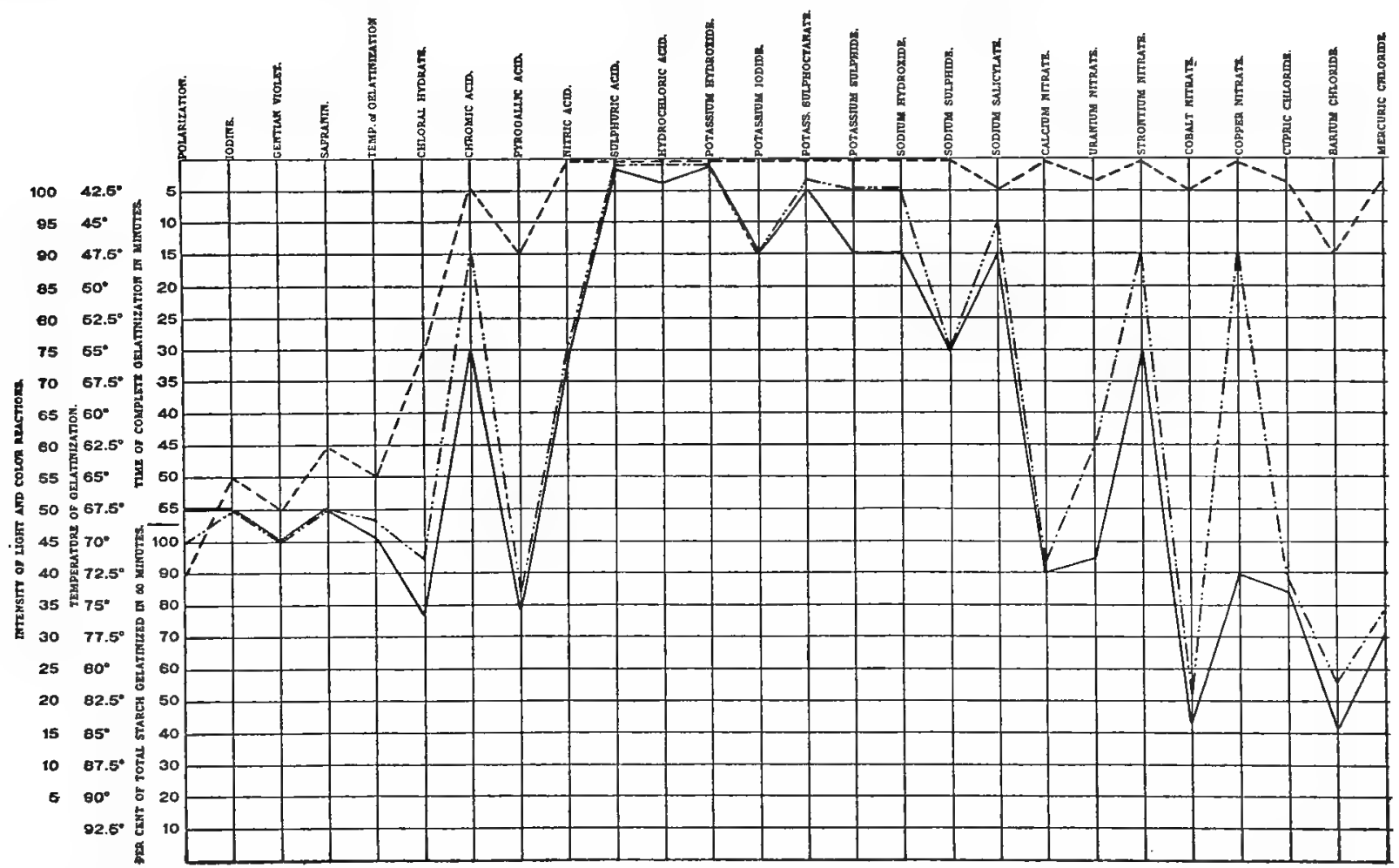

Chart E 41.-Composite Curves of the Starches of Musa arnoldiana (....), Musa gilletii (_.....), and Musa hybrida (-). 
280

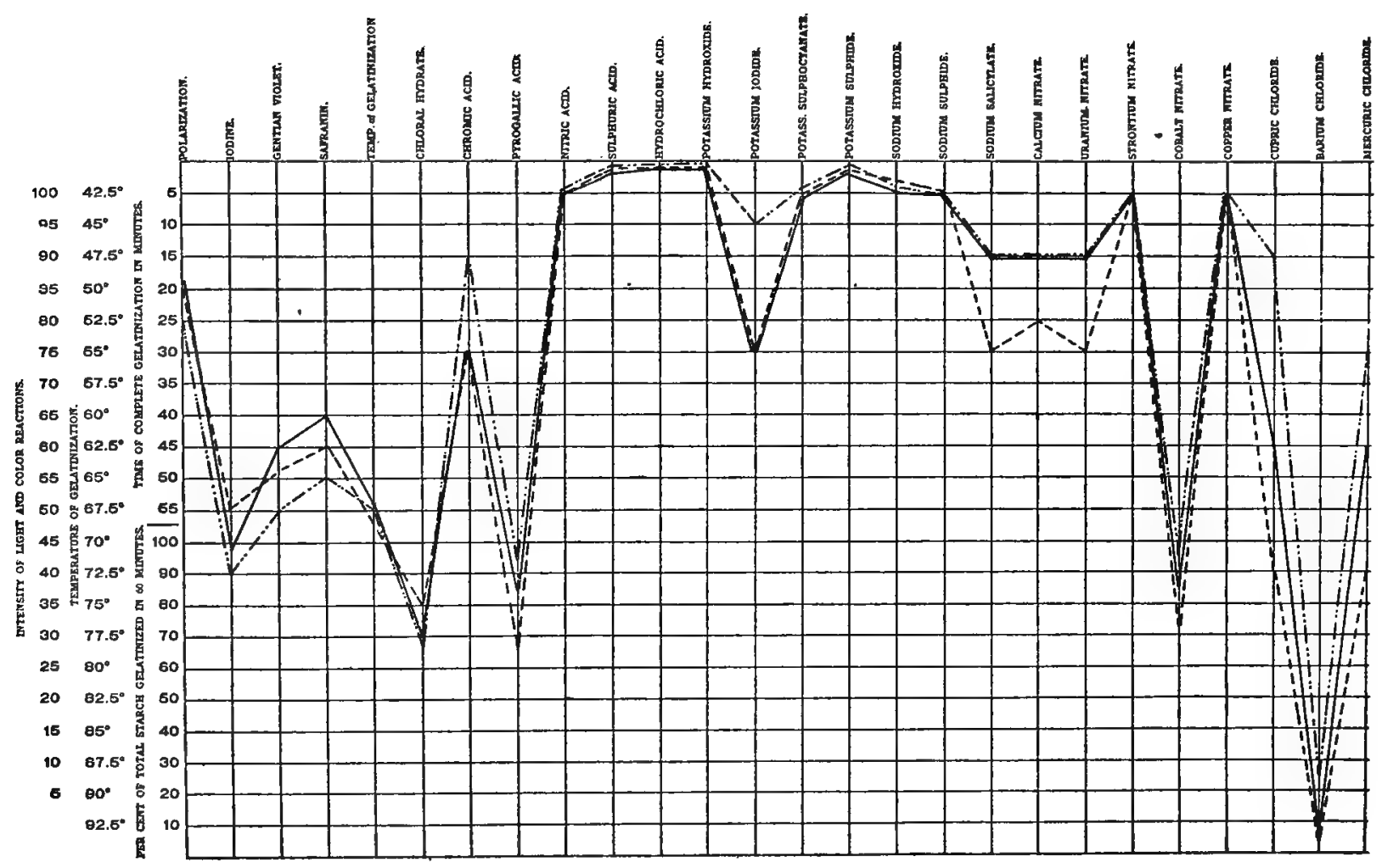

Chart E 42.-Composite Curves of the Starches of Phaius grandifolius (-...), Phaius wallichii (-.....-), and Phaius hybridus (-).

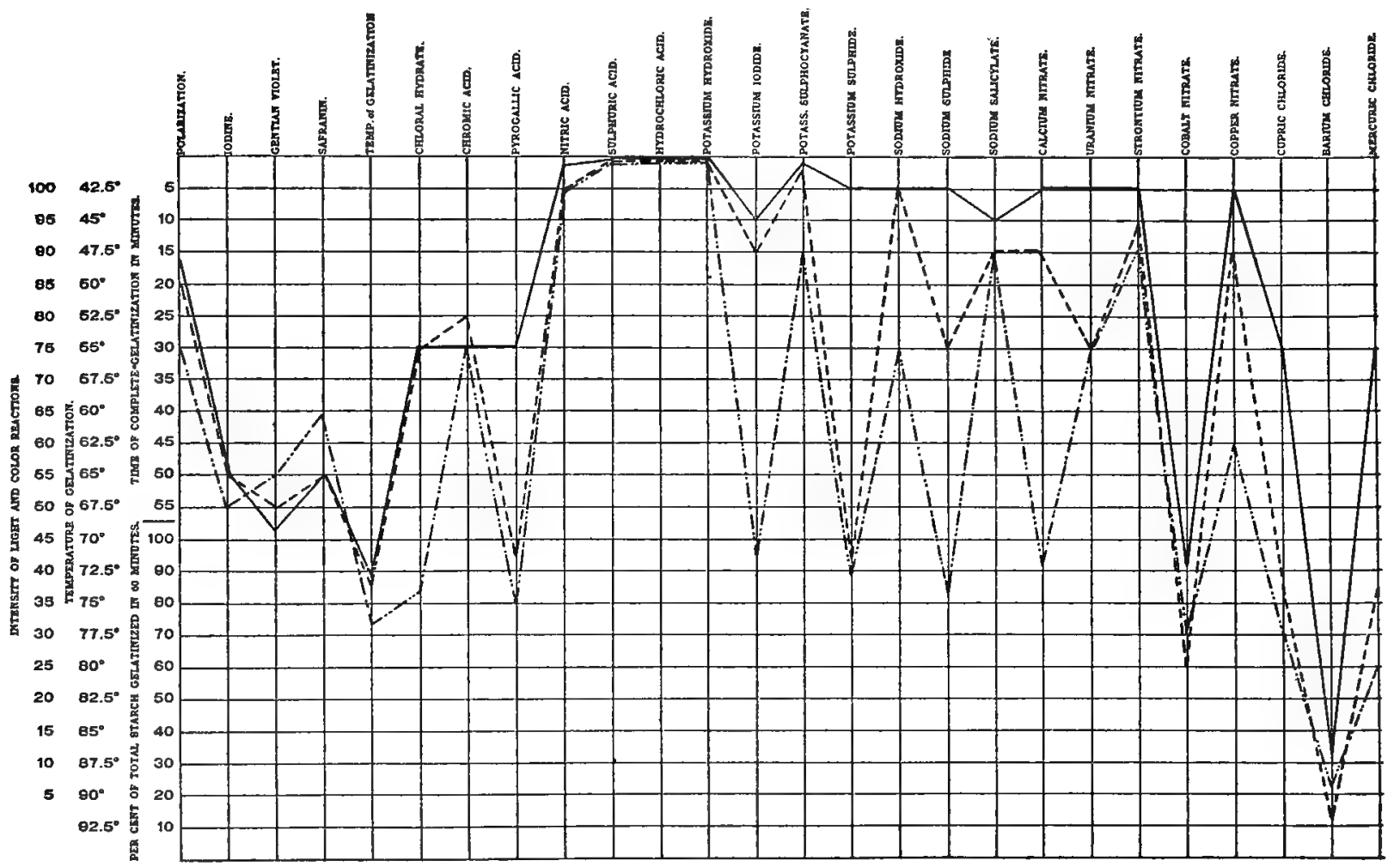

CharT E 43.-Composite Curves of the Starches of Miltonia vexillaria (-....), Miltonia roezlii (.......), and Miltonia bleuana (-). 


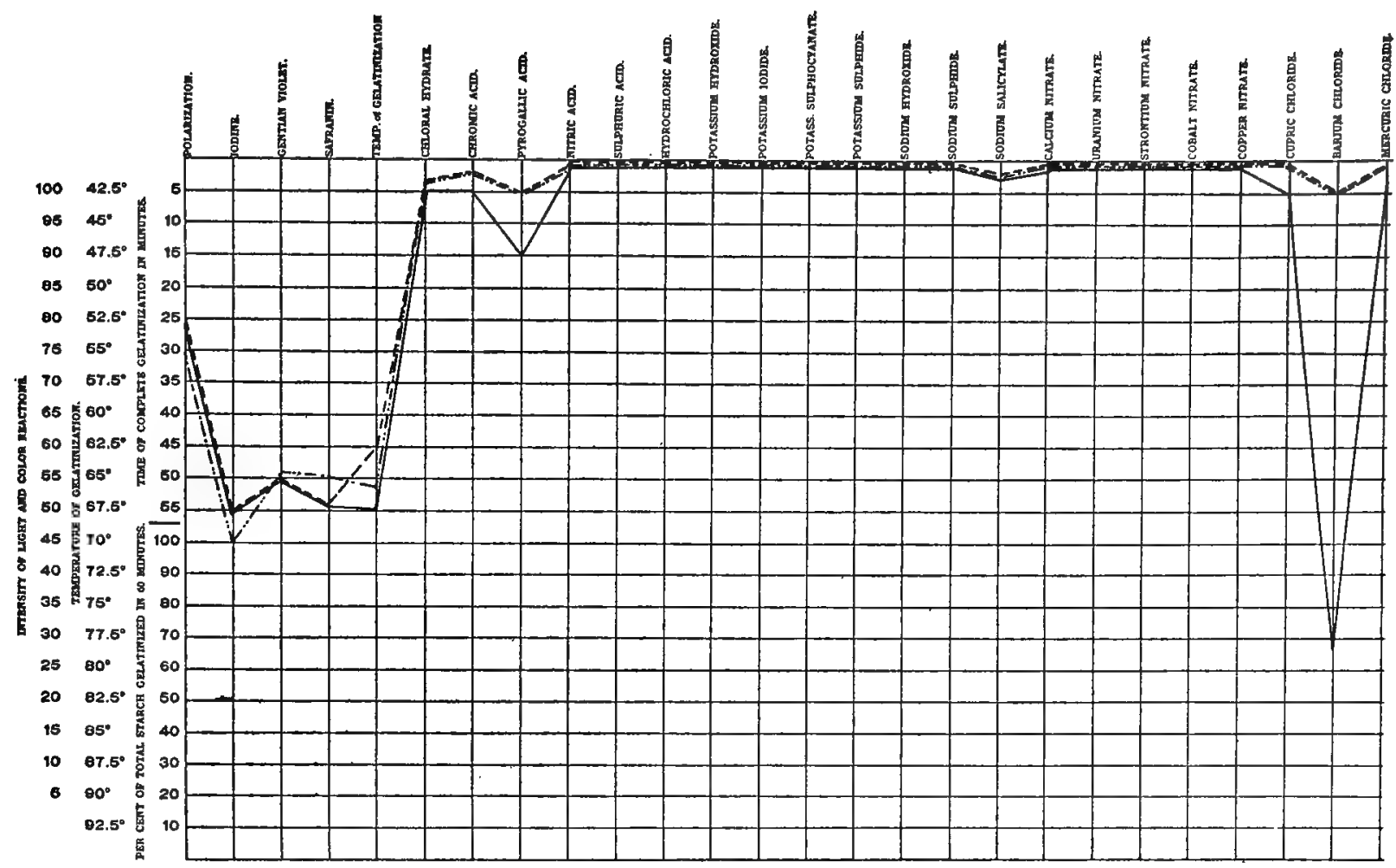

Chart E 44.-Composite Curves of the Starches of Cymbidium lowianum (-....), Cymbidium eburneum (-......), and Cymbidium eburneo-lowianum (-).

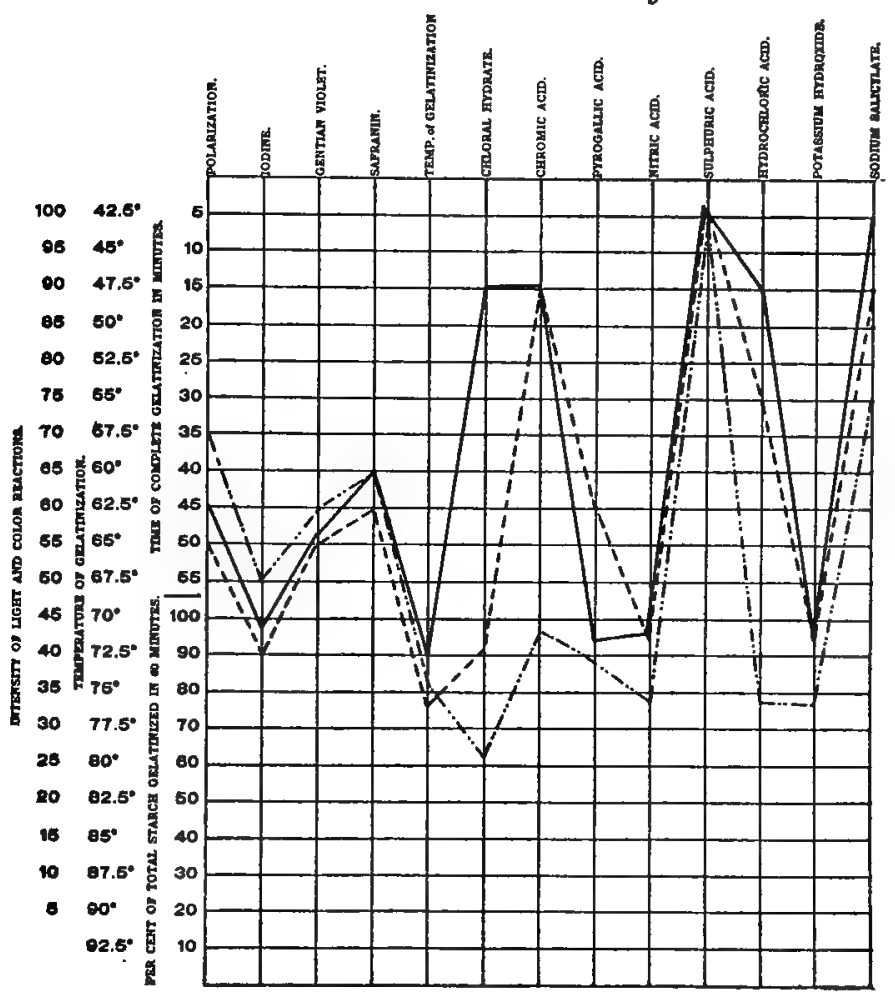

Chart E 45.-Composite Curves of the Starches of Calanthe rosea (...-), Calanthe vestita var. rubro-oculata (......-), and Calanthe veitchii (_).

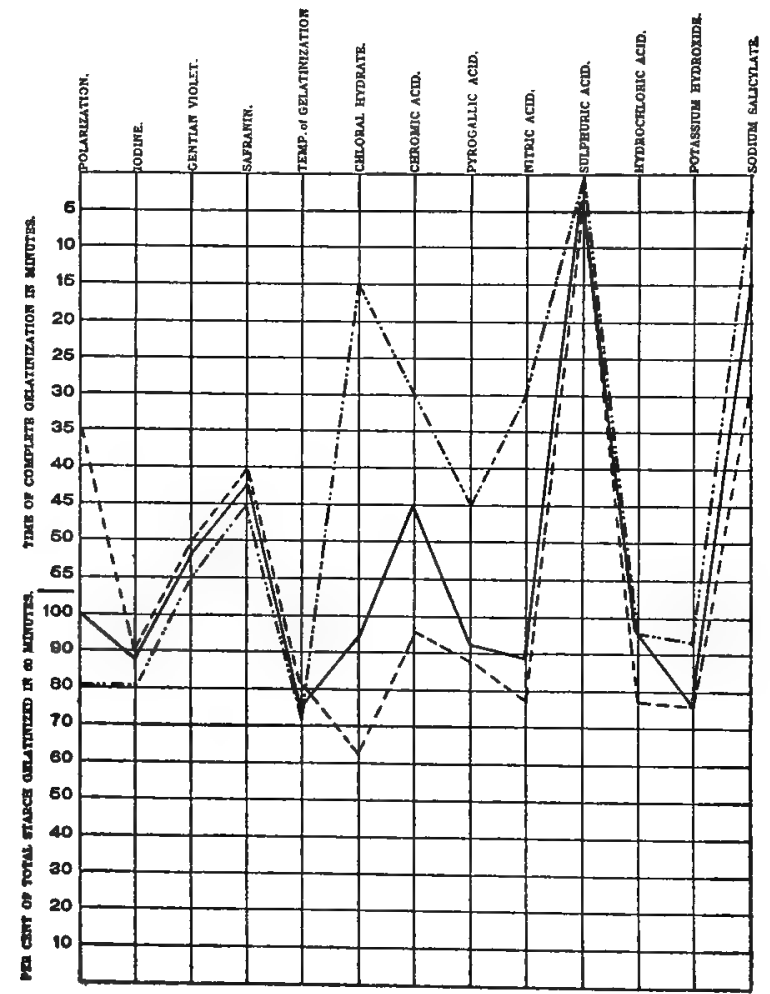

Chart E 46.-Composite Curves of the Starches of Calanthe vestita var. rubro-oculata (-...-), Calanthe regnieri (.....-), and Calanthe bryan (-). 


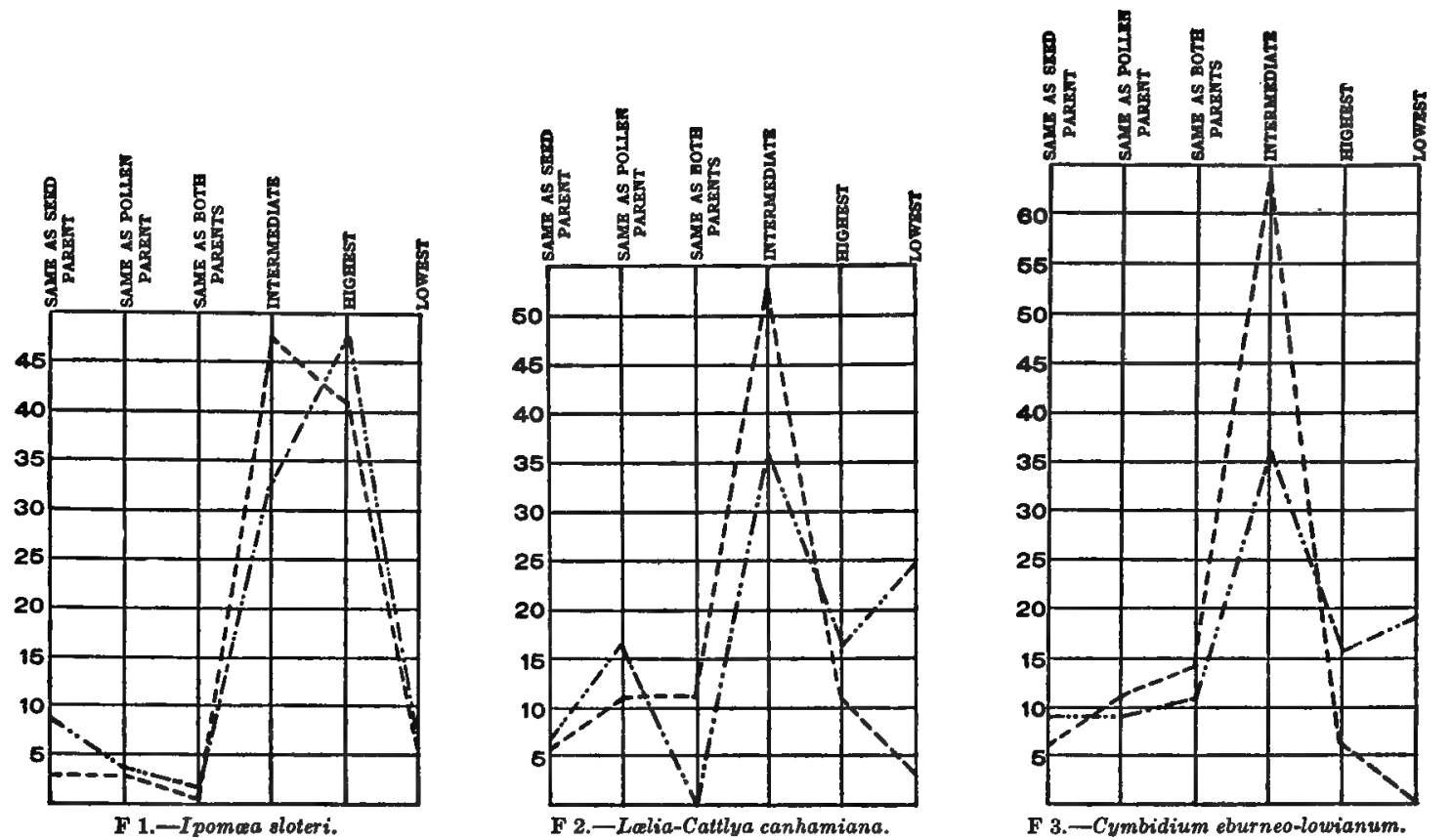

Charts F 1 To F 3.-Percentages of Macroscopic (....) and Mieroscopic (.......) Characters.
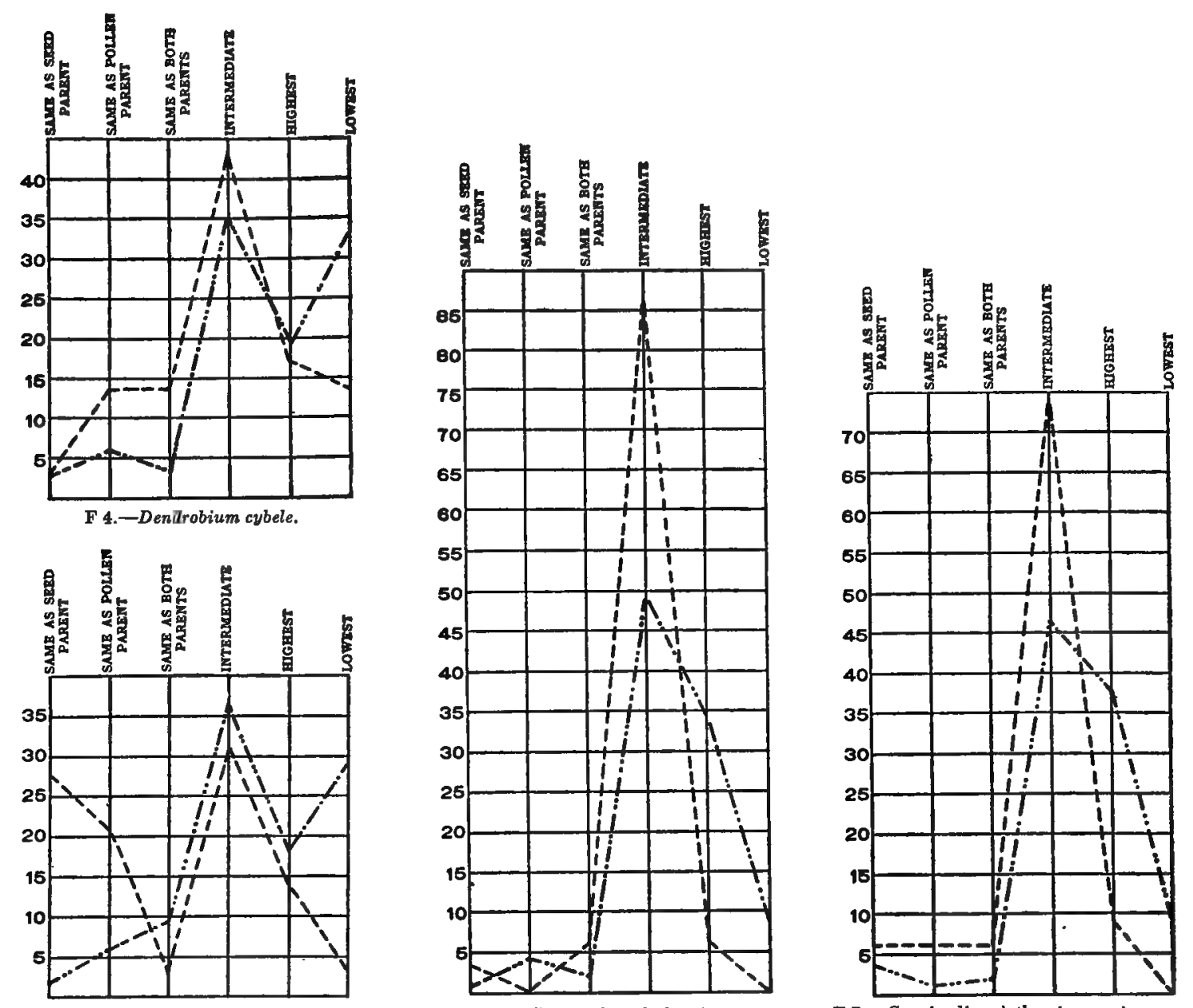

F 5.-Miltonia bleuana.

F 6.-Cypripedium lathamianum.

F 7.-Cypripedium lathamianum inversum.

Charts F 4 To F 7.-Percentages of Macroscopic (....) and Microscopic (...... -) Characters. 


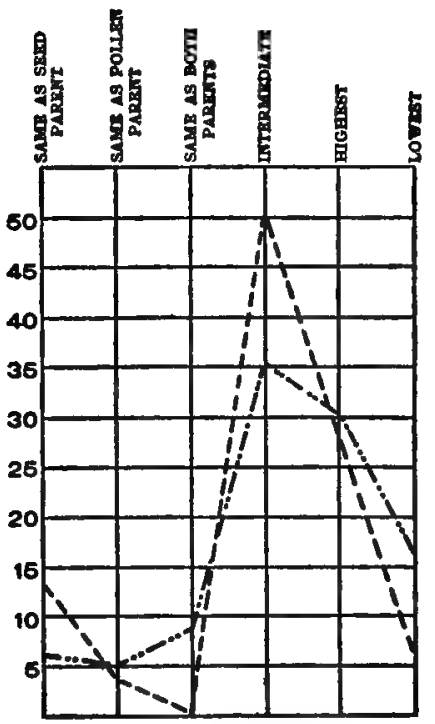

F 8.-Cypripedium nitens.

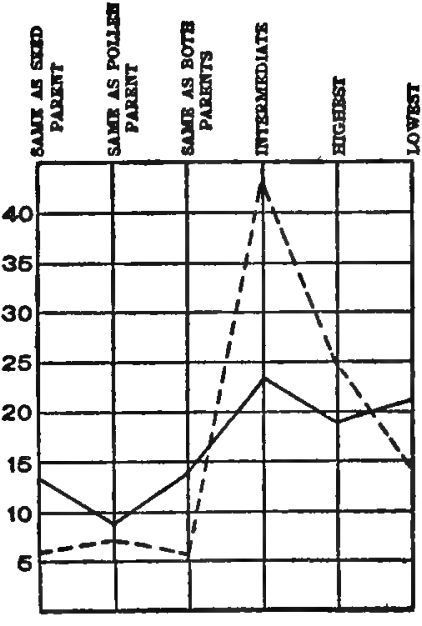

F 9. -Tissues and Starches.

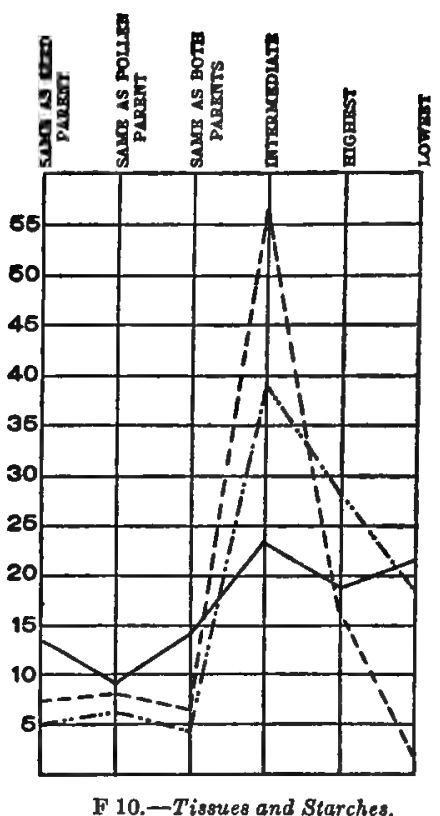

F 10.-Tissues and Starches.

Chart F 8.-Percentages of Macroscopic ( .....) and Microscopic (.......) Characters.

ChART F 9.-Percentages of Macroscopic and Microscopic Characters (-..-.) and Starch Reaction-Intensities $(\longrightarrow)$ of Hybrid-Stocks in regard to Sameness, Intermediateness, and Excess and Deficit of Development in relation to Parent-Stocks.

Chart F 10.-Percentage of Macroscopic (....) and Microscopic (.......) Characters and Starch ReactionIntensities (-) of Hybrid-Stocks in regard to Sameness, Intermediateness, and Excess and Deficit of Development in relation to Parent-Stocks.

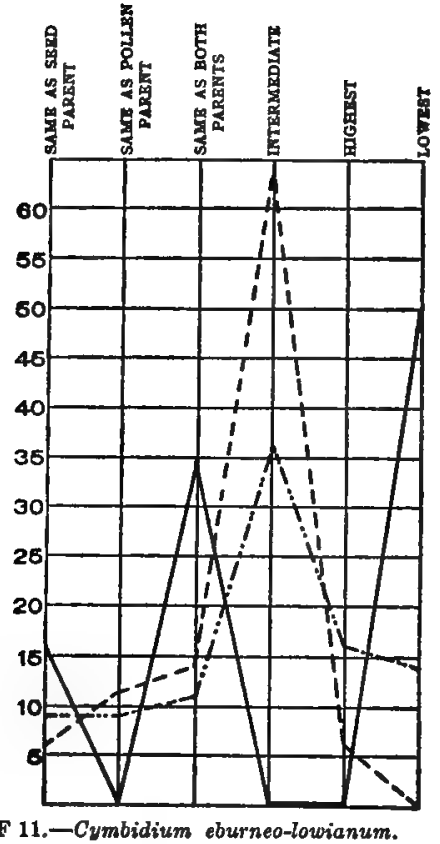

F 11.-Cymbiditum eburneo-lowianum.

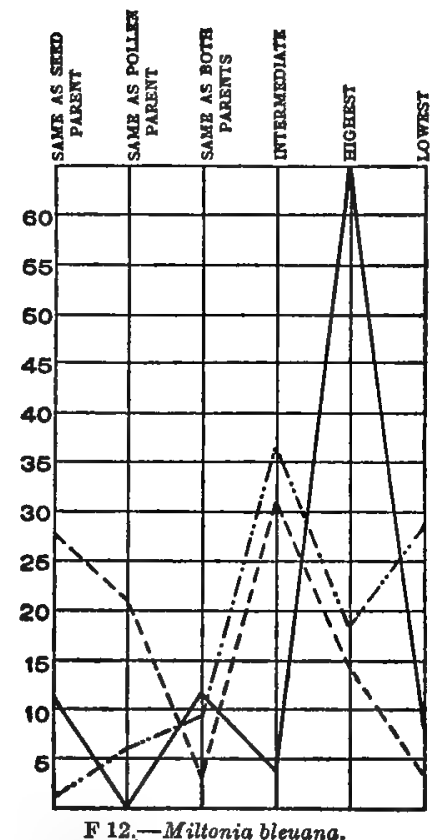

F 12.-Miltonia bleuana.

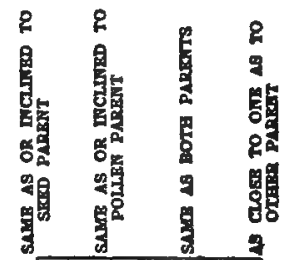

CHARTS F 11 AND F 12-Percentages of Macroscopic ( Chacticters and Starch Reaction-Intensities $(-)$ in regard to Sameness, Intermediateness, and Excess and Deficit of Development in relation to Parent-Stocks.

Charts F 13 AND F 14.-Percentages of Sameness and Inclination of Macroscopic (.....) and Microscopic (......-) Tissue Characters and Starch Reaction-Intensities (-) in relation to those of Parent-Stocks. 


\section{CHAPTER V. \\ SUMMARIES OF THE HISTOLOGIC CHARACTERS, ETC.}

This chapter is devoted to the summaries of the histologic characters and qualitative and quantitative reactions of the starches of hybrid-stocks in relation to the starches of the parent-stocks, and of the microscopic and macroscopic characters of the hybrid-stocks in relation to the parent-stock plants.

\section{THE STARCHES.}

Histologic Characters and Certain Qualitative and Quantitative Reactions.

(Tables C, 1 to $17 ; \mathrm{D} ; \mathrm{E}, 1$ to $22 ; \mathrm{F}, 1$ to $50 ; \mathrm{G} ; \mathrm{H}, 1$ to 26 ; and I, 1 to 8 .)

The methods used in this research in the differentiation of starches are both quantitative and qualitative. From a glance at the large number of charts and tables that set forth quantitative results the impression may be gained that much more importance is to be attached to the former than to the latter method of investigation; but this will be found to be unwarranted by the considerable space that has been given to and the remarkably valuable results that have been recorded under qualitative reactions. In fact, the qualitative method has been found to have far the larger and more varied, and an at least equally important, field of usefulness. Unfortunately very little data included under histologic and qualitative records lend themselves to chart-making, or to such forms of tabulation, as have proven so valuable in the preceding chapter and elsewhere in this memoir. Hence, the records herein summarized are presented in a modified arrangement that is particularly well adapted to set forth only a certain but an important aspect of the comparative peculiarities of hybrid and parental properties.

From the records found in various parts of this work it will be noted that the starch of the hybrid exhibits, histologically, physically, and physico-chemically, not only both uniparental and biparental inheritance, but also individualities that are not observed in either parent; and that any given parental character that appears in the hybrid may be found in quality and quantity to be the same or practically the same as that of one parent or both parents, or of some degree of intermediateness, or developed in excess or deficit of parental extremes. Moreover, each unit character and unit character-phase (see Preface and Chapter I, Section 8) is to such a degree independent of the others that one unit-character or character unit-phase may be identical with or very close to that of one parent, while another bears the same relation to the other parent, etc. Thus, in regard to the unitcharacters (especially the lamellæ), the hybrid may show a very close relationship in the distinctness of the lamellæ to one parent, but in the forms of the lamellæ to the other parent; in fineness or coarseness it may be exactly intermediate; while in variety, or distribution, or number it may be found at the same time to have the most varying relationships. In a word, in the summing up of the parental relationships it is usually recorded in each of the designations of study (hilum, lamellæ, size, polari284 scopic reactions, iodine reactions, and gelatinization reactions with each of the different reagents) that a number of correlated unit-characters or unit-character-phases are separable, and that there is a most remarkable and inexplicable swinging to one or the other parent of unit character-development and unit character-phasedevelopment.

These records show collectively an extraordinary variability in the character relationships of the hybrid to the parents; an independence of each unit-character and unit-character-phase of every other in the direction and degree of its development; an absolute unpredictability at the present embryonic stage of our knowledge of the form, in which, if at all, any given unit-character or unit-character-phase of either or both parents may appear in the hybrid; and the closer relationship usually of the hybrid in the sum-total of the group-characters or character-phases included in every designation, and of these designations collectively, to one or the other parent. For instance, among the data pertaining to the histologic properties of Brunsdonna sanderce alba, under the designation form it will be noted that the starch grains are more like those of Amaryllis belladonna than those of Brunsvigia josephince in that they are usually simple and isolated, in their regularity of outline, and in their conspicuous forms; yet in other respects they are more like those of Brunsvigia josephina because of the presence of a relatively large number of compound grains, of a few small aggregates that consist of 2 or 3 components, and of a peculiar form of compound grain, both of which latter are found in this parent but not in A maryllis belladonna. In the data relating to the lamellæ, the hybrid is closer in form and arrangement to the corresponding parts of the grains of Amaryllis belladonna; but in average number it is closer to the other parent. In the chloral-hydrate reactions the hybrid in its quantitative reactions shows a decidedly greater sensitivity than either parent, but it is distinctly closer to A maryllis belladonna than to Brunsvigia josephino. In other reactions the starch is the same or practically the same as one parent or the other or both parents, or of some degree of intermediateness, or of less or even very decidedly less sensitivity than in either parent, very commonly of the latter category. In the qualitative reactions it is in certain well-defined respects closer to Amaryllis belladonna than to the other parent, and in others the reverse; but on the whole the inclination is distinctly toward Amaryllis belladonna.

Moreover, forms of gelatinization are seen in the hybrids that are individual. In this hybrid it will be found that in the aggregate the gelatinization phenomena recorded under each reagent incline more or less markedly toward Amaryllis belladonna. With other hybrids the greatest variability of parental relationships may be noted, as, for instance, in Hippeastrum, where it will be found that with one reagent the relationship may be closer to one parent and with another to the other, and more or less marked differences may be noted in the 
hybrids from the same cross (see Brunsdonna); but here again in the final summing up there is usually found to be a distinct majority of the reactions leaning to one or the other parent. It is unfortunate that very frequently the data have not been recorded in accordance with the plan adopted at the outstart of the research so as to leave no doubt in each character or characterphase of the parental relationships of the hybrid, such as was pursued in making the quantitative determinations. Owing to this defect it is necessary to present these summaries in a modified tabular form, and with the view particularly of showing the fluctuating relationships of the hybrids to the parents. In the preparation of the tables that follow (Tables C 1 to $\mathrm{C} 17$ ), the properties of the hybrids in their parental relationships have been considered collectively in designations or groups that are indicated by the divisions of the tables, those of form being taken as one designation, those with a given reagent as one designation, and so on. The plus sign is to be interpreted as meaning that in the final summing up of the data of each designation the hybrid in its unitcharacter and unit-character-phase bears, on the whole, a closer relationship to the parent indicated at the head of the column. The minus sign is, of course, the negative correlative of the former; while the plus-minus sign indicates that the hybrid resembles in degree one as much as the other parent. In the last column the terms excess and deficit mean that a unit-character or unit-characterphase is developed in excess or deficit of parental extremes; individual means that a unit-character or unitcharacter-phase has been discovered in the hybrid that was not observed in either parent.

Certain apparently minor peculiarities have been disregarded in this tabulation. In some instances it is entirely arbitrary whether we regard a given property as being developed in excess or deficit of parental extremes. Thus, if the grains of the hybrid be more irregular, or the resistance to reagents greater, than those of the parents, are we to look upon the difference as being an expression of increased or decreased development? Tentatively, such differences have been taken as representing increased development; and, if there be less irregularity or less resistance, the opposite. It is obvious that these tables indicate merely very grossly certain prominent phases of hybrid and parental relationships, and that the context must be studied therewith in order that the qualitative and quantitative fluctuations of the hybrid in relation to each parent can properly be understood. In the several sets of tables that follow, the symbols 우, $\sigma^{7}$ and $q=\delta$ are used as sex designations to indicate nearer the seed parent, nearer the pollen parent, and equally related to both, respectively. The symbol $\oplus$ in Tables $\mathrm{F}, 1$ to 50 , and $\mathrm{H}, 1$ to 26 , indicates that the reactions are too fast or too slow for satisfactory differentiation, or that because of fluctuations in the courses of gelatinization there is either no satisfactory differentiation or sufficiently definite inclination to either parent. The data of the quantitative reactions are taken from the various tables of the reaction-intensities expressed by the percentage of total starch gelatinized at definite timeintervals that constitute the third section of each summary in Chapter III, and also tabulated in modified arrangement in Section 4 of this chapter. These data have also been presented in the form of charts in Chapter IV.

It is important to note that in the studies of the qualitative reactions the reagents selected varied somewhat in number and kind in the different sets of parents and hybrids, and that in the formulation of these tables the quantitative reactions given are limited to those of the reagents used to elicit the qualitative reactions. Hence, in the summing up in these tables of the relationships of the reactions of the hybrids to those of the parents there may seem to be some discrepancies when the figures are compared with those of Tables E, 1 to 22, F, 1 to 50, and $H, 1$ to 26. For instance, in the quantitative reactions of Brunsdonna sanderoe alba it will be noted that of the 8 reactions with the chemical reagents none is like that of the seed parent, pollen parent, or both parents, 1 is intermediate, 1 is higher than that of either parent, and 6 are lower than those of either parent. When, however, all of the 21 reactions are summed up it is found (Table F, 1) that 4 are the same as those of seed parents, none the same as those of the pollen parent, 1 the same as those of both parents, 5 intermediate, 3 higher than those of the parents, and 13 lower than those of the parents.

The limited quantitative data given in Tables $\mathrm{C} 1$ to $\mathrm{C} 17$ are mainly for comparisons with the qualitative reactions with the same reagents, the data of this kind being tabulated in full in tables $\mathrm{E}, \mathrm{F}$, and $\mathrm{H}$. Limited comment only is necessary in explaining this series of tables.

(a) Brunsdonna sanderce alba (same parentage as follouing hybrid).

TABLE C 1.-Brunsdonna sandera alba.

\begin{tabular}{|c|c|c|c|c|}
\hline \multirow{2}{*}{ Designation, agent, and reagent. } & \multicolumn{2}{|c|}{ Closer, as a whole, to the- } & \multirow{2}{*}{$\begin{array}{l}\text { Excess, deficit, or } \\
\text { individual. }\end{array}$} & \multirow{2}{*}{ Quantitative reactions. } \\
\hline & Seed parent. & Pollen parent. & & \\
\hline 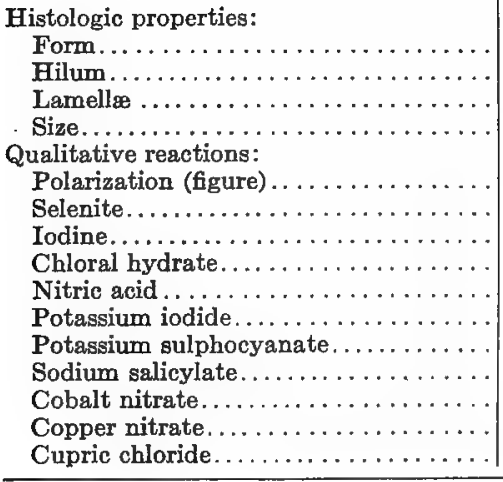 & $\begin{array}{l}+ \\
+ \\
\text { Form, arrange. } \\
+ \\
+ \\
+ \\
+ \\
+ \\
+ \\
+ \\
+ \\
+ \\
+ \\
+\end{array}$ & $\begin{array}{l}\text { - } \\
\text { Number } \\
- \\
- \\
- \\
- \\
- \\
- \\
- \\
- \\
- \\
-\end{array}$ & 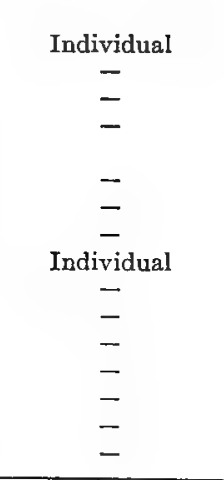 & $\begin{array}{l}\text { Intensity) practically same as } ᄋ \\
\text { Same as o } \\
\text { Much higher than either parent o } \\
\text { Slightly lower than either parent ơ } \\
\text { Very much lower than either parent } ᄋ=\sigma^{7} \\
\text { Very much lower than either parent } ᄋ=\sigma^{7} \\
\text { Intermediate } ᄋ \\
\text { Much lower than either parent } \sigma^{7} \\
\text { Much lower than either parent } \sigma^{7} \\
\text { Much lower than either parent } \sigma^{7}\end{array}$ \\
\hline
\end{tabular}


TABLE C 2.-Hippeastrum.

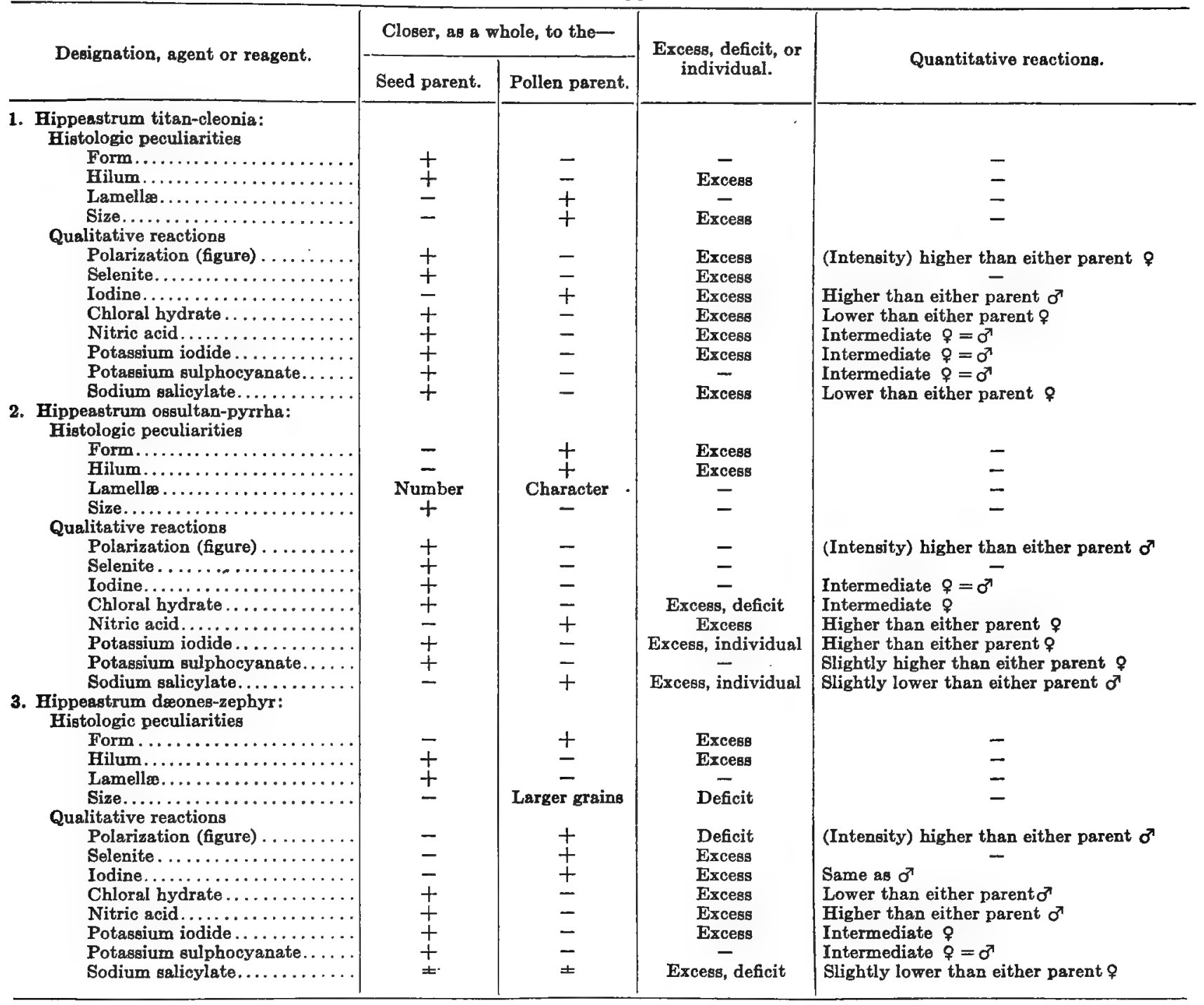

(b) Brunsdonna sanderce (same parentage as preceding hybrid).

The foregoing table is with five differences duplicated by the records of this hybrid. These hybrids differ more in certain particulars (both qualitatively and quantitatively) from each other than do either from their parents or the parents from each other. This hybrid, like its mate, bears, on the whole, a decidedly closer relationship to Amaryllis belladonna than to Brunsvigia josephino, and is closer than the first hybrid to Amaryllis belladonna.

The dissociation of lamellar characteristics (the form and arrangement being closer to one parent, and the number to the other) is very interesting, but by no means an uncommon phenomenon in the starches of hybrids. Moreover, as will be found by reference to the context, similar splitting occurs of the characters of the hilum and in the size of the grains.

That the quantitative and qualitative reactions are also as independent of each other in the direction of their parental relationships is strikingly shown in the table. Throughout the qualitative reactions the hybrids incline to the seed parent, but in the quantitative reactions wide variations are shown in the parental relationships. Thus, in the polarization reactions the first hybrid is the same as the seed parent, while the second is intermediate but closer to the seed parent; in the potassium-iodide reactions both have reactivities lower than those of the parents, the first being closer to the seed parent and the second as close to one as to the other parent; in the sodium-salicylate reactions the first is intermediate but closer to the seed parent, and the second is the same as the seed parent; and in the cobalt reactions both have reactivities lower than those of the parents, but one is closer to the pollen parent while the other is as close to one as to the other parent. Otherwise they are essentially the same in their parental relationships. Curiously, while in the qualitative reactions with chloral hydrate, nitric acid, potassium iodide, potassium sulphocyanate, and sodium salicylate it is closer than the other 
hybrid to Amaryllis belladonna, in the copper-nitrate and cupric-chloride reactions it is not so close as the other hybrid.

Hippeastrum. (Table C 2.)

In comparing these records and keeping in view the botanical closeness of the parents in each case, and also a corresponding closeness of the offspring to the parents, together with the great importance that is commonly attached to intermediateness as a criterion of hybrids, one is struck by (1) the frequency of the development of properties of the hybrid in excess or deficit of parental extremes; (2) the appearance of reactions in the hybrid which were not seen in the parents; and (3) the swinging of hybrid development to one or the other parent in an utterly inexplicable manner. Among the 36 designations of the three sets, in no less than 23 some property or properties were developed in excess of parental extremes, and in 4 there was deficient development. In two instances properties were noted in the hybrid that were not apparent in either parent. The hybrid of the first set is in form closer to the seed parent, but in the second and third sets it is closer to the pollen parent; in hilum, in the first and third sets, closer to the seed parent, but in the second set closer to the pollen parent; in lamellæ, in the first set closer to the pollen parent, in the third set closer to the seed parent, and in the second set closer to the seed parent in number and to the pollen parent in general characters; in size, in the first set closer to the pollen parent, in the second set closer to the seed parent, and in the third set equally like both parents in common size, but like the pollen parent in the larger grains. In polariscopic figures and reactions with selenite, in the first and second sets the hybrids are more like the seed parent, but in the third set the likeness is to the pollen parent. The qualitative reactions with the chemical reagents are full of interest. In the first set, with all five reagents the reactions are, on the whole, closer to those of the seed parent; in the second set those of three. of the reagents (chloral hydrate, potassium iodide, and potassium sulphocyanate) are closer to those of the seed parent, and two (nitric acid and sodium salicylate) closer to those of the pollen parent; and in the third set those of four of the reagents are closer to the seed parent and that of one (sodium salicylate) as close to that of one as to that of the other parent. The relationships, on the whole, are somewhat closer to the seed parent. The quantitative and qualitative reactions show comparatively the most variable relationships.

\section{Hamanthus. (Table C 3.)}

The hybrid in the first set, in form and hilum, is closer to the seed parent; in lamellæ it resembles both parents in equal degree; and in size it is nearer the pollen parent. In the second set, in all four histologic designations, it is nearer the pollen parent. In the polariscopic figures and selenite reactions and in the qualitative reactions with the chemical reagents the resemblance (except the iodine reaction in the second set) is closer to the seed parent. In three instances development in excess of parental extremes, and in one instance individuality, were recorded. The quantitative reactions are most vagarious in their relations to the qualitative reactions. It is of interest to note that the seed parent is the same in both sets and that in both

TABLE C 3.-Homanthus.

\begin{tabular}{|c|c|c|c|c|}
\hline \multirow{2}{*}{ Designation, agent and reagent. } & \multicolumn{2}{|c|}{ Closer, as a whole, to the- } & \multirow{2}{*}{$\begin{array}{l}\text { Excess, deficit, or } \\
\text { individual. }\end{array}$} & \multirow{2}{*}{ Quantitative reactions. } \\
\hline & Seed parent. & Pollen parent. & & \\
\hline 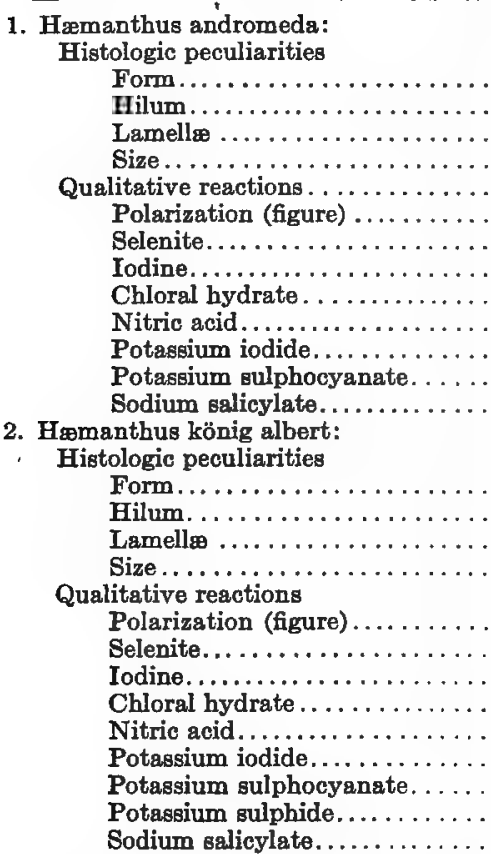 & $\begin{array}{l}+ \\
+ \\
+ \\
+ \\
+ \\
+ \\
+ \\
+ \\
+ \\
+ \\
+ \\
+ \\
- \\
+ \\
+ \\
+ \\
+ \\
+ \\
+ \\
+ \\
+\end{array}$ & $\begin{array}{l}- \\
\overline{-} \\
- \\
+ \\
\bar{t} \\
= \\
\overline{-} \\
=\end{array}$ & $\begin{array}{c}\text { Excese, individual } \\
= \\
= \\
= \\
= \\
= \\
= \\
= \\
- \\
\text { Excess } \\
= \\
- \\
= \\
= \\
= \\
\text { Excess } \\
= \\
= \\
= \\
-\end{array}$ & 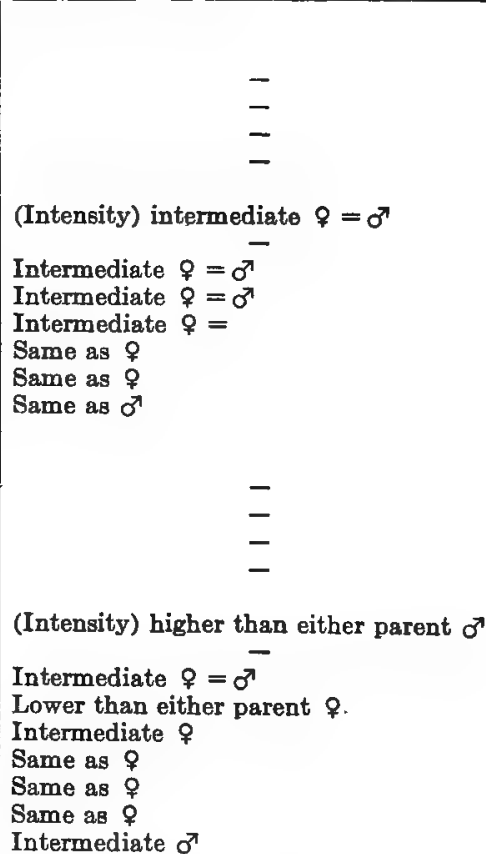 \\
\hline
\end{tabular}


hybrids there is clear evidence of biparental inheritance. The relationships, on the whole, are distinctly closer to the seed parent.

\section{Crinium. (Table C 4).}

The parents in each of these three sets of Crinums are recognized species that belong to the hardy and tender groups-C. moorei and $C$. longifolium to the former and $C$. zeylanicum to the latter. In each set the hybrid shows very markedly in each of the designations biparental inheritance, varying in degree in relation to the various unit-characters and unit-character-phases. Occasional individualities of the hybrids are recorded, and excessive and deficient developments are noted rarely in the first and second sets, but not infrequently in the third set. In the first and third sets $C$. moorei was a parent-in the first the seed parent, and in the third

TaBLE C 4.-Crinum

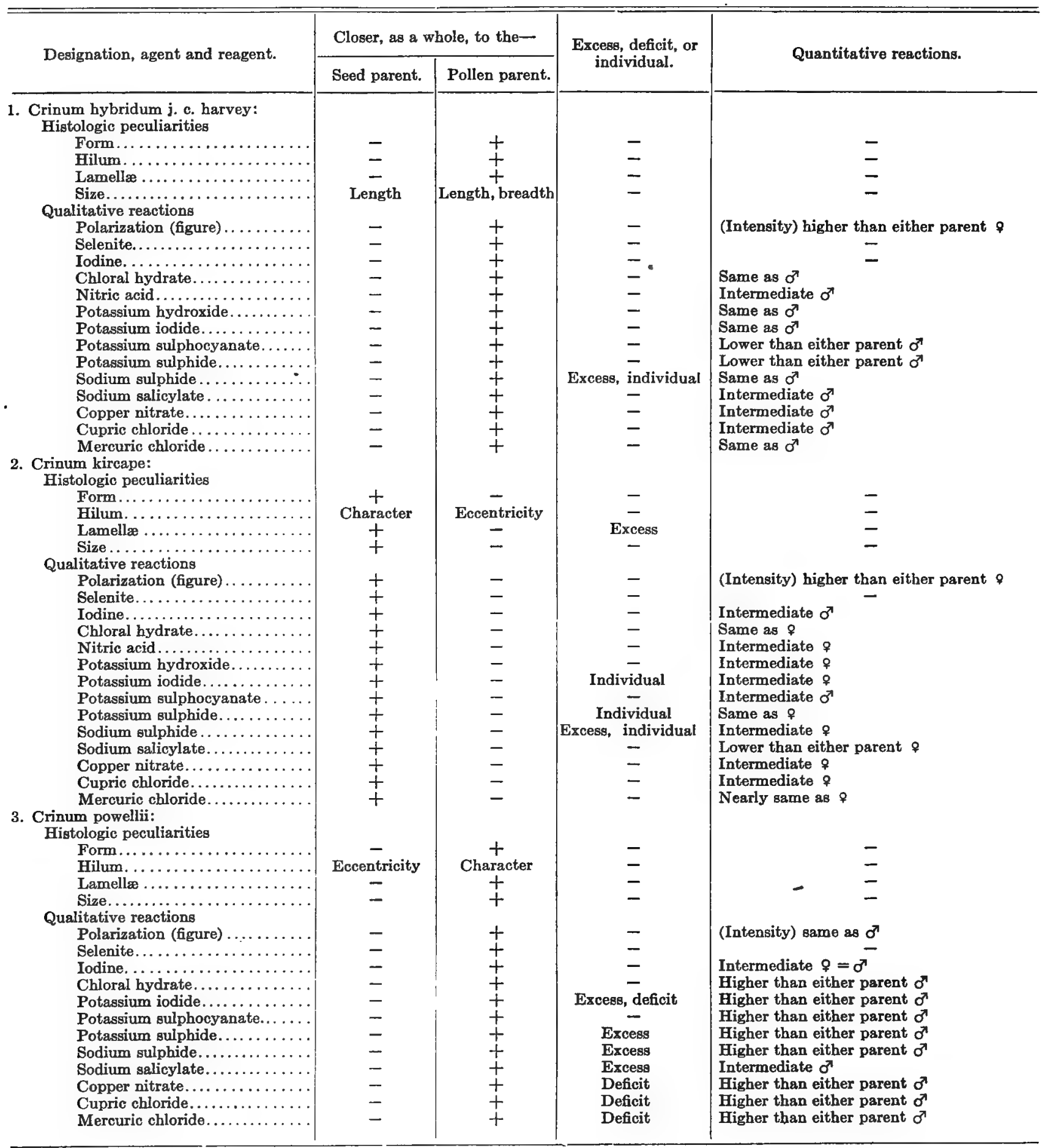


the pollen parent. In the histologic properties and qualitative reactions, in the first set the hybrid shows throughout the designations a markedly closer relationship, on the whole, to $C$. zeylanicum (the pollen parent) than to C. moorei (the seed parent); while in the third set the hybrid shows a closer relationship, on the whole, to $C$. moorei (the pollen parent) than to C. longifolium (the seed parent). In the first set $C$. moorei (hardy) is crossed with C. zeylanicum (tender), the two species being well separated, the hybrid leaning strongly to the pollen parent $C$. zeylanicum. In the second set $C$. zeylanicum (tender) is crossed with C. longifolium (hardy), the species are well separated, the hybrid leaning strongly, but less strongly than in the preceding set, to $C$. zeylanicumi In the third set $C$. longifolium (hardy) is crossed with $C$. moorei (hardy), the species being comparatively close, the hybrid tending to be, on the whole, distinctly closer to $C$. moorei (the pollen parent) than to C. longifolium. The shifting of parental potency in relation to hybrid development is of interest, C. zeylanicum being the more potent as both pollen and seed parent in relation to $C$. moorei and $C$. longifolium, respectively, and $C$. moorei being more potent than C. longifolium. The quantitative in comparison with the qualitative reactions are of great interest. In the first set there is strong leaning to the pollen parent; in the second set to intermediateness and to the seed rather than to the pollen parent; and in the third set almost wholly to the pollen parent, in each the inclinations being in harmony with the leanings, on the whole, of the qualitative reactions.

\section{Nerine. (TABle C 5.)}

The first two hybrids vary in a most interesting manner in their resemblances and differences in regard to each other and to their parents; and they differ from each other almost as much as they do from the parents, or as the parents differ from each other. Biparental inheritance showing varying degrees of influence of each parent is manifest throughout the designations. The hybrid $N$. queen of roses differs in the form of the grains from the other hybrid by a greater resemblance to $N$. crispa because of its grains haring a more regular form, more aggregates, and more compound grains. The hybrids more closely resemble each other than either parent in the character of the hilum, and both are closer in this feature of $N$. elegans than to $N$. crispa. The lamellæ of $N$. queen of roses are closer than those of the other hybrid to those of $N$. crispa, while those of $N$. dainty maid are closer to those of the other parent. The size of the grains of $N$. queen of roses is less than that of the other hybrid, but it is closer to that of the latter than the latter is to either parent, yet not so close as is that of $N$. dainty maid to that of $N$. elegans. In the polari-

TABle C 5.-Nerine.

\begin{tabular}{|c|c|c|c|c|}
\hline \multirow{2}{*}{ Designation, agent and reagent. } & \multicolumn{2}{|c|}{ Closer, as a whole, to the } & \multirow{2}{*}{$\begin{array}{l}\text { Excess, deficit, or } \\
\text { individual. }\end{array}$} & \multirow{2}{*}{ Quantitative reactions. } \\
\hline & Seed parent. & Pollen parent. & & \\
\hline 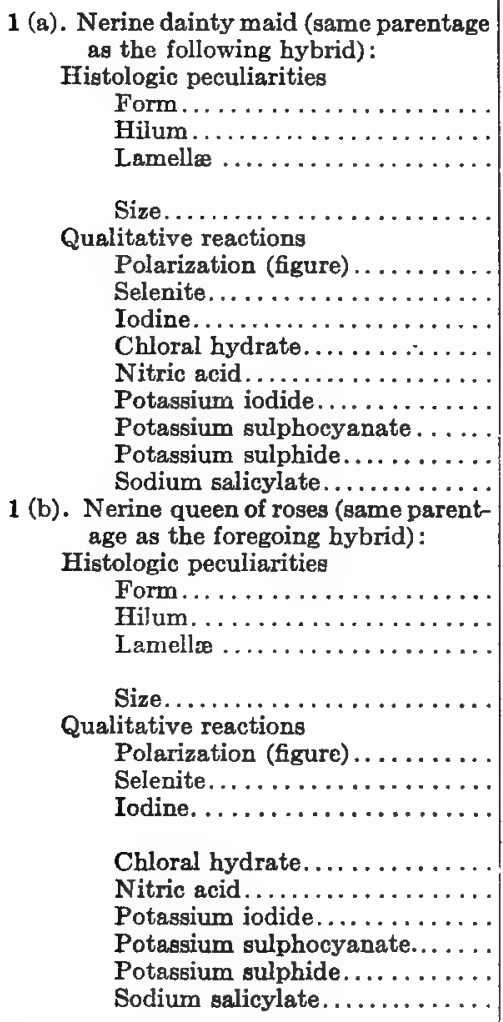 & $\begin{array}{l}- \\
- \\
\text { Character, } \\
\text { arrangement } \\
- \\
- \\
- \\
- \\
- \\
- \\
- \\
- \\
- \\
\\
\text { Distinctness } \\
\text { Character, } \\
\text { arrangement } \\
- \\
\text { - } \\
\text { Gelatinized } \\
\text { grains } \\
+ \\
\text { - } \\
\text { - } \\
\text { + } \\
\text { + }\end{array}$ & $\begin{array}{c}+ \\
+ \\
\text { Fineness } \\
+ \\
+ \\
+ \\
+ \\
+ \\
+ \\
+ \\
+ \\
+ \\
+ \\
\text { Fissuration } \\
\text { Number } \\
+ \\
+ \\
+ \\
+ \\
\text { Raw grains } \\
+ \\
+ \\
+ \\
+ \\
+ \\
+ \\
+ \\
+ \\
+ \\
+ \\
+\end{array}$ & $\begin{array}{c}- \\
- \\
\text { Excesa } \\
- \\
- \\
- \\
- \\
- \\
- \\
- \\
- \\
-\end{array}$ & $\begin{array}{l}\text { Same as } \sigma^{7} \\
\text { (Intensity) lower than either parent ơ } \\
\text { Higher than either parent } \sigma^{7} \\
\text { Intermediate } \%=\sigma^{7} \\
\text { Intermediate } \% \\
\text { Higher than either parent } \% \\
\text { Slightly higher than either parent } \sigma^{7} \\
\text { Higher than either parent } \sigma^{7}\end{array}$ \\
\hline
\end{tabular}


scopic figure and selenite reactions $N$. queen of roses is closer than $N$. dainty maid to $N$. elegans. In the iodine reactions with the raw grains $N$. queen of roses is closer than $N$. dainty maid to $N$. elegans; but with the gelatinized grains they closely resemble those of $N$. crispa, while those of the other hybrid resemble those of the other parent. In the qualitative reactions with chloral hydrate both are closer to $N$. elegans than to $N$. crispa, but $N$. queen of roses is not so close to $N$. crispa as is $N$. dainty maid to $N$. elegans, and there is nearly as much difference between the hybrids as there is between $N$. queen of roses and $N$. elegans. In the reactions of nitric acid, potassium iodide, potassium sulphocyanate, and potassium sulphide the hybrids are close to one another, and $N$. queen of roses is not so close as is $N$. dainty maid to $N$. elegans. In the sodium-salicylate reactions $N$. queen of roses is not so close to $N$. crispa as is $N$. dainty maid to $N$. elegans, and there is nearly as much difference between the hybrids as there is between $N$. queen of roses and $N$. elegans. The reactions of chloral hydrate and sodium salicylate are of especial interest because of the reversal of the hybrid and parental relationships, $N$. queen of roses being closer to $N$. elegans, and $N$. dainty maid closer to $N$. crispa, in both reactions; while both hybrids incline, as a whole, to $N$. elegans, $N$. dainty maid is closer than the other hybrid. The quantitative reactions bear the most variable relationships to the qualitative reactions, showing, as in preceding sets, the independence of qualitative and quantitative reactions with the same agent and reagent.

TABle C 5.-Nerine.-Continued.

\begin{tabular}{|c|c|c|c|c|}
\hline \multirow{2}{*}{ Designation, agent and reagent. } & \multicolumn{2}{|c|}{ Closer, as a whole, to the- } & \multirow{2}{*}{$\begin{array}{l}\text { Excess, deficit, or } \\
\text { individual. }\end{array}$} & \multirow{2}{*}{ Quantitative reactions. } \\
\hline & Seed parent. & Pollen parent. & & \\
\hline 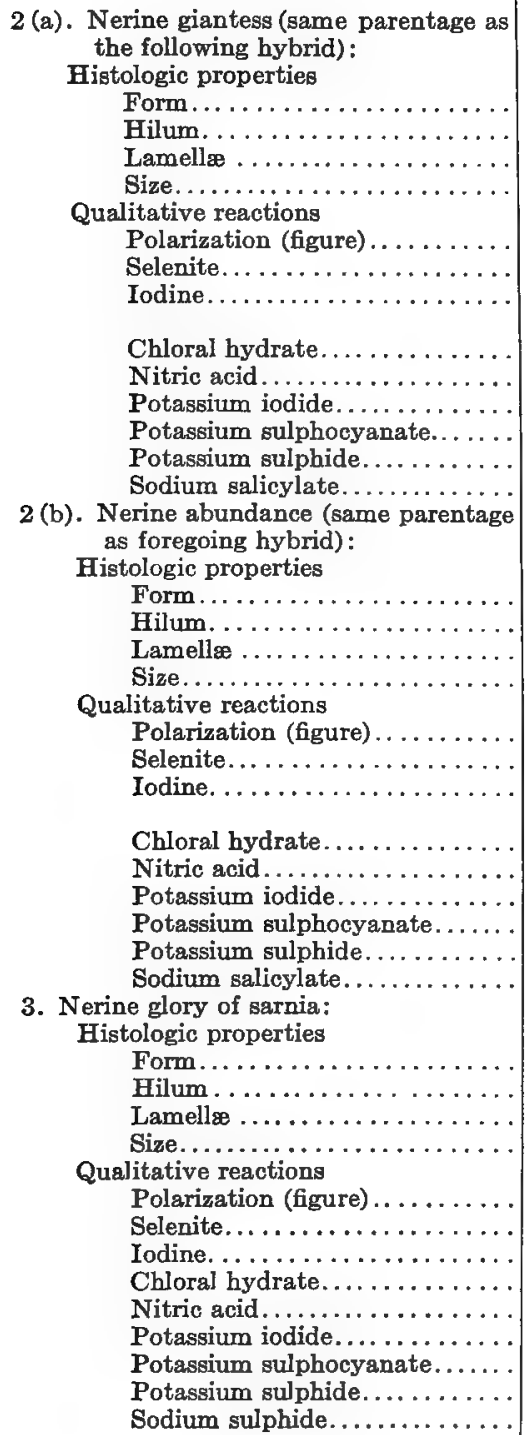 & $\begin{array}{c}- \\
\text { Character } \\
- \\
+ \\
+ \\
+ \\
\text { Raw and gela } \\
\text { grains } \\
+ \\
+ \\
+ \\
+ \\
+ \\
+ \\
+ \\
+ \\
+ \\
+ \\
+ \\
+ \\
+ \\
+ \\
+ \\
+ \\
+ \\
+ \\
+\end{array}$ & 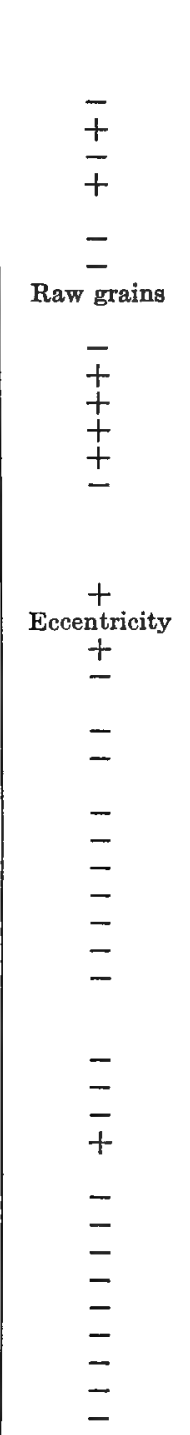 & $\begin{array}{c}- \\
- \\
- \\
- \\
- \\
- \\
- \\
- \\
- \\
- \\
- \\
- \\
\text { Deficit } \\
- \\
\text { Deficit, excess } \\
- \\
- \\
- \\
- \\
- \\
- \\
- \\
- \\
- \\
- \\
- \\
- \\
- \\
- \\
- \\
- \\
- \\
-\end{array}$ & 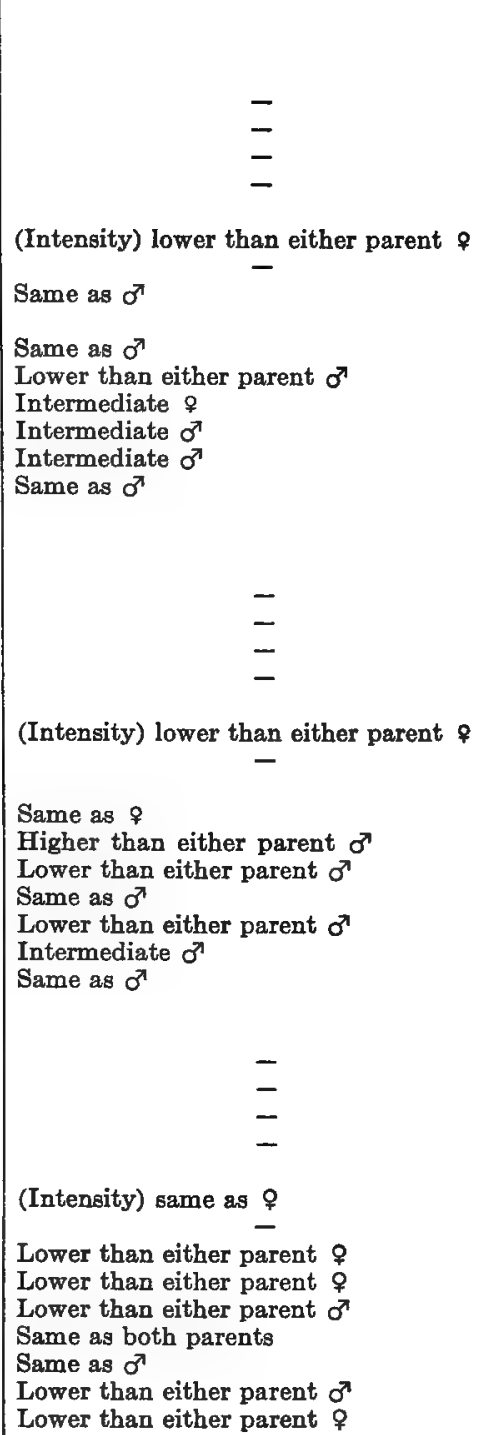 \\
\hline
\end{tabular}


The second two hybrids differ almost as much from | are very close to one another. In hilum, $N$. giantess each other as they do from their parents, or as the parents differ from each other. Biparental inheritance is manifest in all of the designations, varying differences in the degrees of influence of one or the other parent being quite apparent throughout. In form, the grains of $N$. giantess incline to $N$. bowdeni, and those of $N$. abundance to the other parent; but the grains of the hybrids

is closer to $N$. sarniensis var. corusca major; whereas in $N$. abundance it inclines in character to $N$. bowdeni, but in eccentricity to the other parent. In character, $N$. abundance is nearer than $N$. giantess to $N$. bowdeni. In both lamellæ and size there are reversals in both hybrids of parental relationships. In size $N$. abundance is nearer than $N$. giantess to $N$. bowdeni. In the

\begin{tabular}{|c|c|c|c|c|}
\hline \multirow{2}{*}{ Designation, agent and reagent. } & \multicolumn{2}{|c|}{ Closer, as a whole, to the- } & \multirow{2}{*}{$\begin{array}{l}\text { Excess, deficit, or } \\
\text { individual. }\end{array}$} & \multirow{2}{*}{ Quantitative reactions. } \\
\hline & Seed parent. & Pollen parent. & & \\
\hline 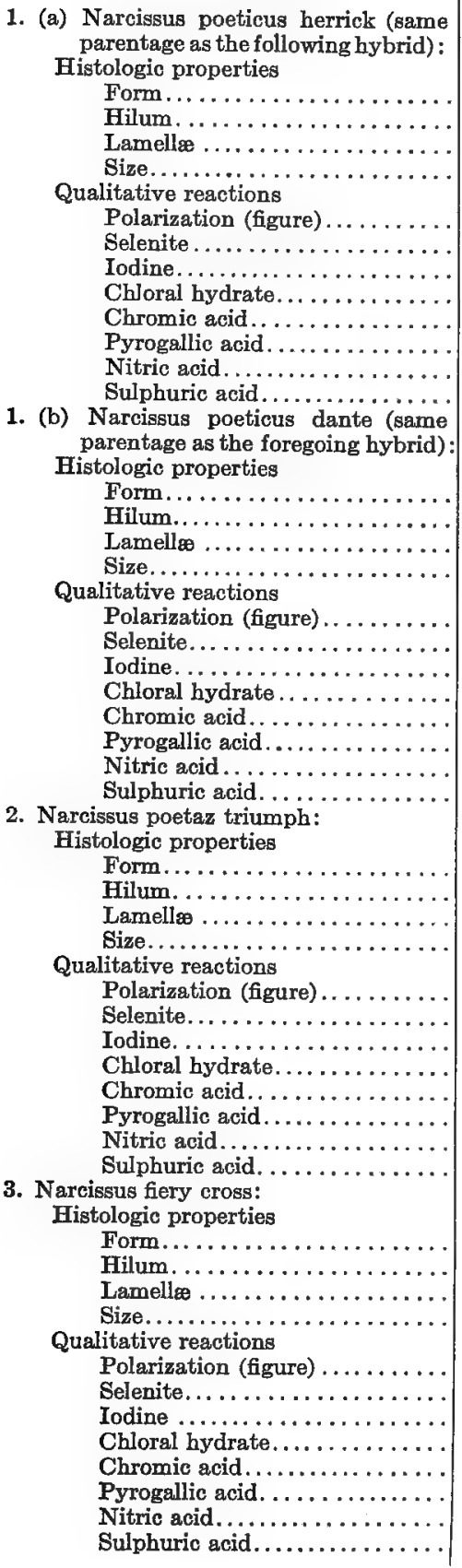 & $\begin{array}{c}+ \\
\text { Character } \\
+ \\
+ \\
= \\
= \\
+ \\
+ \\
+ \\
+ \\
+\end{array}$ & $\begin{array}{c}- \\
\text { Character } \\
- \\
+ \\
+ \\
+ \\
= \\
= \\
= \\
- \\
\text { Eicentricity } \\
= \\
- \\
+ \\
\pm \\
\pm \\
= \\
= \\
= \\
-\end{array}$ & $\begin{array}{c}\text { Deficit } \\
- \\
- \\
= \\
= \\
- \\
\text { Deficit } \\
= \\
- \\
\text { Excess } \\
\text { Excess } \\
\text { Excess } \\
- \\
= \\
= \\
= \\
\overline{-}\end{array}$ & 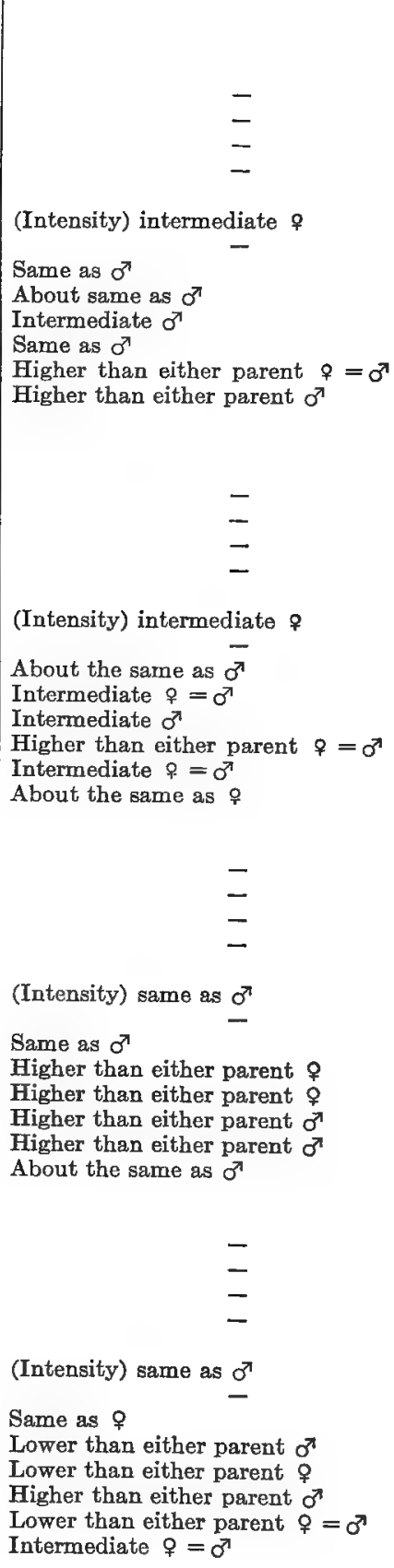 \\
\hline
\end{tabular}


TABLE C 6.-Narcissus.-Continued.

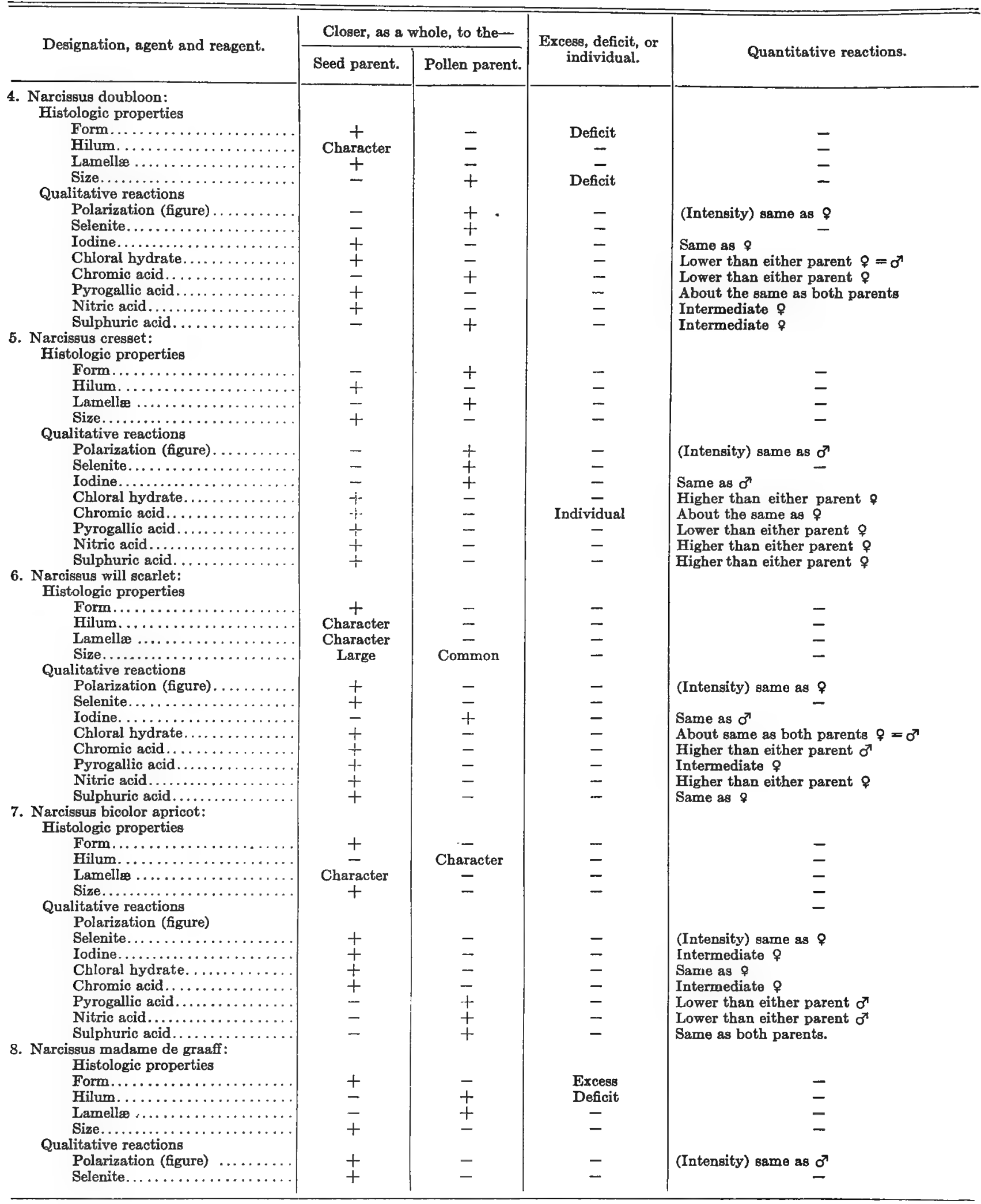


TABle C 6.-Narcissus.-Continued.

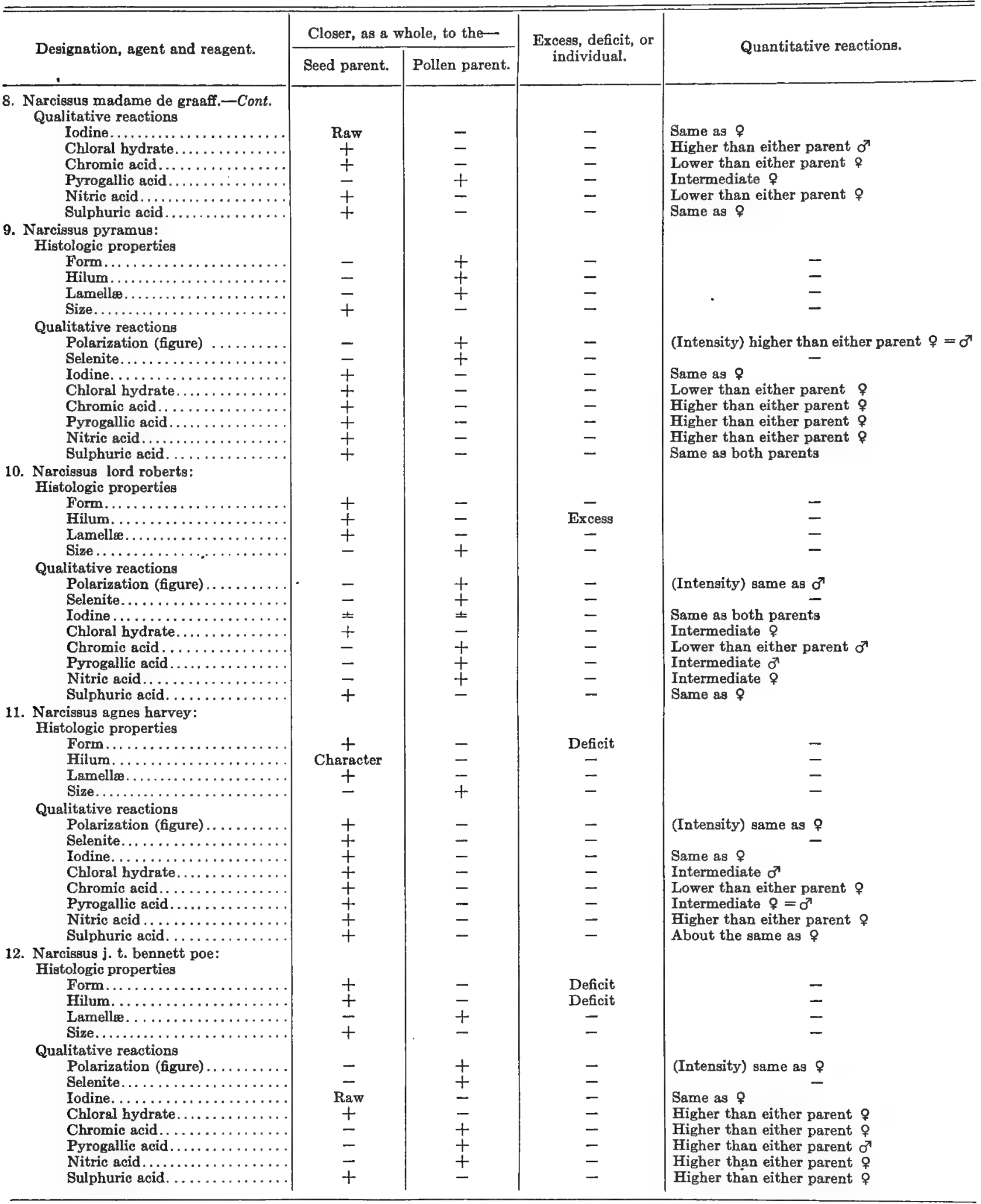


polariscopic reactions both incline to $N$. bowdeni, but $N$. abundance is not so close as $N$. giantess. In the iodine reactions with the raw grains the hybrids are as well separated from each other as they are from the parents. In $N$. giantess the gelatinized grains behave more like those of $N$. bowdeni, while the raw grains lean to the other parent; but in the other hybrid there was not found any difference in the parental inclinations of both gelatinized and raw grains. The qualitative reactions with the chemical reagents show curious differences, $N$. giantess in only two of the six reactions inclining to $N$. bowdeni and in the other four to the other parent; while the other hybrid inclines all six reactions to $N$. bowdeni. In the reactions of chloral hydrate, potassium sulphocyanate, and sodium salicylate $N$. abundance is closer than $N$. giantess to $N$. bowdeni; and in the potassium-sulphocyanate reaction the hybrids are closer to each other than to either parent. In the nitric-acid reaction $N$. giantess is closer to $N$. sarniensis var. corusca major than is $N$. abundance to $N$. bowdeni, but the hybrids themselves are very close. In the potassium-iodide reaction $N$. giant'ess leans to $N$. sarniensis var. corusca major, while the other hybrid inclines to the other parent; but the hybrids are closer to each other than is either to the parent to which it is the more closely related. The quantitative and qualitative reactions show most interesting differences and independence.

It will be seen by an examination of the preceding table how variable and absolutely unpredictable is the shifting of hybrid properties toward one or the other parent. Biparental inheritance in each of the designations is manifest; but in some instances hybrid and parents are very closely alike, and in others the hybrids are more alike or more different than are the parents, or they differ more from the parents or resemble more closely one or the other parent than do the parents themselves appear to be the same or different. With the first pair of hybrids, $N$. dainty masd inclines in the histologic properties and qualitative reactions, with the exception of the character and arrangement of the lamellæ, in every designation to $N$. elegans: while its mate, $N$. queen of roses, leans in only about two-thirds of the designations to the same parent. With the second pair, N. giantess inclines in about one-half of the designations to $N$. bowdeni, while $N$. abundance inclines almost wholly to the same parent. With the last hybrid, $N$. glory of sarnia, the inclination with the exception of a single designation is to $N$. sarniensis var. corusca major. Excess and deficit of development are rarely noted, and no individuality of the hybrid in any case was recorded. In the quantitative reactions there is obvious independence of the qualitative reactions, inasmuch as they may or may not correspond. In $N$. dainty maid, while in both histologic properties and qualitative reactions the inclination is positively to the pollen parent, in the quantitative reactions there is a tendency to intermediateness, and to the pollen parent. In $N$. queen of roses there is an inclination of about two-thirds of the histologic properties and qualitative reactions to the pollen parent, while in the quantitative reactions there is more of a leaning to the pollen than to the seed parent. In $N$. giantess about one-half of the histologic properties and qualitative reactions lean to the seed parent, in the quantitative reactions six of the eight reactions lean to the pollen parent. In $N$. abundance the histologic properties and qualitative reactions incline almost wholly to the seed parent, in the quantitative reactions six of the eight incline to the pollen parent. In $N$. glory of sarnia the histologic properties and qualitative reactions incline almost wholly to the seed parent and the quantitative reactions incline equally to each of the two parents.

\section{Narcissus. (Table C 6.)}

The first two hybrids, while showing throughout the various designations biparental inheritance, usually bear a closer relationship to $N$. poeticus poetarum than to $N$. poeticus ornatus; and on the whole are closer to one another than to either parent. It is strange that while $N$. poeticus herrick is in form, hilum, and lamellæ closer to $N$. poeticus ornatus than to the other parent, the relationship in size and all other designations is closer to $N$. poeticus poetarum. $N$. poeticus dante is in form closer. to $N$. poeticus ornatus, but in all other designations closer to the other parent. In form both hybrids are closer to $N$. poeticus ornatus, but $N$. poeticus herrick. is the closer of the two. In hilum and lamellæ, $N$. poeticus herrick shows as close relationship to $N$. poeticus ornatus as does $N$. poeticus dante to $N$. poeticus poetarum. In size, $N$. poeticus herrick is closer than $N$. poeticus dante to $N$. poeticus poetarum. In both polariscopic figure and selenite reactions both hybrids are closer, and in equal degree, to $N$. poeticus poetarum. In the iodine reactions the hybrids do not differ and are therefore equally close to $N$. poeticus poetarum. Throughout the qualitative chemical reagent designations the hybrids are closer to $N$. poeticus poetarum. In the chloral-hydrate and nitric-acid reactions $N$. poeticus dante is closer than $N$. poeticus herrick to $N$. poeticus poetarum; but in the chromic-acid and pyrogallicacid reactions the reverse. Only rare records of deficient development were recorded; in no instance was there excess of development or individuality. In the quantitative reactions $N$. poeticus herrick is mid-intenmediate or shows a closer relationship to the pollen parent; while $N$. poeticus dante is mid-intermediate in three of the seven reactions and shows a closer relationship in two to the seed parent, and in two to the pollen parent. It is of interest to note that while in the qualitative reactions both hybrids are throughout very much closer to the pollen parent than to the seed parent, in the quantitative reactions the first leans markedly to the pollen parent and the second to one as much as to the other parent.

There is seen throughout the designations of the various sets of Narcissi the same swinging of hybrid development to one or the other parent, the independence of each unit-character and unit-character-phase of every other in its direction and degree of development, the absolute impossibility of forecasting the parental relationship of any designation, and the usually close relationship of the hybrid in its properties, as a whole, to one or the other parent, as is evident in preceding sets. Special features of the Narcissi group are attached to the relative potencies of cerbain of the parents that occur in a number of sets, and to the hybrid $N$. madame de graaff, which in two sets is the pollen parent. $N$. poeticus ornatus is the seed parent in Set 1 and the pollen parent in Sets 2,3 , and 4 . As the seed parent, it exhibits 
Table C 7.-Lilium.

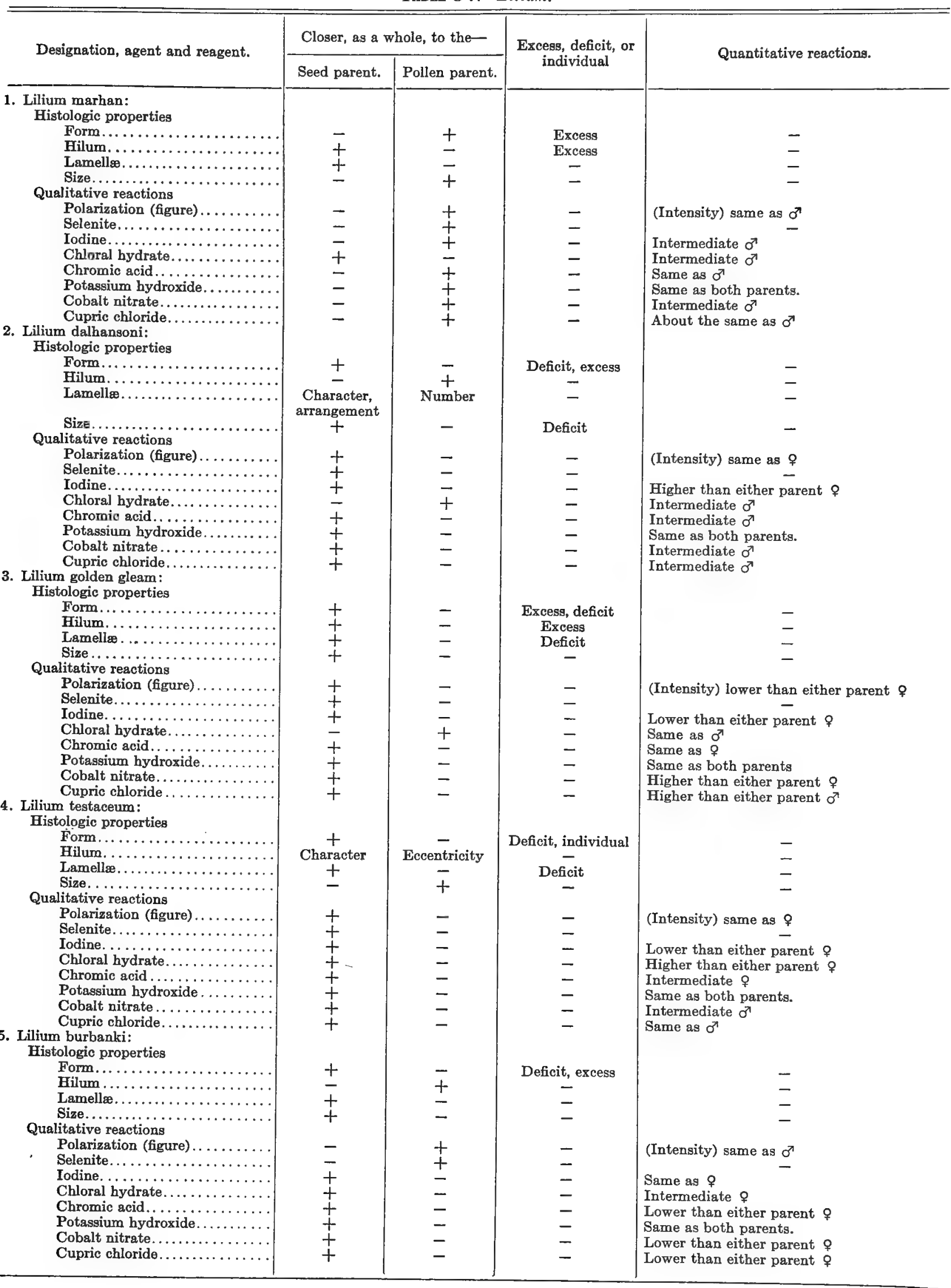


very much less influence on the properties of the hybrids than the pollen parent; in Sets 2 and 3 , as the pollen parent, it is less effective than the seed parent; and in Set 4 it is about equally effective as the seed parent. $N$. poeticus poetarum appears in Sets 1,5 , and 6 as the pollen parent. In Set 1 it greatly dominates the seed parent in its influence; in Set 5 it is of somewhat less potency than the other parent; and in Set 6 it is almost completely dominated by the seed parent. $N$. abscissus is the seed and the pollen parent, respectively, in Sets 7 and 8 . In the former, it somewhat dominates the pollen parent, and in the latter it is distinctly subordinate to the seed parent. $N$. triandrus albus is the pollen parent in Sets 11 and 12, in the former it being almost wholly subordinate, and in the latter of about equal value to the other parent, in influencing the properties of the offspring. $N$. madame de graaff is of especial interest because of its being a hybrid in Set 8 , and the seed

\begin{tabular}{|c|c|c|c|c|}
\hline \multirow{2}{*}{ Designation, agent and reagent. } & \multicolumn{2}{|c|}{ Closer, as a whole, to the- } & \multirow{2}{*}{$\begin{array}{l}\text { Excess, deficit, or } \\
\text { individual. }\end{array}$} & \multirow{2}{*}{ Quantitative reactions. } \\
\hline & Seed parent. & Pollen parent:- & & \\
\hline 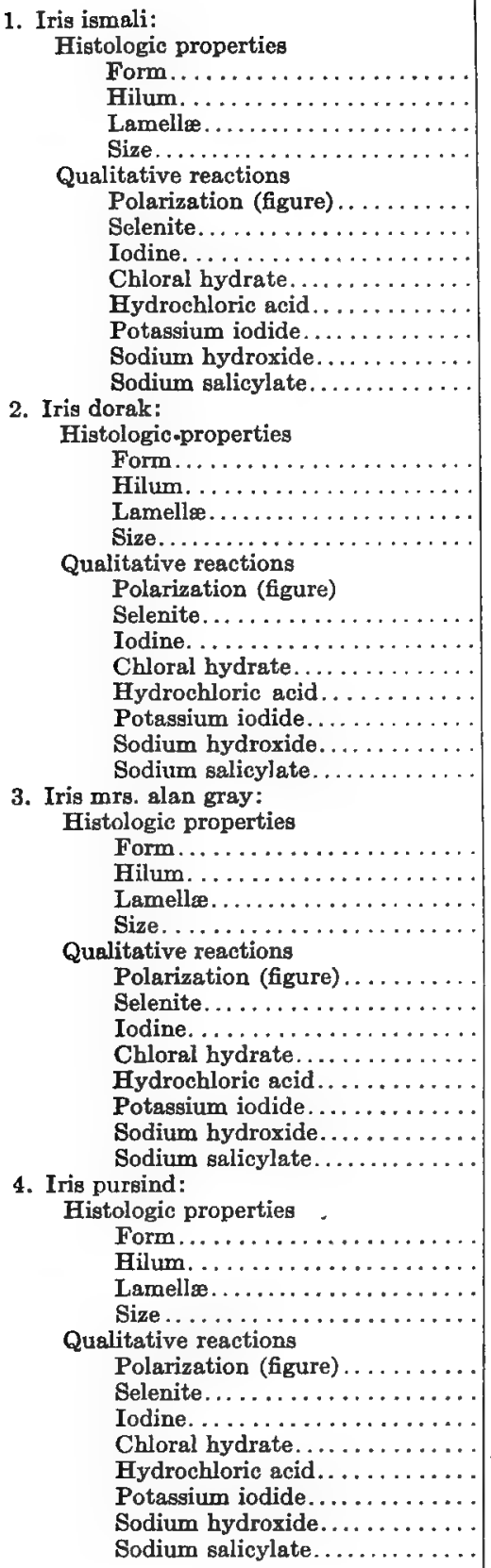 & $\begin{array}{c}- \\
- \\
+ \\
+ \\
+ \\
+ \\
+ \\
+ \\
\text { Character } \\
\text { Number } \\
+ \\
+ \\
+ \\
+ \\
+ \\
+ \\
+ \\
+ \\
+ \\
+ \\
+ \\
+ \\
+ \\
+ \\
+ \\
+ \\
+ \\
+ \\
+ \\
+ \\
+ \\
+ \\
+ \\
+ \\
+ \\
+ \\
+ \\
+ \\
+ \\
+ \\
+ \\
+ \\
+ \\
+ \\
+ \\
+ \\
+ \\
+ \\
+ \\
+ \\
+ \\
+ \\
+ \\
+ \\
+ \\
+ \\
+ \\
+ \\
+ \\
+ \\
+ \\
+\end{array}$ & 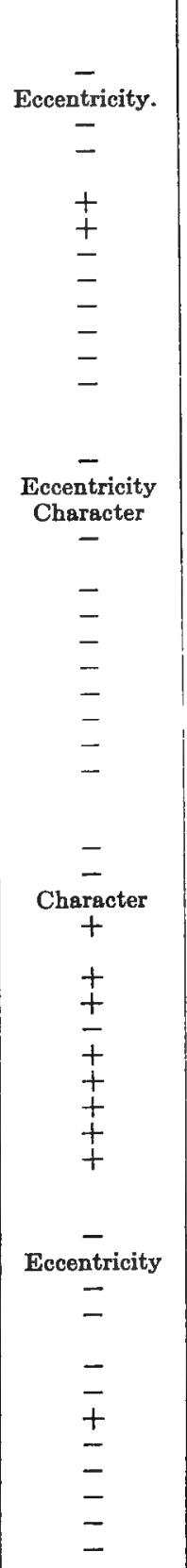 & 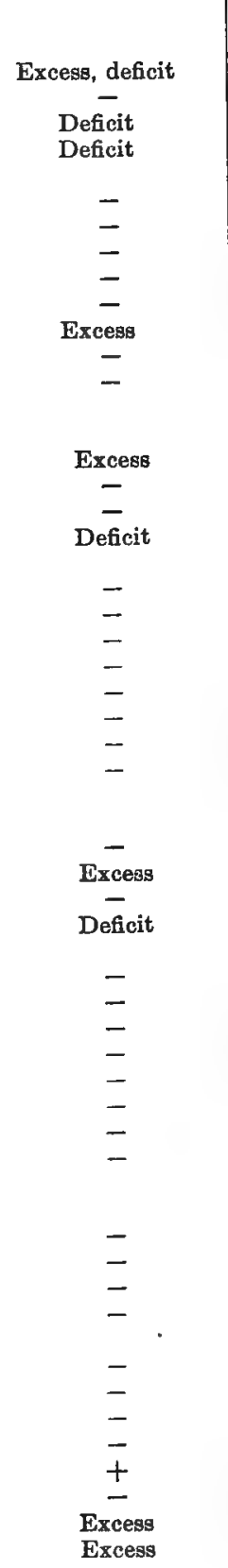 & $\begin{array}{l}\text { (Intengity) lower than either parent } \sigma^{7} \\
\text { Higher than either parent } \% \\
\text { Higher than either parent } \sigma^{7} \\
\text { Lower than either parent } \sigma^{7} \\
\text { Lower than either parent } \sigma^{\top} \\
\text { Lower than either parent }{ }^{7}=\sigma^{\nearrow} \\
\text { Higher than either parent } \sigma^{\top}\end{array}$ \\
\hline
\end{tabular}


TABuE C 9.-Gladiolus.

\begin{tabular}{|c|c|c|c|c|}
\hline \multirow{2}{*}{ Designation, agent and reagent. } & \multicolumn{2}{|c|}{ Closer, as a whole, to the- } & \multirow{2}{*}{$\begin{array}{l}\text { Excess, deficit, or } \\
\text { individual. }\end{array}$} & \multirow{2}{*}{ Quantitative reactions. } \\
\hline & Seed parent. & Pollen parent. & & \\
\hline 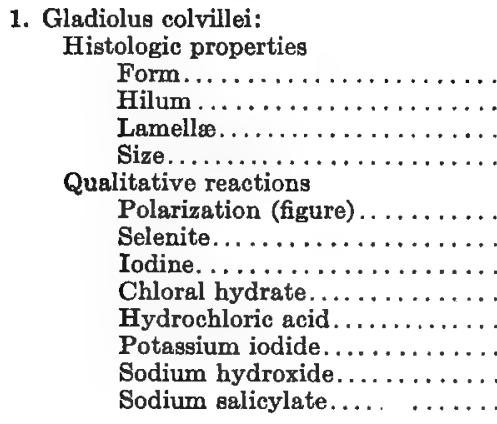 & $\begin{array}{l}+ \\
\text { Character } \\
\pm \\
+ \\
+ \\
+ \\
+ \\
+ \\
+ \\
+ \\
+ \\
+\end{array}$ & $\begin{array}{l}\text { Eccentricity } \\
\pm \\
- \\
- \\
= \\
= \\
= \\
= \\
=\end{array}$ & $\begin{array}{c}- \\
\text { Excess } \\
- \\
- \\
\overline{-} \\
\text { - } \\
\text { Individual } \\
- \\
- \\
-\end{array}$ & $\begin{array}{l}\text { - } \\
\text { (Intensity) intermediate } \% \\
\text { Lower than either parent o } \\
\text { Lower than either parent o } \\
\text { Lower than either parent } \% \\
\text { Lower than either parent o } \\
\text { Lower than either parent } \% \\
\text { Lower than either parent } q\end{array}$ \\
\hline
\end{tabular}

parent in Sets 9 and 10. As a hybrid it exhibits markedly biparental inheritance in all of the designations in varying degrees in relation to one or the other parent, but leaning, on the whole, strongly to the seed parent; not exhibiting any notable peculiarity that is not observed in one or the other parent, nor showing any development in excess or deficit of parental development, except in certain histologic features of minor character. As a seed parent it shows in Set 9 less potency, and in Set 10 about equal potency, compared with the other parent in determining the properties of the hybrid. $N$. madame de graaff shows in its qualitative reactions with the various chemical reagents the peculiar processes of gelatinization that were recorded in the reactions of one parent or both parents; and the processes of this hybrid are manifested in its offspring in a manner not distinguishable from that which on general principles should be expected were it a species or a variety and not a hybrid.

The quantitative reactions bear to the histologic properties and qualitative reactions the most variable relationships in their parental leanings.

\section{Liliom. (Table C 7.)}

In histologic properties and qualitative reactions L. marhan bears in three-fourths of its designations a closer relationship to the pollen parent. In form and size of the grains the relationship is closer to the pollen parent; but in hilum and lamellæ the reverse. Apart from the chloral hydrate reaction, which is closer to the seed parent, all of the qualitative reactions are closer to the pollen parent. L. dalhansoni in form, size, character, and arrangement of the lamellæ is closer to the seed parent, but in hilum and number of the lamellæ is closer to the pollen parent. In only the chloralhydrate reaction is the hybrid closer in the qualitative reactions to the pollen parent, and in the others closer to the seed parent, the opposite to what was noted in the first hybrid. Each of these hybrids has the same pollen parent, but there is an almost entire reversal of the parental relationships in the various designations. In $L$. golden gleam the relationship is, with the single exception of the chloral-hydrate reaction, closer to the seed parent. The pollen parent of $L$. marhan is the same as the seed parent of $L$. golden gleam, the hybrid relationships of each being closer to the seed parent, $L$. maculatum and $L$. tenuifolium, respectively. $L$. testaceum in form and in character of the hilum and lamellæ is closer to the seed parent, but in eccentricity of the hilum and in size it is closer to the pollen parent. In all of the qualitative reactions it is shown to be closer

TaBle C 10.-Tritonia.

\begin{tabular}{|c|c|c|c|c|}
\hline \multirow{2}{*}{ Designation, agent and reagent. } & \multicolumn{2}{|c|}{ Closer, as a whole, to the- } & \multirow{2}{*}{$\begin{array}{l}\text { Excess, deficit, or } \\
\text { individual. }\end{array}$} & \multirow{2}{*}{ Quantitative reactions. } \\
\hline & Seed parent. & Pollen parent. & & \\
\hline 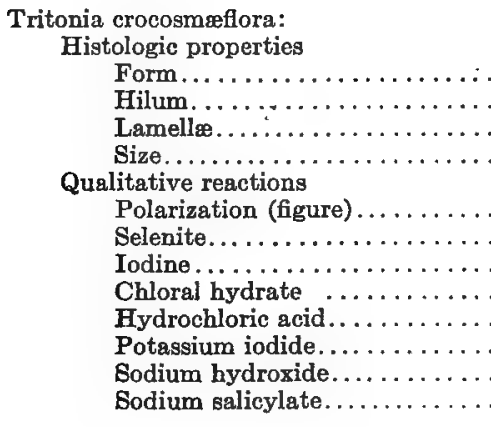 & $\begin{array}{l}- \\
\text { Eccentricity } \\
+ \\
+ \\
+ \\
+ \\
+ \\
= \\
= \\
= \\
-\end{array}$ & $\begin{array}{l}+ \\
\text { Character } \\
- \\
- \\
- \\
= \\
- \\
+ \\
+ \\
+ \\
+ \\
+\end{array}$ & $\begin{array}{l}- \\
\overline{-} \\
- \\
= \\
= \\
= \\
= \\
=\end{array}$ & 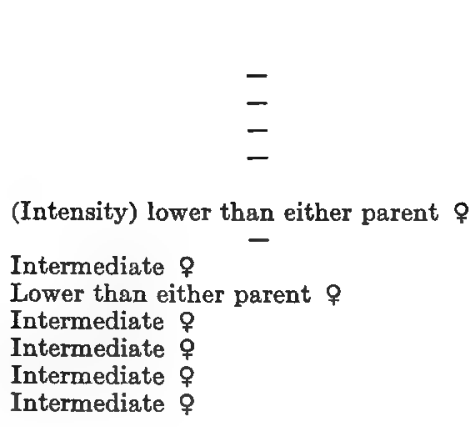 \\
\hline
\end{tabular}


to the seed parent. L. burbanki in form, lamellæ, and size is closer to the seed parent, but in hilum closer to the pollen parent. Except the polariscopic figure and selenite reaction it is closer in all of the qualitative designations to the seed parent. Excess and deficit of development are recorded only among the histologic properties, and no individuality is noted in any of the five hybrids in any of the designations.
The quantitative reactions bear most variable and independent relationships to the qualitative reactions in each of the sets of parents and hybrid.

\section{IRIS. (TABLE C 8.)}

I. ismali inclines to the seed parent in all of the designations of histologic properties and qualitative reactions, except in eccentricity of the hilum, polariscopic

TABLE C 11.-Begonia.

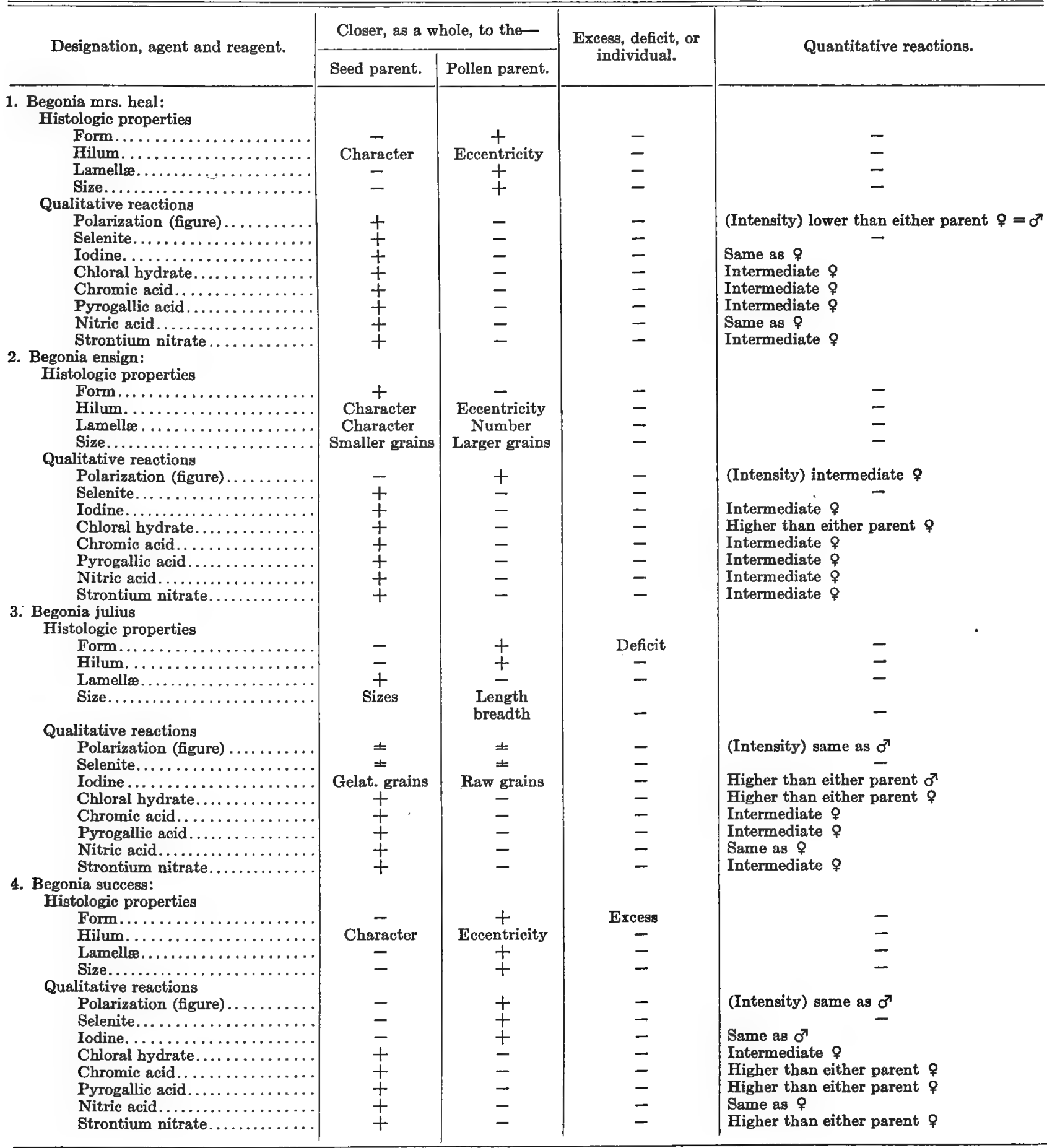


figure, and selenite reactions. I. dorak shows even a stronger leaning to the seed parent, closer resemblances to the pollen parent being recorded in only the eccentricity of the hilum and lamellæ. The seed parent of these hybrids is the same and it shows in both hybrids much greater potency than the other parent. In $I$. mrs. alan grey the form, hilum, and indistinctness of the lamellæ lean to the seed parent, but the general characters of the lamellæ and the size of the grains incline to the pollen parent. Among the qualitative reactions, in those with iodine alone is there greater closeness to the seed parent. I. dorak and I. mrs. alan grey have I. cengialti as their pollen and seed parent, respectively; in each hybrid this parent exhibits the lesser influence on the histologic characters and qualitative reactions of the hybrids. I. pursind shows, with the exception of eccentricity of the hilum and qualitative reactions with iodine, a closer relationship to the seed parent. Deficit and excess of development, mostly in histologic properties, are occasionally noted; but individualities of the hybrids are absent.

The independence and vagariousness of the quantitative reactions in relation to the qualitative reactions are very striking in all of the sets.

\section{Giadiolus. (Table C 9.)}

The seed parent of $G$. colvillei shows throughout the histologic properties and qualitative reactions, the more potent influence on the hybrid, excepting in the eccentricity of the hilum and the lamellæ, in the former respect being subordinate, and in the latter of equal value, to the seed parent. Excess of development of parental extremes was noted in the lamellæ, and individuality was recorded in the hydrochloric-acid reaction.

In the quantitative reactions there is mostly a tendency to sameness as both parents, together with some inclination to excess and deficit of development; but, on the whole, the leaning is rather toward the seed parent.

\section{Tritonia. (Table C 10.)}

This hybrid in its designations shares about equally in closeness to one or the other parent. In eccentricity of the hilum, lamellæ, and size it is closer to the seed parent, but in form and character of the hilum closer to the pollen parent. In the polariscopic figure, and in the selenite and iodine reactions it is closer to the seed parent, but in all the other qualitative reactions it is closer to the pollen parent. Euxcess and deficit of development and individualities were not noted. Curiously, while in the qualitative reactions with the various chemical reagents the leaning of the hybrid is to the pollen parent, in the quantitative reactions the inclination is in all seven reactions to the seed parent. This almost complete reversal of qualitative and quantitative parental relationships is by no means uncommon, as will be seen in other tables.

\section{Begonia. (TABLE C 11.)}

$B$. socotrana is the pollen parent in all four hybrids, it belonging to the semi-tuberous group; the seed parents are horticultural varieties that belong to the tuberous group. In all four hybrids there is among the histological properties a manifest tendency to a splitting of the characters in their parental relationships (except solely in the form of the grains) and to fluctuation of given characters in different hybrids to one parent or the other. The form of the grains in B. mrs. heal, B. jutius, and $B$. success is closer to the pollen parent, but in $B$. ensign closer to the other parent. The hilum in character is in B. mrs. heal, B. ensign, and B. success closer to the seed parent, but in $B$. jutius closer to the pollen parent; while in eccentricity it is closer in all to the pollen parent. The lamellæ in character are in $B$. ensign and $B$. julius closer to the pollen parent, while in number this property is in all four closer to the pollen parent. In size, in common sizes it is in $B . m r s$. heal and $B$. success closer to the seed parent, in the larger grains in $B$. ensign closer to the pollen parent, and in proportion of length to breadth in $B$. julius closer to the pollen parent. The polariscopic figure is in $B$. mrs. heal closer to the seed parent, but in the other three the same as both parents or closer to the pollen parent. The selenite reactions are closer to those of the seed parent in $B$. mrs. heal and $B$. ensign; closer to those of the pollen parent in B. success; and the same as both parents in $B$. julius. The independence of polariscopic figure and selenite reaction is illustrated in $B$. ensign. In the iodine reactions the inclinations may be to one or the other parent, but in $B$. julius there is a splitting so that the reactions of the gelatinized grains are closer to the seed parent,

TABLE C 12.-Richardia.

\begin{tabular}{|c|c|c|c|c|}
\hline \multirow{2}{*}{ Designation, agent and reagent. } & \multicolumn{2}{|c|}{ Closer, as a whole, to the- } & \multirow{2}{*}{$\begin{array}{l}\text { Excess, deficit, or } \\
\text { individual. }\end{array}$} & \multirow{2}{*}{ Quantitative reactions. } \\
\hline & Seed parent. & Pollen parent. & & \\
\hline 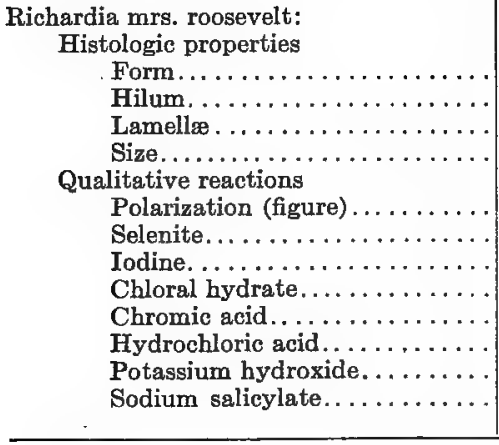 & $\begin{array}{l}\overline{+} \\
\pm \\
+ \\
- \\
- \\
+ \\
+ \\
+ \\
+ \\
+\end{array}$ & $\begin{array}{l}+ \\
\pm \\
- \\
+ \\
+ \\
+ \\
- \\
- \\
-\end{array}$ & $\begin{array}{l}\text { Deficit, excess } \\
\begin{array}{l}- \\
\text { Deficit } \\
- \\
- \\
- \\
- \\
- \\
- \\
-\end{array}\end{array}$ & $\begin{array}{l}\text { Intensity) intermediate } ᄋ=\sigma^{7} \\
\text { Same as } \% \\
\text { Higher than either parent } \sigma^{7} \\
\text { About the same as both parents } \\
\text { Lower than either parent } \sigma^{7} \\
\text { Higher than either parent } \sigma^{7} \\
\text { Same as both parents }\end{array}$ \\
\hline
\end{tabular}


TABLE C 13.-Musa.

\begin{tabular}{|c|c|c|c|c|}
\hline \multirow{2}{*}{ Designation, agent and reagent. } & \multicolumn{2}{|c|}{ Closer, as a whole, to the- } & \multirow{2}{*}{$\begin{array}{l}\text { Excess, deficit, or } \\
\text { individual. }\end{array}$} & \multirow{2}{*}{ Quantitative reactions. } \\
\hline & Seed parent. & Pollen parent. & & \\
\hline 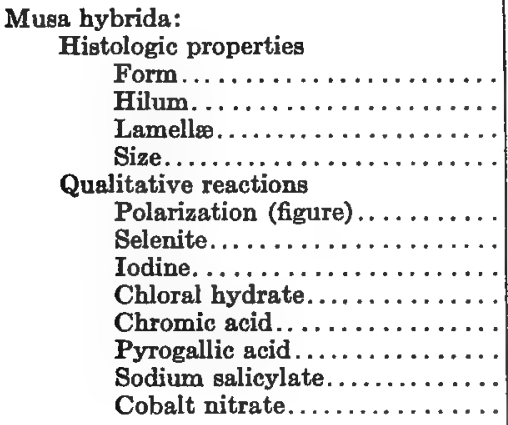 & $\begin{array}{l}- \\
\text { Number } \\
- \\
- \\
- \\
- \\
- \\
- \\
- \\
-\end{array}$ & $\begin{array}{l}+ \\
+ \\
\text { Character } \\
+ \\
+ \\
+ \\
+ \\
+ \\
+ \\
+ \\
+\end{array}$ & $\begin{array}{l}\text { Excess } \\
\text { Excess } \\
\text { Excess } \\
\\
- \\
- \\
- \\
- \\
- \\
- \\
-\end{array}$ & $\begin{array}{l}\text { (Intensity) higher than either parent } \sigma^{7} \\
\text { Same as } \sigma^{7} \\
\text { Lower than either parent } \sigma^{7} \\
\text { Lower than either parent } \sigma^{7} \\
\text { Same as ơ } \\
\text { Lower than either parent } \sigma^{7} \\
\text { Lower than either parent } \sigma^{7}\end{array}$ \\
\hline
\end{tabular}

TABLE C 14.-Phaius.

\begin{tabular}{|c|c|c|c|c|}
\hline \multirow{2}{*}{ Designation, agent and reagent. } & \multicolumn{2}{|c|}{ Closer, as a whole, to the- } & \multirow{2}{*}{$\begin{array}{l}\text { Excess, deficit, or } \\
\text { individual. }\end{array}$} & \multirow{2}{*}{ Quantitative reactions. } \\
\hline & Seed parent. & Pollen parent. & & \\
\hline 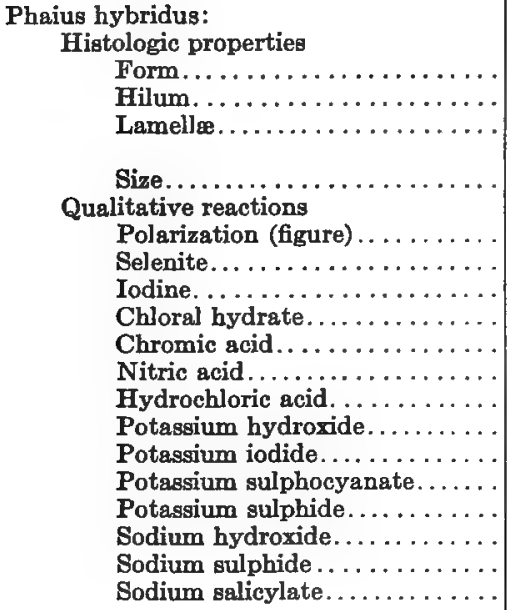 & 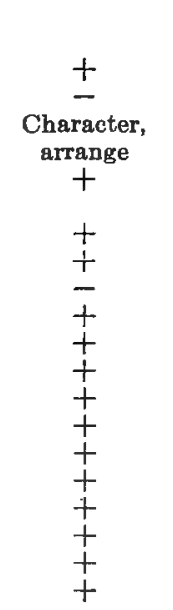 & $\begin{array}{c}\text { Character } \\
- \\
- \\
- \\
- \\
+ \\
- \\
- \\
- \\
- \\
- \\
- \\
- \\
- \\
- \\
-\end{array}$ & $\begin{array}{c}\text { Excess } \\
- \\
- \\
- \\
- \\
- \\
- \\
- \\
- \\
- \\
- \\
- \\
- \\
- \\
- \\
- \\
-\end{array}$ & $\begin{array}{l}\text { Intensity) higher than either parent } \% \\
\text { Intermediate } \sigma^{7} \\
\text { Lower than either parent } \sigma^{7} \\
\text { Intermediate } 9=\sigma^{7} \\
\text { Intermediate } \% \\
\text { Same as ơ } \\
\text { Same as both parente. } \\
\text { Intermediate } 9=\sigma^{7} \\
\text { Same as both parents. } \\
\text { Lower than either parent } ᄋ=\sigma^{7} \\
\text { Lower than either parent } \sigma^{\top} \\
\text { Same as } \sigma^{7} \\
\text { Same as } \sigma^{7}\end{array}$ \\
\hline
\end{tabular}

TABLE C 15.-Miltonia.

\begin{tabular}{|c|c|c|c|c|}
\hline \multirow{2}{*}{ Designation, agent and reagent. } & \multicolumn{2}{|c|}{ Closer, as a whole, to the- } & \multirow{2}{*}{$\begin{array}{l}\text { Excess, deficit, or } \\
\text { individual. }\end{array}$} & \multirow{2}{*}{ Quantitative reactions. } \\
\hline & Seed parent. & Pollen parent. & & \\
\hline 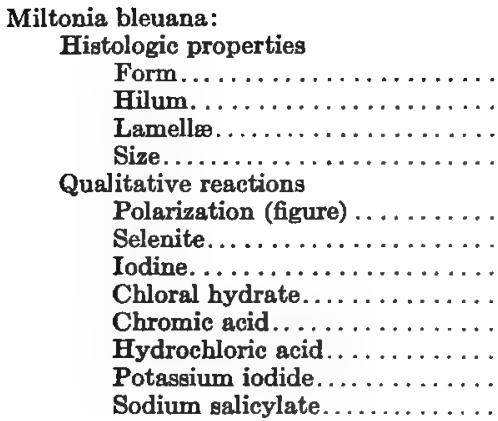 & $\begin{array}{l}+ \\
\text { Character } \\
\text { Character } \\
- \\
+ \\
+ \\
+ \\
+ \\
+ \\
+ \\
+ \\
+\end{array}$ & $\begin{array}{l}\text { Eccentricity } \\
- \\
+ \\
- \\
- \\
- \\
- \\
- \\
- \\
-\end{array}$ & $\begin{array}{l}\text { Excess } \\
- \\
- \\
\text { Excess } \\
- \\
- \\
- \\
- \\
- \\
- \\
-\end{array}$ & $\begin{array}{l}\text { Intensity) higher than either parent } \% \\
\text { Same as } \% \\
\text { Intermediate } \% \\
\text { Higher than either parent } \% \\
\text { Same as both parents } \\
\text { Higher than either parent } \% \\
\text { Higher than either parent } q\end{array}$ \\
\hline
\end{tabular}


TABLE C 16.-Cymbidium.

\begin{tabular}{|c|c|c|c|c|}
\hline \multirow{2}{*}{ Designation, agent and reagent. } & \multicolumn{2}{|c|}{ Closer, as a whole, to the- } & \multirow{2}{*}{$\begin{array}{l}\text { Excess, deficit, or } \\
\text { individual. }\end{array}$} & \multirow{2}{*}{ Quantitative reactions. } \\
\hline & Seed parent. & Pollen parent. & & \\
\hline 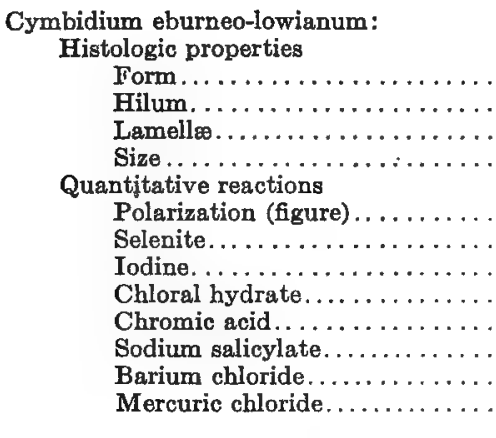 & $\begin{array}{c}+ \\
\text { Character } \\
+ \\
\text { Size } \\
+ \\
+ \\
+ \\
+ \\
+ \\
+ \\
+ \\
+\end{array}$ & $\begin{array}{l}\text { Eccentricity } \\
\text { Length, width } \\
\text { - } \\
- \\
- \\
- \\
- \\
- \\
-\end{array}$ & $\begin{array}{l}\bar{z} \\
\overline{-} \\
= \\
\bar{z} \\
= \\
= \\
= \\
=\end{array}$ & $\begin{array}{l}\text { (Intensity) same as } q \\
\text { Same as } q \\
\text { Lower than either parent } q=\sigma^{7} \\
\text { Lower than either parent } q=\sigma^{7} \\
\text { Lower than either parent } q=\sigma^{7} \\
\text { Lower than either parent } q=\sigma^{7} \\
\text { Lower than either parent } q=\sigma^{7}\end{array}$ \\
\hline
\end{tabular}

while those of the raw grains are closer to the pollen parent. With one exception, in all of the qualitative reactions of all four hybrids the relationship is closer to the seed parent. Excess of qualitative development was noted once, deficit once, and individuality not at all. The quantitative reactions are frequently intermediate, sometimes the same as or higher or lower than both parents; usually very much closer to the seed parent and far separated from the pollen parent, and rarely the same as or closer to the pollen parent.

Richardia. (TABLe C 12.)

In form, polariscopic figure, selenite reaction, and iodine reaction the hybrid inclines to the pollen parent; in lamellæ it is equally related to both parents; and in all other designations closer to the seed parent. Deficit of development was noted twice, excess of development once, and individuality not at all.

The quantitative reactions are quite variable in their parental relationships, and without other than casual correspondence in their bearings with the qualitative reactions.

\section{MUSA. (TABLE C 13.)}

With the exception of the number of the lamellæ, the designations of this hybrid are toward the pollen parent. The quantitative reactions are in all seven designations toward the pollen parent.

TABue C 17.-Calanthe.

\begin{tabular}{|c|c|c|c|c|}
\hline \multirow{2}{*}{ Designation, agent and reagent. } & \multicolumn{2}{|c|}{ Closer, as a whole, to the -} & \multirow{2}{*}{$\begin{array}{l}\text { Excess, deficit, or } \\
\text { individual. }\end{array}$} & \multirow{2}{*}{ Quantitative reactions. } \\
\hline & Seed parent. & Pollen parent. & & \\
\hline 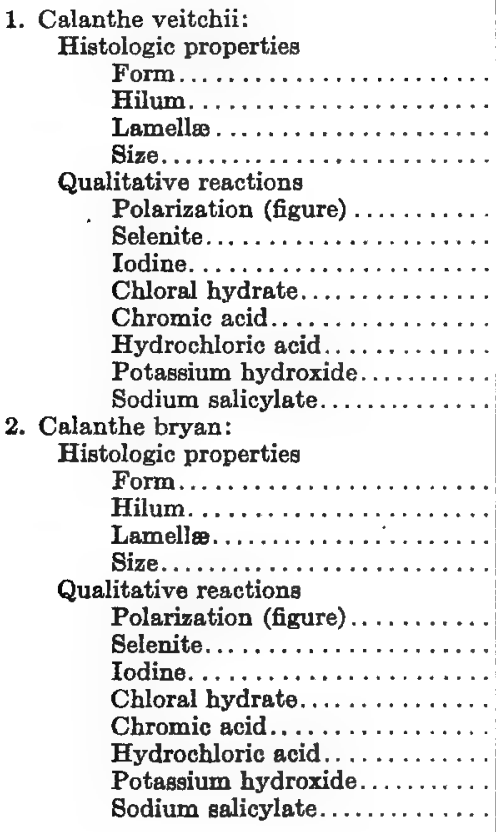 & $\begin{array}{c}\text { Most } \\
+ \\
\pm \\
- \\
+ \\
+ \\
\pm \\
+ \\
\pm \\
- \\
\text { Some } \\
= \\
- \\
\text { Length, width } \\
- \\
\pm \\
+ \\
+ \\
\pm \\
+\end{array}$ & $\begin{array}{c}\text { Some } \\
= \\
\overline{+} \\
= \\
\overline{-} \\
\pm \\
\pm \\
\overline{+} \\
+\end{array}$ & $\begin{array}{c}= \\
= \\
= \\
= \\
= \\
= \\
= \\
= \\
- \\
= \\
\text { Excess } \\
= \\
= \\
= \\
= \\
= \\
-\end{array}$ & $\begin{array}{l}\text { Intensity) intermediate } \sigma^{\top} \\
\text { Intermediate } ᄋ=\sigma^{7} \\
\text { Intermediate } \%=\sigma^{7} \\
\text { Intermediate } \sigma^{7} \\
\text { Higher than either parent } \sigma^{7} \\
\text { Same as } \% \\
\text { Intermediate } \odot=\sigma^{7}\end{array}$ \\
\hline
\end{tabular}


Phatus. (Table C 14.)

With the exception of the character of the hilum and the reaction with iodine the hybrid in its histologic properties and qualitative reactions is closer to the seed parent. Excess of development is noted once; deficit and individuality not at all.

The quantitative reactions are very variable in their parental relationships, exhibiting sameness in relation to one parent or the other or both parents, intermediateness, and excess or deficit in relation to parental extremes, as the case may be.

Miltonia. (TABle C 15.)

Except in the eccentricity of the hilum and size of the grains all of the designations of this hybrid incline toward the seed parent.

The qualitative reactions while variable in their parental relationships tend with one exception to the seed parent, but in none to the pollen parent.

Crmbidium. (Table C 16.)

The hybrid bears a closer relationship to the seed parent in all of the histologic and qualitative designations with the exception of eccentricity of the hilum and of ratio of length to breadth of the grains.

In the quantitative reactions the inclination is, with one exception, to lower reactivity than in either parent, the hybrid being in the latter reactions lower than in either parent but as close to one as to the other parent. The leaning is generally very doubtful because of the great rapidity of the reactions.

\section{Calanthe. (Table C 17.)}

In C. veitchii two-thirds of the designations incline to the seed parent. In form most of the grains are more like those of $C$. rosea, and only some like those of the other parent. In hilum and lamellæ the hybrid is close to the seed parent, but in size closer to the other parent. In the polarization figure, selenite reaction, and iodine reaction it is closer to the seed parent. In the qualitative reactions with chloral hydrate, potassium hydroxide and sodium salicylate it is closer to the pollen parent; but in those with chromic acid and hydrochloric acid it is closer to the seed parent. In the quantitative reactions throughout the hybrid is the same as or closer to the seed parent.

In C. bryan the designations are about equally divided in their parental closeness. In form some of the grains are more like those of the seed parent, but most are like those of the pollen parent-the reverse of what was recorded in the other hybrid (in this set the seed parent is the same as the pollen parent in the preceding set). There is in this hybrid in comparison with the other hybrids reversal of the relations of the hilum and lamellæ to the parents, and there is a splitting of the characters pertaining to size-the grains in ratio of length to breadth being closer to the seed parent, but in size generally closer to the pollen parent. While the polariscopic figure and selenite reaction are in comparison with the foregoing hybrid reversed, the iodine reaction remains closer to the seed parent. The qualitative reactions likewise show curious differences. Here the chloral hydrate, chromic acid, and sodium salicylate reactions are closer to the seed parent, while the hydrochloric acid and potassium hydroxide reactions are closer to the pollen parent (the reactions of chloral hydrate, hydrochloric acid, and sodium salicylate being reversed, but those of chromic acid and potassium hydroxide remaining the same in comparison with those of $C$. veitchii).

The quantitative reactions exhibit a tendency to midintermediateness, and otherwise mostly to closeness to the pollen parent. In only one of the seren quantitative designations is there manifest greater closeness to the seed parent than to the pollen parent.

\section{Histologic Properties of Starches of Hybrids} in Relation to those of the Parents.

In the preceding section, in the consideration of the peculiarities of each starch, reference was made to the remarkable shifting of the various histologic characters in their parental relationships. These peculiarities are of exceptional interest and significance, and they have been presented for the most part in a succinct form in Table D. One would not unnaturally be led to the conclusion that if the grains of the hybrid are closely like those of the seed parent or the pollen parent in form, lamellæ, and size, the same would hold good for the hilum, but such may in fact be far from the case. Moreover, not only may there be different parental relationships of the hybrid starch in form, hilum, lamellæ, and size, but there may also be a splitting of characters in each of these designations, so that in a certain respect the hilum, for instance, may be close in its relationship to one parent, but in another respect equally as close to the other parent. In other words, not only are form, hilum, lamellæ, and size independent characters that may be modified in the starch of any hybrid in their parental relations in like or unlike directions, but each may be split into a variable number of components which in like manner may swing to one or the other parent in an absolutely unpredictable and inexplicable way. It is unfortunate that in making the laboratory records the data pertaining to variations in form were not so systematically made as to make it possible to present in a consistent way the splitting of properties such as was recorded in the properties of the hilum, lamellæ, and size, especially of the two former. Sufficient data were accumulated to show that such splitting is a common phenomenon, as, for instance, where it has been found that the hybrid is close to one parent in the characters and numbers of compound grains, but close to the other parent in the characters and numbers of the aggregates; where a certain type of compound grain or aggregate is closer to that of one parent, but another type closer to that of the other; where the kinds of irregularity of the grains incline to one parent, but the frequency of irregularity to the other, etc. Similarly, only little analytic attention was given to the peculiarities of sizes, but enough to show that a splitting of characters must be quite common. On the other hand, the records of the peculiarities of the hilum and lamellæ, while capable of much and important extension, are rich in instances of splitting. Taking several concrete examples for illustration, we find that both Brunsdonna hybrids are closer to the seed parent in form, hilum, and size, but closer to the pollen parent in the form, arrangement, and number of the lamellæ. Hippeastrum titan-cleonia is closer to the seed parent in form and hilum; but closer to 
- the pollen parent in lamellæ and size. Hippeastrum ossultan-pyrrha is closer to the seed parent in the number of the lamellæ and in size; but closer to the pollen parent in form, hilum, and characters of the lamellæ. Iris dorak is closer to the seed parent in form, size, characters of the hilum, and number of the lamellæ; but closer to the pollen parent in eccentricity of the hilum, and in the character of the lamellæ, etc.

In only two of the hybrids (Homanthus könig albert and Lilium golden gleam) is the parental relationship in all four designations the same, i.e., the hybrid is in form, hilum, lamellæ, and size closer to one parent; the former is closer to the pollen parent, and the latter to the seed parent. In other hybrids, as in Brunsdonna, Crinum hybridum j. c. h., Nerine dainty maid, and Narcissus cresset, as many as three designations may be closer to one parent; but there are seldom more than two, as is seen in Hippeastrum titan-cleonia and Homanthus andromeda. In others, there may be only one, the other three being split in various ways, as in Begonia ensign, in which hybrid the form of the grains is closer to the seed parent, and the character of the hilum closer to the seed parent, but in eccentricity closer to the pollen parent; the character of the lamellæ is closer to the seed

'TABLE D.

\begin{tabular}{|c|c|c|c|c|c|c|c|c|}
\hline \multirow{3}{*}{ Hybrids. } & \multicolumn{2}{|c|}{ Form. } & \multicolumn{2}{|c|}{ Hilum. } & \multicolumn{2}{|c|}{ Lamellæ. } & \multicolumn{2}{|c|}{ Size. } \\
\hline & \multicolumn{2}{|c|}{ Closer, on the whole, to- } & \multicolumn{2}{|c|}{ Closer, on the whole, to- } & \multicolumn{2}{|c|}{ Closer, on the whole, to- } & \multicolumn{2}{|c|}{ Closer, on the whole, to- } \\
\hline & Seed parent. & Pollen parent. & Seed parent. & Pollen parent. & Seed parent. & Pollen parent. & Seed parent. & Pollen parent. \\
\hline B. sanderce alba,$\ldots \ldots \ldots$. & + & - & + & - & Form, arrang. & No. & + & - \\
\hline B. sanderœ ............ & + & - & $\dot{t}$ & - & Form, arrang. & No. & + & - \\
\hline H. titan-cleonia ......... & + & - & + & - & - & + & - & + \\
\hline H. ossult.-pyrh .......... & - & + & - & + & No. & Char. & + & - \\
\hline H. dæon,-zeph $\ldots \ldots \ldots \ldots$ & - & + & + & - & + & - & - & Larger grains \\
\hline Hæmanthus andromeda. . & + & - & + & - & \pm & \pm & - & + \\
\hline Hæmanthus könig albert. . & - & + & - & + & - & + & - & + \\
\hline C. hybridum j. c. h...... & - & + & - & + & - & + & Length & $\begin{array}{l}\text { Length to } \\
\text { breadth }\end{array}$ \\
\hline C. kircape.............. & + & - & Char. & Eccen. & + & - & + & - \\
\hline C. powellii ........... & - & + & Eccen. & Char. & - & + & - & + \\
\hline N. dainty maid. . . . . . . & - & + & - & - & Char., arrang. & Fineness & - & + \\
\hline N. queen of roses......... & - & + & Distinctness & $\begin{array}{c}\text { Fiss., char., \& } \\
\text { eccen. }\end{array}$ & + & - & - & + \\
\hline N. giantess............. & + & - & - & + & + & - & - & + \\
\hline N. abundance............ & - & + & Char. & Eccen. & - & + & + & - \\
\hline N. glory of sarnia. . . . . . & + & - & + & - & - & $\dot{+}$ & - & + \\
\hline N. poeticus herrick ....... & + & - & + & - & + & - & + & + \\
\hline N. poeticus dante ....... & $\dot{+}$ & - & - & + & - & + & - & + \\
\hline N. poetaz triumph . . . . . & $\dot{t}$ & - & - & Char. & + & - & + & - \\
\hline N. fiery cross $\ldots \ldots \ldots \ldots$ & $\dot{t}$ & - & Char. & Eccen. & + & - & + & - \\
\hline N. doubloon........... & + & - & Char. & - & + & - & - & + \\
\hline N. cresset............ & - & + & + & - & + & - & + & - \\
\hline N. will scarlet............ & + & - & Char. & - & Char. & - & Large & Common \\
\hline N. bicolor apricot. ....... & + & - & - & Char. & Char. & - & + & - \\
\hline N. madame de graaff..... & + & - & - & + & - & + & + & - \\
\hline N. pyramus $\ldots \ldots \ldots \ldots$ & - & + & - & + & - & + & + & - \\
\hline N. lord roberts........... & + & - & + & - & + & - & - & + \\
\hline N. agnes harvey . . . . . . . & + & - & Char. & - & + & - & - & + \\
\hline N. j. t. bennett poe...... . & + & - & + & - & - & + & + & - \\
\hline L. marhan $\ldots \ldots \ldots \ldots \ldots$ & - & + & + & - & + & - & - & + \\
\hline L. dalhansoni........... & + & - & - & + & Char., arrang. & No. & + & - \\
\hline L. golden gleam .......... & + & - . & + & - & + & - & + & - \\
\hline L. testaceum $\ldots \ldots \ldots \ldots$ & + & - & Char. & Eccen. & + & - & -1 & + \\
\hline L. burbanki ............ & + & - & - & + & + & - & + & - \\
\hline I. ismali............... & + & - & Char. & Eccen. & + & - & + & - \\
\hline I. dorak $\ldots \ldots \ldots \ldots \ldots \ldots$ & + & - & Char. & Eccen. & No. & Char. & + & - \\
\hline I. mrs. alan gray $\ldots \ldots \ldots$ & + & - & + & - & Indist. & Char. & - & + \\
\hline I. pursind............. & + & - & Char. & Eccen. & + & - & + & - \\
\hline G. colvillei $\ldots \ldots \ldots, \ldots$ & $\dot{t}$ & - & Char. & Eccen. & \pm & \pm & + & - \\
\hline T. crocosmæAlora......... & - & + & Eccen. & Char. & + & - & + & - \\
\hline B. mrs. heal ............ & - & + & Char. & Eccen. & - & + & - & + \\
\hline B. ensign.................. & + & $\frac{1}{2}$ & Char. & Eccen. & Char. & No. & Smaller & Larger \\
\hline B. julius............... & - & + & - & + & + & - & Sizes & Length to \\
\hline B. success............. & - & + & Char. & Eccen. & - & + & - & $\begin{array}{l}\text { breadth } \\
+\end{array}$ \\
\hline R. mrs. roosevelt.......... & - & + & + & - & \pm & \pm & $\overline{+}$ & 士 \\
\hline M. hybrida............. & - & + & - & + & No. & Char., arrang. & - & + \\
\hline P. hybridus ............ & + & - & - & Char. & Char., arrang. & - & + & - \\
\hline M. bleuana.............. & + & - & Char. & Eccen. & Char. & - & - & + \\
\hline C. eburneo-lowianum ..... & $\dot{+}$ & - & Char. & Eccen. & + & - & Sizes & Length to \\
\hline C. veitchii........... & Most & Some & + & - & + & - & - & breadth \\
\hline C. bryan................ & Some & Most & - & + & - & + & Length to & Sizes \\
\hline
\end{tabular}


parent, but in number is closer to the pollen parent; and the smaller sizes are closer to the seed parent, but the larger sizes closer to the pollen parent. A similar splitting and shifting is seen in Miltonia bleuana, in which the form is closer to the seed parent; the character of the hilum closer to the seed parent, but eccentricity is closer to the pollen parent; the character of the lamellæ is closer to the seed parent, but certain other features closer to the pollen parent, or as close to one as to the other parent; and the common sizes are closer to the pollen parent. These last two instances are exceptional, probably, merely because of inadequate data. In over half the hybrid is the same as or closer to one parent in only two designations, and in less than half in three designations. In only two are all four designations alike, and in only two are all four designations different, in their parental relationships.

It is of especial interest to note that in $\mathbf{1 5}$ of the 50 hybrids (nearly one-third) character and eccentricity of the hilum are separated in their parental relationships, character in 12 being closer to the seed parent and in 3 being closer to the pollen parent; while eccentricity in 12 is closer to the pollen parent and in 3 closer to the seed parent (an exact reversal), a most remarkable peculiarity and one that is very suggestive in connection with the processes concerned in the formation of the starch grain. Another of the several forms of splitting is instanced in Nerine queen of roses, where the hilum in distinctness is closer to the seed parent, but in fissuration, character, and eccentricity closer to the pollen parent; and it is very much less often fissured but more eccentric than in either parent. The lamellæ appear to show less tendency to a splitting of their characters in their parental relationships, but this may be merely apparent and not actual, as will probably be brought out by a sufficiently detailed study. In 9 of the hybrids there occurred an obvious splitting of lamellar properties, this being noted in a separation of character and number; but here, unlike in the case of the hilum, there is not a definite inclination generally of one or the other of these features to one or the other parent. In the splitting of the hilum into character and eccentricity, character tends to the seed parent and eccentricity to the pollen parent; but in the lamellæ split, character, and number swing apparently indifferently to one or the other parent. In size, splitting of characters seems to be comparatively uncommon, though here as elsewhere in these studies it is probably not so much an absence of commonness as of careful investigation and analysis. Such splitting as has been recorded under this designation has been manifested chiefly in the ratios of length to breadth of the grains and of the common sizes to other types and different types of grains.

Qualitative and Quantitative Reactions of Starches of Hybrids with Especial RefeRence to Reversal of these Reactions in their Parental Relationships.

(Table E, Parta 1 to 21 and Summary.)

In the first section, in the tabulations of the starches in regard to histologic and polariscopic properties and to the reactions with iodine and various chemical reagents, data were collected to indicate that the characters em- braced under the designations form, hilum, lamellæ, and size, respectively, may in each designation collectively be independently heritable; or that each designation may be split into several independently heritable characters, so that a given hybrid may have a starch that is like that of the seed parent in form, but like that of the other parent in the lamellæ; or that it may be like one parent in the general characters of the hilum, but like the other parent in the eccentricity of the hilum, and so on. In the second section, further consideration was given to these peculiarities with reference to histological inheritance. It was shown, moreover, that each reaction is, in its qualitative and quantitative manifestations, heritable independently of each other, so that while with a given reagent there may be sameness or near sameness in the qualitative reaction to the seed parent, with another reagent the relationship may correspond to the pollen parent; that while a given qualitative reaction may correspond to that of the seed parent, the correlative quantitative reaction may correspond to that of the pollen parent, etc.; and that while with one reagent the relationship may be to the seed parent, with another reagent it may be to the pollen parent, and so on. These parental similarities and dissimilarities are of such interest and suggestiveness in connection with both the constitutional peculiarities of different starches and the mechanism of heredity that it seems desirable to tabulate such data more fully and with especial reference to the reversals of the qualitative and quantitative reactions of each agent and reagent in their parental relationships. Of Table $\mathbf{E}$ it will be noticed that with only three of the agents and reagents were the reactions of all of the 50 hybrids recorded; and that in the others the number of hybrids varied from 1 to 32 (in seven less than 10, and in eleven 10 or more-the restricted numbers being due to the limitations of studies of the qualitative reactions).

The most conspicuous features of these tables, apart from those already referred to, are:

(1) The absence in members of a genus of constancy of both qualitative and quantitative reactions as regards sameness of the reactions in their parental bearings; (2) the tendency to the appearance of a definite ratio in the qualitative reactions in their inclinations to the seed and pollen parent; ( 3 ) the tendency to an absence of such a ratio in the quantitative reactions in their inclinations to the seed and pollen parent; (4) the large percentage of instances of reversal of the parental relationships of qualitative and quantitative reactions with given agents and reagents.

It will be noted that in the reactions with each reagent there does not exist generic constancy or uniformity of either qualitative or quantitative reactions in their parental closeness. For instance, while in the chloral hydrate qualitative reactions of Brunsdonna, Hippeastrum, Hamanthus, and Begonia all of the hybrids belonging to each genus incline to the seed parent, in all other genera represented in which there are two or more members some of the hybrids of each genus incline to one parent and others to the other parent. Thus, in Crinum one hybrid inclines to the seed parent and two to the pollen parent; in Nerine four incline to the seed parent and one to the pollen parent; in Narcissus eleven incline to the seed parent and two to the pollen parent; in Lilium three incline to the seed parent and two to the 
pollen parent; in Iris three incline to the seed parent and one to the pollen parent; and in Calanthe one inclines to the seed parent and one to the pollen parent. In the quantitative reactions this absence of constancy to one or the other parent is much more marked; thus, in only Brunsdonna and Begonia do all of these chloralhydrate reactions tend to the seed parent; but in no genus do all of them incline to the pollen parent. Examining the different generic groups we note that in Hippeastrum in two hybrids the reactions incline to the seed parent and in one to the pollen parent; in Homanthus in one hybrid the reaction inclines to one as much as to the other parent, and in the other to the seed parent; in Crinum one inclines to the seed parent and two to the pollen parent; in Nerine one inclines to the seed parent and four to the pollen parent; in Narcissus five incline to the seed parent, six to the pollen parent, and two incline to one as much as to the other parent; in Lilium two incline to the seed parent and three to the pollen parent; in Iris two incline to one as much as to the other parent, and two incline to the pollen parent; and in Calanthe one inclines to the seed parent and the other inclines to one as much as to the other parent. Of exceptional interest is the fact, several times noted, that in case of any hybrid the qualitative and quantitative reactions may or may not correspond in their parental inclinations. It is certainly remarkable that with a given reagent the qualitative reaction may correspond with that of the seed parent and the quantitative reaction with that of the pollen parent, or vice versa, and so on in other varied relationships.

The tendency in general to a ratio of approximately $2: 1$ in the qualitative reactions in their relations to the seed and pollen parents is well marked. This ratio varies from $4: 0$ to $1: 1$, but in about half of the cases it will be found to be as first stated. Totaling these records, it will be seen that 62.8 per cent of these reactions incline to the seed parent and 35.8 per cent to the pollen parent, a ratio of $1.8: 1$. In other words, there is approximately twice the tendency for the qualitative reaction to be closer to the seed parent than to the pollen parent.

There is not a corresponding tendency to such a common ratio in the quantitative reactions, but to a marked inconstancy. In the qualitative reactions the ratio is always in favor of the seed parent; but in the quantitative reactions it may be in favor of either or of neither parent. Thus, it is found that there may be a ratio of $4: 1$ in favor of the seed parent, or one of $1: 3$ or $1: 4$ in favor of the pollen parent, and intermediate gradations. Summing up these reactions, 44 per cent incline to the seed parent and 40 per cent to the pollen parenta ratio of approximately $1: 1$. In studying the quantitative records the large number of reactions that are recorded as being the same as those of both parents should be taken into consideration, because had these been shown to have had in each case, or even in most cases, definite uniparental inclinations these ratios would of course be subject to more or less modification. Nearly all these reactions showed no difference from the parental reactions because of gelatinization occurring with too great a rapidity or slowness for differentiation. Modified strengths of reagents would doubtless have elicited differences that are wholly obscured by very quick or slow reactions. It is, however, not probable that there would be brought about any important change, as a whole, in these ratios. Why the qualitative ratios should be so different from the quantitative ratios is entirely problematical, very interesting, and very suggestive of stereochemic peculiarities of the starches.

No feature of these records is more remarkable than the reversal of the qualitative and quantitative reactions of a given starch with a given reagent in their parental inclinations. It is of importance to note that this phenomenon is not peculiar to any starch or reagent, but is common, and doubtless common to all starches and to all reagents. With not a single starch was it found that there was not such reversal; and with only four of the reagents (strontium nitrate, barium chloride, and mercuric chloride) was reversal not recorded, the reason for which is doubtless to be found in the small number of qualitative reactions recorded with these reagents (four reactions with the first, one with the second, and four with the third). Not less remarkable than the reversal of the reactions is the frequency with which this phenomenon occurs, the percentages ranging from 6 in the iodine reactions to as high as 50 in the cobalt-nitrate and cupric-chloride reactions with the different starches.' The mean is 22.5 , or close to one-fourth.

TABLE E.

\begin{tabular}{|c|c|c|c|c|}
\hline \multirow[t]{2}{*}{ Hybrids. } & \multicolumn{2}{|c|}{$\begin{array}{l}\text { Qualitative } \\
\text { reactions,* } \\
\text { closer as a } \\
\text { whole to- }\end{array}$} & \multicolumn{2}{|c|}{$\begin{array}{c}\text { Quantitative } \\
\text { reactions, * } \\
\text { closer as a } \\
\text { whole to- }\end{array}$} \\
\hline & $\begin{array}{c}\text { Seed } \\
\text { parent. }\end{array}$ & $\begin{array}{l}\text { Pollen } \\
\text { parent. }\end{array}$ & $\begin{array}{c}\text { Seed } \\
\text { parent }\end{array}$ & $\begin{array}{l}\text { Pollen } \\
\text { parent }\end{array}$ \\
\hline 1. Polarization reactions: & & & & \\
\hline Brunsdonna sanderoe alba ......... & + & - & + & - \\
\hline Brunsdonna sanderce........... & + & - & + & - \\
\hline Hippeastrum titan-cleonia. . . . . . & + & - & + & - \\
\hline Hippeastrum ossult.-pyrh. . . . . . & + & - & - & + \\
\hline Hippeastrum dæon.-zeph......... & - & + & - & + \\
\hline Hæmanthus andromeda......... & + & $\frac{1}{-}$ & \pm & \pm \\
\hline Hæmanthus könig albert......... & - & + & - & + \\
\hline Crinum hybridum j. c. h..... & - & + & - & + \\
\hline Crinum kircape............. & + & - & + & - \\
\hline Crinum powellii.............. & - & + & - & + \\
\hline Nerine dainty maid $\ldots \ldots \ldots \ldots \ldots$ & - & + & - & + \\
\hline Nerine queen of roses. . . . . . . . & - & + & - & + \\
\hline Nerine giantess. . . . . . . . . . & + & - & + & - \\
\hline Nerine abundance....... & + & - & + & - \\
\hline Nerine glory of sarnia..... & + & - & + & - \\
\hline Narcissus poeticus herrick... & - & + & + & - \\
\hline Narcissus poeticus dante.... & - & + & + & - \\
\hline Narcissus poetaz triumph....... & - & + & - & + \\
\hline Narcissus fiery cross. $\ldots \ldots \ldots \ldots \ldots$ & - & + & - & + \\
\hline Narcissus doubloon............. & - & + & + & - \\
\hline Narcissus cresset.............. & - & + & - & + \\
\hline Narcissus will scarlet. . . . . . . . . & + & - & + & $\underline{-}$ \\
\hline Narcissus bicolor apricot......... & + & - & + & - \\
\hline Narcissus madame de graaff....... & + & - & + & - \\
\hline Narcissus pyramus............ & - & + & \pm & \pm \\
\hline Narcissus lord roberts $\ldots \ldots \ldots \ldots$ & - & + & - & + \\
\hline Narcissus agnes harvey. .......... & + & - & + & - \\
\hline Narcissus j. t. bennett poe.... & - & + & + & - \\
\hline Lilium marhan. . . . . . . . . & - & + & 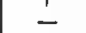 & + \\
\hline Lilium dalhansoni........... & + & - & + & - \\
\hline Lilium golden gleam.......... & + & - & + & - \\
\hline Lilium testaceum $\ldots \ldots \ldots \ldots \ldots \ldots$ & + & - & + & - \\
\hline Lilium burbanki $\ldots \ldots \ldots \ldots \ldots \ldots$ & - & + & - & + \\
\hline Iris ismali $\ldots \ldots \ldots \ldots \ldots \ldots \ldots$ & - & + & - & + \\
\hline
\end{tabular}

*Qualitative reactions $=$ polarization figure; quantitative reactions $=$ polarization intensity. 
TABte E.-Continued.

\begin{tabular}{|c|c|c|c|c|}
\hline \multirow[t]{2}{*}{ Hybrids. } & \multicolumn{2}{|c|}{$\begin{array}{l}\text { Qualitative } \\
\text { reactions, } \\
\text { closer as a } \\
\text { whole to- }\end{array}$} & \multicolumn{2}{|c|}{$\begin{array}{c}\text { Quantitative } \\
\text { reactione, } \\
\text { closer as a } \\
\text { whole to- }\end{array}$} \\
\hline & $\begin{array}{c}\text { Seed } \\
\text { parent. }\end{array}$ & $\begin{array}{l}\text { Pollen } \\
\text { parent. }\end{array}$ & $\begin{array}{c}\text { Seed } \\
\text { parent. }\end{array}$ & $\begin{array}{l}\text { Pollen } \\
\text { parent. }\end{array}$ \\
\hline \multicolumn{5}{|l|}{ 1. Polarization reactions.-Cont.: } \\
\hline 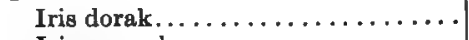 & + & - & + & $\rightarrow$ \\
\hline Iris mrs. alan grey $\ldots \ldots \ldots \ldots \ldots$ & - & + & - & + \\
\hline Iris pursind $\ldots \ldots \ldots \ldots \ldots \ldots \ldots$ & + & - & + & - \\
\hline Gladiolus colvillei............. & + & - & + & - \\
\hline Tritonia crocosmaflora.......... & + & - & + & - \\
\hline Begonia mrs. heal. . . . . . . . . . . . & + & - & \pm & \pm \\
\hline Begonia ensign. . . . . . . . . . & - & + & + & - \\
\hline Begonia julius. . . . . . . . . . . & \pm & \pm & - & + \\
\hline Begonia success. . . . . . . . . . & - & + & - & + \\
\hline Richardia mrs. roosevelt. . . . . . . . & - & + & \pm & \pm \\
\hline Muss hybrida................ & - & + & - & + \\
\hline Phaius hybridus . . . . . . . . . . & + & - & + & - \\
\hline Miltonis bleuana............... & + & - & + & - \\
\hline Cymbidium eburneo-lowianum . . . & + & - & + & - \\
\hline Calanthe veitchii . . . . . . . . . & + & - & + & - \\
\hline Calanthe bryan............. & - & + & - & + \\
\hline \multicolumn{5}{|l|}{ 2. Iodine reactions: } \\
\hline Brunsdonna sanderce alba........ & + & - & + & - \\
\hline Brunsdonna sanderce. . . . . . . . & + & $\rightarrow$ & + & - \\
\hline Hippeastrum titan-cleonia. . . . . . . . & - & + & - & + \\
\hline Hippeastrum ossult.-pyrh. . . . . . . & + & - & \pm & \pm \\
\hline Hippeastrum døon.-zeph. . . . . . & - & + & - & + \\
\hline Hemanthus andromeda . . . . . . & + & - & \pm & \pm \\
\hline Hæmanthus könig albert. . . . . . . . & + & - & \pm & $\Rightarrow$ \\
\hline Crinum hybridum j. c. h........ & - & + & - & + \\
\hline Crinum kircape............. & + & - & - & + \\
\hline Crinum powellii. ............. & - & + & \pm & \pm \\
\hline Nerine dainty maid . . . . . . . . & - & + & - & + \\
\hline Nerine queen of roses. . . . . . . . . & - & + & - & + \\
\hline Nerine giantess. . . . . . . . . . . & $\overline{-}$ & + & - & + \\
\hline Nerine abundance............. & + & - & + & - \\
\hline Nerine glory of sarnia . . . . . . . . & + & - & + & - \\
\hline Narcissus poeticus herrick......... & $\rightarrow$ & + & - & + \\
\hline Narcissus poeticus dante........ & - & + & - & + \\
\hline Narcissus poetaz triumph. . . . . . . & - & + & - & + \\
\hline Narcissug fiery cross. . . . . . . . . . & \pm & $\approx$ & + & - \\
\hline Narcissus doubloon. . . . . . . . . . . & + & - & + & - \\
\hline Narcissus cresset. . . . . . . . . . . & - & + & - & + \\
\hline Narcissus will scarlet. . . . . . . . . . & - & + & - & + \\
\hline Narcissus bicolor apricot. . . . . . . . & + & - & + & - \\
\hline Narciseus madame de graaff....... & & - & + & - \\
\hline Narcissus pyramus............ & + & - & + & - \\
\hline Narcissus lord roberts.......... & \pm & \pm & \pm & \pm \\
\hline Narcissus agnes harvey. . . . . . . . . & + & - & + & - \\
\hline Narcissus j.t. bennett poe....... & + & - & + & - \\
\hline Lilium marhan . . . . . . . . . . & - & + & - & + \\
\hline Lilium dalhansoni. . . . . . . . . . . . & + & - & + & - \\
\hline Lilium golden gleam . . . . . . . . . & + & - & + & - \\
\hline Lilium testaceum . . . . . . . . . . & & - & + & - \\
\hline Lilium burbanki............. & + & $\rightarrow$ & + & - \\
\hline Iris iemali . . . . . . . . . . . . . . & + & - & + & - \\
\hline Iris dorak . . . . . . . . . . . . . & + & - & + & - \\
\hline Iris mrs. alan grey $\ldots \ldots \ldots \ldots \ldots$ & + & 一 & + & - \\
\hline Iris pursind $\ldots \ldots \ldots \ldots \ldots \ldots$ & - & + & - & + \\
\hline Gladiolus colvillei. . . . . . . . . . & + & - & - & + \\
\hline Tritonia crocosmæflora . . . . . . . & + & - & + & - \\
\hline Begonia mrs. heal. . . . . . . . . . & + & - & + & - \\
\hline Begonia ensign ............. & + & - & + & - \\
\hline Begonia julius. . . . . . . . . . . . & - & + & - & + \\
\hline Begonia success. . . . . . . . . . & $\rightarrow$ & + & - & + \\
\hline Richardia mrs. roosevelt. . . . . . . & - & + & + & - \\
\hline Musa hybrida ................ & - & + & - & + \\
\hline Phaius hybridus . . . . . . . . . . & - & + & - & + \\
\hline Miltonia bleuana . . . . . . . . . & + & - & + & - \\
\hline Cymbidium eburneo-lowianum . . . . & + & - & + & - \\
\hline Calanthe veitchii . . . . . . . . . & + & - & + & - \\
\hline Calanthe bryan.............. & + & - & \pm & \pm \\
\hline \multicolumn{5}{|l|}{ 3. Chloral-hydrate reactions: } \\
\hline Brunsdonna sanderce alba....... & + & - & + & - \\
\hline Brunsdonna sanderce. . . . . . . . . & + & - & + & - \\
\hline
\end{tabular}

TABLE E.-Continued.

\begin{tabular}{|c|c|c|c|}
\hline \multirow[t]{2}{*}{ Hybridg. } & \multicolumn{2}{|c|}{$\begin{array}{l}\text { Qualitative } \\
\text { reactions, } \\
\text { closer as a } \\
\text { whole to- }\end{array}$} & $\begin{array}{l}\text { Quantitative } \\
\text { reactions, } \\
\text { closer as a } \\
\text { whole to- }\end{array}$ \\
\hline & \begin{tabular}{|c} 
Seed \\
parent.
\end{tabular} & $\begin{array}{l}\text { Pollen } \\
\text { parent. }\end{array}$ & \begin{tabular}{|c|c} 
Seed & Pollen \\
parent. & parent.
\end{tabular} \\
\hline
\end{tabular}

3. Chloral-hydrate reactions.-Cont. Hippeastrum titan-cleonia........ Hippeastrum ossult.-pyrh. ........

Hippeastrum dæon. zeph.........

Hæmanthus andromeda..........

Hæmanthus könig albert.........

Crinum hybridum j. c. h.........

Crinum kircape................

Crinum powellii................

Nerine dainty maid ............

Nerine queen of roses...........

Nerine giantess. ...............

Nerine abundance................

Nerine glory of sarnia...........

Narcissus poeticus dante.........

Narciseus poetaz triumph. ........

Narcissus fiery cross...............

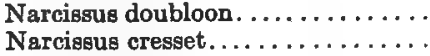

Narcissus will scarlet............

Narcissus bicolor apricot..........

Narcissus madame de graaff......

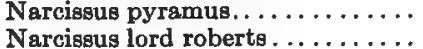

Narcissus lord roberts ...........

Narcissus j. t. bennett poe . . . . . .

Lilium marhan . .............

Lilium dalhansoni..............

Lilium golden gleam............

Lilium testaceum . . . . . . . . .

Lilium burbanki .

Iris ismali.

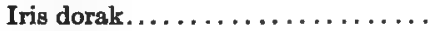

Iris mrs. alan grey ..............

Iris pursind .

Gladiolus colvillei.............

Tritonia crocosmæflora . . . . . . . . .

Begonia mrs. heal..............

Begonia ensign .

Begonia julius.

(n)

Richardia mrs. roosevelt. . . . . . . .

Musa hybrida .................

Phaius hybridus...............

Miltonia bleuana.................

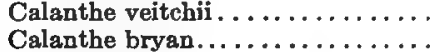

Calanthe bryan ...........

Narcissus poeticus herrick . . . . . . .

Narcissus poeticus dante.........

Narcissus poetaz triumph. ........

Narcissus fiery cross.

Narcissus doubloon . . . . . . . . . .

Narcissus cresset................

Narcissus will scarlet.

Narcissus bicolor apricot.........

Narcissus madame de graaff.......

Narcissus pyramus.............

Narcissus lord roberts.

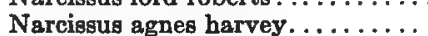

Narciesus j. t. bennett poe........

Lilium marhan.

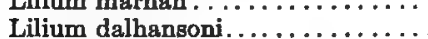

Lilium golden gleam...........

Lilium testaceum.

Lilium burbanki. .

Begonis mrs, hes.

Begonia engign.

Begonia julius. . . . . . ........... 
TABLE E.-Continued.

\begin{tabular}{|c|c|c|c|c|}
\hline \multirow[t]{2}{*}{ - Hybrids. } & \multicolumn{2}{|c|}{$\begin{array}{l}\text { Qualitative } \\
\text { reactions, } \\
\text { closer as a } \\
\text { whole to- }\end{array}$} & \multicolumn{2}{|c|}{$\begin{array}{l}\text { Quantitative } \\
\text { reactions, } \\
\text { closer as a } \\
\text { whole to- }\end{array}$} \\
\hline & $\mid \begin{array}{c}\text { Seed } \\
\text { parent. }\end{array}$ & 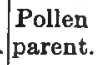 & $\begin{array}{c}\text { Seed } \\
\text { parent. }\end{array}$ & $\begin{array}{l}\text { Pollen } \\
\text { parent. }\end{array}$ \\
\hline $\begin{array}{l}\text { 4. Chromic-acid reactions.-Cont. : } \\
\text { Begonia success.............. }\end{array}$ & & & & \\
\hline $\begin{array}{l}\text { Begonia success..................... } \\
\text { Richardia mrs. roosevelt. . }\end{array}$ & + & $\overline{-}$ & $\underline{t}$ & $\overrightarrow{+}$ \\
\hline Musa hybrida................ & - & + & - & t \\
\hline Phaius hybridus . ............. & + & - & \pm & \pm \\
\hline Miltonia bleuana $\ldots \ldots \ldots \ldots \ldots$ & + & - & + & - \\
\hline Cymbidium eburneo-lowianum... & + & - & \pm & \pm \\
\hline Calanthe veitchii............. & + & - & + & - \\
\hline Calanthe bryan.............. & + & - & - & + \\
\hline 5. Pyrogallic-acid reactions: & & & & \\
\hline Narcissus poeticus herrick........ & - & + & - & + \\
\hline Narcissus poeticus dante......... & - & + & $\neq$ & \pm \\
\hline Narcissus poetaz triumph. ........ & + & - & - & + \\
\hline Narcissus fiery cross............ & + & - & - & + \\
\hline Narcissus doubloon ............ & + & - & \pm & \pm \\
\hline Narcissus cresset............... & + & - & + & - \\
\hline Narcissus will scarlet. .......... & + & - & + & - \\
\hline Narcissus bicolor apricot......... & - & + & - & + \\
\hline Narcissus madame de graaff....... & - & + & + & - \\
\hline Narcissus pyramus............ & + & - & + & - \\
\hline Narcissus lord roberts. .......... & - & + & - & + \\
\hline Narcissus agnes harvey. . . . . . . . . & + & - & \pm & \pm \\
\hline Narcissus j. t. bennett poe........ & $\dot{-}$ & + & - & + \\
\hline Begonia mrs. heal. ............... & + & - & + & - \\
\hline Begonia ensign $\ldots \ldots \ldots \ldots \ldots \ldots$ & + & - & + & - \\
\hline Begonia julius. . . . . . . . . . . & + & - & + & - \\
\hline Begonia success............... & + & - & + & - \\
\hline Musa hybrida..$\ldots \ldots \ldots \ldots \ldots$ & - & + & & + \\
\hline 6. Nitric-acid reactions: & & & & \\
\hline Brunsdonna sanderce alba......... & + & - & - & + \\
\hline Brunsdonna sanderœ. . . . . . . . . & + & - & - & + \\
\hline Hippeastrum titan-cleonia........ & + & - & \pm & \pm \\
\hline Hippeastrum ossult.-pyrh. . . . . . . & - & + & + & - \\
\hline Hippeastrum dæon.-zeph......... & + & - & - & + \\
\hline Hæmanthus andromeda. . . . . . . . . & + & - & + & - \\
\hline Hæmanthus könig albert......... & + & - & + & - \\
\hline Crinum hybridum j. c. b......... & - & + & - & + \\
\hline Crinum kircape............... & + & - & + & - \\
\hline Crinum powellii............... & 0 & 0 & - & + \\
\hline Nerine dainty maid..$\ldots \ldots \ldots \ldots$ & - & + & + & - \\
\hline Nerine queen of roses............. & - & + & \pm & \pm \\
\hline Nerine giantess. $\ldots \ldots \ldots \ldots \ldots \ldots$ & - & + & - & + \\
\hline Nerine abundance.............. & + & - & - & + \\
\hline Nerine glory of sarnia . . . . . . . . & + & - & - & + \\
\hline Narcissus poeticus herrick......... & - & + & - & + \\
\hline Narcissus poeticus dante......... & - & + & \pm & \pm \\
\hline Narcissus poetaz triumph........ & + & - & - & + \\
\hline Narcissus fiery cross. . . . . . . . . & + & - & \pm & \pm \\
\hline Narcissus doubloon............... & + & - & + & - \\
\hline Narcissus cresset............. & + & - & + & - \\
\hline Narcissus will scarlet. . . . . . . . . & + & - & + & - \\
\hline Narcissus bicolor apricot..... & - & + & - & + \\
\hline Narcissus madame de graaff...... & + & - & + & - \\
\hline Narcissus pyramus.............. & + & - & + & - \\
\hline Narcissus lord roberts . . . . . . . . . . & - & + & + & - \\
\hline Narcissus agnes harvey.......... & + & - & + & - \\
\hline Narcissus j. t. bennett poe ........ & - & + & + & - \\
\hline Begonia mrs. heal.............. & + & - & + & - \\
\hline Begonia ensign $\ldots \ldots \ldots \ldots \ldots \ldots$ & + & - & + & - \\
\hline Begonia julius. $\ldots \ldots \ldots \ldots \ldots \ldots$ & + & - & + & - \\
\hline Begonia success............... & + & - & + & - \\
\hline Phaius hybridus . .......... & + & - & $\dot{+}$ & - \\
\hline Sulphuric-acid reactions: & & & & \\
\hline Narcissus poeticus herrick........ & - & + & - & + \\
\hline Narcissus poeticus dante......... & - & + & + & - \\
\hline Narciseus poetaz triumph......... & + & - & - & + \\
\hline Narcissus fiery cross............ & + & - & \pm & \pm \\
\hline Narcissus doubloon... & - & + & + & - \\
\hline Narcissus cresset. . . . . . . . . . & + & - & $\dot{t}$ & - \\
\hline Narciseus will scarlet. . . . . . . . . & + & - & + & - \\
\hline Narcissus bicolor apricot......... & - & + & \pm & \pm \\
\hline
\end{tabular}

TABLE E.-Continued.

\begin{tabular}{l|c|c}
\hline Hybridu. & $\begin{array}{c}\text { Qualitative } \\
\text { reactions, } \\
\text { closer as a } \\
\text { whole to- }\end{array}$ & $\begin{array}{c}\text { Qualitative } \\
\text { reactions, } \\
\text { closer as a } \\
\text { whole to- }\end{array}$ \\
\hline $\begin{array}{c}\text { Seed } / \text { Pollen } \\
\text { parent. }\end{array}$ & $\begin{array}{c}\text { Seed } / \text { Parent. } \\
\text { parent. parent. }\end{array}$
\end{tabular}

7. Sulphuric-acid reactions.-Cont.:

Narcissus madame de graaft ......

Narcissus pyramus ..............

Narcissus lord roberts . ...........

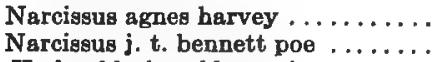

8. Hydrochloric-acid reactions:

Iris ismali

Iris dorak

Iris mrs. glan

Iris pursind

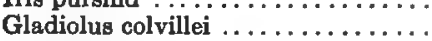

Tritonia crocosmæflora ...........

Richardia mrs. roosevelt ...........

Phaius hybridus . .............

Miltonia bleuana ..............

Calanthe veitchii $\ldots \ldots \ldots \ldots \ldots \ldots \ldots$

Calanthe bryan ...............

Crinum hybridum j. c. h .........

Crinum kircape

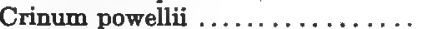

Lilium marhan

Lilium dalhansoni

Lilium golden gleam ...............

Lilium testaceum

Lilium burbanki ...............

Richardia mrs. roosevelt . . . . . . . .

Phaius hybridus

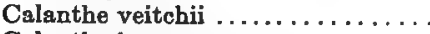

Calanthe bryan ...................

10. Potassium-iodide resctions:

Brunsdonna sanderce alba.

Brunsdonna sanderœ.

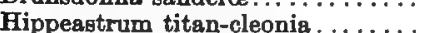

Hippeastrum ossult.-pyrh.........

Hippeastrum dæon.-zeph.

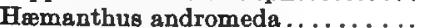

Hæmanthus könig albert. . . . . . . .

Crinum bybridum j. c. h...........

Crinum kircape................

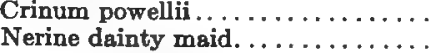

Nerine dainty maid. ............

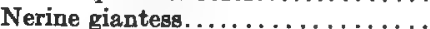

Nerine abundance. ............

Nerine glory of sarnia............

Iris ismali.

Iris dorak....................

Iris mra. alan grey...............

Iris pursind:.

Gladiolus colvillei..............

Tritonia crocosmeflora ...........

Phaius hybridus.

Miltonia bleusna.

11. Potassium-sulphocyanate reactions:

Brunsdonna sanderce alba . . . . . . .

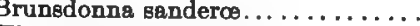

Hippeastrum titan-cleonia . ...........

Hippeastrum ossult.-pyrh... . . . . .

Hippeastrum dæon.-zeph . . .........

Himanthus andromeda . . ............

Hæmanthus könig albert............

Crinum hybridum j. c. h...........

Crinum kircape. .

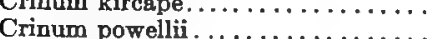

Nerine dainty maid................

Nerine queen of roses. . . . . . . . . . .

Nerine giantess.................

Nerine abundance...............

Nerine glory of sarnia. . . . . . . . .

Phaius hybridus.

+
+
+
+
+
+
+
+
+
+
+
+
+
+
+
+
+
+
+
+
+
+
+
+
+
+
+
+
+
+
+
+
+
+
+
+
+
+
+
+
+
+
+
+

parent. parent. parent. parent. 
TABLE E.-Continued.

\begin{tabular}{|c|c|c|c|c|}
\hline \multirow[t]{2}{*}{ Hybrids. } & \multicolumn{2}{|c|}{$\begin{array}{l}\text { Qualitative } \\
\text { reactions, } \\
\text { closer as a } \\
\text { whole to- }\end{array}$} & \multicolumn{2}{|c|}{$\begin{array}{l}\text { Qualitative } \\
\text { reactions } \\
\text { closer as a } \\
\text { whole to- }\end{array}$} \\
\hline & $\mid \begin{array}{c}\text { Seed } \\
\text { parent. }\end{array}$ & $\begin{array}{l}\text { Pollen } \\
\text { parent. }\end{array}$ & $\begin{array}{c}\text { Seed } \\
\text { parent. }\end{array}$ & $\begin{array}{l}\text { Pollen } \\
\text { parent. }\end{array}$ \\
\hline 12. Potassium-sulphide reactions: & & & & \\
\hline Hæmanthus andromeda......... & 0 & 0 & \pm & \pm \\
\hline Hæmanthus könig albert........ & + & - & + & - \\
\hline Crinum hybridum j. c. h.......... & - & + & - & + \\
\hline Crinum kircape............... & + & - & + & - \\
\hline 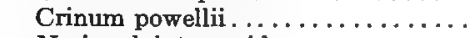 & - & + & - & + \\
\hline Nerine dainty maid. ........... & - & + & \pm & \pm \\
\hline Nerine queen of roses............. & - & + & - & + \\
\hline 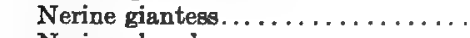 & - & $\dot{t}$ & - & + \\
\hline Nerine abundance $\ldots \ldots \ldots \ldots \ldots$ & + & - & - & $=+$ \\
\hline Nerine glory of sarnia........ & + & - & - & + \\
\hline Phaius hybridus $\ldots \ldots \ldots \ldots \ldots \ldots$ & + & - & \pm & \pm \\
\hline \multicolumn{5}{|l|}{ 13. Sodium-hydroxide reactions: } \\
\hline 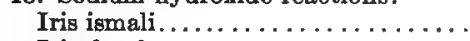 & + & - & \pm & \pm \\
\hline Iris dorak ............... & + & - & + & - \\
\hline Iris mre. alan grey. . . . . . . . & - & + & \pm & \pm \\
\hline Iris pursind. ...... & + & - & \pm & \pm \\
\hline Gladiolus colvillei............ & + & - & + & - \\
\hline Tritonia crocosmæflora . . . . & - & + & + & - \\
\hline Phaius hybridus. ....... & + & - & - & + \\
\hline \multicolumn{5}{|l|}{ 14. Sodium-sulphide reactions: } \\
\hline Crinum hybridum j. c. h...... & - & + & - & + \\
\hline Crinum kircape $\ldots \ldots \ldots \ldots \ldots \ldots$ & + & - & + & - \\
\hline Crinum powellii ........ & - & + & - & + \\
\hline Phaius hybridus . .............. & + & - & - & + \\
\hline \multicolumn{5}{|l|}{ 15. Sodium-salicylate reactions: } \\
\hline Brunsdonna sanderce alba . . . & + & - & + & - \\
\hline Brunsdonna sanderø.............. & + & - & + & - \\
\hline Hippeastrum titan-c & + & - & + & - \\
\hline Hippeastrum ossult.-pyrh.... & - & + & - & + \\
\hline Hippeastrum dæon.-zeph. . . & \pm & \pm & \pm & \pm \\
\hline Hamanthus andromeda . . . . & + & - & - & + \\
\hline Hæmanthus könig albert....... & + & - & - & $\dot{+}$ \\
\hline Crinum hybridum j. c. h...... & - & + & - & + \\
\hline Crinum kircape............ & + & - & + & - \\
\hline Crinum powellii...$\ldots \ldots \ldots$ & - & + & - & + \\
\hline Nerine dainty maid......... & - & + & - & + \\
\hline Nerine queen of roses.. & + & - & - & + \\
\hline Nerine giantess....... & + & - & - & + \\
\hline Nerine abundance . . . . . . . . . & + & - & - & + \\
\hline Nerine glory of sarnia. . . . . . . . & + & - & + & - \\
\hline Iris ismali. . . . . . . . . & + & - & - & + \\
\hline Iris dorak........ & + & - & + & - \\
\hline Iris mrs. alan grey. & - & + & - & + \\
\hline Iris pursind. . & + & - & + & - \\
\hline Gladiolus colvillei....... & + & - & + & - \\
\hline Tritonia crocosmæflora....... & - & + & + & - \\
\hline Richardia mrs. roosevelt...... & + & - & \pm & \pm \\
\hline Musa hybrida...... & - & + & - & + \\
\hline Phaius hybridus. ... . & + & - & - & + \\
\hline Miltonia bleuana. . . . . . . . . . & + & - & + & - \\
\hline Cymbidium eburneo-lowianum ..... & + & - & \pm & \pm \\
\hline Calanthe veitchii $\ldots \ldots \ldots \ldots \ldots$ & - & + & + & - \\
\hline Calanthe bryan................ & + & - & \pm & \pm \\
\hline \multicolumn{5}{|l|}{ 16. Strontium-nitrate reactions: } \\
\hline Begonia mre heal............ & + & - & + & - \\
\hline Begonia ensign .......... & + & - & + & - \\
\hline Begonia julius.......... & + & - & + & - \\
\hline Begonia success............ & + & - & + & - \\
\hline \multicolumn{5}{|l|}{ 17. Cobalt-nitrate reactions: } \\
\hline Brunsdonna sanderœ alba .. & + & - & - & + \\
\hline Brunsdonna sanderœ...... & + & - & - & + \\
\hline Lilium marhan. . ......... & - & + & - & + \\
\hline Lilium dalhansoni.......... & + & - & - & + \\
\hline Lilium golden gleam............. & + & - & + & - \\
\hline Lilium testaceum. . . . . . . . . . . & + & - & - & + \\
\hline Lilium burbanki... & $\dot{+}$ & - & + & - \\
\hline Musa hybrida.......... & - & + & - & + \\
\hline
\end{tabular}

TABLE E.-Continued.

\begin{tabular}{|c|c|c|c|c|}
\hline \multirow[t]{2}{*}{ Hybrids. } & \multicolumn{2}{|c|}{$\begin{array}{l}\text { Qualitative } \\
\text { reactions, } \\
\text { closer as a } \\
\text { whole to- }\end{array}$} & \multicolumn{2}{|c|}{$\begin{array}{l}\text { Qualitative } \\
\text { reactions, } \\
\text { closer as a } \\
\text { whole to- }\end{array}$} \\
\hline & $\begin{array}{c}\text { Seed } \\
\text { parent. }\end{array}$ & $\begin{array}{c}\text { Pollen } \\
\text { parent. }\end{array}$ & $\begin{array}{c}\text { Seed } \\
\text { parent. }\end{array}$ & $\begin{array}{l}\text { Pollen } \\
\text { parent }\end{array}$ \\
\hline 18. Copper-nitrate reactions: & & & & \\
\hline Brunedonna sanderce alba . & + & - & - & + \\
\hline Brunsdonna sanderœ . . . . . & + & - & - & + \\
\hline Crinum hybridum j. c. h.. & - & + & - & + \\
\hline Crinum kircape.......... & + & - & + & - \\
\hline Crinum powellii . . . . . . . . & - & + & - & + \\
\hline 19. Cupric-chloride reactions: & & & & \\
\hline Brunsdonna sanderce alba. . & + & - & - & + \\
\hline Brunsdonna sanderœ...... & + & - & - & + \\
\hline Crinum hybridum j. c. h.... & - & + & - & + \\
\hline Crinum kircape........... & + & - & + & \\
\hline Crinum powellii ........ & - & + & - & $\ddot{+}$ \\
\hline Lilium marhan. . . . . . . . & - & + & - & + \\
\hline Lilium dalhansoni......... & + & - & - & + \\
\hline Lilium golden gleam . ... & + & - & - & + \\
\hline Lilium testaceum....... & + & - & & + \\
\hline Lilium burbanki.......... & + & - & + & \\
\hline 20. Barium-chloride reaction: & & & & \\
\hline Cymbidium eburneo-lowianum . & + & - & \pm & \pm \\
\hline 21. Mercuric-chloride reactions: & & & & \\
\hline Crinum hybridum j. c. h...... & - & + & - & + \\
\hline Crinum kircape........... & + & - & + & - \\
\hline Crinum powellii................... & - & + & - & + \\
\hline Cymbidium eburneo-lowianum . ... . & + & - & \pm & \pm \\
\hline
\end{tabular}

SUMmary OF TABLE E.-Qualitative and Quantitative Reactions of the Starches of Hybrid-stocks in regard to Sameness and Inclination to one or the other or both Parent-stocks.

\begin{tabular}{|c|c|c|c|c|c|c|c|c|}
\hline \multirow{3}{*}{$\begin{array}{l}\text { Agents } \\
\text { and } \\
\text { Reagents. }\end{array}$} & \multirow{3}{*}{ 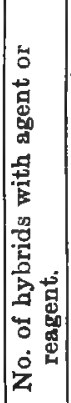 } & \multicolumn{3}{|c|}{$\begin{array}{l}\text { Qualitative } \\
\text { reactions. }\end{array}$} & \multicolumn{3}{|c|}{$\begin{array}{l}\text { Quantitative } \\
\text { reactions. }\end{array}$} & \multirow{3}{*}{ 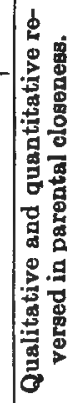 } \\
\hline & & \multicolumn{2}{|c|}{$\begin{array}{l}\text { Closer, } \\
\text { on the } \\
\text { whole, } \\
\text { to the- }\end{array}$} & \multirow{2}{*}{ 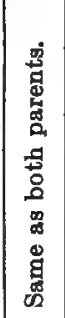 } & \multicolumn{2}{|c|}{$\begin{array}{l}\text { Closer, } \\
\text { on the } \\
\text { whole, } \\
\text { to the- }\end{array}$} & \multirow{2}{*}{ 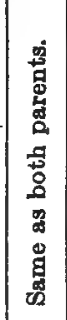 } & \\
\hline & & 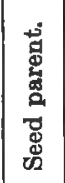 & 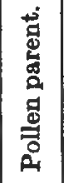 & & 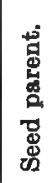 & 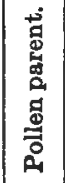 & & \\
\hline & 50 & 24 & 25 & 1 & 27 & 19 & 4 & 6 \\
\hline & 50 & 28 & & 2 & & & & 3 \\
\hline & 50 & 38 & & 0 & 23 & 20 & 7 & 15 \\
\hline & 29 & 2. & 8 & & 18 & & 2 & \\
\hline & 18 & 11 & 7 & 0 & 8 & 7 & 3 & 2 \\
\hline & 32 & 22 & 10 & 1 & 19 & 9 & & 8 \\
\hline Sul & 13 & 9 & 4 & 0 & 8 & 2 & 3 & 3 \\
\hline & 11 & 7 & 4 & 0 & 2 & $\overline{7}$ & & \\
\hline & 11 & 7 & 4 & 0 & 2 & 3 & & 2 \\
\hline & 23 & 15 & 8 & 0 & 10 & 7 & 6 & 6 \\
\hline & 16 & 11 & 5 & 0 & 5 & 6 & & \\
\hline & 10 & 5 & 5 & 0 & 2 & 6 & 2 & 2 \\
\hline & 7 & 4 & 3 & & 2 & & & \\
\hline & 4 & 2 & 2 & 0 & 1 & 3 & 0 & 1 \\
\hline & 28 & 18 & 9 & 1 & 10 & 14 & 4 & 8 \\
\hline & 4 & 4 & 0 & 0 & 4 & 0 & 0 & 0 \\
\hline & 8 & 6 & 2 & 0 & 2 & 6 & 0 & 4 \\
\hline & 5 & 3 & 2 & 0 & 1 & 4 & 0 & 2 \\
\hline & 10 & 7 & 3 & 0 & 3 & 7 & 0 & \\
\hline & 1 & 1 & 0 & 0 & 1 & 0 & 1 & 0 \\
\hline Mercuric chloride......... & 4 & 2 & 2 & 0 & 2 & 1 & 1 & 0 \\
\hline Total num & 374 & 235 & 134 & 5 & 166 & 150 & 59 & 84 \\
\hline Per cent ...... & & 62.8 & 35.8 & 1.34 & 44 & 40 & 15.8 & 22.5 \\
\hline
\end{tabular}


Reaction-intensities of Each Hybrid Starch. (Tables F, Parts 1 to 50 and Summary; G and H, Parts 1 to 26 and Summaries 1 and 2.)

In Chapter I particular reference was made to the recognition of intermediateness as one of the primary criteria of hybrids, this applying not only to macroscopic and microscopic characters of plants, but also to the microscopic characters of starches. Intermediateness of starches was therein shown to have been recorded by MacFarlane (page 7) in Ribes, Bryanthus, and Hedychium, and by Darbyshire (page 8) in Pisum. MacFarlane states that in Ribes grossularia, $R$. culverwellii (hybrid) and $R$. nigrum the starch grains of the three are very variable in size, but in the first the largest are $7 \mu$ and the average $4 \mu$; in the third the largest are $3 \mu$ and the average $11 / 2 \mu$; and in the second the largest are $5 \mu$ and the average $3 \mu$. In Menziesis empertriformis var., Bryanthus erectus (hybrid) and Rhododendron chamocistus he found that in the third the starch grains are $4 \mu$ across the largest, though most are from $2 \mu$ to $3 \mu$; in the first the largest granules are $6 \mu$ across, and in all cases they are larger than in the third; and in the second the size of the granules falls rather toward the third. In Hedychium gardnerianum, $H$. sadlerianum (hybrid), and $H$. coronarium he notes that in the first each starch grain is a small triangular plate, measuring $10 \mu$ to $12 \mu$, from hilum to base, and that the lamination is not very distinct; in the third each grain is ovate, or in some cases tapered rather finely to a point at the hilum, $32 \mu$ to $60 \mu$ long from hilum to base, and the lamination is very marked; in the second "the grains may best be described if we suppose a rather reduced one of the first parent to be set on the reduced basal half of one of the latter. The lamination also is more pronounced than in the first, less so than in the second." Darbyshire records that the round starch grain of the $F_{1}$ generation is a blend between the type of grain of the round pea (the potato-shaped) and the type of grain of the wrinkled pea (the compound) in respect to the three characters: length-breadth-index, distribution of compoundness, and degree of compoundness. While these data are very meager they are concordant and in harmony with the dictum of intermediateness of histologic and naked-eye characters of hybrids.

In the present research it was found in the studies of the histologic peculiarities that in case of every hybrid there are certain characters that are intermediate, the degree of intermediateness varying from mid-intermediateness to almost identity with one or the other parent. Mid-intermediateness was found to be, on the whole, far less common than a degree of intermediateness that closely approached one or the other parent; identity of a given character with that of one or the other parent was quite common; development of a given character or character-phase in excess or deficit of those of both parents quite frequent; and the appearance of individualities in the hybrid that are not seen in either parent was by no means rare. In fact, it seems clear that the more in detail these studies are carried out the farther we are taken from the conception of generality of intermediateness of the properties of the hybrid. The records of the histologic peculiarities of the starches are fully supported by those of the histologic and macroscopic characters of plants as set forth in this chapter and in
Part II, Chapter II, and also by the qualitative and quantitative reactions of the starches throughout the entire range of agents and reagents as shown by the data that are represented especially in Chapter III and Part II, Chapter I. In preceding parts of the present chapter various tabular statements exhibit from different aspects parental relationships of the hybrids. It seems desirable at this point to tabulate the reaction-intensities of the hybrids with reference to sameness to one or the other parent or both parents, intermediateness, and excess and deficit of development in relation to the parents, so that one may see at a glance, as it were, the relative importance of the several phases of parent-character development in regard to the reaction-intensities of: (a) Each hybrid starch with different agents and reagents, which will exhibit particularly the differences in the behavior of each starch in comparison with the reaction of other starches in the presence of the same agents and reagents; $(b)$ each hybrid starch as regards sameness and inclination in its properties in relation to one or the other or both parents, which will exhibit particularly the comparative potencies of the parents in determining the properties of the starch of the hybrid; and $(c)$ all of the hybrid starches with each agent and reagent, which will exhibit particularly the independence of the behavior of each agent and reagent, and also all of the hybrid starches with each agent and reagent, as regards sameness and inclination in the properties to one or the other parent or both parents, which will exhibit particularly the independent tendencies of each agent or reagent to elicit definite and specific parent-phases. While all of these tabulations are most intimately correlated, each brings out certain features with marked accentuation in a form not elicited by the others.

\section{Reaction-intensities of Eadi Hybrid Starch with Different Agents and Reagents.}

(Tables F, Parts 1 to 50 and Summary.)

It is to be noted in an examination of the results formulated in the accompanying table that in only 32 of the 50 hybrids recorded all of the 26 reactions, 16 recorded only 10 reactions, and 2 only 13 reactions. Taking up this table, even a most cursory examination will indicate the very wide variations of the numerical values of the 6 phases of parent-development of the different starches in their parental relationships, and each part of the table is different from every other part and is specifically distinctive of the hybrid, even in the cases of hybrids that have resulted from the same cross, as in Brunsdonna sanderce alba and $B$. sanderce (Table F, 1 and 2), and Narcissus poeticus herrick and $N$. poeticus dante (Table F, 16 and 17). Moreover, in one hybrid intermediateness may be relatively so very conspicuous that the other phases sink into insignificance, while in another this phase may be as markedly conspicuous by its almost or entire absence, and so on in other tables with the other phases. It is also very obvious that the hybrid is less apt to be characterized by a prominence of intermediateness than by a conspicuousness of highest or lowest development or even of other phase of parental relationship.

The several parts of this table may, for convenience of study, be grouped into four classes: (1) those in which one of the phases of development very markedly dominates the others, one-half or more of the reactions being 
included in this phase; (2) those in which two phases are definitely dominant, but which may be quite different in value; (3) those in which three phases are dominant, but which may have different values; and (4) those in which the parental relationships of the hybrid seem to be directed largely indifferently to the several phases. Among the starches that were studied in all of the 26 reactions it is rare, as, for instance in Irils dorak, to find that the assignment is not unmistakable. Where the number of reactions is restricted to 10 to 13 the classification is often indefinite. The grouping in aocordance with the foregoing is as follows:

\begin{tabular}{|c|c|c|c|c|c|c|}
\hline Hybrids. & 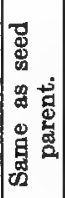 & 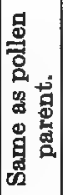 & 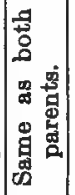 & 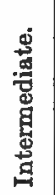 & 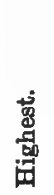 & 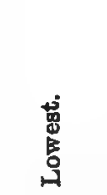 \\
\hline \multicolumn{7}{|l|}{ First class: } \\
\hline Brunsdonna sanderœ alba . . & 4 & 0 & 1 & 5 & 3 & 13 \\
\hline Brunsdonna sanderce . . . . . & 6 & $\mathbf{0}$ & 1 & 2 & $\mathbf{3}$ & 14 \\
\hline Crinum kircape........... & 4 & 1 & 0 & 18 & 2 & 1 \\
\hline Crinum powellii. . . . . . . . & 0 & 3 & $\mathbf{0}$ & 2 & 21 & 0 \\
\hline Narcissus poetaz triumph. . & 2 & 2 & 1 & 0 & 20 & 1 \\
\hline Narcissus j.t. bennett poe. . & 2 & $\mathbf{0}$ & $\mathbf{0}$ & 0 & 8 & $0(10) *$ \\
\hline Lilium burbanki $\ldots \ldots \ldots$. & 2 & 1 & 1 & 6 & 0 & 16 \\
\hline Iris mre. alan grey. ........ & $\mathbf{0}$ & 1 & 3 & 1 & 4 & 17 \\
\hline Tritonia crocosmæflora..... & 2 & 1 & 2 & 16 & 3 & 2 \\
\hline Begonia ensign........... & 0 & 0 & 0 & 7 & 1 & $2(10)$ \\
\hline Musa hybrida............ & 1 & 3 & 0 & 2 & 0 & 20 \\
\hline Miltonia bleuana . . . . . . . & 3 & 0 & 3 & 1 & 17 & 2 \\
\hline Calanthe bryan........ & 1 & 0 & 0 & 11 & 1 & $0(13)^{*}$ \\
\hline \multicolumn{7}{|l|}{ Second class: } \\
\hline Hippeastrum ossult.-pyrha. . & 3 & $\mathbf{0}$ & 8 & 3 & 11 & 1 \\
\hline Hæmanthus könig albert... & 5 & 0 & 0 & 7 & 1 & 3 \\
\hline Nerine queen of roses . . . . . . & 2 & 1 & 7 & 3 & 11 & 2 \\
\hline Nerine abundance . ........ & 3 & 3 & 7 & 3 & 1 & $\boldsymbol{\theta}$ \\
\hline Narcissus poeticus dante.... & $\mathbf{1}$ & 4 & 0 & 4 & 1 & $0(10) *$ \\
\hline Narcissus lord roberts. . . . . . & 3 & 1. & 1 & 4 & 0 & $1(10) *$ \\
\hline Narcissus agnes harvey.... . & 4 & 0 & 1 & $\mathbf{3}$ & 1 & $1(10) *$ \\
\hline Iris ismali $\ldots \ldots \ldots \ldots \ldots \ldots$ & 3 & 2 & 2 & 12 & 1 & 6 \\
\hline Gladiolus colvillei.... & 7 & $\mathbf{0}$ & 1 & 4 & 0 & 14 \\
\hline Begonia mrs. heal.......... & 9 & $\mathbf{0}$ & 2 & 14 & 0 & 1 \\
\hline Begonia julius............ & 1 & 1 & 0 & 4 & 4 & $0(10) *$ \\
\hline Phaius hybridus . . . . . . . . & 1 & 3 & 5 & 11 & 3 & 3 \\
\hline Cymbidium eburneo-lowia- & & & & & & \\
\hline num $\ldots \ldots \ldots \ldots \ldots$ & 4 & 0 & 9 & 0 & $\mathbf{0}$ & 13 \\
\hline Calanthe veitchii.......... & 2 & 1 & 0 & 5 & 4 & $1(13) *$ \\
\hline \multicolumn{7}{|l|}{ Third class: } \\
\hline Hæmanthus andromeda . . . & 8 & $\mathbf{0}$ & 6 & 11 & 0 & 1 \\
\hline Crinum hybridum j. c. h.... & 0 & 12 & 0 & 5 & 2 & 7 \\
\hline Nerine dainty maid........ & 1 & 2 & 7 & 6 & 8 & $\mathbf{2}$ \\
\hline Nerine glory of sarnia. ..... & 1 & 6 & 8 & 1 & 0 & 10 \\
\hline Narcissus doubloon ........ & 2 & 1 & 1 & 4 & 0 & $2(10) *$ \\
\hline Narcissus will scarlet....... & 2 & 1 & 1 & 2 & 4 & $0(10) *$ \\
\hline Lilium dalhansoni...... . & 4 & 1 & 9 & $\theta$ & 2 & 1 \\
\hline \multirow{2}{*}{\multicolumn{7}{|c|}{ Fourth class: }} \\
\hline & & & & & & \\
\hline Hippeastrum titan-cleonia. & 2 & 3 & 8 & 4 & 5 & 4 \\
\hline Hippeastrum dæones-zephyr & $\mathbf{0}$ & 2 & 9 & 6 & 5 & 4 \\
\hline Nerine giantess............ & 2 & 6 & 7 & 6 & 1 & 4 \\
\hline Narcissus poeticus herrick & $\mathbf{0}$ & 3 & 0 & 3 & 3 & $2(10) *$ \\
\hline Narcissus fiery cross . . . . . . & 1 & 2 & 0 & 2 & 2 & $3(10) *$ \\
\hline Narcissus cresset. . . . . . . & 2 & 3 & 0 & 0 & $\mathbf{3}$ & $2(10) *$ \\
\hline Narcissus bicolor apricot.... & 3 & 1 & 1 & 2 & 0 & $3(10) *$ \\
\hline Narcissus madame de graaff & 4 & 1 & 0 & 1 & 1 & $2(10) *$ \\
\hline Narcissus pyramus......... & 1 & $\mathbf{0}$ & 1 & 2 & 4 & $2(10) *$ \\
\hline Lilium marhan ............ & 0 & 5 & 9 & 6 & 1 & $6(10) *$ \\
\hline Lilium golden gleam . . . . . . . & 4 & 4 & $\mathbf{5}$ & 2 & 7 & 4 \\
\hline Lilium testaceum $\ldots \ldots \ldots$. & 4 & 3 & 2 & 7 & 6 & 4 \\
\hline Iris dorak $\ldots \ldots \ldots \ldots \ldots \ldots$ & $\check{\mathbf{z}}$ & 3 & 2 & 1 & 11 & 4 \\
\hline Iris pursind $\ldots \ldots \ldots \ldots \ldots$ & 3 & 2 & 5 & 5 & 5 & 6 \\
\hline Begonia success. . . . . . . . . . & 2 & 3 & $\mathbf{0}$ & 2 & 3 & $0(10) *$ \\
\hline
\end{tabular}

* Number of reactions when less than 26.
The distribution of the hybrids among the four classes is fairly uniform except in the third class, there being 13 (26 per cent) in the first class, 14 (28 per cent) in the second class, 8 ( 6 per cent) in the third class, and 15 ( 30 per cent) in the fourth class. In the first class, 4 of the hybrids are characterized by the conspicuousness of intermediateness, this phase of parental relationship being noted in one hybrid in 18 of the 26 reactions, in another in 16 of 26 reactions, in another in 7 of 10 reactions, and in another in 11 of 13 reactions. In 4 hybrids the characterization is especially in development in excess of parental extremes, this phase being recorded in one in 21 of the 26 reactions, in another in 20 of the 26 reactions, in another in 8 of 10 reactions, and in another in 17 of 26 reactions. In 5 hybrids the characterization is especially by development in deficit of parental extremes, this being found in one in 13 of 26 reactions, in another in 14 of 26 reactions, in another in 16 of 26 reactions, in another in 17 of 26 reactions, and in another in 20 of 26 reactions. In the second class, the dominant figure of the couple is found in 1 hybrid under the phase the same as the seed parent, in 5 under intermediate, in 2 under highest, and in 3 under lowest; in 1 there is duplication of the figures under the phases the same as the pollen parent and intermediate, and in another under intermediate and highest. This coupling is more marked in the instances where 26 reactions were studied than when the number is 10 or 13. In the third class there is not only less tendency to a very marked degree of characterization as regards any one or more of these phases, but also to the characterization being present in three phases usually with slight gradation, as, for instance, in Nerine dainty maid where the values are 7,6 , and 8 under same as both parents, intermediate, and highest, respectively; and in Nerine glory of sarnia, where the values are 6, 8, and 10 under same as pollen parent, same as both parents, and lowest, respectively. Or there may be some duplication, as, for instance, in Lilium dalhansoni, where the values are 4, 9, and 9 under same as seed parent, same as both parents and intermediate, respectively, etc.

From this limited data one may expect that further studies will elicit various combinations of both phases and values. In one hybrid the highest number of the triple is found under same as seed parent, in two under intermediate, in two under highest, and in one, under lowest. In one there is duplication of the highest values under same as both parents and intermediate; and in another under same as both parents and highest. In the three hybrids with which in each only 10 reactions were recorded the grouping of the phases in triplets does not yield the striking comparisons that are observed when the reactions number 26 , or $21 / 2$ times larger. In the fourth class, with 7 of the 15 hybrids only 10 reactions were recorded in each, and in these instances the values are (with possibly two exceptions, Narcissus pyramus and $N$. madame de graaff) so distributed among the different phases that there is not the convincing evidence of a well-defined inclination of the hybrids in their parental relationships that was found in corresponding cases in the preceding classes. Among the remaining 8 there is marked dominance of 1 phase of the 6 in a single hybrid (Iris dorak) in which 11 of the 26 reactions fall under highest, the other values being $5,3,2,1$, 
and 4. This hybrid should perhaps be assigned to the first or second class. In several other instances there is evident tendency to dominance in one phase especially, as in Hippeastrum titan-cleonia, $H$. doones-zephyr, and Lilium marhan.

Apropos of intermediateness as a criterion of hybrids, it is of interest to note that 4 of the hybrids (Narcissus poetaz triumph, N. j. t. bennett poe, $N$. cresset, and Cymbidium eburneo-lowianum) do not in a single reaction exhibit intermediateness. Two of these belong to the first class, both being conspicuous because fourfifths of the reactions of each hybrid are higher than those of the parents. One belongs to the fourth class, and there are no very definite parental leanings. One is found in the third class, with very definite inclinations to activities that are the lowest or the same as those of both parents, especially the first and in the order given $(13,9$, and 4 , respectively).

In recapitulating the totals exhibited by these tables several very interesting points of comparison are elicited (summary of Table F). All together 1,018 reactions were recorded, which are distributed as follows: Same as seed parent 137 (13.4 per cent); same as pollen parent 94 (9.2 per cent); same as both parents 138 (13.6 per cent) ; intermediate 236 ( 23.2 per cent); highest 187 (18.4 per cent); and lowest 226 (22.2 per cent). It is very obvious that there are much more marked tendencies to intermediateness, highness, and lowness than to sameness of development in relation to one or the other parent or both parents, there being somewhat less than two-thirds of the reactions (63.8 per cent) that fall within the first, and 36.2 per cent within the second category. There is about an equal tendency to intermediateness ( 23.2 per cent) as to lowest development (22.2 per cent) and distinctly less tendency to highest development (18.2 per cent) than to either of the former; and there is on an average approximately only about one-half the tendency to sameness to the seed parent (13.4 per cent) and to both parents (13.6 per cent) as there is to intermediateness, the least tendency being shown in sameness to the pollen parent $(9.2$ per cent). Comparing the tendency to intermediateness with the tendencies to highest plus the lowest reactivities, it is found that the latter predominate in the proportion of 23.2 to 40.6 per cent, or approximating 1:2; in other words, there is only a little more than one-half the tendency to an intermediate reaction as there is to one that is above or below parental extremes; and there is an equal tendency to sameness as one or the other parent as there is to intermediateness. If a comparison is made the number of intermediate reactions with the total of other reactions the proportion is found to be 23.2 to 76.8 per cent or approximately $1: 3$, that is, there is in general a likelihood of only 1 reaction in 4 being intermediate. When these intermediate reactions are analyzed only 54 of 236 , or somewhat more than one-fifth and less than one-fourth (23 per cent), are mid-intermediate, the larger' proportion being closer to one or the other parent than to mid-intermediateness.
TABle F.

\begin{tabular}{|c|c|c|c|c|c|c|}
\hline Agent or reagent. & 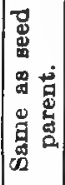 & 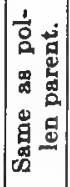 & 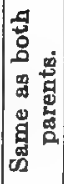 & 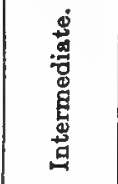 & 总 & $\begin{array}{l}\mathbf{3} \\
\mathbf{g} \\
\stackrel{5}{8}\end{array}$ \\
\hline 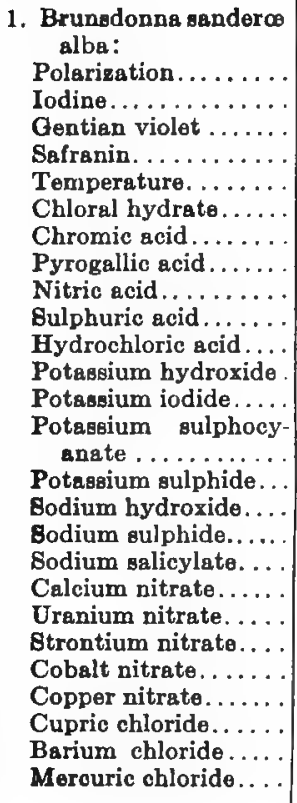 & $\begin{array}{l}t \\
\pm \\
- \\
- \\
- \\
- \\
- \\
\pm \\
- \\
- \\
- \\
- \\
- \\
- \\
- \\
- \\
= \\
\overline{-} \\
\pm\end{array}$ & 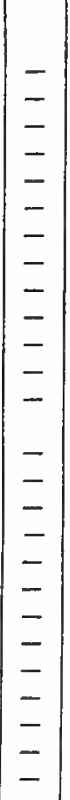 & $\begin{array}{l}\bar{z} \\
- \\
- \\
- \\
= \\
- \\
= \\
\bar{\oplus} \\
- \\
- \\
- \\
- \\
- \\
= \\
= \\
= \\
= \\
-\end{array}$ & $\begin{array}{c}- \\
z \\
- \\
+9 \\
- \\
+8=\sigma^{7} \\
- \\
= \\
- \\
- \\
- \\
- \\
+8=0 \\
- \\
+8 \\
- \\
+9=0 \\
- \\
- \\
- \\
-\end{array}$ & $\begin{array}{l}\overline{-} \\
+0^{7} \\
+\sigma^{7} \\
+0 \\
= \\
= \\
= \\
= \\
= \\
= \\
= \\
= \\
= \\
= \\
= \\
= \\
=\end{array}$ & 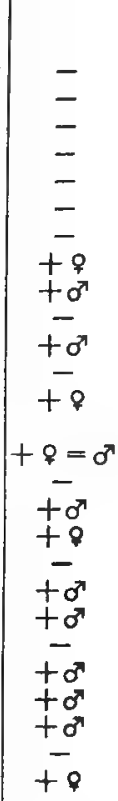 \\
\hline & 4 & 0 & I & B & 3 & 13 \\
\hline 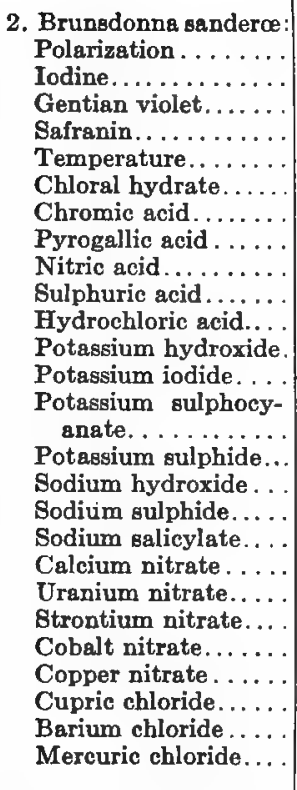 & $\begin{array}{l}\bar{t} \\
\overline{-} \\
\overline{+} \\
- \\
- \\
- \\
- \\
\overline{-} \\
\pm\end{array}$ & $\begin{array}{l}- \\
- \\
- \\
- \\
- \\
- \\
- \\
= \\
- \\
- \\
- \\
- \\
- \\
- \\
- \\
- \\
- \\
- \\
- \\
-\end{array}$ & 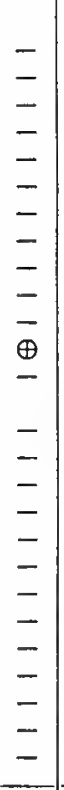 & $\begin{array}{l}+9 \\
= \\
= \\
= \\
= \\
= \\
= \\
= \\
= \\
= \\
= \\
= \\
= \\
= \\
= \\
=\end{array}$ & $\begin{array}{c}- \\
- \\
+0 \\
+8 \\
- \\
+9 \\
= \\
= \\
= \\
= \\
-\end{array}$ & 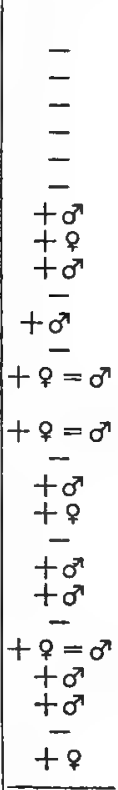 \\
\hline & 6 & 0 & 1 & 2 & $\mathbf{3}$ & 14 \\
\hline
\end{tabular}


TABLE F.-Continued.

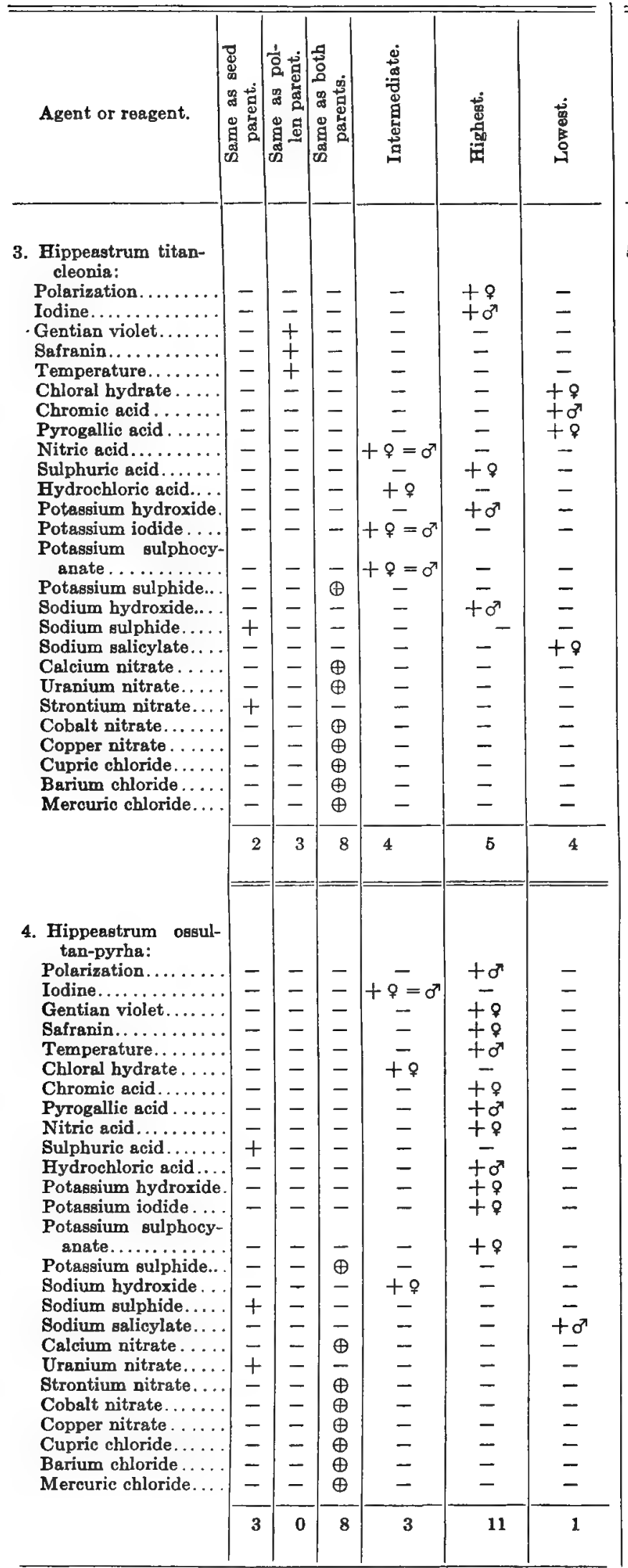

TABLE F.-Continued.

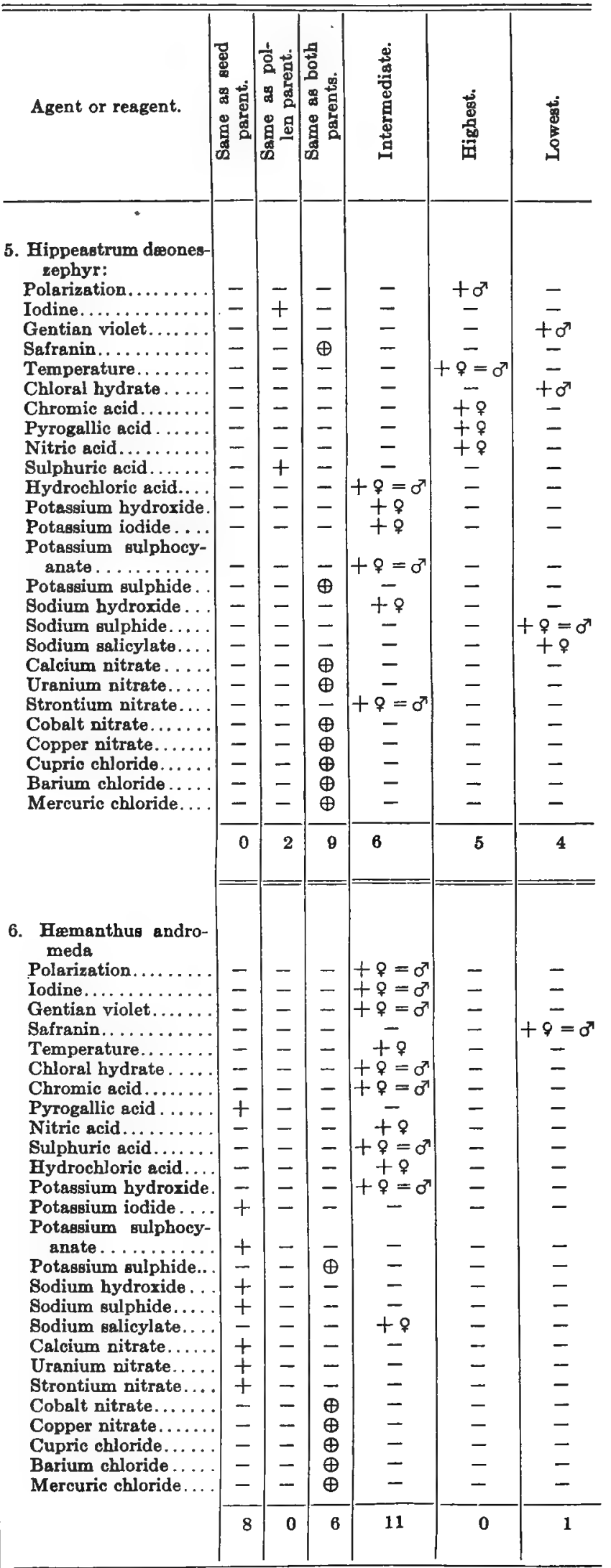


TABLE F.-Continued.

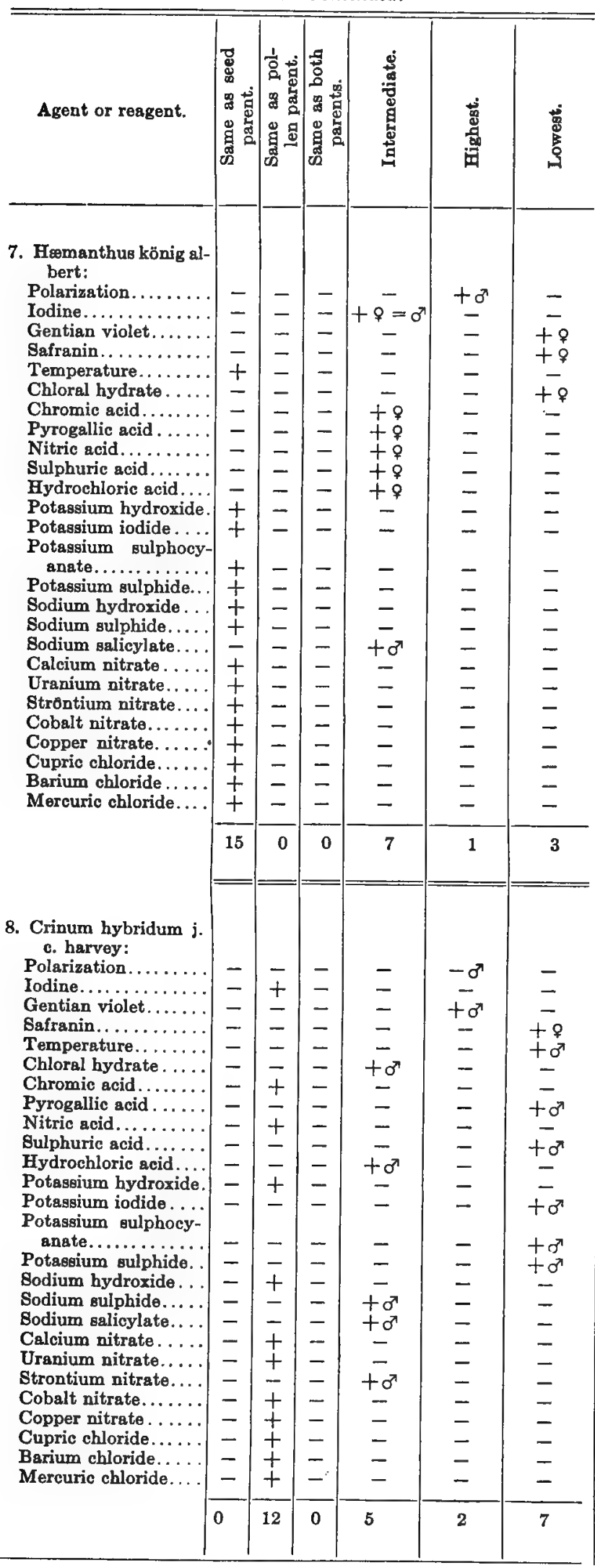

TaBle F.-Continued.

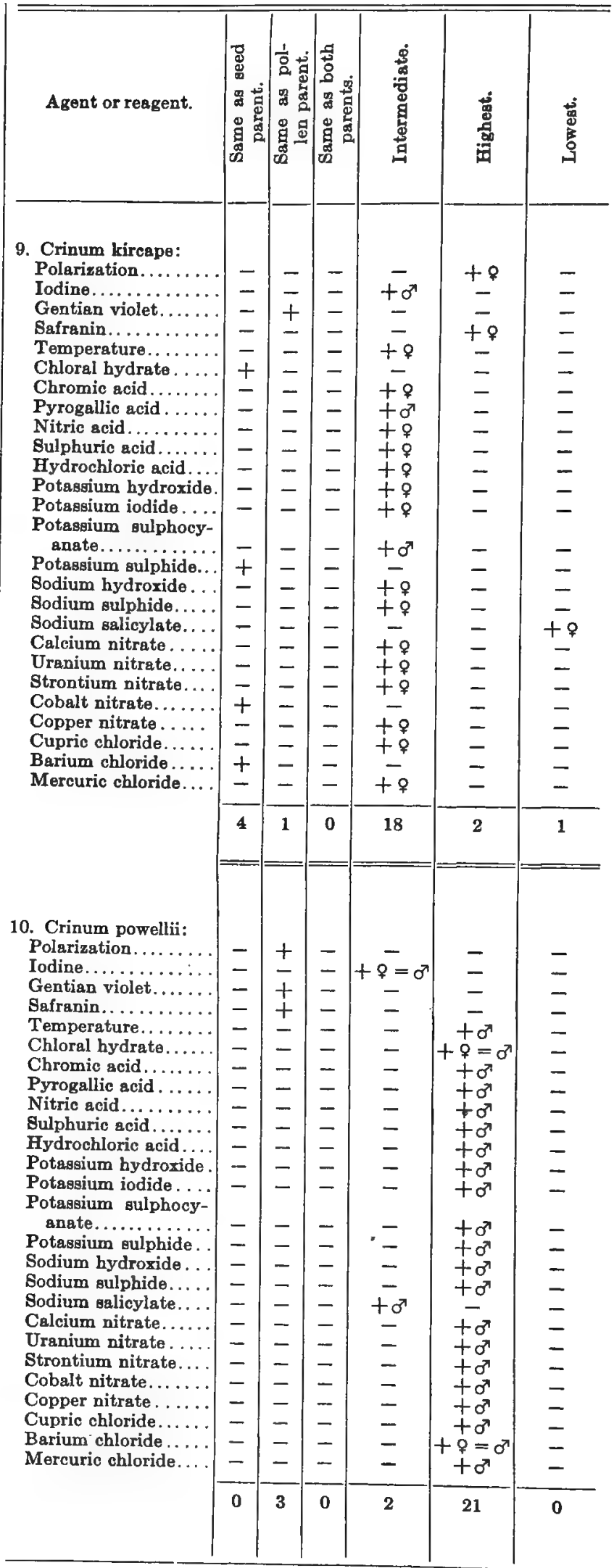


TABLe F.-Continued.

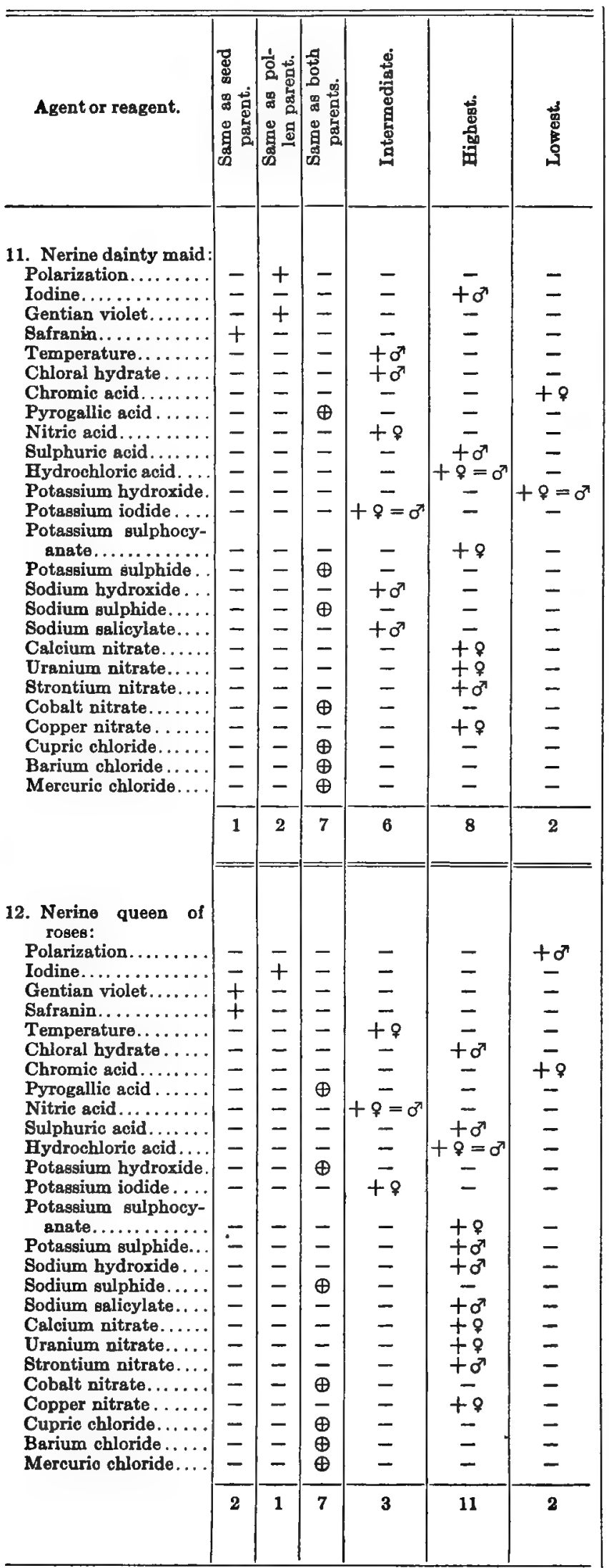

TABLE F.-Continued.

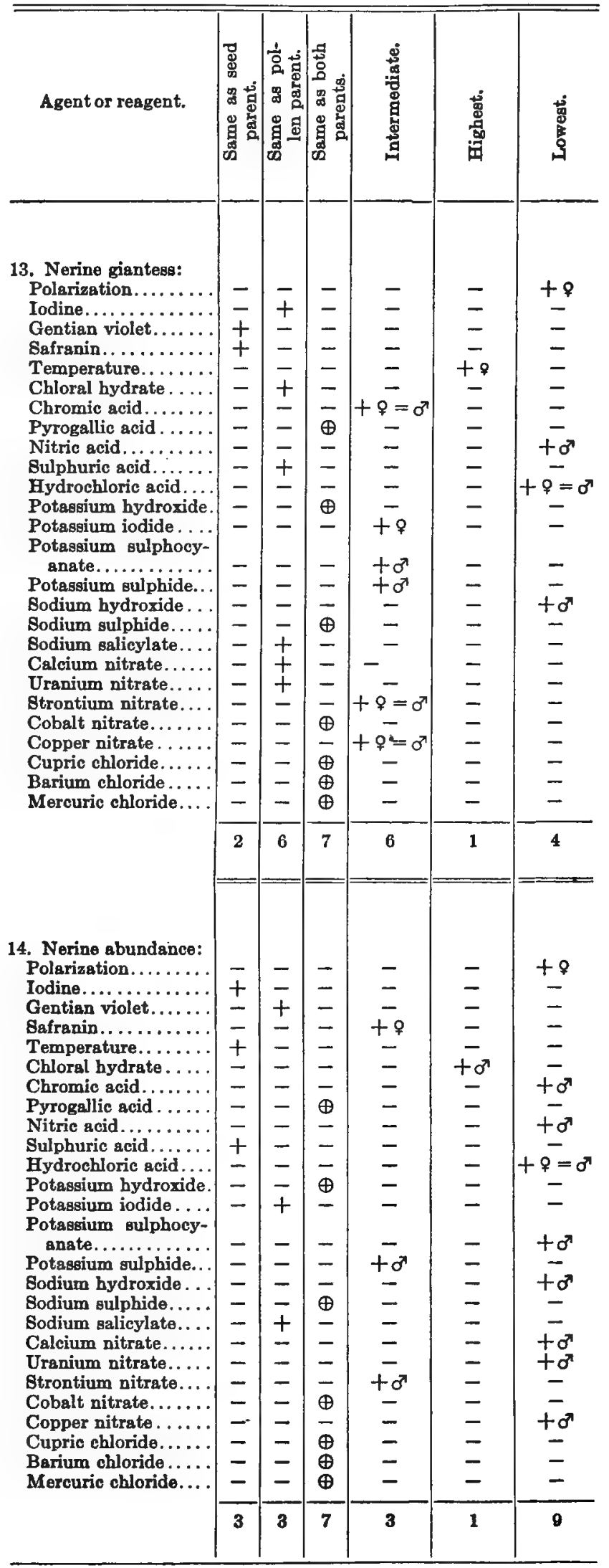


TABLE F-Continued.

\begin{tabular}{|c|c|c|c|c|c|c|}
\hline Agent or reagent. & 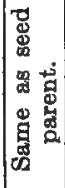 & 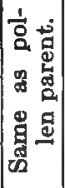 & 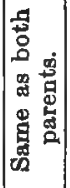 & 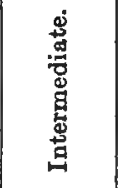 & 兽 & 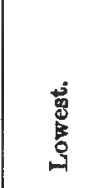 \\
\hline 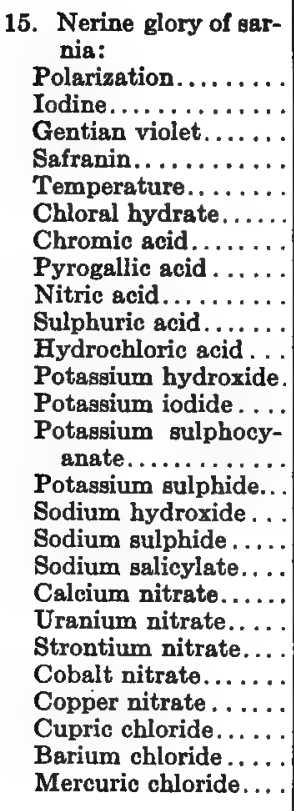 & $\begin{array}{l}+ \\
= \\
= \\
= \\
= \\
= \\
= \\
= \\
= \\
= \\
= \\
= \\
= \\
= \\
=\end{array}$ & $\begin{array}{l}- \\
\pm \\
\pm \\
- \\
- \\
- \\
- \\
- \\
- \\
- \\
\pm \\
\pm \\
\pm \\
\pm \\
\pm \\
\pm \\
- \\
- \\
= \\
-\end{array}$ & $\begin{array}{l}\bar{z} \\
\bar{z} \\
\bar{z} \\
\overline{-} \\
\overline{-} \\
\oplus \\
\overline{-} \\
\bar{\oplus} \\
\oplus \\
\bar{\oplus} \\
\overline{-} \\
\overline{-} \\
\overline{-} \\
\overline{-} \\
\overline{-} \\
\oplus \\
\oplus \\
\oplus \\
\oplus\end{array}$ & $\begin{array}{l}= \\
= \\
\pm 0 \\
= \\
= \\
= \\
= \\
= \\
= \\
= \\
= \\
= \\
=\end{array}$ & $\begin{array}{l}= \\
= \\
= \\
= \\
= \\
= \\
= \\
= \\
= \\
= \\
= \\
= \\
= \\
= \\
= \\
=\end{array}$ & $\begin{array}{c}+8 \\
+8 \\
- \\
+8 \\
+0 \\
- \\
+8=0 \\
+8 \\
- \\
- \\
+\infty \\
= \\
+8 \\
- \\
+\infty \\
= \\
- \\
-\end{array}$ \\
\hline & 1 & 6 & 8 & 1 & D & 10 \\
\hline 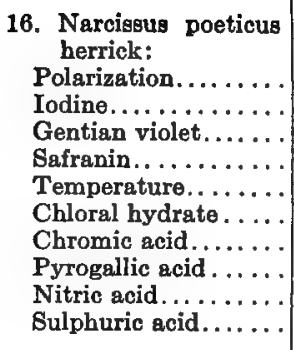 & $\begin{array}{l}= \\
= \\
= \\
= \\
= \\
= \\
=\end{array}$ & $\begin{array}{l}\bar{t} \\
- \\
\overline{-} \\
\pm \\
\pm \\
\pm \\
-\end{array}$ & $\begin{array}{l}- \\
= \\
z \\
z \\
z \\
z \\
-\end{array}$ & $\begin{array}{c}+8 \\
- \\
= \\
+8 \\
+8 \\
= \\
=\end{array}$ & $\begin{array}{c}\bar{z} \\
= \\
= \\
= \\
= \\
+9=0 \\
+\sigma^{\circ}\end{array}$ & $\begin{array}{l}\bar{z} \\
+8 \\
+8 \\
= \\
= \\
=\end{array}$ \\
\hline & 0 & 3 & 0 & 3 & 2 & 2 \\
\hline 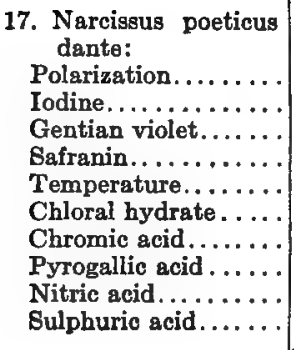 & $\begin{array}{l}- \\
= \\
= \\
= \\
= \\
= \\
\overline{+}\end{array}$ & 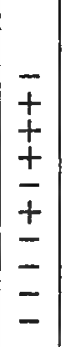 & $\begin{array}{l}- \\
= \\
- \\
- \\
= \\
= \\
-\end{array}$ & $\begin{array}{c}+9 \\
- \\
- \\
+9 \\
-\infty \\
- \\
+9=\sigma^{\circ} \\
-\end{array}$ & $\begin{array}{c}\bar{z} \\
= \\
- \\
\overline{-} \\
+8=\sigma^{3} \\
-\end{array}$ & $\begin{array}{l}\bar{z} \\
\overline{-} \\
\overline{-} \\
\overline{-} \\
= \\
-\end{array}$ \\
\hline - & 1 & 4 & 0 & 4 & I & 0 \\
\hline
\end{tabular}

TabLe F.-Continued.

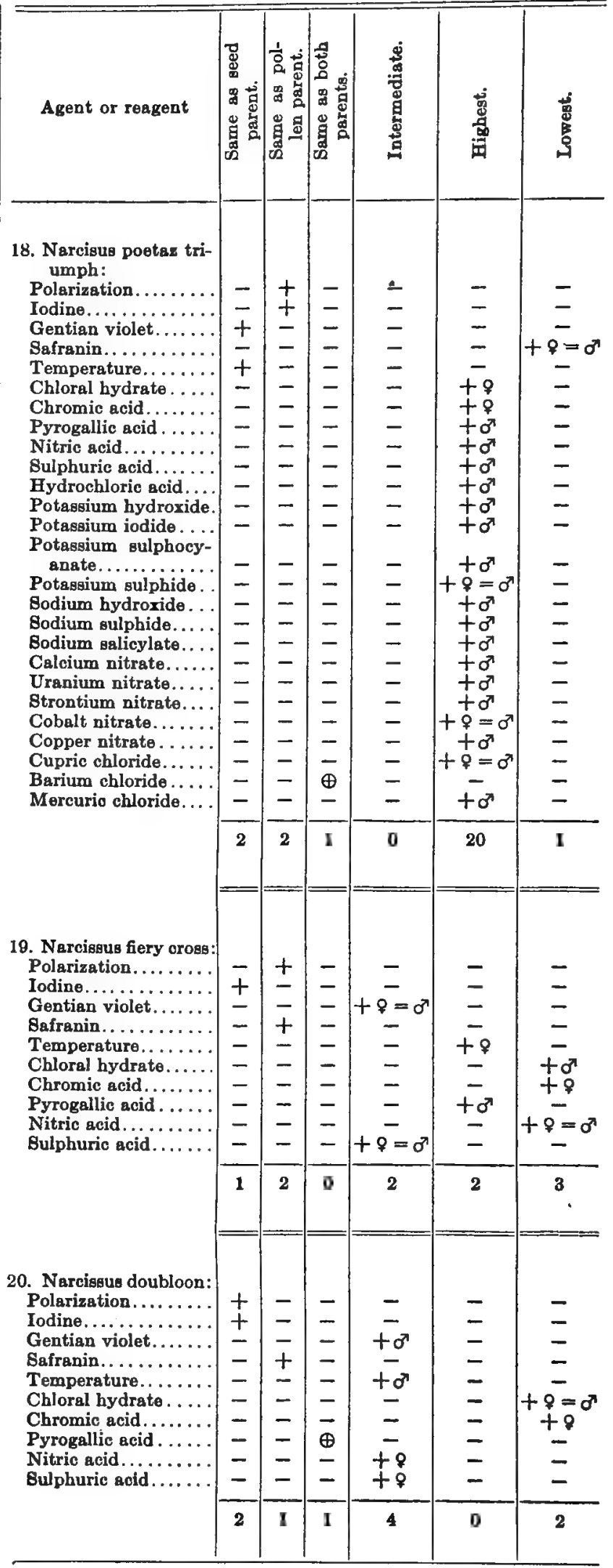


Tabue F-Continued.

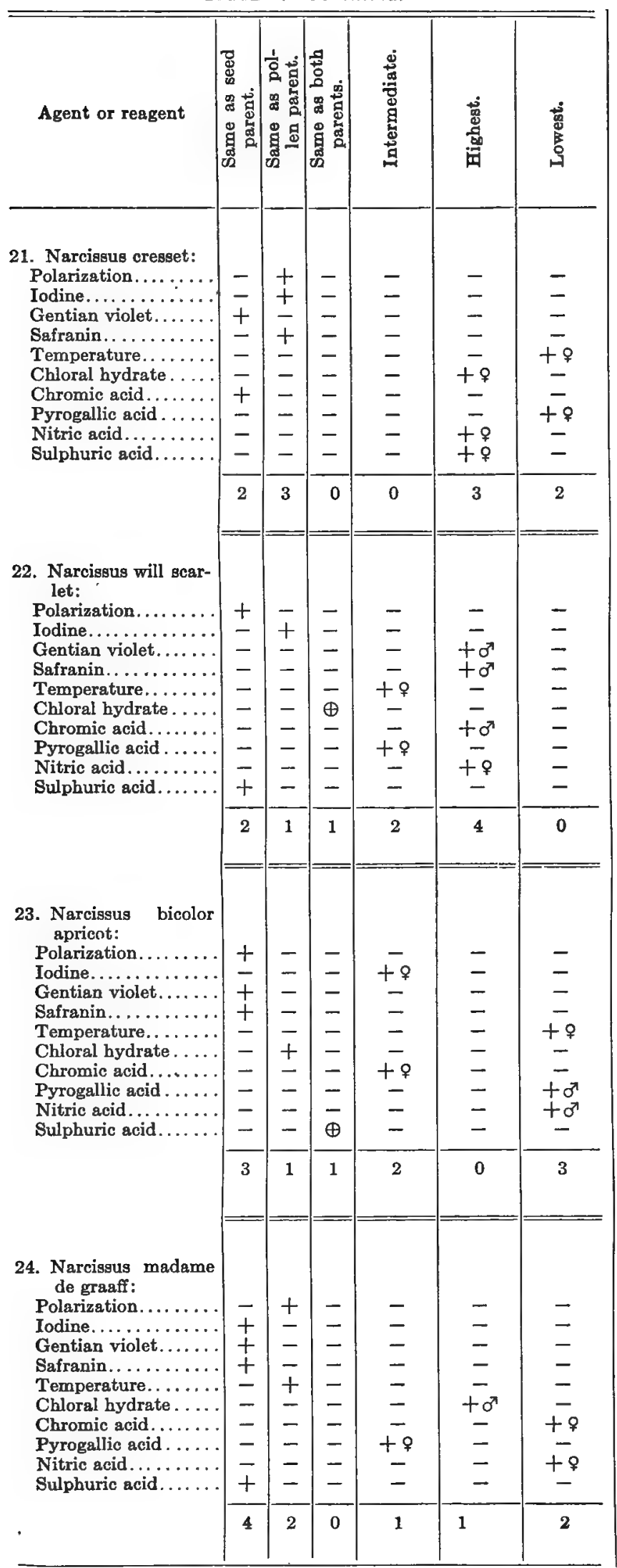

Table F.-Continued.

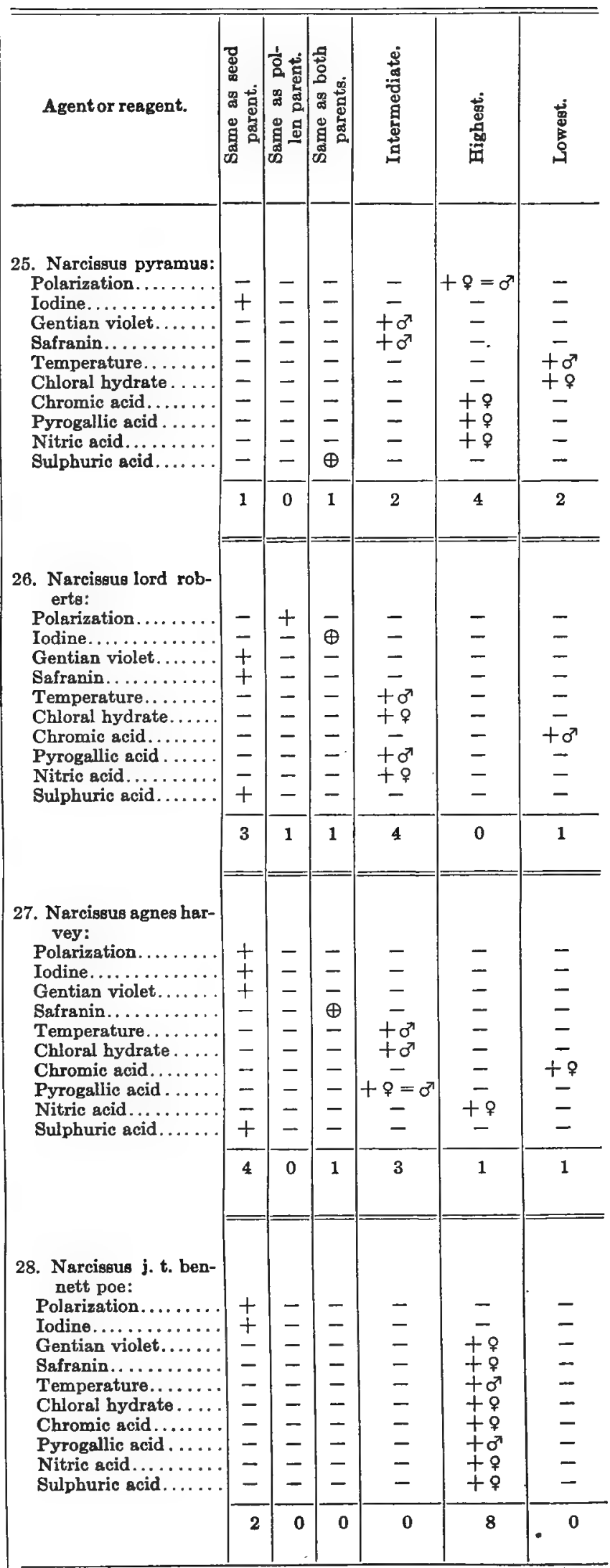


TABLE F.-Continued.

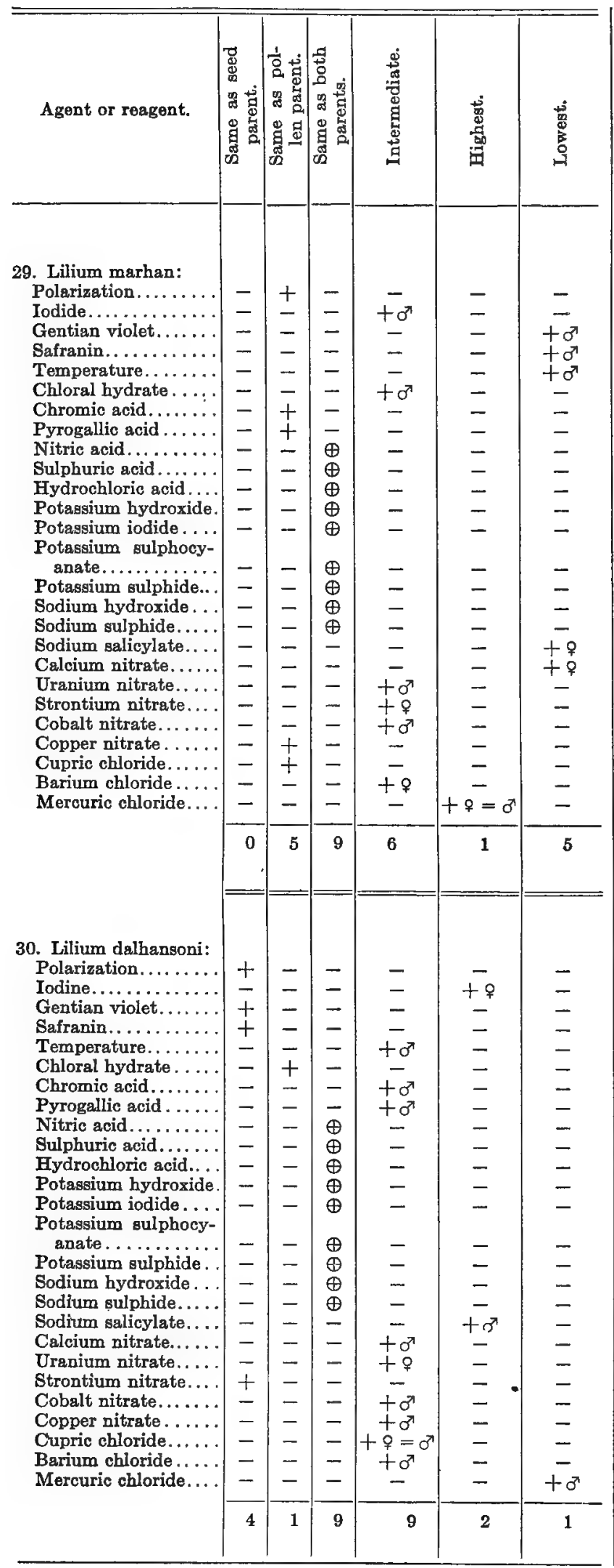

Tabue F.-Continued.

\begin{tabular}{|c|c|c|c|c|c|c|}
\hline Agent or reagent. & 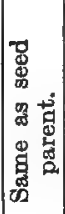 & 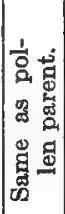 & 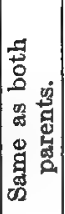 & 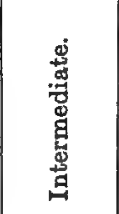 & 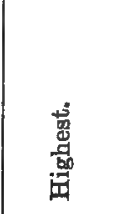 & 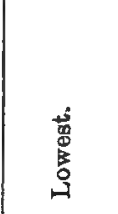 \\
\hline 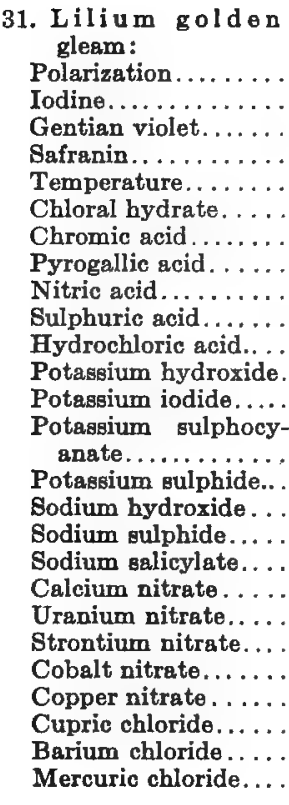 & $\begin{array}{l}\overline{-} \\
\bar{z} \\
\overline{-} \\
\overline{\mathbf{t}} \\
\pm \\
\pm \\
\overline{-} \\
\overline{-} \\
\overline{-}\end{array}$ & $\begin{array}{l}- \\
- \\
- \\
\bar{t} \\
- \\
- \\
- \\
- \\
- \\
- \\
- \\
+ \\
+ \\
\pm \\
- \\
- \\
- \\
- \\
- \\
- \\
-\end{array}$ & $\begin{array}{l}- \\
= \\
- \\
- \\
- \\
- \\
\bar{\oplus} \\
\oplus \\
\oplus \\
\oplus \\
\oplus \\
- \\
= \\
= \\
- \\
= \\
= \\
= \\
= \\
= \\
= \\
-\end{array}$ & $\begin{array}{c}= \\
= \\
= \\
+9 \\
= \\
= \\
= \\
= \\
= \\
- \\
= \\
= \\
= \\
= \\
= \\
\pm \\
= \\
= \\
=\end{array}$ & $\begin{array}{c}- \\
= \\
= \\
= \\
= \\
= \\
= \\
= \\
- \\
- \\
- \\
- \\
+9=0 \\
+0 \\
+9 \\
+9 \\
+0 \\
+0 \\
+0 \\
-\end{array}$ & $\begin{array}{l}+9 \\
+0 \\
+0^{7} \\
+0^{7} \\
= \\
= \\
= \\
= \\
= \\
= \\
= \\
= \\
= \\
= \\
= \\
= \\
= \\
=\end{array}$ \\
\hline Mercuric chloride.... & 4 & 4 & 5 & 2 & 7 & 4 \\
\hline 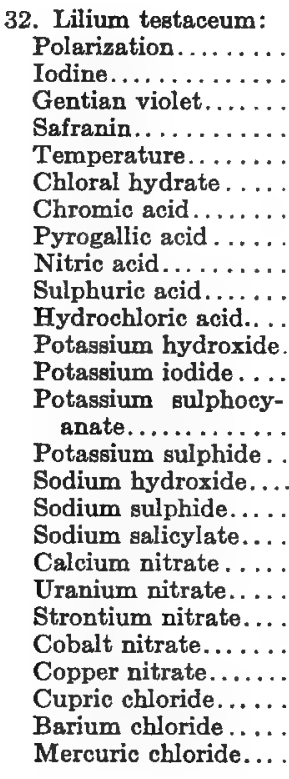 & $\begin{array}{l} \pm \\
= \\
- \\
= \\
= \\
- \\
- \\
- \\
\overline{+}\end{array}$ & $\begin{array}{l}- \\
\overline{+} \\
\pm \\
- \\
- \\
- \\
- \\
- \\
- \\
- \\
- \\
- \\
- \\
- \\
- \\
= \\
= \\
= \\
= \\
= \\
\pm \\
\pm \\
-\end{array}$ & 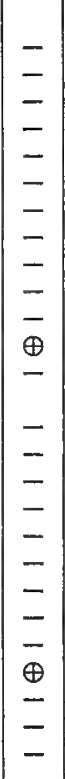 & $\begin{array}{c}- \\
- \\
- \\
- \\
- \\
+9 \\
+o \\
- \\
+9=0^{x} \\
+q \\
- \\
- \\
- \\
- \\
- \\
+9 \\
- \\
+0 \\
- \\
+9 \\
-\end{array}$ & 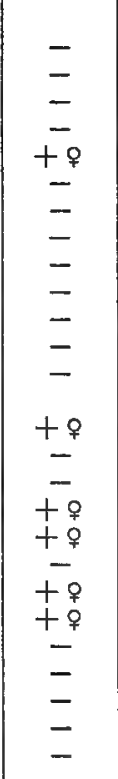 & $\begin{array}{c}- \\
+9 \\
= \\
= \\
+9 \\
= \\
+9=0 \\
= \\
= \\
- \\
= \\
= \\
= \\
= \\
= \\
= \\
= \\
= \\
+0\end{array}$ \\
\hline Mercuric chloride.... & 4 & 3 & 2 & 7 & 6 & 4 \\
\hline
\end{tabular}


Table F-Continued.

\begin{tabular}{|c|c|c|c|c|c|c|}
\hline Agent or reagent. & 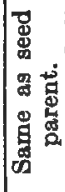 & 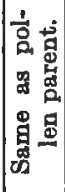 & 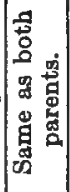 & 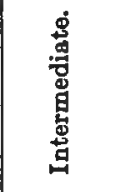 & $\begin{array}{l}\text { 罟 } \\
\text { 点 } \\
\text { 苗 }\end{array}$ & 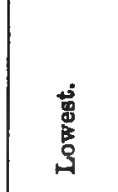 \\
\hline \multicolumn{7}{|l|}{ 33. Lilium burbanki: } \\
\hline Polarization.......... & - & + & - & - & - & - \\
\hline $\begin{array}{l}\text { Iodin }{ } . \ldots \ldots \ldots \ldots \\
\text { Gentian violet. . . . . }\end{array}$ & + & - & - & - & - & - \\
\hline Gentian violet....... & $\therefore$ & - & - & $+\sigma^{x}$ & - & - \\
\hline 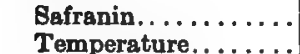 & - & - & - & $+\sigma^{7}$ & - & - \\
\hline Temperature....... & - & - & - & - & - & +9 \\
\hline Chloral hydrate..... & - & - & - & +8 & - & - \\
\hline Chromic acid........ & - & 一 & - & - & 一 & +9 \\
\hline Pyrogallic acid..... . & - & - & - & - & - & +9 \\
\hline Nitric acid $\ldots \ldots \ldots$ & - & - & - & - & - & $+9=\sigma^{7}$ \\
\hline Sulphuric acid...... & - & - & - & $+\infty$ & - & - \\
\hline Hydrochloric acid... & - & - & - & - & - & +9 \\
\hline Potassium hydroxide. & - & - & $\oplus$ & - & - & - \\
\hline Potassium iodide..... & - & - & - & - & - & $+90^{7}$ \\
\hline $\begin{array}{r}\text { Potassium sulphocy- } \\
\text { anate........... }\end{array}$ & - & - & - & - & - & $+9=\sigma^{\prime}$ \\
\hline Potassium sulphide... & - & - & - & - & - & $+\stackrel{+}{+}=\sigma^{2}$ \\
\hline Sodium hydroxide... & - & - & - & - & - & $+q=\sigma^{\prime}$ \\
\hline Sodium sulphide.... & - & - & - & - & - & $+\%=\sigma^{2}$ \\
\hline Sodium salicylate... . & - & - & - & $+\sigma^{\top}$ & - & - \\
\hline Calcium nitrate..... & + & - & - & - & - & - \\
\hline Uranium nitrate.... & - & - & - & - & - & +9 \\
\hline Strontium nitrate... & - & - & - & - & - & +8 \\
\hline Cobalt nitrate....... & - & - & - & - & - & +9 \\
\hline Copper nitrate. . . . . & - & - & - & - & - & $+\varphi=\sigma^{\prime}$ \\
\hline Cupric chloride...... & - & - & - & - & - & +8 \\
\hline Barium chloride..... & - & - & - & +9 & - & - \\
\hline \multirow[t]{2}{*}{ Mercuric chloride... } & - & - & - & - & - & $+q$ \\
\hline & 2 & 1 & 1 & B & $\mathbf{0}$ & 16 \\
\hline \multicolumn{7}{|l|}{ 34. Iris ismali: } \\
\hline Polarization... & - & - & - & - & - & $+\sigma^{7}$ \\
\hline Iodine $\ldots \ldots \ldots \ldots \ldots$ & + & - & - & - & - & - \\
\hline Gentian violet....... & + & - & - & 一 & - & - \\
\hline Bafranin........... & + & - & - & - & - & - \\
\hline Temperature........ & - & - & - & $+q=\sigma^{x}$ & - & - \\
\hline Chloral hydrate..... & - & - & - & $+\infty$ & - & - \\
\hline Chromic acid........ & - & - & - & +9 & - & - \\
\hline Pyrogallic acid. . & - & - & - & $+\%=\sigma^{\pi}$ & - & - \\
\hline Nitric acid...... & - & - & - & $+q=0^{\pi}$ & - & - \\
\hline Sulphuric acid...... & - & - & - & $+q=\sigma^{3}$ & - & - \\
\hline Hydrochloric acid.... & - & - & - & $+\sigma^{7}$ & - & - \\
\hline Potassium hydroxide. & - & - & - & - & $\rightarrow$ & +9 \\
\hline Potassium iodide.... & - & + & - & - & - & - \\
\hline Potassium sulphocy- & & & & & & \\
\hline anate $. . \ldots, \ldots$ & - & - & $\oplus$ & - & - & - \\
\hline Potassium sulphide... & - & - & - & $+\%=\sigma^{2}$ & - & - \\
\hline Sodium hydroxide... & - & - & $\oplus$ & - & - & - \\
\hline Sodium eulphide.... . & - & - & - & $+\%=\sigma^{7}$ & - & - \\
\hline Sodium salicylate... & - & + & - & - & - & - \\
\hline Calcium nitrate...... & - & - & - & $+q$ & - & - \\
\hline Uranium nitrate.... & - & - & - & - & $+\phi^{7}$ & - \\
\hline Strontium nitrate... & - & + & - & +9 & - & - \\
\hline Cobalt nitrate....... & - & - & - & - & - & $+\%=\sigma^{\pi}$ \\
\hline Copper nitrate. . . . . . & - & - & - & +9 & - & - \\
\hline Cupric chloride...... & - & - & - & - & - & +9 \\
\hline \multirow{3}{*}{ Mercuric chloride.... } & - & - & - & - & - & $+9=\sigma^{7}$ \\
\hline & - & $\rightarrow$ & $\rightarrow$ & - & - & +9 \\
\hline & 3 & 2 & 2 & 12 & 1 & 6 \\
\hline
\end{tabular}

Table F.-Contimeed.

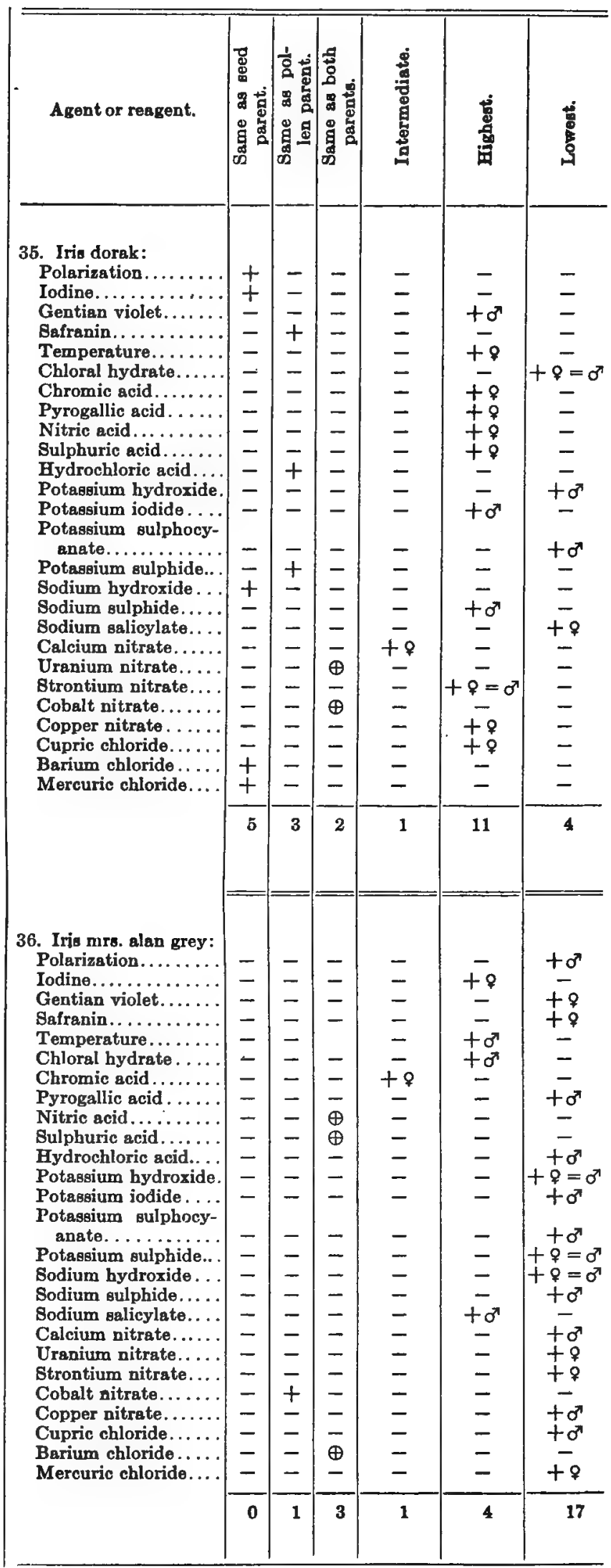


Table F.-Continued.

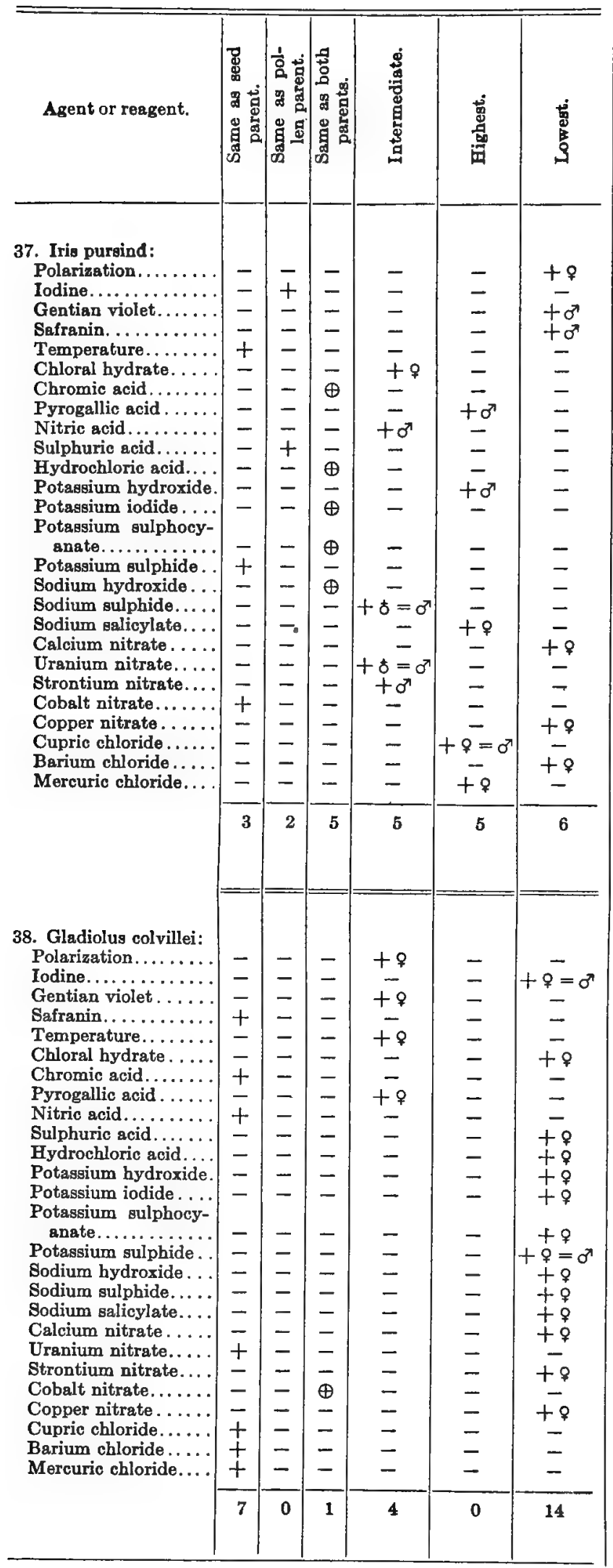

TABLE F.-Continued.

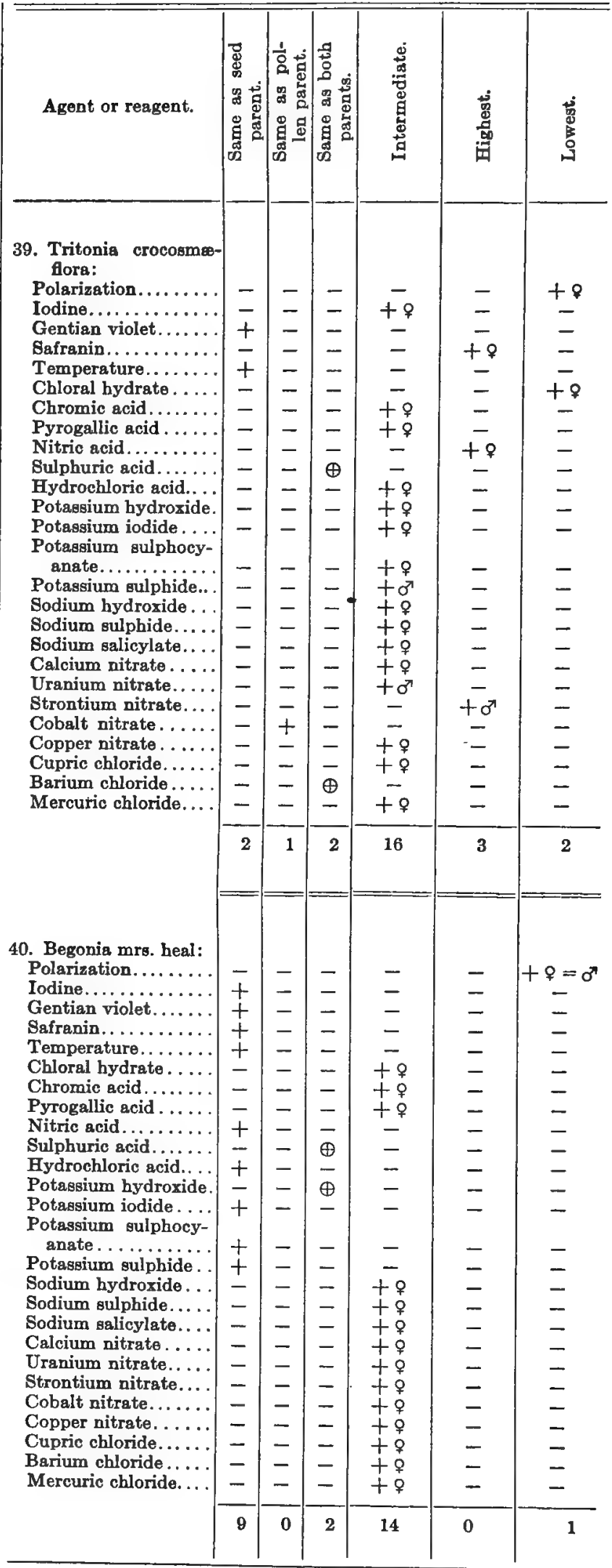


TABLE F.-Continued.

\begin{tabular}{|c|c|c|c|c|c|c|}
\hline Agent or reagent. & 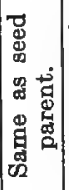 & 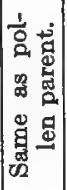 & 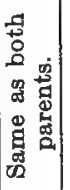 & 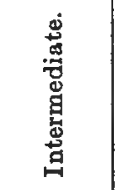 & 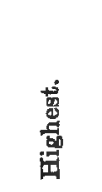 & 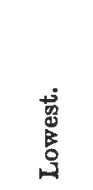 \\
\hline \multicolumn{7}{|l|}{ 41. Begonia ensign: } \\
\hline Polarization......... & - & 一 & 一 & +9 & - & - \\
\hline Iodine............. & - & - & - & +9 & - & - \\
\hline Gentian violet....... & - & - & - & - & - & $+\infty$ \\
\hline Safranin.......... & - & - & - & - & - & $+\sigma^{7}$ \\
\hline Temperature........ & - & - & - & +9 & - & - \\
\hline Chloral hydrate..... & - & - & - & - & +9 & - \\
\hline Chromic acid ........ & - & - & - & +9 & - & - \\
\hline Pyrogallic acid..... & - & 一 & - & +9 & - & - \\
\hline Nitric acid......... & - & - & - & +9 & - & - \\
\hline \multirow[t]{2}{*}{ Strontium nitrate.... } & - & 一 & - & +0 & - & - \\
\hline & 0 & 0 & 0 & 7 & 1 & 2 \\
\hline \multicolumn{7}{|l|}{ 42. Begonia julius: } \\
\hline Polarization........ & -4 & + & - & - & - & - \\
\hline Iodine............ & - & - & - & - & $+\sigma^{7}$ & - \\
\hline Gentian violet....... & - & - & - & - & $+\overline{0}$ & 一 \\
\hline Safranin........... & - & - & - & - & $+\sigma^{\pi}$ & - \\
\hline Temperature........ & - & - & - & + & - & - \\
\hline Chloral hydrate..... & - & - & - & - & +9 & - \\
\hline Chromic acid....... & - & - & - & $+q$ & - & - \\
\hline Pyrogallic acid ...... & - & - & - & $+q$ & - & - \\
\hline Nitric acid........ & + & - & - & - & - & - \\
\hline \multirow[t]{2}{*}{ Strontium nitrate... } & - & - & 一 & +9 & - & - \\
\hline & 1 & 1 & 0 & 4 & 4 & 0 \\
\hline \multicolumn{7}{|l|}{ 43. Begonia success: } \\
\hline Polarization......... & - & + & - & - & - & - \\
\hline Iodine............. & - & + & - & - & - & - \\
\hline Gentian violet....... & - & + & - & - & - & - \\
\hline 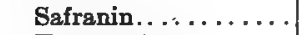 & + & - & - & - & - & - \\
\hline Temperature....... & - & - & - & $+q$ & - & - \\
\hline Chloral hydrate..... & - & - & - & +0 & - & - \\
\hline Chromic acid....... & - & - & - & - & +8 & - \\
\hline Pyrogallic acid . . & $\overrightarrow{1}$ & - & - & - & +9 & - \\
\hline Nitric acid. . . . . . . & + & - & - & - & - & - \\
\hline \multirow[t]{2}{*}{ Strontium nitrate... } & - & - & - & - & +9 & - \\
\hline & 2 & 3 & 0 & 2 & 3 & 0 \\
\hline \multicolumn{7}{|l|}{$\begin{array}{l}\text { 44. } \mathrm{Richardia} \mathrm{mrs.} \\
\text { roosevelt }\end{array}$} \\
\hline Polarization........ & - & - & - & + + $+=\sigma^{-7}$ & 一 & - \\
\hline Iodine............ & + & - & - & - & - & - \\
\hline Gentian violet...... & - & - & - & - & $+0^{x}$ & 一 \\
\hline Safranin.......... & - & - & - & - & $+0^{x}$ & - \\
\hline Temperature....... & - & - & - & $+\%=\sigma^{r}$ & - & - \\
\hline Chloral hydrate. . . . . & - & - & - & - & $+\sigma^{x}$ & - \\
\hline Chromic acid....... & - & - & $\oplus$ & 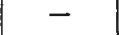 & $=$ & 一 \\
\hline Pyrogallic acid . ..... & - & - & $\oplus$ & - & 一 & - \\
\hline Nitric acid.......... & - & - & - & $+q=\sigma^{\lambda}$ & - & - \\
\hline Sulphuric acid. ...... & - & - & $\oplus$ & - & - & - \\
\hline Hydrochloric acid.... & - & - & - & - & - & $+0^{x}$ \\
\hline Potassium hydroxide. & - & - & - & - & $+d^{x}$ & - \\
\hline \multirow[t]{2}{*}{ Sodium salicylate.... } & - & 一 & $\oplus$ & - & - & - \\
\hline & 1 & 0 & 4 & 3 & 4 & 1 \\
\hline
\end{tabular}

TABLE F.-Continued.

\begin{tabular}{|c|c|c|c|c|c|c|}
\hline Agent or reagent. & 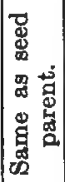 & 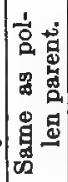 & 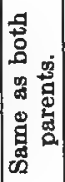 & 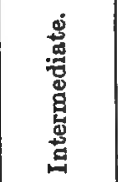 & 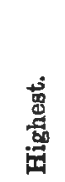 & 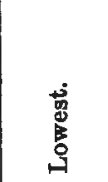 \\
\hline \multicolumn{7}{|l|}{ 45. Musa hybrida: } \\
\hline Polarization......... & - & - & - & - & - & $+\sigma^{x}$ \\
\hline Iodine ........... & - & + & - & - & - & - \\
\hline Gentian violet....... & - & + & - & - & - & - \\
\hline Safranin............ & - & + & - & - & - & - \\
\hline Temperature........ & - & - & - & - & - & $+0^{x}$ \\
\hline Chloral hydrate.... & - & - & - & - & - & $+0^{x}$ \\
\hline Chromic acid........ & - & - & - & - & - & $+0^{x}$ \\
\hline Pyrogallic acid ...... & + & - & - & - & - & - \\
\hline Nitric acid......... & - & - & - & - & - & $+\sigma^{x}$ \\
\hline Sulphuric acid....... & - & - & - & - & - & $+0^{x}$ \\
\hline Hydrochloric acid... & - & - & - & $+\sigma^{x}$ & 一 & - \\
\hline Potassium hydroxide. & - & - & - & $+0=\sigma^{2}$ & - & - \\
\hline Potassium iodide..... & - & - & - & - & 一 & $+\infty^{x}$ \\
\hline $\begin{array}{l}\text { Potassium sulphocy } \\
\text { anate........... }\end{array}$ & - & - & - & - & - & $+\infty^{x}$ \\
\hline Potassium sulphide. & - & - & - & - & - & $+0^{x}$ \\
\hline Sodium hydroxide... & - & - & - & - & - & $+\sigma^{x}$ \\
\hline Sodium sulphide.... & - & - & - & - & - & $+0^{x}$ \\
\hline Sodium salicylate.... & - & - & - & - & - & $+0^{7}$ \\
\hline Calcium nitrate...... & - & - & - & - & - & $+0^{2}$ \\
\hline Uranium nitrate.... & - & - & - & - & - & $+\sigma^{7}$ \\
\hline Strontium nitrate... & - & - & - & - & - & $+0^{x}$ \\
\hline Cobalt nitrate...... & - & - & - & - & - & $+0^{2}$ \\
\hline Copper nitrate..... & - & - & - & - & - & $+\sigma^{x}$ \\
\hline Cupric chloride..... & - & - & - & - & - & $+0^{\pi}$ \\
\hline Barium chloride..... & - & - & - & - & - & $+\sigma^{x}$ \\
\hline \multirow[t]{2}{*}{ Mercuric chloride... } & - & - & - & - & - & \\
\hline & $\mathbf{1}$ & 3 & 0 & 2 & 0 & 20 \\
\hline \multicolumn{7}{|l|}{ 46. Phaius hybridus: } \\
\hline Polarization........ & - & - & - & - & $+\%$ & - \\
\hline Iodine $\ldots \ldots \ldots \ldots$ & - & - & - & $+\sigma^{x}$ & - & - \\
\hline Gentian violet....... & - & - & - & - & $+q$ & - \\
\hline Safranin.......... & - & - & - & - & +0 & - \\
\hline Temperature....... & - & + & - & - & - & - \\
\hline Chloral hydrate..... & - & - & - & - & - & $+\sigma^{\top}$ \\
\hline Chromic acid....... & - & - & - & $+q=\sigma^{7}$ & - & - \\
\hline Pyrogallic acid ..... & - & - & - & $+9=\sigma^{\prime \prime}$ & - & - \\
\hline Nitric acid........ & - & - & - & $+q$ & - & - \\
\hline Sulphuric acid...... & - & - & $\oplus$ & - & - & - \\
\hline Hydrochloric acid... . & - & - & $\oplus$ & - & - & - \\
\hline Potassium hydroxide. & - & - & $\oplus$ & - & - & - \\
\hline Potassium iodide. . . & - & - & - & $+q=\sigma^{7}$ & - & - \\
\hline Potassium sulphocy- & - & _ & $\oplus$ & - & - & - \\
\hline 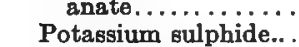 & - & - & - & - & - & $+\%=\sigma$ \\
\hline Sodium hydroxide... & - & - & - & - & - & $+\sigma^{\pi}$ \\
\hline Sodium sulphide.... & - & + & - & - & - & - \\
\hline Sodium salicylate... & - & + & - & - & - & - \\
\hline Calcium nitrate. . . . & - & - & - & $+\%$ & - & - \\
\hline Uranium nitrate.... & - & - & - & $+q$ & - & - \\
\hline Strontium nitrate... & + & - & - & - & - & - \\
\hline Cobalt nitrate..... & - & - & - & $+\sigma^{\pi}$ & - & - \\
\hline Copper nitrate. . . . . . & - & - & $\oplus$ & - & - & - \\
\hline Cupric chloride...... & - & - & - & $+\%=\sigma^{x}$ & - & - \\
\hline Barium chloride.... & - & - & - & +9 & - & - \\
\hline \multirow[t]{2}{*}{ Mercuric chloride... } & - & - & - & $+q=\sigma^{7}$ & - & - \\
\hline & 1 & 3 & 5 & 11 & 3 & 3 \\
\hline
\end{tabular}


Table F.-Continued.

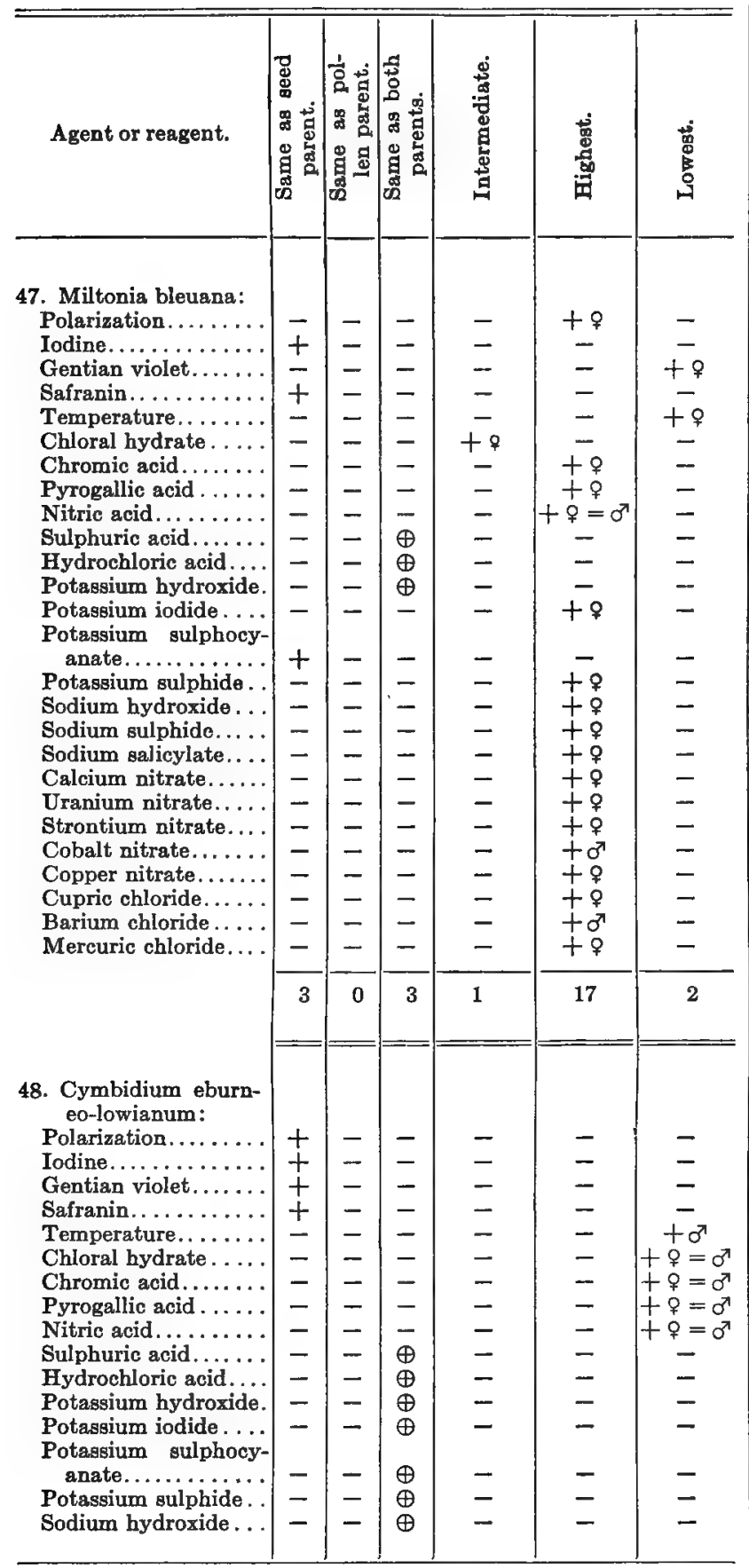

Tarle F.-Concluded.

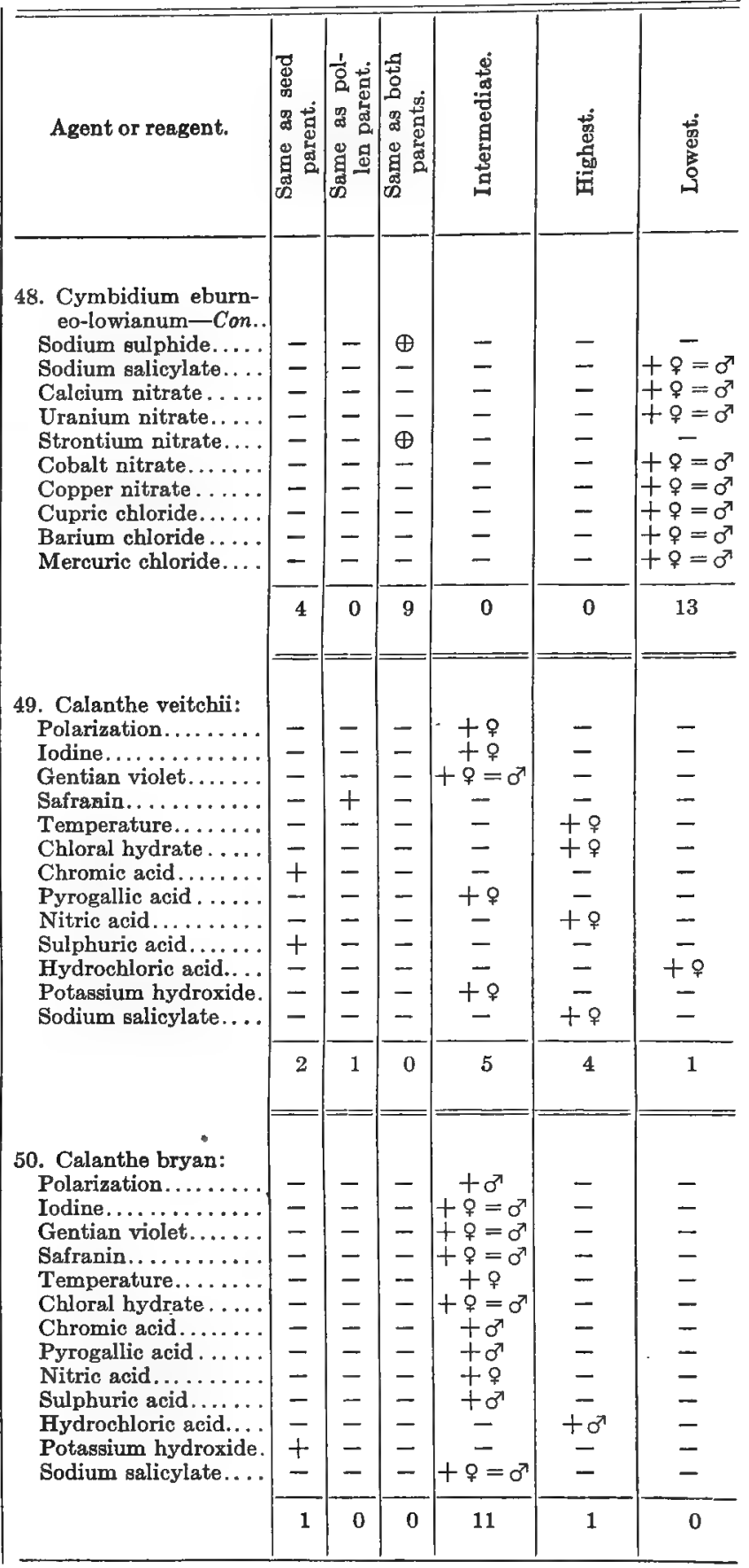


SUmmary OF TABLE F.-Recapitulation of the Sum-totals of the Reaction-intensities of the Starches of all of the Hybrids as regards Sameness, Intermediateness, Excess, and Deficit of Development of Different Hybrids in relation to the Parents.

\begin{tabular}{|c|c|c|c|c|c|c|}
\hline Hybrids. & $\begin{array}{c}\text { Same as } \\
\text { seed } \\
\text { parent. }\end{array}$ & $\begin{array}{c}\text { Same as } \\
\text { pollen } \\
\text { parent. }\end{array}$ & $\begin{array}{c}\text { Same as } \\
\text { both } \\
\text { parents. }\end{array}$ & $\begin{array}{l}\text { Inter- } \\
\text { mediate. }\end{array}$ & Highest. & Lowest. \\
\hline 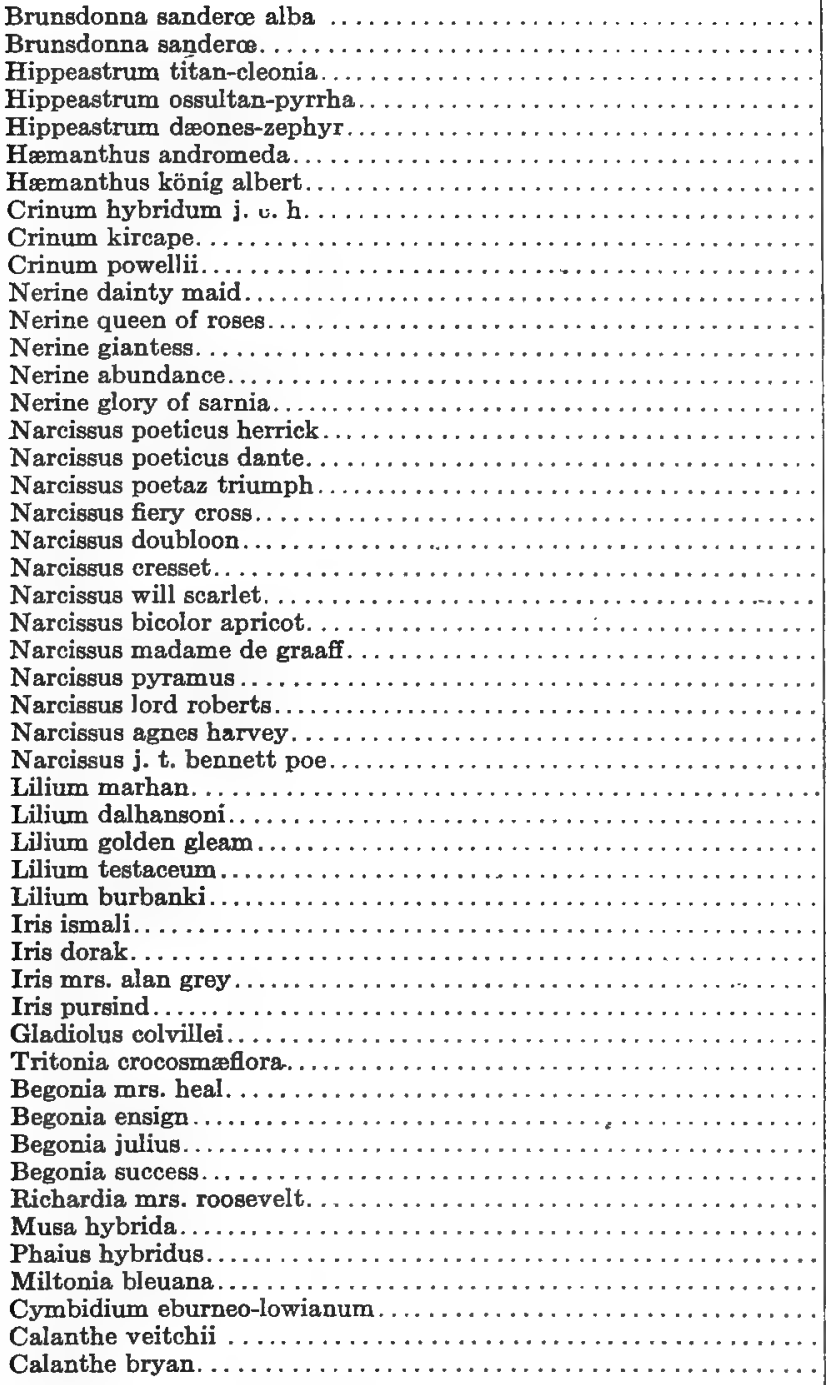 & 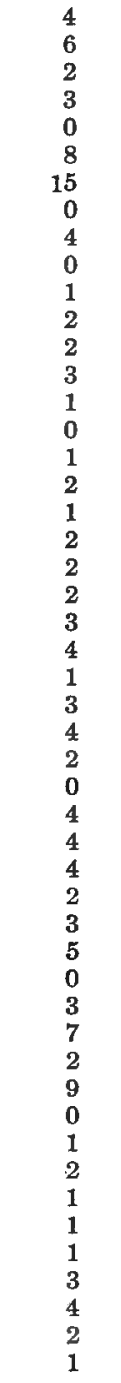 & 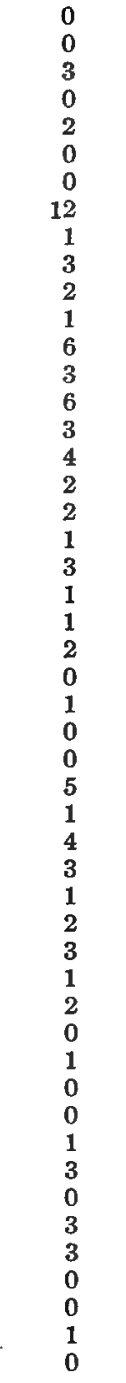 & 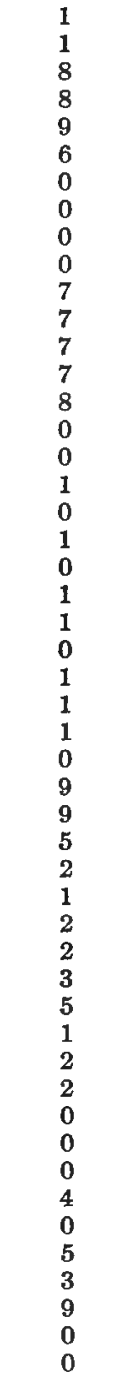 & $\begin{array}{r}5 \\
2 \\
4 \\
3 \\
6 \\
11 \\
7 \\
5 \\
18 \\
2 \\
6 \\
3 \\
6 \\
3 \\
1 \\
3 \\
4 \\
0 \\
2 \\
4 \\
0 \\
0 \\
0 \\
0 \\
2 \\
11\end{array}$ & $\begin{array}{r}3 \\
3 \\
5 \\
11 \\
5 \\
0 \\
1 \\
2 \\
2 \\
21 \\
8 \\
11 \\
1 \\
1 \\
0 \\
2 \\
1 \\
20 \\
2 \\
0\end{array}$ & 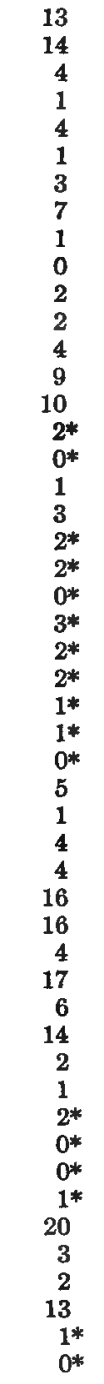 \\
\hline 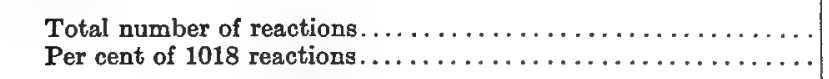 & $\begin{array}{r}137 \\
13.4\end{array}$ & $\begin{array}{r}94 \\
9.2\end{array}$ & $\begin{array}{r}138 \\
13.6\end{array}$ & $\begin{array}{r}236 \\
23.2\end{array}$ & $\begin{array}{r}187 \\
18.4\end{array}$ & $\begin{array}{r}226 \\
22.2\end{array}$ \\
\hline Per cent of sameness and of intermediateness, highest and lowest. & \multicolumn{3}{|c|}{36.2} & \multicolumn{3}{|c|}{63.8} \\
\hline
\end{tabular}

* Number of reactions $=10$ or 13 .

Reaction-Intensities of Each Hybrid Starch in Relation to Sameness and Inclination to mach Parent and Both Parents.

(Table G.)

The data included in Table F, Parts 1 to 50, can be given a setting that will show quite clearly, although somewhat grossly, the comparative degrees of influence that have been exerted by each of the parents on the properties of the starch of the hybrid. Such a presentation will be found in Table G. From the figures here formulated it will be seen that the various hybrids ex- hibit the widest differences in their parental bearings, there being all gradations between one extreme where with the exception of 3 reactions of 26 there is sameness or inclination to the seed parent (as in Homanthus könig albert and Begonia mrs. heal) and the other extreme where with the exception of 1 or 2 reactions of 26 the corresponding relationship is borne to the pollen parent (as in Crinum hybridum j.c. h., C. powellii, Gladiolus colvillei, and Musa hybrida). In most of the hybrids there is a quite definite leaning to one or the other parent. In summing up the total number of reactions in each column it is found that of 1,018 reactions 
434 (42.7 per cent) fall under same as or inclined to seed parent, 330 (32.4 per cent) under same as or inclined to pollen parent, 140 (13.8 per cent) under same as both parents, and 114 (11.1 per cent) under as close to one as to the other parent. Nearly all of the reactions recorded as being the same as those of both parents have been found so because of too rapid or too slow gelatinization, and therefore doubtless misleading and defective in classification. It is of especial interest to note that

TABLE G 1.-Summary of Sameness and Inclination of the Reactionintensities of the Starches of the Hybrids in relation to the Starches of the Parent-Stocks.

\begin{tabular}{|c|c|c|c|c|}
\hline \multirow{2}{*}{ Hybrids. } & \multicolumn{2}{|c|}{$\begin{array}{l}\text { Same as or } \\
\text { inclined to- }\end{array}$} & \multirow{2}{*}{ 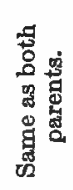 } & \multirow{2}{*}{ 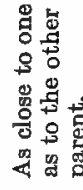 } \\
\hline & 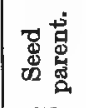 & 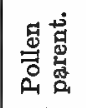 & & \\
\hline Brunsdonna sanderco alba.... & 11 & 10 & 1 & 4 \\
\hline Brunsdonna sanderco........... & 13 & 9 & 1 & 3 \\
\hline Hippeastrum titan-cleonia........ & 8 & 7 & 8 & 3 \\
\hline Hippeastrum ossultan-pyrrha. . . . . & 11 & 6 & 8 & 1 \\
\hline Hippeastrum dæones-zephyr....... & 7 & 5 & 9 & 5 \\
\hline Hæmanthus andromeda........... & 12 & 0 & 6 & 8 \\
\hline Hæmanthus könig albert. . . . . . . & 23 & $\mathbf{2}$ & 0 & 1 \\
\hline Crinum hybridum j. c. h. . . . . . & 1 & 25 & 0 & 0 \\
\hline Crinum kircape $\ldots \ldots \ldots \ldots \ldots \ldots$ & 22 & 4 & 0 & 0 \\
\hline Crinum powellii, $\ldots \ldots \ldots \ldots \ldots \ldots$ & 0 & 24 & 0 & 2 \\
\hline Nerine dainty maid...$\ldots \ldots$ & 7 & 9 & 7 & 3 \\
\hline Nerine queen of roses. . . . . . . . . & 9 & 8 & 7 & 2 \\
\hline Nerine giantess................ & 5 & 10 & 7 & 4 \\
\hline Nerine abundance. .......... & 6 & 12 & 7 & 1 \\
\hline Nerine glory of sarnia ......... & 7 & 10 & 8 & 1 \\
\hline Narcissus poeticus herrick. ... & 4 & 5 & 0 & 1 \\
\hline Narcissus poetious dante. . . . . & 3 & 5 & 0 & 2 \\
\hline Narcissus poetaz triumph..... & 4 & 17 & 1 & 4 \\
\hline Narcissus fiery cross. . . . . . . . & 3 & 4 & 0 & 3 \\
\hline Narcissus doubloon............. & 5 & 3 & 1 & 1 \\
\hline Narcissus cresset. $\ldots \ldots \ldots \ldots \ldots \ldots$ & 7 & 3 & 0 & 0 \\
\hline Narcissus will scarlet. . . . . . . . . & 5 & 4 & 1 & 0 \\
\hline Narcissus bicolor apricot. ......... & 6 & 3 & 1 & 0 \\
\hline Narcissus madame de graaff .. & 7 & 3 & 0 & 0 \\
\hline Narcissus pyramus .......... & $\mathbf{5}$ & 3 & 1 & 1 \\
\hline Narcissuis lord roberts........ & 5 & 4 & 1 & 0 \\
\hline Narcisgus agnes harvey........ & 5 & 3 & $\mathbf{1}$ & 1 \\
\hline Narcissus j. t. bennett poe......... & 10 & 2 & $\mathbf{0}$ & 0 \\
\hline Lilium $\operatorname{marhan} . \ldots \ldots \ldots \ldots \ldots \ldots$ & 4 & 12 & 9 & 1 \\
\hline Lilium dalhansoni. . . . . . . . . . . & 6 & 10 & 9 & 1 \\
\hline Lilium golden gleam............. & 12 & 8 & 5 & 1 \\
\hline Lilium testaceum $\ldots \ldots \ldots \ldots \ldots \ldots$ & 17 & 5 & 2 & 2 \\
\hline Lilium burbanki $\ldots \ldots \ldots \ldots \ldots \ldots$ & 13 & 5 & 1 & 7 \\
\hline Iris ismali $\ldots \ldots \ldots \ldots \ldots \ldots \ldots \ldots$ & 10 & 6 & 2 & 8 \\
\hline Iris dorak $\ldots \ldots \ldots \ldots \ldots \ldots \ldots$, & 13 & 9 & 2 & 2 \\
\hline Iris mrs. alan grey $\ldots \ldots \ldots \ldots \ldots \ldots$ & 7 & 13 & 3 & 3 \\
\hline Iris pursind $\ldots \ldots \ldots \ldots \ldots \ldots$ & 10 & 8 & 5 & 3 \\
\hline Gladiolus colvillei $\ldots \ldots \ldots \ldots \ldots \ldots$ & 23 & 0 & 1 & 2 \\
\hline Tritonia crocosmæflora.......... & 20 & 4 & 2 & 0 \\
\hline Begonia mrs. heal. . . . . . . . . . & 23 & 0 & 2 & 1 \\
\hline Begonia ensign............... & 8 & 2 & 0 & 0 \\
\hline Begonia julius $\ldots \ldots \ldots \ldots \ldots \ldots$ & 6 & 4 & 0 & 0 \\
\hline Begonia success. . . . . . . . . . . & 7 & 3 & 0 & 0 \\
\hline Richardia mrs. roosevelt . . . . . . . . & 1 & 5 & 4 & 3 \\
\hline Musa hybrida.$\ldots \ldots \ldots \ldots \ldots \ldots$ & 0 & 25 & 0 & 1 \\
\hline Phaius hybridus.$\ldots \ldots \ldots \ldots \ldots$ & 8 & 7 & 5 & 6 \\
\hline Miltonia bleuana.............. & 20 & 2 & 3 & 1 \\
\hline Cymbidium eburneo-lowianum... . & 4 & 1 & 9 & 12 \\
\hline Callanthe veitchii. . . . . . . . . . . & 11 & 1 & 0 & 1 \\
\hline Calenthe bryan $\ldots \ldots \ldots \ldots \ldots$ & 3 & 5 & 0 & 5 \\
\hline \multirow{3}{*}{$\begin{array}{l}\text { Total number of reactions. ........ } \\
\text { Per cent of } 1018 \text { reactions...... }\end{array}$} & 434 & 330 & 140 & 114 \\
\hline & 42.7 & 32.4 & 13.8 & 11.1 \\
\hline & \multicolumn{2}{|c|}{75.1} & \multicolumn{2}{|r|}{24.9} \\
\hline
\end{tabular}

764 (75.1 per cent) of the reactions fall under the first two columns, 42.7 per cent of the 75.1 per cent, or distinctly more than one-half, being in favor of the seed parent and the remaining 32.4 per cent being in favor of the pollen parent, showing a distinctly greater influence of the seed parent. The last column includes many of the intermediate, excess, and deficit reactions of the hybrids, some of which will likely be traced by further investigation to closeness to one or the other parent. Thus, when a reaction of the hybrid exceeds parental limits and is as close to one as to the other parent it is as likely that the peculiarity of the hybrid is due to one of the parents as to both. At present we have not the data to permit of this differentiation.

Reaction-Intensities of All the Hrbrid Starches with Each Agent and Reagent and as Regards Sameness and Inclination of Their Properties in Relation to One or the Other or Both Parents.

(Table H, Parts 1 to 26 and Summaries 1 and 2.)

In Table F, 1 to 50 , in a preceding subsection it is shown that combinations of the reactions of starches with different agents and reagents give in the case of each starch a mosaic picture that is specific to the starch, no two tables being the same, or even very much alike, even when the hybrids are of the same cross; and that, as a corollary, each hybrid starch can positively be diagnosed from every other by the peculiarities of the parental relationships. It was also rendered evident that this demonstration of individuality is dependent upon both specificity of the starch and specificity of the agent or reagent, as is manifest by the fact that if one starch be substituted for another or one reagent substituted for another the reactions may be like or unlike. Thus, taking the three Crinums, it will be seen that the iodine reactions of the seed parents are in all three the same or practically the same as those of the corresponding pollen parents. In the temperature reactions one (C. hybridum $j . c . h$.) has a higher reactivity than that of either parent and closer to the pollen parent; another ( $C$. kircape) has an intermediate reactivity and is closer to the seed parent; and another ( $C$. powellii) has a higher reactivity than that of either parent and closer to the pollen parent. In the chloral-hydrate reactions one hybrid is intermediate and closer to the pollen parent; another the same as the seed parent; and another the highest, and as close to one as to the other parent. In the pyrogallic acid reactions one hybrid is the lowest and closer to the pollen parent; another intermediate and closer to the pollen parent; another highest and closer to the pollen parent, etc. In other words, the nature of the reaction is determined by the character of the starch plus the character of the agent or reagent; each starch has inherently potentialities of both parents that are expressed by reactionintensities, either or both of which may be elicited in accordance with conditions; different agents and reagents may behave the same or differently in relation to these potentialities; and either parental potentiality can be developed at will by proper selection of the agent or reagent.

These facts are of such fundamental importance and broadness in their bearings that it seems to be highly 
desirable to inquire somewhat critically into the evidence at hand so as to learn to what extent, if any, each of the various agents and reagents exhibits a definite propensity to elicit one or the other parent-phases. Consequently, the data recorded in the preceding tables have been given a resetting in Table $\mathrm{H}$, Parts 1 to 26 , in each of which division will be found the reactions of all of the hybrid starches with each agent and reagent, thus presenting in a most succinct and striking form the peculiarities manifested by each agent and reagent in the elicitation of such reactions. Each division of the table is, as in the preceding set, so characteristic of the agent or reagent that each is specific and diagnostic-in the former set, specific and diagnostic in relation especially to the starch; in this set, specific and diagnostic in relation especially to the agent or reagent. Even the tables representing the offspring of the same cross (Brunsdonna sandero alba and $\mathcal{B}$. sandero; and Narcissus poeticus herrick and $N$. poeticus dante) can be distinguished from each other at a glance. In the present table of agents and reagents we find parallels in pairs that are similar to the pairs of hybrids in the preceding tables, as, for instance, in potassium hydroxide and sodium hydroxide and potassium sulphide and sodium sulphide which are comparable to two hybrids of the same cross, in each of which pairs the two tables will be found to be so definitely unlike in so many respects as to be as specific and diagnostic as are the tables of the pairs of Brunsdonnoe and Narcissus hybrids, respectively.

It has been pointed out particularly that different starches in their reactions with different agents and reagents exhibit marked variations in both kind and distribution of the reactions among the six parental phases, there being all gradations between one extreme that is characterized by almost universal sameness of the hybrid starch to the starch of the seed parent and the other extreme where a corresponding relationship was found toward the pollen parent; or a striking proneness to intermediateness; or for the reactions to be in excess of deficit of parental extremes. In other words, certain starches show in their reactions marked likeness to the seed or pollen parent, or intermediateness, etc., while others exhibit a two-phase peculiarity which may be manifested in sameness to both parents associated with development in excess of the parental extremes, or in other forms of combination as pointed out in Table C 17 under Calanthe. Inasmuch as the reactions of the different starches were obtained by means of the same agents and reagents, one would naturally be led to the conclusion that with the starch as the rarying factor and the agents and reagents as the constant factor the propensities of different starches to exhibit especially seed or pollen parent propensities, intermediateness, etc., are inherent to the starch molecules, and that the agents and reagents may be inert or indifferent, or in other words, that they do not have any especial propensity of themselves to elicit any given parent-phase in preference to any other. Therefore, in differentiating the part played by starch molecule and reagent, respeotively, when a given parent-phase is developed, it seems that we should take into account in the reaction whether or not the starch molecule has been altered, for if not altered the peculiarity of the reaction would naturally be attributed to the starch alone and would represent an existent phase in contradistinction to a developed phase that is owing to the reagent bringing to light a potential or latent phase.

In some instances as pointed out the starch molecule is either not in the least modified or but extremely slightly modified in the reaction, whereas in others it is partially or completely broken down by presumably simple processes of hydration, or by a process of hydration plus some additional reaction or reactions that depended upon some peculiar component or components of the reagent. Inasmuch as in the polarization reaction the molecules are unchanged the reaction must depend solely upon inherent properties of the molecules and indicate an existent parent-phase, comparable to the obvious parent-phases that are exhibited in the histologic properties of the starch grains; and it might be taken for granted, as a corollary, that any agent or reagent that yields a reaction with the starch molecules without breaking down the molecules, would elicit the same parentphase reaction. That is, if in the polarization reaction sameness to the seed parent is noted the same would be seen in the iodine and aniline reactions; but as this is, in fact, not the case, any parent-phase of this complex may be demonstrated without or with molecular disorganization. Thus, in Crinum kircape, we find that the polarization reaction is higher than in either parent, but closer to the reaction of the seed parent; the iodine reaction is intermediate, but closer to that of the pollen parent; the gentian-violet reaction is the same as that of the pollen parent; and the safranin reaction higher than in either parent, but nearer the reaction of the seed parent, and so on in different starches in varying forms of combination of these reactions. In other words, in the starch molecule as in the albumin molecule the components or potentials are in the form of a complex labile aggregate, so that it is easy to elicit any parent-phase component or potential of the starch molecule. Not only are these parent-phases readily separable and demonstrable by proper agents and reagents, but there is also evidence that different agents and reagents exhibit marked differences in their propensities to elicit a given phase or given phases. This is rendered very obvious by the data as reset in the summaries of Table $H$ (page 336) in which, however, those recorded under " same as both parents" should be omitted because in nearly all instances there was no satisfactory differentiation owing to extremely rapid or extremely slow gelatinization.

It will be seen by the first summary of this Table that while in case of many of the agents and reagents there is no manifest propensity to elicit sameness as the seed parent, or sameness as the pollen parent, or intermediateness, etc., the opposite holds good in varying degree for others. Thus, in the polarization reactions the reactions of the 50 starches are distributed quite equally among the 5 phases. In the iodine reactions there is an obvious increase in the number of reactions that fall in the first column, this being associated particularly with a falling off in the "highest" and "lowest" columns. In the temperatures of gelatinization there is a marked lessening in sameness as the seed parent and sameness as the pollen parent, this being associated with a corresponding increase in the intermediate column, showing that in 21 of the 50 starches heat, in 
causing gelatinization, gives rise to conspicuousness of an intermediate parent-phase. In 10 of the 47 starches sulphuric acid developed sameness as the seed parent, and in only 3 sameness as the pollen parent; potassium sulphocyanate developed sameness as seed parent in 6 of the 32 reactions and sameness as the pollen parent in one only; patassium sulphide, in 5 and 2 , respectively; strontium nitrate, in 5 and 0 , respectively, and so on. Certain other reagents exhibit a reversal of these propensities, as is noted particularly in the reactions of chloral hydrate, sodium salicylate, and cupric chloride, in which are found ratios $1: 6,1: 4$, and $2: 3$, respectively. But in the intermediate, highest, and lowest columns, many reactions are recorded that are closer to one than to the other parent, and when these are added to the first two columns, as in the summary of Table $\mathrm{E}$, the propensities are in some instances practically unaltered, in others accentuated, and in others lessened or reversed. It will be seen by comparing the two summaries that in the first in the polarization reactions 11 are the same as those of the seed parent and 11 the same as those of the pollen parent; and in the second an almost equal division, 26 and 20 , respectively. In the iodine reactions the figures in the two tables are $16: 12$ and $25: 18$, respectively-a ratio of $1: 0.75$ and $1: 0.72$, respectively; in both of these reactions there being no essential difference in the two tables. In the temperature of gelatinization reactions the first table gives $1:: 3$, and the second $29: 18$, or ratios of $1: 0.43$ and $1: 0.62$, which show a slight falling off in the latter. In the chloral-hydrate reactions the first table shows a marked propensity to the pollen parent, and the second a propensity to one about as much as to the other; on the other hand, in the chromicacid reactions in the first table there is shown a ratio of $4: 3$ and in the second table $31: 12$, or in the latter two and a half times the propensity to develop sameness or closeness to the seed parent as to the pollen parent. In other words, it seems that certain reagents, while having definite propensities to develop a seed or pollen phase, show varying degrees in their propensities to elicit sameness or closeness, some tending comparatively largely to sameness and little to closeness, and others the reverse, and so forth. Moreover, while a given reagent may have a propensity to elicit sameness as, one parent, it may have at the same time a marked propensity to develop closeness to the other parent in other starches, so that in the summing up of the reactions with different starches one may counterbalance the other. This is illustrated in the chloral-hydrate reactions, in which it is shown in the two summaries that the propensity to elicit sameness to the pollen parents is 6 times greater than to sameness to the other parent, while it is also shown that because of a propensity to develop closeness to the seed parent the former difference is dissipated and an equal tendency is manifested to develop either the seed or pollen parent phase, the ratio being 23:20. It seems, therefore, that' a better picture is to be obtained of these propensities if those to sameness are included with those to closeness. A cursory examination of the figures of the first two columns of the latter table (the other columns may be omitted to advantage and without leading to misunderstanding), will render it evident that the agents and reagents fall into 3 classes in accord- ance with their propensity to elicit sameness and closeness to the seed parent, sameness or closeness to the pollen parent, or an absence of propensity to elicit either parental relationship in preference to the other, and that the classes merge into each other, as follows:

\begin{tabular}{|c|c|c|}
\hline & \multicolumn{2}{|c|}{$\begin{array}{c}\text { Same as or closer } \\
\text { to the }\end{array}$} \\
\hline & $\begin{array}{c}\text { Seed } \\
\text { parent. }\end{array}$ & $\begin{array}{l}\text { Pollen } \\
\text { parent. }\end{array}$ \\
\hline 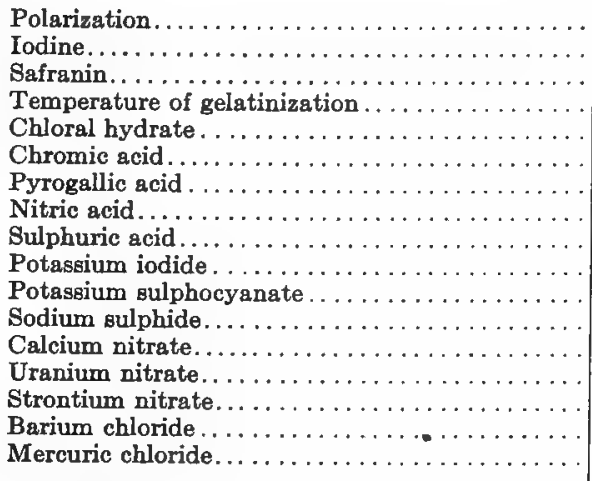 & $\begin{array}{l}26 \\
25 \\
24 \\
29 \\
23 \\
31 \\
23 \\
24 \\
18 \\
13 \\
13 \\
12 \\
16 \\
15 \\
15 \\
13 \\
14\end{array}$ & $\begin{array}{r}20 \\
18 \\
21 \\
18 \\
20 \\
12 \\
15 \\
11 \\
11 \\
8 \\
9 \\
9 \\
12 \\
10 \\
10 \\
4 \\
6\end{array}$ \\
\hline 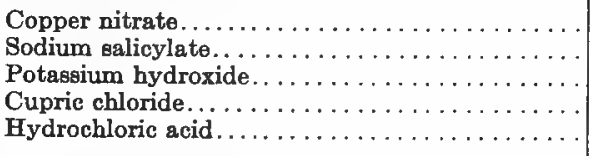 & $\begin{array}{r}12 \\
16 \\
8 \\
9 \\
12\end{array}$ & $\begin{array}{r}10 \\
15 \\
8 \\
9 \\
12\end{array}$ \\
\hline 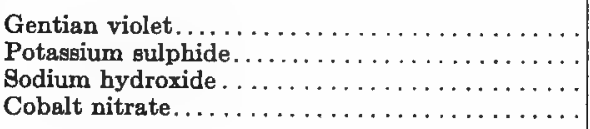 & $\begin{array}{r}21 \\
7 \\
11 \\
6\end{array}$ & $\begin{array}{l}25 \\
10 \\
14 \\
11\end{array}$ \\
\hline
\end{tabular}

With very few exceptions the ratios appear to be such as to make the assignment quite definite. From these groups it will be seen that most of the agents and reagents ( 17 of the 26 ) tend, most of them markedly, to elicit the seed parent phase; somewhat less than one-sixth ( 4 of the 26), seldom markedly, tend to elicit the pollen parent phase; and the remaining less than one-fifth ( 5 of the 26) tend with about or equal propensity to elicit one or the other parent-phase. Perhaps, several that have been assigned to the first group, especially chloral hydrate, should be transferred to the last group, and other redistribution made.

It seems from the foregoing data that the development of the various parent-phases is dependent upon two fundamental factors: One, inherent properties of the starch by virtue of which different starches exhibit with the same agent or reagent specific parent-phase reactions, one starch reacting the same as the seed parent, another the same as the pollen parent, another intermediate between the two parents, etc., as shown in preceding table; and the other, inherent properties of the agents and reagents by virtue of which, in association with the plastic starch molecule, any parent-phase desired may be developed at will in any given starch. Inasmuch as there are thus two factors which may tend in like or unlike directions in the evolution of a parent-phase, it is clear that the greatest variations in these manifestations must be expected in the reactions, both when there is a single starch reacting with various reagents or a single reagent reacting with various starches. 
TABLE $H$.

\begin{tabular}{|c|c|c|c|c|c|c|}
\hline Hybrids. & 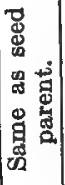 & 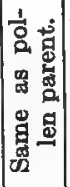 & 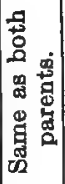 & 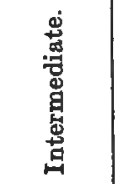 & 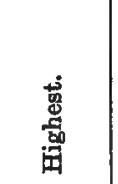 & $\begin{array}{l}\text { 䓵 } \\
\text { 总 } \\
\text { 总 }\end{array}$ \\
\hline \multicolumn{7}{|l|}{$\begin{array}{l}\text { 1. Polarization reactions: } \\
\text { Brunsdonna sanderœ }\end{array}$} \\
\hline & + & - & - & - & - & - \\
\hline $\begin{array}{l}\text { Brunsdonna anderoe. } \\
\text { Hippeastrum titan- }\end{array}$ & - & - & - & +9 & - & - \\
\hline \multicolumn{7}{|l|}{$\begin{array}{c}\text { Hippeastrum ossul- } \\
\text { tan-pyrrha.......... }\end{array}$} \\
\hline $\begin{array}{l}\text { tan-pyrrha ......... } \\
\text { Hippeastrum dæones }\end{array}$ & - & - & - & - & $+\sigma^{x}$ & - \\
\hline Hæmanthus andro- & - & - & - & - & $+\infty$ & - \\
\hline \multicolumn{7}{|l|}{$\begin{array}{r}\text { meda.............. } \\
\text { Hæmanthus könig al- }\end{array}$} \\
\hline Crinum hybridum $\mathrm{j}$. & - & - & - & - & $+\sigma^{\prime}$ & - \\
\hline 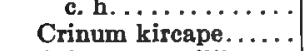 & 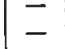 & $\overline{-}$ & $=$ & - & $\begin{array}{l}+\sigma^{7} \\
+8\end{array}$ & $\overline{-}$ \\
\hline Crinum powellii .... & - & + & - & - & - & - \\
\hline Nerine dainty maid. . & - & + & - & - & - & - \\
\hline Nerine queen of roses & - & - & - & - & - & $+\sigma^{7}$ \\
\hline Nerine giantess...... & - & - & - & - & - & +9 \\
\hline Nerine abundance. . . & - & - & - & - & - & +9 \\
\hline \multicolumn{7}{|l|}{$\begin{array}{l}\text { Nerine glory of sarnia } \\
\text { Narcissus poeticus }\end{array}$} \\
\hline herrick............ & - & - & - & +9 & - & - \\
\hline $\begin{array}{c}\text { Narcissus poeticus } \\
\text { dante. } \ldots \ldots \ldots \ldots\end{array}$ & - & - & - & +8 & - & - \\
\hline \multirow{2}{*}{\multicolumn{7}{|c|}{ Narcissus poetaz tri- }} \\
\hline & - & + & - & - & - & - \\
\hline Narcissus fiery cross & - & + & - & - & - & - \\
\hline Narcissus doubloon. . & + & - & - & - & - & - \\
\hline Narcissus cresset. . . . & - & + & - & - & - & - \\
\hline \multirow{2}{*}{\multicolumn{7}{|c|}{$\begin{array}{l}\text { Narcissus will scarlet } \\
\text { Narcissus bicolor apri- }\end{array}$}} \\
\hline & + & - & - & - & - & - \\
\hline \multicolumn{3}{|l|}{ 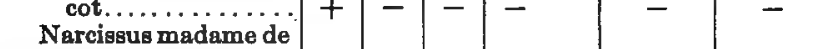 } & & - & - & - \\
\hline Narciseus pyramus... & - & - & - & - & $+\phi=\sigma^{\top}$ & - \\
\hline $\begin{array}{l}\text { Narciseus lord roberts } \\
\text { Narciseus agnes har- }\end{array}$ & - & + & - & - & $1+$ & - \\
\hline \multirow{2}{*}{\multicolumn{7}{|c|}{$\begin{array}{r}\text { vey ..................... } \\
\text { Narcissus j.t. bennett }\end{array}$}} \\
\hline & & & & & & \\
\hline poe $\ldots \ldots \ldots \ldots \ldots$ & + & - & - & - & - & - \\
\hline Lilium marhan. ..... & - & + & - & - & - & - \\
\hline Lilium dalhansoni . . & + & - & - & - & - & - \\
\hline Lilium golden gleam. & - & - & - & - & - & $+q$ \\
\hline Lilium testaceum. ... & + & - & - & - & - & - \\
\hline Lilium burbanki..... & - & + & - & - & - & - \\
\hline Iris ismali . . . . . . . . . & - & - & - & - & - & $+0^{x}$ \\
\hline Iris dorak . . . . . . . . . & + & - & - & - & - & \\
\hline Iris mrs. alan grey... & - & - & - & - & - & $+\infty^{x}$ \\
\hline Iris pursind $\ldots \ldots \ldots$. & - & - & - & - & - & +0 \\
\hline \multirow{2}{*}{\multicolumn{7}{|c|}{$\begin{array}{l}\text { Gladiolus colvillei.... } \\
\text { Tritonia crocosm }{ }^{-}\end{array}$}} \\
\hline & & & & & & \\
\hline flora .............. & - & - & - & - & - & +9 \\
\hline Begonia mrs. hesl.... & - & - & - & - & - & $+q=\sigma^{7}$ \\
\hline Begonia ensign ...... & - & - & - & +9 & - & - \\
\hline Begonia julius....... & - & + & - & - & - & - \\
\hline \multirow{2}{*}{\multicolumn{7}{|c|}{$\begin{array}{l}\text { Begonia success . . .... } \\
\text { Richardia mrs. roose- }\end{array}$}} \\
\hline & - & - & - & $+0=0$ & - & - \\
\hline Musa hybrida........ & - & - & - & $17-0$ & - & $+0^{11}$ \\
\hline Phaius hybridus..... & - & - & - & - & +9 & - \\
\hline \multirow{2}{*}{\multicolumn{7}{|c|}{ Miltonia bleuana .... }} \\
\hline & & & & & & \\
\hline & + & - & - & - & - & - \\
\hline \multirow{3}{*}{$\begin{array}{l}\text { Calanthe veitchii .... } \\
\text { Calanthe bryan ..... }\end{array}$} & - & - & - & +9 & - & - \\
\hline & - & - & - & $+\sigma^{x}$ & - & - \\
\hline & 11 & 11 & 0 & 9 & 9 & 10 \\
\hline
\end{tabular}

TABLE H.-Continued.

\begin{tabular}{|c|c|c|c|c|c|c|}
\hline Hybrids. & 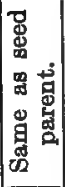 & 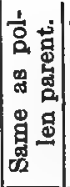 & 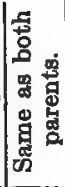 & 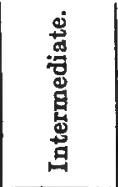 & $\begin{array}{l}\text { 兽 } \\
\text { 总 } \\
\text { 昜 }\end{array}$ & 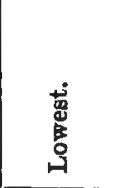 \\
\hline \multicolumn{7}{|l|}{$\begin{array}{l}\text { 2. Iodine reactions: } \\
\text { Brunsdonna sanderce }\end{array}$} \\
\hline & + & - & - & - & - & - \\
\hline $\begin{array}{l}\text { Brunsdonna sanderœ. } \\
\text { Hippeastrum titan- }\end{array}$ & + & - & - & - & - & - \\
\hline \multicolumn{7}{|l|}{ Hippeastrum ossul- } \\
\hline \multicolumn{7}{|l|}{ Hippeastrum-døones- } \\
\hline \multicolumn{7}{|l|}{ Hrmanthus andro- } \\
\hline \multicolumn{7}{|l|}{$\begin{array}{r}\operatorname{meda} . \ldots \ldots \ldots \ldots . . . \\
\text { Hæmanthus könig al- }\end{array}$} \\
\hline \multicolumn{7}{|l|}{$\begin{array}{l}\text { bert............. } \\
\text { Crinum hybridum } \mathbf{j} .\end{array}$} \\
\hline c. h. . . . . . . . & - & + & - & - & - & - \\
\hline Crinum kircape ...... & - & - & - & $+\infty^{x}$ & - & - \\
\hline Crinum powellii .... & - & - & - & $+9=\sigma^{7}$ & - & - \\
\hline Nerine dainty maid. . & - & - & - & - & $+\sigma^{\pi}$ & - \\
\hline Nerine queen of rosee & - & + & - & - & - & - \\
\hline Nerine giantess...... & - & + & - & - & - & - \\
\hline Nerine abundance... . & + & - & - & - & - & - \\
\hline \multirow{2}{*}{\multicolumn{7}{|c|}{$\begin{array}{l}\text { Nerine glory of sarnia } \\
\text { Narcissus poeticus }\end{array}$}} \\
\hline & - & + & - & - & - & - \\
\hline \multicolumn{7}{|l|}{ Narcissus poeticus } \\
\hline \multicolumn{7}{|l|}{ Narcissus poetaz tri- } \\
\hline Narcissus fiery cross. & + & - & - & - & - & - \\
\hline Narcissus doubloon. . & + & - & - & - & - & - \\
\hline Narcissus cresset.... & - & + & - & - & - & - \\
\hline \multirow{2}{*}{\multicolumn{7}{|c|}{$\begin{array}{l}\text { Narcissus will acarlet. } \\
\text { Narcissus bicolor apri- } \\
\text { cot. }\end{array}$}} \\
\hline & - & - & - & $+q$ & - & - \\
\hline \multicolumn{7}{|l|}{ Narcissus madame de } \\
\hline Narcissus pyramua... & + & - & - & - & - & $\bar{z}$ \\
\hline Narcissus lord roberts & - & - & $\oplus$ & - & - & - \\
\hline \multicolumn{7}{|l|}{$\begin{array}{c}\text { Narcissus agnes har- } \\
\text { vey ............. }\end{array}$} \\
\hline \multicolumn{7}{|l|}{$\begin{array}{r}\text { vey ................ } \\
\text { Narciseus j. t. bennett }\end{array}$} \\
\hline & + & - & - & - & - & - \\
\hline Lilium marhan ...... & - & - & - & $+\sigma^{3}$ & - & - \\
\hline Lilium dalhansoni.... & - & - & - & - & +9 & - \\
\hline Lilium golden gleam. & - & - & - & - & - & $+q$ \\
\hline Lilium testaceum... & - & - & - & - & - & $+\stackrel{+}{+}$ \\
\hline Lilium burbanki..... & + & - & - & - & - & - \\
\hline Iris ismali........... & + & - & - & - & - & - \\
\hline Iris dorak. ......... & + & - & - & - & - & - \\
\hline Iria mrs. alan grey... & - & - & - & - & +9 & - \\
\hline Iris purgind......... & - & + & - & - & - & - \\
\hline \multirow{2}{*}{\multicolumn{7}{|c|}{$\begin{array}{l}\text { Gladiolus colvillei.... } \\
\text { Tritonia crocosme- }\end{array}$}} \\
\hline & & & & & & \\
\hline & - & - & - & $+q$ & - & - \\
\hline Begonia mra heal.... & + & - & - & - & - & - \\
\hline Begonia ensign . . . . . . & - & - & - & +9 & - & - \\
\hline Begonia julius....... & - & - & - & - & $+0^{x}$ & - \\
\hline Begonia success...... & - & + & - & - & - & - \\
\hline \multirow{2}{*}{\multicolumn{7}{|c|}{$\begin{array}{l}\text { Richardia mrs. roose- } \\
\text { velt. }\end{array}$}} \\
\hline & + & - & - & - & - & - \\
\hline \multirow{5}{*}{$\begin{array}{l}\text { Musa hybrida........ } \\
\text { Phaius hybridus. .... } \\
\text { Miltonia bleuana.... } \\
\text { Cymbidium eburneo- } \\
\text { lowianum......... }\end{array}$} & - & + & - & - & - & - \\
\hline & - & - & - & $+\infty^{x}$ & - & - \\
\hline & + & - & - & - & - & - \\
\hline & & & & & & \\
\hline & + & - & - & - & - & - \\
\hline \multirow{3}{*}{$\begin{array}{l}\text { Calanthe veitchii .... } \\
\text { Calanthe bryan..... }\end{array}$} & - & - & - & +9 & - & - \\
\hline & - & - & - & $+\phi=\sigma^{x}$ & - & - \\
\hline & 16 & 12 & 1 & 12 & 5 & 4 \\
\hline
\end{tabular}


TABLE H.-Continued.

\begin{tabular}{|c|c|c|c|c|c|c|}
\hline Hybrids. & 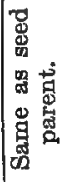 & 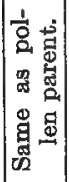 & 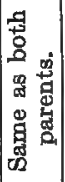 & 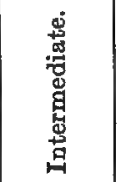 & 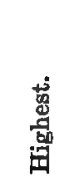 & 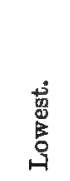 \\
\hline \multicolumn{7}{|l|}{$\begin{array}{l}\text { 3. Gentian-violet reac- } \\
\text { tions: }\end{array}$} \\
\hline & - & - & $+\sigma^{x}$ & - \\
\hline \multirow{2}{*}{\multicolumn{6}{|c|}{ Hippeastrum titan- }} & - \\
\hline & & & & & - & - \\
\hline & \multicolumn{5}{|c|}{ Hippeastrum ossul- } & - \\
\hline $\begin{array}{l}\text { Hippeastrum dæones- } \\
\text { zephyr. . . . . . . . }\end{array}$ & - & - & $\rightarrow$ & - & - & $+\sigma^{x}$ \\
\hline \multicolumn{2}{|l|}{ Hrmanthus andro- } & - & - & $+9=\sigma^{7}$ & - & - \\
\hline $\begin{array}{l}\text { Hemanthus könig al- } \\
\text { bert. . }\end{array}$ & - & - & - & - & - & + 우 \\
\hline 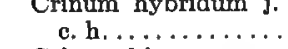 & - & - & - & - & $+\sigma^{x}$ & - \\
\hline Crinum kircape...... & - & + & - & - & - & - \\
\hline Crinum powellii.... & - & + & - & - & - & - \\
\hline Nerine dainty maid. . & - & + & - & - & - & - \\
\hline Nerine queen of roses & + & - & - & - & - & - \\
\hline Nerine giantess... . . & + & - & - & - & - & - \\
\hline Nerine abundance... & - & + & - & - & - & - \\
\hline \multirow{2}{*}{\multicolumn{7}{|c|}{ Narcissus poeticus }} \\
\hline & & & & & & \\
\hline \multicolumn{7}{|l|}{ Narcissus poeticus } \\
\hline \multicolumn{7}{|l|}{$\begin{array}{l}\text { Narcissus poetaz tri- } \\
\text { umph. }\end{array}$} \\
\hline Narcissus fiery cross. & - & - & - & $+0=\sigma^{x}$ & - & - \\
\hline Narcissus doubloon. . & - & - & - & $+0^{\pi}$ & - & - \\
\hline Narcissus cresset. . . . & + & - & - & - & - & - \\
\hline Narcissus will scarlet. & \multicolumn{4}{|c|}{$\begin{array}{l}\text { Narcissus will scarlet. } \\
\text { Narcissus bicolor apri- }\end{array}$} & $+d^{\lambda}$ & \\
\hline \multicolumn{7}{|l|}{$\begin{array}{r}\text { cot. ............... } \\
\text { Narcissus madame de }\end{array}$} \\
\hline $\begin{array}{l}\text { Narcissus madame de } \\
\text { graaff . . . . . . . . . }\end{array}$ & + & - & - & - & - & - \\
\hline Narcissus pyramus... & - & - & - & $+0^{x}$ & - & - \\
\hline \multicolumn{7}{|l|}{$\begin{array}{l}\text { Narcissus lord roberts } \\
\text { Narcissus agnes har- }\end{array}$} \\
\hline \multicolumn{7}{|l|}{$\begin{array}{r}\text { vey .... . . . . . . . } \\
\text { Narcissus j. t. bennett }\end{array}$} \\
\hline poe $\ldots \ldots \ldots \ldots$ & - & - & - & - & $+q$ & - \\
\hline Lilium marhan . . . . . & - & - & - & - & - & $+\sigma^{x}$ \\
\hline Lilium dalhansoni... . & + & - & - & - & - & - \\
\hline Lilium golden gleam. . & - & - & - & - & - & $+\sigma^{\pi}$ \\
\hline Lilium testaceum. . . & - & + & - & - & - & - \\
\hline Lilium burbanki.... . & - & - & - & $+\sigma$ & - & - \\
\hline Iris ismali. ......... & + & - & - & - & - & - \\
\hline Iris dorak. . . . . . . . & - & - & - & - & $+\sigma^{\pi}$ & - \\
\hline Iris mrs. alan grey... & - & - & - & - & - & $+\%$ \\
\hline Iris pursind. . . . . . . & - & - & - & - & - & $+\sigma$ \\
\hline Gladiolus colvillei... . & - & - & - & $+0^{7}$ & - & - \\
\hline \multicolumn{7}{|l|}{ Tritonia crocosmæ } \\
\hline flora. . . . . . . . . & + & - & - & - & - & - \\
\hline Begonia mrs. heal... & + & - & - & - & - & - \\
\hline Begonia ensign . . . . . & - & - & - & - & - & $+0^{x}$ \\
\hline Begonia julius...... & - & - & - & - & $+\sigma^{2}$ & - \\
\hline Begonia success...... & $\div$ & + & - & - & - & - \\
\hline \multirow{2}{*}{\multicolumn{7}{|c|}{ Richardia mrs. roose- }} \\
\hline & - & - & - & - & $+\sigma^{\top}$ & - \\
\hline Musa hybrida. . . . . . & - & + & - & - & - & - \\
\hline Phaius hybridus. . . . . & - & - & - & - & $+q$ & - \\
\hline Miltonia bleuana... & - & - & 一 & - & - & +9 \\
\hline \multicolumn{7}{|l|}{ Cymbidium eburneo- } \\
\hline lowianum........ & + & - & - & - & 一 & - \\
\hline Calanthe veitchii.... & - & - & - & $+0=\sigma^{\lambda}$ & - & - \\
\hline Calanthe bryan...... & - & - & - & $+q=\sigma^{7}$ & - & - \\
\hline & 13 & 9 & 0 & 8 & 10 & 10 \\
\hline
\end{tabular}

TABLE H.-Continued.

\begin{tabular}{|c|c|c|c|c|c|c|}
\hline Hybrids. & 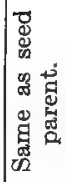 & 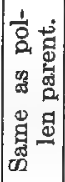 & 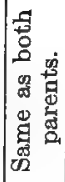 & 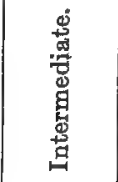 & 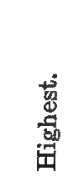 & 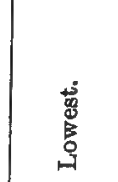 \\
\hline \multicolumn{7}{|l|}{$\begin{array}{l}\text { 4. Safranin reactions: } \\
\text { Brunsdonna sanderoe }\end{array}$} \\
\hline $\begin{array}{c}\text { alba....................... } \\
\text { Brunsdonna sanderœ. }\end{array}$ & $\overline{-}$ & $\overline{-}$ & $\overline{-}$ & $\begin{array}{l}- \\
-\end{array}$ & $\begin{array}{l}+0^{+1} \\
+0\end{array}$ & $\overline{-}$ \\
\hline $\begin{array}{c}\text { Hippeastrum titan- } \\
\text { cleonia........... }\end{array}$ & - & + & - & - & +9 & - \\
\hline $\begin{array}{c}\text { Hippeastrum ossul- } \\
\text { tan-pyrrha. . . . . }\end{array}$ & - & - & - & - & +0 & - \\
\hline $\begin{array}{l}\text { Hippeastrum dæones- } \\
\text { zephyr ........... }\end{array}$ & - & - & $\oplus$ & - & - & - \\
\hline $\begin{array}{l}\text { Hæmanthus andro- } \\
\text { meda } . . . \ldots \ldots\end{array}$ & - & - & - & - & - & $+9=\sigma^{\prime}$ \\
\hline $\begin{array}{c}\text { Hæmanthus könig al- } \\
\text { bert. . . . . . . . }\end{array}$ & - & - & - & - & - & \\
\hline $\begin{array}{l}\text { Crinum hybridum } \mathbf{j} \\
\text { c. h. }\end{array}$ & - & - & - & - & - & +8 \\
\hline Crinum kircape................. & - & - & - & - & +8 & +7 \\
\hline Crinum powellii : ..... & - & + & - & - & T & - \\
\hline Nerine dainty maid. . & + & - & - & - & - & - \\
\hline Nerine queen of roses & + & - & - & - & - & - \\
\hline Nerine giantess...... & + & - & - & - & - & - \\
\hline Nerine abundance... & - & - & - & $+q$ & - & - \\
\hline Nerine glory of sarnia & - & + & - & - & - & - \\
\hline $\begin{array}{c}\text { Narcissus poeticus } \\
\text { herrick ........... }\end{array}$ & - & - & - & - & - & +9 \\
\hline $\begin{array}{l}\text { Narcissus poeticus } \\
\text { dante. . . . . . . }\end{array}$ & - & + & - & - & - & - \\
\hline Narcissus poetaz tri- & & & & & & \\
\hline $\begin{array}{l}\text { umph ............ } \\
\text { Narcissus flery cross. }\end{array}$ & - & $\bar{t}$ & - & - & - & $+\%=\sigma^{7}$ \\
\hline Narcissus doubloon. & - & + & - & - & $\bar{z}$ & $\overline{-}$ \\
\hline Narcissus cresset.... & - & + & - & - & - & - \\
\hline Narcissus will scarlet. & - & $\frac{1}{-}$ & - & - & $+\infty$ & - \\
\hline $\begin{array}{l}\text { Narcissus bicolor apri- } \\
\text { cot. }\end{array}$ & 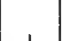 & 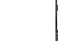 & & & 10 & \\
\hline Narcissus madame de & + & - & - & - & - & - \\
\hline graaff . . . . . . . . . . & + & - & - & - & - & - \\
\hline Narcissus pyramus... & - & - & - & $+\sigma^{7}$ & - & - \\
\hline Narcissus lord roberts & + & - & - & - & - & - \\
\hline 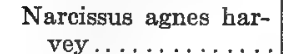 & - & - & $\oplus$ & - & - & - \\
\hline Narcissus j.t. bennett & - & - & - & - & +0 & $\ldots$ \\
\hline Lilium marhan ...... & - & - & - & - & T & $+\sigma^{1}$ \\
\hline dalhansoni... & + & - & - & - & - & - \\
\hline Lilium golden gleam.. & - & - & - & - & - & $+\infty^{7}$ \\
\hline Lilium testaceum.... & - & + & - & - & - & - \\
\hline Lilium burbanki..... & - & - & - & $+\sigma^{\pi}$ & - & - \\
\hline Iris ismali............ & + & - & - & - & - & - \\
\hline Iris dorak ........... & - & + & - & - & - & - \\
\hline Iris mrs. alan grey... & - & - & - & - & - & $+q$ \\
\hline Iris pursind. ......... & - & - & - & - & - & $+\sigma^{3}$ \\
\hline Gladiolus colvillei.... & + & - & - & - & - & - \\
\hline Tritonia crocosmæ- & & & & & & \\
\hline flora $\ldots \ldots \ldots \ldots$ & - & - & - & - & +9 & - \\
\hline Begonia mrs. heal ... & + & - & - & - & - & - \\
\hline Begonia ensign...... & - & - & - & - & - & $+0^{\pi}$ \\
\hline Begonia julius...... & - & - & - & - & $+0^{x}$ & - \\
\hline Begonia success...... & + & - & - & - & - & - \\
\hline $\begin{array}{l}\text { Richardia mrs. roose- } \\
\text { velt. }\end{array}$ & - & - & - & - & $+\pi$ & - \\
\hline Musa hybrida........ & - & + & - & - & +o & $\overline{-}$ \\
\hline Phaius hybridus. .... & - & - & - & - & $+q$ & - \\
\hline Miltonia bleuana.... & + & - & - & - & - & - \\
\hline Cymbidium eburneo- & & & & & & \\
\hline & + & - & - & - & - & - \\
\hline Calanthe veitchii. ... & - & + & - & - & - & - \\
\hline Calanthe bryan...... & - & - & - & $+q=\sigma^{7}$ & - & - \\
\hline & 13 & 11 & 2 & 4 & 10 & 10 \\
\hline
\end{tabular}


Table H.-Continued.

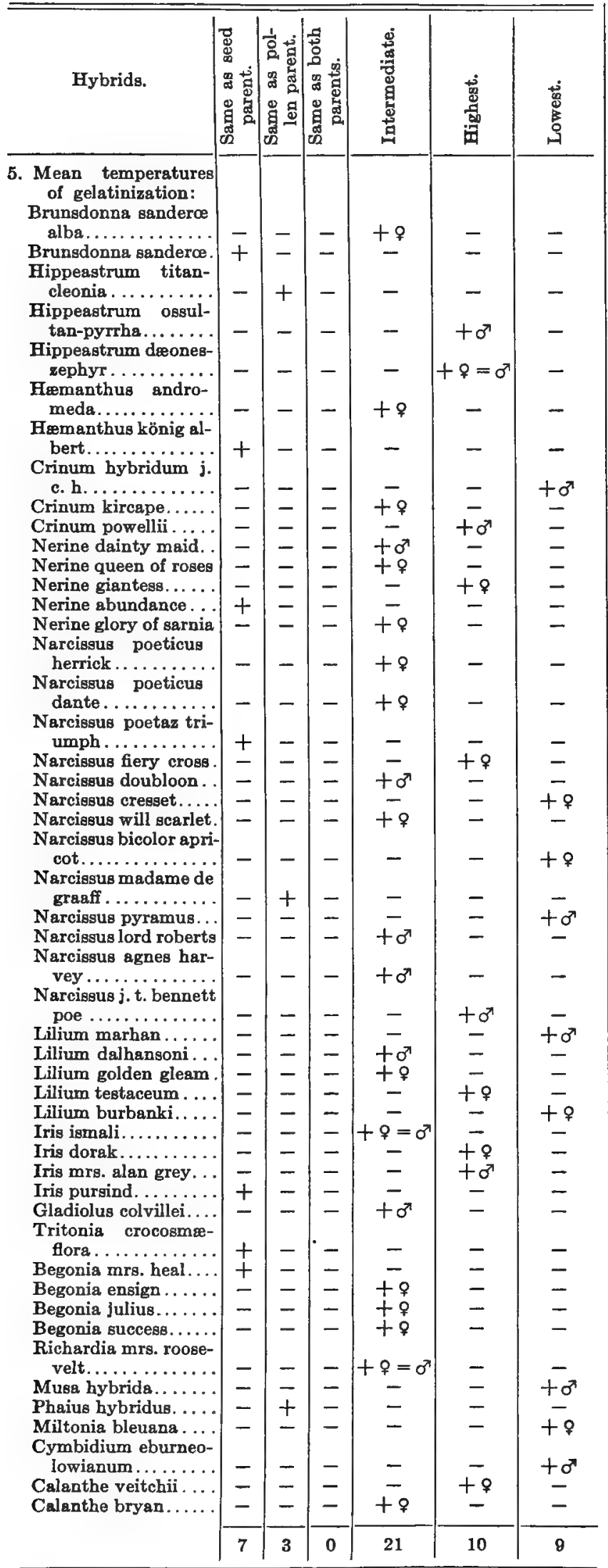

TABLE H.-Continued.

\begin{tabular}{|c|c|c|c|c|c|c|}
\hline Hybrids. & 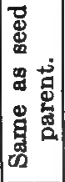 & 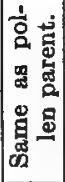 & 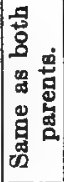 & 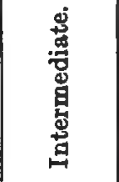 & 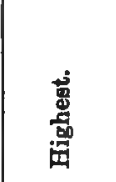 & 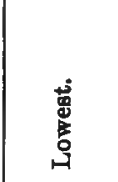 \\
\hline \multicolumn{7}{|l|}{$\begin{array}{l}\text { 6. Chloral-hydrate reac- } \\
\text { tions: }\end{array}$} \\
\hline $\begin{array}{c}\text { Brunsdonna sandero } \\
\text { alba.............. }\end{array}$ & - & - & - & - & $+\%$ & \\
\hline \multicolumn{7}{|l|}{ Brunsdonna sandero. } \\
\hline $\begin{array}{r}\text { cleonia.......... } \\
\text { Hippeastrum ossul- }\end{array}$ & - & - & - & - & - & $+q$ \\
\hline \multicolumn{7}{|l|}{$\begin{array}{c}\text { Hippeastrum dxones- } \\
\text { zephyr. . . . . }\end{array}$} \\
\hline \multicolumn{7}{|l|}{ Hæmanthus andro- } \\
\hline \multicolumn{7}{|l|}{$\begin{array}{r}\text { meda } . . . \ldots \ldots \ldots \ldots \\
\text { Hæmanthus könig al- }\end{array}$} \\
\hline \multicolumn{7}{|l|}{$\begin{array}{l}\text { bert.................. } \\
\text { Crinum hybridum }\end{array}$} \\
\hline c. h. ............. & - & - & - & $+0^{x}$ & - & - \\
\hline Crinum kircape...... & + & - & - & - & - & - \\
\hline Crinum powellii ..... & - & - & - & - & $+\varphi=\sigma^{\top}$ & - \\
\hline Nerine dainty maid. . & - & - & - & $+\sigma^{x}$ & - & - \\
\hline Nerine queen of roses & - & - & - & - & $+\sigma^{\top}$ & - \\
\hline Nerine giantess...... & - & + & - & - & - & - \\
\hline Nerine abundance. . . & - & - & - & - & $+\sigma^{\pi}$ & - \\
\hline \multirow{2}{*}{\multicolumn{7}{|c|}{$\begin{array}{l}\text { Nerine glory of earnia } \\
\text { Narcigsus poeticus }\end{array}$}} \\
\hline & & & & & & \\
\hline \multicolumn{7}{|l|}{$\begin{array}{l}\text { herrick............. } \\
\text { Narcissus poeticus }\end{array}$} \\
\hline \multicolumn{7}{|l|}{$\begin{array}{l}\text { dente . . . . . . . . . } \\
\text { Narcissus poetaz tri- }\end{array}$} \\
\hline umph ............ & - & - & - & - & $+\%$ & - \\
\hline Narcissus fiery cross. & - & - & - & - & - & $+\sigma^{x}$ \\
\hline Narcissue doubloon. . & - & - & - & - & - & $+q=\sigma^{\prime}$ \\
\hline Narcissur cresset ... & - & - & - & - & +9 & - \\
\hline \multicolumn{7}{|l|}{$\begin{array}{l}\text { Narcissus will scarlet. } \\
\text { Nareissus bicolor apri- }\end{array}$} \\
\hline \multirow{2}{*}{\multicolumn{7}{|c|}{$\begin{array}{c}\cot . . . \ldots \ldots \ldots \ldots \\
\text { Narcissus madame de }\end{array}$}} \\
\hline & - & - & - & - & $+\sigma^{7}$ & - \\
\hline Narcissus pyramus... & - & - & - & - & $\rightarrow$ & +9 \\
\hline \multirow{2}{*}{\multicolumn{7}{|c|}{$\begin{array}{l}\text { Narcissus lord roberts } \\
\text { Narcissus agnes har- }\end{array}$}} \\
\hline & & & & & & \\
\hline \multicolumn{7}{|l|}{$\begin{array}{l}\text { vey } \ldots . . . . . . . . . \\
\text { Narcissus j. t. bennett }\end{array}$} \\
\hline & - & - & - & - & +9 & - \\
\hline Lilium mar & - & - & - & $+\sigma^{x}$ & - & - \\
\hline Lilium dalh & - & + & - & - & - & - \\
\hline Lilium golden gleam. & - & + & - & - & - & - \\
\hline Lilium testaceum.... & - & - & - & - & - & +9 \\
\hline Lilium burbanki.... . & - & - & - & +9 & - & - \\
\hline Iris ismali . . . & - & - & - & $+\sigma^{\circ}$ & - & - \\
\hline ris dorak..... & - & - & - & - & - & $+q=0$ \\
\hline Iris mrs. alan grey. .. & - & - & - & - & $+0^{\pi}$ & - \\
\hline Iris pursind. ........ & - & - & - & +9 & - & - \\
\hline Gladiolus colvillei.... & - & - & - & - & - & +9 \\
\hline \multicolumn{7}{|l|}{$\begin{array}{l}\text { Gladiolus colvillei.... } \\
\text { Tritonia crocosmæ- }\end{array}$} \\
\hline & - & - & - & - & - & +9 \\
\hline Begonia mrs heal.... & - & - & - & +9 & - & - \\
\hline Begonia ensig & - & - & - & - & $+q$ & - \\
\hline Begonia julius....... & - & - & - & - & +9 & - \\
\hline \multirow{2}{*}{\multicolumn{7}{|c|}{ Richardia mrs. roose- }} \\
\hline & & & & & & \\
\hline & - & - & - & - & $+\sigma^{x}$ & - \\
\hline Musa hybrida....... & - & - & - & - & - & $+\infty^{*}$ \\
\hline Phaius hybridus..... & - & - & - & - & - & $+\sigma^{7}$ \\
\hline & - & - & - & $+q$. & - & - \\
\hline \multirow{2}{*}{\multicolumn{7}{|c|}{ Cymbidium eburneo- }} \\
\hline & - & - & - & - & - & $+9=$ \\
\hline \multirow{3}{*}{$\begin{array}{l}\text { Calanthe veitchii.... } \\
\text { Calanthe bryan..... }\end{array}$} & - & - & - & - & + ? & - \\
\hline & - & - & - & $+9=\sigma^{7}$ & - & - \\
\hline & 1 & 6 & 1 & 14 & 14 & 14 \\
\hline
\end{tabular}


Table H.-Continued.

\begin{tabular}{|c|c|c|c|c|c|c|}
\hline Hybrids. & 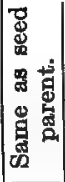 & 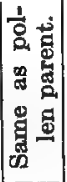 & 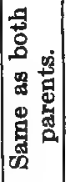 & 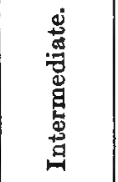 & $\begin{array}{l}\text { 总 } \\
\text { 总 } \\
\text { 蛋 }\end{array}$ & 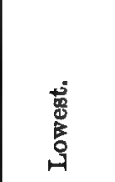 \\
\hline \multicolumn{7}{|l|}{$\begin{array}{l}\text { 7. Chromic-acid reac- } \\
\text { tions: }\end{array}$} \\
\hline \multicolumn{6}{|l|}{ Brunsdonna aanderce } & - \\
\hline \multirow{2}{*}{\multicolumn{7}{|c|}{$\begin{array}{l}\text { Brunsdonna sanderce } \\
\text { Hippeastrum titan- }\end{array}$}} \\
\hline & - & - & & & & $+\infty^{x}-1-1$ \\
\hline \\
\hline \multicolumn{7}{|l|}{$\begin{array}{r}\tan \text {-pyrrha......... } \\
\text { Hippeastrum dæones- }\end{array}$} \\
\hline \multicolumn{7}{|l|}{$\begin{array}{l}\text { zephyr } \ldots \ldots \ldots \ldots \\
\text { Hæmanthus }\end{array}$} \\
\hline $\begin{array}{l}\text { Hæmanthus andro- } \\
\text { meda............. }\end{array}$ & \multicolumn{6}{|c|}{$\begin{array}{l}\text { meda................. } \\
\text { Hæmanthus könig al- }\end{array}$} \\
\hline $\begin{array}{l}\text { Hrmanthus könig al- } \\
\text { bert. } \ldots \ldots \ldots \ldots \ldots\end{array}$ & \multirow{2}{*}{\multicolumn{6}{|c|}{$\begin{array}{l}\text { bert............. } \\
\text { Crinum hybridum }\end{array}$}} \\
\hline $\begin{array}{c}\text { Crinum hybridum j. } \\
\text { e. h. } \ldots \ldots \ldots \ldots \ldots\end{array}$ & & + & - & - & -1 & - \\
\hline Crinum kircape..... & - & - & - & -9 & - & - \\
\hline Crinum powellii..... & - & - & - & - & $-\sigma$. & - \\
\hline Nerine dainty maid. . & - & - & - & - & $=$ & +0 \\
\hline Nerine queen of roses & - & - & - & - & - & +9 \\
\hline Nerine giantess..... & - & - & - & $+\%=\sigma^{7}$ & - & - \\
\hline Nerine abundance... & - & - & - & - & - & $+\sigma^{x}$ \\
\hline \multirow{2}{*}{\multicolumn{7}{|c|}{ Narcissus poeticus }} \\
\hline & - & - & - & $+\infty^{x}$ & & - \\
\hline \multicolumn{7}{|l|}{ 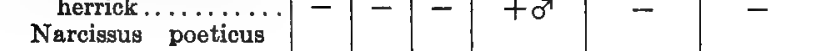 } \\
\hline \multicolumn{7}{|l|}{$\begin{array}{l}\text { dante........... } \\
\text { Narcissus poetaz tri- }\end{array}$} \\
\hline \multicolumn{7}{|l|}{$\begin{array}{c}\text { Narcissus poetaz tri- } \\
\text { umph } . . . \ldots \ldots \ldots \ldots\end{array}$} \\
\hline \multicolumn{7}{|l|}{ Narcissus fiery cross. } \\
\hline \multirow{2}{*}{\multicolumn{7}{|c|}{$\begin{array}{l}\text { Narcissus doubloon. } \\
\text { Narcissus cresset. }\end{array}$}} \\
\hline & + & - & - & - & - & - \\
\hline \multirow{2}{*}{\multicolumn{7}{|c|}{$\begin{array}{l}\text { Narcissus will scarlet. } \\
\text { Narcissus bicolor apri- } \\
\text { cot. }\end{array}$}} \\
\hline & - & - & - & +0 & - & - \\
\hline \\
\hline graaff $\ldots \ldots \ldots \ldots$ & - & - & - & - & - & $+q$ \\
\hline Narcissus pyramus... & - & - & - & - & +9 & - \\
\hline $\begin{array}{l}\text { Narcissus lord roberts } \\
\text { Narcissus agnes har- }\end{array}$ & - & - & - & - & - & $+o^{7}$ \\
\hline vey $\ldots \ldots \ldots \ldots \ldots$ & - & - & - & - & - & +9 \\
\hline Narcissus j.t. bennett & & & & & & \\
\hline poe ............... & - & - & - & - & +9 & - \\
\hline Lilium marhan...... & - & + & - & - & - & - \\
\hline Lilium dalhansoni.... & - & - & - & $+\infty^{x}$ & - & - \\
\hline Lilium golden gleam. & + & - & - & - & - & - \\
\hline Lilium testaceum.... & - & - & - & $+q$ & - & - \\
\hline Lilium burbanki.... . & - & - & - & - & - & $-\%$ \\
\hline Iris ismali........... & - & - & - & $+q$ & - & - \\
\hline Iris dorak ........... & - & - & - & - & $+q$ & - \\
\hline Iris mrs. alan grey... & - & - & - & $+\%$ & - & - \\
\hline Iris pursind. . . . . . . & - & - & $\oplus$ & - & - & - \\
\hline Gladiolus colvillei.... & + & - & - & - & - & - \\
\hline Tritonia crocosmæ- & & & & & & \\
\hline flora $\ldots \ldots \ldots \ldots$ & - & - & - & +우 & - & - \\
\hline Begonia mrs. heal.... & - & - & - & $+\%$ & - & - \\
\hline Begonia ensign ..... . & - & - & - & $+q$ & - & - \\
\hline Begonia julius....... & - & - & - & $+\%$ & - & - \\
\hline Begonia success...... & - & - & - & - & + o & - \\
\hline Richardia mrs. roose- & & & & & & \\
\hline velt.............. & - & - & $\oplus$ & - & - & - \\
\hline Musa hybrida....... & - & - & 一 & $+0-\pi$ & - & $+0^{x}$ \\
\hline Phaius hybridus. .... & - & - & - & $+9=\sigma^{-1}$ & - & - \\
\hline Miltonia bleuana.... & - & - & - & - & $+q$ & - \\
\hline Cymbidium eburneo- & - & - & - & _ & - & $+0=0$ \\
\hline Calanthe veitchii .... & + & - & - & - & - & - \\
\hline Calanthe bryan...... & - & - & - & $+0^{x}$ & - & - \\
\hline & 4 & 2 & 2 & 18 & 10 & 14 \\
\hline
\end{tabular}

TABLE H.Continued.

\begin{tabular}{|c|c|c|c|c|c|c|}
\hline Hybrids. & 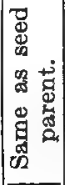 & 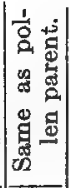 & 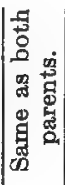 & 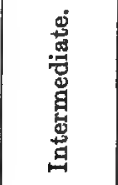 & 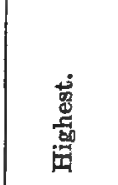 & 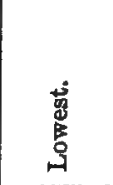 \\
\hline \multicolumn{7}{|l|}{$\begin{array}{l}\text { 8. Pyrogallic-acid reac- } \\
\text { tions: }\end{array}$} \\
\hline $\begin{array}{l}\text { tions: } \\
\text { Brunsdonna sanderœ }\end{array}$ & - & - & - & - & - & +8 \\
\hline \multicolumn{7}{|l|}{$\begin{array}{l}\text { Brunsdonna sanderoe. } \\
\text { Hippeastrum titan- }\end{array}$} \\
\hline $\begin{array}{c}\text { Hippeastrum titan- } \\
\text { cleonia............ }\end{array}$ & - & - & - & - & - & +9 \\
\hline \multicolumn{2}{|l|}{ Hippeastrum ossul- } & - & - & $\leftarrow$ & $+\sigma^{\pi}$ & - \\
\hline $\begin{array}{l}\text { Hippeastrum dæones- } \\
\text { zephyr } . \ldots \ldots \ldots \ldots\end{array}$ & - & - & - & - & $+\%$ & - \\
\hline $\begin{array}{l}\text { Hrmanthus andro- } \\
\text { meda........... }\end{array}$ & + & - & - & - & - & - \\
\hline \multicolumn{7}{|l|}{$\begin{array}{l}\text { bert.............. } \\
\text { Crinum hybridum j. }\end{array}$} \\
\hline $\begin{array}{l}\text { Crinum hybridum j. } \\
\text { c. h. } \ldots \ldots \ldots \ldots \ldots \ldots\end{array}$ & - & - & - & - & - & $+0^{x}$ \\
\hline Crinum kircape..... & $-"$ & - & - & $+0^{\pi}$ & - & - \\
\hline Crinum powellii.... & - & - & - & - & $+0^{x}$ & - \\
\hline Nerine dainty maid. . & - & - & $\oplus$ & - & - & - \\
\hline Nerine queen of roses & - & - & $\oplus$ & - & - & - \\
\hline Nerine giantess...... & - & - & $\oplus$ & - & - & - \\
\hline Nerine abundance... . & - & - & $\oplus$ & - & - & - \\
\hline \multirow{2}{*}{\multicolumn{7}{|c|}{$\begin{array}{l}\text { Nerine glory of sarnia } \\
\text { Narcissus poeticus }\end{array}$}} \\
\hline & \multirow{2}{*}{\multicolumn{4}{|c|}{$\begin{array}{l}\text { herrick............ } \\
\text { Narcissus poeticus }\end{array}$}} & & \\
\hline $\begin{array}{l}\text { Narcissus poeticus } \\
\text { dante................... }\end{array}$ & & - & & & \multicolumn{2}{|c|}{$\begin{array}{r}\text { dante } . . . \ldots \ldots \ldots \\
\text { Narcissus poeta tri- }\end{array}$} \\
\hline $\begin{array}{l}\text { Narcissus poetaz tri- } \\
\text { umph ........... }\end{array}$ & - & - & - & - & $+\sigma^{r}$ & - \\
\hline Narcissus fiery cross. & - & - & - & - & $+0^{7}$ & - \\
\hline Narcissus doubloon... & - & - & $\oplus$ & - & - & - \\
\hline Narcissus cresset.... & - & - & - & - & - & $+q$ \\
\hline \multicolumn{7}{|l|}{$\begin{array}{l}\text { Narcissus will scarlet. } \\
\text { Narcissus bicolor apri- }\end{array}$} \\
\hline \multirow{2}{*}{\multicolumn{7}{|c|}{$\begin{array}{l}\cot . \ldots \ldots \ldots \ldots \ldots \\
\text { Narcissus madame de }\end{array}$}} \\
\hline & - & - & - & +9 & - & - \\
\hline Narcissus pyramus... & - & - & - & - & $+q$ & - \\
\hline \multirow{2}{*}{\multicolumn{4}{|c|}{$\begin{array}{l}\text { Narcissus lord roberts } \\
\text { Narcissus agnes har- }\end{array}$}} & $+\infty^{x}$ & & \\
\hline & & - & - & $+9=8$ & - & - \\
\hline \multicolumn{6}{|l|}{ Narcissus j.t. bennett } & - \\
\hline Lilium marhan...... & - & + & - & - & To & - \\
\hline Lilium dalhansoni.... & - & - & - & $+\sigma^{x}$ & - & - \\
\hline Lilium golden gleam. & + & - & - & - & - & - \\
\hline n testaceum.... & - & - & - & $+o$ & - & - \\
\hline Lilium burbanki.... & - & - & - & - & - & +9 \\
\hline Iris ismali . . & - & - & - & $+\varphi=0^{x}$ & - & - \\
\hline Iris dorak.......... & - & - & - & - & $+q$ & - \\
\hline Iris mrs. alan grey. . & - & - & - & - & - & $+\sigma^{7}$ \\
\hline Iris pursind. . . . . . . & - & - & - & - & $+0^{7}$ & - \\
\hline \multirow{2}{*}{\multicolumn{7}{|c|}{$\begin{array}{l}\text { Gladiolus colvillei.... } \\
\text { Tritonia crocosmæ- }\end{array}$}} \\
\hline & & & & & & \\
\hline flora...$\ldots \ldots$ & - & - & - & +9 & - & - \\
\hline Begonia mrs. heal.... & - & - & - & +9 & - & - \\
\hline Begonia ensign...... & - & - & - & +9 & - & - \\
\hline Begonia julius.... . . . & - & - & - & +9 & - & - \\
\hline \multirow{3}{*}{\multicolumn{7}{|c|}{$\begin{array}{c}\text { Richardia mrs. roose- } \\
\text { velt. }\end{array}$}} \\
\hline & & & & & & \\
\hline & - & - & $\oplus$ & - & - & - \\
\hline Musa hybrida...... & + & - & - & - & - & - \\
\hline Phaius hybridus.... . & - & - & - & $+q=\sigma^{\pi}$ & - & - \\
\hline \multirow{2}{*}{\multicolumn{7}{|c|}{$\begin{array}{l}\text { Miltonia bleuana.... } \\
\text { Cymbidium eburneo- }\end{array}$}} \\
\hline & & & & & & \\
\hline & - & - & - & - & - & $-q=d^{7}$ \\
\hline Calanthe veitchii.... & - & - & - & $+q$ & - & - \\
\hline Calanthe bryan...... & - & - & - & $+\sigma^{r}$ & - & - \\
\hline & 3 & 2 & 7 & 17 & 12 & $\boldsymbol{\theta}$ \\
\hline
\end{tabular}


TABLE H.-Continued.

\begin{tabular}{|c|c|c|c|c|c|c|}
\hline Hybrids. & 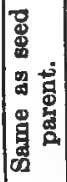 & 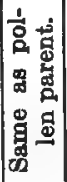 & 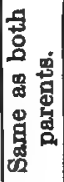 & 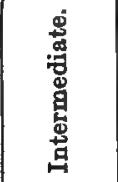 & 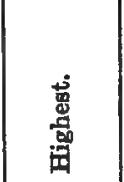 & 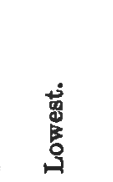 \\
\hline \multicolumn{7}{|l|}{ 9. Nitric-acid reactions: } \\
\hline alba................ & - & - & - & - & - & $+\sigma^{x}$ \\
\hline Brunsdonna sanderce & - & - & - & - & - & $+\infty$ \\
\hline $\begin{array}{c}\text { Hippeastrum titan- } \\
\text { cleonia } \ldots \ldots \ldots \ldots\end{array}$ & - & - & - & $+\%=\sigma^{\pi}$ & - & - \\
\hline $\begin{array}{c}\text { Hippeastrum ossu]- } \\
\text { tan-pyrrha. . . . }\end{array}$ & - & - & - & $10+2$ & +0 & - \\
\hline \multirow{2}{*}{\multicolumn{7}{|c|}{ Hippeastrum døones- }} \\
\hline & - & - & - & - & +8 & - \\
\hline 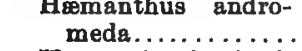 & - & - & - & +9 & - & - \\
\hline \multicolumn{6}{|l|}{ Hæmanthus könig al- } & - \\
\hline \multicolumn{7}{|l|}{ Crinum hybridum $\mathrm{j}$. } \\
\hline Crinum kircape........ & - & - & - & +9 & - & - \\
\hline Crinum powellii..... & - & - & - & - & $+0^{x}$ & - \\
\hline Nerine dainty maid. . & - & - & - & +9 & - & - \\
\hline Nerine queen of roses & - & - & - & $+q=\sigma^{r}$ & - & - \\
\hline Nerine giantess...... & - & - & - & - & - & $+0^{x}$ \\
\hline Nerine abundance. . . & - & - & - & - & - & $+\sigma^{x}$ \\
\hline \multirow{2}{*}{\multicolumn{7}{|c|}{ Narcissug poeticus }} \\
\hline & - & - & - & - & $+9=\sigma^{7}$ & - \\
\hline \multicolumn{7}{|l|}{ Narcissus poeticus } \\
\hline \multirow{2}{*}{\multicolumn{7}{|c|}{$\begin{array}{l}\text { Narcisius poetaz tri- } \\
\text { umph. }\end{array}$}} \\
\hline & - & - & - & - & $+\sigma^{\pi}$ & - \\
\hline Narcissus fiery cross. & - & - & - & - & - & $+9=\sigma^{7}$ \\
\hline Narciseus doubloon. . & - & - & - & +9 & - & - \\
\hline Narciseus cresset. . . . & - & - & - & - & $+q$ & - \\
\hline \multicolumn{7}{|l|}{$\begin{array}{l}\text { Narciasus will scarlet. } \\
\text { Narciasus bicolor apri- }\end{array}$} \\
\hline $\cot \ldots \ldots \ldots \ldots \ldots$ & - & - & - & - & - & $-\sigma^{7}$ \\
\hline \multicolumn{6}{|l|}{ Narcissus madame de } & +9 \\
\hline Narcisgus pyramus... & - & - & - & - & +9 & - \\
\hline Narcissus lord roberts & - & - & - & +8 & - & - \\
\hline \multicolumn{5}{|l|}{ Narcissus agnes har- } & $+\%$ & - \\
\hline \multirow{2}{*}{\multicolumn{7}{|c|}{ Nareissus j. t. bennett }} \\
\hline & - & - & - & - & +9 & - \\
\hline Lilium marhan ...... & - & - & $\oplus$ & - & - & - \\
\hline Lilium dalhansoni. . . & - & - & $\oplus$ & - & - & - \\
\hline Lilium golden gleam. & - & - & $\oplus$ & - & - & - \\
\hline Lilium testaceum.... & - & - & - & - & - & $+q=0^{7}$ \\
\hline Lilium burbanki..... & - & - & - & - & - & $+\%=0^{\prime}$ \\
\hline Iris ismali $\ldots \ldots \ldots$ & - & - & - & $+q=\sigma^{\prime}$ & - & - \\
\hline Iris dorak $\ldots \ldots \ldots \ldots$ & - & - & - & - & +9 & - \\
\hline Iris mrs. alan grey. .. & - & - & $\oplus$ & - & - & - \\
\hline Iris pursind. $\ldots \ldots \ldots$ & - & - & - & $+\sigma^{7}$ & - & - \\
\hline Gladiolus colvillei.... & + & - & - & - & - & - \\
\hline \multicolumn{7}{|l|}{ Tritonia crocosma- } \\
\hline flora...$\ldots \ldots \ldots$ & - & - & - & - & +9 & - \\
\hline Begonia mra. heal.... & + & - & - & - & - & - \\
\hline Begonia ensign ...... & - & - & - & +9 & - & - \\
\hline Begonia julius....... . & + & - & - & - & - & - \\
\hline \multirow{2}{*}{\multicolumn{7}{|c|}{$\begin{array}{l}\text { Begonia success. . . . . } \\
\text { Richardia mrs. roose- }\end{array}$}} \\
\hline & & & & & & $\ldots$ \\
\hline \multirow{2}{*}{$\begin{array}{l}\text { Musa hybrida...... } \\
\text { Phaiue hybridus. .... }\end{array}$} & - & - & - & $1+-$ & - & $+\sigma^{x}$ \\
\hline & - & - & - & $+\%$ & - & - \\
\hline \multirow{2}{*}{\multicolumn{7}{|c|}{$\begin{array}{l}\text { Miltonia bleuana .... } \\
\text { Cymbidium eburneo- }\end{array}$}} \\
\hline & & & & & & \\
\hline lowianum $\ldots . . . \ldots$ & - & - & - & - & - & $+9=0^{8}$ \\
\hline \multirow{3}{*}{$\begin{array}{l}\text { Calanthe veitchii .... } \\
\text { Calanthe bryan.... }\end{array}$} & - & - & - & - & +9 & - \\
\hline & - & - & - & +9 & - & - \\
\hline & 4 & 1 & 4 & 15 & 14 & 12 \\
\hline
\end{tabular}

TABLe H.-Continued.

\begin{tabular}{|c|c|c|c|c|c|c|}
\hline Hybrids. & 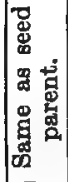 & 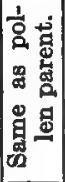 & 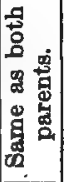 & 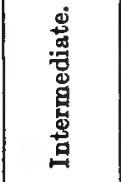 & 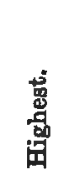 & 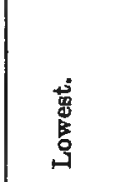 \\
\hline \multicolumn{7}{|l|}{$\begin{array}{l}\text { 10. Sulphuric-acid reac- } \\
\text { tions: }\end{array}$} \\
\hline $\begin{array}{r}\text { Brunsdonna sanderce } \\
\text { alba............. }\end{array}$ & + & - & - & - & - & - \\
\hline $\begin{array}{l}\text { Brunadonna sanderce } \\
\text { Hippeastrum titan- }\end{array}$ & + & - & - & - & - & - \\
\hline \multicolumn{7}{|l|}{$\underset{\text { tan-pyrrha }}{\text { Hippeastrum ossul- }}$} \\
\hline $\begin{array}{r}\text { tan-pyrrha ......... } \\
\text { Hippeastrum dæones- }\end{array}$ & + & - & - & - & - & - \\
\hline $\begin{array}{r}\text { zephyr } \ldots \ldots \ldots \\
\text { Hæmanthus andro }\end{array}$ & - & + & - & - & - & - \\
\hline $\begin{array}{l}\text { meda } \\
\text { Hæmanthus könig al- }\end{array}$ & \multicolumn{3}{|c|}{ Hæmanthus könig al- } & $+\%=\sigma^{\gamma}$ & - & - \\
\hline bert................... & - & - & - & +9 & - & - \\
\hline $\begin{array}{l}\text { Crinum hybridum j. } \\
\text { c. } h . \ldots \ldots \ldots \ldots \ldots\end{array}$ & - & - & - & - & - & $+a^{7}$ \\
\hline Crinum kircape...... & - & - & - & +8 & - & - \\
\hline Crinum powellii..... & - & - & - & - & $+0^{x}$ & - \\
\hline Nerine dainty maid. . & - & - & - & - & $+0^{2}$ & - \\
\hline Nerine queen of roses & - & - & - & - & $+\infty^{x}$ & - \\
\hline Nerine giantess...... & - & + & - & - & - & - \\
\hline Nerine abundance. . . & + & - & - & - & - & - \\
\hline \multicolumn{7}{|l|}{$\begin{array}{l}\text { - Nerine glory of arnia } \\
\text { Narcissus poeticus }\end{array}$} \\
\hline & - & - & - & - & $+o^{x}$ & - \\
\hline $\begin{array}{c}\text { Narcissus poeticus } \\
\text { dante. . . . }\end{array}$ & & & & & . & \\
\hline \multirow{2}{*}{\multicolumn{7}{|c|}{ Narcissus poetaz tri- }} \\
\hline & - & - & - & - & $+0^{x}$ & - \\
\hline Narcissus fiery cross & - & - & - & $+q=\sigma^{7}$ & - & - \\
\hline Narcissus doubloon. . & - & - & - & +9 & - & - \\
\hline Narciseus cresset. . . . & - & - & - & - & +9 & - \\
\hline \multirow{2}{*}{\multicolumn{4}{|c|}{ Narcissus bicolor apri- }} & - & & \\
\hline & & - & $\oplus$ & - & & \\
\hline \multirow{2}{*}{\multicolumn{7}{|c|}{ Narcissus madame de }} \\
\hline & + & - & - & - & - & - \\
\hline Narcissus pyramus... & - & - & $\oplus$ & - & - & - \\
\hline \multirow{2}{*}{\multicolumn{7}{|c|}{$\begin{array}{l}\text { Narcissus agnes har- } \\
\text { vey }\end{array}$}} \\
\hline & + & - & - & - & - & - \\
\hline \multicolumn{7}{|l|}{ Narcissus j.t. bennett } \\
\hline pœ.............. & - & - & - & - & $+\%$ & - \\
\hline Lilium marhan...... & - & - & $\oplus$ & - & - & - \\
\hline Lilium dalhansoni... . & - & - & $\oplus$ & - & - & - \\
\hline Lilium golden gleam. & $\rightarrow$ & - & $\oplus$ & - & - & - \\
\hline Lilium testaceum. ... & - & - & - & $+9=0^{7}$ & - & - \\
\hline Lilium burbanki..... & - & - & - & $+\infty$ & - & - \\
\hline Iris ismali $\ldots \ldots \ldots \ldots$ & - & - & - & $+q=\sigma^{7}$ & - & - \\
\hline Iris dorak.......... & - & - & - & - & $+\%$ & - \\
\hline Iris mra. alan grey. . & - & - & $\oplus$ & - & - & - \\
\hline Iris pursind. . . . . . . & - & + & - & - & - & - \\
\hline \multirow{2}{*}{\multicolumn{7}{|c|}{$\begin{array}{l}\text { Gladiolus colvillei.... } \\
\text { Tritonia crocosmæ- }\end{array}$}} \\
\hline & & & & & & \\
\hline flora...$\ldots \ldots \ldots$ & - & - & $\oplus$ & - & - & - \\
\hline \multirow{3}{*}{$\begin{array}{l}\text { Begonia mrs. heal.... } \\
\text { Richardia mrs. roose- } \\
\text { velt............ }\end{array}$} & - & $\rightarrow$ & $\oplus$ & - & - & - \\
\hline & & & & & & - \\
\hline & $\overline{-}$ & $\overline{-}$ & $\stackrel{\oplus}{-}$ & $\overline{-}$ & $\overline{-}$ & $+0^{x}$ \\
\hline \multirow{4}{*}{$\begin{array}{l}\text { Musa hybrida........ } \\
\text { Phaius hybridus. .... } \\
\text { Miltonia bleuana.... } \\
\text { Cymbidium eburneo- } \\
\text { lowianum........ }\end{array}$} & - & - & $\oplus$ & - & - & - \\
\hline & - & - & $\oplus$ & - & - & - \\
\hline & & & & & & \\
\hline & - & - & $\oplus$ & - & - & - \\
\hline \multirow{3}{*}{$\begin{array}{l}\text { Calanthe veitchii... } \\
\text { Calanthe bryan..... }\end{array}$} & + & - & - & - & - & - \\
\hline & - & - & - & $+0^{7}$ & - & - \\
\hline & 10 & 3 & 12 & 9 & 9 & 4 \\
\hline
\end{tabular}


Table H.-Continued.

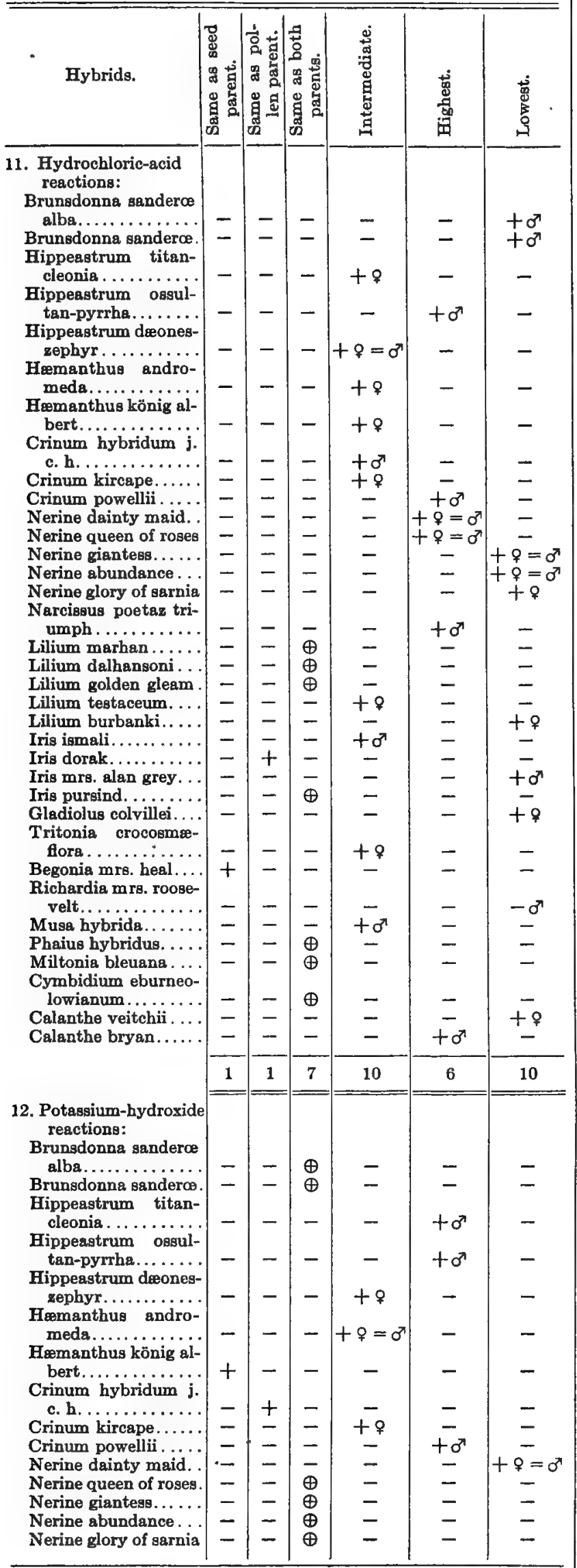

TABLE H.-Continued.

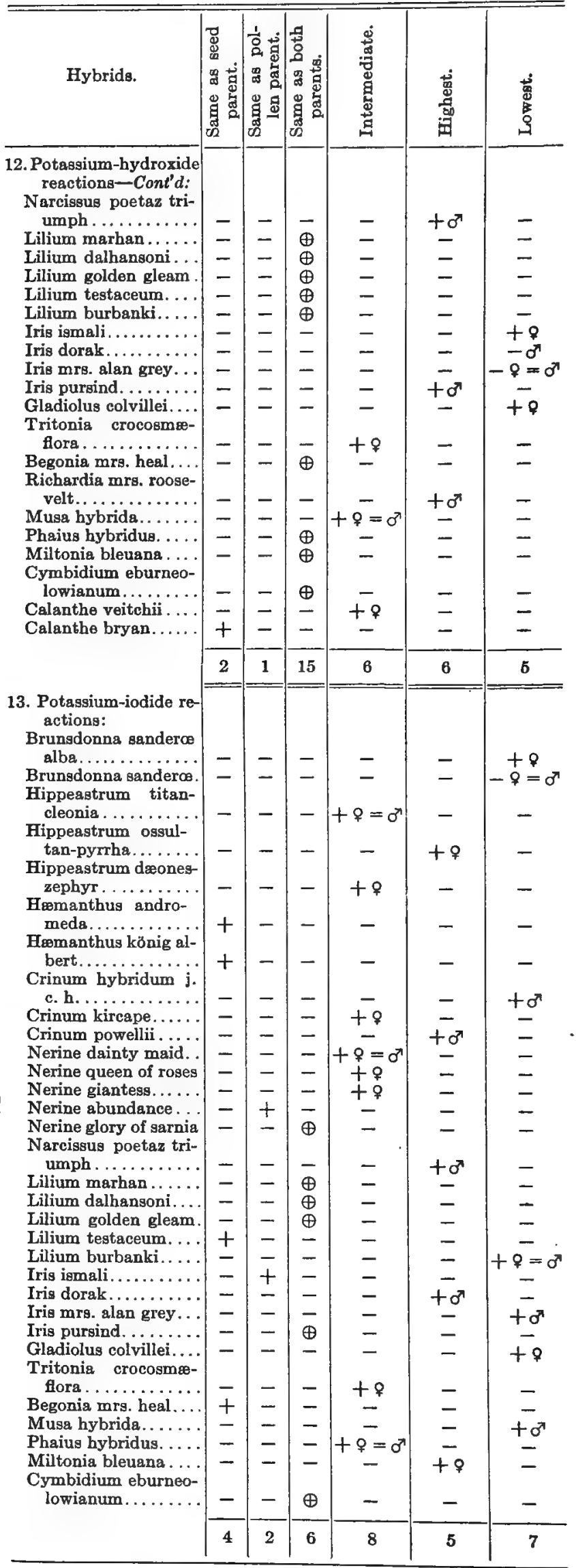


TABLE H.-Continued.

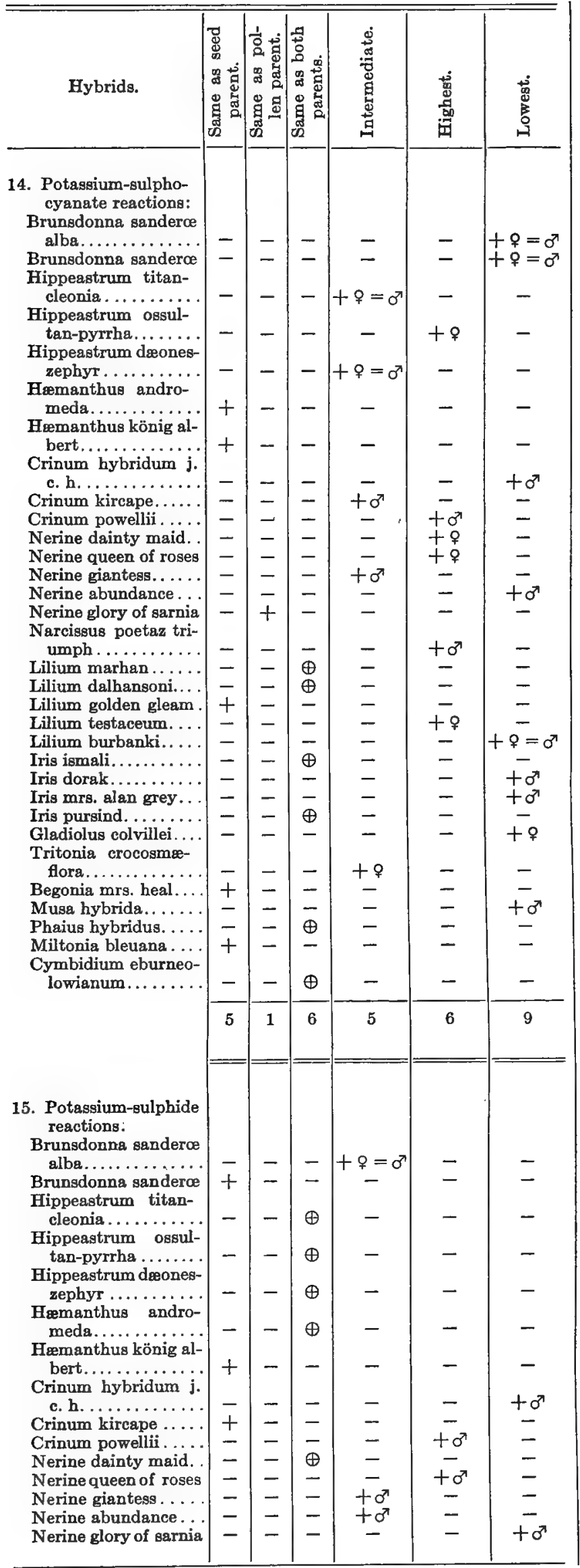

TABLE H.-Continued.

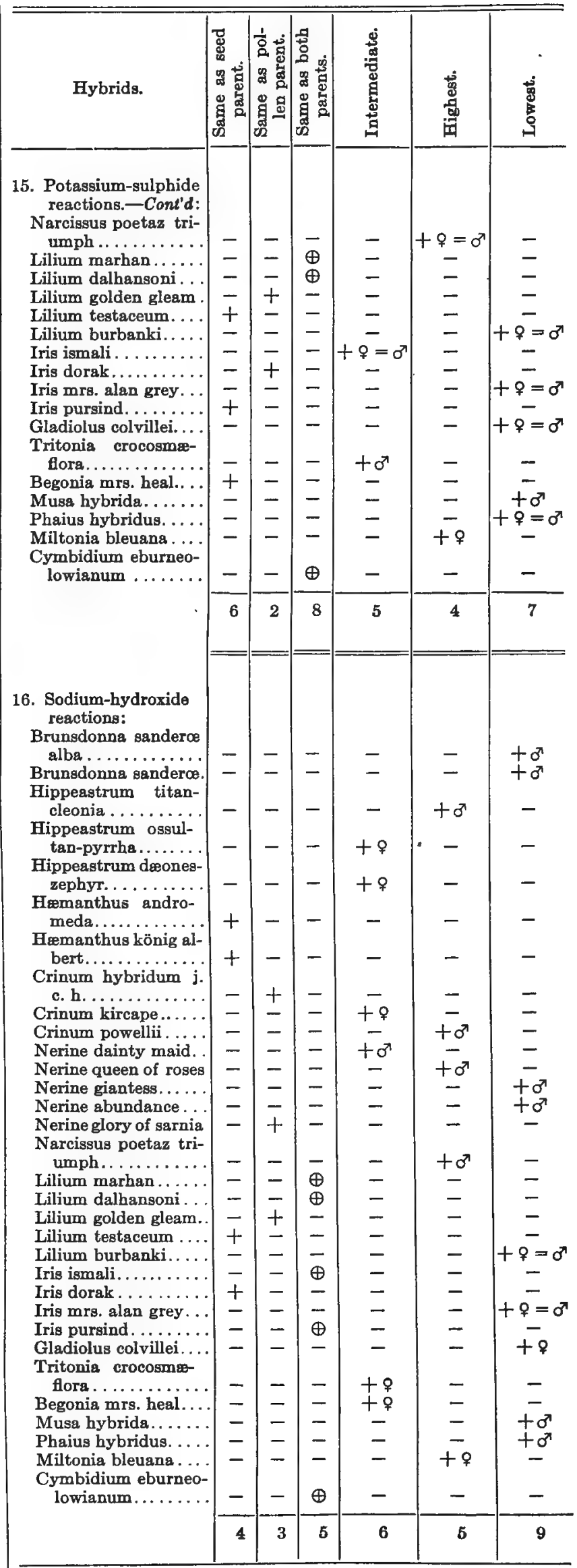


TABLE H.-Continued.

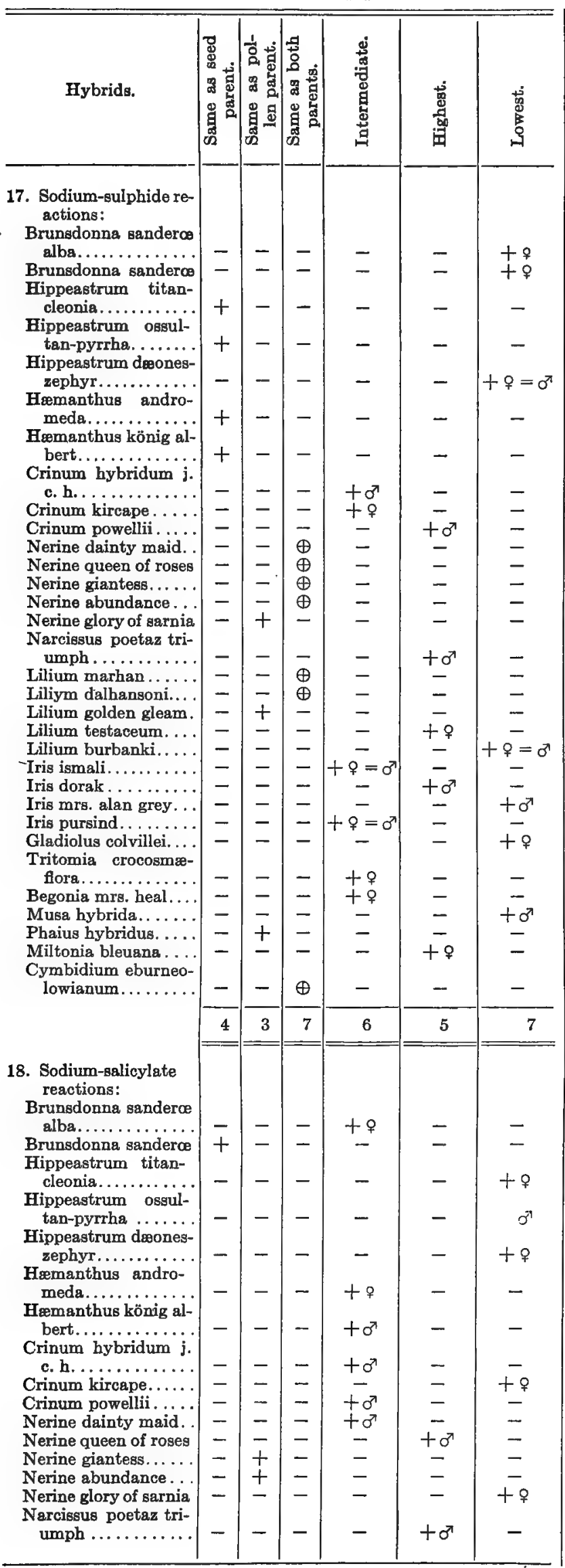

TABLE H.-Continued.

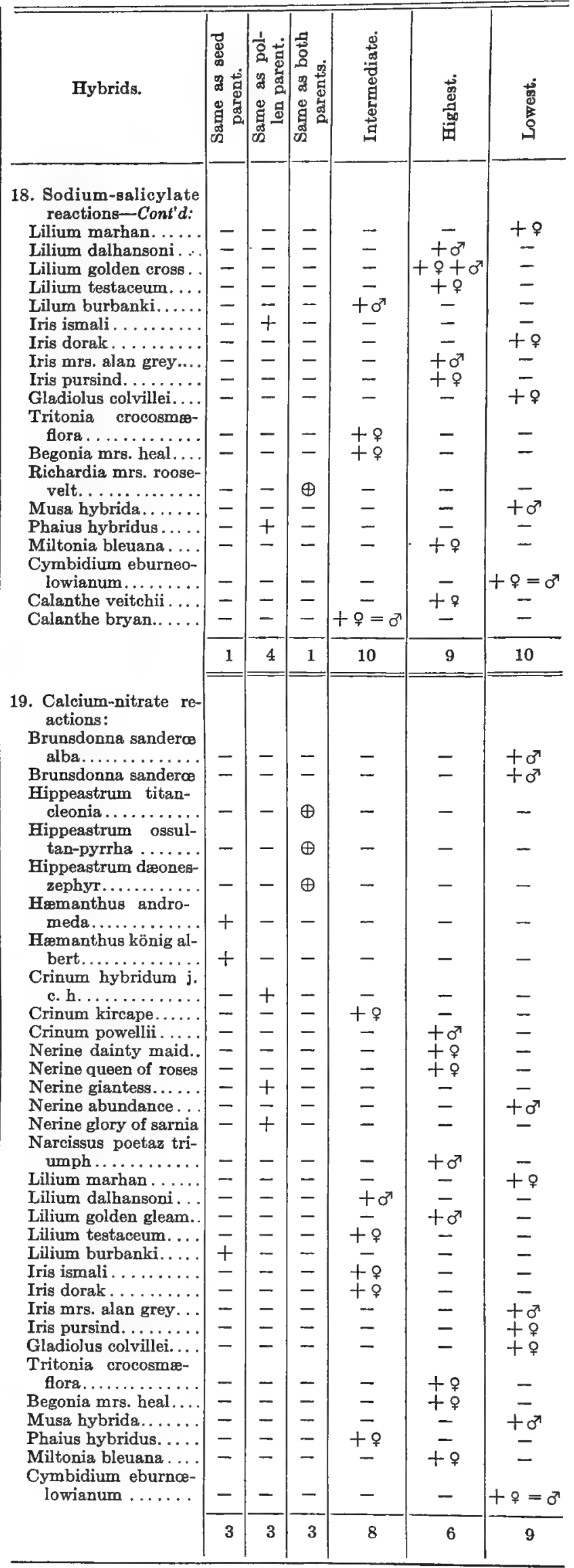


TaBle H.-Continued.

\begin{tabular}{|c|c|c|c|c|c|c|}
\hline Hybrids. & 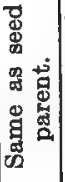 & 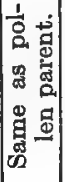 & 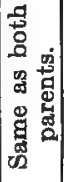 & 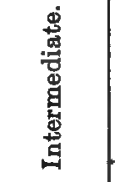 & 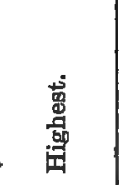 & 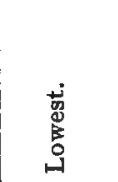 \\
\hline \multicolumn{7}{|l|}{$\begin{array}{l}\text { 20. Uranium-nitrate re- } \\
\text { actions: }\end{array}$} \\
\hline Brunsdonna sanderœe & & & & & & \\
\hline Brunsdonna sanderce. & $\overline{-}$ & $\overline{-}$ & - & $\overline{-}$ & $\overline{-}$ & $\begin{array}{l}+\sigma^{2} \\
+\sigma^{7}\end{array}$ \\
\hline Hippeastrum titan- & - & - & $\oplus$ & - & - & \\
\hline H. ossultan-pyrrha . & + & - & - & - & - & - \\
\hline H. dæones-zephyr ... & - & - & $\oplus$ & - & - & - \\
\hline $\begin{array}{l}\text { Hæmanthus andro- } \\
\text { meda............ }\end{array}$ & + & - & - & - & - & - \\
\hline H. könig albert .... & + & - & - & - & - & - \\
\hline Crinum hyb. j. c. h. & - & + & - & - & - & - \\
\hline Crinum kircape..... & - & - & - & +9 & - & - \\
\hline Crinum powellii.... & - & - & - & - & $+\sigma^{3}$ & - \\
\hline Nerine dainty maid. . & - & - & - & - & $+q$ & - \\
\hline Nerine queen of roses & - & - & - & - & +9 & - \\
\hline Nerine giantess...... & - & + & - & - & - & - \\
\hline Nerine abundance... & - & - & - & - & - & $+\sigma^{7}$ \\
\hline Nerine glory of sarnia & - & + & - & - & - & - \\
\hline Narcissus p. triumph & - & - & - & - & $+\sigma^{7}$ & - \\
\hline Lilium marhan. . ... . & - & - & - & $+\sigma^{x}$ & - & - \\
\hline Lilium dalhansoni... & - & - & - & $+q$ & - & - \\
\hline Lilium golden gleam & - & - & - & - & $+q$ & - \\
\hline Lilium testaceum. . . . & - & - & - & - & $+q$ & - \\
\hline Lilium burbanki..... & - & - & - & - & - & +9 \\
\hline Iris ismali. .......... & - & - & - & - & $+\sigma^{7}$ & - \\
\hline Iris dorak . . . . . . . & - & - & $\oplus$ & - & - & - \\
\hline Iris mrs, alan grey... & - & - & - & - & - & $+\%$ \\
\hline Iris pursind. ......... & - & - & - & $+\varphi=\sigma^{7}$ & - & - \\
\hline Gladiolus colvillei. ... & + & - & - & - & - & - \\
\hline Trit. crocosmæiflora... & - & - & - & $+\sigma^{7}$ & - & - \\
\hline Begonia mrs. heal... . & - & - & - & $+q$ & - & - \\
\hline Musa hybrida....... & - & - & - & - & - & $+\sigma^{\pi}$ \\
\hline Phaius hybridus, .... & - & - & - & +9 & - & - \\
\hline Miltonia bleuana... & - & - & - & - & $+q$ & - \\
\hline \multirow[t]{2}{*}{$\begin{array}{c}\text { Cymbidium eburneo- } \\
\text { lowianum......... }\end{array}$} & - & - & - & - & & \\
\hline & 4 & 3 & 3 & 7 & 8 & 7 \\
\hline \multirow{2}{*}{\multicolumn{7}{|c|}{$\begin{array}{l}\text { 21. Strontium-nitrate } \\
\text { reactions: }\end{array}$}} \\
\hline & & & & & & \\
\hline Brunsdonna sanderœe & & & & & & \\
\hline alba $\ldots \ldots \ldots \ldots$ & - & - & - & $+q=0^{7}$ & - & - \\
\hline Brunsdonna sanderce. & - & - & - & $+q$ & - & - \\
\hline Hippeastrum titan- & & & 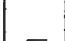 & & & \\
\hline & \pm & $\overline{-}$ & $\bar{\oplus}$ & - & $\overline{-}$ & $\bar{z}$ \\
\hline $\begin{array}{l}\text { H. ossultan-pyrrna .. } \\
\text { H. dæones-zephyr ... }\end{array}$ & - & - & $\underline{\oplus}$ & $+\%=0^{\lambda}$ & - & $\overline{-}$ \\
\hline Hæmanthus andro- & & & & & & \\
\hline meda............. & + & - & - & - & - & - \\
\hline H. könig albert. . . . . . & + & - & - & - & - & - \\
\hline Crinum hyb. j. c. h. & - & - & - & $+\infty^{7}$ & - & - \\
\hline Crinum kircape...... & - & - & - & $+\%$ & - & - \\
\hline Crinum powellii. .... & - & - & - & - & $+0^{x}$ & - \\
\hline Nerine dainty maid. . & - & - & - & - & $+\infty^{7}$ & - \\
\hline Nerine queen of roses & - & - & - & - & $+0^{7}$ & - \\
\hline Nerine giantess...... & - & - & - & $+\%=0^{7}$ & - & - \\
\hline Nerine abundance... & - & - & - & $+0^{x}$ & - & - \\
\hline Nerine glory of sarnia & - & - & - & - & - & $+\sigma^{7}$ \\
\hline Narcissus $p$. triumph & - & - & - & - & $+0^{x}$ & - \\
\hline Lilium marhan...... & - & - & - & + 우 & - & - \\
\hline Lilium dalhansoni ... & + & - & - & - & - & - \\
\hline Lilium golden glow. . & - & - & - & $+q$ & - & - \\
\hline Lilium testaceum. ... & - & - & - & - & $+q$ & - \\
\hline Lilium burbanki.... . & - & - & - & - & - & $+\%$ \\
\hline Iris ismali $\ldots \ldots \ldots \ldots$ & - & - & - & +9 & - & - \\
\hline Iris dorak. . . . . . . . & - & - & - & - & $+\%=\sigma^{\top}$ & - \\
\hline Iris mrs. alan grey. . . & - & - & - & - & - & $+q$ \\
\hline Iris pursind. . . . . . . . & - & - & - & $+\sigma^{7}$ & - & - \\
\hline Gladiolus colvillei... . & - & - & - & - & - & $+q$ \\
\hline Trit. crocosmæflora . & - & - & - & - & $+0^{x}$ & - \\
\hline Begonia mrs. heal.... & - & - & - & $+q$ & - & - \\
\hline
\end{tabular}

TABLE H.-Continued.

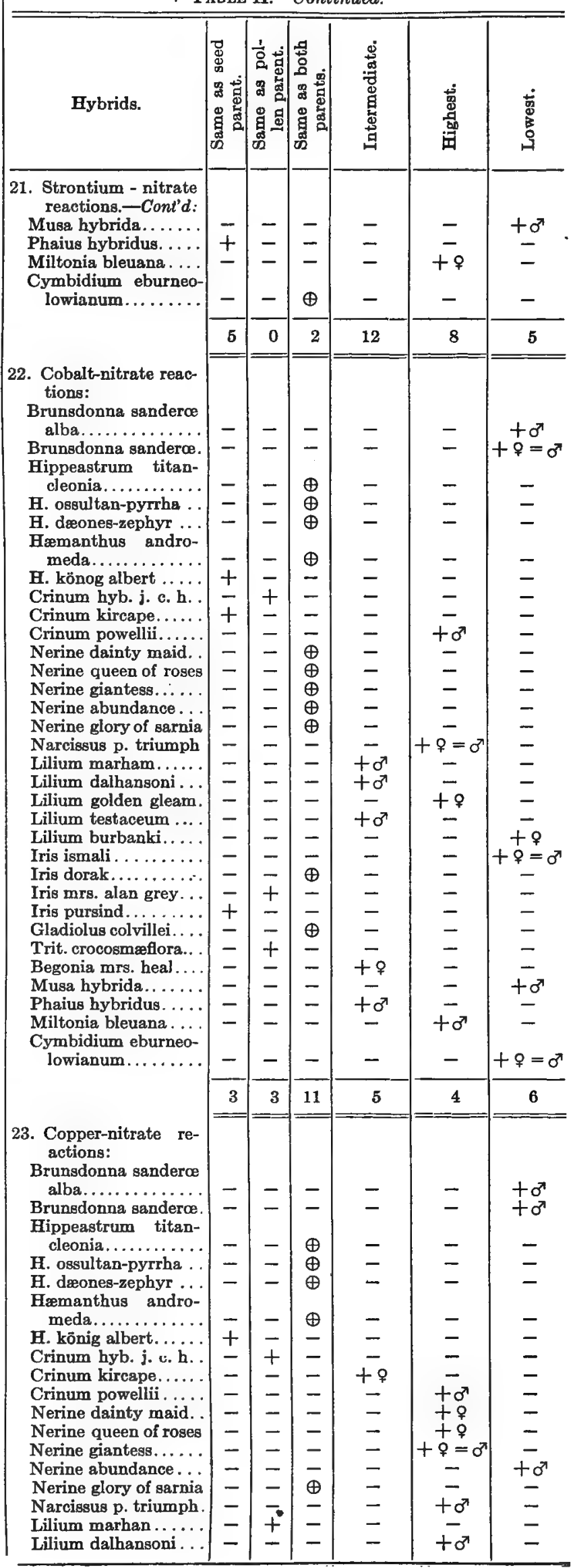


Table H.-Continued.

\begin{tabular}{|c|c|c|c|c|c|c|}
\hline Hybrids. & 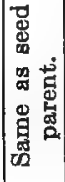 & 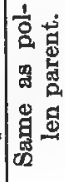 & 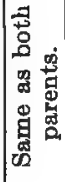 & 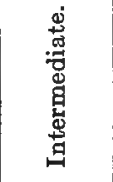 & $\begin{array}{l}\text { 曾 } \\
\text { 晋 }\end{array}$ & 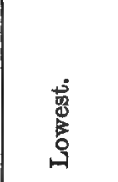 \\
\hline \multicolumn{7}{|l|}{$\begin{array}{l}\text { 23. Copper-nitrate re- } \\
\text { actions-Cont'd: }\end{array}$} \\
\hline Lilium golden gleam. & - & - & - & - & $+\%$ & - \\
\hline Lilium testaceum.... & - & - & $\oplus$ & - & - & - \\
\hline Lilium burbanki..... & - & - & - & - & - & $+\%=\sigma^{\top}$ \\
\hline Iris ismali $\ldots \ldots \ldots \ldots$ & - & - & - & $+q$ & - & - \\
\hline Iris dorak. .......... & - & - & - & - & +9 & - \\
\hline Iris mrs. alan grey. . & - & - & - & - & - & $+\infty^{7}$ \\
\hline Iris pursind........ & - & - & - & - & - & +9 \\
\hline Gladiolus colvillei.... & - & - & - & - & - & $+q$ \\
\hline Trit. crocosmæflora . . & - & - & - & $+q$ & - & - \\
\hline Begonia mrs. heal.... & - & - & - & $+q$ & - & - \\
\hline Musa hybrida....... & - & - & - & - & - & $+\sigma^{7}$ \\
\hline Phaius hybridus..... & - & - & $\oplus$ & - & - & - \\
\hline Miltonia bleuana.... & - & - & - & - & $+o$ & - \\
\hline \multirow[t]{2}{*}{$\begin{array}{l}\text { Cymbidium eburneo- } \\
\text { lowianum ......... }\end{array}$} & - & - & - & - & - & $+q=0^{x}$ \\
\hline & 1 & 2 & 7 & 4 & 9 & 9 \\
\hline \multicolumn{7}{|l|}{$\begin{array}{l}\text { 24. Cupric-chloride re- } \\
\text { actions: }\end{array}$} \\
\hline \multicolumn{7}{|l|}{ Brunsdonna sanderce } \\
\hline \multirow{2}{*}{\multicolumn{7}{|c|}{$\begin{array}{l}\text { Brunsdonna sanderœe } \\
\text { Hippeastrum titan- }\end{array}$}} \\
\hline & & & & & & \\
\hline H. ossultan-pyrrha . & - & - & $\oplus$ & - & - & - \\
\hline H. dæones-zephyr . . . & - & - & $\oplus$ & - & - & - \\
\hline \multicolumn{7}{|l|}{ 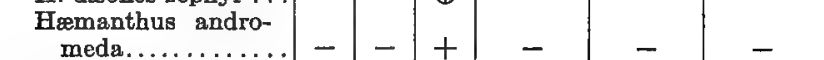 } \\
\hline H. könig albert . .... & + & - & - & - & - & - \\
\hline Crinum hyb. j. c. h. . & - & + & - & - & - & - \\
\hline Crinum kircape...... & - & - & - & + + & - & - \\
\hline Crinum powellii..... & - & - & - & - & $+o^{n}$ & - \\
\hline Nerine dainty maid. . & - & - & $\oplus$ & - & - & - \\
\hline Nerine queen of roses & - & - & $\oplus$ & - & - & - \\
\hline Nerine giantess...... & - & - & $\oplus$ & - & - & - \\
\hline Nerine abundance... & - & - & $\oplus$ & - & - & - \\
\hline Nerine glory of sarnia & - & - & $\oplus$ & - & - & - \\
\hline Narcissus p. triumph & - & - & - & - & $+q=\sigma^{x}$ & - \\
\hline Lilium marhan...... & - & + & - & - & - & - \\
\hline Lilium dalhansoni.... & - & - & - & $+q=0^{\pi}$ & - & - \\
\hline Lilium golden gleam. & - & - & - & - & $+\sigma^{x}$ & - \\
\hline Lilium testaceum.... & - & + & - & - & - & - \\
\hline Lilium burbanki..... & - & - & - & - & - & $+q$ \\
\hline Iris ismali........... & - & - & - & - & - & $+q$ \\
\hline Iris dorak . . . . . . . & - & - & - & - & $+q$ & - \\
\hline Iris mrs. alan grey... & - & - & - & - & - & $+\sigma^{7}$ \\
\hline Iris pursind. ........ & - & - & - & - & $+9=0^{7}$ & - \\
\hline Gladiolus colvillei... & + & - & - & - & - & - \\
\hline Trit. crocosmæflora .. & - & - & - & $+q$ & - & - \\
\hline Begonia mrs. heal... . & - & - & - & +9 & - & - \\
\hline Musa hybrida....... & - & - & - & . - & - & $+\sigma^{x}$ \\
\hline Phaius hybridus. .... & - & - & - & $+q=\sigma^{7}$ & - & - \\
\hline Miltonia bleuana .... & - & - & - & - & $+\%$ & - \\
\hline \multirow[t]{2}{*}{$\begin{array}{l}\text { Cymbidium eburneo- } \\
\text { lowianum......... }\end{array}$} & - & - & - & - & - & $+q=\sigma^{7}$ \\
\hline & 2 & 3 & 9 & 5 & 6 & 7 \\
\hline \multicolumn{7}{|l|}{$\begin{array}{l}\text { 25. Barium-chloride re- } \\
\text { actions: }\end{array}$} \\
\hline $\begin{array}{c}\text { Brunsdonna sanderce } \\
\text { alba } \ldots \ldots \ldots \ldots \ldots\end{array}$ & + & - & - & - & - & - \\
\hline Brunsdonna sanderœ. & + & - & - & - & - & - \\
\hline $\begin{array}{l}\text { Hippeastrum titan- } \\
\text { cleonia............ }\end{array}$ & - & - & $\oplus$ & - & - & - \\
\hline H. ossul tan-pyrrha... & - & - & $\oplus$ & - & - & - \\
\hline H. dæoneঞ-zephyr . . . & - & - & $\oplus$ & - & - & - \\
\hline $\begin{array}{l}\text { Hæmanthus andro- } \\
\text { meda.................. }\end{array}$ & - & - & $\oplus$ & - & - & - \\
\hline
\end{tabular}

Table H.-Continued.

\begin{tabular}{|c|c|c|c|c|c|c|}
\hline Hybrids. & 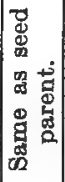 & 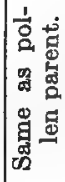 & 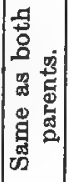 & 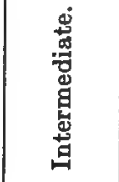 & $\begin{array}{l}\text { 总 } \\
\text { 总 } \\
\text { 夏 }\end{array}$ & 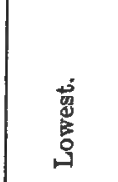 \\
\hline 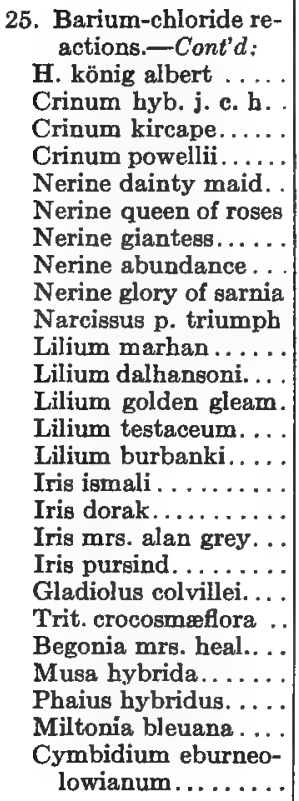 & $\begin{array}{l} \pm \\
\pm \\
- \\
- \\
- \\
- \\
- \\
- \\
- \\
- \\
- \\
- \\
\overline{+} \\
- \\
\overline{+} \\
\pm \\
- \\
- \\
-\end{array}$ & 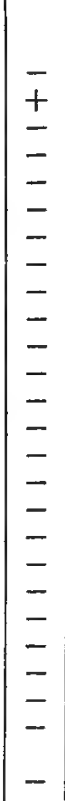 & $\begin{array}{l}\overline{-} \\
- \\
\bar{\oplus} \\
\oplus \\
\oplus \\
\oplus \\
\oplus \\
\oplus \\
- \\
- \\
- \\
- \\
- \\
\bar{\oplus} \\
- \\
\bar{\oplus} \\
- \\
- \\
-\end{array}$ & $\begin{array}{l}= \\
= \\
= \\
= \\
= \\
= \\
+0 \\
+0 \\
+0 \\
+0 \\
= \\
= \\
= \\
= \\
+9 \\
+0 \\
-\end{array}$ & $\begin{array}{c}- \\
- \\
+8=0 \\
= \\
- \\
= \\
= \\
= \\
= \\
+9 \\
= \\
= \\
= \\
= \\
= \\
= \\
= \\
+\infty \\
-\end{array}$ & $\begin{array}{c}- \\
- \\
= \\
- \\
= \\
= \\
= \\
= \\
= \\
= \\
= \\
+9 \\
= \\
+9 \\
- \\
= \\
+0^{7} \\
- \\
+0=0\end{array}$ \\
\hline & 6 & 1 & 12 & 6 & 3 & 4 \\
\hline 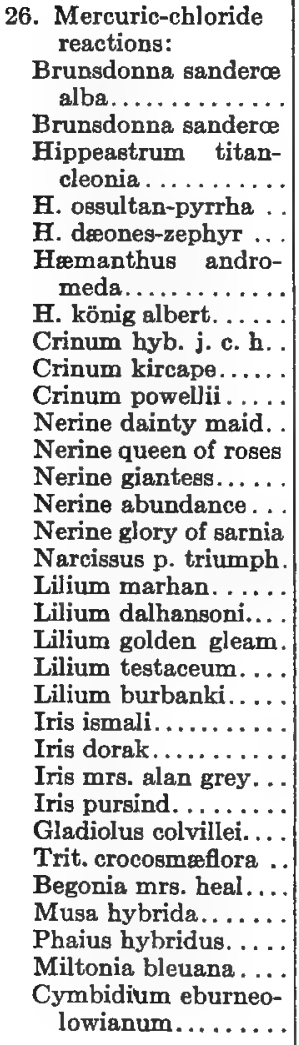 & 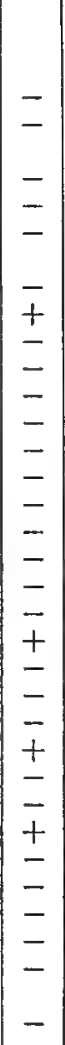 & $\begin{array}{l}- \\
- \\
- \\
- \\
- \\
- \\
= \\
- \\
- \\
- \\
- \\
- \\
- \\
- \\
- \\
- \\
- \\
- \\
- \\
- \\
- \\
- \\
-\end{array}$ & $\begin{array}{l}\oplus \\
- \\
- \\
\overline{-} \\
\oplus \\
\oplus \\
\oplus \\
\oplus \\
\oplus \\
- \\
- \\
- \\
- \\
- \\
- \\
- \\
- \\
- \\
- \\
- \\
- \\
-\end{array}$ & 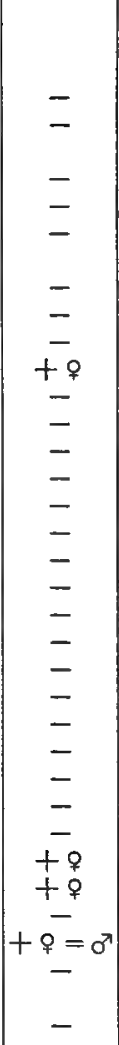 & $\begin{array}{c}- \\
- \\
- \\
- \\
- \\
- \\
- \\
+0 \\
- \\
= \\
- \\
+ \\
+0 \\
+0 \\
= \\
= \\
= \\
- \\
= \\
+9 \\
- \\
- \\
- \\
+9 \\
+ \\
-\end{array}$ & 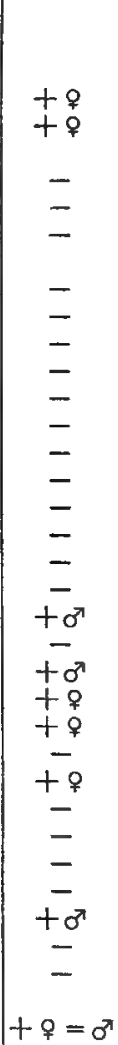 \\
\hline & 4 & 1 & 9 & 4 & 5 & 9 \\
\hline
\end{tabular}


1. Summary of Table H.-Toials of Reaction-intensities of Starches of Hybrids with each Agent and Reagent as regards Sameness, Intermediateness, Excess, and Deficit of Development in relation to the Parents.

\begin{tabular}{|c|c|c|c|c|c|c|c|}
\hline Agents and reagents. & $\begin{array}{c}\text { Same as } \\
\text { seed } \\
\text { parent. }\end{array}$ & $\begin{array}{c}\text { Same as } \\
\text { pollen } \\
\text { parent. }\end{array}$ & $\begin{array}{c}\text { Same as } \\
\text { both } \\
\text { parents. }\end{array}$ & $\begin{array}{l}\text { Inter- } \\
\text { mediate. }\end{array}$ & Highest. & Lowest. & $\begin{array}{l}\text { No. of } \\
\text { starches. }\end{array}$ \\
\hline 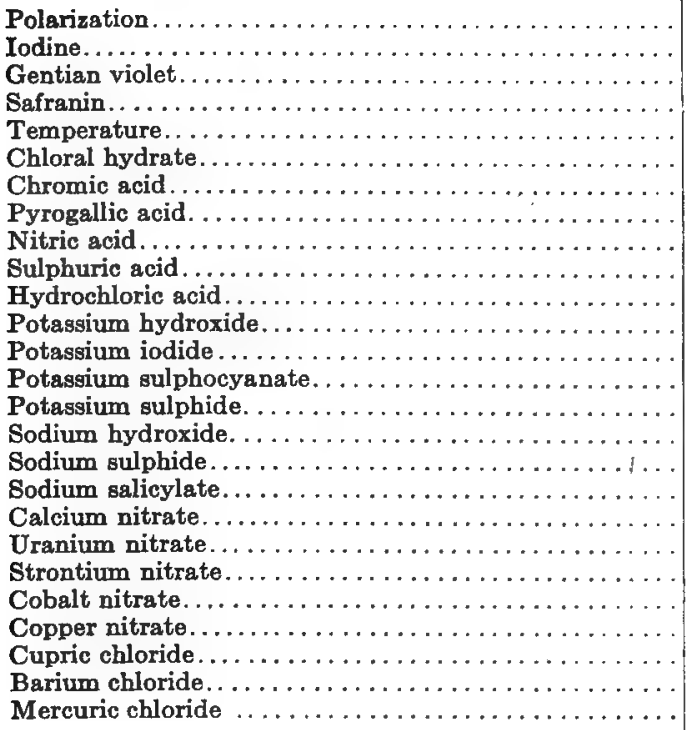 & $\begin{array}{r}11 \\
16 \\
13 \\
13 \\
7 \\
1 \\
4 \\
3 \\
4 \\
10 \\
1 \\
2 \\
4 \\
6 \\
5 \\
4 \\
4 \\
1 \\
3 \\
4 \\
5 \\
3 \\
1 \\
2 \\
6 \\
4\end{array}$ & $\begin{array}{r}11 \\
12 \\
9 \\
11 \\
3 \\
6 \\
3 \\
2 \\
1 \\
3 \\
1 \\
1 \\
2 \\
1 \\
2 \\
3 \\
3 \\
4 \\
4 \\
3 \\
0 \\
3 \\
2 \\
3 \\
1 \\
1\end{array}$ & $\begin{array}{r}0 \\
1 \\
0 \\
2 \\
0 \\
1 \\
2 \\
7 \\
4 \\
12 \\
7 \\
15 \\
6 \\
6 \\
8 \\
5 \\
7 \\
1 \\
3 \\
3 \\
2 \\
11 \\
7 \\
9 \\
12 \\
9\end{array}$ & $\begin{array}{r}9 \\
12 \\
8 \\
4 \\
21 \\
14 \\
18 \\
17 \\
15 \\
9 \\
10 \\
6 \\
8 \\
5 \\
5 \\
6 \\
6 \\
10 \\
8 \\
7 \\
12 \\
5 \\
4 \\
5 \\
6 \\
4\end{array}$ & $\begin{array}{r}9 \\
5 \\
10 \\
10 \\
10 \\
14 \\
10 \\
12 \\
14 \\
9 \\
6 \\
6 \\
5 \\
6 \\
4 \\
5 \\
5 \\
9 \\
6 \\
8 \\
8 \\
4 \\
9 \\
6 \\
3 \\
5\end{array}$ & $\begin{array}{r}10 \\
4 \\
10 \\
10 \\
9 \\
14 \\
14 \\
9 \\
12 \\
4 \\
10 \\
5 \\
7 \\
9 \\
7 \\
9 \\
7 \\
10 \\
9 \\
7 \\
5 \\
6 \\
9 \\
7 \\
4 \\
9\end{array}$ & $\begin{array}{l}\mathbf{5 0} \\
\mathbf{5 0} \\
\mathbf{5 0} \\
\mathbf{5 0} \\
\mathbf{5 0} \\
\mathbf{5 0} \\
\mathbf{5 0} \\
\mathbf{5 0} \\
\mathbf{5 0} \\
\mathbf{4 7} \\
\mathbf{3 5} \\
\mathbf{3 5} \\
\mathbf{3 2} \\
\mathbf{3 2} \\
\mathbf{3 2} \\
\mathbf{3 2} \\
\mathbf{3 2} \\
\mathbf{3 5} \\
\mathbf{3 2} \\
\mathbf{3 2} \\
\mathbf{3 2} \\
\mathbf{3 2} \\
\mathbf{3 2} \\
\mathbf{3 2} \\
\mathbf{3 2} \\
\mathbf{3 2}\end{array}$ \\
\hline
\end{tabular}

2. SUMmary of TABLE H.-Sameness and Inclination of the Reaction-intensities of all of Hybrid Starches with each Agent and Reagent.

\begin{tabular}{|c|c|c|c|c|c|}
\hline \multirow{2}{*}{ Agents and reagents. } & \multicolumn{2}{|c|}{ Same as or closer to $\longrightarrow$} & \multirow{2}{*}{$\begin{array}{c}\text { Same as both } \\
\text { parents. }\end{array}$} & \multirow{2}{*}{$\begin{array}{l}\text { As close to } \\
\text { one as to the } \\
\text { other parent. }\end{array}$} & \multirow{2}{*}{$\begin{array}{c}\text { Number of } \\
\text { starches. }\end{array}$} \\
\hline & Seed parent. & Pollen parent. & & & \\
\hline \multirow[t]{2}{*}{ 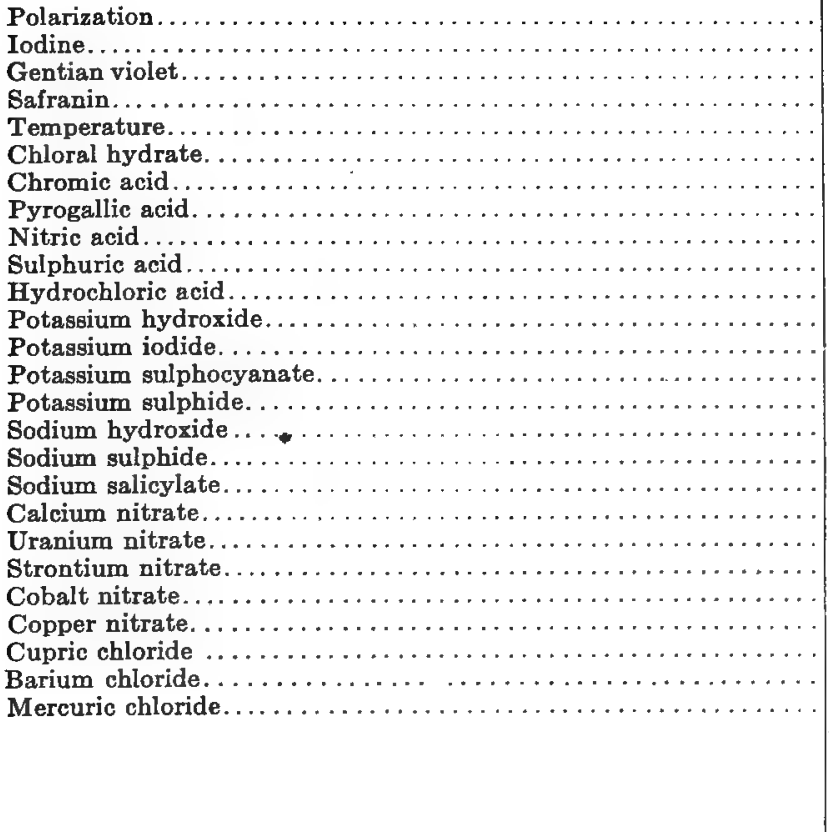 } & $\begin{array}{r}26 \\
25 \\
21 \\
24 \\
29 \\
23 \\
31 \\
23 \\
24 \\
18 \\
11 \\
8 \\
13 \\
13 \\
7 \\
11 \\
12 \\
16 \\
16 \\
15 \\
15 \\
6 \\
12 \\
9 \\
13 \\
14\end{array}$ & $\begin{array}{r}20 \\
18 \\
25 \\
21 \\
18 \\
20 \\
12 \\
15 \\
11 \\
11 \\
12 \\
8 \\
8 \\
9 \\
10 \\
14 \\
9 \\
15 \\
12 \\
10 \\
10 \\
11 \\
10 \\
9 \\
4 \\
6\end{array}$ & $\begin{array}{r}0 \\
1 \\
0 \\
2 \\
0 \\
1 \\
2 \\
7 \\
4 \\
12 \\
7 \\
15 \\
6 \\
6 \\
8 \\
5 \\
7 \\
1 \\
3 \\
3 \\
2 \\
11 \\
7 \\
9 \\
12 \\
9\end{array}$ & $\begin{array}{r}4 \\
6 \\
4 \\
3 \\
3 \\
6 \\
5 \\
5 \\
11 \\
6 \\
5 \\
4 \\
5 \\
4 \\
7 \\
2 \\
4 \\
3 \\
1 \\
2 \\
5 \\
4 \\
3 \\
5 \\
3 \\
3\end{array}$ & $\begin{array}{l}\mathbf{5 0} \\
\mathbf{5 0} \\
\mathbf{5 0} \\
\mathbf{5 0} \\
\mathbf{5 0} \\
\mathbf{5 0} \\
\mathbf{5 0} \\
\mathbf{5 0} \\
\mathbf{5 0} \\
\mathbf{4 7} \\
\mathbf{3 5} \\
\mathbf{3 5} \\
\mathbf{3 2} \\
\mathbf{3 2} \\
\mathbf{3 2} \\
\mathbf{3 2} \\
\mathbf{3 2} \\
\mathbf{3 5} \\
\mathbf{3 2} \\
\mathbf{3 2} \\
\mathbf{3 2} \\
\mathbf{3 2} \\
\mathbf{3 2} \\
\mathbf{3 2} \\
\mathbf{3 2} \\
\mathbf{3 2}\end{array}$ \\
\hline & 437 & 328 & $\underbrace{140}$ & 113 & 1018 \\
\hline
\end{tabular}




\section{THE PLANT TISSUES.}

Macroscopic and Microscopid Characters of Hybrid-stocks In CoMparison with the ReaCtion-Intensities of Starches of HybridStOCKS AS Regards Sameness, Intermediateness, Excess, and Deficit of Development in relation to the Parent-stocks.

(Table I, Parts 1 to 8, and Summaries 1 to 7. Charts F, 1 to 14.)

Inasmuch as the macroscopic and microscopic characters of plants are, like the microscopic characters and reactions of starches, expressions of physico-chemical processes, it follows, as a corollary, if starches exhibit well-defined peculiarities in their parental relationships, such as have been shown very clearly in preceding pages that corresponding characteristics should be manifested by the plant tissues. This is not only what has been found, but also a remarkable congruity of the data considering the exceptional diversity of the methods of investigation in the two entirely distinct although cooperative lines of investigation. In the studies of the starches the records show that each form of starch exhibits in its histologic, polariscopic, and chemical properties varying relationships to the parents, some of these properties (varying in kind and number in different hybrids) being the same or practically the same as the property of the seed parent, or of the pollen parent, or of both parents; others being intermediate between the corresponding properties of the parents; and others showing development in excess or deficit of parental extremes. As exceptionally striking facts it was also observed that the distribution of the data of parental relationship under the six parent-phase divisions varied with the different hybrid starches so markedly and characteristically that each table of the characters of each starch is diagnostic of the starch; that the proportions of intermediate and non-intermediate characters vary within wide limits in different starches; that the development of characters in excess or deficit of parental extremes is more conspicuous than intermediateness or sameness to either parent or both parents; and that the comparative degree of influence of the seed and pollen parents varied within extremes characterized by an almost universal dominance of one or the other parent. Tables $F, G$, and $H$ give recapitulations and summaries of the reaction-intensities of the starches of hybrids which are not only exceptionally well adapted for comparisons of certain fundamental data of the peculiarities of starches, but also for bases of comparison of starch and tissue characteristics.

In Table I the macroscopic and microscopic data of hybrid-stocks are formulated in correspondence with the reaction-intensity data of the starches in Tables $\mathrm{F}$ and $\mathrm{H}$. Comparing in a general way the two sets of tables one gets at first glance the impression of concordance, and of so definite a character that it seems obvious that if the two sets of tables were intermingled, the botanical names having been removed, it would be impossible to distribute them to their proper plant and starch groups. The tissue tables differ from each other as do the starch tables, and each is as individualized and diagnostic of the plant as is each starch table. In comparing the data of Table 1 and its summaries the most con- spicuous features are: The general or gross agreement between the figures of the corresponding columns; the small number of characters and reactions that are the same as one or the other or both parents in comparison with the number that are intermediate, highest, and lowest; the distinctly smaller number that are intermediate in comparison with the combined numbers that are highest and lowest; the comparatively small number that are intermediate (in view of intermediateness being a criterion of hybrids); and the many or less marked dissimilarities in the distribution of the macroscopic and microscopic data among the six parent-phases. In making these comparisons it is preferable to take percentages, inasmuch as the numbers of characters and reactions are not the same.

Referring to the first summary, it will be found that of the 959 tissue characters 17.8 per cent are the same as one or the other parent or both parents, and that 82.2 per cent are intermediate, highest, and lowest; while with the reactions of the starches (Table F) the figures are 36.2 and 63.8 per cent, respectively, the ratio of the former being $1: 4.7$ and of the latter $1: 1.8$. Comparing the figures of the corresponding columns of the two tables, the following percentages will be noted, the first figure being for the tissues and the second for the starches: Same as seed parent 5.8 and 13.4; same as pollen parent 6.8 and 9.2 ; same as both parents 5.2 and 13.6 ; intermediate 43.2 and 23.2 ; highest 24.9 and 18.4; and lowest 14.1 and 22.2. Intermediate characters in the tissue represent 43.2 , and highest and lowest characters 39 , compared with 23.2 and 40.6 in the reactions, showing in both cases that the percentages of characters and reactions developed in excess or deficit of parental extremes are very large, and in the reactions very much larger than the intermediate percentages. It therefore would seem to follow, as a corollary, that if intermediateness is of given value as a criterion of hybrids, development in excess and deficit of parental extremes is a criterion of greater value.

One of the most unexpected features exhibited by these data is the presence or absence of close correspondence in the form of distribution of the macroscopic and microscopic characters among the six parent-phases. One would naturally be led to the assumption that if, for instance, a given percentage of macroscopic characters were the same as those of the seed parent a similar or very closely similar percentage of microscopic characters would fall under the same heading; but, strange enough, there may be a range of relationship between almost or practical identity and very marked divergence, and even inversion, of the percentages of the two groups of characters. Thus, in Ipomoea sloteri (Chart F 1, Table I, Part 1 and Summary 1) there is in general closeness of the two curves, the only marked variation being in the intermediate characters. The percentages of characters that are the same as those of the pollen parent and both parents, and that are developed in deficit of parental extremes, are in each case very close. The percentages of macroscopic characters under each of these parentphases is lower than the corresponding percentages of microscopic characters except in intermediate characters. In the latter the percentages are not only markedly different (macroscopic 47.4 and microscopic 32.6), but there is also an inversion of the percentages, and there- 
fore of the relative positions of the curves. The percentage of microscopic characters developed in excess of parental extremes is precisely the same as the percentage of macroscopic intermediate characters; and the combined percentages of macroscopic and microscopic characters developed in excess and deficit of parental extremes is much larger than the combined percentages of macroscopic and microscopic intermediate characters, the proportions being 51.9 to 36.9 . It is remarkable and inexplicable that the percentage of macroscopic characters should exceed the percentage of microscopic characters among intermediate groups and be the reverse in all of the other five parent-phase groups.

In Loelia-Cattleya canhamiana (Chart F 2, Summary 1 of Table I, Part 2 and Summary 1) there is similar gross correspondence and lack of correspondence in percentages and in curves, but the curves so differ from those of Ipomoca sloteri as to be readily distinguishable. In this hybrid the differences between the macroscopic and microscopic data are, as a whole, distinctly more marked; the percentages' of macroscopic characters are less than those of the microscopic characters in 5 of the 6 parent-phases, the most marked difference being noted among the characters that are developed in deficit of parental extremes, while the percentages of both macroscopic and microscopic characters that are intermediate are notably in excess of the percentages of characters falling under the other 5 parent-phases. Among the intermediate characters, 52.9 per cent are macroscopic and 35.3 per cent microscopic. Taking the characters as a whole, 40.3 per cent are intermediate and 34.4 per cent are developed in excess or deficit of parental extremes.

In Cymbidium eburneo-lowianum (Chart F 3, Table I, Part 3 and Summary 1) the percentages of characters differ, on the whole, only slightly more than in either Ipomoea sloteri or Lotia-Cattleya canhamiana. The percentages of macroscopic characters are higher than those of the microscopic characters in 3 and lower in 3 of the six parent-phases, and the most marked differences are found among the characters that are intermediate and that are developed in excess and deficit of parental extremes. The percentage of macroscopic intermediate characters is very much higher than the percentage of microscopic characters (62.9 and 36 , respectively); the combined percentages of both macroscopic and microscopic intermediate characters is close to one-half ( 44.6 per cent) of the total of all of the characters, and nearly double the combined percentages (25.4 per cent) of characters that are developed in excess and deficit of parental extremes. It is extraordinary that while the ratio of macroscopic characters that are intermediate to those which are developed in excess and deficit of parental extremes is $62.9: 5.7$, the ratio of microscopic characters is $36: 34 . \%$.

In Dendrobium cybele (Chart F 4, Table I, Part 4 and Summary 1) the percentages of characters differ in degree, with one exception, from distinct to well marked, the greatest divergence being noted among the characters that fall under those which are the same as those of the pollen parent, the same as those of both parents, and which are developed in deficit of parental extremes, especially the latter. In 3 of the 6 parentphases the macroscopic characters show higher percent- ages than the microscopic characters, in 2 lower percentages, and in 1 practically the same percentages. The percentages of microscopic characters that are intermediate represent much more than one-third ( 43.3 per cent) of the total characters and distinctly more than the combined percentages (29.9 per cent) of characters that are developed in excess and deficit of parental extremes. The intermediate microscopic characters represent a percentage (37 per cent) somewhat lower than the macroscopic characters and distinctly lower than the combined percentages of characters developed in excess and deficit of parental extremes (52.5 per cent). This inversed relationship of the percentages that are intermediate and developed in excess and deficit in comparison with the macroscopic characters is extremely interesting. The total percentage of intermediate characters is 37 in comparison with 46.6 per cent of characters developed in excess or deficit of parental extremes.

In Miltonia bleuana (Chart F 5, Table I, Part 5 and Summary 1) there is a marked tendency to variation in the distribution of percentages of macroscopic and microscopic characters among the 6 parent-phases, the percentages being close in $\mathbf{3}$ and well apart in 3 . The most marked differences noted are in the percentages that fall under characters that are the same as the seed parent, the same as the pollen parent, and which developed in deficit of parental extremes. The differences are not only well marked, but much accentuated because of the relatively small differences found under the other parent-phases. The macroscopic character percentages are higher than the microscopic percentages in 2 of the 4 parent-phases. The macroscopic characters that are intermediate represent 31 per cent of the total characters, distinctly higher than the combined percentages of characters developed in excess and deficit of parental extremes (17.2 per cent). The microscopic characters that are intermediate show a somewhat higher percentage than the macroscopic characters, but distinctly lower than the combined percentages of characters developed in excess and deficit of parental extremes, the ratio being $36.4: 45.9$, a reversal of values in comparison with the macroscopic characters. The total percentage of intermediate characters is 35.1 compared with the combined percentages ( 38.7 per cent) of characters developed in excess and deficit of parental extremes.

The two Cypripedium hybrids $C$. lathianum and $C$. lathianum inversum are offspring of reversed crosses. In Cypripedium lathamianum (Chart F 6, Table I, Part 6 and Summary 1) the records are remarkable on account chiefly of the comparatively high percentages of characters that are intermediate and that are developed in excess of parental extremes, and the correspondingly low percentages that fall under all of the other parentphases; the very marked differences between the percentages of macroscopic and microscopic characters that are intermediate, and that are developed in excess of parental extremes; and the inversion of the macroscopic and microscopic values in these two phases. The macroscopic percentages are lower than the microscopic percentages among the characters that are the same as those of the pollen parent, developed in excess of parental extremes, and developed in deficit of parental extremes; and lower in the other three phases. Among characters 
that are the same as one or the other parent or both parents the differences are small. Among the macroscopic characters, 85.3 per cent are intermediate, and there is a very small combined percentage of characters developed in excess and deficit of parental extremes (5.9 per cent). Among the microscopic characters 49.4 per cent are intermediate and 42.5 per cent are developed beyond parental extremes. Summing up the percentages of characters that are intermediate and that are developed beyond parental extremes, respectively, it is seen that of the total characters 60 per cent are intermediate and 32.4 per cent developed beyond parental extremes.

In the companion hybrid, Cypripedium lathamianum inversum (Chart F 7, Table I, 1), the macroscopic and microscopic characters are found to be closely in accord in their percentages with those of the C. lathamianum, the most noticeable differences being in the percentages that fall under the characters that are the same as the pollen parent and those that are intermediate. In this hybrid the percentage of macroscopic characters that are the same as those of the pollen parent is larger than the percentage of microscopic characters; but in the other hybrid the reverse. The percentages of both macroscopic and microscopic intermediate characters are less, especially as regards the former. In this hybrid 73.5 per cent and in the other 85.3 per cent of the macroscopic characters are intermediate, while the figures for the microscopic characters are 46.6 and 49.4 , respectively. Summing up the characters that are intermediate and those that are developed beyond parental extremes, respectively, it is seen that of the total characters 54.1 per cent are intermediate and 36.5 per cent developed beyond parental extremes. This gives in this hybrid in comparison with the other a lower percentage of characters that are intermediate and a larger percentage that are developed in excess and deficit of parental extremes. The corresponding percentages and hence the corresponding curves of these hybrids are so closely alike that one should at a glance suspect that the plants are very closely related. In fact, the similarities and dissimilarities noted are generally in accord with what should naturally be expected from the data of hybrids.

The remarkable degree of concordance of the data of these two hybrids is a matter of pre-eminent importance because of the data of one being in the nature of a check-off or test experiment in relation to the other. It is obvious if the data do not agree within limits that have been found by the systematist in his descriptions of the naked-eye characters of plants, that they would be regarded as being undependable, and that if, on the other hand, they do agree that the differences in the corresponding percentages in the macroscopic and microscopic characters are not fallacious. It scarcely seems within the realm of possibility, if the data were not reliable within reasonable or small limits of error of observation, that the two sets of curves would be so nearly alike and differ only to about the degree that should be expected in the case of offspring of reciprocal crosses. There is also, as will be seen, a distinct likeness of the courses of the curves of the chart of Cypripedium nitens to those of the preceding Cypripedium charts, and the differences between the former and the latter are defi- nitely more marked, thus indicating that the parentage in the two cases is not identical. The likeness can be accounted for in part by the fact that one of the parents of $C$. nitens (C. villosum) is also a parent of each of the other hybrids-the pollen parent in the first and the seed parent in the second. The charts of $C$. nitens and $C$. lathamianum inversum are more alike than those of $C$. nitens and C. lathamianum; in both of the former the seed parent is the same; and, as will be pointed out later in sufficient detail, C. villosum is more potent in influencing the characters of the hybrids than is either of the other parents, which in a measure will account for similarities of all three charts.

In Cypripedium nitens (Chart F 8, Table I, 1) the percentages of both macroscopic and microscopic characters that are the same as those of the seed parent and that are developed in excess of parental extremes are distinctly larger, and there are notable lowerings of percentages of both macroscopic and microscopic intermediate characters. There is a more marked difference between the percentages of macroscopic characters that are the same as those of both parents, with, moreover, an inversion of the macroscopic and microscopic values in this phase; and the macroscopic and microscopic percentages of characters that are developed in excess of parental extremes are practically the same, whereas in the other two hybrids they are very different. The macroscopic percentages are higher than the microscopic percentages among the characters that are the same as those of the seed parent and that are intermediate, but lower in the other four sex-phases. Of the total number of macroscopic characters 50 per cent are intermediate and 34.4 per cent are developed in excess and deficit of parental extremes; and of the microscopic characters 35 per cent are intermediate and 47 per cent are developed in excess or deficit of parental extremes. Summing both macroscopic and microscopic characters, 39 per cent are intermediate and 42.4 per cent are developed beyond parental extremes. The corresponding figures for $C$. lathamianum are 60 and 32.4, and for C. lathamianum inversum 54.1 and 36.5 , showing in $C$. nitens an inversion of these sex-phase values compared with the values of the other two hybrids.

By comparing Charts $\mathrm{F} 1$ to $\mathrm{F} 8$ it will be seen that while there are throughout certain well-defined resemblances, no two are so similar, even in the case of the two Cypripedium hybrids that have come from reciprocal crosses, as to lead to one being mistaken for another. A common plan of distribution of percentages of characters among the six parent-phases is evident in all of the charts and is only exceptionally departed from-that is, in general, comparatively low percentages of characters that are the same as one or the other parent or both parents, generally higher percentages of characters that are developed in excess or deficit of parental extremes, and still higher percentages of characters that are intermediate. Departures of modifications of this plan are seen particularly in Ipomoea sloteri, in the higher percentage of characters developed in excess of parental extremes than of intermediate characters; and in Miltonia bleuana in the high percentage of macroscopic characters that are the same as those of the seed and pollen parent. Perhaps there is nothing 
so remarkable among these records as the marked tendencies in the several sets of parents and hybrids to inverted relations of macroscopic and microscopic values; and the tendency for macroscopic values to be higher than the microscopic values in the intermediate characters, and for the reverse in the characters that are developed in excess and deficit of parental extremes.

Recapitulating the sums of both macroscopic and microscopic characters that fall under the six sex-phases (Table I, Summary 1) it is found that of the 959 characters 5.8 per cent are the same as those of the seed parent, 6.8 the same as those of the pollen parent, 5.2 the same as those of both parents, 43.2 intermediate, 24.9 developed in excess of parental extremes, and 14.1 in deficit of parental extremes. It will also be seen that 17.8 per cent are the same as those of one or the other parent or both parents; that 82.2 per cent are intermediate and developed beyond parental extremes; and that 43.2 per cent are intermediate against 39 per cent that are developed beyond parental extremes.

Further studies of the separate percentages of macroscopic and microscopic characters show, as presented in the second summary of Table $I$, in the former as compared with the latter, lower percentages in the characters that are the same as one or the other parent or both parents and that are intermediate, but higher percentages in the characters that are developed beyond parental extremes, especially in those which are developed in deficit of parental extremes. The figures in relation to sameness to one or the other parent or both parents run closely, but in the other three parent-phases they show marked divergence.

The frequent absence of agreement between the distribution of the macroscopic and microscopic data of the hybrids among the six parent-phases is at present inexplicable. As before stated, it seems, if in any hybrid given proportions of macroscopic characters would be found to be the same as those of the seed parent and as those of the pollen parent, that the corresponding proportions of the microscopic characters would be found; but the proportions may not only be quite different but even reversed. The proportions of macroscopic and microscopic characters that are the same as or inclined to the seed and pollen parents, respectively, are approximately in Ipomoea sloteri (Table I, Summary 4) about 2 to 1 and 3 to 1, respectively; in Locia-Cattleya can hamiana, 1 to 2 and 1 to 2 ; in Cymbidium eburneolowianum, 3 to 2 and nearly 1 to 1 respectively; in Denbrobium cybele, 1 to 3 and about 1 to 1 respectively; in Miltonia bleuana, 4 to 3 and 1 to nearly $11 / 2$ respectively; in Cypripedium lathamianum, about 1 to 1 and nearly 1 to $11 / 2$, respectively; in C. Lathamianum inversum, 2 to 1 and $11 / 2$ to 1 respectively, and in $C$. nitens $11 / 3$ to 1 and 1 to $11 / 3$, respectively. With such marked and unaccountable variations of macroscopic and microscopic values, it is to be expected that owing to the great dissimilarity in the methods and characters of the data of the tissue and starch investigations the two sets of data may differ even more widely than the macroscopic and microscopic data just examined; and such is found to be the case, as will be shown in the following section wherein additional consideration of the tissue characters is given.

\section{TISSUES AND STARCHES OF SAME} PARENT- AND HYBRID-STOCKS.

Comparisons of Charagters of the Tissues and of the Histologic and other Properties and Reaction-Intensities of the Starches of Hybrid-Stocks as Regards Sameness, Intermediateness, Excess and Deficit of Development in Relation to the Parent-Stocks.

(Table I, Parts 1 to 8, and Summaries 1 to 9. Charts F 1 to F 14.)

When the present research was planned it was the intention, as stated in the introduction, to make coincident studies of the tissues and starches of each parent and hybrid specimen, with the especial object of showing what relationships, if any, exist between the macroscopic and microscopic characters of the plants and the histological and other properties and reaction-intensities of the starches, but various conditions combined to render this project impracticable. One might be led to the assumption, upon superficial thought, that if, for instance, the macroscopic plant-characters of any hybrid are distributed in certain percentages among the six sex-phase divisions a closely corresponding division of the microscopic characters would be found, and that starch characters, physical and physico-chemical, would be in similar agreement. In other words, a universality of type or plan of distribution of characters, so that if, for example, in Ipomoea sloteri the distribution of macroscopic characters among the six parent-phases be (Table I, Summary 1) 2.6, 2.6, 0, 47.4, 42.1, and 5.3 per cent, respectively, the distribution of the microscopic characters would be essentially or closely the same; but, in fact, there are more or less marked differences, as is evident by the following figures for the latter: $8.4,3.2,2.1,32.6$, 47.4, and 6.3 per cent, respectively. By such comparisons it will be noted that, among the macroscopic characters as compared with the number of microscopic characters, less than one-third will be the same as those of the seed parent (2.6:8.4); a slightly smaller percentage the same as pollen parent $(2.6: 3.2)$; a smaller percentage the same as both parents $(0: 2.1)$; a very much higher percentage intermediate $(47.4: 32.6)$; a smaller percentage developed to excess of parental extremes (42.1:47.4); and a slightly smaller percentage developed in deficit of parental extremes $(5.3: 6.5)$. Such differences vary in the different hybrids in both quantity and direction, and when the percentages for all of the hybrids are summed up, as in Table I, Summary 2 , the macroscopic characters show distinctly higher percentages than the micrascopic characters in regard to sameness as the seed parent, pollen parent, and both parents, and also to intermediateness, especially the latter; and markedly lower percentages in the characters developed beyond parental extremes.

In view of such extraordinary differences in percentages of microscopic and macroscopic characters, interest is at once aroused in regard to the relative peculiarities of the tissues and starches in their parental relationships. On general principles it seems probable that if two groups of characters which are so closely related as the naked eye and microscopic characters differ so notably that the group of characters consisting of reaction-intensities of the starches should differ as much or more from 
the tissue groups as do the latter from each other. Comparing the tissue characters and starch reactivities (Table I, Summary 3), it is found that the former show distinctly lower percentages in regard to sameness as the seed parent, pollen parent, and both parents; markedly higher percentages in regard to intermediateness and characters that are developed in excess of parental extremes; and a distinctly lower percentage developed in deficit of parental extremes. It seems obvious from this that the figures recorded in any one of these modes of investigation can not be taken as an index of what is to be found by another. If the percentages of the tissue characters and starch characters are charted (Chart F 9) it will be seen that there is only a very gross, if any, correspondence between the two curves. If three curves are constructed to show the macroscopic, microscopic, and reaction data respectively (Chart F 10), a modified picture is presented. It will be noted that the macroscopic and microscopic curves show similarities and that neither appears to be related to the starch curve.

The comparative degrees of influence of each of the parents in determining the characters of the hybrid varies not only with the different sets, but also in the percentages of macroscopic and microscopic characters in each set. Table $\mathrm{H}$, Summary 2 , gives a summary of the sameness and inclination of the reaction-intensities of the starches of hybrids to one or the other parent or both parents. Table I, Summary 4, presents similar data of the macroscopic and microscopic plant characters. Taking the macroscopic and microscopic characters together, it will be found that there is marked dominance of the seed parent in Ipomcea sloteri (58:23) and Cypripedium lathamianum inversum $(60: 43)$, and of the pollen parent in Lolia-Cattleya canhamiana (31:61), and that there is little dominance of either parent in Cymbidium eburneo-lowianum (41:35), Miltonia bleuana (39:47), Cypripedium lathamianum (39:48), and Cypripedium nitens $(41: 47)$. In none of these hybrids is there noted in the tissue characters the extreme dominance recorded in the reaction-intensities and histological properties of some of the hybrids in the starch investigation, but such dominance will undoubtedly be brought out in researches with other parents and hybrids.

In summing up the numbers and percentages of the tissue charactens and starch reaction-intensities that are the same as or inclined to the seed parent, the pollen parent, and to both parents, and which are as close to one as to the other parent, respectively, it is found that the different hybrids show the widest variations in direction and degree (Table I, Summary 6, and Table G). Thus, in Ipomcea sloteri the ratio of macroscopic characters that are the same as or inclined to the seed parent to those that are the same as or inclined to the pollen parent is about 2:1, while of the microscopic characters it is almost 3:1. In Lolia-Cattleya canhamiana the ratios are about $1: 2$ and $1: 2$ respectively. In $C y m$ bidium eburneo-lowianum the ratios are $11 / 2: 1$, and $1: 1$, respectively. In Dendrobium cybele the ratios are $1: 3$ and $1: 1$, respectively, and so on. In the case of the starches the ratios are far more varied, ranging from 23: 0 at one extreme to 0:25 at the other extreme, with great variations in between. In summing up the figures and percentages for the tissues and comparing them with the corresponding figures for the starches, it is found that the figures for the combined macroscopic and microscopic characters that are the same as or inclined to the seed parent and the pollen parent, respectively, are 36.8 and 36.9 , while for the starches they are 42.7 and 32.4 . Of characters that are the same as those of both parents the figures for the tissues and starches are 5.2 and 13.8, respectively. In group of characters first stated the figures are almost the same in the first couple, while in the second couple the first figure is about one-third higher than the second. In the second group the first figure is small in comparison with the second, this probably being due to the fact that in the study of the tissue characters many characters that were found in the hybrid to be the same or practically the same as the characters in the parents were not recorded. Of characters that are as close to one as to the other parent the tissue character percentage is 21.1, while that of the starches is 11.1 . Finally, among the tissue characters, 73.7 per cent are the same as or inclined to the seed or the pollen parent; and among the starch characters 75.1 per cent, or practically the same.

In case of two sets of parents and hybrids (Cymbidium and Miltonia), studies were made coincidently of both tissue and starch characters, but unfortunately in one (Cymbidium) the reactions of the starches were with few exceptions so very rapid that satisfactory data. for differential purposes were not obtained. These data are summarized in Tables $I, 3$, and 5, and F, 47 and 48, and also in Charts F $3, F 5, F 11$, and F 12 . Referring to the characters and character-phases of $C y m$ bidium eburneo-lowianum it will be apparent upon comparison of the data pertaining to the several parentalphases (Chart F 3) that the percentages of macroscopic characters are smaller than those of the microscopic characters that are the same as those of the seed parent, and which are developed in excess and in deficit of parental extremes; but larger among those which are the same as those of the pollen parent and of both parents, and which are intermediate. Hence, there are inversions of the curves in the chart. The quantitative differences between the plant and the reaction characters vary in the several parental-phases (Chart F 11), the differences being distinct among the characters that are the same as those of one or the other parent or both parents, marked among those which are developed in excess or deficit of parental extremes, and very marked among those which are intermediate. While there are some correspondences in the percentages and curves of the macroscopic and microscopic data, there is no correspondence between these and the starch reaction-intensity curve. In fact, there seems to be a tendency to inverse rather than direct relationship. In Miltonia bleuana the macroscopic and microscopic figures and curves differ in some respects less and in others more than in Cymbidium eburneo-lowianum (Chart F 12). The percentages of the macroscopic characters are higher than those of the macroscopic characters among the characters that are the same as those of the seed parent and the same as those of the pollen parent, but lower among the characters that fall under the other four parental-phases, so that here also there is inversion of the two curves. The percentages and curves of the starch reaction-intensities bear, as in the foregoing hybrid, apparently no relationship to either macroscopic or 
microscopic character curve, and here also it appears as though there is a tendency to inverse rather than direct relationship. While the starch reaction-intensity data in Cymbidium are of little value, for reasons stated, the data of Miltonia are to be regarded as being quite as dependable as those of either macroscopic or microscopic characters.

In further comparisons to bring out specifically the comparative influences of the seed and the pollen parent on the properties of the hybrids (Table I, Summary 5, Charts F 11 and F 12) it will be found in Cymbidium eburneo-lowianum that the macroscopic and microscopic percentages and curves tend to correspondence in their courses with varying degrees of separation, and also to inversions in their positions. The percentages of macroscopic characters compared with those of microscopic characters are lower among the characters that are the same as those of the seed parent, that are highest and that are lowest; and higher among those that are the same as those of the pollen parent, that are the same as those of both parents and that are intermediate.

Comparing now the starch-reaction data with the foregoing, it will be seen that while the percentages and curves of the tissue data have some correspondence, the starch data and curve appear to be quite independent, the starch curve being higher than the tissue curve in respect to characters that are the same as those of the seed parent, the same as those of both parents and those which are lowest; and zero in characters that are the same as those of the pollen parent, intermediate and highest. In Miltonia bleuana the macroscopic and microscopic values and curves are quite different from the preceding. The curve of the macroscopic characters is higher than that of the microscopic characters among the characters that are the same as those of the seed parent and the same as those of the pollen parent, and lower in the other four parental designations. The starch curve here is also very variant, bearing no relationship to the tissue curves. It is intermediate between the macroscopic and microscopic curves in regard to characters that are the same as those of the seed parent and that are lowest, lower in characters that are the same as those of the pollen parent and that are intermediate, and higher in characters that are the same as those of both parents and that are highest. In Cymbidium eburneolowianum (Table I, Summary 5) 30 per cent of the tissue characters are the same as those of one or the other parent or both parents ; 44.5 per cent intermediate; and 25.4 per cent developed in excess or deficit of parental extremes. The starch reactions show 50.1, 0, and 50 per cent, respectively, the figures in the several columns differing markedly from those of the tissues. In Miltonia bleuana the figures for the tissues are 26.2, 35.1 and 38.6, respectively; and for the starch $23,3.8$, and 73.1 , respectively.

The comparative degrees of influence exerted by each parent on the properties of the hybrid are shown in Table I, Summary 6 , and presented in chart form in
Charts F 14 and F 15. In Cymbidium eburneo-lowianum, in the macroscopic characters the seed parent has exerted a much greater influence than the pollen parent, but in the microscopic characters very little more than the pollen parent. In Miltonia bleuana, in the macroscopic characters the seed parent is distinctly more potent, but in the microscopic characters the pollen parent is the more potent, the values being practically reversed. Summing up the macroscopic and microscopic characters it is found that in Cymbidium eburneo-lowianum the seed parent is but little more potent than the pollen parent (37.3:31.8 per cent), and that in Miltonia blueana the seed parent is decidedly less potent than the pollen parent (34.2:41.2 per cent). As to the starches in Cymbidium eburneo-lowianum the influences of the seed parent are far greater than those of the pollen parent as shown by the ratio of $15.4: 3.8$; and in Miltonia blueana the difference is very much greater, the ratio here being $77: 7.7$-in the former 4 times greater and in the latter almost 10 times greater. Little or no importance, however, is to be attached to the data of the starch of Cymbidium for reasons already given.

In the histological examinations of the starches it was found that the starch of Cymbidium eburneo-lowianum in the form of the grains, character of the hilum, lamellæ, and size is closer, as a whole, to the seed parent; and in eccentricity of the hilum and ratio of length to width of the grains closer, as a whole, to the pollen parent. In the qualitative reactions it is in all respects closer to the seed parent. In Miltonia bleuana the starch is in the form of the grains, character of the hilum, and character of the lamellæ closer, as a whole, to the seed parent; but in eccentricity of the hilum and size of the grains it is closer, as a whole, to the pollen parent. In all of the qualitative reactions it is closer to the seed parent.

Apropos of intermediateness as a criterion of hybrids, it is worth while to compare the percentages of microscopic and macroscopic characters and starch reaction-intensities that are intermediate and non-intermediate. These data are given in Table I, Summary 7 , by which it will be seen that of 264 macroscopic characters recorded 56.4 per cent are intermediate and 43.6 per cent non-intermediate; of the 695 microscopic characters, 38.2 per cent are intermediate and 61.8 per cent non-intermediate ; and of the 1,018 starch reaction-intensities, 23.2 per cent are intermediate and 76.8 per cent non-intermediate.

The data recorded are so numerous and of such a character that considerable space could be devoted to their study, but this seems unnecessary because they have been so thoroughly systematized and clearly presented in tables and charts as to be instantly understood and readily available for any who may be particularly interested in any or all of the various phases represented; nor is it necessary, because such detailed consideration as has been given meets the requirements of the objects of the research. 
TABLE I.

\begin{tabular}{|c|c|c|c|c|c|c|}
\hline & 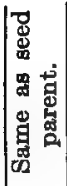 & 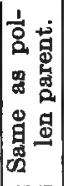 & 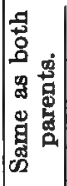 & 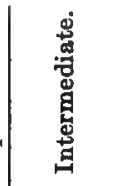 & 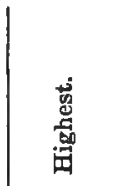 & 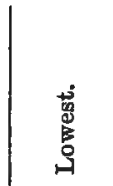 \\
\hline \multicolumn{7}{|l|}{$\begin{array}{l}\text { 1. Ipomoea sloteri, mac- } \\
\text { roscopic charac- } \\
\text { ters: }\end{array}$} \\
\hline $\begin{array}{l}\text { Cotyledons: } \\
\text { Shape.............. }\end{array}$ & & & & & & \\
\hline Length of midrib... & $=$ & $\overline{-}$ & z & $\begin{array}{l}+ \\
+0\end{array}$ & $\overline{-}$ & $\overline{-}$ \\
\hline Length of petiole... & - & - & - & $+\%$ & - & - \\
\hline \multirow{2}{*}{\multicolumn{7}{|c|}{$\begin{array}{l}\text { Root: } \\
\text { Length of primary }\end{array}$}} \\
\hline $\begin{array}{l}\text { Length of primary } \\
\text { root before }\end{array}$ & & & & & & \\
\hline branching........ & + & - & - & - & - & - \\
\hline $\begin{array}{l}\text { Diameter......... } \\
\text { Extent of root iys- }\end{array}$ & - & - & - & - & +9 & - \\
\hline tom................. & - & - & - & - & +9 & - \\
\hline \multicolumn{7}{|l|}{ Stem: } \\
\hline $\begin{array}{l}\text { Diameter........... } \\
\text { Growth.............. }\end{array}$ & $=$ & $\overline{-}$ & $=$ & $\overline{-}$ & $\begin{array}{l}+q \\
+q\end{array}$ & $\overline{-}$ \\
\hline \multicolumn{7}{|l|}{$\begin{array}{c}\text { Distance } \\
\text { ground } \\
\text { from } \\
\text { before }\end{array}$} \\
\hline branching....... & - & - & - & $+\sigma^{7}$ & - & - \\
\hline Length of branches & - & - & - & - & $+q$ & - \\
\hline \multicolumn{7}{|l|}{ Leaf: } \\
\hline Number. ......... & - & - & $\overline{-}$ & $\overline{-}$ & $\left|\begin{array}{l}+\sigma^{x} \\
+8=a^{x}\end{array}\right|$ & $=$ \\
\hline $\begin{array}{l}\text { Duration .......... } \\
\text { Firmness of texture }\end{array}$ & $\bar{z}$ & - & $\overline{-}$ & $\overline{-}$ & $\begin{array}{l}+q=0 \\
+q=\sigma^{\prime}\end{array}$ & $\overline{-}$ \\
\hline $\begin{array}{r}\text { Resistance to in- } \\
\text { sects........... }\end{array}$ & $\pi$ & . & - & - & 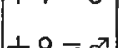 & 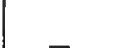 \\
\hline Shape of lamina... & - & $=$ & $\overrightarrow{-}$ & $+q^{-}=\sigma^{\prime}$ & $\mid+7=0$ & $\overline{-}$ \\
\hline Length of lamina.. & - & - & - & +9 & - & - \\
\hline Width of lamina... & - & - & - & & $+\%$ & 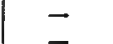 \\
\hline Length of petiole. . & - & - & - & $+q$ & & - \\
\hline \multicolumn{7}{|l|}{ Flower: } \\
\hline $\begin{array}{l}\text { Length of flower } \\
\text { stalk. }\end{array}$ & - & - & - & - & +8 & - \\
\hline $\begin{array}{c}\text { Number of flowers } \\
\text { per flower stalk.. }\end{array}$ & - & - & - & $+\varphi=\sigma^{7}$ & - & - \\
\hline Relationship of pe- & & & & & & \\
\hline $\begin{array}{r}\text { duncle to pedicle } \\
\text { Shape of sepals.... }\end{array}$ & $=$ & $\bar{z}+2 \cdot+x+1$ & $=$ & $\begin{array}{l}+q=0^{7} \\
+q\end{array}$ & $\bar{z}+$ & $\bar{z}$ \\
\hline Shape of corolla & & & & & & \\
\hline $\begin{array}{c}\operatorname{limb} . . . \ldots \ldots \ldots \ldots \\
\text { Diameter of corolla }\end{array}$ & - & - & - & $+q=\sigma^{3}$ & - & - \\
\hline $\begin{array}{r}\text { Diameter of corolla } \\
\text { limb................ }\end{array}$ & - & - & - & - & $+q=\sigma^{3}$ & - \\
\hline $\begin{array}{c}\text { Color of corolla } \\
\text { limb.................. }\end{array}$ & 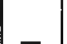 & 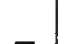 & - & $+0=\pi$ & $1=-1$ & - \\
\hline 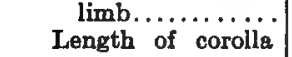 & - & - & - & $T F=0$ & 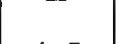 & \\
\hline & - & - & - & - & $+0^{x}$ & - \\
\hline $\begin{array}{c}\text { Diameter of corolla } \\
\text { tube }\end{array}$ & & & - & & $+\sigma^{7}$ & \\
\hline Color of corolla tube & - & - & $=$ & $+q^{-}=0^{x}$ & +o & - \\
\hline Color of anthers... & - & - & - & $+q=0^{2}$ & - & - \\
\hline & & - & - & $+\varphi=\sigma^{\prime \prime}$ & - & - \\
\hline Length of filaments & - & - & - & - & $+\%=\sigma^{7}$ & - \\
\hline \multirow{2}{*}{\multicolumn{7}{|c|}{$\begin{array}{l}\text { Capsule: } \\
\text { Number maturing } \\
\text { on one flower }\end{array}$}} \\
\hline & & & & & & \\
\hline 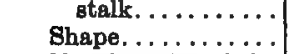 & $\bar{z}$ & \pm & $=$ & $+0^{-}=0^{n}$ & $\bar{z}$ & $\overline{-}$ \\
\hline $\begin{array}{l}\text { Number of seeds in } \\
\text { capsule.......... }\end{array}$ & - & - & - & $D=-1$ & - & $+\sigma^{\pi}$ \\
\hline Proportion of seeds & & & & & & \\
\hline that germinate. & - & - & - & - & - & $+9=\sigma^{x}$ \\
\hline Length of seeds.... & - & - & - & $+\infty^{\circ}$ & - & - \\
\hline Width of geeds.... & - & - & 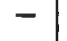 & & +9 & - \\
\hline Total..........38 & 1 & 1 & $\mathbf{0}$ & 18 & 16 & 2 \\
\hline
\end{tabular}

TABLE I-Continued.

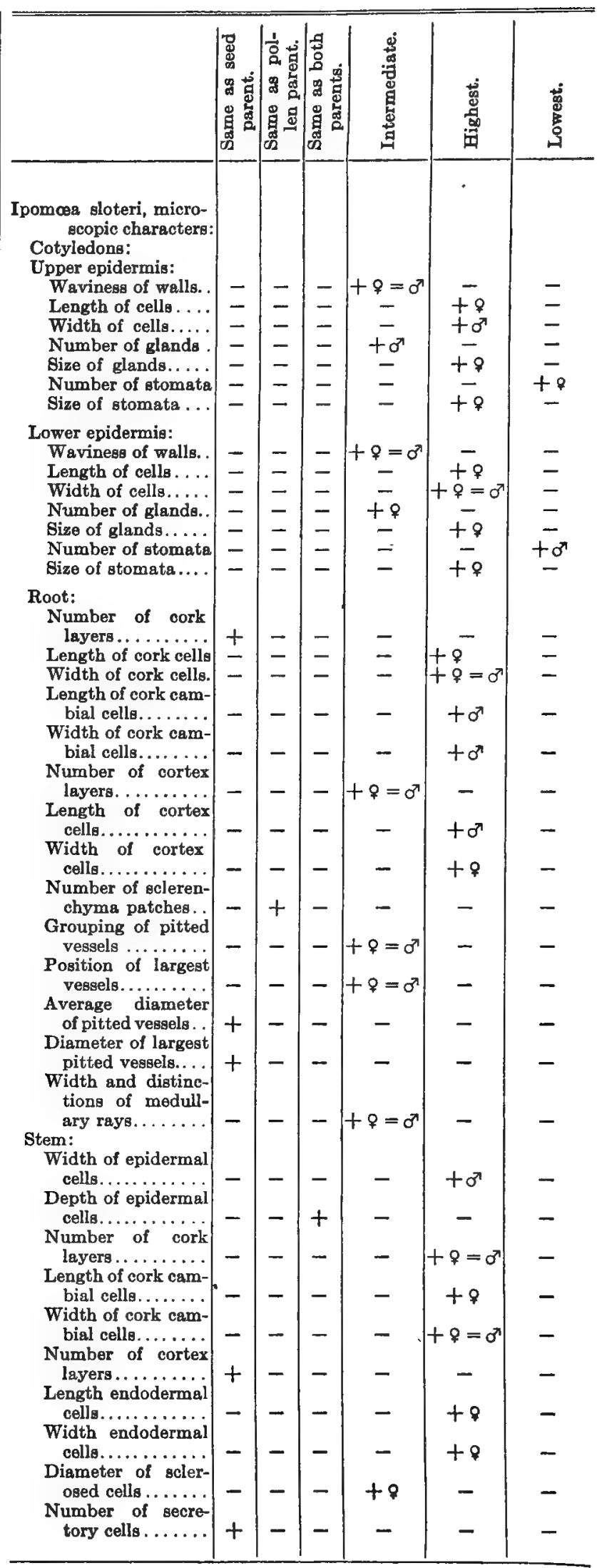


TABLE I.-Continued.

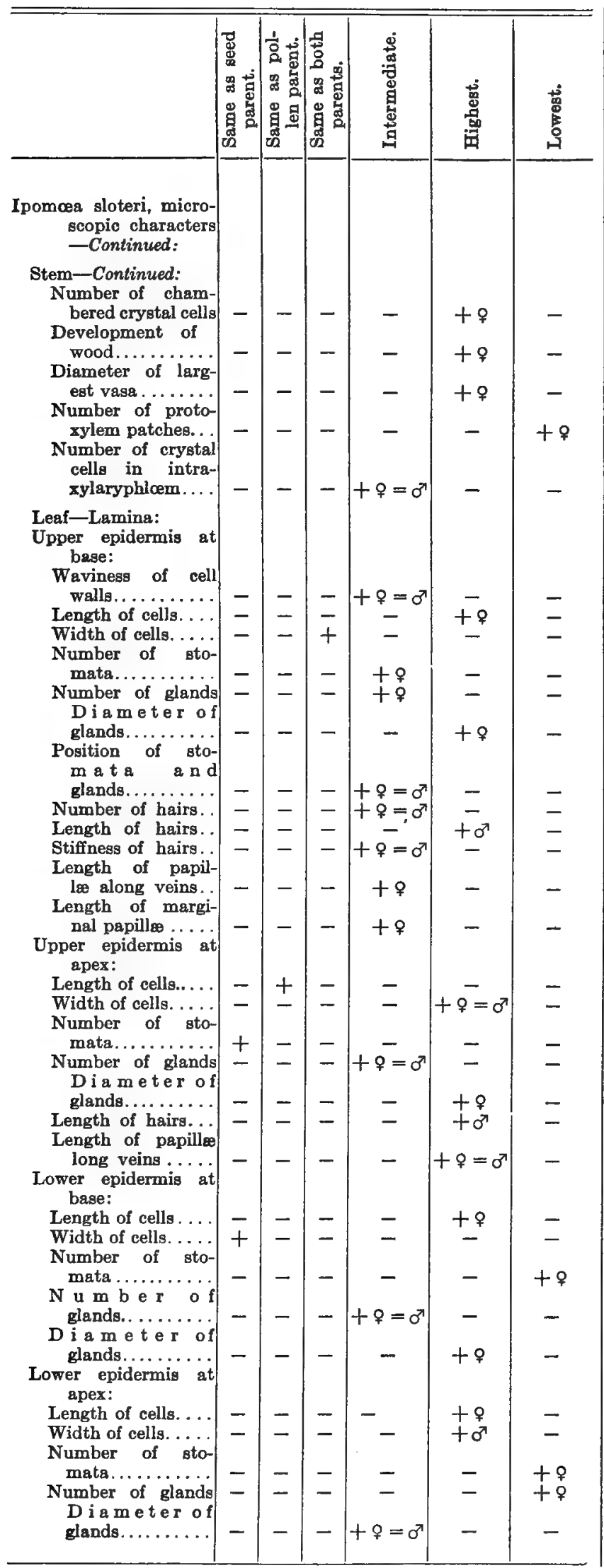

Table I.-Continued.

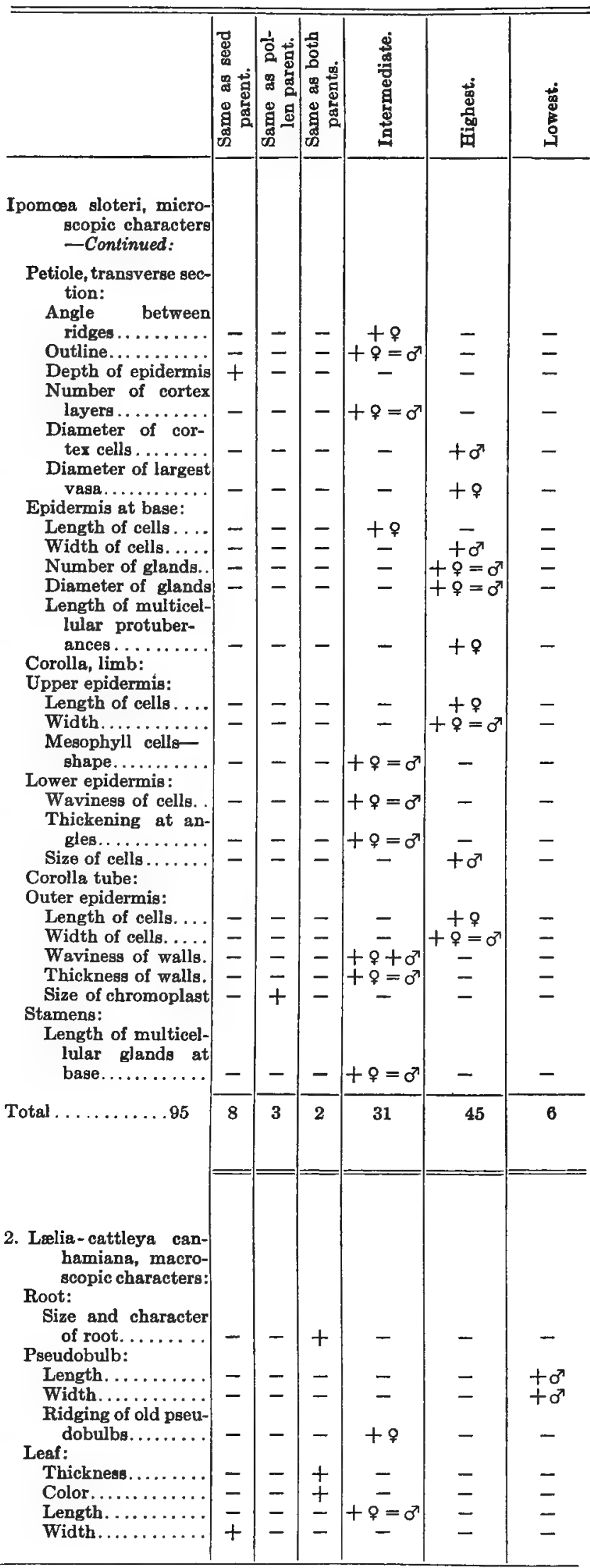


Table I-Continued.

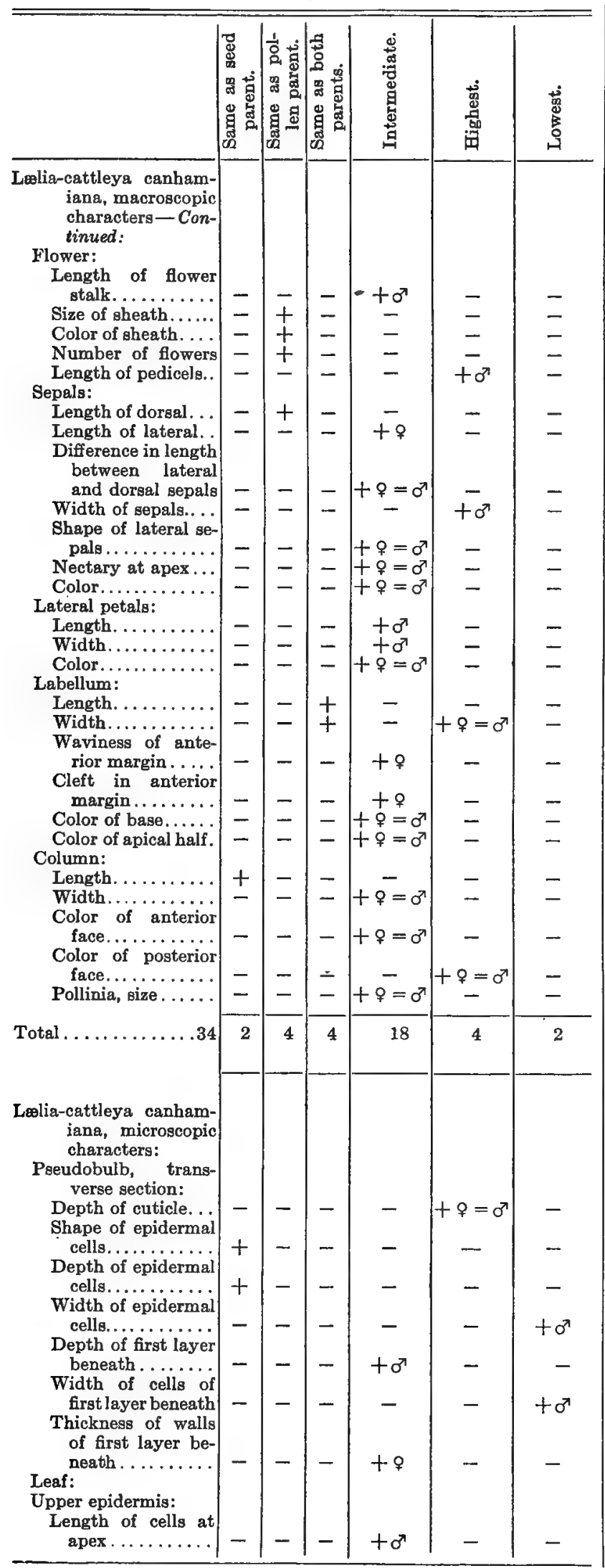

Table I.-Continued.

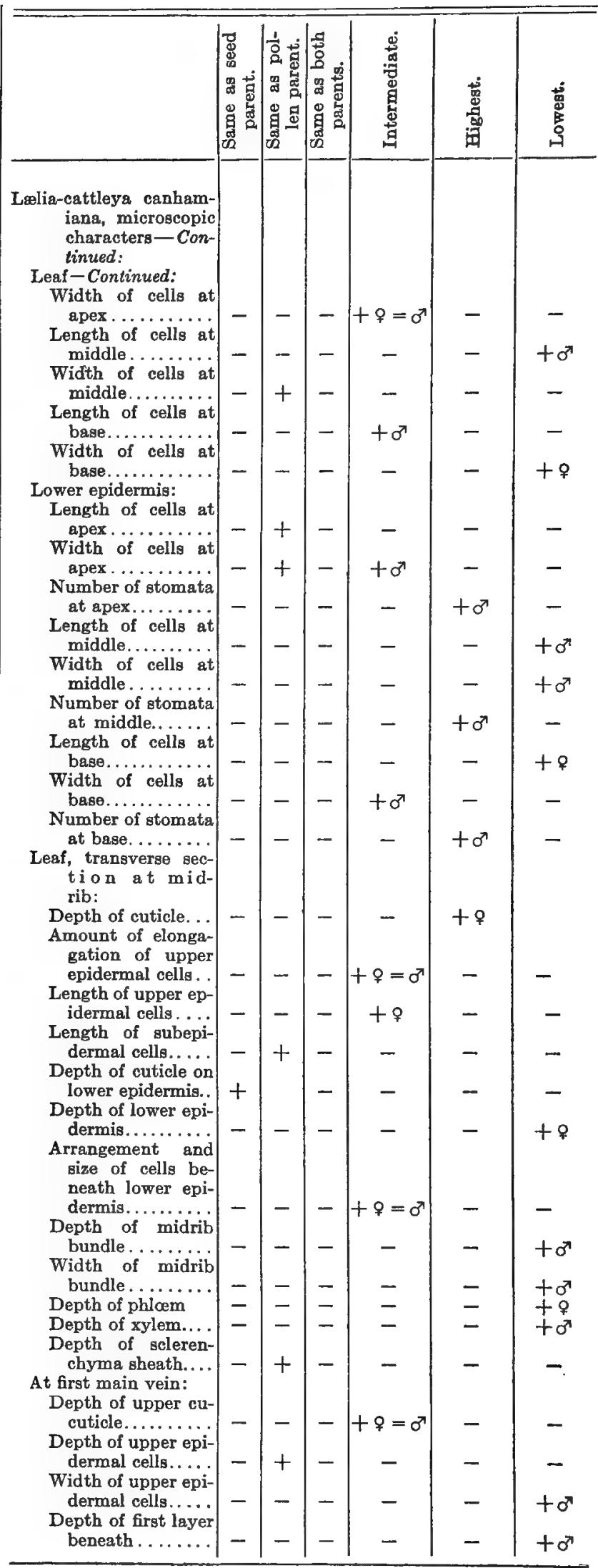


T ABLE I.-Continued.

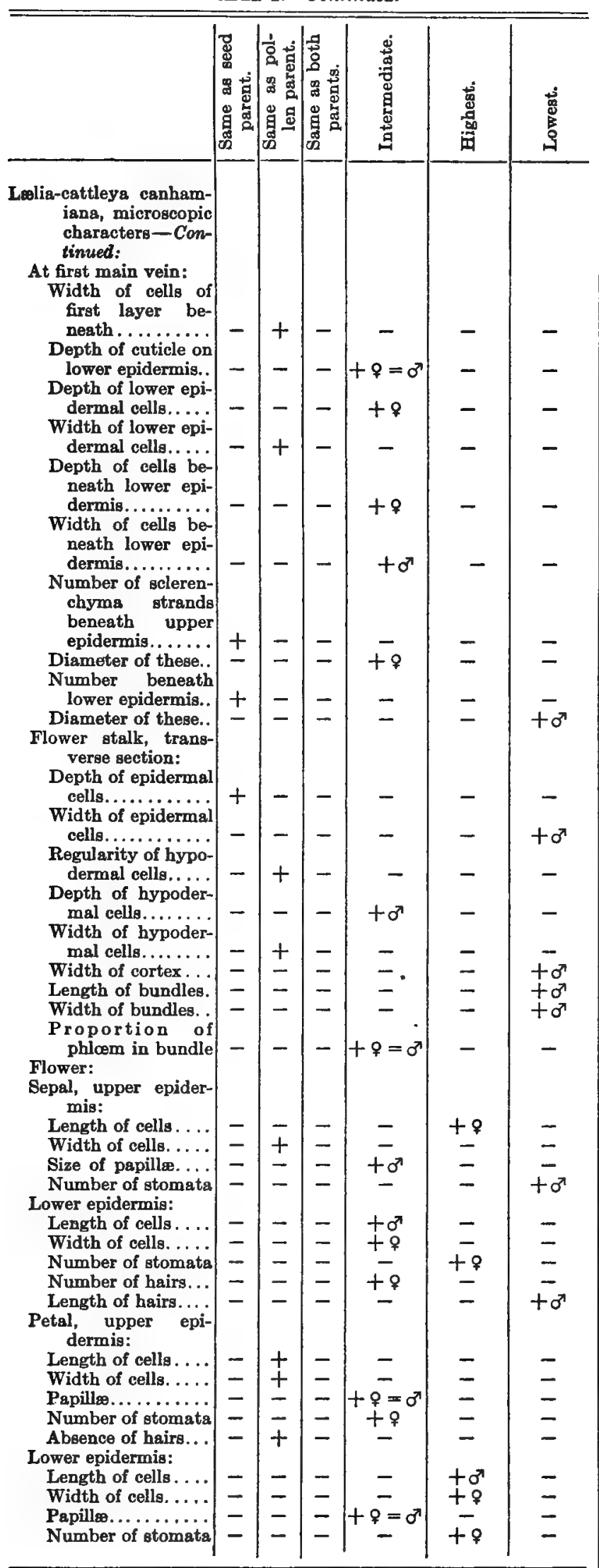

Table I.-Continued.

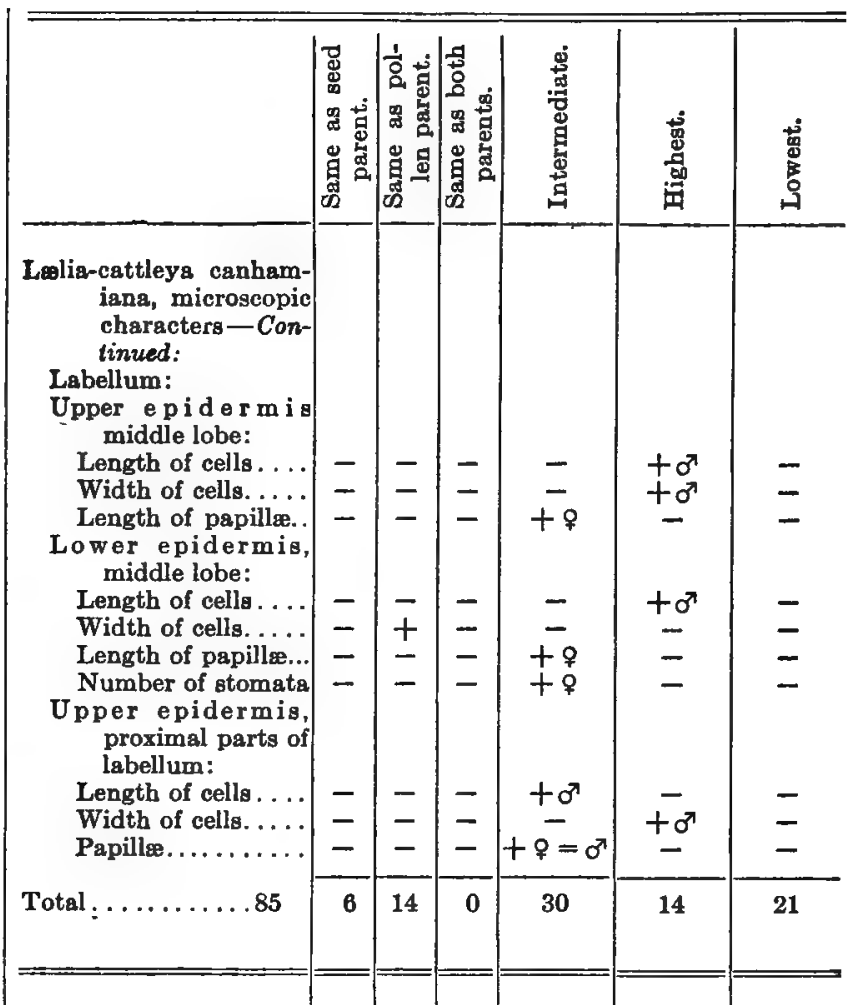

3. Cymbidium eburneolowianum, macroRoot:

Size and character of roots.........

Pseudobulb:

Length.

Leaf:

Amount of drooping previous years..........

Length of old leaves

Width of old leaves

Length of this

Wear's leaves....

Width of this year's leaves....

Number of leaves in growth.......

Flower:

Length of flower stalk.............

Diameter of flower

stalk. ...........

Length of bracts...

Number of flowers.

Dorsal sepal:

Length...........

Width.............

Shape............

Lateral sepals:

Length. ..........

Width............

Color - background

of upper surface

sepals..........

Color lines on upper

surface sepals....

Color lower surface

sepals..........

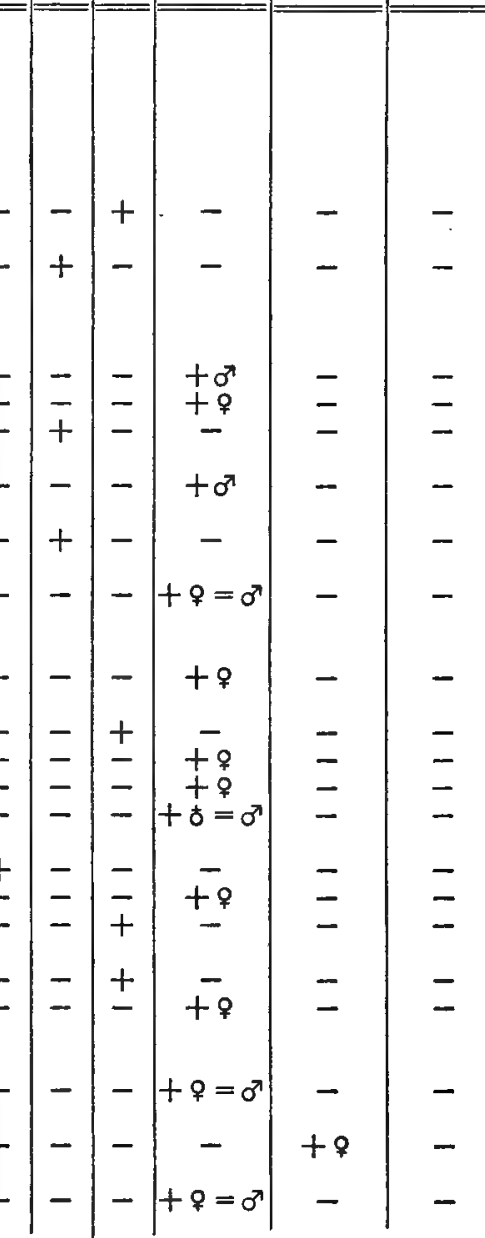


Table 1.-Continued.

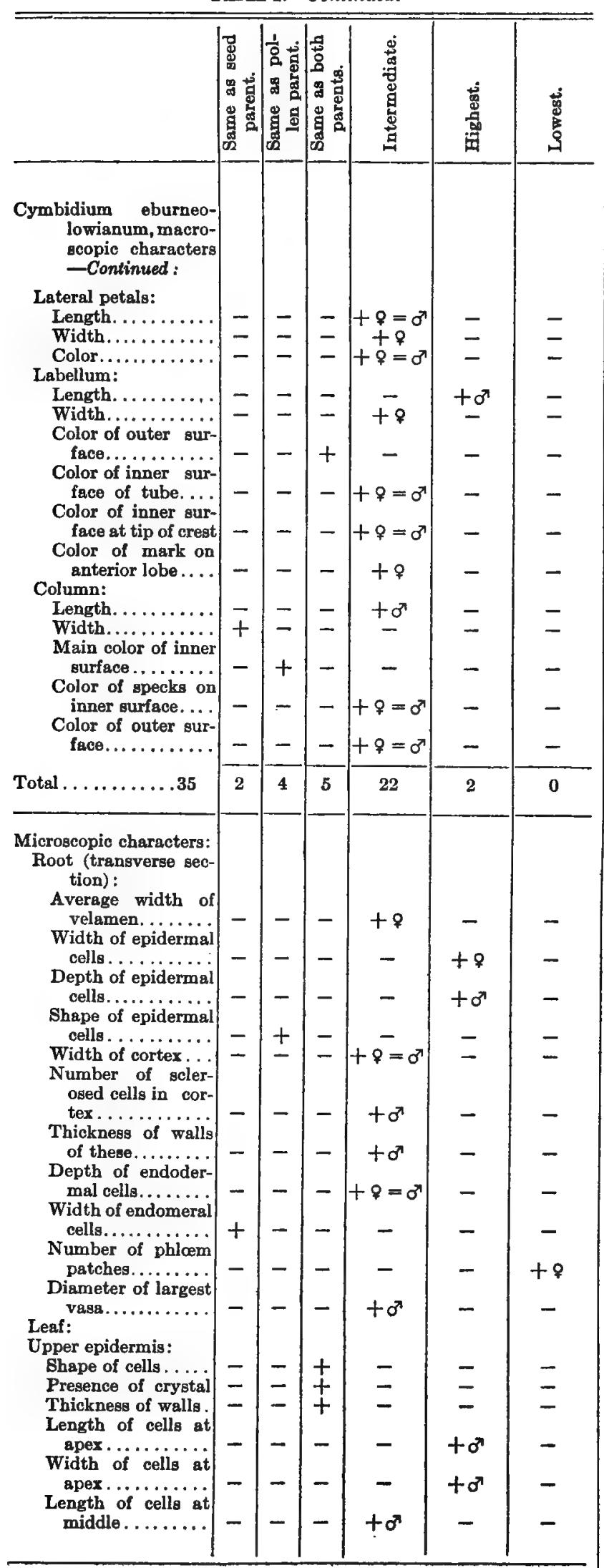

Table I.-Continued.

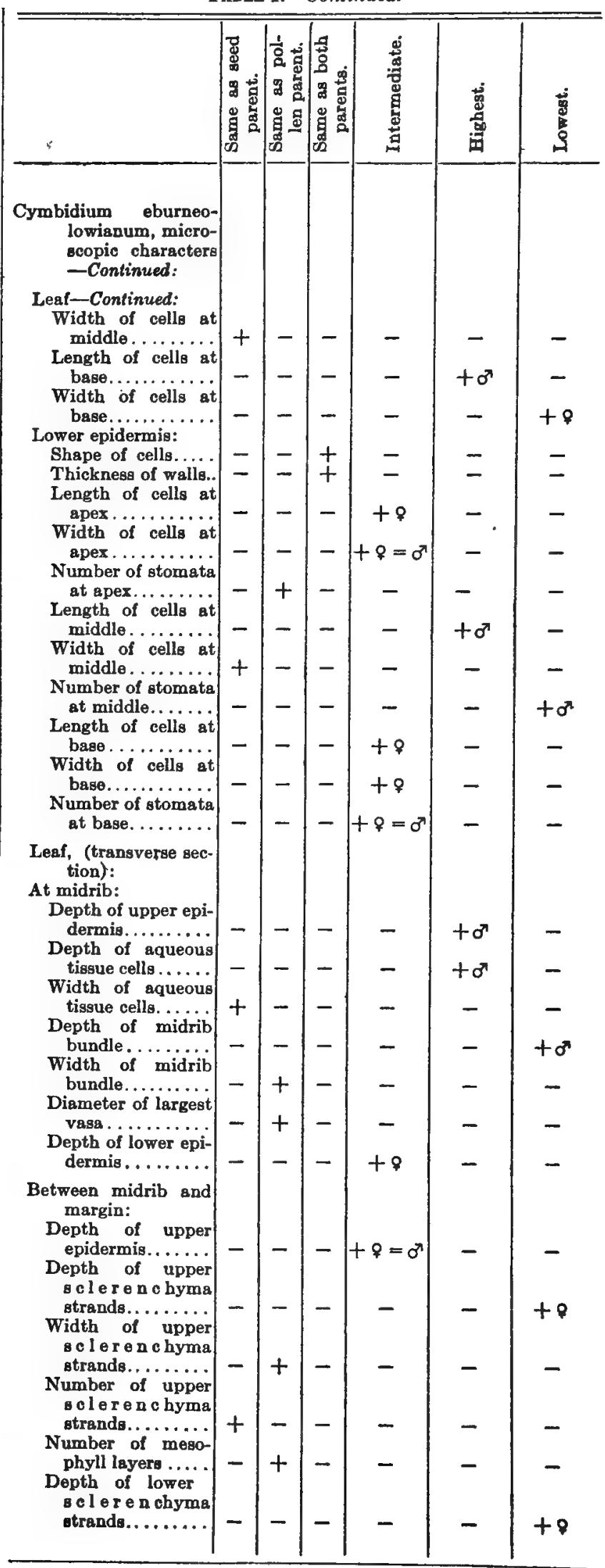


Table I.-Continued.

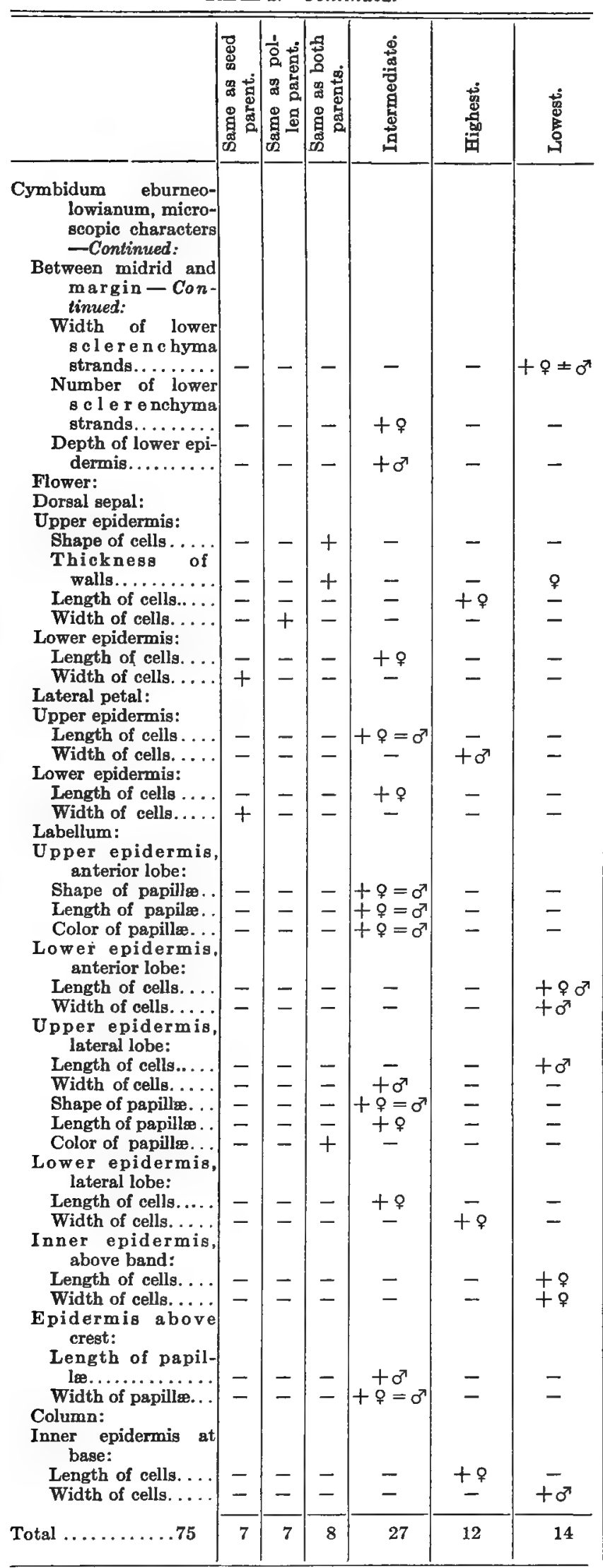

Table I.-Continued.

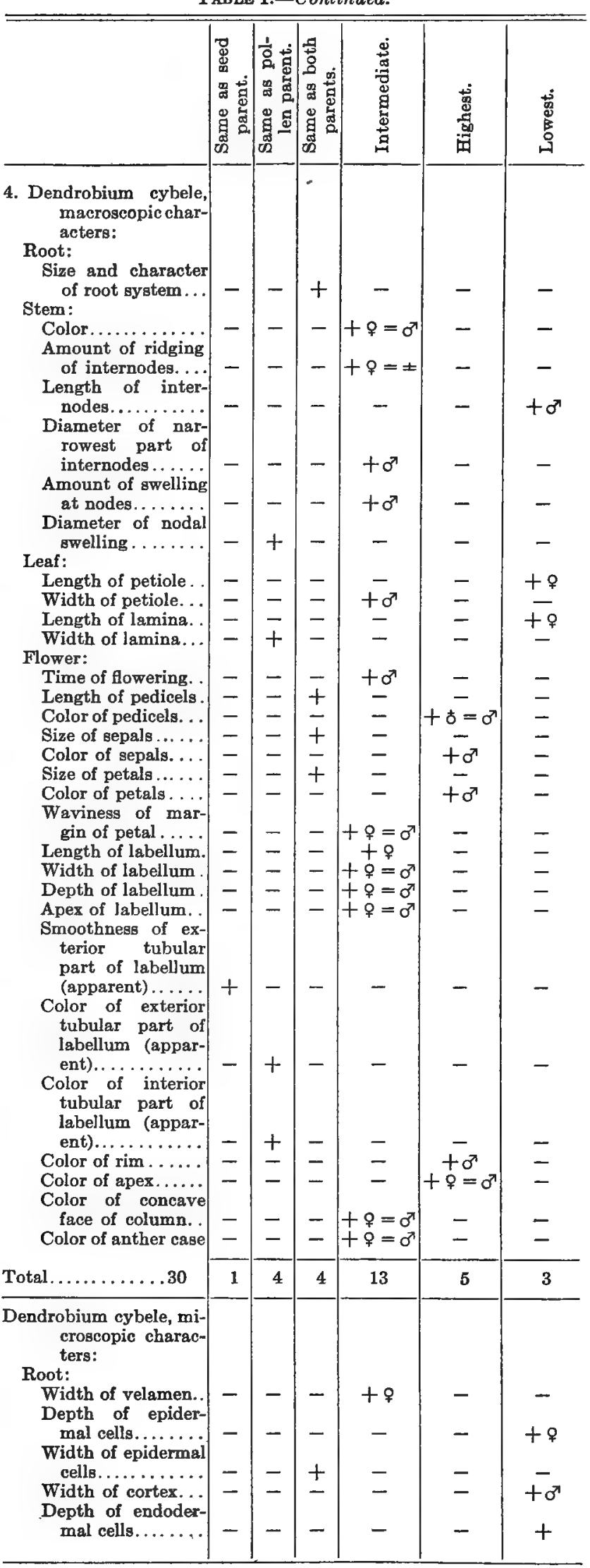


TABr. I-Continued.

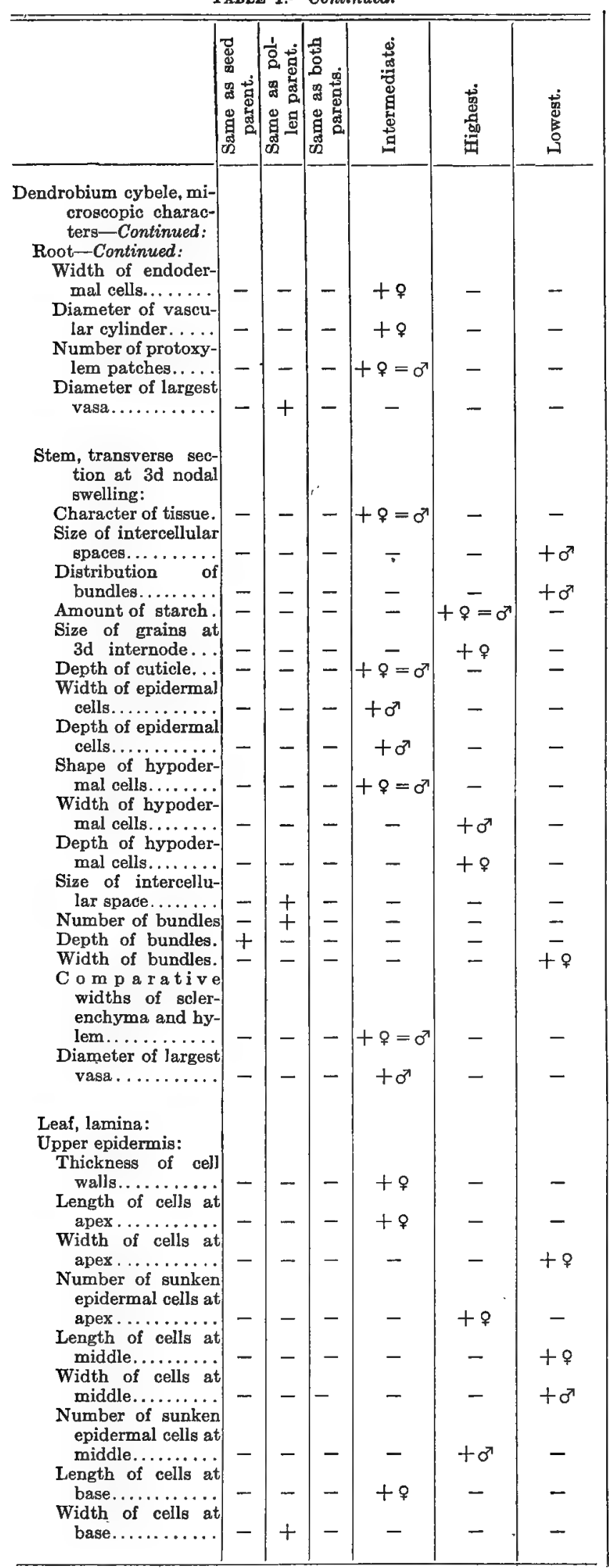

TABLE I.-Continued.

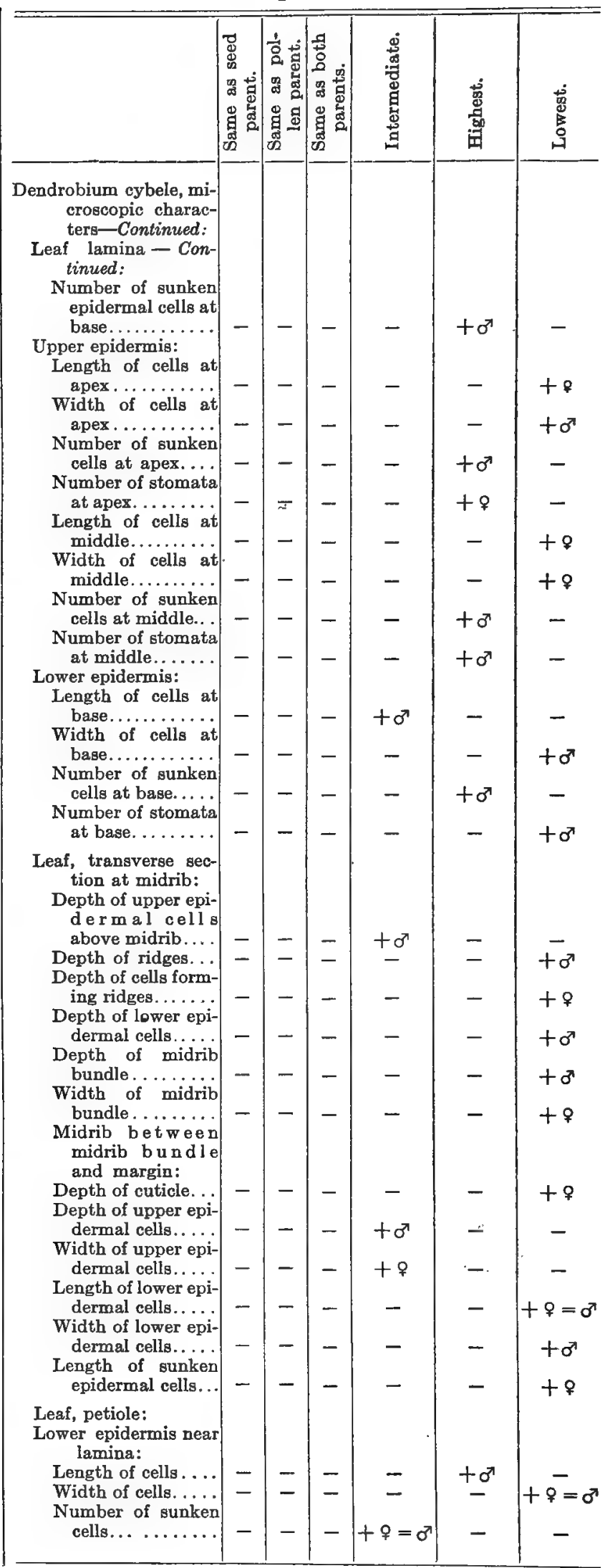


TABLe 1.-Continued.

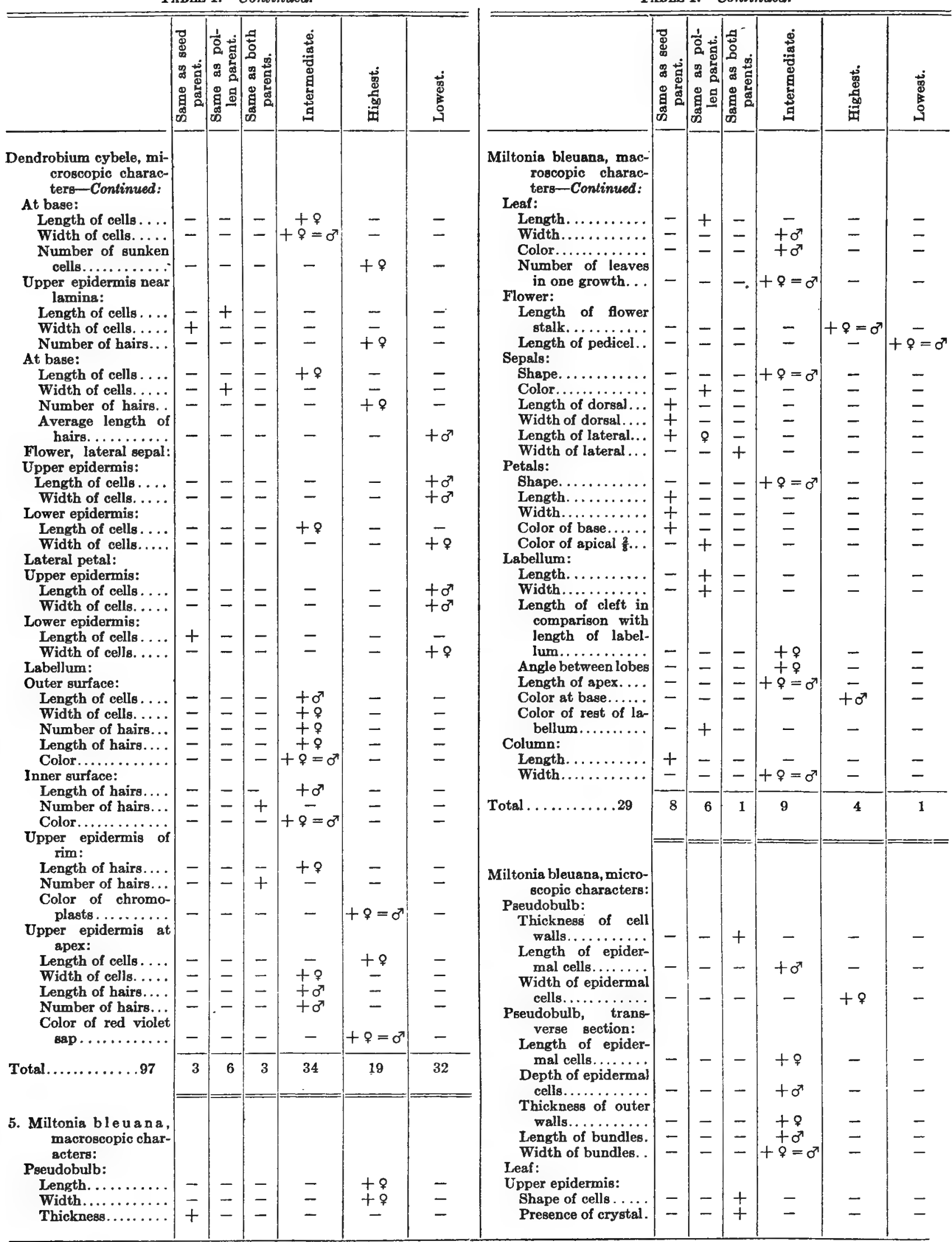

Table I.-Continued. 
Table I.-Continued.

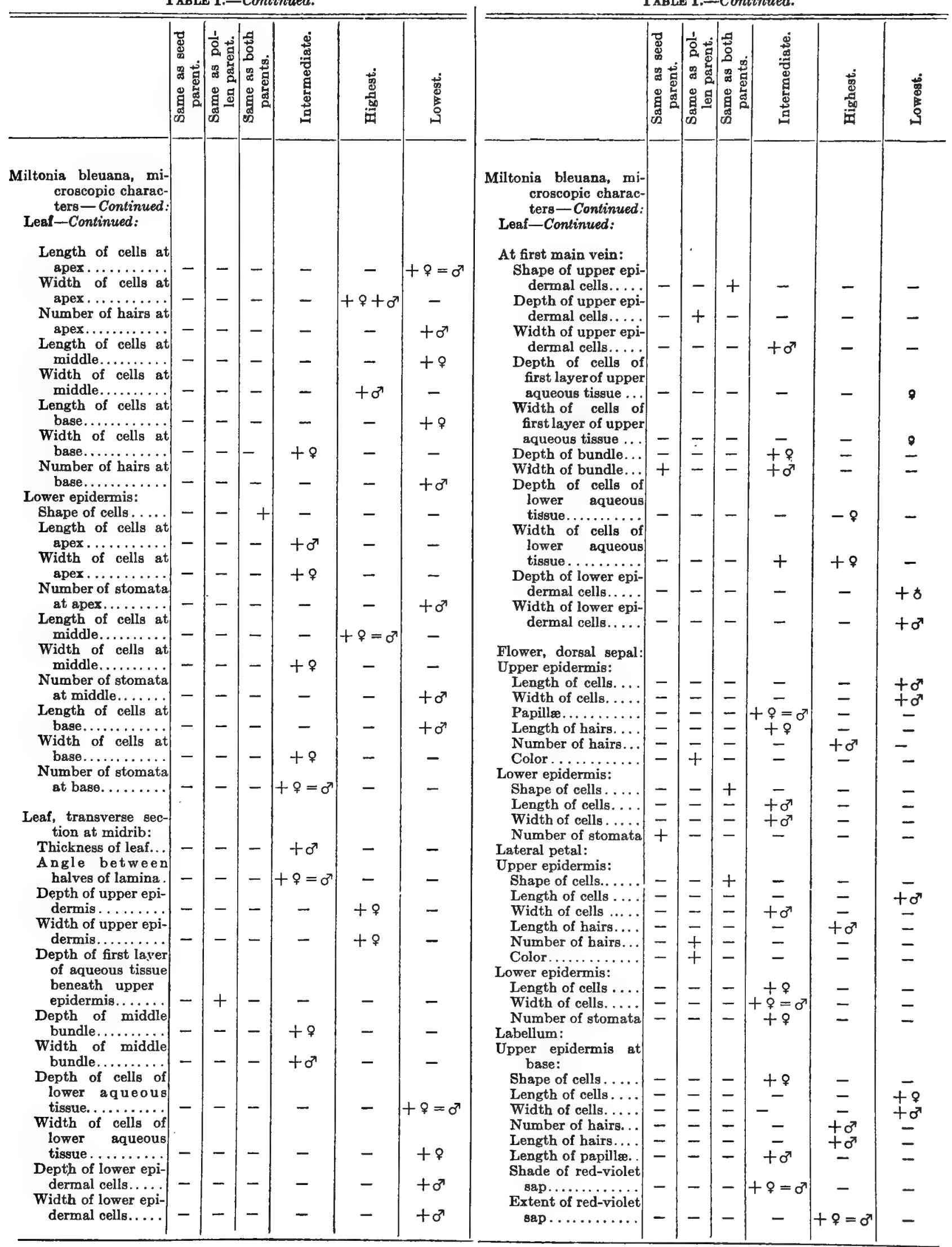


TABLE I.-Continued.

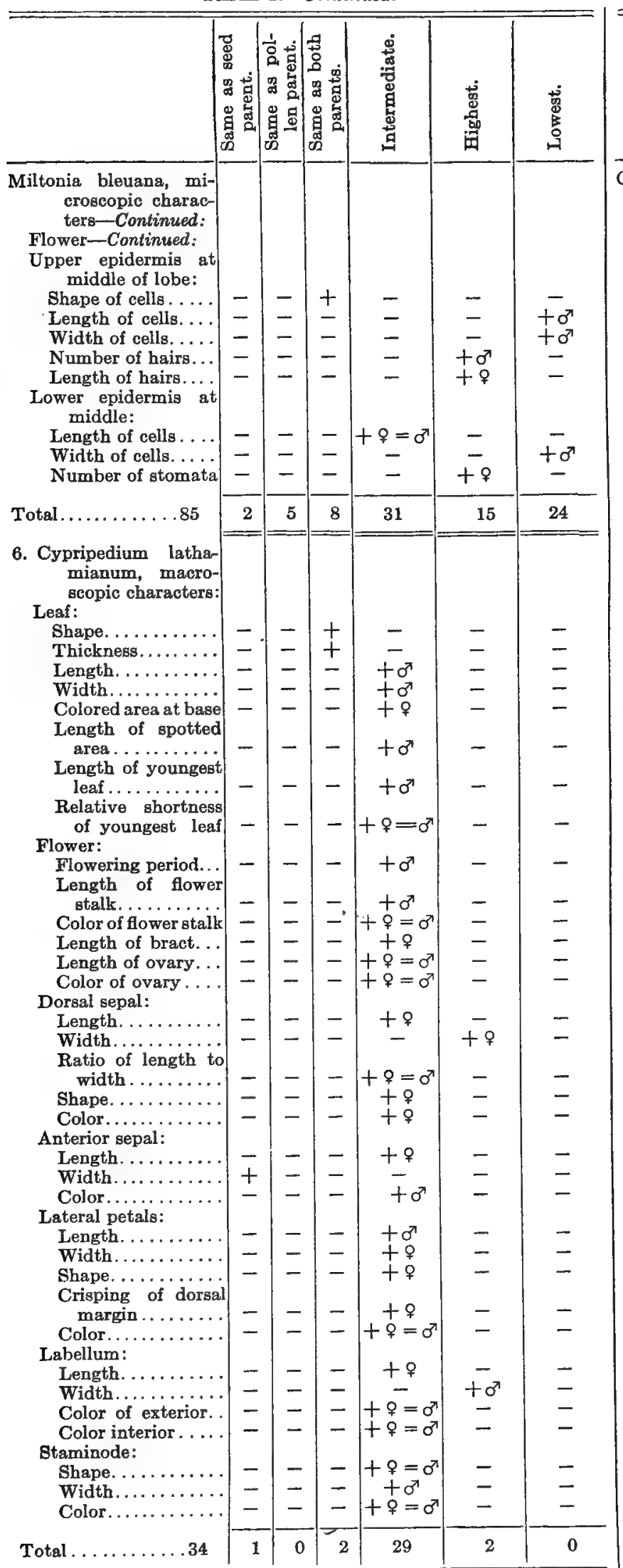

TaBle I.-Continued.

\begin{tabular}{|c|c|c|c|c|c|c|}
\hline & 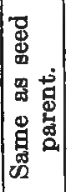 & 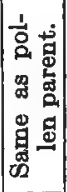 & 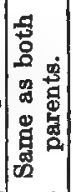 & 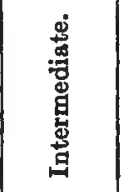 & $\begin{array}{l}\text { 范 } \\
\text { 点 } \\
\text { 苗 }\end{array}$ & 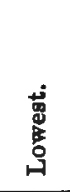 \\
\hline $\begin{array}{l}\text { Cypripedium lathamia- } \\
\text { num, microscopic } \\
\text { characters: }\end{array}$ & & & & & & \\
\hline Leaf: & & & & & & \\
\hline & & & & & & \\
\hline $\begin{array}{r}\text { at apex.......... } \\
\text { Length of cells at }\end{array}$ & - & - & - & +9 & - & - \\
\hline & - & - & - & +9 & - & - \\
\hline $\begin{array}{c}\text { apex................ } \\
\text { Thickness of walls }\end{array}$ & - & - & - & $+\sigma^{7}$ & - & - \\
\hline $\begin{array}{c}\text { middle............ } \\
\text { Length of cells at }\end{array}$ & - & - & + & - & - & - \\
\hline middle .......... & - & - & - & $+\sigma^{x}$ & - & - \\
\hline Width of cells at & - & - & - & +8 & - & - \\
\hline $\begin{array}{c}\text { Length of cells at } \\
\text { base............... }\end{array}$ & - & - & - & - & $+\sigma^{\prime}$ & - \\
\hline $\begin{array}{r}\text { Width of celle at } \\
\text { base ............ } \\
\text { Lower epidermis: }\end{array}$ & - & - & - & - & $+0^{x}$ & - \\
\hline $\begin{array}{r}\text { Length of cells at } \\
\text { apex............ }\end{array}$ & - & - & - & $+\infty$ & - & - \\
\hline $\begin{array}{c}\text { Width of cells at } \\
\text { apex............ }\end{array}$ & - & - & - & $+q$ & - & - \\
\hline Number of stomata & - & - & - & $+\%=\sigma^{\pi}$ & - & - \\
\hline $\begin{array}{l}\text { Length of cells at } \\
\text { middle } \ldots \ldots \ldots\end{array}$ & - & - & - & $+\sigma^{x}$ & - & - \\
\hline $\begin{array}{l}\text { Width of cells at } \\
\text { middle........ }\end{array}$ & - & - & - & - & $+\%=\sigma^{3}$ & - \\
\hline $\begin{array}{l}\text { Number of stomata } \\
\text { at middle....... }\end{array}$ & - & + & - & - & - & - \\
\hline $\begin{array}{r}\text { Length of cells at } \\
\text { base } \ldots \ldots \ldots \ldots .\end{array}$ & - & - & - & - & $+\sigma^{x}$ & - \\
\hline $\begin{array}{c}\text { Width of cells at } \\
\text { base } \ldots . . . \ldots \ldots\end{array}$ & - & - & - & - & $+\sigma^{\pi}$ & - \\
\hline $\begin{array}{r}\text { Number of stomata } \\
\text { at base. ........ }\end{array}$ & - & - & - & $+q=\sigma^{x}$ & - & - \\
\hline $\begin{array}{l}\text { Leaf, transverse sec- } \\
\text { tion: }\end{array}$ & & & & & & \\
\hline $\begin{array}{c}\text { Depth of cuticle } \\
\text { wax.............. }\end{array}$ & - & - & - & - & - & $+\sigma^{\pi}$ \\
\hline $\begin{array}{r}\text { Depth of upper epj- } \\
\text { dermal cells..... }\end{array}$ & - & - & - & - & - & $+0^{x}$ \\
\hline $\begin{array}{r}\text { Depth of cuticle on } \\
\text { lower epidermis.. }\end{array}$ & - & - & - & - & - & +8 \\
\hline $\begin{array}{r}\text { Depth of lower epi- } \\
\text { dermal cells..... }\end{array}$ & - & - & - & - & $+\sigma^{x}$ & - \\
\hline $\begin{array}{l}\text { Width of lower epi- } \\
\text { dermal cells..... }\end{array}$ & - & - & - & $+\sigma^{7}$ & - & - \\
\hline $\begin{array}{c}\text { Depth of midrib } \\
\text { bundle............. }\end{array}$ & - & - & - & $+\sigma^{3}$ & - & - \\
\hline $\begin{array}{c}\text { Width of midrib } \\
\text { bundle........... }\end{array}$ & - & - & - & - & $+\sigma^{7}$ & - \\
\hline $\begin{array}{c}\text { Thickness of trans- } \\
\text { verse section at } \\
\text { midrib......... }\end{array}$ & - & - & - & - & $+\sigma^{7}$ & - \\
\hline Flower stalk: & & & & & & \\
\hline $\begin{array}{l}\text { Epidermis at top: } \\
\text { Length of cells. . . }\end{array}$ & - & - & - & $+\sigma^{7}$ & - & - \\
\hline Width of cells..... & - & - & - & - & $+\infty^{x}$ & - \\
\hline $\begin{array}{r}\text { Kind of hairs pre- } \\
\text { sent............. }\end{array}$ & + & - & - & - & - & - \\
\hline Number of hairs... & - & - & - & $+\sigma^{7}$ & - & - \\
\hline $\begin{array}{r}\text { Length of pointed } \\
\text { hairs } \ldots \ldots \ldots \ldots\end{array}$ & - & - & - & $+q$ & - & - \\
\hline Color................. & - & - & - & $+\phi=0$ & - & - \\
\hline
\end{tabular}


TABLE I.-Continued.

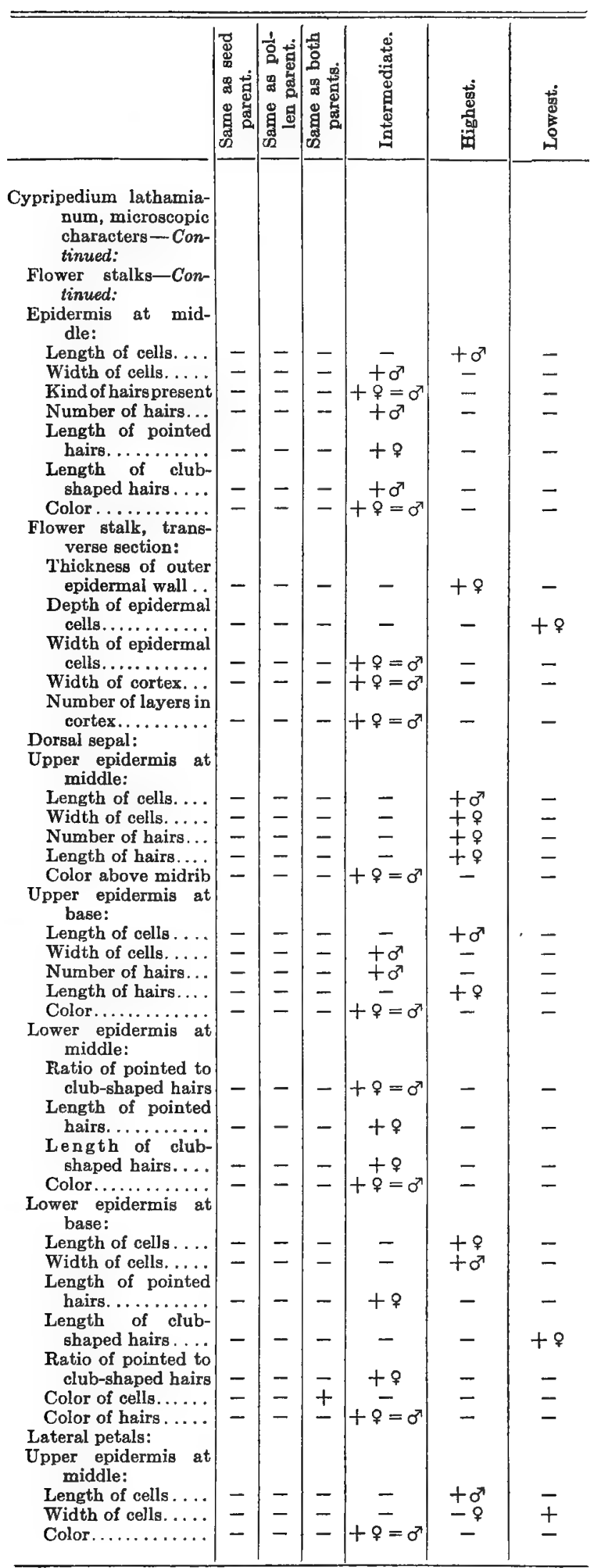

TABLE I-Continued

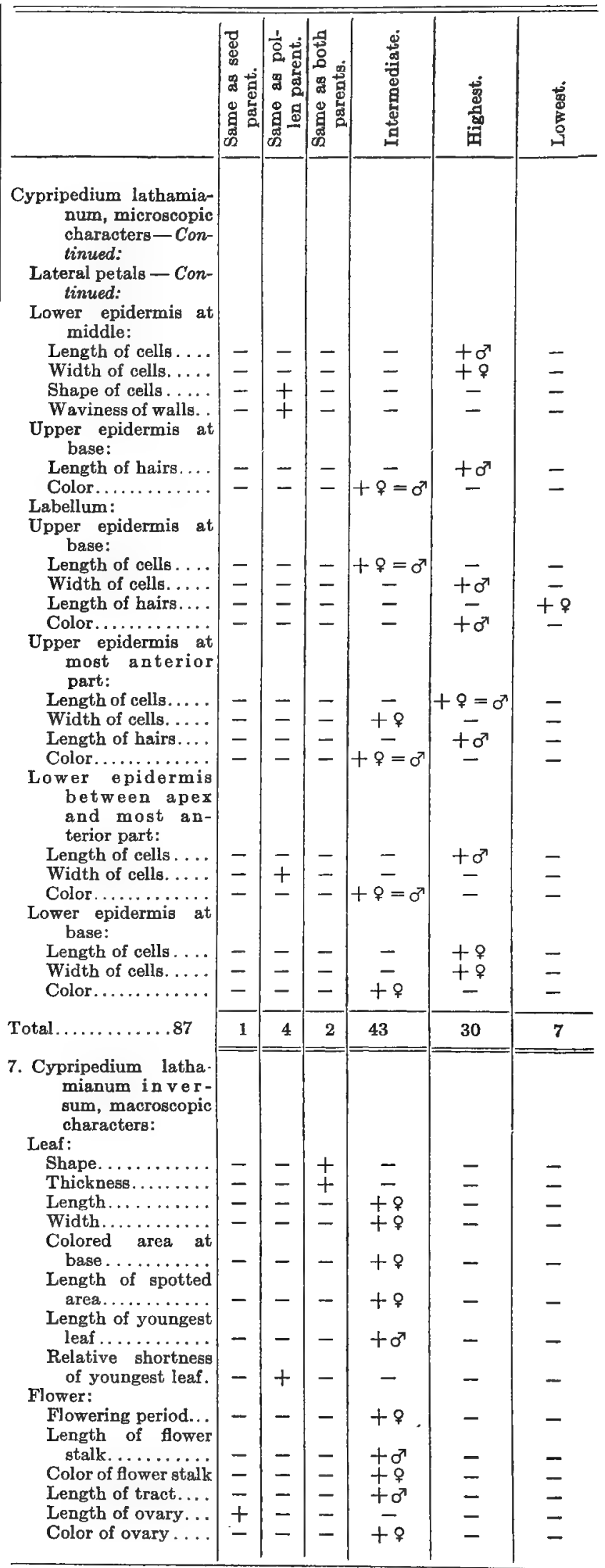


TABLE I-Continued.

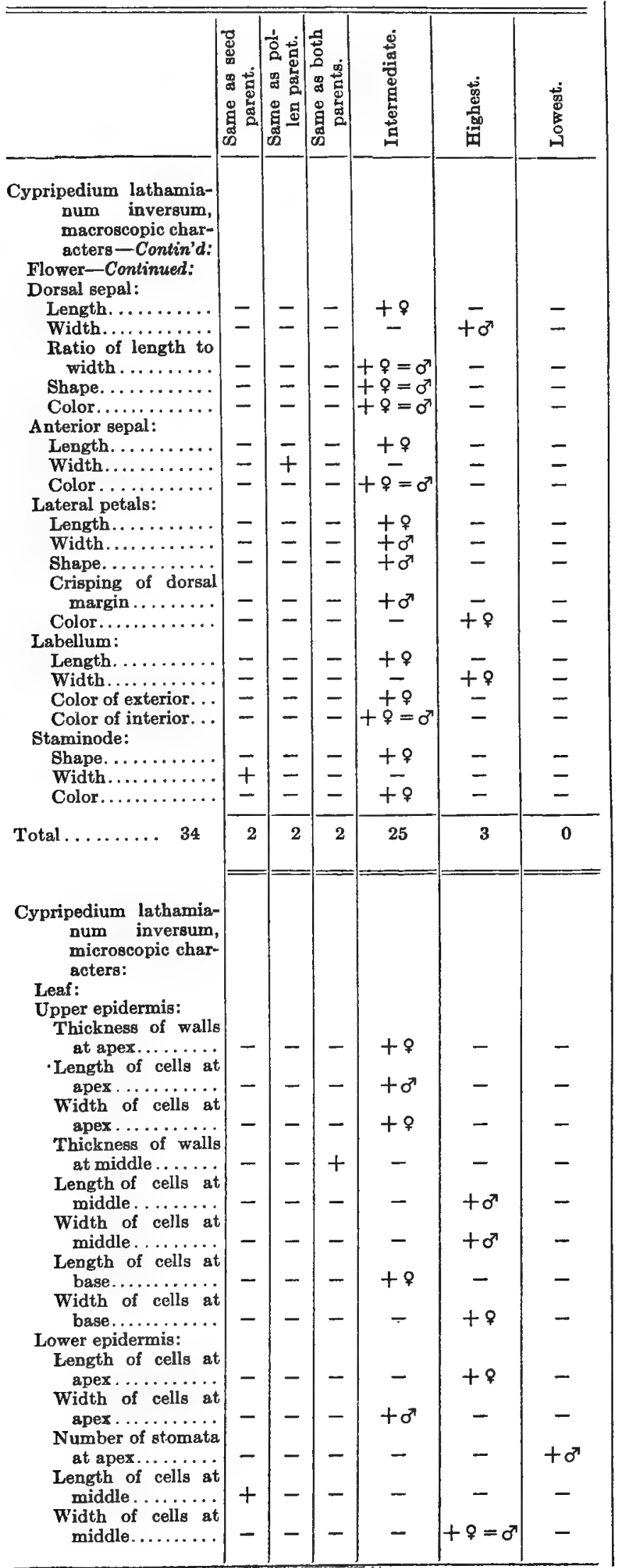

TABle I.-Continued.

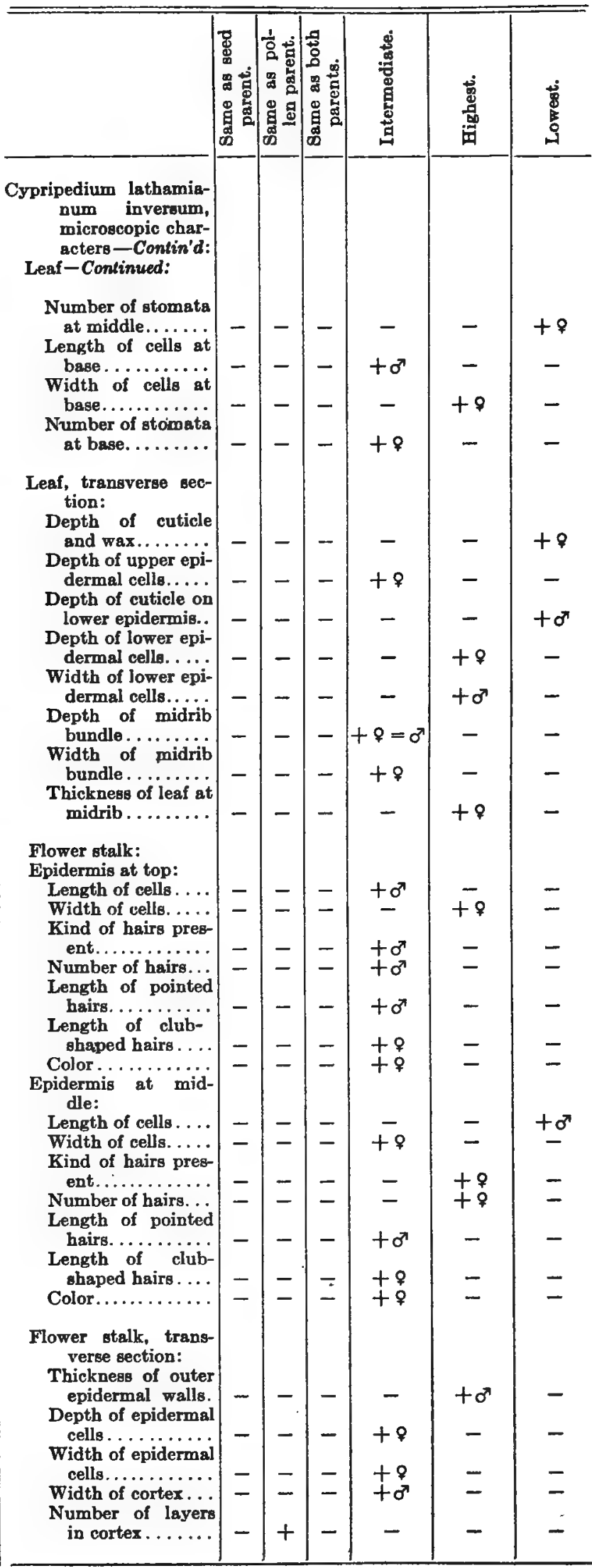


TABLE I-Continued.

\begin{tabular}{|c|c|c|c|c|c|c|}
\hline & 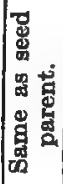 & 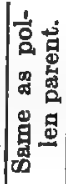 & 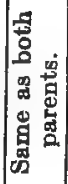 & 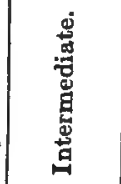 & 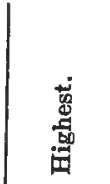 & 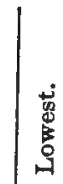 \\
\hline \multicolumn{7}{|l|}{$\begin{array}{r}\text { Cypripedium Iathamianum } \\
\text { inversum, microscopic } \\
\text { characters - Contin'd: }\end{array}$} \\
\hline $\begin{array}{l}\text { Dorsal sepal: } \\
\text { Upper epid e r m is at } \\
\text { middle: }\end{array}$ & & & & & & \\
\hline Length of cells . . . . . . . & 一 & - & 一 & - & $+\%$ & - \\
\hline Width of cells. . . . . . . & - & - & - & - & $+\sigma^{x}$ & - \\
\hline Number of hairs. . . . . . . & - & - & - & - & $+\sigma^{2}$ & - \\
\hline Length of hairs. . . . . . . & - & - & - & - & $+\infty$ & - \\
\hline Color above midrib. ... & - & - & - & $+9=\sigma^{7}$ & - & - \\
\hline \multicolumn{7}{|l|}{ Upper epidermis at base: } \\
\hline Length of cells........ & - & - & - & - & +9 & - \\
\hline Width of cells........ & - & - & - & $+0^{7}$ & - & - \\
\hline Number of hairs..... & - & - & - & +8 & - & 一 \\
\hline Length of hairs....... & 一 & - & - & - & $+\sigma^{x}$ & - \\
\hline & \\
\hline $\begin{array}{l}\text { Lower epidermis at middle: } \\
\text { Length of pointed hairs } \\
\text { Length of club-shaped }\end{array}$ & - & 一 & - & +9 & - & - \\
\hline hairs ............. & - & - & - & - & - & $+\delta_{0}$ \\
\hline $\begin{array}{l}\text { Ratio of pointed to club- } \\
\text { shaped hairs......... }\end{array}$ & - & - & - & $+\%=\sigma^{x}$ & - & - \\
\hline \multicolumn{7}{|l|}{ Lower epidermis at base: } \\
\hline Length of cells. ........ & - & - & - & - & $+\sigma^{x}$ & - \\
\hline Width of cells. . . . . . . & - & - & - & - & $+q$ & - \\
\hline Length of pointed hairs & 一 & 一 & - & $+\sigma^{7}$ & - & - \\
\hline $\begin{array}{l}\text { Length of club-shaped } \\
\text { hairs. } \ldots \ldots \ldots \ldots\end{array}$ & - & - & - & $+\sigma^{\pi}$ & - & - \\
\hline $\begin{array}{l}\text { Ratio of pointed to club- } \\
\text { shaped hairs. ........ }\end{array}$ & - & - & - & + 우 $=\sigma^{7}$ & - & - \\
\hline Color of cells.......... & - & - & + & - & - & - \\
\hline $\begin{array}{l}\text { Color of hairs. . . . . . } \\
\text { Lateral petals: }\end{array}$ & - & - & - & $+9=\sigma^{7}$ & - & - \\
\hline \multicolumn{7}{|l|}{$\begin{array}{l}\text { Lateral petals: } \\
\text { Upper epid e rmis at } \\
\text { middle: }\end{array}$} \\
\hline Length of cells . . . . . . . . & - & - & - & - & 十 9 & - \\
\hline Width of cells......... & - & - & 一 & $+\sigma^{x}$ & - & - \\
\hline Color. . . . . . . . . . & - & - & - & $+0=\sigma^{3}$ & - & - \\
\hline \multicolumn{7}{|l|}{$\begin{array}{l}\text { Lower epidermis at } \\
\text { middle: }\end{array}$} \\
\hline Length of cells. . . . . . . & - & - & - & - & $+q$ & - \\
\hline Width of cells. . . . . . . & - & - & - & - & $+\sigma^{7}$ & - \\
\hline Shape of cells........ & + & - & - & - & - & - \\
\hline Waviness of walls...... & + & - & - & - & - & - \\
\hline \multicolumn{7}{|l|}{ Upper epidermis at base: } \\
\hline Length of hairs. . . . . . . & - & - & 一 & - & +9 & - \\
\hline $\begin{array}{l}\text { Color............ } \\
\text { Labellum: }\end{array}$ & - & - & - & $+\rho=\sigma^{\top}$ & - & - \\
\hline \multicolumn{7}{|l|}{$\begin{array}{l}\text { Labellum: } \\
\text { Upper epidermis at base: }\end{array}$} \\
\hline Length of cells. . . . . . . & 一 & - & - & 一 & +0 & - \\
\hline Width of cells. . . . . . . & - & $一$ & - & - & - & $+\sigma^{x}$ \\
\hline Length of hairs. . . . . . . & $\rightarrow$ & - & - & + 우 & - & - \\
\hline Color . . . . . . . . . . & - & - & - & +8 & 一 & - \\
\hline \multicolumn{7}{|l|}{$\begin{array}{l}\text { Upper epidermis at most } \\
\text { anterior part: }\end{array}$} \\
\hline Length of cells. . . . . . . & - & - & - & + & $+\phi=\sigma^{r}$ & - \\
\hline Width of cells. . . . . . . & - & - & - & - & $+\sigma$ & - \\
\hline Length of hairs....... & - & - & - & $+\sigma^{x}$ & - & - \\
\hline \multirow{2}{*}{\multicolumn{7}{|c|}{$\begin{array}{l}\text { Lower epidermis between } \\
\text { apex and most ante- } \\
\text { rior part: }\end{array}$}} \\
\hline & & & & & & \\
\hline Length of cells. . . . . . . & - & - & - & - & +9 & - \\
\hline Width of cells........ & - & - & - & - & +9 & - \\
\hline Color............... & - & - & - & - & +8 & - \\
\hline \multicolumn{7}{|l|}{$\begin{array}{l}\text { Color. ................ } \\
\text { Lower epidermis at base: }\end{array}$} \\
\hline Length of cells. . . . . . . & - & - & - & 一 & $+\sigma^{7}$ & - \\
\hline Width of cells. ........ & - & - & - & - & - & +8 \\
\hline Color............... & - & - & - & - & $+\sigma^{2}$ & - \\
\hline otal. & 3 & 1 & 2 & 41 & 33 & 8 \\
\hline
\end{tabular}

TABLE I.-Continued

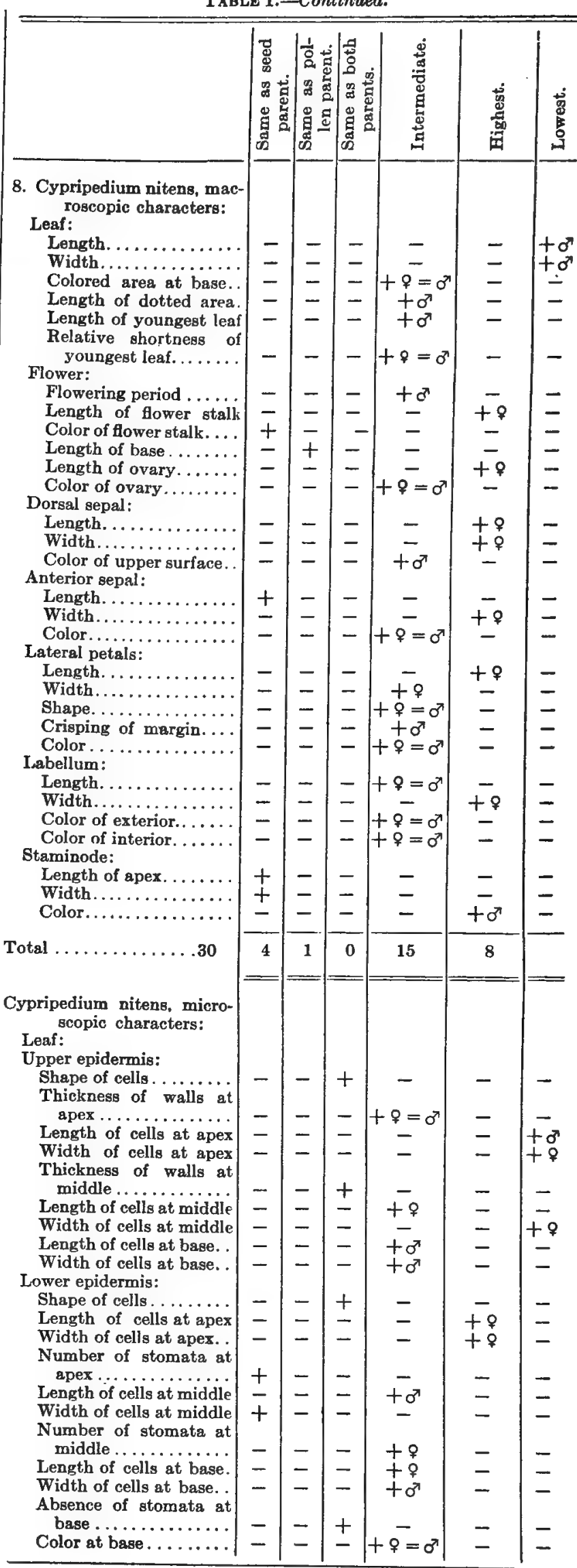


Table I.-Continued.

\begin{tabular}{|c|c|c|c|c|c|c|}
\hline & 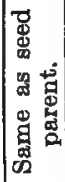 & 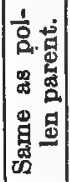 & 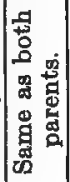 & 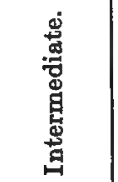 & 苞 & 莒 \\
\hline \multicolumn{7}{|l|}{$\begin{array}{l}\text { Cypripedium nitens, micro- } \\
\text { scopic characters - } \\
\text { Continued: }\end{array}$} \\
\hline $\begin{array}{l}\text { Leai, transverse section: } \\
\text { Depth of cuticle and wax } \\
\text { Depth of upper epider- }\end{array}$ & - & - & - & - & - & $+\sigma^{7}$ \\
\hline mal cells............. & - & - & - & - & - & +9 \\
\hline $\begin{array}{l}\text { lower epidermis....... } \\
\text { Depth of lower epider- }\end{array}$ & - & - & - & - & - & $+\sigma^{7}$ \\
\hline $\begin{array}{r}\text { mal cells.............. } \\
\text { Width of lower epider- } \\
\text { mal cells. }\end{array}$ & - & - & - & - & $\begin{array}{c}+\% \\
-\end{array}$ & $\begin{array}{l}- \\
+0\end{array}$ \\
\hline Depth of midrib bundle & - & - & - & - & $+\%$ & 17 \\
\hline Width of midrib bundle & - & - & - & - & + + & - \\
\hline Thickness of transverse & & & & & & \\
\hline $\begin{array}{l}\text { section at midrib.... } \\
\text { Flower stalk: }\end{array}$ & - & - & - & $+q$ & - & - \\
\hline Epidermis at top: & & & & & & \\
\hline Shape of cells......... & - & - & + & - & - & - \\
\hline Length of cells........ & - & - & - & - & - & $+\infty^{2}$ \\
\hline Width of cells......... & - & - & - & $+0^{7}$ & - & - \\
\hline Thickness of walls..... & - & - & + & - & - & - \\
\hline $\begin{array}{l}\text { Ratio of pointed to club- } \\
\text { shaped hairs. ....... }\end{array}$ & - & - & - & $+8+8$ & - & - \\
\hline Number of hairs........ & - & - & - & $+9=0^{3}$ & - & - \\
\hline Length of pointed hairs & - & - & - & $+\sigma^{\pi}$ & - & - \\
\hline Length of club-shaped & + & - & - & - & - & - \\
\hline Color. ................ & + & - & - & - & - & - \\
\hline Epidermis at middle: & & & & & & \\
\hline Length of cells . . . . . . . & - & - & - & - & - & $+\sigma^{7}$ \\
\hline Width of cells............ & - & - & - & - & $+q$ & - \\
\hline $\begin{array}{l}\text { Ratio of pointed to club- } \\
\text { shaped hairs........ }\end{array}$ & - & - & - & - & $+0=c^{\lambda}$ & - \\
\hline $\begin{array}{l}\text { 8naped hairs.......... } \\
\text { Number of hairs...... }\end{array}$ & - & $\overline{-}$ & - & $+\sigma^{7}$ & $-7=0$ & - \\
\hline Length of pointed hairs & - & - & - & $+\sigma^{7}$ & - & - \\
\hline $\begin{array}{c}\text { Length of club-shaped } \\
\text { hairs } \ldots \ldots \ldots \ldots\end{array}$ & - & - & - & - & - & $+\sigma^{7}-1-1$ \\
\hline $\begin{array}{c}\text { Flower stalk, transverse } \\
\text { section: }\end{array}$ & & & & & & \\
\hline $\begin{array}{c}\text { Thickness of outer epi- } \\
\text { dermal wall.......... }\end{array}$ & - & + & - & - & - & \\
\hline Shape of epidermal cells & - & - & + & - & - & - \\
\hline Depth of epidermal cells & - & - & - & $+q$ & - & - \\
\hline Width of epidermal cells & - & - & - & $+\phi=\sigma^{x}$ & - & - \\
\hline Width of cortex....... & - & - & - & $+\sigma^{\pi}$ & - & - \\
\hline $\begin{array}{r}\text { Number of layers in } \\
\text { cortex............ }\end{array}$ & - & + & - & - & - & - \\
\hline Dorsal sepal: & & & & & & \\
\hline $\begin{array}{l}\text { Upper epidermis at } \\
\text { middle: }\end{array}$ & & & & & & \\
\hline Length of cells........ & - & - & - & - & $+\sigma^{\pi}$ & - \\
\hline Width of cells. . . . . . . & - & - & - & - & $+\sigma^{7}$ & \\
\hline Color $\ldots \ldots \ldots \ldots \ldots$ & - & - & - & $+\sigma^{7}$ & - & - \\
\hline Upper epidermis at base: & & & & & & \\
\hline . Length of cells . . . . . . . & - & $\overline{1}$ & - & - & $+\infty$ & - \\
\hline Width of cells ......... & - & + & - & - & & \\
\hline
\end{tabular}

TABLE I.-Continued.

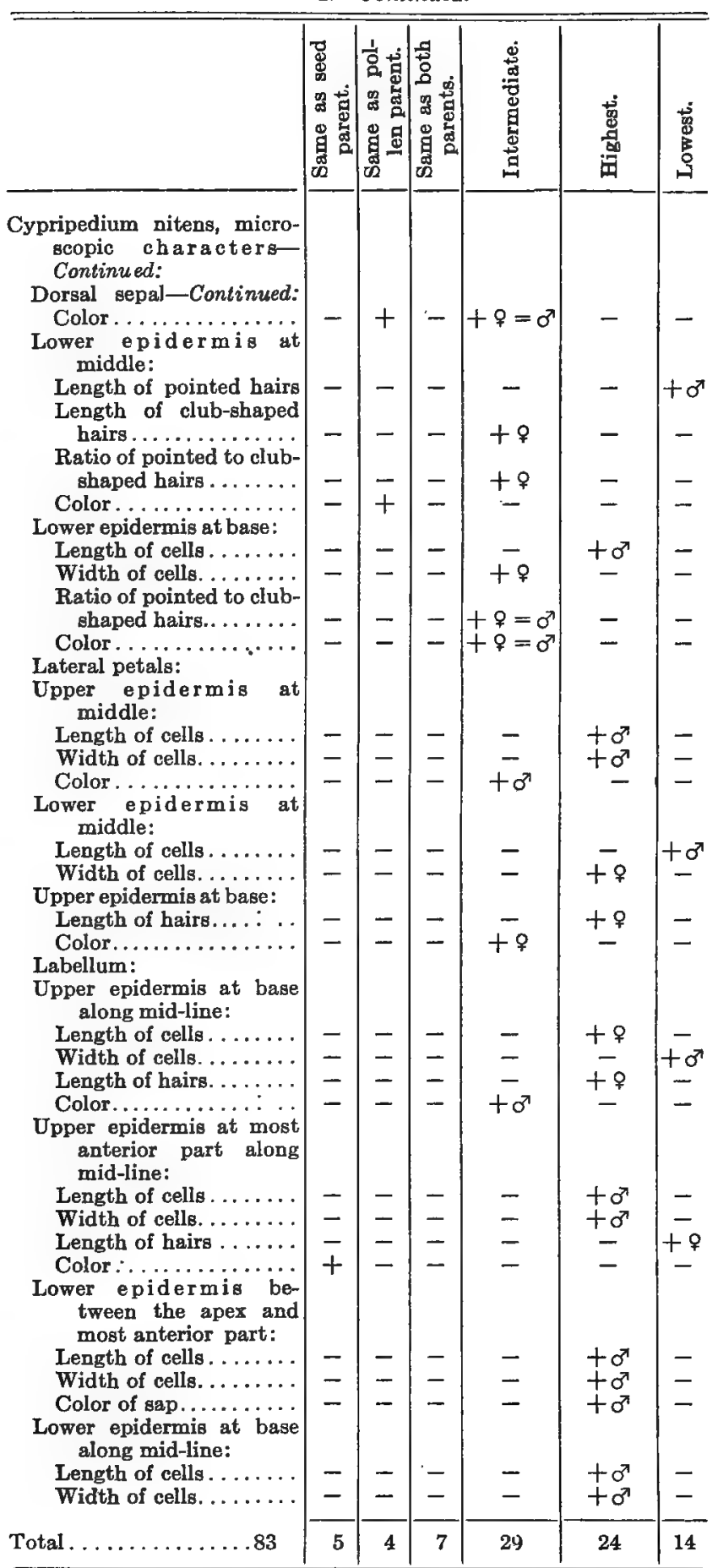


1. Summary of TABle I.-Recapitulation o the totals of the Microscopic and Macroscopic Characters of the Hybrids in relation to the Parents.

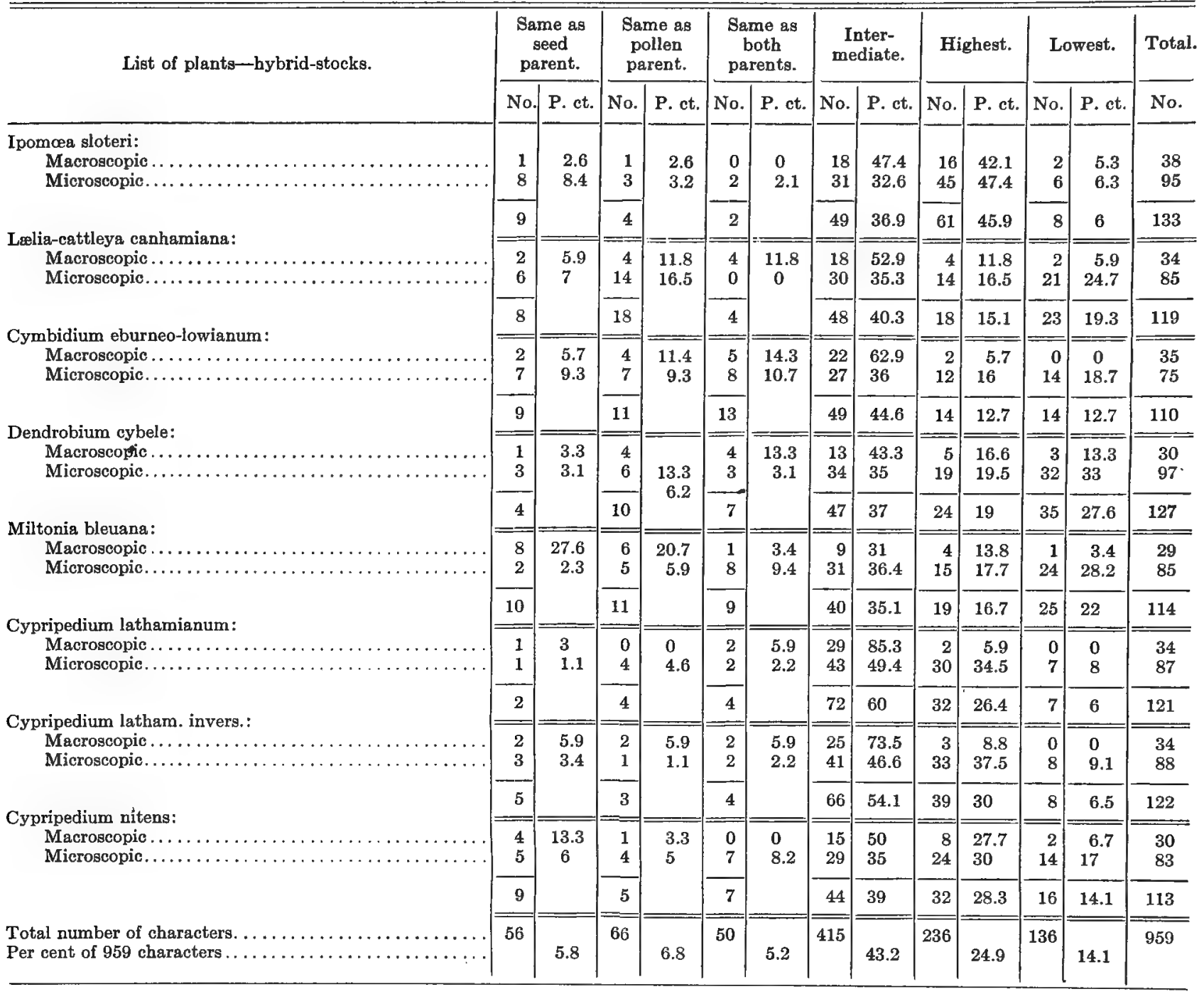

2. Summart of Table I.-Numbers and Percentages of the Macroscopic and Microscopic Characters of Hybrid-stocks as regards Sameness, Intermediateness, Excess, and Deficit of Development in Relation to the Parent-stocks.

\begin{tabular}{|c|c|c|c|c|c|c|c|}
\hline List of plants-hybrid-stocks. & $\begin{array}{l}\text { Same as } \\
\text { seed } \\
\text { parent. }\end{array}$ & $\begin{array}{l}\text { Same as } \\
\text { pollen } \\
\text { parent. }\end{array}$ & $\begin{array}{l}\text { Same as } \\
\text { both } \\
\text { parents. }\end{array}$ & $\begin{array}{l}\text { Inter- } \\
\text { mediate. }\end{array}$ & Highest. & Lowest. & Total. \\
\hline 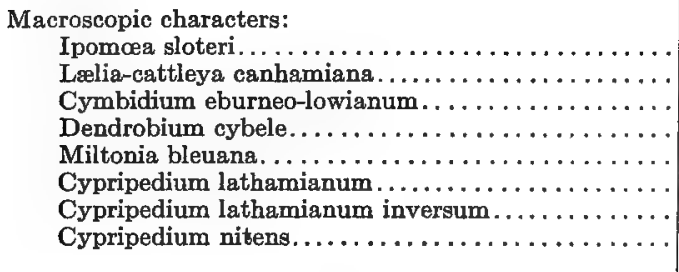 & $\begin{array}{l}1 \\
2 \\
2 \\
1 \\
8 \\
1 \\
2 \\
4\end{array}$ & $\begin{array}{l}1 \\
4 \\
4 \\
4 \\
6 \\
0 \\
2 \\
1\end{array}$ & $\begin{array}{l}0 \\
4 \\
5 \\
4 \\
1 \\
2 \\
2 \\
0\end{array}$ & $\begin{array}{r}18 \\
18 \\
22 \\
13 \\
9 \\
29 \\
25 \\
15\end{array}$ & $\begin{array}{r}16 \\
4 \\
2 \\
5 \\
4 \\
2 \\
3 \\
8\end{array}$ & $\begin{array}{l}2 \\
2 \\
0 \\
3 \\
1 \\
0 \\
0 \\
2\end{array}$ & $\begin{array}{l}38 \\
34 \\
35 \\
30 \\
29 \\
34 \\
34 \\
20\end{array}$ \\
\hline 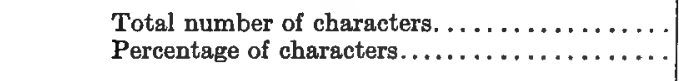 & $\begin{array}{l}21 \\
7.9\end{array}$ & $\begin{array}{l}22 \\
8.7\end{array}$ & $\begin{array}{l}18 \\
6.8\end{array}$ & $\begin{array}{r}149 \\
56.4\end{array}$ & $\begin{array}{c}44 \\
16.6\end{array}$ & $\begin{array}{r}10 \\
3.8\end{array}$ & 264 \\
\hline 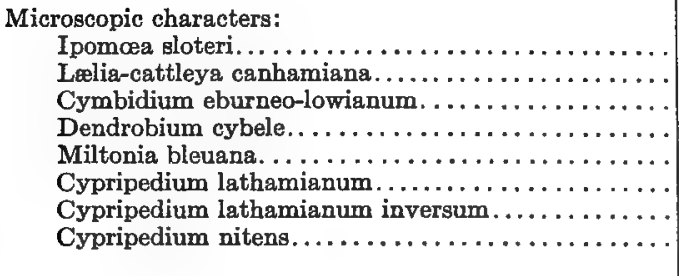 & $\begin{array}{l}8 \\
6 \\
7 \\
3 \\
2 \\
1 \\
3 \\
5\end{array}$ & $\begin{array}{r}3 \\
14 \\
7 \\
6 \\
5 \\
4 \\
1 \\
4\end{array}$ & $\begin{array}{l}2 \\
0 \\
8 \\
3 \\
8 \\
2 \\
2 \\
7\end{array}$ & $\begin{array}{l}31 \\
30 \\
27 \\
34 \\
31 \\
43 \\
41 \\
29\end{array}$ & $\begin{array}{l}45^{-} \\
14 \\
12 \\
19 \\
15 \\
30 \\
33 \\
24\end{array}$ & $\begin{array}{r}6 \\
21 \\
14 \\
32 \\
24 \\
7 \\
8 \\
14\end{array}$ & $\begin{array}{l}95 \\
85 \\
75 \\
97 \\
85 \\
87 \\
88 \\
83\end{array}$ \\
\hline 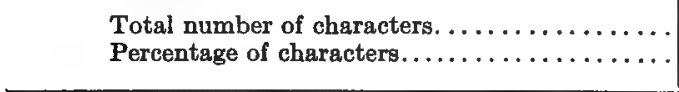 & $\begin{array}{r}35 \\
5 .\end{array}$ & $\begin{array}{l}44 \\
6.5\end{array}$ & $\begin{array}{l}32 \\
4.7\end{array}$ & $\begin{array}{r}266 \\
38.2\end{array}$ & $\begin{array}{r}192 \\
27.6\end{array}$ & $\begin{array}{r}126 \\
18.1\end{array}$ & 695 \\
\hline
\end{tabular}


3. Summary of Table I.-Numbers and Percentages of Tiseue Characters and Starch Reaction-intensities of the Hybrid-stocks in regard to Sameness, Intermediateness, and Excess, and Deficit of Development in Relation to the Parent-stocks. Charts F 9 and F 10.

\begin{tabular}{c} 
Parent-relationships. \\
\hline
\end{tabular}

4. Summary or Tabe I.-Summary of Sameness and Inclination of the Macroscopic and Microscopic Characters of the Hybridstocks in Relation to the Parent-stocks.

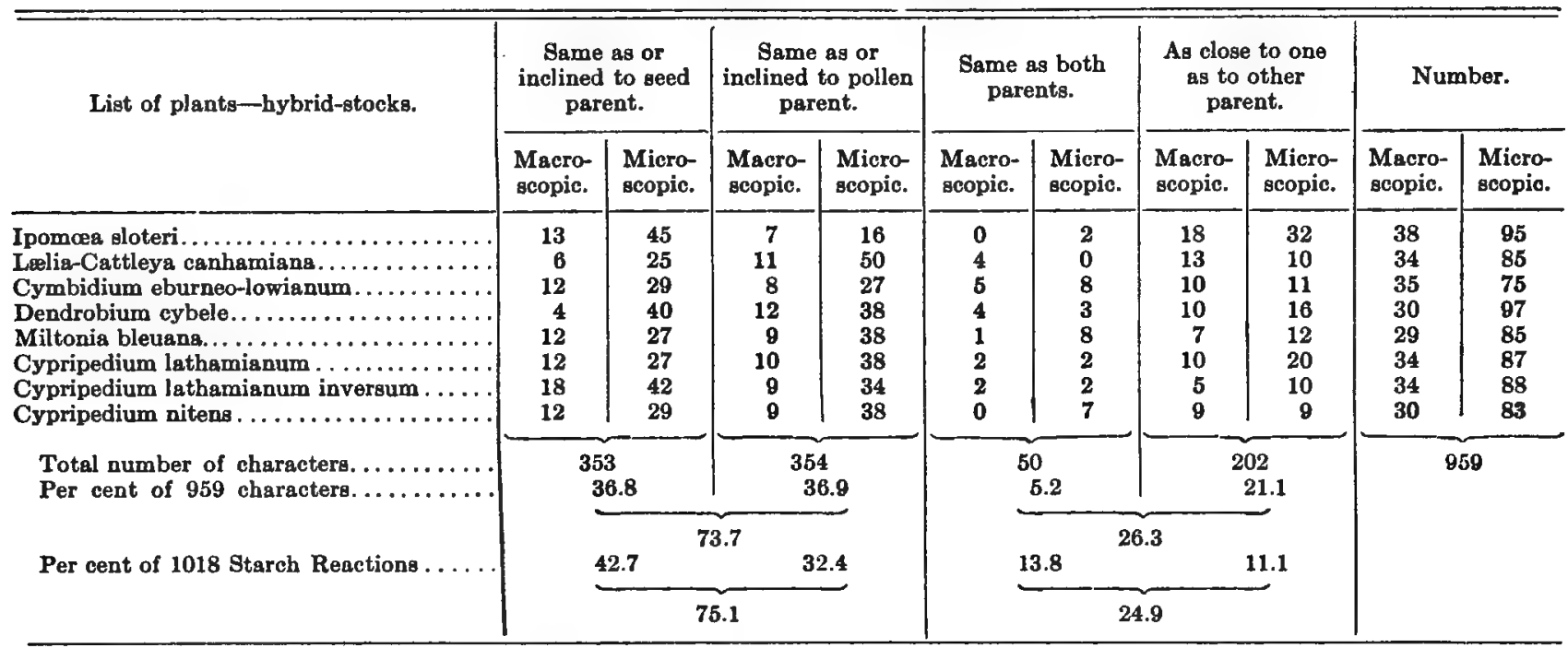

5. SUMmary or TABLE I.-Summary of the Macroscopic and Microscopic Characters and of the Starch Reaction-Intensities of Cymbidium eburneo-lowianum and Millonia blewana in regard to Sameness, Intermediateness, and Excess and Deficit of Development in relation to the Parent-Slocks. Charts F 11 and F 12.

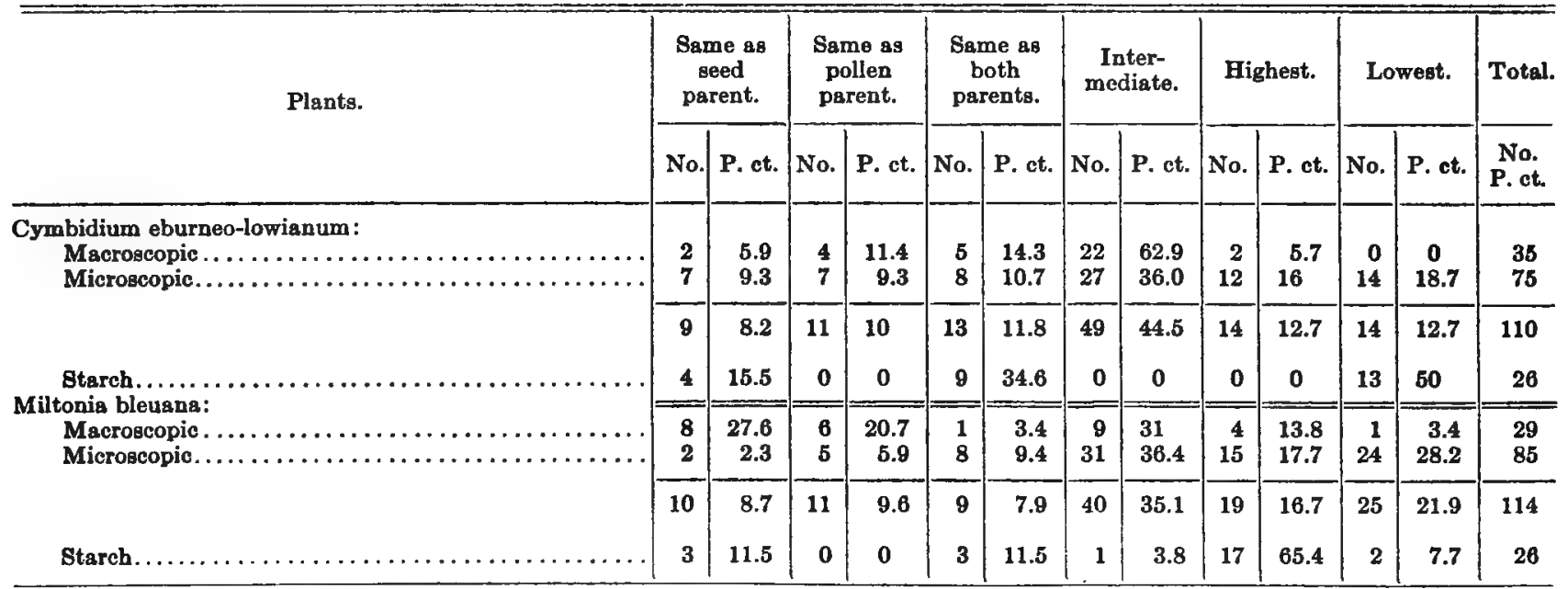


6. Summary or TABte I.-Summary af Sameness and Inclination of the Macroscopic and Microscopic Characters and of the Starch Reaction-Intensities of Cymbidium eburneo-lowianum and Miltonia bleuana in relation to the Parent-Stocks. Charls F 13 and F 14.

\begin{tabular}{|c|c|c|c|c|c|c|c|c|c|}
\hline \multirow[t]{2}{*}{ Plants. } & \multicolumn{2}{|c|}{$\begin{array}{l}\text { Same as or } \\
\text { inclined to } \\
\text { geed parent. }\end{array}$} & \multicolumn{2}{|c|}{$\begin{array}{l}\text { Same as or } \\
\text { inclined to } \\
\text { pollen parent. }\end{array}$} & \multicolumn{2}{|c|}{$\begin{array}{l}\text { Same as or } \\
\text { inclined to } \\
\text { both parents. }\end{array}$} & \multicolumn{2}{|c|}{$\begin{array}{l}\text { As close to one } \\
\text { as to the other } \\
\text { parent. }\end{array}$} & \multirow{2}{*}{$\begin{array}{l}\text { Total. } \\
\text { No. } \\
\text { P. ct. }\end{array}$} \\
\hline & No. & P. ct. & No. & P. ct. & No. & P. ct. & No. & P. ct. & \\
\hline \multirow[t]{2}{*}{ 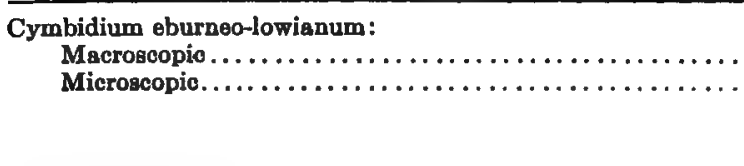 } & $\begin{array}{l}12 \\
29\end{array}$ & $\begin{array}{l}34.3 \\
38.6\end{array}$ & $\begin{array}{r}8 \\
27\end{array}$ & $\begin{array}{l}29.9 \\
36\end{array}$ & $\begin{array}{l}5 \\
8\end{array}$ & $\begin{array}{l}14.3 \\
10.7\end{array}$ & $\begin{array}{l}10 \\
11\end{array}$ & $\begin{array}{l}28.6 \\
14.7\end{array}$ & $\begin{array}{l}35 \\
75\end{array}$ \\
\hline & 41 & 37.3 & 35 & 31.8 & 13 & 11.8 & 21 & 19.1 & 110 \\
\hline \multirow{4}{*}{ 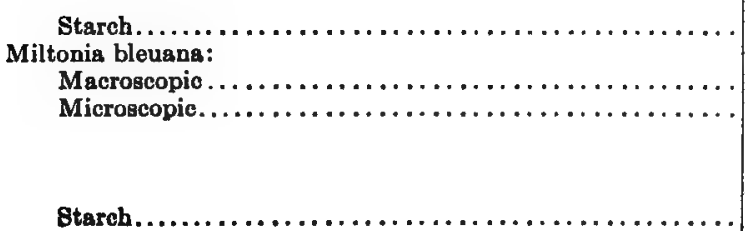 } & 4 & 15.4 & 1 & 3.8 & 9 & 34.6 & 12 & 46.2 & 26 \\
\hline & $\begin{array}{l}12 \\
27\end{array}$ & $\begin{array}{l}41.4 \\
31.8\end{array}$ & $\begin{array}{r}0 \\
38\end{array}$ & $\begin{array}{l}31 \\
44.7\end{array}$ & $\begin{array}{l}1 \\
8\end{array}$ & $\begin{array}{l}3.5 \\
9.4\end{array}$ & $\begin{array}{r}7 \\
12\end{array}$ & $\begin{array}{l}24.1 \\
14.1\end{array}$ & $\begin{array}{l}29 \\
85\end{array}$ \\
\hline & 39 & 34.2 & 47 & 41.2 & 9 & 7.9 & 19 & 16.7 & 114 \\
\hline & 20 & 77 & 2 & 7.7 & 3 & 11.6 & 1 & 3.8 & 26 \\
\hline
\end{tabular}

7. SUMMARY OF TABLE I-Tissue Characters and Starch Reactions as Regards Intermediateness and Non-Intermediateness of the Hybrids.

\begin{tabular}{|c|c|c|c|c|}
\hline \multirow{2}{*}{ Characters. } & \multicolumn{2}{|c|}{$\begin{array}{l}\text { Intermediate- } \\
\text { ness. }\end{array}$} & \multicolumn{2}{|c|}{$\begin{array}{c}\text { Non-inter- } \\
\text { mediatenegs. }\end{array}$} \\
\hline & No. & P. ct. & No. & P. ct. \\
\hline $\begin{array}{l}\text { Tissue characters: } \\
\text { Macroscopic } \ldots \ldots \ldots \ldots \ldots \ldots \\
\text { Microscopic } \ldots \ldots \ldots \ldots \ldots \ldots\end{array}$ & $\begin{array}{l}149 \\
266\end{array}$ & $\begin{array}{l}56.4 \\
38.2\end{array}$ & $\begin{array}{l}115 \\
429\end{array}$ & $\begin{array}{l}43.6 \\
61.8\end{array}$ \\
\hline & 415 & 43.2 & 544 & 56.8 \\
\hline Btarch reactions $\ldots \ldots \ldots \ldots \ldots$ & 236 & 23.2 & 782 & 76.8 \\
\hline
\end{tabular}




\section{CHAPTER VI. APPLICATIONS OF RESULTS OF RESEARCHES.}

In considering the applications of the results of these researches to the explanation of the developmental changes in the germplasm, and of variations, fluctuations, sports, mutations, Mendelism, the genesis of species, etc., it must be borne in mind that the investigations (Publications Nos. 116, 173, and the present) have been of a purely exploratory character and no serious attempt has been made to do more than lay a substantial foundation for future investigation, theoretical and practical. Hence, in the present chapter nothing more than mere suggestions will be offered in the applications of the results of fundamental problems of biology; nor would more here be possible, if for no other reason than the enormity of the field to be covered.*

\section{Specificity of Stereorsomerides in Relation to Geniera, Species, Etc.}

These researches have as their essential basis the conception that in different organisms corresponding complex organic substances that constitute the supreme structural components of protoplasm and the major synthetic products of protoplasmic activity are not in any case absolutely identical in chemical constitution, and that each such substance may exist in countless modifications, each modification being characteristic of the form of protoplasm, the organ, the individual, the sex, the species, and the genus. This conception was supported not only by the extraordinary differences noted between the albuminous substances of venom and those of other parts of the serpent, $\uparrow$ but also by the results of the investigations of Hanriot, who described marked differences in the properties of the lipases of the pancreatic juice and the blood; of Hoppe-Seyler and others who stated that the pepsins of cold- and warm-blooded animals are not identical; of Wróblewsky and others who recorded differences in the pepsins of mammals; of Kossell and his students who found that the protamins obtained from the spermatozoa of different species of fish are not identical; and of various observers who have noted that the erythrocytes of one species when injected into the blood of another are in the nature of foreign bodies and rapidly destroyed. During subsequent years, and especially very recently, data have been rapidly accumulating along many and diverse lines of investigation which collectively indicate that every individual is a chemical entity that differs in characteristic particulars from every other. To any one familiar with the advances of biochemistry and with the trend of scientific progress toward the explanation of vital phenomena on a physico-chemical basis, it will be obvious that if the conception of the non-uniform constitution of

*The first three sections of this chapter are reproduced, with some alteration and addition, from an article that was published in Science, 1914, n.s., XI., 649-661.

tResearches upon the Venoms of Poisonous Serpents. By S. Weir Mitchell and Edward T. Reichert. Smithsonian Contributions to Knowledge, Publication No.647, 1886. 360 corresponding proteins and other corresponding complex organic substances in different organisms and parts of organisms were found to be justified by the results of laboratory investigation a bewildering field of speculation, reasoning, and investigation would be laid opena field so extensive as to include every domain of biological science, and seemingly to render possible, and even probable, a logical explanation of the mechanisms underlying the differentiations of individuals, sex, varieties, species, and genera; of the causes of fluctuations and mutations; of the phenomena of Mendelism and heredity in general; of the processes of fecundation and sex-determination; of the tolerance of certain organisms to organic poisons that may be extremely virulent to other forms of life ; of tumor formation, reversions, malformations, and monsters; of anaphylaxis, certain toxemias, immunities, etc.; and of a vast number of other phenomena of normal and abnormal life which as yet are partially or wholly clothed in mystery.

Some years previous to the discovery of the nature of the lethal constituents of venoms, Pasteur found that there exist three kinds of tartaric acid which, because of different effects on the ray of polarized light, are distinguished as the dextro-, lævo- and racemic-tartaric acids, the dextro form rotating the ray to the right, the lævo form to the left, and the racemic form not at all. When these acids were subjected in separate solutions to the actions of Penicillium glaucum fermentation proceeded in the dextro form, but not in the lævo form, while in the solution of the racemic acid, which is a mixture of the dextro and lævo acids, the dextro form disappeared, leaving the lævo moiety unaffected. All three acids have the same chemical composition and chemical properties, but differ strikingly in their effects on polarized light and in nutritive properties. Identical or corresponding peculiarities have since been recorded in relation to a large number of substances. Thus, of the twelve known forms of hexoses, or glucoses, only the dextro forms are fermentable, that is, capable of being used by certain low organisms as food, but not all are thus available, and, moreover, those which are show marked differences in the degrees of fermentability. In the case of other substances Penicillium may consume the lævo form, but not the dextro form. Other organisms show similar selectivities, using either dextro or lævo form, or both, but in the latter case in unequal degree. Even more striking instances have been recorded in the actions of poisons, as, for instance, dextro-nicotine is only half as toxic as the lævo form; dextro-adrenalin has only one-twelfth the power of the lævo form; racemic-cocaine has a quicker and more intense but less lasting action than the lævo form; the asparagines, hyoscines, hyoscyamines and other substances have been found to exhibit marked differences in accordance with variations in their optical properties. With other bodies belonging to this category it may be 
found that one form is sweet while another is tasteless; another may be odorous, but its enantiomorphous form without odor.

To the foregoing there may be added examples of other substances that exist in several forms, but which physico-chemically belong to a different class. Thus, nitroglycerine may exist in forms that are so different that under given conditions of temperature and percussion one is explosive and the other non-explosive. Differences in substances which are found in allotropic forms may be as marked as in any of the preceding illustrations, as, for instance, in the case of phosphorus, which is farmiliar as the yellow, white, black, and red varieties, all of which with the exception of red phosphorus are exceedingly poisonous, while the latter is inert. The ortho, meta, and para forms of a given substance may exhibit more or less marked physiological and toxicological variations, and so on.

The explanation of the remarkable differences shown by these substances, which differences are paralleled by those manifested by the lethal and inocuous proteins of the serpent, the pepsins, the protamins and the red-blood corpuscles, is to be found in the results of two independent but intimately related lines of physioo-chemical research : (1) The investigations of Van't Hoff and LeBel and subsequent observers which have laid the foundation of a new, and to the biologist and physician an extraordinarily important, development of chemistry known as stereochemistry-a department that treats of the arrangements of the atoms, groups and masses of molecules, or in other words of intramolecular arrangement or configuration of molecular components in the three dimensions of space. (2) The investigations of Willard Gibbs and others which have given us the "phase rule," which defines the phases or forms in which a given substance or combination of substances may exist owing to differences in intramolecular and extramolecular arrangements and concentration of their components in relation to temperature and pressure.

According to stereochemistry a given substance may exist in multiple forms dependent upon differences in the configuration of the molecule, all of which forms have in common the fundamental chemical characteristics of a given prototype, yet each may have certain properties which positively distinguish it from the others. Theoretically, such substances as serum albumin, serum globulin, hemoglobin, starch, glycogen, and chlorophyl may be produced by nature in countless modified forms, owing to differences in intramolecular arrangements. Miescher has estimated that the serum globulin molecule may exist in a thousand million forms. Substances that exist in such multiple forms of a prototype are distinguished as stereoisomers. The remarkable fact has been noted by Fischer and others that stereoisomers may exhibit as great or even greater differences in their properties than those manifested by even closely related isomers, which latter in comparison with stereoisomers are distantly if at all chemically related. As already instanced, so slight a change in molecular configuration as gives rise to dextro and lævo forms may be sufficient to cause definite and characteristic and even profound differences in physical, nutritive, and physiological properties.

In accordance with the "phase rule" a substance or a combination of substances may exist in the form of heterogeneous or homogeneous systems, a heterogeneous system consisting of a number of homogeneous systems, each of which latter is a manifestation of an individual phase and distinguishable from the others by physical, mechanical, chemical, or physiological properties. The number of phases of a heterogeneous system increases with the number of component systems and the number of the latter is in direct relationship to the number of independent variable constituents. Therefore, by means of variations of either or both intramolecular or extramolecular arrangement the number of forms of a substance or combination of substances may range from few to infinite.

Our means of differentiating stereoisomers are, on the whole, limited, and for the most part crude, and while it has been found that differences so marked as those referred to may be detected by the ordinary procedures, it seems obvious that the inherent limitations of such methods render them inadequate where a large number of stereoisomerides or related bodies which may exhibit only obscure modifications are to be definitely differentiated, so that other and more sensitive methods must be sought, or at least special methods that are adapted to exceptional conditions. The results of much preliminary investigation in this direction led in one research to the adoption of the crystallographic method, especially the use of the polarizing microscope, which in its very modern developments of analysis has demonstrated that substances which have different molecular structures exhibit corresponding differences in crystalline form and polariscopic properties; and, moreover, that the "optical reactions" may be found to be as distinctive and as exact analytically as the reactions obtained by the conventional methods of the chemist. Furthermore, the necessities of the hypothesis demanded the selection of a substance for study of a character which upon theoretical grounds might be expected to exist in nature widely distributed and readily procurable, and, as a consequence, hemoglobin was selected.

In the study of the hemoglobins the author had as a co-worker Professor Amos Peaslee Brown.* Hemoglobins were examined from over 100 animals, representing a large variety of species, genera, and families. From the data recorded certain facts are especially conspicuous, among which may be mentioned the following:

1. The constant recurrence of certain angles, plane and dihedral, in the hemoglobins of various species, even when the species are widely separated and the crystals belong to various crystal systems. This feature indicates a common structure of the hemoglobin molecules, whatever their source.

2. The constant recurrence of certain types of twinning in the hemoglobins, and the prevalence of mimosie. This has the same significance as the foregoing.

3. The constancy of generic characters in the crystals. The crystals of the various species of any genus belong to a crystallographic group. When their characters are tabulated they at once recall crystallographic groups of inorganic compounds. The crystals of the genus Felis constitute an isomorphous group which is as strictly isomorphous as the groups of rhombohedral and orthorhombic carbonates among minerals, or the more

* Carnegie Inst. Wash. Pub. No. 116. 
complex molecules of the members of the group of monosymmetric double sulphates.

4. The crystallographic specificity in relation to species. The crystals of each species of a genus, when they are favorably developed for examination in the polarizing microscope, can usually be distinguished from each other by definite angles and other properties, while preserving the isomorphous character belonging to the genus. Where, on account of difficulty of measurement, the differences can not be given a quantitative. value, variations in habit and mode of growth of the crystals often show specific differences.

5. The occurrence of several types' of oxy-hemoglobin in members of certain genera. In some species the oxyhemoglobin is dimorphous and in others trimorphous. Where several types of crystals occur in this way in the species of a genus the crystals of each type may be arranged in an isomorphous series. In other words, certain genera as regards the hemoglobins are isodimorphous and others isotrimorphous.

6. When orders, families, genera, or species are well separated the hemoglobins are correspondingly markedly differentiated. For instance, so different are the hemoglobins of Aves, Marsupiatia, Ungulata, and Rodentia that there would be no more likelihood of confounding the hemoglobins than there would be of mistaking the animals themselves. Even where there is much less zoological separation, as in the case of the genera of a given family, but where there is well-marked zoological distinction, the hemoglobins are so different as to permit readily of positive diagnosis. When, however, the relationships are close the hemoglobins are correspondingly close, so that in instances of an alliance such as in Canis, Vulpes, and Urocyon, which genera years ago were included in one genus (and doubtless correctly) the hemoglobins are very much alike, and in these cases they may exhibit closer resemblances than may be found in general in specimens obtained from well-separated species of a genus.

So distinctive zoologically are these modified forms of hemoglobins that we had no difficulty in recognizing that the common white rat is the albino of Mus norvegicus (Mus norvegicus albus Hatai) and not of Mus rattus, as almost universally stated, and that Ursidæ are related to Phocidæ (as suggested by Mivart 30 years ago), but not to Canidæ, as stated in modern works on zoology. Moreover, we were quick to detect errors in labeling, as, for instance, when a specimen marked as coming from a species of Papio was found to belong to one of the Felidx. Generic forms of hemoglobin when obtained from well-separated genera are, in fact, so different in their molecular structures that when any two are together in solution they do not fuse to form a single kind of hemoglobin or a homogeneous solution, but continue as discrete disunited particles, so that when crystallization occurs each crystallizes independently of the other and without modification other than that which is dependent upon such incidental conditions as are to be taken into account ordinarily during crystallization. Thus, the hemoglobin of the dog crystallizes in rhombic prisms which have a diamond-shaped cross-section; that of the guinea-pig in tetrahedra; that of the squirrel in hexagonal plates; and that of the rat in elongated sixsided plates. When any two of these hemoglobins are together in solution and crystallization occurs, each appears in its own form. Such phenomena indicate that the structures of the hemoglobin molecules are quite different; in fact, more differentiated than the molecules of members of an isomorphous group of simple carbonates, such as the carbonates of calcium and magnesium, which in separate solutions crystallize in rhombohedrons whose corresponding angles differ $2^{\circ} 1^{\prime}$, but in molecular union, as in the mineral dolomite, crystallize as a single substance which has an intermediate angle.

Upon the basis of our data it is not going too far to assume that it has been satisfactorily demonstrated theoretically, inferentially, and experimentally that at least this one substance (hemoglobin) may exist in an inconceivable number of stereoisomeric forms, ${ }^{*}$ each form being peculiar to at least genus and species and so decidedly differentiated as to render the "hemoglobin crystal test" more sensitive in the recognition of animals and animal relationships than the "zooprecipitin test."

Subsequent to the research referred to, investigations have been pursued in the study of hemoglobins from various additional sources, especially from representatives of Primates, with the result in the latter case of finding indubitable evidence of an ancestral alliance of man and the man-like apes.

More or less elaborate studies by crystallographic and other methods have also been made with other albuminous substances and with starches, glycogens, phytocholesterins, chlorophyls, and other complex synthetic products of animal and plant life, especially with starches, of which over 300 specimens were examined, obtained from representatives of a considerable number of families, genera, species, varieties, and hybrids. In all of these investigations the results are not only in full accord with those of the hemoglobin researches but, in some instances of broader significance, because by better methods of differentiation it was found possible to recognize not only peculiarities as regards genus or species, but also varieties and hybrids, and even to trace in hybrids with marked definiteness the transmission of parental characteristics.

Summing up the results of these independent but interwoven researches, we find that the modified forms of each of these substances lend themselves to a very definite system of classification, and to one that is in general accord with that of the botanist and zoologist, that is, each genus is characterized by a distinctive type of hemoglobin, albumin, starch, etc., as the case may be, which may be designated the generic-type; every species of the genus will have a modification of this type, which is a species-type, or generic primary sub-type; and every variety of a species will have a modification of the speciestype, that is a variety-type, or generic secondary subtype, or species sub-type. In fact, it seems clear that with revisions of present classifications that are certain to come there will be found definite family types; and, moreover, that with improved methods of differentiation there will be discovered positively distinctive sex- and

*Even if we assume that the different forms are not, strictly speaking, stereoisomers it must be admitted that hemoglobin exists in forms that are specifically modified in relation to genera and species. 
individual-types. This last statement already has support in the results of collateral lines of research which bear upon the specificities of enzymes, anaphylaxis, precipitin reactions, immune sera, etc.

From the foregoing data it seems obvious that the complex organic substances which may be assumed to constitute the essential fundamental constituents of protoplasm and the immediate complex synthetic products of protoplasmic activity may exist in exceedingly numerous or even countless stereoisomeric forms, each form being peculiarly and specifically modified in relation to genus, species, variety, race, sex, individual, or - even part of an individual.

Protoplasm a Complex Stereoisomeric System.

The next logical step in our investigation is manifestly the study of the bearings of these stereoisomers, as such and in their variable combinations and associations, upon the structure, processes, and products of protoplasm. Protoplasm, according to the modern developments of biochemistry, is to be regarded as being in the nature of an extremely complex, labile aggregate of proteins, fats, carbohydrates, and other substances that are peculiarly associated to constitute a physico-chemical mechanism. The possible number of "phases" in which such a system can exist varies with the forms of the stereoisomerides and in general with the number and independent variability of the components. In such a mechanism we conceive that the number of variables is inconceivably great. From analogy we believe that such mechanisms are so extremely sensitive that the properties and processes may be modified by even so slight a change as the substitution of one form of stereoisomeride for another of the same prototype. Were it practicable to examine all of the most complex of the organic structural components of protoplasm, it doubtless would be found that every one exists in a form peculiar to the individual and his position in classification. Moreover, we must conceive that the components of protoplasm are as specific in relation to the form of protoplasm as are the peculiar forms of stereoisomers, so that different forms of protoplasm are characterized physico-chemically (1) by the peculiarities of the stereoisomerides, and (2) by the peculiarities of the kinds, combinations, associations, and arrangements of the components in the three dimensions of space.

In accordance with the foregoing the human organism may be regarded as being a highly organized composite of heterogeneous physico-chemical systems that are composed of a vast number of parts, each such part representing a particular "phase" of the system and being physically, mechanically, chemically, and functionally an individual interacting unit of the aggregate. Hence, it follows that the sum or totality of these peculiarly modified stereoisomers per se, and of their arrangements with the associated components, constitutes a "stereochemic system" peculiar to the cell; that the sum of the cell-systems is peculiar to the tissue; that the sum of the tissue-systems is peculiar to the organ; and that the sum of the organ-systems is peculiar to the individual.

While the living organism had been for years recognized as being in the nature of an exceedingly complex physico-chemical aggregate of interacting independent and interdependent parts that constitute a single working unit in only recent years have the mechanisms that bring about co-operative activities of the various parts been made clear. The governing influences of the nervous system were found inadequate even in the highest organisms, not to speak of forms of life in which such actions occur, but in which there is apparently a total absence of nervous matter. As an associate of the nervous system, and doubtless far antedating it in organic evolution, is a correlative mechanism of a chemical character of the greatest importance, and doubtless equally so throughout the whole range of living organisms from the lowest to the highest. Every living cell, whether it be in the form of a unicellular organism or a component of a multicellular organism, is undoubtedly in the nature of a heterogeneous stereochemic system, each of the component parts of the system forming substances which may affect directly or indirectly the activities of the processes of the other parts; likewise, every cell of a multicellular organism is not only in itself a heterogeneous system, but a part of a number of associated heterogeneous systems and which by virtue of certain of its products, with or without the agency of the bloodvascular or lymph-vascular systems, may exercise influences upon other structures, which structures may have or seemingly not have either structural or physiological relationship. Thus we find that a secretin formed in the pyloric glands of the gastric mucosa may excite the glands of the cardia; that growth is determined by some product or products of the pituitary body that are carried to the various structures; that the liver, pancreas and intestinal glands are excited to secretory activity by a peculiar substance formed in the duodenal and jejunal mucosæ; that carbohydrate metabolism in the liver and muscles is influenced to a profound degree by hormones that are formed in the pancreas; that lactation is determined essentially by substances derived from the corpus luteum, placenta, and involuting womb; that the periods of ovulation and menstruation are inhibited by secretions of the corpus luteum; that vitally important states of activity of the generative organs are directly associated with functions of the adrenal and other glands ; and that normal development, especially of secondary sexual characters, is intimately related to the ovaries and testicles. To these extraordinary correlations might be added many others. Some of the bodily structures are in this way so definitely associated in their activities as to constitute co-operating or interacting systems, so that the tissue products are complementary, supplementary, synergistic, or antagonistic in their influences upon given structures. Such correlations must be, for perfectly obvious reasons, one of the most primitive forms of interprotoplasmic correlation, and we are justified, upon the basis of our present knowledge, in the conclusion that each active part of a cell, each cell, each tissue and each organ contributes products which may affect the activities of functionally related or unrelated parts. Hence would follow the dictum that not only is every part of a cell, every cell, every tissue, and every organ an individualized stereochemic unit, but also that its operations, and hence the nature of it's products, must be subject directly or indirectly to the influence of every other active part of the organism, however different the structures and functions may $b e$. 
The Germplasm a Stereochemio Ststem.

The Germplasm is a Stereochemic System-that is, a Physico-chemical System Particularized by the Characters of its Stereoisomers and the Arrangements of its Components in the Three Dimensions of Space.

If during the progress of development there arise the multiple forms of differentiated protoplasm that are represented in the nerve cells, muscles, glands, etc., which exhibit such diversity of form, functions, composition, and products, each part being correlated to other parts by the agency of tissue products, it is logical to assume that in the development of the ovaries and testicles these organs have been so specialized as to endow them with the attribute of producing a form of protoplasm that embodies in a germinal state the fundamental peculiar stereoisomerides and the peculiar arrangements or phases of the associated proteins, fats, carbohydrates, and other substances which inherently characterize the organism; and, moreover, that owing to the influences of the products of activity of the various tissues upon these organs, such changes in the organism as give rise to acquired characters may through the actions of modified or new tissue products or foreign substances affect the operations of these organs and thus alter the germplasm and consequently become manifested in some form in the offspring. The ovule in its incipiency is conceived to be comparable to a complex unequilibrated solution in which changes go on until the attainment of full development, at which time it is equilibrated and remains inactive because of the absence of some disturbing influence, but in which energy-reactions may be initiated physically, mechanically, or chemically, and proceed according to definite physico-chemical laws in definite directions to a definite end. For instance, when a solution of boiled starch and diastase is at a temperature below the minimal of activity and the temperature is raised, causing immediate developmental activation; or when the equilibrated molecules of nitroglycerine are exploded by percussion; or when an equilibrated maltose-dextrose-glucase solution is rendered active by dilution with water.

The nature of the germplasm or transmissive material that serves as the bridge of continuity between parents and offspring has been the subject of speculation from time immemorial. Such hypotheses and theories as have been advanced have had reference almost wholly to its physical constitution or ultimate morphological structure. Most of them are micromeric, that is, they hold that the germplasm is made up of an infinite number of discrete ultramicroscopic particles which are endowed with both determinate structural and vital attributes. A considerable degree of ingenuity has been displayed in their formulation. Thus, we have the "organic molecules" of Buffon, the "microzymes" of Bèchamp, the "life units" of Spencer, the "plastidules" of Maggi, the "bioplasts" of Altmann, the "stirps" of Galton, the "gemmules" of Darwin, the "biophors" of Weismann, the "pangens" of DeVries, etc., each author attributing to the units certain inherent peculiarities. To the foregoing might be added particularly the conceptions that belong to the chemical category, such as the "chemism" of Le Dantec and the "physico-chemical" theory of Delage. Some of these conceptions are so fan- ciful in the light of modern science as to be unworthy of more than passing consideration, while none of them has led anywhere beyond the field of speculation and reasoning. Even the very recent and extremely interesting and important additions to our knowledge of the histological phenomena of the developing ovum, especially of the chromosomes, have not taken us appreciably nearer the ultimate constitution or mechanism of the germplasm, or even to the nature of the reactions which occur immediately antecedent to and cause the formation of the chromosomes.

A theory to be ideal must not only have as its basis well-defined principles that are consistent with facts, but also be capable of substantiation by laboratory investigation. Given as the basis of scientific study a germplasm that has inherently the power of development, that is in the form of a stereochemic system that is peculiar to the organism, that is highly impressionable to stimuli, and that has the marked plasticity inherent to organic colloidal matter, we have all the postulates that are needed as a foundation upon which, according to the laws of physical chemistry, can be built a logical explanation of the essential fundamental elements of the mechanism of heredity.

The inherent potentiality that determines the development of the egg along a line of definite sequential processes must be recognized as being common to both animate and inanimate matter and subject to the same laws, so that the phenomena of living and dead matter are inseparably linked and reciprocally explanatory. The typical condition of matter of definite composition is crystalline, and the crystalline form is the result of development that becomes manifested in a separation and orderly and progressive arrangements of components in the three dimensions of space. Having a homogeneous solution of various selected crystalline substances of appropriate chemical composition and constitution, and given conditions attendant to crystallization, the successive stages of crystalline development will proceed along fixed and definitely recognized lines, and the interactions and interaction-relationships between the various substances constituting the physico-chemical mechanism become obvious to a greater or less extent in the peculiarities of form, composition, and other properties of the crystals. Having in the germplasm an analogous physicochemical system, but one which is markedly different especially because of its organic and colloidal character and infinitely greater molecular complexity and sensitivity, the phenomena of development likewise proceed in conformity with the same laws along definite lines, but they are for perfectly manifest reasons more complex and varied, more difficult of analysis, and necessarily in many very important respects quite different. Each step in this orderly development leads not merely to changes of the physico-chemical mechanism by the modification, rearrangement, or splitting off of component parts, but also to alterations which automatically determine the characters of the next succeeding step, and so on to the establishment of physico-chemical equilibrium and the consequent termination of the reactions.

In living matter the chemical processes are dependent to a preëminent degree upon enzymes that are formed by the different kinds of protoplasm to serve as 
implements to carry out operations that are essential to their existence, and such enzymes are modifiable in quantity and quality in accordance with changes in internal and external conditions. The nature of both reactions and products of enzymic action depends upon the constitution and composition of the physico-chemical mechanism of which the enzyme is an integral part. Whether or not at each step of serial reactions a portion of pre-existing enzyme is merely modified or a new enzyme is formed which constitutes an essential part of the particular phase of the reactions is not known, but that one or the other occurs is apparently without question. It has long been established that some of the lower organisms, such as the yeast plant, have the property of modifying the characters of the enzymes produced in relation to varying conditions; recent studies of the animal organism show that the same phenomenon occurs in both tissues and blood; and our knowledge of the processes concerned in the catabolism and anabolism of complex substances, such as starch, is fully in support of such a conception. In other words, as each step of development is reached the alterations which occur in the physico-chemical mechanism absolutely automatically predetermine the characters of the changes of the next succeeding step, and so on to the end. Hence it follows that the peculiarities of any given physicochemical mechanism predetermine the characters of the phenomena which ensue under given conditions.

An illustration of the probable modus operandi of such a mechanism is found in the phenomena of the synthesis and analysis of starch: During the production of starch through the agency of the chloroplast or leucoplast we conceive that there are instituted a predetermined, orderly, independent and interdependent series of reactions, the first of which is manifested in an interaction between water and carbon dioxide through the agency of an enzyme in the form of an oxidase to form formaldehyde. During this process there is formed another enzyme, which tentatively may be designated an aldehydase, that reacts with formaldehyde and by polymerization and condensation of six molecules gives rise to a simple sugar, such as dextrose. At the same time another enzyme appears in the form of maltase, which, reacting with the dextrose causes the formation of maltose, during which reaction another enzyme, a dextrinase, is produced which reacts with the maltose to yield dextrin. Going on with this reaction, another enzyme which may be designated an amylase appears, which, reacting with the dextrin, forms soluble starch. During this stage there arises another enzyme, a coagulase, which converts the starch from the soluble to the insoluble form or ordinary starch. At this stage the series of reactions have reached their end because a state of physico-chemical equilibrium has become established, the ultimate purpose of the processes being attained, that is, a form of pabulum of extremely high nutritive value and of extremely low molecular pressure, even in soluble form, so that it may entirely and rapidly disappear without disturbance of physico-chemical equilibrium in the starch-bearing cells. The mechanism concerned in starch-formation is without doubt paralleled in the synthesis of proteins, fats, and other complex organic substances, and it is but a step from the individual serial processes concerned in the formation of each of these substances to associated processes whereby there are formed and combined the various substances that constitute the organic structural components of protoplasm. Moreover, such serial processes are reversible at any stage, and so simple a modification as a change in the percentage of water may, as in the maltosedextrose-glucase reaction, cause a synthetic change.

In vitro in both synthetic and analytic processes like those which constitute serial steps in the building up and breaking down of starch, protein, fat, and other complex organic substances, there does not occur in any reaction, as far as known, either a transformation or a production of enzyme such as occurs in vivo, hence, when a single enzyme is present it carries out but one step of the reactions, but when, as in the case of diastases as ordinarily prepared, the enzyme is not a single substance or unit body but a composite of a number of enzymes or modifications of a given basic enzyme, serial steps may occur as in vivo. Thus, if only a single enzyme be present formaldehyde may be converted into a monosaccharose, or a monosaccharose into a disaccharose, or a disaccharose into a polysaccharose such as dextrin, or a dextrin into a higher form of polysaccharose such as soluble starch, according to the enzyme or modified enzyme and initial substance present; or the reverse of any one of these processes may occur if proper conditions are present, but never do any two successive progressive or regressive steps occur unless through the agency of two different enzymes or modified forms of one enzyme which are present.

It will thus be apparent that the first step of synthesis is determined by the character of the initial physico-chemical mechanism and that all subsequent reactions under given conditions are definitely predetermined; in other words, the entire train of reactions depends inherently upon the nature of the initial physicochemical mechanism of which the enzyme that starts the serial changes is an integral part.

Having a specific stereochemic system, such a system in accordance with the laws of physical-chemistry can exist in either a latent or active state, and that when in an active state the reaction or reactions are always in the direction of the establishment of equilibrium of solution, every reaction or series of reactions being as definitely predetermined as is every reaction familiar to the inorganic chemist. The germplasm in the form in which it is secreted may be regarded as being in the nature of an exceedingly complex stereochemic system which is from its incipiency, or very soon is in a state of physico-chemical unequilibrium, and in which, as a consequence, reactions are set up which are manifested especially in histological developments that ultimately characterize the fully developed ovule, at which time a state of physico-chemical equilibrium is established, as is evident by the arrested developmental activities. This state of physico-chemical equilibrium of the matured ovule may be instantly changed to one leading to serial definitely predetermined reactions by means of an activating substance or condition, such as certain ions or inorganic salts, a spermatozoon, or a needle prick, by initiating the first step of the reactions, the nature of the succeeding reactions being predetermined primarily by the inherent nature of the physico-chemical system 
and secondarily by the factor that activates it. In other words, from this initial stereochemic system there arises a complex heterogeneous system that ultimately is morphologically expressed in the histology of the matured ovule and from which are formed a composite of correlated, independent, interdependent, and differentiated masses which represent different phases of the components of the initial system which have been modified not only physico-chemically as expressed by changes in physical, mechanical, and chemical properties, but also in developmental energies; and from this composite are developed successively other systems.

Owing to the great impressionability and plasticity of such an exceedingly complex stereochemic system as the germplasm, it follows that the germplasm must be extremely sensitive to changes in internal and external conditions, and that its operations and products may be so materially modified by changes in its molecular arrangements or components as to give rise to variables that are manifested in the transmutability of sex, variations, fluctuations, mutations, deformities, retrogressions, tumor formation, immunities, etc.

Assuming in accordance with our conception that the germplasm is in its incipiency an unequilibrated stereochemic system that is characteristic of the inherent, fundamental stereochemic system of the parent, it follows, as a corollary, that having a highly specialized form of parental structural material with peculiar energy-properties, the offspring must of necessity possess essentially the same fundamental characteristics as the parents when normal fecundation has occurred, and that it would be quite as impossible to have any other result than in ordinary chemical reactions under given conditions of experiment. The essential characters of the building material as regards substances, arrangements, and energy-properties are definitely fixed within narrow limits of variation.

That the peculiar forms of stereoisomerides or intimately related bodies that are inherent in the parent are conveyed in the germplasm to the offspring, ancl hence of necessity serve to distinguish a given form of germplasm from that of any other species or genus, 'and that the stereochemic conception of the nature of the germplasm is capable of laboratory demonstration, are instanced in the results of the investigations of Kossell and his students who found that simple forms of protein, known as protamins, obtained from the spermatozoa of different species of fish are different, each being apparently of a form peculiar to the source. Here is one substance at least that seems to be in specific stereoisomeric forms in the sperm of different species, which obviously must affect the properties of the germplasm, and which when brought in contact with the germplasm of the egg plays its part in determining the phenomena of development. Moreover, by the "precipitin reaction" method Blakeslee and Gortner have found evidence that is consistent with the conclusion that there are not only "species proteins" but also "sex proteins," and this receives support in a number of very recent investigations, especially those of Steinach, who found that the corresponding hormones secreted by the ovaries and testicles are different, and that by virtue of these differences the secondary sexual characters, female and male, are determined. Thus he found in castrated young males, in which transplantation of ovaries had been practised, that the development of masculine peculiarities is inhibited and female traits substituted, so that the individuals tend to assume the female type and become to a striking degree feminized-males, as shown in bodily form, in a development of the mammary glands, in lactation, and in an alteration of psycho-sexual characters, Lillie, in studies of the explanation of the sterility of females of opposite-sexed twins, has presented evidence of the existence of sex hormones, and both Lipschiitz and Morgan have recorded facts to justify the belief that the testicular hormone furthers the development of male characters and inhibits the development of female characters, while the ovarian hormone favors the development of female characters and inhibits the development of male characters. This dual property is obviously of great fundamental importance in the explanation of various sex phenomena which have been quite inexplicable. Furthermore, Riddle has found that the ora of the pigeon are dimorphic, one-half having an inherent tendency to produce males and the other half females; that eggs with the male tendency have a higher percentage of water, a smaller size, and a lower percentage of potential energy; and that the "sex-foundation" of the germplasm is transmutable, so that an egg that has inherently the male tendency may become female, and that such females exhibit secondary male sexual characters. The transmutability of the germplasm is comparable in its physico-chemical mechanism to the reversion of the maltose-dextrose-glucase reaction caused by a change in concentration of the solution, the dextrose being reverted into isomaltose and not to the antecedent maltose-the male egg is not changed into a female egg, but into a modified or feminized-male egg.

In considering the transmissibility of parental substances it is essential to distinguish positively between the stereoisomerides and intimately related bodies that are inherent in the parent and those which are acquired through infection or otherwise. Thus antibodies acquired by the mother may be without influence upon the ovary during the formation of the germplasm and not even become a constituent of the latter. On the other hand, an immunity may be established in the mother that may be conveyed to the offspring, yet, curiously enough, such an immunity may not be transmitted by the immunized male. In processes of the production of the germplasm the ovary may be as insensitive to the presence of many acquired substances of the blood as are some or all other organs, and there is no more reason in general for expecting the ovary and its product to be affected by such bodies or conditions than there is for the pancreas and the pancreatic juice or any other secretory structure and its product to be affected. Every acquired substance must in its relations to the ovaries be governed by the same physico-chemical laws as determine specific selectivities or reactivities in connection with the tissues generally. Hence, any such substance may be reactive in relation to one structure, but not to another.

Plasticity as regards sex-determination has been demonstrated in the studies of the development of a male (drone) bee from the unfertilized egg, and of a female 
from the fertilized egg. Moreover, the developing female bee when fed on ordinary food becomes a common female "worker," but when fed on royal food develops into a queen. (See also pages 375 and 376 .)

The continuity of the building material between parent and offspring is seen in its simplest manifestations in reproduction among protozoa by binary fission and budding, by which the part separated from the parent mass is in all essential respects like the parent, having the same fundamental physico-chemical composition and constitution. That in such instances the offspring should be a segmental counterpart of the parent mass seems as obvious as that halves of a cube of sugar should be alike. Similarly, if we have in the ovule and sperm forms of protoplasm which as stereochemic systems are in all fundamental respects counterparts of those from which the parents were developed, it follows that the offspring must under normal conditions in accordance with the laws of physical chemistry have the same fundamental parental characteristics, as much so as separated portions of any complex stereochemic system must possess the properties of the initial mass. Moreover, if the stereochemic systems of germplasms of the female and male differ, as must be admitted, it is manifest that the stereochemic system of the egg that has been activated artificially or naturally, as the case may be, must be different and hence undergo development differences that will be obvious in the offspring. In the first instance, the serial reactions which lead to the formation of the different tissues, etc., are activated by a mere disturbance of physico-chemical equilibrium, which may be due to the conversion of a proenzyme into enzyme or a prosecretin to a secretin, or in other words of an inactive body into an active one. In the second instance, there is not only activation, but the extremely important addition of the male stereochemic system which by admixture with the female system constitutes a female-male system. Therefore, in the first place the offspring is developed solely from the female stereochemic system, and in the second place from the combined female and male systems, one or the other of which may be wholly or in part accountable in determining certain peculiarities in the developmental changes. Moreover, owing to the transmutability of stereoisomerides and the multiphase transmutability of stereochemic systems, coupled with the reversibility of metabolic processes which may be due to even the simplest of changes in physico-chemical mechanisms, we have a logical basis for the explanation of the phenomena of sexual dimorphism that is expressed in the so-called male and female ova, and male and female spermatozoa; of primary and secondary hermaphroditism; of paradoxical sex developments where the unfertilized egg develops into either male or female offspring ; and of sexual transmutability of the inherently male or female ovule.

It follows upon the basis of our theory that because of the inherent peculiarities of the stereochemic systems of the germplasms and the definitely predetermined nature of the entire series of reactions in accordance with the laws of physical chemistry that "like begets like" because like every other physico-chemical phenomenon, individual or serial, single or complex, under given conditions, it is a physico-chemical fatality.
Protoplasmic Stereochemic System Applied to the Explanation of the Mechanism of Variations, Sports, Fluctuations, Etc.

Among the most constant phenomena of living matter is inconstancy or variation. The fundamental reasons for this peculiarity are to be found in the extreme complexity, impressionability, and plasticity of the molecules of protoplasm in association with unceasing and varying kinds and degrees of environmental changes. Plasticity is a property that is doubtless common to every form of matter, the degree varying within wide limits in different substances and under varying conditions. Oxygen, nitrogen, carbon, sulphur, selenium, phosphorrus, arsenic, tin, iridium, palladium, and other elements have long been known to be allotropic; calcium nitrate and metaphosphate, ammonium nitrate and fluosilicate, silver nitrate and iodide, calcium carbonate, silica, copper sulphate, iron sulphate, magnesium sulphate, mercuric chloride and iodide, zinc chloride, arsenious and antimonious oxides, potassium bichromate and ammonium paratungstate, are only a few of the simple inorganic compounds that have been found to be dimorphous or polymorphous; and the known organic or carbon compounds that exist in multiple forms are so numerous as to make an exceedingly large list. In some instances the differences in form are said to indicate merely differences in physical nature, thers being variations in color, hardness, density, meltingpoint, crystalline form, etc., without change in chemical properties; but in others the differences are both physical and chemical and the latter may completely overshadow the former. Perhaps, there is no more remarkable or suggestive instance of difference in properties that is associated with differences in molecular form than that of strychnine in ordinary and colloidal states, the latter having only one-fourth the toxicity of the former; and one wonders, apart from anything else, what changes have occurred in the properties of the various non-colloidal substances such as inorganic salts when they have become an integral part of the molecule of the most complex of all colloids--protoplasm. Moreover, change from one state or phase into another is usually brought about by very simple means, such as mere solution, heat, sunlight, repeated recrystallization, gelation, chemical reagents, etc. (See Publication No. 173, Introduction, page 9.)

Water, while among the simplest substances of nature, is endowed with most extraordinary properties, especially in connection with living matter. It exhibits a remarkable degree of plasticity in its molecular structure. The universal conception up to very recent years that water is correctly represented by the symbol $\mathrm{H}_{2} \mathrm{O}$ has been shown to be untenable excepting under very limited conditions, and it seems clear that the molecule must be looked upon as being in the form of a molecular system that consists of $\mathrm{H}_{2} \mathrm{O}$ (monohydrol), $\left(\mathrm{H}_{2} \mathrm{O}\right)_{2}$ (dihydrol), and $\left(\mathrm{H}_{2} \mathrm{O}\right)_{:}$(trihydrol), which vary in proportions in relation to temperature and pressure, and which are readily convertible from one form into another by changes in attendant conditions. It is assumed that when polymerization occurs there takes place a chemical combination of the simple molecules and that with this combination changes occur in properties, such, 
for instance, as has been referred to in the synthesis of starch (see Publication 173, page 156), when six molecules of formaldehyde are polymerized and condensed to form dextrose. Moreover, it is to be assumed that the molecular system consists of these three forms of molecules in chemical combination, and therefore if the proportions vary the system will vary in its properties. The chief component of this system when water is in the form of ice is $\left(\mathrm{H}_{2} \mathrm{O}\right)_{3}$ and of steam $\left(\mathrm{H}_{2} \mathrm{O}\right)$, while in the form of liquid water it is $\left(\mathrm{H}_{2} \mathrm{O}\right)_{2}$.

Each of these forms of water is, therefore, a ternary mixture of molecules in chemical combination, the proportions of the three kinds of molecules differing, and alterable in relation to changes in temperature and pressure, and in the direction of the maintenance of physico-chemical equilibrium. It is also probable that there may be higher polymers, and that each polymer may exist in more than one form, thus indicating a further and by no means unimportant degree of plasticity in stereochemic phenomena, especially in relation to vital processes. Even the proportions of these molecules in ice prepared under varied conditions are almost certainly different, inasmuch as some forms of ice are heavier and other forms lighter than water, and as one form crystallizes in the hexagonal system, another in the tetragonal system, and another in the regular system.

Further evidence of the plasticity of water is seen in the variety of forms of snow crystals, all of which are said to belong to the hexagonal system. It is easy to account for these different forms if, as is indicated, the proportions of these three kinds of molecules vary with temperature; if water in vapor form in the clouds has like steam a maximum proportion of the $\left(\mathrm{H}_{2} \mathrm{O}\right)$ molecules, and if cooling to the freezing-point brings about (as the temperature falls) progressive changes in the proportions of the molecules, and hence of the molecular system, so that at any given temperature the composition of the system is different from that at any other temperature; if these changes in proportions may be further influenced by the rapidity of the fall of temperature, the velocity of the change not keeping pace with the temperature change; and if crystallization may be influenced by incidental conditions, as is manifested in the variety of crystalline figures when ice forms on a window pane. It has recently been found that when condensation takes place in highly supersaturated ascending air, and the air temperature is much below freezingpoint, both snow crystals and rain-drops are formed. If such plasticity is to be found in substances so simple as water it seems that almost any conceivable degree is to be expected in complex substances, such as the proteins, fats, carbohydrates, and other organic metabolites, and to the very ultimate degree in protoplasm. The plasticity of proteins has been demonstrated in the modifications of the hemoglobins in specific relationship to the source; and of carbohydrates in the starches in the same respect, and especially in the diversified reactions in which properties are elicited that are the same as those of one or the other parent, or both parents, or which are not exhibited by either parent, and which are therefore peculiar to the hybrid, and in all the phases of the reactions seem to be limited only by the number of reagents.
Having now in protoplasm a molecular system of extreme complexity, affectibility, and plasticity, unceasing changes in internal and external conditions and a knowledge of the fundamentals of biochemistry such as is indicated in preceding sections, it requires no more effort of the imagination, than in the reactions of organic substances generally, to picture the underlying factors and processes that become expressed in the differences in form, structure, and vital characteristics that are manifested in variations, sports, fluctuations, and kindred phenomena, and in individuals, varieties, species, and genera. It seems that the mechanisms of Mendelian inheritance and sex have striking analogies in the evolution of $\alpha$ and $\beta$ forms of stereoisomers, as, for instance, in the case of $\alpha$ - and $\beta$-glucose, as was pointed out in the preceding memoir, page 10.

\section{Protoplasmic Stereochemic System Applied to the Genesis of Specrms.}

The importance of hybridization in the genesis of species has undoubtedly been greatly underestimated, chiefly because of a false valuation that has been placed upon intermediateness as a criterion of hybrids and the belief that the hybrids between species are very commonly infertile. But it seems obvious from the records of this research that such characters of a hybrid as may be intermediate may be overshadowed by others, some of which are the same as those of one or the other parent or both parents, or developed beyond parental extremes, or which may be peculiar to the hybrid. De Vries, in his exposition of the laws of mutation of Oenothera, states as follows:

"The mutations to which the origin of new elementary species is due appear to be indefinite, that is to say, the changes may affect all organs and seem to take place in almost every conceivable direction. The plants become stronger (gigas) or weaker (albida), with broader or with smaller leaves. The flowers become larger (gigas) and darker yellow (rugrinervis), or smaller (oblonga and scintillans) and paler (albida). The fruits become longer (rubrinervis) or shorter (gigas, albida, lata). The epidermis becomes more uneven (albida) or smoother (lavifolia); the crumples on the leaves cither increase (lata) or diminish (scintillans). The production of pollen is either increased (rubrinervis) or diminished (scintillans); the seeds become larger (gigas) or smaller (scintil lans), more plentiful (rubrimervis) or more scanty (lata). The plant becomes female (lata) or almost entirely male (brevistylis); many forms which are not described here were almost entirely sterile, some almost destitute of flowers. 0 . gigas, $O$. scintillans, $O$. oblongata tends to become biennial more than 0 . lamarcliana; and 0 . lata tends to become less so; whilst $O$. nanella cultivated in the usual way scarcely ever runs into the second year. This list could easily be extended, but for the present it may suffice. To regard the new forms from another point of view, some of them are fitter, come unfitter, than the parent form and others neither the one nor the other."

In reference to $O$. lamarckiana, he states that nearly all organs and all characters mutate, and in almost every conceivable direction and combination. The foregoing quotation is of especial interest at the present juncture because the data are applicable to hybrids, and as it seems to have been satisfactorily established that these mutants are actually hybrids. Moreover, when they are taken in connection with the data quoted from Focke in the Introduction, we have facts that are in entire accord with the results of the studies of the physico-chemical properties of the starches. Again, Ipomca sloteri, one 
of the hybrids studied in this research in respect to its macroscopic and microscopic characters, has been found to so differ from its parents that were it not known to be a hybrid there would be ample justification to regard it as a species (see Ipomoea, Part II). It is well known to the botanist that many of the hybrids included among the hundreds referred to by Focke are so individualized as to warrant their assignment as species or subspecies. Finally, it seems from the present state of our knowledge that the difficulty of hybridization, the tendency to infertility of the offspring, the tendency to the development of characters in the hybrid in excess of parental extremes, and the tendency to develop new characters in the hybrid, bear usually an inverse relationship to the nearness of the parents, while the tendency to intermediateness bears usually a direct relationship. Owing, however, to the extreme plasticity of protoplasm the most variable results in hybridization are to be expected, as is indicated by the results of the studies of the starches, as presented particularly in Table H, Parts 1 to 26 , and summaries.

The study of the genesis of species is without doubt a study of the evolution of chemical compounds, and essentially of interactions, rearrangements, and combinations of stereochemic systems and their components. In the origin of species by hybridization there is, according to the conception stated in the penultimate section, a union of two stereoisomeric systems of varying plasticities, female and male, in each of which there are assumed to be potentially every or practically every character and character-phase of the parent. Moreover, this variability of plasticity applies not only to the system, as a whole, but also to each of the integral stereochemic units. Having extremely complex, plastic, interacting systems, and applying thereto a fundamental knowledge of physical chemistry, especially of organic colloids, as is indicated, it seems that there should be no more difficulty than in the reactions of organic substances generally in reaching satisfactory theoretic understandings of the diverse developmental changes that occur in the hybrid-that is, why some characters are like those of one or the other parent or both parents, or developed beyond parental extremes, or new characters appear; or why one parent may be of equal or greater potency in influencing the development of the characters of the hybrid; or why species of remote genera can not be crossed, or, on the other hand, why varieties of the same species may readily be crossed; or why characters that may have existed in ancestral generations, but which are not apparent in the parents, may appear in the offspring; or why there may or may not be Mendelian inheritance; or why mutations can be induced artificially by the injection of certain substances into the ovaries, etc., etc. Unfortunately this subject is so vast that a detailed consideration of such points would take us far beyond the possible limits of space of this report, and therefore, as previously stated, nothing more can be offered at present than mere suggestions. 


\section{CHAPTER VII. NOTES AND CONCLUSIONS.}

\section{Hypothesis Underlying These Restarches.}

These investigations (Publications Nos. 116, 173, and the present) have as their essential basis the conception that in different organisms corresponding complex organic substances that constitute the supreme structural elements of protoplasm and the major synthetic products of protoplasmic activity are not in any case absolutely identical in chemical constitution, and that each substance may exist in countless modifications, each modification being characteristic of the form of protoplasm, the organ, the individual, the sex, the species, the genus, etc., and that the possible number of modified forms of each substance is in direct relationship to the complexity of the molecules.

\section{Exploratory Character-Evidence in Support of the Нypothesis, Етс.}

These inquiries have for certain reasons been practically of a purely exploratory character and therefore no serious attempt has been made to do more than gather sufficiently convincing evidence to amply sustain the hypothesis and thus lay a satisfactory foundation for subsequent inquiries. It is obvious, from the results of each of these studies, that considering the difficulties met in pioneer investigations the measure of success has been beyond that which should reasonably have been expected.

Hemoglobins from 107 species were examined, mostly from mammals, including representatives of Pisces, Batrachia, Ares, Marsupialia, Edenta, Sirenia, Ungulata, Rodentia, Otariidia, Phocidæ, Mustelidæ, Procyonidæ, Ursidæ, Canidæ, Felidæ, Viveridæ, Insectivora, Chiroptera, and Primates. The number seems large in comparison with the numbers studied by various previous investigators, yet it is an insignificant fraction of the number existent in vertebrates and invertebrates. Moreover, in antecedent investigations the crystallographic examinations were, with scarcely an exception of a single hemoglobin, limited to geometric form, while in the studies embraced in this series of researches both geometric form and optic reactions were recorded, the latter being here very important and often as distinctive and as exact in differentiation as chemical reactions.

The starches studied have been so numerous as to cover a far broader field, including in the preceding research 300 that represent 105 genera and 35 families, and in the present research 47 sets of parent- and hybridstocks, and representing 17 genera and 7 families. The total number examined compared with those available for similar investigation is, as in the hemoglobins, an exceedingly small or almost negligible fraction.

Not only have the hemoglobins and starches been scarcely more than touched, but there remains an enormous list of complex metabolites included among the proteins, fats, carbohydrates, enzymes, coloring matters, cholesterols, organic acids, alkaloids, etc., and also a very large number of compounds, which as yet have been 370 subjected to extremely little or absolutely no investigation in regard to their constitutional properties in relation to biological source. Some or even many of these metabolites are not unit substances-that is, they are combinations, physical or chemical, of like or unlike substances. Moreover, there are derivatives of many of these primary or initial substances-for instance, the crystalline chlorophyls (ethylchlorophylides) - that are most promising for such investigations. An unlimited field of investigation in both material and promise is opened by the facts that probably every substance, elementary and compound, may exist in more than one form; that when molecules are associated during polymerization there is chemical combination, and that in these combinations the arrangements of the components in the three dimensions of space may yield different forms of the same substance (as in water), or entirely different substances (as in the polymerization of formaldehyde to form dextrose); that the possible number of stereoisomeric forms increases directly with the complexity of the molecular organization; and that in all probability these various stereoisomeric forms of substances produced by protoplasmic activity are specifically modified in relation to biologic origin.

\section{Methods Employed and Recommended.}

The crystallographic method used in the investigations of the hemoglobins is, in so far as the requirements of these investigations are concerned, not only exact but also a very sensitive means of differentiation of different forms of these substances. Differences in chemical constitution can readily be demonstrated which as yet are too obscure for detection by any known chemical procedures; differences have been shown that can not be brought out by any of the biologic tests; repeated experiments with the hemoglobins from different individuals of the same species have yielded practically or absolutely the same results; biologic differences elicited by this means are in accord with the data of the systematist wherever the latter is not open to question; and these records have had confirmation in the results of anaphylactic reactions. The methods for differentiating stereoisomers are with rare exceptions quite crude, but even those which are inexact may be not only checks upon each other but also collectively and even individually be of much usefulness in such investigations. It was pointed out that differences had been recorded in the hemoglobins from different species in their solubilities, crystallizabilities, water of crystallization, extinction coefficients and quotients, and decomposability; and it is evident, inasmuch as differences that may be exhibited by one method may not be brought out by another, or in varying degree, that much is to be gained by the use of many or all methods. Very much is possible by means of further development of biologic tests.

In the differentiation of starches, both in the preceding and present researches, the methods employed 
are the same but modified in their applications in certain important respects. In both investigations the histologic properties, the iodine and aniline reactions, and the gelatinization reactions with heat and various chemical reagents were studied, the chief differences being in the method of recording the reactions with the chemical reagents, and in the kinds and concentrations of the reagents. In the former research the quantitative differentiations by means of the chemical reagents were made by determining the time of the occurrence of complete or practically complete gelatinization, and the preparations of starch with the reagent were not adequately protected from the air and evaporation. It was found during the progress of this work that fictitious values may be recorded owing to the existence in nearly every form of starch of different kinds of grains which vary in proportions and gelatinizabilities, together with varying degrees of influence of the air (probably chiefly or solely differences in oxidation), and effects that are due to varying rapidity and degrees of evaporation. Such sources of fallacy have been practically eliminated in the present research by making records of the progress of gelatinization in regard to both the entire number of grains completely gelatinized and the percentage of the total starch gelatinized at definite time-intervals; and by the prevention of oxidation and evaporation by sealing the preparations. In nearly every form of starch there are grains, usually very small, and also parts of grains, that are quite resistant to reagents. The former commonly represent much less than 5 per cent of the total quantity of starch, and it has been assumed that gelatinization is practically complete when 95 per cent of the total starch has been gelatinized. The methods used and their values in the differentiation of starches have been set forth in full in the preceding memoir on pages 305 to 313 , and supplementary statements are to be found in the present memoir in Chapters II, IV, and V.

The histologic method employed in this research is the same in all respects as in the preceding investigation, in the report of which it has been discussed with sufficient fulness (page 307). Its value has not only been substantiated but accentuated by the results of the present study of the starches of parent- and hybrid-stocks.

The polariscopic, iodine, and aniline methods are so crude that the personal equation enters largely into the determination of the values recorded, and while they have proved of unquestionable usefulness they are so inferior to the gelatinization method that they should be given a very subordinate place. The polarization and aniline methods are by far the least useful of all of those used, yet the anilines will be found of much value in the differentiation of different lamellæ of individual grains, as has been shown by the work of Denniston (see previous Memoir, page 56). Iodine, like the anilines, can be used to great advantage in the study of the structure of the starch grain. It is also of usefulness by showing by variations in the color reactions differences in the constitution of starches from different sources; of different kinds of grains of the same starch; of the capsular and intracapsular parts of the grains; and of the capsules themselves. The method used in determining the temperature of gelatinization is practically exact, as has been shown by the fact that when the experiments are made with proper care the figures recorded are quite as uniform as those obtained in the determination of the melting-points of various substances.

The gelatinization method by means of various chemical reagents as here pursued has proven to be so near exact that the records of repeated experiments have, except very rarely, been found to be exactly or practically exactly the same, even though made at widely different periods and with varying temperature and humidity. Very rarely, for some inexplicable reason, a more or less markedly aberrant record has been made. In every instance this error was detected because of the absence of agreement with what was positively indicated by conditions. In fact, as was found in practice and as will be obvious by the context, the records of the reactions obtained by means of the various methods employed are in the case of each agent and reagent, and of all collectively, in a very large measure checks upon each other. In other words, the values for the starch of a given species serve as prototype or generic standard with which the records of all other species and varieties of the genus must conform, unless there are represented members of subgenera or other subgeneric divisions. The closer botanically the species or the varieties the closer will the records collectively agree with the given standard. Varieties of a species exhibit remarkable closeness, and their values represent a species type. When members of subgenera or other form of subgeneric division are represented they may exhibit differences that are as marked, and even more marked, than those of members of closely related genera.

It is to be borne in mind that the method of classification of the systematist is of an arbitrary character, as is evident, for instance, in the shifting of species from one to another genus, the remodeling of genera, families, etc. This classifying and reclassifying that has been in progress for generations continues at the present time, and even now the most generally accepted classification can not be accepted as being more than tentative. If, therefore, the results of these investigations seem to be or are not in accord in isolated instances with the classification of the systematist it does not follow that the former are wrong. As evidence of the mutual checking of the records one need examine only the very similar curves of the starches of the closely related members of

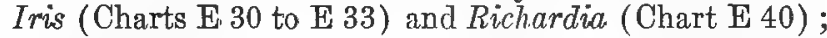
the dissimilar curves of the starches of members of subgeneric divisions, such as the hardy and tender species of Crinum (Charts E 7 to E 9); the dissimilar curves of the starches of members of subgenera of $\mathrm{Be}$ gonia (Charts $\mathrm{N} 36$ to $\mathrm{E} 39$ ); the similar curves of the starches of the closely related genera Amaryllis and Brunsvigia (Chart E 1), and of Gladiolus and Tritonia (Charts E 34 and E 35) ; and the dissimilar curves, usually highly characteristic, of the starches of various genera of the same and different families that are shown in this series of charts (E 1 to $E$ 46), as a whole. These similarities and dissimilarities are in degree variable in accordance with what in general should be expected, or what is at least in accord with unquestionable botanical classification.

The differentiation of starches by heat, as in the temperature of gelatinization method, is to be recommended as being of much value, both quantitatively and qualitatively. It was shown in the preceding investi- 
gation that the temperatures of gelatinization of starches from different sources vary within a range of over $40^{\circ}$ C.; and that the figures for the starches of different members of a genus usually tend to keep within limits of about $5^{\circ}$, the closer the plant sources the closer the temperatures. Moreover, qualibative differences similar to those elicited by the various chemical reagents have been observed, and they are worthy of detailed study. These it seems will be found to differ not only in different starches, but also to differ from the reactions elicited by the chemical reagents and to differ as much from them as they do from each other. These qualitative reactions have been found, as a whole, to have such values as to recommend them for extensive use. In the present research these reactions with heat and chemical reagents have yielded records that are of especial interest in the differentiation of the starches of the hybrid- and parent-stocks, and they have not only shown peculiarities of the hybrid that are the same as those of one or the other parent or both parents, but also individualities not observed in either parent and corresponding to what was found in the records of the histologic and other characters and character-phases. The extraordinary plasticity and complexity of the starch molecules and its character and character-phase potentialities offer endless opportunities in this form of investigation.

The quantitative data appeal more to both experimenter and reader because they lend themselves so admirably to reduction to tables and charts. The possibilities for additions to our knowledge of this kind are unlimited. As previously indicated, the number of starches available for such investigations is enormous and the number of the reagents can be considerably amplified. Moreover, there can often be used, to much advantage, several concentrations of the same reagent and also combinations of certain reagents.

These various reagents differ markedly in their values in the quantitative and qualitative reactions, respectively, and some are better for the former than the latter and vice versa; moreover, a reagent that may be particularly good for qualitative reactions with one form of starch may be inferior for another form, and so on. Recognition of these points will be of great advantage in subsequent investigations.

\section{Starch Substances as Non-Unit Substances.}

Starch from any given plant is a heterogeneous collection of grains which vary in microscopical and molecular properties; even the individual grains, except perhaps the very small embryonic, spherical, and seemingly amorphous forms, are likewise of non-uniform composition. The differences in the behavior of the inner and outer parts or (according to general ideas) of the so-called amylose and cellulose can be demonstrated with the greatest ease and in ways to show that these parts represent different forms of starch-substance. As already repeatedly pointed out, the individualities of these two parts are markedly shown in their different behavior towards various reagents. As a rule, the outer part is the more resistive, but toward some reagents it is the less resistive. In relation to moist heat, when the grains are boiled in water the outer part is always the last to disappear, sometimes resisting boiling for many minutes, appearing in suspension in the form of empty capsules from which the less resistive inner starch has escaped in semi-liquid form and passed into a pseudo solution.

The different lamellæ of the mature starch-grain are of less and less density from without inward. These peculiar variations are, it seems clear, not owing to an increase in the density of each additional lamella as it is deposited, but to a gradual transition of the molecular states of the inner or older lamellæ to a less dense condition. Such a change is explicable in the light of the ready transmutability of one stereoisomeric form into another owing to slight differences in attendant conditions. (See preceding memoir-Publication No. 173, page 9.) The mere separation of the starch from direct contact with the plastid or the cell-sap by the later-deposited starch, age, and other incidental conditions, are of themselves doubtless sufficient to satisfactorily account for this transmutation. Likewise, differences in other parts, such as in primary and secondary lamella, protuberances, etc., in relation to other parts of the grains, may be explained in the same way.

\section{Each Starch Property an Independent Physico- Chemical Unit-character.}

Each starch property, whether it be manifested in peculiarities in size, form, hilum, lamellation, or fissuration, or in reactions to light, or in color reactions with iodine or anilines, or in gelatinization reactions with heat or chemical reagents, is an expression of an independent physico-chemical unit-character that is an index of specific peculiarities of intramolecular configuration, the sum of which is in turn an index which expresses specific peculiarities of the constitution of the protoplasm that synthetized the starch molecule. The unitcharacter represented by the form of the starch grain is independent of that size; that of lamellation independent of that of fissuration, etc. This is evident in the fact that in different starches variations in one may not be associated with variation in another, and that when variations in different properties are coincidently observed they may be of like or unlike character. Gelatinizability is one of the most conspicuous properties of starch and it represents a primary physico-chemical unit-character, which character may be studied in as many quantitative and qualitative phases as there are kinds of starches and kinds of gelatinizing reagents, the phenomena of gelatinization by heat being distinguishable from those by a given chemical reagent, and those by one reagent from those by another, and those of one starch by a given reagent from those of another starch. The gelatinization of the starch grain is not only a very definite chemical process but one that must vary in character in accordance with the reagent entering into the reaction. It follows, as a corollary, that the property of gelatinizability of any specimen of starch may be expressed in as many independent physico-chemical unitcharacter-phases as there are reagents to elicit them.

\section{Individuality or Specificity of Each Agent and REAGENT.}

The methods employed in the research, all microscopic, have, as stated, included inquiries into histologic characters; polariscopic, iodine and aniline reactions; temperatures of gelatinization; and quantitative 
and qualitative gelatinization reactions with a variety of chemical reagents which represent a wide range of differences in molecular composition. In some instances the starch molecules alone or largely determine the reaction, while in others both starch and reagent play important parts, as in chemical reactions generally. Thus, in the crystallographic studies of the hemoglobin crystals and in the polarization reactions with starch the molecules undergo no change; hence the reactions express peculiarities that are inherent to the molecules. In other starch reactions, in the gentianviolet and safranin reactions, the organization of the molecules is either unaffected or affected to an undetectable degree, the reactions being presumably adsorption phenomena; in the iodine reactions there is probably a feeble chemical combination of the iodine and starch, but without apparent intermolecular disorganization; in the temperature and chemical-reagent reactions there is an intermolecular breaking down by a process of hydration, with which process there may be associated reactions that vary in character and number in accordance with peculiarities in the compasition of the reagents. If the molecules of the starches from different sources are in the form of stereoisomers, it follows, as a corollary, that they must exhibit differences in their behavior with different agents and reagents, and show differences that are related to variation in the kind of agent and in the composition and concentration of the reagents. In other words, the reaction in each case is conditioned by the kind of starch and the kind of agent or reagent.

\section{Reliability of Methods as Shown by Charts and} Conformity of Results Collectively.

It is obvious that tests of the reliability of the methods employed in the differentiation of starches from various sources are to be found in the agreement of the results of repeated experiments and in the conformity of the results with established data of the systematist. As stated in preceding paragraphs, the polarization, iodine, and aniline methods are, notwithstanding their crudity and limitations, reliable if the experiments are carried out with sufficient care; the temperature of gelatinization method is accurate within very narrow limits of error; and the gelatinization method used in the present research by means of chemical reagents is practically exact. The first three methods are, owing to their usually very restricted range of values, of very much more usefulness in the differentiation of members of a genus than of different genera, and this applies, although to a less degree, to the temperature of gelatinization method; while the chemical reagent method has unlimited application to both intrageneric and intergeneric differentiation, though the different reagents have widely varying values. In comparing these records with those of the systematist it is important to recognize that a slight change in molecular constitution may give rise to very marked changes in properties and that distinction must be made between that which is definitely established and that which is tentative in even the most advanced taxonomic system. All things considered, it is remarkable how close in general is the agreement of the data of these exceedingly dissimilar methods of investigation. In fact, they are evidently mutually corrective and confirmatory, and where seeming or actual disagreements exist it doubtless will be found that further applications of the physico-chemical method will demonstrate the reasons.

Certain of the several forms of charts are of especial value in showing the reliability of the methods used, particularly those which are included in the groups D 1 to $\mathrm{D} 691$ and $\mathrm{E} 1$ to $\mathrm{E} 46$. These charts have been given somewhat detailed discussion in Sections 2 and 3 of Chapter IV. Even a most cursory examination separately and together will demonstrate their taxonomic values. In group $\mathrm{D} 1$ to $\mathrm{D} 691$, in which are presented the progress of gelatinization at definite time-intervals, it is obvious from the characters of the curves, both in courses in the individual charts and in the parent-hybrid and the generic groups, that they are quite as dependable as the data of the systematist. Were these records not reliable, it seems clear that the curves would not take regular but irregular or zigzag circumlinear courses, or instead of being straight or practically straight lines be irregular, etc.; moreover, there would not be the conformity of the curves of the reactions with each reagent that is found in each set of parent-and hybrid-stocks, or in the sets belonging to each genus, excepting in the latter when subgeneric divisions are represented. The more or less marked subgeneric differences attest the value of the method, and if in some instances they may seem to be disproportionate to the differences of the systematist, this may be and doubtless is owing to a greater sensitivity of the physico-chemical method.

The plan adopted in the preparation of Charts $\mathrm{E} 1$ to E 46, in which composite curves of the reaction-intensities are exhibited, has proved in a very large measure successful in eliciting varietal, species, subgeneric, and generic peculiarities, but its essential defect is to be found in the neglect of differences that were found during the earlier periods of experiment. In the formulation of these charts terminal data were used-that is, the time of complete or practically complete gelatinization in an hour or of the percentage of total starch gelatinized within the same period. In many instances such figures may be the same, yet there may have been more or less marked differences in the progress of gelatinization during the early periods of the experiments. Notwithstanding such defects, there is in general a remarkable degree of conformity of these curves with taxonomic data. There should be considered with the foregoing the figures presented in Table B 1 which give the numbers of very high, high, moderate, low, and very low reactions; the sums of reaction-intensities; and average reaction-intensities of each starch and each parent-hybrid set of starches.

\section{General Conclusions drawn from Results of the Hemoglobin Researches.}

The results of the crystallographic studies of the hemoglobins indicate: that there is a common structure of the hemoglobin molecule, whatsoever the source of the hemoglobin; that the crystals of the species of a genus belong to a crystallographic group which represents a generic type; that the crystals of each species of a genus when favorably developed can be distinguished from those of another species of the genus; that in some species there may be found one, two, or three forms of hemoglobin, and that this seems to be a generic peculiarity, 
inasmuch as if in one species there be found, say, three forms the same number will exist in other members of the genus; that the crystals of different genera differ as definitely and specifically as those of crystalline groups of mineral substances differ chemically and as generic groups differ zoologically or botanically; and that by means of peculiarities of the hemoglobins phylogenetic relationships can be traced, as has been found in the case of the bear and seal and other animals.

\section{General Conclusions drawn from the Starch RESEARCHES.}

The results of the hemoglobin and starch researches are mutually confirmatory in support of the existence of stereoisomeric forms of complex organic substances that are specifically modified in relation to varieties, species, subgenera, and genera, and that these specificities indicate corresponding peculiarities of the protoplasms in which the substances are formed. The records of the starch researches indicate: that each starch property is an independent physico-chemical unit-charaeter, and that the unit-character represented by the property of gelatinizability may be manifested in an indefinite number of quantitative and qualitative unit-character-phases, the number varying with the form of starch and the number of gelatinizing reagents employed; that qualitative reactions are as distinctive and important as the quantitative reactions; that the reactions of different starches with a given reagent vary within wide limits, and that those of each starch vary with each reagent independently of the variations of other starches; that the reactions of varieties of a species very closely correspond to those of the species and are in aocord with botanical characters; that the reactions of members of a genus are in general in close accord with taxonomic data and constitute a generic type, the varieties and species tending to exhibit closeness or separation in their relationships in close accord with botanical peculiarities; that when a genus is represented by subgenera or other form of subgeneric division (such as rhizomatous and tuberous plants, or hardy and tender species, etc.), the reactions may exhibit as many different groupings as there are subgeneric divisions, and that these divisions may show very marked differences, even more marked than what may be noted in the case of closely related genera; that the reactions of closely related genera tend to be similarly close; that in hybrids any one of the six parent-phases (the same as the seed parent, the same as the pollen parent, the same as both parents, intermediate, higher than either parent, and lower than either parent) can be developed at will by the selection of the proper reagent; that the tendencies of different reagents to elicit in the hybrid any given parent-phase varies with reagent and starch, certain reagents tending to develop sameness to the seed parent or to the pollen parent, etc., and a given reagent may elicit one phase with one starch and another phase with another starch, etc., so that by the selection of the reagent any parent-phase can be developed in any given starch; that the starches of hybrids tend to show marked closeness to the properties of the parental starches when the parents are closely related, and to exhibit a tendency to more and more divergence as the parents are more and more distantly related, in some instances tending by comparatively numerous intermediate characters to bridging the parental characters and in others to be particularly characterized by being very closely related to one parent, or in others (by excess or deficit of development) to be quite variant from the parental types, etc.; that the starches of different hybrids show a very wide range in their parental relationships, some being almost throughout very close to the seed parent, others very close to the pollen parent, others for the most part intermediate, etc.; that the starches of hybrids of reciprocal crosses and of the same cross, respectively, are different, the former differing from each other far more than the latter from each other; that the relationships of the properties of starches of hybrids to the properties of the parents are in harmony with the data of the macroscopic characters collected by Focke, with the data of DeVries mutants (hybrids), and with the macroscopic and microscopic tissue characters recorded in this research, in showing that in any given hybrid the development of different characters may take on different directions so that some properties are like those of one or the other parent or both parents, or developed in excess or deficit of parental extremes, and also that new characters and character-phases may appear.

\section{General Conclusions drawn from Investiga-} tions of the Macroscopic and Microscopio Characters of the Plant.

The results of the studies of macroscopic and microscopic tissue characters are in harmony with those recorded by Focke and of the researches with the starches in showing that in any given hybrid certain characters may be the same as those in one or the other parent or both parents, intermediate, or developed in excess or deficit of parental extremes, and that the distribution of these directions of character development is most variable. A surprising result is found in a common lack of correspondence between the percentages of macroscopic and microscopic characters of any given hybrid that are the same as those of the seed parent or pollen parent, or intermediate, etc. Why, for instance, in any hybrid the percentage of macroscopic characters that are the same as those of the seed parent are relatively large in comparison with the percentage of microscopic characters or vice versa is as yet inexplicable. What pertains to one of the six parent-phases applies equally to all. Moreover, there is not a constant quantitative agreement between the macroscopic and microscopic characters, separately or combined, and between either of these and the starch characters of the same plant in the percentage distributions among the parent-phases.

\section{The Relative Potentialities of the Seed} Parent and the Pollen Parent in Influencing the Characters of the Hybrid.

The relative potentialities of the parents in determining the characters of the hybrids and in the distribution of characters among the six parent-phases varies within wide limits. In the starch reactions it is shown that in some hybrids the influences of one parent are almost or practically negligible, in others they appear to be about equally divided, and in others there are various gradations in degree and kind between these extremes. In the tissue characters concordant results were recorded, but here the variations were found to be very much restricted, 
doubtless because chiefly of the small number and the kinds of hybrids studied. In summing up the characters that are the same as or inclined to the seed parent and the pollen parent, respectively, it was found in the 1,018 starch reactions that the seed parent is, on the whole, distinctly more potent than the pollen parent, while in 959 tissue characters the parental influences are equal.

\section{Species Parents versus Sex Parents.}

The parental properties referred to in the preceding section are, in an important sense, illusory, because they indicate sexual instead of species characters. The terms seed parent and pollen parent have been used in this research in the conventional sense of the botanist and horticulturist, that is, without necessarily implying or even inferring unisexuality of the plants. This usage, together with the employment of the signs $o+$ and $\delta$, may carry the impression that the parents of the hybrids are correspondingly female and male, but all of the parents are flowering plants in which in each individual there are produced both female and male gametes. Each plant is, therefore, female or male in reproduction in accordance with whether it furnishes the seed or the pollen, irrespective of the actual sex of the organism. A concrete illustration of this paradoxical statement is found, for instance, in Cypripedium spencerianum and C. villosum, which have been reciprocally crossed, yielding the hybrids $C$. lathamianum and $C$. lathiamianum inversum, these hybrids not being identical but very closely resembling each other (page 338 et seq.). In the first cross the seed of $C$. spencerianum was fertilized by the pollen of $C$. villosum, and in the second cross the pollen of $C$. spencerianum fertilized the seed of $C$. vitlosum, thus reversing the parentage. Inasmuch as each plant is precisely the same in both crosses, it is evident that the properties ascribed to $C$. spencerianum as the seed parent and the pollen parent, respectively, are identical and therefore that they are, as far as we can discern, peculiarities of species and not of sex. However, the differences in the offspring of reciprocal crosses show that while the seed and the pollen carry species-characters they also transmit certain obscure properties that are peculiar to each of the sex elements.

All living tissues have without question species-types of metabolism, and, as a corollary, species-types of complex organic metabolites (see preceding memoir, Carnegie Institution of Washington, Pub. No. 173, page 12) ; and if the tissues are further characterized by femaleness or maleness, they must have the corresponding sex-types. In bisezual or monocious organisms, such as the plants used in this research for the sources of the starches and tissues, the structures, processes, and products, with the exception of those belonging to the primary sex organs, are without determinable sex characters, yet for wellknown reasons it is certain that they possess inherently potentialities of both sexes. In unisexual organisms, as in certain plants and in all normal mammalia, there must be both species-types and sex-types. Therefore, in the first group of the properties are broadly speaking or preeminently those of species, and in the second those of species and sex.

That there are species-types is convincingly shown by the distinguishing features of species; and that there are very definite sex-types has been rendered positive, especially. by recent investigations. For instance, in gynandromorphs (as noted in a bullfinch by Poll, in a chaffinch by Weber, in a pheasant by Bond, and in men, dogs, guinea-pigs, crabs, bees, ants, butterflies, and moths by various writers) the structures of the two sides or of the anterior and posterior parts of the body, or of different organs or of parts of an organ are oppositely sexed. Geoffrey Smith found that the bloods of female and male spider crabs differ, and Stecke in investigations with moths noted that not only do the bloods of the sexes differ but also are as much unlike as are thase of individuals of the same sex of different species. The bloods of woman and man, and of the sexes of certain other mammals, are not identical. The ovaries and testicles are specifically female and male organs, and the egg, spermatozoon, and sex hormones are similarly sexed. Moreover, during the existence of the germplasm, and even in some organisms long after development has proceeded, there is a period of sexual plasticity during which various factors may be directly operative on the egg or indirectly through the parent, or directly on the metabolic processes of the individual, to lead to the development of either sex or of either female or male secondary characters, as the case may be, and hence to corresponding female or male types of metabolism and metabolites. In studies of the pupa of butterflies, Standfuss found that by the influence of temperature the female can be made to assume the male type. Geoffrey Smith noted that the sacculinated male spider crab (that is partially or completely parasitically castrated) becomes markedly feminized, even to the extent of rudimentary eggs being formed in the testes. Riddle records in studies of pigeon eggs a transmutability so marked that eggs having one sex tendency may be caused to become oppositely sexed. Steinach and others in ovarian and testicular transplantation experiments have shown that the female can be masculinized and the male feminized. Moreover, the potent influences of food, of an excess or deficit of water in the egg, of the energy of oxidative metabolism, and of light on sex control are well known. And in the human being indications of female and male types of metabolism and metabolites are to be found among differences in the sexes in bodily structures, in the composition of the blood and certain other parts, in the actions of a number of medicinal substances and certain internal secretions, in the properties of the sex hormones and of some other substances that are produced by sex organs other than the ovaries and testes, in basal metabolism, in psychic phenomena, etc.

The factor or factors that determine species-types are not known, nor have we much definite knowledge of those which control sex-types, but it may justly be assumed that what is learned of one is applicable in principle to the other. Since the discovery of the sex hormones there has been a tendency generally to attribute to them the determination of secondary sex characters, but there are reasons for believing that other substances, as yet unknown, may be similarly potent. Thus, Meisenheimer showed by the results of experiments with the larvæ of the gypsy moth that secondary sex characters are developed without material modification after the removal of the ovaries and testes; and it is evident that in gynandromorphs both sex hormones circulate throughout the organism, and thus reach every tissue, yet some parts 
become specifically female and others male. Moreover, in addition to these sex hormones and hypothetical substances there are the influences of environmental conditions which are effective in unknown ways.

If, as seems manifest, there are species-types of metabolism, if these types are undoubtedly modifiable by environmental conditions, if these types give rise to corresponding species-types of metabolites, and if these metabolites have inherently the potentialities of both parents that can, as has been shown, be elicited in any one or more of the six parent-phases by the selection of the proper agent or reagent, it seems to follow, as a corollary, that corresponding properties should be manifested by sex-types. These statements suggest that in artificial parthenogenesis and artificial fertilization the selection of a proper agent or reagent may render it possible to give rise to either sex, or before or after development has begun, to gynandromorphism. In a word, from present knowledge and indications (and all that they imply), species, parthenogenesis, fertilization, sex, secondary sex characters, and sex control are problems of physical chemistry.

\section{Intermediateness as a Criterion of Hybrids.}

Whether or not intermediateness is a criterion of hybrids depends upon the sense in which these two terms are used-that is, whether or not intermediateness is to be taken as meaning mid-intermediateness, and where the line is to be drawn where intermediateness in either a broad or a narrow sense is or is not a criterion. Some authorities, as is evident by references in the introduction, look upon intermediateness in the sense of midintermediateness or " exact intermediateness," and upon this developmental peculiarity as being a criterion when all or nearly all of the characters of the hybrid are midintermediate; but it is manifest that such a conception is not justified by literature and is untenable. Viewing intermediateness from a broad point of view-that is, to include all characters which show stages of character development between those of the parents, it is an open question as to whether a character that is intermediate but exhibits almost identity with that of one parent should be classified as intermediate or as being the same as the character of the parent. Many of both the starchreaction and the tissue characters that herein have been classified as intermediate have been so close in their development to the parent characters that it is questionable if they should not have been assigned to the characters that are the same or practically the same as those of the parent. Then again, what percentage of intermediate characters must be intermediate to justify the application of the term criterion? Among the 1,018 starch reactions, 236 were recorded as being intermediate, while 53 were mid-intermediate. Among the 959 macroscopic and microscopic tissue characters 415 were intermediate, and 160 were mid-intermediate. The differences in the figures of the starch and tissue records are probably due chiefly to differences in both number and kind of material. Moreover, the percentages of characters developed beyond parental extremes are very high, those in the starch reactions exceeding (nearly doubling) the percentage in intermediate characters $(40.6: 23.2)$, and in the tissue characters being almost as high as the latter $(39: 43.2)$. It seems from these data that if intermedi- ateness is a criterion, development in excess and deficit of parental extremes may or should have an equal or greater degree of importance, and even a far greater value if only mid-intermediate characters are taken as the criterion.

\section{Gramplasm a Stereochemic System.}

The recognition that the germplasm is a stereochemic system that is characterized by the kinds and arrangements of its stereoisomers in the three dimensions of space; that it is of great complexity, impressionability, and plasticity; that it presumably possesses potentially the characters and character-phases of the parent; that the germplasms of the sexes are different, varying in plasticity, etc.; and that in normal fecundation there occurs a union of the two sex systems with interactions, rearrangements, and combinations, and therefore a new physico-chemical state is developed that possesses the potentialities of both sexes; that stereoisomerides are readily transmuted with attendant change of properties, and that the directions and propensities of the reactions are determined by peculiarities of the compounds and attendant conditions; and, finally, that we hare, in a word, in the germplasm a form of protoplasm that must like all colloidal substances be studied upon the basis of physical chemistry, opens up a unique and promising field for investigation of the laws that determine organic growth, form, and function.

Applications to the Explanations of the Occurrence of Vartations, Spokits, Fluctuations AND the Genesis of Species.

The characters of the germplasm and of protoplasm, and incidentally the extraordinary plasticity of the starch molecule, as set forth by the results of this research, seem readily to induce clear conceptions of the mechanisms that underlie variations, sports, fluctuations, Mendelism, reversions, monstrosities, etc., and also the genesis of strains, subspecies, and species by gradual and progressive changes and ultimate fixation. And it also seems, from the data presented in conjunction with biological literature, that we have all of the postulates that are necessary to warrant the assumption that probably the chief method in the genesis of species is by hybridization.

Scientific Basis for Classification of Plants and Amimals and for the Study of ProtoPLASM.

The discovery of the existence of highly specialized stereoisomers that are specifically modified in relation to genera, species, varieties, etc., has brought to light one of the most extraordinary phenomena of living matter, and it not only gives us a strictly scientific basis for the classification of all forms of life, but also leads us to the varying constitutions of protoplasm of the same and of different organisms, and to the differences in vital phenomena that are dependent upon these variations. The dictum set forth in the hemoglobin investigation that "vital peculiarities may be resolved to a physicochemical basis" has been most substantially supported, and it may be safely predicted that important and even epochal advances in the elucidation of many of the great problems of biology will be made in the near future along such or closely related lines of investigation as have been pursued in these researches. 

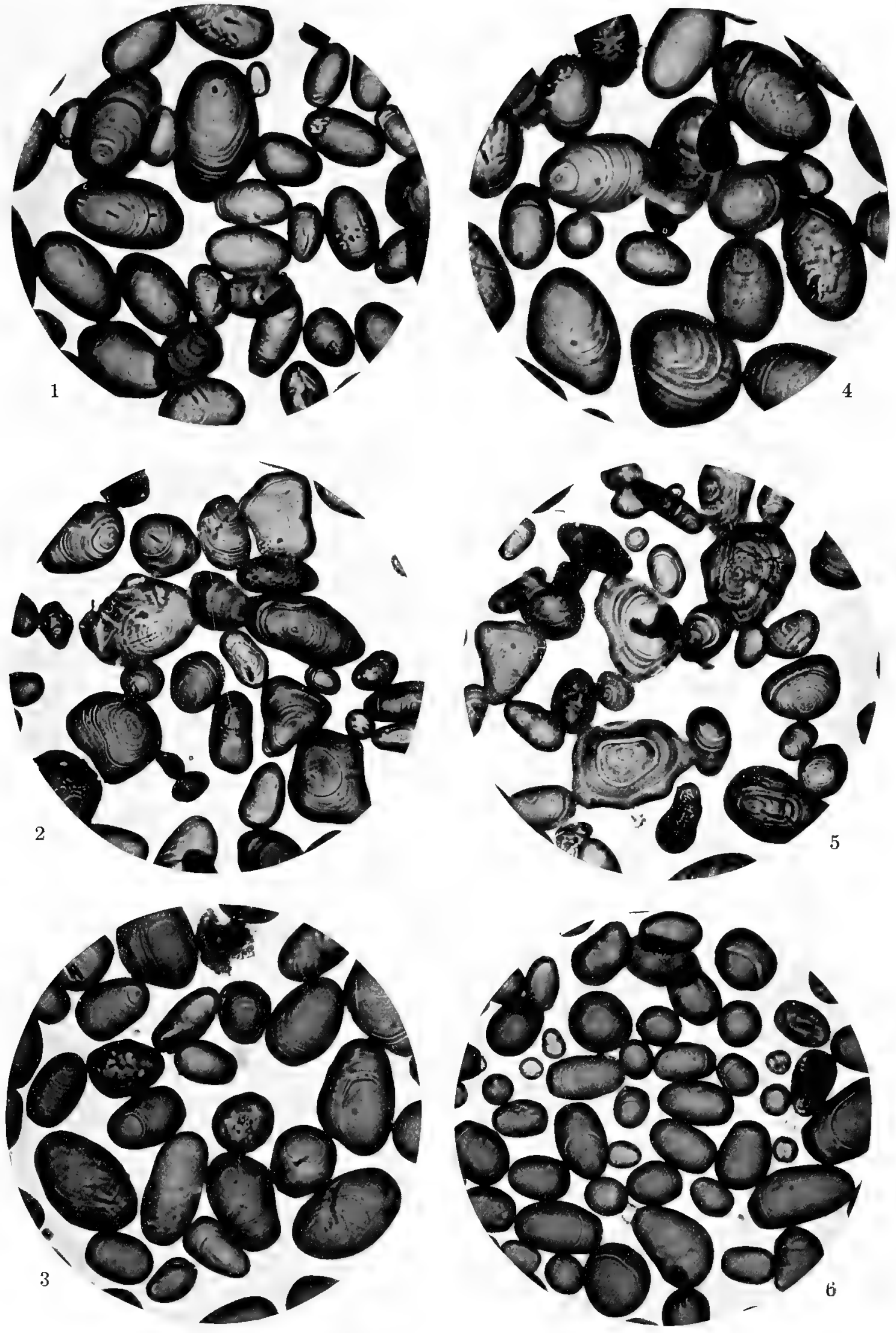

1 and 4. Amaryllis belladonna. 2 and 5. Brunsvigia josephince.

3. Brunsdonna sanderce alba. 6. Brunsdonna sandero, 


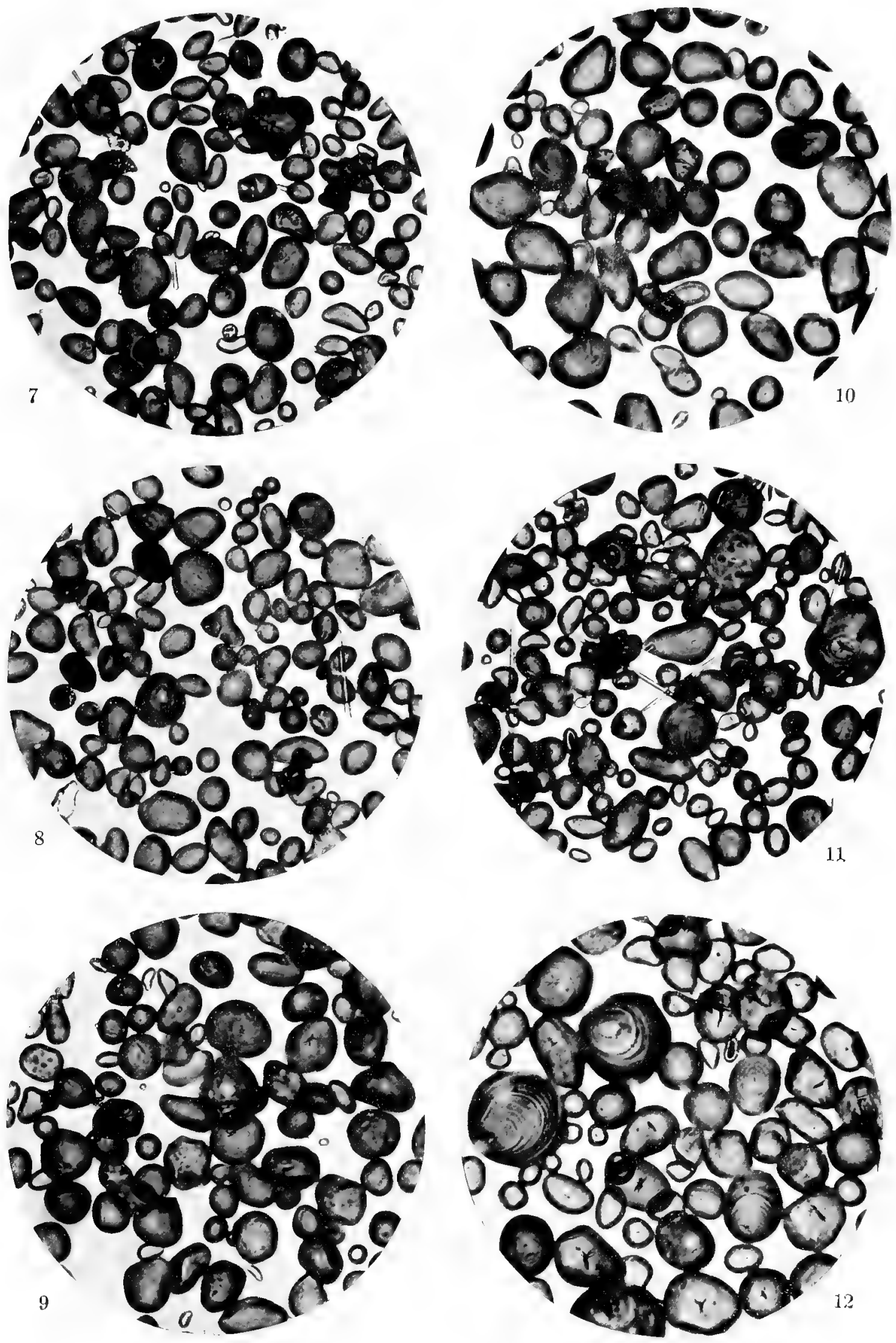

7. Hippeastrum titan.

8. Hippeastrum cleonia.

9. Hippeastrum titan-cleonia.

ippeastrum ossultan.

11. Hippeastrum pyrrha.

12. Hippeastrum ossultan-pyrrha. 

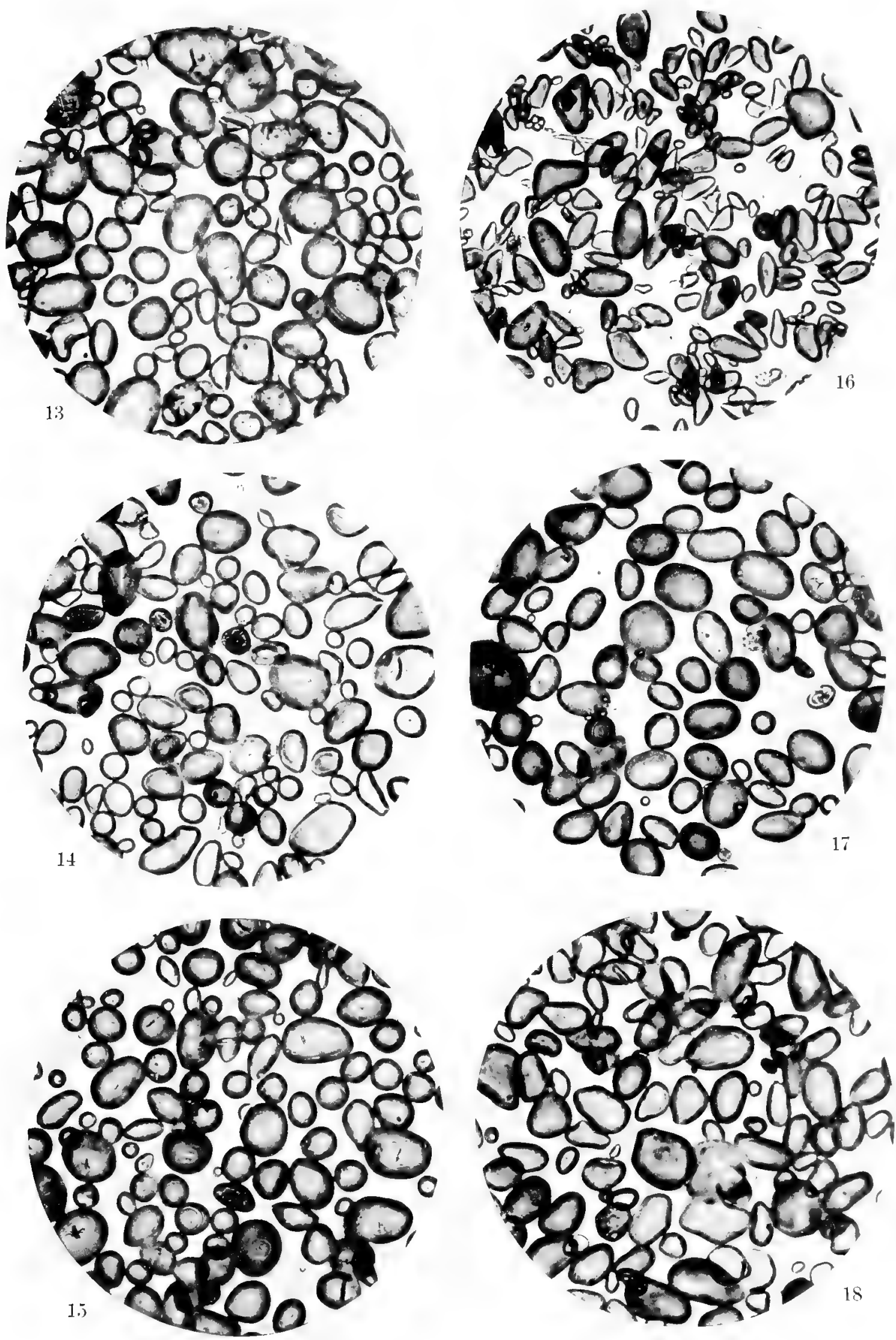

13. Hippeastrum doones

14. Hippeastrum zephyr.

15. Hippeastrum doones-zephyr.

16. Homanthus katherince.

17. Homanthus magnificus.

18. Hamanthus andromeda, 

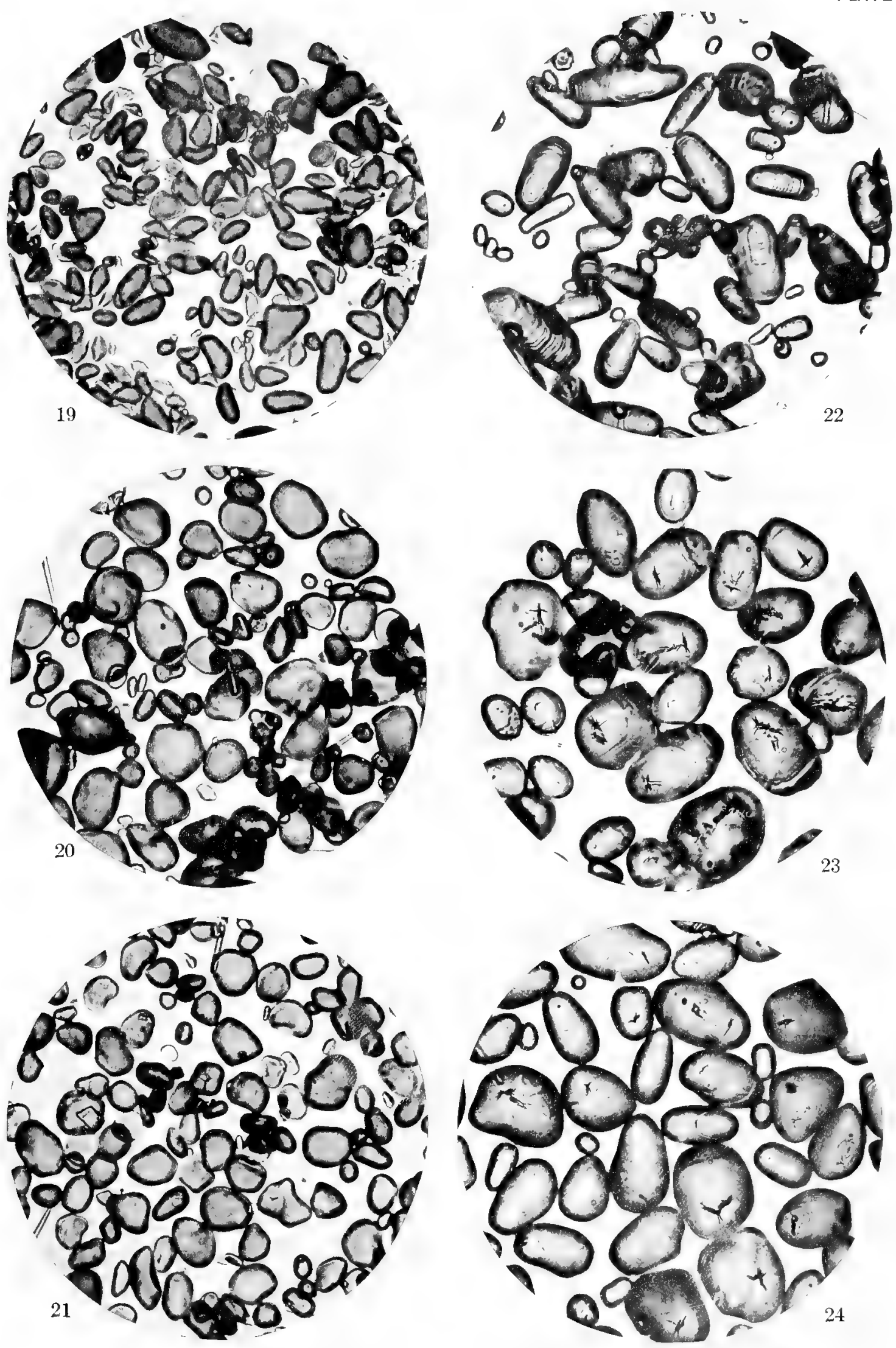

19. Homanthus katherince.

20. Homanthus puniceus.

21. Homanthus könig albert.

22. Crinum moorei

23. Crinum zeylanicum.

24. Crinum hybridum j. c. harvey. 

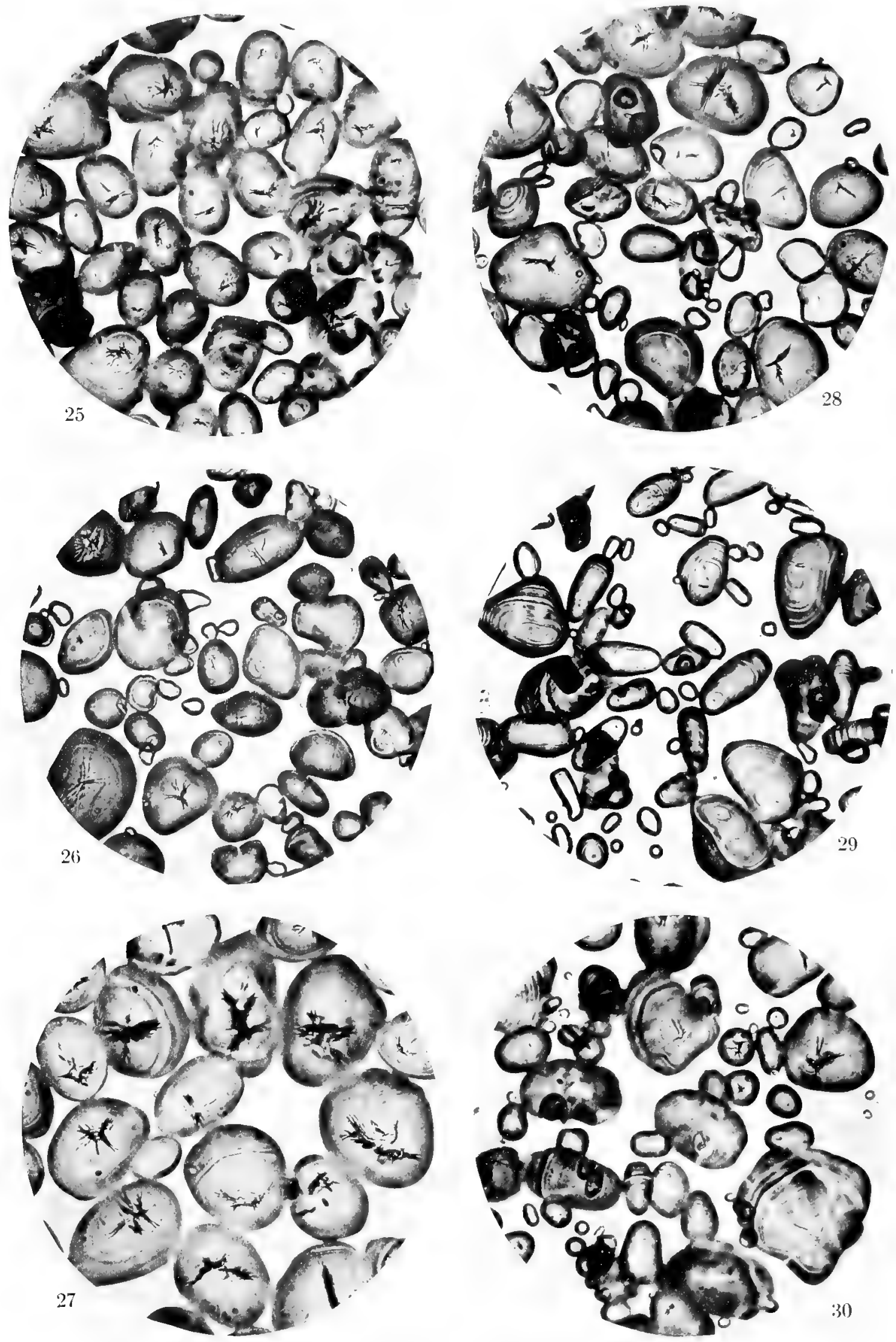

25. Crinum zeylanicum. 


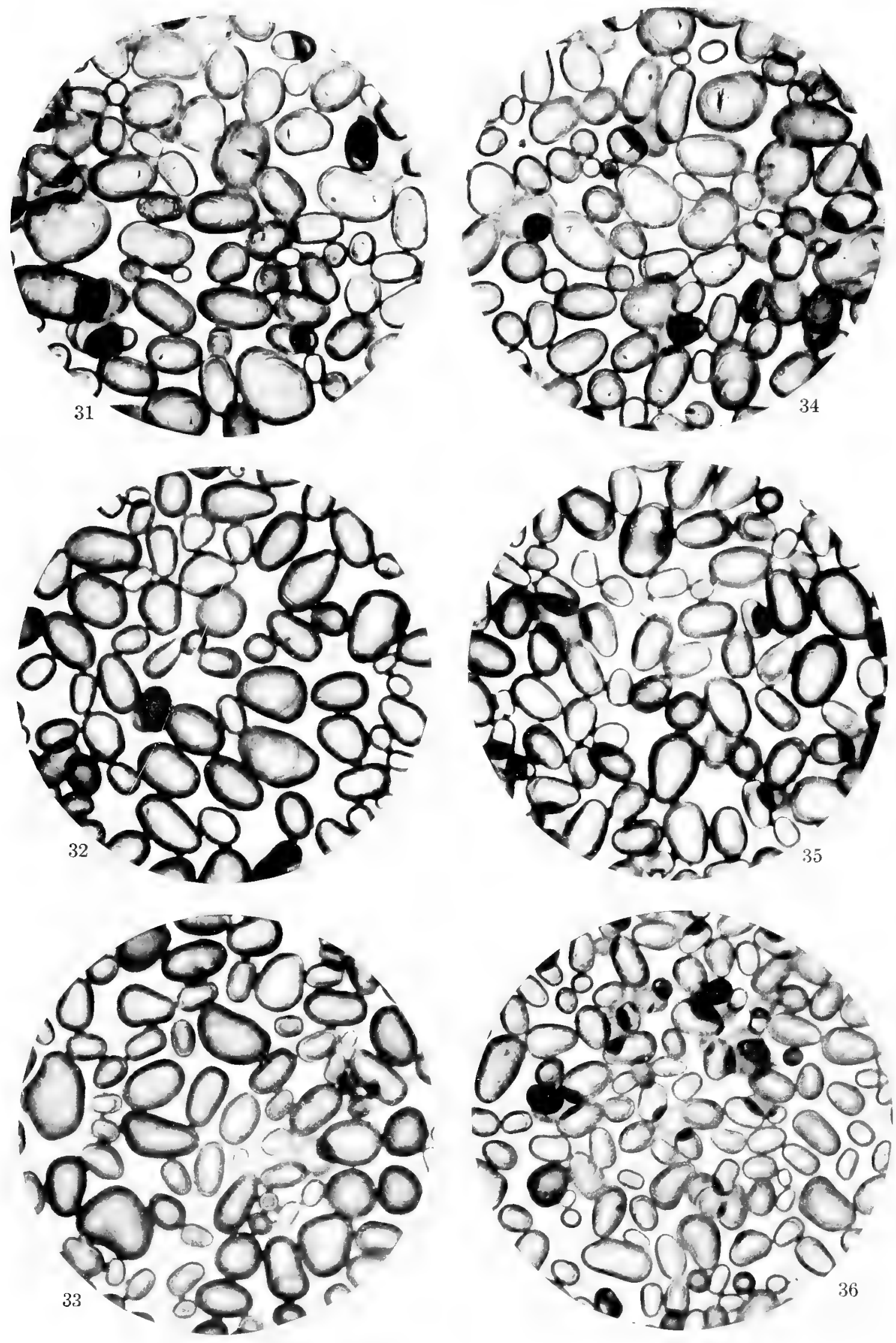


$$
\text { - }
$$



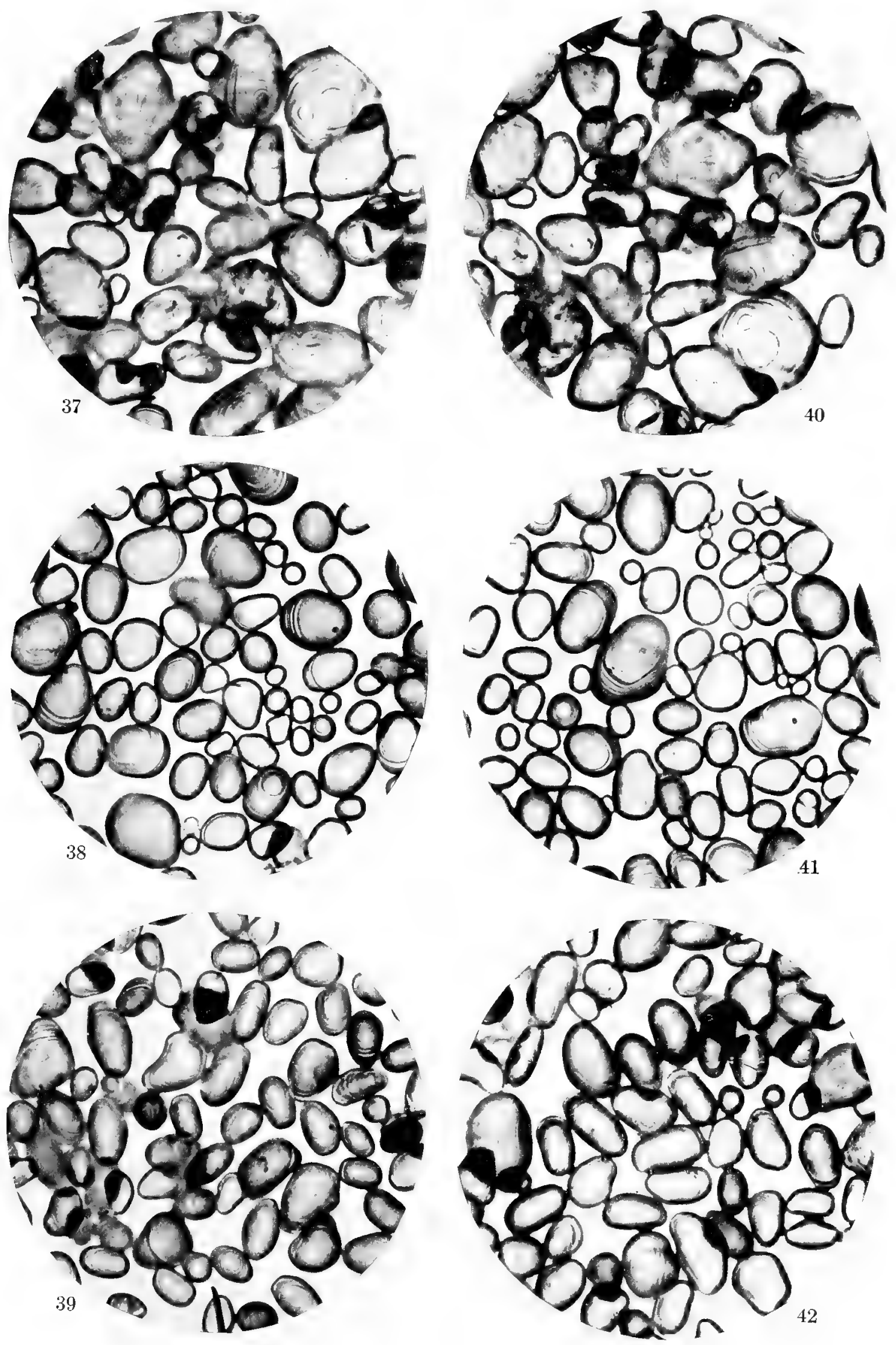

37 and 40 . Nerine bowdeni.

38 and 41. Nerine sarniensis var. corusca major.

39. Nerine giantess.

42. Nerine abundance. 

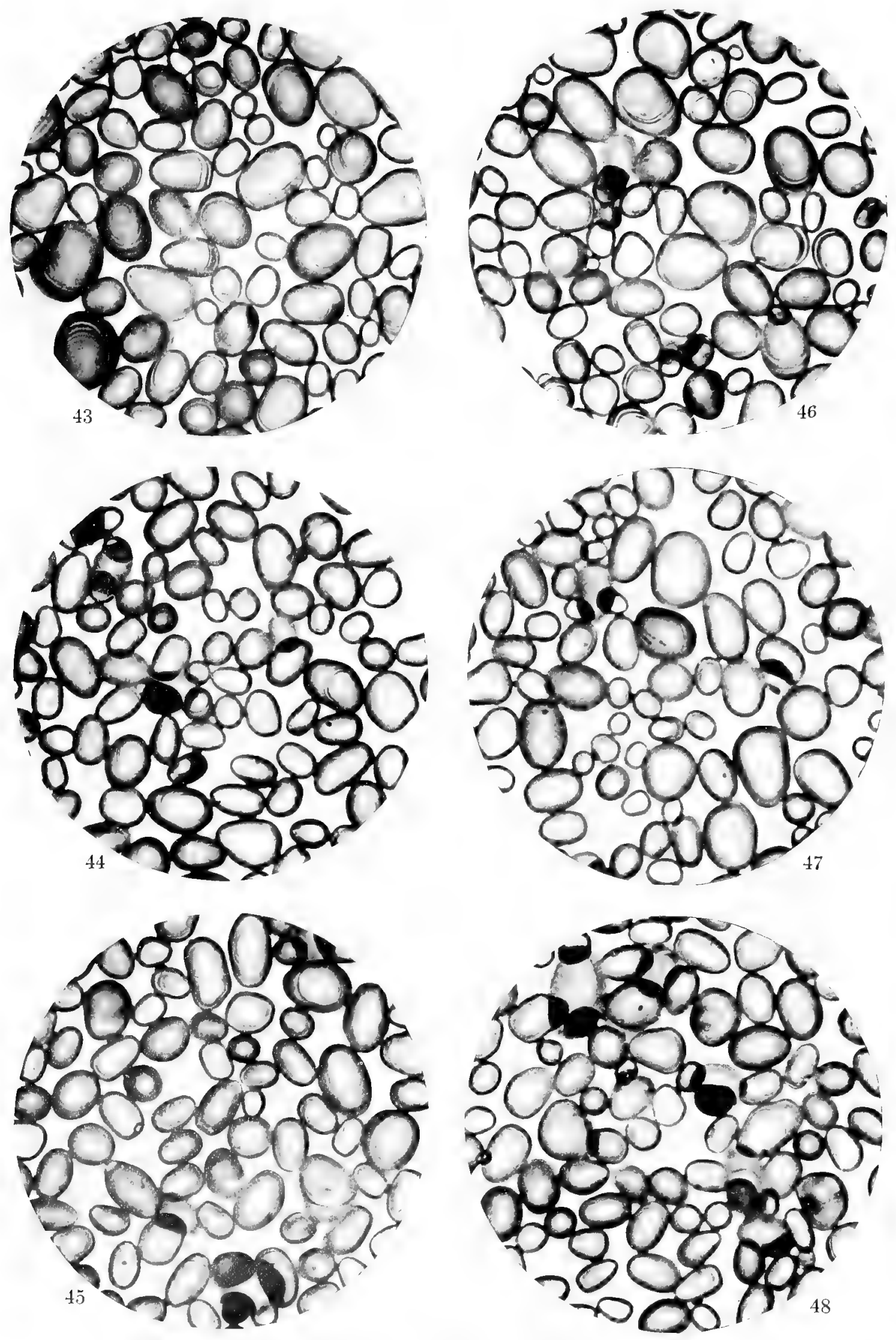

43 and 46. Nerine sarniensis var. corusca major.

45 and 48. Nerine glory of sarnia. 

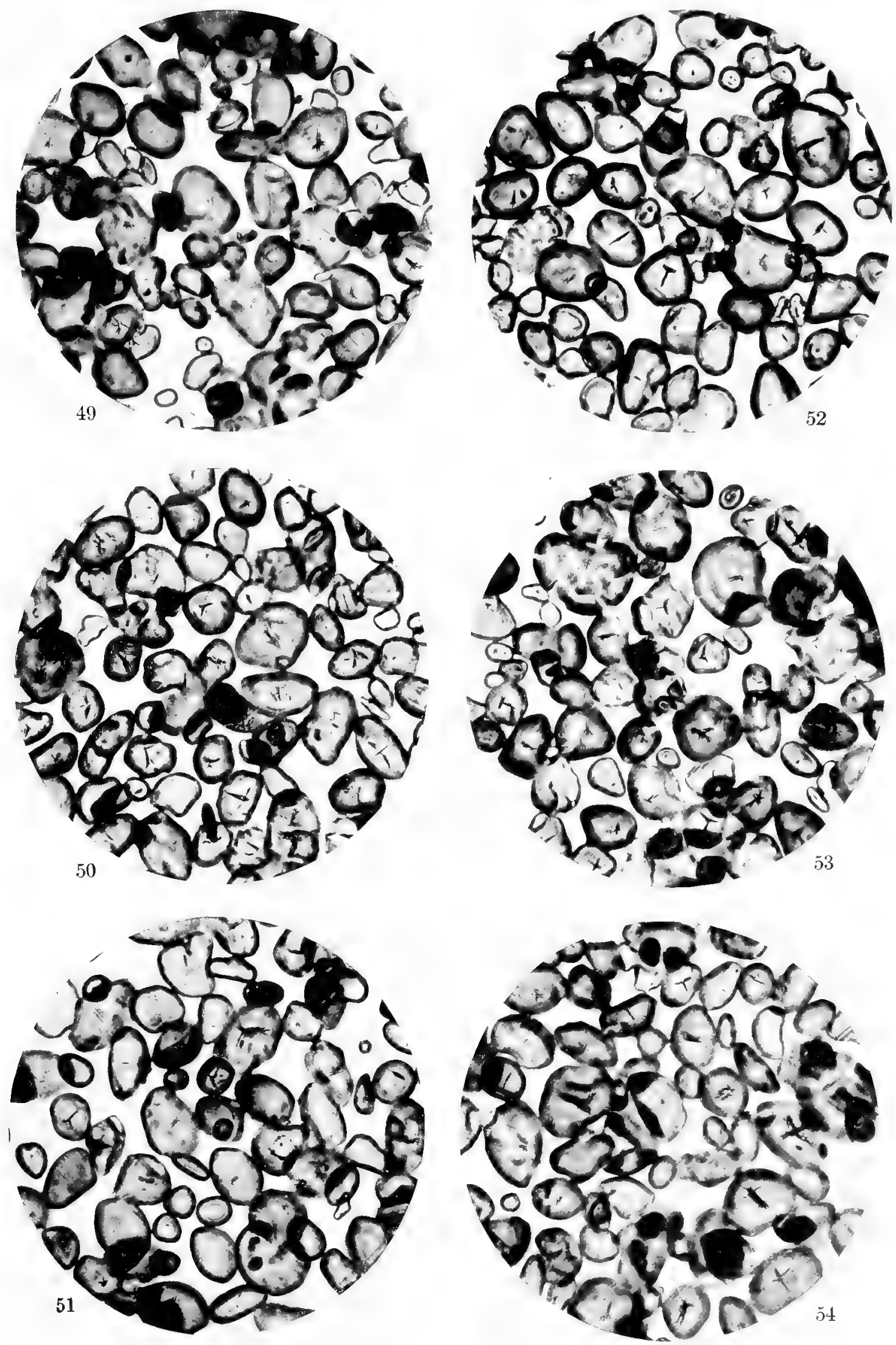

50 and 53. Narcissus poeticus poetarum.

51. Narcissus poeticus herrick.

54. Narcissus poeticus dante. 


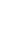



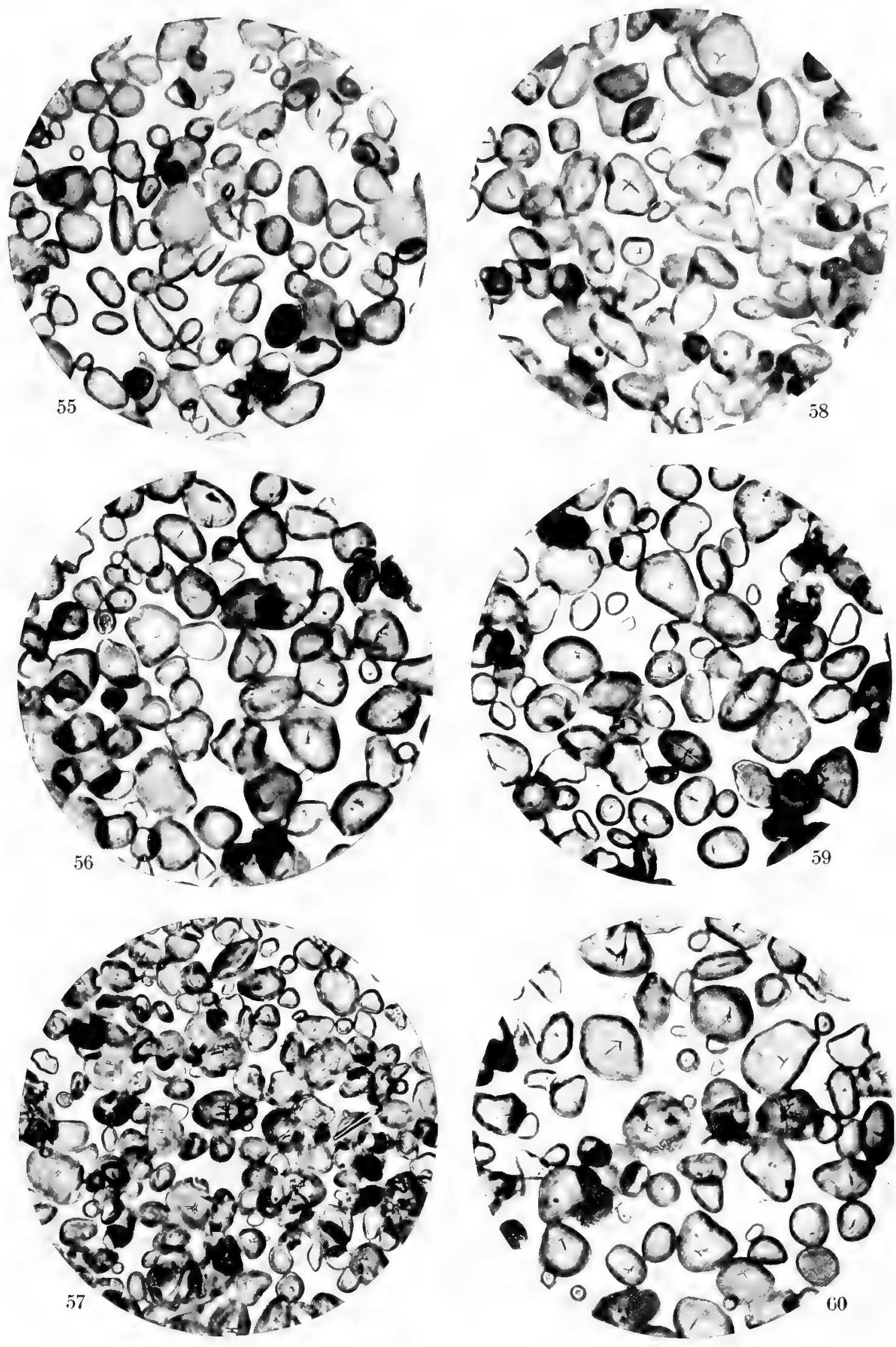

55. Narcissus tazetin grand monarque. 56. Narcissus poeticus ornatus. 57. Narcissus poetaz triumph. 

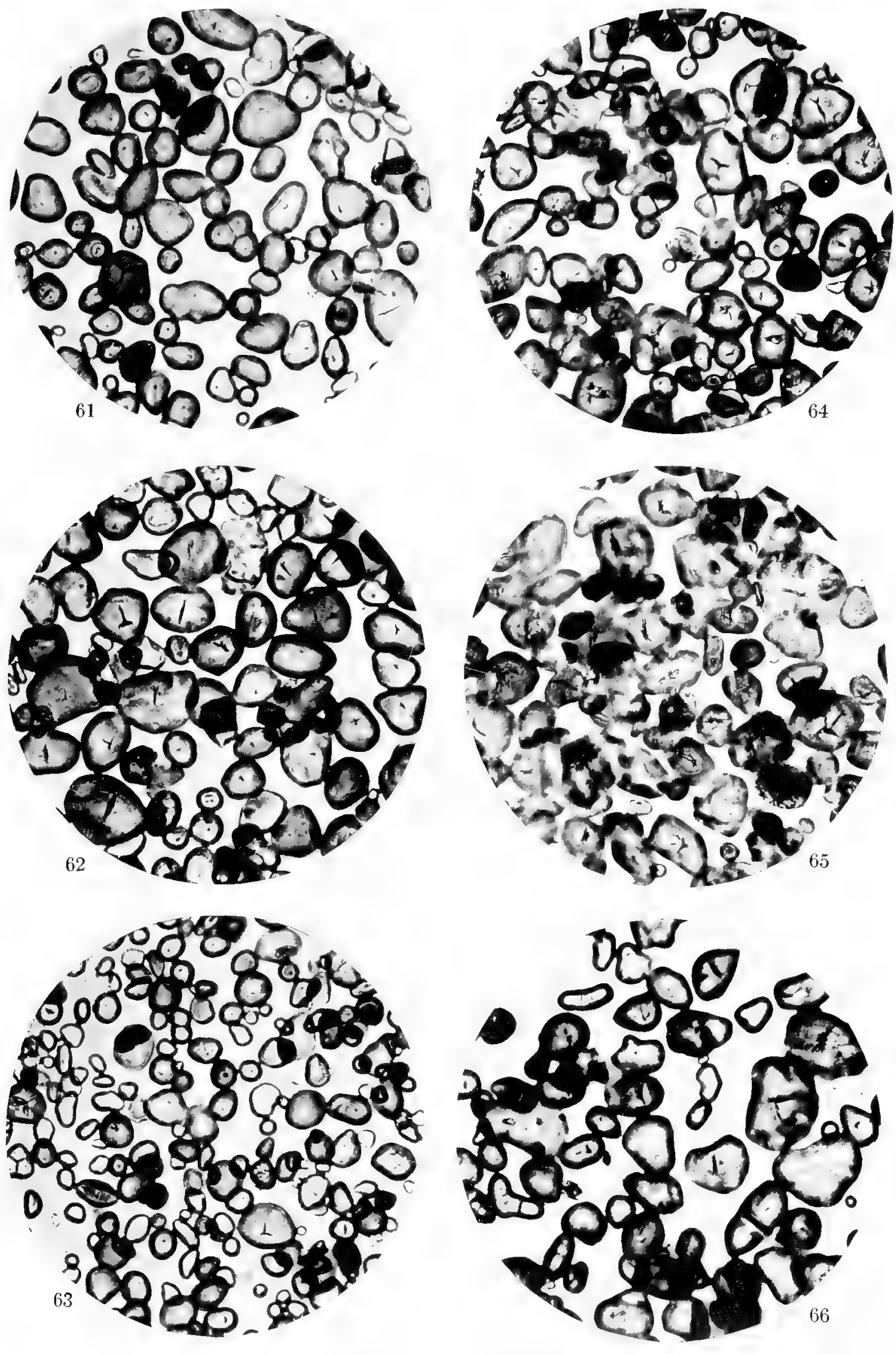

61. Narcissus telamonius plenus. 62. Narcissus poeticus ornatus. 63. Narcissus doubloon. 


\section{.}



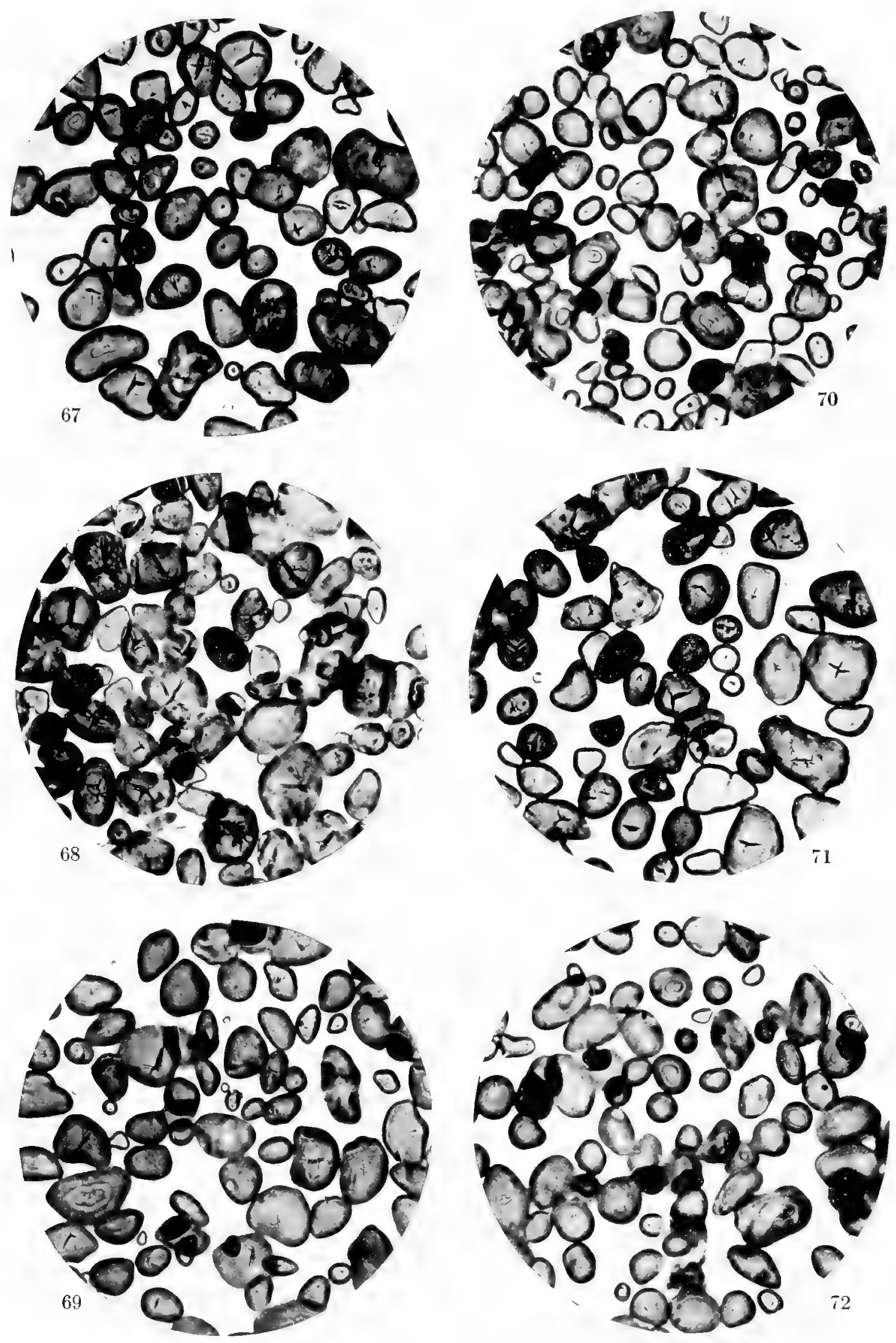

67. Narcissus abscissus.
68. Narcissus poeticus poetarım. 69. Narcissus will scurlet.

70. Narciswus allicans.

71. Narcisists absisioles.

72. Nurcissus bicolor apricol. 
$$
\text { , }
$$ 

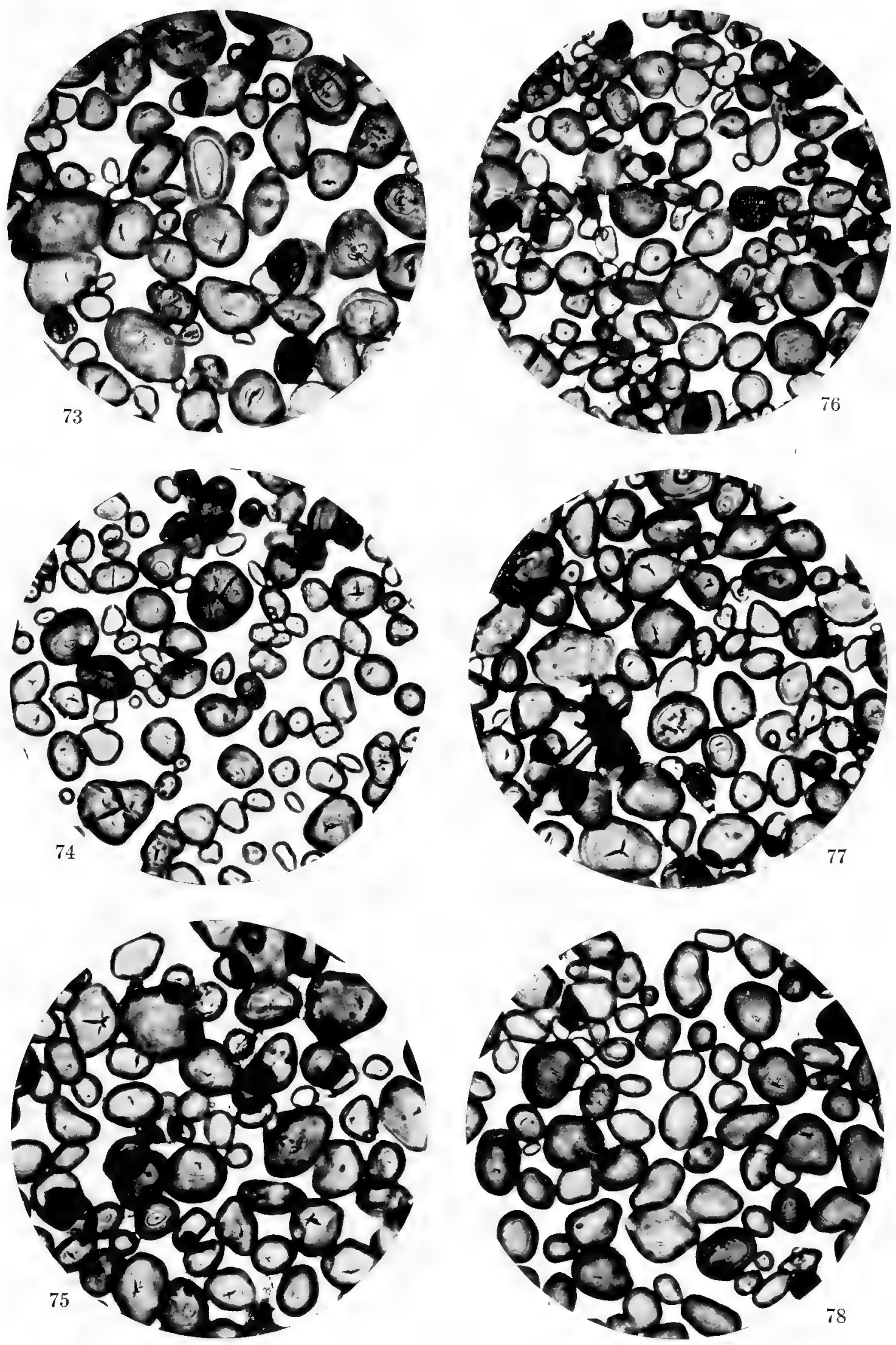

73. Narcissus empress.

74. Narcissus albicans.

75. Narcissus madame de graaff.

76. Narcissus weardale perfection.

77. Narcissus madame de graaff.

78. Narcissus pyramus. 

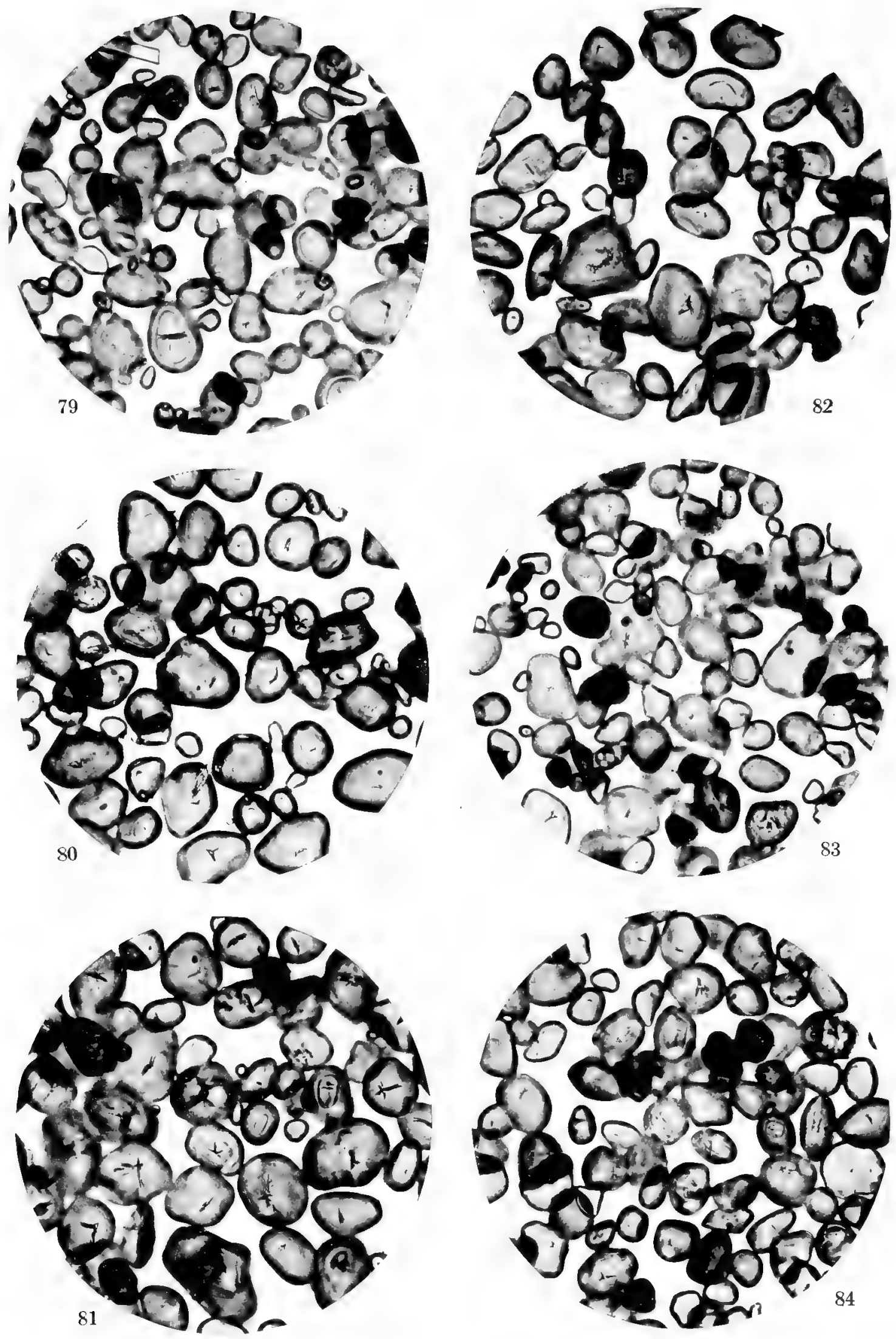

79. Narcissus monarch. 80. Narcissus madame de grat
81. Narcissu: lord roberts.

82. Narcissus leedsii minnie hume

83. Narcisins triandrus albus.

84. Narcissus agnes harrey. 


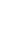



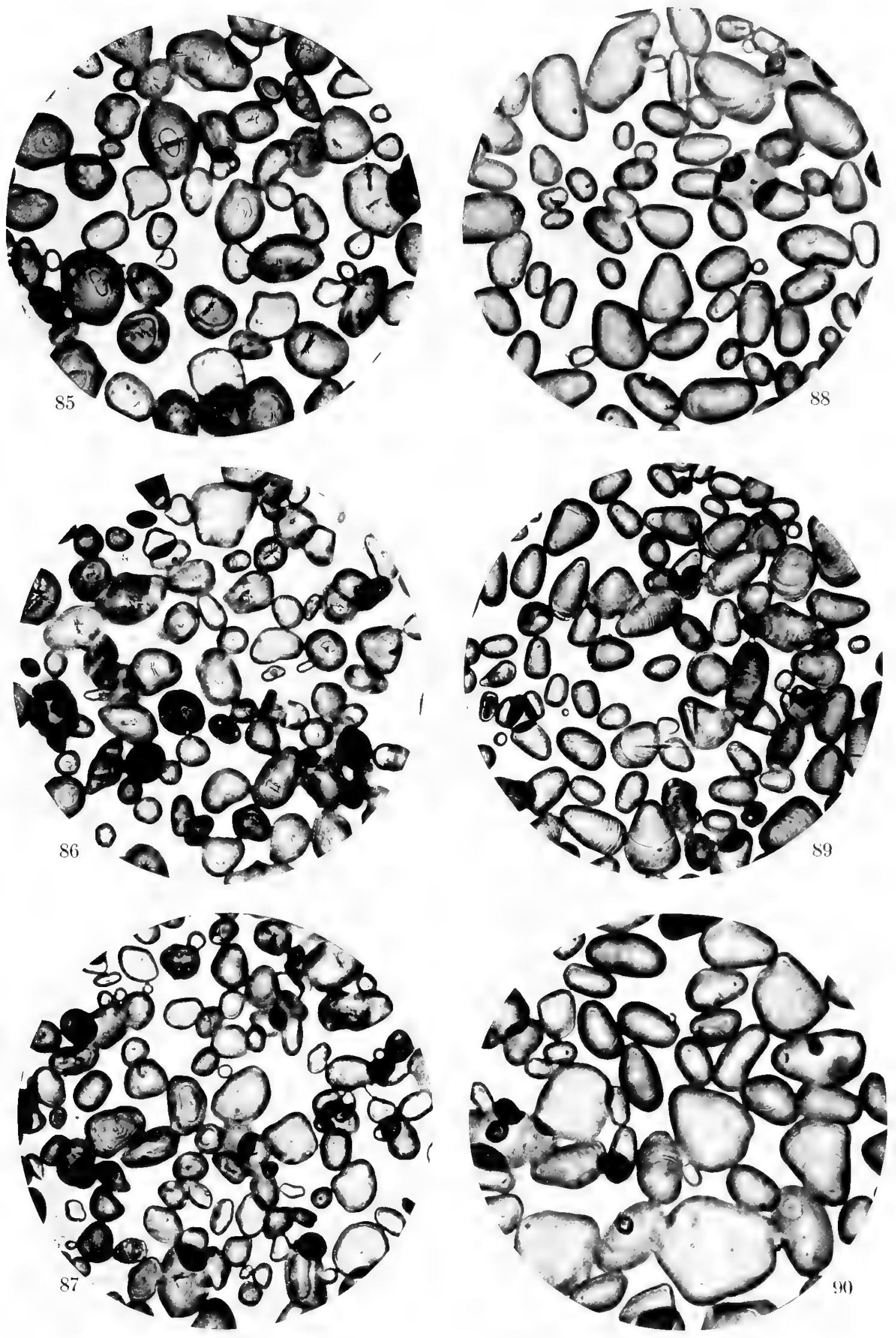

85. Narcissus emperor.

86. Narcissus triandrus albus.

87. Narcissus j. t. bennett poe.

88. Lilium martagon album.

89. Lilium maculatum.

90. Lilium marhan. 


$$
\text { - }
$$



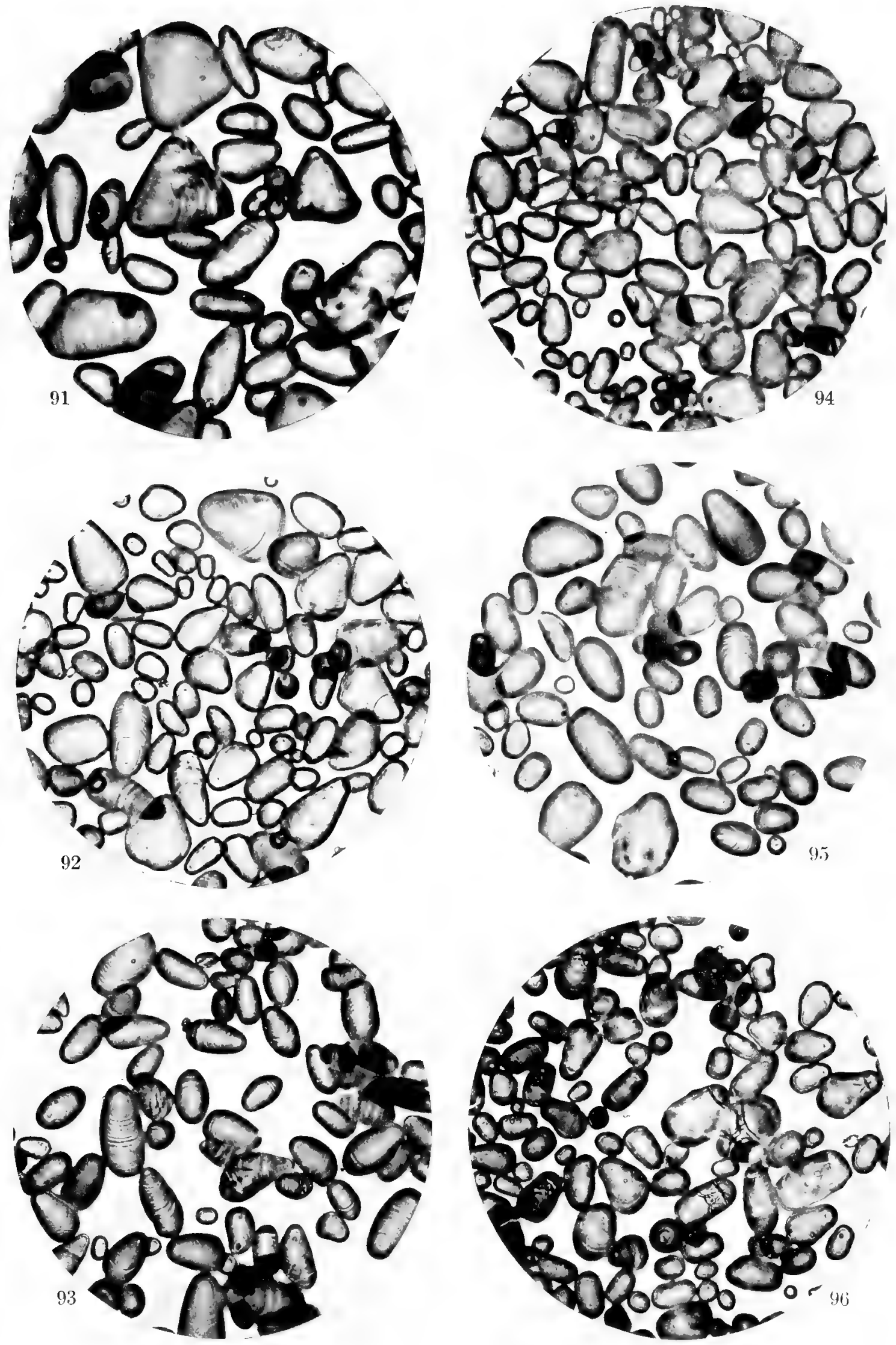

91. Lilium marlagon.

92. Lilium maculatum.

94. Lilium tenuifolium.

93. Lilium dalhansoni.

95. Lilium martagon album.

96. Lilium golden gleam. 


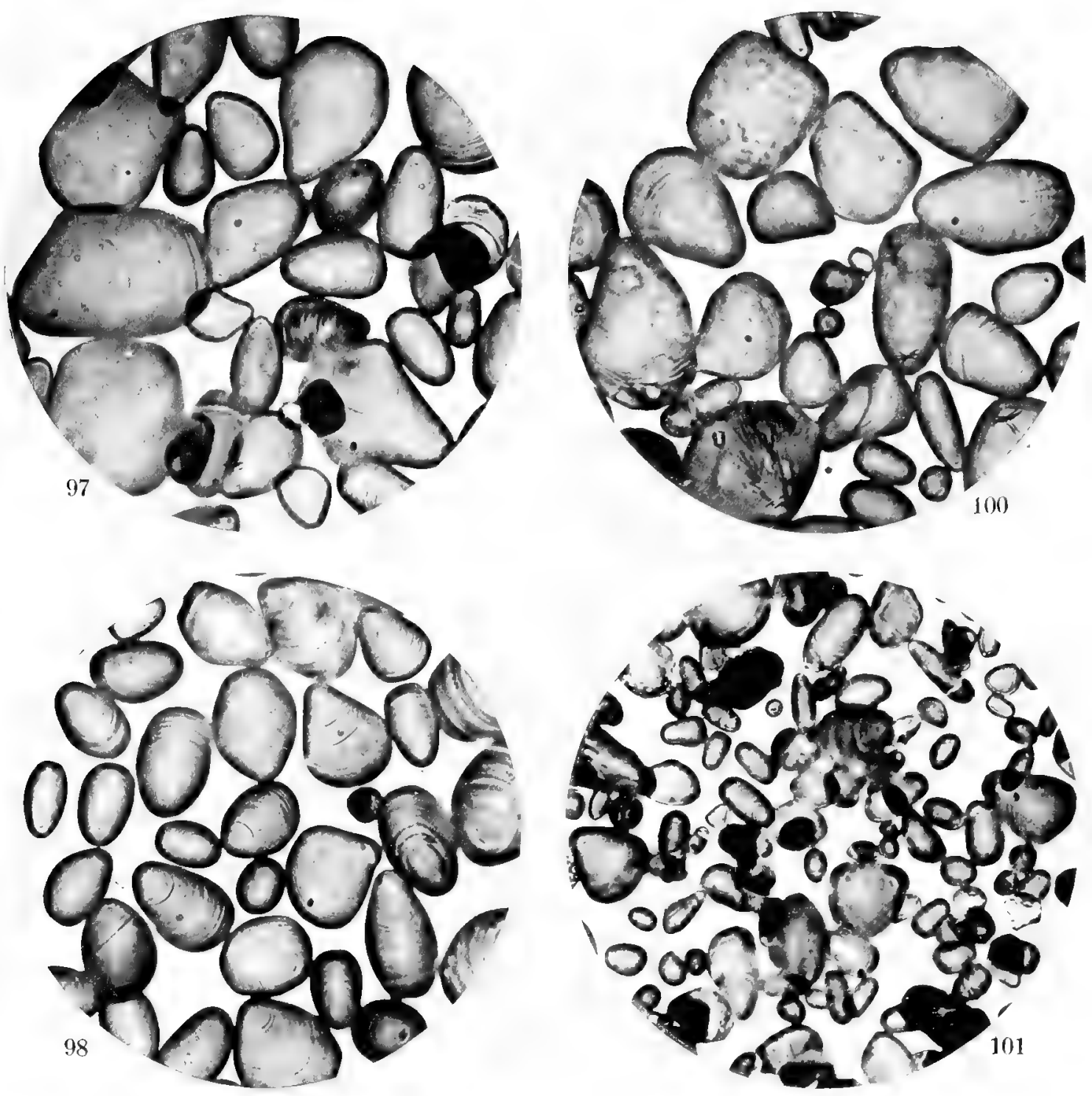

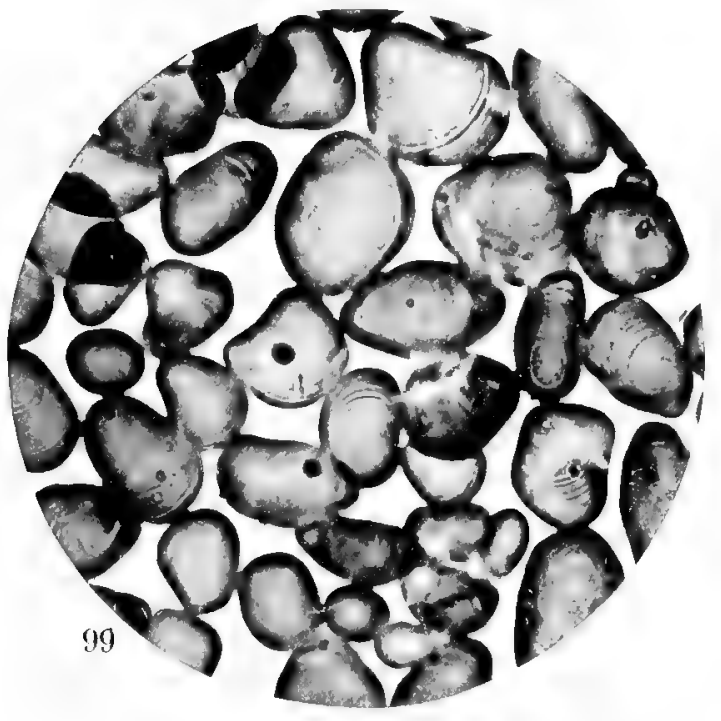

97. Lilium chalcedonicum 98. Lilium candidum. 99. Lilium testaceum.

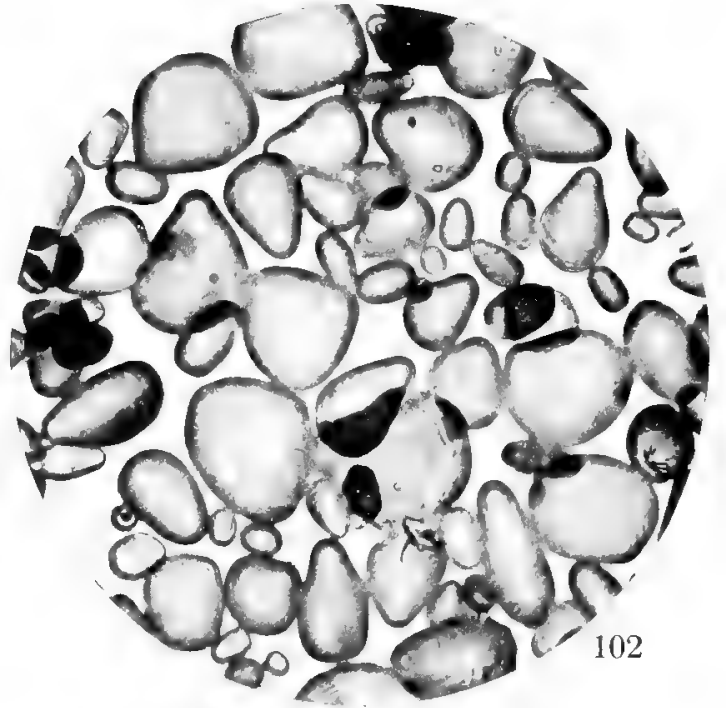

100. Lilium pardalinum

101. Lilium parryi.

102. Lilium burbanki. 


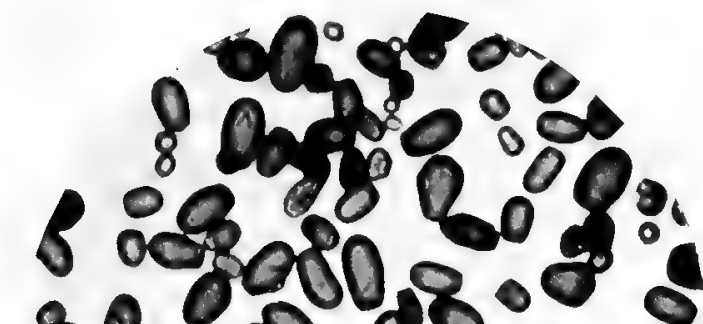

30.0908 .8

$1388^{\circ} 8 \%$

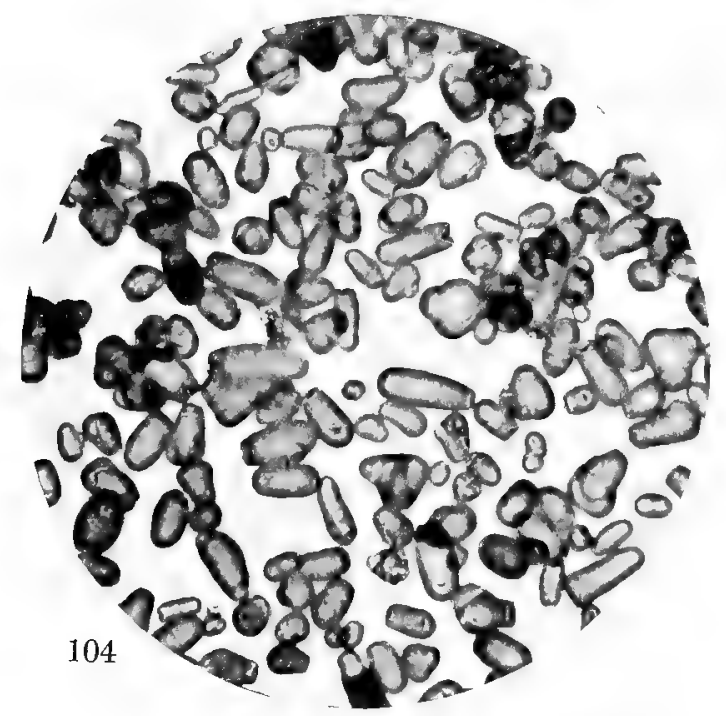

80009

So 092 \& 60.75

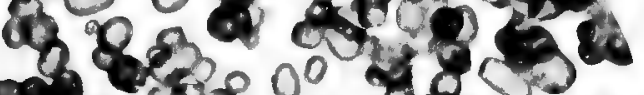

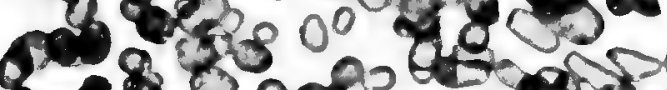

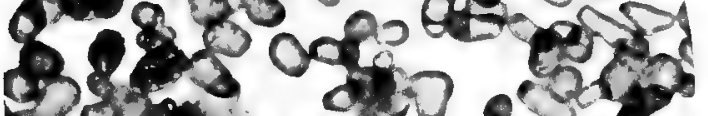

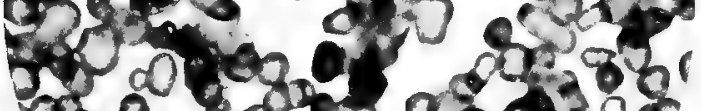

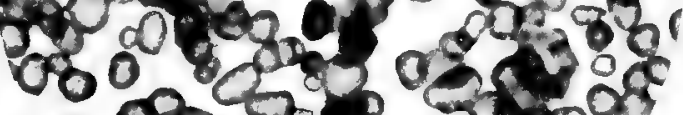

2059 की 80 o

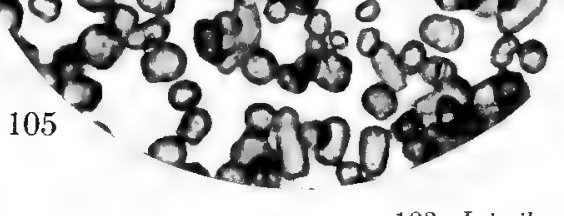

103. Iris iberica.

104. Iris trojana.
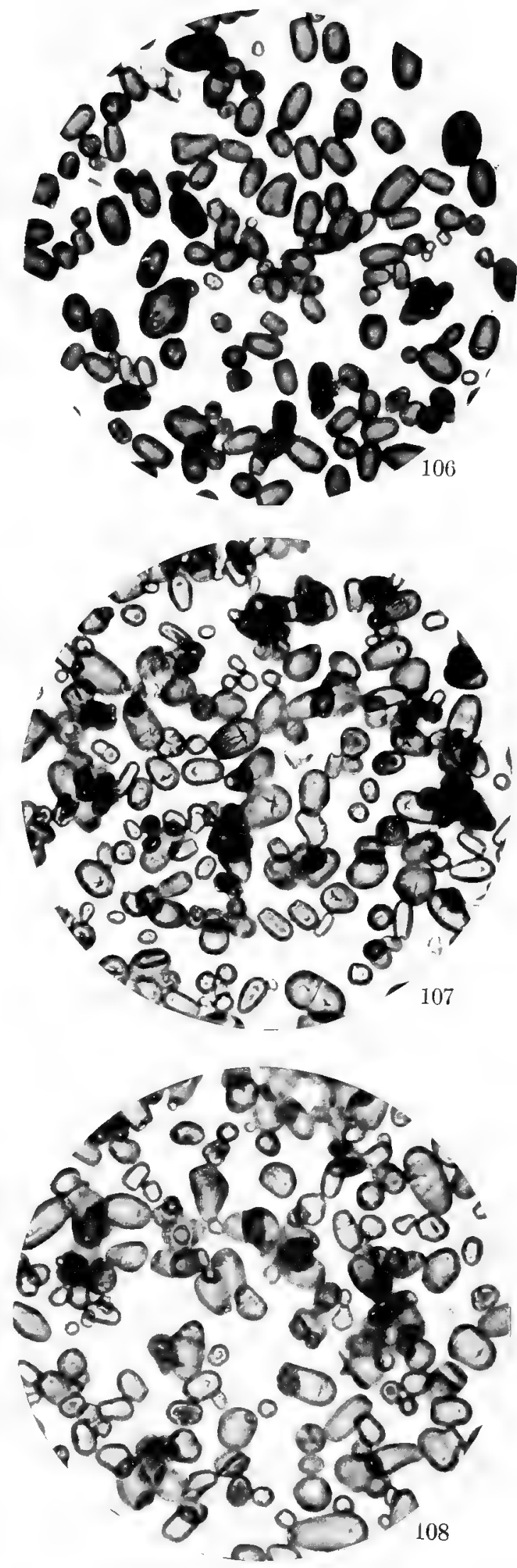

106. Iris iberica.

107. Iris cengialti.

108. Iris dorak. 
$+$ 

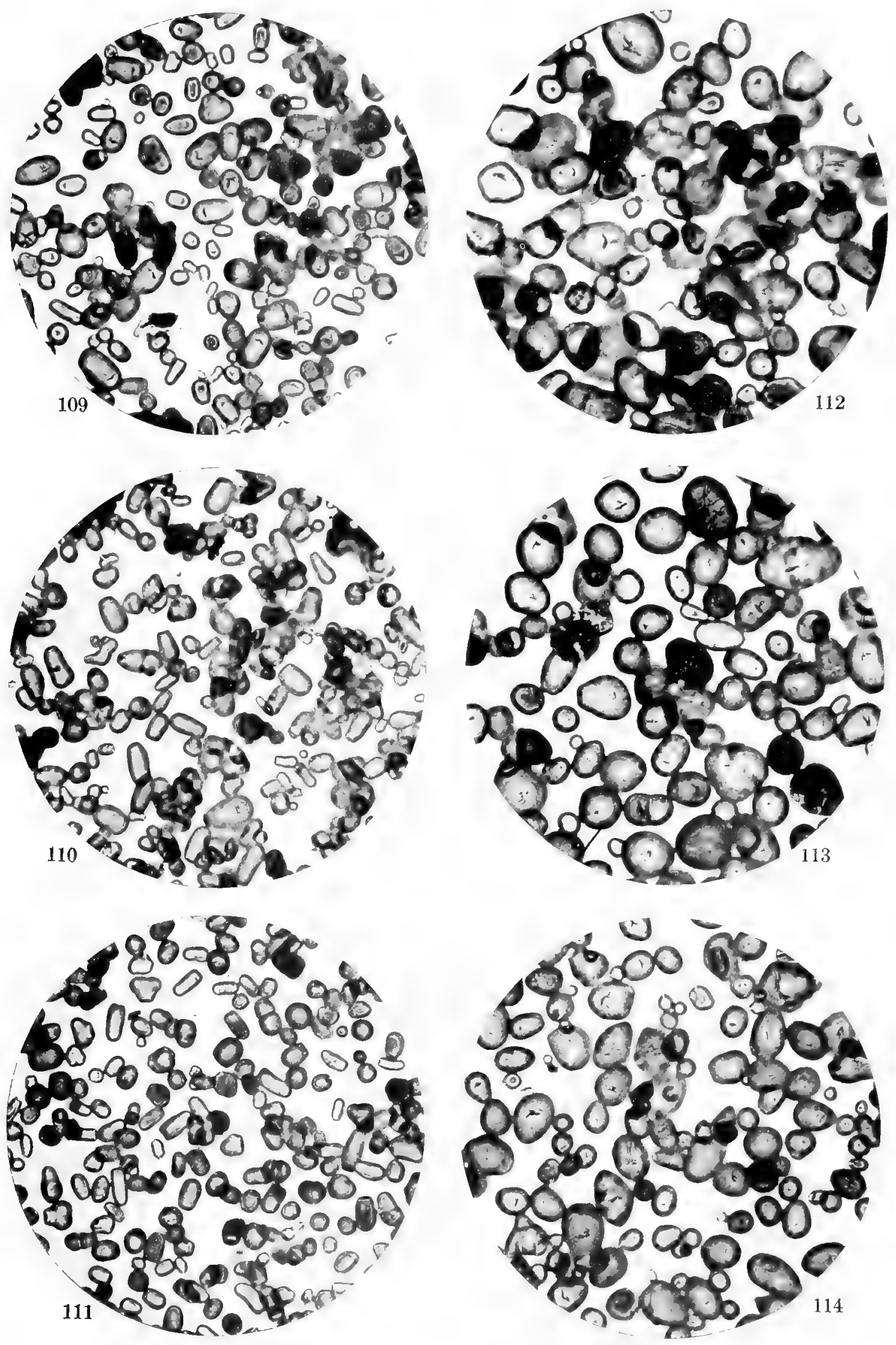

109. Iris cengialti

110. Iris pallida queen of may.

111. Iris mrs. alan grey.

112. Iris persica var, purpurea.

113. Iris simljarensis.

114. Iris pursind. 

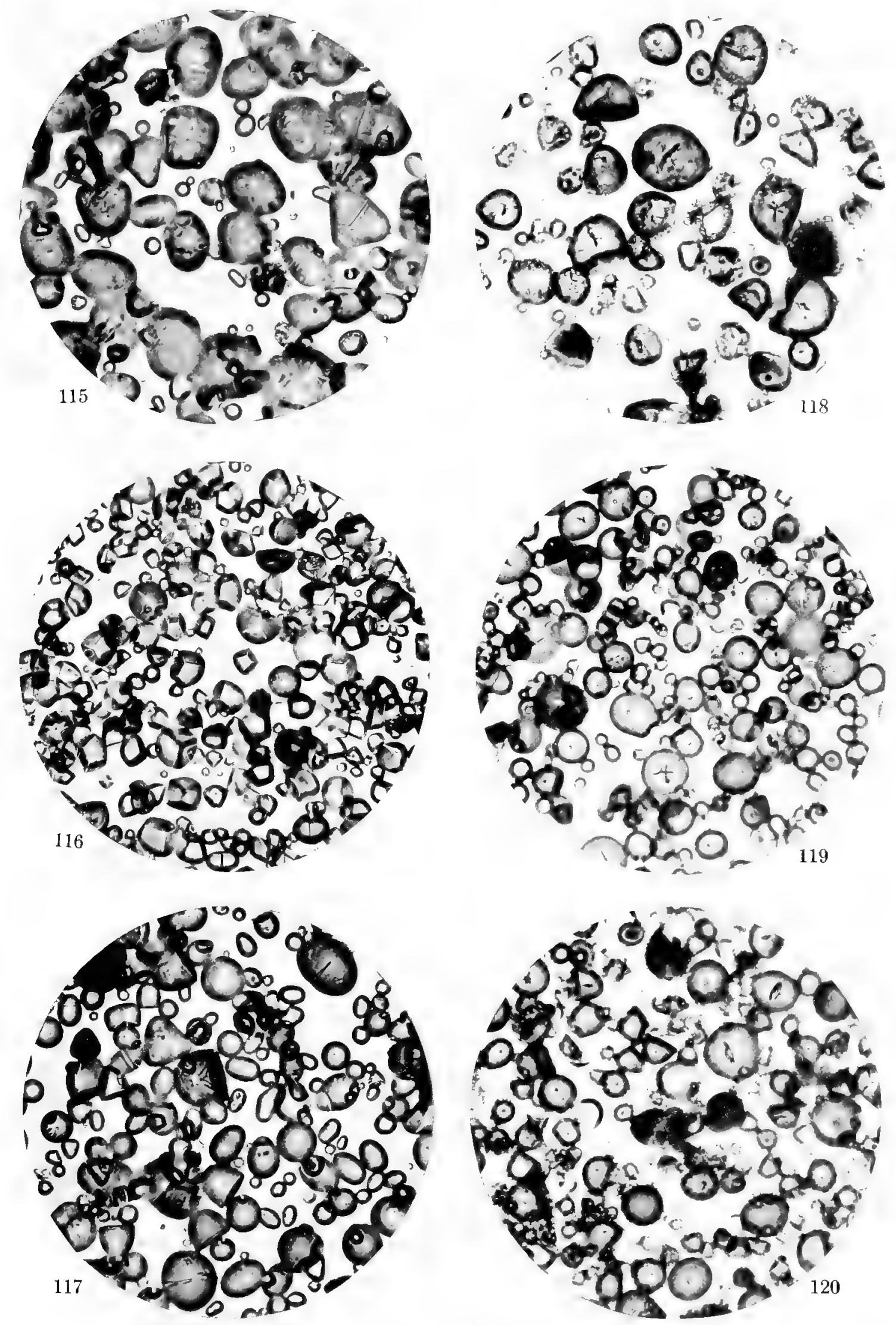

115. Gladiolus cardinalis 116. Gladiolus tristis. 117. Gladiolus colvillei.

118. Tritonia pottsii

119. Tritonia crocosmia aurea.

120. Tritonia crocosmoftora. 

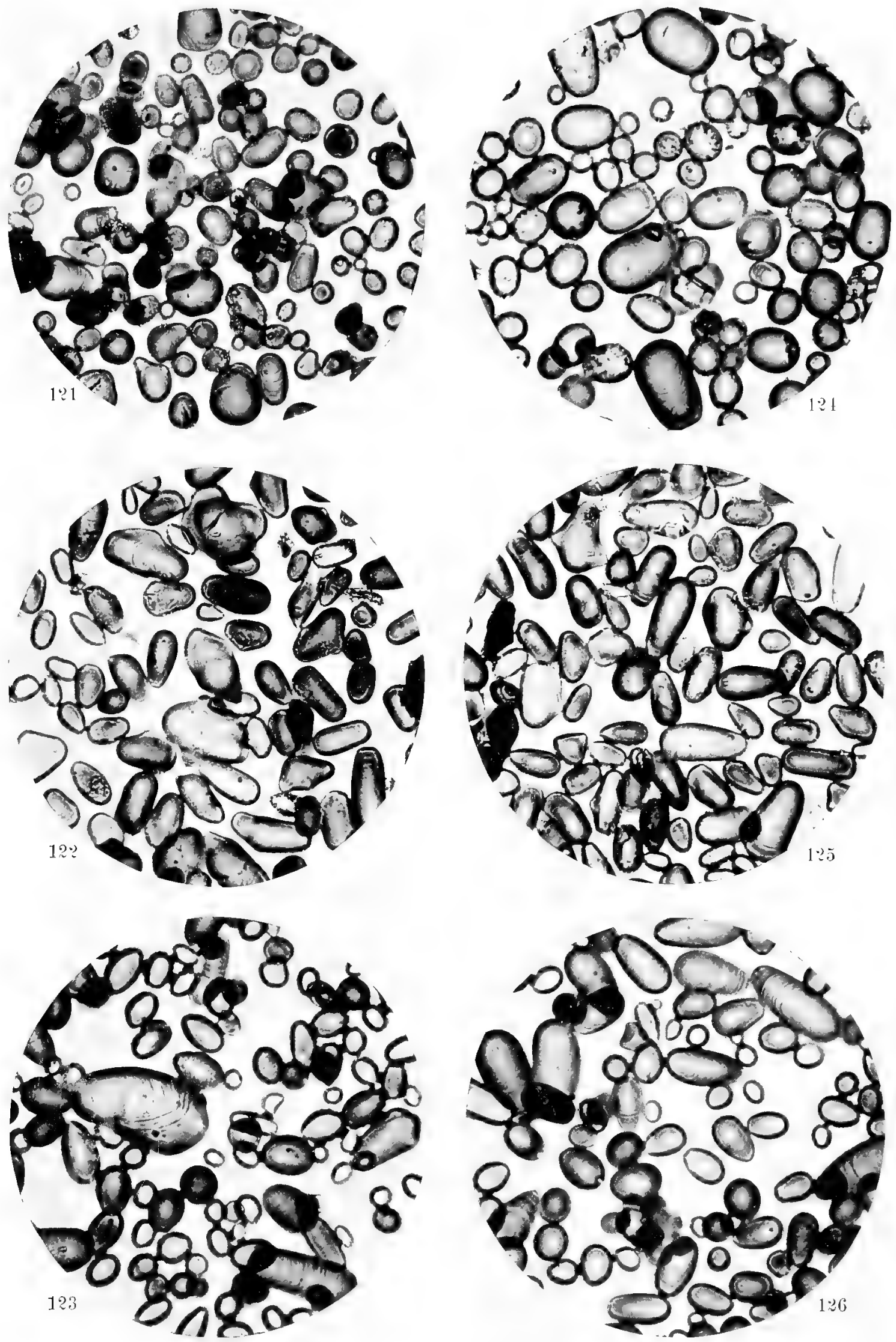

121. Begonia single crimson scarlet.

122. Begonia socotrana.

123. Begonia mrs, heal.

124. Begonia double light rose.

125. Begonia socotrana.

126. Begonia ensign. 


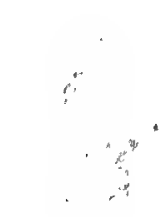



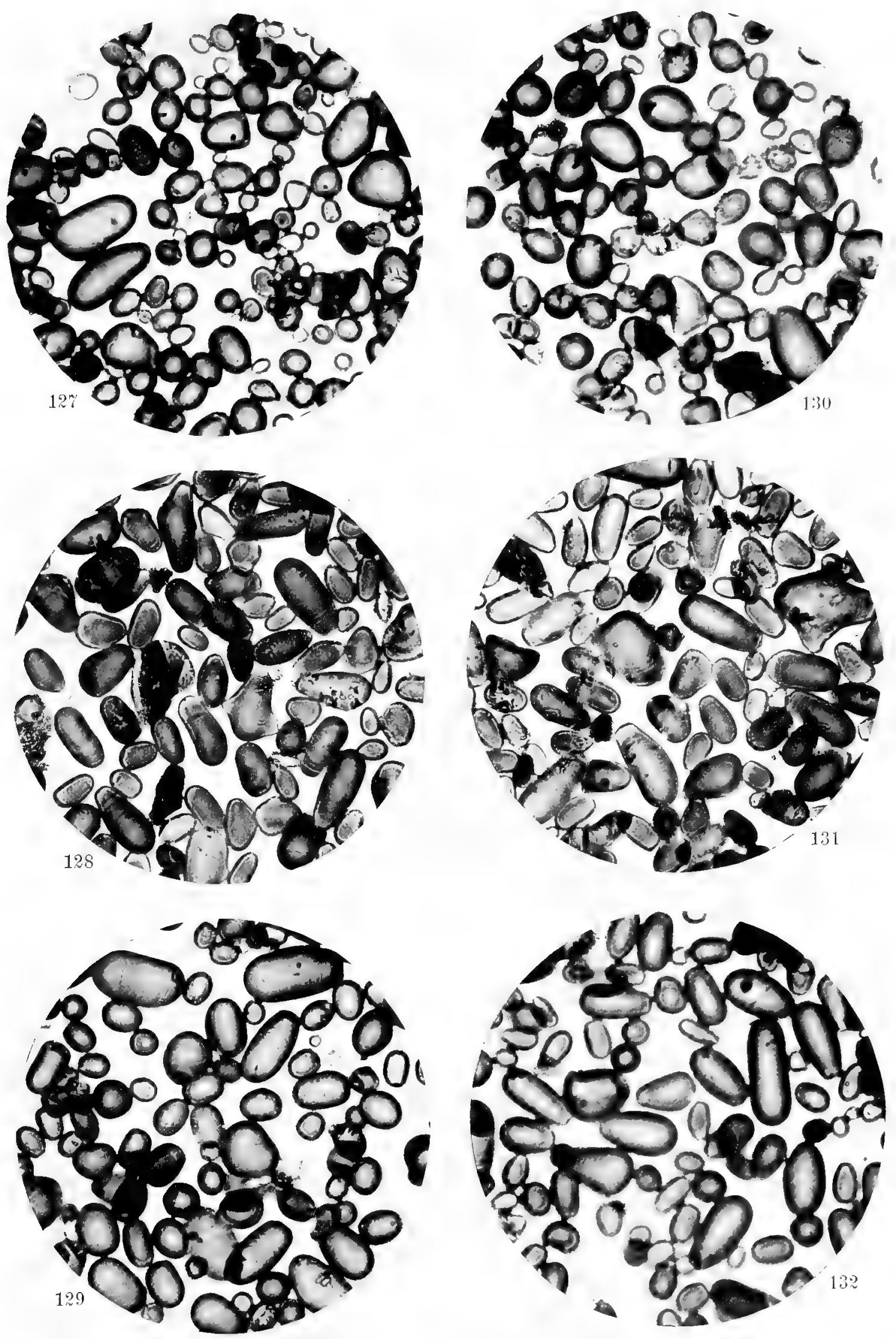

127. Begonia double white 128. Begonia socotrana. 129. Begonia julius.

130. Begonia double decp rose.

131. Begonia socotrana,

132. Begonia success. 

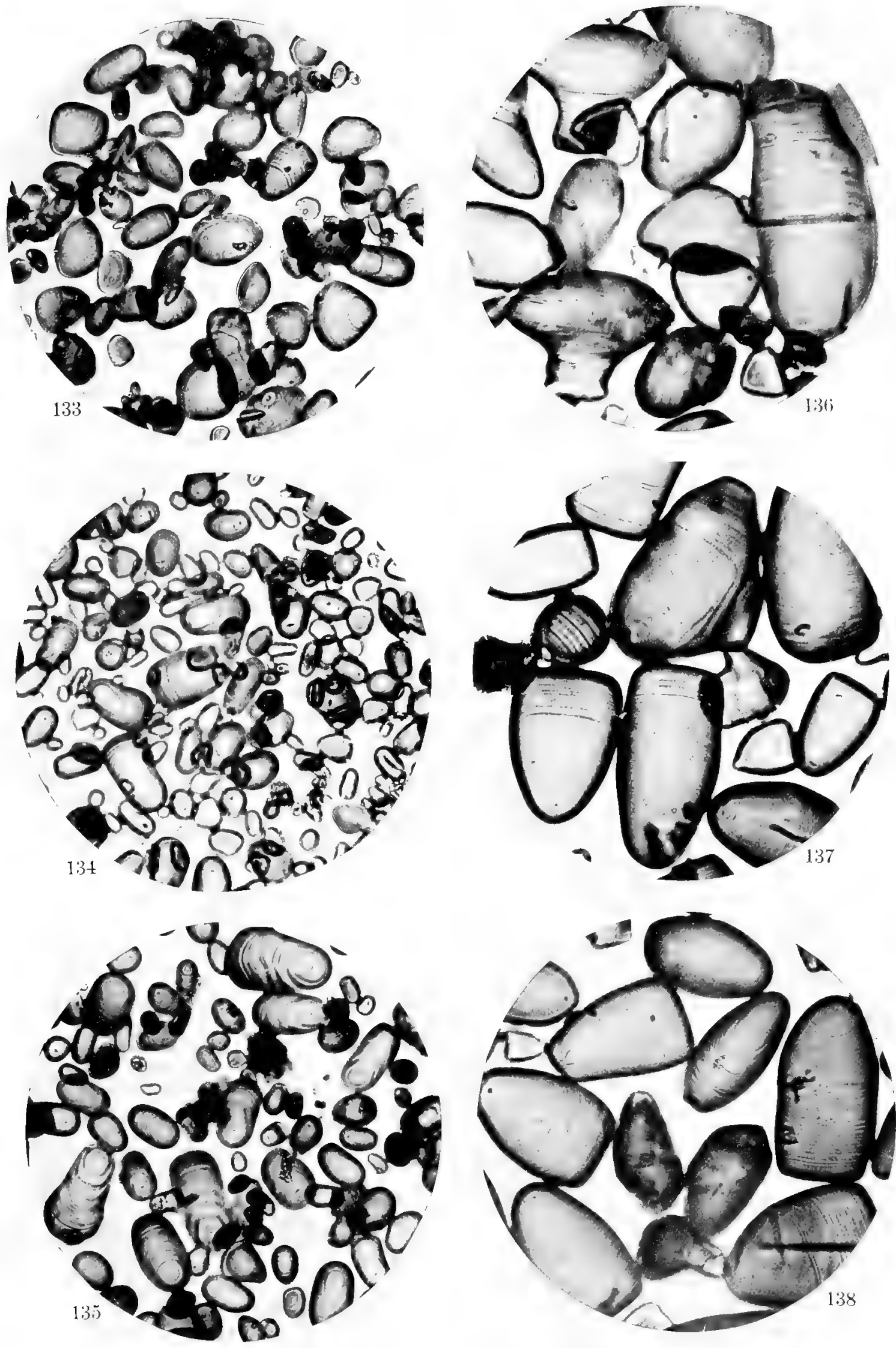

133. Musa arnoldiana 134. Musa gilletii.
135. Musa hybrida.

136. Phaius grandifolius

137. Phaius wallichii. 

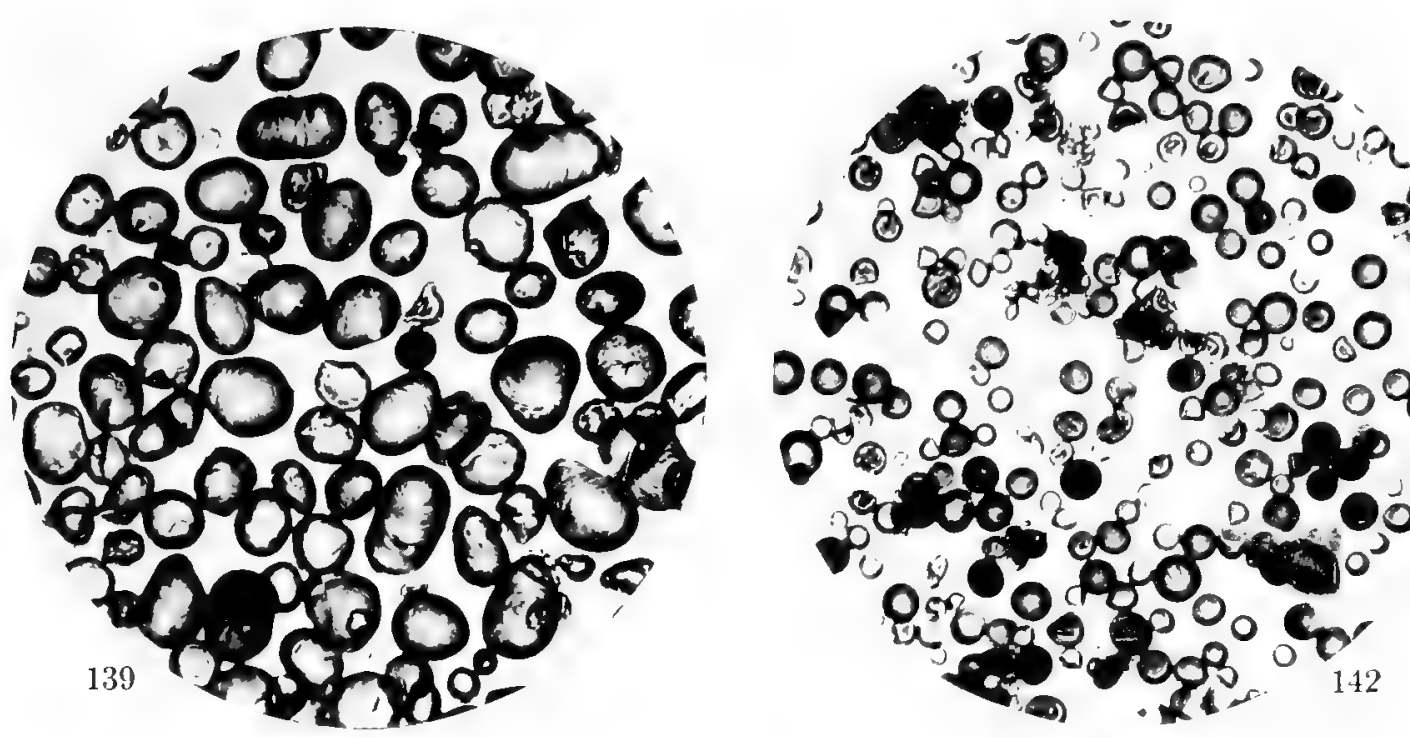

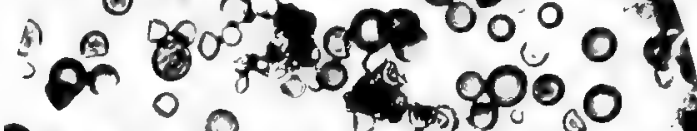

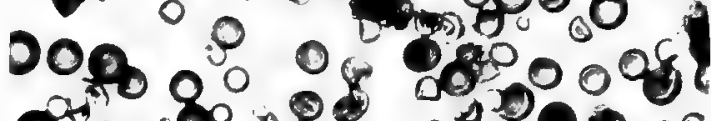
(4)

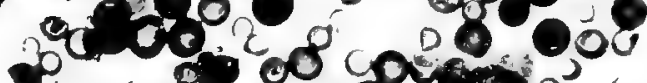

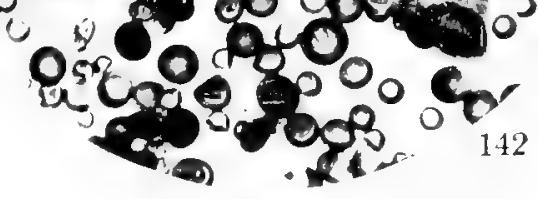

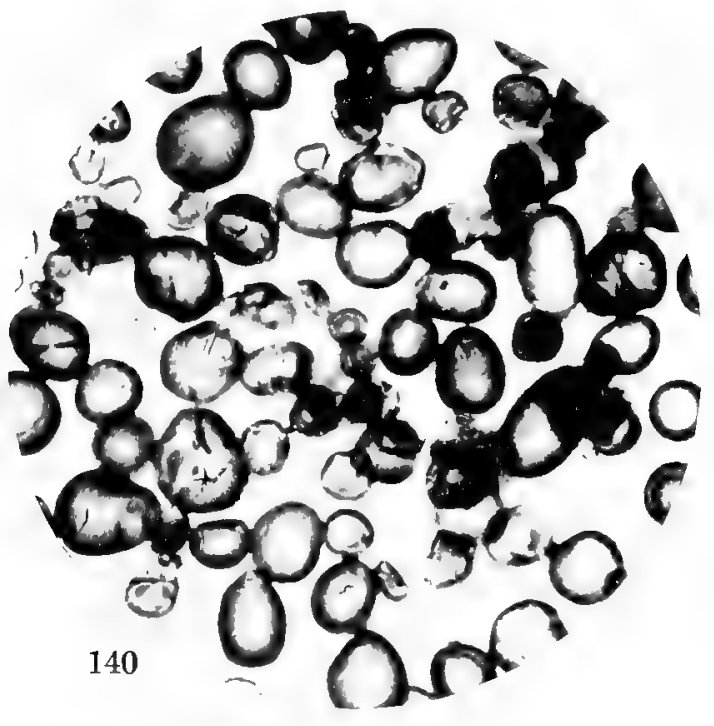

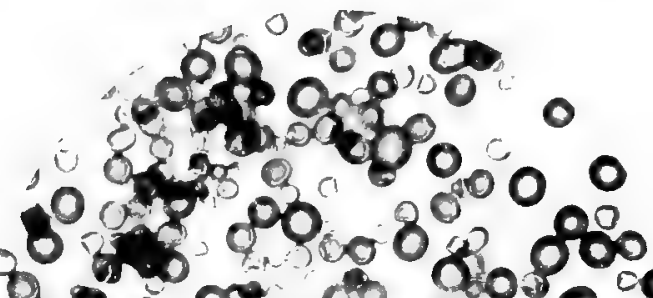

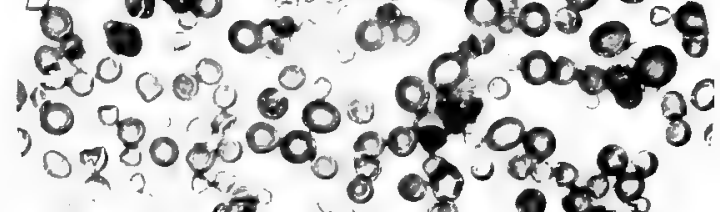

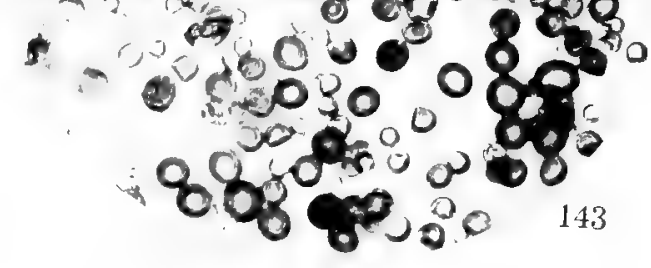

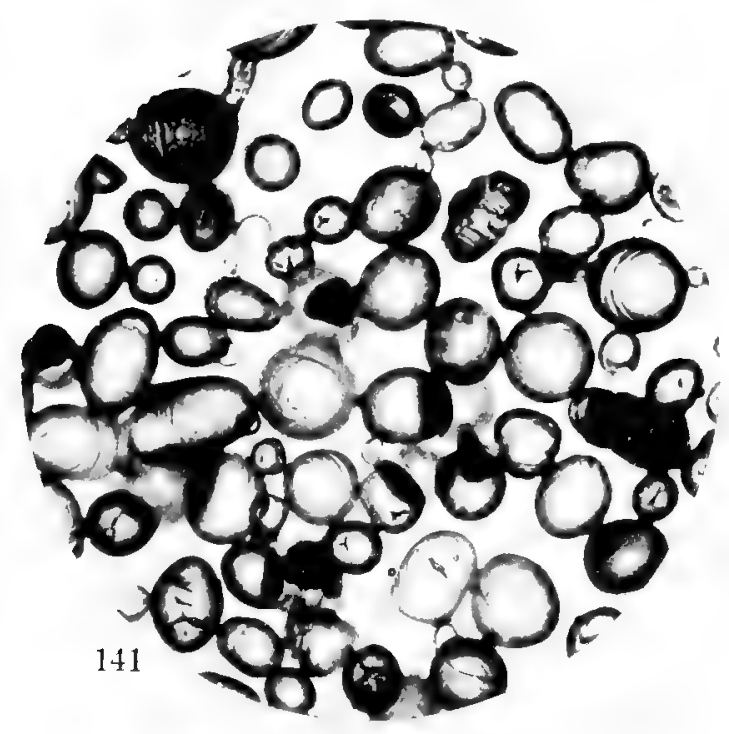

139. Miltonia vexillaria.

140. Miltonia rozalii.

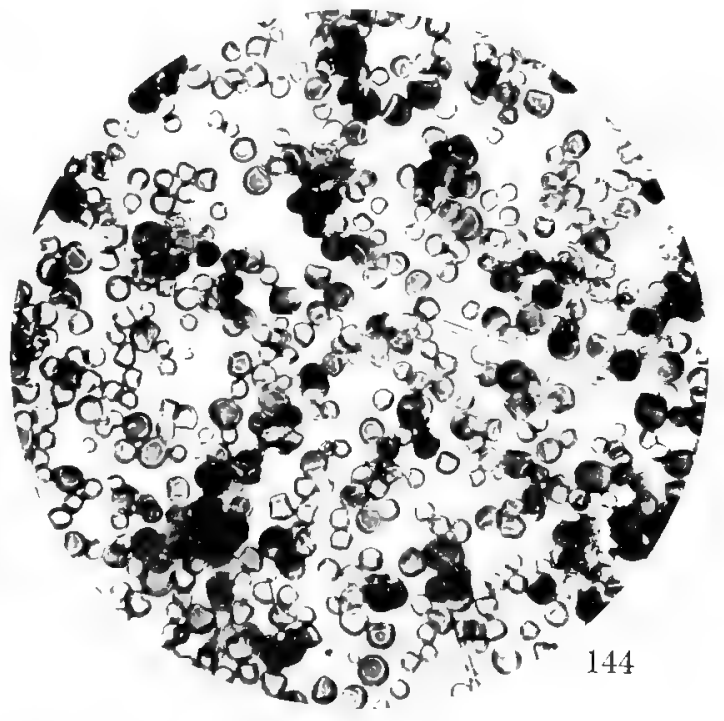

142. Cymbidium lowianum.

143. Cymbidium eburneum.

144. Cymbidium eburneo-lowianum. 
. 


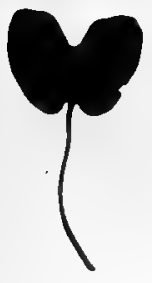

145

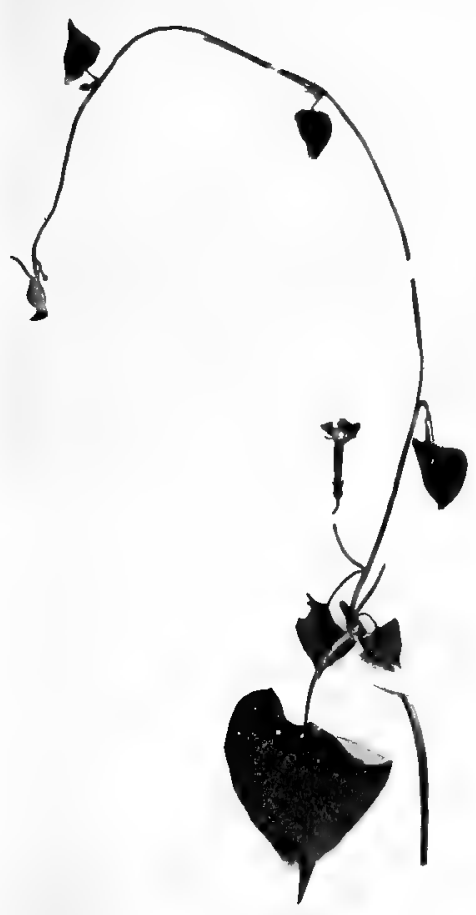

146

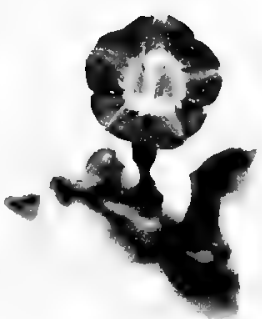

$\overline{14} 7$

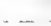

148

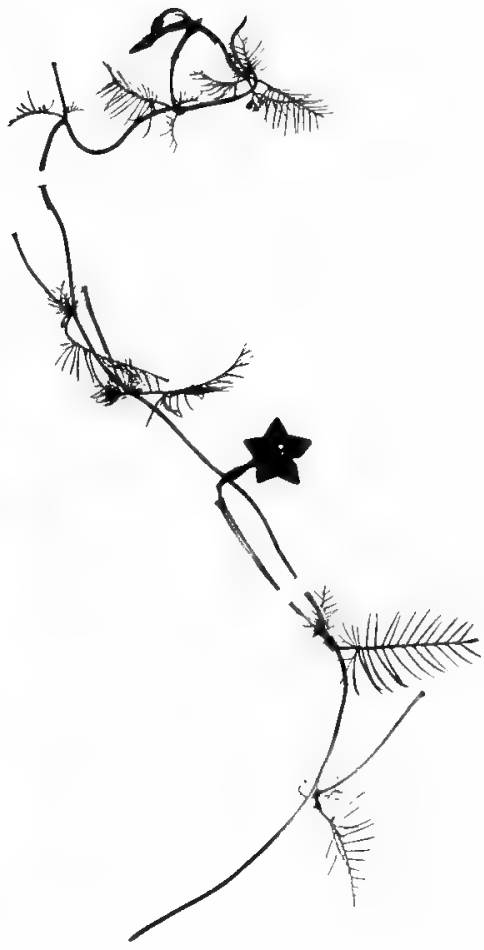

149

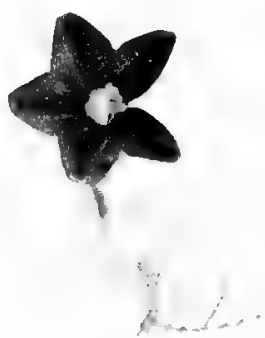

150

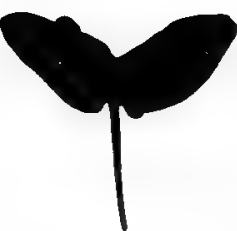

151

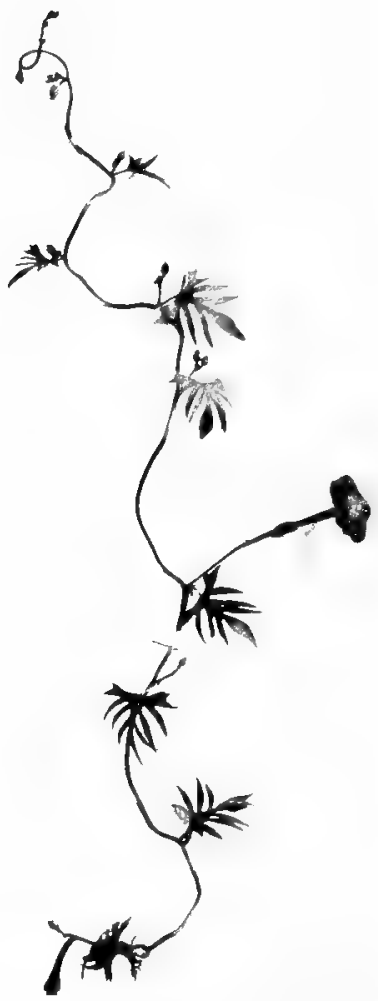

152

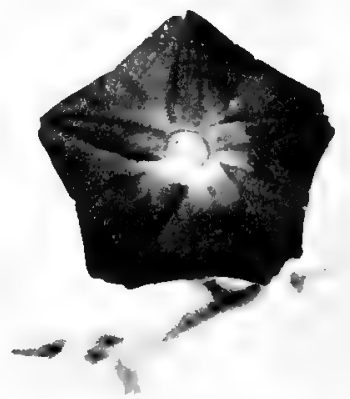

145. Ipomoa coccinea. Cotyledon, showing long petiole, long midrib, blunt wide lobes, with an angle of $90^{\circ}$ between

148. Ipomoe quamoclit. The same, showing short petiole, short midrib, long narrow pointed lobes, and an angle of $150^{\circ}$ between lobes.

151. Ipomcea sloteri. The same, showing medium length petiole and midrib, lobes of medium width and somewhat tapering, and an angle of $120^{\circ}$ between lobes.

146. Ipomcea coccinea. Lateral branch, showing entire leaves.

149. I pomoea quamoclit. The same, showing pinnate leaves.

152. I pomaa sloteri. The same, showing deeply lobed leaves.

147. I pomaa coccinea. Flower, showing rounded lobes.

150. I pomcea quamoclit. The same, showing pointed lobes.

153. I pomcea sloteri. The same, showing slightly pointed hexagonal outline.

Figs. 145, 148, and 151 are slightly, but equally, reduced. Figs. 146 and 149 are reduced equally, and Fig. 152 is reduced more than the former. Figs. 147, 150, and 153 are natural size. 

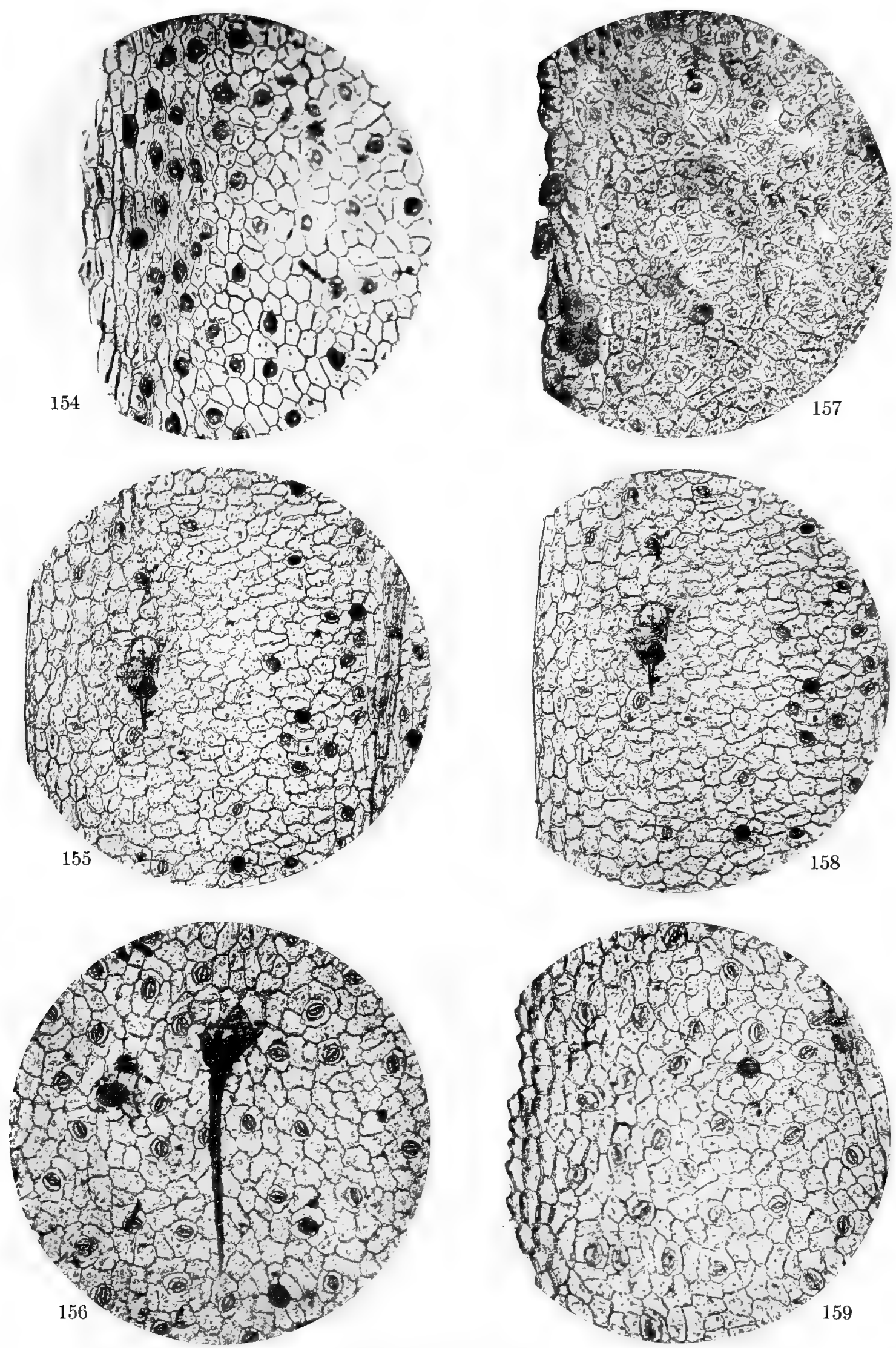

154. I pomaa coccinea. Section of upper epidermis at base of mature leaf, showing numerous stomata, regular distribution of stomata, straight-walled cells, and no hairs.

155. I pomce quamoclit. The same, showing fewer stomata; stomata grouped mainly at veins, wavy-walled cells and short dagger-like hairs.

156. I pomea sloteri. The same, showing more stomata than in I. quamoclit, but fewer than in $I$. coccinea, moderately wavy-walled cells, longer hairs, and larger cells and stomata.

157. Ipomos coccinea. Section of same at margin of leaf, showing protuberances from marginal cells.

158. Ipomcea quamoclit. The same, showing flat marginal cells, no protuberances.

159. I pomoca sloteri. The same, showing smaller protuberances from marginal cells. 


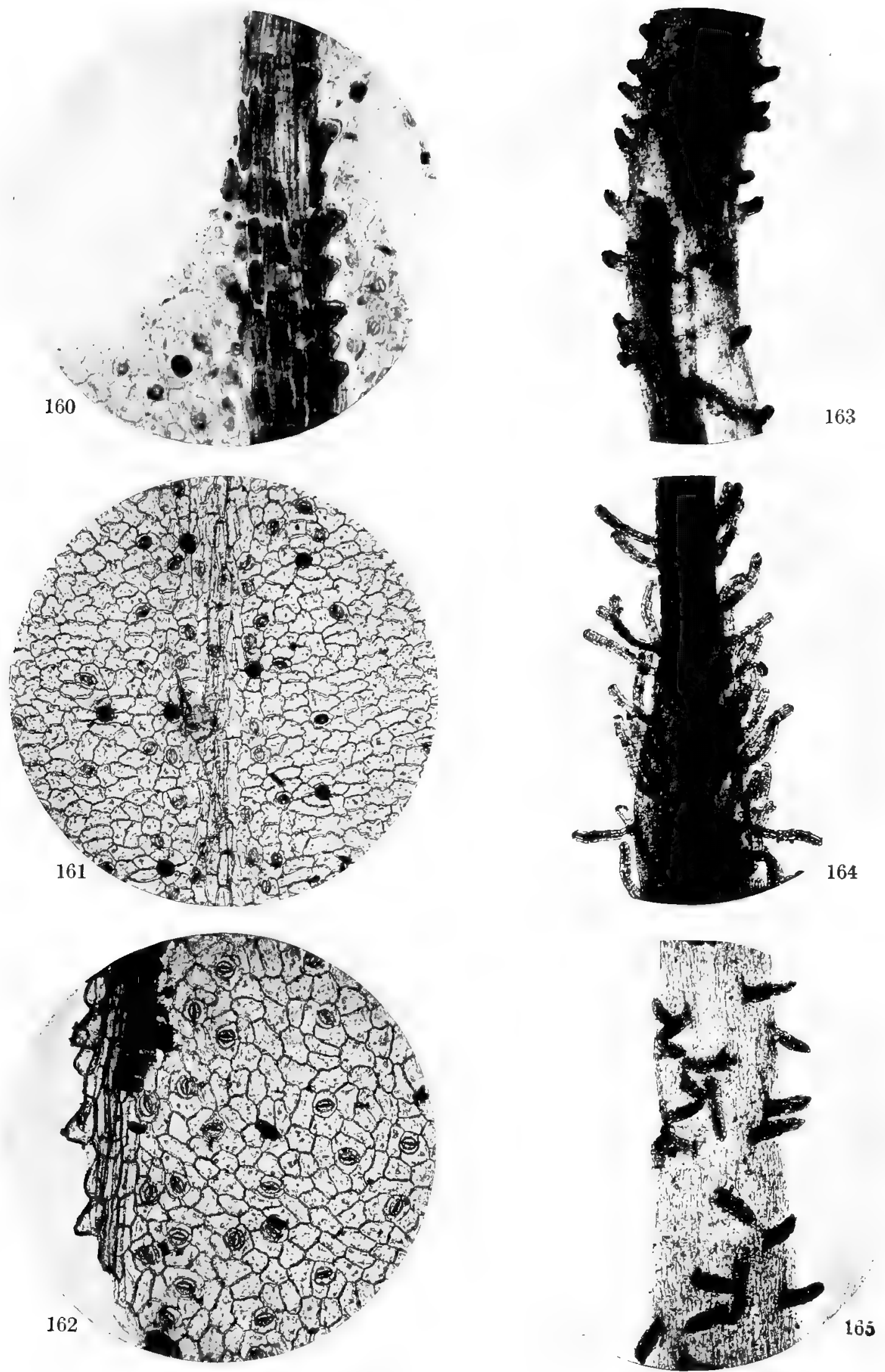

160. I pomcea coccinea. Section of upper epidermis at base of mature leaf; over a vein, showing long papillæ along veins.

161. I pomoea quamoclit. The same, showing no papillæ over veins.

162. Ipomcea sloteri. The same, showing smaller papillæ along vein and that the stomata are slightly more numerous at the veins.

163. I pomea coccinea. Section of epidermis at base of filament showing short glandular shaggy hairs.

164. I pomoa quamoclit. The same, showing much longer glandular shaggy hairs.

165. I pomoe sloteri. The same, showing glandular shaggy hairs of intermediate length. 


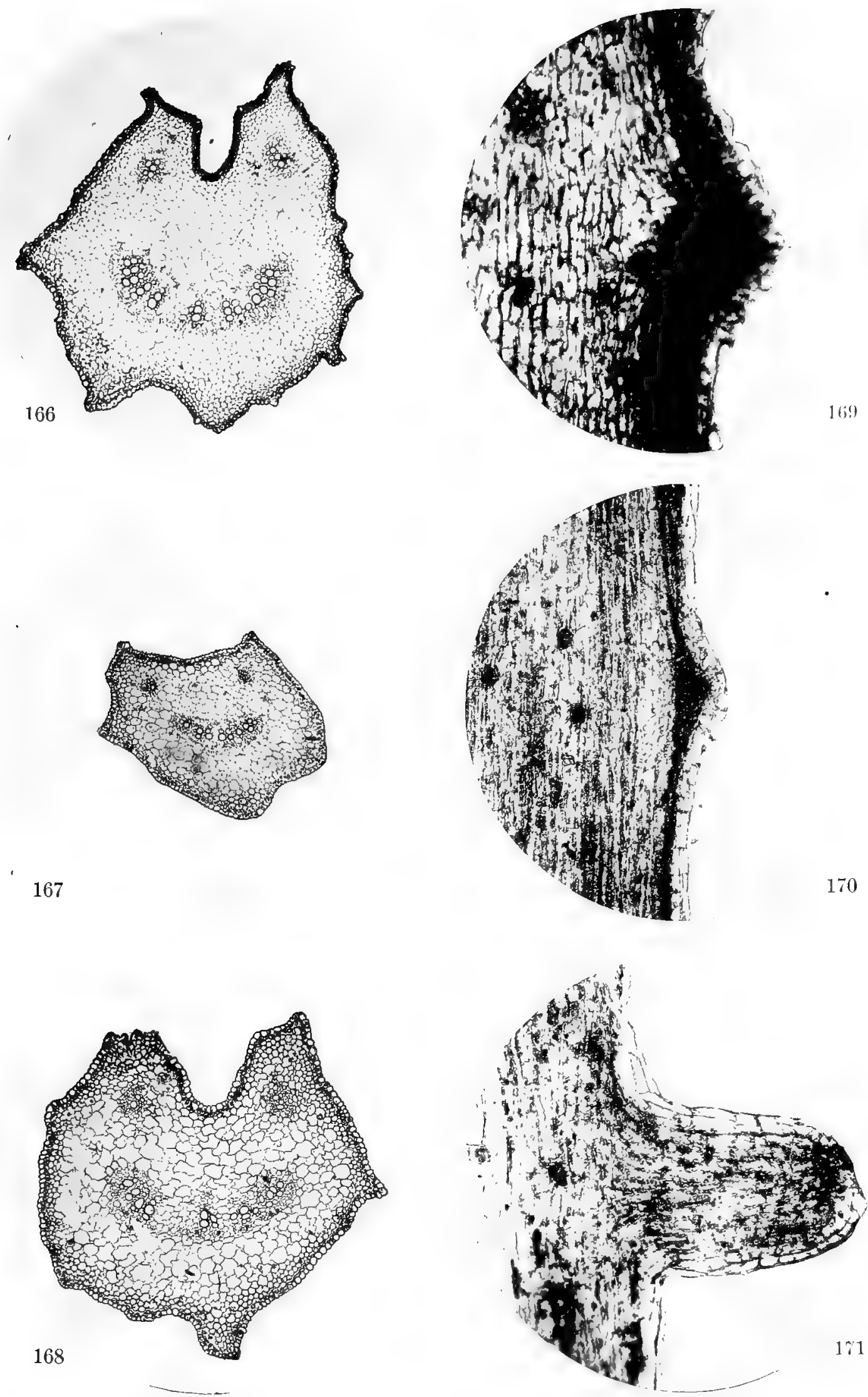

168

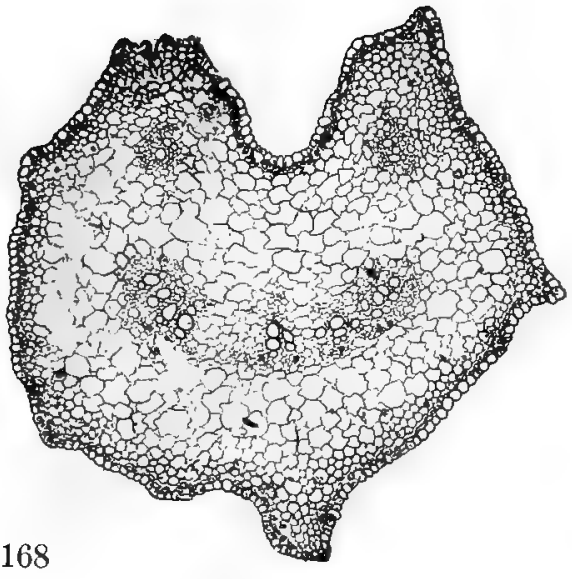

166. Ipomoe coccinea. Transverse section; petiole of mature leaf equidistant from the lamina and the base.

167. Ipomoea quamoclit. The same.

168. I pomoea sloteri. The same.

169. I pomcea coccinea. Multicellular protuberances at base of petiole.

170. Ipomee quamoclit. The same.

171. I pomoa sloteri. The same. 


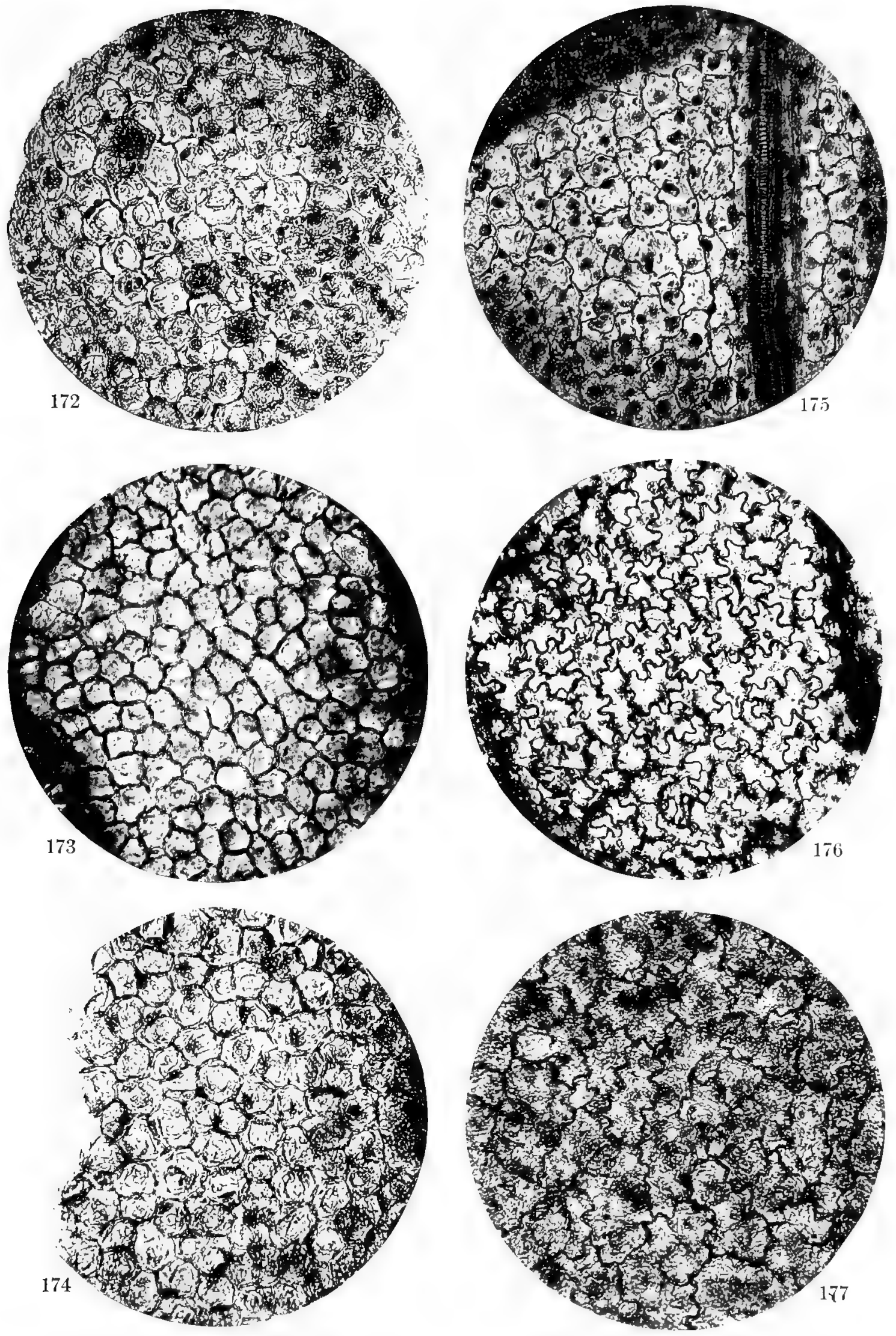

172. Ipomcea coccinea. Upper epidermis, limb of corolla.

173. I pomce quamoclit. The same.

174. I pomcea sloteri. The same, showing larger cells than in either I. coccinea or I. quamoclit.

175. I pomoea coccinea. Lower epidermis, limb of corolla showing slightly wavy cell walls.

176. I pomoea quamoclit. The same, showing very wavy cell walls.

177. I pomae sloteri. The same, showing waviness between that of the two parents. 

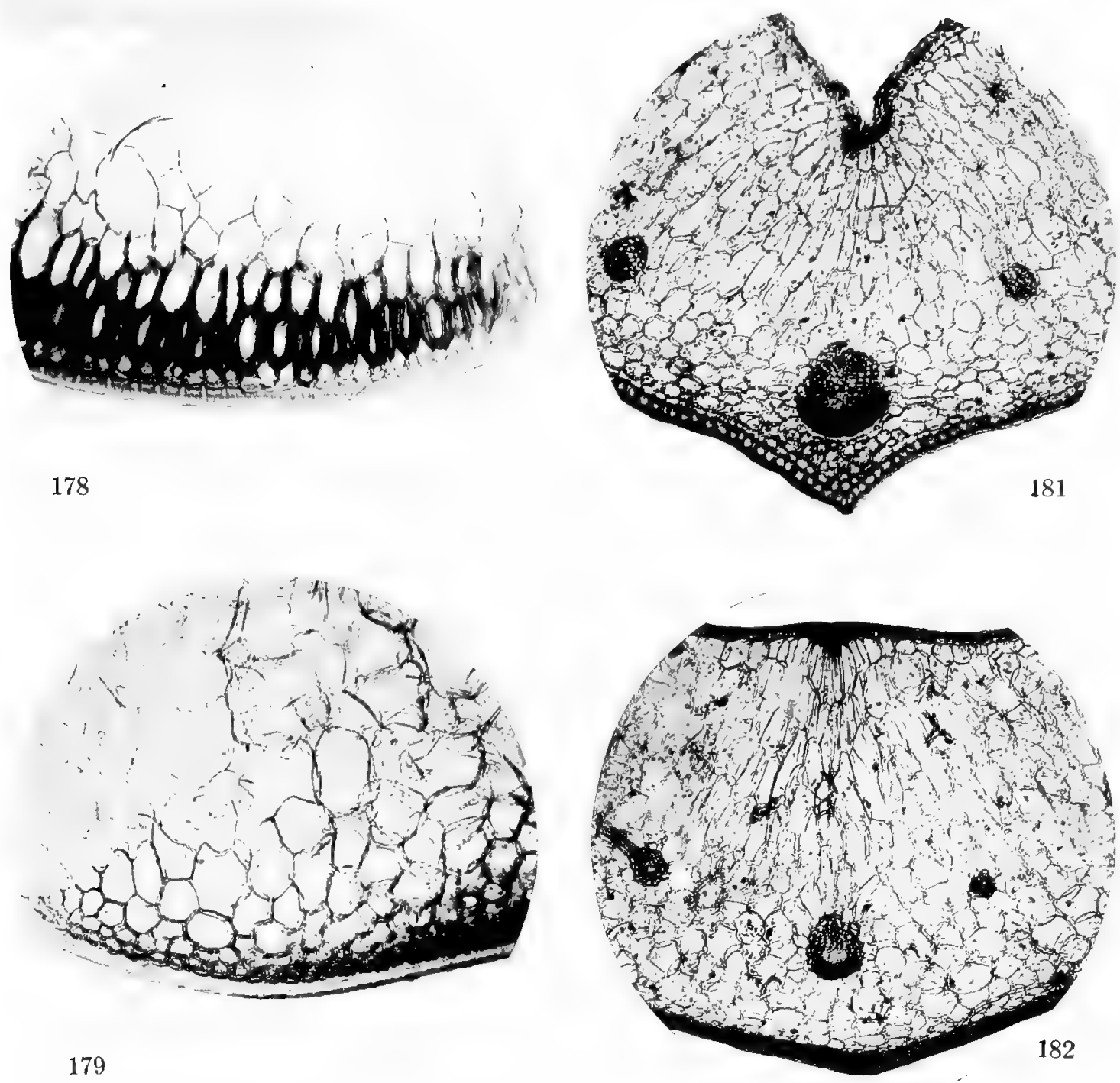

179
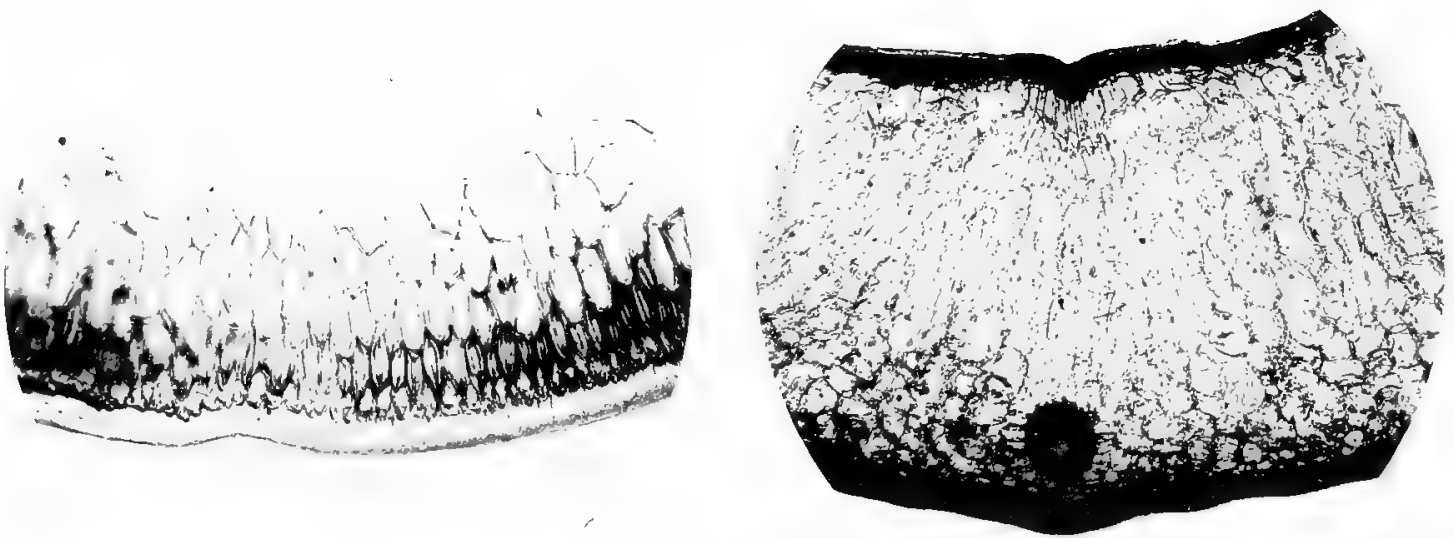

183

178. Laelia purpurata. Transverse section of pseudobulb at middle, showing deep epidermis, deep cuticle, elongated thick-walled cells of two layers beneath epidermis.

179. Cattleya mossioe. The same, showing shallower epidermal cells, cuticle as deep as in $L$. purpurata, cells of two layers beneath the epidermis not elongated and only those of first layer have thickened walls.

180. Lølia-Cattleya canhamiana. The same, showing epidermal cells, deeper than those in C. mossice but not quite as deep as those in $L$. purpurata; cuticle, deeper than in either $C$. mossia or $L$. purpurata; and two layers beneath the epidermis not as elongated nor as thick-walled as in $L$. purpurata, but distinctly more elongated and thicker walled than in C. mossice.

181. Lalia purpurata. Transverse section of leaf near apex, showing slightly elongated cells of first layer of aqueous tissue at midrib, and large bundle.

182. Cattleya mossice. The same, showing more elongated cells of aqueous tissue and rather small bundle.

183. Lolia-Cattleya canhamiana. The same, showing cells of aqueous tissue of same length as in C. mossia and smaller bundle than in either C. mossio or L. purpurata. 


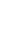



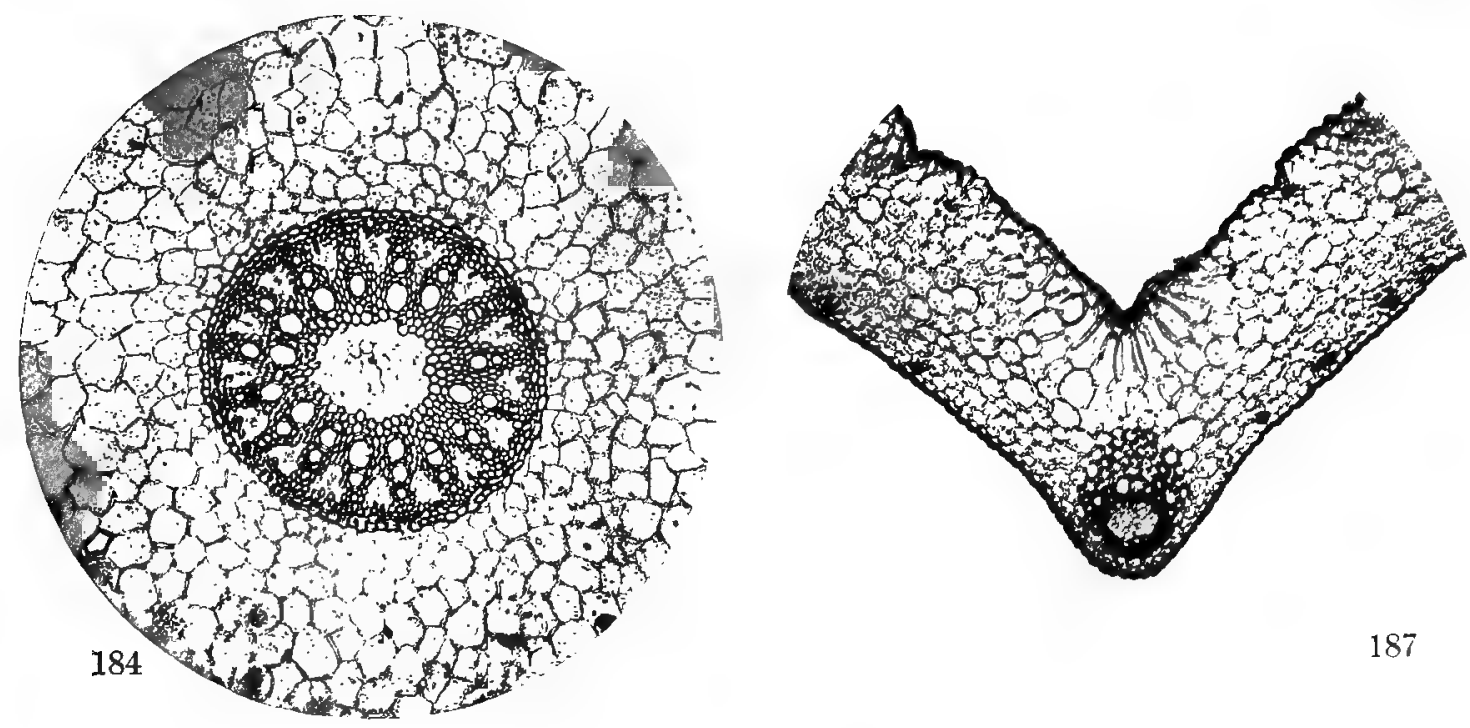

187
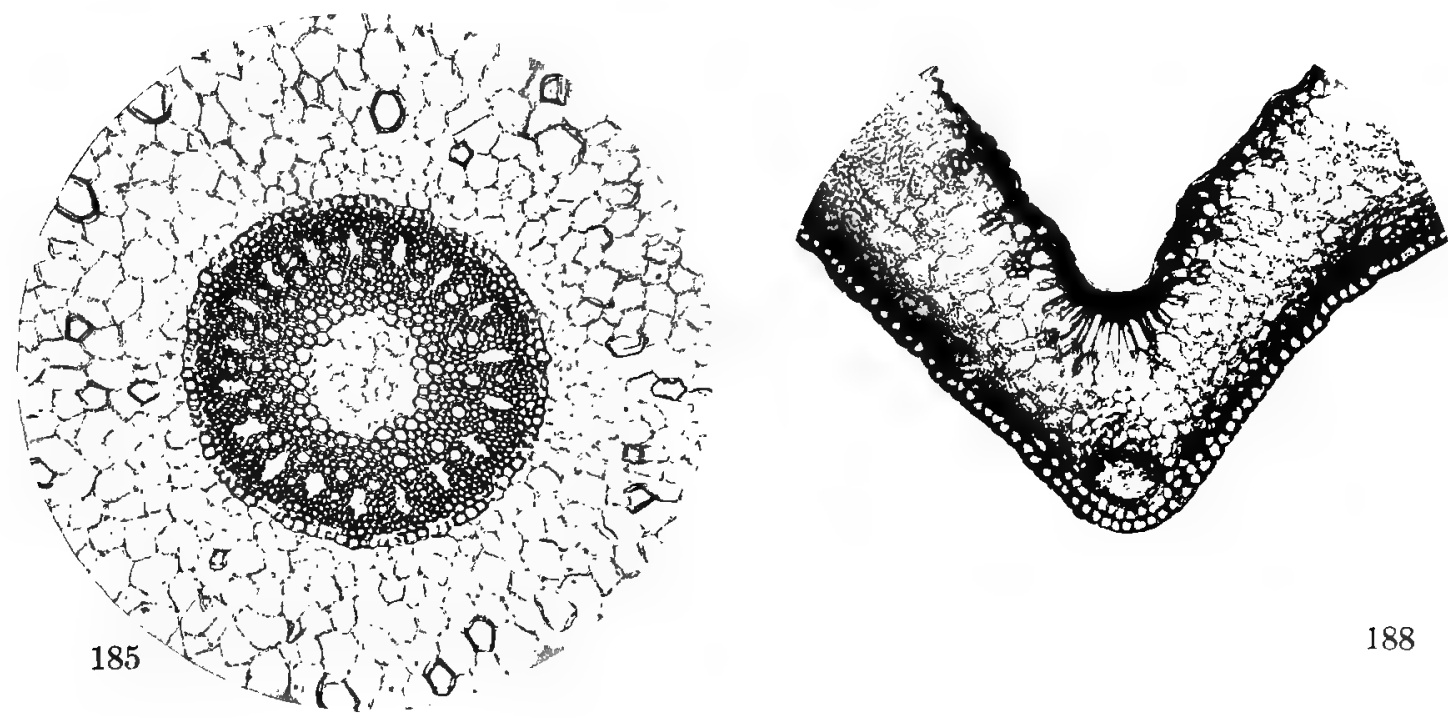

188
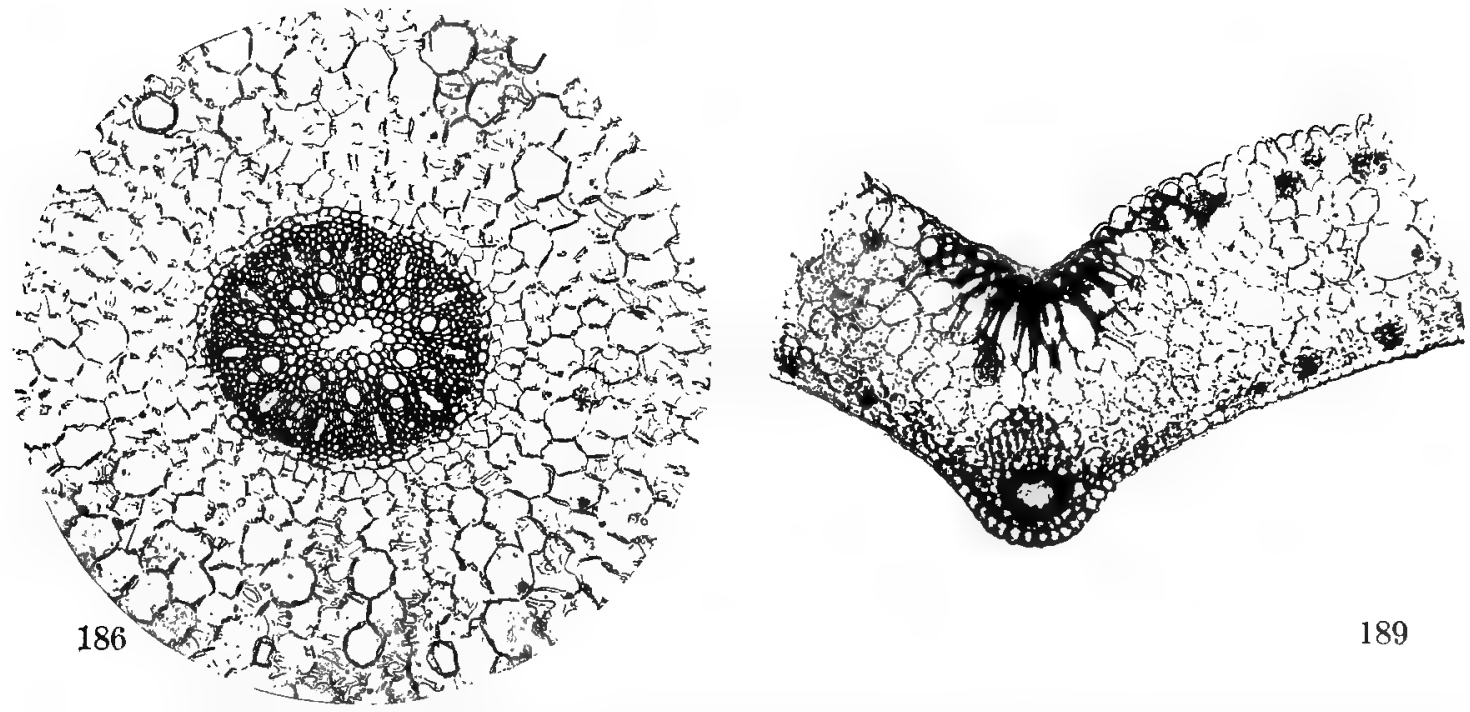

189

184. Cymbidium lowianum. Transverse section of root, showing vascular cylinder and part of surrounding cortex, showing one very rare, slightly sclerosed cell in cortex, narrow endodermal cells, 16 phloem patches, and large vasa.

185. Cymbidium eburneum. The same, showing numerous thickly sclerosed cells, deeper endodermal cells, $18 \mathrm{phl} \wp \mathrm{m}$

patches, and small vasa.
186. Cymbidium eburneo-lowianum. The same, showing sclerosed rells not as thick-walled or as numerous as in $C$. eburneum but more numerous than in C. lowianum; endodermal cells exactly mid-intermediate between the two parents in depth, 11 phlom patches and vasa in size between those of two parents.

187. Cymbidium lowianum. Transverse section of leaf near apex, showing comparatively shallow upper epidermal cells, short cells of layer beneath upper epidermis.

188. Cymbidium eburneum. The same, showing deeper upper epidermal cells, long cells of layer beneath upper epidermis.

189. Cymbidium eburneo-lowianum. The same, showing deeper epidermal cells than in either $C$. lowianum or $C$. eburneum; cells of layer beneath upper epidermis longer than in either $C$. Lowianum or $C$. eburneum. 


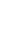



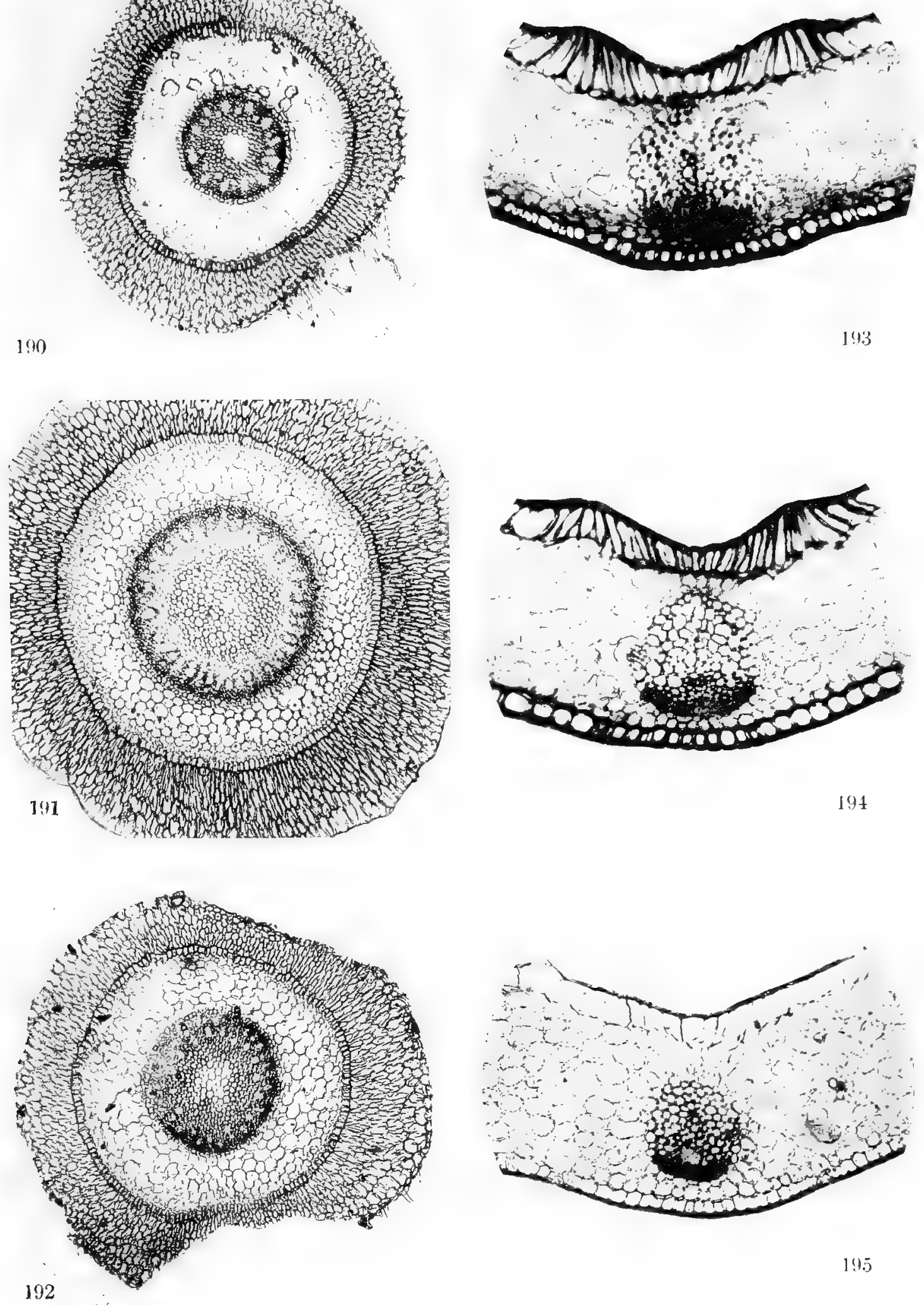

190. Dendrobium findlayanum. Transverse section of root, showing narrow velamen and small vascular cylinder.

191. Dendrobium nobile. The same, showing wide velarcen and wide vascular cylinder.

192. Dendrobium cybele. The same, showing velamen and vascular cylinder in width between the two parents.

193. Dendrobium findlayanum. Transverse section of leaf nidway between apex and base, showing deep ridges, large lower epidermal cells and large bundle.

194. Dendrobium nobile. The same, showing slightly larger ridges, large lower epidermal cells, and slightly smaller bundle.

195. Dendrobium cybele. The same, showing faint ridges, smaller epidermal cells, and smaller bundle than in either parent. 
. 

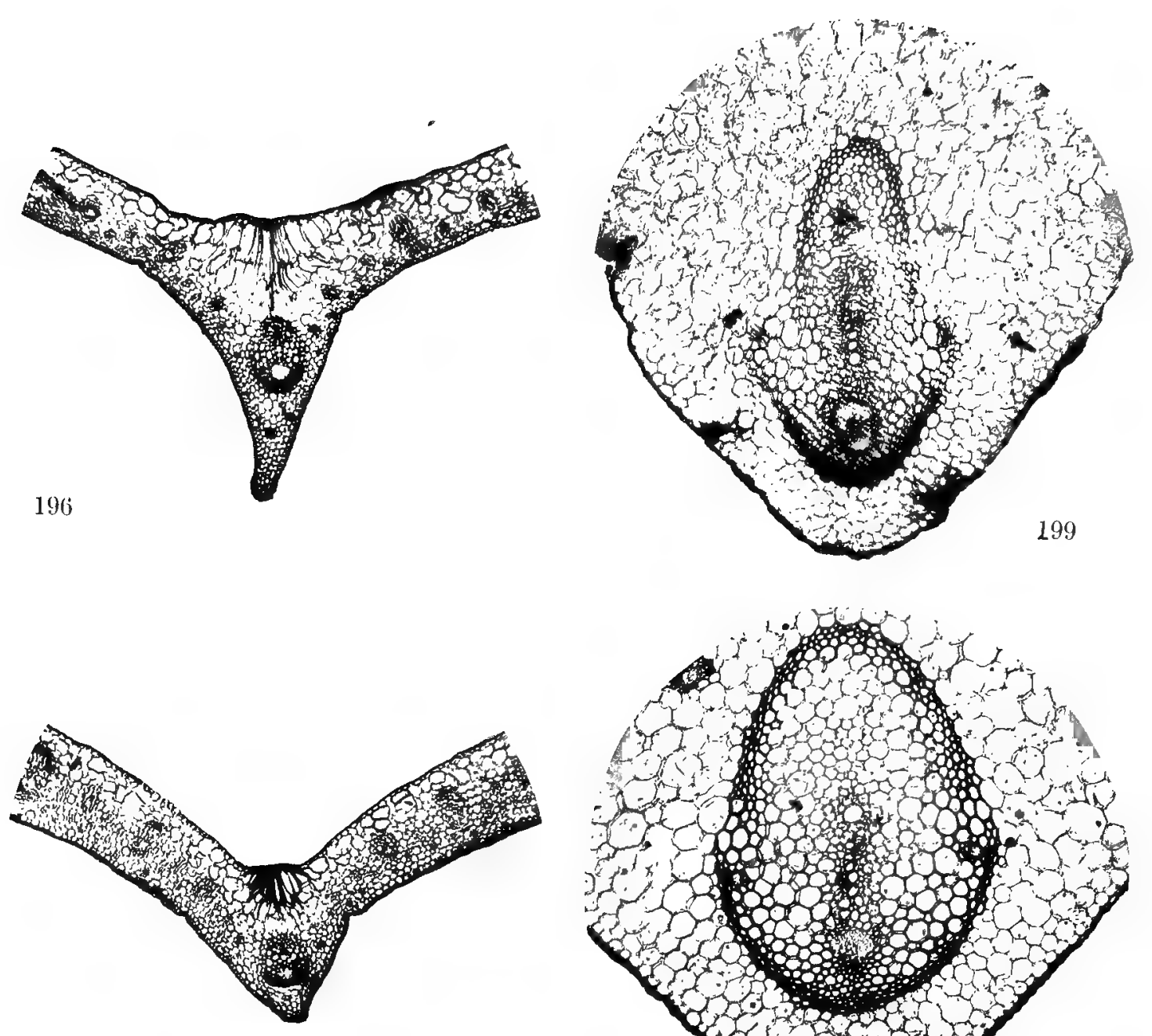

197
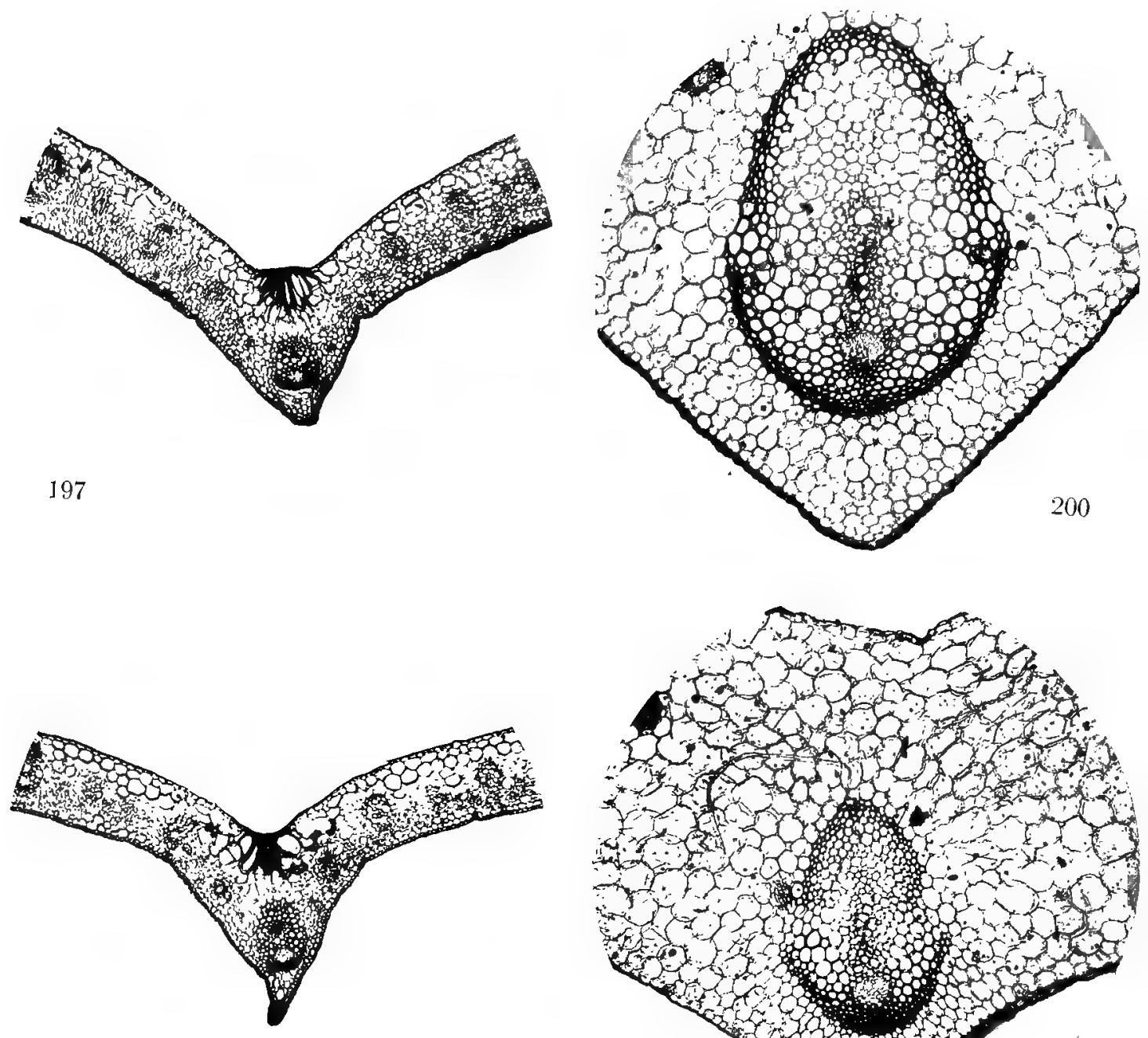

198

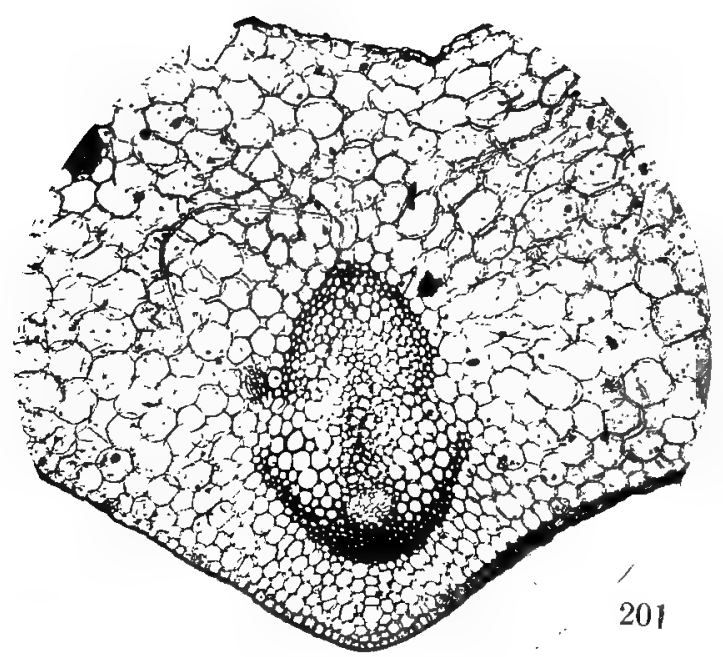

196. Miltonia vexillaria. Transverse section of leaf at equal distances from apex and base, showing elongated keel, elongated cells below upper epidermis, large oval bundle.

197. Miltonia roclii. The same, showing much shorter keel, more acute angle at midrib, less elongated cells below the upper epidermis, and a small almost circular bundle.

198. Mitionia bleuana. The same, showing keel fairly intermediate, also angle at midrib fairly intermediate, elongated cells of layer beneath upper epidermis as long as in $M$. rozlii, oval bundle nearly as Jarge as in M. vexillaria.

199. Phaius grandifolius. Transverse section petiole of mature leaf, showing midrib bundle with upper and lower sclerenchyma sheaths.

200. Phaius wallichii. The same, showing midrib bundle with continuous sclerenchyma sheath.

201. Phaius hybridus. The same, showing midrib bundle with upper and lower sclerenchyma sheaths, but more nearly joining each other than in $P$. grandifolius, an approximate mean between the two parents. 


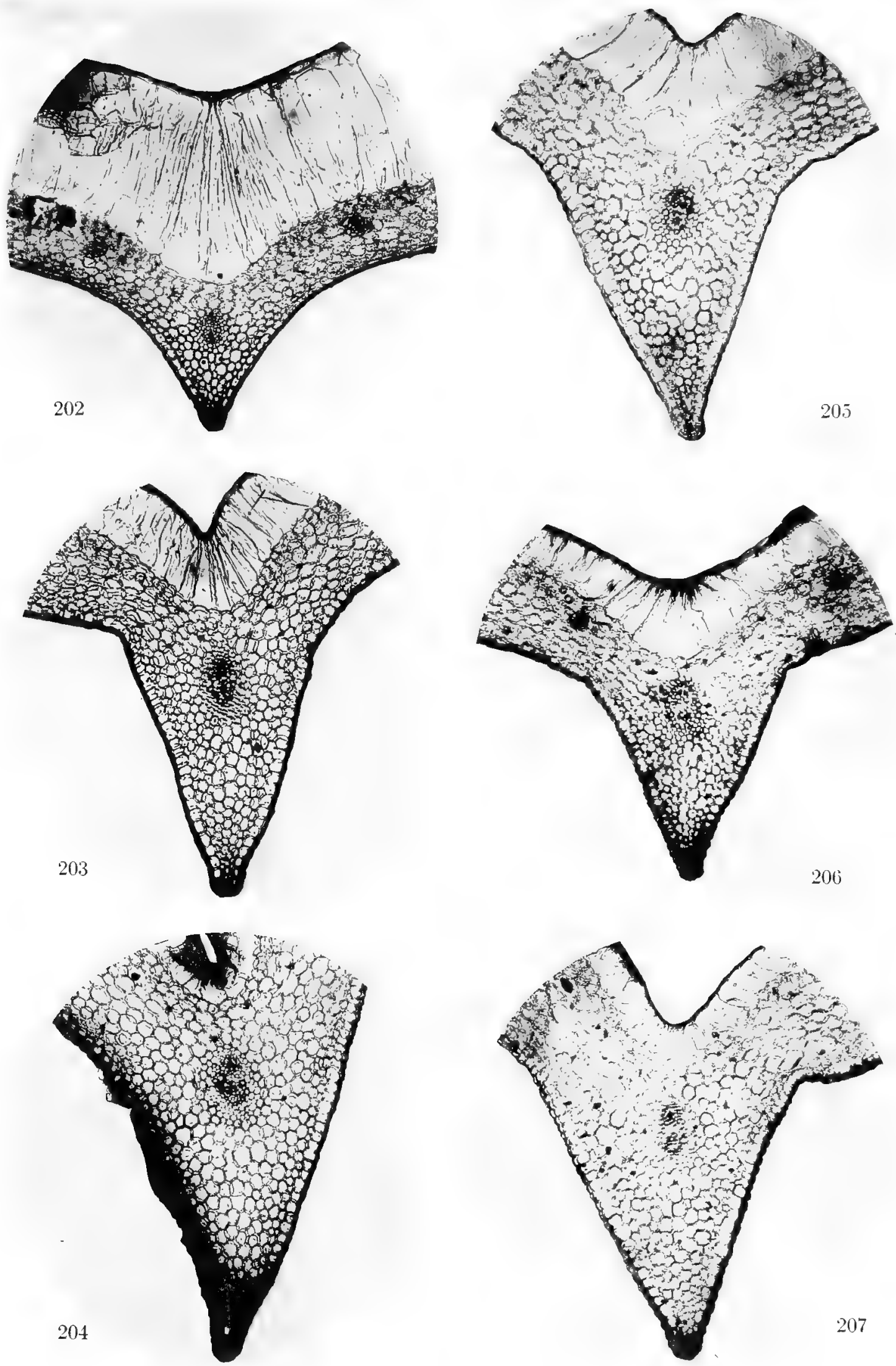

202. Cypripedium spicerianum. Transverse section of leaf midway between apex and base, showing deep aqueous tissue and narrow leaf at midrib region.

203. Cypripedium villosum. The same, showing narrow aqueous tissue and wide leaf at midrib region.

204. Cypripedium lathamianum. The same, showing narrower aqueous tissue and leaf wider at midrib than in either parent.

205. Cypripedium lathamianum inversum. The same, showing aqueous tissue in width between the two parents, and wider leaf than in either parent.

206. Cypripedium insigne maulei. The same, showing aqueous tissue almost same width as in $C$. villosum, but narrower leaf at midrib region.

207. Cypripedium nitens. The same, showing narrower aqueous tissue than in either parent, and width of leaf between the two parents. 




